\title{
Post-Closure Nuclear Criticality Safety Evaluations for Disposition of Criticality Control Overpacks at the Waste Isolation Pilot Plant
}

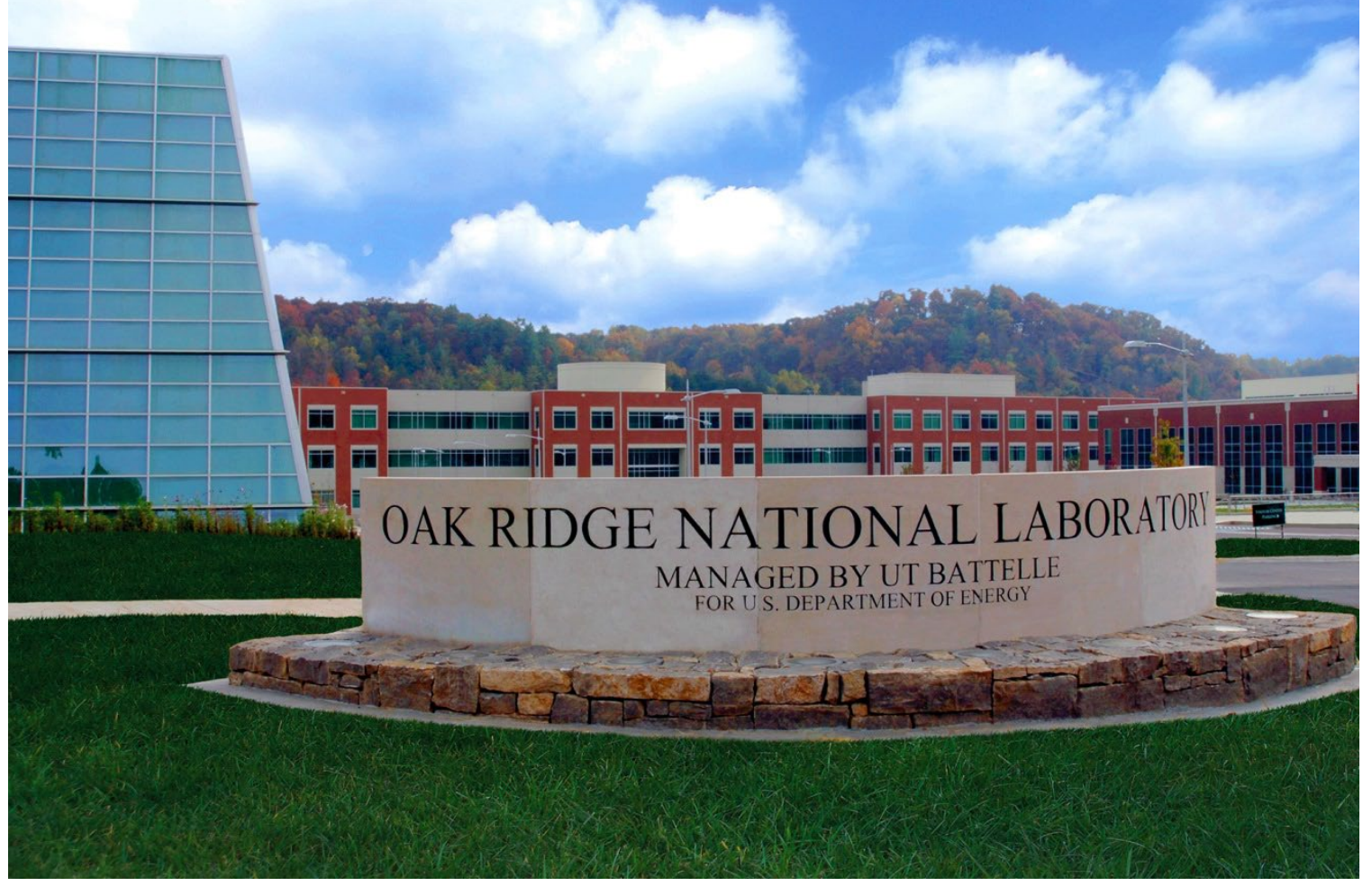

Bret D. Brickner

Riley Cumberland

Richard Reed

Alex Lang

February 2022

Approved for public release.

Distribution is unlimited. 


\section{DOCUMENT AVAILABILITY}

Reports produced after January 1, 1996, are generally available free via US Department of Energy (DOE) SciTech Connect.

Website http://www.osti.gov

Reports produced before January 1, 1996, may be purchased by members of the public from the following source:

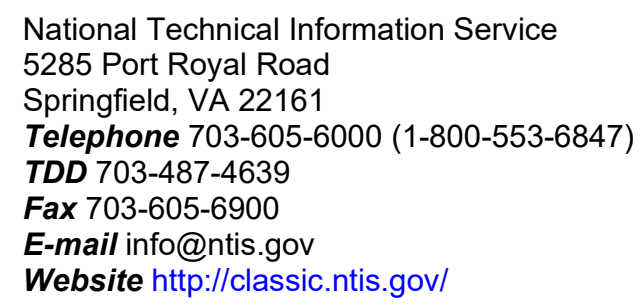

Reports are available to DOE employees, DOE contractors, Energy Technology Data Exchange representatives, and International Nuclear Information System representatives from the following source:

Office of Scientific and Technical Information

PO Box 62

Oak Ridge, TN 37831

Telephone 865-576-8401

Fax 865-576-5728

E-mail reports@osti.gov

Website https://www.osti.gov/

This report was prepared as an account of work sponsored by an agency of the United States Government. Neither the United States Government nor any agency thereof, nor any of their employees, makes any warranty, express or implied, or assumes any legal liability or responsibility for the accuracy, completeness, or usefulness of any information, apparatus, product, or process disclosed, or represents that its use would not infringe privately owned rights. Reference herein to any specific commercial product, process, or service by trade name, trademark, manufacturer, or otherwise, does not necessarily constitute or imply its endorsement, recommendation, or favoring by the United States Government or any agency thereof. The views and opinions of authors expressed herein do not necessarily state or reflect those of the United States Government or any agency thereof. 
Nuclear Energy and Fuel Cycle Division

\title{
POST-CLOSURE NUCLEAR CRITICALITY SAFETY EVALUATIONS FOR DISPOSITION OF CRITICALITY CONTROL OVERPACKS AT THE WASTE ISOLATION PILOT PLANT
}

\author{
Bret D. Brickner \\ Riley Cumberland \\ Richard Reed \\ Alex Lang
}

February 2022

Prepared by

OAK RIDGE NATIONAL LABORATORY

Oak Ridge, TN 37831-6283

managed by

UT-BATTELLE, LLC

for the

US DEPARTMENT OF ENERGY

under contract DE-AC05-00OR22725 
This page is intentionally blank 


\section{CONTENTS}

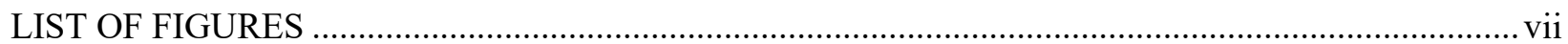

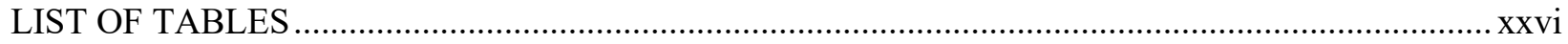

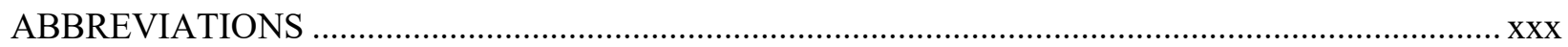

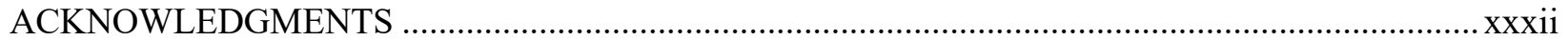

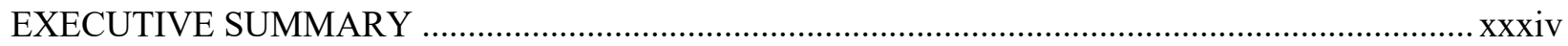

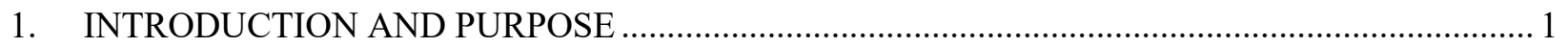

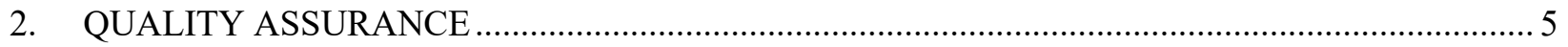

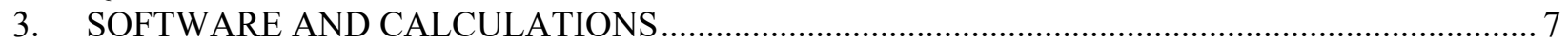

4. DEVELOPMENT OF ANALYSIS INPUTS AND DATA ….............................................. 9

4.1 QUALITATIVE CHARACTERISTICS OF THE WIPP CCO CONFIGURATION ............... 9

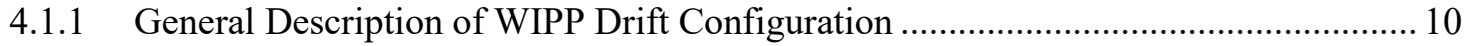

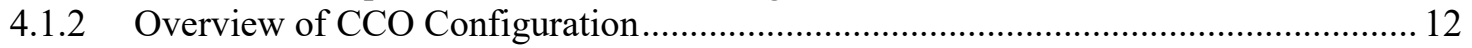

4.1.3 Waste Form Geometry and Composition Representative of the CCO in the

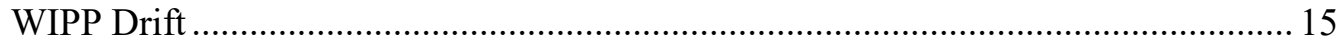

4.2 SELECTION OF PARAMETERS TO CHARACTERIZE CCO SYSTEMS AT WIPP......... 15

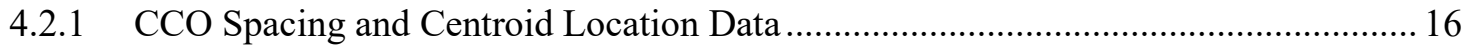

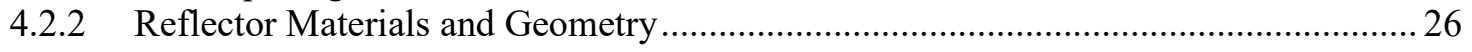

4.2.3 WASTE FORM MATERIALS, COMPOSITION AND GEOMETRY ...................... 31

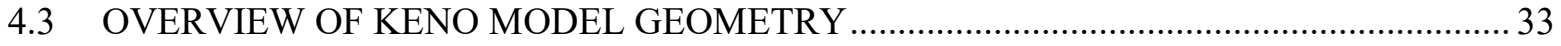

4.4 GENERAL INPUT DATA TABLES AND MATERIAL COMPOSITIONS ........................ 38

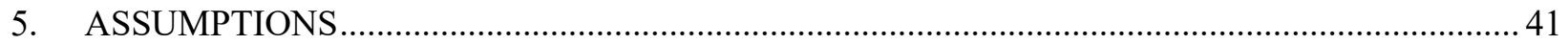

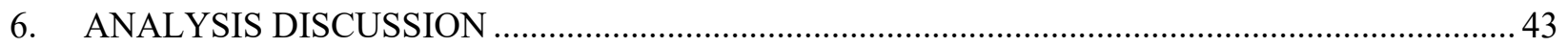

6.1 DISCUSSION OF THE THREE-HIGH UNIFORM ARRAY PARAMETRIC SWEEPS...... 47

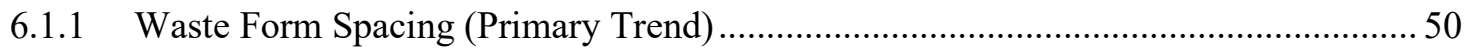

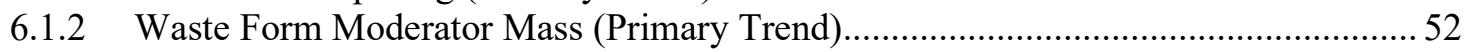

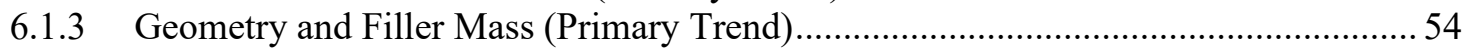

6.1.4 Discrete Reflector Material and Thickness (Secondary Trend) ..................................5 54

6.1.5 Difference in Generic Filler Material and the Graphite Filler Material

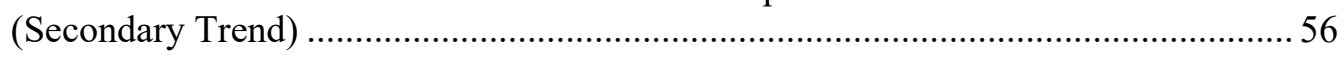

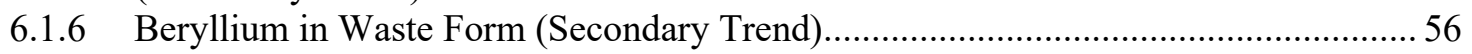

6.2 DISCUSSION OF THE SIX-HIGH UNIFORM ARRAY PARAMETRIC SWEEPS ............. 56

6.3 DISCUSSION OF THE NONUNIFORM ARRAY PARAMETRIC SWEEPS .................... 62

6.4 ADDITIONAL NONUNIFORM ARRAY ANALYSIS ..................................................... 71

6.5 SENSITIVITY OF RESULTS TO PARAMETERS VARIED .......................................... 82

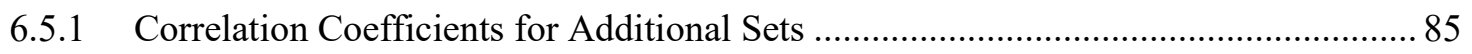

6.6 UPPER SUBCRITICAL MODERATOR MASSES ….......................................................... 87

6.6.1 Set-1 Uniform Array of Waste Forms Stacked Three High with Closer Spacing ....... 87

6.6.2 Set-1a Uniform Array of Waste Forms Stacked Three High with Wider Spacing ...... 89

6.6.3 Set-2 Nonuniform Array of Waste Forms............................................................... 91

6.6.4 Set-3 Uniform Array of Waste Forms Stacked Six High with Closer Spacing ........... 93

6.6.5 Set-3a Uniform Array of Waste Forms Stacked Six High with Wider Spacing .......... 94

6.7 EFFECT OF WASTE FORM HETEROGENEITY ........................................................ 96

6.8 STUDY OF NEUTRON TRANSMISSION FROM CCO SOURCE THROUGH

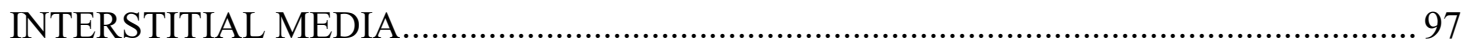

6.9 STUDIES ON THE REACTIVITY EFFECT OF BRINE AND THE ASSUMPTIONS RELATED TO INTERSTITIAL REFLECTOR MATERIAL DENSITY AND COMPOSITION 98 
6.10 STUDIES WITH ADDITIONAL CENTROID LOCATIONS FOR VARIOUS CONTAINER DESIGNS AND INITIAL SPATIAL ARRANGEMENTS (Appendix M) ..... 99

6.11 STUDIES TO EVALUATE THE IMPACT OF LARGER DISCRETE REFLECTOR THICKNESSES (APPENDIX O)... 103

6.12 STUDIES TO EVALUATE VARIOUS AMOUNTS OF $\mathrm{B}_{4} \mathrm{C}$ MIXED UNIFORMLY IN THE WASTE FORM (Appendix N). 104

6.13 DISCUSSION REGARDING THE EXTENSION OF THE TSUNAMI VALIDATION

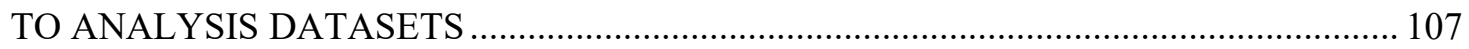

7. CONCLUSIONS 113

8. REFERENCES 115

APPENDIX A. SET-1: RESULTS OF THE THREE-HIGH UNIFORM ARRAY WITH BOUNDING SPACING CALCULATIONS A-1

APPENDIX B. SET-1A: RESULTS OF THE THREE-HIGH UNIFORM ARRAY WITH ALTERNATIVE SPACING CALCULATIONS B-1

APPENDIX C. SET-2: RESULTS OF THE NONUNIFORM ARRAY FOR THE UPPER HORIZON CALCULATIONS

APPENDIX D. SET-2-LH: RESULTS OF THE NONUNIFORM ARRAY FOR THE LOWER HORIZON CALCULATIONS

APPENDIX E. SET-2-TD: RESULTS OF THE TIME DEPENDENT NONUNIFORM ARRAY CALCULATIONS

APPENDIX F. SET-3: RESULTS OF THE SIX-HIGH UNIFORM ARRAY WITH BOUNDING SPACING CALCULATIONS

APPENDIX G. SET-2 UPPER HORIZON CENTROID LOCATION AND ORIENTATION STUDIES

APPENDIX H. CALCULATIONAL VALIDATION H-1

APPENDIX I. SET-3a: RESULTS OF THE SIX-HIGH UNIFORM ARRAY WITH ALTERNATIVE SPACING CALCULATIONS ... I-1 APPENDIX J. SET-4: RESULTS OF HETEROGENOUS WASTE FORM MIXING STUDIES ........... J-1 APPENDIX K. EVALUATION OF NEUTRON TRANSPORT FROM A CCO IN 50\%MGO 50\% NACL MIXTURE

APPENDIX L. SET-5: RESULTS OF THE NONUNIFORM ARRAY FOR THE UPPER HORIZON CALCULATIONS WITH ALTERNATIVE INTERSTITIAL REFLECTOR MATERIALS

APPENDIX M. SET-6: RESULTS OF THE TRIANGULAR PITCHED CCO NONUNIFORM ARRAY CENTROID LOCATION STUDIES AND THE POP HEXAGONAL AND TRIANGULAR PITCHED NONUNIFORM ARRAY CENTROID LOCATION STUDIES M-1 APPENDIX N. SET-7: RESULTS OF THE CALCULATIONS TO SHOW THE REACTIVITY EFFECT OF VARIOUS AMOUNTS OF B $_{4}$ C UNIFORMLY MIXED IN THE WASTE FORM N-1

APPENDIX O. SET-8: RESULTS OF THE CALCULATIONS TO SHOW THE REACTIVITY EFFECT OF GREATER THICKNESSES OF THE DISCRETE REFLECTOR MATERIAL AROUND THE WASTE FORM. 


\section{LIST OF FIGURES}

Figure 4.1. Schematic of WIPP repository, used with permission from Rechard [11] ........................... 10

Figure 4.2. Diagram showing cross-drifts, used with permission [18] ................................................ 11

Figure 4.3. Photograph of WIPP room loaded with waste containers, used with permission from

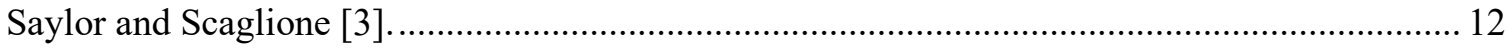

Figure 4.4. Criticality control container (CCC), and criticality control overpack (CCO), used with

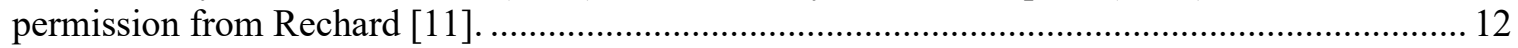

Figure 4.5. Diagram of the surplus Pu can-bag-can process, .............................................................. 14

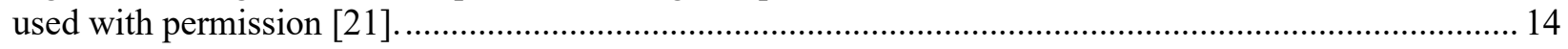

Figure 4.6. Representation of the SNL compaction data used with permission from Reedlunn [25]

Figure 4.7. Illustration of the room closure in the vertical and horizonal directions, used with permission [8].

Figure 4.8. Illustration of the lower and upper horizons at WIPP used with permission from Reedlunn and Bean [8].

Figure 4.9. Salt compaction of CCOs in WIPP disposal room: (a) lower strata horizon in southern portion of repository, and (b) upper strata horizon in northern portion of repository, used with permission [11].

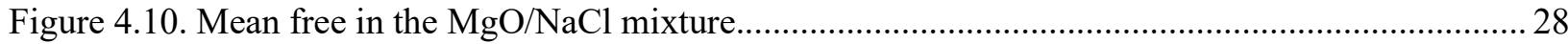

Figure 4.11. Macroscopic total interaction cross section for the the $\mathrm{MgO} / \mathrm{NaCl}$ mixture components.

Figure 4.12. Macroscopic capture cross section for the the $\mathrm{MgO} / \mathrm{NaCl}$ mixture components .................. 30

Figure 4.13. Diagram showing how the nonuniform array model is constructed with cylinders. .............. 36

Figure 4.14. Diagram showing how a uniform array model is constructed with spheres......................... 37

Figure 6.1. Diagram of the triangular pitch for the three-high uniform arrays....................................... 45

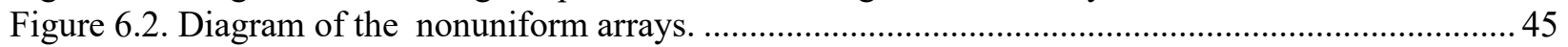

Figure 6.3. Diagram of the triangular pitch for the six-high uniform arrays two represent two

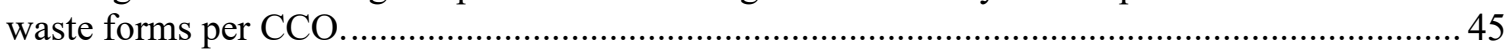

Figure 6.4. Full set of results for set-1 compared to various representative curves................................. 48

Figure 6.5. Full set of results for set-1a compared to various representative curves. ............................... 49

Figure 6.6. Comparison of the representative curves between set-1 and set-1a (set-1-set-1a)..................51

Figure 6.7. Comparison of various cases for both water and polyethylene waste form moderator

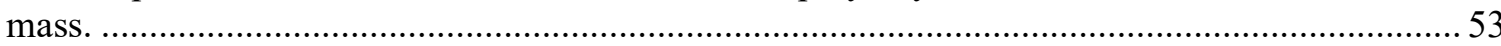

Figure 6.8. Comparison of the discrete reflector material and filler material on reactivity for

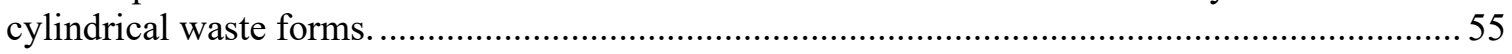

Figure 6.9. Comparison of the six-high and three-high arrays. ........................................................ 57

Figure 6.10 . Full set of results for set-3 compared to various representative curves............................... 58

Figure 6.11. Full set of results for set- 1 compared to set-3 with various representative curves (set-

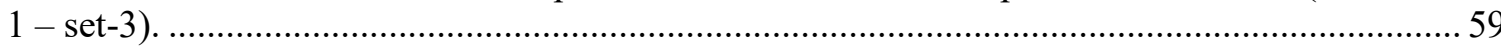

Figure 6.12. Reactivity effect of $25 \mathrm{~g} \mathrm{~B}_{4} \mathrm{C}$ compared to representative curves. ................................... 61

Figure 6.13. An example representation of the Reedlunn and Bean [8] data in the form of

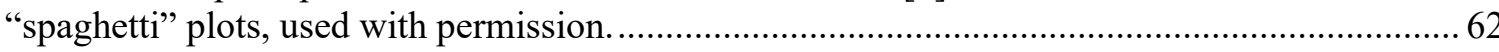

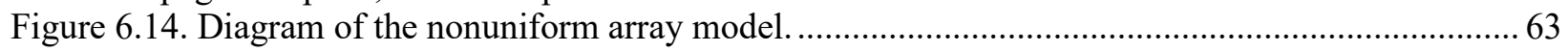

Figure 6.15. Full set of results for set-2-uh compared to various representative curves.......................... 65

Figure 6.16. Full set of results for set-2-lh compared to various representative curves. ........................... 66

Figure 6.17. Delta k of set-2-uh compared to set-2-lh with various representative curves (set-2-uh - set-2-lh).

Figure 6.18. Full set of results for set-1 compared to set-2-uh with various representative curves (set-1 - set-2-uh). 
Figure 6.19. Full set of results for set-1a compared to set-2-uh with various representative curves (set-1a- set-2-uh).

Figure 6.20. Full set of results for set-1 compared to set-2-1h with various representative curves

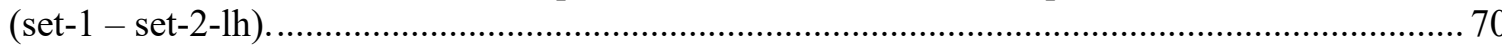

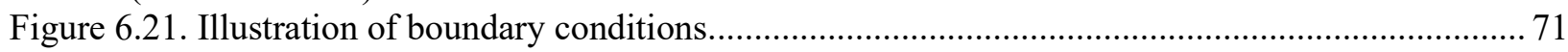

Figure 6.22. Diagram of flux for time-dependent cases.................................................................... 73

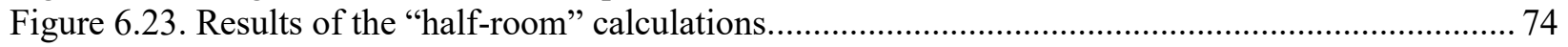

Figure 6.24. Results of the time-dependent calculations (set-2-4 with no filler) .................................... 76

Figure 6.25. Comparison between the 1-unit model and the 2-unit model (set-2-4 with no filler)........... 77

Figure 6.26. Comparison of various boundary conditions (set-2-4 with no filler). ................................ 78

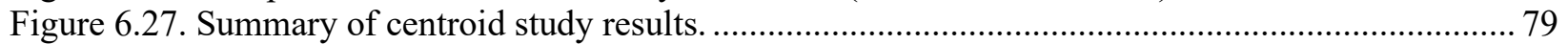

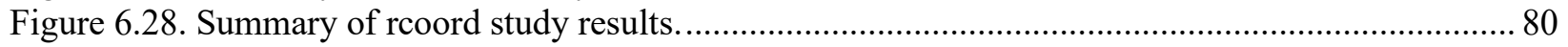

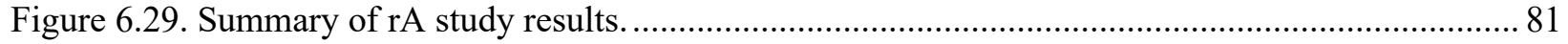

Figure 6.30. Absolute values of linear correlation coefficients for set-1 cylinders showing the

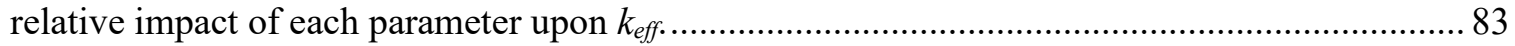

Figure 6.31. Differences in linear correlation constant terms relative to set-1 values.............................. 84

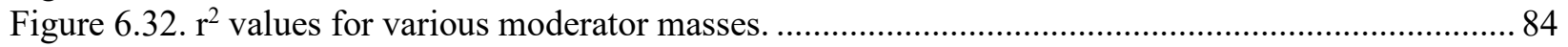

Figure 6.33. Absolute values of linear correlation coefficients for set-1 spheres showing the

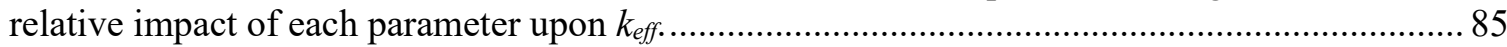

Figure 6.34. Absolute values of linear correlation coefficients for set 2-lh cylinders showing the

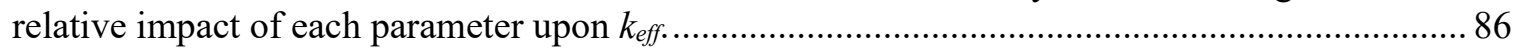

Figure 6.35. Absolute values of linear correlation coefficients for set-2lh spheres showing the

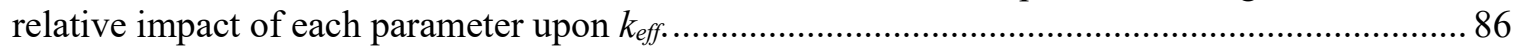

Figure 6.36. Subcritical moderator masses for evaluations in set-1 without filler vs. cylinder

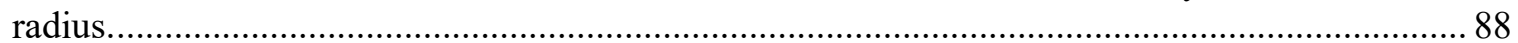

Figure 6.37. Subcritical moderator masses for evaluations in set-1 with filler vs. cylinder radius. ........... 88

Figure 6.38. Subcritical moderator masses for evaluations in set-1a without filler vs. cylinder

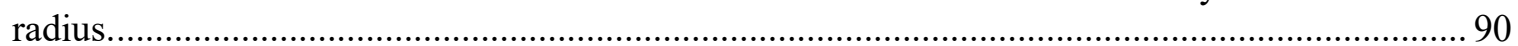

Figure 6.39. Subcritical moderator masses for evaluations in set-1a with filler vs. cylinder radius........... 90

Figure 6.40. Subcritical masses for evaluations in set-2 without filler vs. cylinder radius....................... 92

Figure 6.41. Subcritical masses for evaluations in set-2 with filler vs. cylinder radius............................ 92

Figure 6.42. Subcritical masses for evaluations in set-3 without filler vs. cylinder radius....................... 94

Figure 6.43. Subcritical masses for evaluations in set-3 with filler vs. cylinder radius............................ 94

Figure 6.44. Subcritical masses for evaluations in set-3a without filler vs. cylinder radius..................... 95

Figure 6.45. Subcritical masses for evaluations in set-3a with filler vs. cylinder radius.......................... 96

Figure 6.46. Diagram of the various heterogenous sphere cases and spacings...................................... 96

Figure 6.47. Delta- $k_{\text {eff }}$ when comparing highest reactivity three-sphere case to a uniform case (positive values correspond to cases in which the highest reactivity three-sphere

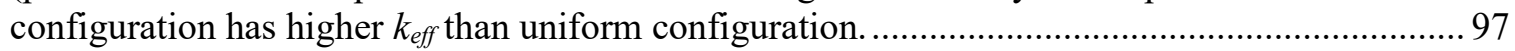

Figure 6.48. Flux per source particle at various distances in the salt form the source (linear scale)......... 98

Figure 6.49. Reactivity of set-5e compared to a single set- 2 representative curve to evaluate the effect of brine (no $\mathrm{MgO}$ ) as interstitial reflective material. ....................................................... 99

Figure 6.50. Diagrams of the CCO compared with the POCs evaluated in Appendix M (used with permission from Reedlunn and Bean [46]).

Figure 6.51. Max $k_{\text {eff }}$ of all subsets of all CCO and POC centroid configurations at time $=1,000$ years compared with max $k_{\text {eff }}$ of all subsets of set-2-uh hexagonal pitch (mirror boundary conditions) as $k_{\text {eff }} \mathrm{vs}$. moderator mass, time $=1,000$ years.

Figure 6.52. Comparison of the effect of FGE on the maximum $k_{\text {eff }}$ of all subsets of all CCO and POC centroid configurations at time $=1,000$ years (mirror boundary conditions)...

Figure 6.53. Set-8a and set-1 maximum $k_{\text {eff }}$ results (three-high uniform array model) overall sublistings as a function of discrete reflector thickness. 
Figure 6.54. Set-8b and set-2-uh maximum $k_{\text {eff }}$ results (nonuniform array model) overall sublistings as a function of discrete reflector thickness.

Figure 6.55. Set-7a results (three-high uniform array model) for 10, 15, 20, 25, 30, and $50 \mathrm{~g}$ of

$\mathrm{B}_{4} \mathrm{C}$ for all subcases. 105

Figure 6.56. Set-7c results (nonuniform array model) for $10,15,20,25,30$, and $50 \mathrm{~g}$ of $\mathrm{B}_{4} \mathrm{C}$ for up to a $6 \mathrm{~kg}$ moderator. 105

Figure 6.57. Set-7a results (three-high array model) for 10, 15, 20, 25, 30, and $50 \mathrm{~g}$ of $\mathrm{B}_{4} \mathrm{C}$ for up to a $6 \mathrm{~kg}$ moderator. 106

Figure 6.58. Set-7c results (nonuniform array model) for $10,15,20,25,30$, and $50 \mathrm{~g}$ of $\mathrm{B}_{4} \mathrm{C}$ for up to a $6 \mathrm{~kg}$ moderator.

Figure 6.59. Comparison of mean chord length for the different geometric configurations.

Figure 6.60. EALF of set-1 (all cases), set-2-uh (subcase 10 only), and set-1 subcase 2 (cylinder radius fixed at $7.7 \mathrm{~cm})$

Figure 6.61. Trend of bias and bias uncertainty as $c_{k}$ approaches 1 for set-1-2-uac1_uh_m2_graphite_cyl_ps_1000_yr_para_00444 for $c_{k} \geq 0.5$.

Figure 6.62. Trend of bias and bias uncertainty as $c_{k}$ approaches 1 for set-1-2-uac1_uh_m2_graphite_cyl_ps_1000_yr_para_00444 for $c_{k} \geq 0.6$.

Figure 6.63. Trend of bias and bias uncertainty as $c_{k}$ approaches 1 for set-1-2-uac1_uh_m2_graphite_cyl_ps_1000_yr_para_00480 for $c_{k} \geq 0.6$. 


\section{APPENDIX A: LIST OF FIGURES}

Figure A-1. Diagram of the uniform array three-high model.

Figure A-2. Set-1 results, plot 1: reactivity effect of cylinder radius, pipe steel, no filler, no Be, water moderated.

Figure A-3. Set-1 results, plot 2: reactivity effect of cylinder radius, pipe steel, no filler, no Be, poly moderated.

Figure A-4. Set-1 results, plot 3: reactivity effect of cylinder radius, pipe poly, no filler, no Be, water moderated.

Figure A-5. Set-1 results, plot 4: reactivity effect of cylinder radius, pipe poly, no filler, no Be, poly moderated.

Figure A-6. Set-1 results, plot 5: reactivity effect of cylinder radius, pipe steel, $5 \mathrm{~kg}$ graphite/can, no Be, water moderated.

Figure A-7. Set-1 results, plot 6: reactivity effect of cylinder radius, pipe steel, $5 \mathrm{~kg}$ graphite/can, no Be, poly moderated.

Figure A-8. Set-1 results, plot 7: reactivity effect of cylinder radius, pipe poly, $5 \mathrm{~kg}$ graphite/can, no Be, water moderated.

Figure A-9. Set-1 results, plot 8: reactivity effect of cylinder radius, pipe poly, $5 \mathrm{~kg}$ graphite/can, no Be, poly moderated.

Figure A-10. Set-1 results, plot 9: reactivity effect of various parameters with $7.7 \mathrm{~cm}$ cylinder radius, graphite filler, poly moderated.

Figure A-11. Set-1 results, plot 10: reactivity effect of various parameters with $7.7 \mathrm{~cm}$ cylinder radius, generic filler, poly moderated.

Figure A-12. Set-1 results, plot 11: comparison of graphite and generic filler with $7.7 \mathrm{~cm}$ cylinder radius, no Be, poly moderated, thick discrete reflector.

Figure A-13. Set-1 results, plot 12: reactivity effect of various parameters with spherical waste form geometry, graphite filler, water moderated.

Figure A-14. Set-1 results, plot 13: reactivity effect of various parameters with spherical waste form geometry, graphite filler, poly moderated.

Figure A-15. Set-1 results, plot 14: comparison of spherical and cylindrical geometries $(\mathrm{h} / \mathrm{x})$.

Figure A-16. Set-1 results, plot 15: comparison of water and poly $\mathrm{h} / \mathrm{x}$.

Figure A-17. Set-1 results, plot 16: comparison of spherical and cylindrical geometries (mod mass).

Figure A-18. Set-1 results, plot 17: comparison of $50 \mathrm{~g} \mathrm{~B}_{4} \mathrm{C}$ vs. no $\mathrm{B}_{4} \mathrm{C}$ for spherical and cylindrical geometries (mod mass). 


\section{APPENDIX B: LIST OF FIGURES}

Figure B-1. Diagram of the uniform array three-high model. This is not an actual analysis model: it is a diagram of a model used by SAMPLER to generate the analysis models...

Figure B-2. Set-1a results, plot 1: reactivity effect of cylinder radius, pipe steel, no filler, no Be, water moderated.

Figure B-3. Set-1a results, plot 2: reactivity effect of cylinder radius, pipe steel, no filler, no Be, poly moderated.

Figure B-4. Set-1a results. plot 3: reactivity effect of cylinder radius, pipe poly, no filler, no Be, water moderated.

Figure B-5. Set-1a results, plot 4: reactivity effect of cylinder radius, pipe poly, no filler, no Be, poly moderated.

Figure B-6. Set-1a results, plot 5: reactivity effect of cylinder radius, pipe steel, $5 \mathrm{~kg}$ graphite/can, no Be, water moderated.

Figure B-7. Set-1a results, plot 6: reactivity effect of cylinder radius, pipe steel, $5 \mathrm{~kg}$ graphite/can, no Be, poly moderated.

Figure B-8. Set-1a results, plot 7: reactivity effect of cylinder radius, pipe poly, $5 \mathrm{~kg}$ graphite/can, no Be, water moderated.

Figure B-9. Set-1a results, plot 8: reactivity effect of cylinder radius, pipe poly, $5 \mathrm{~kg}$ graphite/can, no Be, poly moderated.

Figure B-10. Set-1a results, plot 9: reactivity effect of various parameters with $7.7 \mathrm{~cm}$ cylinder radius, graphite filler, poly moderated.

Figure B-11. Set-1a results, plot 10: reactivity effect of various parameters with $7.7 \mathrm{~cm}$ cylinder radius, generic filler, poly moderated.

Figure B-12. Set-1a results, plot 11: comparison of graphite and generic filler with $7.7 \mathrm{~cm}$ cylinder radius, no Be, poly moderated, thick discrete reflector.

Figure B-13. Set-1a results, plot 12: reactivity effect of various parameters with spherical waste form geometry, graphite filler, water moderated.

Figure B-14. Set-1a results, plot 13: reactivity effect of various parameters with spherical waste form geometry, graphite filler, poly moderated.

Figure B-15. Set-1a results, plot 14: comparison of spherical and cylindrical geometries $(\mathrm{h} / \mathrm{x})$........ B-20

Figure B-16. Set-1a results, plot 15: comparison of water and poly $\mathrm{h} / \mathrm{x}$

Figure B-17. Set-1a results, plot 16: comparison of spherical and cylindrical geometries (mod mass).

Figure B-18. Set-1a results, plot 17: comparison of $50 \mathrm{~g} \mathrm{~B}_{4} \mathrm{C}$ vs. no $\mathrm{B}_{4} \mathrm{C}$ for spherical and cylindrical geometries (mod mass). 


\section{APPENDIX C: LIST OF FIGURES}

Figure C-1. Diagram of the nonuniform array model.

Figure C-2. Set-2-uh results, plot 1: reactivity effect of cylinder radius, pipe steel, no filler, no

$\mathrm{Be}$, water moderated.

Figure C-3. Set-2-uh results, plot 2: reactivity effect of cylinder radius, pipe steel, no filler, no

Be, poly moderated

Figure C-4. Set-2-uh results, plot 3: reactivity effect of cylinder radius, pipe poly, no filler, no Be, water moderated.

Figure C-5. Set-2-uh results, plot 4: reactivity effect of cylinder radius, pipe poly, no filler, no Be, poly moderated

Figure C-6. Set-2-uh results, plot 5: reactivity effect of cylinder radius, pipe steel, $5 \mathrm{~kg}$ graphite/can, no Be, water moderated.

Figure C-7. Set-2-uh results, plot 6: reactivity effect of cylinder radius, pipe steel, $5 \mathrm{~kg}$ graphite/can, no Be, poly moderated.

Figure C-8. Set-2-uh results, plot 7: reactivity effect of cylinder radius, pipe poly, $5 \mathrm{~kg}$ graphite/can, no Be, water moderated

Figure C-9. Set-2-uh results, plot 8: reactivity effect of cylinder radius, pipe poly, $5 \mathrm{~kg}$ graphite/can, no Be, poly moderated.

Figure C-10. Set-2-uh results, plot 9: reactivity effect of various parameters with $7.7 \mathrm{~cm}$ cylinder radius, graphite filler, poly moderated.

Figure C-11. Set-2-uh results, plot 10: reactivity effect of various parameters with $7.7 \mathrm{~cm}$ cylinder radius, generic filler, poly moderated.

Figure C-12. Set-2-uh results, plot 11: comparison of graphite and generic filler with $7.7 \mathrm{~cm}$ cylinder radius, no Be, poly moderated, thick discrete reflector.

Figure C-13. Set-2-uh results, plot 12: reactivity effect of various parameters with spherical waste form geometry, graphite filler, water moderated.

Figure C-14. Set-2-uh results, plot 13: reactivity effect of various parameters with spherical waste form geometry, graphite filler, poly moderated.

Figure C-15. Set-2-uh results, plot 14: comparison of spherical and cylindrical geometries $(\mathrm{h} / \mathrm{x})$.

Figure C-16. Set-2-uh results, plot 15: comparison of water and poly $\mathrm{h} / \mathrm{x}$. C-21

Figure C-17. Set-2-uh results, plot 16: comparison of spherical and cylindrical geometries (mod mass).

Figure C-18. Set-2-uh results, plot 17: comparison of $50 \mathrm{~g} \mathrm{~B}_{4} \mathrm{C}$ vs. no $\mathrm{B}_{4} \mathrm{C}$ for spherical and cylindrical geometries (mod mass). 


\section{APPENDIX D: LIST OF FIGURES}

Figure D-1. Diagram of the nonuniform array three-high model. D-5

Figure D-2. Set-2-lh results, plot 1: reactivity effect of cylinder radius, pipe steel, no filler, no

Be, water moderated.

Figure D-3. Set-2-lh results, plot 2: reactivity effect of cylinder radius, pipe steel, no filler, no

Be, poly moderated.

Figure D-4. Set-2-lh results, plot 3: reactivity effect of cylinder radius, pipe poly, no filler, no Be, water moderated

Figure D-5. Set-2-lh results, plot 4: reactivity effect of cylinder radius, pipe poly, no filler, no Be, poly moderated.

Figure D-6. Set-2-lh results, plot 5: reactivity effect of cylinder radius, pipe steel, $5 \mathrm{~kg}$ graphite/can, no Be, water moderated.

Figure D-7. Set-2-lh results, plot 6: reactivity effect of cylinder radius, pipe steel, $5 \mathrm{~kg}$ graphite/can, no Be, poly moderated.

Figure D-8. Set-2-lh results, plot 7: reactivity effect of cylinder radius, pipe poly, $5 \mathrm{~kg}$ graphite/can, no Be, water moderated.

Figure D-9. Set-2-1h results, plot 8: reactivity effect of cylinder radius, pipe poly, $5 \mathrm{~kg}$ graphite/can, no Be, poly moderated.

Figure D-10. Set-2-1h results, plot 9: reactivity effect of various parameters with $7.7 \mathrm{~cm}$ cylinder radius, graphite filler, poly moderated.

Figure D-11. Set-2-lh results, plot 10: reactivity effect of various parameters with $7.7 \mathrm{~cm}$ cylinder radius, generic filler, poly moderated.

Figure D-12. Set-2-1h results, plot 11: comparison of graphite and generic filler with $7.7 \mathrm{~cm}$ cylinder radius, no Be, poly moderated, thick discrete reflector.

Figure D-13. Set-2-lh results, plot 12: reactivity effect of various parameters with spherical waste form geometry, graphite filler, water moderated. .

Figure D-14. Set-2-1h results, plot 13: reactivity effect of various parameters with spherical waste form geometry, graphite filler, poly moderated.

Figure D-15. Set-2-1h results, plot 14: comparison of spherical and cylindrical geometries (h/x)....... D-20

Figure D-16. Set-2-lh results, plot 15: comparison of water and poly $\mathrm{h} / \mathrm{x}$.

Figure D-17. Set-2-1h results, plot 16: comparison of spherical and cylindrical geometries (mod mass).

Figure D-18. Set-2-lh results, plot 17: comparison of $50 \mathrm{~g} \mathrm{~B} \mathrm{~B}_{4} \mathrm{C}$ vs. no $\mathrm{B}_{4} \mathrm{C}$ for spherical and cylindrical geometries (mod mass). 


\section{APPENDIX E: LIST OF FIGURES}

Figure E-1. 3D Isometric view (top) and 2D top view (bottom) representations of the 1-unit

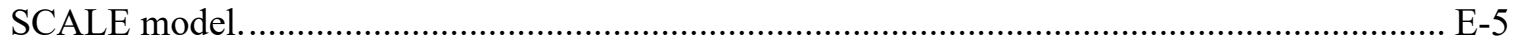

Figure E-2. 3D isometric (top) and 2D front (bottom) representations of the 2-unit SCALE model...... E-6

Figure E-3. Layout of the 1-unit, 0-year CCO locations in 3D isometric (top left), top view (right)

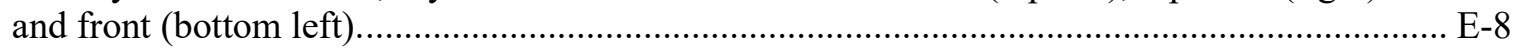

Figure E-4. Distribution of the flux for the $\mathrm{x}-\mathrm{z}$ direction (top and bottom) with contour count of 10 for 0 years for the SNL upper horizon compaction results............................................. E-9

Figure E-5. Layout of the 1-unit, 50-year CCO locations in 3D isometric (top left), top view (right) and front (bottom left).................................................................................... E-10

Figure E-6. Distribution of the flux for the x-z direction (top and bottom) with contour count of 10 for 50 years for the SNL upper horizon compaction results............................................ E-11

Figure E-7. Layout of the 1-unit, 100-year CCO locations in 3D isometric (top left), top view

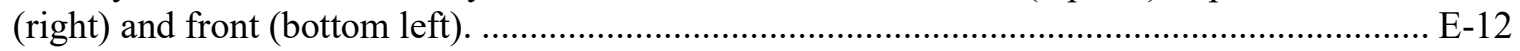

Figure E-8. Distribution of the flux for the $\mathrm{x}-\mathrm{z}$ direction (top and bottom) with contour count of 10 for 100 years for the SNL upper horizon compaction results........................................ E-13

Figure E-9. Layout of the 1-unit, 200-year CCO locations in 3D isometric (top left), top view

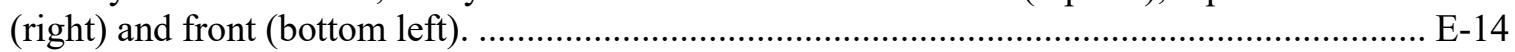

Figure E-10. Distribution of the flux for the $\mathrm{x}-\mathrm{z}$ direction (top and bottom) with contour count of 10 for 200 years for the SNL upper horizon compaction results......................................... E-15

Figure E-11. Layout of the 1-unit, 300-year CCO locations in 3D isometric (top left), top view (right) and front (bottom left). ...................................................................................... E-16

Figure E-12. Distribution of the flux for the $\mathrm{x}-\mathrm{z}$ direction (top and bottom) with contour count of 10 for 300 years for the SNL upper horizon compaction results........................................ E-17

Figure E-13. Layout of the 1-unit, 400-year CCO locations in 3D isometric (top left), top view (right) and front (bottom left). ...................................................................................... E-18

Figure E-14. Distribution of the flux for the $x-z$ direction (top and bottom) with contour count of 10 for 400 years for the SNL upper horizon compaction results........................................ E-19

Figure E-15. Layout of the 1-unit, 1,000-year CCO locations in 3D isometric (top left), top view (right) and front (bottom left)................................................................................... E-20

Figure E-16. Distribution of the flux for the $\mathrm{x}-\mathrm{z}$ direction (top and bottom) with contour count of 10 for 1,000 years for the SNL upper horizon compaction results. ...................................... E-21

Figure E-17. Comparison of various small-step time-dependent compaction steps for the SNL

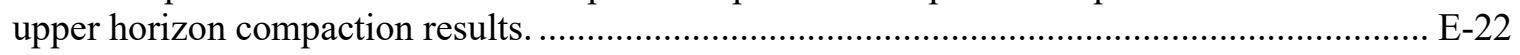

Figure E-18. Comparison of various large-step time-dependent compaction steps for the SNL

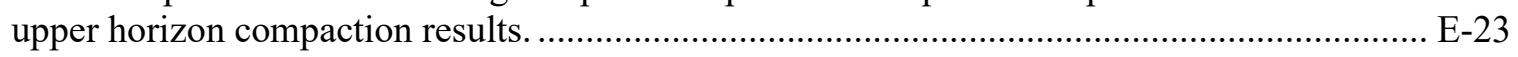

Figure E-19. Comparison of various small-step time-dependent compaction steps between 100 and 200 years for the SNL upper horizon compaction results................................................ E-24

Figure E-20. Comparison of the 1- (-1u-) to 2-unit SCALE models for various time dependent cases for the SNL upper horizon compaction results...................................................... E-25

Figure E-21. Comparison of the sphere and cylinder models for various time-dependent cases for the SNL upper horizon compaction results................................................................. E-26

Figure E-22. Comparison of the reactivity effect of location of the reflective boundary conditions, KENO parametric block parameters for the 1- and 2-unit SCALE sphere, and cylinder models for the 1,000-year time-dependent case for the SNL upper horizon compaction results.

Figure E-23. Comparison of the reactivity effect of various boundary conditions for the 1- and 2unit SCALE sphere and cylinder models for the 1,000-year time-dependent case for the SNL upper horizon compaction results. 


\section{APPENDIX F: LIST OF FIGURES}

Figure F-1. Diagram of the uniform array six-high model. ..................................................................F-5

Figure F-2. Set-3 results, plot 1: reactivity effect of cylinder radius, pipe steel, no filler, no Be,

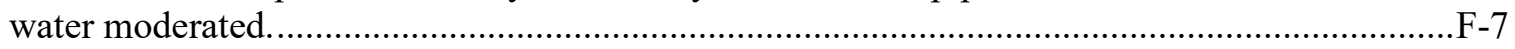

Figure F-3. Set-3 results, plot 2: reactivity effect of cylinder radius, pipe steel, no filler, no Be,

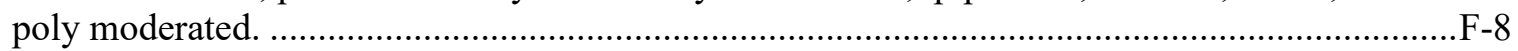

Figure F-4. Set-3 results, plot 3: reactivity effect of cylinder radius, pipe poly, no filler, no Be,

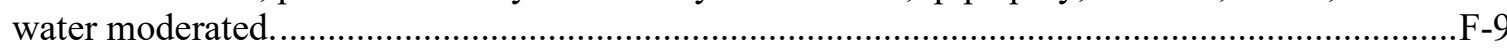

Figure F-5. Set-3 results, plot 4: reactivity effect of cylinder radius, pipe poly, no filler, no Be,

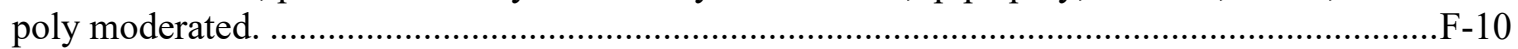

Figure F-6. Set-3 results, plot 5: reactivity effect of cylinder radius, pipe steel, $5 \mathrm{~kg}$ graphite/can, no Be, water moderated. ...............................................................................................

Figure F-7. Set-3 results, plot 6: reactivity effect of cylinder radius, pipe steel, $5 \mathrm{~kg}$ graphite/can,

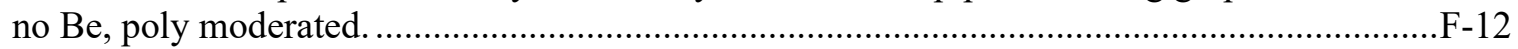

Figure F-8. Set-3 results, plot 7: reactivity effect of cylinder radius, pipe poly, $5 \mathrm{~kg}$ graphite/can,

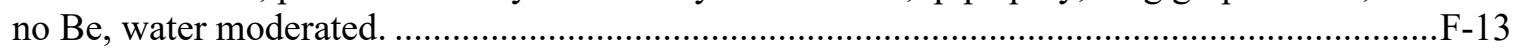

Figure F-9. Set-3 results, plot 8: reactivity effect of cylinder radius, pipe poly, $5 \mathrm{~kg}$ graphite/can,

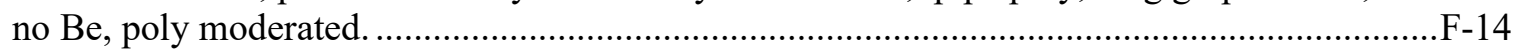

Figure F-10. Set-3 results, plot 9: reactivity effect of various parameters with $7.7 \mathrm{~cm}$ cylinder

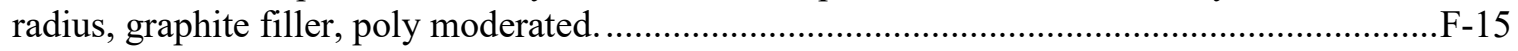

Figure F-11. Set-3 results, plot 10: reactivity effect of various parameters with $7.7 \mathrm{~cm}$ cylinder radius, generic filler, poly moderated. ...........................................................................

Figure F-12. Set-3 results, plot 11: comparison of graphite and generic filler with $7.7 \mathrm{~cm}$ cylinder radius, no $\mathrm{Be}$, poly moderated, thick discrete reflector.......................................................... 17

Figure F-13. Set-3 results, plot 12: reactivity effect of various parameters with spherical waste form geometry, graphite filler, water moderated....................................................................

Figure F-14. Set-3 results, plot 13: reactivity effect of various parameters with spherical waste form geometry, graphite filler, poly moderated.................................................................. -19

Figure F-15. Set-3 results, plot 14: comparison of spherical and cylindrical geometries (h/x).............F-20

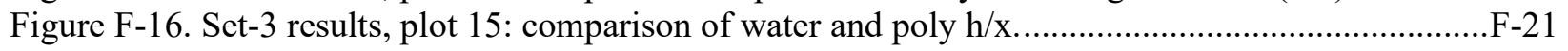

Figure F-17. Set-3 results, plot 16: comparison of spherical and cylindrical geometries (mod

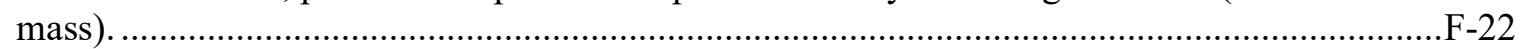

Figure F-18. Set-3 results, plot 17: Reactivity effect of $25 \mathrm{~g} \mathrm{~B}_{4} \mathrm{C}$ per waste form (2 per CCO) ............... -23 


\section{APPENDIX G: LIST OF FIGURES}

Figure G-1. Representation of the compacted CCO pipe centerline "spaghetti model" drawn from

13 centroids per pipe centerline from [28] with permission.

Figure G-2. Example of centroid locations within compacted pipes. .................................................. G-6

Figure G-3. 3D representation of the Appendix C and Appendix D 2-unit analysis model illustrating how pipe center centroid data were used to orient vertical cylinders.

Figure G-4. A 3D python-generated illustration of how cylinder orientations follow pipe centerline orientations.

Figure G-5. Comparison of local and global orientations for centroid 1 using the isometric, top, and front view angles.

Figure G-6. Comparison of different visualizations of Centroid 6 shifted with global orientation case, $\mathrm{x}$-y plane (top view).

Figure G-7. Comparison of different visualizations of the Centroid 6 shifted with global

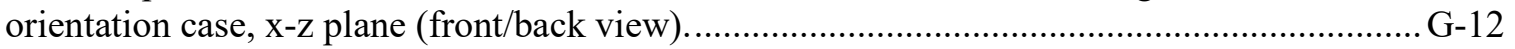

Figure G-8. Results of the centroid studies for centroid 1 ................................................................

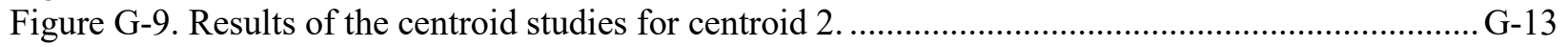

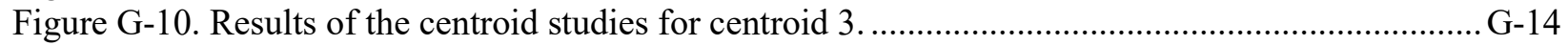

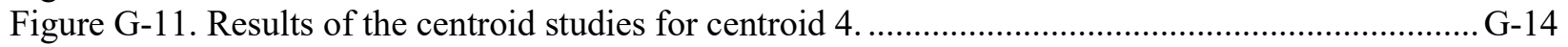

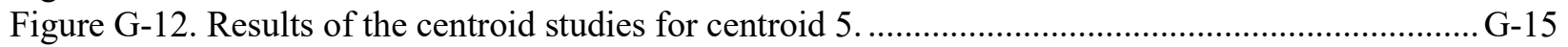

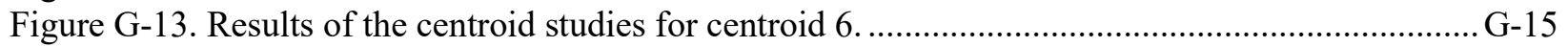

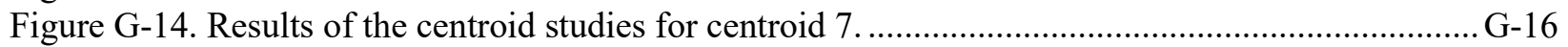

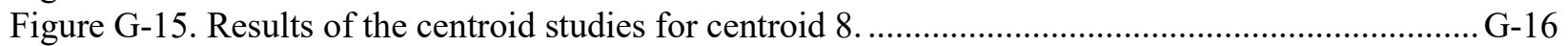

Figure G-16. Results of the centroid studies for centroid 9 ..............................................................

Figure G-17. Results of the centroid studies for centroid 10 .............................................................. 17

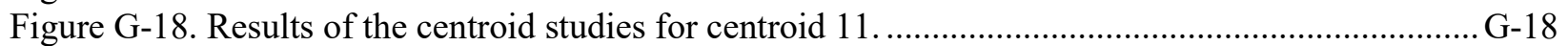

Figure G-19. Results of the centroid studies for centroid 12 ......................................................... G-18

Figure G-20. Results of the centroid studies for centroid 13..............................................................

Figure G-21. Results of the centroid studies for the "midpoint" centroid. .......................................... G-19

Figure G-22 Comparison of all centroids and midpoint to various representative curves..................... G-20

Figure G.23. Set-2-4 orientation study, random coordinate studies, results for 100 randomly selected centroid locations for each subset. ...................................................................... G-22

Figure G.24. Set-2-4 orientation study, random angle studies, results for 20 randomly selected angles for the 13 centroids plus the midpoint for each subset. ................................................. G-24

Figure G.25. Set-2-4 orientation study, random angle studies, results for 10 randomly selected angles for the 10 randomly selected centroids plus midpoint for each subset. ........................ G-25 


\section{APPENDIX H: LIST OF FIGURES}

Figure H-1. Top view of modified configuration.

Figure H-2. Set-1-1 cases, water moderator, graphite filler, stainless steel pipe................................. H-8

Figure H-3. Set-1-2 cases, poly moderator, graphite filler, stainless steel pipe ................................. H- 8

Figure H-4. Set-1-3 cases, water moderator, graphite filler, poly pipe ............................................... H-9

Figure H-5. Set-1-4 cases, poly moderator, graphite filler, poly pipe.............................................. H-9

Figure H-6. Set-1-5 cases, water moderator, generic filler, stainless steel pipe. .................................. H-10

Figure H-7. Set-1-6 cases, poly moderator, generic filler, stainless steel pipe ................................. H-10

Figure H-8. Set-1-7 cases, water moderator, generic filler, poly pipe .................................................. H-11

Figure H-9. Set-1-8 cases, poly moderator, generic filler, poly pipe ................................................ H-11

\section{APPENDIX I: LIST OF FIGURES}

Figure I-1. Diagram of the uniform array six-high model.

Figure I-2. Set-3a results, plot 1: reactivity effect of cylinder radius, pipe steel, no filler, no Be, water moderated.

Figure I-3. Set-3a results, plot 2: reactivity effect of cylinder radius, pipe steel, no filler, no Be, poly moderated.

Figure I-4. Set-3a results, plot 3: reactivity effect of cylinder radius, pipe poly, no filler, no Be, water moderated.

Figure I-5. Set-3a results, plot 4: reactivity effect of cylinder radius, pipe poly, no filler, no Be, poly moderated.

Figure I-6. Set-3a results, plot 5: reactivity effect of cylinder radius, pipe steel, $5 \mathrm{~kg}$ graphite/can, no Be, water moderated.

Figure I-7. Set-3a results, plot 6: reactivity effect of cylinder radius, pipe steel, $5 \mathrm{~kg}$ graphite/can, no Be, poly moderated.

Figure I-8. Set-3a results, plot 7: reactivity effect of cylinder radius, pipe poly, $5 \mathrm{~kg}$ graphite/can, no Be, water moderated.

Figure I-9. Set-3a results, plot 8: reactivity effect of cylinder radius, pipe poly, $5 \mathrm{~kg}$ graphite/can, no Be, poly moderated.

Figure I-10. Set-3a results, plot 9: reactivity effect of various parameters with $7.7 \mathrm{~cm}$ cylinder radius, graphite filler, poly moderated.

Figure I-11. Set-3a results, plot 10: reactivity effect of various parameters with $7.7 \mathrm{~cm}$ cylinder radius, generic filler, poly moderated.

Figure I-12. Set-3a results, plot 11: comparison of graphite and generic filler with $7.7 \mathrm{~cm}$ cylinder radius, no Be, poly moderated, thick discrete reflector.

Figure I-13. Set-3a results, plot 12: reactivity effect of various parameters with spherical waste form geometry, graphite filler, water moderated.

Figure I-14. Set-3a results, plot 13: reactivity effect of various parameters with spherical waste form geometry, graphite filler, poly moderated.

Figure I-15. Set-3a results, plot 14: comparison of spherical and cylindrical geometries $(\mathrm{h} / \mathrm{x})$.

Figure I-16. Set-3a results, plot 15: comparison of water and poly $\mathrm{h} / \mathrm{x}$.

Figure I-17. Set-3a results, plot 16: comparison of spherical and cylindrical geometries (mod mass).

Figure I-18. Set-3a results, plot 17: Reactivity effect of $25 \mathrm{~g} \mathrm{~B}_{4} \mathrm{C}$ per waste form (2 per CCO) 


\section{APPENDIX J: LIST OF FIGURES}

Figure J-1. Diagram of the various heterogenous sphere cases and spacings........................................ J-4

Figure J-2. Geometry diagram for a three-sphere case with spheres in centermost position..................... J-6

Figure J-3. Comparison of 1 -sphere $k_{\text {eff }}$ to uniform $k_{\text {eff }}$ for cylinderical wasteforms of $7.7 \mathrm{~cm}$ diameter.

Figure J-4. Delta-k_eff when comparing highest reactivity 1 sphere case to a uniform case (positive values correspond to cases where the highest reactivity 1-sphere configuration has higher keff than uniform configuration.

Figure J-5. Comparison of 2-sphere $k_{\text {eff }}$ to uniform $k_{\text {eff }}$ for cylinderical wasteforms of $7.7 \mathrm{~cm}$ diameter.

Figure J-6 Delta-k_eff when comparing highest reactivity 2-sphere case to a uniform case (positive values correspond to cases where the highest reactivity 2-sphere configuration has higher $\mathrm{k}_{\mathrm{eff}}$ than uniform configuration.

Figure J-7. Comparison of three-sphere $\mathrm{k}_{\mathrm{eff}}$ to uniform $\mathrm{k}_{\mathrm{eff}}$ for cylinderical wasteforms of $7.7 \mathrm{~cm}$ diameter.

Figure J-8 Delta-k $\mathrm{k}_{\mathrm{eff}}$ when comparing highest reactivity three-sphere case to a uniform case (positive values correspond to cases where the highest reactivity three-sphere configuration has higher $\mathrm{k}_{\mathrm{eff}}$ than uniform configuration.

Figure J-9. Plot showing frequency of each radial position factor producing worst k-eff plotted for a given uniform case, plotted against moderator mass.

Figure J-10. Plot showing frequency of each sphere radius producing worst k-eff plotted for a given uniform case, plotted against moderator mass.

Figure $\mathrm{J}-11$. Plot showing upper values of $\mathrm{k}_{\mathrm{eff}}$ for various cylinder radii and polyethylene reflector combinations.

Figure $\mathrm{J}$-12. Plot showing upper values of $\mathrm{k}_{\text {eff }}$ for spheres with different polyethylene reflector thicknesses.

Figure J-13. Comparing $\mathrm{k}_{\text {eff }}$ computed using multigroup and continuous energy. $\mathrm{J}-15$

\section{APPENDIX K: LIST OF FIGURES}

Figure K-1. Geometry of MAVRIC model with vacuum boundary conditions. K-5

Figure K-2. Neutron flux tallies at locations in 50/50 NaCl/MgO mixture. Values in neutrons $/ \mathrm{cm}^{2}$-s per source particle.

Figure K-3. Flux per source particle at various distances in the salt form the source (linear scale)......... K-7

Figure K-4. Flux per source particle at various distances in the salt form the source (log scale). ... K-7

Figure K-5. Flux spectra (n/ $\mathrm{cm}^{2} \mathrm{~s}$ per source particle) at $10 \mathrm{~cm}$ from source (blue), $50 \mathrm{~cm}$ from source (red) and $100 \mathrm{~cm}$ from source (green). 


\section{APPENDIX L: LIST OF FIGURES}

Figure L-1. Delta-k between set-2 and set-5 base case (set-2 minus set-5) to evaluate location of reflective boundary conditions.

Figure L-2. Delta-k between set-5 base case and set-5a (set-5 minus set-5a) to evaluate the effect of half density interstitial reflective material. ..................................................................... L-10

Figure L-3. Delta-k between set- 5 base case and set-5b (set-5 minus set-5b) to evaluate the effect of zero density (void) interstitial reflective material............................................................. L-11

Figure L-4. Delta-k between set-5 base case and set-5c to evaluate the effect of pure salt as

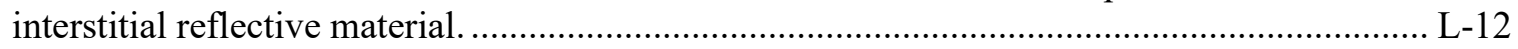

Figure L-5. Delta-k between set- 5 base case and set- $5 \mathrm{~d}$ to evaluate the effect of brine (no $\mathrm{MgO}$ )

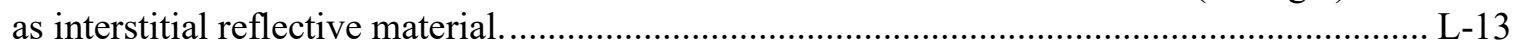

Figure L-6. Reactivity of set-5d compared to a single set-2 representative curve to evaluate the effect of brine (no $\mathrm{MgO}$ ) as interstitial reflective material. .................................................... L-14

Figure L-7. Delta-k between set-2 and set-5e to evaluate the effect of brine (no $\mathrm{MgO}$ ) as interstitial reflective material but with the additional $50 \mathrm{~cm}$ of space to locate the $y-$ direction reflective boundary conditions removed (similar to set-2).

Figure L-8. Reactivity of set-5e compared to a single set-2 representative curve to evaluate the effect of brine (no $\mathrm{MgO}$ ) as interstitial reflective material but with the additional $50 \mathrm{~cm}$ of space to locate the y-direction reflective boundary conditions removed (similar to set-2)....... L-16

Figure L-9. Delta-k between set-2 and set- $5 \mathrm{f}$ to evaluate the effect of brine (no $\mathrm{MgO}$ ) as interstitial reflective material but with the additional $50 \mathrm{~cm}$ of space to locate the $y-$ direction reflective boundary conditions removed (similar to set-2) and brine in the external salt box.

Figure L-10. Delta-k between set-2 and set-5g to evaluate the effect of brine in a $50 \%$ mixture with $\mathrm{MgO}$ as interstitial reflective material but with the additional $50 \mathrm{~cm}$ of space to locate the y-direction reflective boundary conditions removed (similar to set- 2 ) and brine in the external salt box.

Figure L-11. Delta-k between set- 2 and set- $5 \mathrm{~h}$ to evaluate the effect of brine in a $50 \%$ mixture with $\mathrm{MgO}$ as interstitial reflective material but with the additional $50 \mathrm{~cm}$ of space to locate the y-direction reflective boundary conditions removed (similar to set-2) and brine in the external salt box. The graphite filler, when present, is replaced by an equivalent mass of brine in a $50 \%$ mixture with $\mathrm{MgO}$.

Figure L-12. $\mathrm{k}_{\text {eff }}$ of set-5h compared to a single set-2 representative curve to evaluate the effect of brine in a $50 \%$ mixture with $\mathrm{MgO}$ as interstitial reflective material but with the additional $50 \mathrm{~cm}$ of space to locate the y-direction reflective boundary conditions removed (similar to set-2) and brine in the external salt box. The graphite filler, when present, is replaced by an equivalent mass of brine in a $50 \%$ mixture with $\mathrm{MgO}$.

\section{APPENDIX M: LIST OF FIGURES}

Figure M-1. Comparison of CCO upper horizon datasets, set-2-uh hexagonal pitch results (mirror boundary conditions) with set-6-uh-tp triangular pitch results (mirror boundary conditions) as delta- $k_{\text {eff }}$ vs. moderator mass, time $=0$ years.

Figure M-2. Comparison of CCO upper horizon datasets, set-2-uh hexagonal pitch results (mirror boundary conditions) with set-6-uh-tp triangular pitch results (mirror boundary conditions) as delta- $k_{\text {eff }}$ vs. moderator mass, time $=1,000$ years. 
Figure M-3. Comparison of CCO lower horizon datasets, set-2-lh hexagonal pitch results (mirror boundary conditions) with set-6-lh-tp triangular pitch results (mirror boundary conditions) as delta- $k_{e f f}$ vs. moderator mass, time $=1,000$ years.

Figure M-4. Comparison of CCO upper horizon datasets, set6-uh-tp triangular pitch results (mirror boundary conditions) results with set6a-uh-tp triangular pitch results (periodic boundary conditions) as delta- $k_{\text {eff }}$ vs. moderator mass, time $=0$ years.

Figure M-5. Comparison of CCO upper horizon datasets, set6-uh-tp triangular pitch results (mirror boundary conditions) results with set6a-uh-tp triangular pitch results (periodic boundary conditions) as delta- $k_{\text {eff }}$ vs. moderator mass, time $=1,000$ years.

Figure M-6. Comparison of CCO lower horizon datasets, set6-lh-tp triangular pitch results (mirror boundary conditions) results with set6a-lh-tp triangular pitch results (periodic boundary conditions) as delta- $k_{\text {eff }}$ vs. moderator mass, 1,000 years.

Figure M-7. Summary of CCO upper horizon datasets, set6a-uh-tp triangular pitch results (periodic boundary conditions) as $k_{\text {eff }} \mathrm{vs}$. moderator mass, time $=0$ years.

Figure M-8. Summary of CCO upper horizon datasets, set6a-uh-tp triangular pitch results (periodic boundary conditions) as $k_{\text {eff }}$ vs. moderator mass, time $=1,000$ years.

Figure M-9. Summary of 6 in. POP upper horizon datasets, set6-uhpop6-tp triangular pitch results (mirror boundary conditions) as $k_{\text {eff }} \mathrm{vs.} \mathrm{moderator} \mathrm{mass,} \mathrm{time}=0$ years.

Figure M-10. Summary of 6 in. POP upper horizon datasets, set6-uhpop6-tp triangular pitch results (mirror boundary conditions) as $k_{\text {eff }} \mathrm{vs}$. moderator mass, time $=1,000$ years.

Figure M-11. Summary of 6 in. POP lower horizon datasets, set6-lhpop6-hp hexagonal pitch results (mirror boundary conditions) as $k_{\text {eff }} \mathrm{vs.} \mathrm{moderator} \mathrm{mass,} \mathrm{time}=0$ years.

Figure M-12. Summary of 6 in. POP lower horizon datasets, set6-lhpop6-hp hexagonal pitch results (mirror boundary conditions) as $k_{\text {eff }}$ vs. moderator mass, time $=1,000$ years.

Figure M-13. Comparison of 6 in. POP upper horizon datasets set6-uhpop6-tp triangular pitch results (mirror boundary conditions) results with set6a-uhpop6-tp triangular pitch results (periodic boundary conditions) as delta- $k_{\text {eff }} \mathrm{vs}$. moderator mass, time $=1,000$ years.

Figure M-14. Comparison of 6 in. POP lower horizon datasets set6-lhpop6-hp hexagonal pitch results (mirror boundary conditions) results with set6a-lhpop6-hp hexagonal pitch results (periodic boundary conditions) as delta- $k_{\text {eff }} \mathrm{vs.}$ moderator mass, time $=1,000$ years.

Figure M-15. Comparison of 6 in. POP lower horizon datasets set6-lhpop6-hp hexagonal pitch results (mirror boundary conditions) results with upper horizon set6-uhpop6-tp triangular pitch results (mirror boundary conditions) as delta- $k_{\text {eff }}$ vs. moderator mass, time $=1,000$ years.

Figure M-16. Summary of 12 in. POP upper horizon datasets, set6-uhpop12-tp triangular pitch results (mirror boundary conditions) as $k_{\text {eff }} \mathrm{vs.} \mathrm{moderator} \mathrm{mass,} \mathrm{time}=0$ years. M-46

Figure M-17. Summary of 12 in. POP upper horizon datasets, set6-uhpop12-tp triangular pitch results (mirror boundary conditions) as $k_{\text {eff }} \mathrm{vs}$. moderator mass, time $=1,000$ years.

Figure M-18. Summary of 12 in. POP lower horizon datasets, set6-lhpop12-hp hexagonal pitch results (mirror boundary conditions) as $k_{\text {eff }}$ Vs. moderator mass, time $=0$ years.

Figure M-19. Summary of 12 in. POP lower horizon datasets, set6-lhpop12-hp hexagonal pitch results (mirror boundary conditions) as $k_{\text {eff }} \mathrm{Vs}$. moderator mass, time $=1,000$ years.

Figure M-20. Comparison of 12 in. POP upper horizon datasets set6-uhpop12-tp triangular pitch results (mirror boundary conditions) results with set6a-uhpop12-tp triangular pitch results (periodic boundary conditions) as delta- $k_{\text {eff }}$ vs. moderator mass, time $=1,000$ years.

Figure M-21. Comparison of 12 in. POP lower horizon datasets set6-lhpop12-hp hexagonal pitch results (mirror boundary conditions) results with set6a-lhpop12-hp hexagonal pitch results (periodic boundary conditions) as delta- $k_{\text {eff }}$ vs. moderator mass, time $=1,000$ years.

Figure M-22. Comparison of 12 in. POP lower horizon datasets set6-lhpop12-hp hexagonal pitch results (mirror boundary conditions) results with upper horizon set6-uhpop12-tp 
triangular pitch results (mirror boundary conditions) as delta- $k_{\text {eff }}$ vs. moderator mass, time

$=1,000$ years.

Figure M-23. Max $k_{e f f}$ of all subsets of all CCO and POP centroid configurations at time $=1,000$ years compared with max $k_{\text {eff }}$ of all subsets of set-2-uh hexagonal pitch (mirror boundary conditions) as $k_{\text {eff }}$ vs. moderator mass, time $=1,000$ years.

Figure M-24. Max $k_{\text {eff }}$ of all subcase- 1 of all $\mathrm{CCO}$ and POP centroid configurations at time = 1,000 years compared with max $k_{\text {eff }}$ of all subsets of set-2-uh hexagonal pitch (mirror boundary conditions) as $k_{\text {eff }} \mathrm{vs}$. moderator mass, time $=1,000$ years.

Figure M-25. Max $k_{\text {eff }}$ of all subcase-2 of all CCO and POP centroid configurations at time = 1,000 years compared with max $k_{\text {eff }}$ of all subsets of set-2-uh hexagonal pitch (mirror boundary conditions) as $k_{\text {eff }} \mathrm{Vs}$. moderator mass, time $=1,000$ years.

Figure M-26. Max $k_{\text {eff }}$ of all subcase- 3 of all CCO and POP centroid configurations at time = 1,000 years compared with max $k_{\text {eff }}$ of all subsets of set-2-uh hexagonal pitch (mirror boundary conditions) as $k_{\text {eff }} \mathrm{vs}$. moderator mass, time $=1,000$ years.

Figure M-27. Max $k_{\text {eff }}$ of all subcase- 4 of all $\mathrm{CCO}$ and POP centroid configurations at time $=$ 1,000 years compared with max $k_{\text {eff }}$ of all subsets of set-2-uh hexagonal pitch (mirror boundary conditions) as $k_{\text {eff }} \mathrm{vs}$. moderator mass, time $=1,000$ years.

Figure M-28. Max $k_{\text {eff }}$ of all subcase-5 of all CCO and POP centroid configurations at time = 1,000 years compared with max $k_{\text {eff }}$ of all subsets of set-2-uh hexagonal pitch (mirror boundary conditions) as $k_{\text {eff }} \mathrm{vs}$. moderator mass, time $=1,000$ years.

Figure M-29. Max $k_{\text {eff }}$ of all subcase- 6 of all CCO and POP centroid configurations at time = 1,000 years compared with max $k_{\text {eff }}$ of all subsets of set-2-uh hexagonal pitch (mirror boundary conditions) as $k_{\text {eff }} \mathrm{vs}$. moderator mass, time $=1,000$ years.

Figure M-30. Max $k_{\text {eff }}$ of all subcase-7 of all CCO and POP centroid configurations at time = 1,000 years compared with max $k_{\text {eff }}$ of all subsets of set-2-uh hexagonal pitch (mirror boundary conditions) as $k_{\text {eff }} \mathrm{Vs}$. moderator mass, time $=1,000$ years.

Figure M-31. Max $k_{\text {eff }}$ of all subcase- 8 of all CCO and POP centroid configurations at time = 1,000 years compared with max $k_{\text {eff }}$ of all subsets of set-2-uh hexagonal pitch (mirror boundary conditions) as $k_{\text {eff }} \mathrm{vs}$. moderator mass, time $=1,000$ years.

Figure M-32. Max $k_{\text {eff }}$ of all subcase- 9 of all CCO and POP centroid configurations at time = 1,000 years compared with max $k_{\text {eff }}$ of all subsets of set-2-uh hexagonal pitch (mirror boundary conditions) as $k_{\text {eff }} \mathrm{vs}$. moderator mass, time $=1,000$ years.

Figure M-33. Max $k_{\text {eff }}$ of all subcase-10 of all CCO and POP centroid configurations at time = 1,000 years compared with max $k_{\text {eff }}$ of all subsets of set-2-uh hexagonal pitch (mirror boundary conditions) as $k_{\text {eff }} \mathrm{vs}$. moderator mass, time $=1,000$ years.

Figure M-34. Max $k_{\text {eff }}$ of all subcase-11 of all CCO and POP centroid configurations at time = 1,000 years compared with max $k_{\text {eff }}$ of all subsets of set-2-uh hexagonal pitch (mirror boundary conditions) as $k_{\text {eff }} \mathrm{vs}$. moderator mass, time $=1,000$ years.

Figure M-35. Max $k_{\text {eff }}$ of all subcase-12 of all CCO and POP centroid configurations at time $=$ 1,000 years compared with max $k_{\text {eff }}$ of all subsets of set-2-uh hexagonal pitch (mirror boundary conditions) as $k_{\text {eff }} \mathrm{vs}$. moderator mass, time $=1,000$ years.

Figure M-36. Max $k_{\text {eff }}$ of all subcase-13 of all CCO and POP centroid configurations at time $=$ 1,000 years compared with max $k_{\text {eff }}$ of all subsets of set-2-uh hexagonal pitch (mirror boundary conditions) as $k_{\text {eff }} \mathrm{vs}$. moderator mass, time $=1,000$ years.

Figure M-37. Max $k_{\text {eff }}$ of all subcase-14 of all CCO and POP centroid configurations at time $=$ 1,000 years compared with max $k_{\text {eff }}$ of all subsets of set-2-uh hexagonal pitch (mirror boundary conditions) as $k_{\text {eff }} \mathrm{vs}$. moderator mass, time $=1,000$ years.

Figure M-38. Max $k_{\text {eff }}$ of all subcase-15 of all CCO and POP centroid configurations at time $=$ 1,000 years compared with max $k_{\text {eff }}$ of all subsets of set-2-uh hexagonal pitch (mirror boundary conditions) as $k_{\text {eff }} \mathrm{vs}$. moderator mass, time $=1,000$ years. 
Figure M-39. Max $k_{\text {eff }}$ of all subcase-16 of all CCO and POP centroid configurations at time = 1,000 years compared with max $k_{\text {eff }}$ of all subsets of set-2-uh hexagonal pitch (mirror boundary conditions) as $k_{\text {eff }} \mathrm{vs}$. moderator mass, time $=1,000$ years.

Figure M-40. Diagram of the neutron flux for subcase-10 in the CCO with no filler material and thin SS discrete reflector (sweep 470), upper horizon with hexagonal pitch (set-2-uh) with a maximum $k_{\text {eff }}$ of 1.0134 and moderator mass of 2,084 g, comparable to Figure 29 in [46]....

Figure M-41. Diagram of the neutron flux for subcase-10 in the CCO with $4 \mathrm{~kg}$ graphite filler material and thin SS discrete reflector (sweep 494), upper horizon with hexagonal pitch (set-2-uh) with a maximum $k_{\text {eff }}$ of 0.9649 and moderator mass of $2,084 \mathrm{~g}$, comparable to Figure 29 in [46].

Figure M-42. Diagram of the neutron flux for subcase-10 in the CCO with no filler material and thick SS discrete reflector (sweep 472), upper horizon with hexagonal pitch (set-2-uh) with a maximum $k_{\text {eff }}$ of 1.047 and moderator mass of 2,084 g, comparable to Figure 29 in [46].....

Figure M-43. Diagram of the neutron flux for subcase-10 in the CCO with $4 \mathrm{~kg}$ graphite filler material and thick SS discrete reflector (sweep 496), upper horizon with hexagonal pitch (set-2-uh) with a maximum $k_{\text {eff }}$ of 1.047 and moderator mass of $2,084 \mathrm{~g}$, comparable to Figure 29 in [46].

Figure M-44. Diagram of the neutron flux for subcase-10 in the CCO with no filler material and thin SS discrete reflector (sweep 470), lower horizon with hexagonal pitch (set-2-1h) with a maximum $k_{\text {eff }}$ of 1.0428 and moderator mass of $2,084 \mathrm{~g}$, comparable to Figure 27 in [46]

Figure M-45. Diagram of the neutron flux for subcase-10 in the 12 in. POP with no filler material and thick SS discrete reflector (sweep 688), lower horizon with hexagonal pitch with a maximum $k_{\text {eff }}$ of 0.9816 and moderator mass of 3,000 g, comparable to Figure 37 in [46]...... M-75

Figure M-46. Diagram of the neutron flux for subcase-10 in the CCO with no filler material and thin SS discrete reflector (sweep 470), lower horizon with triagonal pitch with a maximum $k_{\text {eff }}$ of 1.0227 and moderator mass of 2,084 g, comparable to Figure 33 in [46]..... M-76

Figure M-47. Diagram of the neutron flux for subcase-10 in the 6 in. POP with no filler material and thin SS discrete reflector (sweep 470), lower horizon with hexagonal pitch with a maximum $k_{\text {eff }}$ of 1.0227 and moderator mass of 2,084 g, comparable to Figure 35 in [46]

\section{APPENDIX N: LIST OF FIGURES}

Figure N-1. Set-7a results (three-high uniform array model) for 10, 30, and $50 \mathrm{~g}$ of $\mathrm{B}_{4} \mathrm{C}$ for all subcases.

Figure N-2. Set-7b results (six-high uniform array model) for 10,30 , and $50 \mathrm{~g}$ of $\mathrm{B}_{4} \mathrm{C}$ for all subcases.

Figure N-3. Set-7c results (three-high uniform array model) for 10, 30, and $50 \mathrm{~g}$ of $\mathrm{B}_{4} \mathrm{C}$ for all subcases.

Figure N-4. Set-7a results (three-high uniform array model) for $0,10,30$, and $50 \mathrm{~g}$ of $\mathrm{B}_{4} \mathrm{C}$ for all subcases.

Figure N-5. Set-7b results (six-high uniform array model) for $0,10,30$, and $50 \mathrm{~g}$ of $\mathrm{B}_{4} \mathrm{C}$ for all subcases.

Figure N-6. Set-7c results (nonuniform upper horizon array model) for $0,10,30$, and $50 \mathrm{~g}$ of $\mathrm{B}_{4} \mathrm{C}$ for all subcases. 
Figure N-7. Set-7a results (three-high uniform array model) for 10, 30, and $50 \mathrm{~g}$ of $\mathrm{B}_{4} \mathrm{C}$ for all subcases.

Figure N-8. Set-7b results (six-high uniform array model) for 10, 30, and $50 \mathrm{~g}$ of $\mathrm{B}_{4} \mathrm{C}$ for all subcases.

Figure N-9. Set-7c results (nonuniform array model) for 10,30 , and $50 \mathrm{~g}$ of $\mathrm{B}_{4} \mathrm{C}$ for all subcases....... N-17

Figure N-10. Set-7a results (three-high uniform array model) for 10, 15, 20, 25, 30, and $50 \mathrm{~g}$ of $\mathrm{B}_{4} \mathrm{C}$ for subcase- 10 only.

Figure N-11. Set-7b results (six-high uniform array model) for 10, 15, 20, 25, 30, and $50 \mathrm{~g}$ of $\mathrm{B}_{4} \mathrm{C}$ for subcase-10 only.

Figure N-12 Set-7c results (nonuniform array model) for 10, 15, 20, 25, 30, and $50 \mathrm{~g}$ of $\mathrm{B}_{4} \mathrm{C}$ for subcase- 10 only.....

Figure N-13. Set-7a results (three-high uniform array model) for 10, 15, 20, 25, 30, and $50 \mathrm{~g}$ of $\mathrm{B}_{4} \mathrm{C}$ for all subcases.

Figure N-14. Set-7b results (six-high uniform array model) for 10, 15, 20, 25, 30, and $50 \mathrm{~g}$ of $\mathrm{B}_{4} \mathrm{C}$ for all subcases.

Figure N-15. Set-7c results (nonuniform array model) for 10, 15, 20, 25, 30, and $50 \mathrm{~g}$ of $\mathrm{B}_{4} \mathrm{C}$ for up to a $6 \mathrm{~kg}$ moderator.

\section{APPENDIX O: LIST OF FIGURES}

Figure O-1. Set-8a and set- $1 k_{\text {eff }}$ results (three-high uniform array model) for the cylindrical waste forms with a poly discrete reflector and a discrete reflector thickness comparison between the sublisting parameters, which yield a maximum subcritical moderator mass for a discrete reflector thickness of $0.001 \mathrm{~cm}$

Figure O-2. Set-8a and set- $1 k_{\text {eff }}$ results (three-high uniform array model) for the spherical waste forms with a poly discrete reflector, and a discrete reflector thickness comparison between the sublisting parameters, which yield a maximum subcritical moderator mass for a discrete reflector thickness of $0.001 \mathrm{~cm}$.

Figure O-3. Set-8a and set- $1 k_{\text {eff }}$ results (three-high uniform array model) for the cylindrical waste forms with a stainless-steel discrete reflector and a discrete reflector thickness comparison between the sublisting parameters, which yield a maximum subcritical moderator mass for a discrete reflector thickness of $0.001 \mathrm{~cm}$.

Figure O-4. Set-8a and set- $1 k_{\text {eff }}$ results (three-high uniform array model) for the spherical waste forms with a stainless-steel discrete reflector and a discrete reflector thickness comparison between the sublisting parameters, which yield a maximum subcritical moderator mass for a discrete reflector thickness of $0.001 \mathrm{~cm}$.

Figure O-5. Set-8a and set-1 maximum $k_{\text {eff }}$ results (three-high uniform array model) overall sublistings as a function of discrete reflector thickness.

Figure O-6. Set-8b and set-2-uh $k_{\text {eff }}$ results (nonuniform array model) for the cylindrical waste forms with a poly discrete reflector and a discrete reflector thickness comparison between the sublisting parameters, which yield a maximum subcritical moderator mass for a discrete reflector thickness of $0.001 \mathrm{~cm}$.....

Figure O-7. Set-8b and set-2-uh $k_{\text {eff }}$ results (nonuniform array model) for the spherical waste forms with a poly discrete reflector and a discrete reflector thickness comparison between the sublisting parameters, which yield a maximum subcritical moderator mass for a discrete reflector thickness of $0.001 \mathrm{~cm}$ 
Figure O-8. Set-8b and set-2-uh $k_{\text {eff }}$ results (nonuniform array model) for the cylindrical waste forms with a stainless-steel discrete reflector and a discrete reflector thickness comparison between the sublisting parameters, which yield a maximum subcritical moderator mass for a discrete reflector thickness of $0.001 \mathrm{~cm}$.

Figure 0-9. Set-8b and set-2-uh $k_{\text {eff }}$ results (nonuniform array model) for the spherical waste forms with a stainless-steel discrete reflector and a discrete reflector thickness comparison between the sublisting parameters, which yield a maximum subcritical moderator mass for a discrete reflector thickness of $0.001 \mathrm{~cm}$.

Figure O-10. Set- $8 \mathrm{~b}$ and set-2-uh maximum $k_{\text {eff }}$ results (nonuniform array model) overall sublistings as a function of discrete reflector thickness.

Figure O-11. Set-8a sublisting-1 results (three-high uniform array model): $k_{\text {eff }}$ for set-1 and set-8a by discrete reflector thicknesses.

Figure 0-12. Set-8a sublisting-2 results (three-high uniform array model): $k_{\text {eff }}$ for set- 1 and set-8a by discrete reflector thicknesses.

Figure O-13. Set-8a sublisting-3 results (three-high uniform array model): ): $k_{\text {eff }}$ for set-1 and set8 a by discrete reflector thicknesses.

Figure O-14. Set-8a sublisting-4 results (three-high uniform array model): $k_{\text {eff }}$ for set-1 and set-8a by discrete reflector thicknesses.

Figure O-15. Set-8a sublisting-5 results (three-high uniform array model): $k_{\text {eff }}$ for set- 1 and set- $8 \mathrm{a}$ by discrete reflector thicknesses.

Figure O-16. Set-8a sublisting-6 results (three-high uniform array model): $k_{\text {eff }}$ for set- 1 and set-8a by discrete reflector thicknesses.

Figure 0-17. Set-8a sublisting-7 results (three-high uniform array model): $k_{\text {eff }}$ for set- 1 and set-8a by discrete reflector thicknesses.

Figure O-18. Set-8a sublisting-8 results (three-high uniform array model): $k_{\text {eff }}$ for set- 1 and set-8a by discrete reflector thicknesses.

Figure O-19. Set-8a sublisting-9 results (three-high uniform array model): $k_{\text {eff }}$ for set-1 and set-8a by discrete reflector thicknesses.

Figure O-20. Set-8a sublisting-10 results (three-high uniform array model): $k_{\text {eff }}$ for set-1 and set8 a by discrete reflector thicknesses.

Figure O-21. Set-8a sublisting-11 results (three-high uniform array model): $k_{\text {eff }}$ for set-1 and set8 a by discrete reflector thicknesses.

Figure O-22. Set-8a sublisting-12 results (three-high uniform array model): $k_{\text {eff }}$ for set-1 and set8 a by discrete reflector thicknesses.

Figure O-23. Set-8a sublisting-13 results (three-high uniform array model): $k_{\text {eff }}$ for set-1 and set8 a by discrete reflector thicknesses.

Figure O-24. Set-8a sublisting-14 results (three-high uniform array model): $k_{\text {eff }}$ for set-1 and set8 a by discrete reflector thicknesses.

Figure O-25. Set-8a sublisting-15 results (three-high uniform array model): $k_{\text {eff }}$ for set- 1 and set8 a by discrete reflector thicknesses.

Figure O-26. Set-8a sublisting-16 results (three-high uniform array model): $k_{\text {eff }}$ for set- 1 and set8 a by discrete reflector thicknesses.

Figure O-27. Set-8b sublisting-1 results (nonuniform array model): $k_{\text {eff }}$ for set-2-uh and set-8a by discrete reflector thicknesses.

Figure O-28. Set-8b sublisting-2 results (nonuniform array model): $k_{\text {eff }}$ for set-2-uh and set-8a by discrete reflector thicknesses.

Figure O-29. Set-8b sublisting-3 results (nonuniform array model): $k_{\text {eff }}$ for set-2-uh and set-8a by discrete reflector thicknesses.

Figure O-30. Set-8b sublisting-4 results (three-high uniform array model): $k_{\text {eff }}$ for set-2-uh and set-8a by discrete reflector thicknesses. 
Figure O-31. Set-8b sublisting-5 results (nonuniform array model): $k_{\text {eff }}$ for set-2-uh and set-8a by discrete reflector thicknesses.

Figure 0-32. Set-8b sublisting-6 results (nonuniform array model): $k_{\text {eff }}$ for set-2-uh and set- 8 a by discrete reflector thicknesses.

Figure 0-33. Set-8b sublisting-7 results (nonuniform array model): $k_{\text {eff }}$ for set-2-uh and set-8a by discrete reflector thicknesses.

Figure 0-34. Set-8b sublisting-8 results (nonuniform array model): $k_{\text {eff }}$ for set-2-uh and set-8a by discrete reflector thicknesses.

Figure O-35. Set-8a sublisting-9 results (nonuniform array model): $k_{\text {eff }}$ for set-2-uh and set-8a by discrete reflector thicknesses.

Figure O-36. Set-8a sublisting-10 results (nonuniform array model): $k_{\text {eff }}$ for set-2-uh and set-8a by discrete reflector thicknesses.

Figure O-37. Set-8b sublisting-11 results (nonuniform array model): $k_{\text {eff }}$ for set-2-uh and set-8a by discrete reflector thicknesses.

Figure O-38. Set-8b sublisting-12 results (nonuniform array model): $k_{\text {eff }}$ for set-2-uh and set-8a by discrete reflector thicknesses.

Figure O-39. Set-8b sublisting-13 results (nonuniform array model): $k_{\text {eff }}$ for set-2-uh and set-8a by discrete reflector thicknesses.

Figure O-40. Set-8b sublisting-14 results (nonuniform array model): $k_{\text {eff }}$ for set-2-uh and set-8a by discrete reflector thicknesses.

Figure O-41. Set-8b sublisting-15 results (nonuniform array model): $k_{\text {eff }}$ for set-2-uh and set-8a by discrete reflector thicknesses.

Figure O-42. Set-8b sublisting-16 results (nonuniform array model): $k_{\text {eff }}$ for set-2-uh and set-8a by discrete reflector thicknesses. 


\section{LIST OF TABLES}

Table E.S-1. Example of possible post-closure criticality safety mass limits for waste content in

CCOs to maintain a subcritical limit of 1.0 for spheres with a thick steel pipe reflector.

Table 4.1. Evaluated array spacings.

Table 4.2. Comparison of the SNL closure data used for uniform arrays.............................................. 19

Table 4.3. Horizonal closure percentage used for $\mathrm{x}$-direction spacing reductions ................................... 20

Table 4.4. Reedlunn and Bean [8] compaction study of centroid locations (in $\mathrm{cm}$ ) for upper and lower horizons

Table 4.5. Comparison of the moderator power and moderating ratio of several materials ...................... 31

Table 4-6. Evaluated parameters and corresponding rationale for evaluating $k_{\text {eff }}$ effect........................... 34

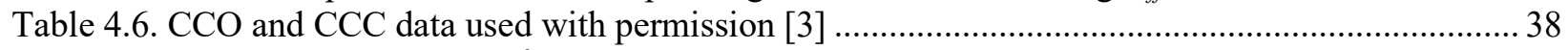

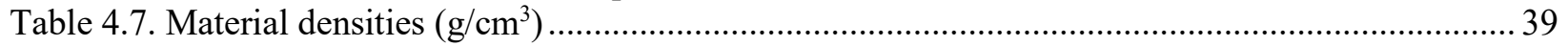

Table 6.1. Description of the cases to evaluate reactivity trends .......................................................... 46

Table 6.2. Summary of parametric sweeps for both cylindrical and spherical waste forms (values

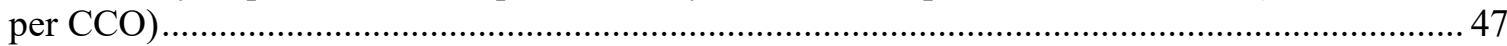

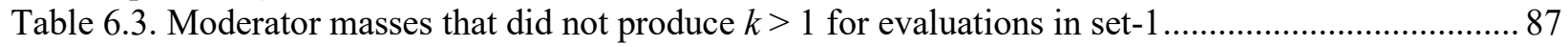

Table 6.4. Moderator masses that did not produce $k>1$ for evaluations in set-1a.................................. 89

Table 6.5. Masses that did not produce $k>1$ for evaluations in set-2 .................................................. 91

Table 6.6. Masses that did not produce $k>1$ for evaluations in set-3 ................................................... 93

Table 6.7. Masses that did not produce $k>1$ for evaluations in set-3a ................................................ 95

Table 6.8. Comparison of set-2-10-uh parameters with set-1-2 parameters.......................................... 107

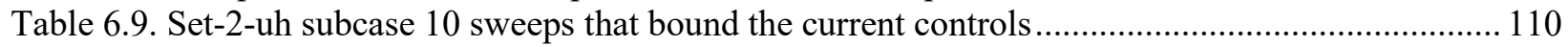

Table 6.10. Number of benchmark experiments in Appendix $H$ with various $c_{k}$ criteria ...................... 110

\section{APPENDIX A: LIST OF TABLES}

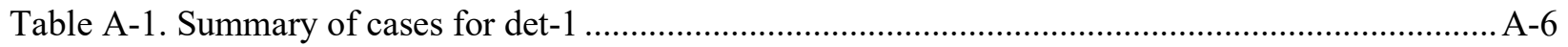

\section{APPENDIX B: LIST OF TABLES}

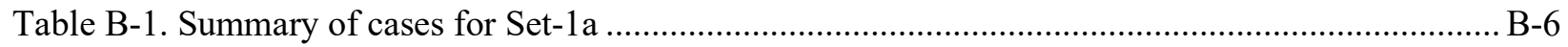

\section{APPENDIX C: LIST OF TABLES}

Table C-1. Summary of cases for set-2-uh for the upper horizon compaction data C-6

\section{APPENDIX D: LIST OF TABLES}

Table D-1. Summary of cases for Set-2-uh for the lower horizon compaction data. D-6 


\section{APPENDIX E: LIST OF TABLES}

Table E-1. SAMPLER sweeps for the time-dependent studies E-7

\section{APPENDIX F: LIST OF TABLES}

Table F-1. Summary of cases for set-3 F-6

\section{APPENDIX H: LIST OF TABLES}

Table H-1. Set-1-1 application cases $\mathrm{H}-12$

Table H-2. Set-1-2 application cases $\mathrm{H}-12$

Table H-3. Set-1-3 application cases $\mathrm{H}-12$

Table H-4. Set-1-4 application cases $\mathrm{H}-13$

Table H-5. Set-1-5 application cases $\mathrm{H}-13$

Table H-6. Set-1-6 application cases $\mathrm{H}-13$

Table H-7. Set-1-7 application cases $\mathrm{H}-14$

Table H-8. Set-1-8 application cases $\mathrm{H}-14$

Table H-9. Set-1-1 $k_{\text {eff }}$ values for comparison. $\mathrm{H}-15$

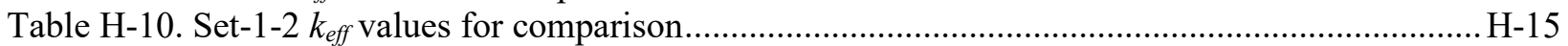

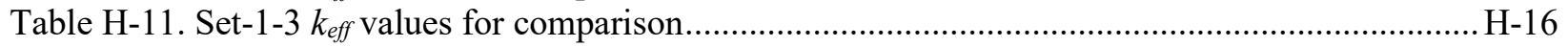

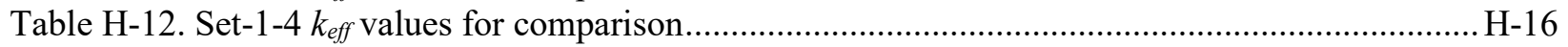

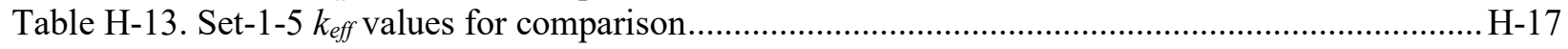

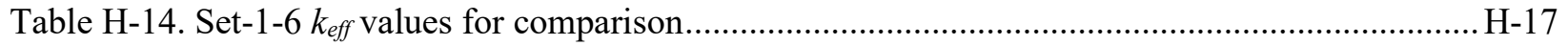

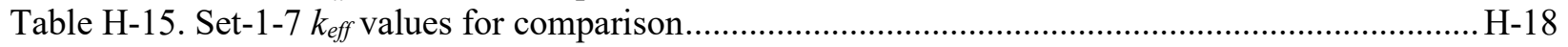

Table H-16. Set-1-8 $k_{\text {eff }}$ values for comparison..................................................................................

Table H-17. Nuclides with the highest sensitivities for set-1-1 cases...................................................19

Table H-18. Nuclides with the highest sensitivities for set-1-2 cases.................................................. H-20

Table H-19. Nuclides with the highest sensitivities for set-1-3 cases..............................................

Table H-20. Nuclides with the highest sensitivities for set-1-4 cases............................................... H-22

Table H-21. Nuclides with the highest sensitivities for set-1-5 cases..............................................23

Table H-22. Nuclides with the highest sensitivities for set-1-6 cases............................................... H-24

Table H-23. Nuclides with the highest sensitivities for set-1-7 cases...............................................25

Table H-24. Nuclides with the highest sensitivities for set-1-8 cases...............................................26

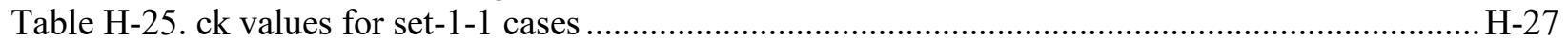

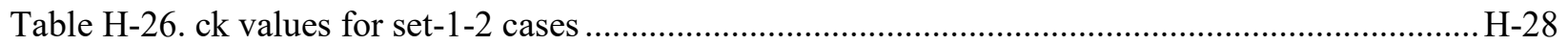

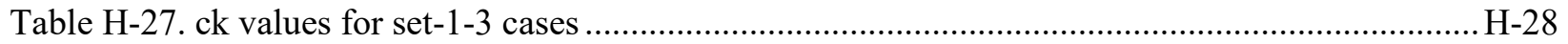

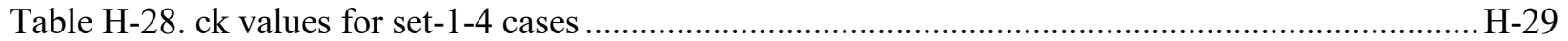

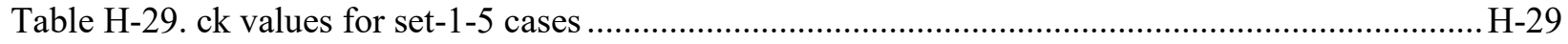

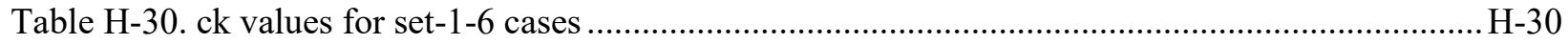

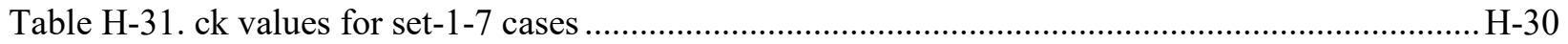

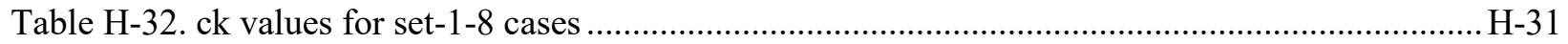

Table H-33. Bias, bias uncertainty, and data-induced uncertainty for set-1-1 cases ............................

Table H-34. Bias, bias uncertainty, and data-induced uncertainty for set-1-2 cases ............................

Table H-35. Bias, bias uncertainty, and data-induced uncertainty for set-1-3 cases .............................

Table H-36. Bias, bias uncertainty, and data-induced uncertainty for set-1-4 cases .............................

Table H-37. Bias, bias uncertainty, and data-induced uncertainty for set-1-5 cases .............................

Table H-38. Bias, bias uncertainty, and data-induced uncertainty for set-1-6 cases ..............................-35 
Table H-39. Bias, bias uncertainty, and data-induced uncertainty for set-1-7 cases H-35

\section{APPENDIX I: LIST OF TABLES}

Table I-1. Summary of cases for set-3a $. \mathrm{I}-6$

\section{APPENDIX J: LIST OF TABLES}

Table J-1. Parameters varied in the heterogenous study $\mathrm{J}-5$

\section{APPENDIX K: LIST OF TABLES}

Table K-1. Parameters of the CCO modeled. K-4

Table K-2. Flux per source particle $\left({ }^{0} \mathrm{n} / \mathrm{cm}^{2} \mathrm{~s}\right)$ at various distances in the salt form the source. K-9

\section{APPENDIX L: LIST OF TABLES}

Table L-1. Summary of cases for set-5. L-5

Table L-2. Summary of parametric sweeps for set-5 (applicable to set-5 and versions a through h)....... L-6

\section{APPENDIX M: LIST OF TABLES}

Table M-1. Summary of cases for all set-6 models. M-8

Table M-2. Centroid locations from [46] for the CCO containers in a triangular pitch. ...................... M-9

Table M-3. Centroid locations from [46] for the 6 in. POP containers. .................................................

Table M-4. Centroid locations from [46] for the 12 in. POP containers................................................23

Table M-5. Summary of the calculations in this appendix. .............................................................

\section{APPENDIX N: LIST OF TABLES}

Table N-1. Summary of cases for set-7a for the three-high uniform array model................................. N-6 Table N-2. Summary of cases for set-7b for the six-high uniform array model. .................................... N-7

Table N-3. Summary of cases for set-7c for the nonuniform array model. ........................................... N-8

Table N-4. Summary of results for the addition of 10,30, or $50 \mathrm{~g}$ of $\mathrm{B}_{4} \mathrm{C}$ with a waste form moderator mass up to $3 \mathrm{~kg}$ for all subcases. N-8

\section{APPENDIX O: LIST OF TABLES}

Table O-1. Summary of cases for set-8a for three-high uniform array model. O-7

Table O-2. Summary of cases for set- $8 \mathrm{~b}$ for the nonuniform array model. O-8 
This page is intentionally blank

xxix 


\section{ABBREVIATIONS}

$\begin{array}{ll}\text { ANS } & \text { American Nuclear Society } \\ \text { ANSI } & \text { American National Standards Institute } \\ \text { CBFO } & \text { Carlsbad Field Office } \\ \text { cCC } & \begin{array}{l}\text { criticality control container } \\ \text { criticality control overpack }\end{array} \\ \text { CCO } & \text { US Code of Federal Regulations } \\ \text { CFR } & \text { contact-handled } \\ \text { CH } & \text { US Department of Energy } \\ \text { DOE } & \text { energy of average lethargy causing fission } \\ \text { EALF } & \text { Evaluated Nuclear Data File } \\ \text { ENDF } & \text { US Environmental Protection Agency } \\ \text { EPA } & \text { Energy Research and Development Administration } \\ \text { ERDA } & \text { feature, event, and process } \\ \text { FEP } & \text { fissile gram equivalent } \\ \text { FGE } & \text { Monte Carlo N-Particle code } \\ \text { MCNP } & \text { mixed oxide } \\ \text { MOX } & \text { metric ton } \\ \text { MT } & \text { Nuclear Energy Agency } \\ \text { NEA } & \text { Nuclear Energy and Fuel Cycle Division } \\ \text { NEFCD } & \text { Nuclear Waste Partnership LLC } \\ \text { NWP } & \text { Oak Ridge National Laboratory } \\ \text { ORNL } & \text { pipe overpack container } \\ \text { POC OR POP } & \text { safety analysis report } \\ \text { SAR } & \text { Systems-Based Management System } \\ \text { SBMS } & \text { Sandia National Laboratories } \\ \text { SNL } & \text { Savanah River Site } \\ \text { SRS } & \text { transuranic } \\ \text { TRU } & \text { TRU Package Transporter } \\ \text { TRUPACT } & \text { waste acceptance criteria } \\ \text { WAC } & \text { Waste Isolation Pilot Plant } \\ \text { WIPP } & \end{array}$


This page is intentionally blank 


\section{ACKNOWLEDGMENTS}

This work would not have been possible without the excellent communication and collaboration within the of the extended multi-laboratory technical team: Ron Livingston (Omega Technical Services), Paul Shoemaker (SNL), Benjamin Reedlunn (SNL), Robert Rechard (SNL), Brad Day (WIPP), Steve Wagner (SNL) and Ross Kirkes (SNL). Special thanks to Brad and Ben for never shying away from asking the hard questions. The authors are also grateful for the engagement, support, and excellent feedback

provided by Rose Montgomery (ORNL), Rose Rainey (ORNL), Kathy Jones (ORNL), and Molly Smith (ORNL). 
This page is intentionally blank 


\section{EXECUTIVE SUMMARY}

The Waste Isolation Pilot Plant (WIPP) is a geological repository in southern New Mexico that provides for disposal of transuranic (TRU) wastes from atomic energy defense activities. The Sandia National Laboratories (Sandia) Report, Consideration of Nuclear Criticality When Disposing of Transuranic Waste at the Waste Isolation Pilot Plant [1], addresses nuclear criticality safety based on the projected inventory characteristics for the initial compliance certification application of WIPP in 1996 [1]. As the inventory, waste forms, and disposal package designs change, revised or new analyses are necessary to demonstrate acceptability for these configurations within the WIPP safety basis [2] and compliance with 10,000-year post-closure standards of the US Environmental Protection Agency (EPA). Saylor and Scaglione evaluated criticality control overpacks (CCOs) in 2017 [3] based on conservative assumptions for postclosure repository structural conditions with resulting effects on containers and container spacing,

The Saylor and Scaglione evaluation of CCOs addressed a single waste configuration that represents the Surplus Plutonium Disposition Program's dilute and dispose waste form and composition. This initial $\mathrm{CCO}$ study demonstrated that 50 grams of boron carbide $\left(\mathrm{B}_{4} \mathrm{C}\right)$ per $\mathrm{CCO}$ is sufficient to ensure postclosure criticality safety based on a well-mixed waste composition, and Oak Ridge National Laboratory (ORNL) subsequently determined that this amount of $\mathrm{B}_{4} \mathrm{C}$ does not require constraints on moisture or plastic present as moderator [29]. The Saylor and Scaglione [3] analysis conservatively assumes repository room closure that eliminates all space between fissile gram equivalent (FGE) ${ }^{239} \mathrm{Pu}$ masses. The close-packed array was selected based on limited availability of repository salt creep modeling results at that time. In 2019, Brickner [4] provided additional evaluations for pipe overpack containers (POCs), building on the conservative basis provided by Saylor and Scaglione. Brickner's 2019 analysis made use of new geomechanical data for post-closure spacing that rely on advances in repository modeling as documented in the work by Reedlunn and Bean [7].

This current $\mathrm{CCO}$ evaluation for generic waste materials expands on earlier work performed at ORNL and includes evaluation of CCOs across a much broader range of possible waste compositions and geometries. This evaluation is intended to provide input for the required feature, event and process (FEP) screening to determine if post-closure criticality must be included as an event in the 10,000-year regulatory evaluation. As such, the approach to modeling post-closure criticality presented in this report has been coordinated with the Sandia team responsible for FEP screening. The resulting analysis supports disposition of fissile materials in the $\mathrm{CCO}$ containing up to $380 \mathrm{FGE}{ }^{239} \mathrm{Pu}$ and expands conditions acceptable for disposal of fissile material in CCOs [6]. This evaluation builds on the methodology of Saylor and Scaglione [3] and Brickner [4], using the most recently available geomechanical data for CCO spacing under salt creep compaction scenarios provided by Reedlunn and Bean [8]. The broad range of fissile material configurations analyzed in this report are intended to account for configurations that may occur during the post-closure disposal time period, and it also includes waste configurations that are not physically possible to support analysis of conditions that influence neutron fluence.

Given the mechanical deformation resulting from room collapse per the recent analysis of Reedlunn and Bean [8], the extent of compaction in a room is significantly less than that used in the initial ORNL bounding evaluation performed by Saylor and Scaglione [3]. Consequently, the results of this analysis support addition of other options for TRU waste disposal in CCOs that do not include $\mathrm{B}_{4} \mathrm{C}$, as the results indicate strong dependence on the size of the waste form (as calculated from mass of materials present in the fissile region) and the amount of moderator from hydrogen-bearing materials within the waste form. Results of this analysis are presented in $\mathrm{H} /{ }^{239} \mathrm{Pu}$ curves that are used for FEP screening to evaluate the effective neutron multiplication factor $\left(k_{e f f}\right)$ for disposal of CCO payload containers within the WIPP repository. These $\mathrm{H} /{ }^{239} \mathrm{Pu}$ curves are used to evaluate rooms filled with CCOs and provide the neutron multiplication as a function of various system parameters for the CCOs at the maximum authorized $\mathrm{FGE}{ }^{239} \mathrm{Pu}$ loading 
of $380 \mathrm{~g}$. Without $\mathrm{B}_{4} \mathrm{C}$, subcriticality is shown to be highly dependent upon (1) the mass of $\mathrm{H}$ in the fissile region of the waste form, (2) the mass of nonfissile, non-H materials within the waste form, and (3) the assumed final geometry of the waste form resulting from post-closure conditions.

Parametric evaluations were performed to facilitate analysis of the limits for moderator (i.e., the maximum mass limit of $\mathrm{H}$ containing materials) and the benefits of including nonfissile filler materials that may be present in the waste (i.e., a minimum mass limit). The parametric variations reported herein demonstrate that $380 \mathrm{FGE}^{239} \mathrm{Pu}$ may be safely dispositioned using the $\mathrm{CCO}$ without requiring the use of $\mathrm{B}_{4} \mathrm{C}$, given the appropriate constraints on water and plastic as long as the assumptions provided as the basis of the safety evaluation are maintained (i.e. well mixed system). This generic approach accounts for the range of parameters allowed for transport to WIPP in the CCO [9], which is also sufficient to demonstrate the $k_{\text {eff }}$ associated with disposal of dilute surplus $\mathrm{Pu}$ in CCOs. Therefore, this analysis supports the disposal of any waste form that complies with the CCO payload characteristics credited in the FEP evaluation and correspondingly specified in the WIPP waste acceptance criteria (WAC) [5].

This analysis was divided into three sets of calculations. The first set provides evaluation of regular, uniformly compacted arrays in a three-high stack of spheres or cylinders, with an equivalent spacing based on Sandia geomechanical compaction results. The first set includes two different spacing evaluations, both with waste forms stacked directly on top of one another. The second set of calculations supports evaluation of an irregular, nonuniformly compacted array with spacing determined by results from Sandia geomechanical modeling. These data include evaluation for the upper and lower horizons of the WIPP repository. The third dataset analyzes the effect of splitting the TRU waste into smaller handling containers within the CCO. In total, more than 20 parameters were evaluated using approximately 28,800 combinations per calculation set (155,520 total combinations) to assess the impact on $\mathrm{k}_{\text {eff. }}$ The complete list of results for these datasets is included in Addendum 1.

Table E.S-1 provides the most restrictive moderator mass that may be present in the generic waste forms when utilizing maximum fissile loading, beryllium content based on transportation limits, and the constraints from the safety evaluation using irregular, nonuniformly compacted array results from set-2 calculations. This ensures a conservative assessment of $k_{\text {eff }}$ without $\mathrm{B}_{4} \mathrm{C}$ present in the waste composition with an array spacing based on geomechanical modeling presented in set-2. Thus, Table ES-1 provides limits for the system to remain subcritical for an irregular array of waste a conservative assumption that waste obtains a spherical geometry postulated as a result of post-closure changes in the material geometry. The table illustrates the ability to increase moderator content when inorganic, non-hydrogenous filler materials are included in the waste to increase the size of the fissile region (i.e., dilution of the fissile material contents with filler material). The set two results include many other conservatisms necessary to support a rigorous safety analysis when allowing for potential uncertainties in the post-closure WIPP environment.

Results from sets one and three provide additional valuable insights into the factors influencing postclosure criticality, and they support continuity with prior analysis to inform the FEP screening for improbability of WIPP post-closure criticality. Based on evaluations of set two data, three components of the waste form content should be considered when prescribing disposal limits. The three controlling parameters for criticality safety evaluation of $\mathrm{CCO}$ waste forms - assuming maximum fissile mass and $\mathrm{Be}$ contents - include mass of hydrogenous moderator (water plus plastic), mass of non-hydrogenous filler material, and mass of $\mathrm{B}_{4} \mathrm{C}$. Note that the mass of $\mathrm{B}_{4} \mathrm{C}$ is provided for comparison purposes based on work performed by Saylor and Scaglione [3] and support reduction in the quantity of $\mathrm{B}_{4} \mathrm{C}$ required for fissile material discarded in the CCO. Other conditions that are subcritical and that support FEP screening can be ascertained using the data provided in the present report. 
Table E.S-1. Example of possible post-closure criticality safety mass limits for waste content in CCOs to maintain a subcritical limit of 1.0 for spheres with a thick steel pipe reflector.

\begin{tabular}{|c|c|c|c|}
\hline \multirow{2}{*}{ Option $^{(\mathbf{1})}$} & $\mathbf{B}_{\mathbf{4}} \mathbf{C}^{(\mathbf{2})}$ & Hydrogenous content $^{(\mathbf{3})}$ & Miscellaneous filler $^{(\mathbf{4})}$ \\
\cline { 2 - 4 } & $\mathbf{( g )}$ & $\mathbf{( g )}$ & $\mathbf{( g )}$ \\
\hline $\mathrm{A}$ & $\geq 10$ & $<3,900$ & - \\
\hline $\mathrm{B}$ & - & $<1,690$ & - \\
\hline $\mathrm{C}$ & - & $<1,880$ & $\geq 2,000$ \\
\hline
\end{tabular}

1. To maintain a subcritical limit of 1.0 for CCOs subject to long-term emplacement at WIPP, waste packaged in each CCO shall adhere to the limits specified under Options A, B, or C.

2. The $\mathrm{B}_{4} \mathrm{C}$ shall be well mixed with the ${ }^{239} \mathrm{Pu}$ FGE and remain so during transportation, storage, and handling operations. The $\mathrm{B}_{4} \mathrm{C}$ mass is based on the natural abundance of ${ }^{10} \mathrm{~B}$ (i.e., 19.9 wt $\%$ ${ }^{10} \mathrm{~B}$ ). The $\mathrm{B}_{4} \mathrm{C}$ mass requirement shall apply to (a) each criticality control container (CCC) that contains directly loaded TRU waste with ${ }^{239} \mathrm{Pu}$ FGE, or (b) any convenience containers used to load a CCC that contain ${ }^{239} \mathrm{Pu}$ FGE. For example, if a CCC is directly loaded with TRU waste containing ${ }^{239} \mathrm{Pu} \mathrm{FGE}$ and also loaded with two cans containing ${ }^{239} \mathrm{Pu}$ FGE, the directly-loaded TRU waste in the CCC and each can in the CCC shall include at least 10-g of well mixed $\mathrm{B}_{4} \mathrm{C}$.

3. Mass of hydrogenous content shall include mass of any organic material (e.g., mass of plastic, cellulose, foam) and mass of water associated with any inorganic material (e.g., mass of adsorbed water on zeolite, water of hydration in concrete and clay, or water in hydrate such as hydrated metal ion).

4. Only the non-hydrogenous portion of the miscellaneous filler mass that is well mixed with the ${ }^{239} \mathrm{Pu}$ FGE mass shall meet the miscellaneous filler mass requirement. The miscellaneous filler shall remain well mixed with ${ }^{239} \mathrm{Pu}$ FGE during transportation, storage, and handling operations. If several convenience containers are used to load a CCC, then each convenience container shall independently meet the miscellaneous filler criteria.

5. The values of mass of hydrogenous content for Option A, B and C, and the mass of miscellaneous filler for Option $\mathrm{C}$, are selected as an example using the spheres with thick steel pipes as reflectors in the nonuniform array models. The value of hydrogenous content for Option A can be seen in Figure 6.58, for Option B it can be seen in Table 6.5 (rounded) and for both the hydrogenous content and miscellaneous filler for Option $\mathrm{C}$ it can be interpolated from the results presented in Table 6.5 or utilizing the raw data (see Addendum 1).

6. FGE should only include fissile isotopes, but the fissile material does not have to be uniformly mixed with the waste form or have a specific geometric size or shape.

7. It is allowable to utilize a separate Option for individual CCOs since each Option is based on the same maximum reactivity subcritical limit. 
This page is intentionally blank 


\section{INTRODUCTION AND PURPOSE}

The Waste Isolation Pilot Plant (WIPP) provides for safe permanent disposal of government-owned transuranic (TRU) and TRU mixed wastes. Receipt and disposal of waste at the WIPP site began in March of 1999. The Sandia Report, Consideration of Nuclear Criticality When Disposing of Transuranic Waste at the Waste Isolation Pilot Plant [1], addresses potential nuclear criticality safety issues based on the projected inventory characteristics known at the time of its 2,000 publication date [1]. As the inventory, waste forms, and disposal package designs have changed, new analyses have been performed to address any potential effects to the performance assessment. More recent evaluations have considered post-closure effects on container spacing. In 2017, Saylor and Scaglione [3] evaluated criticality control overpacks (CCOs) and pipe overpack containers (POCs), and in 2019, Brickner [4] evaluated POCs. The evaluation presented in this document provides additional information for CCOs containing 380 fissile gram equivalent (FGE) ${ }^{239} \mathrm{Pu}$ to provide a generic analysis that was specifically performed to support the use of CCOs for the disposal of materials in addition to dilute surplus Pu in CCOs [6].

The current WAC for CCO waste forms with $380 \mathrm{FGE}{ }^{239} \mathrm{Pu}$ is supported by the 2017 analysis of Saylor and Scaglione [3], which determined that $50 \mathrm{~g} \mathrm{~B}_{4} \mathrm{C}$ per (CCO) was sufficient to preclude criticality. The Saylor and Scaglione [3] analysis assumes that all space between FGE masses is eliminated due to salt creep. Brickner's 2019 analysis [4] made use of geomechanical analysis data for post-closure spacing from Sandia National Laboratories (SNL) as documented by Reedlunn and Bean in 2019 [7]; Brickner developed a methodology for using the data [4] to demonstrate that no restrictions (waste acceptance limits) were required for $200 \mathrm{FGE}{ }^{239} \mathrm{Pu}$ in POCs.

This analysis builds on the post-closure criticality safety methodology developed by Saylor, Scaglione [3], and Brickner [4], using the most recently available (2020) geomechanical data for CCO spacing under salt creep compaction scenarios as provided by Reedlunn and Bean [8]. The main objective of this evaluation is to determine the $k_{\text {eff }}$ of disposed CCOs loaded with $380 \mathrm{FGE}{ }^{239} \mathrm{Pu}$ as a function of moderator content for various parametric combinations of reflector, non-hydrogenous filler, waste form shape and spacing configurations related to room compaction scenarios. The upper edge of the envelope of the curves representing plausible configurations could inform the FEP process for various CCO contents. The results of the analysis provide a full range of $k_{\text {eff }}$ Vs. moderator curves to evaluate the $k_{\text {eff }}$ effect of the most important system parameters for the CCO's allowable payload per the CCO TRUPACTII Safety Analysis Report [9], and sufficient information to demonstrate $k_{\text {eff }}$ associated with disposal of dilute surplus $\mathrm{Pu}$ in CCOs [10].

While the waste stream for the disposal of dilute surplus $\mathrm{Pu}$ in CCOs is not specifically addressed, the results remain applicable because the dilute surplus $\mathrm{Pu}$ is shipped in the $\mathrm{CCO}$ and must meet the $\mathrm{CCO}$ TRUPACT-II Safety Analysis Report [9] limits. To avoid specifying allowable waste forms, the analysis herein examines the potential for criticality in CCOs containing generic waste forms. This approach requires a more general analysis using criticality conditions that are based primarily on CCO transportation limits.

To certify the compliance ${ }^{1}$ of a geologic repository for radioactive waste, the US Environmental Protection Agency (EPA) requires estimates of the range of future behavior through models that capture the disposal system's essential FEPs. At the WIPP, it may be possible for sufficient fissile mass and concentration to cause a self-sustained neutron chain reaction — or criticality. In the past, concern about criticality in TRU waste has been low because of the low fissile mass limits in contact-handled containers, the neutronic isolation associated with the disposal of remote-handled containers, and the natural tendency of fissile solute to disperse after release from degraded containers. This low concern is

\footnotetext{
${ }^{1}$ Much of the discussion in this section is directly from Rechard [11].
} 
still valid for the majority of TRU waste disposed at WIPP. However, waste destined for WIPP has expanded to include TRU waste disposed in CCOs with larger fissile mass limits per transportation cask. Hence, the likelihood of assembling a critical mass and concentration of CCOs in WIPP after its closure is being reevaluated.

The EPA uses a probabilistic approach to evaluation of criticality that has previously been applied to WIPP as part of prior FEP screening [1], [14], [15], [30]. Because EPA does not designate post-closure criticality for special consideration, the post-closure criticality potential is evaluated within the probabilistic regulatory framework for disposal as defined by EPA in 40 CFR 191 [12], [13].

Section 2 discuses quality assurance specifications and describes the process used to develop this report. The software used to perform these calculations is described in Section 3. Direct inputs that were used in the development of this technical product are documented in Section 4. Section 5 describes the assumptions used in the absence of direct confirming data or evidence to perform the modeling and analyses documented herein. A description of the different analyses performed is provided in Section 6, as well as the systems, processes, and phenomena considered to assess criticality potential over the WIPP post-closure period.

This report documents a large, complicated analysis, and thus some key terms, concepts, and assumptions are listed as follows for technical clarification purposes.

\section{Emplacement and Compaction}

The CCOs are placed in the repository at time $=0$ during emplacement. The geometry of the emplaced configuration is technically equivalent to the transport condition with respect to the physical configuration and arrangement of the various CCO components.

The CCOs are at the final resting location after room closure at a time between 0 and 10,000 years after compaction. The geometry of the compacted configuration is variable, and the material composition and arrangement of the various $\mathrm{CCO}$ components is variable.

\section{Waste Form}

The mass/volume of anything placed within the CCC. This does not include the CCC pipe itself at emplacement. However, the waste form always includes the FGE and moderator and may also include the filler, $\mathrm{Be}$, and/or $\mathrm{B}_{4} \mathrm{C}$ at emplacement. It may also include other materials present in the repository after compaction (e.g., packaging materials).

\section{3.}

The mass of fissile isotopes. It does not include any other elements, such as $\mathrm{O}$, which may be chemically bound. Therefore, the calculation of the FGE should only include fissile isotopes. It does not have to be uniformly mixed with the waste form or have a specific geometric shape or size.

\section{Waste Form Moderator}

The H-bearing compounds in the $\mathrm{CCC}$ represented as either pure water or polyethylene $\left(\mathrm{CH}_{2}\right)$. This includes water, polyethylene, the moisture content of inorganic materials (e.g., cement) and materials of unknown organic content (should be conservatively considered to be water or plastic). Organics are typically excluded from the CCO based on other WIPP operational constraints. It is always assumed to be uniformly mixed with the waste form. 


\section{Waste Form Moderator Mass}

Mass of water or polyethylene in the CCC. It always includes the mass of every H-bearing molecule within the CCC and uniformly mixed with the waste form. Therefore, the calculation of the moderator mass at emplacement should include the total mass of all H-bearing molecules within the CCC as the equivalent mass of water or plastic. Only the moisture content of an inorganic materials (e.g., cement) is included as part of the moderator, not the full mass of the materials. Materials of unknown organic content should be represented as plastic in evaluating moderator content. Organics are typically excluded from the CCO based on other WIPP operational constraints.

\section{Filler Material}

Any material placed within the CCC that is non-fissile and inorganic may be considered as filler, Beryllium metal and compounds are excluded as a special reflector material. Filler material is always assumed to be uniformly mixed with the fissile material during transportation, handling and emplacement.

\section{Filler Material Mass}

The total mass of filler material compounds within the CCC that is credited toward a filler mass limit at emplacement. It does not include the mass of H-bearing compounds, FGE, or special reflector materials at emplacement.

For a filler material that contains moisture or releases water vapor during thermal decomposition, the filler mass calculation should exclude the mass loss associated with the water. For example, concrete present in as filler may include $20 \%$ water yet appear dry. This water must be included in the waste form moderator mass calculation. However, the balance of dry cement ingredients associated with concrete (e.g., gravel and sand) may be included as part of the filler mass.

Any additional material that could be characterized as a filler material and that is not being credited toward a filler mass is not required to be uniformly mixed with the waste form.

\section{Be Mass}

The total mass of special reflector material (modeled as $\mathrm{Be}$ ) that is uniformly mixed with the waste form in the CCC at emplacement. It is always assumed to be uniformly mixed with the waste form.

\section{9. $\underline{B}_{4} \mathrm{C}$ Mass}

The total mass of neutron absorber material that is uniformly mixed with the waste form at emplacement. Therefore, the calculation of the waste form neutron absorber material mass must include the neutron absorption cross section equivalent of $\mathrm{B}_{4} \mathrm{C}$. It is always assumed to be small particles (e.g., less than $3.3 \mathrm{~mm}$ diameter or 6 mesh) and uniformly mixed with the waste form.

\section{0. $\underline{\text { Reflector }}$}

In this evaluation, the term interstitial reflector, or discrete reflector, is used to describe materials physically external to the waste form, and the term waste form is used to describe anything placed inside the CCC prior to emplacement. 
This page is intentionally blank 


\section{QUALITY ASSURANCE}

This report was prepared in accordance with Oak Ridge National Laboratory (ORNL) procedures meeting DOE Order 414.1D, Admin Change 1, Quality Assurance. Procedures, policies, and guidelines can be found in the Publications and Other Scientific Communications subject area of the ORNL StandardsBased Management System (SBMS) under the Integrated Performance Management system. 
This page is intentionally blank 


\section{SOFTWARE AND CALCULATIONS}

The calculations for this investigation were predominately performed using the SCALE code system (ORNL 2016 [16]), version 6.2.3. The Criticality Safety Analysis Sequences (CSAS) with KENO-VI (CSAS6) were used to calculate neutron multiplications factors ( $k_{\text {eff }}$ values). The sequences have different geometry packages, but the solutions they provide are statistically identical.

All cases were performed with Evaluated Nuclear Data File (ENDF)/B-VII.1 cross section data in the 252-group library. CENTRM was used to provide problem-dependent multigroup cross section processing on the Remus or Artemis computer cluster. Remus and Artemis are maintained under the configuration control of ORNL's Fusion and Fission Energy and Science Division staff.

All calculations were run with sufficient numbers of neutron histories (generations, neutrons per generation, and generations skipped) to yield converged results that passed the appropriate statistical checks. The plots showing $k_{\text {eff }}$ by generation and $k_{\text {eff }}$ by generation skipped provided in the output files showed that the $k_{\text {eff }}$ eigenvalue was essentially flat for all active generations for all cases. Fission source convergence was verified using the Shannon entropy tests or other means. The results are reported as $k_{e f f}$.

Sampler [see Section 6.7 of the SCALE Manual] is referred to as a super-sequence within SCALE because it wraps around other sequences (e.g., CSAS) and perturbs inputs. Sampler has two modes: (1) uncertainty quantification via random sampling, and (2) parametric analysis via sweeping through parameter space. In this work, the second option was used extensively to explore parameter space for moderator mass, along with many other variables impacting system reactivity. These additional variables include the size and geometry of the waste form, constituents, and spacing. Sampler creates entire SCALE inputs - in this case CSAS6 inputs - with each combination of variable parameters, thus allowing for a single input to generate a large number of SCALE inputs covering the entire desired space. Further discussion of similar analyses using the Sampler parametric capability for nuclear criticality analyses is presented in Marshall et al. [27].

As with any computer code/calculation used for safety analysis and assessment, the ability of the calculation methodology to prove a configuration to be subcritical is realized through a validation process. This safety analysis utilized SCALE with TSNUAMI sequence (see Appendix H) for validation.

The SCALE MAVRIC sequence was used to perform neutron transport calculations. MAVRIC accelerates convergence of Monte Carlo transport calculations using variance reduction techniques. The MAVRIC results were successfully compared to experimental measurements that tested photon and neutron transmission through various shielding materials [32]. Therefore, MAVRIC is appropriate for modeling highly attenuating shielding. SCALE 6.2 development practices follow various quality standards, enabling compliance with American Society of Mechanical Engineers (ASME) Nuclear Quality Assurance (NQA)-1 [33]. 
This page is intentionally blank 


\section{DEVELOPMENT OF ANALYSIS INPUTS AND DATA}

In this section, the physical system being modeled is first considered from a repository-wide perspective, then focusing down to waste form level. The general characteristics of the WIPP CCO configuration are outlined in this subsection, beginning with a large drift-scale description, and narrowing in scale to a description of the waste form itself. Then the data supporting values for parameter sweeps are identified and discussed. Finally, an overview of the layout for the system models is provided.

This work is focused on ${ }^{239} \mathrm{Pu}$ from atomic energy defense activities in general and surplus weapons $\mathrm{Pu}$ in particular, rather than fissile uranium. $\mathrm{Pu}$ was modeled as $\mathrm{PuO}_{2}$ instead of metallic $\mathrm{Pu}$ or $\mathrm{Pu}$ with water of hydration or hydroxyl groups (i.e., $\mathrm{PuO}_{2}(\mathrm{OH})_{2} \cdot \mathrm{H}_{2} \mathrm{O}$ or $\left.\mathrm{Pu}(\mathrm{OH})_{4}\right)$. For highly enriched ${ }^{239} \mathrm{Pu}$, the mineral form only influences criticality limits when the mixture is severely under moderated [11].

The CCO FGE loading is set at the transportation maximum limit of $380 \mathrm{FGE}{ }^{239} \mathrm{Pu}$. During a transportation accident, a CCO is designed to maintain fissile separation, so each CCO in a shipment can be at the maximum $380 \mathrm{FGE}{ }^{239} \mathrm{Pu}$, which is not the case for other TRU waste containers except for the similar POC. Although some TRU waste containers, such as the standard waste box and the 55-, 85-, and 100 -gallon drums, slightly increase the transportation maximum mass limit if the ${ }^{240} \mathrm{Pu}$ mass is specified, the $\mathrm{CCO}$ is fixed at $380 \mathrm{FGE}{ }^{239} \mathrm{Pu}$ in all cases [11].

Other isotopes mixed with ${ }^{239} \mathrm{Pu}$, such as ${ }^{240} \mathrm{Pu}$, have an important influence on criticality. Therefore, the transportation limit is actually set at 380 FGE ${ }^{239} \mathrm{Pu}$ rather than ${ }^{239} \mathrm{Pu}$. The ${ }^{239} \mathrm{Pu}$ FGE is the mass of ${ }^{239} \mathrm{Pu}$ plus a factor for other fissionable masses: specifically, $0.113 \cdot{ }^{238} \mathrm{Pu}, 0.0225 \cdot{ }^{240} \mathrm{Pu}, 2.25 \cdot{ }^{241} \mathrm{Pu}, 0.0075 \cdot{ }^{242} \mathrm{Pu}$, $0.9 \cdot{ }^{233} \mathrm{U}, 0.643 \cdot{ }^{235} \mathrm{U}, 0.015 \cdot{ }^{237} \mathrm{~Np}, 0.0187 \cdot{ }^{241} \mathrm{Am}, 34.6 \cdot{ }^{242 \mathrm{~m}} \mathrm{Am}, 0.0129 \cdot{ }^{243} \mathrm{Am}, 15 \cdot{ }^{245} \mathrm{Cm}, 0.5 \cdot{ }^{247} \mathrm{Cm}$, $45 \cdot{ }^{245} \mathrm{Cf}$, and $90 \cdot{ }^{251} \mathrm{Cf}[11]$. In general, the contact-handled TRU waste disposed at WIPP is typically only $90 \%$ enriched in ${ }^{239} \mathrm{Pu}$, and remote-handled TRU waste is typically only $78 \%$ enriched. However, some of the surplus weapons Pu may have higher enrichment (>95\%). In this work the waste form is modeled as $100 \%{ }^{239} \mathrm{Pu}$ to accommodate this possibility. This assumption adds conservatism, but it avoids requiring that the waste form composition be defined precisely for the criticality analysis [10].

\subsection{QUALITATIVE CHARACTERISTICS OF THE WIPP CCO CONFIGURATION}

Determination of whether a fissile region or an assembly of fissile regions is critical depends upon the fissile region neutron generation rate and interaction of neutrons with matter within and outside the fissile regions. For a finite heterogenous system ${ }^{2}$, this depends upon the individual package configuration and its contents:

(1) The type of fissile material,

(2) The fissile mass,

(3) Mass of other materials intermingled in the fissile material region,

(4) Mass of materials in the non-fissile regions,

(5) The shape and distribution of the materials in the fissile and non-fissile regions

\footnotetext{
${ }^{2}$ The WIPP system is considered a heterogenous system of packages. The neutron generation rate of a homogenous system depends on the fissile material concentration.
} 
and the array of packages with the facility and distribution and concentration of materials within the array:

(1) Mass of materials between, above, and below individual packages

(2) The overall distribution and concentration of the materials, and

(3) The location and contents of other packages disposed within the same drift at WIPP (not covered in this analysis)

Because of the robust capability of geologic disposal in general and the WIPP salt repository in particular, characteristics of waste packages important to criticality are primarily based on technical constraints for transportation as specified in the WAC [5]. That is, the transportation mass limits in the CCO TRUPACTII Safety Analysis Report [9] on waste packages set the primary boundary conditions for modeling potential criticality at WIPP: if the waste can be shipped, it can be disposed if the social-political agreements in the WIPP Land Withdrawal Act are met [21]).

\subsubsection{General Description of WIPP Drift Configuration}

The WIPP underground disposal repository consists of multiple salt panels mined from the Salado formation, a series of salt beds that are 2,000 feet thick. A typical underground panel includes several rooms, each of which is approximately 33 feet wide by 13 feet high by 300 feet long [11].

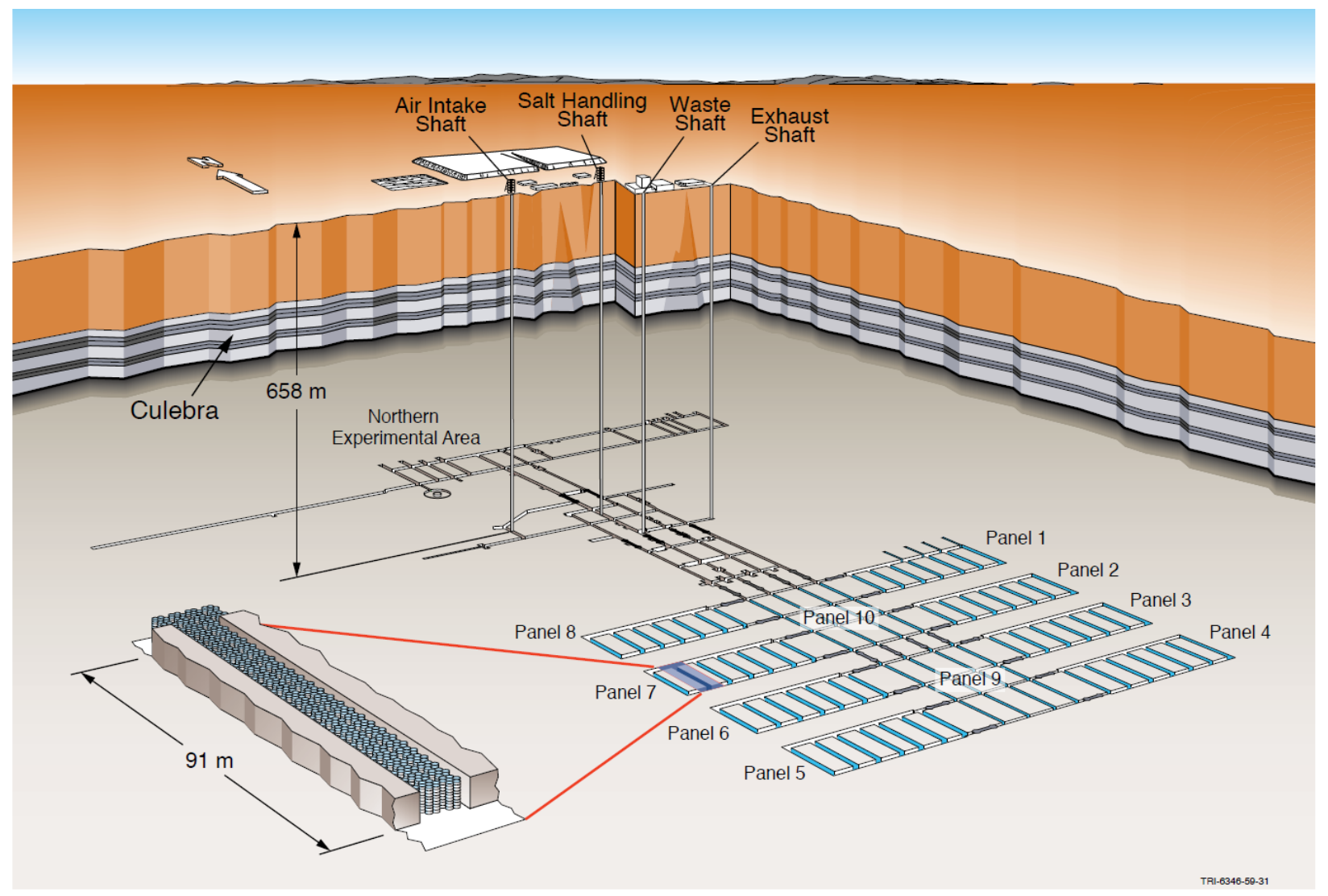

Figure 4.1. Schematic of WIPP repository, used with permission from Rechard [11]. 
The area shown in Figure 4.1 represents the boundaries for both the geomechanical and criticality analysis models. Panels cross-connect via drifts, as shown in Figure 4.2. The cross-connect drifts are not included in either the geomechanical analysis or the criticality analysis because it is assumed that they do not change the results. For the criticality analysis, the array models neglect the cross-connects on either end because the area of interest is in the center, where compaction is greatest, and where neutrons have the lowest chance of escaping in the horizontal direction due to the large number CCOs in that direction. Furthermore, since the major compaction effects are oriented vertically and horizontally along the shortest horizontal dimension of the room, the reactivity effect of the cross-connects is insignificant to the effect in the center of the room. However, future work could investigate the effect using results from alternative compaction models which include the cross-connects when those models are available.

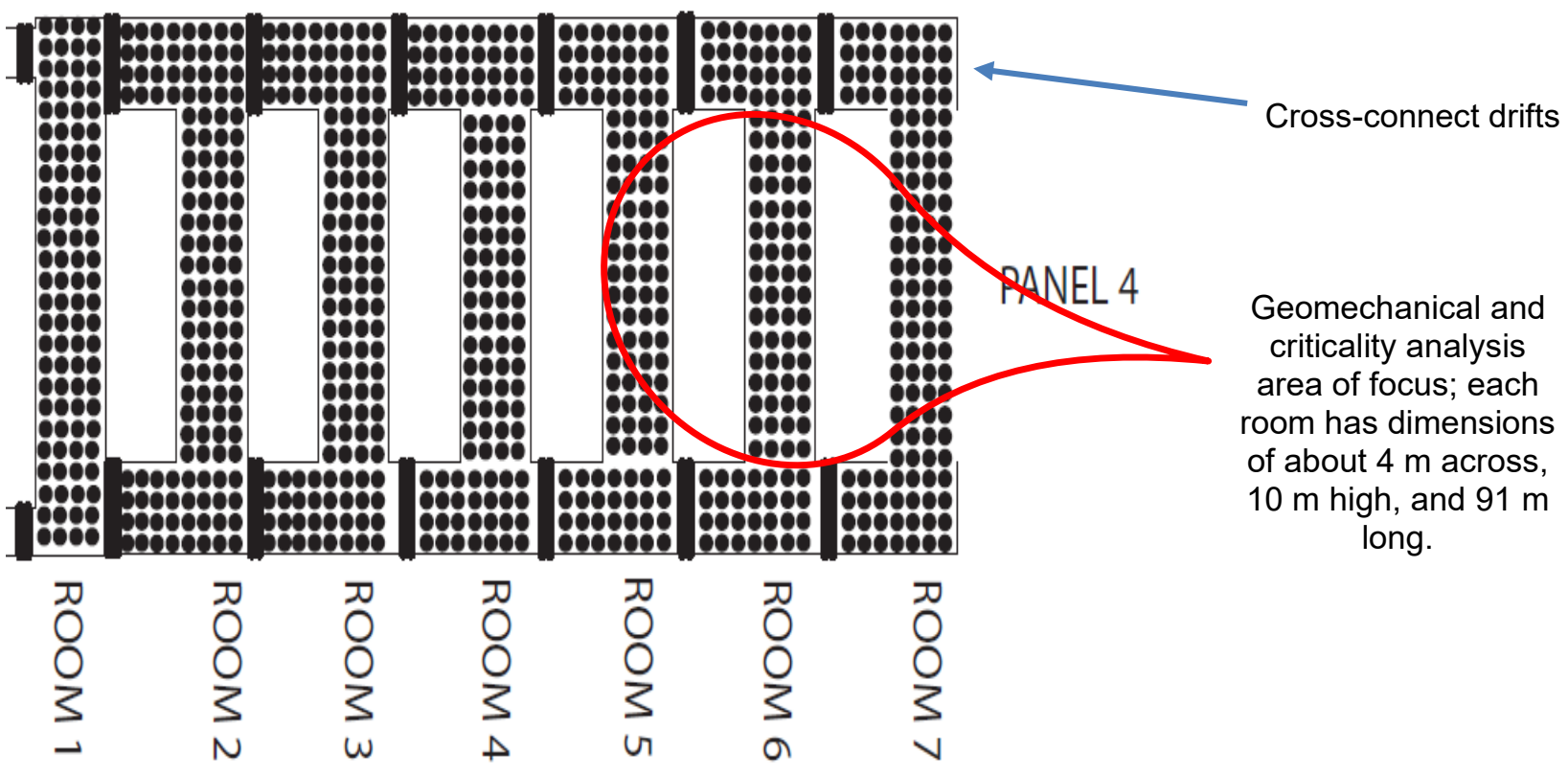

Figure 4.2. Diagram showing cross-drifts, used with permission [18].

The emplaced configuration of the waste form consists of 7-packs of COCs stacked no more than three high in a closely packed hexagonal array. $\mathrm{MgO}$ Supersacks are placed on top of the stacks of waste containers for approximately every other stack. A representative photograph showing how a room is loaded at WIPP is provided in Figure 4.3. 


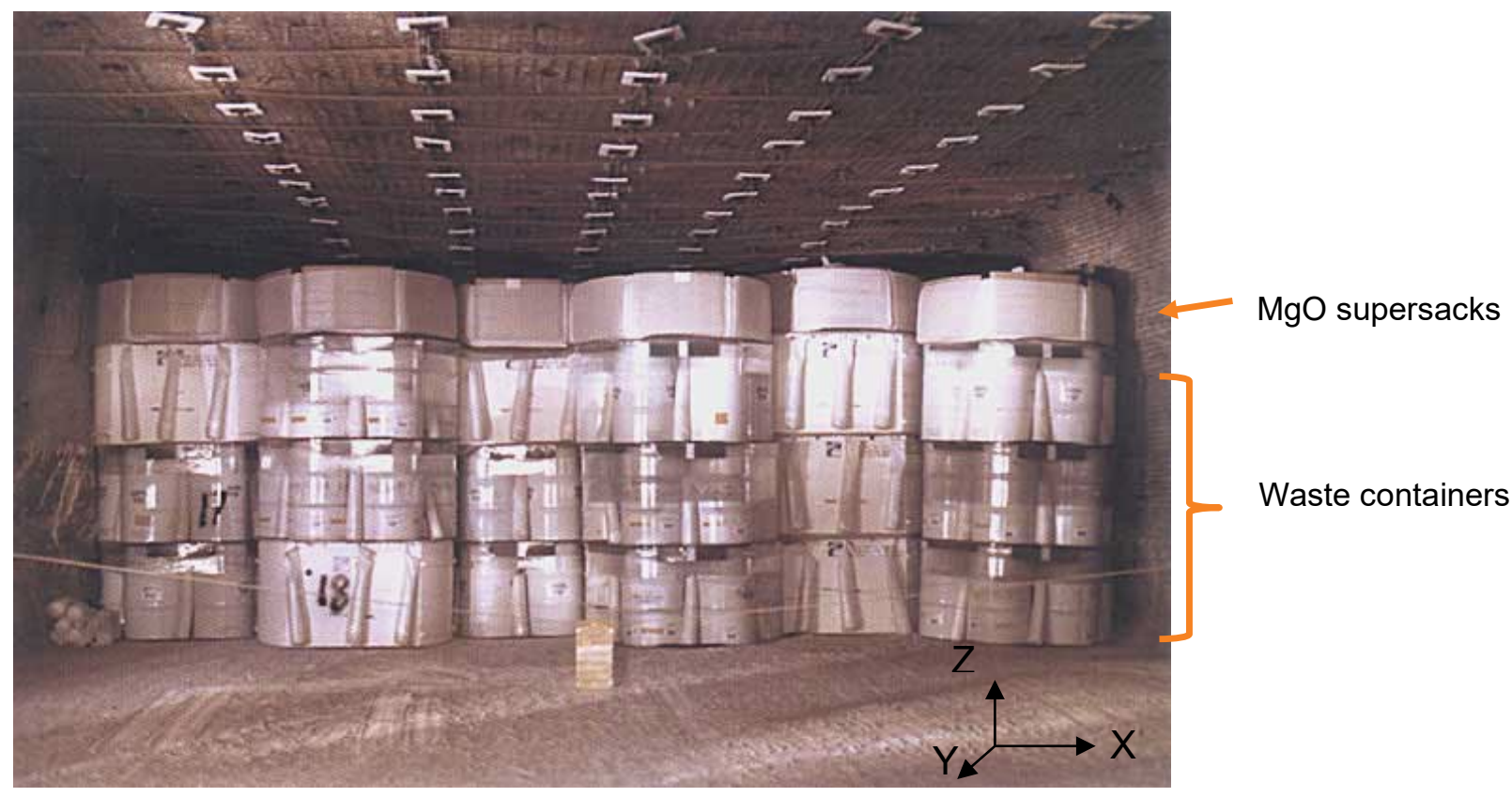

Figure 4.3. Photograph of WIPP room loaded with waste containers, used with permission from Saylor and Scaglione [3].

The WIPP panel rooms were modeled as single-room models surrounded by an infinite salt reflector $[3,4]$. The three-high hexagonal stacks of containers with MgO Supersacks have been modeled within the single-room model as individual containers in a conservatively tight fitting triangular-pitched array ${ }^{3}$ filling the room space (approximately 7,000 containers), with $\mathrm{MgO}$ either as a thick reflector above the array [3] or intermixed uniformly with the salt between the packages [4].

\subsubsection{Overview of CCO Configuration}

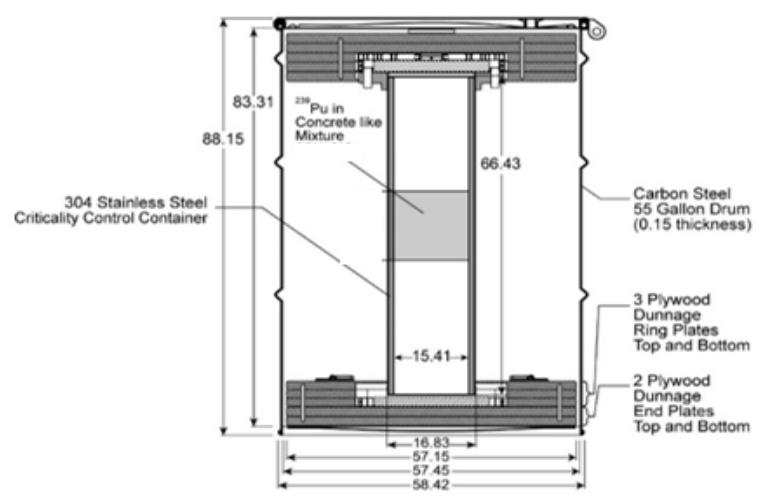

Figure 4.4. Criticality control container (CCC), and criticality control overpack (CCO), used with permission from Rechard [11].

\footnotetext{
${ }^{3}$ Triangular-pitched arrays are more closely packed than hexagonally-pitched arrays and are therefore conservative because the fissile masses are closer together.
} 
Based on the CCC information presented in Figure 4.4, the thickness of the CCC pipe wall is modeled as $0.7112 \mathrm{~cm}$, which is a measurement used specifically in the models as a discrete reflector. Reflector geometry is discussed in more detail in Section 4.2.2. Previous analyses by Saylor, Scaglione [3], and Brickner [4] evaluated the impact of the plywood (as cellulose) and other container material remnants. The results of those analyses show that the reactivity effect of interstitial mixing of the CCO materials between the waste forms with the salt and $\mathrm{MgO}$ is inconsequential. Therefore, the compaction of plywood around the waste forms is better evaluated by using the discrete reflector models in this analysis. ${ }^{4}$

The material composition of the waste form is based on a combination of the TRUPACT-II safety analysis report (SAR) limits [9], which is the approach used by Saylor and Scaglione [3] to evaluate the surplus Pu waste form. Waste form materials and geometry are discussed in more detail in Sections 4.1.3 and 4.2.3.

The WIPP repository may be subject to various types of geologic, environmental, and material degradation, as well as biological transmutation processes, over the regulatory timeframe. Because the final amount and configuration of any emplaced materials cannot be known, care is taken to avoid an approach which attempts to credit any specific amount of material either as a waste form or as reflector. Instead, known compositions and quantities at emplacement are used to establish the most likely material compositions and configurations post-emplacement. This information is then used to evaluate system $k_{\text {eff }}$ under various scenarios, and a conservative and bounding approach is then taken with materials and geometry to account for the unknown nature of the repository over 10,000 years.

In this evaluation, the term interstitial reflector, or discrete reflector, is used to describe compositions physically external to the waste form, and the term waste form is used to describe anything placed inside the CCC prior to emplacement.

During the initial post-closure timeframe, the waste form will retain its original geometry. For example, the surplus $\mathrm{Pu}$ waste form is placed within the CCC in what is termed a can-bag-can configuration [20] (Figure 4.5).

\footnotetext{
${ }^{4}$ Specifically the polyethylene discrete reflector is a surrogate and bounding for materials like cellulose.
} 


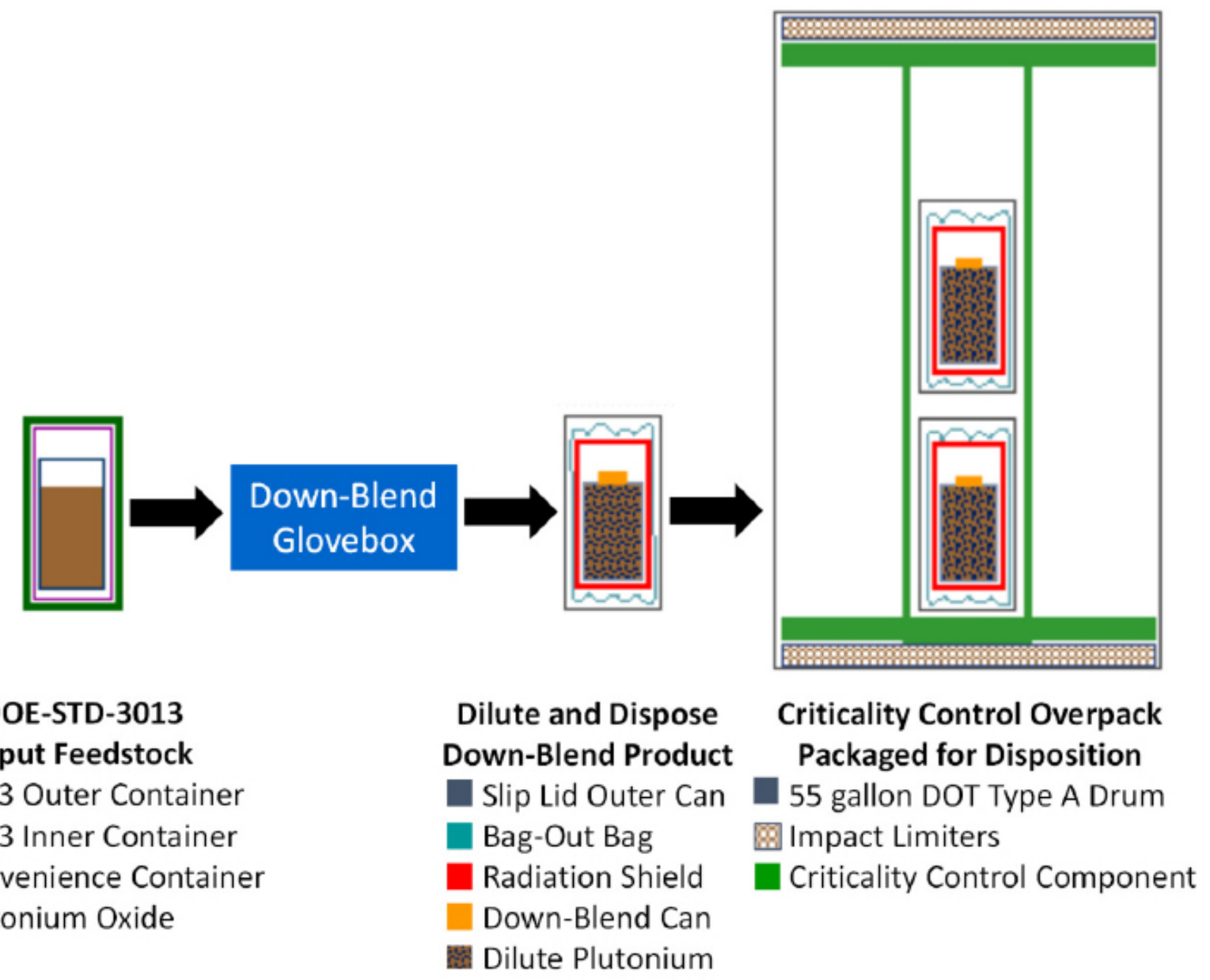

Note: Illustration is not to scale.

Figure 4.5. Diagram of the surplus Pu can-bag-can process, used with permission [21].

The can-bag-can configuration illustrated in the center of Figure 4-5 contains the waste form within a single can, which is wrapped in plastic and then placed in another can. The entire configuration is then placed in a CCC, and sometimes these are stacked two high (right image in Figure 4-5). The slip lid outer can and down-blend cans are referred to in this work as convenience cans and are modeled as a uniformly mixed mass (Section 4.2.3). At emplacement, this configuration illustrates how the waste form may be surrounded by layers of material that can act as a discrete reflector. Additionally, in the two-per-CCC configuration, the materials between the waste forms may act to thermalize neutrons as they pass from one can, through these materials, and into the adjacent waste form. This moderation by interstitial material could increase total system $k_{\text {eff }}$ when compared to a case in which an interstitial material has reduced moderating power.

The materials physically external to the waste form inside the inner can may compact around the waste form during salt intrusion and room compaction. During the later stages of the repository timeframe, the materials which were once discrete external reflectors could degrade and migrate within the waste form, mixing with the waste form and becoming some unknown configuration. Therefore, variations of known (steel from the pipe or can) and conservative (graphite) materials are used as filler in the KENO cases constituting this study. 
The scenarios requiring evaluation involve (1) the steel from the CCC and from the convenience can that is used to directly hold the waste forms and (2) how that material impacts $k_{\text {eff }}$ either as a discrete reflector (initial emplacement), as a material mixed within the waste form (long timeframes), or both together (intermediate timeframes). Likewise, other scenarios to be considered involve (1) the polyethylene from the convenience can's bag-out bag or from other external sources such as other package designs, and (2) how that polyethylene material impacts $k_{\text {eff }}$ either as a discrete reflector (initial emplacement), as a material mixed within the waste form (long timeframes), or both together (intermediate timeframes). Furthermore, these two material types being considered should also be considered coincidently. The masses of the stainless steel from the CCC or convenience can types of containers and polyethylene considered in this calculation sufficiently bound any realistic amount anticipated to be present.

For the initial post-emplacement timeframe, the full CCC wall thickness of stainless-steel material surrounding the waste form, as well as the waste form containers themselves, are known to be present. The known thickness of the CCC is sufficient to determine the effect that a thick discrete reflector has on $k_{\text {eff. }}$ Like the surplus Pu can-bag-can configuration, under compaction, the wall thicknesses of the CCC and waste form containers are likely to be reduced by the pressures of salt intrusion during room closure. Therefore, the discrete reflector's thickness is alternatively set to be very thin so that the $k_{\text {eff }}$ trend associated with the thin discrete reflector thickness can be compared to a thick discrete reflector with a thickness of $0.7112^{5} \mathrm{~cm}$.

\subsubsection{Waste Form Geometry and Composition Representative of the CCO in the WIPP Drift}

As a generic analysis, the evaluation considers parametric variations on both waste form geometry (i.e., cylinders and spheres) and materials (i.e., amounts and compositions). The inclusion of both cylinders and spheres provides additional information for the FEP screening process that can be used to determine the potential $k_{\text {eff }}$ of the system as a function of time, especially for waste forms where either geometry is especially appropriate. This is particularly applicable for the CCC because it is cylindrical in shape and is constructed from steel. The CCC is not expected to change its shape for some significant period of time after disposal and it may not decompose at all unless the system chemistry changes significantly. If it does decompose, a sphere may be more appropriate due to the uncertainty associated with the shape over the passage of time. Under flooding and post-flooding (dryout) conditions, the CCC geometry may no longer be cohesive or contiguous, and the material composition may have changed as a result of oxidation or other processes. The introduction of brine interstitially into the system dramatically reduces reactivity [4]. Migration of materials subsequent to dryout and during that process all but preclude any ideal spherical shape.

\subsection{SELECTION OF PARAMETERS TO CHARACTERIZE CCO SYSTEMS AT WIPP}

The methodology contains sets of evaluations that include (1) cases with masses of pure water or polyethylene uniformly mixed with the waste form in part to account for long timeframes, (2) pure stainless steel homogeneously mixed with the waste form from the CCC or waste form containers like the surplus Pu can-bag-can material to account for very long timeframes, and (3) combinations of those two to represent intermediate timeframes. Polyethylene as a discrete reflector is treated in the same manner, so it is important to evaluate the reactivity effect of the bag in the surplus Pu type of can-bag-can scenario, as well as other external sources of plastics which may migrate around the waste forms.

Approaching the evaluation in this manner also allows for evaluation of waste form containers like the surplus Pu can-bag-can configuration in its emplaced geometry, with the can walls considered discretely, and with those materials uniformly mixed with the waste form or neglected. This is an important

\footnotetext{
${ }^{5}$ Additional thicker discrete reflector thicknesses are evaluated in Appendix O.
} 
component of the evaluation, because the initial geometry of waste forms like the surplus Pu can-bag-can configurations are known to be cylindrical in geometry, and the radius of the cylindrical geometry waste form has a significant effect on $k_{\text {eff. }}$ Therefore, the acceptability of the waste form could be determined by the geometry using, for example, the inner radius of the inner can in the surplus Pu can-bag-can configuration, to define the waste form radius. The results from such an analysis could be used to demonstrate an acceptable FEP screening for the early repository timeframes. In this scenario, any material associated with the can-bag-can from the surplus Pu can-bag-configuration may be used or neglected as uniformly mixed with the waste form, or it could be evaluated as part of the discrete reflector. Specifically, the sets of results representing the desired configuration are used. Flexibility is therefore also allowed in the calculations with the CCC pipe wall thickness as a discrete reflector to be considered as part of the waste form if that is deemed appropriate for waste form processing.

Additionally, providing such a range of results and possible ways to interpret those results allows for alternative waste form situations to be evaluated as acceptable. For example, Saylor and Scaglione [3] provide calculations to show the acceptability of having 1 in $7 \mathrm{CCOs}$ with no $\mathrm{B}_{4} \mathrm{C}$. An alternative approach using the results in this report would be to show that due to the unique geometry and materials inherent in the surplus $\mathrm{Pu}$ waste form, no $\mathrm{B}_{4} \mathrm{C}$ is required to be subcritical. While some requirement for $\mathrm{B}_{4} \mathrm{C}$ in the surplus $\mathrm{Pu}$ waste form may exist [5] for various reasons, situations in which CCOs are not required to utilize $\mathrm{B}_{4} \mathrm{C}$ could be evaluated as acceptable based on the results herein.

The WIPP Project uses administrative controls for (1) safely moving TRU waste within the WIPP facility, (2) positioning TRU waste containers in the disposal room, (3) placing the $\mathrm{MgO}$ engineered barrier, and (4) recording the emplacement location of TRU containers for auditing and potential retrieval. The administrative controls on positioning TRU packages relate to the stability of waste package types when they are stacked on top of each other to promote operational safety. For example, 4-packs of 85-gallon drums can only be stacked on top of each other or placed on the top tiers of other container stacks. The proximity of various types of packages to each other is not specified (e.g., any number of CCOs can be placed next to each other in a room). Placing administrative controls on CCO proximity could complicate CCO disposal if a large campaign of CCOs must be stored while waiting for other waste streams to be shipped in order to mix them within a disposal room. Therefore, the criticality analysis evaluates the possibility of a room filled entirely with CCOs during the post-closure period [11].

\subsubsection{CCO Spacing and Centroid Location Data}

Location of the CCOs in the post-closure configuration is a key factor when evaluating post-closure criticality. Table 4.1 shows a summary of the array spacings evaluated. Set- 1 and set- 3 are assumed to be uniform array cases. Set-1 has one waste form per CCO. Because three CCOs are stacked, this results in three waste forms stacked on top of each other with no spacing aside from the discrete reflectors. Set-3 evaluates a variant configuration having two waste forms per CCO. This results in set-3 consisting of six waste forms stacked on top of each other with no spacing aside from discrete reflectors. Set-2 is a nonuniform case using best-estimate data for locations of waste forms after 1,000 years from Reedlunn and Bean [8], so the waste forms are typically not in direct contact. 
Table 4.1. Evaluated array spacings

\begin{tabular}{|c|c|c|}
\hline Case & Description & Location of results \\
\hline set-1 & $\begin{array}{l}\text { Uniform array with three-high spheres and } \\
\text { cylinders, with the most conservative spacing based } \\
\text { on the SNL compaction data. Waste forms are } \\
\text { stacked directly on top of each other. }\end{array}$ & $\begin{array}{l}\text { Appendix A } \\
\text { Addendum } 1\end{array}$ \\
\hline set- $1 \mathrm{a}$ & $\begin{array}{l}\text { Uniform array with three-high spheres and } \\
\text { cylinders, with an alternative spacing based on the } \\
\text { SNL compaction data. Waste forms are stacked } \\
\text { directly on top of each other. }\end{array}$ & $\begin{array}{l}\text { Appendix B } \\
\text { Addendum } 1\end{array}$ \\
\hline set-2 & $\begin{array}{l}\text { Nonuniform array with spacing based on SNL } \\
\text { compaction data. Two sets are included: one set for } \\
\text { the upper horizon (uh), and one set for the lower } \\
\text { horizon (lh). }\end{array}$ & $\begin{array}{l}\text { Appendix C } \\
\text { Addendum } 1 \\
\text { Appendix D } \\
\text { Addendum } 1\end{array}$ \\
\hline set-3 & $\begin{array}{l}\text { Uniform array with six-high spheres and cylinders, } \\
\text { with the most conservative spacing based on the } \\
\text { SNL compaction data. FGE limited to } 180 \text { per } \\
\text { waste form. Waste forms are stacked directly on top } \\
\text { of each other. }\end{array}$ & $\begin{array}{l}\text { Appendix F } \\
\text { Addendum } 1\end{array}$ \\
\hline set-3a & $\begin{array}{l}\text { Uniform array with six-high spheres and cylinders, } \\
\text { with an alternative spacing based on the SNL } \\
\text { compaction data. FGE limited to } 180 \text { per waste } \\
\text { form. Waste forms are stacked directly on top of } \\
\text { each other. }\end{array}$ & $\begin{array}{l}\text { Appendix I } \\
\text { Addendum } 1\end{array}$ \\
\hline
\end{tabular}

\subsubsection{Uniform arrays}

The parametric evaluations based on the uniform arrays use a horizontal, center-to-center spacing which conservatively bounds the reduction of the horizontal pitch based on the greatest overall room closure for the upper and lower horizons. The use of tightly packed, triangular pitched arrays filling a large single room is expected to be modeled on a scale large enough to preclude some other combination of room and cross-drift configuration being more reactive. For both uniform and nonuniform arrays, the portion of the model external to the panel is an infinite salt reflector that mimics the WIPP repository geology.

Two sets of cases are evaluated with the three-high model: set-1 and set-1a. The purpose of set-1a is to evaluate the reactivity effect of the spacing. The only difference between these two sets is the distance between waste forms in the x-direction (as shown in Figure 4.3), where set-1a has an increase in spacing. The SNL data used to determine the spacing for both sets are presented in Table 4.3 below. 

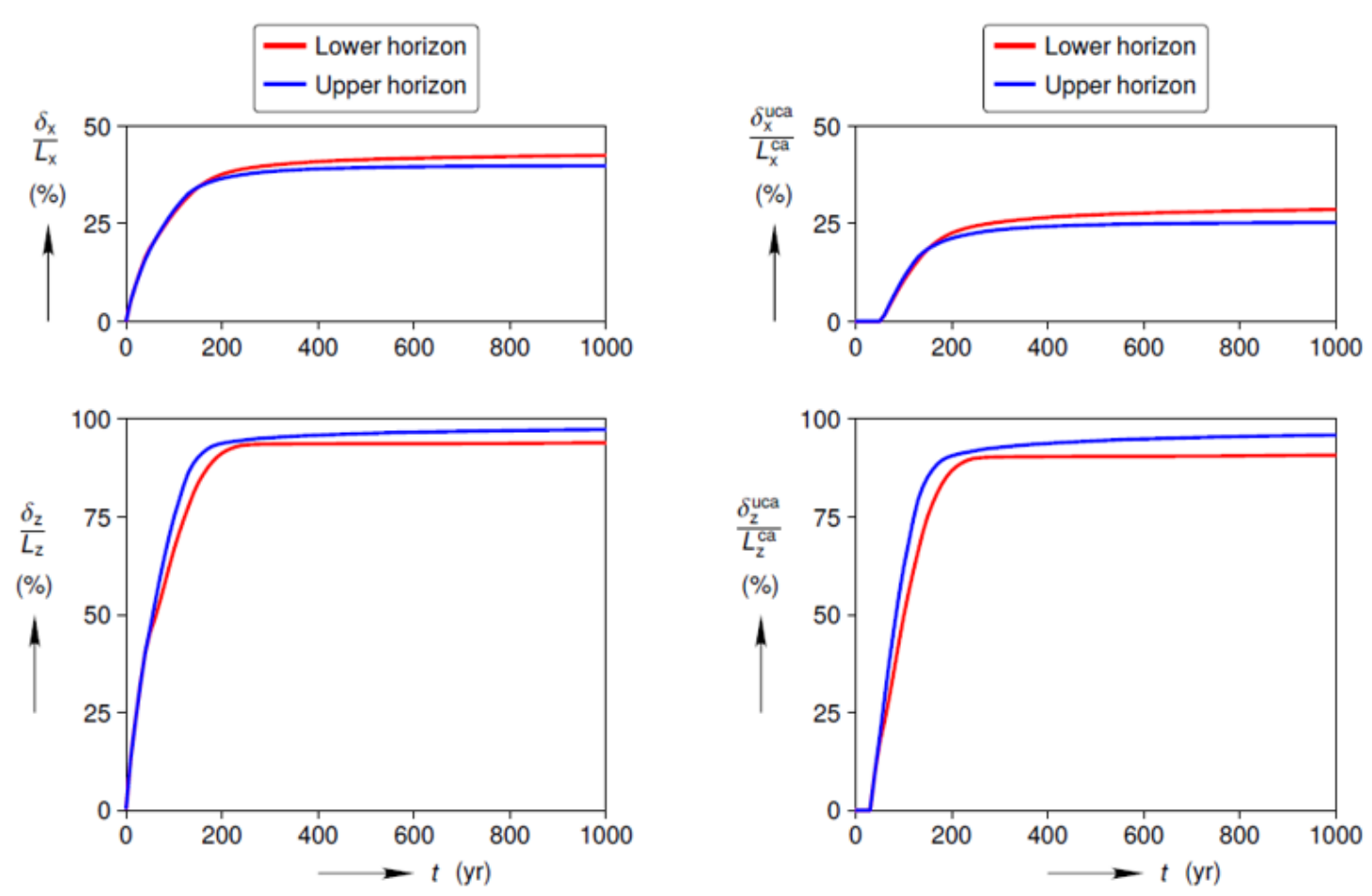

(a) Room closure

(b) Uniform container array compaction

Figure 4.6. Representation of the SNL compaction data used with permission from Reedlunn [25].

The data presented in Figure 4.6 above show that there are two ways to view the compaction of the room with CCOs where the top diagram represents the $\mathrm{x}$ direction closure and the bottom diagram represents the $\mathrm{z}$ direction closure. The first factor assumes that the compaction of the array is proportional to the overall room closure (see also Figure 4.7 and Table 4.2), as opposed to the compaction of the uniform array of containers only. Sets 1 and 3 assume that the $\mathrm{x}$-direction compaction is proportional to room closure, which provides a high estimate of the level of compaction. The second approach (an assumption used for the $x$ direction in Sets 1a and 3a) does not penalize the value with the gaps in the room where the ingress of the salt simply fills in the space that is not used during emplacement. The room closure values used in set-1 serve as a value for spacing, which conservatively bounds the SNL data for both upper horizon and lower horizon for overall room closure (i.e., including the closure of the empty space). The other approach is to consider how the containers themselves are compacted. In other words: the approach of sets 1a and $3 \mathrm{a}$ assume that for the $\mathrm{x}$ direction, only space is removed within the confines of the containers during compaction. Note that for both methods, the models are constructed such that each waste form is stacked on top of the one below with zero space at the point where they touch in the zdirection, and although there is little to no compaction in the y-direction, a value of $10 \%$ compaction is used. A summary of these approaches for using the SNL data is shown in Table 4.2 below. 

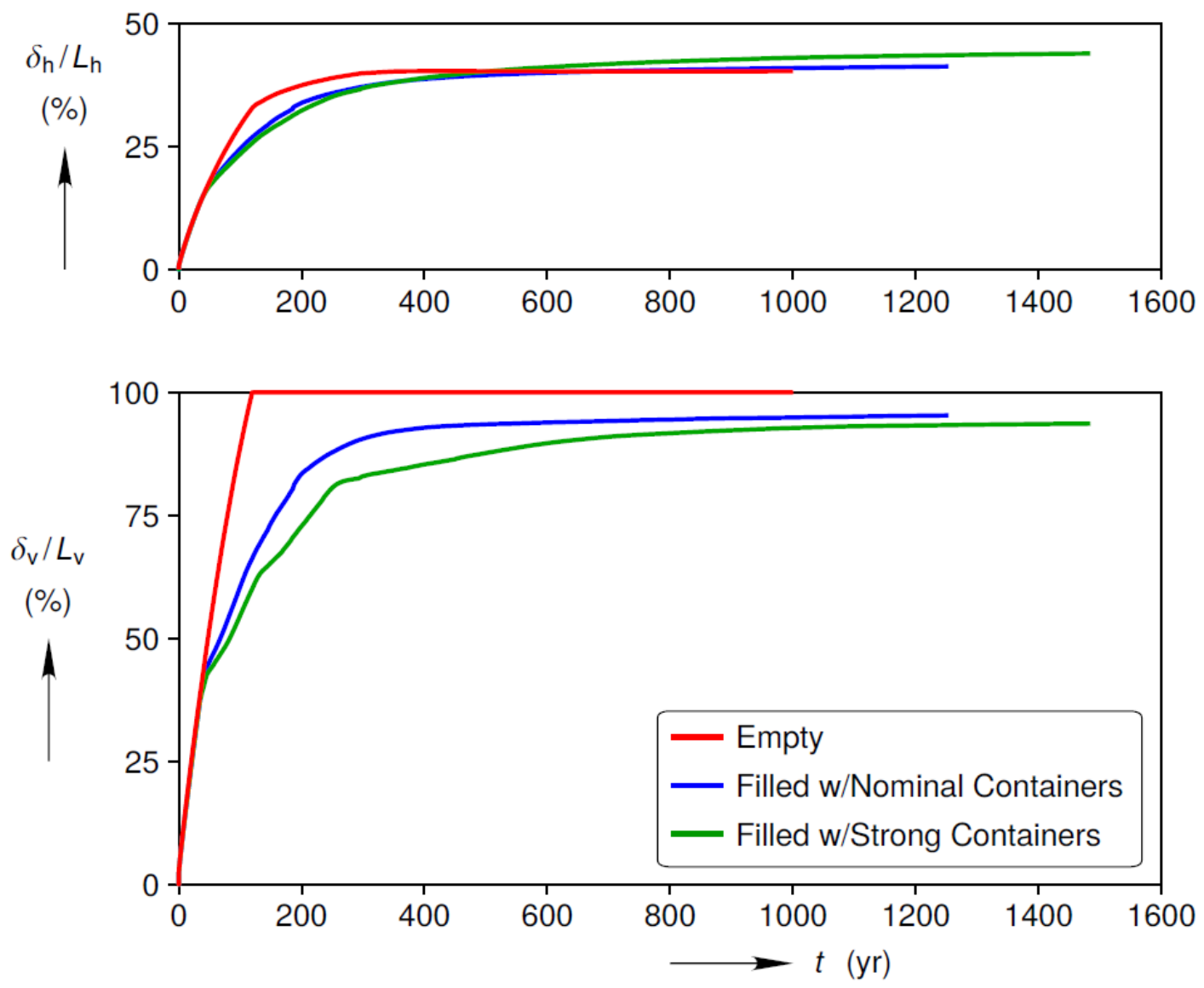

Figure 4.7. Illustration of the room closure in the vertical and horizonal directions, used with permission [8].

Table 4.2. Comparison of the SNL closure data used for uniform arrays

\begin{tabular}{|c|c|c|c|c|}
\hline $\begin{array}{c}\text { Horizon } \\
\text { (at 1,000 years) }\end{array}$ & $\begin{array}{c}\text { Horizontal } \\
\text { room closure } \\
\mathbf{( \% )}\end{array}$ & $\begin{array}{c}\text { Vertical room } \\
\text { closure (\%) }\end{array}$ & $\begin{array}{c}\text { Horizontal } \\
\text { uniform } \\
\text { container array } \\
\text { compaction (\%) }\end{array}$ & $\begin{array}{c}\text { Vertical uniform } \\
\text { container array } \\
\text { compaction (\%) }\end{array}$ \\
\hline Upper & 39.77 & 97.32 & 25.25 & 95.94 \\
\hline Lower & 42.45 & 93.94 & 28.58 & 90.81 \\
\hline $\begin{array}{c}\text { Empty room } \\
\text { (maximum horizon) }\end{array}$ & $44.5 \%$ & - & - & - \\
\hline
\end{tabular}

The SNL data were used to select values for x-direction pitch reduction. These values provide the level of conservatism desired in the results. For set-1, the purpose of the case is to bound all analysis results in the range of interest. For a best-estimate approach, the set-1a value is used as shown in Table 4.3 below. These pitch reduction factors are used to reduce the assumed triangular pitch spacing from the CCO's $57.45 \mathrm{~cm}$ diameter shown in Figure 4.4. 
Table 4.3. Horizonal closure percentage used for $x$-direction spacing reductions

\begin{tabular}{|c|c|c|}
\hline Case & $\begin{array}{c}\text { Horizonal pitch } \\
\text { reduction \% }\end{array}$ & Basis for closure percentage \\
\hline set-1 & 50 & $\begin{array}{c}\text { Spacing reductions that include empty } \\
\text { room space closing }\end{array}$ \\
\hline set-1a & 25 & $\begin{array}{c}\text { Spacing reductions that include CCO } \\
\text { compaction only }\end{array}$ \\
\hline set-3 & 50 & $\begin{array}{c}\text { Spacing reductions that include empty } \\
\text { room space closing }\end{array}$ \\
\hline set-3a & 25 & reductions that include CCO \\
\hline
\end{tabular}

\subsubsection{Nonuniform arrays}

The parametric evaluations with nonuniform arrays use the CCO centroid locations data from Reedlunn and Bean [8] for the upper and lower horizons. The difference between the two horizons is illustrated in Figure 4.8. Panels mined on the lower horizon have different stratigraphy from those carved on the upper horizon, so room compaction may proceed differently. The methodology to use geomechanical postclosure data was developed by Brickner [4]. This previous analysis evaluating POCs used earlier results from Reedlunn and Bean [7]. The parametric evaluation of the CCOs documented herein uses the most recent simulations from Reedlunn and Bean [8], in which CCO post-compaction behavior data are used to evaluate the lower and upper horizon scenarios.

The Reedlunn and Bean [8] analysis used the CCO center-to-center pitch information (outer diameter in Figure 4.4) as an initial condition in two ways: (1) as a uniform orthogonal reduction in the pitch between containers for the uniform arrays, and (2), as the location of container centroids post compaction in nonuniform arrays. Model spacing is discussed in more detail in Section 4.1. 


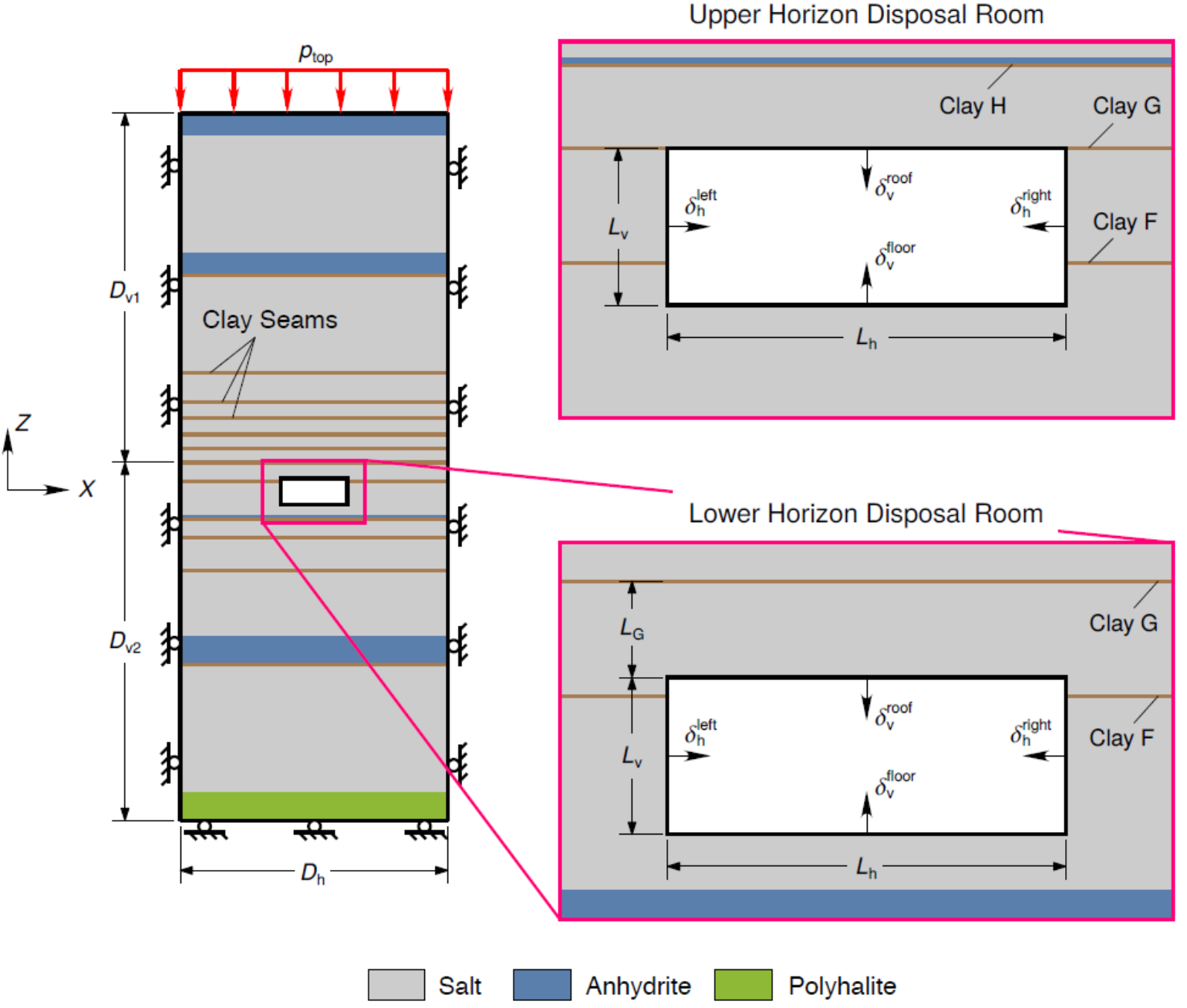

Figure 4.8. Illustration of the lower and upper horizons at WIPP used with permission from Reedlunn and Bean [8].

Although the post-closure compaction behavior between the lower and upper horizons is similar [8], the centroid compaction data from Reedlunn and Bean [8] is used in two separate sets of nonuniform array models to evaluate the potential impact on criticality because the data are available, and it allows for comparison of the ways that geologic variations may impact $k_{\text {eff. }}$.

Figure 4.9 below provides an illustration of the nonuniform array compaction simulation data from Reedlunn and Bean [8], and Table 4.4 provides the centroid-specific locations derived by SNL using a weighted center of mass average. The 153 CCOs occupy a space much smaller than a full panel, so the front and back have reflective boundary conditions to approximate the room as being infinitely long. Thus, neutrons leaving the boundary are re-introduced, resulting in the front and back dimension appearing infinite in extent, thus bounding the length of the full panel. 

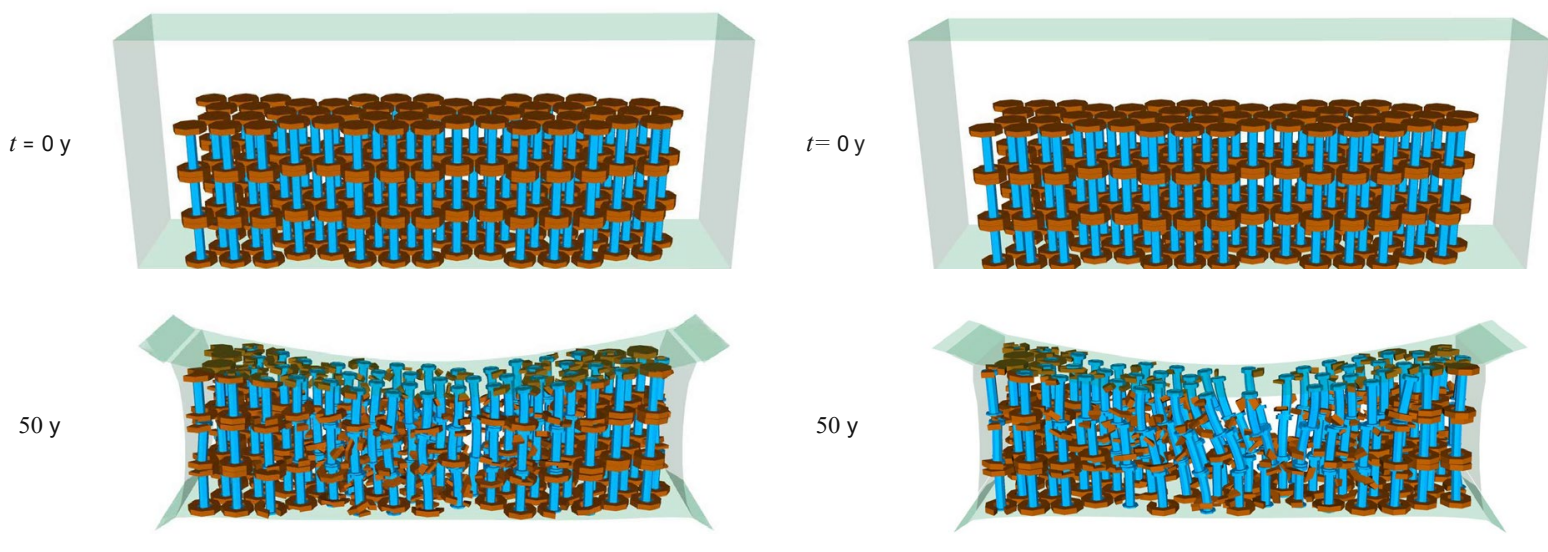

$100 \mathrm{y}$
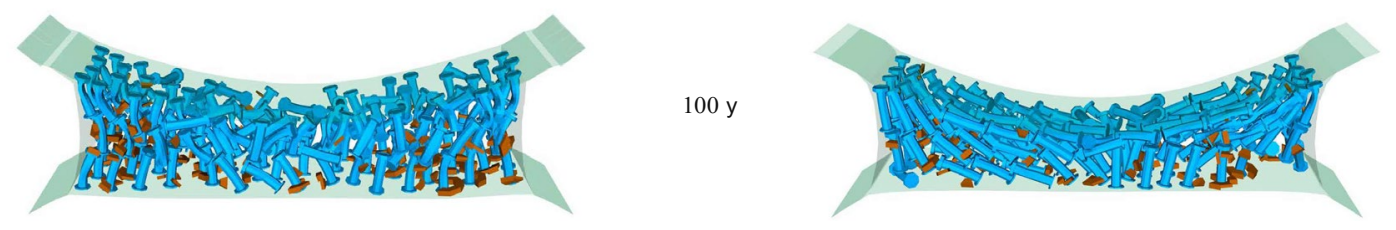

$200 y$
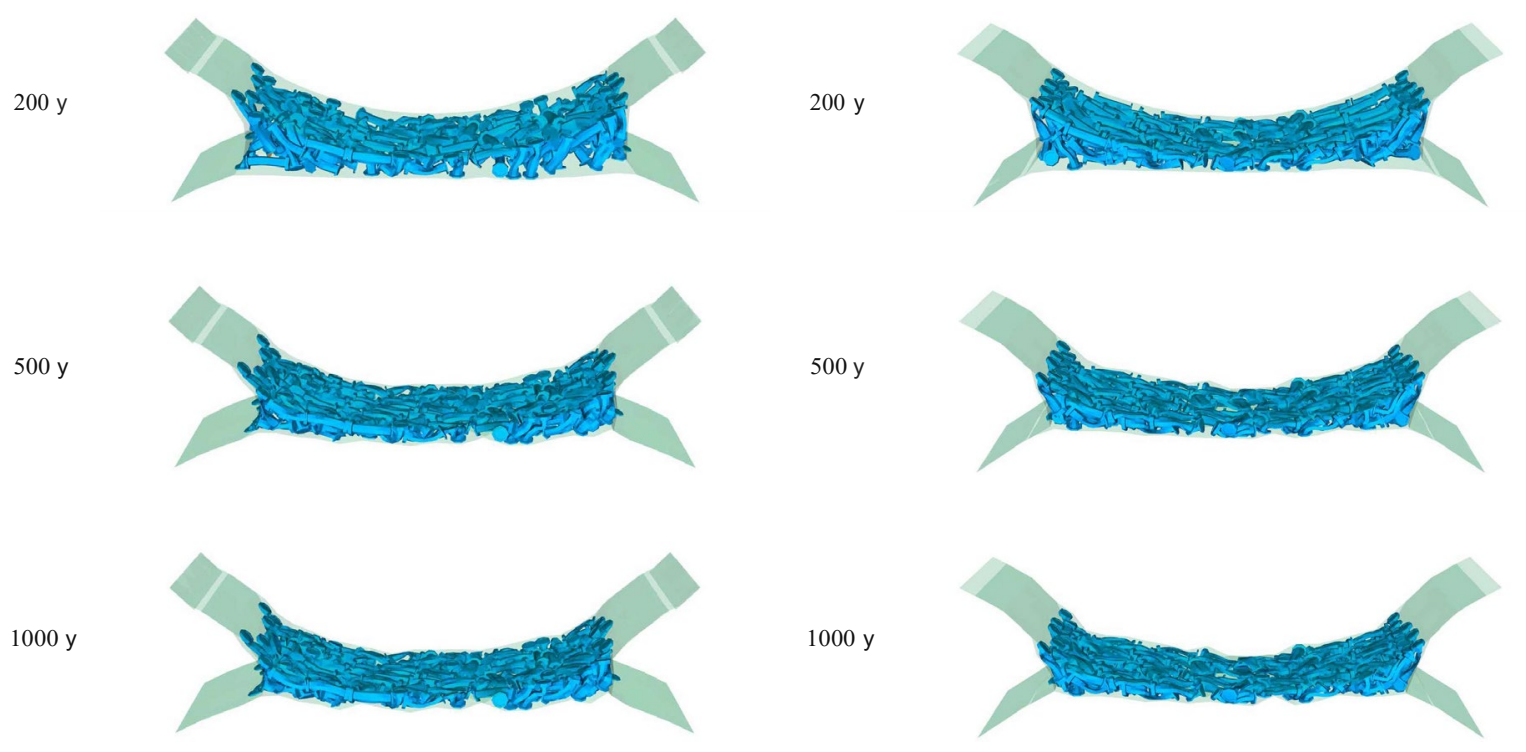

(a) Lower horizon

(b) Upper horizon

Figure 4.9. Salt compaction of CCOs in WIPP disposal room: (a) lower strata horizon in southern portion of repository, and (b) upper strata horizon in northern portion of repository, used with permission [11]. 
Table 4.4. Reedlunn and Bean $[8]^{6}$ compaction study of centroid locations (in $\mathrm{cm}$ ) for upper and lower horizons

\begin{tabular}{|c|c|c|c|c|c|c|}
\hline \multirow{2}{*}{ Centroid } & \multicolumn{3}{|c|}{ Lower horizon } & \multicolumn{3}{|c|}{ Upper horizon } \\
\hline & $x$-coordinate & $y$-coordinate & z-coordinate & $\mathrm{x}$-coordinate & $y$-coordinate & z-coordinate \\
\hline 1 & -195.6072 & -8.5814 & -566.9087 & -213.4896 & -2.0576 & -284.4357 \\
\hline 2 & -168.3766 & 38.4815 & -551.902 & -163.8594 & -20.9648 & -300.5868 \\
\hline 3 & -206.4903 & 68.5531 & -568.2877 & -187.2823 & 47.4028 & -298.7615 \\
\hline 4 & -232.1483 & 48.1034 & -537.9253 & -225.6460 & 60.1507 & -300.7980 \\
\hline 5 & -211.7788 & -27.3806 & -549.6381 & -232.8352 & 8.4008 & -286.8479 \\
\hline 6 & -189.8944 & -64.4194 & -529.0411 & -218.9769 & -60.0147 & -295.0244 \\
\hline 7 & -187.1205 & -6.4709 & -549.6133 & -191.5388 & -72.1002 & -291.3473 \\
\hline 8 & 102.0811 & -29.7449 & -552.5645 & 137.3855 & 20.8934 & -300.5575 \\
\hline 9 & 174.8612 & -47.1992 & -538.3002 & 184.3125 & 18.9962 & -292.9760 \\
\hline 10 & 127.9292 & 26.2153 & -550.6479 & 169.3188 & 64.0558 & -297.1980 \\
\hline 11 & 24.2554 & 27.7693 & -567.3369 & 87.8555 & 43.2948 & -308.5008 \\
\hline 12 & 9.6590 & 38.3789 & -563.5939 & 23.8535 & 13.5799 & -311.4196 \\
\hline 13 & 24.5192 & -68.4402 & -562.3103 & 101.8339 & -64.3174 & -302.3304 \\
\hline 14 & 99.7466 & -82.1888 & -555.3967 & 142.7472 & -40.0338 & -297.0448 \\
\hline 15 & 252.7654 & 10.0135 & -548.1866 & 257.5228 & -43.9402 & -292.7001 \\
\hline 16 & 282.4657 & -35.1615 & -538.2288 & 288.9069 & -3.1321 & -296.7938 \\
\hline 17 & 282.5897 & 93.3085 & -536.9157 & 281.6632 & 69.4535 & -287.4882 \\
\hline 18 & 278.5696 & 51.3496 & -509.8827 & 271.7254 & 38.1149 & -286.9477 \\
\hline 19 & 225.9322 & 37.0264 & -527.9882 & 236.5810 & -16.8224 & -292.4982 \\
\hline 20 & 273.4185 & -63.8927 & -518.9328 & 246.1724 & -76.1062 & -298.3139 \\
\hline 21 & 276.9387 & -88.4429 & -542.0559 & 269.1834 & -67.0046 & -300.5688 \\
\hline 22 & -163.4146 & 3.6923 & -554.6168 & -146.6181 & -4.9767 & -306.9386 \\
\hline 23 & -123.7791 & 2.3381 & -558.0016 & -101.9203 & -35.8322 & -312.8182 \\
\hline 24 & -171.4336 & 49.8568 & -558.1559 & -120.5979 & 40.8157 & -312.6055 \\
\hline 25 & -213.1447 & 26.1211 & -547.1646 & -157.1704 & 64.5581 & -311.4320 \\
\hline 26 & -242.8409 & -1.6661 & -563.6853 & -170.6807 & 3.5146 & -308.9558 \\
\hline 27 & -202.9402 & -57.4155 & -555.2846 & -131.5732 & -50.6890 & -310.5286 \\
\hline 28 & -135.2804 & -50.4475 & -565.4454 & -127.1361 & -90.6006 & -312.9752 \\
\hline 29 & 66.9308 & 10.2665 & -565.9355 & 95.4716 & -34.4232 & -311.6265 \\
\hline 30 & 128.1795 & 5.1647 & -556.7833 & 117.1502 & -3.5771 & -313.4797 \\
\hline 31 & 64.3827 & 46.9144 & -567.4066 & 102.1670 & 46.7216 & -313.6667 \\
\hline 32 & 28.2069 & 42.5161 & -573.6335 & 74.4089 & 8.8337 & -310.4222 \\
\hline 33 & 13.5157 & -8.1312 & -569.7059 & 53.4156 & -31.5836 & -315.0150 \\
\hline 34 & 38.3705 & -35.6255 & -560.2751 & 71.1694 & -65.0879 & -314.3208 \\
\hline 35 & 110.8944 & -65.1957 & -568.2821 & 83.0034 & -90.7105 & -307.6107 \\
\hline 36 & 281.2500 & 8.4249 & -548.2412 & 277.9888 & 20.4604 & -299.2442 \\
\hline 37 & 309.8546 & 3.7029 & -554.3904 & 292.3153 & 58.1228 & -290.9107 \\
\hline 38 & 301.6371 & 47.6465 & -541.9669 & 309.5932 & 95.1232 & -300.8653 \\
\hline 39 & 274.1711 & 37.5543 & -554.4294 & 226.7347 & 33.0839 & -300.4133 \\
\hline
\end{tabular}

${ }^{6}$ Used with permission 
Table 4-4. Reedlunn and Bean [8] compaction study of centroid locations for the upper and lower horizons (continued)

\begin{tabular}{|c|c|c|c|c|c|c|}
\hline \multirow{2}{*}{ Centroid } & \multicolumn{3}{|c|}{ Lower horizon } & \multicolumn{3}{|c|}{ Upper horizon } \\
\hline & $\mathrm{x}$-coordinate & y-coordinate & z-coordinate & $x$-coordinate & $y$-coordinate & z-coordinate \\
\hline 40 & 242.5638 & -9.9588 & -554.2952 & 188.8528 & -26.9823 & -305.3414 \\
\hline 41 & 241.4997 & -48.9540 & -547.1007 & 231.7606 & -33.3423 & -300.5899 \\
\hline 42 & 295.4058 & -59.4042 & -552.7902 & 294.5557 & -18.5864 & -306.8297 \\
\hline 43 & -217.8443 & 6.5048 & -544.1930 & -265.4261 & -12.0709 & -268.1155 \\
\hline 44 & -153.4009 & -53.6609 & -548.4302 & -235.0770 & 33.0402 & -280.8256 \\
\hline 45 & -197.8117 & 80.2423 & -550.1229 & -248.4038 & 68.3274 & -280.9737 \\
\hline 46 & -232.0676 & 61.1887 & -517.6337 & -264.8788 & 31.5113 & -273.7527 \\
\hline 47 & -247.7792 & 16.2919 & -511.9121 & -287.8335 & 13.9891 & -264.7854 \\
\hline 48 & -236.5811 & -64.0944 & -531.9523 & -271.9484 & -38.8405 & -275.7612 \\
\hline 49 & -200.4602 & -32.9123 & -530.9564 & -252.7562 & -60.1307 & -271.3269 \\
\hline 50 & 64.5087 & 31.0070 & -557.3813 & 121.3443 & 69.8568 & -300.9824 \\
\hline 51 & 143.1706 & 54.1248 & -546.8746 & 254.0710 & 9.4251 & -271.9287 \\
\hline 52 & 117.8685 & 61.7223 & -563.2603 & 235.1526 & 61.7990 & -280.1688 \\
\hline 53 & 87.5655 & 74.3758 & -564.3316 & 34.4203 & 59.9985 & -310.1965 \\
\hline 54 & 6.3309 & 4.0348 & -558.7176 & -55.9454 & 19.2380 & -306.0426 \\
\hline 55 & 83.3233 & -48.3772 & -560.6623 & 11.9535 & -72.6694 & -308.7388 \\
\hline 56 & 157.9896 & -21.4957 & -540.4430 & 191.609 & -21.0098 & -289.4311 \\
\hline 57 & 288.3405 & -26.3997 & -500.3040 & 288.4938 & -33.9050 & -261.8424 \\
\hline 58 & 305.0526 & 2.0657 & -488.7934 & 304.4687 & -40.9056 & -253.1890 \\
\hline 59 & 304.9013 & 60.9769 & -488.0990 & 297.569 & 83.2448 & -257.1121 \\
\hline 60 & 297.5841 & 21.8671 & -508.6516 & 302.8779 & 2.2301 & -256.4747 \\
\hline 61 & 263.3753 & -3.1083 & -512.8440 & 290.4659 & -8.2906 & -263.7739 \\
\hline 62 & 302.8147 & -88.9794 & -499.9142 & 293.9906 & -70.7845 & -265.8125 \\
\hline 63 & 305.0992 & -54.2145 & -495.0503 & 304.2196 & -91.7415 & -255.0902 \\
\hline 64 & -244.4525 & -77.1758 & -542.6508 & -267.1711 & -91.3518 & -296.7223 \\
\hline 65 & -188.4417 & -86.3376 & -559.4857 & -237.3205 & -95.7182 & -293.8821 \\
\hline 66 & -238.8877 & -42.5505 & -530.0501 & -259.8012 & -30.0669 & -290.1501 \\
\hline 67 & -268.6379 & -3.5462 & -518.8964 & -280.3122 & -34.0868 & -296.0446 \\
\hline 68 & -277.0882 & -55.4640 & -524.5588 & -295.7452 & -53.2454 & -289.8989 \\
\hline 69 & -107.8975 & -95.9258 & -552.7917 & -96.6793 & -94.2065 & -303.8164 \\
\hline 70 & -37.0657 & -77.8336 & -557.0315 & 6.4047 & -95.7134 & -315.0579 \\
\hline 71 & -63.3461 & 2.6602 & -559.7408 & -31.5007 & -36.5975 & -309.4587 \\
\hline 72 & -131.9347 & -29.8631 & -558.0907 & -125.6904 & -25.7994 & -299.5687 \\
\hline 73 & -164.3553 & -86.6192 & -556.3458 & -148.8238 & -89.1546 & -305.4559 \\
\hline 74 & 210.3606 & -96.2887 & -543.9088 & 212.9723 & -77.3391 & -289.1568 \\
\hline 75 & 251.8139 & -86.6179 & -518.6836 & 231.9386 & -95.7353 & -293.4954 \\
\hline 76 & 236.0352 & 7.2155 & -519.1767 & 227.2816 & -38.7678 & -293.8478 \\
\hline 77 & 185.3908 & -46.9649 & -548.4143 & 195.7941 & -16.1381 & -294.3386 \\
\hline 78 & 152.8983 & -69.4795 & -555.7515 & 172.8191 & -64.4003 & -294.0295 \\
\hline 79 & -243.7385 & 95.3014 & -538.6218 & -268.6026 & 94.9990 & -298.3608 \\
\hline 80 & -212.0347 & 55.9720 & -546.1598 & -236.3573 & 94.7920 & -287.0739 \\
\hline 81 & -271.4215 & 82.2483 & -526.1757 & -303.7671 & 92.0952 & -295.0585 \\
\hline 82 & -265.7712 & 24.3046 & -527.1078 & -276.2057 & 5.1702 & -294.8271 \\
\hline 83 & -239.3240 & 18.0546 & -538.6019 & -255.3651 & 23.1740 & -299.7947 \\
\hline 84 & -112.4733 & 52.6610 & -562.5813 & -100.8276 & 95.6871 & -303.3531 \\
\hline
\end{tabular}


Table 4-4. Reedlunn and Bean [8] compaction study of centroid locations for the upper and lower horizons (continued)

\begin{tabular}{|c|c|c|c|c|c|c|}
\hline \multirow{2}{*}{ Centroid } & \multicolumn{3}{|c|}{ Lower horizon } & \multicolumn{3}{|c|}{ Upper horizon } \\
\hline & $x$-coordinate & $y$-coordinate & z-coordinate & $x$-coordinate & $y$-coordinate & z-coordinate \\
\hline 85 & 39.2405 & 87.0530 & -562.6270 & -40.6567 & 55.1499 & -309.9441 \\
\hline 86 & -190.4090 & 98.7541 & -550.0681 & -145.5982 & 91.8613 & -305.8976 \\
\hline 87 & -115.5552 & 24.8619 & -559.7710 & -138.6377 & 24.8523 & -300.1584 \\
\hline 88 & -65.4479 & 23.4106 & -554.6334 & -79.5500 & 33.5978 & -307.6583 \\
\hline 89 & 232.1739 & 64.9417 & -566.4858 & 222.0002 & 93.3458 & -298.1699 \\
\hline 90 & 272.3040 & 76.7184 & -508.9081 & 263.9811 & 88.5637 & -289.5813 \\
\hline 91 & 126.5840 & 75.7607 & -546.2491 & 186.6790 & 87.7788 & -291.2231 \\
\hline 92 & 167.1547 & 56.4320 & -562.3259 & 206.7590 & 43.7193 & -296.1452 \\
\hline 93 & 213.2109 & 61.6758 & -536.6561 & 235.5210 & 11.2188 & -292.1886 \\
\hline 94 & -277.9814 & -83.0493 & -558.7073 & -216.7393 & -70.5421 & -303.6883 \\
\hline 95 & -231.3040 & -65.2024 & -565.1735 & -178.8628 & -73.2675 & -302.1743 \\
\hline 96 & -265.0780 & -24.3620 & -550.0945 & -198.7380 & -35.8451 & -297.7332 \\
\hline 97 & -301.0789 & -28.1433 & -544.9897 & -232.9369 & -31.2563 & -300.6552 \\
\hline 98 & -306.6950 & -63.7129 & -549.7599 & -289.7868 & -44.1079 & -303.7186 \\
\hline 99 & -62.8465 & -64.9939 & -571.2789 & -28.9445 & -62.4375 & -313.2632 \\
\hline 100 & -16.6897 & -91.2152 & -571.0237 & 37.9474 & -73.1966 & -312.4582 \\
\hline 101 & -11.3818 & -26.1744 & -567.7898 & -4.0034 & -28.3581 & -315.3801 \\
\hline 102 & -69.6733 & -31.5698 & -569.2037 & -50.3881 & -9.1844 & -317.6224 \\
\hline 103 & -118.9749 & -76.3456 & -555.6463 & -81.3406 & -74.7552 & -313.1018 \\
\hline 104 & 185.5332 & -77.3915 & -549.2069 & 154.2222 & -96.4896 & -309.3883 \\
\hline 105 & 212.6486 & -66.1513 & -547.1550 & 188.9751 & -73.9651 & -303.9341 \\
\hline 106 & 200.9535 & -42.1123 & -557.7798 & 160.2633 & -46.2455 & -302.7995 \\
\hline 107 & 160.5359 & -20.8914 & -553.3461 & 127.2591 & -31.9314 & -310.4759 \\
\hline 108 & 142.7483 & -94.4954 & -557.1335 & 124.9580 & -87.5420 & -311.3654 \\
\hline 109 & -274.7585 & 73.1582 & -550.0604 & -202.1909 & 91.6333 & -308.3082 \\
\hline 110 & -254.6001 & 78.0200 & -560.7598 & -174.4730 & 92.2439 & -307.3053 \\
\hline 111 & -313.6393 & 74.4106 & -553.5117 & -294.6694 & 27.0406 & -299.4978 \\
\hline 112 & -308.0409 & 26.9075 & -541.9002 & -217.6602 & 10.3411 & -304.9176 \\
\hline 113 & -268.5852 & 28.7860 & -553.2664 & -194.0969 & 29.7966 & -303.6382 \\
\hline 114 & -15.4739 & 83.9212 & -570.1639 & -20.3665 & 80.1401 & -312.0258 \\
\hline 115 & -5.6954 & 71.9634 & -566.3322 & 71.7688 & 69.8459 & -312.6709 \\
\hline 116 & -150.2334 & 77.8732 & -558.4633 & -75.0437 & 76.8131 & -315.8768 \\
\hline 117 & -57.4474 & 45.4806 & -562.1516 & -64.8536 & 13.1179 & -313.9009 \\
\hline 118 & -38.0596 & 19.6168 & -570.9484 & -9.0724 & 21.3081 & -313.7538 \\
\hline 119 & 170.7607 & 51.2304 & -567.7764 & 154.6926 & 83.8294 & -310.0220 \\
\hline 120 & 240.4769 & 93.9066 & -547.7869 & 204.8655 & 64.2860 & -302.7075 \\
\hline 121 & 135.4575 & 94.3221 & -562.2151 & 121.9991 & 95.3049 & -310.6237 \\
\hline 122 & 179.6678 & 3.7066 & -555.4600 & 147.7968 & 26.9379 & -306.9927 \\
\hline 123 & 212.8767 & 5.7786 & -562.4326 & 175.2278 & 16.1506 & -305.3567 \\
\hline 124 & -285.5110 & -93.6461 & -511.9963 & -289.4428 & -49.6838 & -259.6640 \\
\hline 125 & -247.9282 & -88.8492 & -519.8870 & -278.0818 & -69.9646 & -260.4170 \\
\hline 126 & -283.9506 & -13.9425 & -488.5638 & -284.8696 & -9.5179 & -267.4761 \\
\hline 127 & -302.9387 & -32.0402 & -494.2332 & -302.7002 & -6.3955 & -254.7843 \\
\hline 128 & -311.4812 & -76.1218 & -472.8971 & -299.8982 & -94.4408 & -253.5523 \\
\hline 129 & -69.9875 & -91.8442 & -567.3457 & -166.0907 & -88.0975 & -292.5673 \\
\hline
\end{tabular}


Table 4-4. Reedlunn and Bean [8] compaction study of centroid locations for the upper and lower horizons (continued)

\begin{tabular}{ccccccc}
\hline \multirow{2}{*}{ Centroid } & \multicolumn{3}{c}{ Lower horizon } & \multicolumn{3}{c}{ Upper horizon } \\
\cline { 2 - 7 } & x-coordinate & y-coordinate & z-coordinate & x-coordinate & y-coordinate & z-coordinate \\
\hline 130 & 37.6640 & -92.6135 & -563.6414 & -91.2889 & -61.8661 & -303.2903 \\
131 & -48.5633 & -57.9711 & -565.9090 & -124.5097 & -0.6922 & -299.9205 \\
132 & -89.8459 & -42.1439 & -554.3143 & -205.2035 & -47.2042 & -287.0985 \\
133 & -180.4832 & -96.3225 & -535.7100 & -245.0691 & -96.7256 & -276.4592 \\
134 & 239.5603 & -52.4520 & -514.3432 & 267.7152 & -87.6572 & -273.3308 \\
135 & 282.6324 & -95.1450 & -506.5219 & 277.9787 & -96.8531 & -271.5817 \\
136 & 256.7301 & -32.4694 & -506.6623 & 278.1862 & -57.5666 & -267.7829 \\
137 & 207.8105 & 12.4602 & -527.3900 & 260.0825 & -18.3410 & -268.9344 \\
138 & 167.7973 & -95.8046 & -552.2154 & 243.7051 & -68.0402 & -274.1189 \\
139 & -283.8441 & 75.4922 & -492.0842 & -287.7614 & 72.6029 & -268.1458 \\
140 & -233.1853 & 72.6945 & -530.3853 & -279.8825 & 56.6815 & -276.2016 \\
141 & -309.8401 & 81.3561 & -480.5229 & -293.8302 & 90.5573 & -251.1806 \\
142 & -302.5714 & 28.8674 & -484.8186 & -303.7403 & 31.0081 & -254.5943 \\
143 & -278.3352 & 50.5453 & -504.1470 & -288.1789 & 38.3306 & -266.2597 \\
144 & -56.0811 & 92.8100 & -559.7273 & -182.8970 & 74.7467 & -291.7416 \\
145 & -9.0936 & 66.5283 & -559.0231 & -111.8636 & 66.8251 & -303.0716 \\
146 & -111.5024 & 79.3568 & -553.5037 & -219.6363 & 89.8358 & -280.4688 \\
147 & -185.2346 & -4.5709 & -535.3569 & -205.6745 & 45.3883 & -286.7643 \\
148 & -81.4054 & -16.8762 & -552.1512 & -146.6689 & 50.7662 & -296.4557 \\
149 & 211.6919 & 92.4383 & -546.4770 & 276.1441 & 73.9273 & -268.7344 \\
150 & 279.3761 & 94.6427 & -491.6647 & 290.6271 & 59.9997 & -259.4299 \\
151 & 175.3590 & 94.0269 & -552.1094 & 258.1187 & 91.9249 & -269.4827 \\
152 & 185.9292 & 46.1741 & -542.5692 & 263.9302 & 36.0571 & -268.6708 \\
153 & 248.2810 & 48.6150 & -526.1100 & 288.0774 & 22.5152 & -261.3250 \\
\hline & & & & & &
\end{tabular}

\subsubsection{Reflector Materials and Geometry}

The reflector materials are those materials in the model located outside of the waste form-specifically, any material which is outside the outer diameter of the waste form. The reflector material has two possible configurations that may exist together in the same model: interstitial and discrete. The interstitial reflector materials are based on the work by Brickner [4] and are described in Section 4.2.1.1below. The discrete reflector described in Section 4.2.2.3 is directly adjacent to the waste form and has a thickness chosen to be very small $(0.001 \mathrm{~cm})$, or the thickness of a CCC $(0.7112 \mathrm{~cm})$, which was chosen to cover the existing arrangement, and for convenience. Additionally, the discrete thickness is used to evaluate the $k_{\text {eff }}$ effect of polyethylene (an appropriate material because of its neutron-moderating/reflecting properties) from external sources that could be pressed around the waste stream under post-closure conditions.

For each waste form parametric sweep, the total mass and volume of the waste form define the waste form geometry dimensions (see Section 4.3 below). The discrete reflector has a fixed thickness which is applied to every parametric sweep over all the waste forms. Every calculation with the same waste form mass and volume has a consistent discrete-reflector-volume-to-waste-form-volume ratio. Parametric sweeps with a mix of waste form mass and volumes have varying discrete-reflector-volume-to-wasteform-volume ratios. Therefore, smaller waste form volumes with the $0.7112 \mathrm{~cm}$ thickness reflector have a much larger discrete-reflector-thickness-volume-to-waste-form-volume ratio than the largest waste form volume. In this manner, the source of external discrete reflector material is irrelevant, and each set of 
parametric sweeps can establish the effect of the discrete reflector for its mass and volume combinations. Therefore, any combination that represents an actual waste form may be applicable. Since a very thin reflector and a thick discrete reflector are used, the effect of values in between can be estimated or bounded.

\subsubsection{Salt and MgO for the interstitial reflector material}

The interstitial reflector materials consist of material between CCCs. Because the material is between the waste forms, it reflects neutrons back into the waste forms. $\mathrm{MgO}$ and $\mathrm{NaCl}$ have very low moderating power. The treatment of the interstitial reflector here follows on the work by Brickner [4] and is described in detail below.

Salt is the most common and abundant material in the system and is considered in both the interstitial reflector and in the infinitely thick external reflector. The salt in the region of the waste forms is denoted as an interstitial reflector here because it lies between the waste forms, and it reflects some neutrons back into the waste forms. Salt can act either as a reflector or an absorber, depending on the neutron energy. In Brickner's work [4], the salt was evaluated as dry and modeled as $\mathrm{NaCl}$. As saturated or brine it was modeled as $\mathrm{NaCl}$ and $\mathrm{H}_{2} \mathrm{O}$. The negative reactivity effect of the neutron absorptions in the brine precludes the necessity of repeating those studies here. The dry salt is assumed to always be present in the reflector.

$\mathrm{MgO}$ is present in Supersacks placed on top of about half of the stacks of three 7-packs. The $\mathrm{MgO}$ is used to absorb $\mathrm{CO}_{2}$ produced by the decay of carbon-based materials such as wood, paper, plastic, or rubber. However, as noted in the High Bridge Associates report [23], the $\mathrm{MgO}$ has a large neutron scattering cross section and can therefore serve as a neutron reflector and/or moderator. Saylor and Scaglione [3] evaluated the impact of the $\mathrm{MgO}$ as a reflector in its as-emplaced configuration, which is essentially a layer of $\mathrm{MgO}$ on top of the stacks of three 7-packs (albeit an exaggeration of the total mass available). Brickner's 2019 [4] evaluations showed that increasing the amount of $\mathrm{MgO}$ that was uniformly mixed with salt increased the $k_{\text {eff. }}$. Because of the far greater amount of salt compared to the MgO, Brickner [4] conservatively placed an upper limit of $50 \%$ on the amount of $\mathrm{MgO}$ compared to salt. The same approach is used here.

The mean free path of the salt/MgO mixture is shown below in Figure 4.10 as the solid orange line. The dotted line indicates the average distance that a neutron would travel between scattering events if no other interactions were to occur, and the dashed line shows the average distance that a neutron would travel before being captured - again assuming no other interactions. Above approximately $0.03 \mathrm{eV}$, scattering dominates the total interaction probability. However, because multiple scatters can occur, absorption can become important, even in the range of hundreds of $\mathrm{eV}$, due to absorption resonances. These lengths were computed using the ENDF-7.1 252 group scale neutron cross sections. Total and capture microscopic cross sections were multiplied by the elemental abundances listed in the SCALE output for a Sampler case to obtain macroscopic cross sections. Mean free path is the multiplicative inverse of the total macroscopic cross section for a particular reaction. 


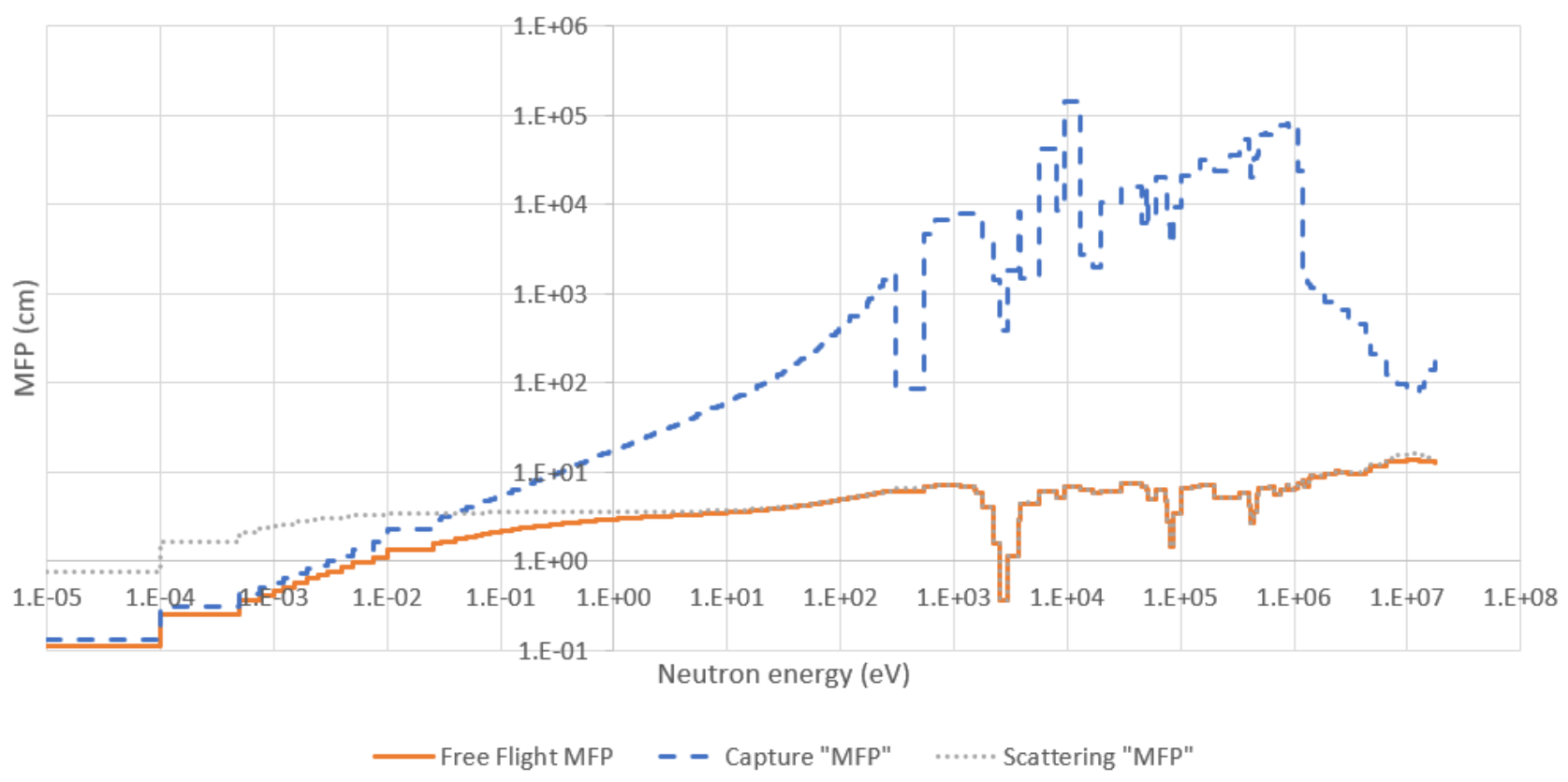

Figure 4.10. Mean free in the $\mathrm{MgO} / \mathrm{NaCl}$ mixture.

Figure 4.11 below plots the macroscopic scattering cross sections of the major constituents of the salt/MgO mixture. As the figure shows, $100 \mathrm{eV}^{35} \mathrm{Cl}$ contributes heavily to the total scattering cross section. In the $\mathrm{keV}$ range, there is a large scattering resonance for ${ }^{23} \mathrm{Na}$. $\mathrm{Mg}$ and $\mathrm{O}$ play more prominent roles at higher energies, but they never truly dominate except at a few energy resonances. For convenience, scattering cross sections were taken as the difference between the total cross section and the capture cross section. 


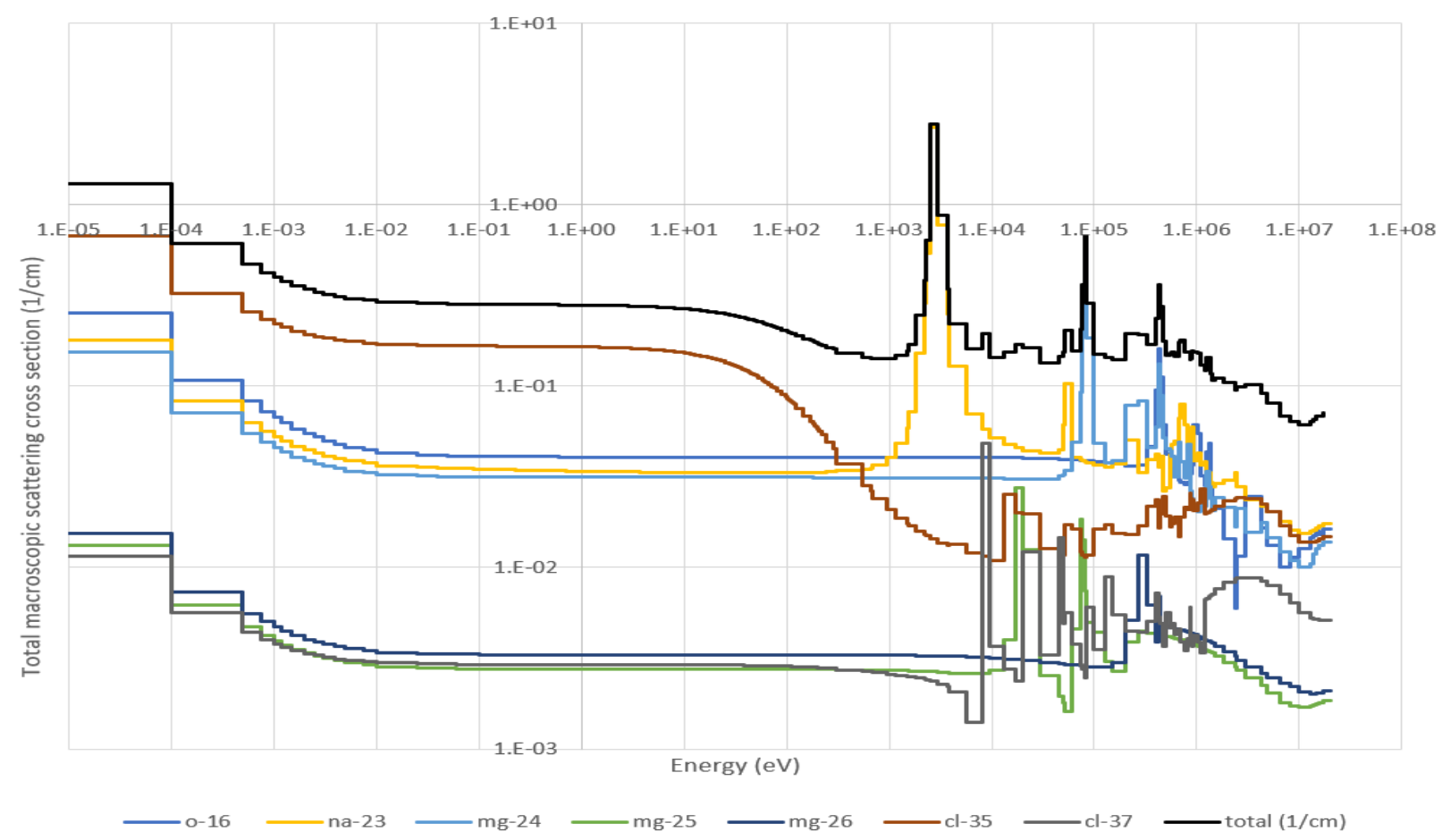

Figure 4.11. Macroscopic total interaction cross section for the the $\mathrm{MgO} / \mathrm{NaCl}$ mixture components.

In Figure 4.12 below, capture cross sections of the salt/ $\mathrm{MgO}$ mixture are dominated by ${ }^{35} \mathrm{Cl}$ at energies below $1 \mathrm{keV}$. Above $1 \mathrm{keV},{ }^{35} \mathrm{Cl}$ remains a heavy contributor to the overall cross section with ${ }^{23} \mathrm{Na}$. 

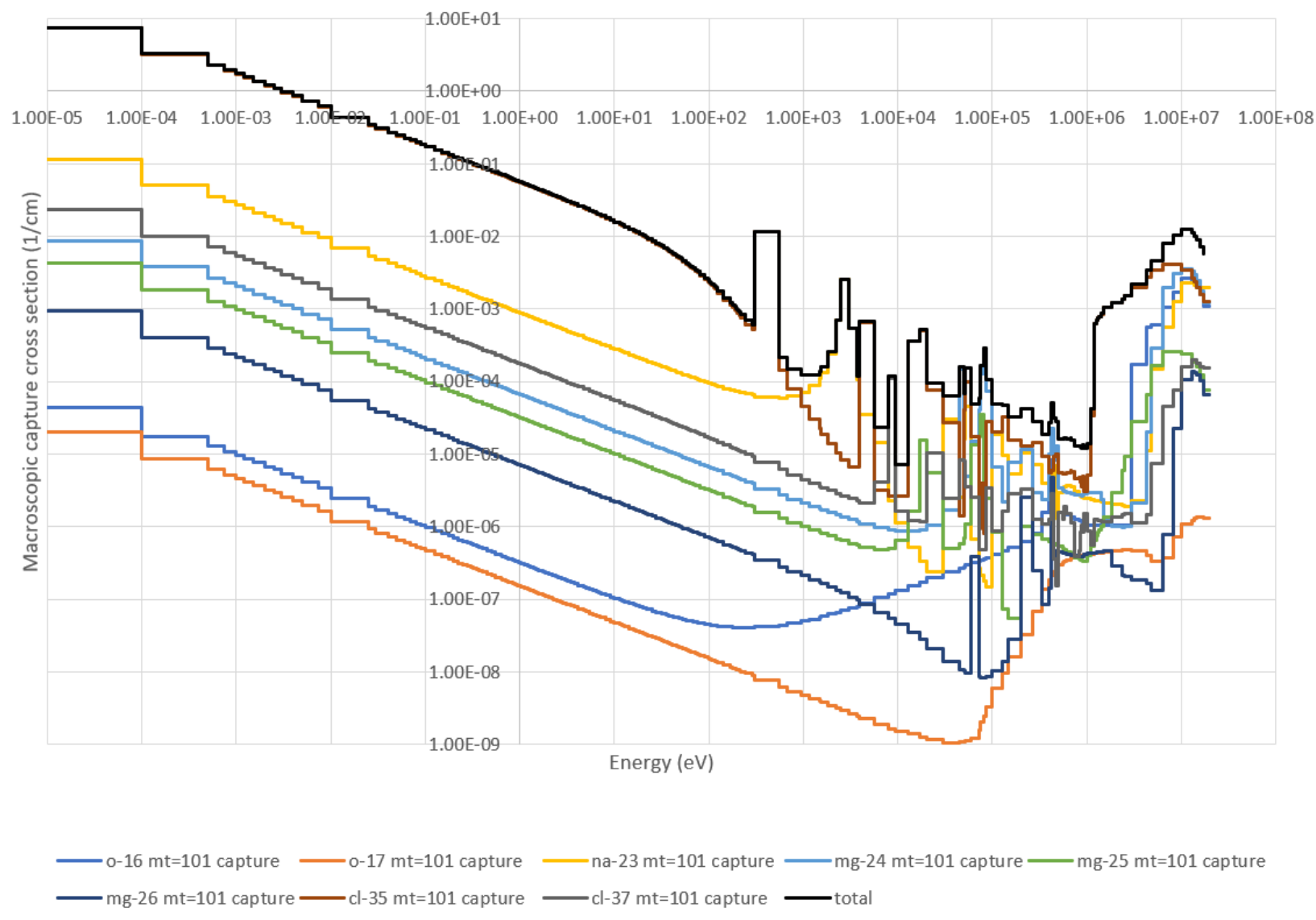

Figure 4.12. Macroscopic capture cross section for the the $\mathrm{MgO} / \mathrm{NaCl}$ mixture components

\subsubsection{Beryllium in the interstitial reflector}

Because the special reflector as defined in the CCO TRUPACT-II Safety Analysis Report [9] can be transported using most container designs for shipment to WIPP, it is possible that Be may be present outside of the CCOs and could become intermixed with other materials. Calculations have been performed with Be uniformly mixed in the interstitial reflector material and are presented in publications by Saylor, Scaglione [3] and Brickner [4]. Those results show little or no difference in the presence of Be in the reflector vs. the waste form. For the present analysis, the Be is evaluated in the waste form only.

\subsubsection{Steel and polyethylene for the discrete reflector material}

The CCC is made of stainless steel and can function to reflect neutrons back into the CCC. The CCC is denoted as a discrete reflector in this document because it is not continuously distributed in the same manner as the salt/MgO mixture comprising the interstitial reflector. The stainless steel may be from the $\mathrm{CCO}$, the $\mathrm{CCC}$, or the packaging cans, and it can act as a reflector. Polyethylene is also used in packaging. Polyethylene is highly moderating, but it is also capable of scattering thermalized neutrons back into the CCO. Pu's fission cross section is orders of magnitude larger for thermal neutrons, which should produce a relatively large effect compared to the effect that would be achieved by simply reflecting faster neutrons back into the waste form. Polyethylene has a high moderating power, so it could bound other materials that may agglomerate into a discrete reflector around the waste form (i.e., cellulose from plywood) over long periods of time. For simplicity, the material card provided by the SCALE code package for 304 stainless steel and polyethylene is used. 


\subsubsection{WASTE FORM MATERIALS, COMPOSITION AND GEOMETRY}

The CCO waste form materials are any materials that may be placed within the $\mathrm{CCC}$ of the $\mathrm{CCO}$ based on the defined limits contained in the CCO TRUPACT-II Safety Analysis Report [9] or otherwise limited by the WAC [5]. The CCO waste form materials include any possible materials which may be used as part of any waste stream processing. Generically, the waste form may potentially have up to $380 \mathrm{FGE}{ }^{239} \mathrm{Pu}$, water and/or plastic materials, $585 \mathrm{~g}$ of special reflector material as defined in the CCO TRUPACT-II Safety Analysis Report [9] like Be, and no limits on any additional filler materials other than FGE and Be. These values bound the WAC limit. In this evaluation, H-bearing materials from any source are bounded by the $\mathrm{H} /{ }^{239} \mathrm{Pu}$ curves as water or polyethylene. In this report, all materials included in the waste form geometry are uniformly mixed. Any waste form which uses a configuration or process that creates a significant deviation in the waste form such that a uniform mixture may not be appropriate must be evaluated to determine the applicability of the results in this report. However, previous work has shown that the uniformly mixed case is an adequate approach to bound nonuniform mixing [24].

For this generic parametric evaluation of the $\mathrm{CCO}$ waste form, two filler materials ${ }^{7}$ were evaluated separately: (1) a cementitious mixture herein termed a generic material, which was used by Saylor and Scaglione [3] to specifically evaluate the dilute surplus Pu waste stream, and (2) a graphite material, which was selected because it is expected to bound most if not all non-hydrogenous materials due to its neutron-moderating capabilities. The energy absorbed by nuclei in elastic collisions with neutrons decreases as atomic mass increases. A comparison of the moderating power of common moderator materials, as defined in Duderstadt and Hamilton [15], shows that lighter nuclei tend to have higher moderating powers (except for helium because it is a gas), so graphite is appropriate to consider, as shown in Table 4.5. Although other materials listed have higher moderator powers, they are either evaluated as moderators elsewhere in this study, or in the case of Be, they are restricted by the WAC.

Table 4.5. Comparison of the moderator power and moderating ratio of several materials

\begin{tabular}{cc}
\hline Material & Moderating power \\
\hline water & 1.28 \\
heavy water & 0.18 \\
He & 0.00001 \\
Be & 0.16 \\
graphite & 0.064 \\
polyethylene & 3.26 \\
\hline
\end{tabular}

In addition to the generic and graphite filler materials, another material is included to provide a means to evaluate the impact of waste form processing cans (i.e., convenience cans) or other types of waste form container configurations. For this purpose, stainless steel is used for these cans because it is a likely candidate for such containers.

\footnotetext{
${ }^{7}$ A filler material is any material in the waste form that is not FGE, special reflector like beryllium as defined in the CCO TRUPACT-II Safety Analysis Report [9], or H-bearing (i.e., water or polyethylene). For subsets of the parametric evaluations, stainless steel is included as a mass of material which represents a uniform mixing of the waste form and the stainless steel from either the convenience can type of configuration, as in the surplus plutonium from the CCC, or from some other external source. This context is considered part of the filler material. Specifically, when the stainless steel is mixed uniformly with the waste form, it is considered filler material that either replaces or is in addition to the other components of the filler material.
} 
The reactivity effect of various filler materials (and the ingress of the can material) is expected to be dependent on the neutron spectrum and the size and shape of the waste form. The reactivity trend for filler materials is established in Section 6.1 below, but no effort is made to evaluate variations in the effect on reactivity for variations of the material components of the waste form, because the inclusion of filler materials shows a reduction in reactivity, and the materials considered are sufficiently conservative.

The waste form is modeled using two geometries: spherical and cylindrical. The use of spherical geometries to model long-term waste configurations is justified in Section 4 above. Additionally, Brickner [4] shows that using optimally moderated spheres (spheres that have moderator added to maximize reactivity) is extremely conservative compared to using other neutronically isolated shapes, and it provides ample technical justification for addressing the complexities of unknown configurations over the 10,000-year regulatory timeframe. However, to achieve optimal moderation for a sphere, the radius of the sphere must grow without bound until the optimum point is found. The radius of the optimally moderated sphere is seen to be well beyond the radius of the CCC for most cases; therefore, it is not realistic. However, the CCC is a cylindrical steel pipe which is expected to maintain its geometry for some significant period of time. Thus, results for calculations with sphere radii larger than the CCC should be viewed as information only and should not be considered likely. Furthermore, waste streams may be expected to use processes which result in rigid, fixed cylindrical shapes like the dilute surplus $\mathrm{Pu}$ can-bag-can configuration (see Figure 4.5, above). The dilute surplus Pu can-bag-can configuration is generically known to include an inner can wrapped in some form of plastic bagging which is then placed in another cylindrical can. This can-bag-can configuration is then placed in the cylindrical CCC pipe. If these cylindrical shapes are a part of any generic waste stream, then they should also be evaluated so that the FEP process may, at a minimum, consider the $k_{\text {eff }}$ effect of the cylindrical geometry for the time periods in which they are expected to maintain their original geometry.

For both the spherical and cylindrical models, the waste forms are modeled for the parametric evaluations as follows:

1. The FGE volume is calculated from the FGE modeled based on one FGE equaling one gram ${ }^{239} \mathrm{Pu}$ in the form of $\mathrm{PuO}_{2}$.

2. If $\mathrm{Be}$ is present, then the $\mathrm{Be}$ mass is used to calculate the volume of the $\mathrm{Be}$.

3. The volume of moderator (water or polyethylene) is determined from the moderator mass at the point in the parametric sweep over moderator mass.

4. Each case, at a minimum, has FGE and moderator, while the Be is case dependent. Some additional cases include variations in filler mass and stainless steel for the can(s). For the spherical models, the total volume of these materials forms the basis for the radius of the sphere.

5. For the cylindrical models, the total volume of these materials is used to determine the cylinder height, based on the fixed radius for that case. Multiple cylindrical radii are considered because the radius and height have a direct impact on $k_{\text {eff. }}$. Furthermore, the $k_{\text {eff }}$ effects seen for the various parameter sweeps may be radii-dependent because of differing leakage for larger radii.

The waste form parametric sweeps consider FGE (380 or 190 for set-3), Be ( 0 and $585 \mathrm{~g}$ ), and moderator (water and polyethylene) masses in the range of 100 to 3,000 $\mathrm{g}$ to establish a clear representation of the $\mathrm{H} /{ }^{239} \mathrm{Pu}$ curve. The sweeps also consider filler material consisting of generic or graphite $(0,1,000,2,000$, 3,000 and 4,000 g), with and without the steel for the convenience can $(0,500$ and 1,000 g). For all the variations of masses within the uniformly mixed waste form cylinders or spheres, the variation of the moderator generates $k_{\text {eff }}$ curves as a function of the parametric sweeps. 


\subsection{OVERVIEW OF KENO MODEL GEOMETRY}

The parametric evaluations described herein are based on the methodology in Saylor, Scaglione [3], and Brickner [4], as well as new methods that were developed to address emerging concerns. These evaluations consider two separate ways of modeling the system with CCOs: (1) uniform arrays of CCOs, and (2) nonuniform arrays of CCOs. The uniform arrays allow for the $k_{\text {eff }}$ of the system to be evaluated in an idealized manner with 3 or 6 waste forms in close contact, whereas the nonuniform arrays are a bestestimate calculation using specific post-closure centroid locations based on the Reedlunn and Bean [8] data, with very few waste forms in close contact.

For both the spherical and cylindrical models, the discrete reflectors are modeled for the parametric evaluations as follows:

1. Both a thin $0.001 \mathrm{~cm}$ layer and a thick $0.7112 \mathrm{~cm}$ layer are modeled directly adjacent to the waste form shape.

2. The total mass and volume of the discrete reflector is allowed to vary based on the total volume of the shape for that sweep.

3. Sweeps are made over two materials: stainless steel and polyethylene.

Table 4-6 summarizes the parameters evaluated and the supporting rationales. The effects of these parameters on $k_{\text {eff }}$ were evaluated using approximately 28,800 combinations per calculation set $(155,520$ total for six sets of calculations). Each set of calculations evaluated the array spacing variations listed in Table 6-1. 
Table 4-6. Evaluated parameters and corresponding rationale for evaluating $\boldsymbol{k}_{\text {eff }}$ effect

\begin{tabular}{|c|c|c|c|}
\hline PARAMETER & DESCRIPTION & VALUES USED & $\begin{array}{l}\text { RATIONALE FOR } \\
\text { EVALUATION }\end{array}$ \\
\hline MODERATOR TYPE & $\begin{array}{l}\text { Moderator uniformly } \\
\text { mixed with waste form }\end{array}$ & Water, polyethylene & $\begin{array}{l}\text { Both are powerful } \\
\text { moderators (see Section } \\
6.1 \text { ). Furthermore, results } \\
\text { show more moderation in } \\
\text { the waste form increases } k_{\text {eff }}\end{array}$ \\
\hline FILLER MASS & $\begin{array}{l}\text { Filler uniformly mixed } \\
\text { with waste form }\end{array}$ & $0,2,4 \mathrm{~kg}$ & Range of interest \\
\hline CYLINDER RADIUS & $\begin{array}{l}\text { Radius of cylindrical } \\
\text { waste form }\end{array}$ & $4.8,6.25,7.7 \mathrm{~cm}$ & $\begin{array}{l}7.7 \mathrm{~cm} \text { is the inner diameter } \\
\text { of the CCC; smaller radii } \\
\text { are listed to show a trend }\end{array}$ \\
\hline CYLINDER HEIGHT & $\begin{array}{l}\text { Height of cylindrical } \\
\text { waste form }\end{array}$ & Set by waste form content & $\begin{array}{l}\text { Dependent upon total } \\
\text { volume }\end{array}$ \\
\hline SPHERE RADIUS & $\begin{array}{l}\text { Radius of spherical waste } \\
\text { form }\end{array}$ & Set by waste form content & $\begin{array}{l}\text { Dependent upon total } \\
\text { volume: also provides some } \\
\text { coverage of the space with } \\
\text { radii }>7.7 \mathrm{~cm}\end{array}$ \\
\hline MODERATOR MASS & $\begin{array}{l}\text { Mass of moderator mixed } \\
\text { with waste form }\end{array}$ & $0.1-3 \mathrm{~kg}$ & $\begin{array}{l}\text { Range of interest to } \\
\text { establish trend }\end{array}$ \\
\hline $\begin{array}{l}\text { STEEL PIPE OR } \\
\text { POLYETHYLENE PIPE }\end{array}$ & $\begin{array}{l}\text { Material of outer layer } \\
\text { overlaying waste form }\end{array}$ & $\begin{array}{l}\text { Stainless pipe or } \\
\text { polyethylene }\end{array}$ & $\begin{array}{l}\text { To show relative effect of } \\
\text { bag, the CCC, and other } \\
\text { materials that may } \\
\text { accumulate like the } \\
\text { polyethylene; polyethylene } \\
\text { is expected to be an } \\
\text { especially impactful } \\
\text { material because of its high } \\
\text { moderating power }\end{array}$ \\
\hline PIPE THICKNESS & $\begin{array}{l}\text { Outer layer overlaying } \\
\text { waste form }\end{array}$ & $\begin{array}{l}0.001 \mathrm{~cm} \\
0.7112 \mathrm{~cm}\end{array}$ & $\begin{array}{l}\text { Thickness of CCC is the } \\
\text { default condition: thin layer } \\
\text { is included to study the } \\
\text { effect of removing the } \\
\text { CCC, and the thick layer is } \\
\text { included to study the effect } \\
\text { of a layer of moderator } \\
\text { immediately outside the } \\
\text { waste form and the effect of } \\
\text { any bagging material }\end{array}$ \\
\hline $\begin{array}{l}\text { CAN MASS MIXED } \\
\text { WITH FILLER }\end{array}$ & $\begin{array}{l}\text { Mass of steel convenience } \\
\text { canister }\end{array}$ & $0,0.5,1 \mathrm{~kg}$ & Values to establish trend \\
\hline $\begin{array}{l}\text { GRAPHITE OR } \\
\text { DEFAULT }\end{array}$ & & Graphite and cement & $\begin{array}{l}\text { Cement is used; graphite is } \\
\text { a good moderator but is not } \\
\text { used elsewhere in the study; } \\
\text { increased moderation tends } \\
\text { to increase } k_{\text {eff }}\end{array}$ \\
\hline
\end{tabular}


Table 4-6. Evaluated parameters and corresponding rationale for evaluating $\boldsymbol{k}_{\text {eff }}$ effect (continued)

\begin{tabular}{|c|c|c|c|}
\hline PARAMETER & DESCRIPTION & VALUES USED & $\begin{array}{l}\text { RATIONALE FOR } \\
\text { EVALUATION }\end{array}$ \\
\hline $\begin{array}{l}\text { GEOMETRY OF } \\
\text { WASTE FORM }\end{array}$ & Shape of waste form & Sphere vs. cylinder & $\begin{array}{l}\text { The cylinder is the initial } \\
\text { condition of the system, and } \\
\text { the sphere is a worst-case } \\
\text { condition from the standpoint } \\
\text { of single-waste form } \\
\text { geometry }\end{array}$ \\
\hline BE MASS & Mass of Be & $0,585 \mathrm{~g}$ & Transport limit \\
\hline $\begin{array}{l}\text { UNIFORM VS. } \\
\text { NONUNIFORM }\end{array}$ & $\begin{array}{l}\text { Uniform vs. nonuniform } \\
\text { case }\end{array}$ & $\begin{array}{l}\text { Triangular pitched 3- and } \\
\text { six-high uniform arrays } \\
\text { and centroid-specific } \\
\text { nonuniform arrays }\end{array}$ & $\begin{array}{l}\text { Uniform arrays are more } \\
\text { conservative because they } \\
\text { limit neutron leakage; } \\
\text { nonuniform arrays are best- } \\
\text { estimate arrays based on } \\
\text { compaction data }\end{array}$ \\
\hline $\begin{array}{l}\text { PERTURBED CCO } \\
\text { COORDINATES }\end{array}$ & & $\begin{array}{l}\text { Nominal, random sub- } \\
\text { centroid, every sub } \\
\text { centroid }\end{array}$ & $\begin{array}{l}\text { In Appendix G, off-nominal } \\
\text { CCO coordinates used to } \\
\text { examine position sensitivity; } \\
\text { the extent of CCO in the } \\
\text { Reedlunn and Bean [8] model } \\
\text { is taken as a reasonable range } \\
\text { of uncertainty }\end{array}$ \\
\hline $\begin{array}{l}\text { PERTURBED } \\
\text { ORIENTATION }\end{array}$ & & $\begin{array}{l}\text { Vertical orientation, } \\
\text { random orientation, } \\
\text { Reedlunn and Bean [8] } \\
\text { best estimate }\end{array}$ & $\begin{array}{l}\text { In Appendix G, off-nominal } \\
\text { CCO orientation is used to } \\
\text { examine sensitivity }\end{array}$ \\
\hline $\mathrm{B}_{4} \mathrm{C}$ CONTENT & $\begin{array}{l}\mathrm{B}_{4} \mathrm{C} \text { uniformly mixed with } \\
\text { waste form }\end{array}$ & $\begin{array}{c}0 \mathrm{~g}, \\
50 \mathrm{~g}(\mathrm{set}-1,1 \mathrm{a}, 2) \\
25 \mathrm{~g}(\text { set-3) }\end{array}$ & $\begin{array}{l}0 \mathrm{~g} \text { is the worst-case value; } \\
\text { other values were included } \\
\text { for comparison }\end{array}$ \\
\hline MGO DENSITY & $\begin{array}{l}\text { Density of } \mathrm{MgO} \text { within } \\
\text { envelope of CCOs }\end{array}$ & $50 \%$, Unperturbed & $\begin{array}{l}\mathrm{MgO} \text { is not placed on every } \\
\text { stack and varies by what is in } \\
\text { the stack of packages }\end{array}$ \\
\hline MGO EXTENT & Size of $\mathrm{MgO}$ envelope & Around CCOs only & $\begin{array}{l}\text { More than 1-2 mean free } \\
\text { paths outside of envelope; } \\
\text { neutrons leak or are absorbed } \\
\text { by } \mathrm{NaCl}\end{array}$ \\
\hline $\begin{array}{l}\text { PACKAGE LOCAL } \\
\text { DENSITY }\end{array}$ & & Unperturbed & $\begin{array}{l}\text { Groups of } 3 \text { immediately } \\
\text { adjacent waste forms } \\
\text { evaluated in uniform array } \\
\text { cases considered an unlikely } \\
\text { condition }\end{array}$ \\
\hline $\begin{array}{l}\text { TIGHTER RUN } \\
\text { PARAMETERS }\end{array}$ & & & Examined in Appendix E \\
\hline
\end{tabular}

Variations in array assumptions were achieved by grouping cases into different sets, as seen in Table 6-1. The full listing of results for these datasets is provided in Addendum 1.

The following diagrams (Figure 4.13 and Figure 4.14) provide additional information to illustrate the construction of the models and the use of the terminology. 


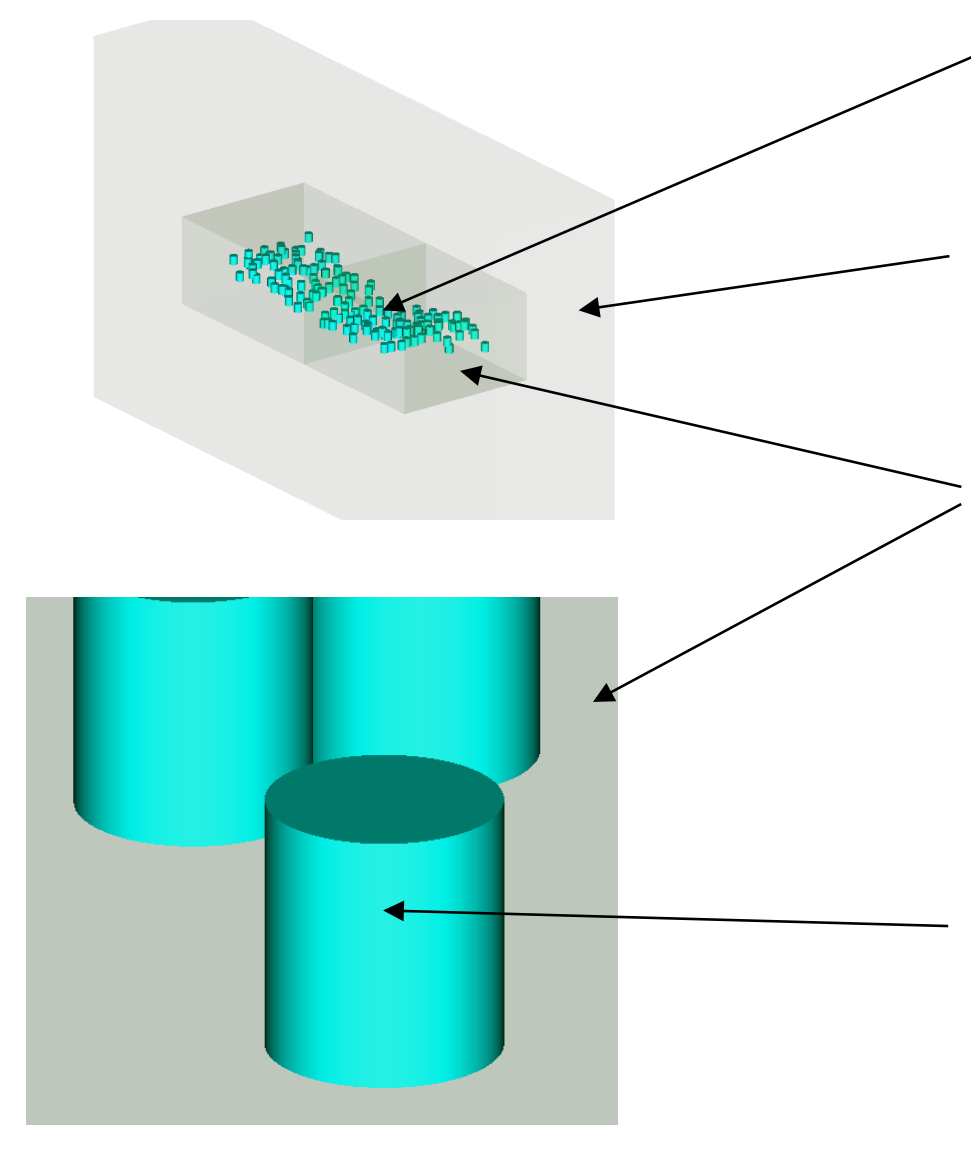

Nonuniform array of vertically oriented cylinders (set-2 cases), representing the waste forms placed by coordinate.

WIPP repository salt, the naturally occurring environment from which the rooms are formed

50/50 mixture of $\mathrm{MgO}$ and salt. The material interstitial to the waste forms is called the interstitial reflector material because it is external to the waste forms and uniformly mixed, yet it is separate from the outer salt material, which is the natural part of the WIPP repository.

Waste forms (as cylinders in this example), showing outer pipe as solid color. The outer pipe may be a thin $(0.001 \mathrm{~cm})$ or thick $(0.7112 \mathrm{~cm})$ stainless steel pipe or poly material layer.

The outer pipe is called the discrete reflector (shown here as transparent) because it is external to the waste form and surrounds it, forming a reflector which is directly adjacent to the waste form and separate from the material which is interstitial to the waste forms.

Waste form inside the discrete reflector (dark solid color) showing outer discrete reflector as transparent. The waste form is a uniformly mixed material that always consists of $\mathrm{PuO}_{2}$ and moderator (water or poly) and that sometimes consists of a filler material (graphite or generic with and without stainless steel from the convenience can) and/or Be.

Figure 4.13. Diagram showing how the nonuniform array model is constructed with cylinders. 


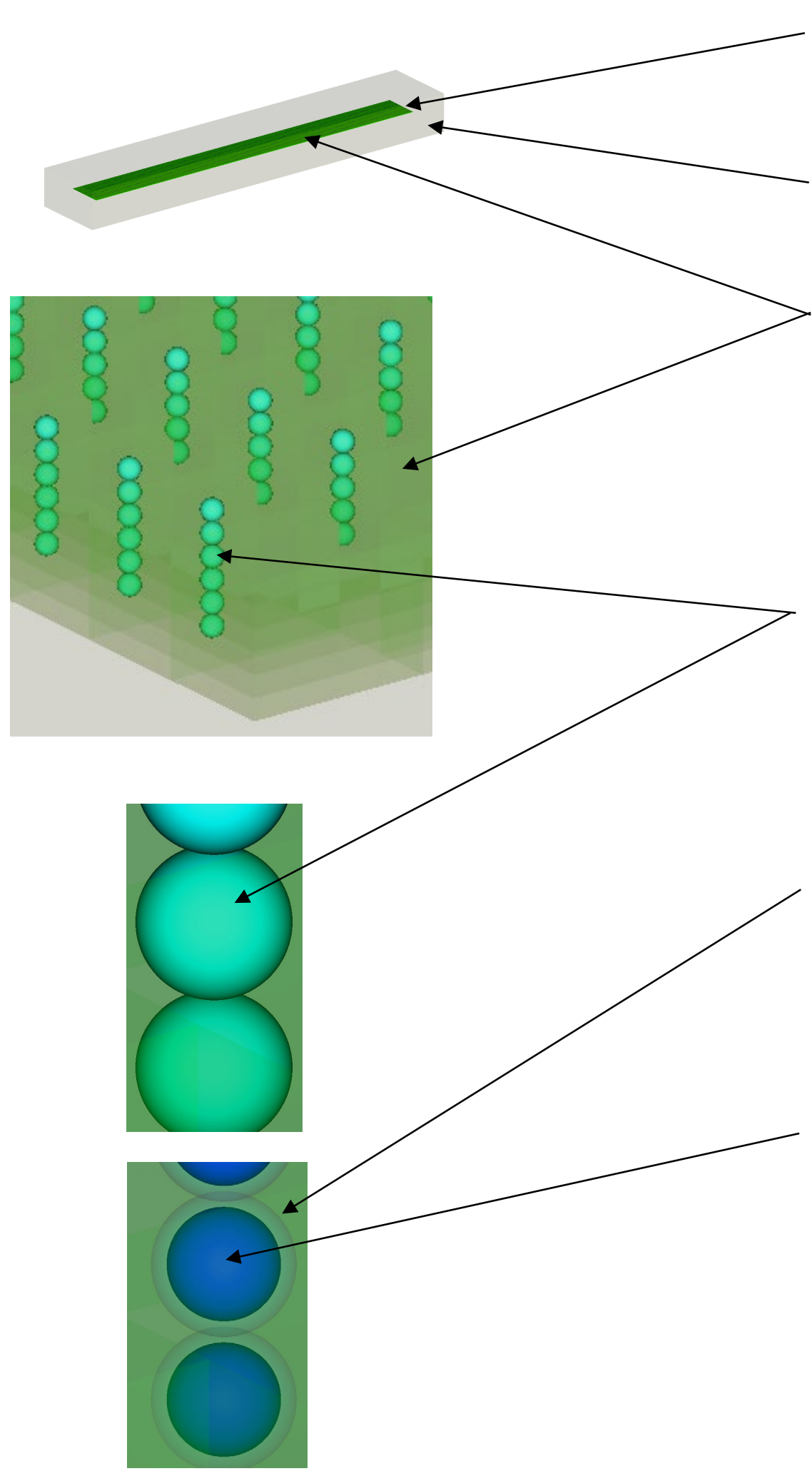

Uniform array representing the waste forms placed in a triangular pitch in six-high stacks of spheres (set-3 cases, detail shown in the following subfigures).

WIPP repository salt, the naturally occurring environment from which the rooms are formed.

50/50 mixture of $\mathrm{MgO}$ and salt. The material interstitial to the waste forms is called the interstitial reflector material because it is external to the waste forms and uniformly mixed, yet it is separate from the outer salt material, which is the natural part of the WIPP repository.

Waste forms (as spheres in this example) showing outer pipe as solid color. The outer pipe may be a thin $(0.001 \mathrm{~cm})$ or thick $(0.7112 \mathrm{~cm})$ stainless steel pipe or a poly material layer.

The outer pipe is called the discrete reflector, here shown as transparent, because it is external to the waste form and surrounds it, forming a reflector which is directly adjacent to the waste form and separate from the material which is interstitial to the waste forms.

Waste form inside the discrete reflector (dark solid color). The waste form is a uniformly mixed material that always consists of $\mathrm{PuO}_{2}$ and moderator (water or poly) and that sometimes consists of a filler material (graphite or generic with and without stainless steel from the convenience can) and/or Be.

Figure 4.14. Diagram showing how a uniform array model is constructed with spheres. 


\subsection{GENERAL INPUT DATA TABLES AND MATERIAL COMPOSITIONS}

Additional data used in the analysis are presented in Table 4.6 and Table 4.7.

Table 4.6. CCO and CCC data used with permission [3]

\begin{tabular}{|c|c|c|c|}
\hline & Inches & Centimeters & Notes \\
\hline \multicolumn{4}{|l|}{ CCO dimensions } \\
\hline Outer diameter & 22.618 & 57.45 & Inner diameter plus wall thickness from CQ5508A5 ${ }^{a}$ \\
\hline Outer radius & 11.309 & 28.725 & $(0.15 \mathrm{~cm} / 0.0590 \mathrm{in}$ [16 gauge $])$ \\
\hline Inner diameter & 22.50 & 57.15 & From CCO-DWG-0001R3 \\
\hline Inner radius & 11.25 & 28.575 & \\
\hline Wall/top/bottom thickness & .059 & 0.15 & $\begin{array}{l}\text { From CQ5508A5, } 16 \text { gauge (drawing gives range of } 0.0543 \\
-0.0590 \mathrm{in} \text { ) }\end{array}$ \\
\hline $\begin{array}{l}\text { Outside height (including bolt } \\
\text { ring) }\end{array}$ & $34.75^{b}$ & 88.265 & $\begin{array}{l}\text { Outside height of drum from CQ5508A5 } 5^{a}(34.25 \mathrm{in})+0.5 \text { in } \\
\text { (conservative underestimate of height added from bolt ring) }\end{array}$ \\
\hline $\begin{array}{l}\text { Outside height (drum only, } \\
\text { not including additional } \\
\text { height from bolt ring) }\end{array}$ & 34.25 & 86.995 & $\begin{array}{l}\text { From CQ5508A5, includes additional height from curvature } \\
\text { of top and bottom of drum (underestimate of } 0.691 \text { in for } \\
\text { each) and top and bottom thickness }\end{array}$ \\
\hline $\begin{array}{l}\text { Inner height (at outermost } \\
\text { edge) }\end{array}$ & 32.75 & 83.185 & $\begin{array}{l}\text { From CCO-DWG-0001R3 (does not include drum top and } \\
\text { bottom thickness) }\end{array}$ \\
\hline Material & & & Carbon Steel \\
\hline \multicolumn{4}{|l|}{$\mathrm{CCC}$ dimensions } \\
\hline Outer diameter & 6.625 & 16.8275 & From CCO-DWG-0001R3, nominal pipe size (NPS) 6, \\
\hline Outer radius & 3.3125 & 8.4138 & $\mathrm{SCH} 40$ \\
\hline Wall thickness & 0.28 & 0.7112 & \\
\hline Inner diameter & 6.065 & 15.4051 & Outer dimension minus wall thickness \\
\hline Inner radius & 3.0325 & 7.7026 & \\
\hline Flange thickness & 1.0 & 2.54 & From CCO-DWG-0001R3, CLASS 150 , NPS 6, top and \\
\hline Flange diameter & 11.02 & 27.9908 & bottom (diameter/radius may be modeled same as CCC pipe \\
\hline Flange radius & 5.51 & 13.9954 & outer dimension) \\
\hline Ring gasket & $1 / 16$ & 0.1588 & From CCO-DWG-0001R3, NPS 6, 1/16 THK \\
\hline Cavity height & 26.9425 & 68.4340 & $\begin{array}{l}\text { From CCO-DWG-0001R3 }(26.50+0.38+(1 / 16)) \text {, includes } \\
\text { additional space from gasket }\end{array}$ \\
\hline Material & & & $\begin{array}{l}\text { From CCO-DWG-0001R3, stainless steel 304, use code } \\
\text { standard composition }\end{array}$ \\
\hline \multicolumn{4}{|l|}{ Dunnage } \\
\hline Thickness & $3 / 4$ & 1.905 & From CCO-DWG-0001R3, all plates (total of 10 plates -2 \\
\hline Outer diameter & 22.0 & 55.88 & end and 3 ring plates on bottom and 2 end and 3 ring plates \\
\hline Outer radius & 11.0 & 27.94 & on top \\
\hline Ring plate inner diameter & 11.5 & 29.21 & From CCO-DWG-0001R3 \\
\hline Ring plate inner radius & 5.75 & 14.605 & \\
\hline Material & & & $\begin{array}{l}\text { Plywood with density of } 0.387 \mathrm{~g} / \mathrm{cm}^{3} \text { (SCALE redwood } \\
\text { standard composition) }\end{array}$ \\
\hline
\end{tabular}


Table 4.7. Material densities $\left(\mathrm{g} / \mathrm{cm}^{3}\right)$

\begin{tabular}{|l|c|l|}
\hline \multicolumn{1}{|c|}{ Parameter } & Value $\left(\mathbf{g} / \mathrm{cm}^{3}\right)$ & \multicolumn{1}{c|}{ Source } \\
\hline $\mathrm{PuO}_{2}$ & $11.46^{8}$ & $\begin{array}{l}\text { Compendium of Material Composition Data for } \\
\text { Radiation Transport Modeling [16] }\end{array}$ \\
\hline Polyethylene $\left(\mathrm{CH}_{2}\right)$ & 0.92 & Saylor and Scaglione [3] \\
\hline $\mathrm{H}_{2} \mathrm{O}$ & 0.9982 & $\begin{array}{l}\text { Compendium of Material Composition Data for } \\
\text { Radiation Transport Modeling [16] }\end{array}$ \\
\hline $\mathrm{Be}$ & 1.848 & Saylor and Scaglione [3] \\
\hline $\mathrm{MgO}$ & 1.45 & Saylor and Scaglione [3] \\
\hline $\mathrm{Salt}(\mathrm{NaCl})$ & 2.165 & Saylor and Scaglione [3] \\
\hline graphite & 2.3 & $\begin{array}{l}\text { Compendium of Material Composition Data for } \\
\text { Radiation Transport Modeling [16] }\end{array}$ \\
\hline generic & 2.84375 & Saylor and Scaglione [3] \\
\hline $50 \%$ mixture $\mathrm{MgO} / \mathrm{salt}$ & 1.737 & calculated \\
\hline brine & 1.1602 & calculated \\
\hline
\end{tabular}

${ }^{8}$ This value is slightly higher than the actual theoretical density. 
This page is intentionally blank 


\section{ASSUMPTIONS}

1. The WIPP repository accepts waste packages of varying designs and payloads [5], whereas this analysis is specific to CCOs. No analytical attempt is made to ascertain the reactivity impact of a room of CCOs mixed with other containers. Rather, the assumption being made is that the CCO with 380 FGE that was evaluated under very conservative assumptions is the bounding configuration of packages to date, so any other packages would have less reactivity from a FGE perspective. The geomechanical impacts are less well known. However, various justifications have been made when using the Reedlunn and Bean data [8].

2. The waste streams are assumed to be uniformly mixed. A significant precedence exists for assuming that uniform mixtures are appropriate to use when significant variations in particle sizes or lumping of constituents may be possible or expected. The ability of neutrons to travel and interact between heterogenous regions of fissile material is typically negated by the heterogeneity. Significant justification exists in the literature, and some analytical justification is also provided. 
This page is intentionally blank 


\section{ANALYSIS DISCUSSION}

The analysis presented herein is a parametric evaluation of the most important system parameters and provides results covering a large range of $\mathrm{H} /{ }^{239} \mathrm{Pu}$ variations. A large range of variations is included to inform an FEP evaluation regarding the conditions under which criticality may be possible, but it does not define the probability of such an occurrence. The very conservative nature of the analysis method does allow an FEP evaluation some latitude in that regard. Many of the analyzed configurations greatly exceed conditions and established controls defined for dilute and dispose waste stream in an attempt to establish a generic approach that may be used for packaging other waste streams using the CCO container.

The generic parametric evaluation that is presented in this report provides results for the broad range of system parameters. Not all of these parameters are expected to be part of any actual waste stream, nor are all configurations physically possible, but these results provide useful information to better understand repository behavior and to help define the unique neutron physics at work in the repository environment.

The generic parametric evaluation presented in this report is based on the previous post-closure criticality methods described by Saylor, Scaglione [3], and Brickner 2019 [4]. Saylor's work forms the basis for the generic waste form filler material (but without the $50 \mathrm{~g} \mathrm{~B}_{4} \mathrm{C}$ included by Saylor), evaluated as an appropriate representation of the dilute surplus $\mathrm{Pu}$ waste from. The methodology in Brickner's document [4] forms the basis for modeling the waste from spacing and centroid locations according to geomechanical analysis from Reedlunn and Bean [7]. Brickner's work [4] also forms the basis for the interstitial reflector material considerations. Additional methods are introduced in this analysis, but they are extensions of the previous work.

Each model used in this analysis considers the waste forms based on a physically realistic representation of the masses of materials evaluated. Specifically, each variation of the waste form considers the theoretical density of the pure material and the mass for each case to determine the constituent's volume. An alternative approach would be to consider the initial volume of the waste form container and then to distribute the constituents over that volume. Because the main interest in this work is to evaluate the effect of compaction, it is desirable to eliminate spacing where possible. Therefore, removing the overall initial dimensions from consideration allows the analysis to proceed conservatively in that regard. Therefore, for each parametric sweep, the uniform waste form volume is calculated as the sum of individual components using the maximum or theoretical density for each constituent from Table 4.2. In this manner, the one constant in all cases is the FGE mass of ${ }^{239} \mathrm{Pu}$ per CCO so changes in the $k_{\text {eff }}$ associated waste form composition can be evaluated for the many system parameters considered.

There are two waste form geometries evaluated: spherical and cylindrical. For the spherical geometry, as the parametric sweeps over waste form constituent masses are performed, the total volume defines the sphere radius. For the cylindrical cases, three radii are arbitrarily selected for evaluation-4.8, 6.25, and $7.7 \mathrm{~cm}$ - with 7.7 as the maximum because it defines the inner diameter of the CCC, and the total volume defines the cylinder height.

In general, the waste form's cylinder radius has a strong effect on $k_{\text {eff }}$, as shown in Section 6.1 (correlation study). The cylinder radius is related to how the other system parameters affect $k_{\text {eff }}$, especially at the extreme ends of the sweeps, and more particularly in moderation sweeps. Therefore, the parametric sweeps cover these various generic radii to (1) evaluate possible options for waste form processing, and (2) to determine the various system $k_{\text {eff }}$ effects. For example, the dilute surplus Pu waste stream is known to use a can-bag-can configuration for which the inner diameter of the inner can or the outer diameter of either can could be used to define the radius of the waste form. The results for the desired radius case could be used to evaluate this waste stream geometry. Furthermore, the results from the equivalent volume sphere waste form models could also be used to evaluate this waste stream. Both geometries may 
be used, for example, if the cylindrical geometry is used for early timeframes and the spherical geometry is used for later timeframes. Additional value is gained in allowing future modifications to be made to waste form geometries, as informed by the results of the additional radii sweeps. Generically, enough information is intended to be provided so that it is generically applicable to a wide range of waste streams.

For both waste form geometries, a discrete reflector is included so that the reactivity effect of material that is directly adjacent to the waste form can be evaluated. At emplacement, the CCC pipe wall is the discrete reflector and is modeled as stainless steel. Later, this may degrade or otherwise change, and other materials may collect around the waste forms. Therefore, polyethylene is also considered as a discrete reflector material. The stainless steel from the pipe or the cans represents a material known to be present at least initially and which should have some impact on the reflection of neutrons for low moderator cases. The polyethylene represents a thermalizing reflector which should have an impact along the entire neutron energy spectrum. These two materials are sufficient to cover the known and predominate quantities of materials in the system (see Section 4.2.2.3). No attempt is made to evaluate how much discrete reflector material from sources external to the $\mathrm{CCO}$, from the $\mathrm{CCO}$ itself, or from $\mathrm{CCO}$ components may be present. This is intentional because such an evaluation could result in an unnecessary restriction or limit, and neither is necessary since this approach covers multiple scenarios over the long regulatory time frame. Therefore, the thickness of the CCC is used to evaluate the $k_{\text {eff }}$ effect of a thick discrete reflector that may be directly adjacent to the waste form, and $0.001 \mathrm{~cm}$ is arbitrarily selected to evaluate the $k_{\text {eff }}$ effect of a thin discrete reflector that may be directly adjacent to the waste form.

In this manner, the CCC thickness is essentially a surrogate for material forming a discrete reflector. Also, this allows waste stream analysis to consider all material present in the repository system during compaction as also being present in the actual waste form and its container(s) to be part of the uniformly mixed constituents for volumes up to the inner diameter of the CCC. For example, for the Savanah River Site (SRS) waste form, the can may or may not be included in the waste form. The thickness of the can is unknown, but it is expected to be adequately represented by either the thick or thin stainless steel discrete reflector. On the other hand, to cover the possibility of the can or stainless steel from another external source mixing with the waste form, sweeps with stainless steel as filler material are also considered (see Section 4.2.3). In that case, the waste form evaluation may use the full thickness of the CCC as a discrete reflector to evaluate the outside sources of discrete reflector materials, or the evaluation may use the known thickness of packaging inside the CCC such as the convenience can, or a combination of the two. Using the discrete reflector in this way allows for flexibility in how a waste form may be evaluated with respect to treating container material as either uniformly mixed with the waste form, or discreetly, or both, if the difference depends on how the configuration changes over time. It also allows for flexibility in future changes to waste form processing and packaging.

The analysis includes flexibility based on to the wide range of material sweeps for each configuration. For example, for the surplus Pu configuration, there are waste form sweeps with only filler material (generic or graphite) mixed uniformly with the waste form (no stainless steel from the convenience can), cases with only material from the convenience can mixed uniformly with the waste form (no filler), and cases with both mixed uniformly with the waste form. Therefore, if it is desirable to consider the waste form as a cylinder within a can, then those results are available with no can material uniformly mixed with the waste form. On the other hand, if it is desirable to consider the waste form with a radius equal to the outer diameter of either the inner can or the outer can, then there are cases with a range of steel masses mixed uniformly with the waste form and with discrete reflectors to account for the CCC or material from other outside sources.

The discrete reflector is evaluated at a very small thickness $(0.001 \mathrm{~cm})$ and at the CCC pipe thickness $(0.7112 \mathrm{~cm})$ for both steel and polyethylene. The use of a very small reflector allows the trend in $k_{\text {eff }}$ due 
to discrete reflector thickness to be analyzed, and it also provides results from the very thin discrete reflector in case these results are more conservative than those of the thick discrete reflector. The use of polyethylene allows for the consideration of external plastic sources which may be present and come to be compacted around the waste forms or for plastics known to reside adjacent to the waste form due to packaging. Because it is very unlikely that a large quantity of plastic from outside sources would collect around every waste form in the system, these results are extremely conservative and provide an upper bound of the possible scenarios.

For the waste form filler materials-i.e., nonfissile, non-hydrogenous, non-special reflector as defined in the CCO TRUPACT-II Safety Analysis Report [9], and non-H bearing materials - two materials are selected to be bounding materials (see Section 4.2.3).

The SCALE models used in this generic evaluation are based on the WIPP repository room layout. Uniform and nonuniform arrays are the two model types used to evaluate a WIPP room full of CCOs. The uniform arrays mimic the as-loaded configuration at time zero with a triangular pitch, as seen in Figure 6.1. Although the WIPP panel rooms are actually currently loaded with 7-packs that have a hexagonal spacing in a three-high uniform array of waste forms, a three-high triangular pitch is used in the SCALE model as a conservatism from Saylor and Scaglione [3] and is consistent with Brickner [4]. The magnitude of the triangular pitch is based on the overall room closure from Reedlunn and Bean [8] (see

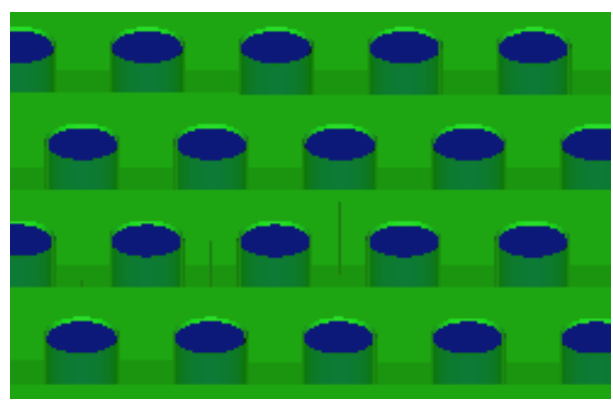

Figure 6.1. Diagram of the triangular pitch for the three-high uniform arrays. Sections 4.2.1).

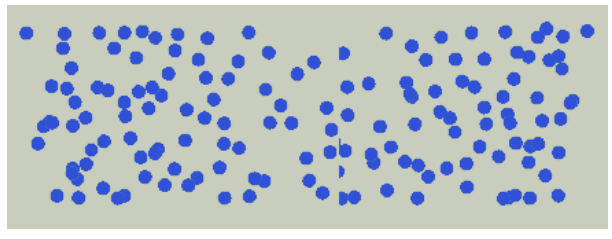

Figure 6.2. Diagram of the nonuniform arrays.
The nonuniform arrays are based on the actual centroid locations for both the lower and upper horizon calculations from Reedlunn and Bean [8], as shown in Figure 6.2.

One additional variation of the uniform array model is used to evaluate the $k_{\text {eff }}$ effect of placing two waste forms per $\mathrm{CCO}$ [20]; this model is similar to the three-high uniform array, except it is a six-high configuration in which each waste form has half the allowable 380 FGE separated physically only by the discrete reflector thickness, as shown in Figure 6.3.

For all uniform and nonuniform array models, both waste form geometries are evaluated for all parametric sweeps listed in Table 6.2. Where necessary, additional sweeps are added so that the neutron physics of the system can be evaluated.

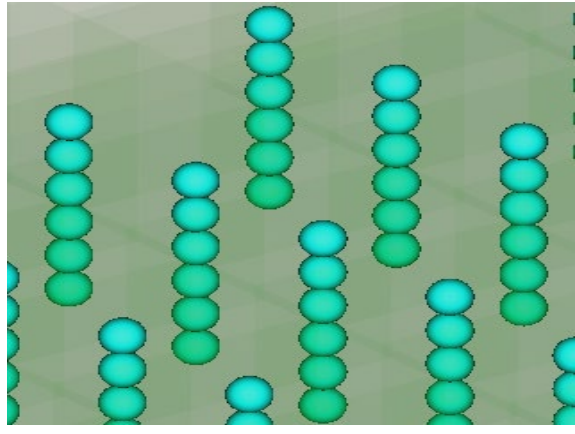

Figure 6.3. Diagram of the triangular pitch for the six-high uniform arrays two represent two waste forms per CCO.

As specified by Brickner [4], all evaluations use the bounding uniform mixture of $50 \% \mathrm{MgO}$ and $50 \%$ salt for the interstitial reflector material. An interstitial reflector material of $50 \%$ salt and $50 \% \mathrm{MgO}$ bounds pure salt, as previously shown by Brickner [4]. It is likely that as the Supersacks of MgO break during roof fall or otherwise decompose, the granules of $\mathrm{MgO}$ will fall down between the as-yet uncompacted CCOs and will form piles on the room floor. When the final compaction condition is 
reached, it is most likely that the $\mathrm{MgO}$ granules will be underneath most waste forms. The final compaction configuration is a relatively high neutron leakage configuration, so the majority of the neutrons escaping the waste forms will not likely contact $\mathrm{MgO}$. Therefore, considering the $\mathrm{MgO}$ uniformly mixed with salt in the interstitial reflector ensures that any neutron escaping the waste form will encounter $\mathrm{MgO}$ and will be less likely to be captured than if it had seen salt. This approach is conservative and bounding, indicating that it is appropriate to avoid unnecessary restrictions or constraints of limits due to use of $\mathrm{MgO}$.

The principal criticality calculations of this study are categorized into the cases shown in Table 6.1 and the subcases shown in Table 6.2. Cases are defined based on array shape. Subcases are defined as parameter sweeps.

Table 6.1. Description of the cases to evaluate reactivity trends

\begin{tabular}{|c|c|c|}
\hline Case & Description & Location of results \\
\hline set-1 & $\begin{array}{l}\text { Uniform array with three-high spheres and } \\
\text { cylinders, with the most conservative spacing based } \\
\text { on the SNL compaction data. Waste forms are } \\
\text { stacked directly on top of each other. }\end{array}$ & $\begin{array}{l}\text { Appendix A } \\
\text { Addendum } 1\end{array}$ \\
\hline set-1a & $\begin{array}{l}\text { Uniform array with three-high spheres and } \\
\text { cylinders, with an alternative spacing based on the } \\
\text { SNL compaction data. Waste forms are stacked } \\
\text { directly on top of each other. }\end{array}$ & $\begin{array}{l}\text { Appendix B } \\
\text { Addendum } 1\end{array}$ \\
\hline set-2 & $\begin{array}{l}\text { Nonuniform array with spacing based on SNL } \\
\text { compaction data. Two sets are included: one set for } \\
\text { the upper horizon (uh), and one set for the lower } \\
\text { horizon (lh). }\end{array}$ & $\begin{array}{l}\text { Appendix C } \\
\text { Addendum } 1 \\
\text { Appendix D } \\
\text { Addendum } 1\end{array}$ \\
\hline set-3 & $\begin{array}{l}\text { Uniform array with six-high spheres and cylinders, } \\
\text { with the most conservative spacing based on the } \\
\text { SNL compaction data. FGE limited to } 180 \text { per } \\
\text { waste form. Waste forms are stacked directly on top } \\
\text { of each other. }\end{array}$ & $\begin{array}{l}\text { Appendix F } \\
\text { Addendum } 1\end{array}$ \\
\hline set-3a & $\begin{array}{l}\text { Uniform array with six-high spheres and cylinders, } \\
\text { with an alternative spacing based on the SNL } \\
\text { compaction data. FGE limited to } 180 \text { per waste } \\
\text { form. Waste forms are stacked directly on top of } \\
\text { each other. }\end{array}$ & $\begin{array}{l}\text { Appendix I } \\
\text { Addendum } 1\end{array}$ \\
\hline
\end{tabular}

For each of the cases listed in Table 6.1 above, the full set of parametric sweeps is provided in Table 6.2 below. 
Table 6.2. Summary of parametric sweeps for both cylindrical and spherical waste forms (values per CCO)

\begin{tabular}{|c|c|c|c|c|c|c|c|c|}
\hline Subcase & $\begin{array}{c}\text { Waste } \\
\text { form } \\
\text { geometry }\end{array}$ & FGE & $\begin{array}{l}\text { Waste form } \\
\text { moderator }\end{array}$ & \begin{tabular}{|c|} 
Waste \\
form \\
filler \\
material
\end{tabular} & $\begin{array}{c}\text { Discrete reflector } \\
\text { material }\end{array}$ & $\begin{array}{l}\text { Waste } \\
\text { form Be } \\
\text { mass (g) }\end{array}$ & $\begin{array}{c}\text { Interstitial } \\
\text { reflector } \\
\text { material }\end{array}$ & $\mathrm{B}_{4} \mathrm{C}(\mathrm{g})$ \\
\hline 1 & cylinder & 380 & water & graphite & SS (from the can) & 0,585 & $50 / 50 \mathrm{salt} / \mathrm{MgO}$ & 0 \\
\hline 2 & cylinder & 380 & polyethylene & graphite & $\mathrm{SS}$ (from the can) & 0,585 & $50 / 50 \mathrm{salt} / \mathrm{MgO}$ & 0 \\
\hline 3 & cylinder & 380 & water & graphite & polyethylene & 0,585 & $50 / 50 \mathrm{salt} / \mathrm{MgO}$ & 0 \\
\hline 4 & cylinder & 380 & polyethylene & graphite & polyethylene & 0,585 & $50 / 50 \mathrm{salt} / \mathrm{MgO}$ & 0 \\
\hline 5 & cylinder & 380 & water & generic & SS (from the can) & 0,585 & $50 / 50$ salt $/ \mathrm{MgO}$ & 0 \\
\hline 6 & cylinder & 380 & polyethylene & generic & SS (from the can) & 0,585 & $50 / 50 \mathrm{salt} / \mathrm{MgO}$ & 0 \\
\hline 7 & cylinder & 380 & water & generic & polyethylene & 0,585 & $50 / 50$ salt $/ \mathrm{MgO}$ & 0 \\
\hline 8 & cylinder & 380 & polyethylene & generic & polyethylene & 0,585 & $50 / 50$ salt $/ \mathrm{MgO}$ & 0 \\
\hline 9 & sphere & 380 & water & graphite & SS (from the can) & 0,585 & $50 / 50$ salt $/ \mathrm{MgO}$ & 0 \\
\hline 10 & sphere & 380 & polyethylene & graphite & SS (from the can) & 0,585 & $50 / 50$ salt $/ \mathrm{MgO}$ & 0 \\
\hline 11 & sphere & 380 & water & graphite & polyethylene & 0,585 & $50 / 50$ salt/MgO & 0 \\
\hline 12 & sphere & 380 & polyethylene & graphite & polyethylene & 0,585 & $50 / 50$ salt $/ \mathrm{MgO}$ & 0 \\
\hline 13 & sphere & 380 & water & generic & SS (from the can) & 0,585 & $50 / 50$ salt/MgO & 0 \\
\hline 14 & sphere & 380 & polyethylene & generic & SS (from the can) & 0,585 & $50 / 50 \mathrm{salt} / \mathrm{MgO}$ & 0 \\
\hline 15 & sphere & 380 & water & generic & polyethylene & 0,585 & $50 / 50$ salt $/ \mathrm{MgO}$ & 0 \\
\hline 16 & sphere & 380 & polyethylene & generic & polyethylene & 0,585 & $50 / 50$ salt $/ \mathrm{MgO}$ & 0 \\
\hline 17 & cylinder & 380 & polyethylene & graphite & polyethylene & 0,585 & $50 / 50$ salt/MgO & 50 \\
\hline 18 & sphere & 380 & polyethylene & graphite & polyethylene & 0,585 & $50 / 50$ salt/MgO & 50 \\
\hline
\end{tabular}

\subsection{DISCUSSION OF THE THREE-HIGH UNIFORM ARRAY PARAMETRIC SWEEPS}

The three-high uniform array results for set-1 are provided in Appendix A, and the results for set-1a are provided in Appendix B. The results presented in Appendix A and Appendix B provide $\mathrm{H} /{ }^{239} \mathrm{Pu}$ curves which show the $k_{\text {eff }}$ trend, with the following parameters associated with the waste form using $380 \mathrm{FGE}$ as ${ }^{239} \mathrm{Pu}$ mass:

- waste form triangular pitch (the reactivity difference between set-1 and set-1a)

- waste form moderator material and mass

- waste form filler material and mass

- waste form Be mass

- discrete reflector material and thickness

The system's $k_{\text {eff }}$ trends are divided into two categories: primary and secondary. Primary categories are $k_{\text {eff }}$ trends which may have a direct, significant impact on a FEP. Secondary $k_{\text {eff }}$ trends are important, but they may not have a significant impact on a FEP. Each trend is reviewed in third-tier headings of this subsection.

The full set of parametric sweeps provide information about the $k_{\text {eff }}$ trends associated with the system, and the full set of results are found in Appendix A and Appendix B.

All of the results for set- 1 and set-1a are plotted below in Figure 6.4 and Figure 6.5, respectively. For each set, representative curves for the cylinder and spheres are accentuated for both discrete reflector thicknesses. The representative curves are provided to show which curves could be selected for limits if appropriate. These curves typically bound their sub-set of results at the moderator mass, resulting in a $k_{\text {eff }}$ of 1 . 


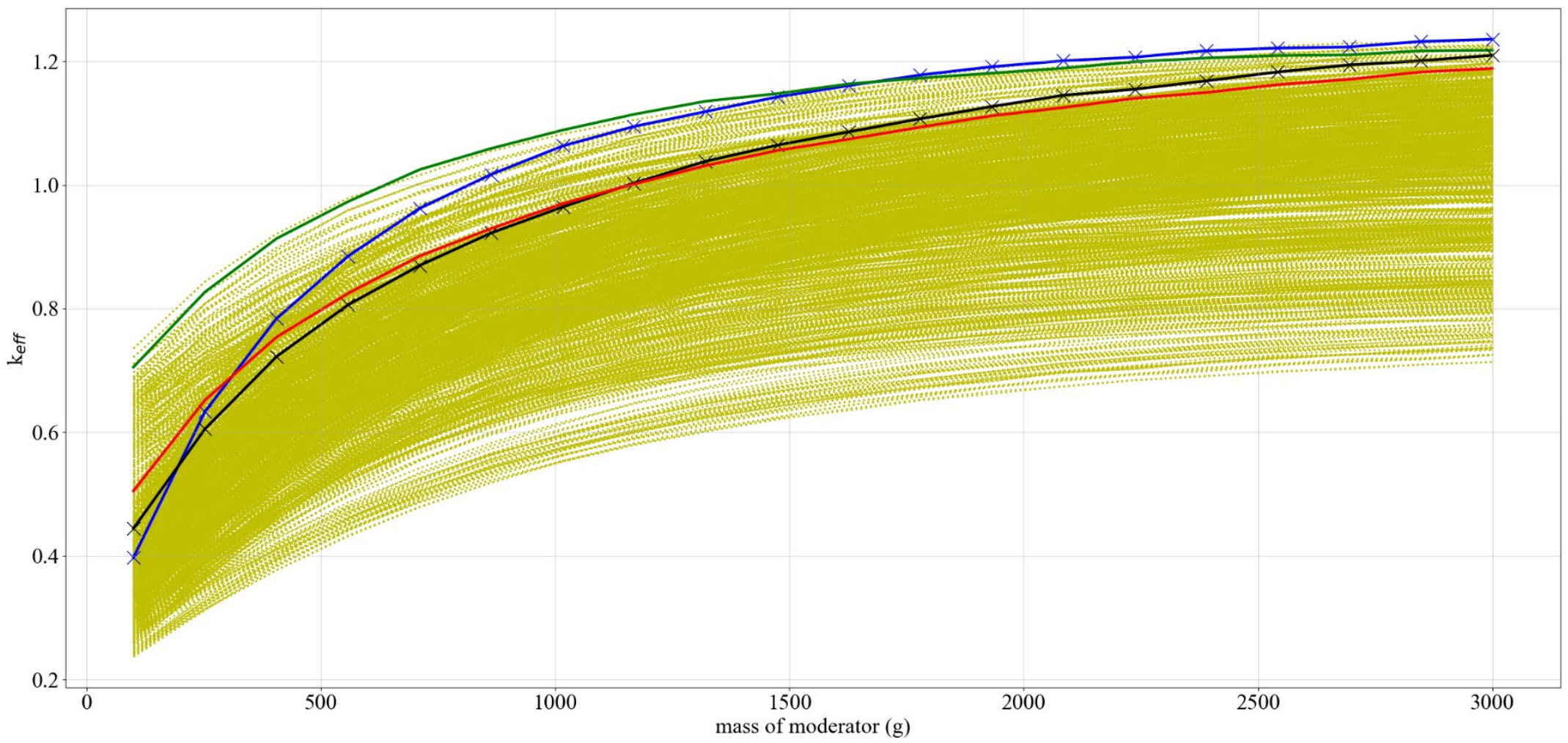

set-1 all data

* set-1-12, sphere, FGE+poly mod only, thin poly discrete reflector

$*$ set-1-4, cylinder, FGE+ poly mod only, thin poly discrete reflector
- set-1-4, cylinder, FGE+poly mod only, thick poly discrete reflecto

et-1-12, sphere, FGE+poly mod only, thick poly discrete reflector

Figure 6.4. Full set of results for set-1 compared to various representative curves. 


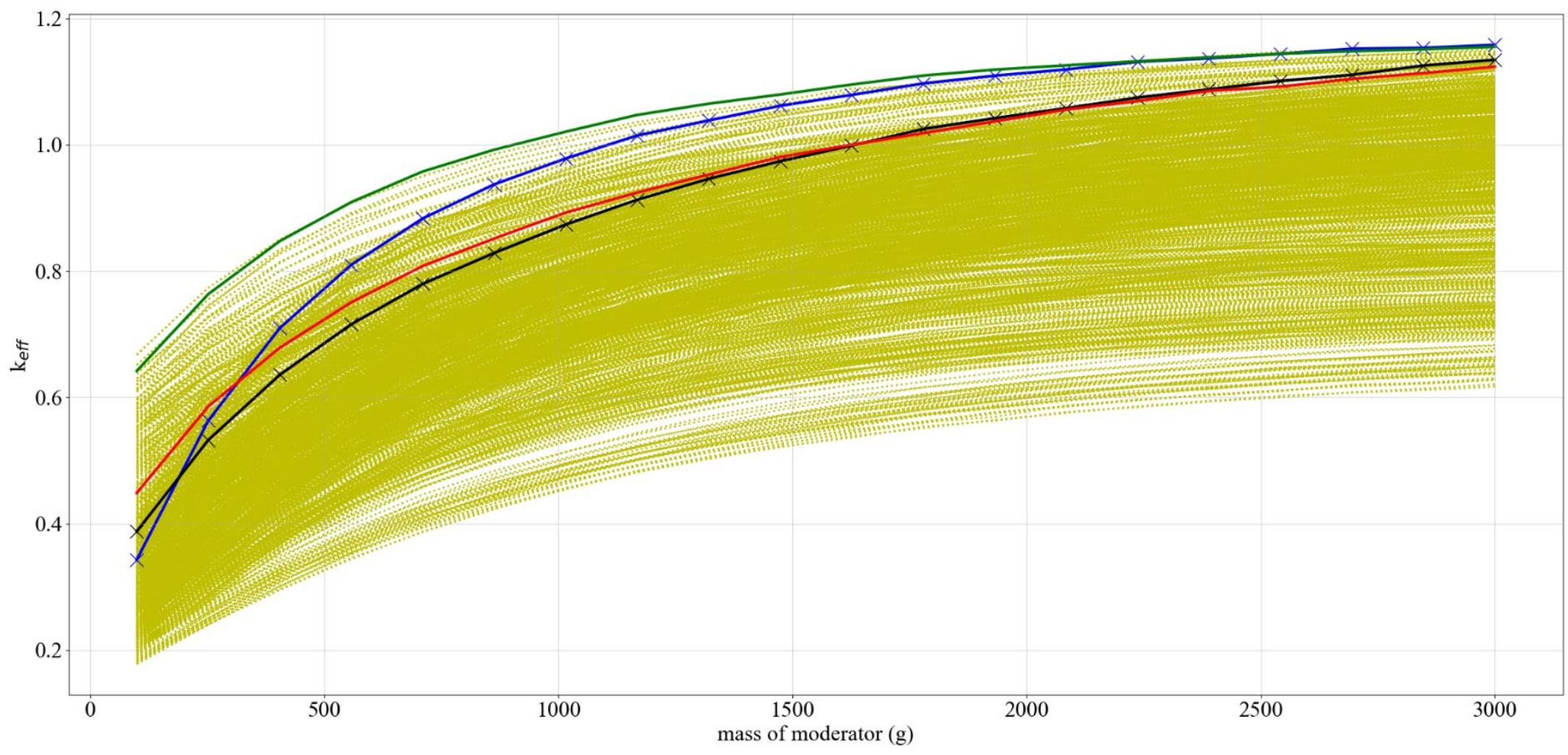

set-1a all data

$\rightarrow$ set-1a-4, cylinder, FGE+poly mod only, thin poly discrete reflector set-1a-4, cylinder, FGE+poly mod only, thick poly discrete reflector
$*$ set-1a-12, sphere, FGE+poly mod only, thin poly discrete reflector set-1a-12, sphere, FGE+poly mod only, thick poly discrete reflector

Figure 6.5. Full set of results for set-1a compared to various representative curves. 
The primary $k_{\text {eff }}$ trends of the system are highly dependent upon the spacing, moderator mass, geometry, and filler mass. These are discussed in more detail below.

\subsubsection{Waste Form Spacing (Primary Trend)}

The predominate reactivity effect of this system is driven by the spacing between the arrays of waste forms, and this effect is the primary motivation for the work described in this report. The previous analysis from Saylor and Scaglione [3] did not consider any spacing in triangular pitched uniform arrays, and the result was a requirement for $50 \mathrm{~g} \mathrm{~B}_{4} \mathrm{C}$ per CCO. In this work, the spacing between the CCOs is evaluated based on the SNL compaction results from Reedlunn and Bean [8]. While the SNL compaction results yield a physically realistic nonuniform distribution of spacing between CCOs, the data can also be used to determine spacing for use in triangular pitched uniform arrays. The purpose for using uniform arrays is based on several objectives. First, is the objective to cover the entire regulatory timeframe, from emplacement to 10,000 years. At time zero, the CCOs are emplaced in a uniform array, and after 10,000 years, the final configuration of the CCOs is very nonuniform. Second, it is beneficial to use a uniform array to enable comparisons to previous work from Saylor, Scaglione [3], and Brickner [4] in which uniform arrays were also used. This will allow for comparison among all of the results. Third, the neutron physics of the system is highly dependent upon neutron leakage, and in the uniform array, the reactivity trends are therefore magnified by the uniform arrays (more sensitive). Furthermore, the lower leakage of the uniform arrays is expected to bound the nonuniform arrays in the moderator range of interest. Therefore, while the uniform arrays are not physically realistic at 10,000 years, they may be appropriate to use as bounding results over the nonuniform arrays so that unwarranted importance is not placed on the high uncertainty associated with the SNL compaction data.

Every case in set-1a has a corresponding case in set-1. The reactivity effect of the difference in spacing is shown by comparing the results of set- 1 to set- $1 \mathrm{a}$ as a delta-k in Figure 6.6 for every curve in the sets. The reactivity effect of the closer spacing for set- 1 is in the range of $10-15 \%$ delta-k for the representative curves, and it is consistent across the range of moderator mass sweeps, showing that there is a large conservatism in using the set- 1 dataset. Furthermore, the comparison which shows the delta-k between the representative curves illustrates how the spacing impacts the representative curves. 


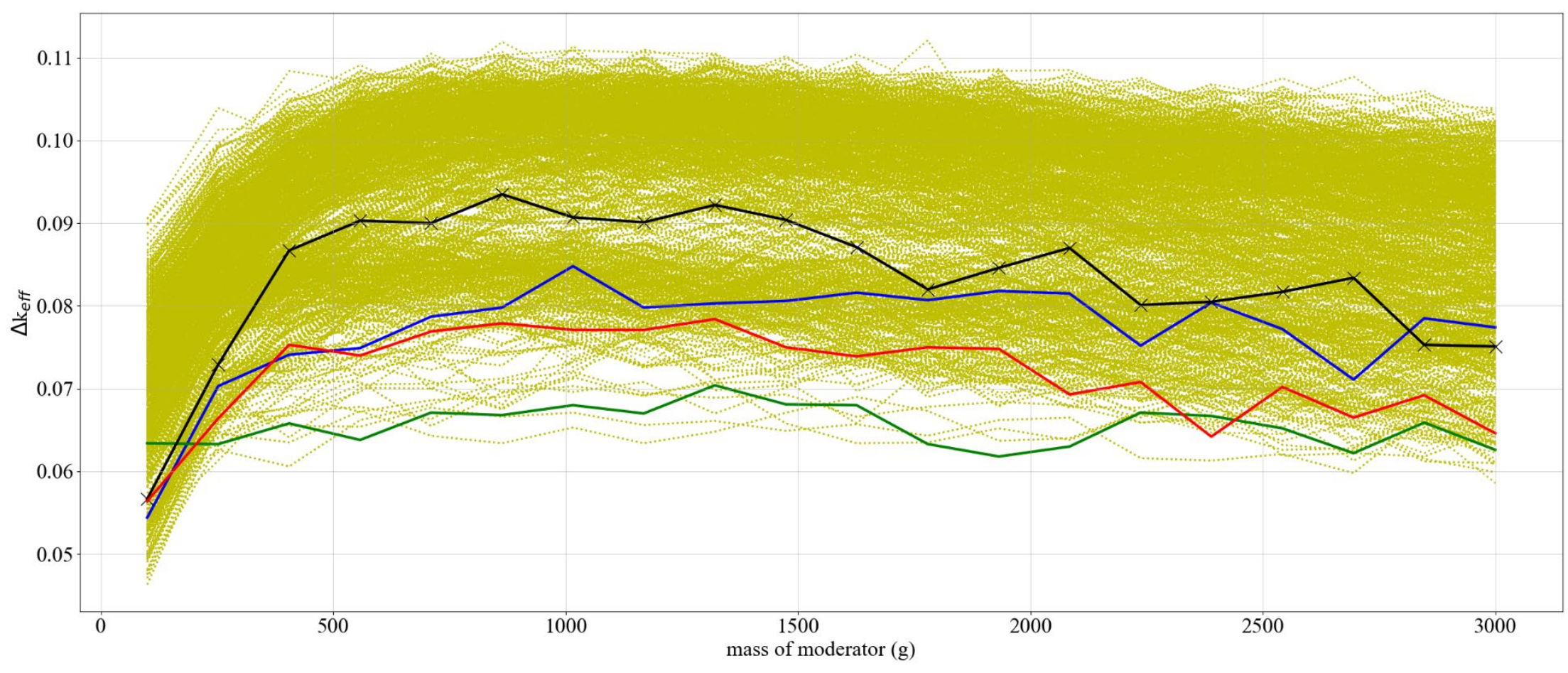

delta all data

- delta set-1-4 to set-1a-4, cylinder, FGE+poly mod only, thick poly discrete reflector delta set-1-4 to set-1a-4, cylinder, FGE+poly mod only, thin poly discrete reflector

* delta set-1-12 to set-1a-12, sphere, FGE+poly mod only, thin poly discrete reflector

_ delta set-1-12 to set-1a-12, sphere, FGE+poly mod only, thick poly discrete reflector

Figure 6.6. Comparison of the representative curves between set-1 and set-1a (set-1-set-1a). 


\subsubsection{Waste Form Moderator Mass (Primary Trend)}

The sweeps over the mass of moderator in the waste form provide a variety of results depending on the other system parameters being swept over. In general, increasing moderator mass increases $k_{\text {eff }}$, but the magnitude and behavior of the trend is dependent on the subcase, the study of which is the purpose to this analysis. For the moderator material itself, the polyethylene bounds the water. These trends are expected because the system is very under-moderated initially, and polyethylene is more H-dense than water. While it is not likely to be physically realistic to have pure polyethylene as the moderator, the curves which use pure polyethylene are expected to conservatively bound other materials since polyethylene is a powerful moderator. Figure 6.7 below shows how the reactivity compares between water and polyethylene for various cases.

The results of the specific cases, presented in detail in APPENDIX A, show how reactivity changes as a function of moderation is reached varies according to the other system parameters for the various sweeps. Fissile mass mixtures are highly dependent on internal moderation, and this effect is clear in the results presented in this parametric evaluation. While most waste form processes are expected to be relatively dry, the CCO TRUPACT-II Safety Analysis Report [9] did not initially limit the mass of H-bearing materials such water or polyethylene. If actual waste forms are known (due to processing) to have masses of $\mathrm{H}$-bearing materials within some range, then that range could be used to select results to limit moderator in the waste form. 


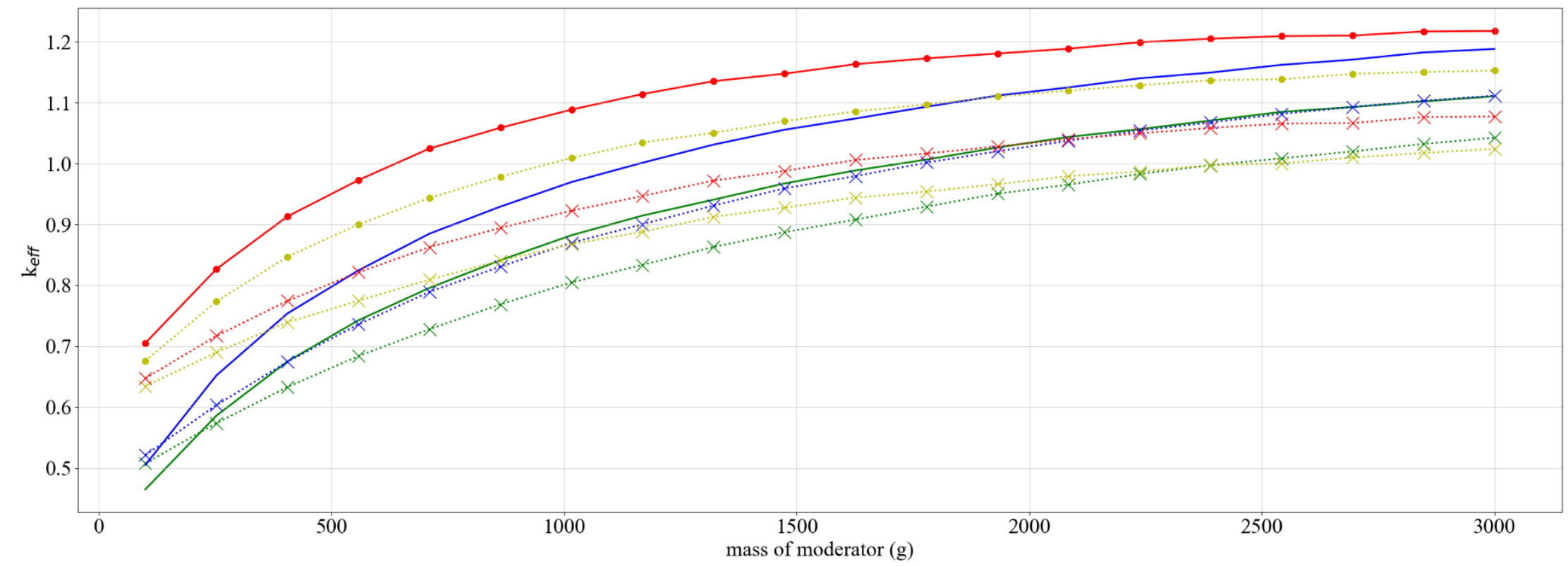

set-1, sphere, no filler, no Be, thick poly discrete reflector, water moderator

…… set-1, sphere, $5 \mathrm{~kg}$ graphite/can, $585 \mathrm{~g} \mathrm{Be}$, thick poly discrete reflector, water moderator

- set-1, sphere, no filler, no Be, thick poly discrete reflector, poly moderator

… $\ldots$ set-1, sphere, $5 \mathrm{~kg}$ graphite/can, $585 \mathrm{~g} \mathrm{Be}$, thick poly discrete reflector, poly moderator

set- 1 , cylinder with radius $=7.7 \mathrm{~cm}$, no filler, no Be, thick poly discrete reflector, water moderator

set-1, cylinder with radius $=7.7 \mathrm{~cm}, 5 \mathrm{~kg}$ graphite $/ \mathrm{can}, 585 \mathrm{~g} \mathrm{Be}$, thick poly discrete reflector, water moderator

$\longrightarrow$ set-1, cylinder with radius $=7.7 \mathrm{~cm}$, no filler, no Be, thick poly discrete reflector, poly moderator

… $\ldots$ set-1, cylinder with radius $=7.7 \mathrm{~cm}, 5 \mathrm{~kg}$ graphite $/ \mathrm{can}, 585 \mathrm{~g} \mathrm{Be}$, thick poly discrete reflector, poly moderator

Figure 6.7. Comparison of various cases for both water and polyethylene waste form moderator mass. 


\subsubsection{Geometry and Filler Mass (Primary Trend)}

The results summarized in Figure 6.7 demonstrate that it is necessary to restrict H-bearing materials (i.e., a moderator mass limit) because the waste form spacing and moderator mass have such a primary impact on system $k_{\text {eff. }}$. While it is likely that such a moderator mass limit is realistic for many waste streams there may be some waste streams which will not meet that limit. Secondary to this type of requirement, it is also clear that most, if not all, actual waste forms also include filler material.

The sweeps over waste form geometry and filler mass trend typically indicate a trend of decreasing $k_{\text {eff }}$ with expanding geometry for a fixed moderator content. This occurs as a consequence of the increasing filler mass. While the reactivity effect of geometry and filler mass is not as large as that caused by spacing or moderator mass, listing the impact as a primary effect is appropriate because it is a significant system component which is highly expected to be present in large mass quantities.

The reactivity effect of cylindrical shape is very dependent on size, whereas the spherical shape is less so. These trends are expected, especially for the cylindrical geometry (for cases with the same radius). For the cylinder shape, generally, decreasing radius and/or increasing height decreases $k_{\text {eff }}$ for a fixed volume. For both shapes, increasing the mass of filler material decreases $k_{\text {eff }}$ substantially, although it is much more pronounced for the cylindrical shape than the spherical shape.

The results shown in Figure 6.7 above provide some examples of the reactivity effect of geometry and filler material.

The secondary $k_{e f f}$ trends of the system are moderately dependent upon the discrete reflector material and thickness, the difference in generic filler material, and the graphite filler material and Be. These factors are discussed in more detail below.

\subsubsection{Discrete Reflector Material and Thickness (Secondary Trend)}

The trend associated with the discrete reflector material and thickness shows a strong dependence on the other waste form parameters. The dependence is very strong for the cylindrical waste form shape, whereas the dependence is greatly reduced for the spherical shape. The overall magnitude of the trends is small enough to be considered a secondary $k_{\text {eff }}$ trend and to provide additional flexibility in determining how to consider packaging. Figure 6.8 below shows an example of the effect of discrete reflector material for thick discrete reflectors. 


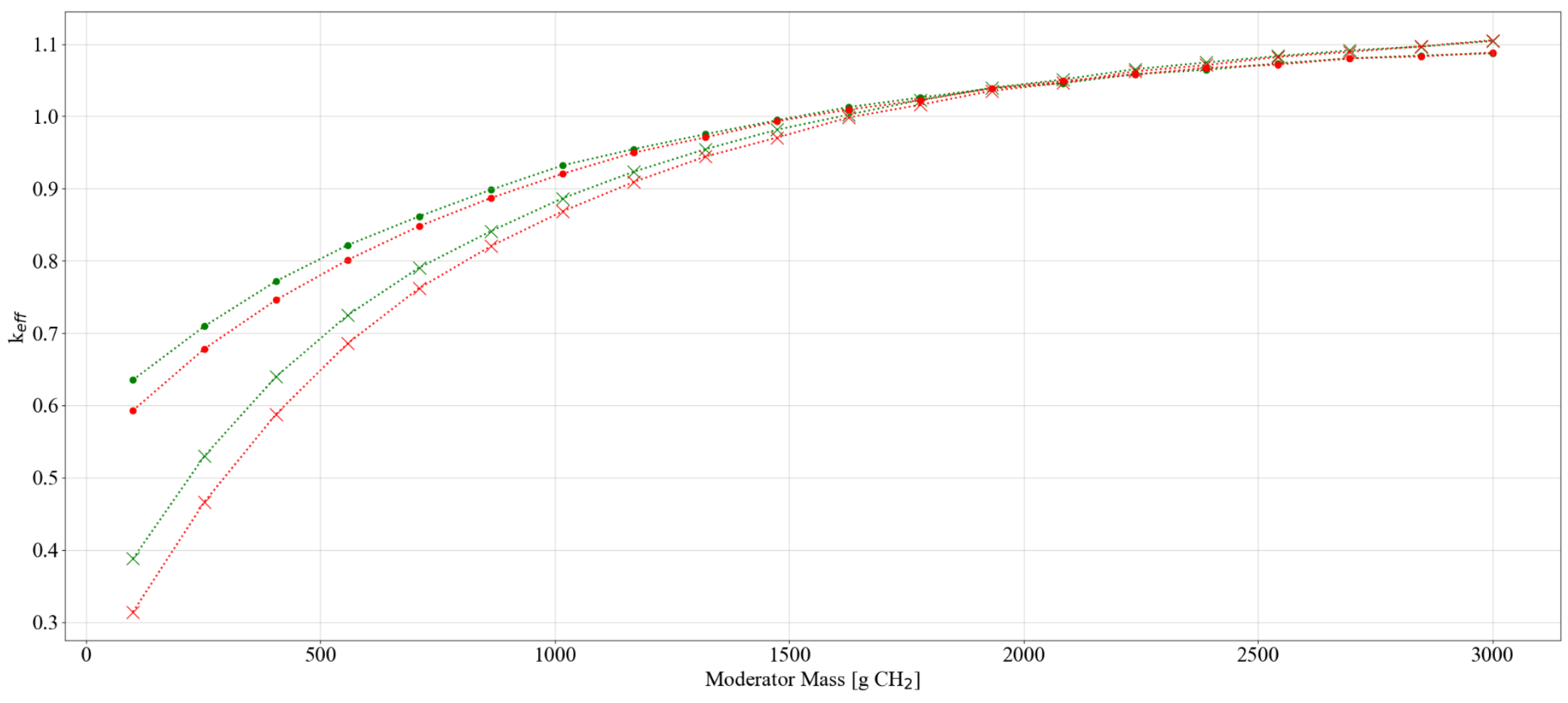

×... set-1, cylinder radius $=7.7 \mathrm{~cm}$, poly moderator, $5 \mathrm{~kg}$ graphite $/ \mathrm{can}$, no $\mathrm{Be}$, thick SS discrete reflector

•.... set-1, cylinder radius $=7.7 \mathrm{~cm}$, poly moderator, $5 \mathrm{~kg}$ graphite $/$ can, no Be, thick poly discrete reflector

……. set-1, cylinder radius $=7.7 \mathrm{~cm}$, poly moderator, $5 \mathrm{~kg}$ generic $/$ can, no $\mathrm{Be}$, thick SS discrete reflector

$\cdots \cdot \ldots$ set-1, cylinder radius $=7.7 \mathrm{~cm}$, poly moderator, $5 \mathrm{~kg}$ generic $/$ can, no Be, thick poly discrete reflector

Figure 6.8. Comparison of the discrete reflector material and filler material on reactivity for cylindrical waste forms. 


\subsubsection{Difference in Generic Filler Material and the Graphite Filler Material (Secondary Trend)}

The trend associated with the comparison between the generic filler material and the graphite filler material shows that the graphite filler material bounds the generic filler material for the most undermoderated cases. Both materials are essentially equivalent for well-moderated cases with generic filler perhaps bounding the graphite by a tiny amount. While the additional moderation effects from the carbon in the graphite material do increase $k_{\text {eff, }}$, the overall effect of the change in volume of the waste form has a greater impact.

\subsubsection{Beryllium in Waste Form (Secondary Trend)}

The trend associated with the increasing the mass of Be is not significant and varies between the two waste form geometries. For the cylindrical waste form, the sensitivity of the reactivity of the system is high, depending on the overall mass of the system (nonfissile, non-H materials). The addition of Be to the system increases the reactivity of the system because it is a special moderator, but the magnitude of the increase is offset by the decrease in reactivity due to the additional mass (volume change). For the cylindrical waste forms, the decrease in reactivity is greater than the increase due to the special moderator. For the spherical geometry, the two competing factors are essentially offset.

\subsection{DISCUSSION OF THE SIX-HIGH UNIFORM ARRAY PARAMETRIC SWEEPS}

As discussed in Section 6.1, an alternative uniform array model is used to address the specific issues caused by having two waste forms per CCO. Additional sweeps are added to address the impact of separating the $50 \mathrm{~g} \mathrm{~B}_{4} \mathrm{C}$ equally into those two waste forms per $\mathrm{CCO}$. The set- 3 cases evaluate this configuration by using a six-high uniform array with the same triangular pitch spacing as in the set-1 cases, as shown in Figure 6.9 below. The set-3 sweeps are the same as the set-1 sweeps. All set- 3 cases have 380 total FGE per CCO and 190 FGE per waste form. For the $\mathrm{B}_{4} \mathrm{C}$ cases, each waste form has $25 \mathrm{~g}$ $\mathrm{B}_{4} \mathrm{C}$ and $50 \mathrm{~g} \mathrm{~B}_{4} \mathrm{C}$ total for the $\mathrm{CCO}$. The sweeps considered are summarized in APPENDIX F.

Figure 6.9 below shows the difference between the three-high and six-high models.

The full set of results for set- 3 are found in APPENDIX F and are summarized in Figure 6.10 below. Additionally, a comparison of set- 1 to set- 3 by delta-k is presented in Figure 6.11 below. 

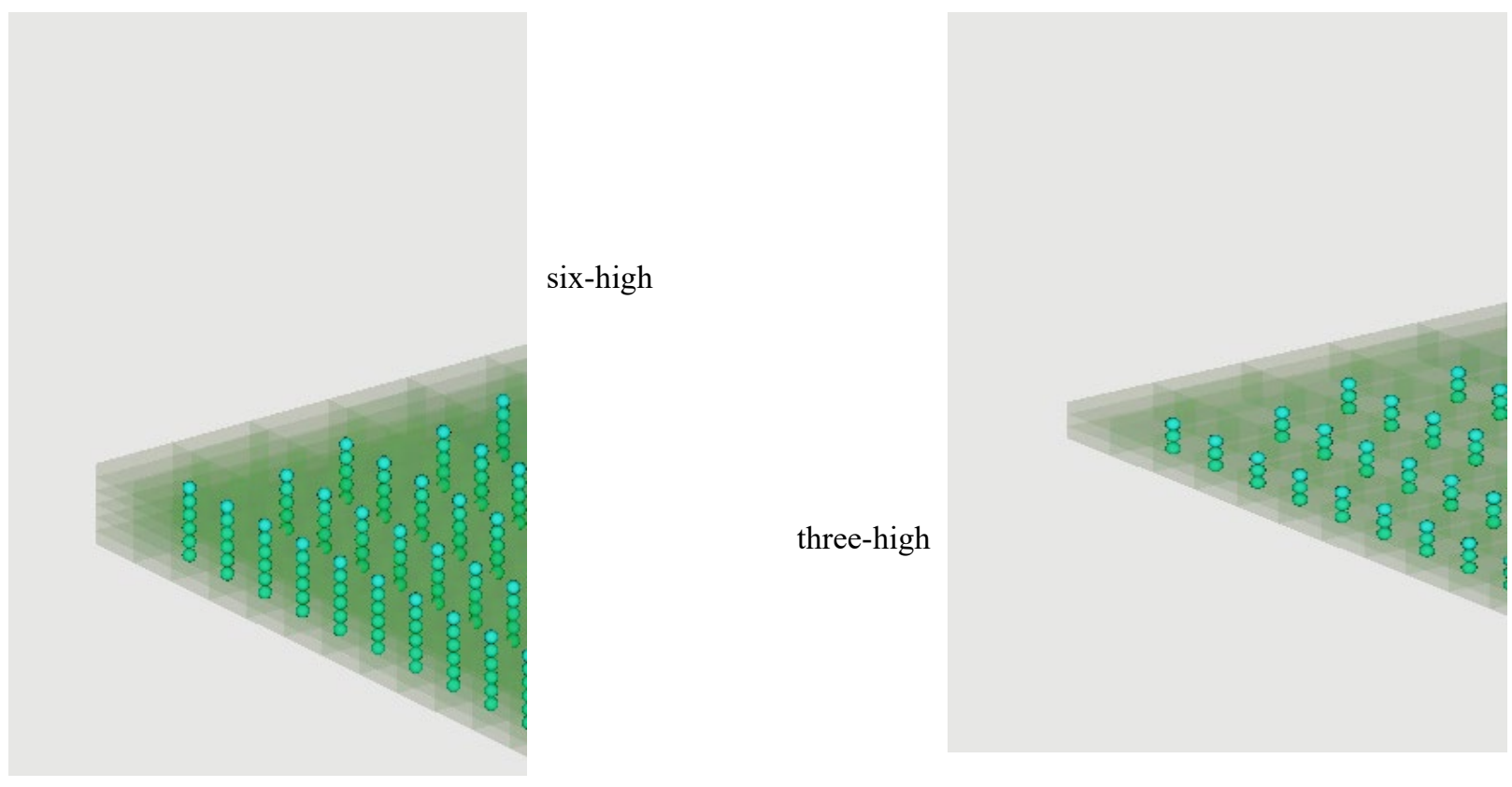

Figure 6.9. Comparison of the six-high and three-high arrays. 


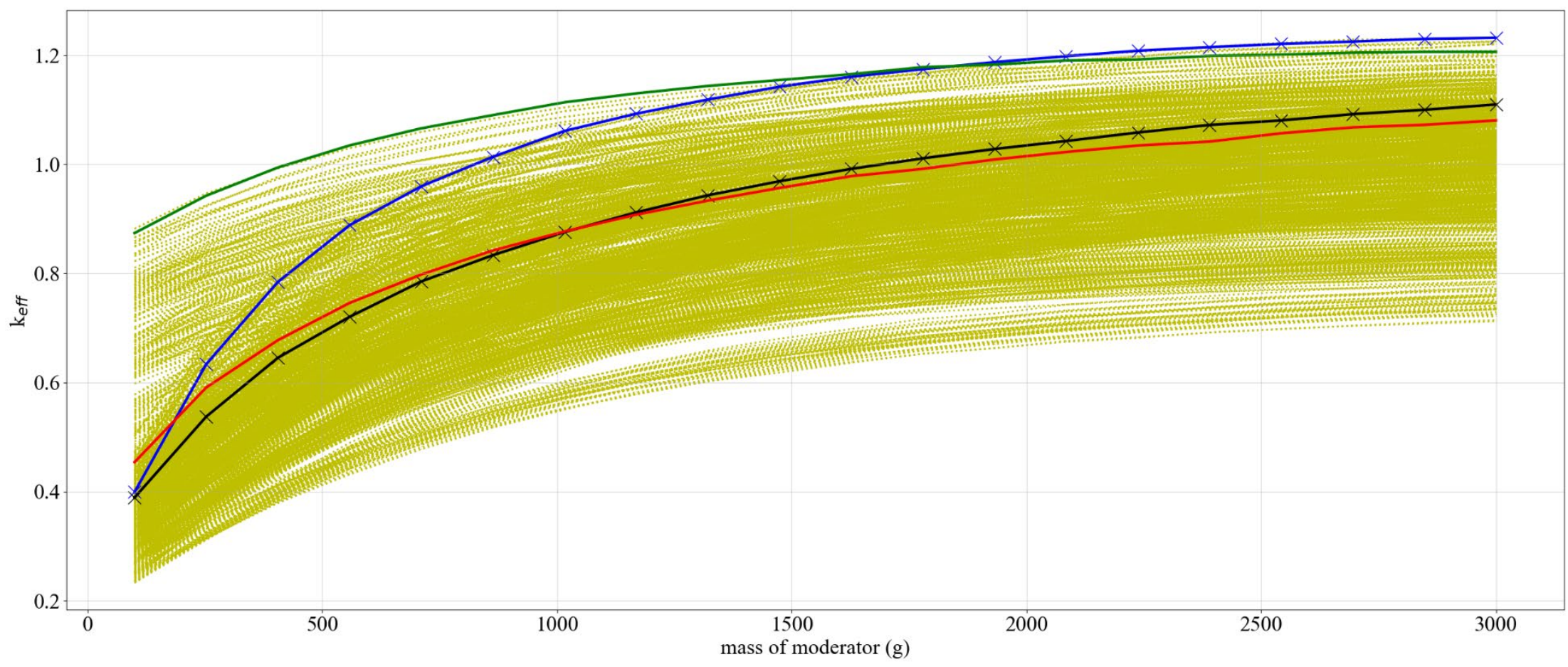

set-3 all data

$\leftarrow$ set-3-4, cylinder, FGE+poly mod only, thin poly discrete reflector

— set-3-4, cylinder, FGE+ poly mod only, thick poly discrete reflector

$\star$ set-3-12, sphere, FGE+ poly mod only, thin poly discrete reflector

— set-3-12, sphere, FGE+poly mod only, thick poly discrete reflector

Figure 6.10. Full set of results for set-3 compared to various representative curves. 


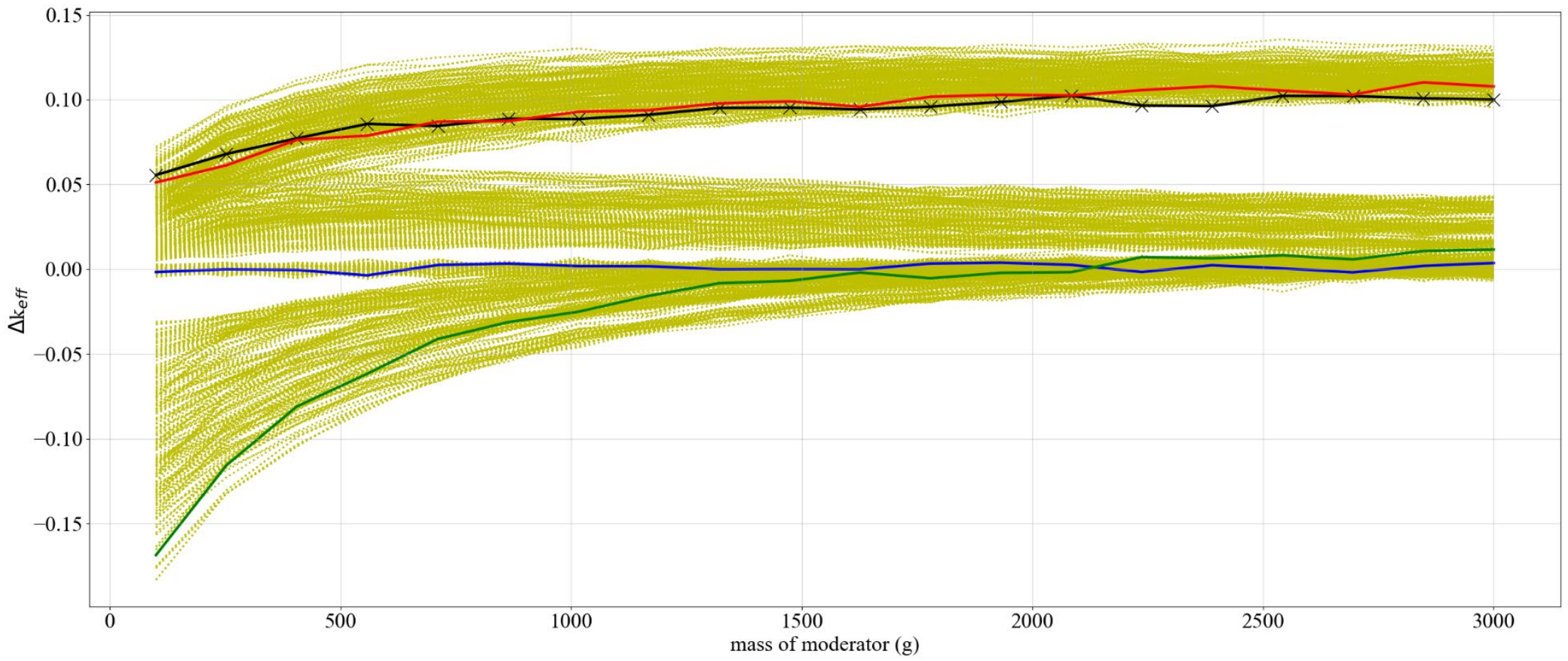

delta all data

- delta set-1-4 to set-3-4, cylinder, FGE+poly mod only, thick poly discrete reflector

- delta set-1-4 to set-3-4, cylinder, FGE+poly mod only, thin poly discrete reflector

$*$ delta set-1-12 to set-3-12, sphere, FGE+poly mod only, thin poly discrete reflector

— delta set-1-12 to set-3-12, sphere, FGE+ poly mod only, thick poly discrete reflector

Figure 6.11. Full set of results for set-1 compared to set-3 with various representative curves (set-1 - set-3). 
The results presented in Figure 6.10 and Figure 6.11 above and in APPENDIX F show that in general, all the reactivity trends evaluated in set-1 and set-1a remain consistent with those evaluated in set-3.

The set- 3 results are also used to show that $25 \mathrm{~g}$ of $\mathrm{B}_{4} \mathrm{C}$ per waste form $\left(50 \mathrm{~g} \mathrm{~B} \mathrm{~B}_{4} \mathrm{C}\right.$ per $\left.\mathrm{CCO}\right)$ is adequate to reduce reactivity consistent with the Saylor and Scaglione [3] analysis. The issue addressed in the studies is that Saylor and Scaglione [3] used a single waste form per CCO. The use of two waste forms with a maximum $190 \mathrm{FGE}$ per waste form had not been considered. This $\mathrm{B}_{4} \mathrm{C}$ study was conducted using the same six-high uniform array model and SAMPLER sweeps as those used for set-3-4 and set-3-12 (see Table F.1 in APPENDIX F), albeit with $25 \mathrm{~g}$ of $\mathrm{B}_{4} \mathrm{C}$ per waste form. These sweeps represent the most reactive sweeps in the range of interest for moderator mass. The results are summarized in Figure 6.12 below. The red bounding curve is for a radius of $7.7 \mathrm{~cm}$, no filler, no Be, thick poly discrete reflector, and poly moderator has the largest value of $k_{e f f}$. The purple curve is exactly the same, except with $25 \mathrm{~g}$ of $\mathrm{B}_{4} \mathrm{C}$ added, and it can be seen that $k_{\text {eff }}$ is reduced substantially. The results provide sufficient data to show that $25 \mathrm{~g} \mathrm{~B}_{4} \mathrm{C}$ per waste form $\left(50 \mathrm{~g} \mathrm{~B}_{4} \mathrm{C}\right.$ per $\left.\mathrm{CCO}\right)$ is acceptable and meets the requirement of $50 \mathrm{~g}$ per $\mathrm{CCO}$ as stipulated by Saylor and Scaglione [3].

Additionally, as was done for set- 1 and set-1a, set-3a results are for cases with the alternative spacing (see Table 6.1). The results for set-3a are provided in APPENDIX I. 


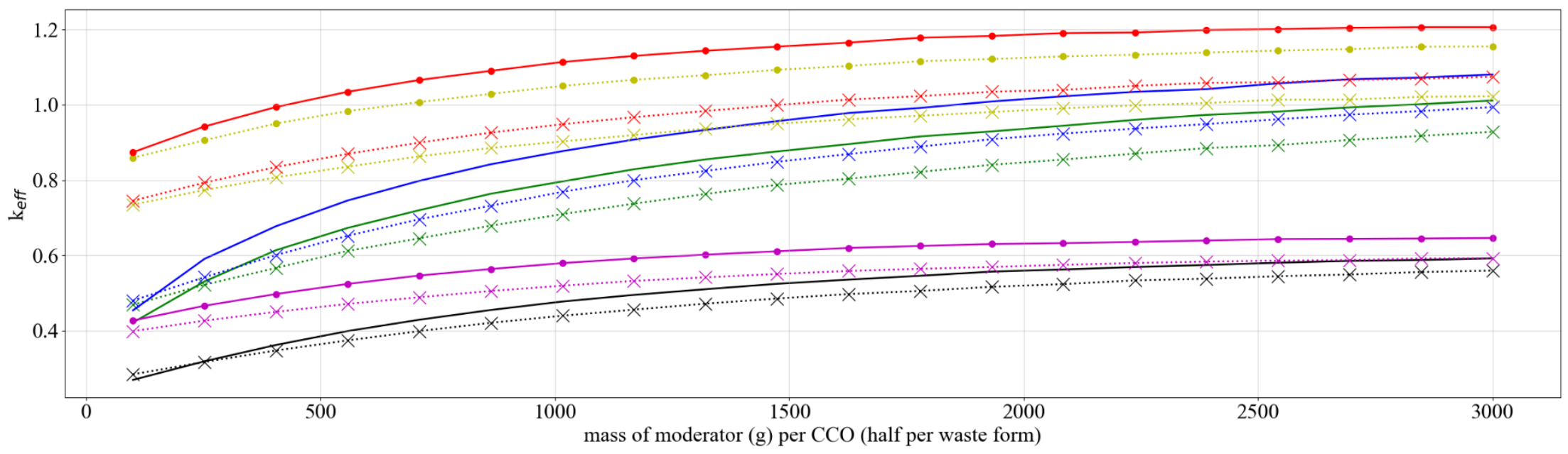

- set-3, sphere, no filler, no Be, thick poly discrete reflector, water moderator

…… set-3, sphere, $5 \mathrm{~kg}$ graphite/can, $585 \mathrm{~g} \mathrm{Be}$, thick poly discrete reflector, water moderator

- set-3, sphere, no filler, no Be, thick poly discrete reflector, poly moderator

…… set-3, sphere, $5 \mathrm{~kg}$ graphite/can, $585 \mathrm{~g} \mathrm{Be}$, thick poly discrete reflector, poly moderator

$\cdots \cdot$ set- 3 , cylinder with radius $=7.7 \mathrm{~cm}$, no filler, no Be, thick poly discrete reflector, water moderator

......... set- 3 , cylinder with radius $=7.7 \mathrm{~cm}, 5 \mathrm{~kg}$ graphite $/ \mathrm{can}, 585 \mathrm{~g} \mathrm{Be}$, thick poly discrete reflector, water moderator

$\ldots$ set- 3 , cylinder with radius $=7.7 \mathrm{~cm}$, no filler, no Be, thick poly discrete reflector, poly moderator

........ set-3, cylinder with radius $=7.7 \mathrm{~cm}, 5 \mathrm{~kg}$ graphite $/ \mathrm{can}, 585 \mathrm{~g} \mathrm{Be}$, thick poly discrete reflector, poly moderator

— set-3, sphere, $25 \mathrm{~g} \mathrm{~B} 4 \mathrm{C}$, no filler, no Be, thick poly discrete reflector, poly moderator

........ set-3, sphere, $25 \mathrm{~g} \mathrm{~B} 4 \mathrm{C}, 5 \mathrm{~kg}$ graphite/can, $585 \mathrm{~g} \mathrm{Be}$, thick poly discrete reflector, poly moderator

- - set-3, cylinder with radius $=7.7 \mathrm{~cm}, 25 \mathrm{~g} \mathrm{~B} 4 \mathrm{C}$, no filler, no Be, thick poly discrete reflector, poly moderator

$\cdots \times \cdots$ set-3, cylinder with radius $=7.7 \mathrm{~cm}, 25 \mathrm{~g} \mathrm{~B} 4 \mathrm{C}, 5 \mathrm{~kg}$ graphite/can, $585 \mathrm{~g} \mathrm{Be}$, thick poly discrete reflector, poly moderator

\section{Figure 6.12. Reactivity effect of $25 \mathrm{~g} \mathrm{~B}_{4} \mathrm{C}$ compared to representative curves.}




\subsection{DISCUSSION OF THE NONUNIFORM ARRAY PARAMETRIC SWEEPS}

As discussed in Section 6.1 and Section 6.2, the Reedlunn and Bean [8] data may be used in various ways for uniform array models. The data which provide the room closure information used in those uniform array models are based on the calculated compaction results which yield orthogonal locations for the CCC (pipes). The data generated by the compaction calculations are a set of data in which each pipe in the compaction model yields nonuniform location datasets, as shown in Figure 6.13 below.
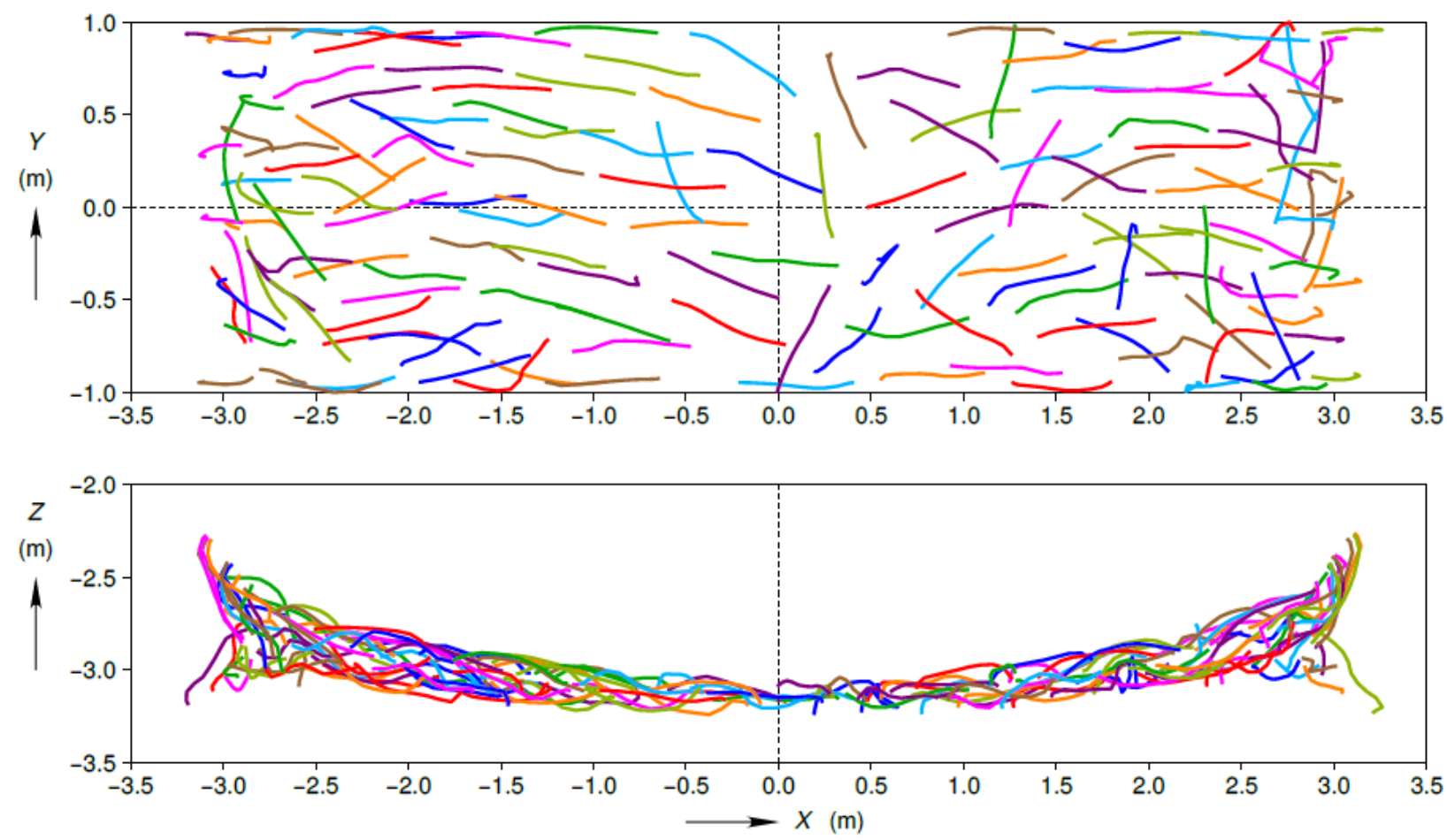

Figure 6.13. An example representation of the Reedlunn and Bean [8] data in the form of "spaghetti" plots, used with permission.

The utilization of the Reedlunn and Bean [8] data for the nonuniform cases (i.e., set-2-uh and set-2-lh) is consistent with the use of similar data in Brickner [4]: that is, that the location of the center of the compacted pipe is used to define a centroid location. The Reedlunn and Bean [8] data provide these results for both the upper and lower horizon, as discussed in Section 4.2.1. To show how the reactivity trends in the system behave for nonuniform arrays, the same sweeps that were used for the uniform arrays (see Section 6.1 and Section 6.2) are also evaluated. A complete description of the sweeps is provided in APPENDIX C (set-2-uh for upper horizon cases) and APPENDIX D (set-2-lh for lower horizon cases).

The nonuniform array model construction is the same as that used previously in Brickner [4]. The 153 data points provided by Reedlunn and Bean [8], shown in Table 4.4, are used to define the center of the vertically oriented cylinder base or the center of a sphere. The nonuniform array model is constructed in a manner similar to that used for the uniform array model in that the waste form geometry is defined by the amount of mass associated with each constituent. Consistent with the method used by Brickner [4], the centroid locations are set in the interstitial reflector material volume of the model (i.e., the "reflector box"). The reflector box is a rectangular volume for which the sides and top are fitted directly adjacent to the outer edge of the outer waste form, and the y-direction reflective boundary conditions are set just outside the outermost waste form in both y-directions. The reflective boundary conditions result in a room 
that is effectively infinite in the y-direction. A diagram of the model construct is shown in Figure 6.14 below. Additional discussions about this model and the assumptions used to construct it are provided in APPENDIX E.

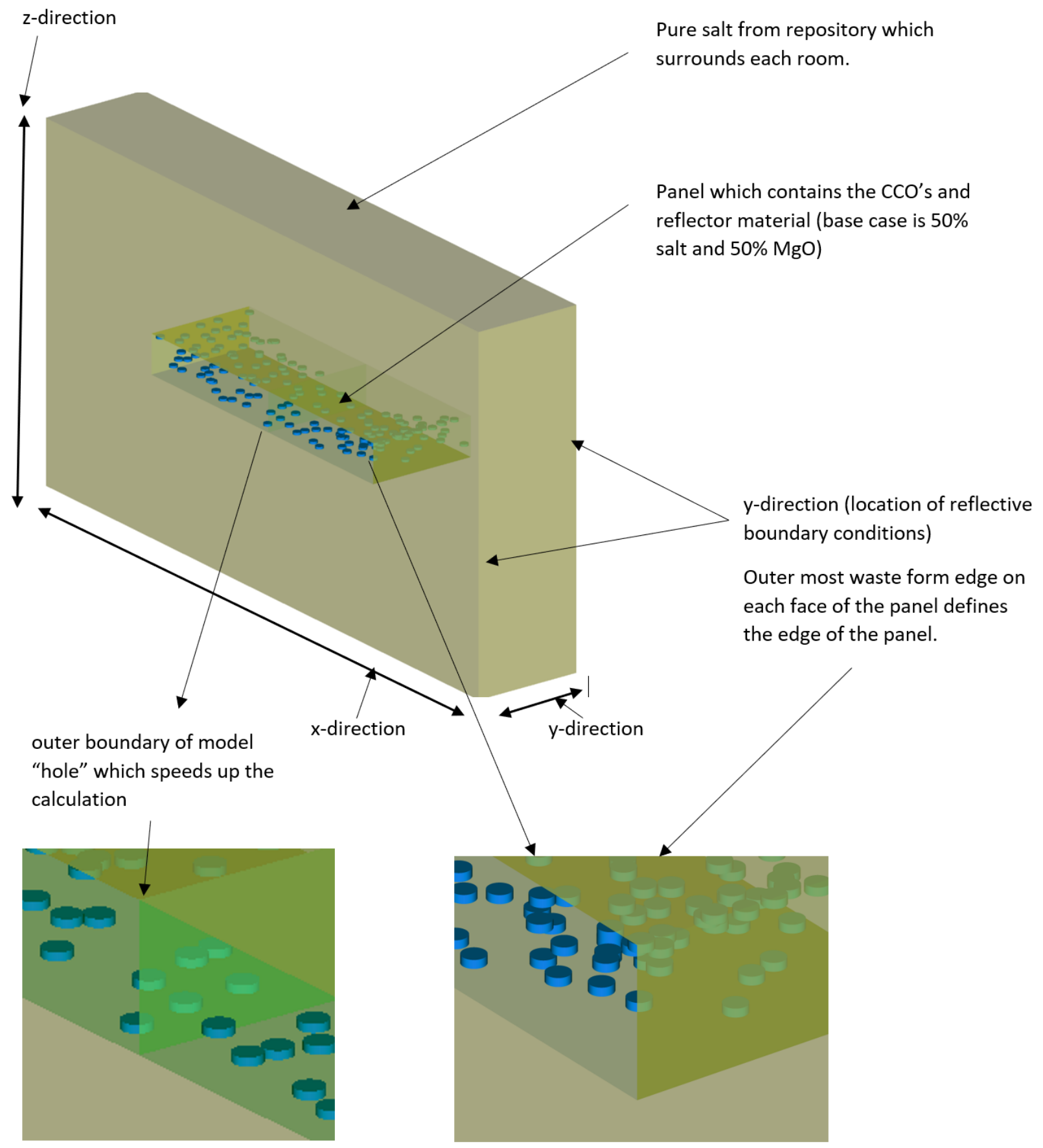

Figure 6.14. Diagram of the nonuniform array model.

The full set of results for the nonuniform array cases is presented in APPENDIX C (set-2-uh for upper horizon cases) and APPENDIX D (set-2-lh for lower horizon cases). The same set of plots shown in 
APPENDIX A and APPENDIX B are shown in APPENDIX C and Appendix D so that comparisons can be made for the FEP analysis. The full set of results with the representative curves are provided in Figure 6.15 and Figure 6.16 below for the upper and lower horizons, respectively, whereas Figure 6.17 provides the delta-k comparison between the upper and lower horizons. The results show that the upper horizon is always more reactive than the lower horizon.

The most meaningful comparisons for these results are shown as delta-k comparisons between set- 1 and set-2-uh and set-1a and set-2-uh in Figure 6.18 and Figure 6.19, respectively. These delta-k comparisons show that set- 1 is significantly more reactive than the other sets, and they also show that the important reactivity trends discussed in Section 6.1 remain consistent between set- 1 and set- 2 cases, with some important exceptions as discussed further below. Figure 6.20 compares set-1 to set-2-lh.

The reactivity of the nonuniform spacing is dramatically lower in $k_{\text {eff }}$ than in set-1. CCO centroid locations in their compacted configurations have significantly lower neutronic coupling than the uniform array. This is likely because in the compacted configuration, neutrons escaping a CCO can only cause fission in other CCOs if they travel in a relatively horizontal direction. However, in the uniform direction, neutrons can travel in more non-horizontal directions and still cause fission. Furthermore, the tangential impact is that the spherical shape is more reactive than the cylinder shape because now that the waste forms are more neutronically isolated from each other, the geometric impact of the surface-to-volume ratio dominates. It should be noted also that many of these differences in trend effectively cancel out at very high moderator mass. 


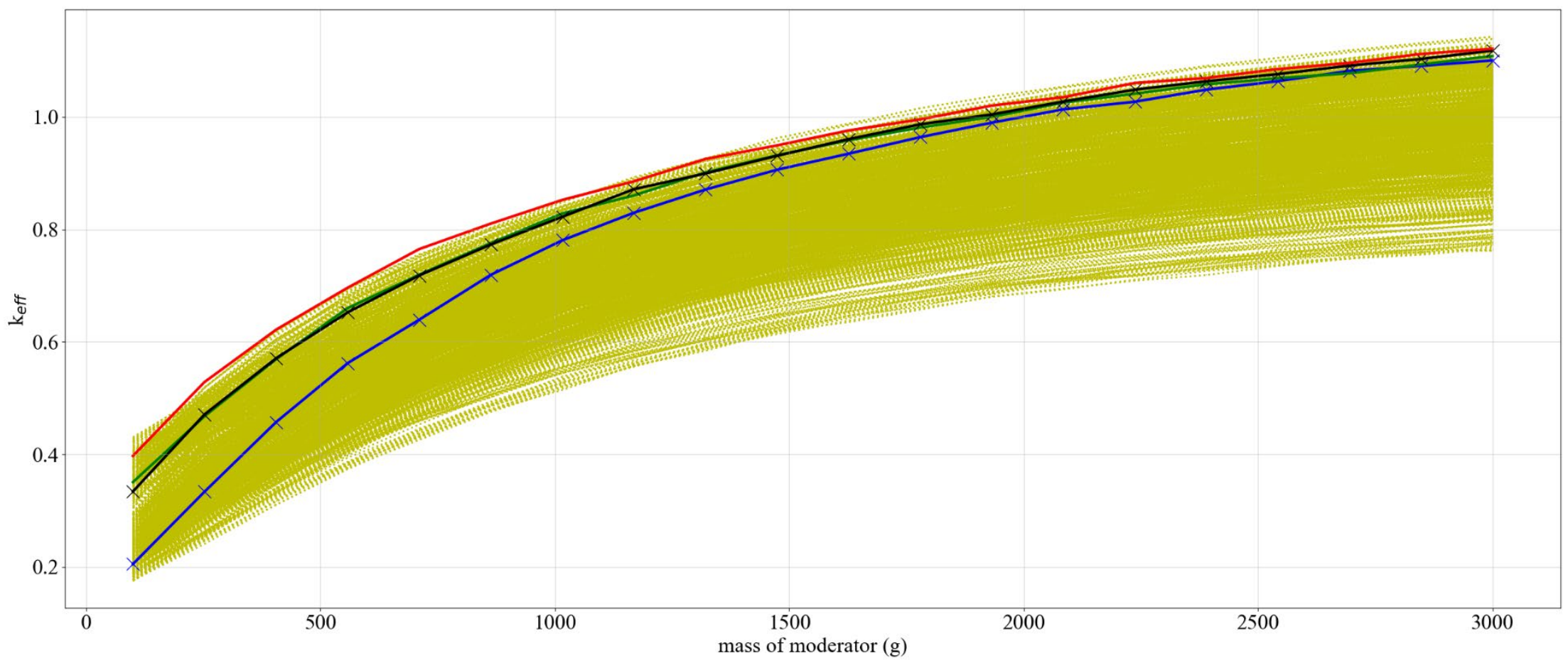




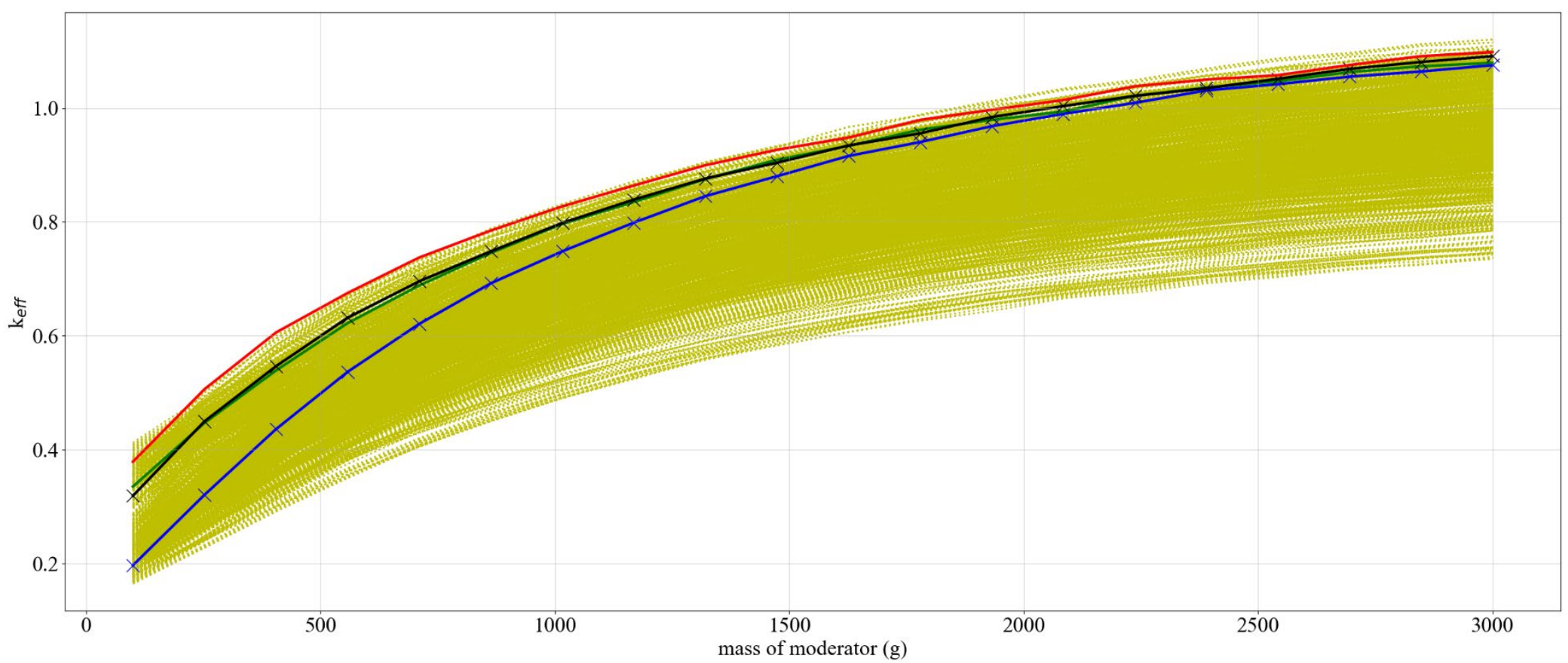

set-2-4-lh all data

* set-2-lh-4, cylinder, FGE+poly mod only, thin poly discrete reflector

- set-2-lh-4, cylinder, FGE+ poly mod only, thick poly discrete reflector

* set-2-lh-12, sphere, FGE+poly mod only, thin poly discrete reflector

- set-2-lh-12, sphere, FGE+ poly mod only, thick poly discrete reflector

Figure 6.16. Full set of results for set-2-lh compared to various representative curves. 


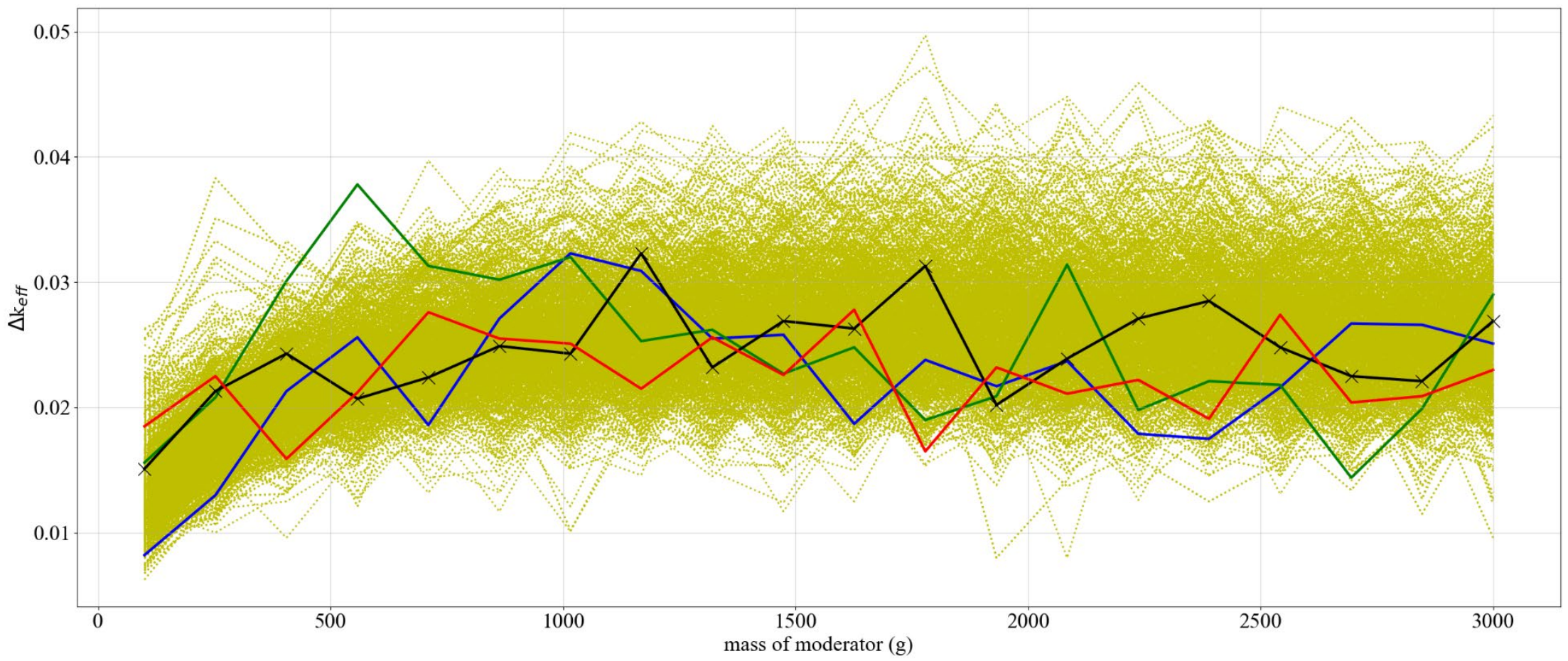

delta all data

- delta set-2-uh-4 to set-2-lh-4, cylinder, FGE+poly mod only, thick poly discrete reflector

- delta set-2-uh-4 to set-2-lh-4, cylinder, FGE+poly mod only, thin poly discrete reflector

$*$ delta set-2-uh-12 to set-2-lh-12, sphere, FGE+poly mod only, thin poly discrete reflector

— delta set-2-uh-12 to set-2-lh-12, sphere, FGE+poly mod only, thick poly discrete reflector

Figure 6.17. Delta $k$ of set-2-uh compared to set-2-lh with various representative curves (set-2-uh - set-2-lh). 


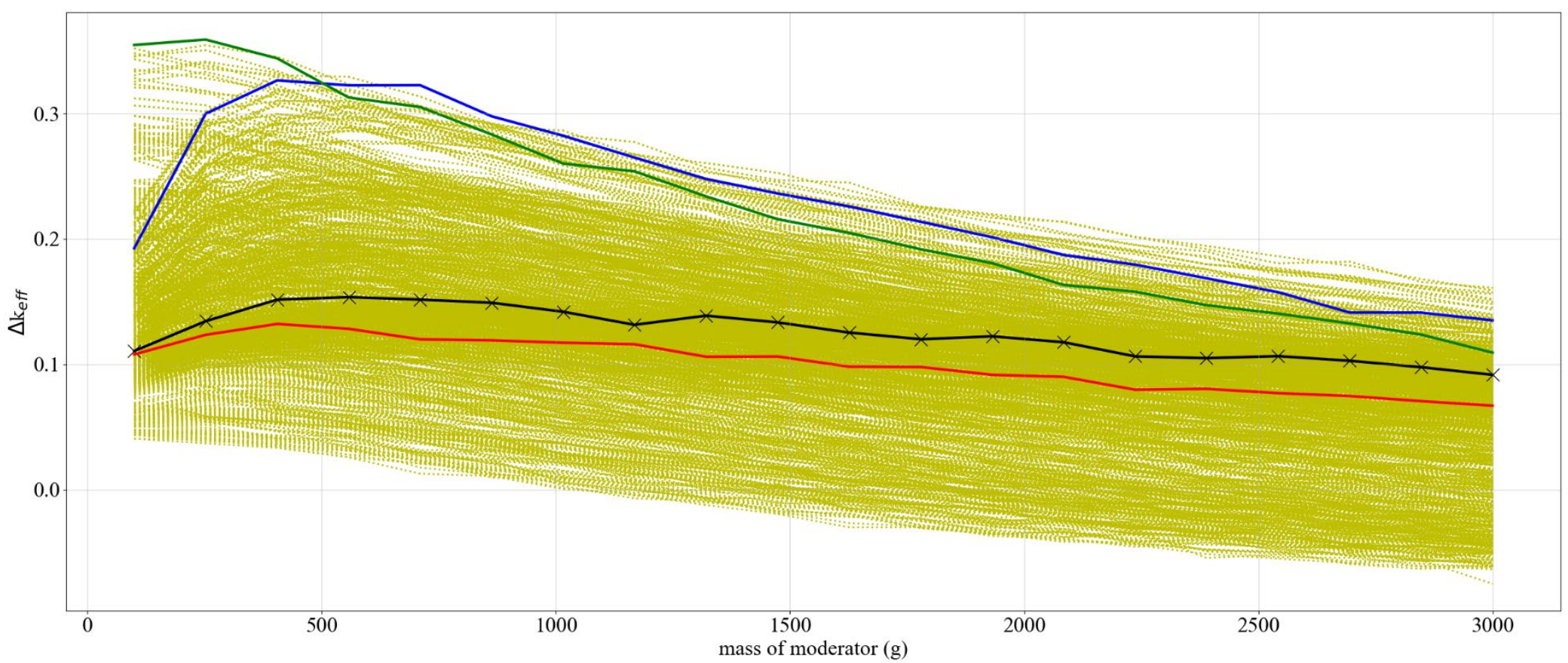

delta all data

delta set-1-4 to set-2-uh-4, cylinder, FGE+poly mod only, thick poly discrete reflector

- delta set-1-4 to set-2-uh-4, cylinder, FGE+poly mod only, thin poly discrete reflector

$\leftarrow$ delta set-1-12 to set-2-uh-12, sphere, FGE+poly mod only, thin poly discrete reflector

— delta set-1-12 to set-2-uh-12, sphere, FGE+poly mod only, thick poly discrete reflector

Figure 6.18. Full set of results for set-1 compared to set-2-uh with various representative curves (set-1 - set-2-uh). 


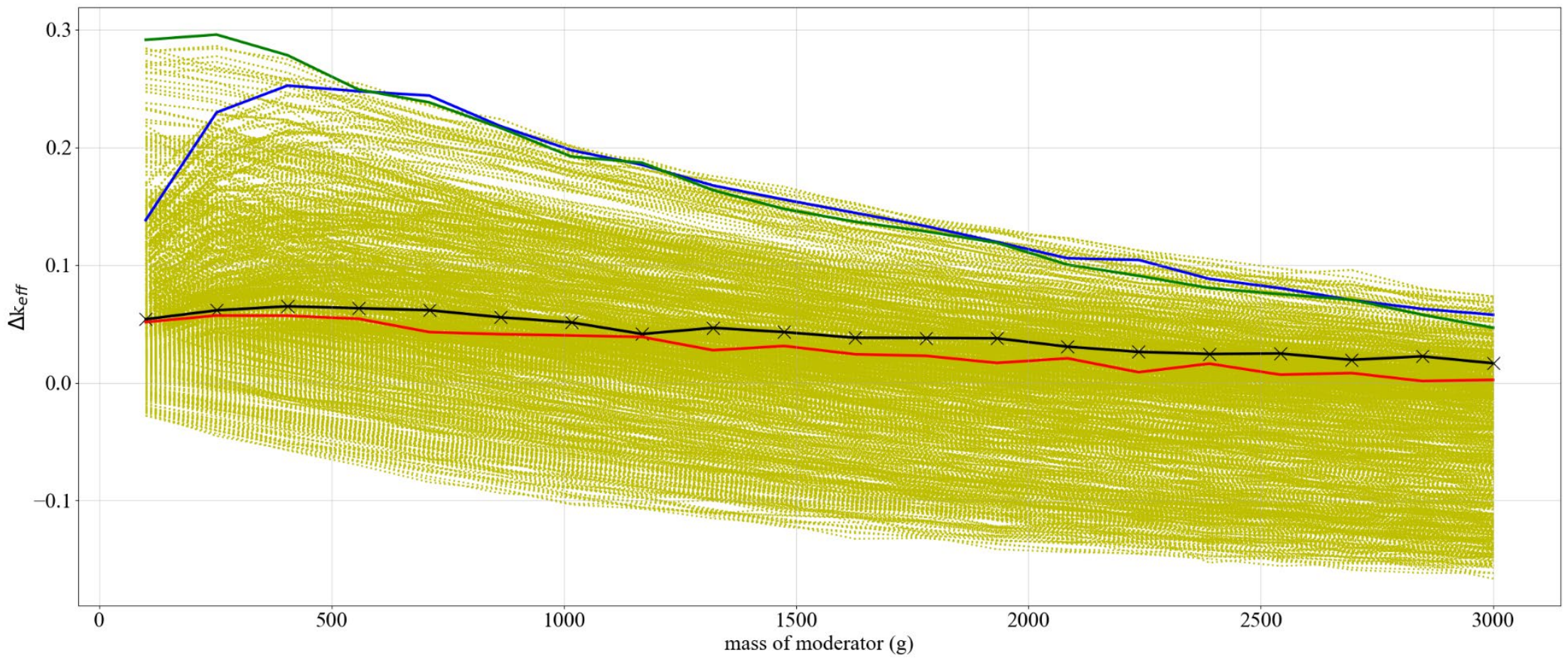

delta all data

- delta set-1a-4 to set-2-uh-4, cylinder, FGE+poly mod only, thick poly discrete reflector

- delta set-1a-4 to set-2-uh-4, cylinder, FGE+poly mod only, thin poly discrete reflector

$\star$ delta set-1a-12 to set-2-uh-12, sphere, FGE+poly mod only, thin poly discrete reflector

—_ delta set-1a-12 to set-2-uh-12, sphere, FGE+poly mod only, thick poly discrete reflector

Figure 6.19. Full set of results for set-1a compared to set-2-uh with various representative curves (set-1a - set-2-uh). 


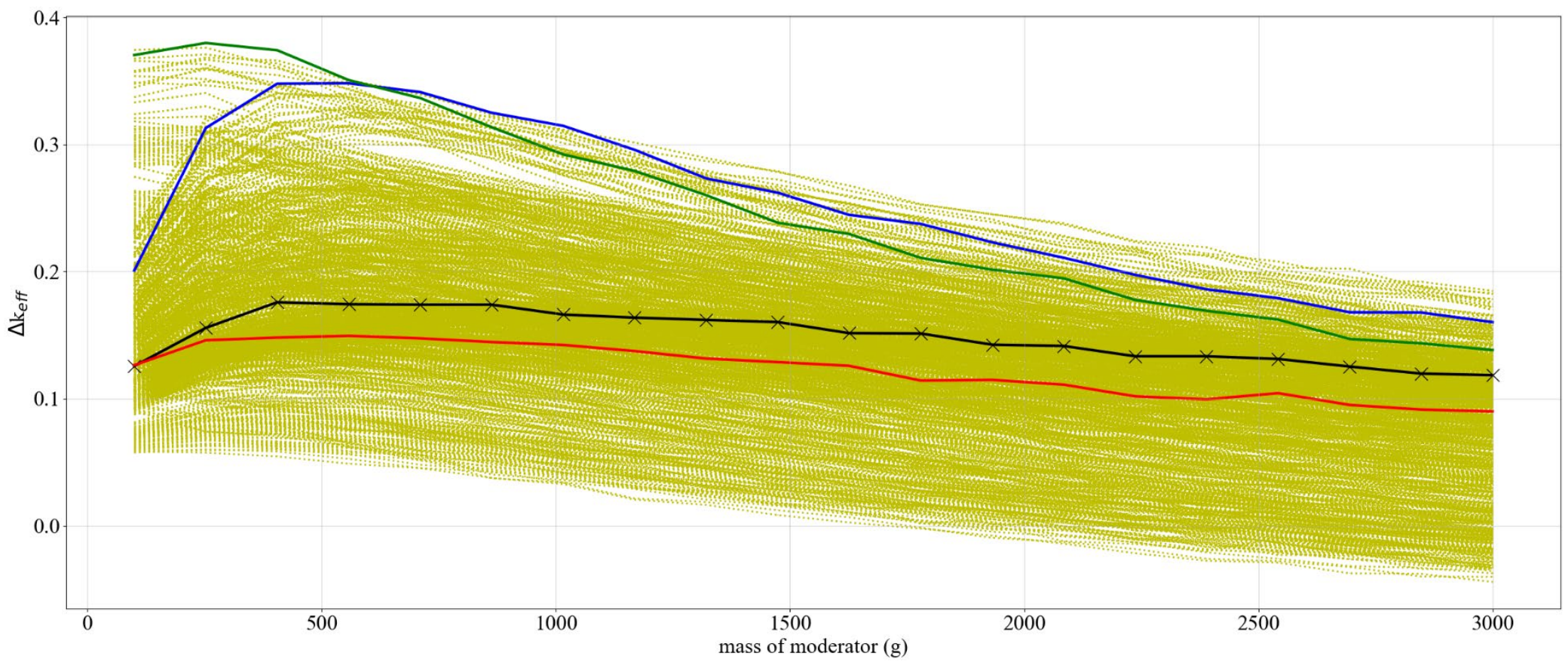

delta all data

- delta set-1-4 to set-2-lh-4, cylinder, FGE+poly mod only, thick poly discrete reflector

delta set-1-4 to set-2-lh-4, cylinder, FGE+poly mod only, thin poly discrete reflector

$*$ delta set-1-12 to set-2-lh-12, sphere, FGE+poly mod only, thin poly discrete reflector

- delta set-1-12 to set-2-1h-12, sphere, FGE+poly mod only, thick poly discrete reflector

Figure 6.20. Full set of results for set-1 compared to set-2-lh with various representative curves (set-1 - set-2-lh). 


\subsection{ADDITIONAL NONUNIFORM ARRAY ANALYSIS}

As is discussed in Section 6.3, the nonuniform array models for set-2-uh and set-2-1h are used to provide a comparison of reactivity between using the Reedlunn and Bean [8] data with uniform arrays (set-1 and set-1a) vs. nonuniform arrays. The set-2 -uh and set-2-lh results are considered appropriate for that purpose and could also be used as best-estimate guidance if needed.

To provide additional technical justification for the appropriateness of specific aspects of the nonuniform array model for set-2 -uh and set-2-lh calculations, additional analysis is provided. Specifically, the following items are addressed:

1. Compaction is a time-dependent process in which the location of the compacted CCOs changes over time. The results in Section 6.3 are for the "final" location at 10,000 years, with 1,000 years being used because there is little change after the first few hundred years. Additional analysis evaluated if there is an intermediate time step that could be more reactive between 0 years and 1,000 years. It also evaluates whether 1,000 years is the most reactive time and if it would be appropriate to use to represent 10,000 years.

2. Satisfactory computational performance is essential to any modeling effort. Additional cases were run to verify that the reactivity difference between a 1-unit KENO model vs. the 2-unit KENO model (used for nonuniform arrays throughout this report) is small. Furthermore, cases with more stringent control parameters examined whether cases are properly converged. The convergence of Monte Carlo criticality calculations is an important feature for providing meaningful results. The nature of the calculational model for the nonuniform array is a computationally intensive calculation for Monte Carlo codes like KENO in the SCALE system because of the nonuniform nature of the location of the fissile regions with multiple surfaces. While the long historic precedence in running criticality calculations provides sufficient support for the Monte Carlo calculations' control parameters used in these KENO inputs, the use of a model enhancement described in Marshall and Brickner [26] motivated this inquiry.

3. The sensitivity of nonuniform models upon boundary conditions was evaluated. Specifically, the reactivity effect of using the reflective boundary conditions vs. a thick layer of reflector box material (salt $+\mathrm{MgO}$ ) or periodic boundary conditions was examined. The Reedlunn and Bean [8] data are based on geomechanical models that utilize boundary conditions to limit the overall calculational effort and runtime. The underlying effect of such little movement in the y-direction (down the length of the room) supports the use of boundary conditions in that direction for the compaction studies. However, the use of boundary conditions is not as clear in criticality models of such nonuniform distributions of fissile regions. Three options are available: (1) no boundary conditions but instead a thick layer of reflecting media, (2) reflective boundary conditions, and (3) periodic boundary conditions. Brickner's original

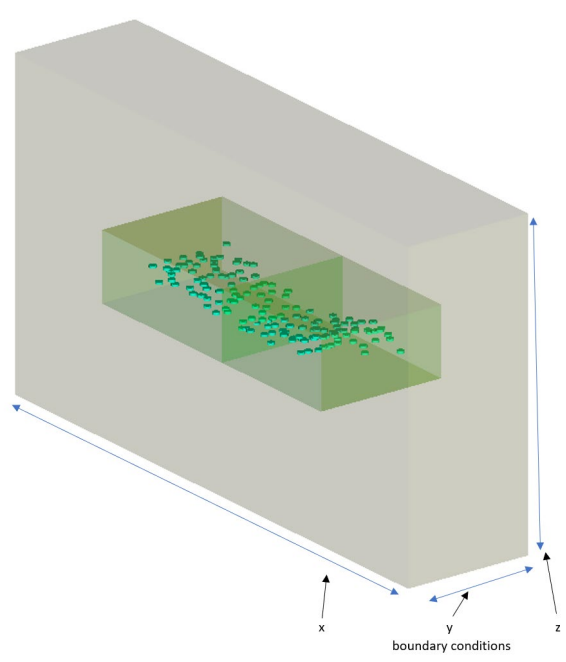

Figure 6.21. Illustration of boundary conditions. approach [4] was to assume that due to the crushing of the salt during creep, all other materials 
would be very closely packed against the waste forms. Therefore, it would be appropriate for the reflector box to be defined as directly adjacent to the waste forms. In the z-direction and $\mathrm{x}$ direction, a thick salt layer was then modeled, whereas in the y-direction, reflective boundary conditions were applied.

4. The original approach for modeling cylinders from Brickner [4] was to use the Reedlunn and Bean [7] data centroid (as determined to be a weighted average center pipe locations) to locate the center of the base of upright cylinders. An in-depth evaluation was performed to examine this assumption. Some cases evaluated if the centroid was located in the physical center of the cylinder instead of the base. In another group of cases, the centroid was oriented according to the pipe orientation at 1,000 years as calculated by Reedlunn and Bean [8]. In more cases, centroid location was varied along the length of the pipe was a more reactive configuration than the center. Also, a random distribution of centroid locations along the lengths of the cylinders was evaluated in search of a more reactive configuration. In addition, random distribution of cylinder orientations was evaluated. Finally, a combination of random distributions of both centroid location and orientations was evaluated.

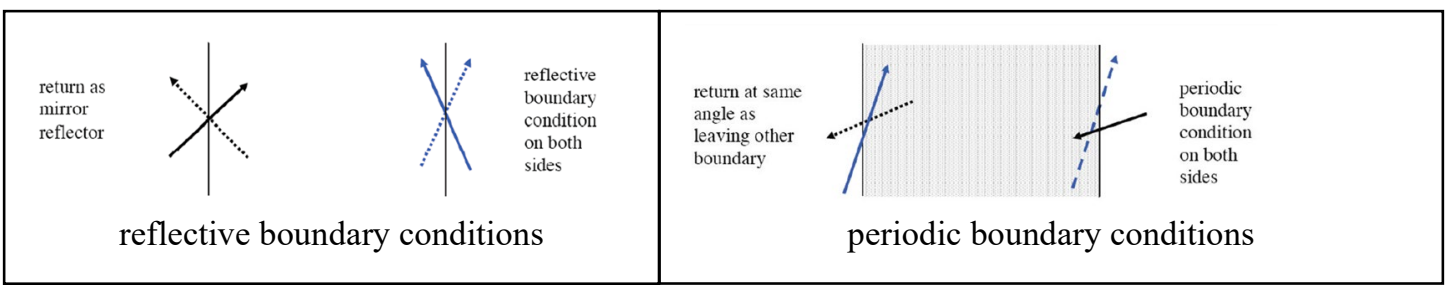

The calculations presented in APPENDIX E address items 1-3, and the calculations presented in APPENDIX G address item 4. Additional discussions are provided below.

In APPENDIX E, time-dependent models are created which use a 1-unit model rather than the 2-unit model, the Monte Carlo calculations control parameters are modified, and variations in boundary condition use are included (see Figure 6.26). The time dependence is for the locations of CCO centroid coordinates only; it does not include decay of ${ }^{239} \mathrm{Pu}$ to ${ }^{235} \mathrm{U}$, corrosion, mass transport, or any other geometric or compositional change. Calculations are performed for the upper horizon dataset from Reedlunn and Bean [8] in 10-year increments up to 300 years and in 100-year increments up to 1,000 years. The full set of time-dependent results is summarized in Figure 6.24 below (see APPENDIX E for more information). The time-dependent results show that there is an expected time-dependent trend in reactivity (reactivity goes up over time due to compaction) and that there are no intermediate time steps which show any significant increase in reactivity. The results also show that after 300 years, there is little change in reactivity because there is little-to-no further movement of the fissile regions due to compaction. 
For all the set- 2 calculations in APPENDIX E which use the 2-unit model and are compared to 1 -unit model, the comparison includes the reactivity effect of the change in Monte Carlo calculations control parameters to verify convergence in the set-2 1-unit model (e.g., larger number of cycles, neutron histories), as seen in Table E.1 in APPENDIX E). The difference in reactivity shown in Figure 6.255 below for the comparison between the 1-unit and 2-unit models shows that the Monte Carlo calculations control parameters used in set- 1 and set- 2 calculations are appropriate because the cases with much larger parameters do not change the result. The cases to verify convergence and to demonstrate adequate sampling use time step data from Reedlunn and Bean [8] (i.e., centroid datasets in 10year increments). For the Monte Carlo cases with the alternative control parameters to verify convergence, flux distribution data were visualized using FULCRUM in the SCALE system, as shown in Figure 6.22 (presented in detail in APPENDIX E). The meaning and significance of these plots is discussed below.

The quantity of interest in this entire study is $k_{\text {eff. }}$. By definition, $k_{\text {eff }}$ is the number of child neutrons produced per parent neutron in a system. Any number of systems or configurations which are very different from each other and which have very different flux distributions may have the same $k_{\text {eff }}$ value. Ultimately, $k_{\text {eff }}$ is a system characteristic independent of flux distribution. However, flux information in $\left(\mathrm{n} / \mathrm{cm}^{2}\right)$ is provided for these KENO calculations to (1) show that the geometry is adequately sampled, and (2) show the location of maximum reactivity which is of interest since several geometry configurations from Reedlunn and Bean [8] are evaluated (see examples from APPENDIX E in the diagram to the right). The flux computed in KENO is non-physical and is only used as a means to analyze the system. Due to the way Monte Carlo computations proceed, where neutrons in higher multiplication areas reproduce more, the areas with larger fluxes contribute more multiplication to the system and have a higher reactivitydefined as $(\mathrm{k}-1) / \mathrm{k})$ - than areas with smaller fluxes. However, because $k_{\text {eff }}$ is a system characteristic independent of flux distribution, there is no unique function that maps a value of flux to a value of reactivity in a region, so flux magnitudes only indicate whether an area has a higher or lower reactivity than another region.

The KENO calculation is time-independent, and it proceeds by providing an assumed fission source defined by the control parameters (here the increased values, see Table E.1 in APPENDIX E), neglecting the intrinsic source of neutrons (radioactive decay-based reactions) in the system from the waste forms. 
The initial generation source distribution is a guess that is uniform across the fissionable material in the model, and it is updated in each generation based on the location's fissions that occurred in the previous generation. The number of neutrons in each generation is forced to remain constant, despite the actual system $k_{\text {eff. }}$ This process leads to the term "iterated source" for a $k_{\text {eff }}$ calculation in a Monte Carlo code such as KENO. As the calculation proceeds, the source distribution should stabilize into a converged distribution representative of the fission source, and thus relative reactivities, in all regions of the problem. The number of skipped generations in the calculation should be selected to be greater than the number of generations necessary to achieve this convergence, and several outputs from the simulation are provided to help the user assess that this source convergence has been achieved (all of which has been done and verified by these comparisons). In each active generation, the tallied flux is a result of the fission sites from the previous generation and the multiplication of each portion of the system. As the calculation proceeds, the iterative nature of the simulation causes the flux to become higher and higher in the most reactive portion of the system. This means that less and less attention is paid to the lower value regions. Therefore, for the 1,000-year case (see bottom image in Figure 6.22), the lack of shading on the left side of the room indicates a very high degree of convergence, whereas for the 0 -year case (top image in Figure 6.22), both sides remain equally important because of the symmetry of the fissile region distribution.

It is worth noting that in the subcritical configurations considered, the real flux in the room would be quite low. The intrinsic source from the waste forms would provide a constant small source of neutrons (from radioactive decay) that would be multiplied in the surrounding fissionable material. The real, timedependent flux distribution would look sparser and more sporadic with occasional peaks that quickly faded away. This sort of calculation is extremely expensive and is not necessary for the analysis presented in this report.

Results are plotted for a number of time steps in APPENDIX E (summarized in Figure 6.22 above) and can be used to observe the following trends:

- At time 0 years, the hexagonally arranged fissile regions show how the calculation converges on the locations where the most fissions occur.

- At time 100 years, the location where the flux is greatest has shifted to the left side of the room, indicating that the compacted arrangement yields a configuration where the calculation focuses to converge its solution.

At time 1,000 years, the location in the model which yields the maximum $k_{\text {eff }}$ has shifted to the far-right side, and the flux indicates that the calculation has determined that this location is of much more importance than the left side. To support this statement, another set of calculations was performed in which one half of the room has no FGE. As can be seen in Figure 6.233, the "half-room" calculations support these conclusions.

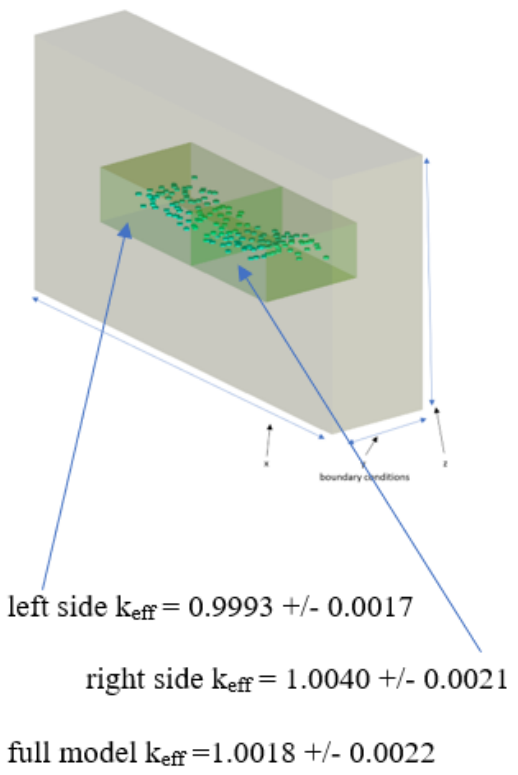

Figure 6.23. Results of the "half-room" calculations.

The results of the calculations to address item 4 (above) are found in APPENDIX G and are summarized in Figure 6.27 through Figure 6.29 below. Three sets of evaluations were performed: centroid studies, rcoord studies, and $r$ A studies. Each set includes multiple subsets, as explained in detail in APPENDIX G and summarized below. 
The centroid studies evaluate the effect of using each of the 13 SNL centroids independently, along with a midpoint. For these cases, each pipe in the model uses the same centroid for that case. For example, if the case is centroid1, then each pipe in the model uses the centroid 1 coordinates. The results are summarized in Figure 6.27.

The rcoord studies evaluate the effect of randomly selecting the 13 centroid coordinates to be used. Each pipe in the model has its own unique, random selection. For these studies, 100 rcoord cases were generated. The results are summarized in Figure 6.28.

The rA studies evaluate the effect of randomly selecting the cylinder orientation. These studies are performed independently and in conjunction with rcoord sets. The results are summarized in Figure 6.29.

The results show that the set-2 approach for using upright cylinders with the Reedlunn and Bean [8] centroid data for the average central centroid defining the center of the bottom of the cylinder is an acceptable approach. 


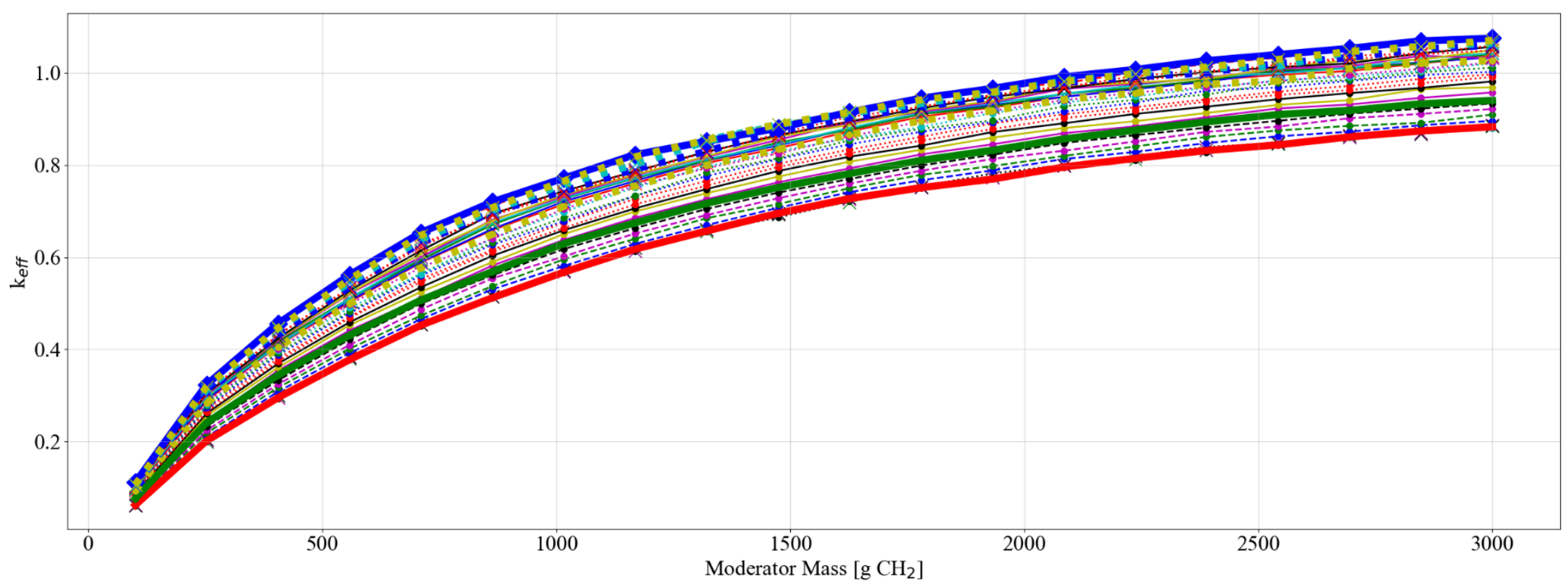

\begin{tabular}{|c|c|c|c|c|c|c|c|}
\hline & set-2-uh-td-0-yr & $\cdots \bullet \cdots$ & set-2-uh-td-150-yr & $\star$ & set-2-uh-td-220-yr & $\cdots \times \cdots \quad$ set-2-uh-td-290-yr & $--\bullet \quad$ set-2-uh-td-600-yr \\
\hline & set-2-uh-td-1000-yr & $\cdots \bullet \cdots$ & set-2-uh-td-160-yr & $\leftarrow$ & set-2-uh-td-230-yr & - s set-2-uh-td-300-yr & $--\bullet$ set-2-uh-td-60-yr \\
\hline & set-2-uh-td-100-yr & $\cdots \bullet \cdots$ & set-2-uh-td-170-yr & $\leftarrow$ & set-2-uh-td-240-yr & $\cdots \times \cdots \quad$ set-2-uh-td-30-yr & $--\bullet \quad$ set-2-uh-td-70-yr \\
\hline$\rightarrow$ & set-2-uh-td-10-yr & $\ldots \bullet \cdots$ & set-2-uh-td-180-yr & $\star$ & set-2-uh-td-250-yr & $=$ - set-2-uh-td-400-yr & --- set-2-uh-td-800-yr \\
\hline$\longrightarrow$ & set-2-uh-td-110-yr & $\ldots \bullet \bullet$ & set-2-uh-td-190-yr & 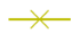 & set-2-uh-td-260-yr & $\cdots \times \cdots \quad$ set-2-uh-td-40-yr & $--\bullet \quad$ set-2-uh-td-80-yr \\
\hline$\rightarrow$ & set-2-uh-td-120-yr & $=$ " " & set-2-uh-td-200-yr & $\leftarrow$ & set-2-uh-td-270-yr & $=-$ " set-2-uh-td-500-yr & $--\quad$ set-2-uh-td-900-yr \\
\hline$\rightarrow$ & set-2-uh-td-130-yr & $\cdots \bullet \cdots$ & set-2-uh-td-20-yr & $\cdots \times \cdots$ & set-2-uh-td-280-yr & $\cdots \times \cdots \quad$ set-2-uh-td-50-yr & $--\bullet--$ set-2-uh-td-90-yr \\
\hline$\cdot \cdots$ & set-2-uh-td-140-yr & $\star$ & set-2-uh-td-210-yr & & & & \\
\hline
\end{tabular}

Figure 6.24. Results of the time-dependent calculations (set-2-4 with no filler). 


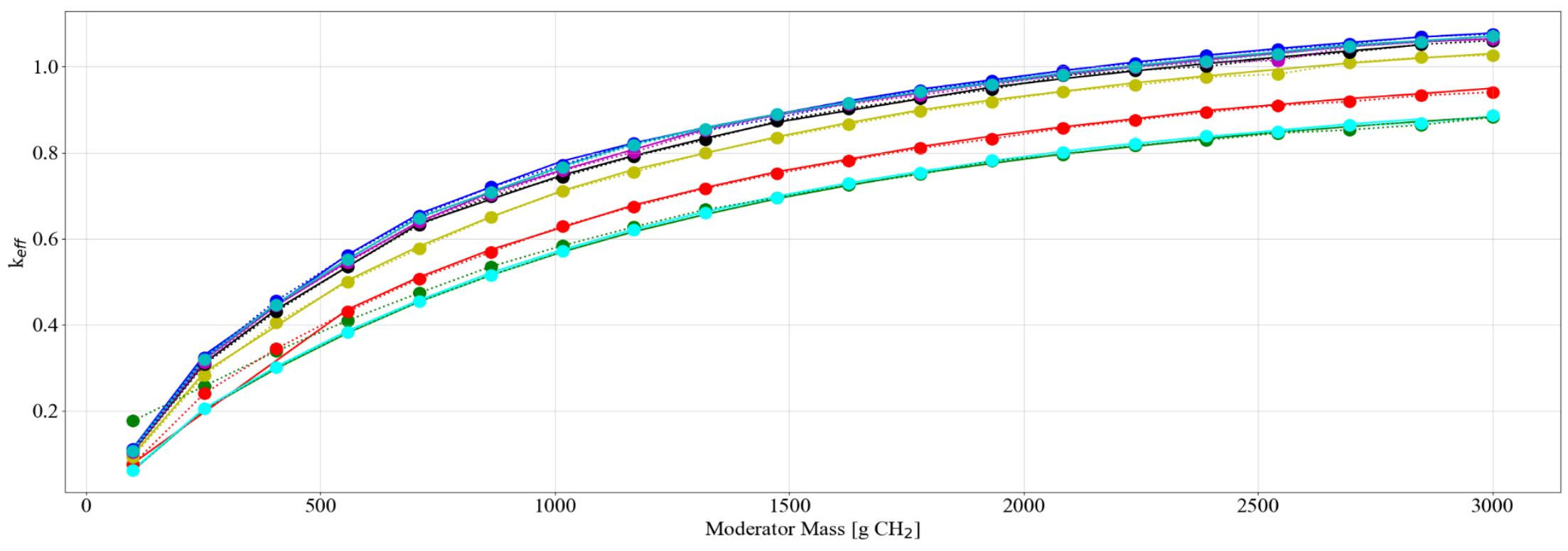

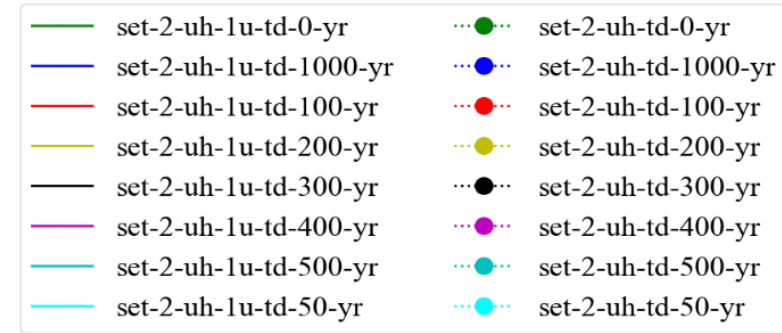

Figure 6.25. Comparison between the 1-unit model and the 2-unit model (set-2-4 with no filler). 


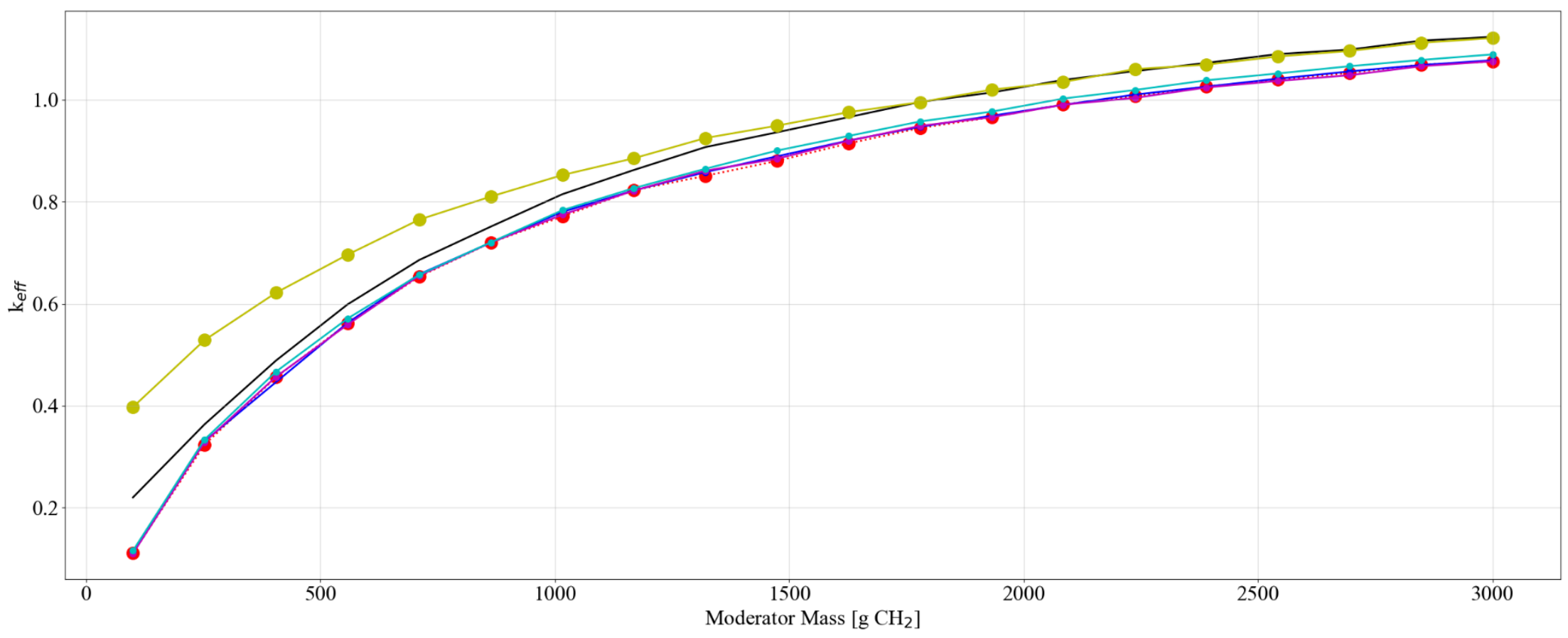

-... set-2-uh-td-1000-yr, 2-unit, $50 \mathrm{~cm}$ before reflective boundary conditions

- - set-2-uh-td-1u-1000-yr, 1-unit, $50 \mathrm{~cm}$ before reflective boundary conditions

- set-2-uh-1000-yr, 2-unit, $0 \mathrm{~cm}$ before reflective boundary conditions

- set-2-uh-1000-yr, 2-unit, $0 \mathrm{~cm}$ before reflective boundary conditions, spheres

$\longrightarrow$ set-2-uh-td-2up-1000-yr, 1-unit, $50 \mathrm{~cm}$ before periodic boundary conditions

$\longrightarrow$ set-2-uhp-1000-yr, 2-unit, set 2 model with periodic boundary conditions

Figure 6.26. Comparison of various boundary conditions (set-2-4 with no filler). 


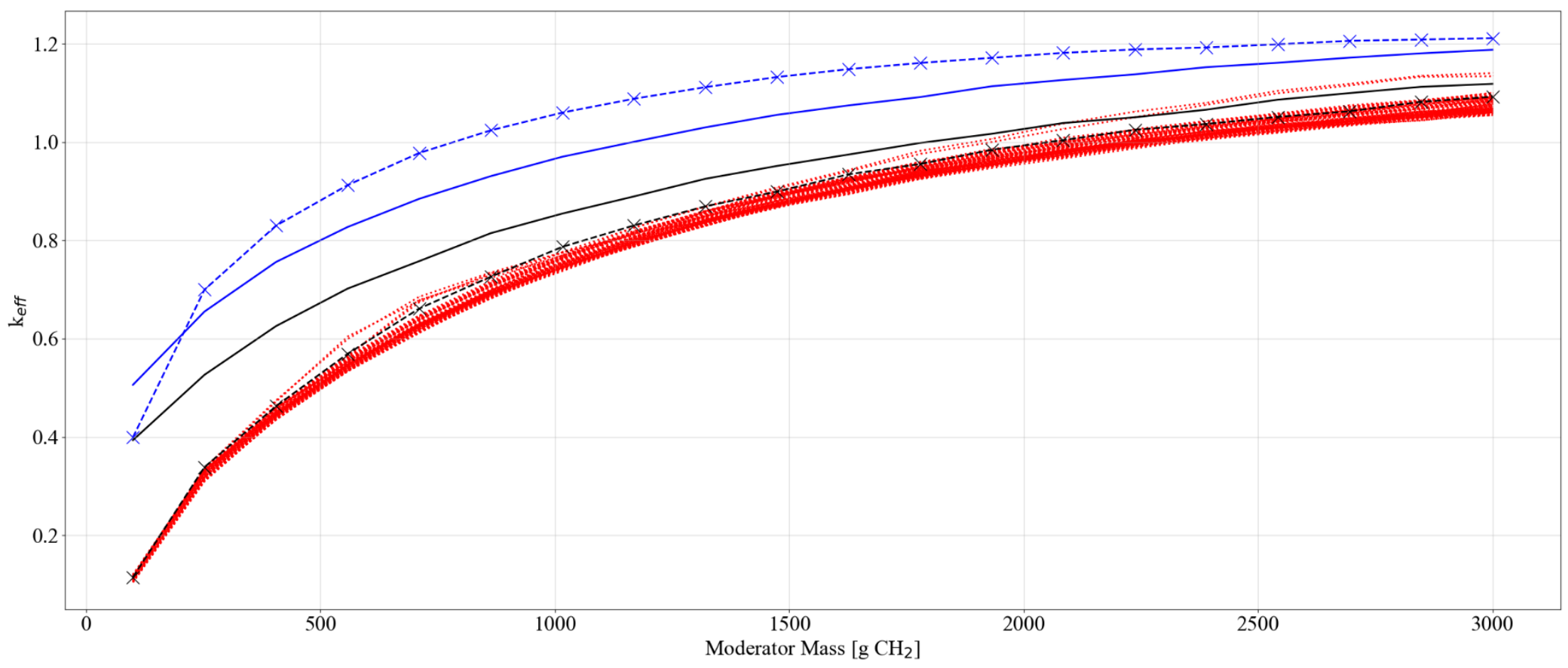

- set-1, sphere, no filler, no Be, thick poly discrete reflector, poly moderator

--׳ -- set-1, cylinder with radius $=7.7 \mathrm{~cm}$, no filler, no Be, thick poly discrete reflector, poly moderator

- set-2-uh, sphere, no filler, no Be, thick poly discrete reflector, poly moderator

--`Ł-- set-2-uh, cylinder with radius $=7.7 \mathrm{~cm}$, no filler, no Be, thick poly discrete reflector, poly moderator all centroids and midpoint

Figure 6.27. Summary of centroid study results. 


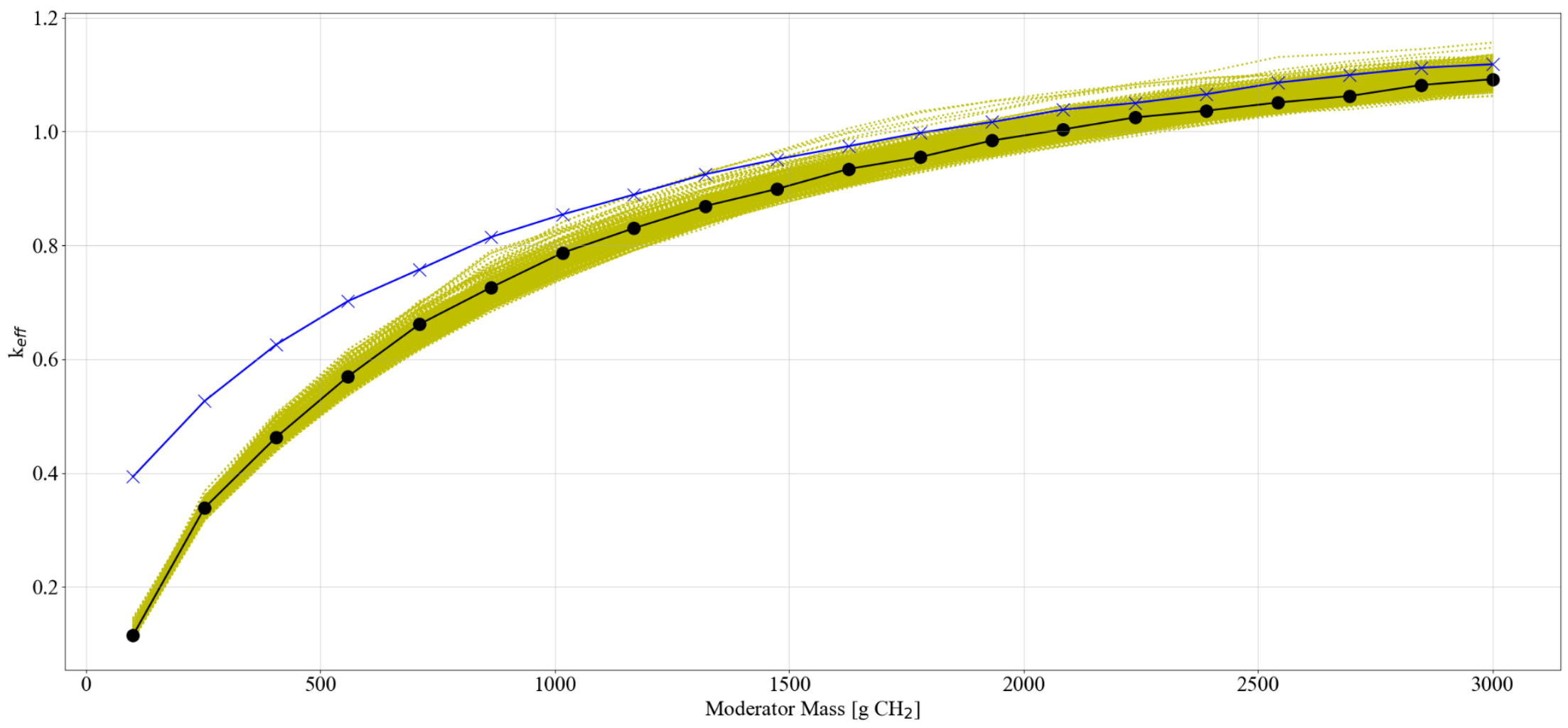

rcoord all

$*$ set-2-uh, sphere, no filler, no Be, thick poly discrete reflector, poly moderator

- set-2-uh, cylinder with radius $=7.7 \mathrm{~cm}$, no filler, no Be, thick poly discrete reflector, poly moderator

Figure 6.28. Summary of rcoord study results. 


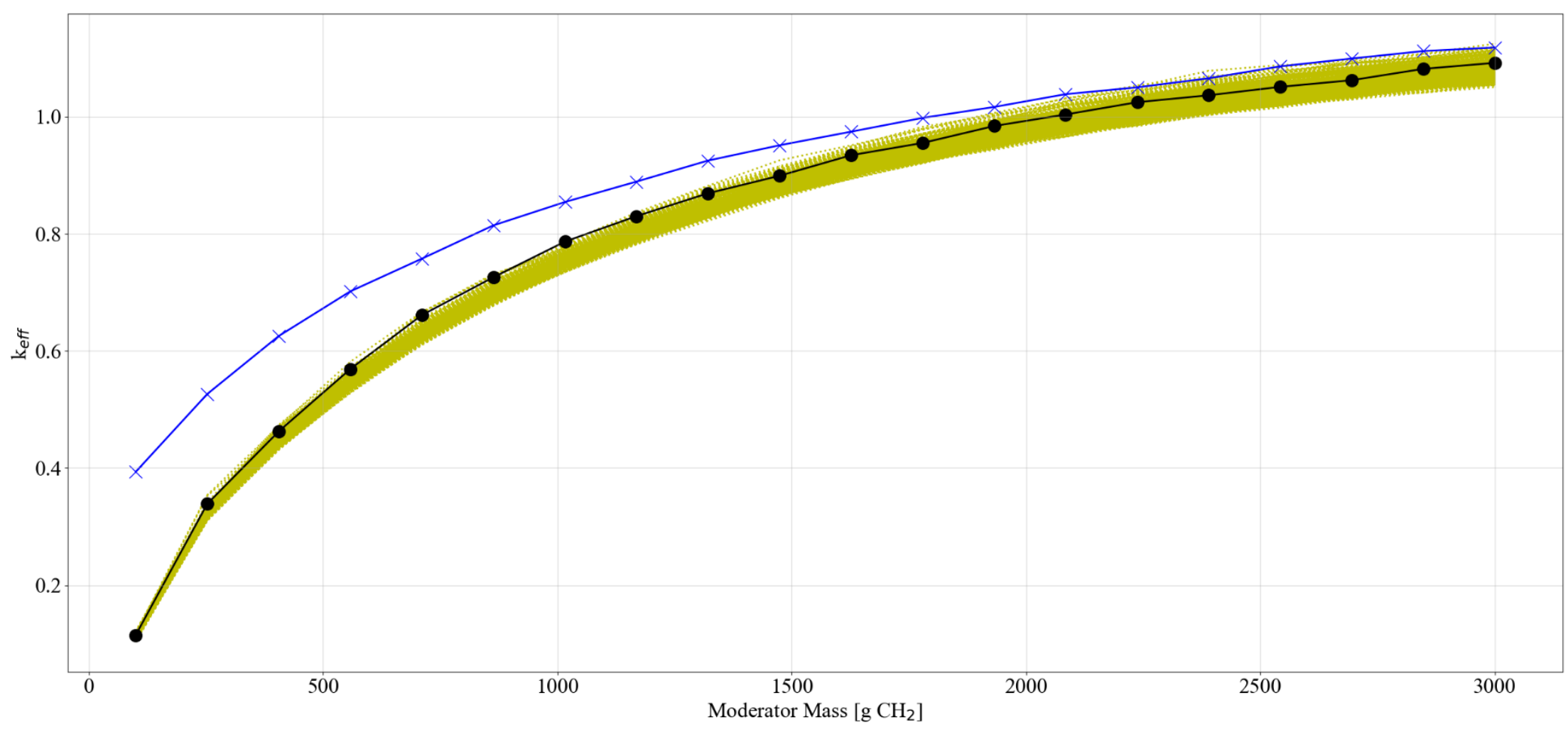

rA all

$*$ set-2-uh, sphere, no filler, no Be, thick poly discrete reflector, poly moderator

$\longrightarrow$ set-2-uh, cylinder with radius $=7.7 \mathrm{~cm}$, no filler, no Be, thick poly discrete reflector, poly moderator

Figure 6.29. Summary of rA study results. 


\subsection{SENSITIVITY OF RESULTS TO PARAMETERS VARIED}

While manual examination played a large part in the interpretation of the resulting $k_{\text {eff }}$ trends, a multivariate linear regression was used to determine the importance of each parameter for a subset of $k_{\text {eff }}$ models. In the production of the linear model, each parameter $(p)$ was rescaled to a range of -1 to 1 to represent "low" and "high," as shown in Eq. (6-1) below

$$
x_{i, j}=\frac{2 \cdot p_{i, j}}{\max _{j}\left(p_{i, j}\right)-\min _{j}\left(p_{i, j}\right)}-1
$$

where $x$ is the rescaled parameter, $i$ is the parameter type, and $j$ indexes the value for the $j$-th output. Parameters having a high covariance with parameters included in the correlation must be excluded. For example, sphere radius and cylinder height cannot be included because they are correlated to moderator mass. If they were included, then the impact of both sphere radius and moderator mass would become arbitrary. Some of the studied parameters such as moderator content, array spacing, and waste form shape were not linearizable, so a correlation was produced for each combination of those parameters instead.

After rescaling, correlation coefficients $(m)$ and a correlation constant $(c)$ were found by fitting the functional form in Eq. (6-2) below.

$$
k_{e f f, j}=\sum_{i} m_{i} x_{i, j}+c
$$

The resulting regression coefficients indicate the parameters driving system behavior. The correlations suggest the typical behavior of the system, but it is not a substitute for physics simulations.

Figure 6.310 shows the absolute value of the linear correlation coefficient (the relative importance) of each parameter for various moderator masses evaluated for a set of cylinder cases in a uniform array (set1). It is clear that variations in $\mathrm{B}_{4} \mathrm{C}$ content have the largest effect on $k_{\text {eff; }}$ filler mass, radius, pipe material, and pipe thickness are less impactful on $k_{\text {eff }}$ over the ranges in which the parameters were varied. Although the correlations are not definitive, they can quickly summarize trends in the data. Since the correlation parameters were normalized to a range of -1 to 1 , multiplying the correlation coefficient by 2 gives the typical delta $\mathrm{k}$ introduced by a given parameter within the evaluation ranges. Thus, $50 \mathrm{~g}$ per $\mathrm{CCO}$ of $\mathrm{B}_{4} \mathrm{C}$ leads to a 0.4 change in $k_{\text {eff }}$ at higher moderator masses. 


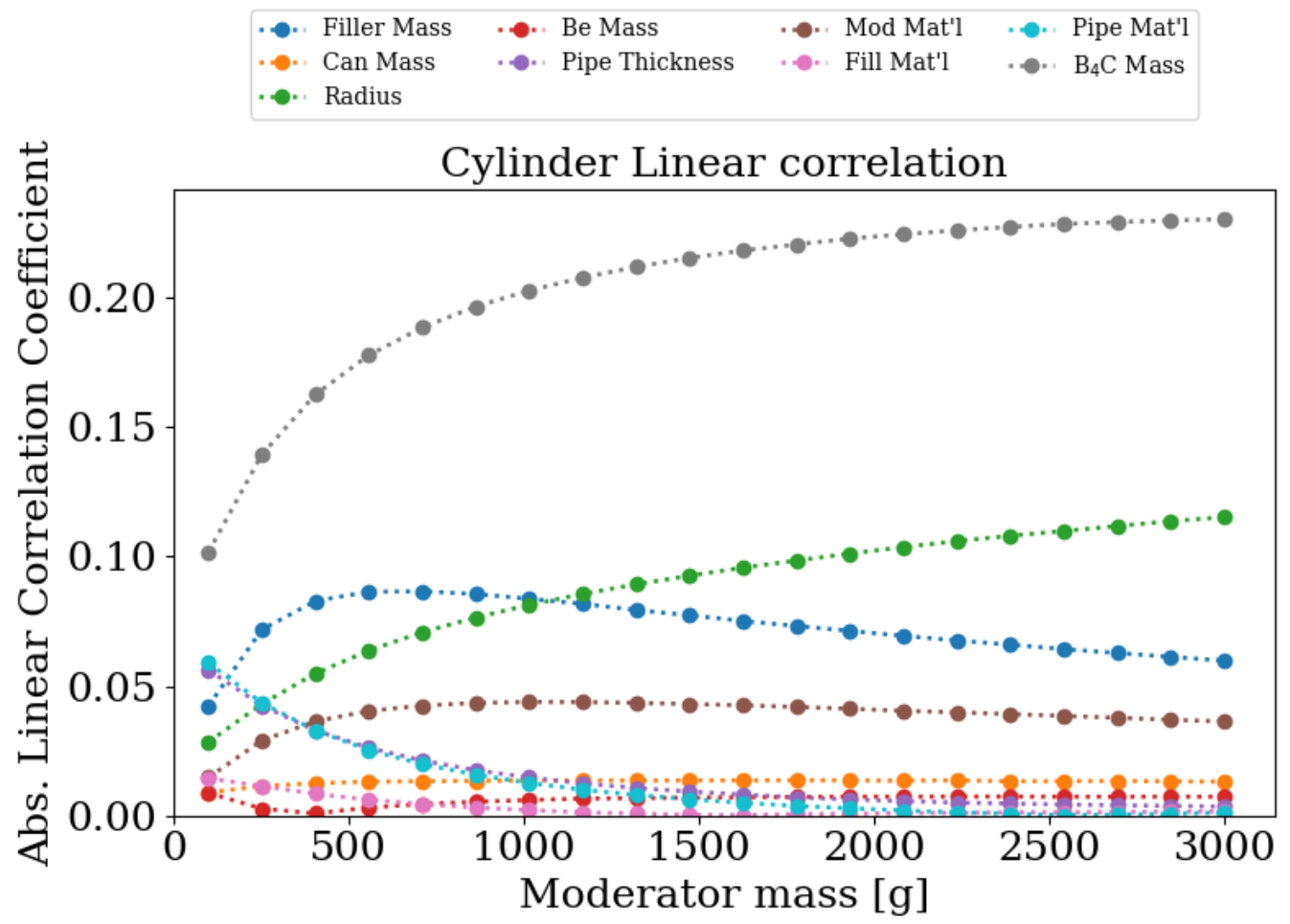

Figure 6.30. Absolute values of linear correlation coefficients for set-1 cylinders showing the relative impact of each parameter upon $k$ eff.

Because each parameter was normalized to center the range about zero, the correlation constants reflect the average behavior of the system (i.e., when all parameters are set to their midpoints). Since the real system is nonlinear, the linear approximation is only useful to gather general trends. By exploring the differences between correlation constants for different sets of parameter sweeps, the approximate average impact on $k_{\text {eff }}$ that results from changing between sets (array spacings in this case) can be observed. Figure 6.321 shows the differences in correlation constants (approximately the change in $k_{e f f}$ ) between set- 1 and set-3 cylinder cases (both uniform arrays) and the other datasets. In the figure, sphere cases were compared to sphere cases, and cylinder cases to cylinder cases. As all of the values in Figure 6.321 are negative, it is clear that set 1 and set-3 cylinders are the most reactive array configurations. Set-3 and 3a subdivide the waste forms in sets 1 and 1a, respectively, into two per CCO, so set- 3 waste forms have half the volume of set- 1 waste forms. The cylinder radii are set parameters, and because the cylinders are stacked in set-1, and because total mass is the same between both sets, set- 3 cylinders have roughly the same stack height as set-1 cylinders. However, they are not identical because of discrete reflector usage. The spheres do not make an equivalent configuration in set- 3 and set- 1 because the differing volume produces differing radii. 


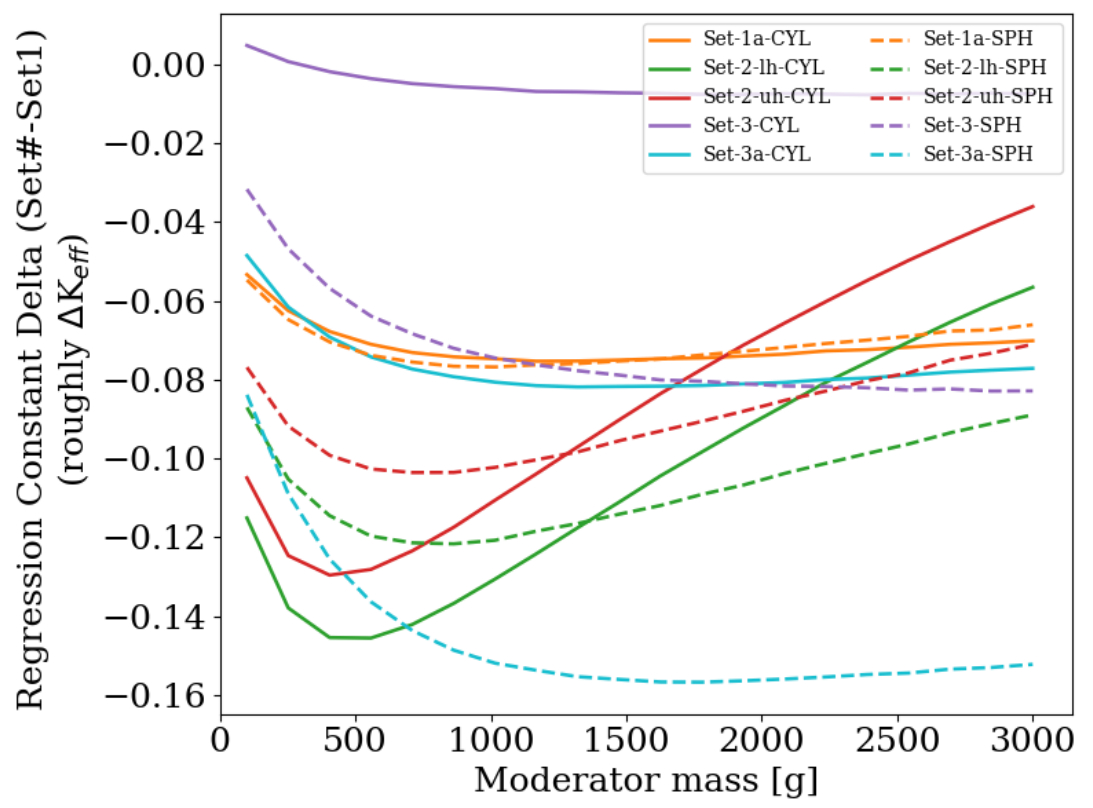

Figure 6.31. Differences in linear correlation constant terms relative to set-1 values.

To show that the correlations are applicable, and there are few nonlinearities or interaction terms, $\mathrm{r}^{2}$ values are plotted in Figure 6-32 below. These scores indicate that the correlations are sufficiently good to identify the primary system drivers.

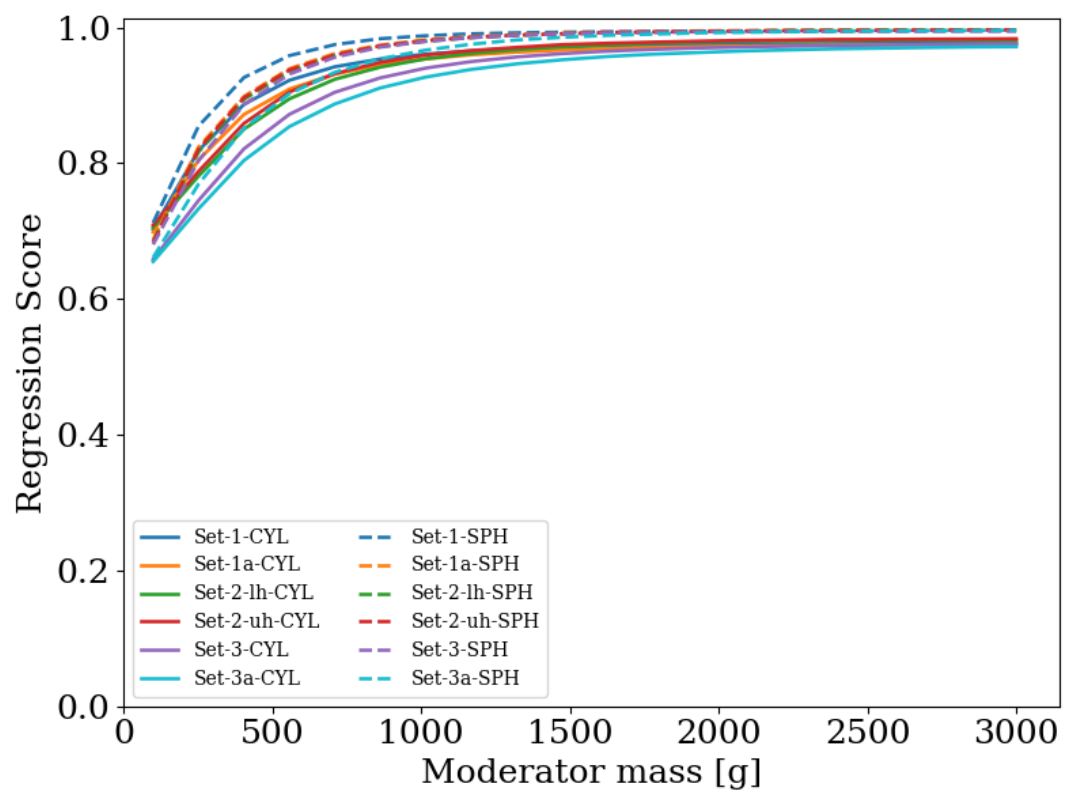

Figure 6.32. $r^{2}$ values for various moderator masses. 


\subsubsection{Correlation Coefficients for Additional Sets}

Correlation coefficients for set-1 spheres are shown in Figure 6-33 below. The coefficients are roughly similar in trend in magnitude to set-1 cylinders.

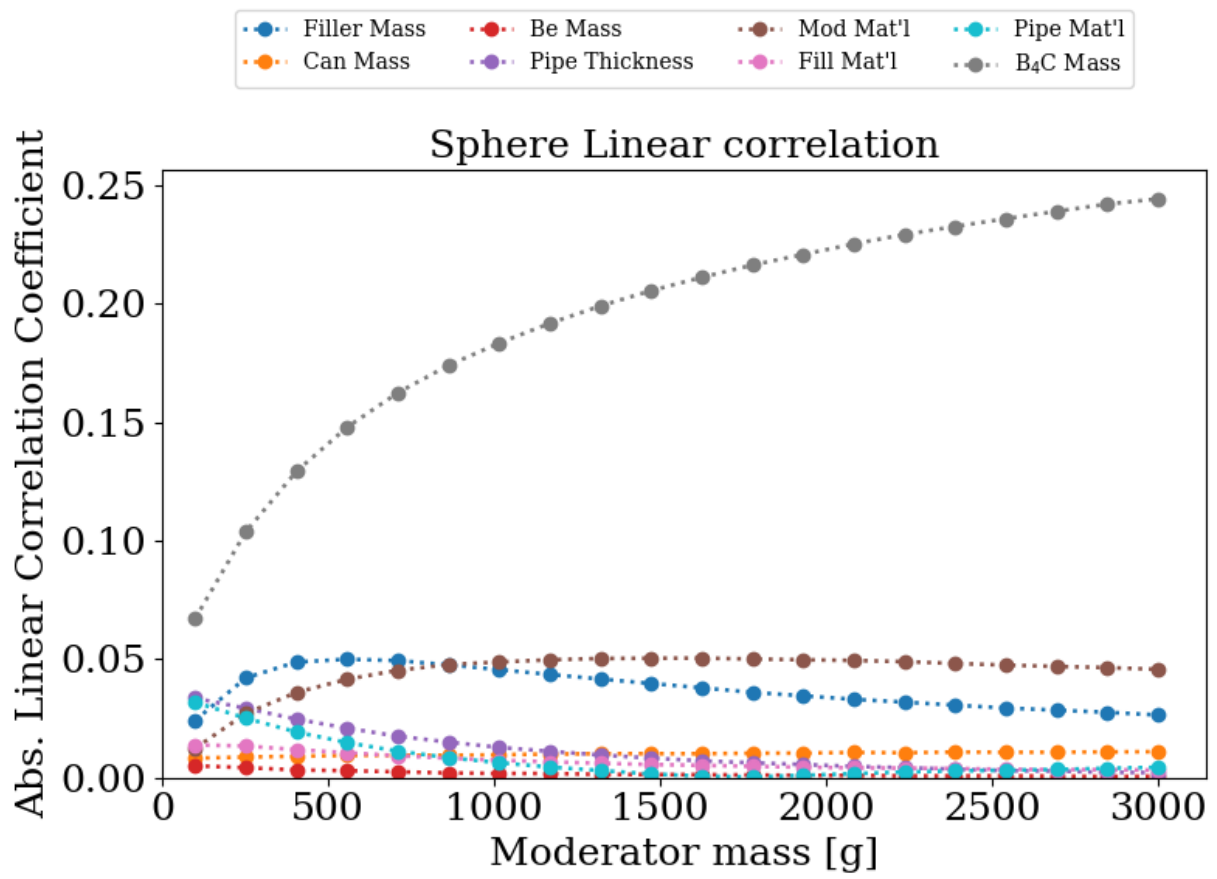

Figure 6.33. Absolute values of linear correlation coefficients for set-1 spheres showing the relative impact of each parameter upon $\boldsymbol{k}_{\text {eff. }}$.

Correlation coefficients for set-2 lower horizon data are shown in Figure 6-34 below. The radius coefficient is particularly affected as compared to that of set- 1 . This may be caused by the set- 2 cylinders being separated, as opposed to cylinders in set-1 being stacked three high in direct contact. This results in different stack height and leakage for a given radius vs. that in a group of three-stacked cylinders. 


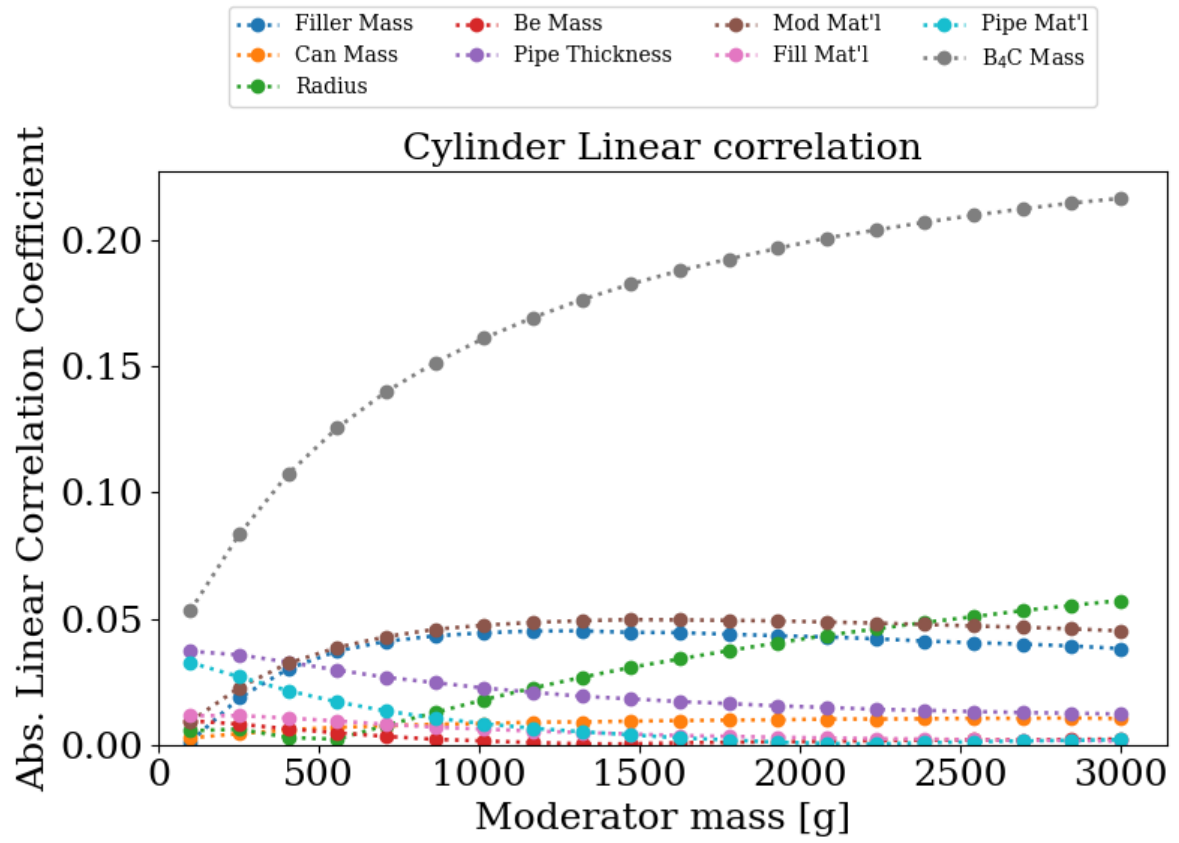

Figure 6.34. Absolute values of linear correlation coefficients for set 2-lh cylinders showing the relative impact of each parameter upon $k_{e f f}$.

Correlation coefficients for set-2-1h spheres are shown in Figure 6-35 below. The trends are roughly similar to those in the cylinder case.

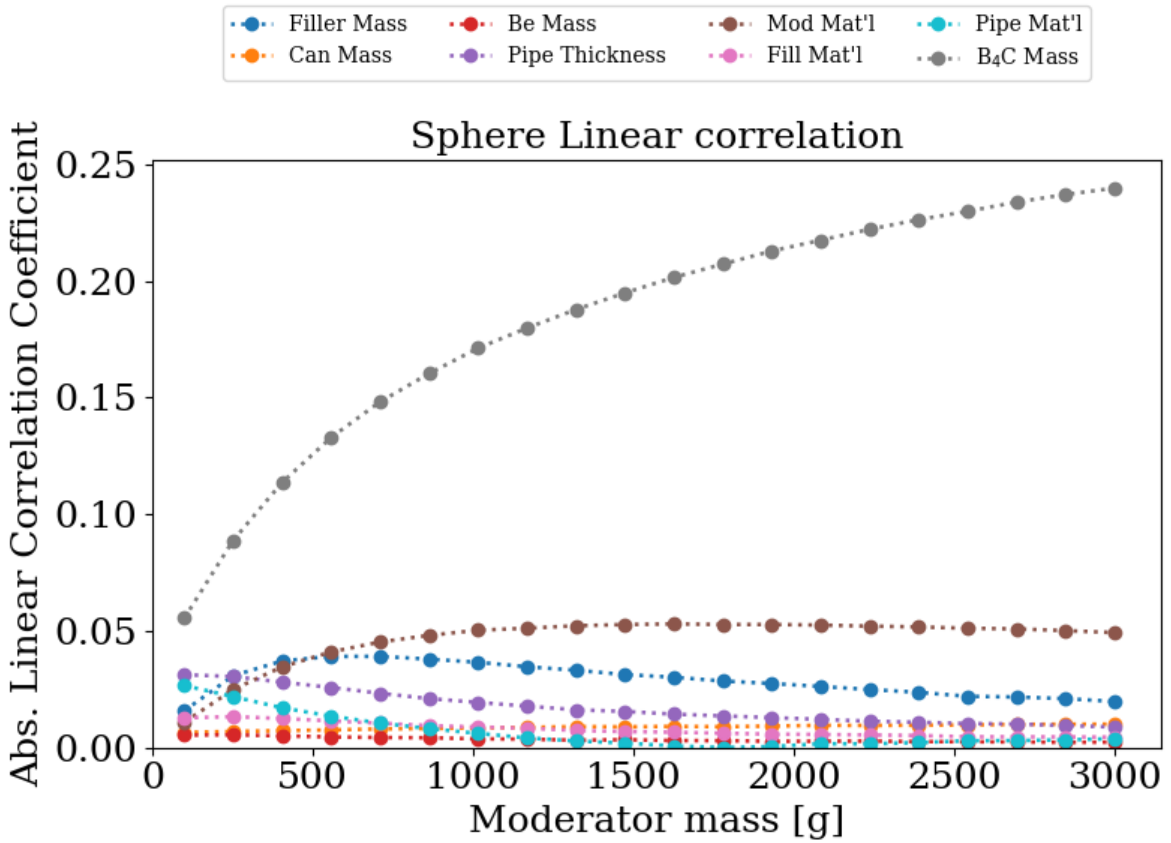

Figure 6.35. Absolute values of linear correlation coefficients for set-2lh spheres showing the relative impact of each parameter upon $\boldsymbol{k}_{\text {eff }}$. 


\subsection{UPPER SUBCRITICAL MODERATOR MASSES}

Upper subcritical masses were evaluated for various configurations of each set of cases evaluated in this work. They are presented here. Note that in these analyses the upper interpolation point was $3000 \mathrm{~g}$ of moderator. For points at $3000 \mathrm{~g}$ of moderator in plots in this subsection, this indicates that no upper value for subcritical moderator mass was found. The lines indicating sphere do not correspond to the radius listed in the plot. The lines for spheres instead simply indicate the upper subcritical mass for all spheres.

\subsubsection{Set-1 Uniform Array of Waste Forms Stacked Three High with Closer Spacing}

Table 6-3, Figure 6-36 and Figure 6-37 below shows the masses that were always associated with subcriticality in this study and did not produce $\mathrm{k}>1$ for various types of scenarios evaluated in set- 1 . These masses were determined by interpolating between datapoints. Addition of filler and reduction of cylinder radius substantially increases the calculated maximum subcritical mass. To a lesser degree, the thick polyethylene pipe (i.e., discrete reflector) reduced the maximum subcritical mass. No such polyethylene geometry exists in the initial waste form configuration, and this case was examined to identify the effect of moderator directly outside of the waste form.

Because the polyethylene is modeled as sandwiched between three cylindrical waste forms, the stack obtains higher reactivity. A similar configuration may be conceivable assuming no decay of the plywood dunnage in the CCOs and perfect stacking in compaction.

Table 6.3. Moderator masses that did not produce $k>1$ for evaluations in set-1

\begin{tabular}{|c|c|c|c|c|c|c|}
\hline \multicolumn{7}{|c|}{ Set-1 interpolated mass of waste form moderator (g) } \\
\hline \multicolumn{7}{|c|}{ No filler mass } \\
\hline Pipe material & Pipe thickness (cm) & Be (g) & cyl $R=4.8$ & cyl $R=6.25$ & cyl $\mathbf{R}=7.7$ & sph \\
\hline Polyethylene & 0.001 & 0 & 1,626 & 900 & 816 & 1,156 \\
\hline Polyethylene & 0.7112 & 0 & 1,497 & 776 & 637 & 1,161 \\
\hline Polyethylene & 0.001 & 585 & No upper bound & 1,013 & 821 & 1,171 \\
\hline Polyethylene & 0.7112 & 585 & No upper bound & 860 & 646 & 1,169 \\
\hline Steel & 0.001 & 0 & 1,631 & 907 & 823 & 1,152 \\
\hline Steel & 0.7112 & 0 & 1,441 & 932 & 876 & 1,093 \\
\hline Steel & 0.001 & 585 & 3,000 & 997 & 820 & 1,164 \\
\hline Steel & 0.7112 & 585 & 2,068 & 1,000 & 880 & 1,107 \\
\hline \multicolumn{7}{|c|}{$4 \mathrm{~kg}$ filler mass } \\
\hline Pipe material & Pipe thickness (cm) & Be (g) & cyl $R=4.8$ & cyl $R=6.25$ & cyl $R=7.7$ & sph \\
\hline Polyethylene & 0.001 & 0 & No upper bound & 2,432 & 1,441 & 1,561 \\
\hline Polyethylene & 0.7112 & 0 & No upper bound & 2,288 & 1,301 & 1,617 \\
\hline Polyethylene & 0.001 & 585 & No upper bound & 2,758 & 1,497 & 1,549 \\
\hline Polyethylene & 0.7112 & 585 & No upper bound & 2,628 & 1,344 & 1,593 \\
\hline Steel & 0.001 & 0 & No upper bound & 2,403 & 1,450 & 1,566 \\
\hline Steel & 0.7112 & 0 & No upper bound & 2,181 & 1,445 & 1,471 \\
\hline Steel & 0.001 & 585 & No upper bound & 2,688 & 1,508 & 1,538 \\
\hline Steel & 0.7112 & 585 & No upper bound & 2,379 & 1,485 & 1,448 \\
\hline
\end{tabular}




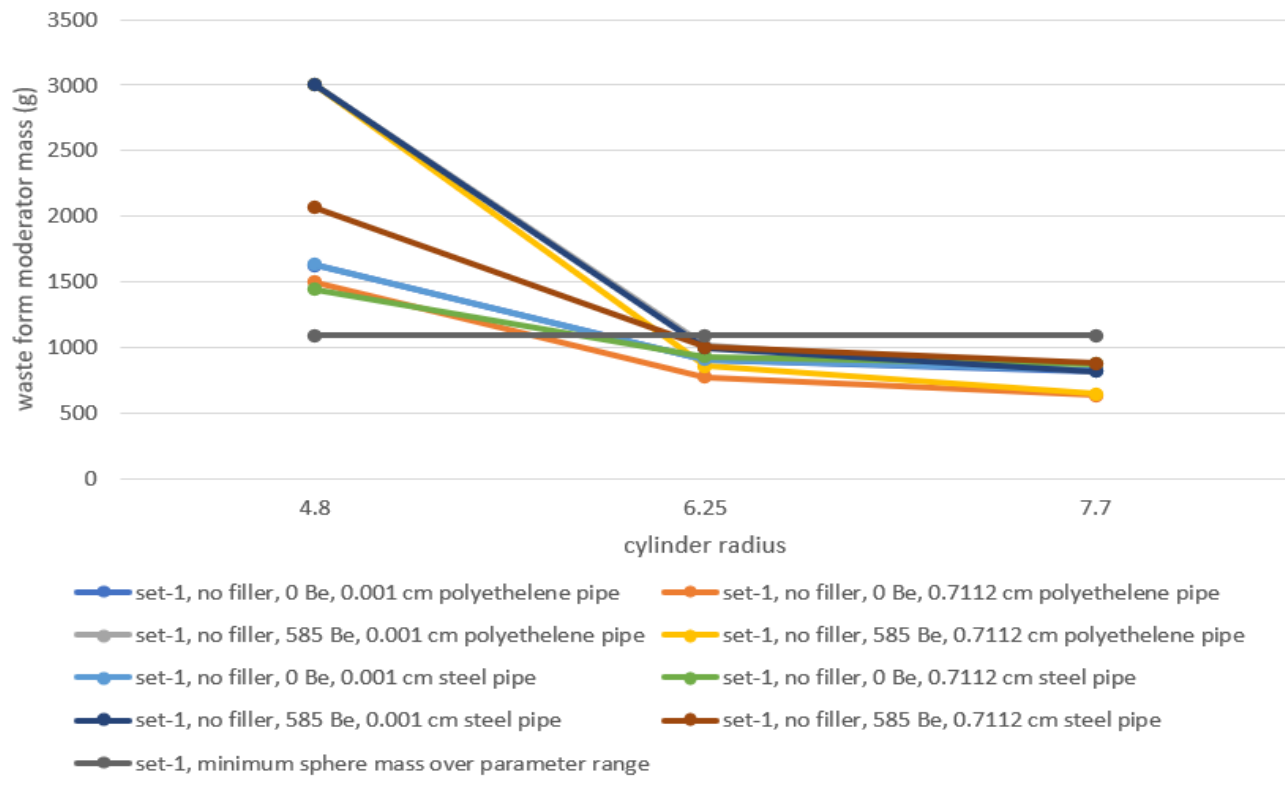

Figure 6.36. Subcritical moderator masses for evaluations in set-1 without filler vs. cylinder radius.

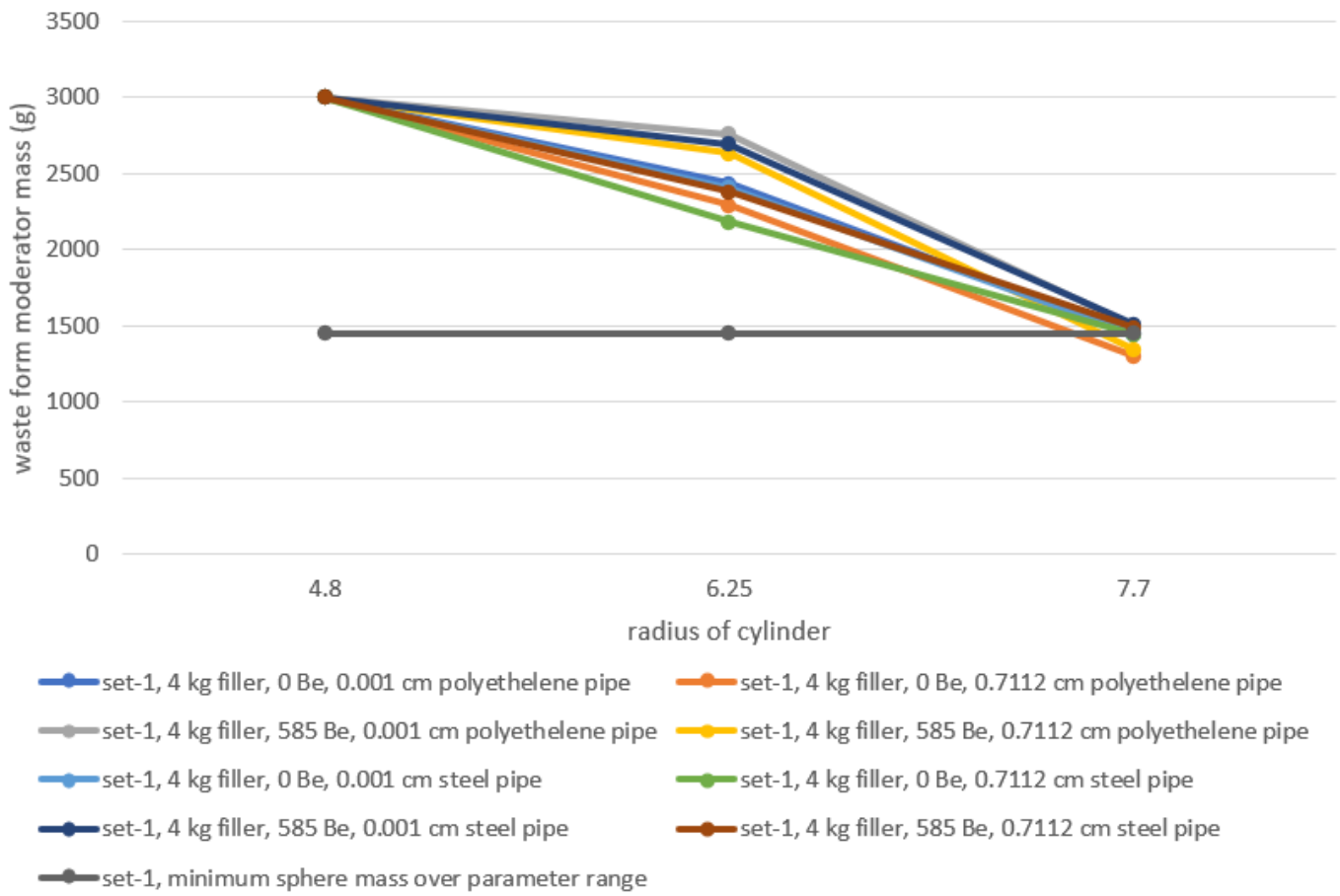

Figure 6.37. Subcritical moderator masses for evaluations in set-1 with filler vs. cylinder radius. 


\subsubsection{Set-1a Uniform Array of Waste Forms Stacked Three High with Wider Spacing}

Table 6-4, Figure 6-38 and Figure 6-39 below shows the masses that were always associated with subcriticality in this study and did not produce $\mathrm{k}>1$ for various types of scenarios evaluated in set- $1 \mathrm{a}$. These were determined in the same manner as the plot in Section 6.6.1, and they have similar trends and caveats overall. Due to the increased spacing in set-1a, upper subcritical masses are larger than those in set-1. This is due to the less restrictive assumption regarding horizontal compaction in the $\mathrm{x}$-direction of the room.

Table 6.4. Moderator masses that did not produce $k>1$ for evaluations in set-1a

\begin{tabular}{|c|c|c|c|c|c|c|}
\hline \multicolumn{7}{|c|}{ Set-1a interpolated mass of waste form moderator (g) } \\
\hline \multicolumn{7}{|c|}{ No filler mass } \\
\hline Pipe material & Pipe thickness (cm) & Be (g) & cyl $R=4.8$ & cyl $R=6.25$ & cyl $R=7.7$ & sph \\
\hline Polyethylene & 0.001 & 0 & No upper bound & 1,448 & 1,107 & 1,634 \\
\hline Polyethylene & 0.7112 & 0 & No upper bound & 1,216 & 905 & 1,625 \\
\hline Polyethylene & 0.001 & 585 & No upper bound & 1,683 & 1,160 & 1,639 \\
\hline Polyethylene & 0.7112 & 585 & No upper bound & 1,468 & 948 & 1,649 \\
\hline Steel & 0.001 & 0 & No upper bound & 1,438 & 1,110 & 1,650 \\
\hline Steel & 0.7112 & 0 & No upper bound & 1,458 & 1,189 & 1,560 \\
\hline Steel & 0.001 & 585 & No upper bound & 1,719 & 1,165 & 1,658 \\
\hline Steel & 0.7112 & 585 & No upper bound & 1,672 & 1,251 & 1,576 \\
\hline \multicolumn{7}{|c|}{$4 \mathrm{~kg}$ filler mass } \\
\hline Pipe material & Pipe thickness (cm) & Be (g) & cyl $R=4.8$ & cyl $R=6.25$ & cyl $R=7.7$ & sph \\
\hline Polyethylene & 0.001 & 0 & No upper bound & No upper bound & 2,233 & 2,182 \\
\hline Polyethylene & 0.7112 & 0 & No upper bound & No upper bound & 2,023 & 2,196 \\
\hline Polyethylene & 0.001 & 585 & No upper bound & No upper bound & 2,372 & 2,169 \\
\hline Polyethylene & 0.7112 & 585 & No upper bound & No upper bound & 2,152 & 2,184 \\
\hline Steel & 0.001 & 0 & No upper bound & No upper bound & 2,264 & 2,186 \\
\hline Steel & 0.7112 & 0 & No upper bound & No upper bound & 2,254 & 2,057 \\
\hline Steel & 0.001 & 585 & No upper bound & No upper bound & 2,389 & 2,174 \\
\hline Steel & 0.7112 & 585 & No upper bound & No upper bound & 2,377 & 2,058 \\
\hline
\end{tabular}




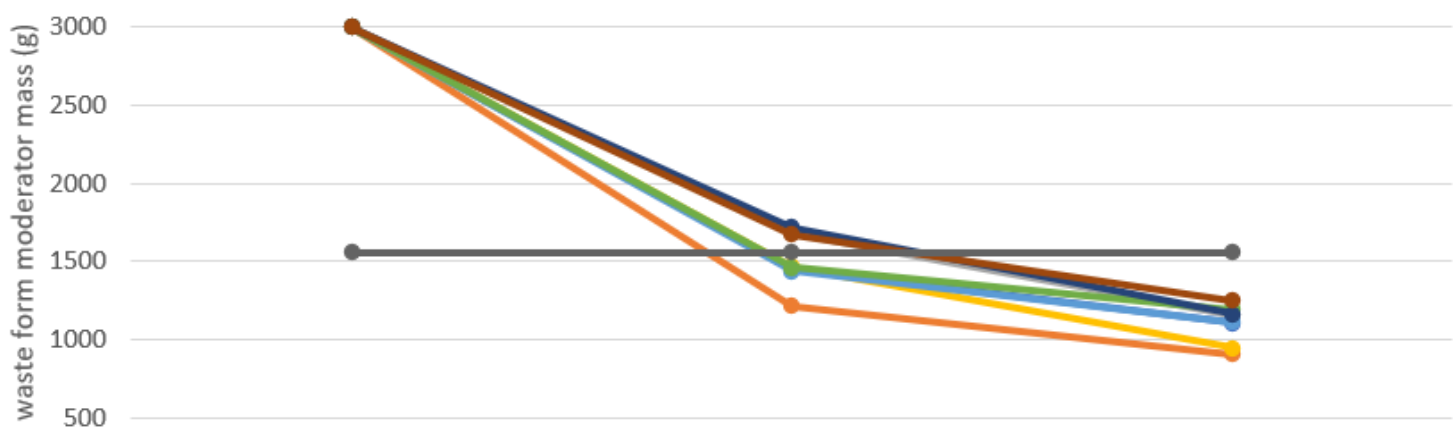

0

cylinder radius

$\longrightarrow$ set-1a, no filler, $0 \mathrm{Be}, 0.001 \mathrm{~cm}$ polyethelene pipe $\longrightarrow$ set-1a, no filler, $0 \mathrm{Be}, 0.7112 \mathrm{~cm}$ polyethelene pipe

$\longrightarrow$ set-1a, no filler, $585 \mathrm{Be}, 0.001 \mathrm{~cm}$ polyethelene pipe

—set-1a, no filler, 0 Be, $0.001 \mathrm{~cm}$ steel pipe

— set-1a, no filler, $585 \mathrm{Be}, 0.7112 \mathrm{~cm}$ polyethelene pipe

$\sim$ set-1a, no filler, $585 \mathrm{Be}, 0.001 \mathrm{~cm}$ steel pipe

set-1a, no filler, $0 \mathrm{Be}, 0.7112 \mathrm{~cm}$ steel pipe

_ set-1a, minimum sphere mass over parameter range

Figure 6.38. Subcritical moderator masses for evaluations in set-1a without filler vs. cylinder radius.

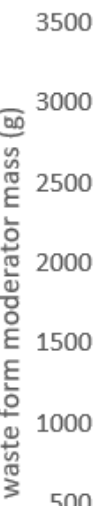

_ set-1a, no filler, 585 Be, $0.7112 \mathrm{~cm}$ steel pipe

500

0

4.8

$$
6.25
$$

7.7

radius of cylinder

— set-1a, $4 \mathrm{~kg}$ filler, $0 \mathrm{Be}, 0.001 \mathrm{~cm}$ polyethelene pipe radius of cylinder $\mathrm{set}-1 \mathrm{a}, 4 \mathrm{~kg}$ filler, $0 \mathrm{Be}, 0.7112 \mathrm{~cm}$ polyethelene pipe

— set-1a, $4 \mathrm{~kg}$ filler, $585 \mathrm{Be}, 0.001 \mathrm{~cm}$ polyethelene pipe

- set-1a, 4 kg filler, 0 Be, $0.001 \mathrm{~cm}$ steel pipe

set-1a, 4 kg filler, $585 \mathrm{Be}, 0.7112 \mathrm{~cm}$ polyethelene pipe

ـset-1a, 4 kg filler, $585 \mathrm{Be}, 0.001 \mathrm{~cm}$ steel pipe

—set-1a, 4 kg filler, 0 Be, $0.7112 \mathrm{~cm}$ steel pipe

—set-1a, minimum sphere mass over parameter range

Figure 6.39. Subcritical moderator masses for evaluations in set-1a with filler vs. cylinder radius. 


\subsubsection{Set-2 Nonuniform Array of Waste Forms.}

Table 6-5, Figure 6-40, and Figure 6-41 show upper subcritical moderator masses for set-2. Because individual waste forms are spaced farther apart compared to sets 1, 1a, 3, and 3a, the mass limits are higher. Other trends are roughly similar to set-1. This set has limited close contact between waste forms and is based on a sampling of 153 CCOs. It constitutes a best estimate array spacing assumption for the worst-case time step for the simulation at 1,000 years after emplacement.

Given that the neutron flux from a package tapers off quickly over half a meter (see Appendix K), the closest grouping of CCOs in a nonuniform array would be the most reactive. Assuming fair odds and representative sampling, roughly one stack of three waste forms would occur in a collection of $153^{2}$ (over $20,000)$ waste forms. Thus, for such a case, set-1 or set1 a may provide $k_{\text {eff }}$ values and mass limits that better approximate a realistic configuration that includes a triple-stack of compacted CCOs.

Table 6.5. Masses that did not produce $k>1$ for evaluations in set-2

\begin{tabular}{|c|c|c|c|c|c|c|}
\hline \multicolumn{7}{|c|}{ Set-2 interpolated mass of waste form moderator (g) } \\
\hline \multicolumn{7}{|c|}{ No filler mass } \\
\hline Pipe material & Pipe thickness $(\mathrm{cm})$ & Be (g) & cyl $R=4.8$ & cyl $R=6.25$ & cyl $R=7.7$ & sph \\
\hline Polyethylene & 0.001 & 0 & 2849 & 2,015 & 1,997 & 1,893 \\
\hline Polyethylene & 0.7112 & 0 & 2702 & 1,947 & 1,930 & 1,806 \\
\hline Polyethylene & 0.001 & 585 & No upper bound & 2,072 & 1,936 & 1,876 \\
\hline Polyethylene & 0.7112 & 585 & 2994 & 1,954 & 1,871 & 1,792 \\
\hline Steel & 0.001 & 0 & 2805 & 2,059 & 2,018 & 1,897 \\
\hline Steel & 0.7112 & 0 & 2262 & 1,791 & 1,811 & 1,722 \\
\hline Steel & 0.001 & 585 & No upper bound & 2,073 & 1,980 & 1,867 \\
\hline Steel & 0.7112 & 585 & 2457 & 1,849 & 1,774 & 1,693 \\
\hline \multicolumn{7}{|c|}{$4 \mathrm{~kg}$ filler mass } \\
\hline Pipe material & Pipe thickness (cm) & Be (g) & cyl $R=4.8$ & cyl $R=6.25$ & cyl $R=7.7$ & sph \\
\hline Polyethylene & 0.001 & 0 & No upper bound & No upper bound & 2,481 & 2,259 \\
\hline Polyethylene & 0.7112 & 0 & No upper bound & No upper bound & 2,384 & 2,198 \\
\hline Polyethylene & 0.001 & 585 & No upper bound & No upper bound & 2,546 & 2,230 \\
\hline Polyethylene & 0.7112 & 585 & No upper bound & No upper bound & 2,394 & 2,132 \\
\hline Steel & 0.001 & 0 & No upper bound & No upper bound & 2,520 & 2,271 \\
\hline Steel & 0.7112 & 0 & No upper bound & 2,704 & 2,207 & 2,059 \\
\hline Steel & 0.001 & 585 & No upper bound & No upper bound & 2,496 & 2,229 \\
\hline Steel & 0.7112 & 585 & No upper bound & 2,727 & 2,235 & 2,022 \\
\hline
\end{tabular}




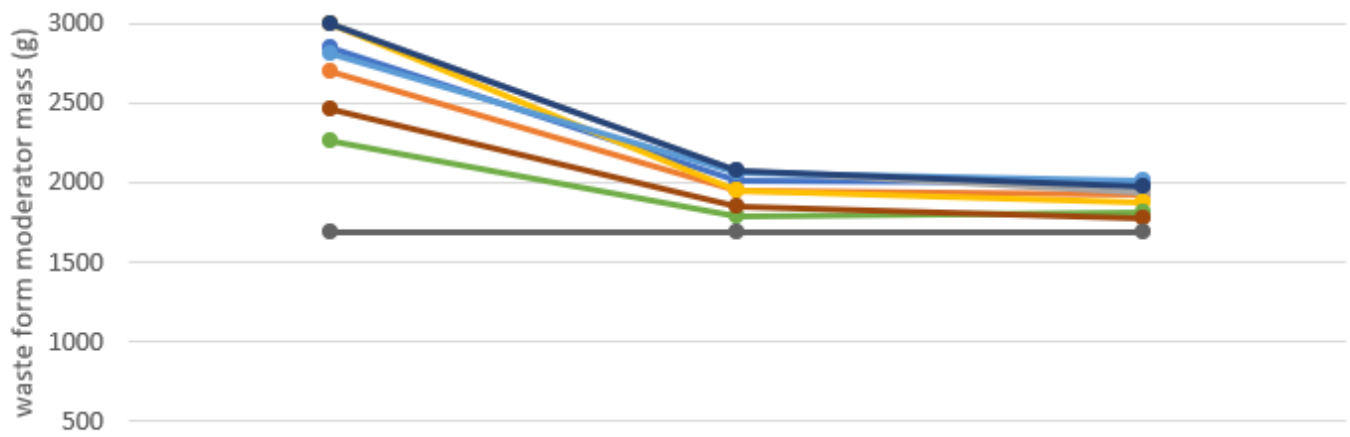

500

4.8

6.25

7.7

cylinder radius

$\longrightarrow$ set-2, no filler, $0 \mathrm{Be}, 0.001 \mathrm{~cm}$ polyethelene pipe — set-2, no filler, $585 \mathrm{Be}, 0.001 \mathrm{~cm}$ polyethelene pipe

— set-2, no filler, $585 \mathrm{Be}, 0.7112 \mathrm{~cm}$ polyethelene pipe

$\longrightarrow$ set-2, no filler, $0 \mathrm{Be}, 0.001 \mathrm{~cm}$ steel pipe

—set-2, no filler, $0 \mathrm{Be}, 0.7112 \mathrm{~cm}$ steel pipe

$\multimap$ set-2, no filler, 585 Be, $0.001 \mathrm{~cm}$ steel pipe

$\longrightarrow$ set-2, no filler, $585 \mathrm{Be}, 0.7112 \mathrm{~cm}$ steel pipe

$\longrightarrow$ - 2 et-2, minimum sphere mass over parameter range

Figure 6.40. Subcritical masses for evaluations in set-2 without filler vs. cylinder radius.

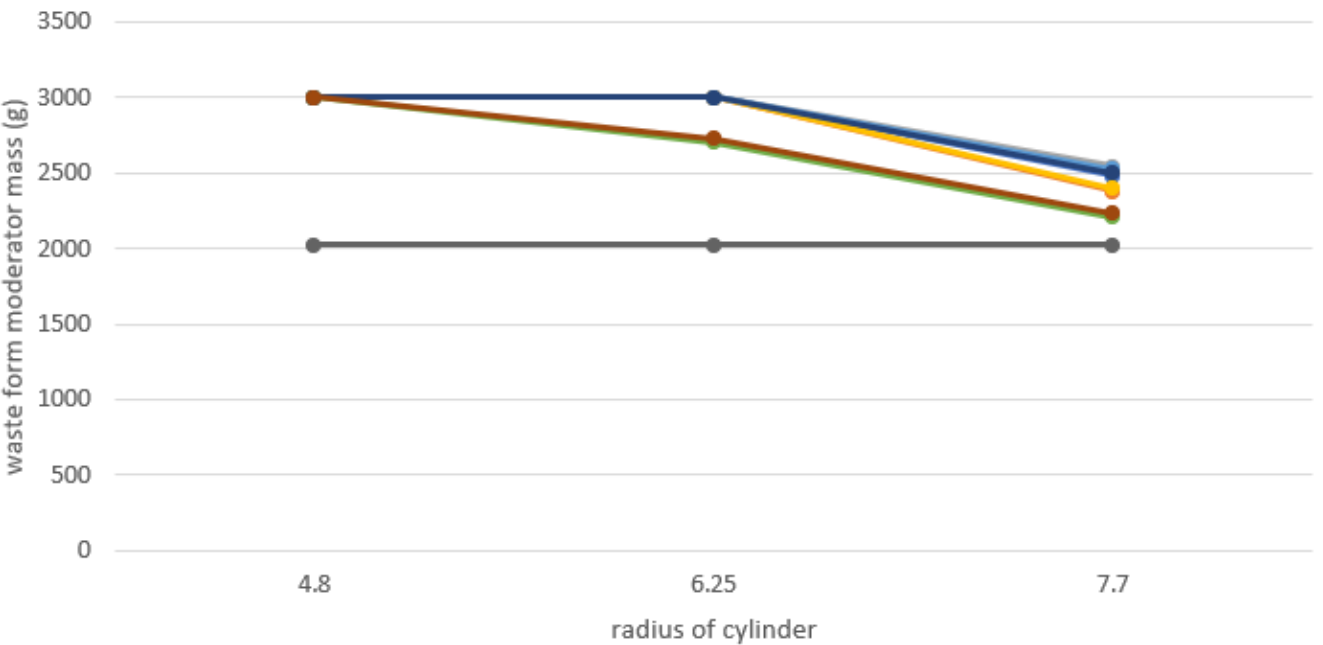

\footnotetext{
—set-2, $4 \mathrm{~kg}$ filler, $0 \mathrm{Be}, 0.001 \mathrm{~cm}$ polyethelene pipe $\quad \longrightarrow$ set- $2,4 \mathrm{~kg}$ filler, $0 \mathrm{Be}, 0.7112 \mathrm{~cm}$ polyethelene pipe — set-2, $4 \mathrm{~kg}$ filler, $585 \mathrm{Be}, 0.001 \mathrm{~cm}$ polyethelene pipe — - set-2, $4 \mathrm{~kg}$ filler, $585 \mathrm{Be}, 0.7112 \mathrm{~cm}$ polyethelene pipe — set-2, $4 \mathrm{~kg}$ filler, 0 Be, $0.001 \mathrm{~cm}$ steel pipe — set-2, $4 \mathrm{~kg}$ filler, $0 \mathrm{Be}, 0.7112 \mathrm{~cm}$ steel pipe

— set-2, 4 kg filler, 585 Be, $0.001 \mathrm{~cm}$ steel pipe — set-2, 4 kg filler, 585 Be, $0.7112 \mathrm{~cm}$ steel pipe

— set-2, minimum sphere mass over parameter range
}

Figure 6.41. Subcritical masses for evaluations in set-2 with filler vs. cylinder radius. 


\subsubsection{Set-3 Uniform Array of Waste Forms Stacked Six High with Closer Spacing}

Table 6-6, Figure 6-42, and Figure 6-431 show upper subcritical moderator masses for set-3. Set-3 is similar to Set-1 with the exception that it subdivides the contents of each CCO into two waste forms. Masses are listed per CCO. Dividing by 2 gives masses per waste form. The trends are roughly similar to those for set-1, especially for cylinders, which stack to form a similar geometry to set-1, with the exception that there are six cylinders rather than three, and therefore more discrete reflector material.

Table 6.6. Masses that did not produce $k>1$ for evaluations in set-3

\begin{tabular}{|c|c|c|c|c|c|c|}
\hline \multicolumn{7}{|c|}{ Set-3 interpolated mass of waste form moderator (g) per CCO ( 2 waste forms per CCO) } \\
\hline \multicolumn{7}{|c|}{ No filler mass } \\
\hline Pipe material & Pipe thickness $(\mathrm{cm})$ & $\operatorname{Be}(\mathrm{g})$ & cyl $R=4.8$ & cyl $R=6.25$ & cyl $R=7.7$ & sph \\
\hline Polyethylene & 0.001 & 0 & 1,599 & 906 & 825 & 1,692 \\
\hline Polyethylene & 0.7112 & 0 & 1,569 & 675 & 427 & 1,853 \\
\hline Polyethylene & 0.001 & 585 & No upper bound & 1,006 & 826 & 1,736 \\
\hline Polyethylene & 0.7112 & 585 & No upper bound & 765 & 436 & 1,907 \\
\hline Steel & 0.001 & 0 & 1,633 & 906 & 823 & 1,700 \\
\hline Steel & 0.7112 & 0 & 1,813 & 1,140 & 1,076 & 1,655 \\
\hline Steel & 0.001 & 585 & No upper bound & 992 & 825 & 1,765 \\
\hline Steel & 0.7112 & 585 & No upper bound & 1,226 & 1,095 & 1,717 \\
\hline \multicolumn{7}{|c|}{$4 \mathrm{~kg}$ filler mass } \\
\hline Pipe material & Pipe thickness (cm) & Be (g) & cyl $R=4.8$ & cyl $R=6.25$ & cyl $R=7.7$ & sph \\
\hline Polyethylene & 0.001 & 0 & No upper bound & 2,398 & 1,447 & 2,445 \\
\hline Polyethylene & 0.7112 & 0 & No upper bound & 2,316 & 1,131 & 2,697 \\
\hline Polyethylene & 0.001 & 585 & No upper bound & 2,691 & 1,474 & 2,488 \\
\hline Polyethylene & 0.7112 & 585 & No upper bound & 2,689 & 1,205 & 2,775 \\
\hline Steel & 0.001 & 0 & No upper bound & 2,385 & 1,449 & 2,443 \\
\hline Steel & 0.7112 & 0 & No upper bound & 2,613 & 1,710 & 2,332 \\
\hline Steel & 0.001 & 585 & No upper bound & 2,728 & 1,493 & 2,484 \\
\hline Steel & 0.7112 & 585 & No upper bound & 2,928 & 1,762 & 2,368 \\
\hline
\end{tabular}




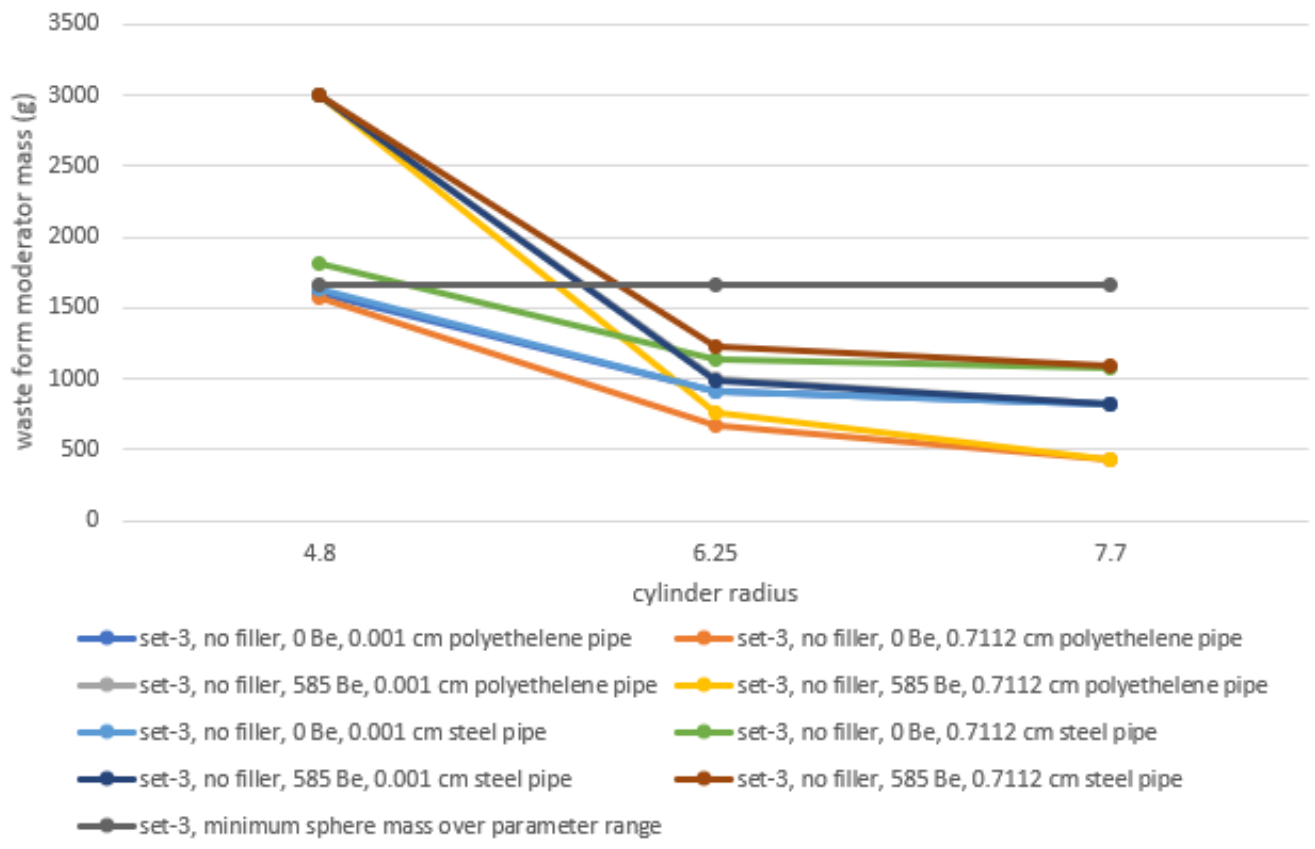

Figure 6.42. Subcritical masses for evaluations in set-3 without filler vs. cylinder radius.

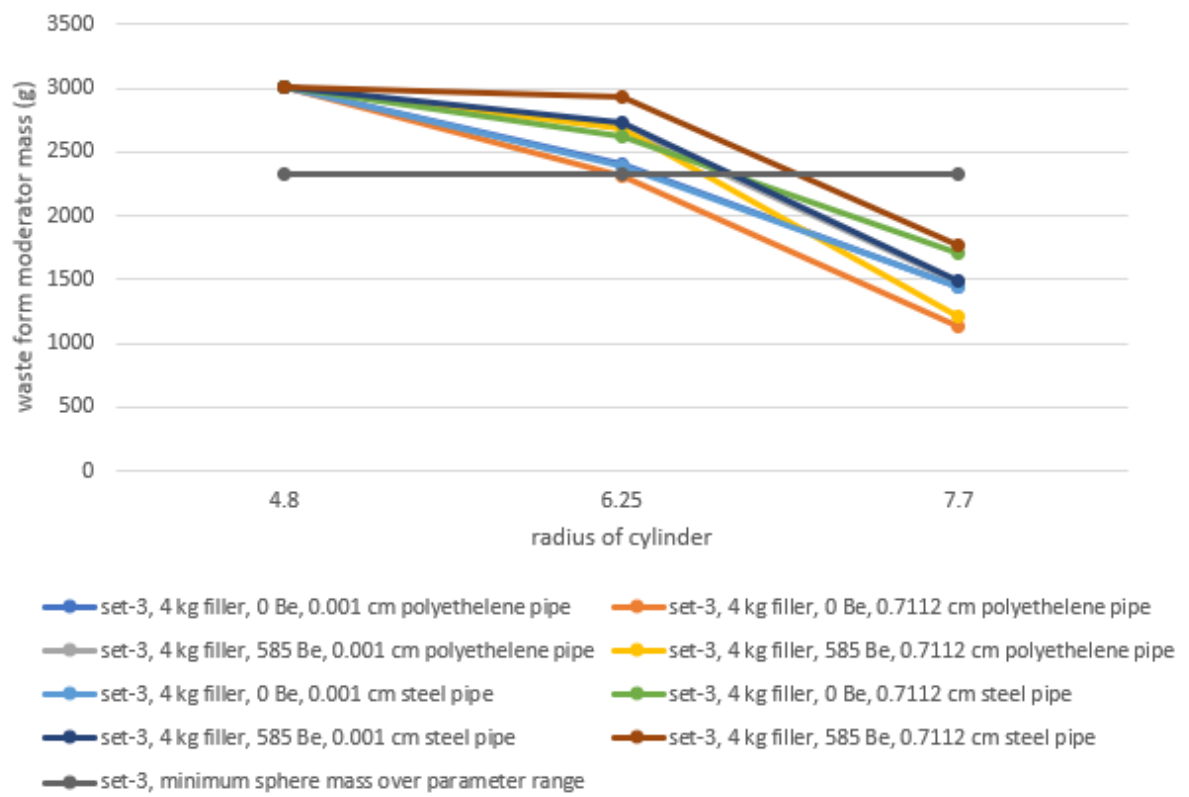

Figure 6.43. Subcritical masses for evaluations in set-3 with filler vs. cylinder radius.

\subsubsection{Set-3a Uniform Array of Waste Forms Stacked Six High with Wider Spacing}

Table 6-7, Figure 6-44, and Figure 6-45 show upper subcritical moderator masses for set-3a. Set-3a is similar to Set-1a with the exception that it subdivides the contents of each CCO into two waste forms. Masses are listed per CCO. Dividing by 2 gives masses per waste form. The trends are roughly similar to set-1a, especially for cylinders, which stack to form a similar geometry to set-1a, with the exception that 
there are six cylinders rather than three, and therefore more discrete reflector material. Set-3a has a larger spacing than set-3, so it has higher upper moderator masses.

Table 6.7. Masses that did not produce $k>1$ for evaluations in set-3a

\begin{tabular}{|c|c|c|c|c|c|c|}
\hline \multicolumn{7}{|c|}{ Set-3a interpolated mass of waste form moderator (g) per $\mathrm{CCO}$ ( 2 waste forms per $\mathrm{CCO})$} \\
\hline \multicolumn{7}{|c|}{ No filler mass } \\
\hline Pipe material & Pipe thickness (cm) & Be (g) & cyl $R=4.8$ & cyl $R=6.25$ & cyl $R=7.7$ & sph \\
\hline Polyethylene & 0.001 & 0 & No upper bound & 1,447 & 1,106 & 2,596 \\
\hline Polyethylene & 0.7112 & 0 & No upper bound & 1,187 & 704 & 2,785 \\
\hline Polyethylene & 0.001 & 585 & No upper bound & 1,694 & 1,158 & 2,691 \\
\hline Polyethylene & 0.7112 & 585 & No upper bound & 1,430 & 767 & 2,903 \\
\hline Steel & 0.001 & 0 & No upper bound & 1,450 & 1,103 & 2,628 \\
\hline Steel & 0.7112 & 0 & No upper bound & 1,890 & 1,523 & 2,560 \\
\hline Steel & 0.001 & 585 & No upper bound & 1,701 & 1,149 & 2,720 \\
\hline Steel & 0.7112 & 585 & No upper bound & 2,131 & 1,597 & 2,662 \\
\hline \multicolumn{7}{|c|}{$4 \mathrm{~kg}$ filler mass } \\
\hline Pipe material & Pipe thickness $(\mathrm{cm})$ & Be (g) & cyl $R=4.8$ & cyl $R=6.25$ & cyl $R=7.7$ & sph \\
\hline Polyethylene & 0.001 & 0 & No upper bound & No upper bound & 2,256 & No upper bound \\
\hline Polyethylene & 0.7112 & 0 & No upper bound & No upper bound & 1,940 & No upper bound \\
\hline Polyethylene & 0.001 & 585 & No upper bound & No upper bound & 2,389 & No upper bound \\
\hline Polyethylene & 0.7112 & 585 & No upper bound & No upper bound & 2,091 & No upper bound \\
\hline Steel & 0.001 & 0 & No upper bound & No upper bound & 2,278 & No upper bound \\
\hline Steel & 0.7112 & 0 & No upper bound & No upper bound & 2,767 & No upper bound \\
\hline Steel & 0.001 & 585 & No upper bound & No upper bound & 2,374 & No upper bound \\
\hline Steel & 0.7112 & 585 & No upper bound & No upper bound & 2,949 & No upper bound \\
\hline
\end{tabular}

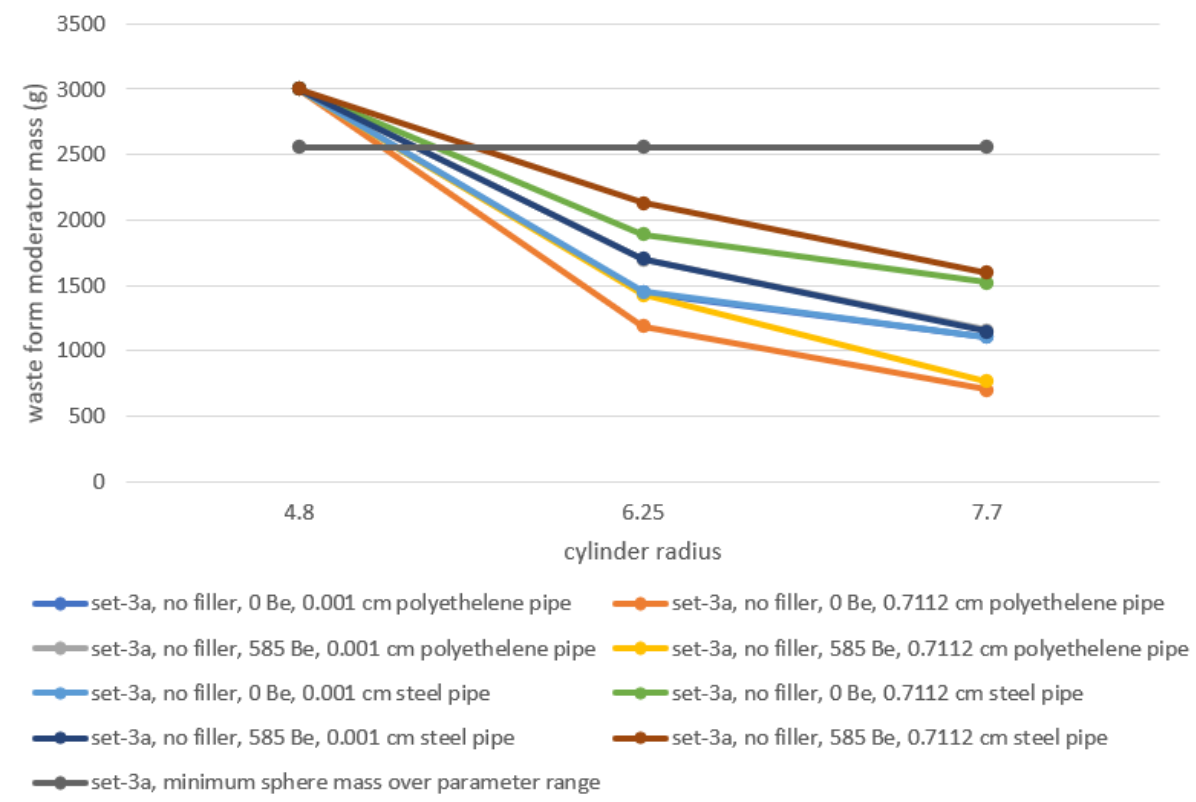

Figure 6.44. Subcritical masses for evaluations in set-3a without filler vs. cylinder radius. 


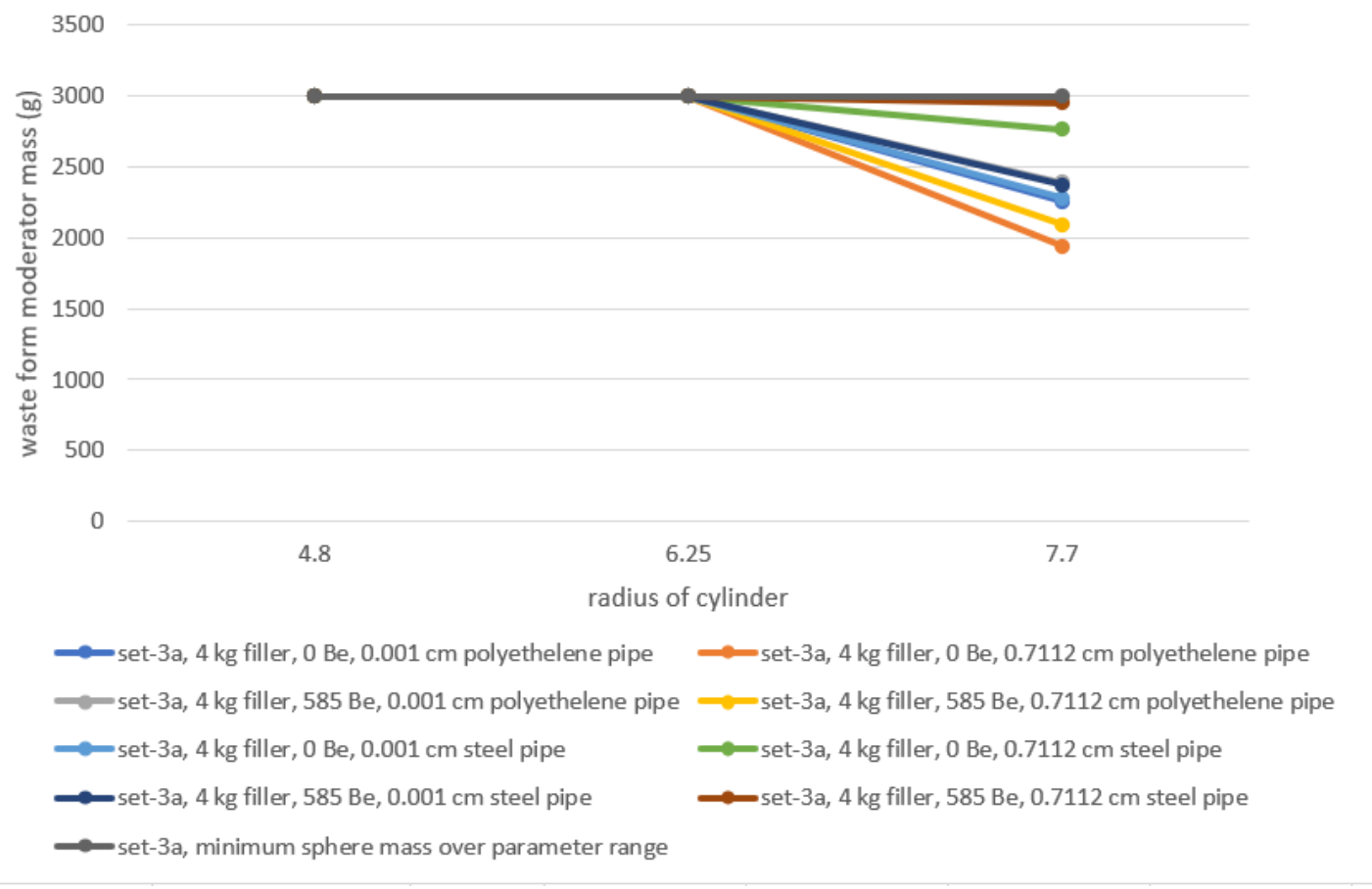

Figure 6.45. Subcritical masses for evaluations in set-3a with filler vs. cylinder radius.

\subsection{EFFECT OF WASTE FORM HETEROGENEITY}

The criticality safety analysis presented in this report uses the same assumption regarding waste form mixtures being uniformly mixed that was used by Saylor [3] and Brickner [4]. To provide additional technical justification for that uniform mixing assumption, studies are presented in Appendix J which constitute set-4. Specifically, the purpose of Appendix $\mathrm{J}$ is to evaluate the validity of the assumption that the $\mathrm{PuO}_{2}$ is uniformly mixed within the waste form. This evaluation examines the effect of locally increased fissile concentration in the waste from in terms of $k_{\text {eff. }}$ This appendix is not intended to address all possible heterogeneous configurations, but only a selection of cases that correspond to the analyses performed and which cover a range of sizes which would allow larger particle sizes within a mixture to be acceptable.

Appendix $\mathrm{J}$ evaluates the assumption that a uniform mixture for the waste form is acceptable by evaluating a unit cylindrical waste form (with set-2-uh subset 2-4 parameters, i.e., polyethylene waste form moderator, polyethylene discrete reflector, and graphite filler material) with variations in the number, size, and location of pure $\mathrm{PuO}_{2}$ spheres (heterogenous spheres) embedded in a mixture of filler and $\mathrm{PuO}_{2}$. Spheres are used because the neutron leakage is bounding compared to similar sized chunks of FGE. Geometries evaluated are shown in Figure 6.46.
1 heterogenous sphere

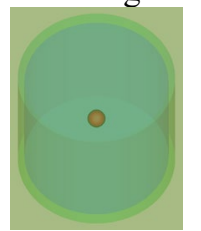

2 heterogenous spheres

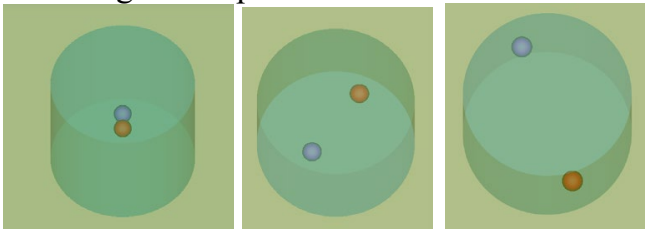

3 heterogenous spheres

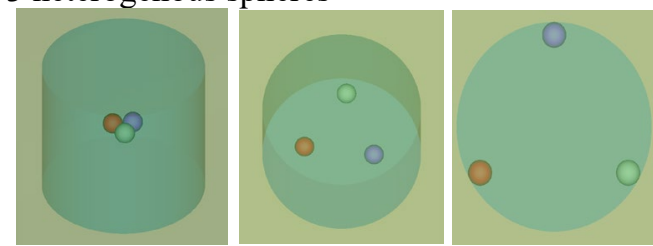

Figure 6.46. Diagram of the various heterogenous sphere cases and spacings. 
The results presented in Appendix J and summarized in Figure 6.47 below for the worst-case configuration show that the heterogeneity may have some positive reactivity impact for waste form cases which are under-moderated and in which the impact to $k_{\text {eff }}$ is not significant because these cases are very subcritical.

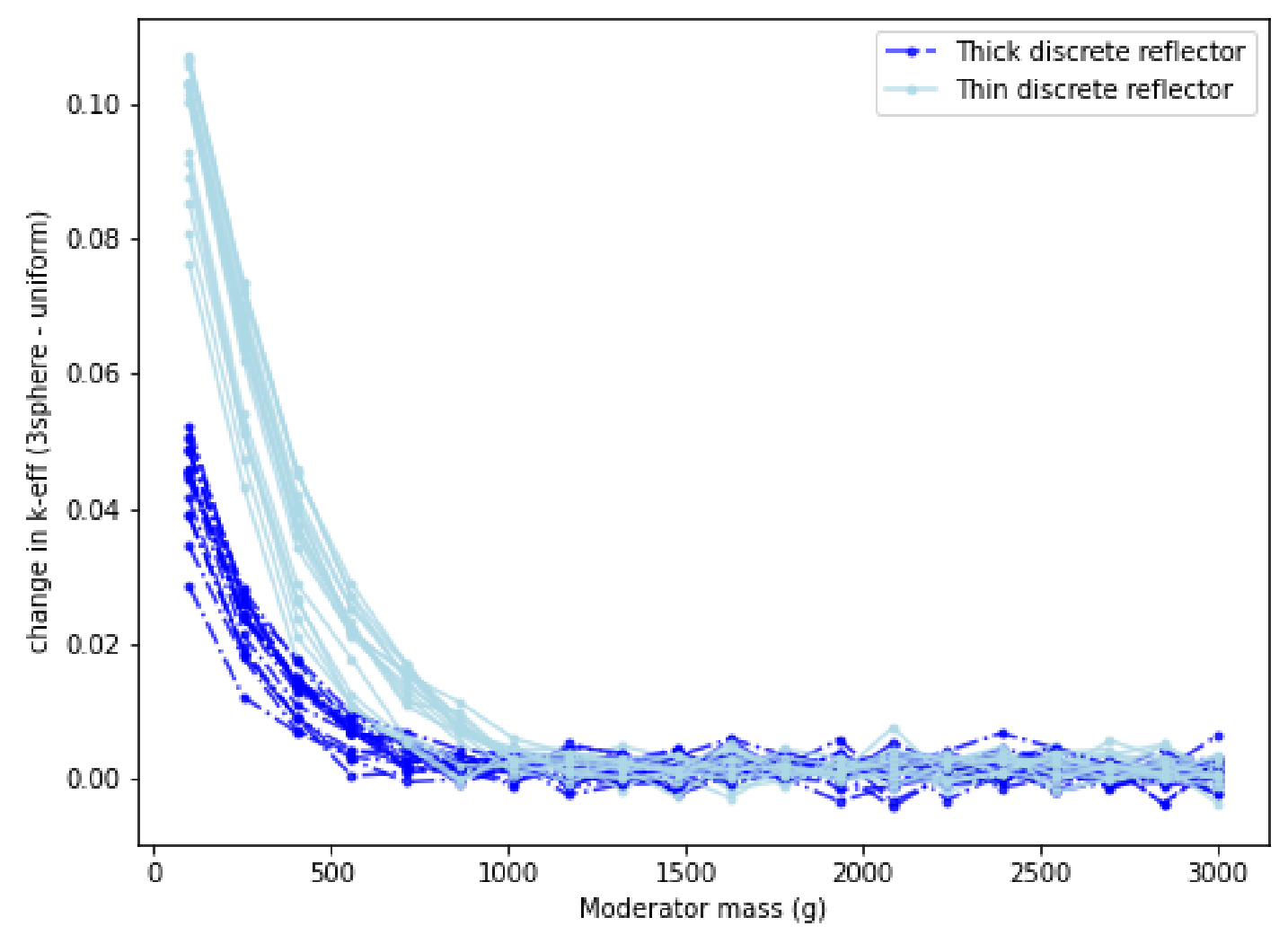

Figure 6.47. Delta- $\boldsymbol{k}_{\text {eff }}$ when comparing highest reactivity three-sphere case to a uniform case (positive values correspond to cases in which the highest reactivity three-sphere configuration has higher $k_{\text {eff }}$ than uniform configuration.

\subsection{STUDY OF NEUTRON TRANSMISSION FROM CCO SOURCE THROUGH INTERSTITIAL MEDIA}

The interstitial material between the waste forms plays a significant part in overall system reactivity. Given the predominance of salt and $\mathrm{MgO}$ in that interstitial mixture, study results are presented in Appendix $\mathrm{K}$ which evaluate the transmission of neutrons from the waste form through this media. Specifically, the results in Appendix K evaluate the sphere of influence of a waste form assuming a waste form in a cuboid of material. The waste form itself is not of particular importance here because the primary concern is the rate at which neutron flux tapers (magnitude goes down) in the salt/MgO mixture.

The results in Appendix K provide neutron intensities at various distances for various types of interstitial reflector media and are shown in Figure 6.48 below. When neutrons escape from a waste form, the neutron intensity decreases roughly exponentially, which is consistent with the solution to the diffusion equation for a point source in non-multiplying media [15]. Note that the waste form used in this study is assumed to be a sphere with a $10 \mathrm{~cm}$ diameter. 
As seen in Figure 6.48 below, the flux at the waste form's surface decreases by a factor of 10 in the first $20 \mathrm{~cm}$ outside the waste form. An important conclusion to be drawn from this result is that $\mathrm{t}$ a few closely spaced packages may locally produce higher values of reactivity.

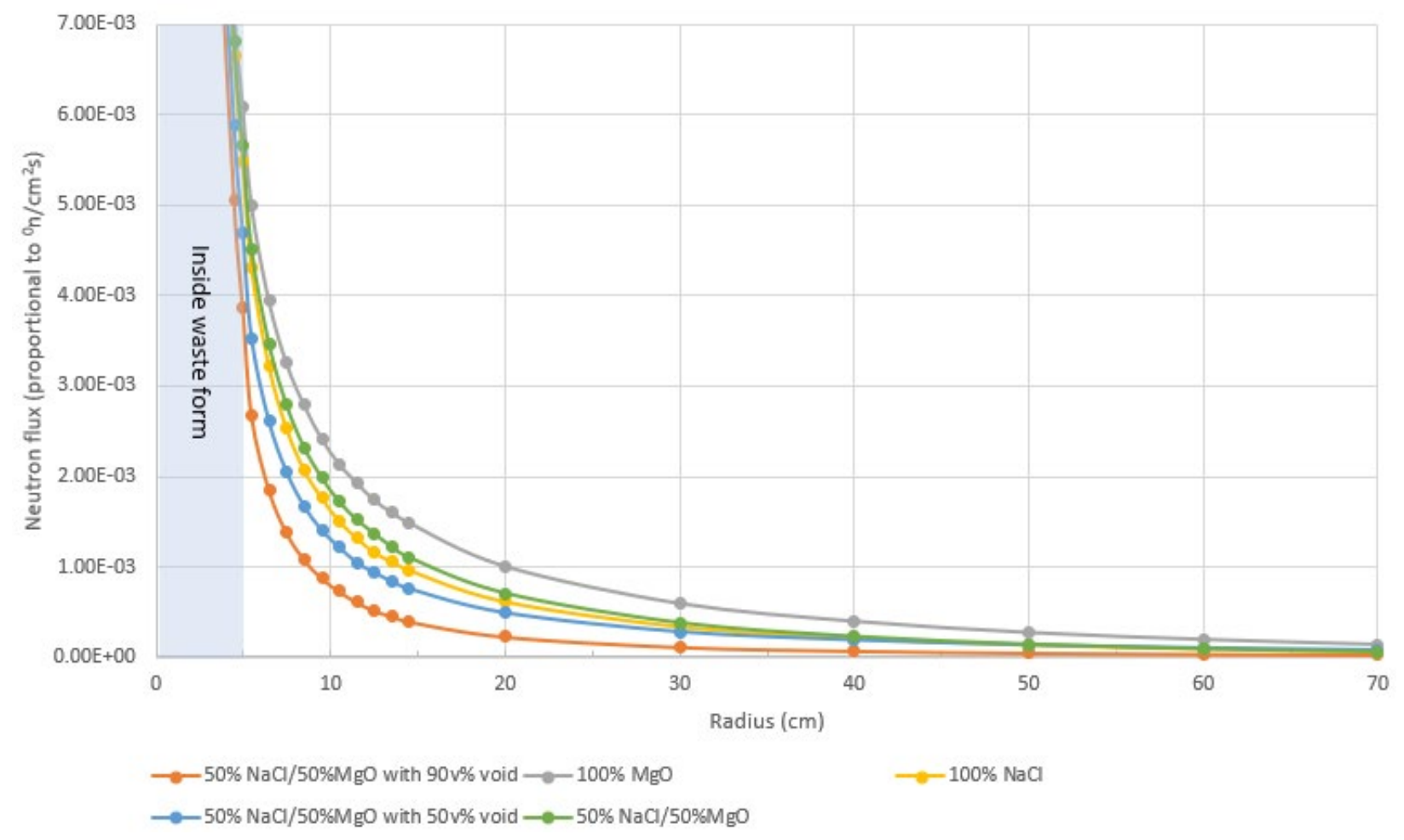

Figure 6.48. Flux per source particle at various distances in the salt form the source (linear scale).

\subsection{STUDIES ON THE REACTIVITY EFFECT OF BRINE AND THE ASSUMPTIONS RELATED TO INTERSTITIAL REFLECTOR MATERIAL DENSITY AND COMPOSITION}

The criticality safety analysis in this report uses a specific mixture of salt and $\mathrm{MgO}$ for the interstitial material between waste forms. To provide additional technical justification for that assumption and to include studies with brine as an interstitial material, the reactivity effects of various interstitial material assumptions were examined using set-5 nomenclature, as shown in Appendix L.

For the results presented in Appendix L, the compositions interstitial material were perturbed. This included altering the material densities, modifying the mixing ratio of salt and $\mathrm{MgO}$, and evaluating the reactivity effect of a brine intrusion. Full sets of parametric sweeps were evaluated for nine different interstitial reflector types. For the case with the brine intrusion into the waste form mixture itself (case $5 \mathrm{~h}$ ), the brine mixture replaces the filler mass (graphite is replaced by brine). Set-2-uh, the nonuniform array for the upper horizon calculations, provided the baseline configuration.

The results in Appendix $\mathrm{L}$ show that the assumptions for the salt and $\mathrm{MgO}$ mixture density used in the criticality safety analysis in this report are conservative for moderator loadings in excess of $200 \mathrm{~g}$. The results in Appendix L also show that the effect of brine replacing the interstitial material was seen to largely decrease $\mathrm{k}_{\text {eff, }}$ summarized below in Figure 6.49. However, this trend does not hold for undermoderated waste forms. For cases in which the brine cases exceeded $\mathrm{k}_{\text {eff }}$ values of set- 2 , the moderator 
contents were at moderator masses below approximately $200 \mathrm{~g}$, and they did not increase $k_{\text {eff }}$ above that of set-2 at $200 \mathrm{~g}$ of moderator.

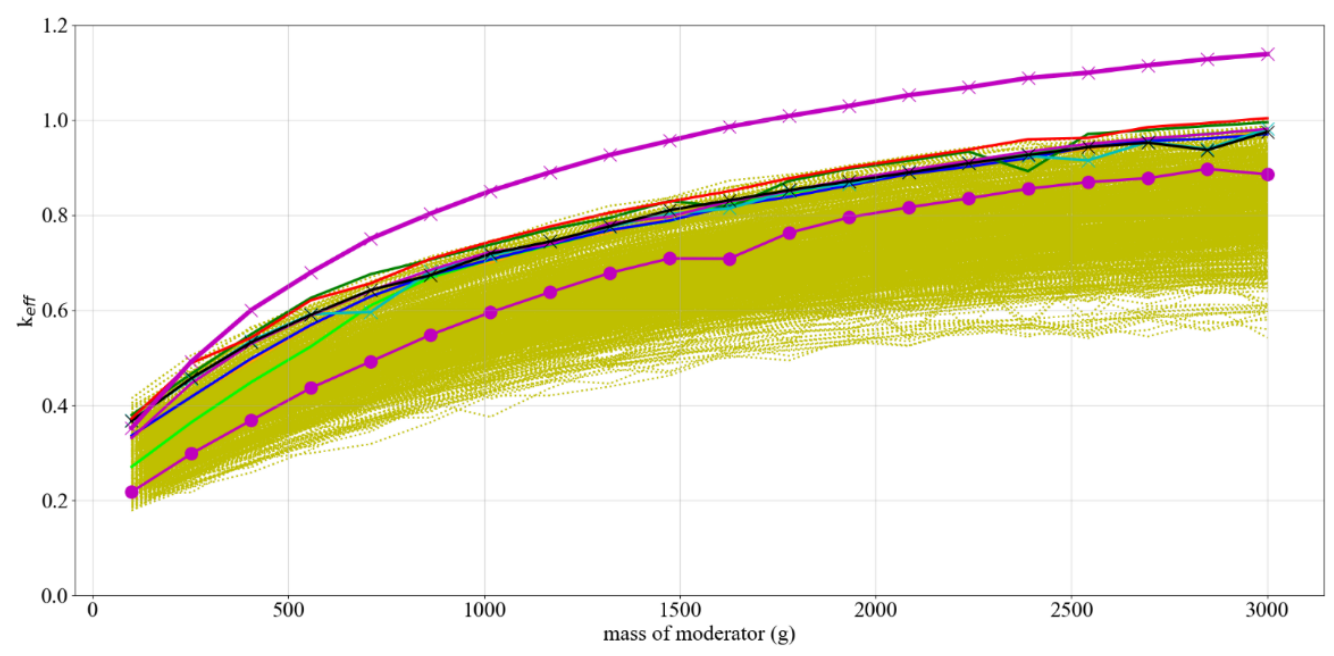

— waste form sphere, FGE+poly mod only, thick steel discrete reflector

$\longrightarrow$ waste form sphere, FGE+poly mod $+4 \mathrm{~kg}$ graphite, thick steel discrete reflector $\rightarrow$ waste form sphere, FGE+poly mod only, thin poly discrete reflector

— waste form sphere, $\mathrm{FGE}+$ poly mod only, thick poly discrete reflector

$\leftarrow$ set-2, waste form sphere, FGE+poly mod only, thick steel discrete reflector
}

Figure 6.49. Reactivity of set-5e compared to a single set-2 representative curve to evaluate the effect of brine (no $\mathrm{MgO}$ ) as interstitial reflective material.

Of the set- 5 cases, the effect of brine intrusion into waste forms had the largest positive effect on $k_{\text {eff. }}$ It increased $k_{\text {eff }}$ at moderator compositions below $600 \mathrm{~g}$, but at no time did the $k_{\text {eff }}$ values exceed the set- $2 k_{\text {eff }}$ at $600 \mathrm{~g}$ of moderator. In other words, $k_{\text {eff }}$ was shown to increase only when the system was already deeply subcritical.

The effect of replacing $\mathrm{MgO}$ with $\mathrm{NaCl}$ slightly decreased $k_{\text {eff }}$ in all cases evaluated, supporting the assumption that the interstitial material is at $100 \%$ density.

Decreasing the density of interstitial material decreased $k_{\text {eff }}$ in all cases evaluated, supporting the assumption that the interstitial material is at $100 \%$ density.

\subsection{STUDIES WITH ADDITIONAL CENTROID LOCATIONS FOR VARIOUS CONTAINER DESIGNS AND INITIAL SPATIAL ARRANGEMENTS (APPENDIX M)}

As previously discussed, the CCO arrive for emplacement in seven-packs, which resemble a hexagonal array (shown in Figure 4-3). For conservatism, the uniform arrays arrange the packages in a triangular pitch, as discussed in Section 4.2.1.1, which conservatively adds more waste forms to the system. The compaction studies from Reedlunn and Bean [8] that provide the centroid locations of the compacted containers considered hexagonal pitched arrays at time $=0$ as a starting point for the simulations. Of some interest is the potential impact of beginning those same compaction studies with the containers in a triangular pitch at time $=0$ because then the comparison between the uniform arrays and nonuniform arrays have more technical commonality, and the additional number of containers in the compacted 
models (167 vs. 152) provides results that have more mass (i.e., more centroids) for the set-2 studies in nonuniform arrays.

Of additional interest is the evaluation of centroid data for other container designs, specifically the POCs evaluated in Brickner [4], using the same methodology outlined in this report - that is, not modifying the parametric sweeps to be specific to the POC designs but applying the CCO sweeps to the centroids provided by the other container while using the lower FGE for the POC (200 g). The main interest is to provide more technical justification that the reactivity of the system using the compaction data centroids in Table 4-4 is not highly susceptible to variations in initial starting conditions for the compaction analysis.

Furthermore, the evaluation provided in Appendix K was used in Reedlunn and Bean [46] to define a method that predicts maximum reactivity locations using the centroid datasets directly. Therefore, a small subset of the calculations presented in Appendix $\mathrm{M}$ is used to generate flux maps and maximum reactivity orthogonal locations in the model for comparison purposes to that method in Reedlunn and Bean [46]. Finally, the additional datasets provide additional means to further evaluate (Appendix E) the studies that were performed to show the effect of the two boundary conditions for these models.

To address these interests, additional centroid datasets were provided in Reedlunn and Bean [46]. These data include CCO centroid locations for simulations that begin in a triangular pitch (yields 167 centroids) and POCs for both hexagonal and triangular pitch simulations scenarios. The differences between the $\mathrm{CCO}$ and POC are presented generically in Figure 6.50. 


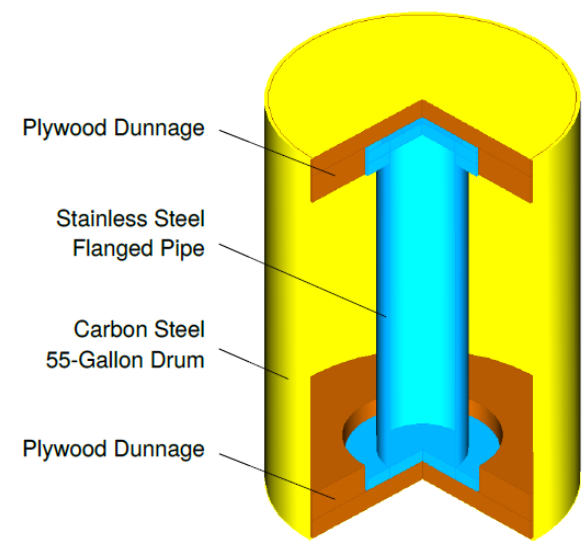

(a) $\mathrm{CCO}$

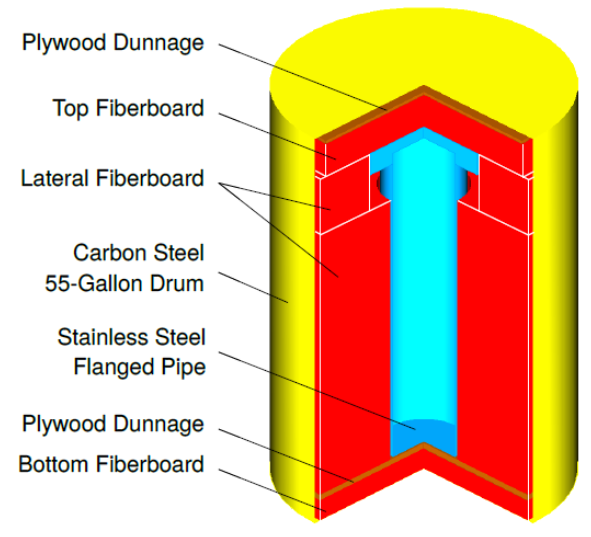

(b) 6-inch POP

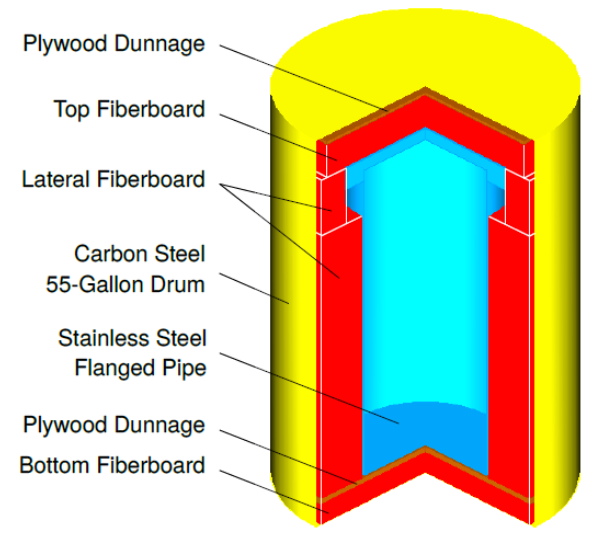

(c) 12-inch POP

Figure 6.50. Diagrams of the CCO compared with the POCs evaluated in Appendix M (used with permission from Reedlunn and Bean [46]).

Although the $\mathrm{CCO}$ and POC are similar, design differences exist. Additional POC design information is available in Brickner [4]. Although the various POC package dimensions and materials are different, the actual values fall within the range of the parametric sweeps used.

The data provided and additional information, including results, are presented in Appendix $\mathrm{M}$ and summarized in Figure 6.51 and Figure 6.52. 


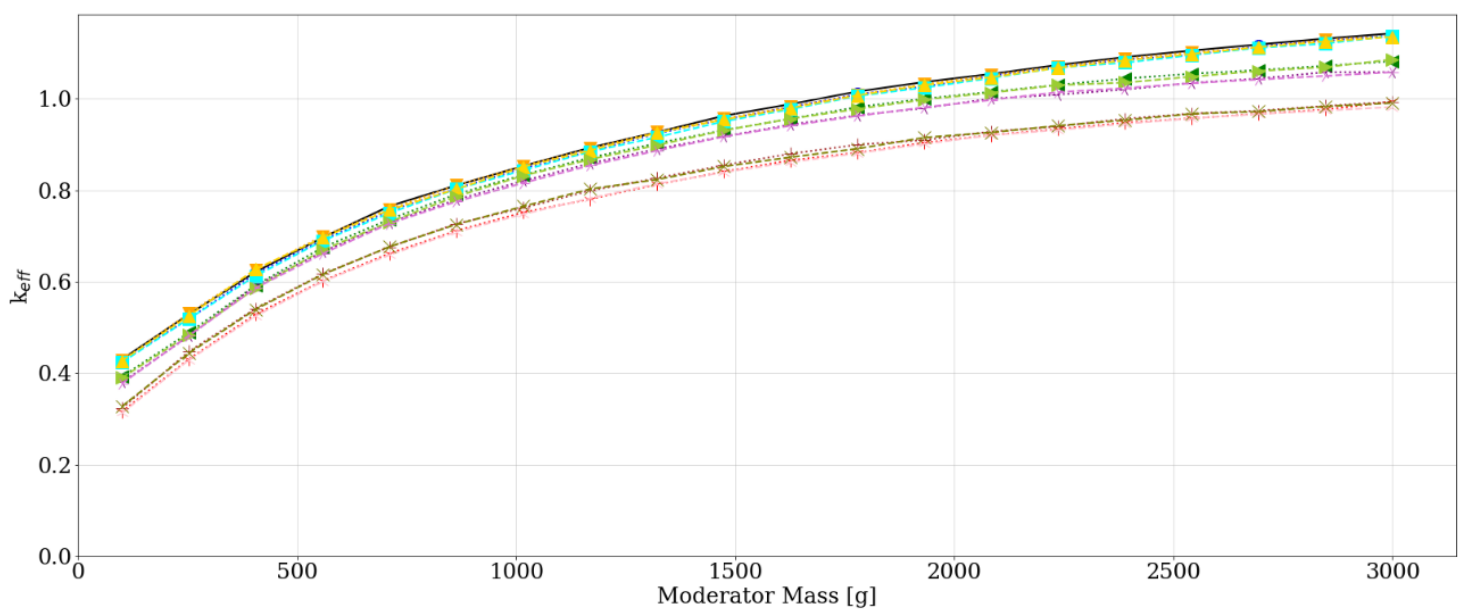

- Max Upper Horizon Reference (Set-2)

-.. Max Lower Horizon, CCO Tri Pitch Array, Mirror BC

$\checkmark$... Max Upper Horizon, CCO Tri Pitch Array, Mirror BC

4. Max Lower Horizon, 6" POP Hex Pitch Array, Mirror BC

... Max Lower Horizon, 12" POP Hex Pitch Array, Mirror BC

.... Max Upper Horizon, 6" POP Tri Pitch Array, Mirror BC

...... Max Upper Horizon, 12" POP Tri Pitch Array, Mirror BC
--1-- Max Lower Horizon, CCO Tri Pitch Array, Periodic BC

--- Max Upper Horizon, CCO Tri Pitch Array, Periodic BC

$\rightarrow$ Max Lower Horizon, 6" POP Hex Pitch Array, Periodic BC Max Lower Horizon, 12" POP Hex Pitch Array, Periodic BC Max Upper Horizon, 6" POP Tri Pitch Array, Periodic BC

--׳-- Max Upper Horizon, 12" POP Tri Pitch Array, Periodic BC

Figure 6.51. Max $\boldsymbol{k}_{\text {eff }}$ of all subsets of all CCO and POC centroid configurations at time $=1,000$ years compared with max $k_{\text {eff }}$ of all subsets of set-2-uh hexagonal pitch (mirror boundary conditions) as $k_{\text {eff }}$ vs. moderator mass, time $=\mathbf{1 , 0 0 0}$ years.

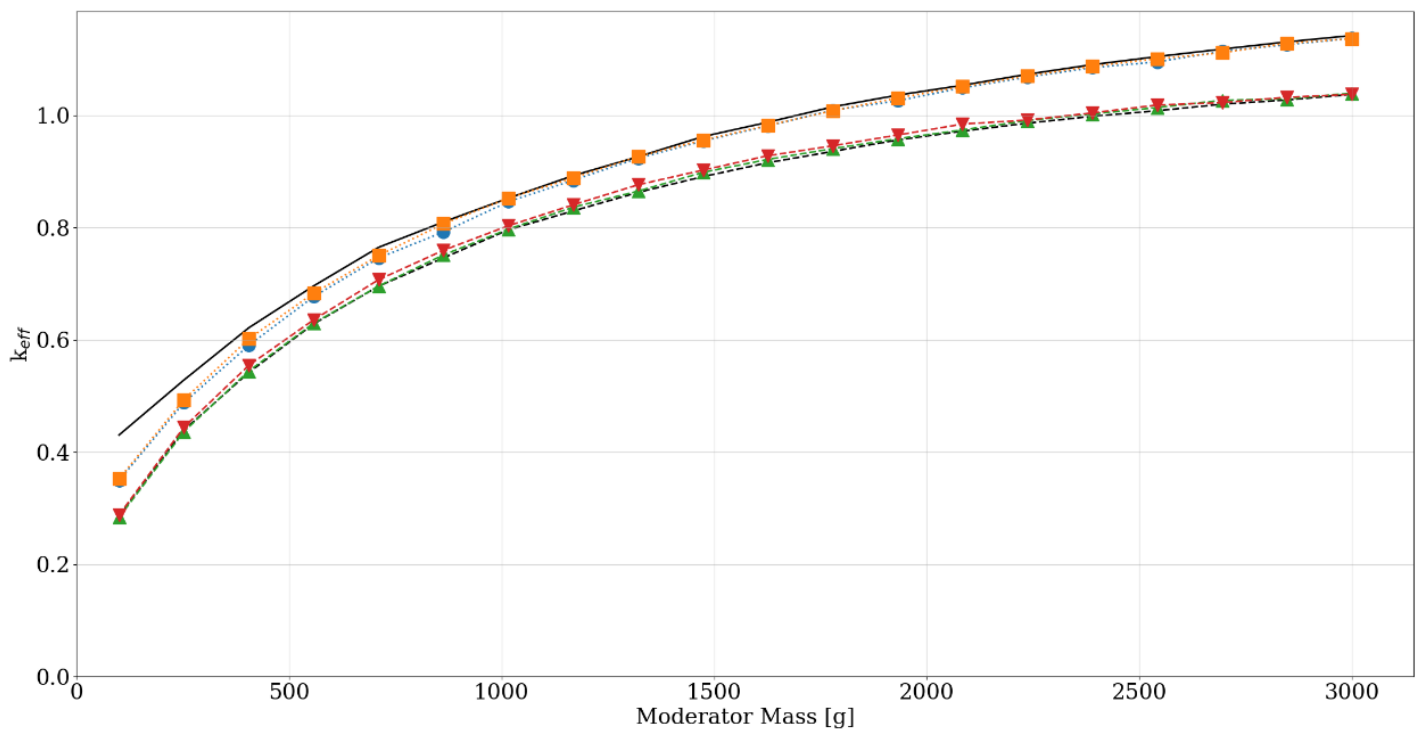

- Max Upper Horizon Reference (Set-2)

---- Max Upper Horizon Reference (Set-2: 200 g FGE)

-... Max Lower Horizon, Tri Pitch Array, Mirror BC (380 g FGE)
…… Max Upper Horizon, Tri Pitch Array, Mirror BC (380 g FGE)

--^-- Max Lower Horizon, Tri Pitch Array, Mirror BC (200 g FGE)

---- Max Upper Horizon, Tri Pitch Array, Mirror BC (200 g FGE)

Figure 6.52. Comparison of the effect of FGE on the maximum $\boldsymbol{k}_{\text {eff }}$ of all subsets of all CCO and POC centroid configurations at time $=1,000$ years (mirror boundary conditions). 


\subsection{STUDIES TO EVALUATE THE IMPACT OF LARGER DISCRETE REFLECTOR THICKNESSES (APPENDIX O)}

As previously discussed in Section 4.2.2, two discrete reflector thicknesses were used in the analysis in this report to evaluate the reactivity impact of various materials. Of interest is the evaluation of thicker discrete reflectors because the discrete reflector model allows materials that may be compacted directly adjacent to the waste form to be evaluated. These studies are presented in Appendix $\mathrm{O}$ and summarized in Figure 6.53 and Figure 6.54.

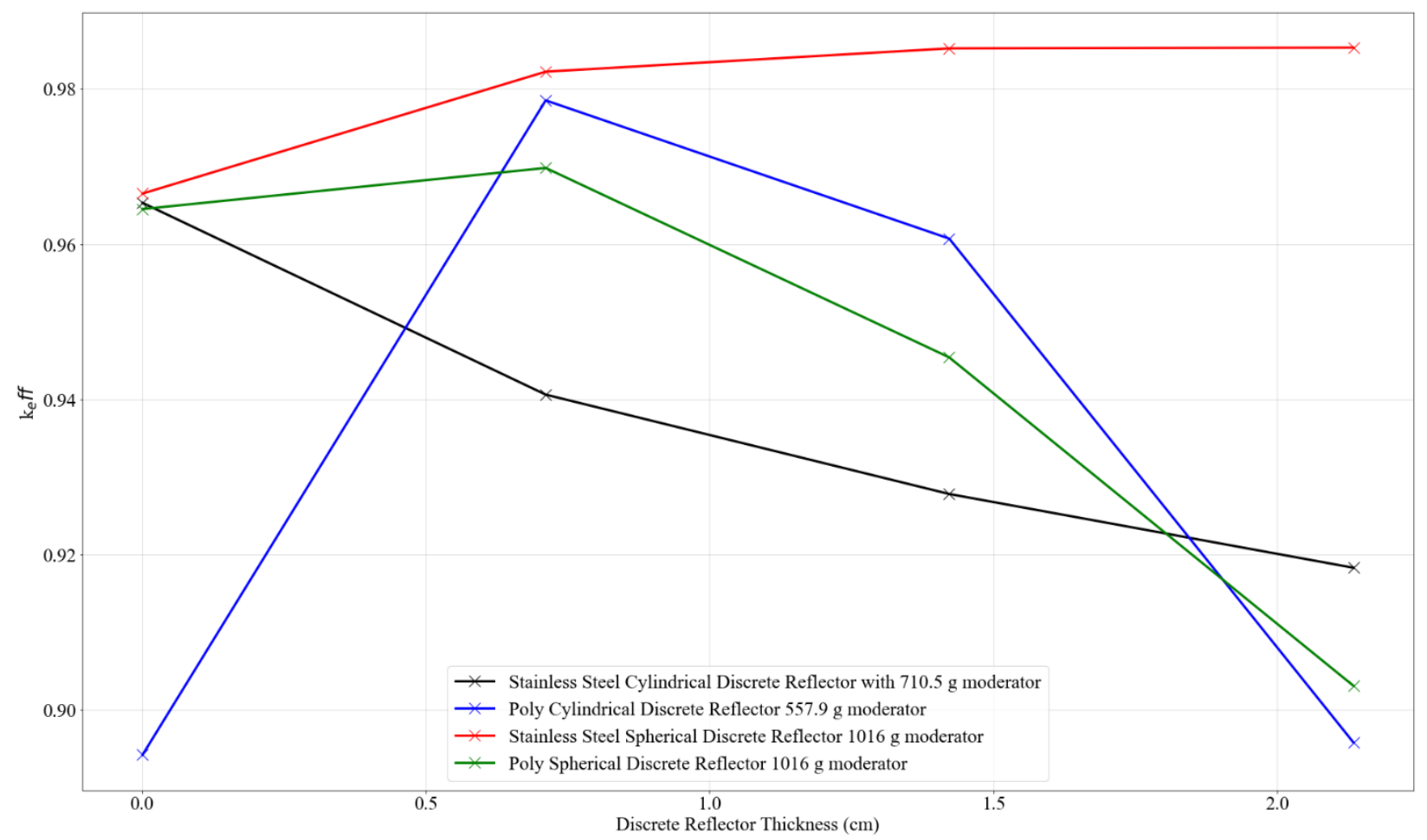

Figure 6.53. Set-8a and set-1 maximum $\boldsymbol{k}_{\text {eff }}$ results (three-high uniform array model) overall sublistings as a function of discrete reflector thickness. 


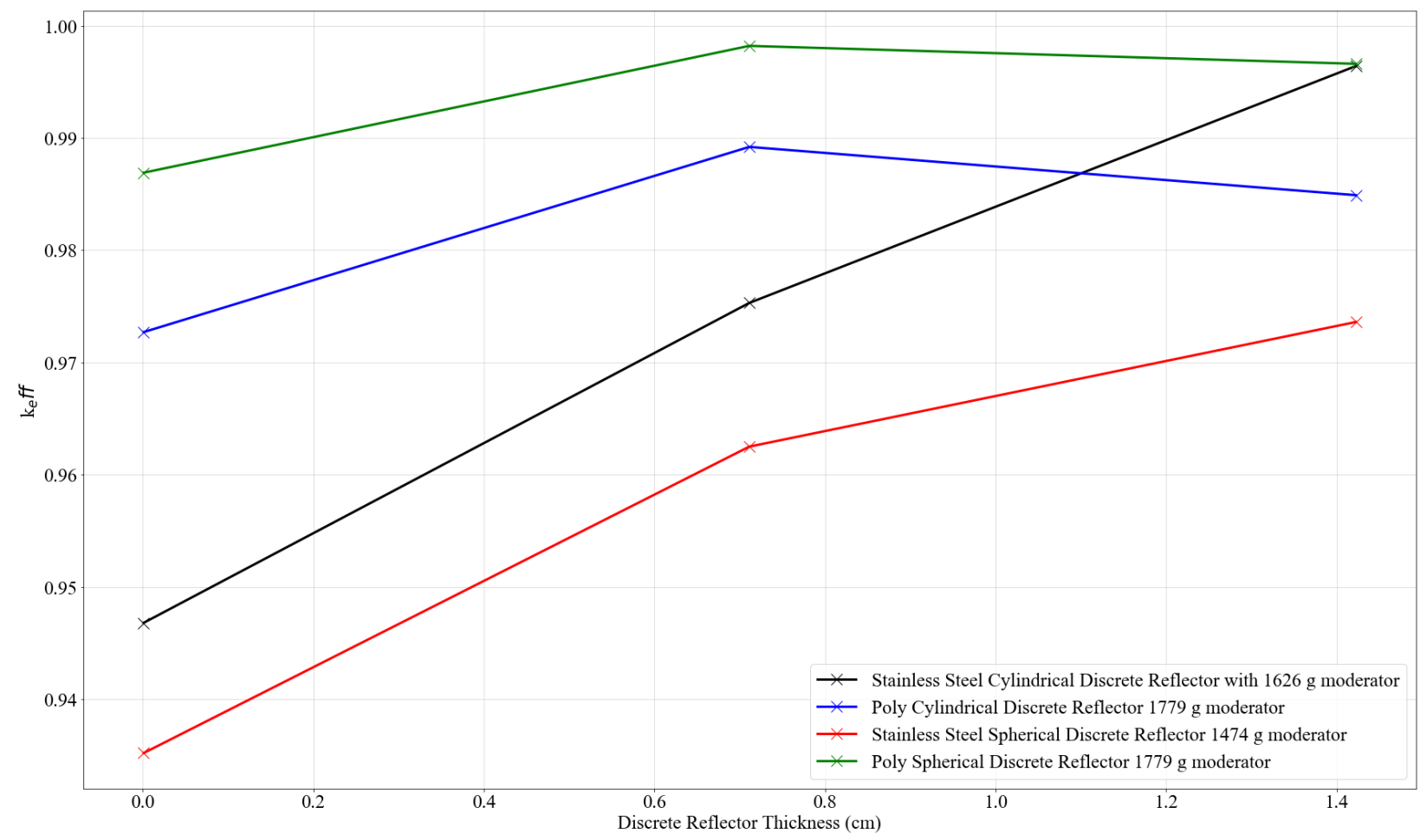

Figure 6.54. Set-8b and set-2-uh maximum $k_{\text {eff }}$ results (nonuniform array model) overall sublistings as a function of discrete reflector thickness.

\subsection{STUDIES TO EVALUATE VARIOUS AMOUNTS OF $B_{4}$ C MIXED UNIFORMLY IN THE WASTE FORM (APPENDIX N).}

As previously discussed in Section 6 and shown in Table 6-2, a subset of calculations in this report considered $50 \mathrm{~g}$ of $\mathrm{B}_{4} \mathrm{C}$ mixed uniformly with the waste form. Additional calculations are provided in Appendix $\mathrm{O}$ to show the complete set of parametric sweeps with various amounts of $\mathrm{B}_{4} \mathrm{C}$ to provide more technical justification for using $\mathrm{B}_{4} \mathrm{C}$ as control. These studies are presented in Appendix $\mathrm{N}$ and summarized in Figure 6.55 through Figure 6.58. 


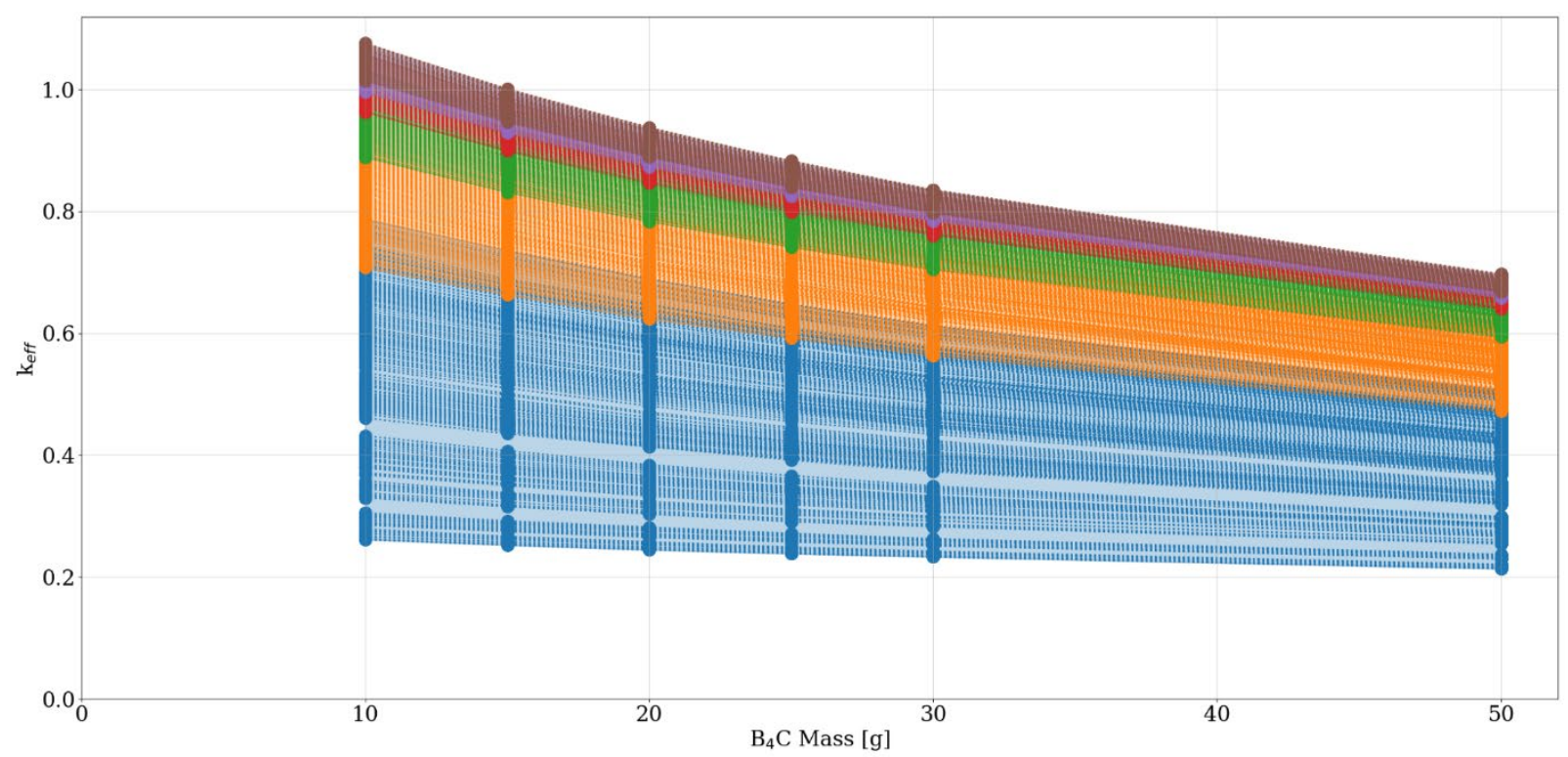

Figure 6.55. Set-7a results (three-high uniform array model) for $10,15,20,25,30$, and $50 \mathrm{~g}$ of $\mathrm{B}_{4} \mathrm{C}$ for all subcases. Reactivity trends of all subcase- 10 as $k_{\text {eff }}$ as a function of $\mathrm{B}_{4} \mathrm{C}$ mass.

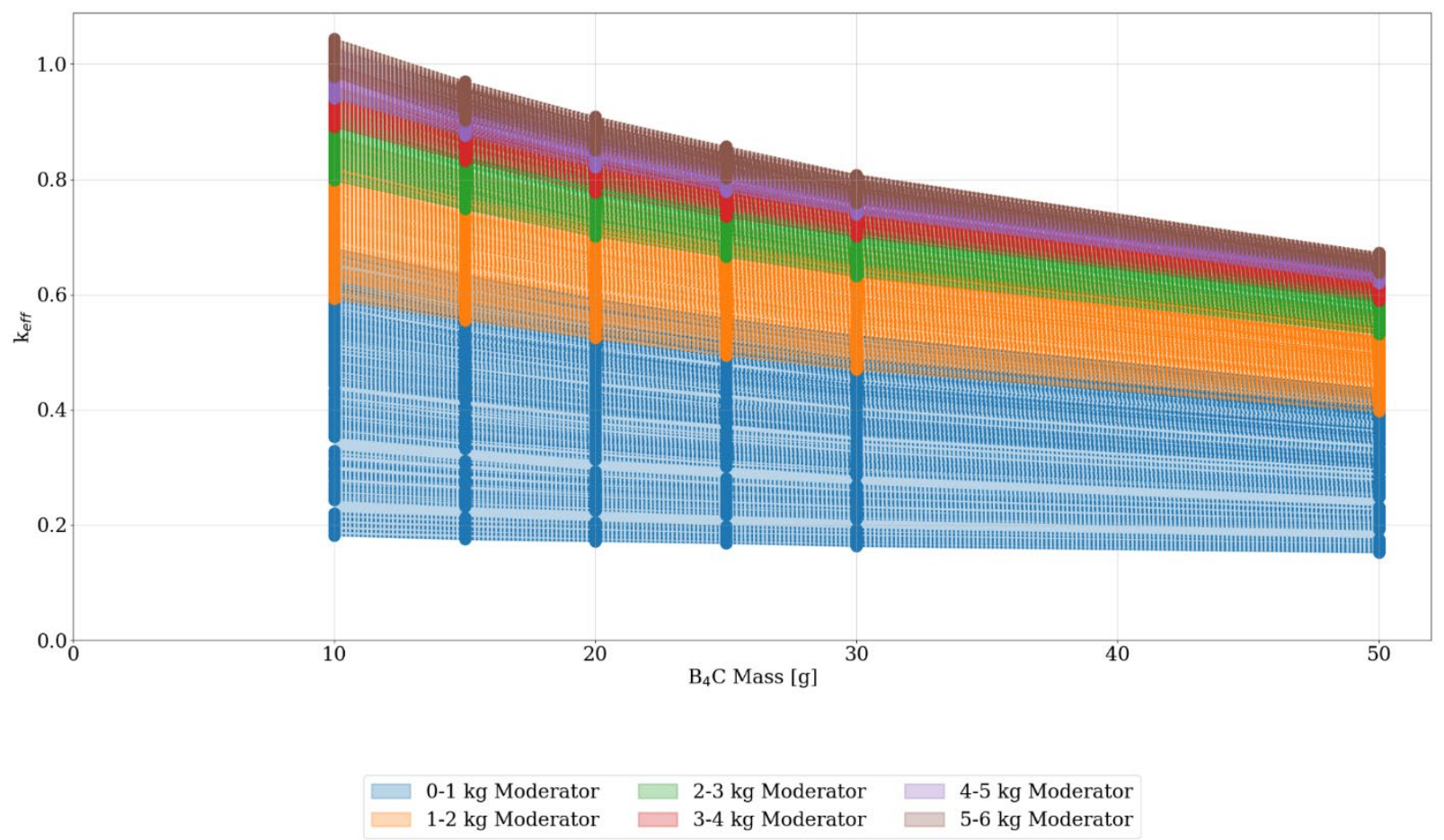

Figure 6.56. Set-7c results (nonuniform array model) for 10, 15, 20, 25, 30, and $50 \mathrm{~g} \mathrm{of} \mathrm{B}_{4} \mathrm{C}$ for up to a $6 \mathrm{~kg}$ moderator. Reactivity trends of subcase-10 as $k_{\text {eff }}$ as a function of $\mathrm{B}_{4} \mathrm{C}$ mass. 


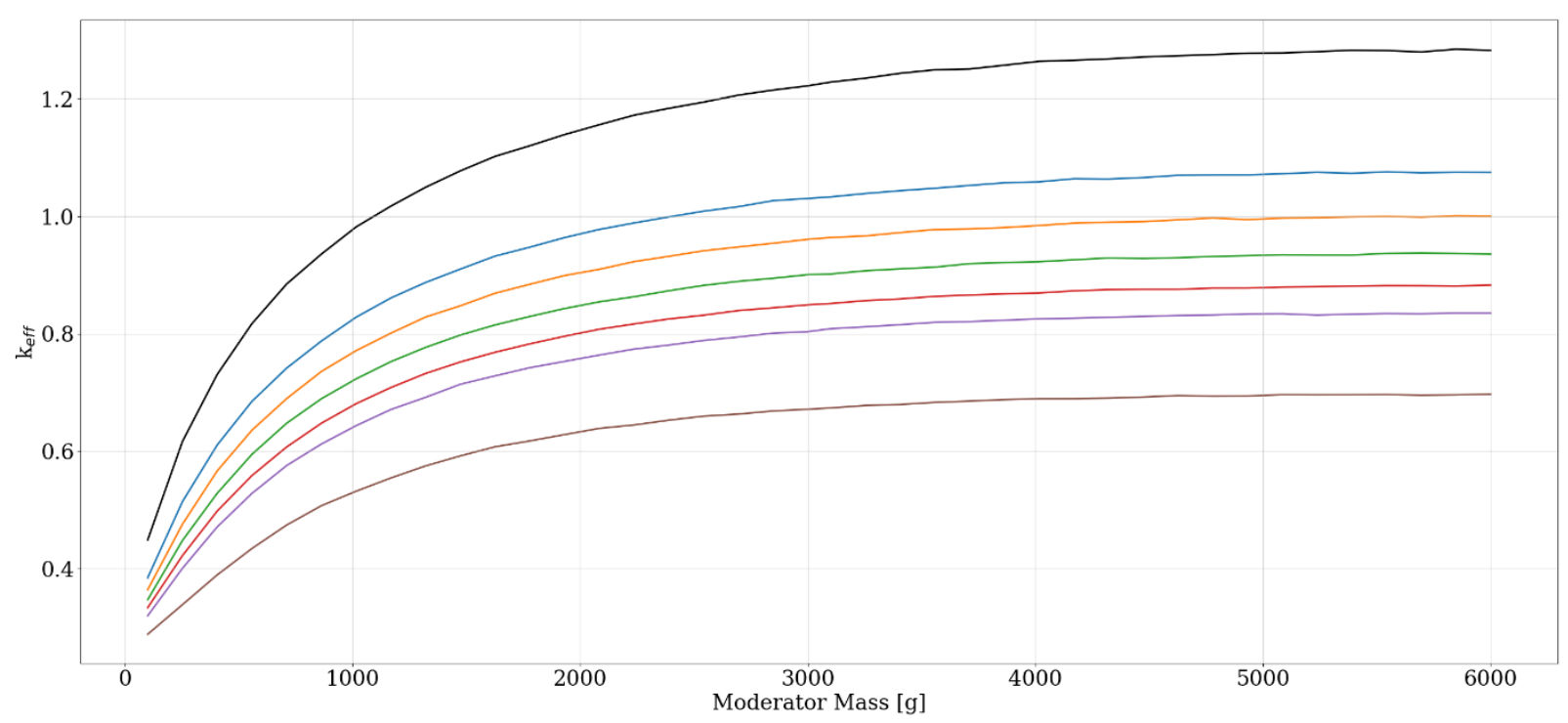

$-0 \mathrm{~g} \mathrm{~B} \mathrm{~B}_{4} \mathrm{C}$
$-20 \mathrm{~g} \mathrm{~B}_{4} \mathrm{C}$
$10 \mathrm{~g} \mathrm{~B}_{4} \mathrm{C}$
$15 \mathrm{~g} \mathrm{~B}_{4} \mathrm{C}$

Figure 6.57. Set-7a results (three-high array model) for $10,15,20,25,30$, and $50 \mathrm{~g}$ of $\mathrm{B}_{4} \mathrm{C}$ for up to a $6 \mathrm{~kg}$ moderator. Reactivity trends of subcase- 14 as $k_{\text {eff }}$ as a function of $\mathrm{B}_{4} \mathrm{C}$ mass.

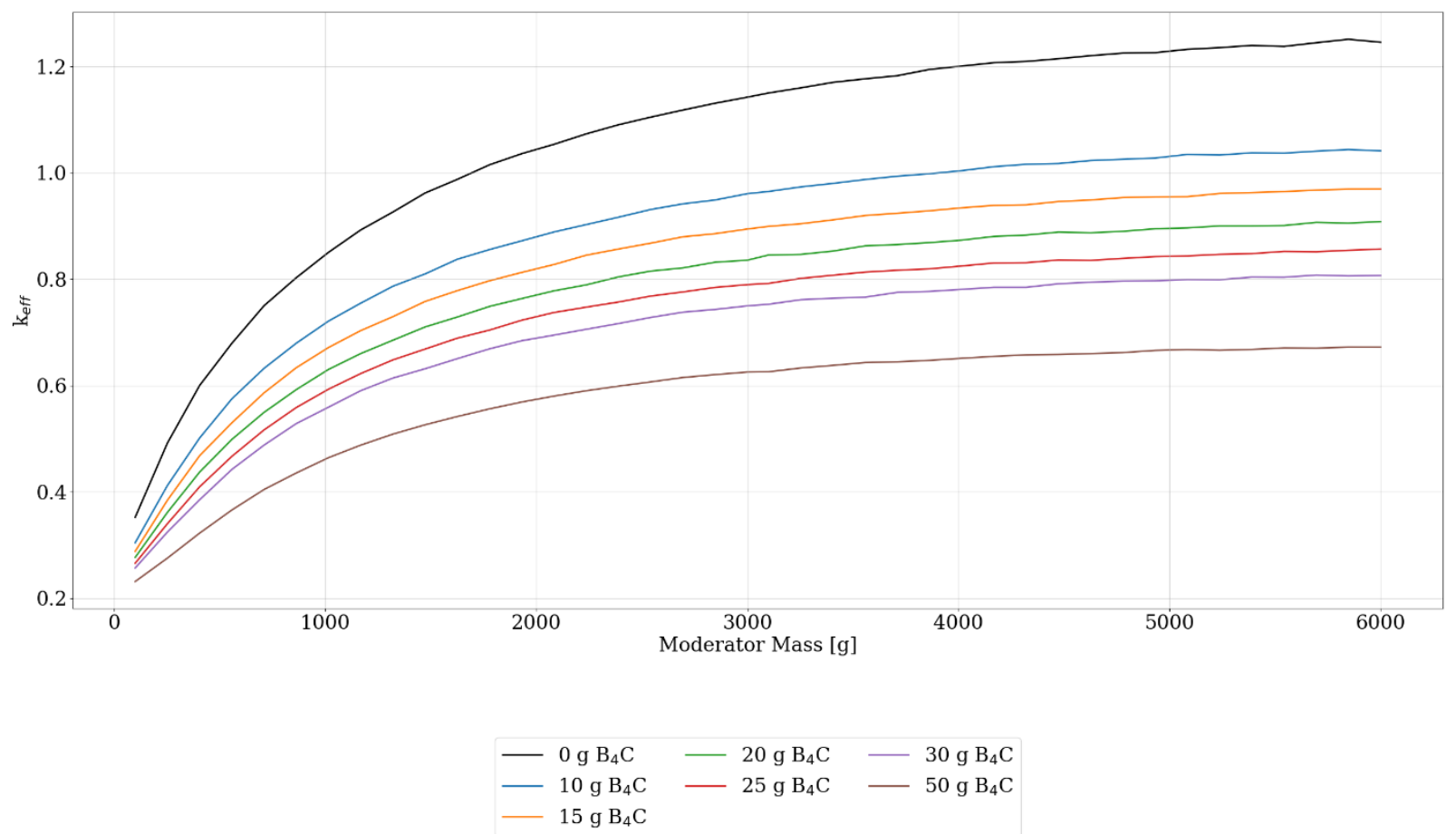

Figure 6.58. Set-7c results (nonuniform array model) for $10,15,20,25,30$, and $50 \mathrm{~g}$ of $\mathrm{B}_{4} \mathrm{C}$ for up to a $6 \mathrm{~kg}$ moderator. Reactivity trends of subcase- 14 as $k_{\text {eff }}$ as a function of $\mathrm{B}_{4} \mathrm{C}$ mass. 


\subsection{DISCUSSION REGARDING THE EXTENSION OF THE TSUNAMI VALIDATION TO ANALYSIS DATASETS}

The criticality calculations in this report were validated using the TSUNAMI sequence in the SCALE code package. Because of the many calculations performed and the resource-consuming nature of the TSUNAMI method, the validation documented in Appendix H considers a small subset of cases entirely from set-1 (subcase 1-8). This section discusses how the Appendix $\mathrm{H}$ validation may be extended to all the results in this analysis. As an example, set-2-uh subcase 10 (set-2-10-uh) is used in the following discussion.

When performing a validation, one important step is determining the "area of applicability" of the validation and limitations. NUREG/CR-6698, Guide for Validation of Nuclear Criticality Safety Calculational Methodology [47], provides insight into parameters to consider for determining the area of applicability. This is typically used to select nuclear criticality experiments in which bias and bias uncertainty are determined for nuclear criticality applications.

The key physical parameters when defining the area of applicability include the materials, geometry, and neutron energy. Table 2.3 of NUREG/CR-6698 [47] describes these three parameters. If set-2-10-uh is within the area of applicability of set-1-2 per NUREG/CR-6698, then this would imply that the bias and bias uncertainty determined for set-1-2 would be applicable for set-2-10-uh. First, the parameters evaluated for set-2-uh-10 compared with the closest set-1 case (Appendix H) that parameterizes the same materials were consider.

Table 6.8. Comparison of set-2-10-uh parameters with set-1-2 parameters.

\begin{tabular}{|l|l|l|l|l|l|l|}
\hline Case & \multicolumn{1}{|c|}{ Model type } & \multicolumn{1}{|c|}{$\begin{array}{c}\text { Waste form } \\
\text { shape }\end{array}$} & $\begin{array}{l}\text { Maste form } \\
\text { moderator }\end{array}$ & $\begin{array}{c}\text { Filler } \\
\text { material } \\
\text { filler } \\
\text { discrete } \\
\text { reflector }\end{array}$ & $\begin{array}{l}\text { Discrete } \\
\text { reflector }\end{array}$ \\
\hline $\begin{array}{l}\text { Set-2- } \\
10 \text {-uh }\end{array}$ & $\begin{array}{l}\text { Nonuniform array with } \\
\text { centroids based on upper } \\
\text { horizon data. Centroid from } \\
\text { pipe center is used as the } \\
\text { point in center of base of } \\
\text { cylinders or the center of } \\
\text { spheres. }\end{array}$ & $\begin{array}{l}\text { Sphere (radius } \\
\text { defined by } \\
\text { volume of mass) }\end{array}$ & Poly & Graphite & $\begin{array}{l}\text { Stainless } \\
\text { steel }\end{array}$ & Steel \\
\hline Set-1-2 & $\begin{array}{l}\text { Uniform array stacked three } \\
\text { high with CCO pitch } \\
\text { reduction. } x=50 \%, y=10 \%, \\
\text { and } z=\text { no space. }\end{array}$ & $\begin{array}{l}\text { Cylinder (radius } \\
\text { range } 4.8,6, \text { and } \\
7.7 \text { and height } \\
\text { defined by total } \\
\text { volume of mass) }\end{array}$ & Poly & Graphite & $\begin{array}{l}\text { Stainless } \\
\text { steel }\end{array}$ & Steel \\
\hline
\end{tabular}

Because the physical parameters within Table 2.3 of NUREG/CR-6698 [47] show sufficient agreement between set-1-2 and set-2-10-uh for the fissionable materials, moderation material within fuel, interstitial moderation, reflector material, and absorber material (i.e., all the parameters and materials are evaluated the same), the only sections that need additional discussion are the geometry and neutron energy.

For geometry, NUREG/CR-6698 [47] notes that the geometry should be as similar as possible. The figures provided in Appendix A and Appendix $\mathrm{C}$ shows the uniform array arrangement of set- 1 and the placement of waste forms in set-2-uh, respectively. The geometry is sufficiently different to warrant an examination of the waste form shape. 
The area of applicability guide refers to shape comparison by the mean cord length. There is an allowable $+/-50 \%$ variation on mean cord length for non-reentrant bodies and $a+/-25 \%$ variation for reentrant bodies. Reentrant bodies have a condition in which a particle leaving the body could possibly reenter without any additional events occurring. An example of a reentrant body would be an annulus geometry. The cylinder and spherical waste forms modeled are considered non-reentrant bodies.

Volumes are determined by the amount of material within the waste form and are the same regardless of the geometric shape of the waste form. The range of volume is 146 to $5,480 \mathrm{~cm}^{3}$ with the primary driver of change being the moderation mixed with the waste. Set-1-2 models the waste form as cylinders that are fixed with a radius of $4.8,6$, and $7.7 \mathrm{~cm}$, whereas set-2-10-uh models the waste form as a sphere. Figure 6.59 compares the mean chords lengths of these waste forms.

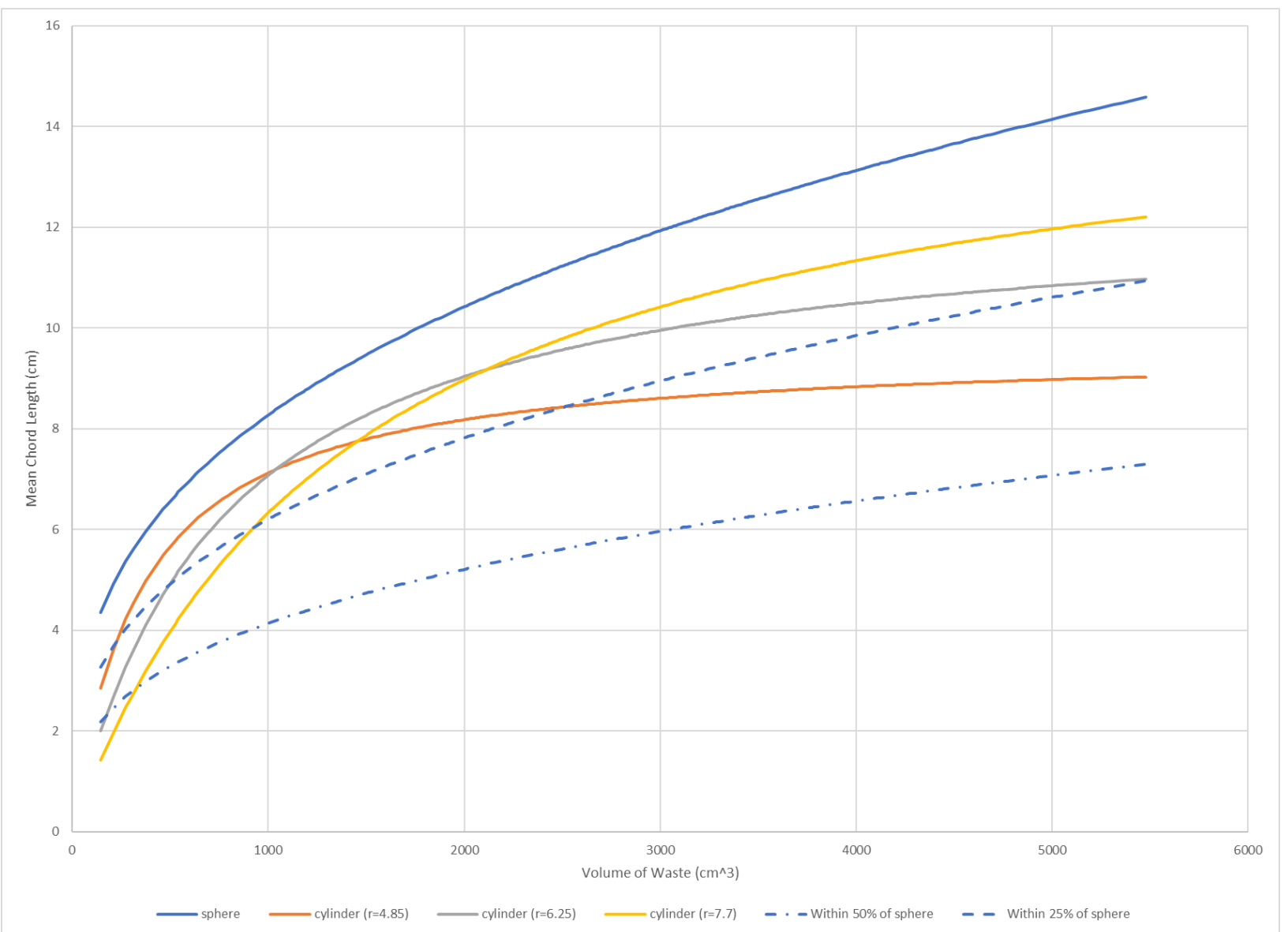

Figure 6.59. Comparison of mean chord length for the different geometric configurations.

The comparison presented in Figure 6.59 shows that, as expected, the mean chord length for the sphere is the largest. Set-1-2 cylinders with radii of 6.25 and $7.7 \mathrm{~cm}$ are essentially within $25 \%$ of the spherical mean cord length for set-2-10-uh when volumes of the waste are around $525 \mathrm{~cm}^{3}$ and $1,000 \mathrm{~cm}^{3}$, respectively. The set-1-2 cylinders with a radius of $4.85 \mathrm{~cm}$ remains within $50 \%$ of the sphere mean chord length. This indicates that the waste form shapes are similar enough that set-2-10-uh is within set-1-2. The set-1-2 models used for validation all use a cylinder radius with $7.7 \mathrm{~cm}$.

For neutron energy, NUREG/CR-6698 [47] notes that the neutron energy spectra should cover the same energy range. A wide tolerance is given based on thermal $(0-0.1 \mathrm{eV})$, intermediate $(1-100 \mathrm{KeV})$, or fast $(100 \mathrm{KeV}-20 \mathrm{MeV})$. The energy of average lethargy causing fission (EALF) is a provided value that 
gives a good indication of the neutron energy spectrum. Neutrons produced from fission are typically in the megaelectron volt range and lose fractions of its energy through scattering before it causes another fission. Figure 6.60 shows how the EALF changes as a function of moderator mass within the waste.
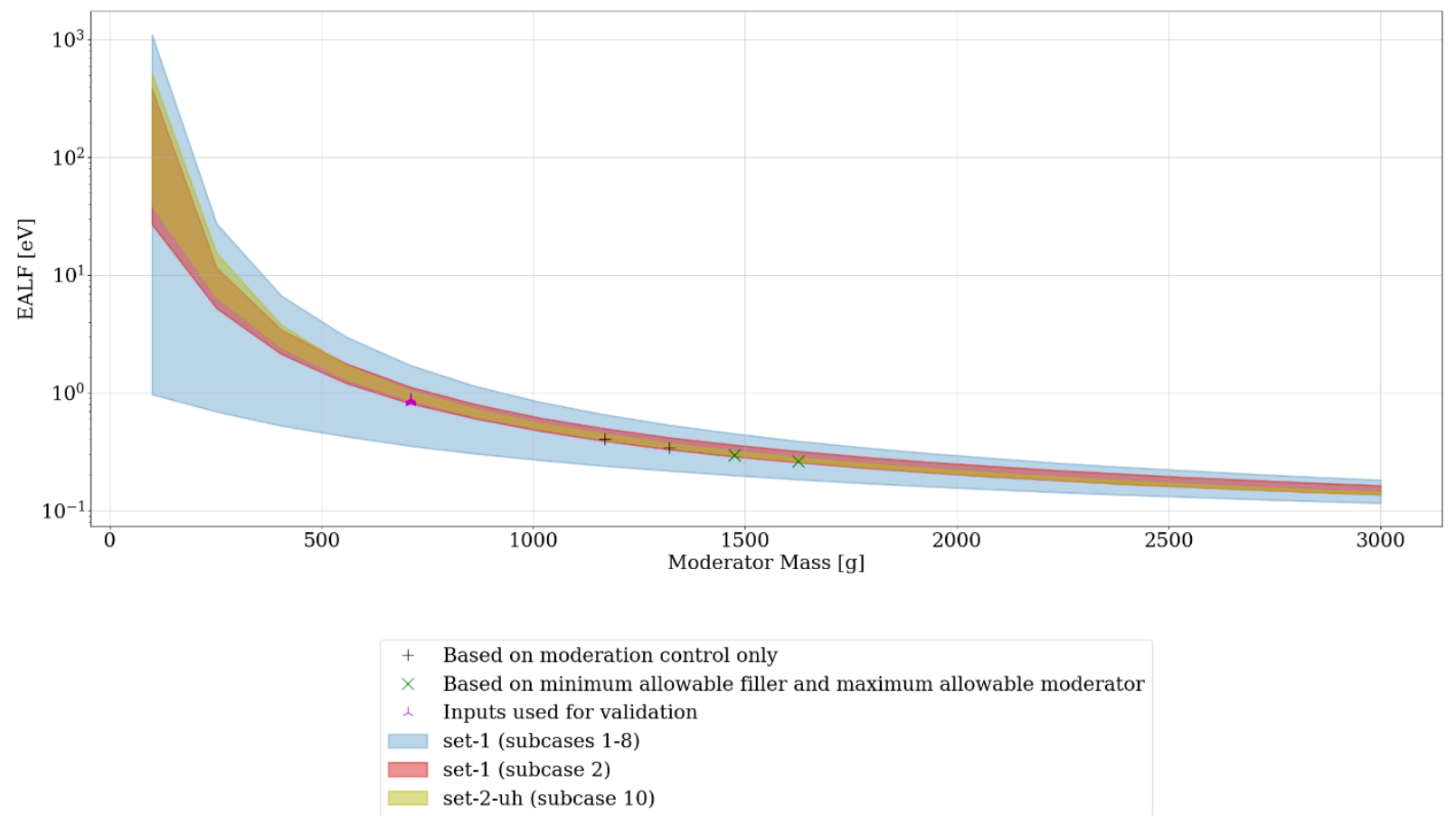

Figure 6.60. EALF of set-1 (all cases), set-2-uh (subcase 10 only), and set-1 subcase 2 (cylinder radius fixed at $7.7 \mathrm{~cm}$ )

The comparison presented in Figure 6.60 shows that, as expected, the EALF dramatically reduces as moderator is added to the waste forms. These also indicate that the EALF for set-2-10-uh is within the boundaries of the EALF for set-1-2. The individual points indicate the location of (1) where the set-1-2 models are used for validation, (2) the bounding set-2-uh subcase 10 models for moderation control (1.3 kg moderator in this example), and (3) the bounding set-2-uh subcase 10 models for controls with a minimum filler content $(6 \times$ FGE mass $)$ and maximum allowable moderator $(1.5 \mathrm{~kg}$ moderator in this example).

The above discussion provides sufficient justification to conclude that the validation in the Appendix $\mathrm{H}$ area of applicability covers the other results in this analysis with set-2-10-uh as an example.

An additional discussion is required to address the use of the results in Appendix $\mathrm{H}$ because of the lack of applicable benchmark experiments and the way that TSUNAMI was used for them.

For this discussion, the same example controls on moderator mass for set-2-10-uh are used: $1.3 \mathrm{~kg}$ with no filler mass requirement, and with $6 \times$ FGE mass as a filler mass requirement, the moderator control is $1.5 \mathrm{~kg}$.

The analysis models with the maximum $k_{\text {eff }}$ that occur with a moderator mass of $1.168 \mathrm{~kg}$ and $1.321 \mathrm{~kg}$ of moderator in the waste were selected for this example for the control of limiting the moderator within the waste to $1.300 \mathrm{~kg}$ (i.e., an interpolation was performed). These models are presented in Table 6.9. 
Table 6.9. Set-2-uh subcase 10 sweeps that bound the current controls

\begin{tabular}{|l|l|}
\hline Control & Bounding inputs \\
\hline Moderator in waste limited to $1.300 \mathrm{~kg}$ & set-2-10-nuac_uh_m2_graphite_sph_ps_1000_yr_para_00256 \\
\cline { 2 - 2 } & set-2-10-nuac_uh_m2_graphite_sph_ps_1000_yr_para_00292 \\
\hline \multirow{3}{*}{ Filler in waste is a minimum of $2280 \mathrm{~kg}$} & set-2-10-nuac_uh_m2_graphite_sph_ps_1000_yr_para_00340 \\
\cline { 2 - 2 } Moderator in waste limited to $1.500 \mathrm{~kg}$ & set-2-10-nuac_uh_m2_graphite_sph_ps_1000_yr_para_00376 \\
\cline { 2 - 2 } & set-2-10-nuac_uh_m2_graphite_sph_ps_1000_yr_para_00352 \\
\cline { 2 - 2 } & set-2-10-nuac_uh_m2_graphite_sph_ps_1000_yr_para_00388 \\
\hline
\end{tabular}

Selecting the most appropriate validation models was done by matching the parameters as closely as possible. All the set-2-uh inputs selected in Appendix $\mathrm{H}$ have no filler mass or can mass, use a thick discrete reflector (i.e., $0.7112 \mathrm{~cm}$ thick), and contain $585 \mathrm{~g}$ Be. The sweeps used for validation that match these criteria are selected from Table H-2. These are:

- set-1-2-uac1_uh_m2_graphite_cyl_ps_1000_yr_para_00444 and

- set-1-2-uac1_uh_m2_graphite_cyl_ps_1000_yr_para_00480.

TSUNAMI-IP determines the similarity value, $c_{k}$, of benchmarks to the selected validation inputs. The correlation coefficient, $c_{k}$, is used to determine the similarity between an application (e.g., a model within set-1-2) and a nuclear criticality benchmark experiment. A $c_{k} \geq 0.8$ is considered to have a high degree of similarity between application and experiment. No experiments with a $c_{k} \geq 0.7$ were evaluated in this analysis (Appendix H). However, a trend can be determined and a bias and bias uncertainty can be applied regardless. It is not unusual to have difficulty in finding experiments with a high $c_{k}$, and usually some compensatory action may be applicable, if desired. The number of benchmarks that met the $c_{k}$ criteria in are listed in Table 6.10 .

Table 6.10. Number of benchmark experiments in Appendix $\mathrm{H}$ with various $\boldsymbol{c}_{k}$ criteria

\begin{tabular}{|c|c|c|}
\hline \multirow{2}{*}{ Input } & \multicolumn{2}{c|}{ Number of benchmark models } \\
\cline { 2 - 3 } & $\boldsymbol{C}_{\boldsymbol{k}}>\boldsymbol{0 . 6}$ & $\boldsymbol{C}_{\boldsymbol{k}}>\boldsymbol{0 . 5}$ \\
\hline set-1-2-uac1_uh_m2_graphite_cyl_ps_1000_yr_para_00444 & 68 & 131 \\
\hline set-1-2-uac1_uh_m2_graphite_cyl_ps_1000_yr_para_00480 & 16 & 92 \\
\hline
\end{tabular}

The various bias and bias uncertainty values for the sets of $c_{k}$ criteria can be used via the approach provided by USLstats, which takes the $c_{k}$ values and trends the bias and bias uncertainty as $c_{k}$ approaches 1. There are two methods for USL: a confidence band with an administrative margin method and uniform width closed interval technique (single-sided tolerance). The latter cannot be used to extrapolate, only the first method can be used for $c_{k}$ trending. This is because the USL is determined when the similarity matches the application (i.e., $c_{k}=1$ ). The first method is discussed as follows [31].

Confidence band with administrative margin:

$$
\begin{gathered}
U S L_{1}(x)=\underset{(f \circ \beta(x)<0)}{1+\beta(x)-W-\Delta k_{m}} \\
U S L_{1}(x)=1-W-\Delta k_{m} \\
(\text { for } \beta(x)>0)
\end{gathered}
$$


Where

$\beta(x)$ is the bias;

$\mathrm{W}$ is the confidence band interval [New reference];

And $\Delta \mathrm{k}_{\mathrm{m}}$ is additional administrative margin applied to cover validation gaps.

Figure 6.61 through Figure 6.63 are plots of the USL trends where set-1-2-00480 with a $c_{k} \geq 0.6$ was not included because there are not enough benchmarks for an appropriate statistical analysis. The trends in each plot show a positive bias. When there is a positive, the bias is assumed to be zero. Therefore, the bias and bias uncertainty in Table H-34 is only the bias uncertainty for set-1-2-para_00444 and set-1-2para_00480. When extrapolating the confidence band interval, $\mathrm{W}$ is a curvilinear function that considers the number of experiments, the confidence level desired (e.g., $95 \%$ confidence level), and extrapolation beyond the range of experimental data points. The values in Table $\mathrm{H}-34$ are the applicable bias and bias uncertainty to use when extending the validation to set-2-10-uh. Additional margin to cover validation gaps. The absolute data-induced uncertainty in Table H-34 could be the applicable margin applied.

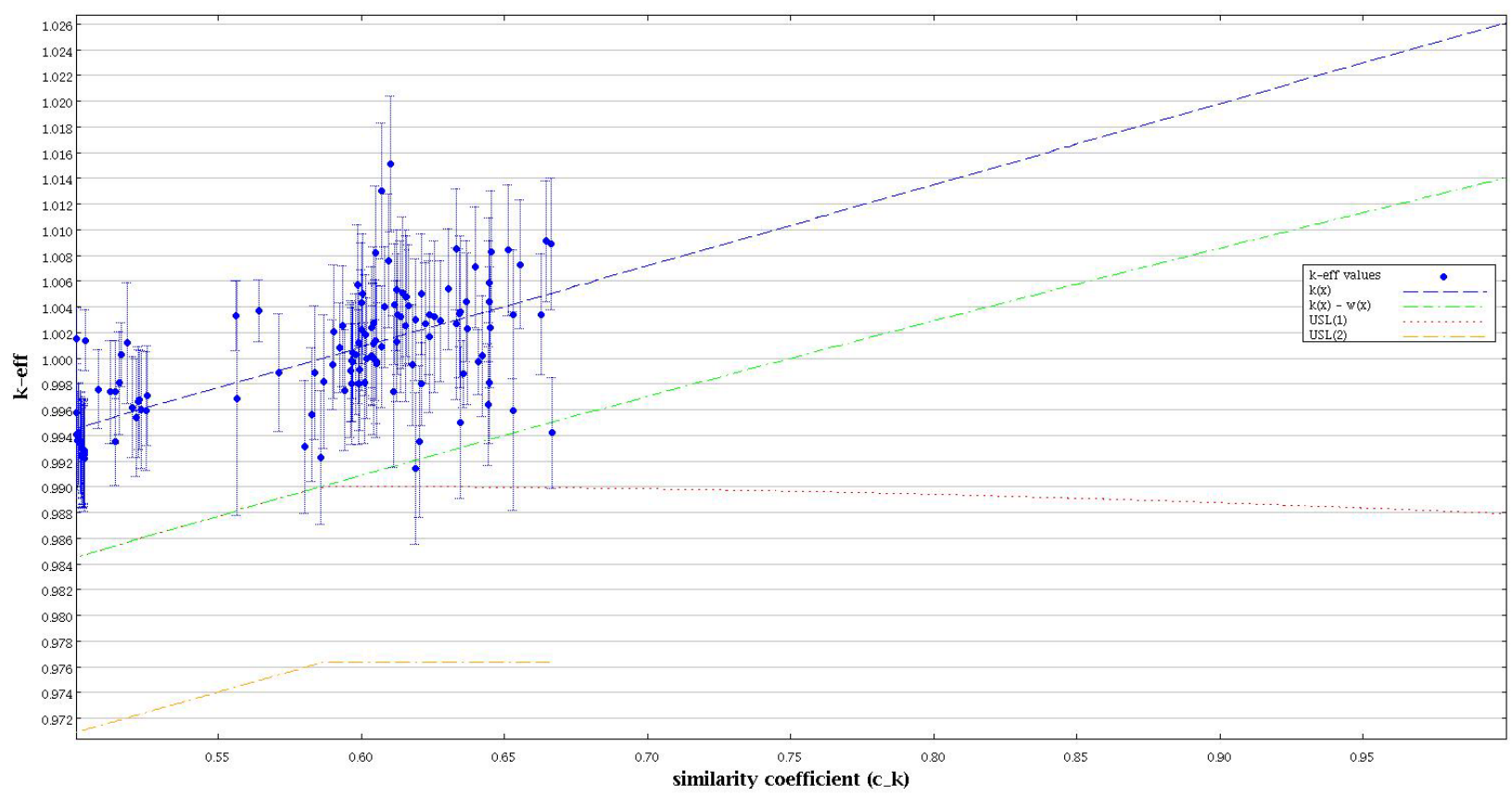

Figure 6.61. Trend of bias and bias uncertainty as $c_{k}$ approaches 1 for set-1-2-uac1_uh_m2_graphite_cyl_ps_1000_yr_para_00444 for $c_{k} \geq 0.5$. 


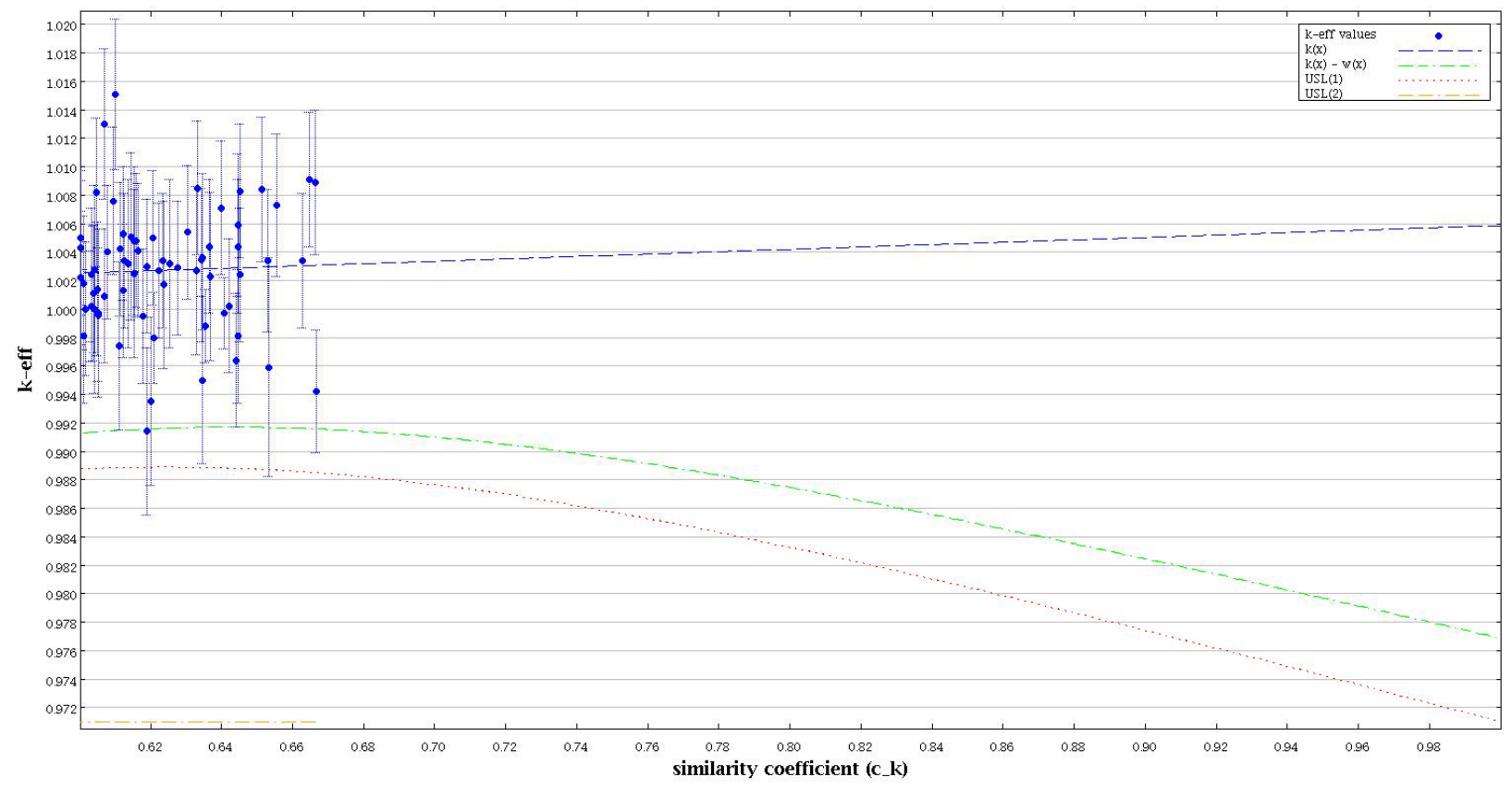

Figure 6.62. Trend of bias and bias uncertainty as $c_{k}$ approaches 1 for set-1-2-uac1_uh_m2_graphite_cyl_ps_1000_yr_para_00444 for $c_{k} \geq 0.6$.

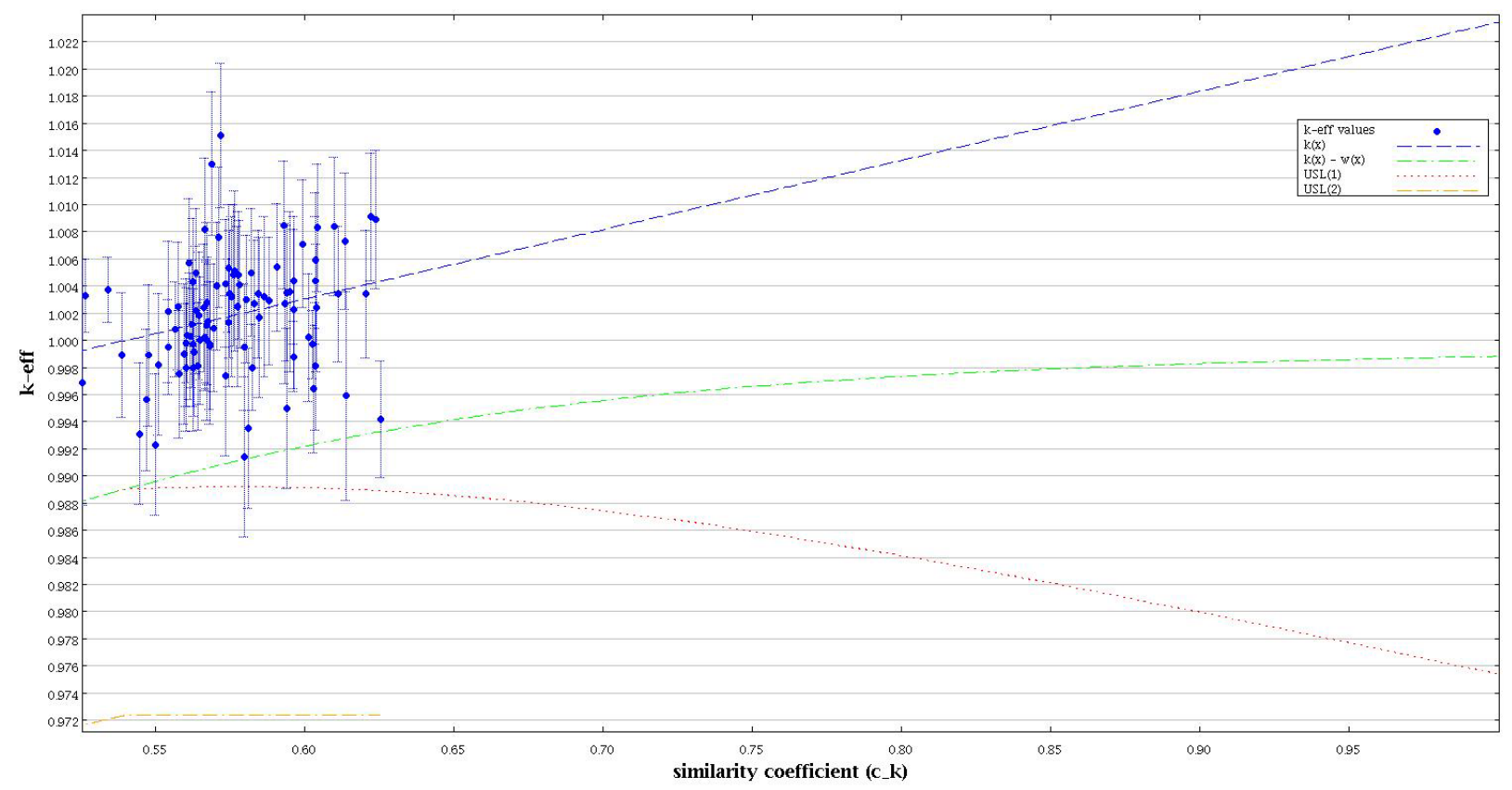

Figure 6.63. Trend of bias and bias uncertainty as $c_{k}$ approaches 1 for set-1-2-uac1_uh_m2_graphite_cyl_ps_1000_yr_para_00480 for $c_{k} \geq 0.6$. 


\section{CONCLUSIONS}

By evaluating $k_{\text {eff }}$ for multiple arrays and CCO configurations and establishing the $\mathrm{H} /{ }^{239} \mathrm{Pu}$ curves related to them, this work establishes a relationship between the controlling parameters and the associated criticality-based system reactivity $\left(k_{e f f}\right)$ during the post-closure repository performance period spanning 10,000 years, given the results of Sandia geomechanical analysis provided by Reedlunn and Bean [8]. Building upon the methodology developed by Brickner [4], parametric sweeps were performed to gauge possible $k_{\text {eff }}$ values over the performance period. The parametric sweeps demonstrated the primary reactivity impact of waste form moderator material and mass, array spacing, fissile region geometry, filler material and mass, and external reflector effects; other parameters studied introduced variation into the $k_{\text {eff }}$ results but did not dominate the system.

Moderator content, spacing, waste form volume and $\mathrm{B}_{4} \mathrm{C}$ content are the principal drivers of system behavior. Therefore, subcriticality can be maintained by managing these parameters. Waste form moderator mass is the primary means to limit system reactivity. As a secondary means, non-hydrogenous waste form filler mass can be used to increase the volume of the waste form to limit the reactivity of the system. Additionally, as previously established, $\mathrm{B}_{4} \mathrm{C}$ can be added to maintain subcriticality. Finally, applicable combinations of these three parameters can be used together to maintain subcriticality with the post-closure repository environment.

The results provided also allow other combinations of parameters to be used as control limits. Although the report documents a small subset of the results, the full set of results applicable to potential control limits are provided in Addendum 1.

Although this report provides results that are potential $\mathrm{B}_{4} \mathrm{C}$ mass limits (i.e., $30 \mathrm{~g}$ is sufficient up to at least $6 \mathrm{~kg}$ moderator) lower than previously provided in Saylor and Scaglione [3], the difference is primarily due to the differences in how the two analyses model the compaction of the CCOs. Therefore, both remain valid and either analysis may be used to provide technical justification for $\mathrm{B}_{4} \mathrm{C}$ mass limits.

Of additional interest is the implication of the results provided for the potential heterogeneity of the FGE. The analysis provides studies that show the behavior of the system - excluding the very low waste form moderator cases, which are also very low reactivity systems - is consistent with historical evaluations of FGE heterogeneity. As such, the well-mixed or heterogenous assumption for distribution of FGE within the waste form is a conservative assumption. Consequently, the inclusion of fissile materials that are not well mixed does not increase $\mathrm{k}_{\text {eff. }}$ Because the analysis is generic, it is intended to cover a wide range of potential scenarios. For example, a waste form may be identified from unused commercial nuclear fuel assembly fuel rods or other noncommercial fuel-related activities that manufactured cylindrical fuel components. As long as the component limits are met (e.g., FGE mass, moderator mass), the shape, size, and number of the FGE pieces is of no concern.

Finally, this report provides a very large set of results (see Addendum 1) that may be utilized to define limits in various ways for various parameters. The mixing of CCOs at emplacement which are qualified under different limits is acceptable as long as the subcritical limit used to define the various limits is similar because using the same subcritical limit to determine a set of limits for different parameters means that the CCOs would all have essentially the same maximum reactivity. 
This page is intentionally blank 


\section{REFERENCES}

[1] Sandia National Laboratories, Consideration of Nuclear Criticality When Disposing of Transuranic Waste at the Waste Isolation Pilot Plant, SAND99-2898, 2,000.

[2] Nuclear Waste Partnership, LLC, Nuclear Criticality Safety Evaluation for Contact-Handled Transuranic Waste Containers at the Waste Isolation Pilot Plant, WIPP-016, Revision 6, 2021.

[3] Saylor, Ellen M. and John M. Scaglione, Nuclear Criticality Safety Assessment of Potential Disposition at the Waste Isolation Plant, ORNL/TM-2017/751/R1, 2017.

[4] Brickner, Bret D., Post Placement Nuclear Criticality Evaluations Involving 6- and 12-Inch Pipe Overpack TRU Waste Containers at the Waste Isolation Pilot Plant, Oak Ridge National Laboratory, ORNL/TM-2019/1222/R0, 2019.

[5] DOE/WIPP-02-3122, Transuranic Waste Acceptance Criteria for the Waste Isolation Pilot Plant, Revision 8, July 5, 2016.

[6] PO 1990300

[7] Reedlunn, B., and J. Bean. Simulations of Pipe Overpack Container Compaction at the Waste Isolation Pilot Plant; Memorandum to Distribution, July 18, 2019. Sandia National Laboratories, 2019.

[8] Reedlunn, B. and J. Bean. Further Simulations of Criticality Overpack Container Compaction at the Waste Isolation Pilot Plant; Memorandum to Distribution, May 14, 2020. Sandia National Laboratories, 2020.

[9] US Department of Energy Carlsbad Field Office, TRUPACT-II Safety Analysis Report, Revision 23, Carlsbad, New Mexico, 2013a.

[10] US Department of Energy, "Surplus Plutonium Disposition, Record of Decision," Federal Register, 81(65), 19588-19594 (2016).

[11] R. P. Rechard, Improbability of Nuclear Criticality in TRU Waste in Criticality Control Overpacks at WIPP after Salt Creep Compaction.

[12] US Environmental Protection Agency, “40 CFR Part 191: Environmental Radiation Protection Standards for the Management and Disposal of Spent Nuclear Fuel, High-Level and Transuranic Radioactive Wastes, Final Rule," Federal Register, 58(242), 66398-66416, 1993.

[13] US Environmental Protection Agency, “40 CFR Part 191: Environmental Standards for the Management and Disposal of Spent Nuclear Fuel, High-Level and Transuranic Radioactive Wastes: Final Rule," Federal Register, 50(182), 38066-38089, 1985.

[14] US Environmental Protection Agency, "40 CFR Part 194: Criteria for the Certification and ReCertification of the Waste Isolation Pilot Plant's Compliance with the 40 CFR Part 191 Disposal Regulations; Final Rule," Federal Register, 61(28), 5224-5245, 1996.

[15] Duderstadt, J. J. and L. J. Hamilton, Nuclear Reactor Analysis, John Wiley \& Sons (1976).

[16] Marshall, W. J., et al., "Validation of SCALE 6.2 Criticality Calculations Using KENO V.a and KENO-VI," In: Proceedings, International Conference on Nuclear Criticality Safety (ICNC 2015), Charlotte, North Carolina, Sept. 13-17, 2015.

[17] American National Standards Institute, An American National Standard for Nuclear Criticality Safety in Operations with Fissionable Materials Outside Reactors, ANSI/ANS-8.1-2014, 2014.

[18] Geotechnical Analysis Report for July 2015 - June 2016 
[19] Reedlunn, B., et al., Initial Simulations of Empty room Collapse and Reconsolidation at the Waste Isolation Pilot Plant, Sandia National Laboratories, SAND2019-15351, date TBD

[20] Williamson, B. M., ANSI/ANS-8.7 Applications for the Storage of Criticality Control Overpacks, Savannah River Nuclear Solutions, Aiken, South Carolina.

[21] National Academies of Sciences, Engineering, and Medicine. Disposal of Surplus Plutonium at the Waste Isolation Pilot Plant: Interim Report. Washington, DC: The National Academies Press, 2018. https://doi.org/10.17226/25272.

[22] PUB. L. 102-579, "Waste Isolation Pilot Plant Land Withdrawal Act” (106 Stat. 4777), 1992.

[23] High Bridge Associates, Inc., Comparison of Plutonium Disposition Alternatives: WIPP Diluted Plutonium Storage and MOX Fuel Irradiation, 2016.

[24] Clark, H. K., et al. 1965, "Effect of Distribution of Fissile Material on Critical Mass," Nuclear Science and Engineering, 24, 133-141, 1966.

[25] Reedlunn, B. and Bean, J. (May 2020). Further Simulations of Criticality Control Overpack Container Compaction at the Waste Isolation Pilot Plant. Tech. rep. SAND2020-5105 CTF. Sandia National Laboratories.

[26] W.J. Marshall and B.D. Brickner, "Improved Runtime Performance in KENO-VI Models Using Arrays and Holes," Trans. Am. Nucl. Soc. 123, 937-940 (2020).

[27] W.J. Marshall, T.M. Greene, B.D. Brickner, and R.A. Hall, "Description and Use of SCALE Sampler Parametric Capability for Engineering Analysis and Optimization," Trans. Am. Nucl. Soc. 122, 471-474 (2020).

[28] Email from Ben Reedlunn to Bret Brickner et al, 2/3/21, "Re: [EXTERNAL] RE: CCO Postclosure Criticality Safety," and attachments.

[29] Saylor, Ellen M., Nuclear Criticality Safety Assessment of Criticality Control Containers without Moderation Control at the Waste Isolation Pilot Plant, ORNL/TM-2020/1713, 2020.

[30] DOE/WIPP-19-3609, Title 40 CFR Part 191 Subparts B and C Compliance Recertification Application 2019 for the Waste Isolation Pilot Plant, Appendix PA-2019 Performance Assessment, US Department of Energy, Carlsbad Field Office, Carlsbad, New Mexico, 2020

[31] Christopher M. Perfetti \& Bradley T. Rearden (2019) Estimating Code Biases for Criticality Safety Applications with Few Relevant Benchmarks, Nuclear Science and Engineering, 193:10, 1090-1128 https://doi.org/10.1080/00295639.2019.1604048.

[32] D. E. Peplow, "Monte Carlo Shielding Analysis Capabilities with MAVRIC," Nucl. Technol. 174(2), 289-313 (2011).

[33] B. T. Rearden, M. T. Sieger, S. M. Bowman, and J. P. Lefebvre, Quality Assurance Plan for the SCALE Code System, SCALE-QAP-005, Rev. 4, May 2013.

[34] American Nuclear Society, Nuclear Criticality Safety in Operations with Fissionable Materials Outside Reactors, ANSI/ANS-8.1-2014, R2018, La Grange Park, IL (2018).

[35] American Nuclear Society, Validation of Neutron Transport Methods for Nuclear Criticality Safety Calculations, ANSI/ANS-8.24-2017, American Nuclear Society, La Grange Park, IL (2017).

[36] International Handbook of Evaluated Criticality Safety Benchmark Experiments, NEA/NSC/DOC(95)03, NEA Nuclear Science Committee (2020). 
[37] W. A. Wieselquist, R. A. Lefebvre, and M. A. Jessee, Eds., SCALE Code System, ORNL/TM2005/39, Version 6.2.4, Oak Ridge National Laboratory, Oak Ridge, TN (2020).

[38] B. T. Rearden, M. L. Williams, M. A. Jessee, D. E. Mueller, and D. A. Wiarda, "Sensitivity and Uncertainty Analysis Capabilities and Data in SCALE," Nucl. Technol. 174 (2), pp. 236-288 (2011).

[39] ORNL. 2013. The SCALE Verified, Archived Library of Inputs and Data - VALID, Oak Ridge National Laboratory, transactions of ANS NCSD 2013, Wilmington, NC, September 29 October 31, 2013.

[40] Division of Spent Fuel Storage and Transportation, Interim Staff Guidance-8, Revision 3, Burnup Credit in the Criticality Safety Analyses of PWR Spent Fuel in Transportation and Storage Casks, US Nuclear Regulatory Commission (2012).

[41] W. J. Marshall, B. J. Ade, S. M. Bowman, I. C. Gauld, G. Ilas, U. Mertyurek, and G. Radulescu, Technical Basis for Peak Reactivity Burnup Credit for BWR Spent Nuclear Fuel in Storage and Transportation Systems, NUREG/CR-7194 (ORNL/TM-2014/240), prepared for the US Nuclear Regulatory Commission by Oak Ridge National Laboratory, Oak Ridge, TN (2015).

[42] W. J. Marshall, J. B. Clarity, and S. M. Bowman, Validation of $k_{\text {eff }}$ Calculations for Extended BWR Burnup Credit, NUREG/CR-7252 (ORNL/TM-2018/797), prepared for the US Nuclear Regulatory Commission by Oak Ridge National Laboratory, Oak Ridge, TN (2018).

[43] J. M. Scaglione, D. E. Mueller, J. C. Wagner, and W. J. Marshall, An Approach for Validating Actinide and Fission Product Burnup Credit Criticality Safety Analyses-Criticality ( $\left.k_{\mathrm{eff}}\right)$ Predictions, NUREG/CR-7109 (ORNL/TM-2011/514), prepared for the US Nuclear Regulatory Commission by Oak Ridge National Laboratory, Oak Ridge, TN (2012).

[44] K. S. Krane, Introductory Nuclear Physics, John Wiley \& Sons (1988).

[45] International Atomic Energy Agency Nuclear Data Section, Livechart - Table of Nuclides, Online, Accessed July 2021, https://www-nds.iaea.org/relnsd/vcharthtml/VChartHTML.html.

[46] B. Reedlunn and J. Bean, Additional Studies of Criticality Control Overpack and Pipe Overpack Container Compaction at the Waste Isolation Pilot Plant. Sandia Memorandum. SAND202111268 CTF (2021).

[47] NUREG/CR-6698, Guide for Validation of Nuclear Criticality Safety Calculational Methodology 
This page is intentionally blank 
APPENDIX A. SET-1: RESULTS OF THE THREE-HIGH UNIFORM ARRAY WITH BOUNDING SPACING CALCULATIONS 
This page is intentionally blank 


\section{APPENDIX A. SET-1: RESULTS OF THE THREE-HIGH UNIFORM ARRAY WITH BOUNDING SPACING CALCULATIONS}

The analysis methodology for the uniform arrays is discussed in detail in Section 6.1 of the main report.

This appendix serves as a repository of those results for the set-1 calculations.

The complete results for all SAMPLER sweeps are provided in ADDENDUM 1.

The analysis model use for the calculations in this appendix is shown in Figure A-1 below.

The SAMPLER case sweeps presented in this appendix are summarized in Table A-1 below.

\section{LIST OF FIGURES}

Figure A-1. Diagram of the uniform array three-high model.

Figure A-2. Set-1 results, plot 1: reactivity effect of cylinder radius, pipe steel, no filler, no Be, water moderated.

Figure A-3. Set-1 results, plot 2: reactivity effect of cylinder radius, pipe steel, no filler, no Be, poly moderated.

Figure A-4. Set-1 results, plot 3: reactivity effect of cylinder radius, pipe poly, no filler, no Be, water moderated

Figure A-5. Set-1 results, plot 4: reactivity effect of cylinder radius, pipe poly, no filler, no Be, poly moderated.

Figure A-6. Set-1 results, plot 5: reactivity effect of cylinder radius, pipe steel, $5 \mathrm{~kg}$ graphite/can, no Be, water moderated.

Figure A-7. Set-1 results, plot 6: reactivity effect of cylinder radius, pipe steel, $5 \mathrm{~kg}$ graphite/can, no Be, poly moderated.

Figure A-8. Set-1 results, plot 7: reactivity effect of cylinder radius, pipe poly, $5 \mathrm{~kg}$ graphite/can, no Be, water moderated.

Figure A-9. Set-1 results, plot 8: reactivity effect of cylinder radius, pipe poly, $5 \mathrm{~kg}$ graphite/can, no Be, poly moderated.

Figure A-10. Set-1 results, plot 9: reactivity effect of various parameters with $7.7 \mathrm{~cm}$ cylinder radius, graphite filler, poly moderated.

Figure A-11. Set-1 results, plot 10: reactivity effect of various parameters with $7.7 \mathrm{~cm}$ cylinder radius, generic filler, poly moderated.

Figure A-12. Set-1 results, plot 11: comparison of graphite and generic filler with $7.7 \mathrm{~cm}$ cylinder radius, no Be, poly moderated, thick discrete reflector.

Figure A-13. Set-1 results, plot 12: reactivity effect of various parameters with spherical waste form geometry, graphite filler, water moderated.

Figure A-14. Set-1 results, plot 13: reactivity effect of various parameters with spherical waste form geometry, graphite filler, poly moderated.

Figure A-15. Set-1 results, plot 14: comparison of spherical and cylindrical geometries $(\mathrm{h} / \mathrm{x})$............ A-20

Figure A-16. Set-1 results, plot 15: comparison of water and poly $\mathrm{h} / \mathrm{x}$.

Figure A-17. Set-1 results, plot 16: comparison of spherical and cylindrical geometries (mod mass).

Figure A-18. Set-1 results, plot 17: comparison of $50 \mathrm{~g} \mathrm{~B} \mathrm{~B}_{4} \mathrm{C}$ vs. no $\mathrm{B}_{4} \mathrm{C}$ for spherical and cylindrical geometries (mod mass). 


\section{LIST OF TABLES}

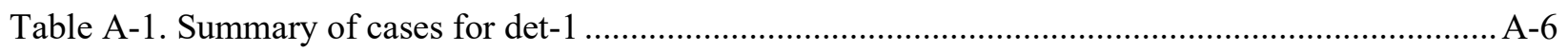




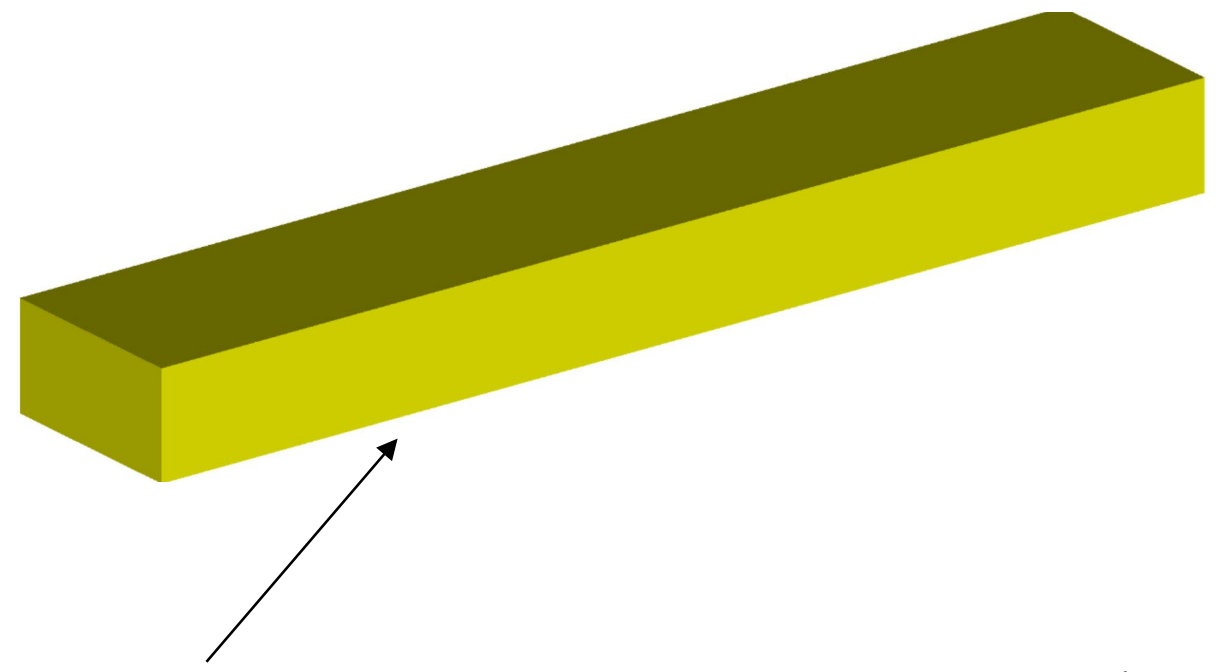

WIPP salt panel, pure salt

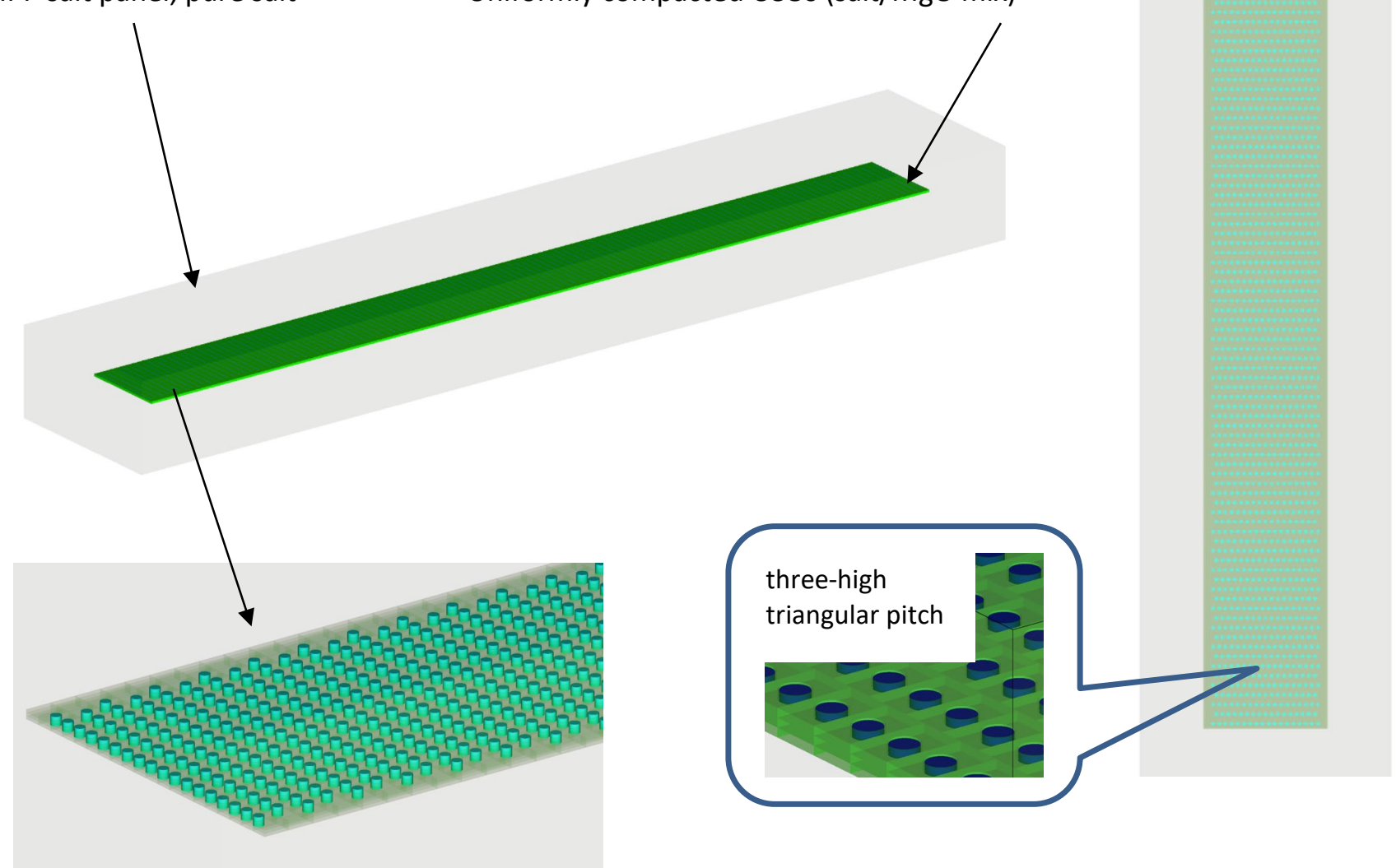

Figure A-1. Diagram of the uniform array three-high model. 
Table A-1. Summary of cases for det-1

\begin{tabular}{|c|c|c|c|c|c|c|c|c|}
\hline Case & Model type & Waste form shape & $\begin{array}{l}\text { Waste form } \\
\text { moderator }\end{array}$ & $\begin{array}{c}\text { Filler material } \\
(\mathbf{0}, \mathbf{2 , 0 0 0}, \\
\mathbf{4 , 0 0 0} \mathbf{g})\end{array}$ & Metal in filler & $\begin{array}{l}\text { Discrete reflector } \\
\text { (thin } 0.001 \text { and } \\
\text { thick } 0.7112 \mathrm{~cm} \text { ) }\end{array}$ & $\operatorname{Be}(\mathrm{g})$ & Subcase \\
\hline \multirow{18}{*}{ Set-1 } & \multirow{18}{*}{$\begin{array}{l}\text { Uniform array stacked three } \\
\text { high with CCO pitch } \\
\text { reduction } x=50 \%, y=10 \%, \\
\qquad z=\text { no space }\end{array}$} & \multirow{8}{*}{$\begin{array}{l}\text { Cylinder (radius range } \\
4.8,6,7.7 \text { and height } \\
\text { defined by total } \\
\text { volume of mass) }\end{array}$} & water & $\mathrm{c} 12$ & \multirow{18}{*}{$\begin{array}{c}\text { SS from can } \\
(0,500,1,000 \mathrm{~g})\end{array}$} & steel & \multirow{18}{*}{$\begin{array}{l}0 \text { to } \\
585\end{array}$} & set-1-1 \\
\hline & & & poly & $\mathrm{c} 12$ & & steel & & set- $1-2$ \\
\hline & & & water & $\mathrm{c} 12$ & & poly & & set-1-3 \\
\hline & & & poly & $\mathrm{c} 12$ & & poly & & set-1-4 \\
\hline & & & water & generic & & steel & & set-1-5 \\
\hline & & & poly & generic & & steel & & set-1-6 \\
\hline & & & water & generic & & poly & & set-1-7 \\
\hline & & & poly & generic & & poly & & set- $1-8$ \\
\hline & & \multirow{10}{*}{$\begin{array}{l}\text { Sphere (radius defined } \\
\text { by total volume of } \\
\text { mass) }\end{array}$} & water & $\mathrm{c} 12$ & & steel & & set-1-9 \\
\hline & & & poly & $\mathrm{c} 12$ & & steel & & set-1-10 \\
\hline & & & water & $\mathrm{c} 12$ & & poly & & \begin{tabular}{|l|} 
set-1-11 \\
\end{tabular} \\
\hline & & & poly & $\mathrm{c} 12$ & & poly & & set-1-12 \\
\hline & & & water & generic & & steel & & \begin{tabular}{|l|} 
set-1-13 \\
\end{tabular} \\
\hline & & & poly & generic & & steel & & set-1-14 \\
\hline & & & water & generic & & poly & & \begin{tabular}{|l|} 
set-1-15 \\
\end{tabular} \\
\hline & & & poly & generic & & poly & & set-1-16 \\
\hline & & & $\begin{array}{c}\text { poly }+50 \mathrm{~g} \mathrm{~B} \mathrm{~B}_{4} \mathrm{C} \\
(\text { cyl) }\end{array}$ & $\mathrm{c} 12$ & & poly $(0.7112)$ & & set-1-17 \\
\hline & & & $\begin{array}{c}\text { poly }+50 \mathrm{~g} \mathrm{~B} \mathrm{~B}_{4} \mathrm{C} \\
(\mathrm{sph})\end{array}$ & $\mathrm{c} 12$ & & poly $(0.7112)$ & & set-1-18 \\
\hline
\end{tabular}




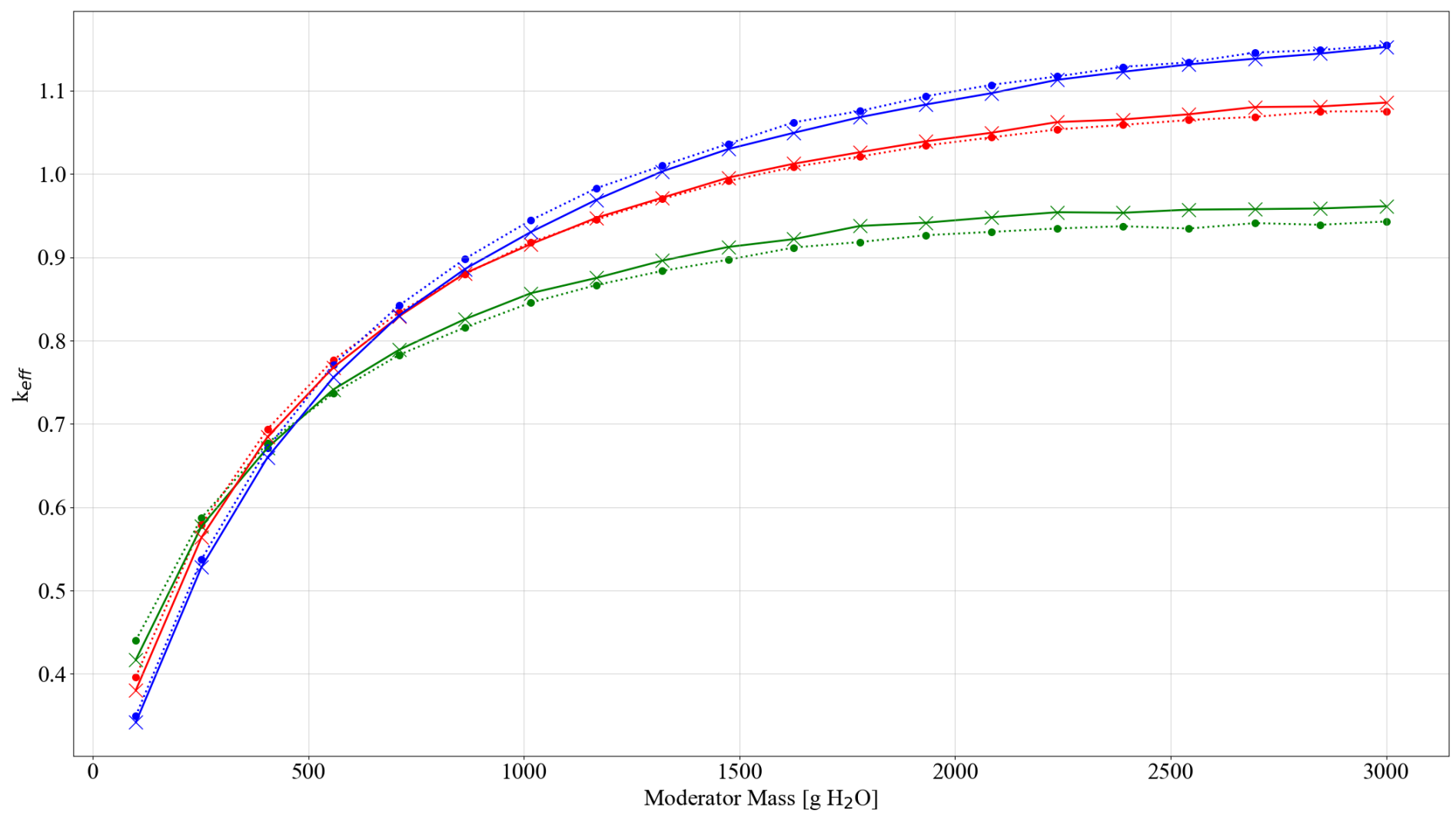

set- 1 , cyl radius $=4.8 \mathrm{~cm}$, thin discrete reflector set- 1 , cyl radius $=6.25 \mathrm{~cm}$, thin discrete reflector
- $\cdots$ set- 1 , cyl radius $=7.7 \mathrm{~cm}$, thin discrete reflector set- 1 , cyl radius $=4.8 \mathrm{~cm}$, thick discrete reflector set- 1 , cyl radius $=6.25 \mathrm{~cm}$, thick discrete reflector set- 1 , cyl radius $=7.7 \mathrm{~cm}$, thick discrete reflector

Figure A-2. Set-1 results, plot 1: reactivity effect of cylinder radius, pipe steel, no filler, no Be, water moderated. 


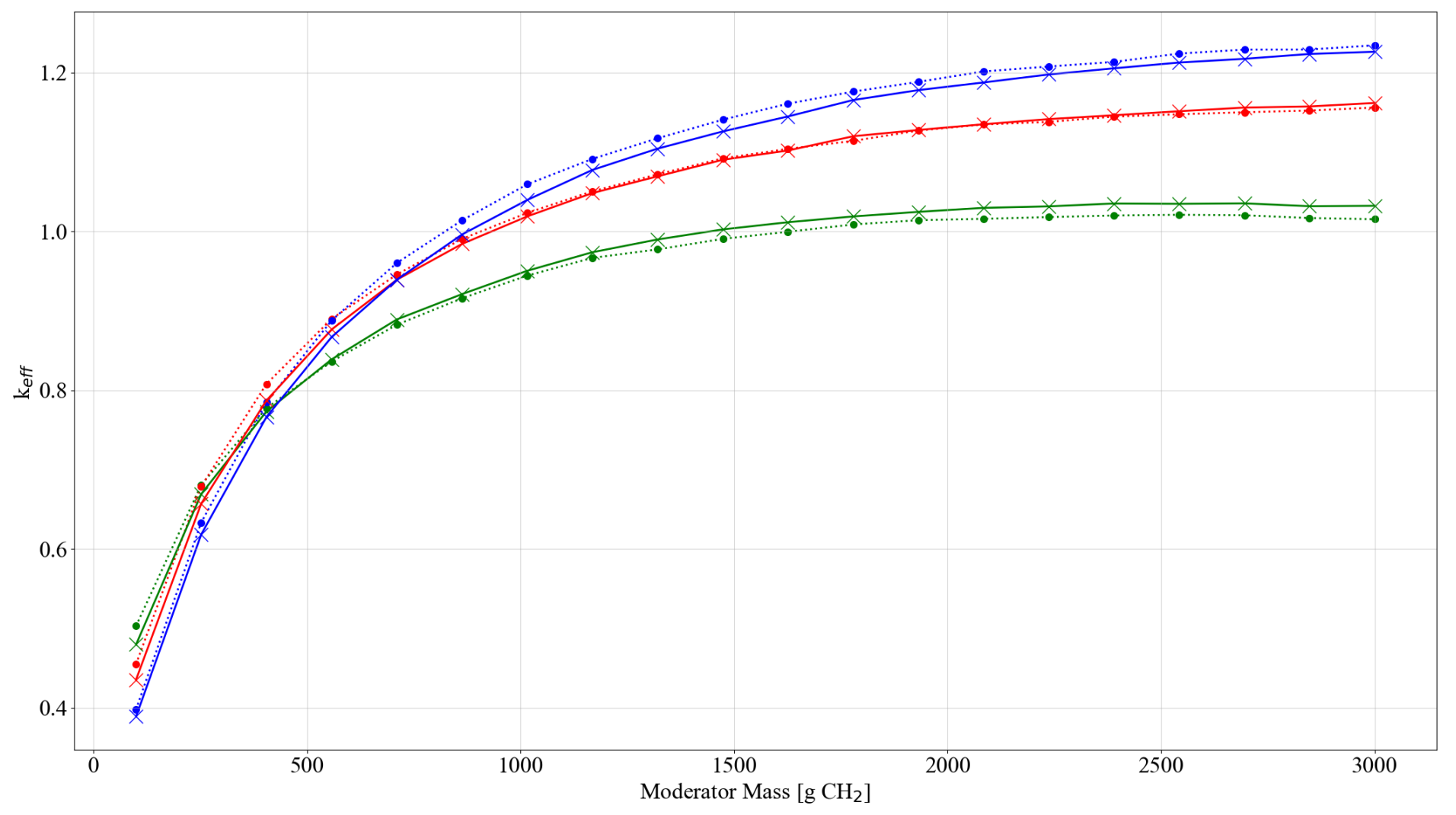

set- 1 , cyl radius $=4.8 \mathrm{~cm}$, thin discrete reflecto set- 1 , cyl radius $=6.25 \mathrm{~cm}$, thin discrete reflector
- $\cdots$ set- 1 , cyl radius $=7.7 \mathrm{~cm}$, thin discrete reflector set -1 , cyl radius $=4.8 \mathrm{~cm}$, thick discrete reflector set- 1 , cyl radius $=6.25 \mathrm{~cm}$, thick discrete reflector set- 1 , cyl radius $=7.7 \mathrm{~cm}$, thick discrete reflector

Figure A-3. Set-1 results, plot 2: reactivity effect of cylinder radius, pipe steel, no filler, no Be, poly moderated. 


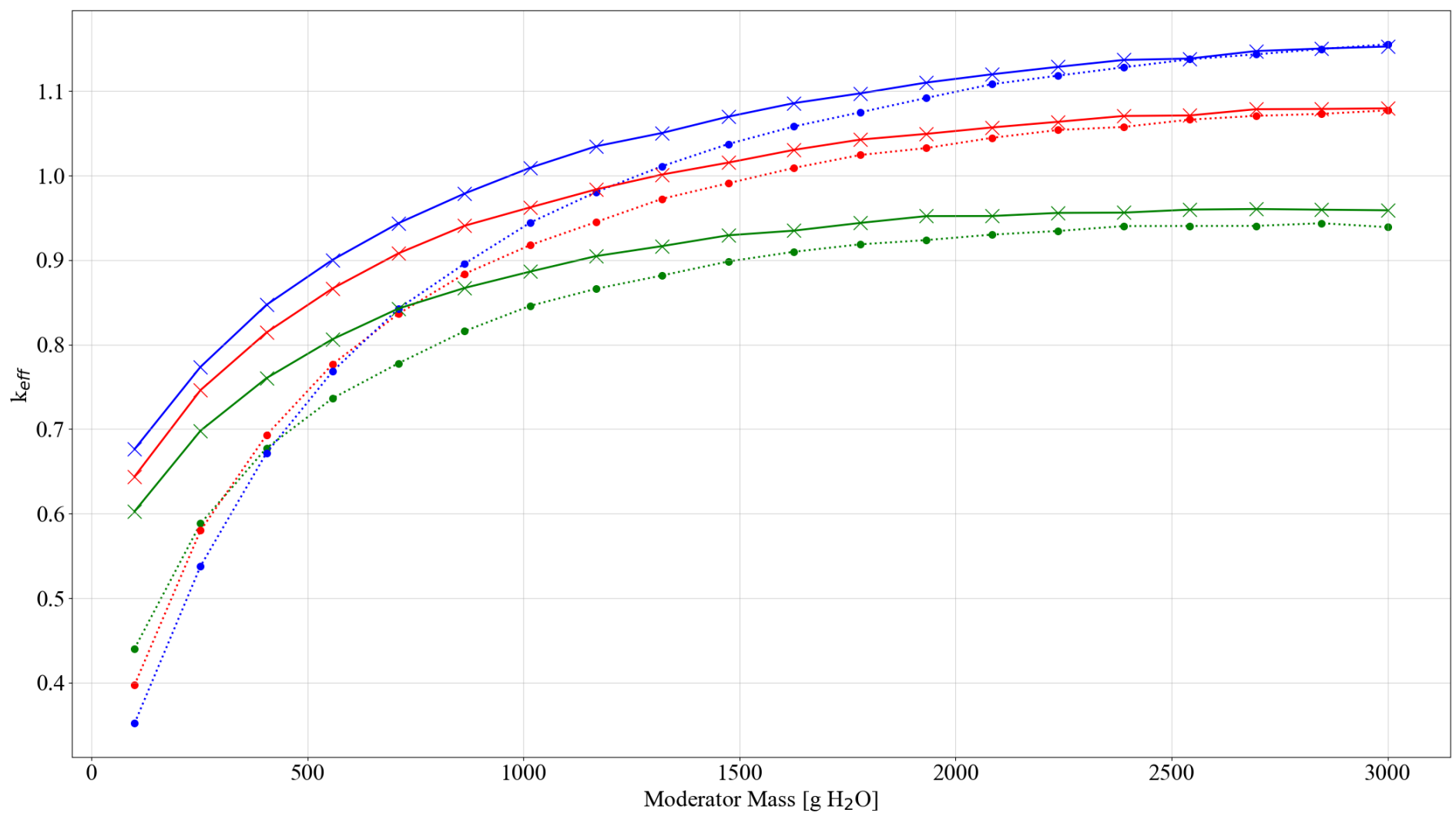

set- 1 , cyl radius $=4.8 \mathrm{~cm}$, thin discrete reflector set- 1 , cyl radius $=6.25 \mathrm{~cm}$, thin discrete reflector
- $\cdots$ set 1 , cyl radius $=7.7 \mathrm{~cm}$, thin discrete reflector set- 1 , cyl radius $=4.8 \mathrm{~cm}$, thick discrete reflector set- 1 , cyl radius $=6.25 \mathrm{~cm}$, thick discrete reflector set- 1 , cyl radius $=7.7 \mathrm{~cm}$, thick discrete reflector

Figure A-4. Set-1 results, plot 3: reactivity effect of cylinder radius, pipe poly, no filler, no Be, water moderated. 


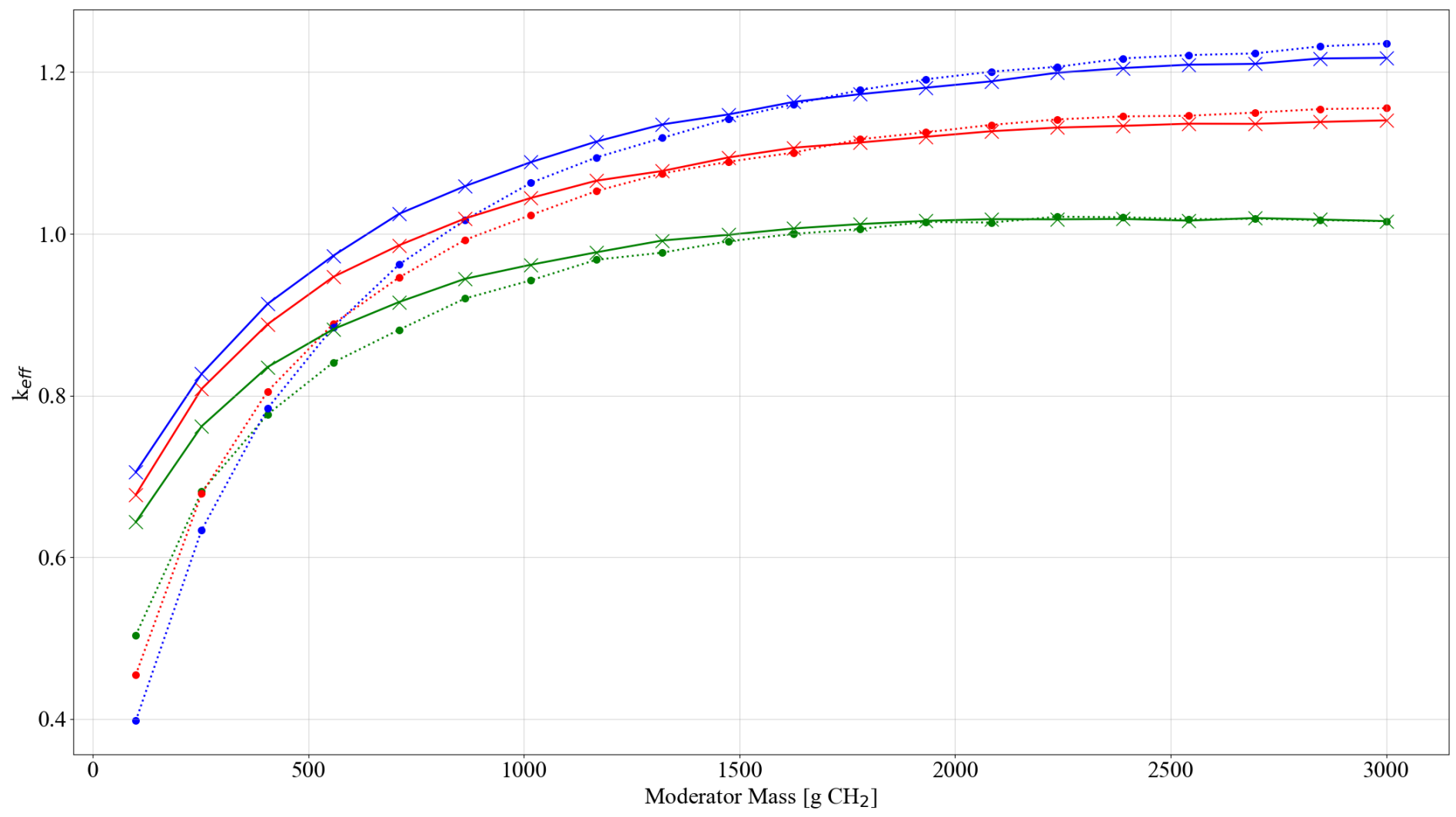

set- 1 , cyl radius $=4.8 \mathrm{~cm}$, thin discrete reflecto

-... set- 1 , cyl radius $=7.7 \mathrm{~cm}$, thin discrete reflector

set-1, cyl radius $=6.25 \mathrm{~cm}$, thick discrete reflector set- 1 , cyl radius $=6.25 \mathrm{~cm}$, thin discrete reflector set- 1 , cyl radius $=4.8 \mathrm{~cm}$, thick discrete reflector

$*$ set- 1 , cyl radius $=7.7 \mathrm{~cm}$, thick discrete reflector

Figure A-5. Set-1 results, plot 4: reactivity effect of cylinder radius, pipe poly, no filler, no Be, poly moderated. 


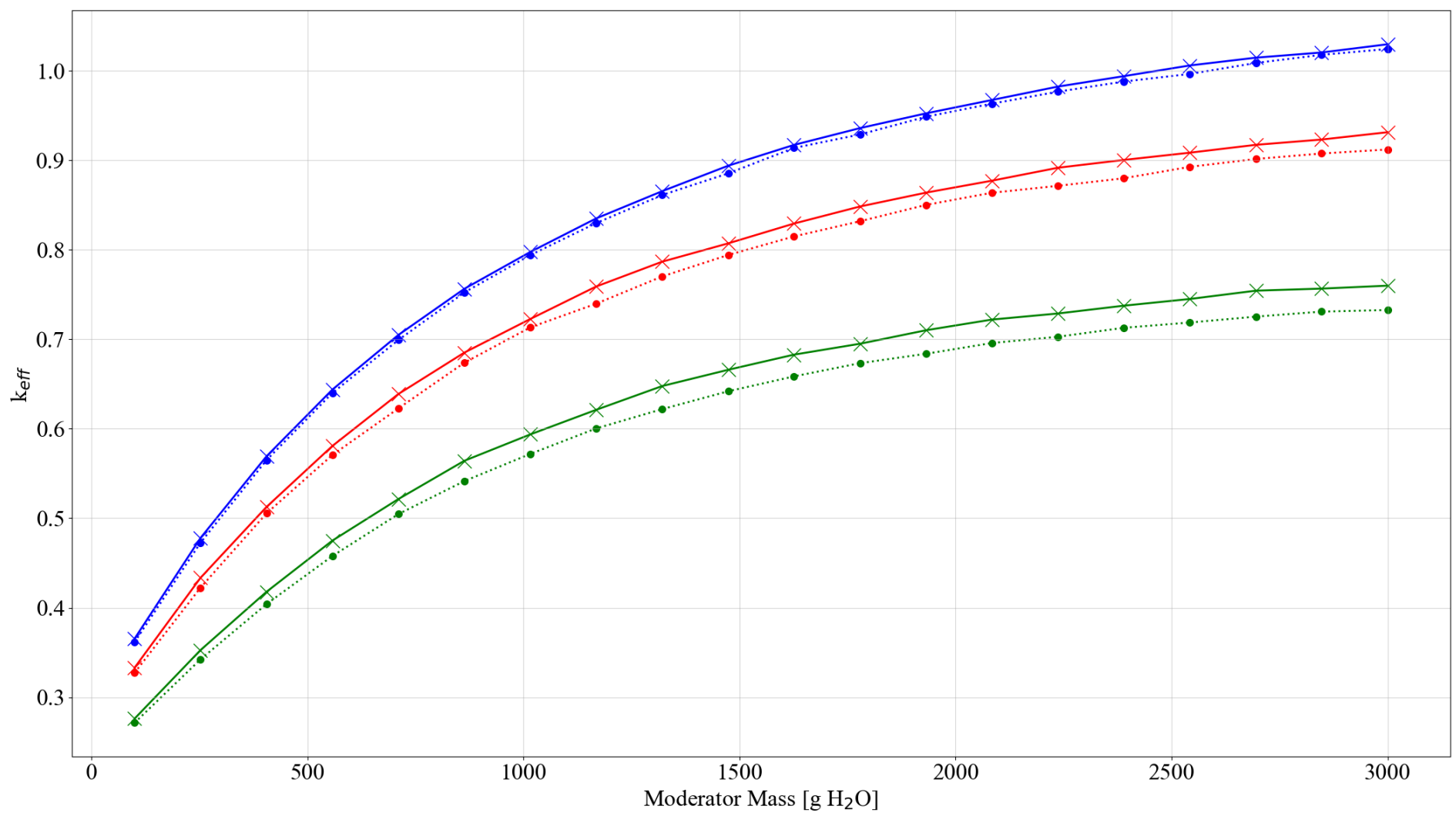

- ... set- 1 , cyl radius $=4.8 \mathrm{~cm}$, thin discrete reflector

$\cdots \bullet \cdots \quad$ set- 1 , cyl radius $=7.7 \mathrm{~cm}$, thin discrete reflector

$\nsucc$ set- 1 , cyl radius $=6.25 \mathrm{~cm}$, thick discrete reflector

-... set-1, cyl radius $=6.25 \mathrm{~cm}$, thin discrete reflector

set- 1 , cyl radius $=4.8 \mathrm{~cm}$, thick discrete reflector

et- 1 , cyl radius $=7.7 \mathrm{~cm}$, thick discrete reflector

Figure A-6. Set-1 results, plot 5: reactivity effect of cylinder radius, pipe steel, $5 \mathrm{~kg}$ graphite/can, no Be, water moderated. 


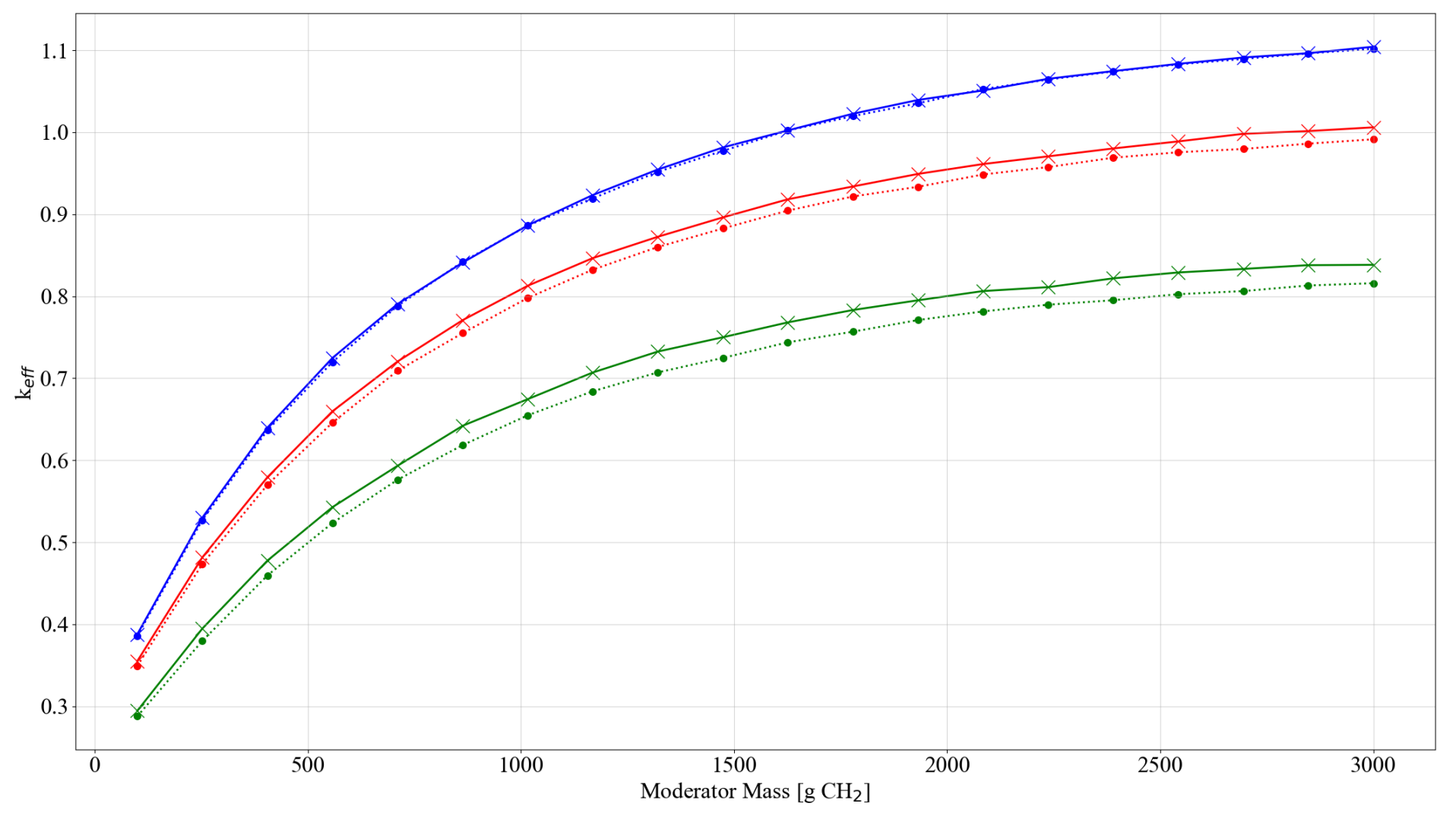

-... set-1, cyl radius $=4.8 \mathrm{~cm}$, thin discrete reflecto

-... set- 1 , cyl radius $=7.7 \mathrm{~cm}$, thin discrete reflector

× set-1, cyl radius $=6.25 \mathrm{~cm}$, thick discrete reflector set- 1 , cyl radius $=6.25 \mathrm{~cm}$, thin discrete reflector set- 1 , cyl radius $=4.8 \mathrm{~cm}$, thick discrete reflector * set-1, cyl radius $=7.7 \mathrm{~cm}$, thick discrete reflector

Figure A-7. Set-1 results, plot 6: reactivity effect of cylinder radius, pipe steel, $5 \mathrm{~kg}$ graphite/can, no Be, poly moderated. 


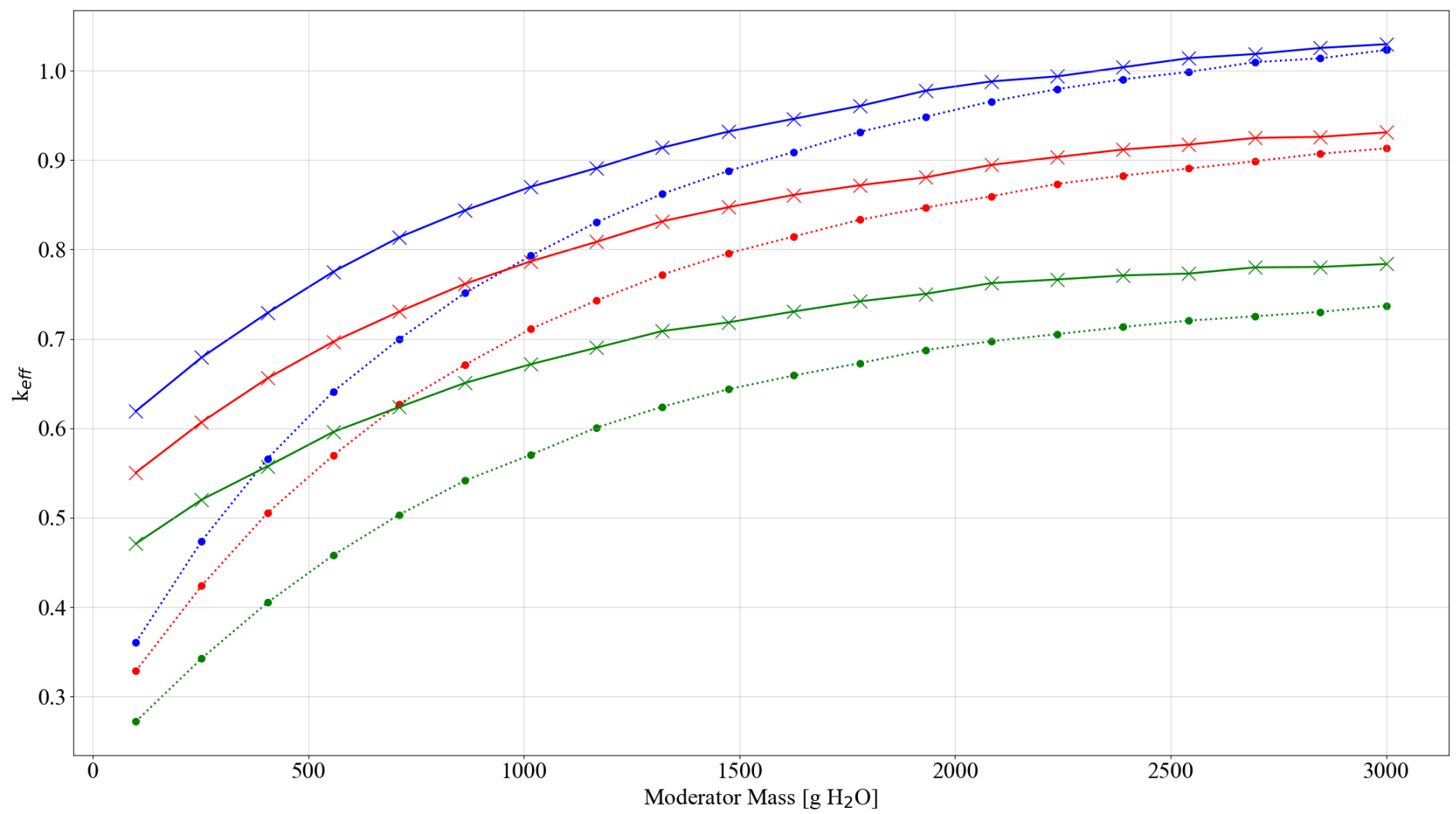

set- 1 , cyl radius $=4.8 \mathrm{~cm}$, thin discrete reflecto

$\cdots \bullet$ set- 1 , cyl radius $=7.7 \mathrm{~cm}$, thin discrete reflector

× set-1, cyl radius $=6.25 \mathrm{~cm}$, thick discrete reflector set- 1 , cyl radius $=6.25 \mathrm{~cm}$, thin discrete reflector set- 1 , cyl radius $=4.8 \mathrm{~cm}$, thick discrete reflector

$\star$ set- 1 , cyl radius $=7.7 \mathrm{~cm}$, thick discrete reflector

Figure A-8. Set-1 results, plot 7: reactivity effect of cylinder radius, pipe poly, $5 \mathrm{~kg}$ graphite/can, no Be, water moderated. 


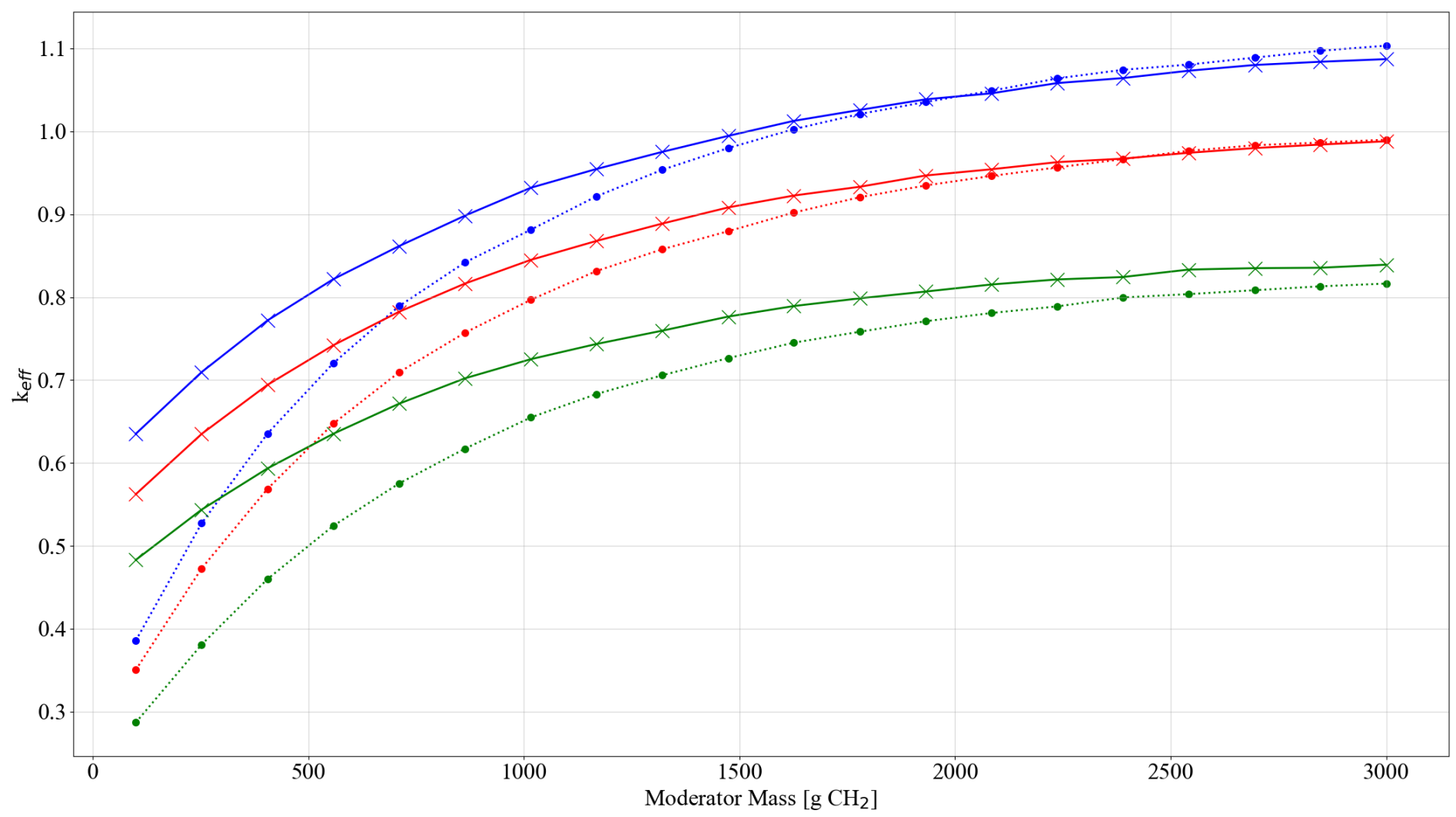

-... set-1, cyl radius $=4.8 \mathrm{~cm}$, thin discrete reflector

$\cdots \bullet \cdots \quad$ set- 1 , cyl radius $=7.7 \mathrm{~cm}$, thin discrete reflector

$\star$ set-1, cyl radius $=6.25 \mathrm{~cm}$, thick discrete reflector

..... set- 1 , cyl radius $=6.25 \mathrm{~cm}$, thin discrete reflector

set- 1 , cyl radius $=4.8 \mathrm{~cm}$, thick discrete reflector

set- 1 , cyl radius $=7.7 \mathrm{~cm}$, thick discrete reflector

Figure A-9. Set-1 results, plot 8: reactivity effect of cylinder radius, pipe poly, $5 \mathrm{~kg}$ graphite/can, no Be, poly moderated. 


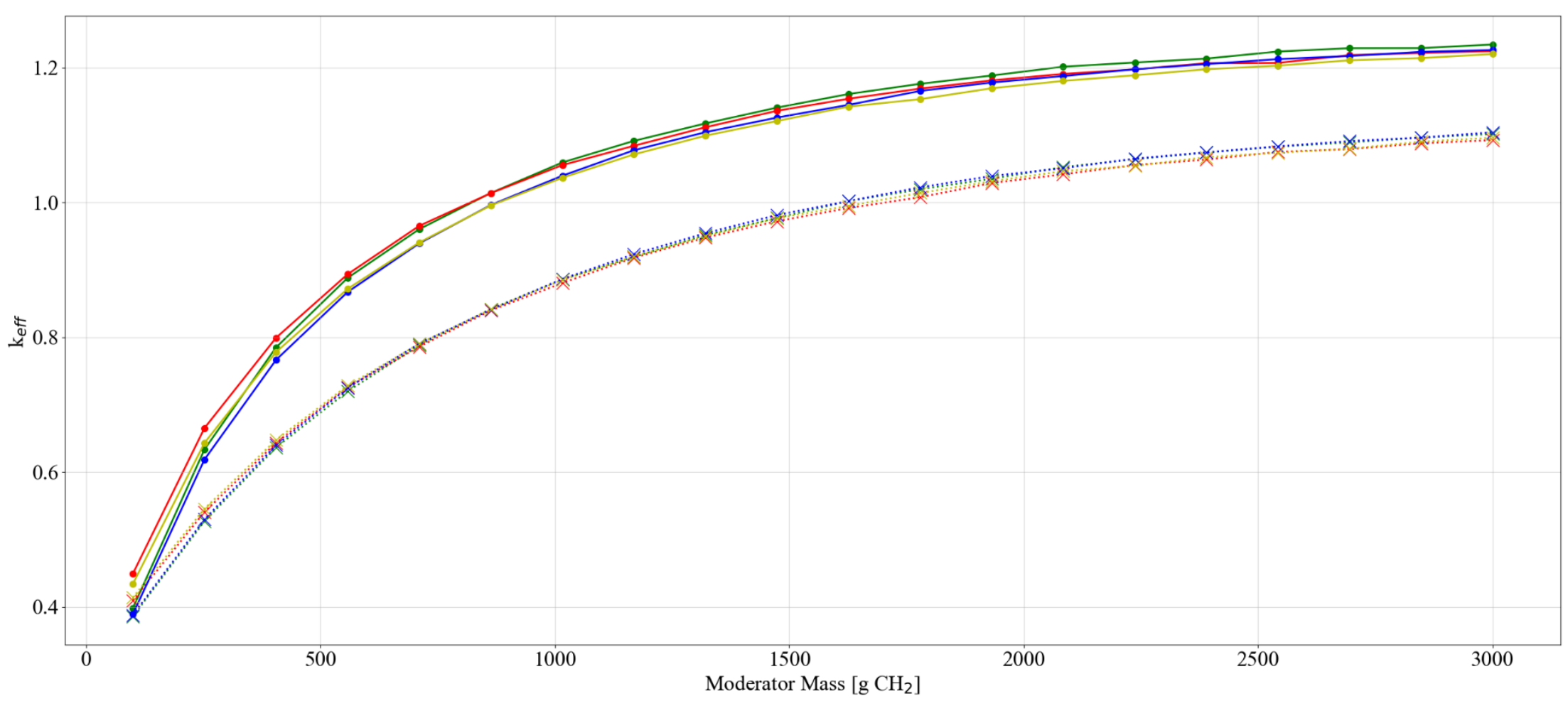

$\longrightarrow$ set-1, no filler, no Be, thin SS discrete reflector

…… set-1, $5 \mathrm{~kg}$ graphite/can, no $\mathrm{Be}$, thin SS discrete reflector

$\longrightarrow$ set-1, no filler, $585 \mathrm{~g} \mathrm{Be}$, thin SS discrete reflector

- - set-1, no filler, no Be, thick SS discrete reflector

…… set-1, $5 \mathrm{~kg}$ graphite/can, no Be, thick SS discrete reflector

$\cdots$ set-1, $5 \mathrm{~kg}$ graphite/can, $585 \mathrm{~g} \mathrm{Be}$, thin SS discrete reflector

- set-1, no filler, $585 \mathrm{~g} \mathrm{Be}$, thick SS discrete reflector

........ set-1, $5 \mathrm{~kg}$ graphite/can, $585 \mathrm{~g} \mathrm{Be}$, thick SS discrete reflector

Figure A-10. Set-1 results, plot 9: reactivity effect of various parameters with $7.7 \mathrm{~cm}$ cylinder radius, graphite filler, poly moderated. 


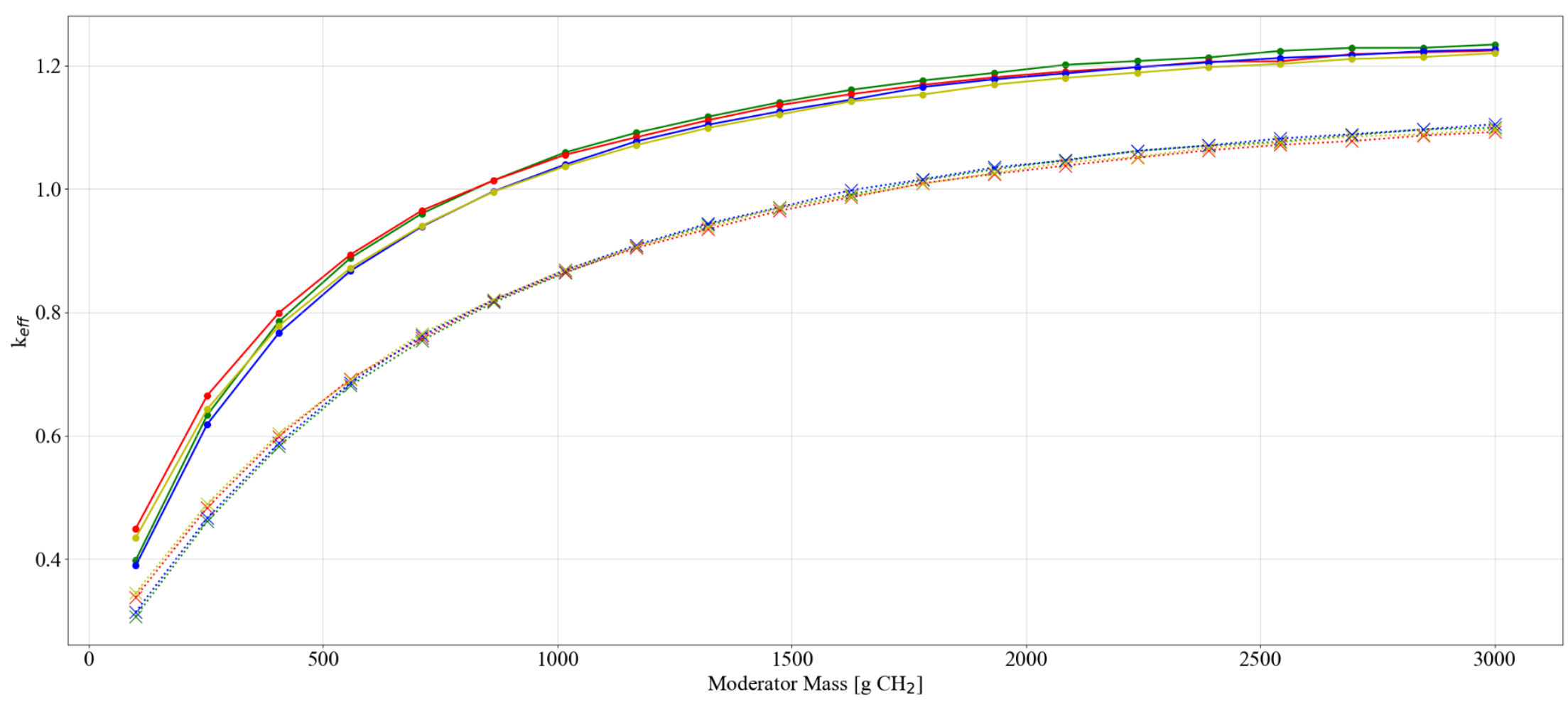

$\longrightarrow$ set-1, no filler, no Be, thin SS discrete reflector

........ set- $1,5 \mathrm{~kg}$ generic/can, no $\mathrm{Be}$, thin $\mathrm{SS}$ discrete reflector

$\longrightarrow$ set-1, no filler, no Be, thick SS discrete reflector

$\ldots$ set-1, no filler, $585 \mathrm{~g} \mathrm{Be}$, thin SS discrete reflector

…… set-1, $5 \mathrm{~kg}$ generic/can, no Be, thick SS discrete reflector

........ set- $1,5 \mathrm{~kg}$ generic/can, $585 \mathrm{~g} \mathrm{Be}$, thin SS discrete $\mathrm{r}$ reflector

$\ldots$ set-1, no filler, $585 \mathrm{~g} \mathrm{Be}$, thick SS discrete reflector

$\cdots$..... set-1, $5 \mathrm{~kg}$ generic/can, $585 \mathrm{~g} \mathrm{Be}$, thick SS discrete reflector

Figure A-11. Set-1 results, plot 10: reactivity effect of various parameters with $7.7 \mathrm{~cm}$ cylinder radius, generic filler, poly moderated. 


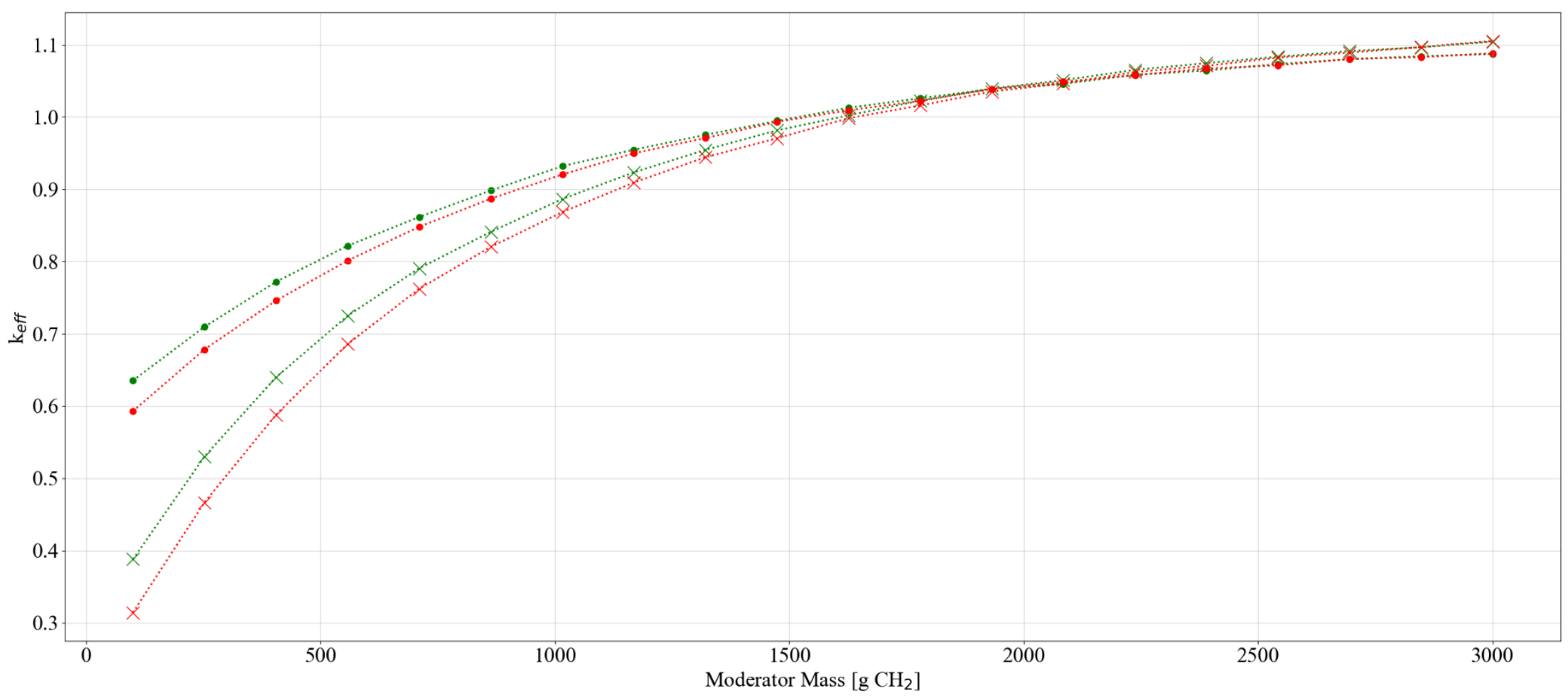

$\Varangle \cdots$ set-1, cylinder radius $=7.7 \mathrm{~cm}$, poly moderator, $5 \mathrm{~kg}$ graphite $/ \mathrm{can}$, no $\mathrm{Be}$, thick SS discrete reflector

•... set-1, cylinder radius $=7.7 \mathrm{~cm}$, poly moderator, $5 \mathrm{~kg}$ graphite $/$ can, no $\mathrm{Be}$, thick poly discrete reflector

…. set-1, cylinder radius $=7.7 \mathrm{~cm}$, poly moderator, $5 \mathrm{~kg}$ generic/can, no Be, thick SS discrete reflector

-... set-1, cylinder radius $=7.7 \mathrm{~cm}$, poly moderator, $5 \mathrm{~kg}$ generic $/ \mathrm{can}$, no $\mathrm{Be}$, thick poly discrete reflector

Figure A-12. Set-1 results, plot 11: comparison of graphite and generic filler with $7.7 \mathrm{~cm}$ cylinder radius, no Be, poly moderated, thick discrete reflector. 


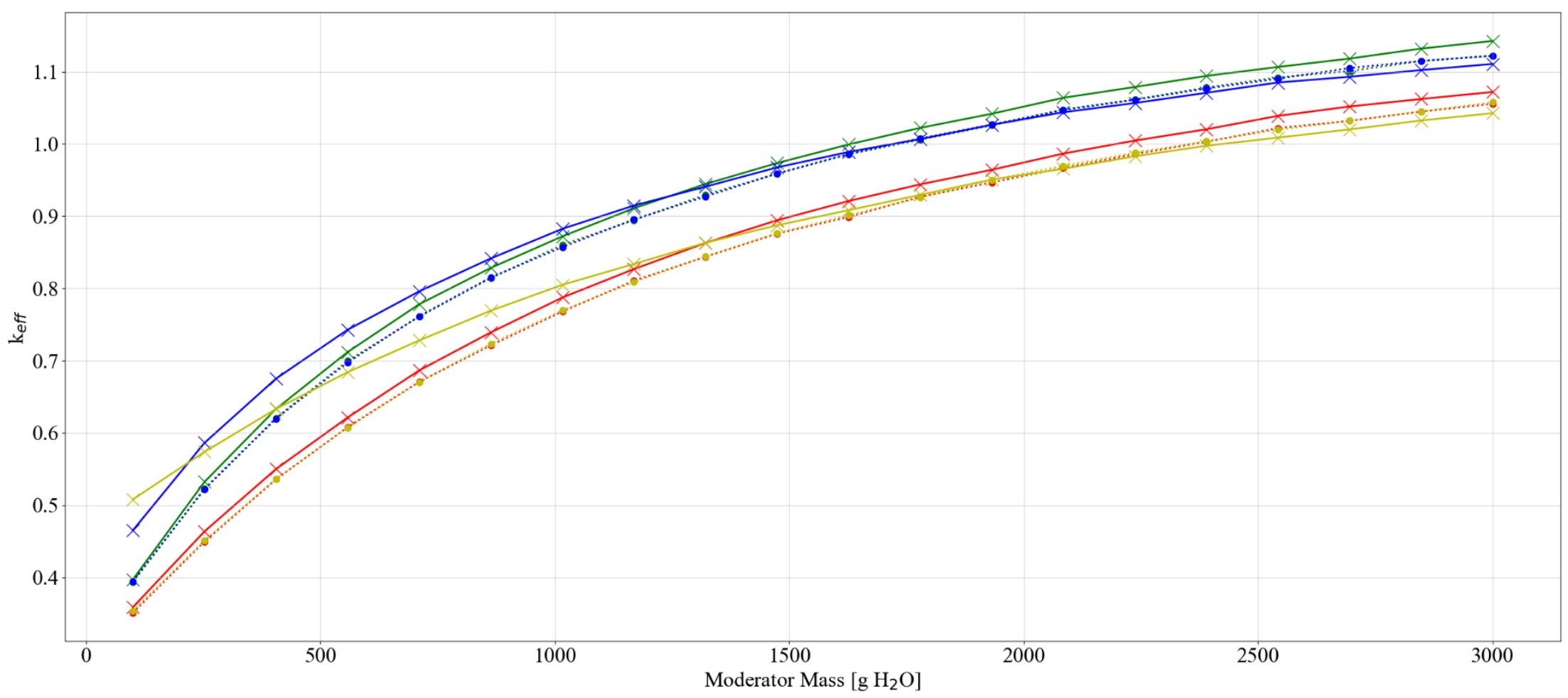

….. set-1, no filler, no Be, thin SS discrete reflector

…... set-1, no filler, no Be, thin poly discrete reflector

- set-1, no filler, no Be, thick SS discrete reflector

$\star$ set-1, no filler, no Be, thick poly discrete reflector

…. set-1, $5 \mathrm{~kg}$ graphite/can, $585 \mathrm{~g} \mathrm{Be}$, thin SS discrete reflector

-... set-1, $5 \mathrm{~kg}$ graphite/can, $585 \mathrm{~g} \mathrm{Be}$, thin poly discrete reflector set-1, $5 \mathrm{~kg}$ graphite/can, $585 \mathrm{~g} \mathrm{Be}$, thick SS discrete reflector

* set-1, $5 \mathrm{~kg}$ graphite/can, $585 \mathrm{~g}$ Be, thick poly discrete reflecto

Figure A-13. Set-1 results, plot 12: reactivity effect of various parameters with spherical waste form geometry, graphite filler, water moderated. 


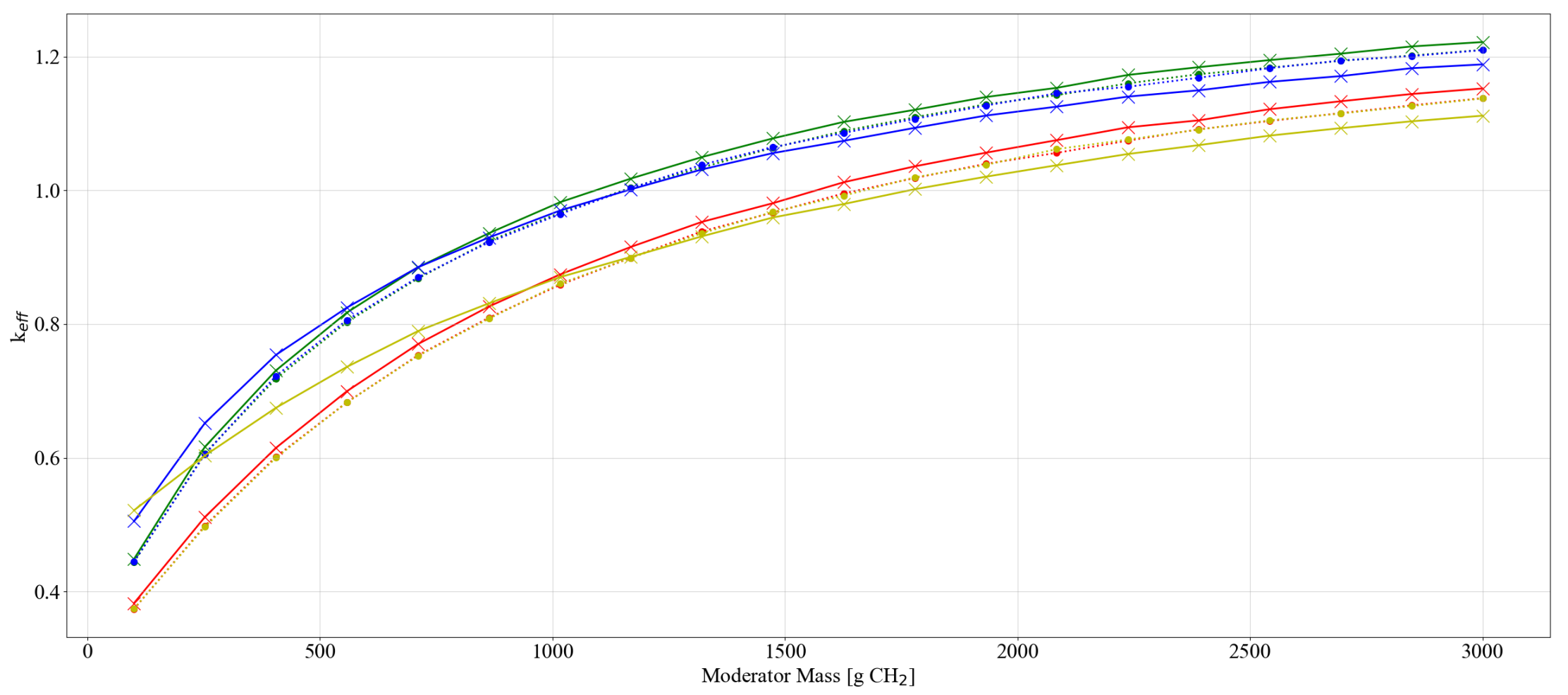

….. set-1, no filler, no Be, thin SS discrete reflector

…... set- 1 , no filler, no $\mathrm{Be}$, thin poly discrete reflector

- set-1, no filler, no Be, thick SS discrete reflector

* set-1, no filler, no Be, thick poly discrete reflector

…... set-1, $5 \mathrm{~kg}$ graphite/can, $585 \mathrm{~g} \mathrm{Be}$, thin SS discrete reflector

-... set-1, $5 \mathrm{~kg}$ graphite/can, $585 \mathrm{~g} \mathrm{Be}$, thin poly discrete reflector

set-1, $5 \mathrm{~kg}$ graphite/can, $585 \mathrm{~g}$ Be, thick SS discrete reflecto

set-1, $5 \mathrm{~kg}$ graphite/can, $585 \mathrm{~g} \mathrm{Be}$, thick poly discrete reflector

Figure A-14. Set-1 results, plot 13: reactivity effect of various parameters with spherical waste form geometry, graphite filler, poly moderated. 


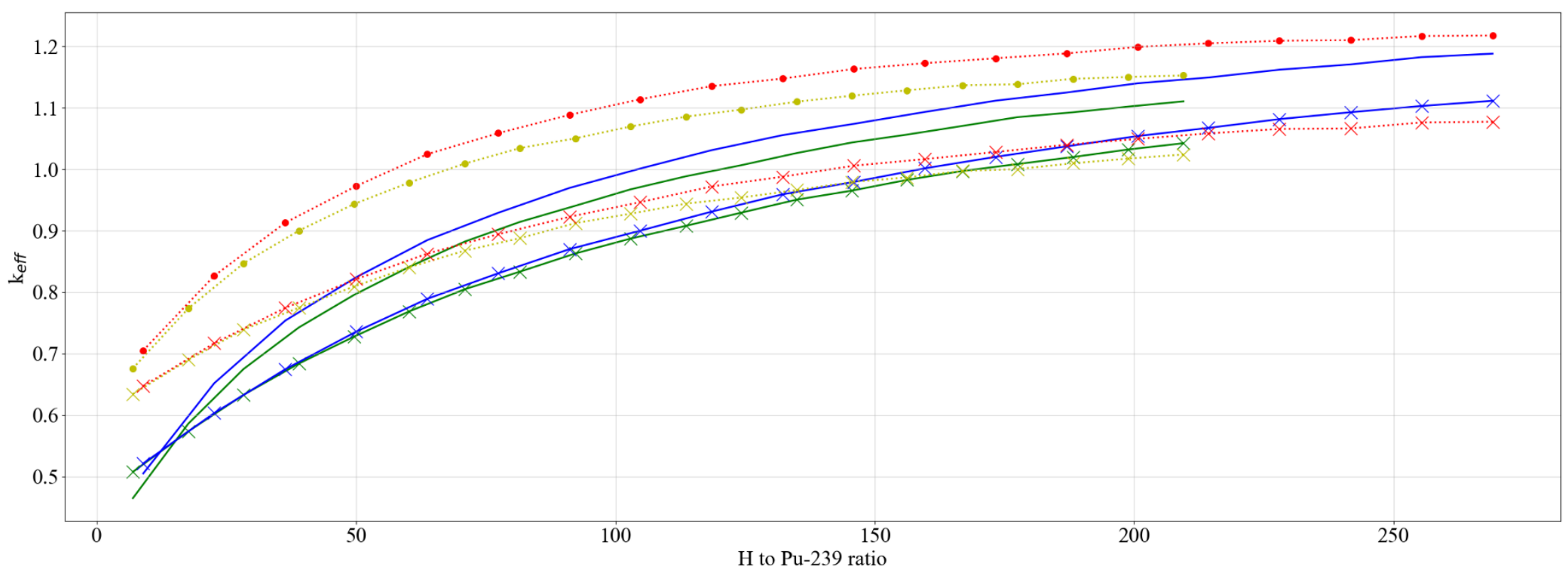

- set-1, sphere, no filler, no Be, thick poly discrete reflector, water moderator

$\leftarrow$ set-1, sphere, $5 \mathrm{~kg}$ graphite/can, $585 \mathrm{~g} \mathrm{Be}$, thick poly discrete reflector, water moderator

- set-1, sphere, no filler, no Be, thick poly discrete reflector, poly moderator

$*$ set-1, sphere, $5 \mathrm{~kg}$ graphite/can, $585 \mathrm{~g} \mathrm{Be}$, thick poly discrete reflector, poly moderator

$\cdots \cdot$ set-1, cylinder with radius $=7.7 \mathrm{~cm}$, no filler, no $\mathrm{Be}$, thick poly discrete reflector, water moderator

set-1, cylinder with radius $=7.7 \mathrm{~cm}, 5 \mathrm{~kg}$ graphite $/ \mathrm{can}, 585 \mathrm{~g}$ Be, thick poly discrete reflector, water moderator

$\cdots \cdot \cdots$ set-1, cylinder with radius $=7.7 \mathrm{~cm}$, no filler, no Be, thick poly discrete reflector, poly moderator

... ‘... set-1, cylinder with radius $=7.7 \mathrm{~cm}, 5 \mathrm{~kg}$ graphite $/ \mathrm{can}, 585 \mathrm{~g} \mathrm{Be}$, thick poly discrete reflector, poly moderator

Figure A-15. Set-1 results, plot 14: comparison of spherical and cylindrical geometries $(\mathbf{h} / \mathbf{x})$. 


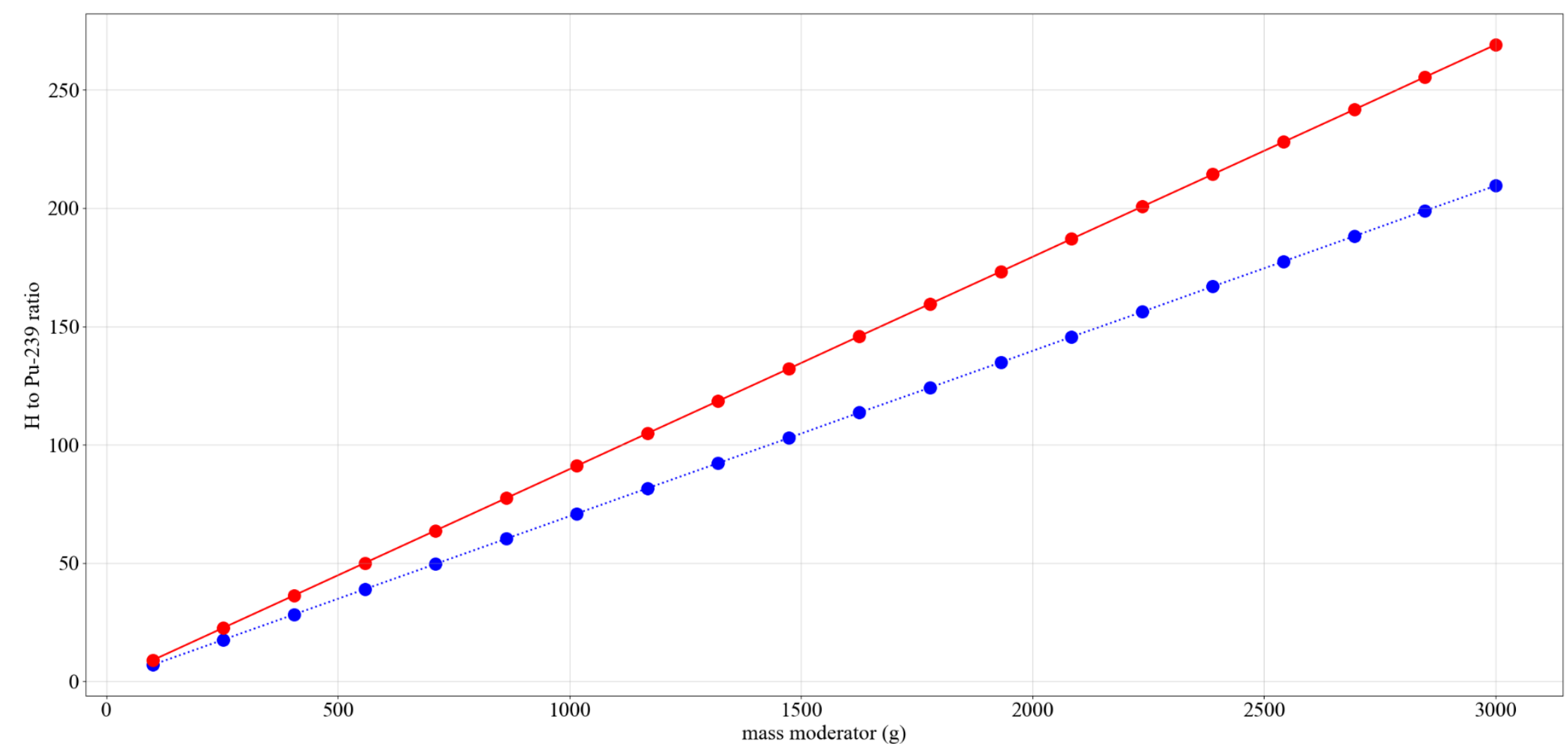

-.... water moderator

- - poly moderator

Figure A-16. Set-1 results, plot 15: comparison of water and poly $\mathbf{h} / \mathbf{x}$. 


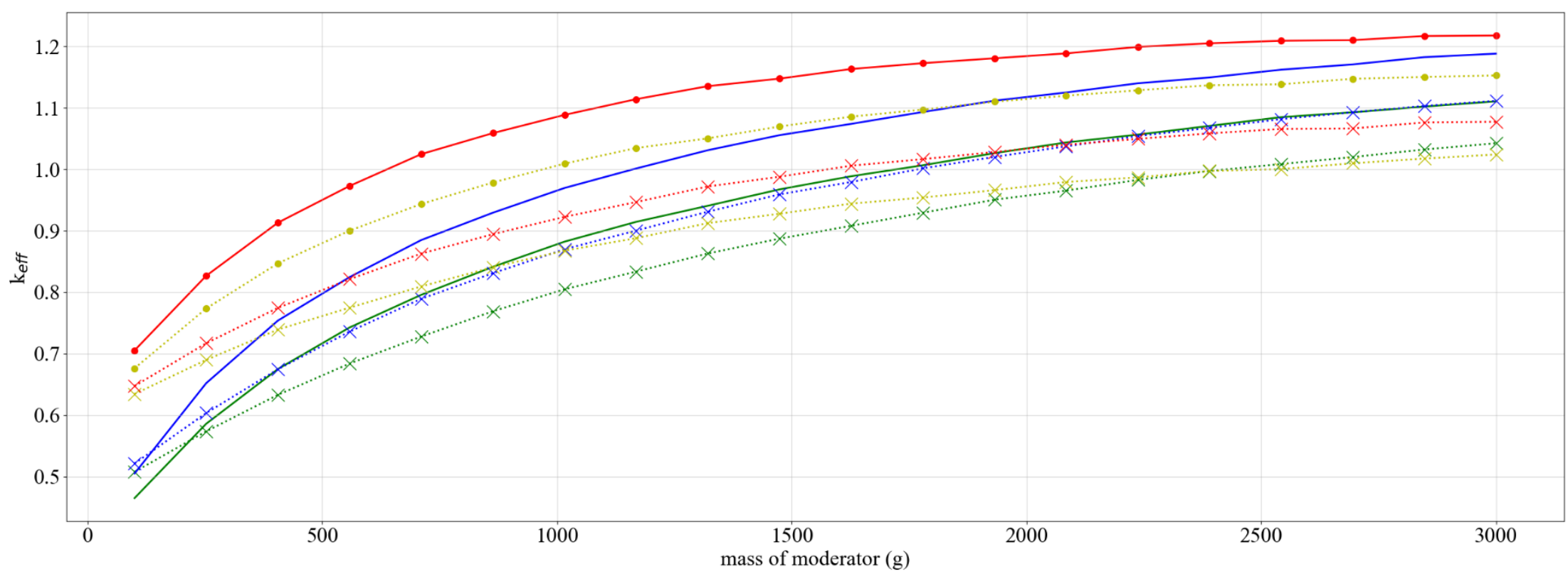

- set-1, sphere, no filler, no Be, thick poly discrete reflector, water moderator

.... $\ldots$ set-1, sphere, $5 \mathrm{~kg}$ graphite/can, $585 \mathrm{~g} \mathrm{Be}$, thick poly discrete reflector, water moderator

- set-1, sphere, no filler, no Be, thick poly discrete reflector, poly moderator

… $\ldots$ set-1, sphere, $5 \mathrm{~kg}$ graphite/can, $585 \mathrm{~g} \mathrm{Be}$, thick poly discrete reflector, poly moderator

$\cdots \cdot$ set-1, cylinder with radius $=7.7 \mathrm{~cm}$, no filler, no $\mathrm{Be}$, thick poly discrete reflector, water moderator

K... set-1, cylinder with radius $=7.7 \mathrm{~cm}, 5 \mathrm{~kg}$ graphite/can, $585 \mathrm{~g}$ Be, thick poly discrete reflector, water moderator

$\longrightarrow$ set- 1 , cylinder with radius $=7.7 \mathrm{~cm}$, no filler, no Be, thick poly discrete reflector, poly moderator

... ‘... set-1, cylinder with radius $=7.7 \mathrm{~cm}, 5 \mathrm{~kg}$ graphite/can, $585 \mathrm{~g} \mathrm{Be}$, thick poly discrete reflector, poly moderator

Figure A-17. Set-1 results, plot 16: comparison of spherical and cylindrical geometries (mod mass). 


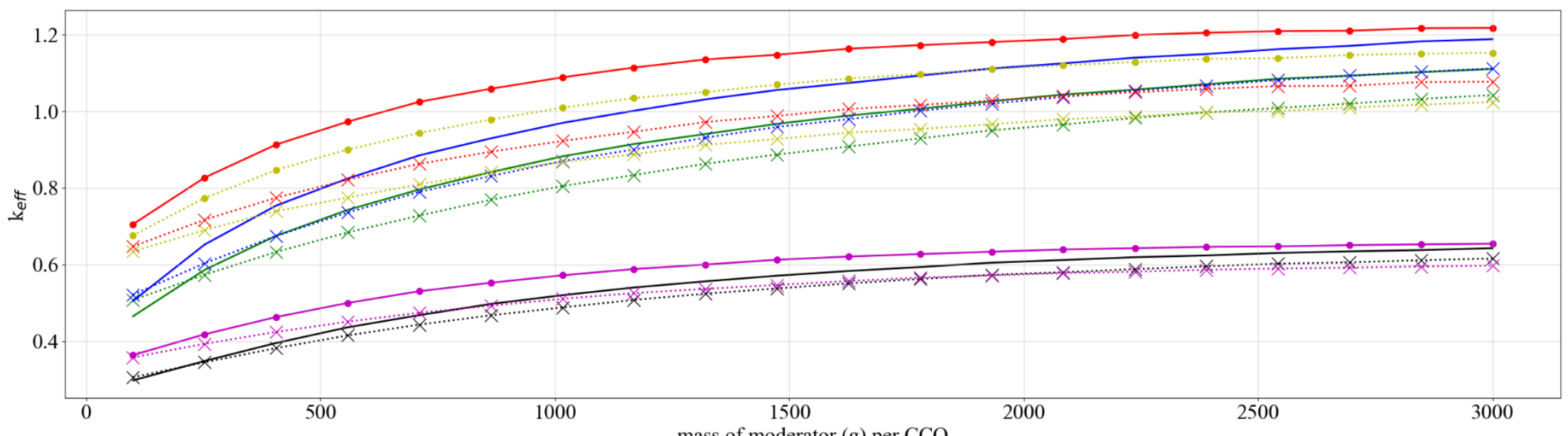

- set-1, sphere, no filler, no Be, thick poly discrete reflector, water moderator

........ set-1, sphere, $5 \mathrm{~kg}$ graphite/can, $585 \mathrm{~g} \mathrm{Be,} \mathrm{thick} \mathrm{poly} \mathrm{discrete} \mathrm{reflector,} \mathrm{water} \mathrm{moderator}$

- set-1, sphere, no filler, no Be, thick poly discrete reflector, poly moderator

....... set-1, sphere, $5 \mathrm{~kg}$ graphite/can, $585 \mathrm{~g} \mathrm{Be}$, thick poly discrete reflector, poly moderator

$\cdots \cdot$ set-1, cylinder with radius $=7.7 \mathrm{~cm}$, no filler, no Be, thick poly discrete reflector, water moderator

(x.... set-1, cylinder with radius $=7.7 \mathrm{~cm}, 5 \mathrm{~kg}$ graphite $/ \mathrm{can}, 585 \mathrm{~g}$ Be, thick poly discrete reflector, water moderator

$\longrightarrow$ set-1, cylinder with radius $=7.7 \mathrm{~cm}$, no filler, no Be, thick poly discrete reflector, poly moderator

…… set-1, cylinder with radius $=7.7 \mathrm{~cm}, 5 \mathrm{~kg}$ graphite $/$ can, $585 \mathrm{~g} \mathrm{Be}$, thick poly discrete reflector, poly moderator

— set-1, sphere, $50 \mathrm{~g} \mathrm{~B} 4 \mathrm{C}$, no filler, no Be, thick poly discrete reflector, poly moderator

........ set-1, sphere, $50 \mathrm{~g} \mathrm{~B} 4 \mathrm{C}, 5 \mathrm{~kg}$ graphite/can, $585 \mathrm{~g} \mathrm{Be}$, thick poly discrete reflector, poly moderator

$\longrightarrow$ set-1, cylinder with radius $=7.7 \mathrm{~cm}, 50 \mathrm{~g} \mathrm{~B} 4 \mathrm{C}$, no filler, no Be, thick poly discrete reflector, poly moderator

$\ldots \times \cdots$ set-1, cylinder with radius $=7.7 \mathrm{~cm}, 50 \mathrm{~g} \mathrm{~B} 4 \mathrm{C}, 5 \mathrm{~kg}$ graphite $/ \mathrm{can}, 585 \mathrm{~g} \mathrm{Be}$, thick poly discrete reflector, poly moderator

Figure A-18. Set-1 results, plot 17: comparison of $50 \mathrm{~g} \mathrm{~B} \mathrm{~B}_{4} \mathrm{C}$ vs. no $\mathrm{B}_{4} \mathrm{C}$ for spherical and cylindrical geometries (mod mass). 
This page is intentionally blank 
APPENDIX B. SET-1A: RESULTS OF THE THREE-HIGH UNIFORM ARRAY WITH ALTERNATIVE SPACING CALCULATIONS 
This page is intentionally blank 


\section{APPENDIX B. SET-1A: RESULTS OF THE THREE-HIGH UNIFORM ARRAY WITH ALTERNATIVE SPACING CALCULATIONS}

The analysis methodology for the uniform arrays is discussed in detail in Section 6.2 of the main report.

This appendix serves as a repository of those results for the set-1a calculations.

The complete results for all SAMPLER sweeps are provided in ADDENDUM 1.

The analysis model use for the calculations in this appendix is shown in Figure B-1 below.

The SAMPLER case sweeps presented in this appendix are summarized in Table B-1 below.

\section{LIST OF FIGURES}

Figure B-1. Diagram of the uniform array three-high model. This is not an actual analysis model: it is a diagram of a model used by SAMPLER to generate the analysis models.

Figure B-2. Set-1a results, plot 1: reactivity effect of cylinder radius, pipe steel, no filler, no Be, water moderated.

Figure B-3. Set-1a results, plot 2: reactivity effect of cylinder radius, pipe steel, no filler, no Be, poly moderated.

Figure B-4. Set-1a results. plot 3: reactivity effect of cylinder radius, pipe poly, no filler, no Be, water moderated.

Figure B-5. Set-1a results, plot 4: reactivity effect of cylinder radius, pipe poly, no filler, no Be, poly moderated.

Figure B-6. Set-1a results, plot 5: reactivity effect of cylinder radius, pipe steel, $5 \mathrm{~kg}$ graphite/can, no Be, water moderated.

Figure B-7. Set-1a results, plot 6: reactivity effect of cylinder radius, pipe steel, $5 \mathrm{~kg}$ graphite/can, no Be, poly moderated.

Figure B-8. Set-1a results, plot 7: reactivity effect of cylinder radius, pipe poly, $5 \mathrm{~kg}$ graphite/can, no Be, water moderated.

Figure B-9. Set-1a results, plot 8: reactivity effect of cylinder radius, pipe poly, $5 \mathrm{~kg}$ graphite/can, no Be, poly moderated.

Figure B-10. Set-1a results, plot 9: reactivity effect of various parameters with $7.7 \mathrm{~cm}$ cylinder radius, graphite filler, poly moderated.

Figure B-11. Set-1a results, plot 10: reactivity effect of various parameters with $7.7 \mathrm{~cm}$ cylinder radius, generic filler, poly moderated.

Figure B-12. Set-1a results, plot 11: comparison of graphite and generic filler with $7.7 \mathrm{~cm}$ cylinder radius, no Be, poly moderated, thick discrete reflector.

Figure B-13. Set-1a results, plot 12: reactivity effect of various parameters with spherical waste form geometry, graphite filler, water moderated.

Figure B-14. Set-1a results, plot 13: reactivity effect of various parameters with spherical waste form geometry, graphite filler, poly moderated.

Figure B-15. Set-1a results, plot 14: comparison of spherical and cylindrical geometries $(\mathrm{h} / \mathrm{x}) \ldots \ldots \ldots \ldots . \mathrm{B}-20$

Figure B-16. Set-1a results, plot 15: comparison of water and poly $\mathrm{h} / \mathrm{x}$.

Figure B-17. Set-1a results, plot 16: comparison of spherical and cylindrical geometries (mod mass).

Figure B-18. Set-1a results, plot 17: comparison of $50 \mathrm{~g} \mathrm{~B}_{4} \mathrm{C}$ vs. no $\mathrm{B}_{4} \mathrm{C}$ for spherical and cylindrical geometries (mod mass). 


\section{LIST OF TABLES}

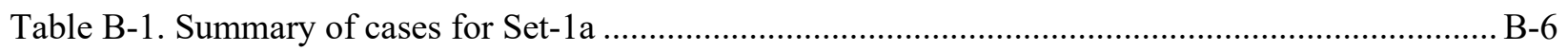



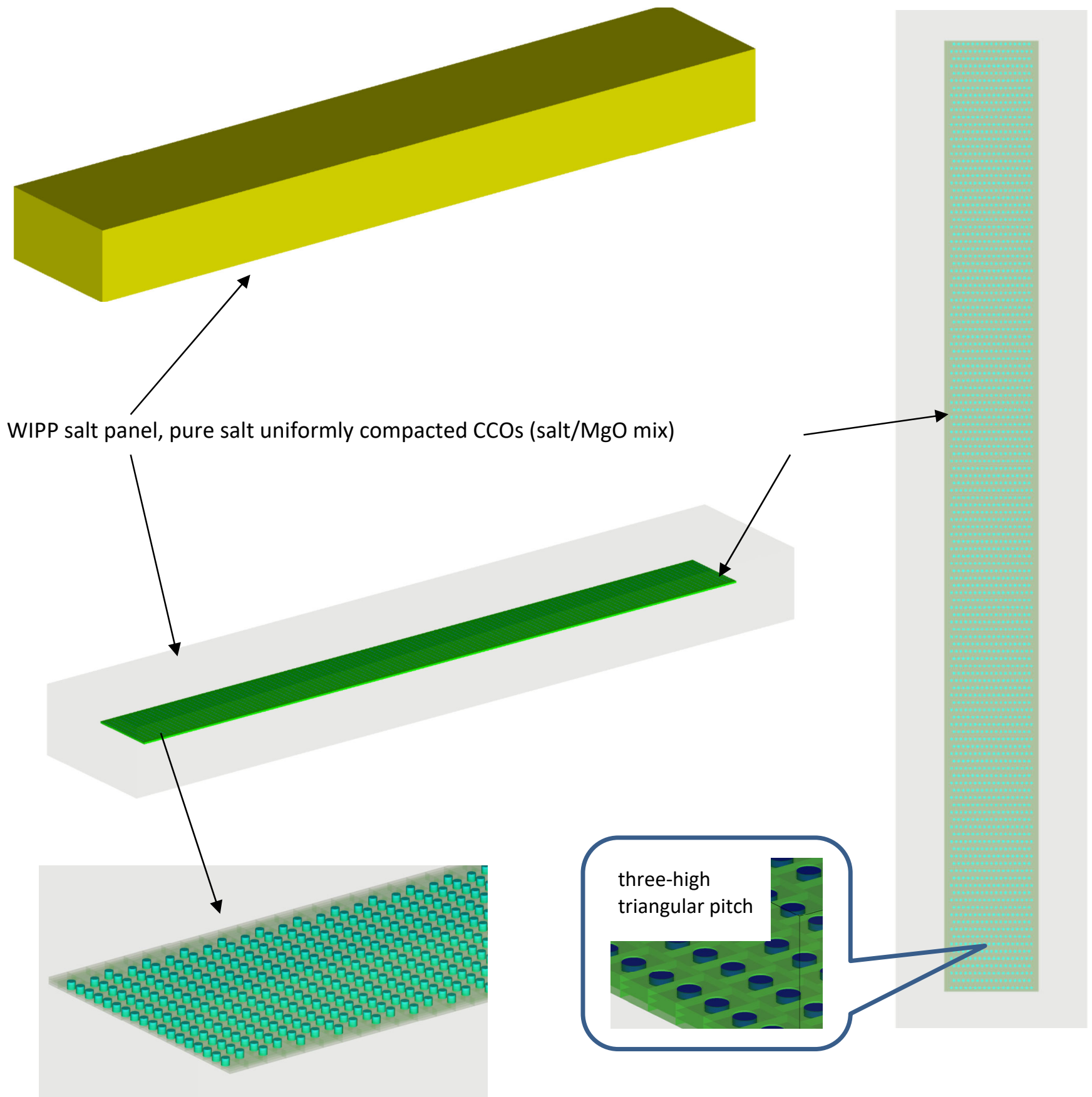

Figure B-1. Diagram of the uniform array three-high model. This is not an actual analysis model: it is a diagram of a model used by SAMPLER to generate the analysis models. 
Table B-1. Summary of cases for Set-1a

\begin{tabular}{|c|c|c|c|c|c|c|c|c|}
\hline Case & Model type & Waste form shape & $\begin{array}{l}\text { Waste form } \\
\text { moderator }\end{array}$ & $\begin{array}{c}\text { Filler material } \\
(0,2,000,4,000 \\
\text { g) }\end{array}$ & Metal in filler & $\begin{array}{c}\text { Discrete reflector } \\
\text { (thin } 0.001 \text { and } \\
\text { thick } 0.7112 \mathrm{~cm} \text { ) }\end{array}$ & $\begin{array}{l}\mathrm{Be} \\
(\mathrm{g})\end{array}$ & Subcase \\
\hline \multirow{18}{*}{ Set-1a } & \multirow{18}{*}{$\begin{array}{l}\text { Uniform array stacked three } \\
\text { high with CCO pitch } \\
\text { reduction } \mathrm{x}=25 \%, \mathrm{y}= \\
10 \%, \mathrm{z}=\text { no space }\end{array}$} & \multirow{8}{*}{$\begin{array}{l}\text { Cylinder (radius range } \\
4.8,6,7.7 \text { and height } \\
\text { defined by total } \\
\text { volume of mass) }\end{array}$} & water & $\mathrm{c} 12$ & \multirow{18}{*}{$\begin{array}{c}\text { SS from can } \\
(0,500,1,000 \mathrm{~g})\end{array}$} & steel & \multirow{18}{*}{$\begin{array}{l}0 \text { to } \\
585\end{array}$} & set-1a-1 \\
\hline & & & poly & $\mathrm{c} 12$ & & steel & & set-1a-2 \\
\hline & & & water & $\mathrm{c} 12$ & & poly & & set-1a-3 \\
\hline & & & poly & $\mathrm{c} 12$ & & poly & & set-1a-4 \\
\hline & & & water & generic & & steel & & set-1a-5 \\
\hline & & & poly & generic & & steel & & set-1a-6 \\
\hline & & & water & generic & & poly & & set-1a-7 \\
\hline & & & poly & generic & & poly & & set-1a-8 \\
\hline & & \multirow{10}{*}{$\begin{array}{c}\text { Sphere (radius defined } \\
\text { by total volume of } \\
\text { mass) }\end{array}$} & water & $\mathrm{c} 12$ & & steel & & set-1a-9 \\
\hline & & & poly & $\mathrm{c} 12$ & & steel & & set-1a-10 \\
\hline & & & water & $\mathrm{c} 12$ & & poly & & set-1a-11 \\
\hline & & & poly & $\mathrm{c} 12$ & & poly & & set-1a-12 \\
\hline & & & water & generic & & steel & & set-1a-13 \\
\hline & & & poly & generic & & steel & & set-1a-14 \\
\hline & & & water & generic & & poly & & set-1a-15 \\
\hline & & & poly & generic & & poly & & set-1a-16 \\
\hline & & & $\begin{array}{c}\text { poly }+50 \mathrm{~g} \\
\mathrm{~B}_{4} \mathrm{C}(\mathrm{cyl})\end{array}$ & $\mathrm{c} 12$ & & poly $(0.7112)$ & & set-1a-17 \\
\hline & & & $\begin{array}{c}\text { poly }+50 \mathrm{~g} \\
\mathrm{~B}_{4} \mathrm{C}(\mathrm{sph})\end{array}$ & $\mathrm{c} 12$ & & poly $(0.7112)$ & & set- $1 \mathrm{a}-18$ \\
\hline
\end{tabular}




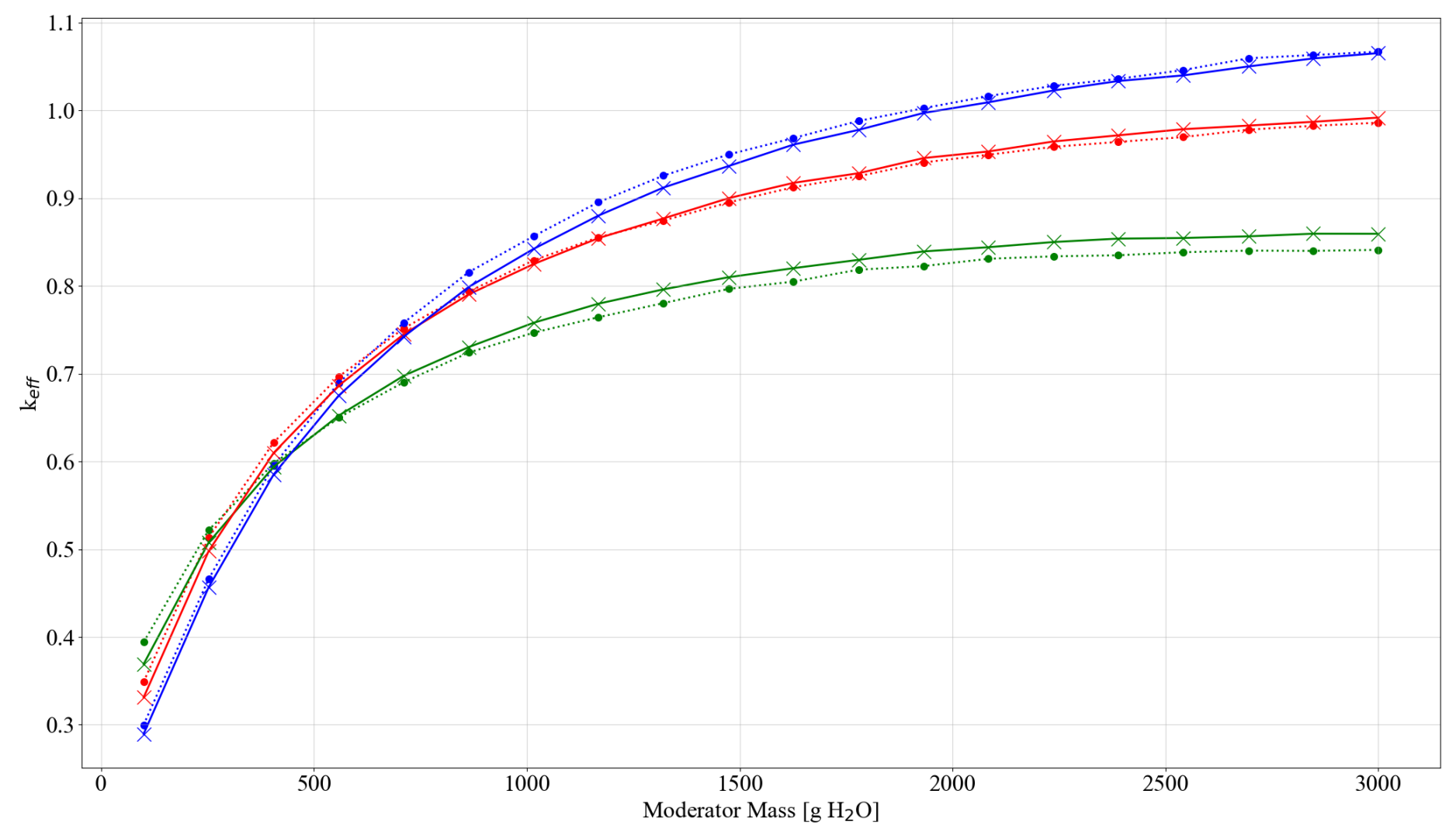

set-1a, cyl radius $=4.8 \mathrm{~cm}$, thin discrete reflecto set-1a, cyl radius $=6.25 \mathrm{~cm}$, thin discrete reflector set- $1 \mathrm{a}$, cyl radius $=7.7 \mathrm{~cm}$, thin discrete reflector set- $1 \mathrm{a}$, cyl radius $=4.8 \mathrm{~cm}$, thick discrete reflector set- 1 a, cyl radius $=6.25 \mathrm{~cm}$, thick discrete reflector set- $1 \mathrm{a}$, cyl radius $=7.7 \mathrm{~cm}$, thick discrete reflector

Figure B-2. Set-1a results, plot 1: reactivity effect of cylinder radius, pipe steel, no filler, no Be, water moderated. 


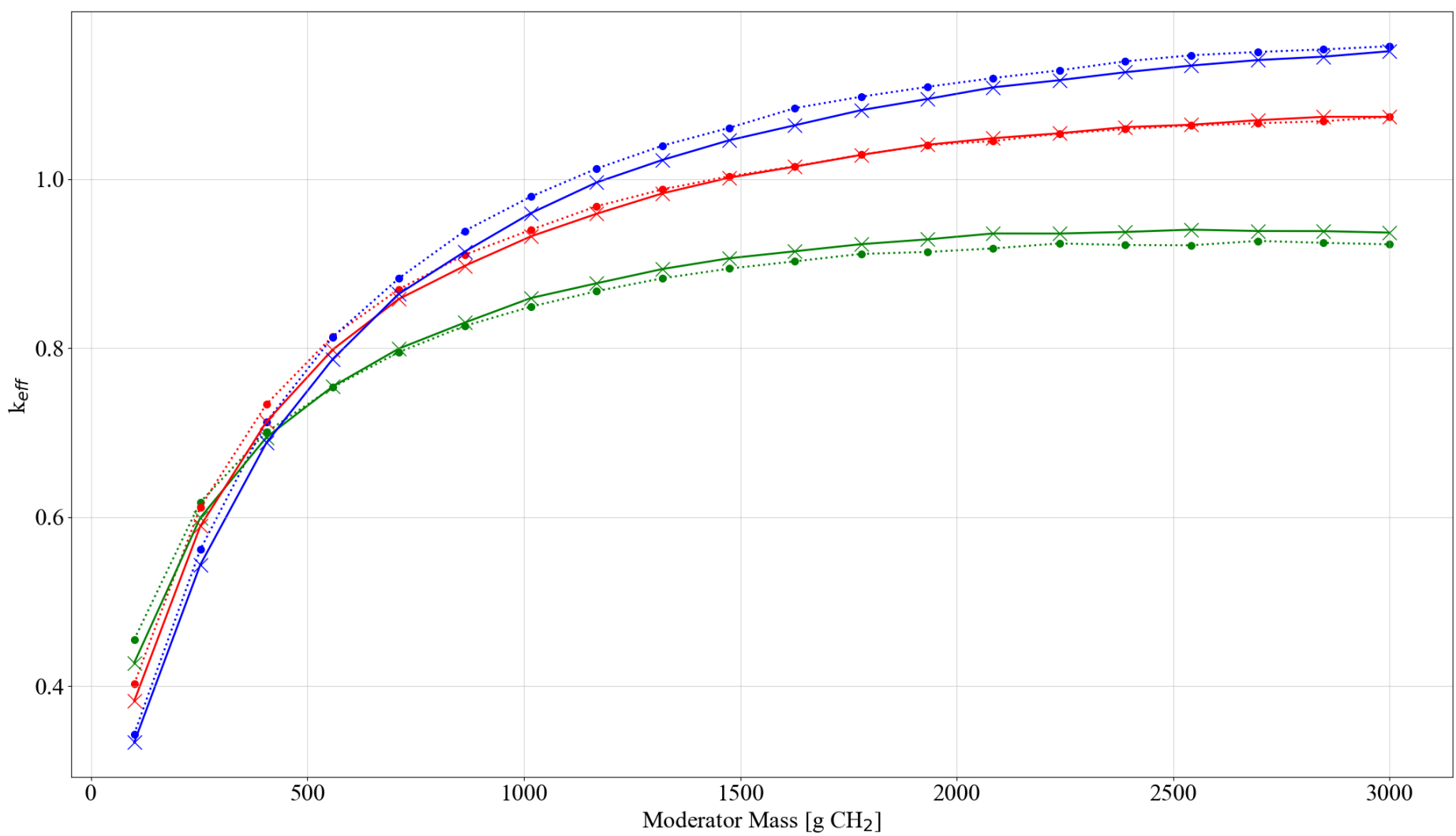

set- $1 \mathrm{a}$, cyl radius $=4.8 \mathrm{~cm}$, thin discrete reflecto set- 1 a, cyl radius $=6.25 \mathrm{~cm}$, thin discrete reflector
-... set-1a, cyl radius $=7.7 \mathrm{~cm}$, thin discrete reflector set- 1 , cyl radius $=4.8 \mathrm{~cm}$, thick discrete reflector set- 1 a, cyl radius $=6.25 \mathrm{~cm}$, thick discrete reflector set- 1 a, cyl radius $=7.7 \mathrm{~cm}$, thick discrete reflector

Figure B-3. Set-1a results, plot 2: reactivity effect of cylinder radius, pipe steel, no filler, no Be, poly moderated. 


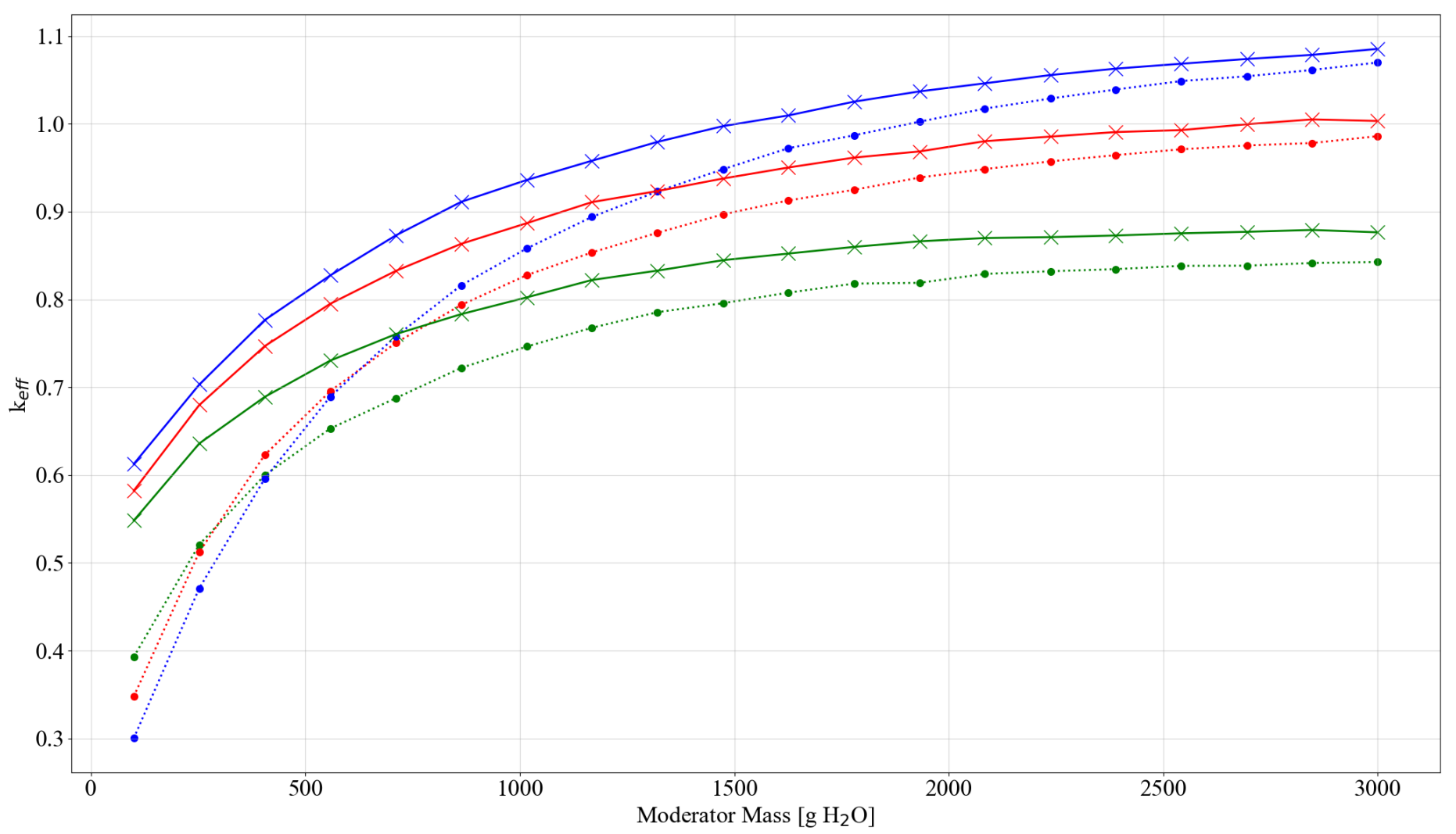

set-1a, cyl radius $=4.8 \mathrm{~cm}$, thin discrete reflecto set- 1 a, cyl radius $=6.25 \mathrm{~cm}$, thin discrete reflector
-... set-1a, cyl radius $=7.7 \mathrm{~cm}$, thin discrete reflector set- 1 , cyl radius $=4.8 \mathrm{~cm}$, thick discrete reflector $\star$ set- 1 a, cyl radius $=6.25 \mathrm{~cm}$, thick discrete reflector $\star$ set-1a, cyl radius $=7.7 \mathrm{~cm}$, thick discrete reflector

Figure B-4. Set-1a results. plot 3: reactivity effect of cylinder radius, pipe poly, no filler, no Be, water moderated. 


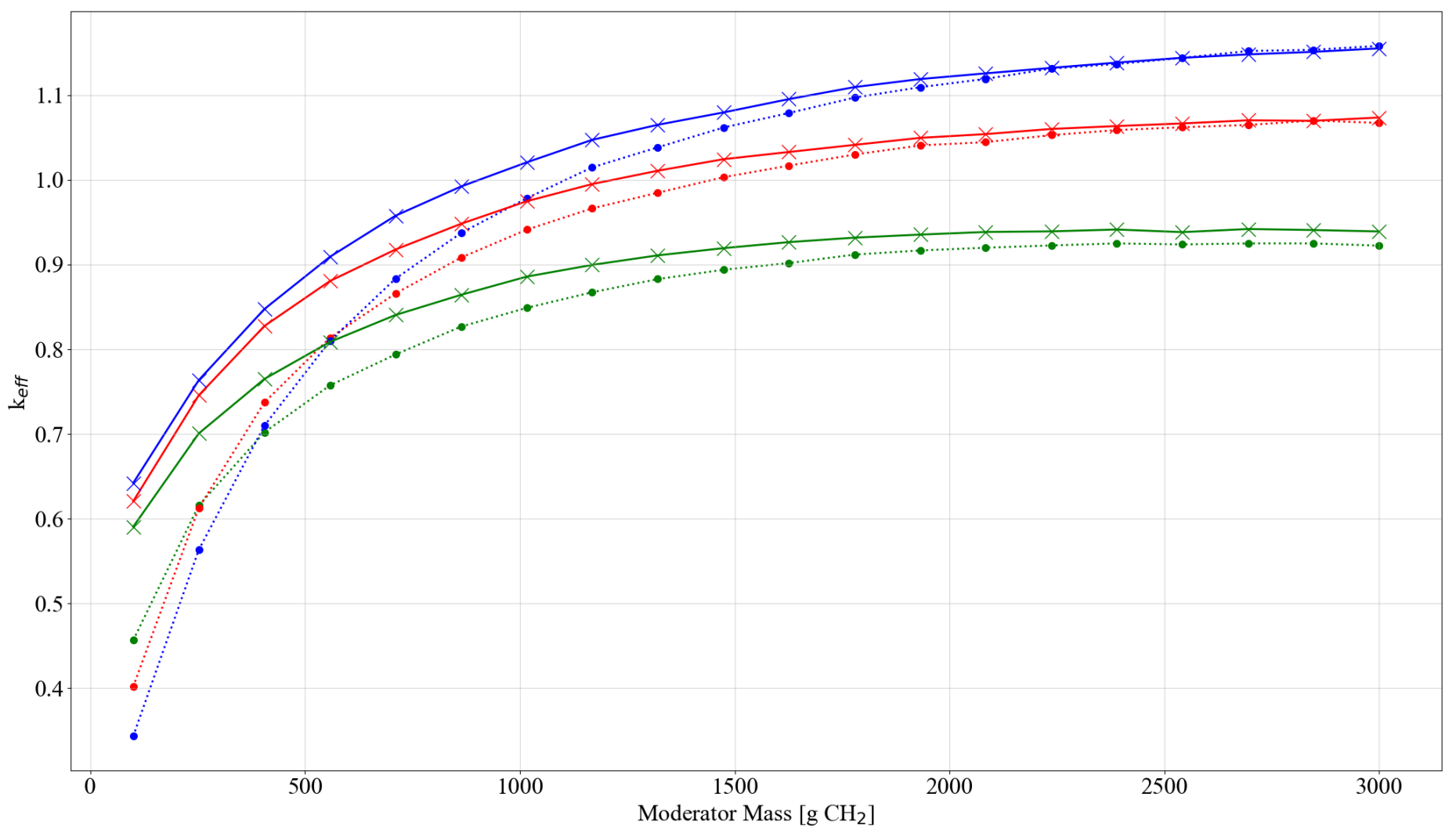

set-1a, cyl radius $=4.8 \mathrm{~cm}$, thin discrete reflecto set- 1 a, cyl radius $=6.25 \mathrm{~cm}$, thin discrete reflector set-1a, cyl radius $=7.7 \mathrm{~cm}$, thin discrete reflector set- 1 , cyl radius $=4.8 \mathrm{~cm}$, thick discrete reflector set- $1 \mathrm{a}$, cyl radius $=6.25 \mathrm{~cm}$, thick discrete reflector set $-1 \mathrm{a}$, cyl radius $=7.7 \mathrm{~cm}$, thick discrete reflector

Figure B-5. Set-1a results, plot 4: reactivity effect of cylinder radius, pipe poly, no filler, no Be, poly moderated. 


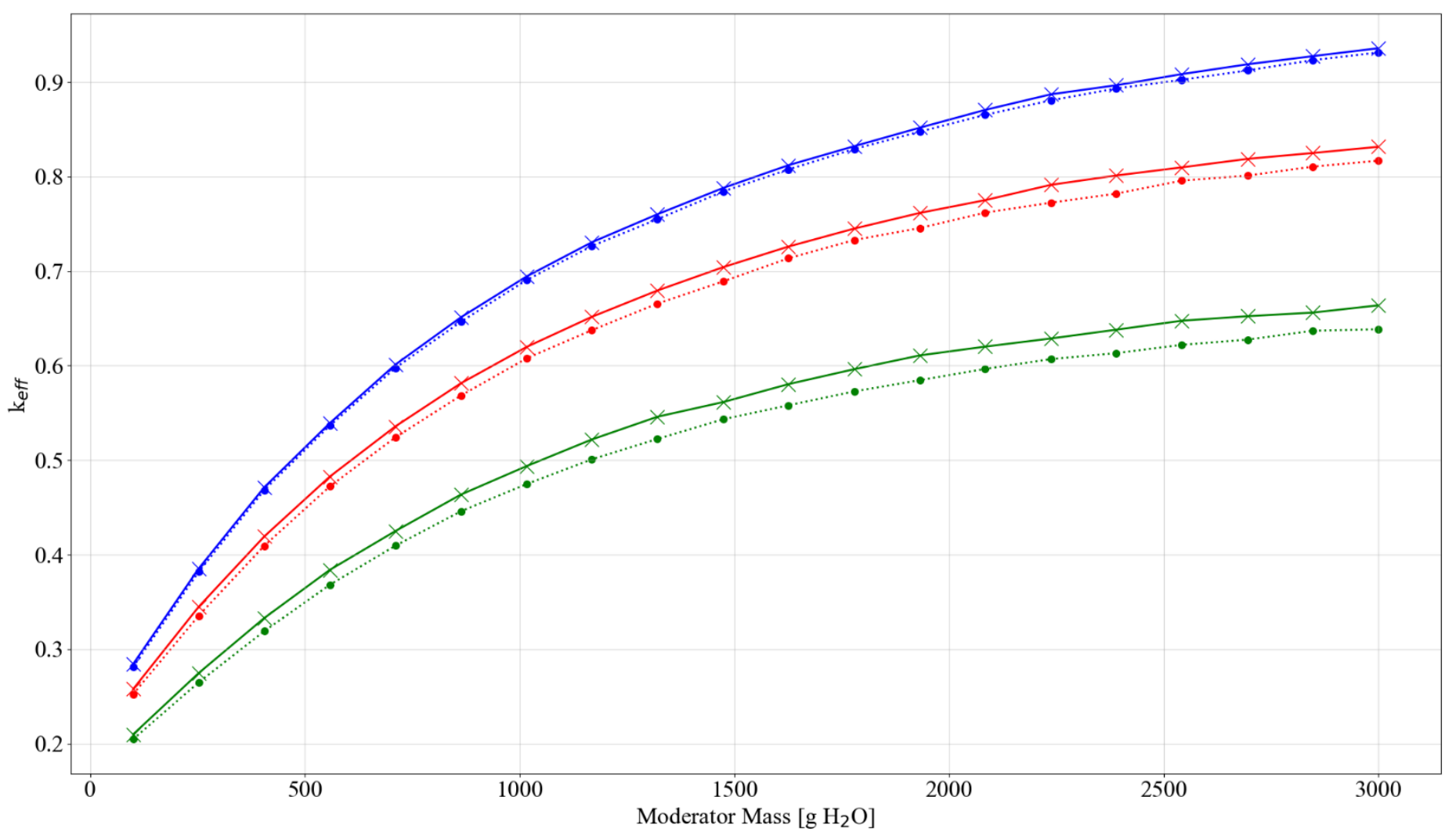

set- $1 \mathrm{a}$, cyl radius $=4.8 \mathrm{~cm}$, thin discrete reflector

$\cdots$ set-1a, cyl radius $=7.7 \mathrm{~cm}$, thin discrete reflector

set-1a, cyl radius $=6.25 \mathrm{~cm}$, thick discrete reflecto set- $1 \mathrm{a}$, cyl radius $=6.25 \mathrm{~cm}$, thin discrete reflector set- $1 \mathrm{a}$, cyl radius $=4.8 \mathrm{~cm}$, thick discrete reflector set- 1 a, cyl radius $=7.7 \mathrm{~cm}$, thick discrete reflector

Figure B-6. Set-1a results, plot 5: reactivity effect of cylinder radius, pipe steel, $5 \mathrm{~kg}$ graphite/can, no Be, water moderated. 


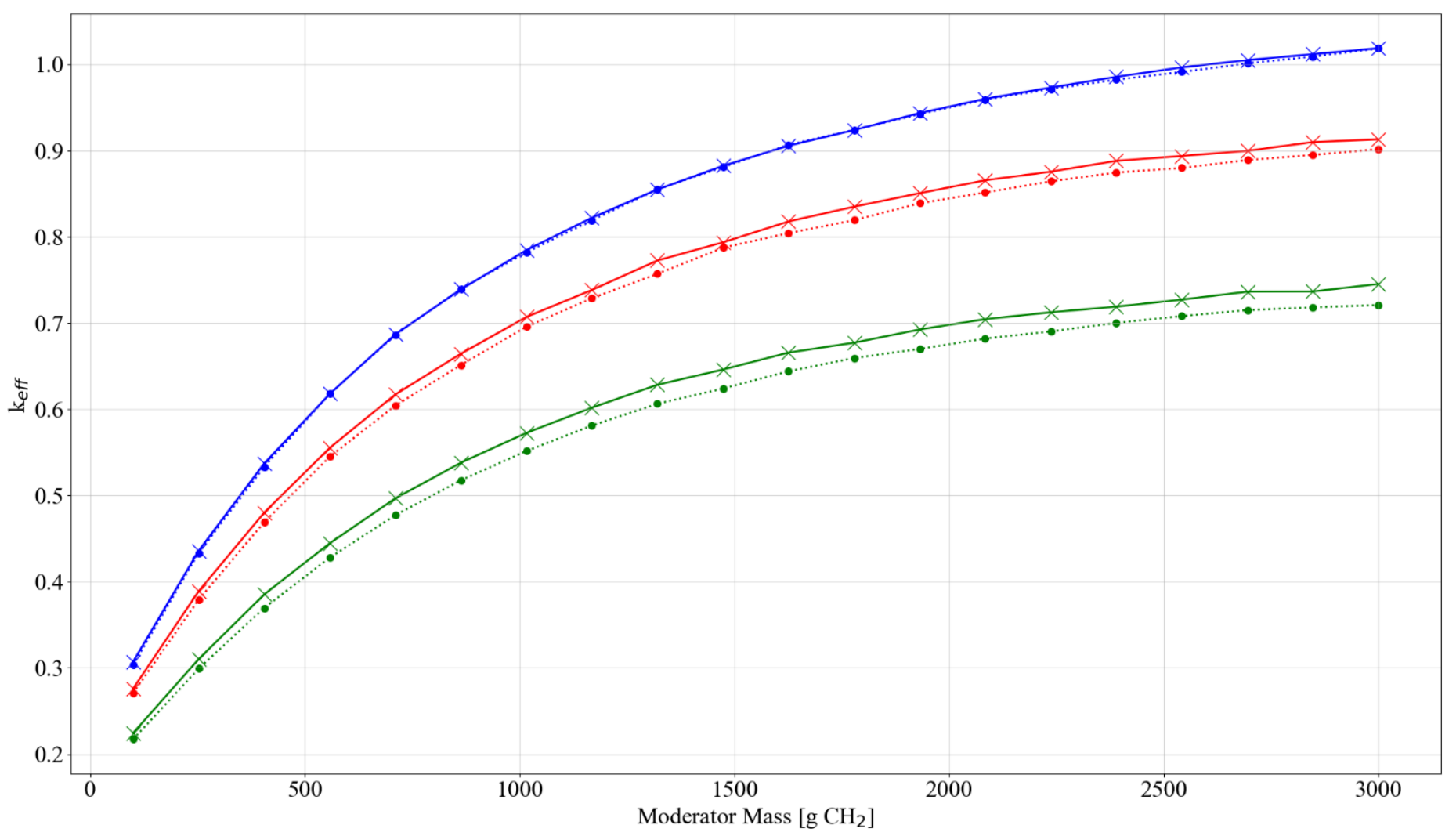

set- $1 \mathrm{a}$, cyl radius $=4.8 \mathrm{~cm}$, thin discrete reflector

$\cdots$ set-1a, cyl radius $=7.7 \mathrm{~cm}$, thin discrete reflector

set-1a, cyl radius $=6.25 \mathrm{~cm}$, thick discrete reflecto set- $1 \mathrm{a}$, cyl radius $=6.25 \mathrm{~cm}$, thin discrete reflector set- $1 \mathrm{a}$, cyl radius $=4.8 \mathrm{~cm}$, thick discrete reflector set- 1 a, cyl radius $=7.7 \mathrm{~cm}$, thick discrete reflector

Figure B-7. Set-1a results, plot 6: reactivity effect of cylinder radius, pipe steel, $5 \mathrm{~kg}$ graphite/can, no Be, poly moderated. 


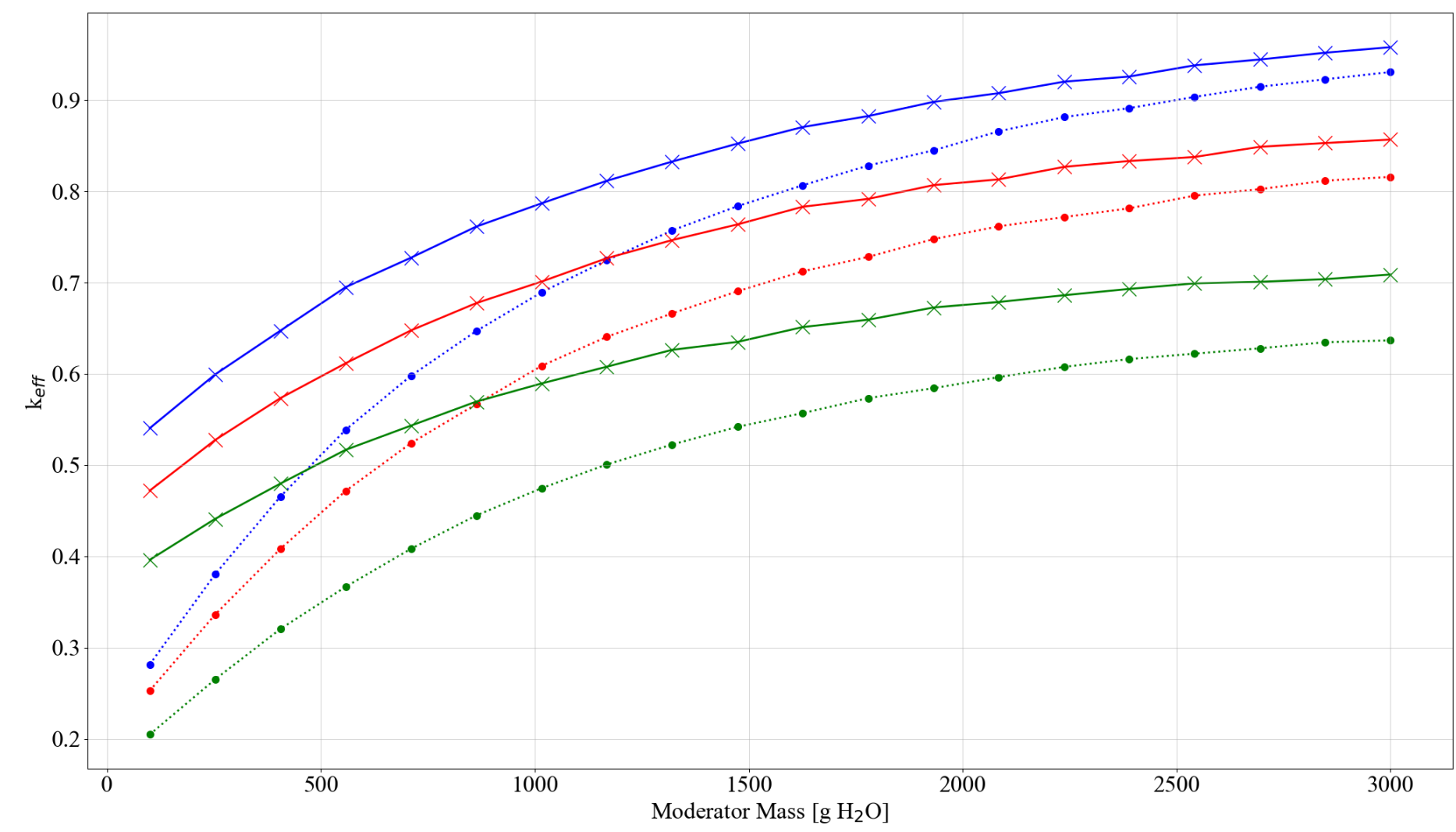

•... set-1a, cyl radius $=4.8 \mathrm{~cm}$, thin discrete reflector

$\cdots \quad$ set-1a, cyl radius $=7.7 \mathrm{~cm}$, thin discrete reflector

$\leftarrow$ set- 1 a, cyl radius $=6.25 \mathrm{~cm}$, thick discrete reflector set-1a, cyl radius $=6.25 \mathrm{~cm}$, thin discrete reflector

Figure B-8. Set-1a results, plot 7: reactivity effect of cylinder radius, pipe poly, $5 \mathrm{~kg}$ graphite/can, no Be, water moderated. 


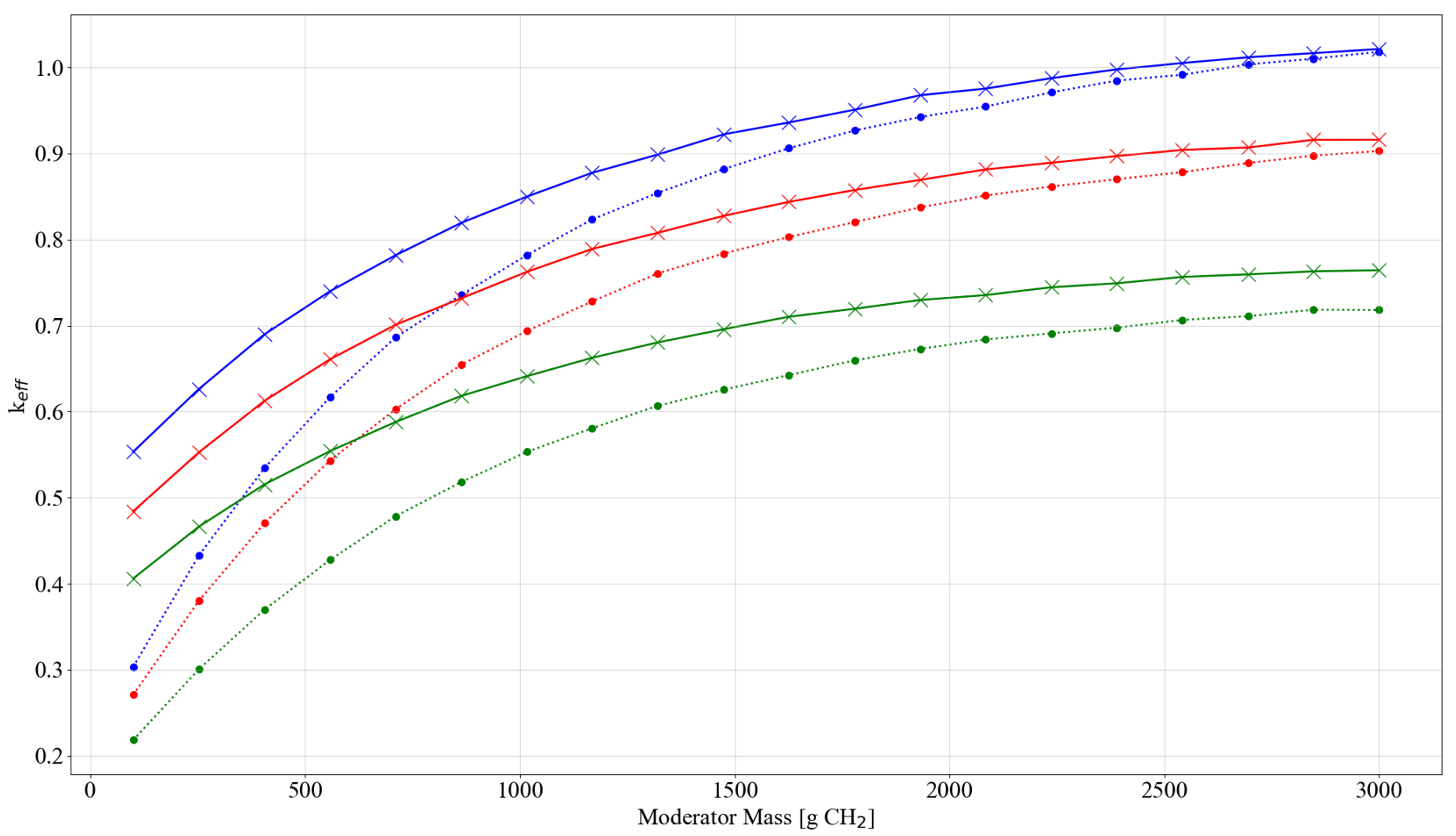

set- $1 \mathrm{a}$, cyl radius $=4.8 \mathrm{~cm}$, thin discrete reflecto set- $1 \mathrm{a}$, cyl radius $=6.25 \mathrm{~cm}$, thin discrete reflector $\cdots \cdots$ set-1a, cyl radius $=7.7 \mathrm{~cm}$, thin discrete reflector set- 1 a, cyl radius $=4.8 \mathrm{~cm}$, thick discrete reflector set- $1 \mathrm{a}$, cyl radius $=6.25 \mathrm{~cm}$, thick discrete reflector set- 1 a, cyl radius $=7.7 \mathrm{~cm}$, thick discrete reflector

Figure B-9. Set-1a results, plot 8: reactivity effect of cylinder radius, pipe poly, $5 \mathrm{~kg}$ graphite/can, no Be, poly moderated. 


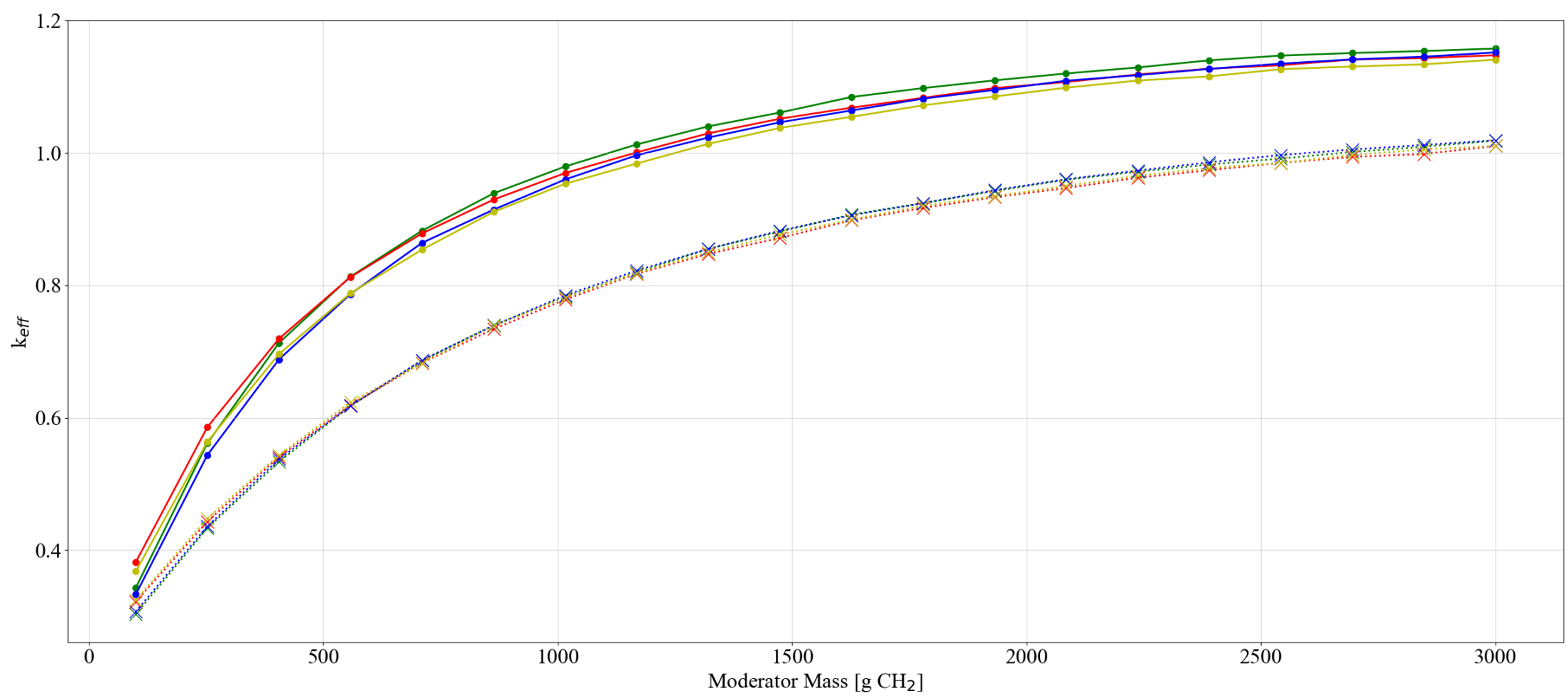

$\longrightarrow$ set-1a, no filler, no Be, thin SS discrete reflector

......... set-1a, $5 \mathrm{~kg}$ graphite/can, no Be, thin SS discrete reflector

$\longrightarrow$ set-1a, no filler, $585 \mathrm{~g} \mathrm{Be}$, thin SS discrete reflector

- - set-1a, no filler, no Be, thick SS discrete reflector

…… set-1a, $5 \mathrm{~kg}$ graphite/can, no Be, thick SS discrete reflector

…… set-1a, $5 \mathrm{~kg}$ graphite/can, $585 \mathrm{~g} \mathrm{Be}$, thin SS discrete reflector

$\longrightarrow$ set-1a, no filler, $585 \mathrm{~g} \mathrm{Be}$, thick SS discrete reflector

s....... 1 set- $5 \mathrm{~kg}$ graphite/can, $585 \mathrm{~g} \mathrm{Be}$, thick SS discrete reflector

Figure B-10. Set-1a results, plot 9: reactivity effect of various parameters

with $7.7 \mathrm{~cm}$ cylinder radius, graphite filler, poly moderated. 


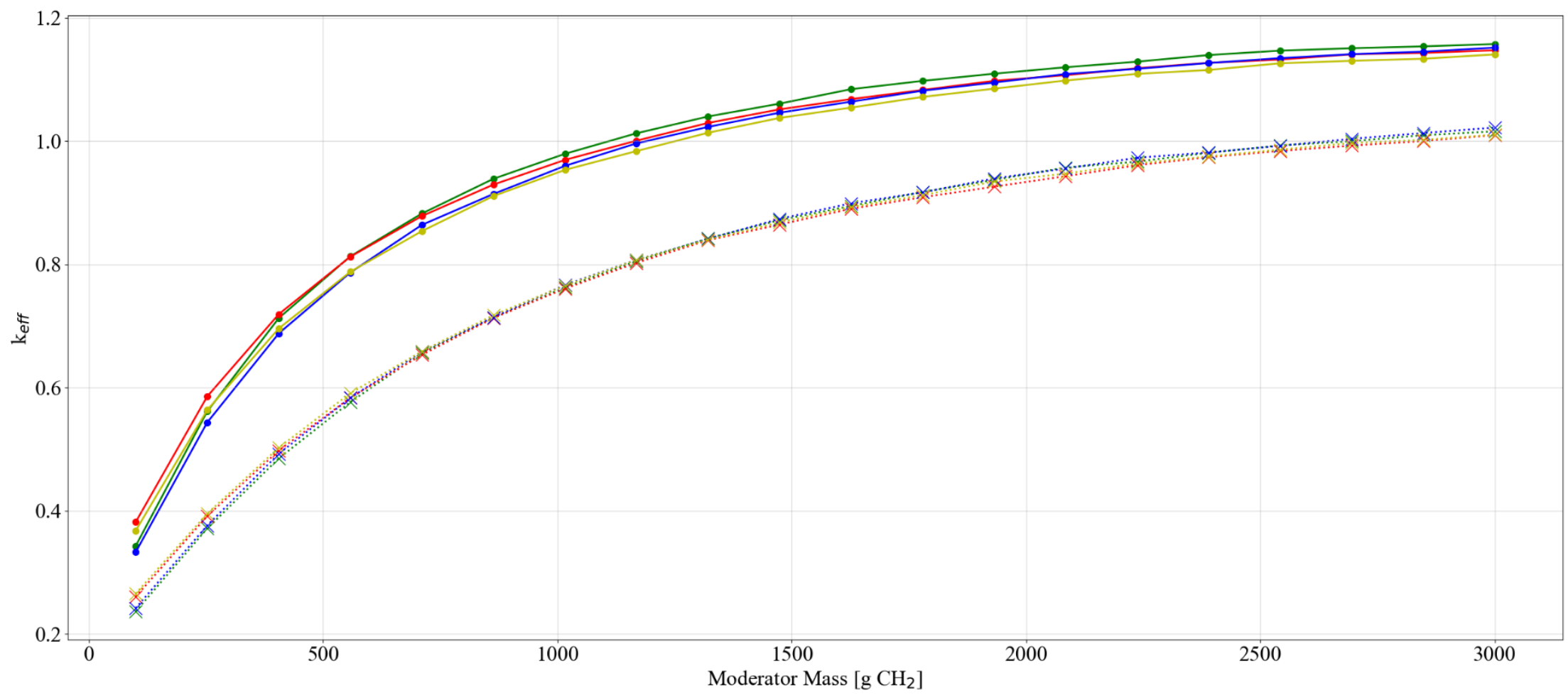
set-1a, $5 \mathrm{~kg}$ generic/can, no Be, thin SS discrete reflector set-1a, no filler, $585 \mathrm{~g} \mathrm{Be}$, thin SS discrete reflector $\longrightarrow$ set-1a, no filler, no Be, thick SS discrete reflector

........ set-1a, $5 \mathrm{~kg}$ generic/can, no Be, thick SS discrete reflector

$\cdots$ set-1a, no filler, $585 \mathrm{~g} \mathrm{Be}$, thick SS discrete reflector

… $\Varangle \cdots$ set-1a, $5 \mathrm{~kg}$ generic/can, $585 \mathrm{~g} \mathrm{Be}$, thin SS discrete $\mathrm{r}$ reflector

…… set-1a, $5 \mathrm{~kg}$ generic/can, $585 \mathrm{~g} \mathrm{Be}$, thick SS discrete reflector

Figure B-11. Set-1a results, plot 10: reactivity effect of various parameters with $7.7 \mathrm{~cm}$ cylinder radius, generic filler, poly moderated. 


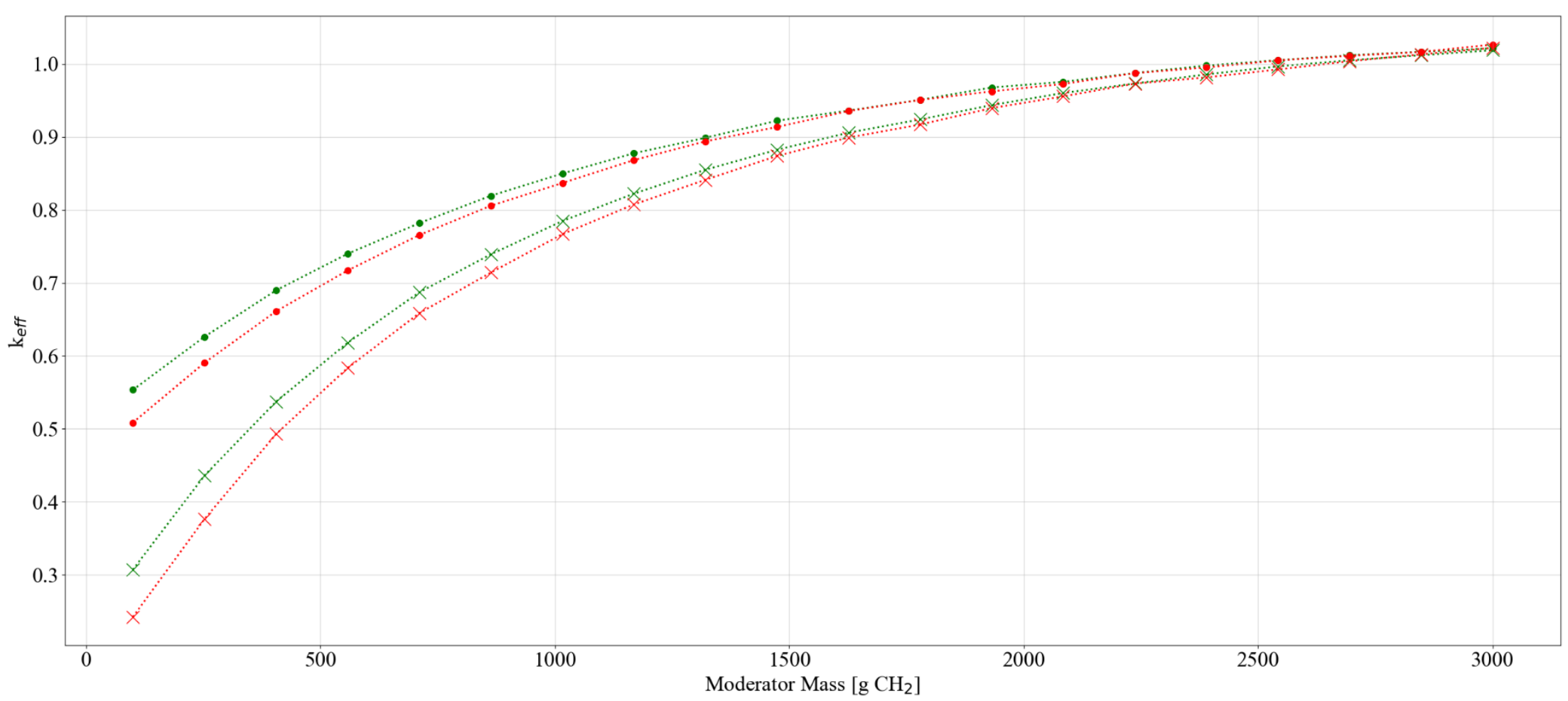

… set-1a, cylinder radius $=7.7 \mathrm{~cm}$, poly moderator, $5 \mathrm{~kg}$ graphite $/$ can, no $\mathrm{Be}$, thick $\mathrm{SS}$ discrete reflector

$\cdots \bullet$ set-1a, cylinder radius $=7.7 \mathrm{~cm}$, poly moderator, $5 \mathrm{~kg}$ graphite $/ \mathrm{can}$, no $\mathrm{Be}$, thick poly discrete reflector

$\cdots \times$ set-1a, cylinder radius $=7.7 \mathrm{~cm}$, poly moderator, $5 \mathrm{~kg}$ generic $/ \mathrm{can}$, no $\mathrm{Be}$, thick SS discrete reflector

$\cdots \bullet$ set-1a, cylinder radius $=7.7 \mathrm{~cm}$, poly moderator, $5 \mathrm{~kg}$ generic $/ \mathrm{can}$, no $\mathrm{Be}$, thick poly discrete reflector

Figure B-12. Set-1a results, plot 11: comparison of graphite and generic filler with $7.7 \mathrm{~cm}$ cylinder radius, no Be, poly moderated, thick discrete reflector. 


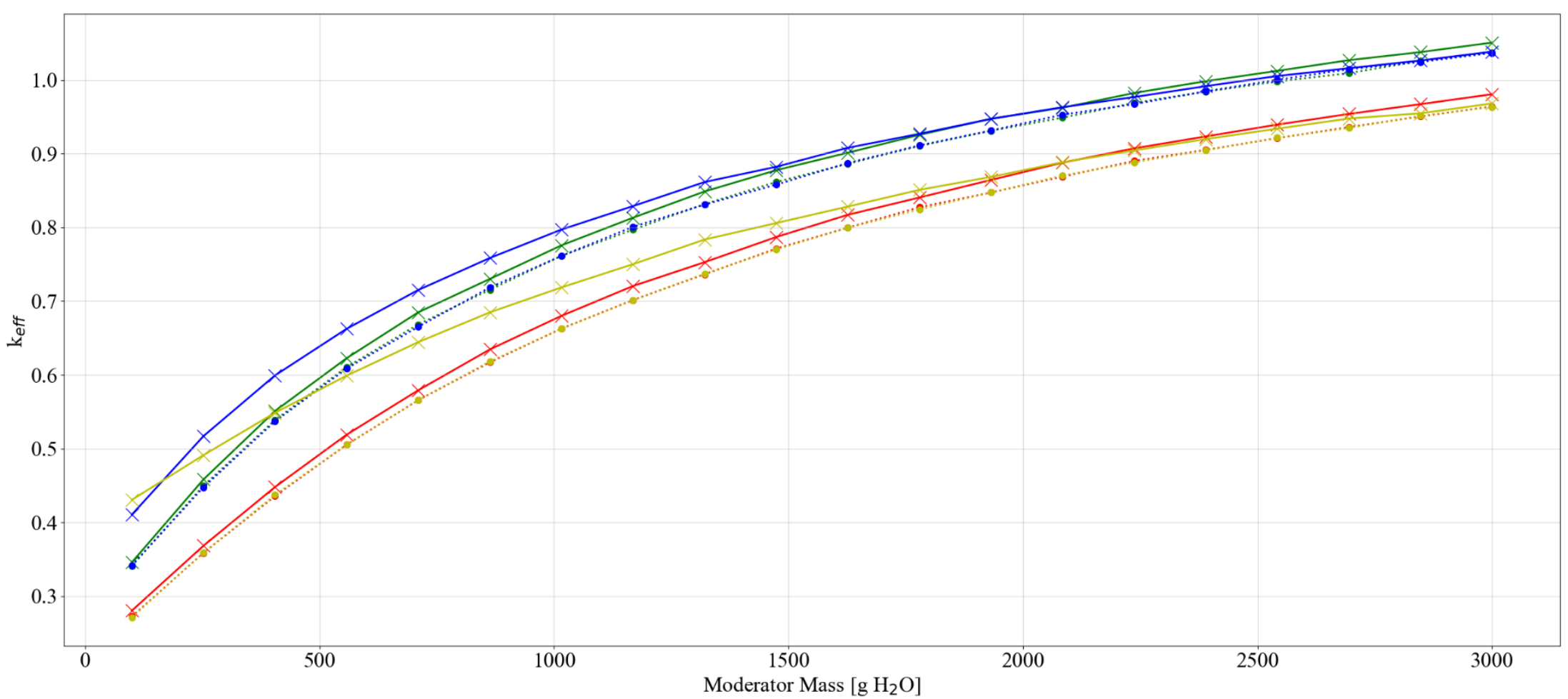

….. set-1a, no filler, no Be, thin SS discrete reflector

- set-1a, no filler, no Be, thick SS discrete reflector

..... set-1a, $5 \mathrm{~kg}$ graphite/can, $585 \mathrm{~g} \mathrm{Be}$, thin SS discrete reflector

set-1a, $5 \mathrm{~kg}$ graphite/can, $585 \mathrm{~g} \mathrm{Be}$, thick SS discrete reflector

…... set-1a, no filler, no Be, thin poly discrete reflector

$\star$ set-1a, no filler, no Be, thick poly discrete reflector

….. set-1a, $5 \mathrm{~kg}$ graphite/can, $585 \mathrm{~g} \mathrm{Be}$, thin poly discrete reflector

set-1a, $5 \mathrm{~kg}$ graphite/can, $585 \mathrm{~g} \mathrm{Be}$, thick poly discrete reflector

Figure B-13. Set-1a results, plot 12: reactivity effect of various parameters with spherical waste form geometry, graphite filler, water moderated. 


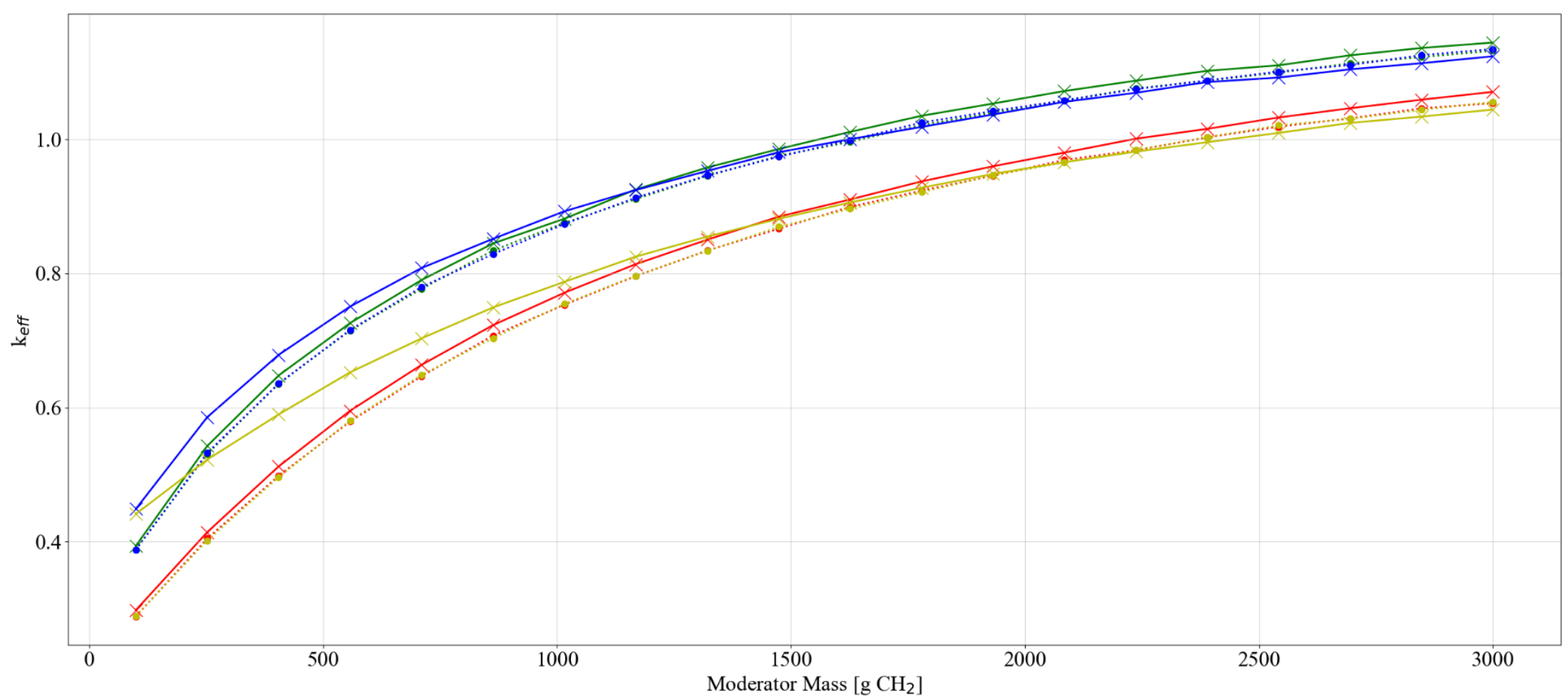

….. set-1a, no filler, no Be, thin SS discrete reflector

- set-1a, no filler, no Be, thick SS discrete reflector

..... set-1a, $5 \mathrm{~kg}$ graphite/can, $585 \mathrm{~g} \mathrm{Be}$, thin SS discrete reflector

set-1a, $5 \mathrm{~kg}$ graphite/can, $585 \mathrm{~g} \mathrm{Be}$, thick SS discrete reflector

…... set-1a, no filler, no Be, thin poly discrete reflector

$*$ set-1a, no filler, no Be, thick poly discrete reflector

-... set-1a, $5 \mathrm{~kg}$ graphite/can, $585 \mathrm{~g} \mathrm{Be}$, thin poly discrete reflector

set-1a, $5 \mathrm{~kg}$ graphite/can, $585 \mathrm{~g} \mathrm{Be}$, thick poly discrete reflector

Figure B-14. Set-1a results, plot 13: reactivity effect of various parameters with spherical waste form geometry, graphite filler, poly moderated. 


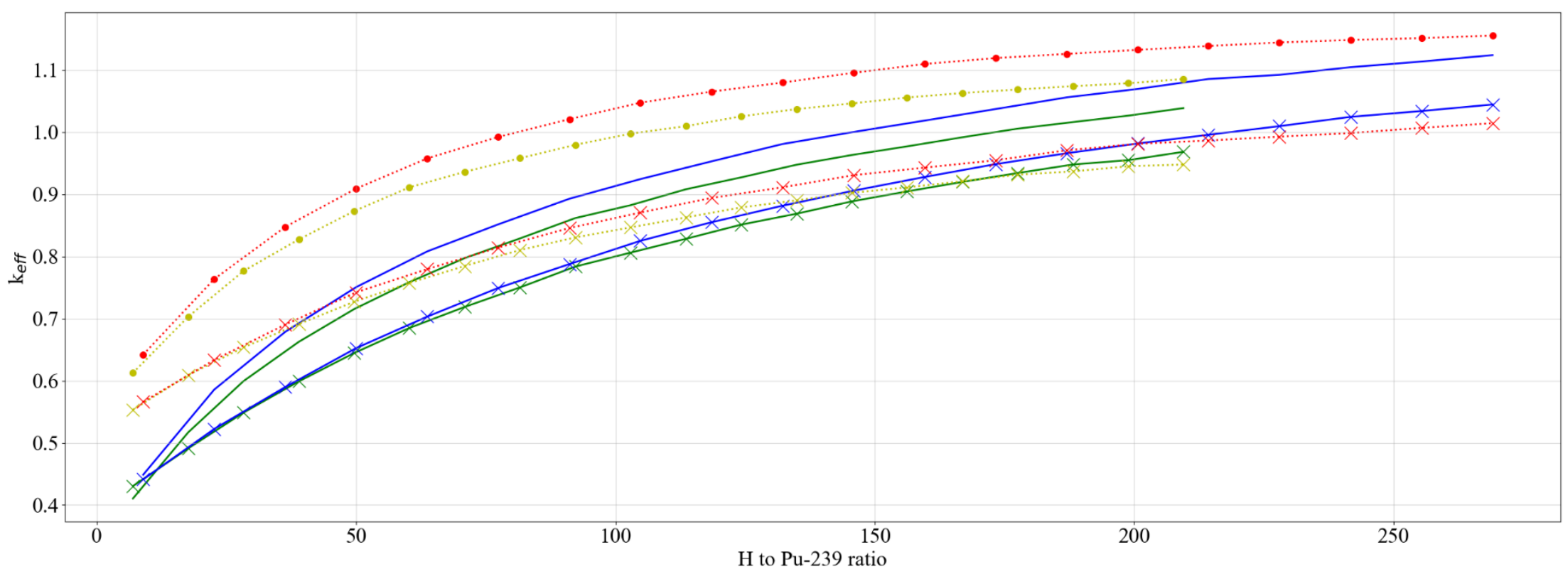

t-1a, sphere, no filler, no Be, thick poly discrete reflector, water moderator

$\leftarrow$ set-1a, sphere, $5 \mathrm{~kg}$ graphite/can, $585 \mathrm{~g} \mathrm{Be}$, thick poly discrete reflector, water moderator

— set-1a, sphere, no filler, no Be, thick poly discrete reflector, poly moderator

$\star$ set-1a, sphere, $5 \mathrm{~kg}$ graphite/can, $585 \mathrm{~g} \mathrm{Be}$, thick poly discrete reflector, poly moderator

$\cdots \cdot$ set-1a, cylinder with radius $=7.7 \mathrm{~cm}$, no filler, no Be, thick poly discrete reflector, water moderator

set-1a, cylinder with radius $=7.7 \mathrm{~cm}, 5 \mathrm{~kg}$ graphite $/ \mathrm{can}, 585 \mathrm{~g}$ Be, thick poly discrete reflector, water moderator

…… set-1a, cylinder with radius $=7.7 \mathrm{~cm}$, no filler, no Be, thick poly discrete reflector, poly moderator

$\ldots . \cdots$ set-1a, cylinder with radius $=7.7 \mathrm{~cm}, 5 \mathrm{~kg}$ graphite $/ \mathrm{can}, 585 \mathrm{~g} \mathrm{Be}$, thick poly discrete reflector, poly moderator

Figure B-15. Set-1a results, plot 14: comparison of spherical and cylindrical geometries $(\mathbf{h} / \mathbf{x})$. 


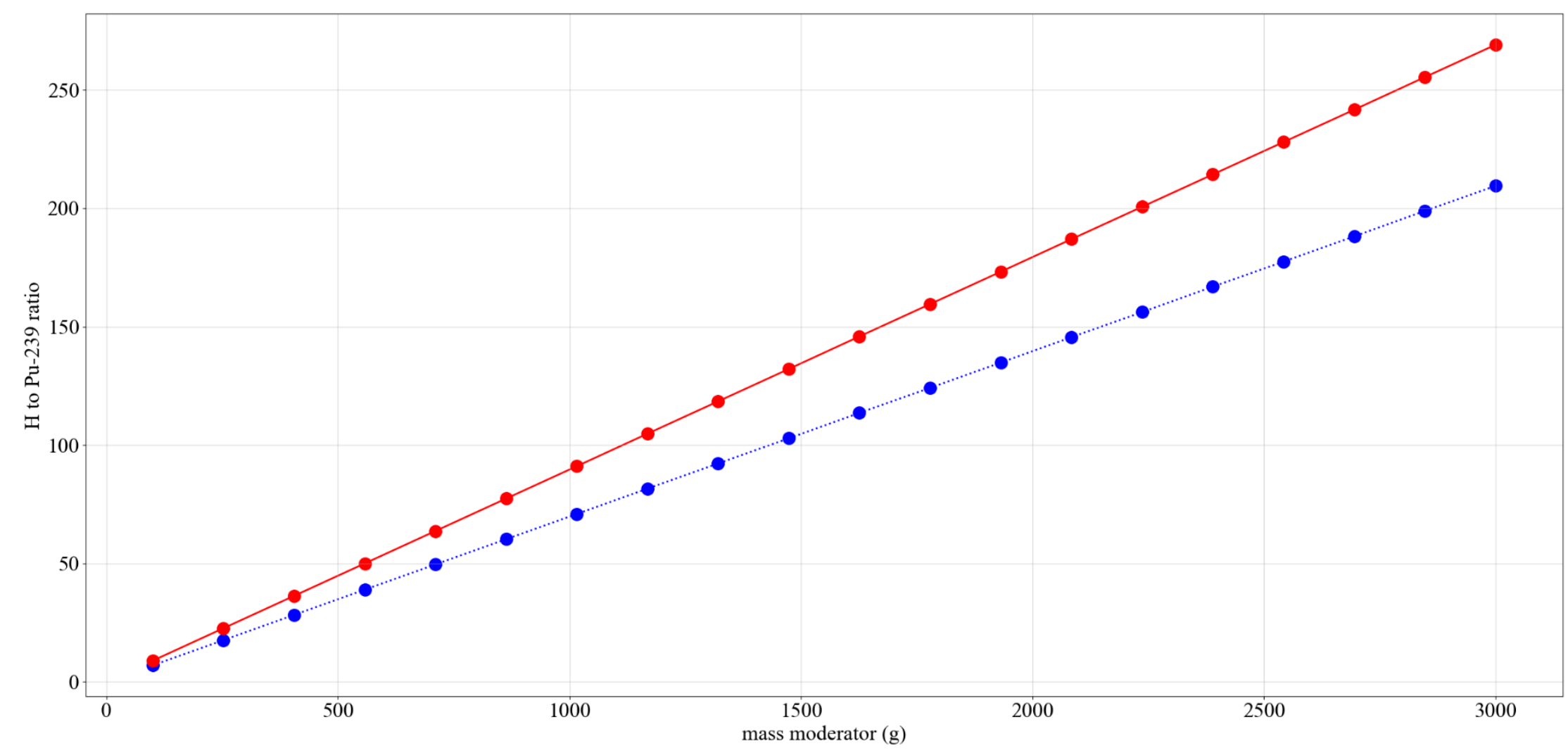

-... water moderator

$\longrightarrow$ poly moderator

Figure B-16. Set-1a results, plot 15: comparison of water and poly $h / x$. 


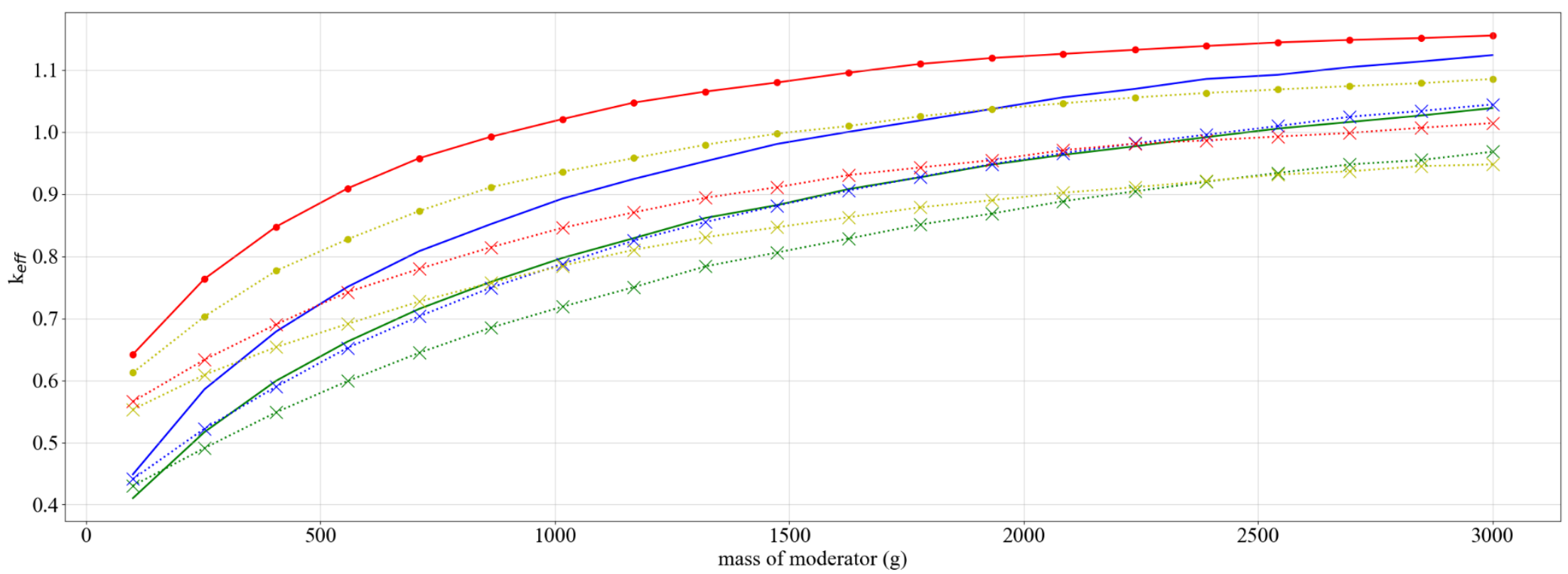

- set-1a, sphere, no filler, no Be, thick poly discrete reflector, water moderator

........ set-1a, sphere, $5 \mathrm{~kg}$ graphite/can, $585 \mathrm{~g} \mathrm{Be}$, thick poly discrete reflector, water moderator

- set-1a, sphere, no filler, no Be, thick poly discrete reflector, poly moderator

… $\Varangle \cdots$ set-1a, sphere, $5 \mathrm{~kg}$ graphite/can, $585 \mathrm{~g} \mathrm{Be}$, thick poly discrete reflector, poly moderator

$\cdots \cdot \cdots$ set-1a, cylinder with radius $=7.7 \mathrm{~cm}$, no filler, no Be, thick poly discrete reflector, water moderator

set-1a, cylinder with radius $=7.7 \mathrm{~cm}, 5 \mathrm{~kg}$ graphite $/ \mathrm{can}, 585 \mathrm{~g} \mathrm{Be}$, thick poly discrete reflector, water moderator

- - set-1a, cylinder with radius $=7.7 \mathrm{~cm}$, no filler, no Be, thick poly discrete reflector, poly moderator

……. set-1a, cylinder with radius $=7.7 \mathrm{~cm}, 5 \mathrm{~kg}$ graphite/can, $585 \mathrm{~g} \mathrm{Be}$, thick poly discrete reflector, poly moderator

Figure B-17. Set-1a results, plot 16: comparison of spherical and cylindrical geometries (mod mass). 


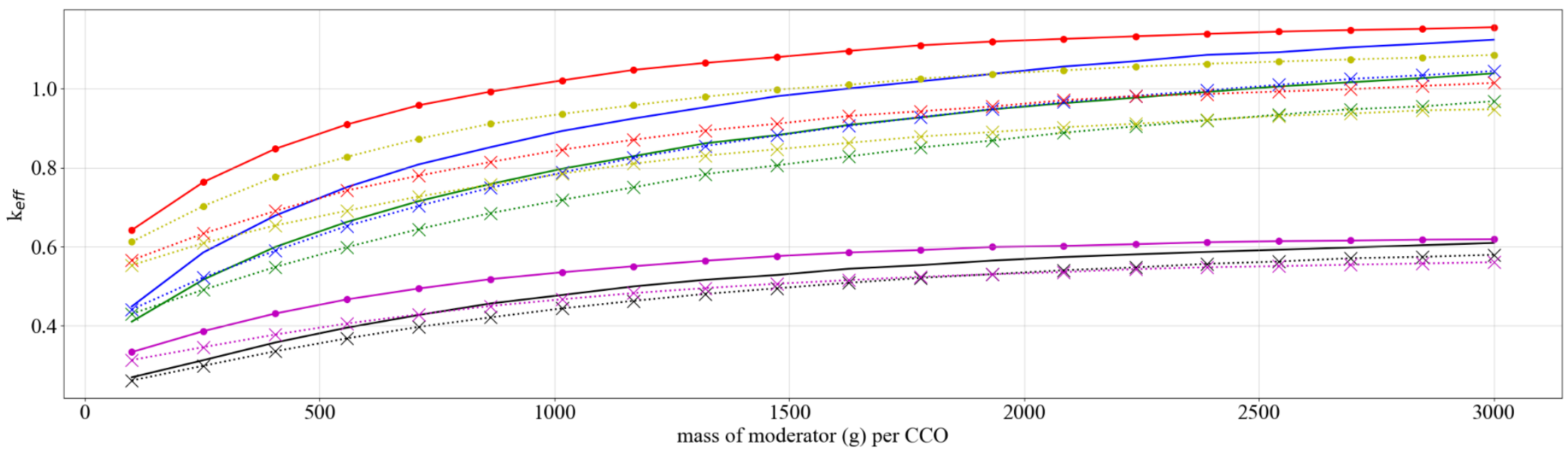

t-1a, sphere, no filler, no Be, thick poly discrete reflector, water moderator

........ set-1a, sphere, $5 \mathrm{~kg}$ graphite/can, $585 \mathrm{~g} \mathrm{Be,} \mathrm{thick} \mathrm{poly} \mathrm{discrete} \mathrm{reflector,} \mathrm{water} \mathrm{moderator}$

set-1a, sphere, no filler, no Be, thick poly discrete reflector, poly moderator

…… set-1a, sphere, $5 \mathrm{~kg}$ graphite/can, $585 \mathrm{~g} \mathrm{Be}$, thick poly discrete reflector, poly moderator

- ... set-1a, cylinder with radius $=7.7 \mathrm{~cm}$, no filler, no Be, thick poly discrete reflector, water moderator

… $\times$ set-1a, cylinder with radius $=7.7 \mathrm{~cm}, 5 \mathrm{~kg}$ graphite $/ \mathrm{can}, 585 \mathrm{~g} \mathrm{Be}$, thick poly discrete reflector, water moderator

$\longrightarrow$ set-1a, cylinder with radius $=7.7 \mathrm{~cm}$, no filler, no Be, thick poly discrete reflector, poly moderator

… $\ldots$ s.. set-1a, cylinder with radius $=7.7 \mathrm{~cm}, 5 \mathrm{~kg}$ graphite $/ \mathrm{can}, 585 \mathrm{~g} \mathrm{Be}$, thick poly discrete reflector, poly moderator

- set-1a, sphere, $50 \mathrm{~g} \mathrm{~B} 4 \mathrm{C}$, no filler, no Be, thick poly discrete reflector, poly moderato

... ※... set-1a, sphere, $50 \mathrm{~g} \mathrm{B4C,} 5 \mathrm{~kg}$ graphite/can, $585 \mathrm{~g} \mathrm{Be,} \mathrm{thick} \mathrm{poly} \mathrm{discrete} \mathrm{reflector,} \mathrm{poly} \mathrm{moderator}$

$\longrightarrow$ set-1a, cylinder with radius $=7.7 \mathrm{~cm}, 50 \mathrm{~g} \mathrm{~B} 4 \mathrm{C}$, no filler, no Be, thick poly discrete reflector, poly moderator

$\cdots \times$ set-1a, cylinder with radius $=7.7 \mathrm{~cm}, 50 \mathrm{~g} \mathrm{~B} 4 \mathrm{C}, 5 \mathrm{~kg}$ graphite $/ \mathrm{can}, 585 \mathrm{~g} \mathrm{Be}$, thick poly discrete reflector, poly moderator

Figure B-18. Set-1a results, plot 17: comparison of $50 \mathrm{~g} \mathrm{~B} \mathrm{~B}_{4} \mathrm{C}$ vs. no $\mathrm{B}_{4} \mathrm{C}$ for spherical and cylindrical geometries (mod mass). 
This page is intentionally blank 
APPENDIX C. SET-2: RESULTS OF THE NONUNIFORM ARRAY FOR THE UPPER HORIZON CALCULATIONS 
This page is intentionally blank 


\section{APPENDIX C. SET-2: RESULTS OF THE NONUNIFORM ARRAY FOR THE UPPER HORIZON CALCULATIONS}

The analysis methodology for the uniform arrays is discussed in detail in Section 6.3 of the main report.

This appendix serves as a repository of those results for the set-2-uh calculations.

The complete results for all SAMPLER sweeps are provided in ADDENDUM 1.

The analysis model use for the calculations in this appendix is shown in Figure C-1 below.

The SAMPLER case sweeps presented in this appendix are summarized in Table C-1 below.

\section{LIST OF FIGURES}

Figure C-1. Diagram of the nonuniform array model. C-5

Figure C-2. Set-2-uh results, plot 1: reactivity effect of cylinder radius, pipe steel, no filler, no

Be, water moderated. C-7

Figure C-3. Set-2-uh results, plot 2: reactivity effect of cylinder radius, pipe steel, no filler, no Be, poly moderated.

Figure C-4. Set-2-uh results, plot 3: reactivity effect of cylinder radius, pipe poly, no filler, no $\mathrm{Be}$, water moderated.

Figure C-5. Set-2-uh results, plot 4: reactivity effect of cylinder radius, pipe poly, no filler, no Be, poly moderated C-10

Figure C-6. Set-2-uh results, plot 5: reactivity effect of cylinder radius, pipe steel, $5 \mathrm{~kg}$ graphite/can, no Be, water moderated

Figure C-7. Set-2-uh results, plot 6: reactivity effect of cylinder radius, pipe steel, $5 \mathrm{~kg}$ graphite/can, no Be, poly moderated.

Figure C-8. Set-2-uh results, plot 7: reactivity effect of cylinder radius, pipe poly, $5 \mathrm{~kg}$ graphite/can, no Be, water moderated.

Figure C-9. Set-2-uh results, plot 8: reactivity effect of cylinder radius, pipe poly, $5 \mathrm{~kg}$ graphite/can, no Be, poly moderated.

Figure C-10. Set-2-uh results, plot 9: reactivity effect of various parameters with $7.7 \mathrm{~cm}$ cylinder radius, graphite filler, poly moderated.

Figure C-11. Set-2-uh results, plot 10: reactivity effect of various parameters with $7.7 \mathrm{~cm}$ cylinder radius, generic filler, poly moderated.

Figure C-12. Set-2-uh results, plot 11: comparison of graphite and generic filler with $7.7 \mathrm{~cm}$ cylinder radius, no Be, poly moderated, thick discrete reflector.

Figure C-13. Set-2-uh results, plot 12: reactivity effect of various parameters with spherical waste form geometry, graphite filler, water moderated.

Figure C-14. Set-2-uh results, plot 13: reactivity effect of various parameters with spherical waste form geometry, graphite filler, poly moderated.

Figure C-15. Set-2-uh results, plot 14: comparison of spherical and cylindrical geometries $(\mathrm{h} / \mathrm{x})$.

Figure C-16. Set-2-uh results, plot 15: comparison of water and poly $\mathrm{h} / \mathrm{x}$.

Figure C-17. Set-2-uh results, plot 16: comparison of spherical and cylindrical geometries (mod mass).

Figure C-18. Set-2-uh results, plot 17: comparison of $50 \mathrm{~g} \mathrm{~B}_{4} \mathrm{C}$ vs. no $\mathrm{B}_{4} \mathrm{C}$ for spherical and cylindrical geometries (mod mass). 


\section{LIST OF TABLES}

Table C-1. Summary of cases for set-2-uh for the upper horizon compaction data .............................. C-6 


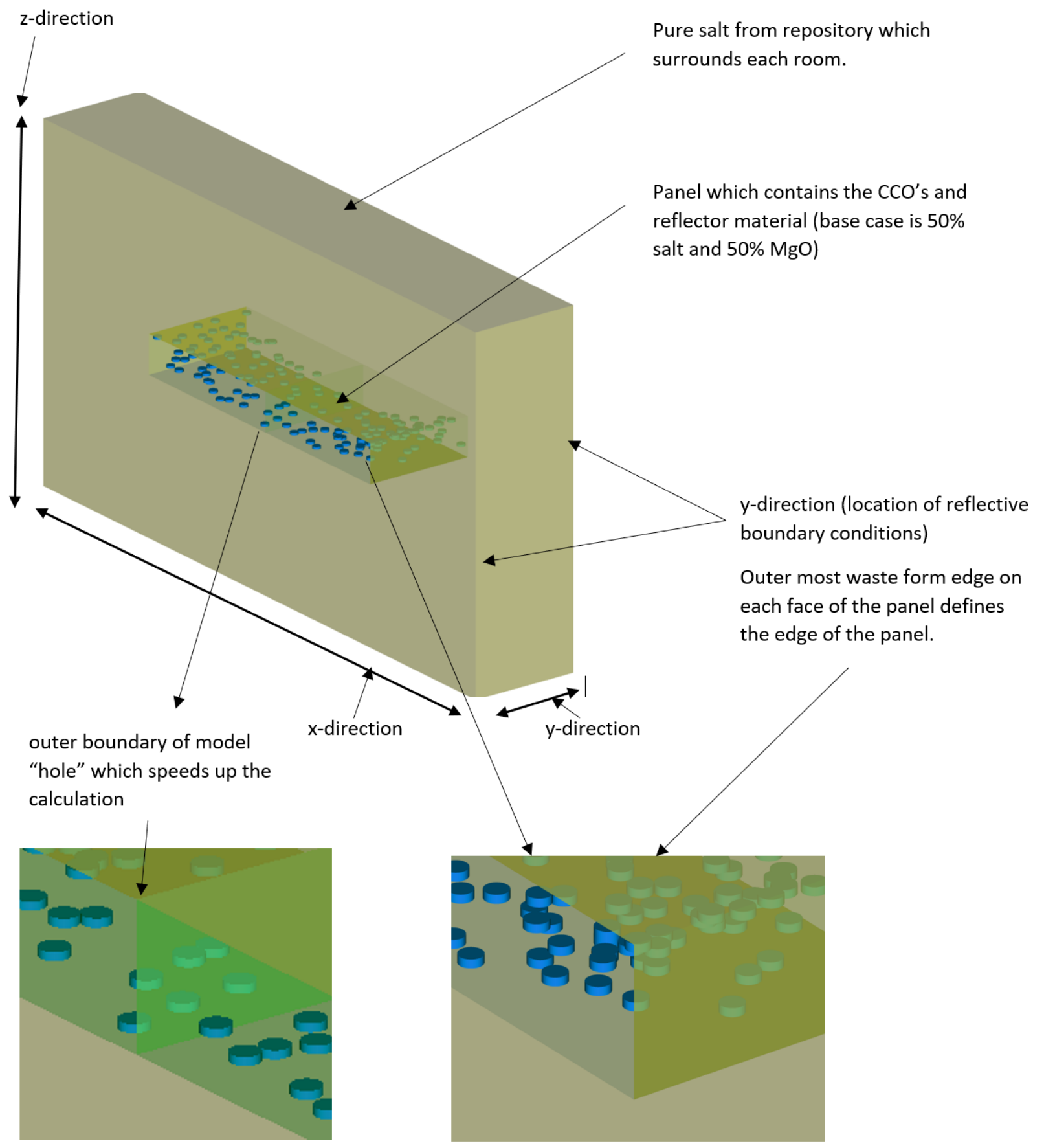

Figure C-1. Diagram of the nonuniform array model. 
Table C-1. Summary of cases for set-2-uh for the upper horizon compaction data

\begin{tabular}{|c|c|c|c|c|c|c|c|c|}
\hline Case & Model type & Waste form shape & $\begin{array}{l}\text { Waste form } \\
\text { moderator }\end{array}$ & $\begin{array}{c}\text { Filler material } \\
(\mathbf{0}, \mathbf{2 , 0 0 0}, \mathbf{4 , 0 0 0} \mathrm{g})\end{array}$ & Metal in filler & $\begin{array}{c}\text { Discrete reflector } \\
\text { (thin } 0.001 \text { and } \\
\text { thick } 0.7112 \mathrm{~cm} \text { ) }\end{array}$ & be (g) & Subcase \\
\hline \multirow{18}{*}{ Set-2-uh } & \multirow{18}{*}{$\begin{array}{l}\text { Nonuniform array with } \\
\text { centroids based on } \\
\text { upper horizon data. } \\
\text { Centroid from pipe } \\
\text { center is used as the } \\
\text { point in center of base } \\
\text { of cylinders or the } \\
\text { center of spheres. }\end{array}$} & \multirow{8}{*}{$\begin{array}{l}\text { Cylinder (radius range } \\
4.8,6,7.7 \text { and height } \\
\text { defined by total } \\
\text { volume of mass) }\end{array}$} & water & $\mathrm{c} 12$ & \multirow{18}{*}{$\begin{array}{c}\text { SS from can } \\
(0,500,1,000 \mathrm{~g})\end{array}$} & steel & \multirow{18}{*}{0 to 585} & set-2-uh-1 \\
\hline & & & poly & $\mathrm{c} 12$ & & steel & & set-2-uh-2 \\
\hline & & & water & $\mathrm{c} 12$ & & poly & & set-2-uh-3 \\
\hline & & & poly & $\mathrm{c} 12$ & & poly & & set-2-uh-4 \\
\hline & & & water & generic & & steel & & set-2-uh-5 \\
\hline & & & poly & generic & & steel & & set-2-uh-6 \\
\hline & & & water & generic & & poly & & set-2-uh-7 \\
\hline & & & poly & generic & & poly & & set-2-uh-8 \\
\hline & & \multirow{10}{*}{$\begin{array}{l}\text { Sphere (radius defined } \\
\text { by total volume of } \\
\text { mass) }\end{array}$} & water & $\mathrm{c} 12$ & & steel & & set-2-uh-9 \\
\hline & & & poly & $\mathrm{c} 12$ & & steel & & set-2-uh-10 \\
\hline & & & water & $\mathrm{c} 12$ & & poly & & set-2-uh-11 \\
\hline & & & poly & $\mathrm{c} 12$ & & poly & & set-2-uh-12 \\
\hline & & & water & generic & & steel & & set-2-uh-13 \\
\hline & & & poly & generic & & steel & & set-2-uh-14 \\
\hline & & & water & generic & & poly & & set-2-uh-15 \\
\hline & & & poly & generic & & poly & & set-2-uh-16 \\
\hline & & & $\begin{array}{c}\text { poly }+50 \mathrm{~g} \\
\mathrm{~B}_{4} \mathrm{C}(\mathrm{cyl})\end{array}$ & $\mathrm{c} 12$ & & poly $(0.7112)$ & & set-2-uh-17 \\
\hline & & & $\begin{array}{c}\text { poly }+50 \mathrm{~g} \\
\mathrm{~B}_{4} \mathrm{C}(\mathrm{sph})\end{array}$ & $\mathrm{c} 12$ & & poly (0.7112) & & set-2-uh-18 \\
\hline
\end{tabular}




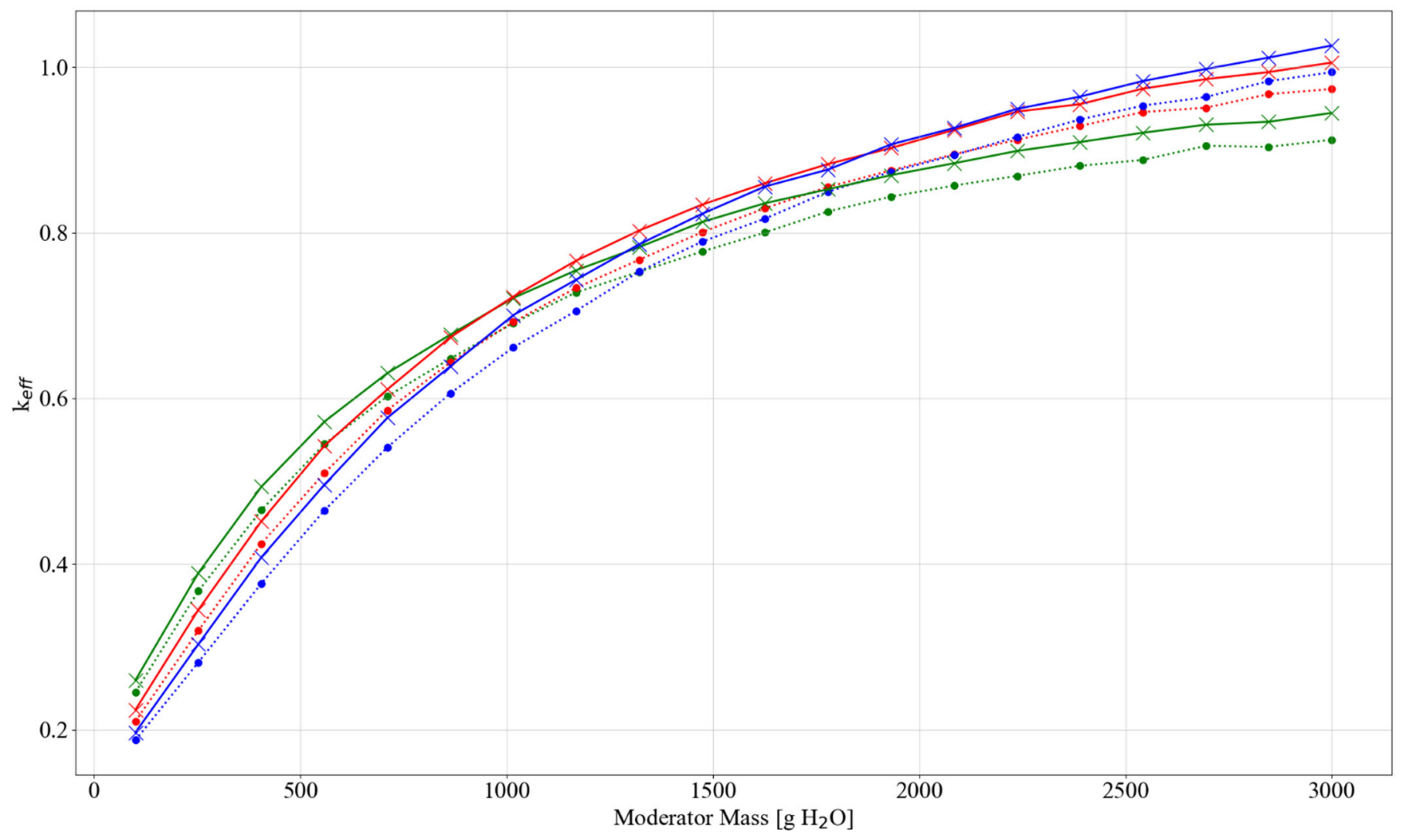

-... set-2-uh, cyl radius $=4.8 \mathrm{~cm}$, thin discrete reflector

•... set-2-uh, cyl radius $=7.7 \mathrm{~cm}$, thin discrete reflector

$\leftarrow$ set-2-uh, cyl radius $=6.25 \mathrm{~cm}$, thick discrete reflecto set- 2 -uh, cyl radius $=6.25 \mathrm{~cm}$, thin discrete reflector set- 2 -uh, cyl radius $=4.8 \mathrm{~cm}$, thick discrete reflector

Figure C-2. Set-2-uh results, plot 1: reactivity effect of cylinder radius, pipe steel, no filler, no Be, water moderated. 


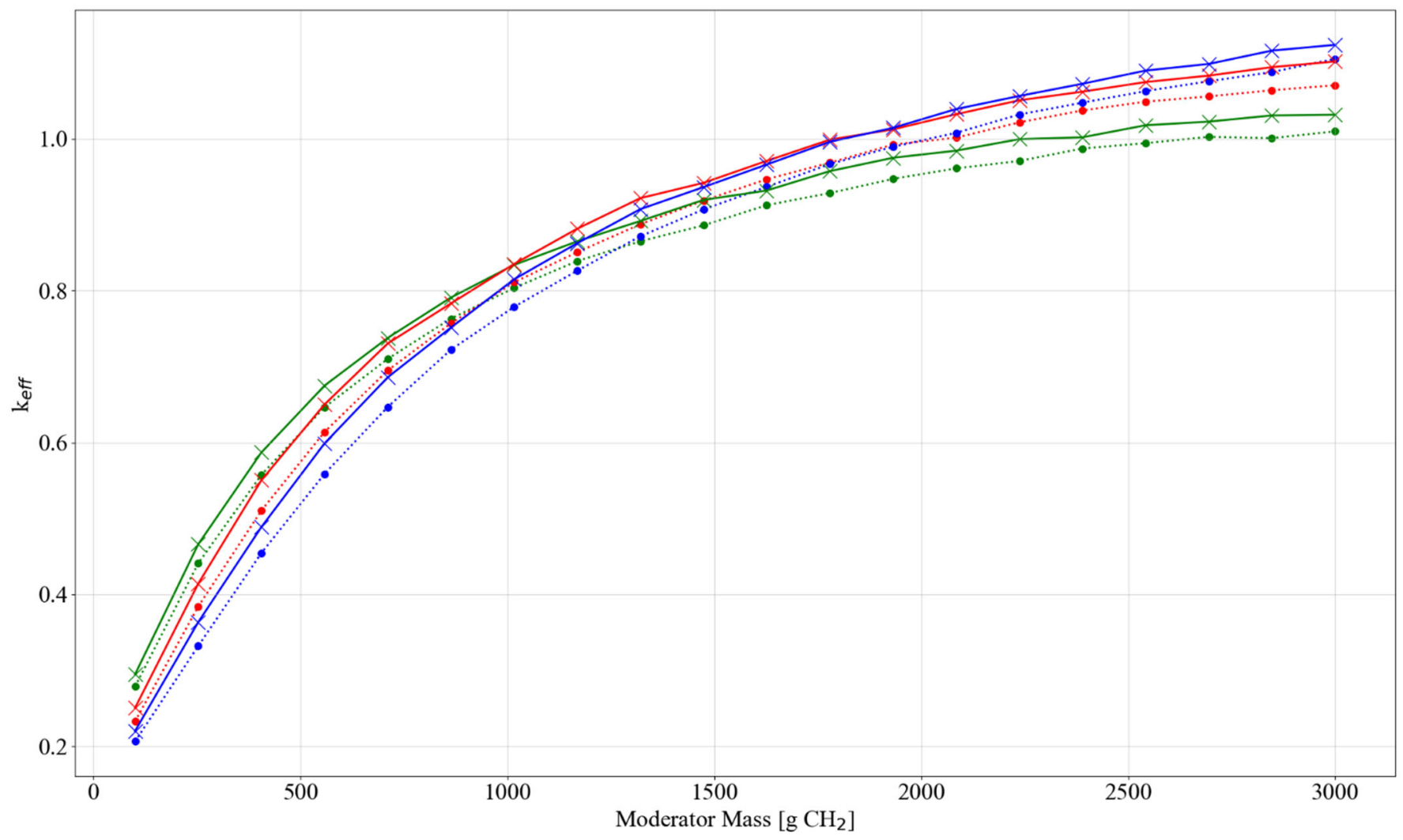

set-2-uh, cyl radius $=4.8 \mathrm{~cm}$, thin discrete reflector

- $\cdots$ set-2-uh, cyl radius $=7.7 \mathrm{~cm}$, thin discrete reflector

set- 2 -uh, cyl radius $=6.25 \mathrm{~cm}$, thick discrete reflecto set- 2 -uh, cyl radius $=6.25 \mathrm{~cm}$, thin discrete reflector set- 2 -uh, cyl radius $=4.8 \mathrm{~cm}$, thick discrete reflector set-2-uh, cyl radius $=7.7 \mathrm{~cm}$, thick discrete reflector

Figure C-3. Set-2-uh results, plot 2: reactivity effect of cylinder radius, pipe steel, no filler, no Be, poly moderated. 


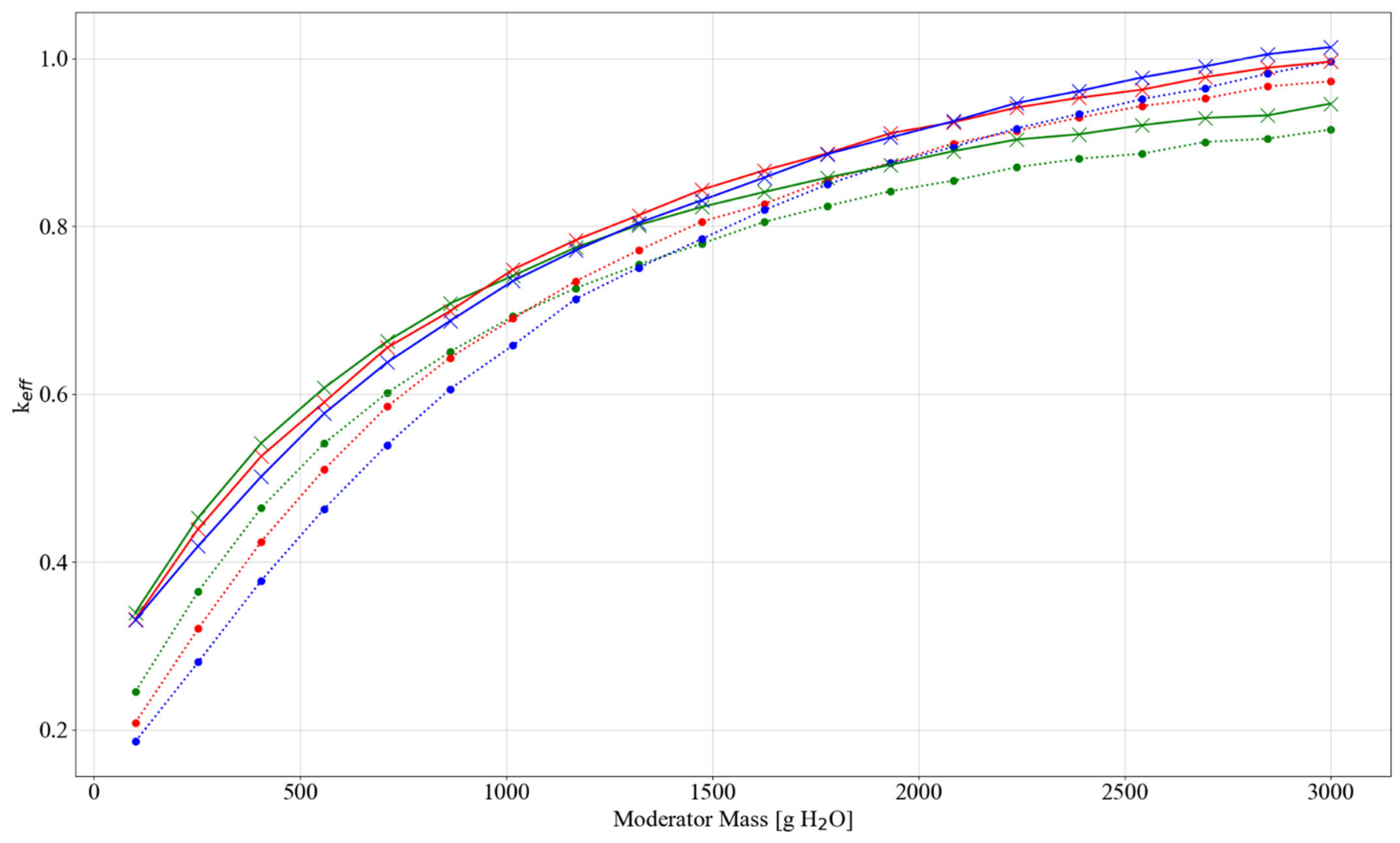

-... set-2-uh, cyl radius $=4.8 \mathrm{~cm}$, thin discrete reflector

•... set-2-uh, cyl radius $=7.7 \mathrm{~cm}$, thin discrete reflector

$\star$ set- 2 -uh, cyl radius $=6.25 \mathrm{~cm}$, thick discrete reflector set- 2 -uh, cyl radius $=6.25 \mathrm{~cm}$, thin discrete reflector set- 2 -uh, cyl radius $=4.8 \mathrm{~cm}$, thick discrete reflector

Figure C-4. Set-2-uh results, plot 3: reactivity effect of cylinder radius, pipe poly, no filler, no Be, water moderated. 


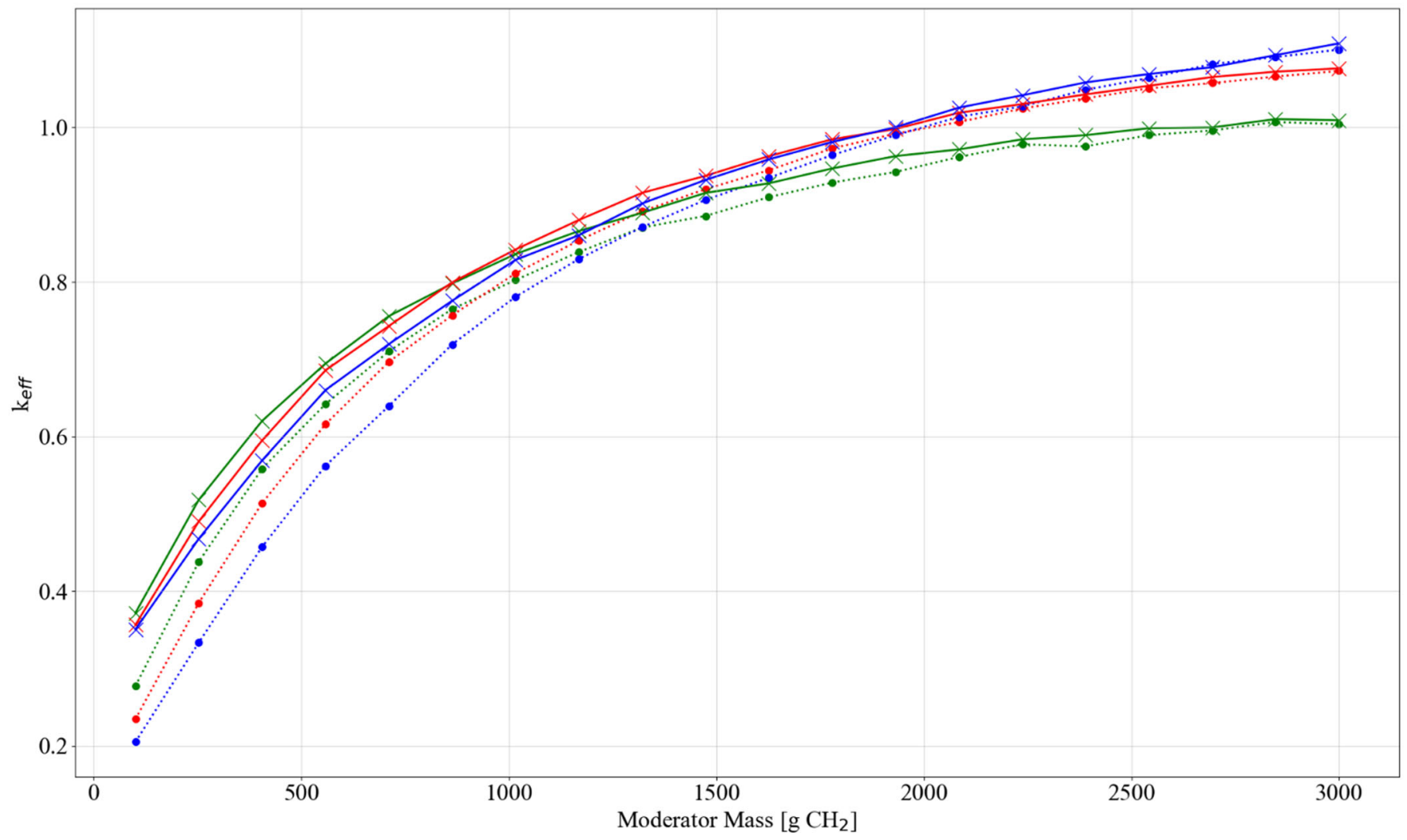

set-2-uh, cyl radius $=4.8 \mathrm{~cm}$, thin discrete reflector

-... set-2-uh, cyl radius $=7.7 \mathrm{~cm}$, thin discrete reflector

$\leftarrow$ set-2-uh, cyl radius $=6.25 \mathrm{~cm}$, thick discrete reflector

....... set-2-uh, cyl radius $=6.25 \mathrm{~cm}$, thin discrete reflector

$*$ set- 2 -uh, cyl radius $=4.8 \mathrm{~cm}$, thick discrete reflector

$*$ set-2-uh, cyl radius $=7.7 \mathrm{~cm}$, thick discrete reflector

Figure C-5. Set-2-uh results, plot 4: reactivity effect of cylinder radius, pipe poly, no filler, no Be, poly moderated . 


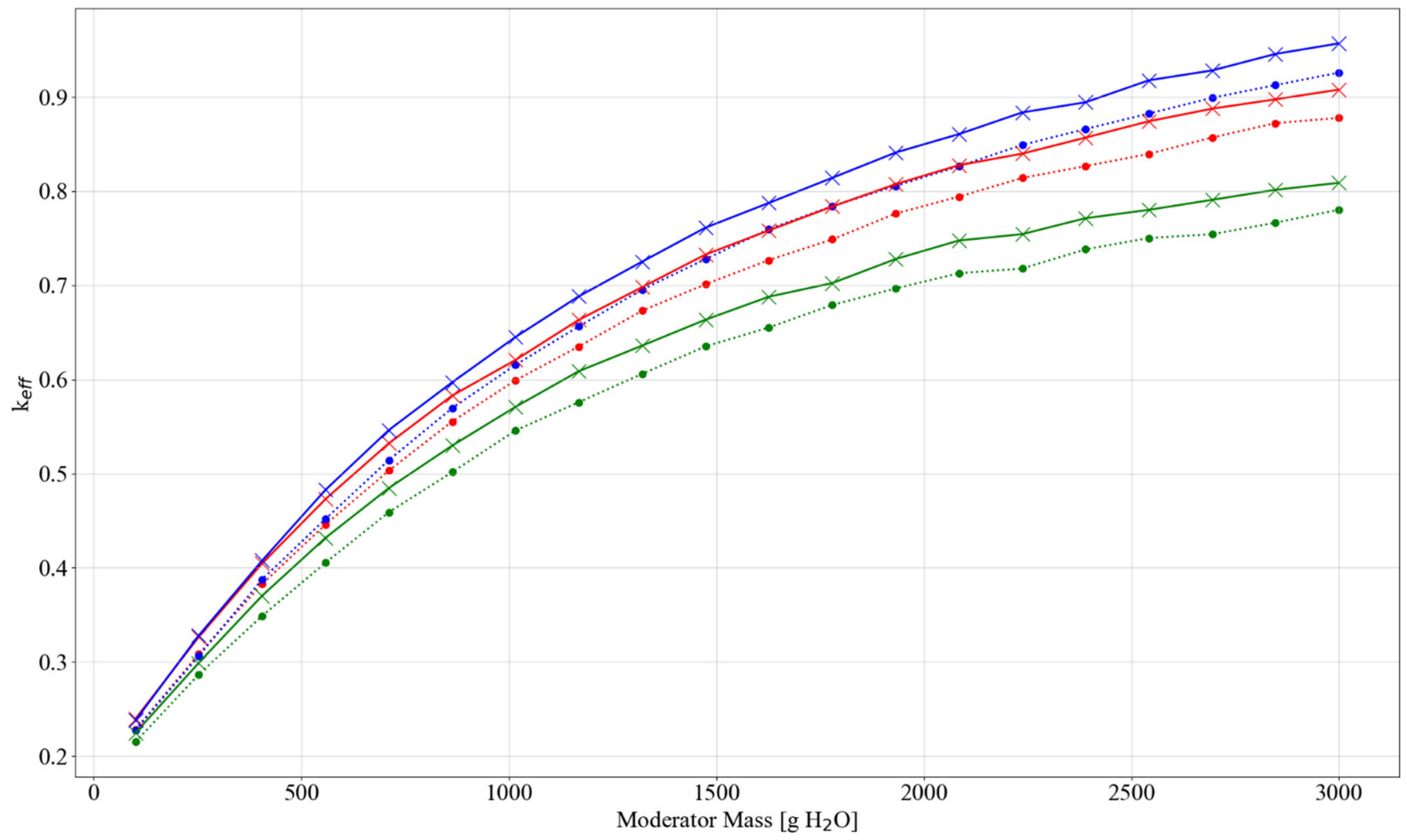

set- 2 -uh, cyl radius $=4.8 \mathrm{~cm}$, thin discrete reflector

- $\cdots$ set-2-uh, cyl radius $=7.7 \mathrm{~cm}$, thin discrete reflector

$\nVdash$ set- 2 -uh, cyl radius $=6.25 \mathrm{~cm}$, thick discrete reflecto
$\star \quad$ set-2-uh, cyl radius $=7.7 \mathrm{~cm}$, thick discrete reflector set-2-uh, cyl radius $=6.25 \mathrm{~cm}$, thin discrete reflector set- 2 -uh, cyl radius $=4.8 \mathrm{~cm}$, thick discrete reflector

Figure C-6. Set-2-uh results, plot 5: reactivity effect of cylinder radius, pipe steel, $5 \mathrm{~kg}$ graphite/can, no Be, water moderated. 


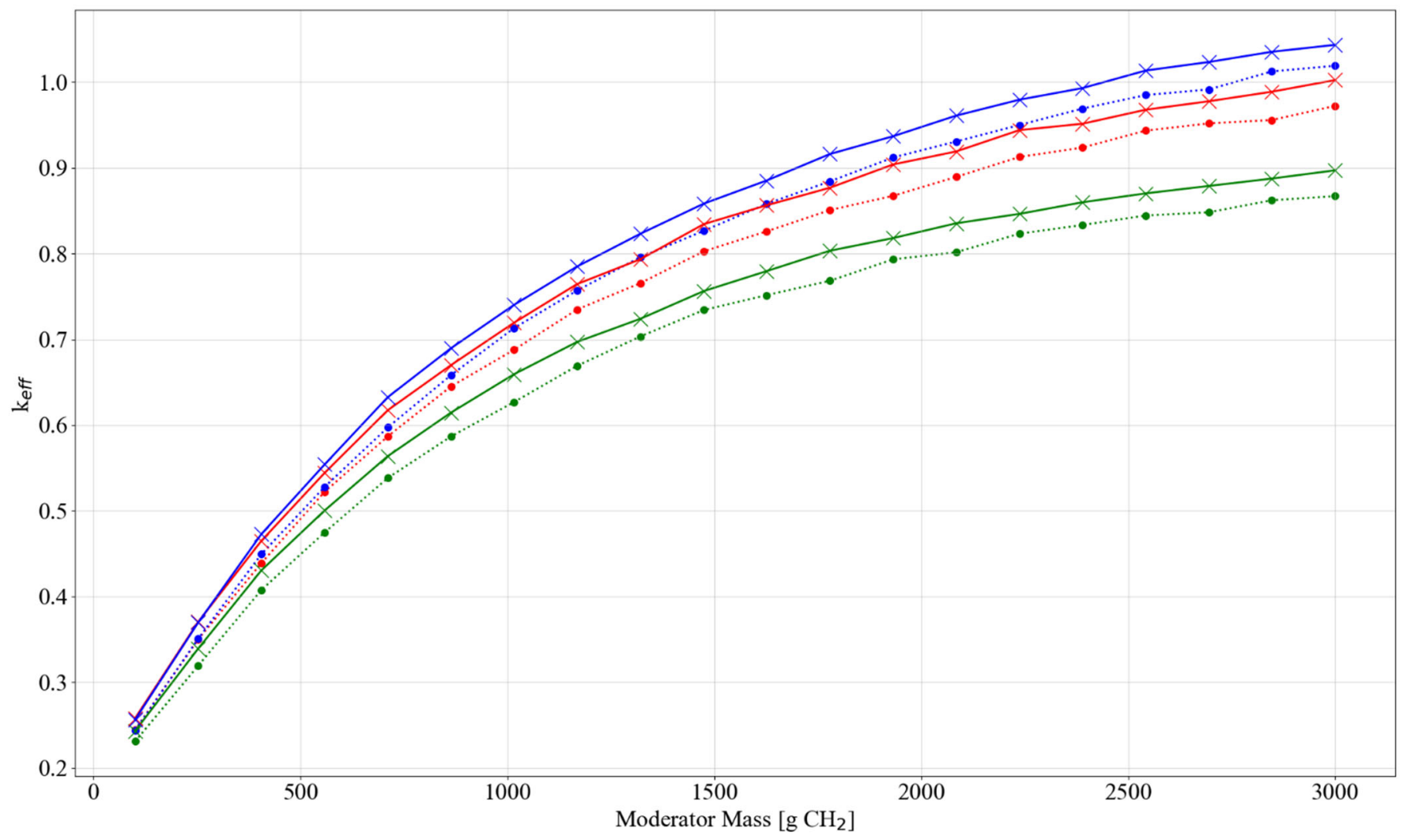

set-2-uh, cyl radius $=4.8 \mathrm{~cm}$, thin discrete reflector

-... set-2-uh, cyl radius $=7.7 \mathrm{~cm}$, thin discrete reflector

$\nVdash$ set- 2 -uh, cyl radius $=6.25 \mathrm{~cm}$, thick discrete reflector
$\star \quad$ set- 2 -uh, cyl radius $=7.7 \mathrm{~cm}$, thick discrete reflector set-2-uh, cyl radius $=6.25 \mathrm{~cm}$, thin discrete reflector set- 2 -uh, cyl radius $=4.8 \mathrm{~cm}$, thick discrete reflector

Figure C-7. Set-2-uh results, plot 6: reactivity effect of cylinder radius, pipe steel, $5 \mathrm{~kg}$ graphite/can, no Be, poly moderated. 


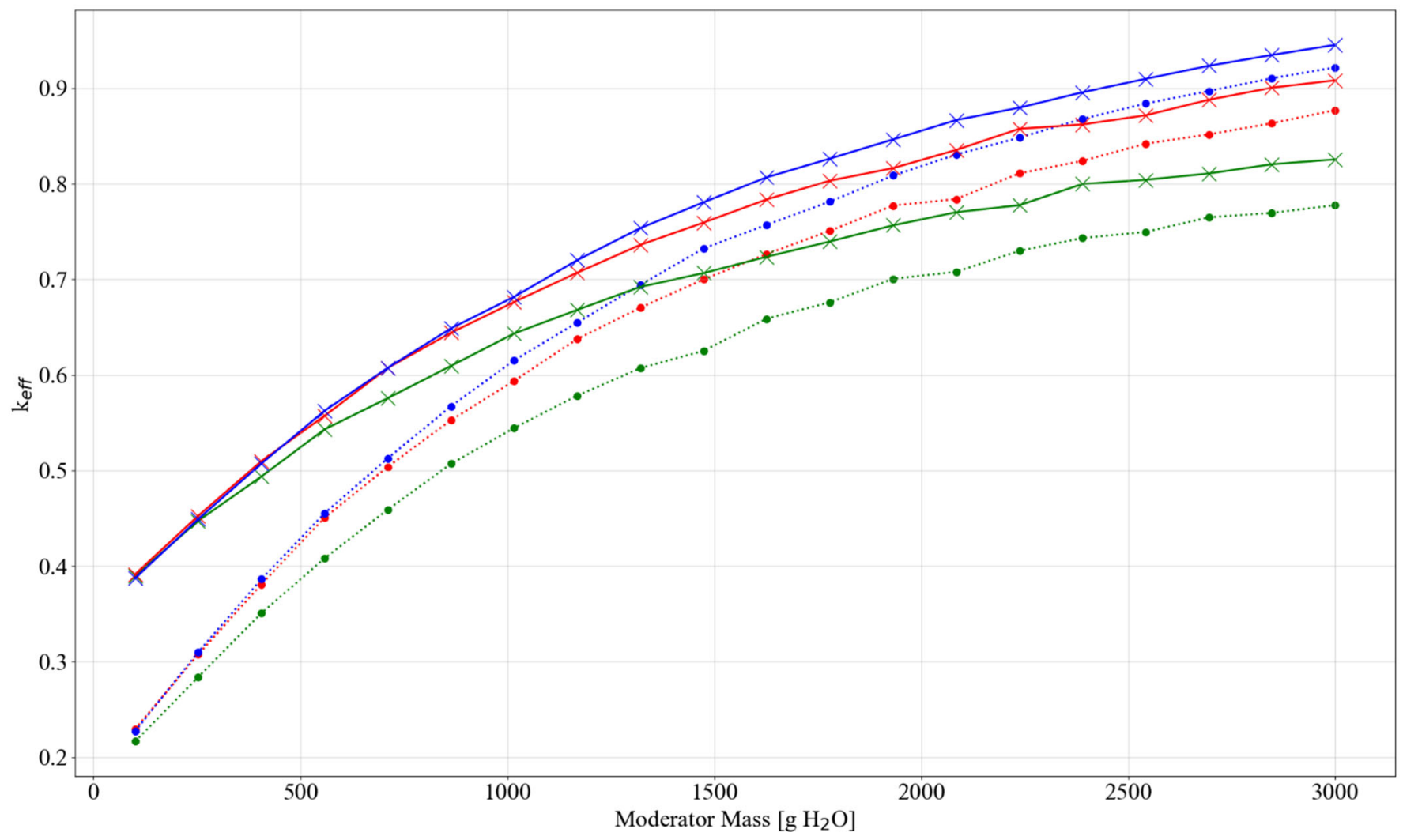

set-2-uh, cyl radius $=4.8 \mathrm{~cm}$, thin discrete reflector

•... set-2-uh, cyl radius $=7.7 \mathrm{~cm}$, thin discrete reflector

set- 2 -uh, cyl radius $=6.25 \mathrm{~cm}$, thick discrete reflecto set-2-uh, cyl radius $=6.25 \mathrm{~cm}$, thin discrete reflector set- 2 -uh, cyl radius $=4.8 \mathrm{~cm}$, thick discrete reflector set-2-uh, cyl radius $=7.7 \mathrm{~cm}$, thick discrete reflector

Figure C-8. Set-2-uh results, plot 7: reactivity effect of cylinder radius, pipe poly, $5 \mathrm{~kg}$ graphite/can, no Be, water moderated. 


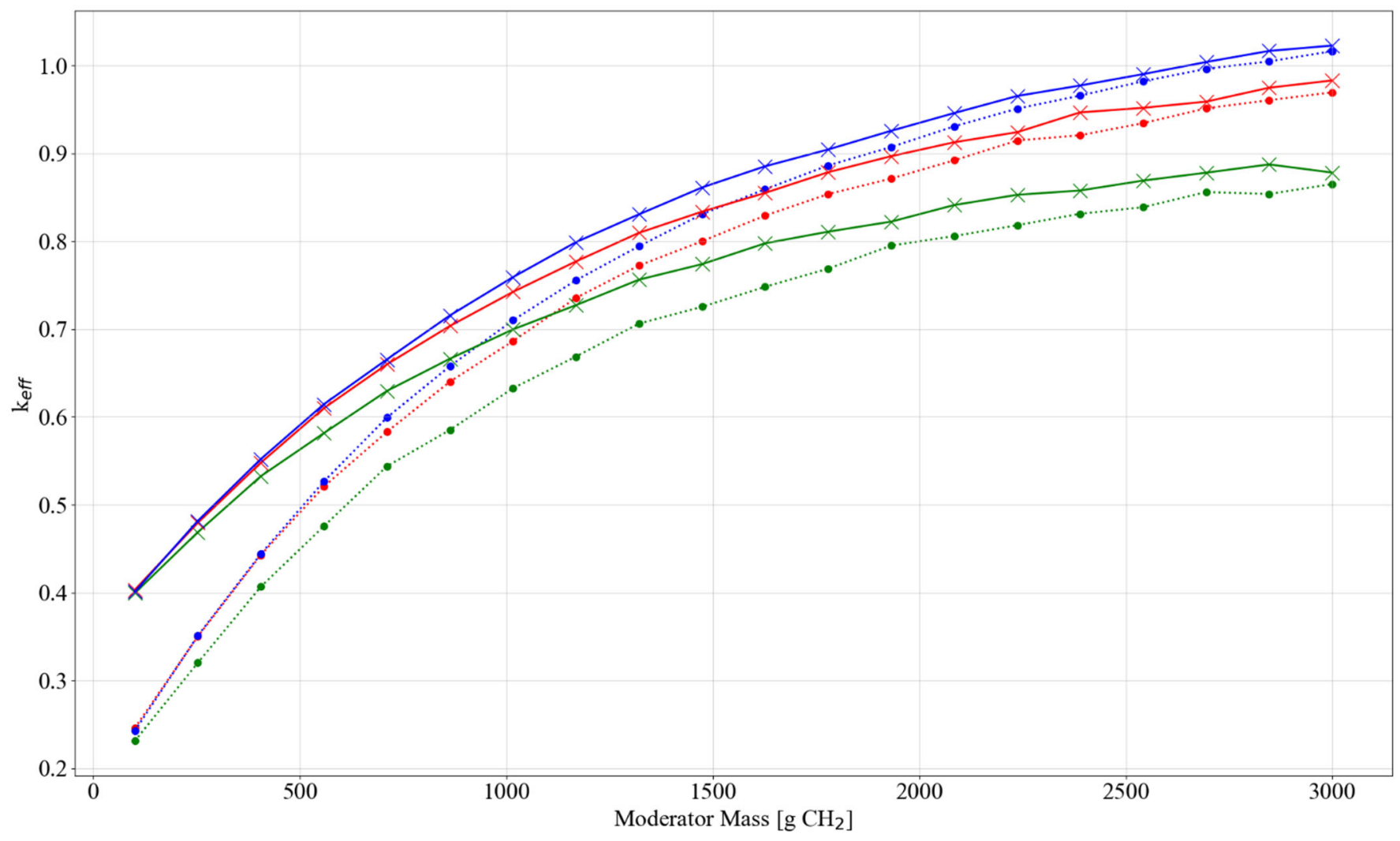

set-2-uh, cyl radius $=4.8 \mathrm{~cm}$, thin discrete reflector

-... set-2-uh, cyl radius $=7.7 \mathrm{~cm}$, thin discrete reflector

$\nVdash$ set- 2 -uh, cyl radius $=6.25 \mathrm{~cm}$, thick discrete reflecto
$\star \quad$ set-2-uh, cyl radius $=7.7 \mathrm{~cm}$, thick discrete reflector set-2-uh, cyl radius $=6.25 \mathrm{~cm}$, thin discrete reflector set- 2 -uh, cyl radius $=4.8 \mathrm{~cm}$, thick discrete reflector

Figure C-9. Set-2-uh results, plot 8: reactivity effect of cylinder radius, pipe poly, $5 \mathrm{~kg}$ graphite/can, no Be, poly moderated. 


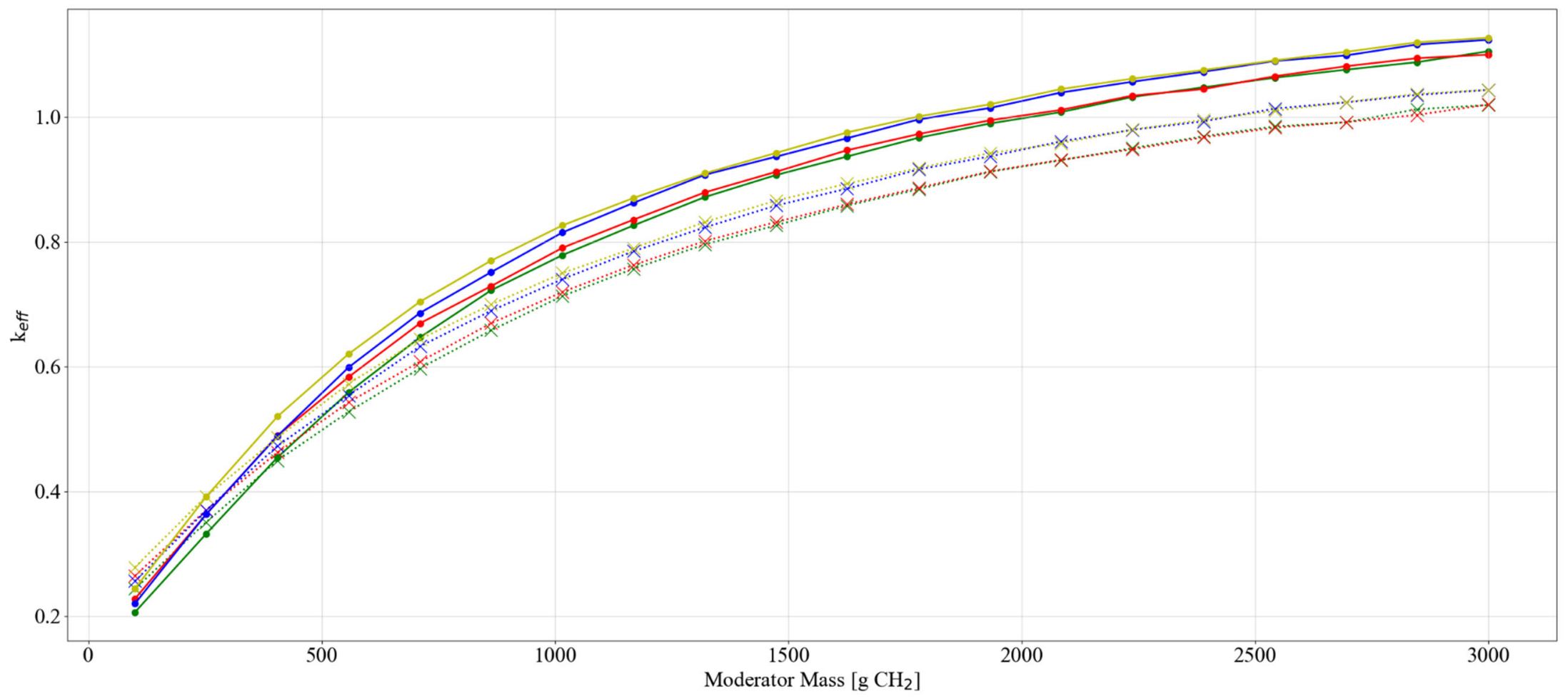

$\rightarrow$ set-2-uh, no filler, no Be, thick SS discrete reflector set-2-uh, $5 \mathrm{~kg}$ graphite/can, no Be, thin SS discrete reflector

- set-2-uh, no filler, $585 \mathrm{~g} \mathrm{Be}$, thin SS discrete reflector

…… set-2-uh, $5 \mathrm{~kg}$ graphite/can, no Be, thick SS discrete reflector

$\longrightarrow$ set-2-uh, no filler, $585 \mathrm{~g} \mathrm{Be}$, thick SS discrete reflector

…... set-2-uh, $5 \mathrm{~kg}$ graphite/can, $585 \mathrm{~g} \mathrm{Be}$, thin SS discrete reflector

Figure C-10. Set-2-uh results, plot 9: reactivity effect of various parameters with $7.7 \mathrm{~cm}$ cylinder radius, graphite filler, poly moderated. 


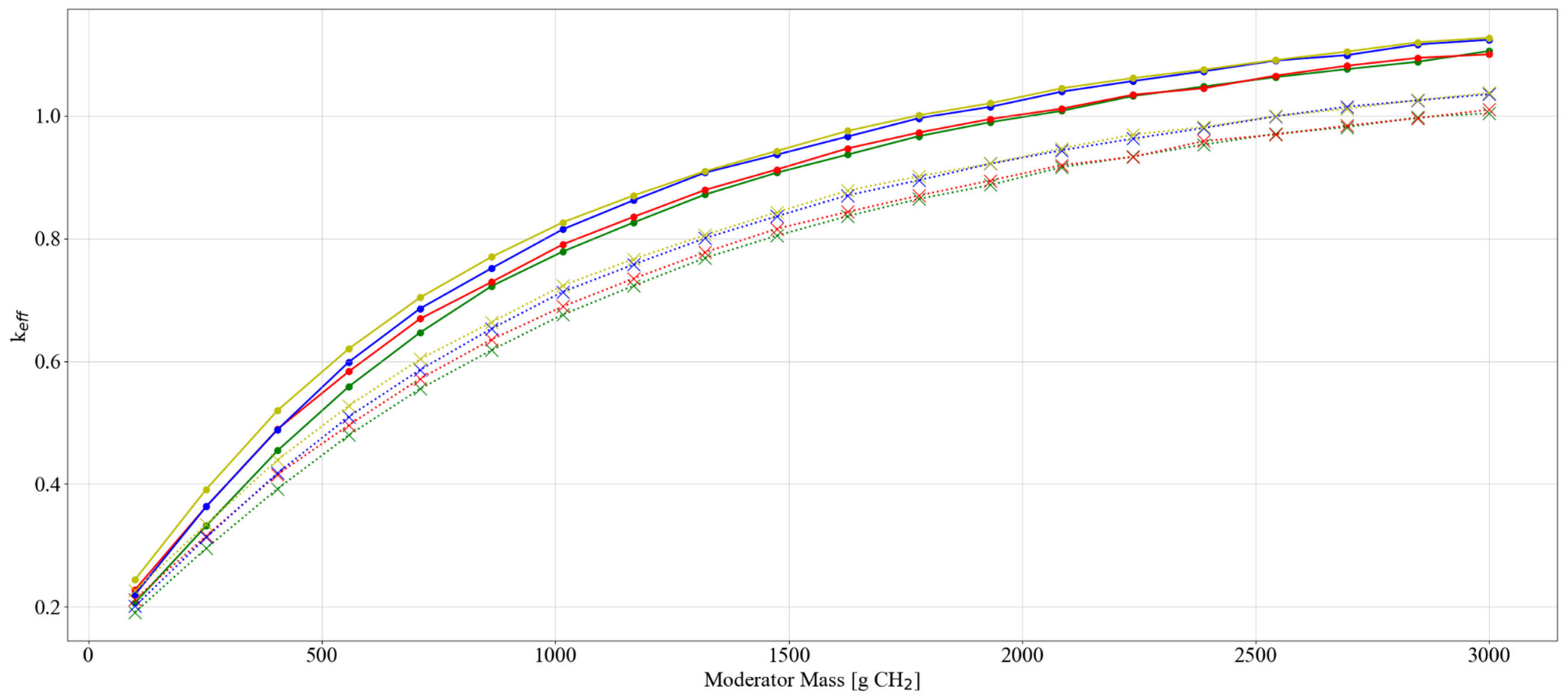

… set-2-uh, $5 \mathrm{~kg}$ generic/can, no Be, thin SS discrete reflector

$\longrightarrow$ set-2-uh, no filler, $585 \mathrm{~g} \mathrm{Be}$, thin SS discrete reflector

$\longrightarrow$ set-2-uh, no filler, no Be, thick SS discrete reflector

........ set-2-uh, $5 \mathrm{~kg}$ generic/can, no Be, thick SS discrete reflector

$\longrightarrow$ set-2-uh, no filler, $585 \mathrm{~g} \mathrm{Be}$, thick SS discrete reflector

…... set-2-uh, $5 \mathrm{~kg}$ generic/can, $585 \mathrm{~g} \mathrm{Be}$, thin SS discrete $\mathrm{r}$ reflector

(x). set-2-uh, $5 \mathrm{~kg}$ generic/can, $585 \mathrm{~g} \mathrm{Be}$, thick SS discrete reflector

Figure C-11. Set-2-uh results, plot 10: reactivity effect of various parameters with $7.7 \mathrm{~cm}$ cylinder radius, generic filler, poly moderated. 


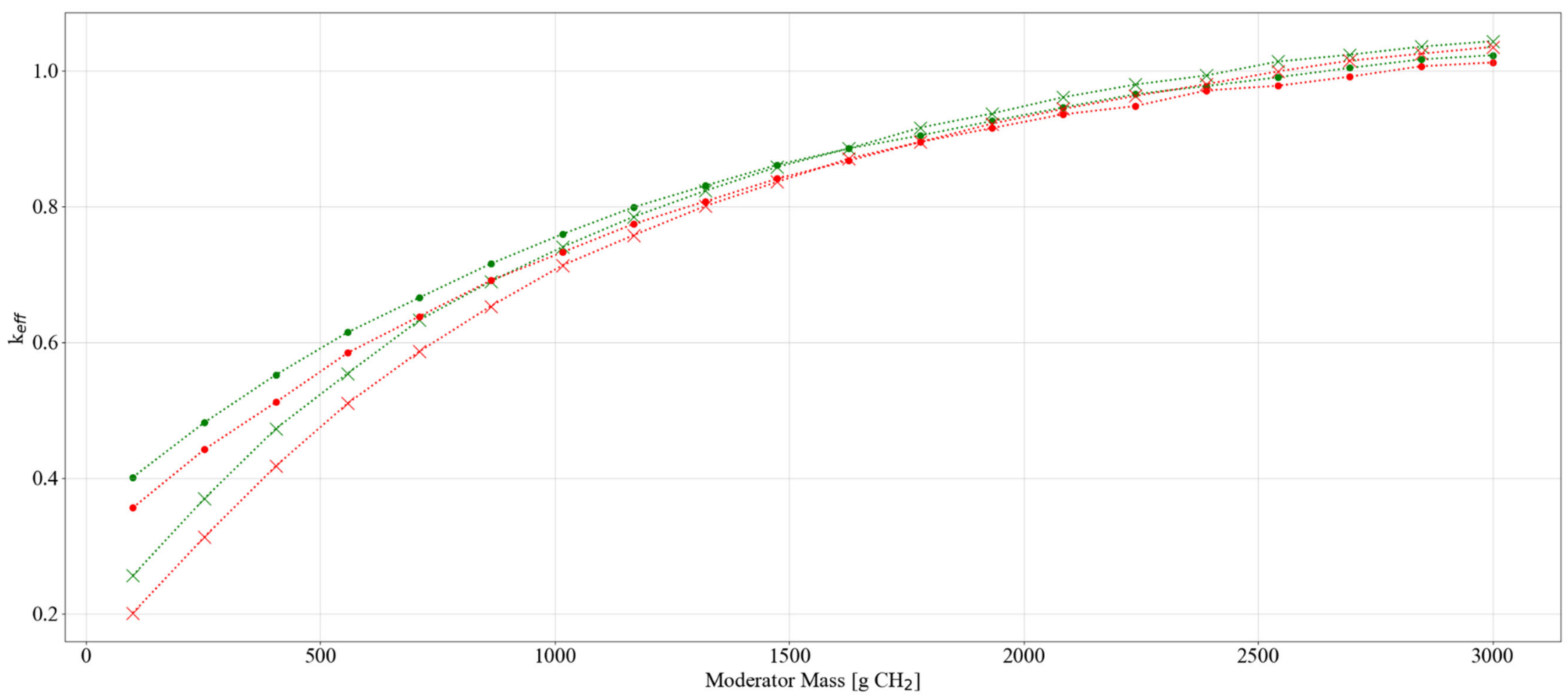

…... set-2-uh, cylinder radius $=7.7 \mathrm{~cm}$, poly moderator, $5 \mathrm{~kg}$ graphite $/ \mathrm{can}$, no Be, thick SS discrete reflector

$\cdots \cdot \cdots$ set-2-uh, cylinder radius $=7.7 \mathrm{~cm}$, poly moderator, $5 \mathrm{~kg}$ graphite $/ \mathrm{can}$, no Be, thick poly discrete reflector

$\cdots \times$ set-2-uh, cylinder radius $=7.7 \mathrm{~cm}$, poly moderator, $5 \mathrm{~kg}$ generic $/ \mathrm{can}$, no Be, thick $\mathrm{SS}$ discrete reflector

$\cdots \cdots$ set-2-uh, cylinder radius $=7.7 \mathrm{~cm}$, poly moderator, $5 \mathrm{~kg}$ generic $/ \mathrm{can}$, no Be, thick poly discrete reflector

Figure C-12. Set-2-uh results, plot 11: comparison of graphite and generic filler with $7.7 \mathrm{~cm}$ cylinder radius, no Be, poly moderated, thick discrete reflector. 


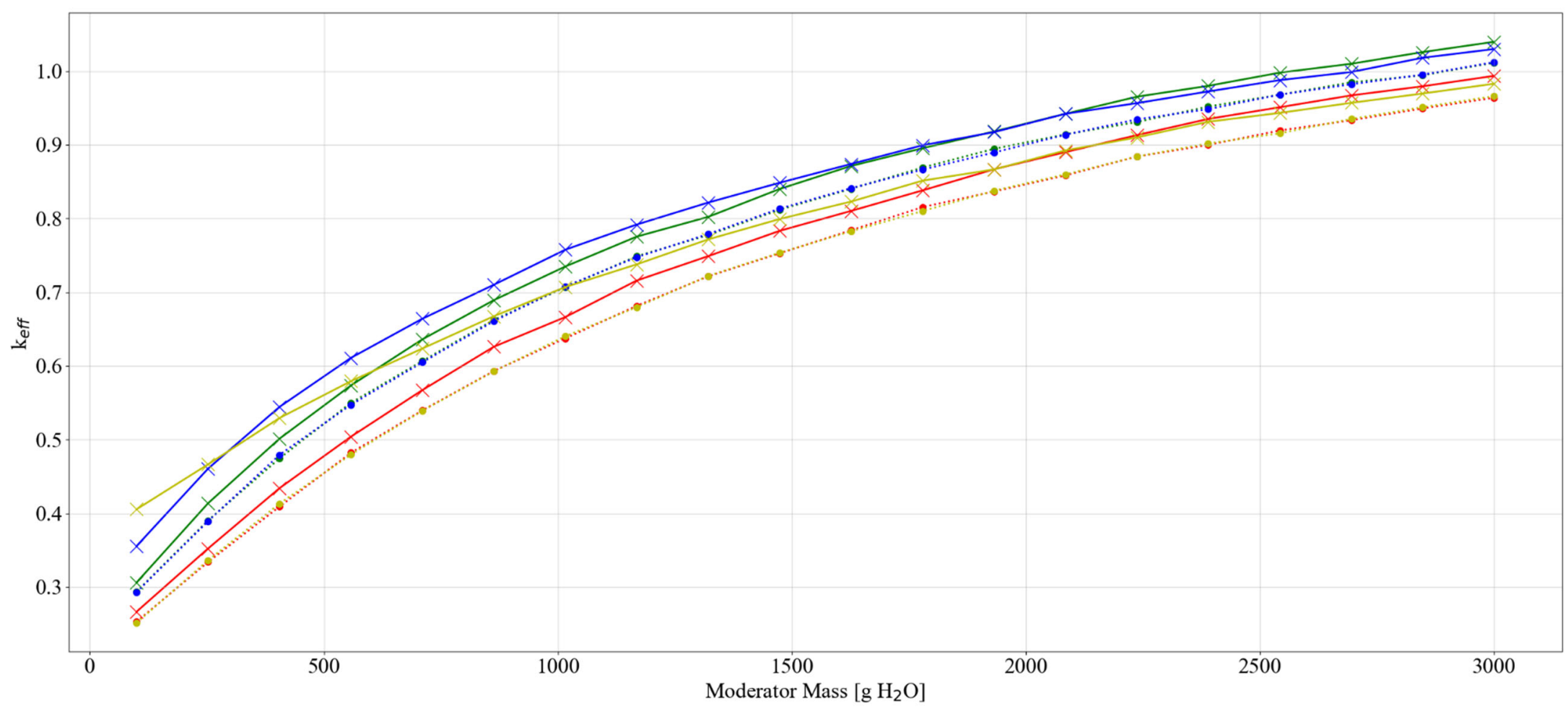

…*... set-2-uh, no filler, no Be, thin SS discrete reflector

…... set-2-uh, no filler, no Be, thin poly discrete reflector

- set-2-uh, no filler, no Be, thick SS discrete reflecto

$\star$ set-2-uh, no filler, no Be, thick poly discrete reflector

….. set-2-uh, $5 \mathrm{~kg}$ graphite/can, $585 \mathrm{~g} \mathrm{Be}$, thin SS discrete reflector

$\succ$ set-2-uh, $5 \mathrm{~kg}$ graphite/can, $585 \mathrm{~g} \mathrm{Be}$, thick SS discrete reflector

-... set-2-uh, $5 \mathrm{~kg}$ graphite/can, $585 \mathrm{~g} \mathrm{Be}$, thin poly discrete reflector

$\times$ set-2-uh, $5 \mathrm{~kg}$ graphite/can, $585 \mathrm{~g} \mathrm{Be}$, thick poly discrete reflector

Figure C-13. Set-2-uh results, plot 12: reactivity effect of various parameters with spherical waste form geometry, graphite filler, water moderated. 


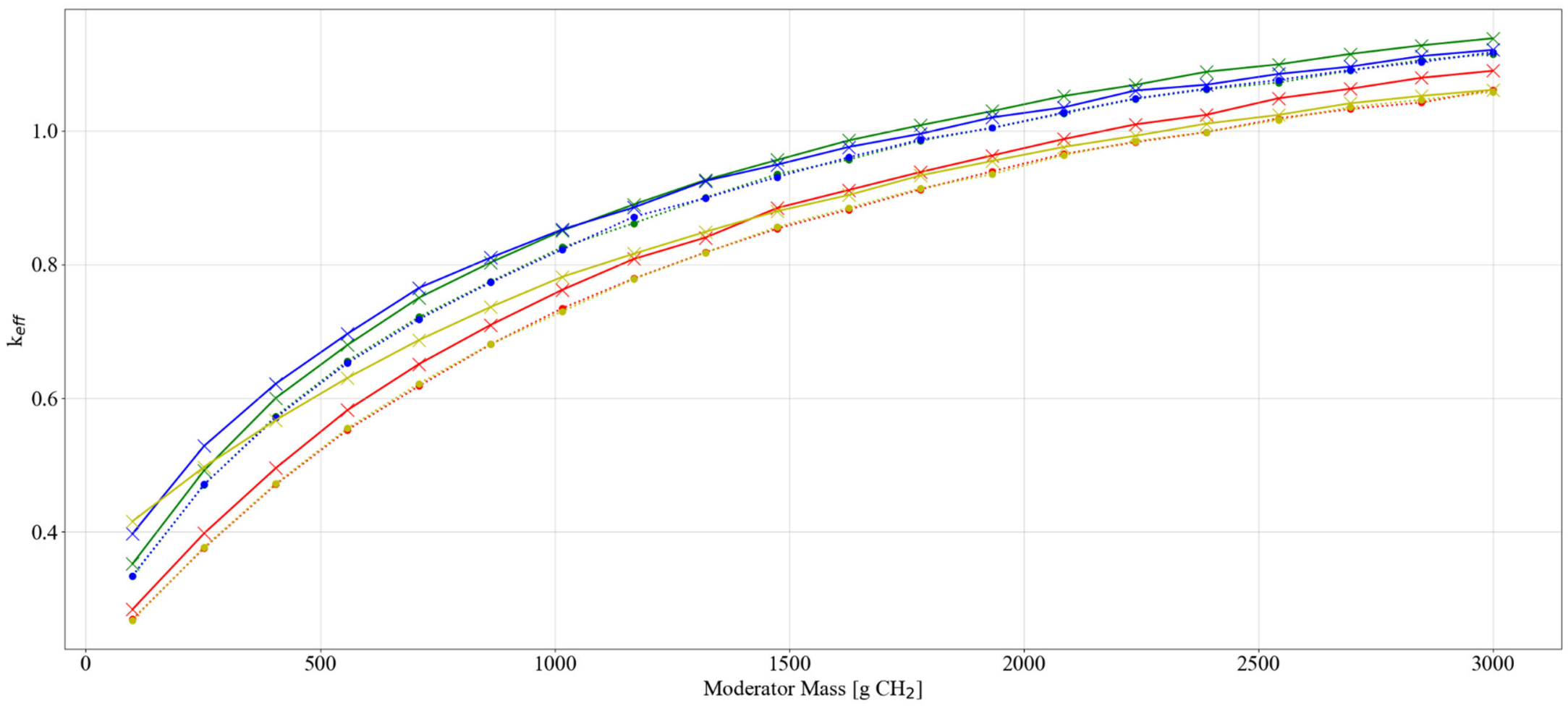

…... set-2-uh, no filler, no Be, thin SS discrete reflector

…... set-2-uh, no filler, no Be, thin poly discrete reflector

set-2-uh, no filler, no Be, thick SS discrete reflecto

* set-2-uh, no filler, no Be, thick poly discrete reflector

-... set-2-uh, $5 \mathrm{~kg}$ graphite/can, $585 \mathrm{~g} \mathrm{Be}$, thin SS discrete reflector

set-2-uh, $5 \mathrm{~kg}$ graphite/can, $585 \mathrm{~g} \mathrm{Be}$, thick SS discrete reflector

- s... set-2-uh, $5 \mathrm{~kg}$ graphite/can, $585 \mathrm{~g} \mathrm{Be}$, thin poly discrete reflector

$\times$ set-2-uh, $5 \mathrm{~kg}$ graphite/can, $585 \mathrm{~g} \mathrm{Be}$, thick poly discrete reflector

Figure C-14. Set-2-uh results, plot 13: reactivity effect of various parameters with spherical waste form geometry, graphite filler, poly moderated. 


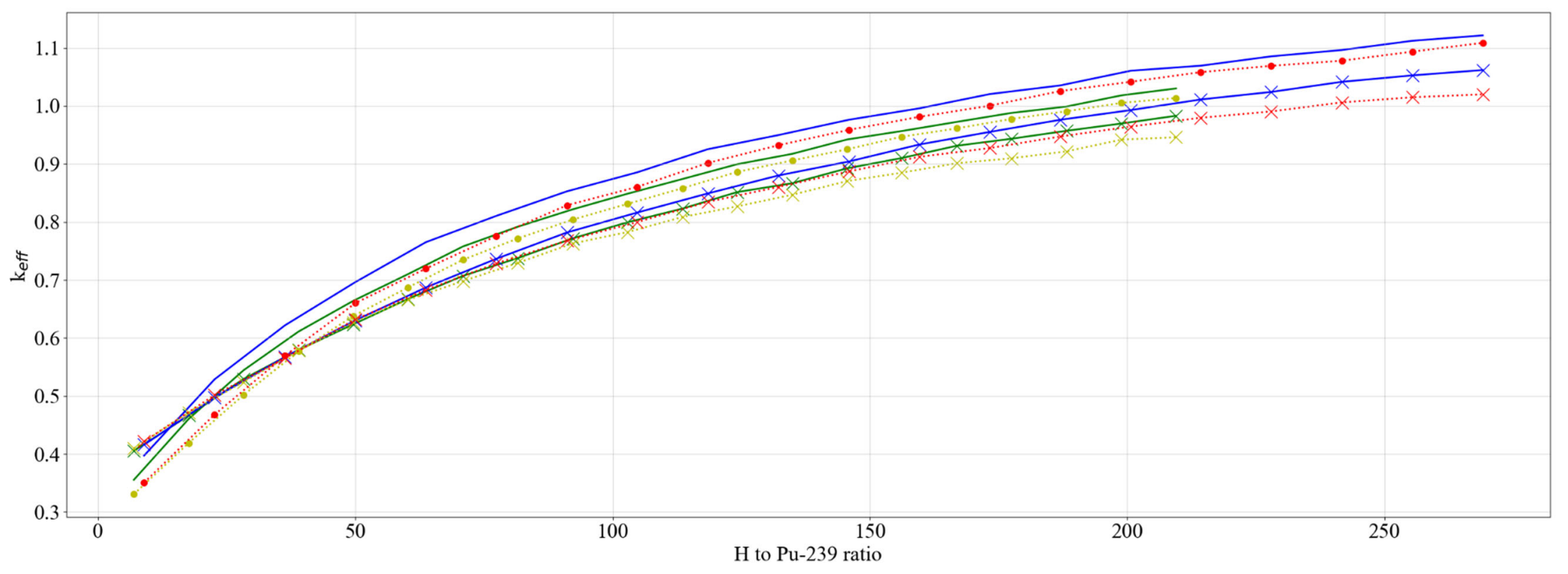

- set-2-uh, sphere, no filler, no Be, thick poly discrete reflector, water moderator

$\star$ set-2-uh, sphere, $5 \mathrm{~kg}$ graphite/can, $585 \mathrm{~g} \mathrm{Be}$, thick poly discrete reflector, water moderator

- set-2-uh, sphere, no filler, no Be, thick poly discrete reflector, poly moderator

$*$ set-2-uh, sphere, $5 \mathrm{~kg}$ graphite/can, $585 \mathrm{~g} \mathrm{Be}$, thick poly discrete reflector, poly moderator

$\cdots$ set-2-uh, cylinder with radius $=7.7 \mathrm{~cm}$, no filler, no Be, thick poly discrete reflector, water moderator

(X... set-2-uh, cylinder with radius $=7.7 \mathrm{~cm}, 5 \mathrm{~kg}$ graphite $/ \mathrm{can}, 585 \mathrm{~g} \mathrm{Be}$, thick poly discrete reflector, water moderator

…*.. set-2-uh, cylinder with radius $=7.7 \mathrm{~cm}$, no filler, no Be, thick poly discrete reflector, poly moderator

… $\ldots \cdots$ set-2-uh, cylinder with radius $=7.7 \mathrm{~cm}, 5 \mathrm{~kg}$ graphite $/ \mathrm{can}, 585 \mathrm{~g} \mathrm{Be}$, thick poly discrete reflector, poly moderator

Figure C-15. Set-2-uh results, plot 14: comparison of spherical and cylindrical geometries $(\mathbf{h} / \mathbf{x})$. 


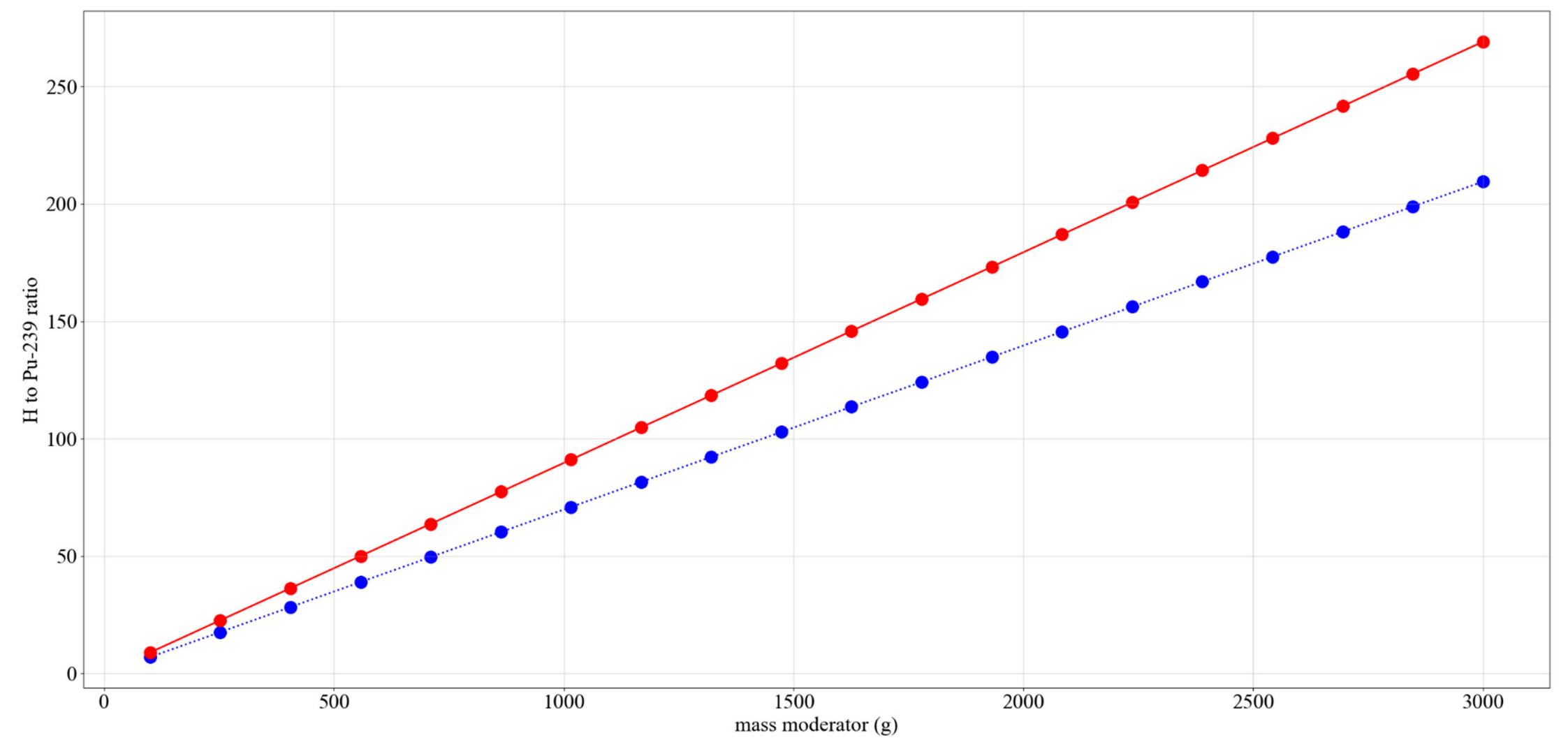

$\cdots$
$-\cdots$
$-\quad$ poly moderator

Figure C-16. Set-2-uh results, plot 15: comparison of water and poly $\mathbf{h} / \mathbf{x}$. 


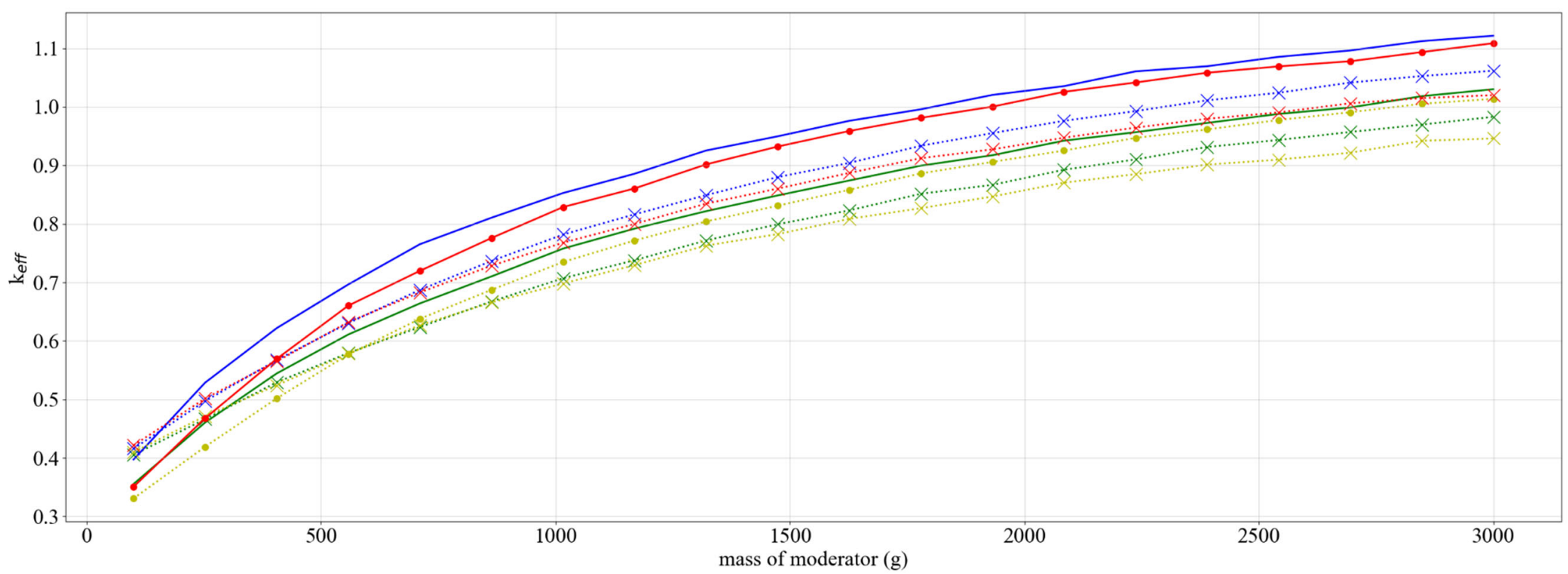

- set-2-uh, sphere, no filler, no Be, thick poly discrete reflector, water moderator

$\cdots \times \cdots$ set-2-uh, sphere, $5 \mathrm{~kg}$ graphite/can, $585 \mathrm{~g} \mathrm{Be}$, thick poly discrete reflector, water moderator

- set-2-uh, sphere, no filler, no Be, thick poly discrete reflector, poly moderator

...... set-2-uh, sphere, $5 \mathrm{~kg}$ graphite/can, $585 \mathrm{~g} \mathrm{Be}$, thick poly discrete reflector, poly moderator

$\cdots \cdots$ set-2-uh, cylinder with radius $=7.7 \mathrm{~cm}$, no filler, no Be, thick poly discrete reflector, water moderator

(x... set-2-uh, cylinder with radius $=7.7 \mathrm{~cm}, 5 \mathrm{~kg}$ graphite $/ \mathrm{can}, 585 \mathrm{~g} \mathrm{Be}$, thick poly discrete reflector, water moderato

$\longrightarrow$ set-2-uh, cylinder with radius $=7.7 \mathrm{~cm}$, no filler, no $\mathrm{Be}$, thick poly discrete reflector, poly moderator

$\cdots \times \cdots$ set-2-uh, cylinder with radius $=7.7 \mathrm{~cm}, 5 \mathrm{~kg}$ graphite $/ \mathrm{can}, 585 \mathrm{~g} \mathrm{Be}$, thick poly discrete reflector, poly moderator

\section{Figure C-17. Set-2-uh results, plot 16: comparison of spherical and cylindrical geometries (mod mass).}




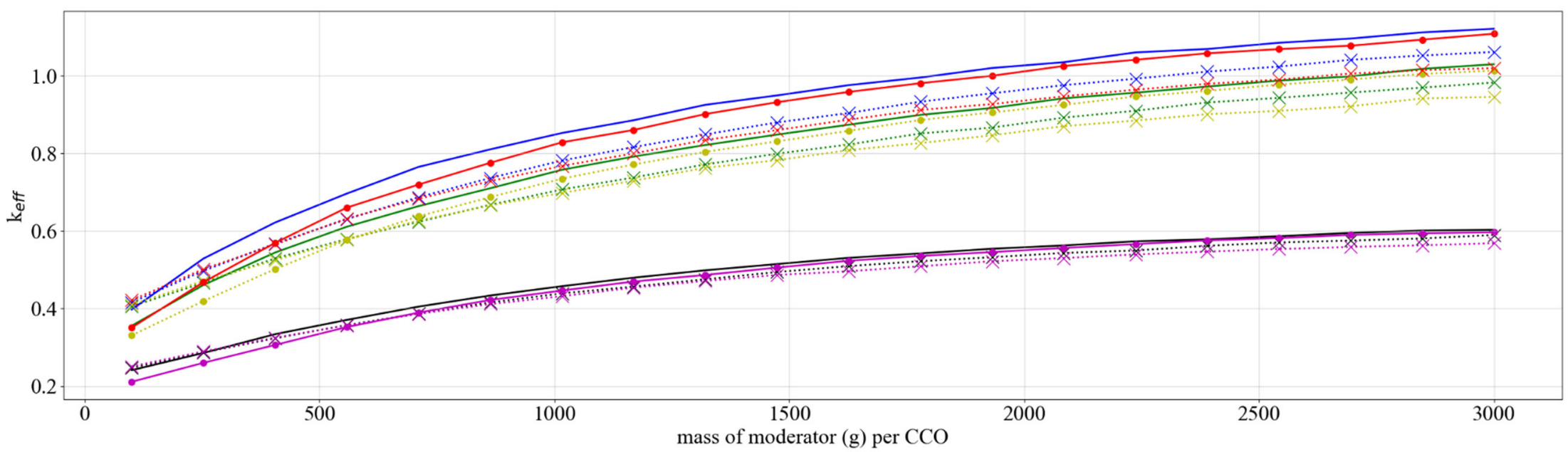

- set-2-uh, sphere, no filler, no Be, thick poly discrete reflector, water moderator

…… set-2-uh, sphere, $5 \mathrm{~kg}$ graphite/can, $585 \mathrm{~g} \mathrm{Be}$, thick poly discrete reflector, water moderator

- set-2-uh, sphere, no filler, no Be, thick poly discrete reflector, poly moderator

… $\ldots$ set-2-uh, sphere, $5 \mathrm{~kg}$ graphite/can, $585 \mathrm{~g} \mathrm{Be}$, thick poly discrete reflector, poly moderator

$\ldots \cdots$ set-2-uh, cylinder with radius $=7.7 \mathrm{~cm}$, no filler, no $\mathrm{Be}$, thick poly discrete reflector, water moderator

… $\times$ set-2-uh, cylinder with radius $=7.7 \mathrm{~cm}, 5 \mathrm{~kg}$ graphite $/ \mathrm{can}, 585 \mathrm{~g} \mathrm{Be}$, thick poly discrete reflector, water moderator

$\rightarrow$ set-2-uh, cylinder with radius $=7.7 \mathrm{~cm}$, no filler, no Be, thick poly discrete reflector, poly moderator

$\cdots \times \cdots$ set-2-uh, cylinder with radius $=7.7 \mathrm{~cm}, 5 \mathrm{~kg}$ graphite $/ \mathrm{can}, 585 \mathrm{~g} \mathrm{Be}$, thick poly discrete reflector, poly moderator

- set-2-uh, sphere, $50 \mathrm{~g} \mathrm{~B} 4 \mathrm{C}$, no filler, no Be, thick poly discrete reflector, poly moderator

…… set-2-uh, sphere, $50 \mathrm{~g} \mathrm{B4C,} 5 \mathrm{~kg}$ graphite/can, $585 \mathrm{~g} \mathrm{Be}$, thick poly discrete reflector, poly moderator

$\longrightarrow$ set-2-uh, cylinder with radius $=7.7 \mathrm{~cm}, 50 \mathrm{~g} \mathrm{~B} 4 \mathrm{C}$, no filler, no Be, thick poly discrete reflector, poly moderator

…… set-2-uh, cylinder with radius $=7.7 \mathrm{~cm}, 50 \mathrm{~g} \mathrm{~B} 4 \mathrm{C}, 5 \mathrm{~kg}$ graphite $/ \mathrm{can}, 585 \mathrm{~g} \mathrm{Be}$, thick poly discrete reflector, poly moderator

Figure C-18. Set-2-uh results, plot 17: comparison of $50 \mathrm{~g} \mathrm{~B}_{4} \mathrm{C}$ vs. no $\mathrm{B}_{4} \mathrm{C}$ for spherical and cylindrical geometries (mod mass). 
APPENDIX D. SET-2-LH: RESULTS OF THE NONUNIFORM ARRAY FOR THE LOWER HORIZON CALCULATIONS 
This page is intentionally blank 


\section{APPENDIX D. SET-2-LH: RESULTS OF THE NONUNIFORM ARRAY FOR THE LOWER HORIZON CALCULATIONS}

The analysis methodology for the uniform arrays is discussed in detail in Section 6.3 of the main report.

This appendix serves as a repository of those results for the set-2-lh calculations.

The complete results for all SAMPLER sweeps are provided in ADDENDUM 1.

The analysis model use for the calculations in this appendix is shown in Figure D-1 below.

The SAMPLER case sweeps presented in this appendix are summarized in Table D-1 below.

\section{LIST OF FIGURES}

Figure D-1. Diagram of the nonuniform array three-high model. D-5

Figure D-2. Set-2-lh results, plot 1: reactivity effect of cylinder radius, pipe steel, no filler, no $\mathrm{Be}$, water moderated.

Figure D-3. Set-2-lh results, plot 2: reactivity effect of cylinder radius, pipe steel, no filler, no Be, poly moderated.

Figure D-4. Set-2-lh results, plot 3: reactivity effect of cylinder radius, pipe poly, no filler, no Be,

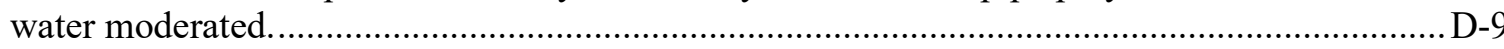

Figure D-5. Set-2-lh results, plot 4: reactivity effect of cylinder radius, pipe poly, no filler, no Be,

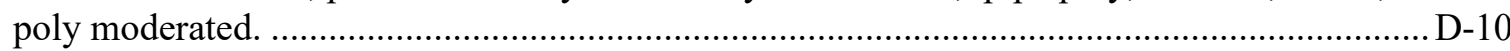

Figure D-6. Set-2-lh results, plot 5: reactivity effect of cylinder radius, pipe steel, $5 \mathrm{~kg}$

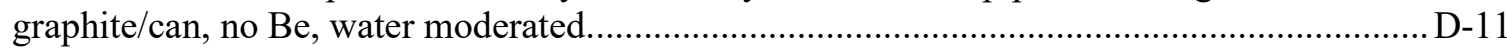

Figure D-7. Set-2-1h results, plot 6: reactivity effect of cylinder radius, pipe steel, $5 \mathrm{~kg}$ graphite/can, no Be, poly moderated.

Figure D-8. Set-2-lh results, plot 7: reactivity effect of cylinder radius, pipe poly, $5 \mathrm{~kg}$ graphite/can, no Be, water moderated

Figure D-9. Set-2-lh results, plot 8: reactivity effect of cylinder radius, pipe poly, $5 \mathrm{~kg}$ graphite/can, no Be, poly moderated.

Figure D-10. Set-2-1h results, plot 9: reactivity effect of various parameters with 7.7 cm cylinder radius, graphite filler, poly moderated.

Figure D-11. Set-2-lh results, plot 10: reactivity effect of various parameters with $7.7 \mathrm{~cm}$ cylinder radius, generic filler, poly moderated.

Figure D-12. Set-2-1h results, plot 11: comparison of graphite and generic filler with $7.7 \mathrm{~cm}$ cylinder radius, no Be, poly moderated, thick discrete reflector.

Figure D-13. Set-2-lh results, plot 12: reactivity effect of various parameters with spherical waste form geometry, graphite filler, water moderated....

Figure D-14. Set-2-lh results, plot 13: reactivity effect of various parameters with spherical waste form geometry, graphite filler, poly moderated.

Figure D-15. Set-2-1h results, plot 14: comparison of spherical and cylindrical geometries $(\mathrm{h} / \mathrm{x}) \ldots \ldots \ldots . \mathrm{D}-20$

Figure D-16. Set-2-lh results, plot 15: comparison of water and poly $\mathrm{h} / \mathrm{x}$.....

Figure D-17. Set-2-lh results, plot 16: comparison of spherical and cylindrical geometries (mod mass).

Figure D-18. Set-2-lh results, plot 17: comparison of $50 \mathrm{~g} \mathrm{~B} \mathrm{~B}_{4} \mathrm{C}$ vs. no $\mathrm{B}_{4} \mathrm{C}$ for spherical and cylindrical geometries (mod mass). 


\section{LIST OF TABLES}

Table D-1. Summary of cases for Set-2-uh for the lower horizon compaction data.............................. D-6 


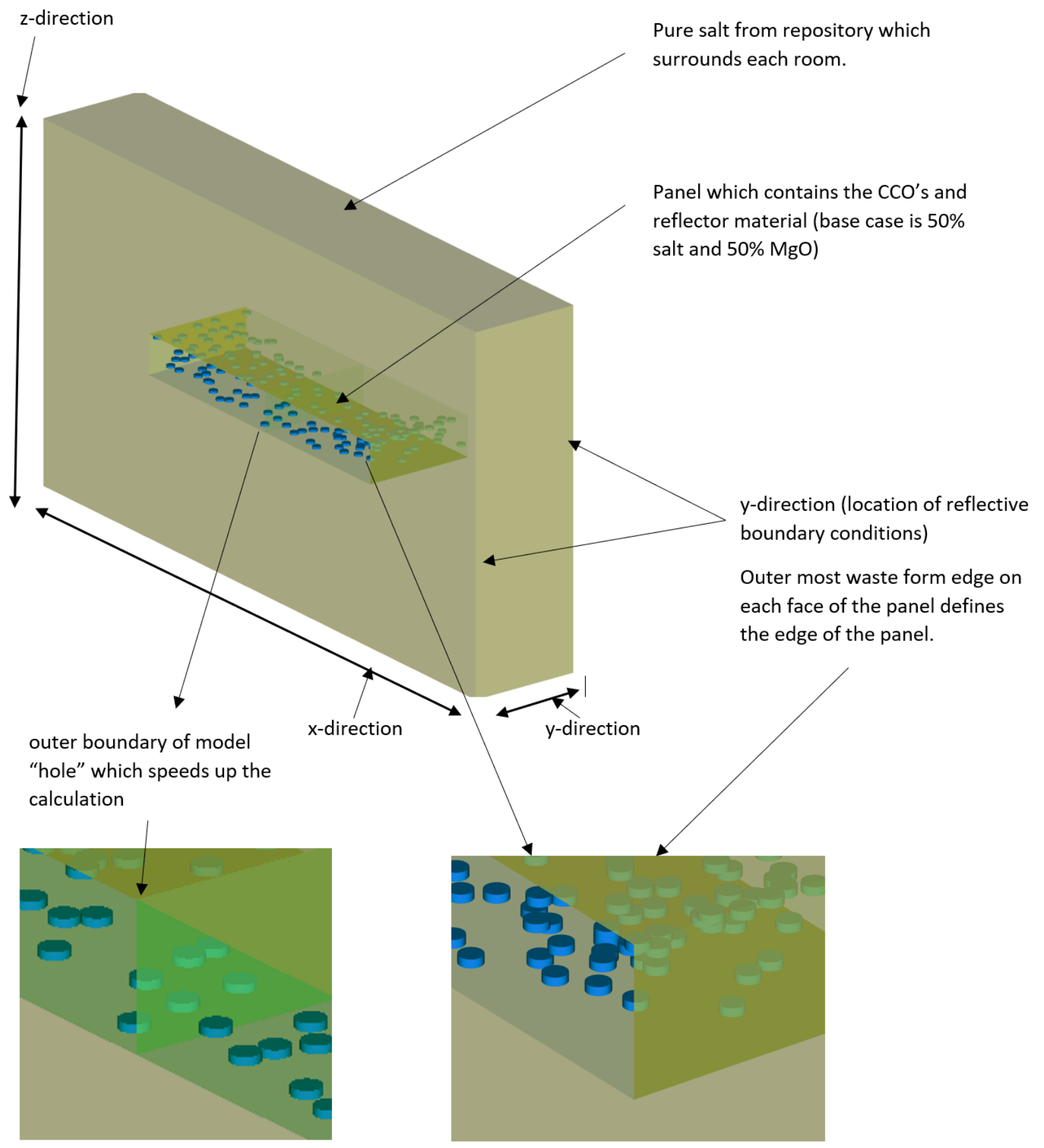

Figure D-1. Diagram of the nonuniform array three-high model. 
Table D-1. Summary of cases for Set-2-uh for the lower horizon compaction data

\begin{tabular}{|c|c|c|c|c|c|c|c|c|}
\hline Case & Model type & Waste form shape & $\begin{array}{l}\text { Waste form } \\
\text { moderator }\end{array}$ & $\begin{array}{c}\text { Filler } \\
\text { material }(0, \\
2,000,4,000 \\
\text { g) } \\
\end{array}$ & Metal in filler & $\begin{array}{c}\text { Discrete reflector } \\
\text { (thin } 0.001 \text { and } \\
\text { thick } 0.7112 \mathrm{~cm} \text { ) }\end{array}$ & $\operatorname{Be}(g)$ & Subcase \\
\hline \multirow{18}{*}{ Set-2-lh } & \multirow{18}{*}{$\begin{array}{l}\text { Nonuniform array with } \\
\text { centroids based on upper } \\
\text { horizon data. Centroid } \\
\text { from pipe center is used } \\
\text { as the point in the center } \\
\text { of the base of cylinders } \\
\text { or the center of the } \\
\text { spheres. }\end{array}$} & \multirow{8}{*}{$\begin{array}{l}\text { Cylinder (radius range } \\
4.8,6,7.7 \text { and height } \\
\text { defined by total volume } \\
\text { of mass) }\end{array}$} & water & $\mathrm{c} 12$ & \multirow{18}{*}{$\begin{array}{c}\text { SS from can } \\
(0,500,1,000 \mathrm{~g})\end{array}$} & steel & \multirow{18}{*}{$\begin{array}{l}0 \text { to } \\
585\end{array}$} & set-2-lh-1 \\
\hline & & & poly & $\mathrm{c} 12$ & & steel & & set-2-lh-2 \\
\hline & & & water & $\mathrm{c} 12$ & & poly & & set-2-lh-3 \\
\hline & & & poly & $\mathrm{c} 12$ & & poly & & \begin{tabular}{|l|} 
set-2-lh-4 \\
\end{tabular} \\
\hline & & & water & generic & & steel & & set-2-lh-5 \\
\hline & & & poly & generic & & steel & & set-2-lh-6 \\
\hline & & & water & generic & & poly & & \begin{tabular}{|l|} 
set-2-1h-7 \\
\end{tabular} \\
\hline & & & poly & generic & & poly & & \begin{tabular}{|l|} 
set-2-lh-8 \\
\end{tabular} \\
\hline & & \multirow{10}{*}{$\begin{array}{c}\text { Sphere (radius defined } \\
\text { by total volume of mass) }\end{array}$} & water & $\mathrm{c} 12$ & & steel & & set-2-lh-9 \\
\hline & & & poly & $\mathrm{c} 12$ & & steel & & \begin{tabular}{|l} 
set-2-lh-10 \\
\end{tabular} \\
\hline & & & water & $\mathrm{c} 12$ & & poly & & \begin{tabular}{|l} 
set-2-lh-11 \\
\end{tabular} \\
\hline & & & poly & $\mathrm{c} 12$ & & poly & & \begin{tabular}{|l} 
set-2-lh-12 \\
\end{tabular} \\
\hline & & & water & generic & & steel & & set-2-lh-13 \\
\hline & & & poly & generic & & steel & & \begin{tabular}{|l} 
set-2-lh-14 \\
\end{tabular} \\
\hline & & & water & generic & & poly & & \begin{tabular}{|l} 
set-2-lh-15 \\
\end{tabular} \\
\hline & & & poly & generic & & poly & & set-2-lh-16 \\
\hline & & & $\begin{array}{c}\text { poly }+50 \mathrm{~g} \mathrm{~B} \mathrm{~B}_{4} \mathrm{C} \\
(\mathrm{cyl})\end{array}$ & $\mathrm{c} 12$ & & poly $(0.7112)$ & & set-2-lh-17 \\
\hline & & & $\begin{array}{c}\text { poly }+50 \mathrm{~g} \mathrm{~B} \mathrm{~B}_{4} \mathrm{C} \\
(\mathrm{sph})\end{array}$ & $\mathrm{c} 12$ & & poly $(0.7112)$ & & set-2-lh-18 \\
\hline
\end{tabular}




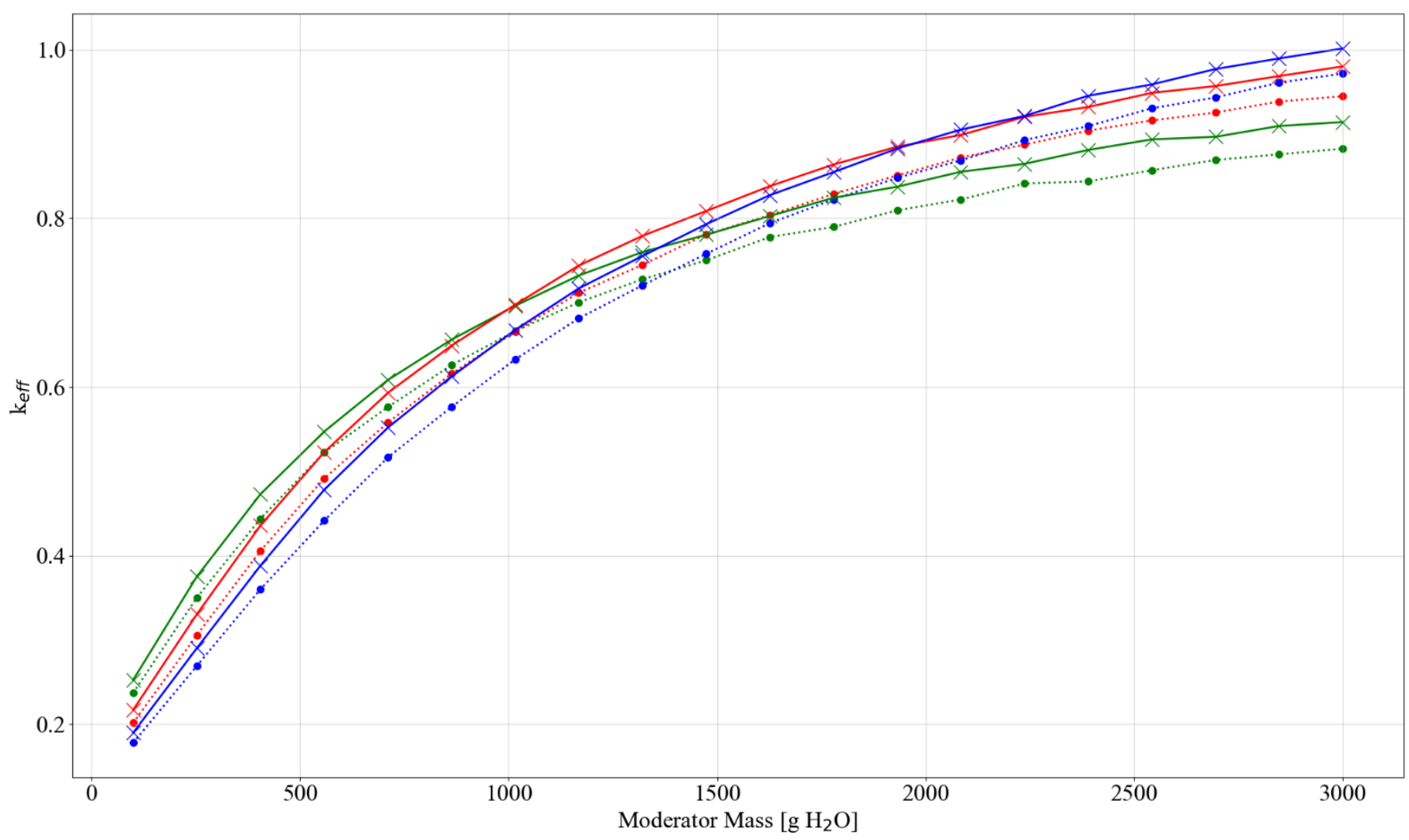

- ... set-2-lh, cyl radius $=4.8 \mathrm{~cm}$, thin discrete reflector

$\cdots \cdots \quad$ set-2-lh, cyl radius $=7.7 \mathrm{~cm}$, thin discrete reflector

set-2-lh, cyl radius $=6.25 \mathrm{~cm}$, thick discrete reflector set-2-lh, cyl radius $=6.25 \mathrm{~cm}$, thin discrete reflector set- 2 -lh, cyl radius $=4.8 \mathrm{~cm}$, thick discrete reflector set- 2 - $\mathrm{lh}$, cyl radius $=7.7 \mathrm{~cm}$, thick discrete reflector

Figure D-2. Set-2-lh results, plot 1: reactivity effect of cylinder radius, pipe steel, no filler, no Be, water moderated. 


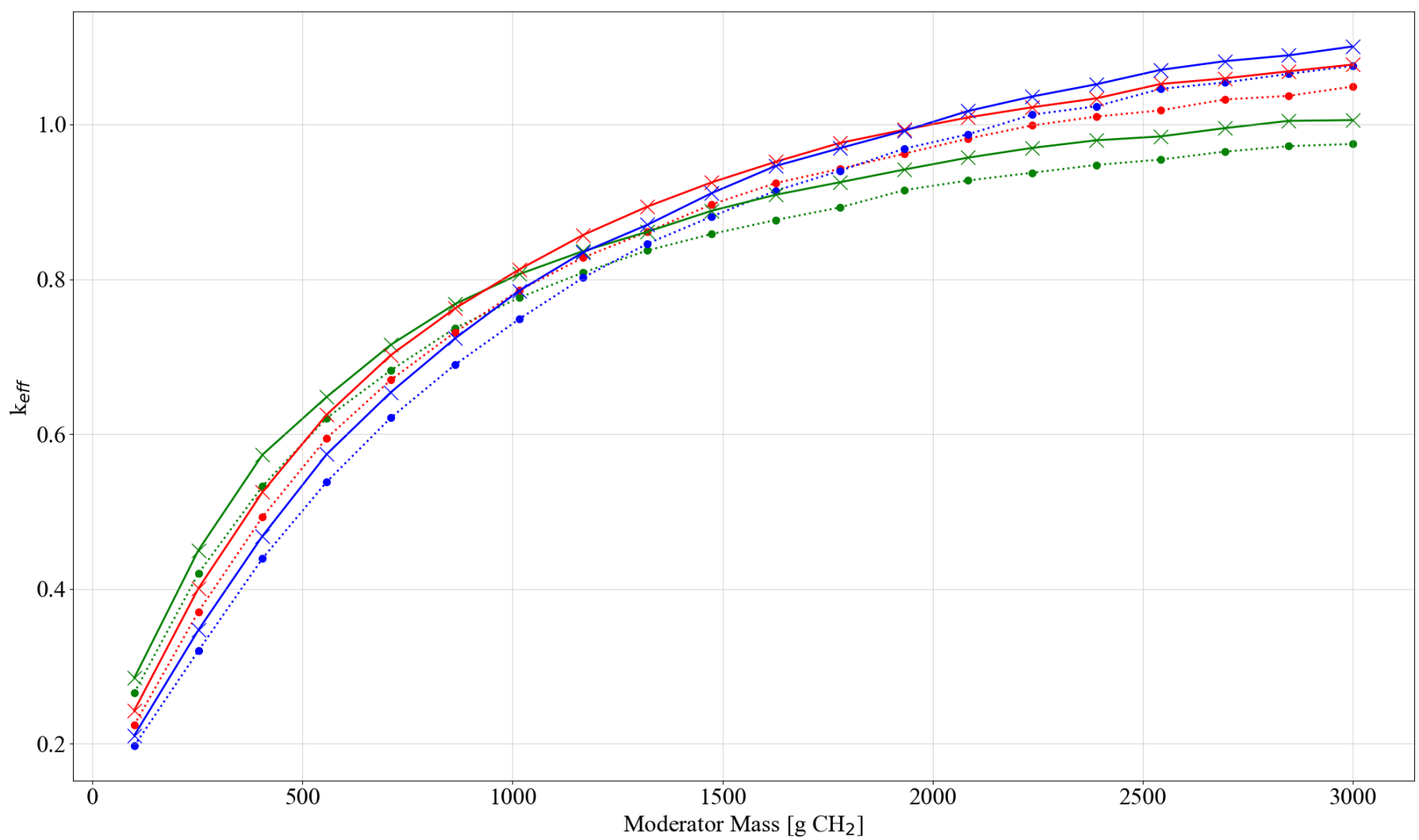

- ... set-2-lh, cyl radius $=4.8 \mathrm{~cm}$, thin discrete reflector

-.. set-2-lh, cyl radius $=7.7 \mathrm{~cm}$, thin discrete reflector

$*$ set-2-lh, cyl radius $=6.25 \mathrm{~cm}$, thick discrete reflector set-2-lh, cyl radius $=6.25 \mathrm{~cm}$, thin discrete reflector

$\star$ set-2-lh, cyl radius $=4.8 \mathrm{~cm}$, thick discrete reflector

set-2-lh, cyl radius $=7.7 \mathrm{~cm}$, thick discrete reflector

Figure D-3. Set-2-lh results, plot 2: reactivity effect of cylinder radius, pipe steel, no filler, no Be, poly moderated. 


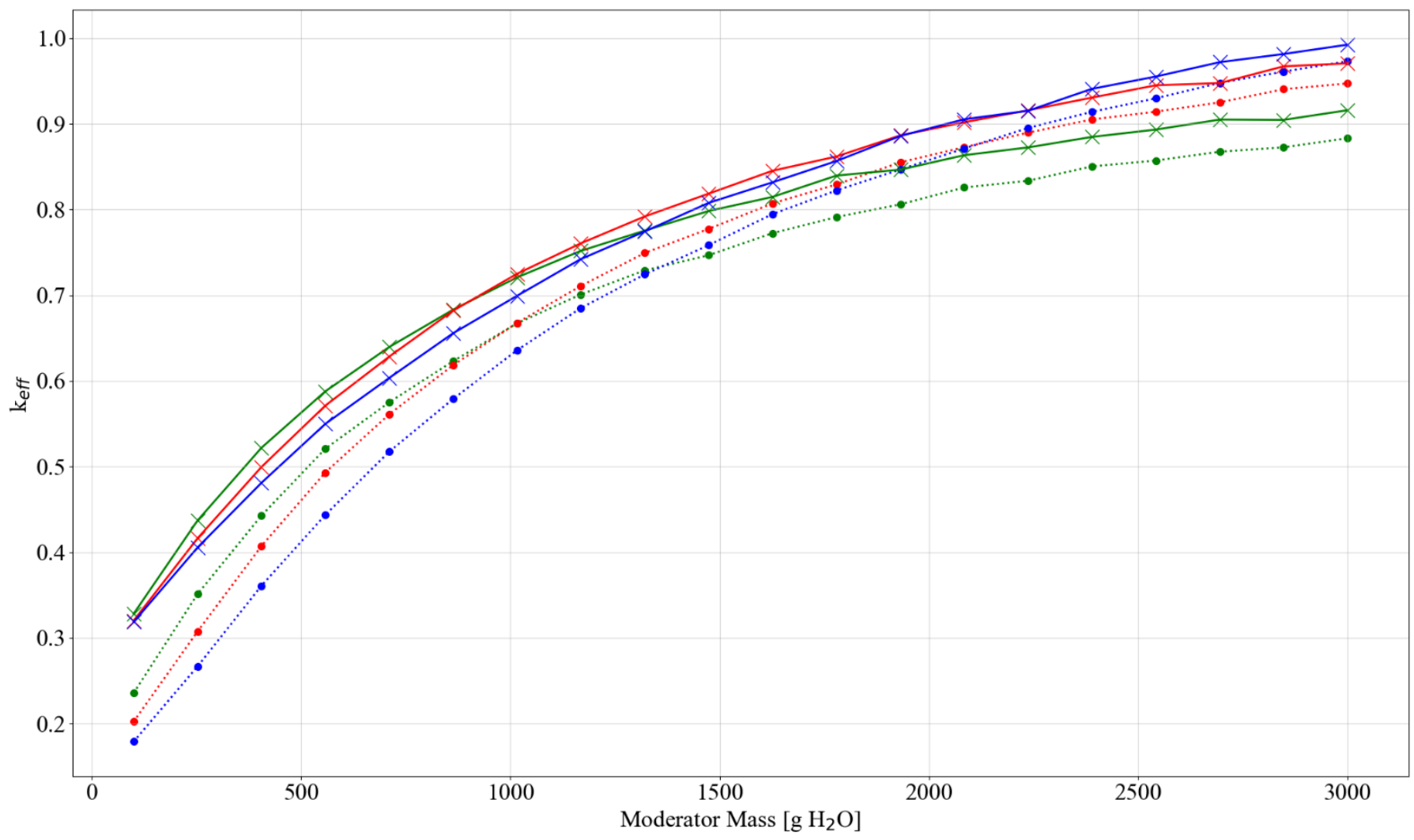

- ... set-2-lh, cyl radius $=4.8 \mathrm{~cm}$, thin discrete reflector

$\cdots \cdot$ set-2-lh, cyl radius $=7.7 \mathrm{~cm}$, thin discrete reflector

set-2-lh, cyl radius $=6.25 \mathrm{~cm}$, thick discrete reflector set-2-lh, cyl radius $=6.25 \mathrm{~cm}$, thin discrete reflector set-2-lh, cyl radius $=4.8 \mathrm{~cm}$, thick discrete reflector set- 2 - $\mathrm{lh}$, cyl radius $=7.7 \mathrm{~cm}$, thick discrete reflector

Figure D-4. Set-2-Ih results, plot 3: reactivity effect of cylinder radius, pipe poly, no filler, no Be, water moderated. 


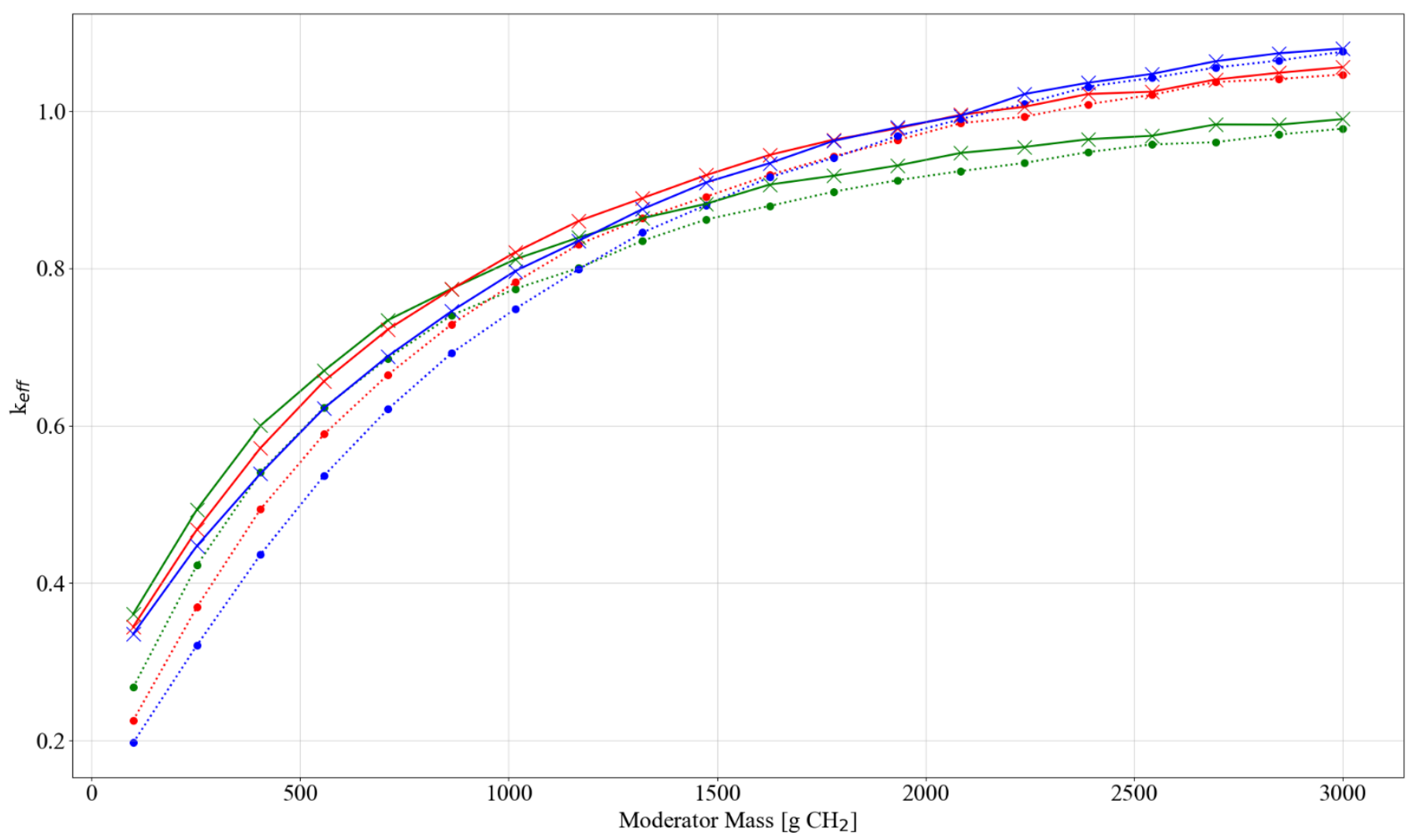

- ... set-2-lh, cyl radius $=4.8 \mathrm{~cm}$, thin discrete reflector

- $\cdots \quad$ set-2-lh, cyl radius $=7.7 \mathrm{~cm}$, thin discrete reflector

set-2-lh, cyl radius $=6.25 \mathrm{~cm}$, thick discrete reflector set-2-lh, cyl radius $=6.25 \mathrm{~cm}$, thin discrete reflector set-2-lh, cyl radius $=4.8 \mathrm{~cm}$, thick discrete reflector set-2-lh, cyl radius $=7.7 \mathrm{~cm}$, thick discrete reflector

Figure D-5. Set-2-lh results, plot 4: reactivity effect of cylinder radius, pipe poly, no filler, no Be, poly moderated. 


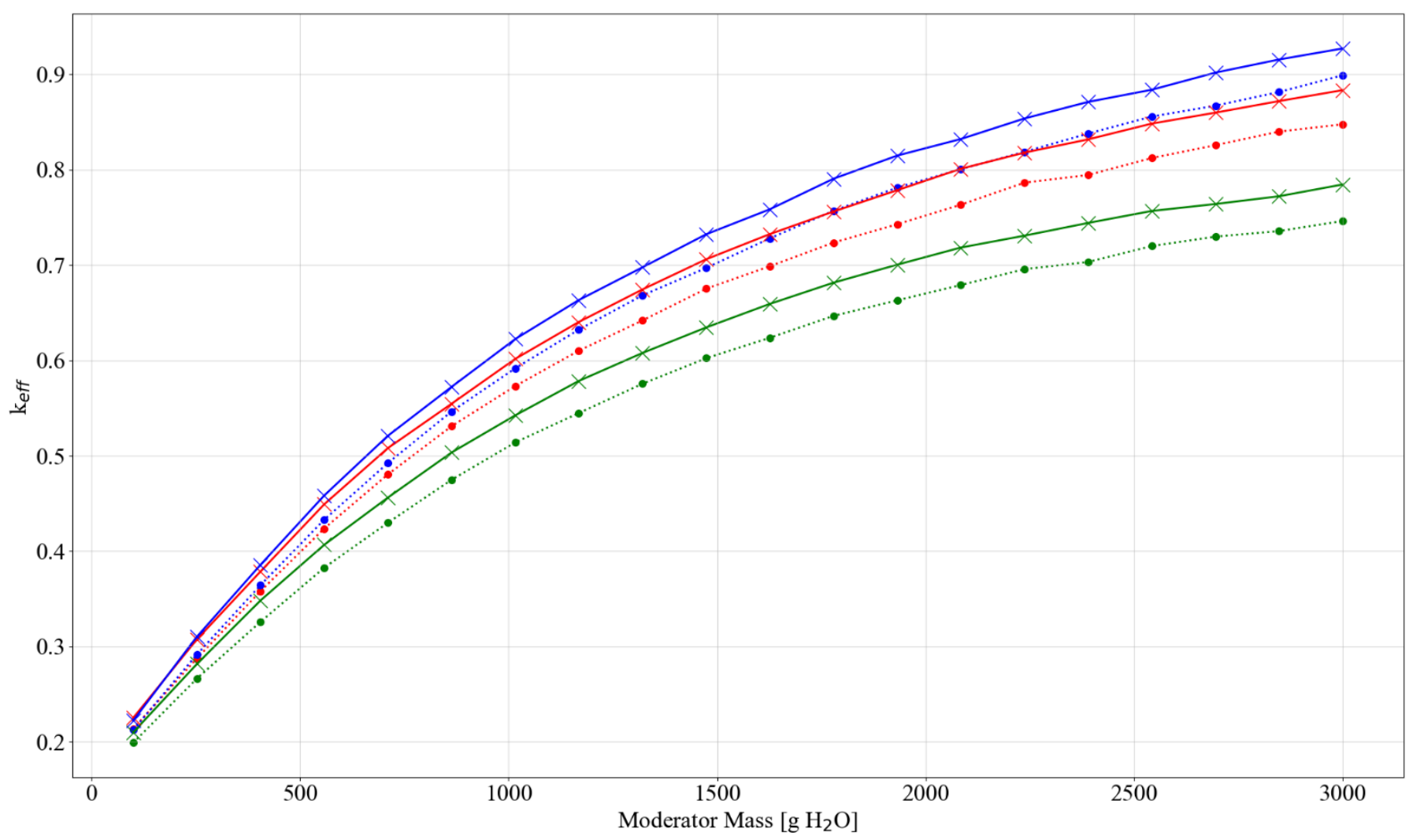

-... set-2-lh, cyl radius $=4.8 \mathrm{~cm}$, thin discrete reflector

$\cdots \cdot$ set-2-lh, cyl radius $=7.7 \mathrm{~cm}$, thin discrete reflector

set-2-lh, cyl radius $=6.25 \mathrm{~cm}$, thick discrete reflector set-2-lh, cyl radius $=6.25 \mathrm{~cm}$, thin discrete reflector set-2-lh, cyl radius $=4.8 \mathrm{~cm}$, thick discrete reflector set- 2 - $\mathrm{lh}$, cyl radius $=7.7 \mathrm{~cm}$, thick discrete reflector

Figure D-6. Set-2-lh results, plot 5: reactivity effect of cylinder radius, pipe steel, $5 \mathrm{~kg}$ graphite/can, no Be, water moderated. 


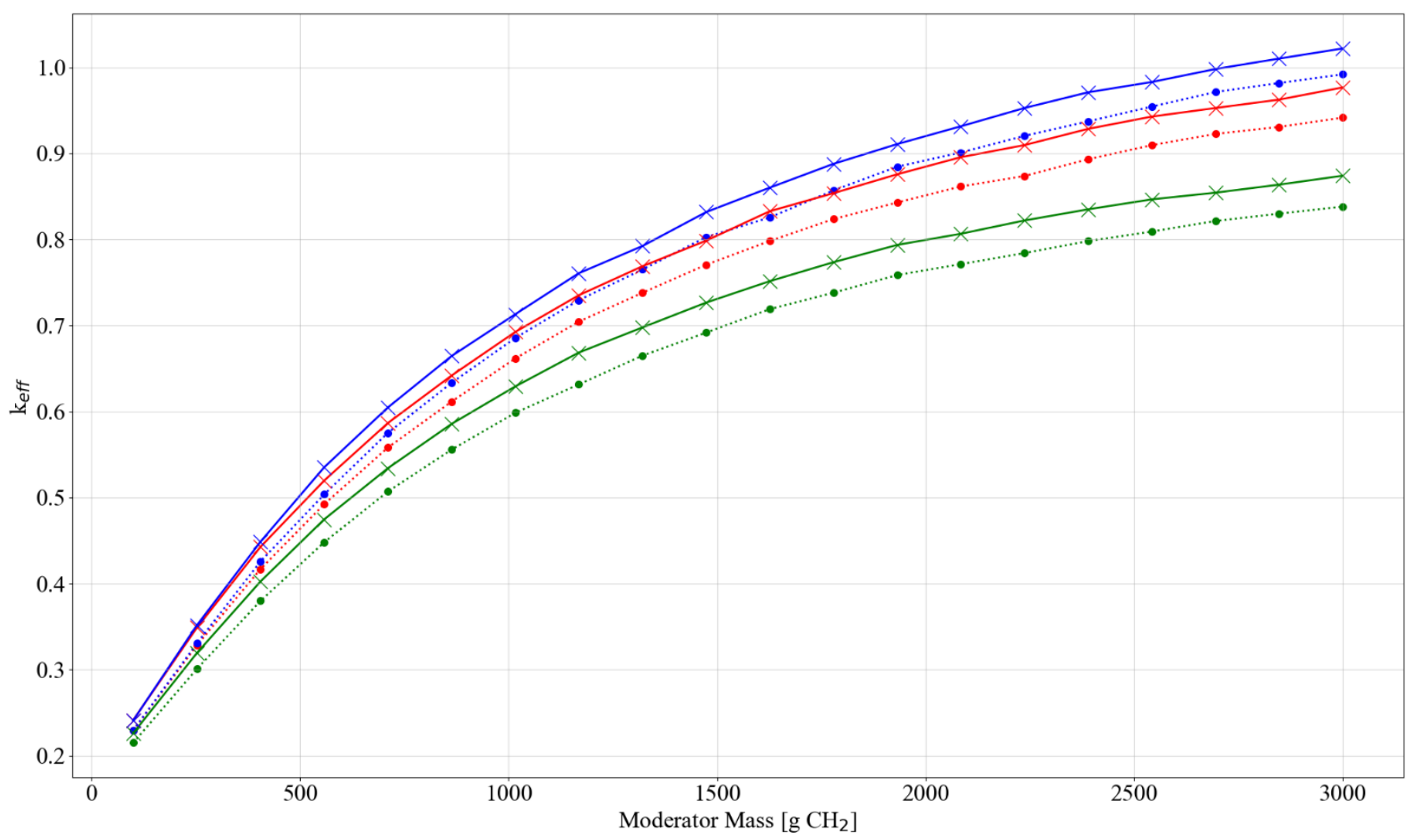

-... set-2-lh, cyl radius $=4.8 \mathrm{~cm}$, thin discrete reflector

$\cdots \cdot$ set-2-lh, cyl radius $=7.7 \mathrm{~cm}$, thin discrete reflector

set-2-lh, cyl radius $=6.25 \mathrm{~cm}$, thick discrete reflector set-2-lh, cyl radius $=6.25 \mathrm{~cm}$, thin discrete reflector set-2-lh, cyl radius $=4.8 \mathrm{~cm}$, thick discrete reflector set- 2 - $\mathrm{lh}$, cyl radius $=7.7 \mathrm{~cm}$, thick discrete reflector

Figure D-7. Set-2-lh results, plot 6: reactivity effect of cylinder radius, pipe steel, $5 \mathrm{~kg}$ graphite/can, no Be, poly moderated. 


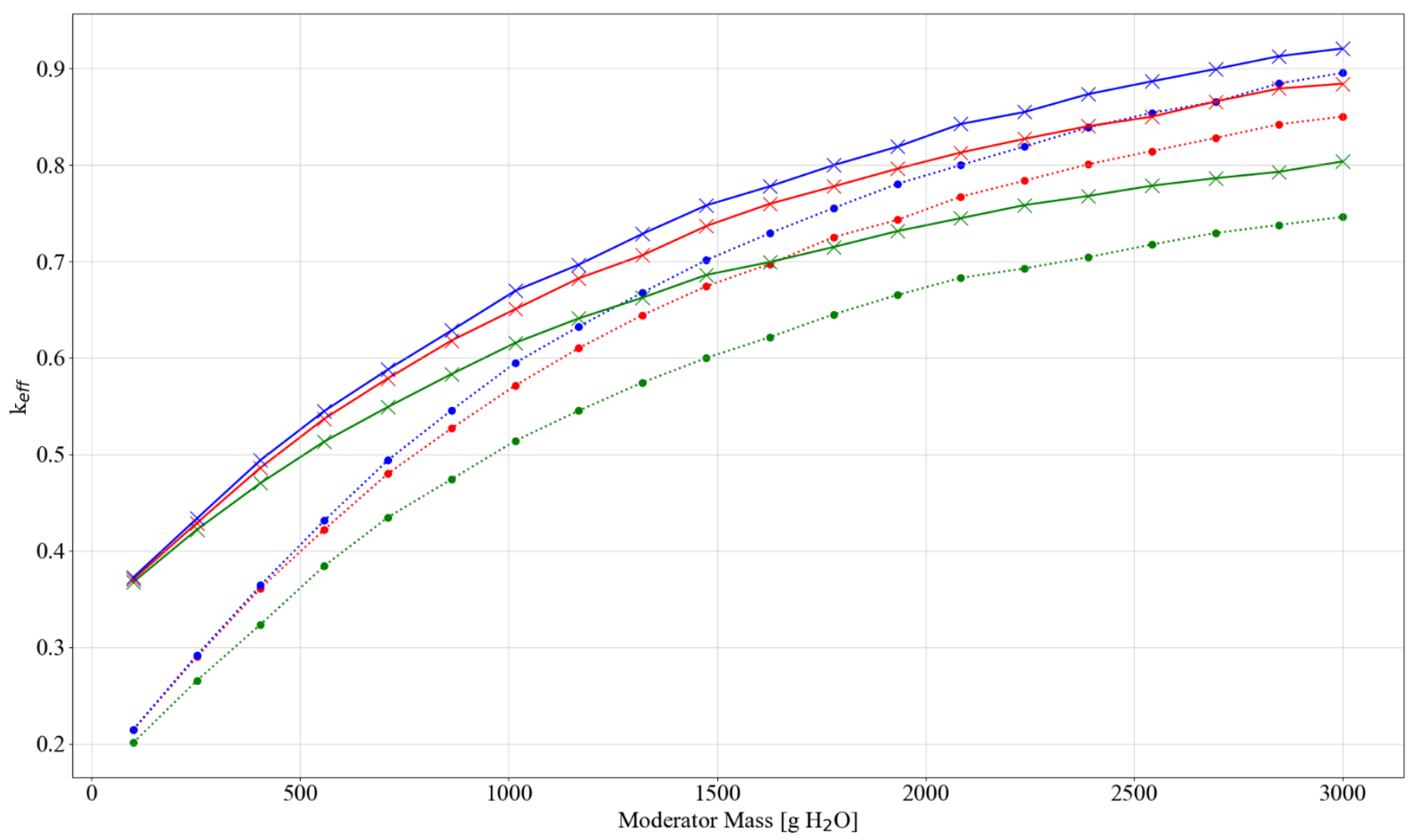

-... set-2-lh, cyl radius $=4.8 \mathrm{~cm}$, thin discrete reflector

$\cdots \cdot$ set-2-lh, cyl radius $=7.7 \mathrm{~cm}$, thin discrete reflector

set-2-lh, cyl radius $=6.25 \mathrm{~cm}$, thick discrete reflector set-2-lh, cyl radius $=6.25 \mathrm{~cm}$, thin discrete reflector set-2-lh, cyl radius $=4.8 \mathrm{~cm}$, thick discrete reflector set- 2 - $\mathrm{lh}$, cyl radius $=7.7 \mathrm{~cm}$, thick discrete reflector

Figure D-8. Set-2-lh results, plot 7: reactivity effect of cylinder radius, pipe poly, $5 \mathrm{~kg}$ graphite/can, no Be, water moderated. 


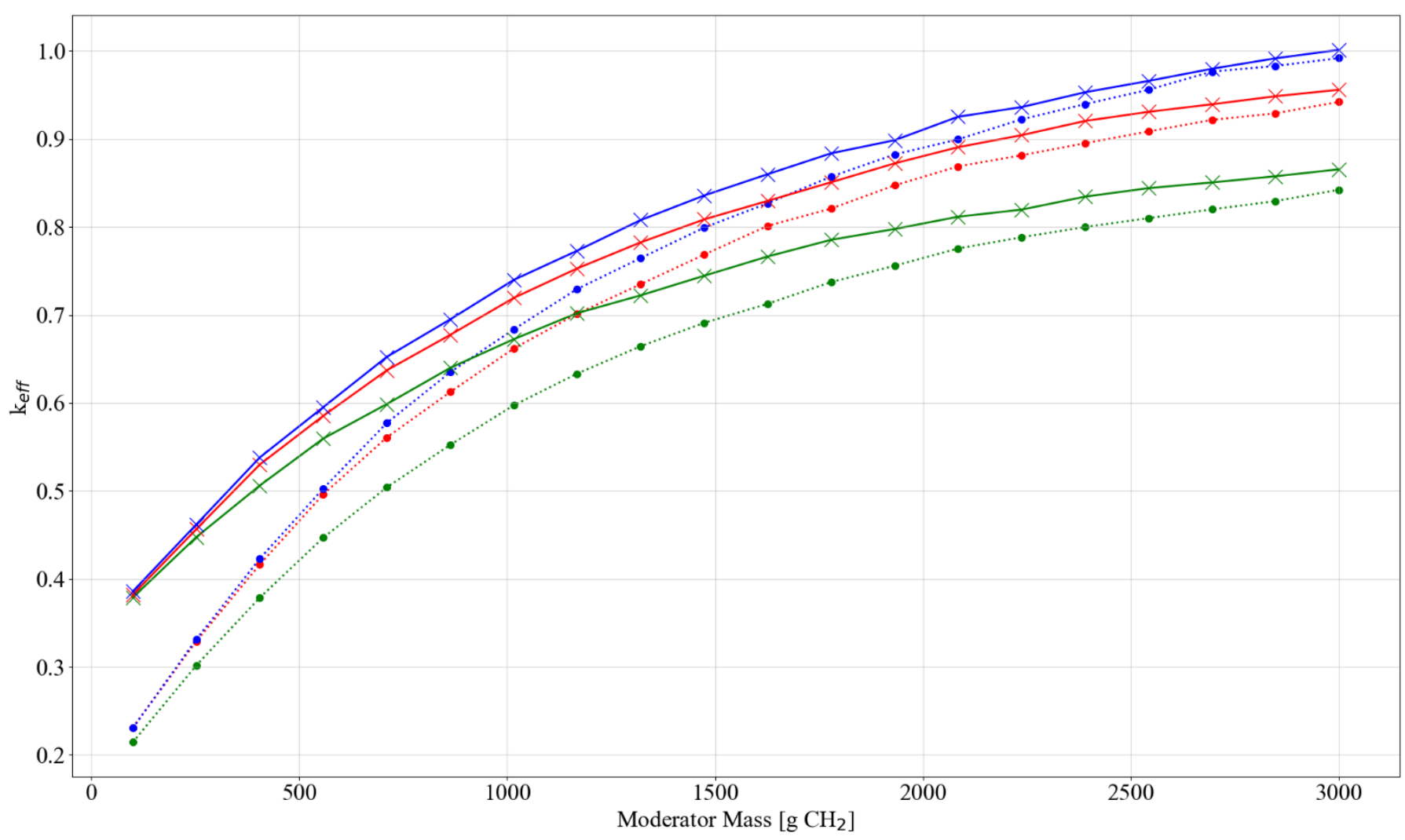

-... set-2-lh, cyl radius $=4.8 \mathrm{~cm}$, thin discrete reflector

$\cdots \cdot$ set-2-lh, cyl radius $=7.7 \mathrm{~cm}$, thin discrete reflector

set-2-lh, cyl radius $=6.25 \mathrm{~cm}$, thick discrete reflector set-2-lh, cyl radius $=6.25 \mathrm{~cm}$, thin discrete reflector set-2-lh, cyl radius $=4.8 \mathrm{~cm}$, thick discrete reflector set- 2 - $\mathrm{lh}$, cyl radius $=7.7 \mathrm{~cm}$, thick discrete reflector

Figure D-9. Set-2-lh results, plot 8: reactivity effect of cylinder radius, pipe poly, $5 \mathrm{~kg}$ graphite/can, no Be, poly moderated. 


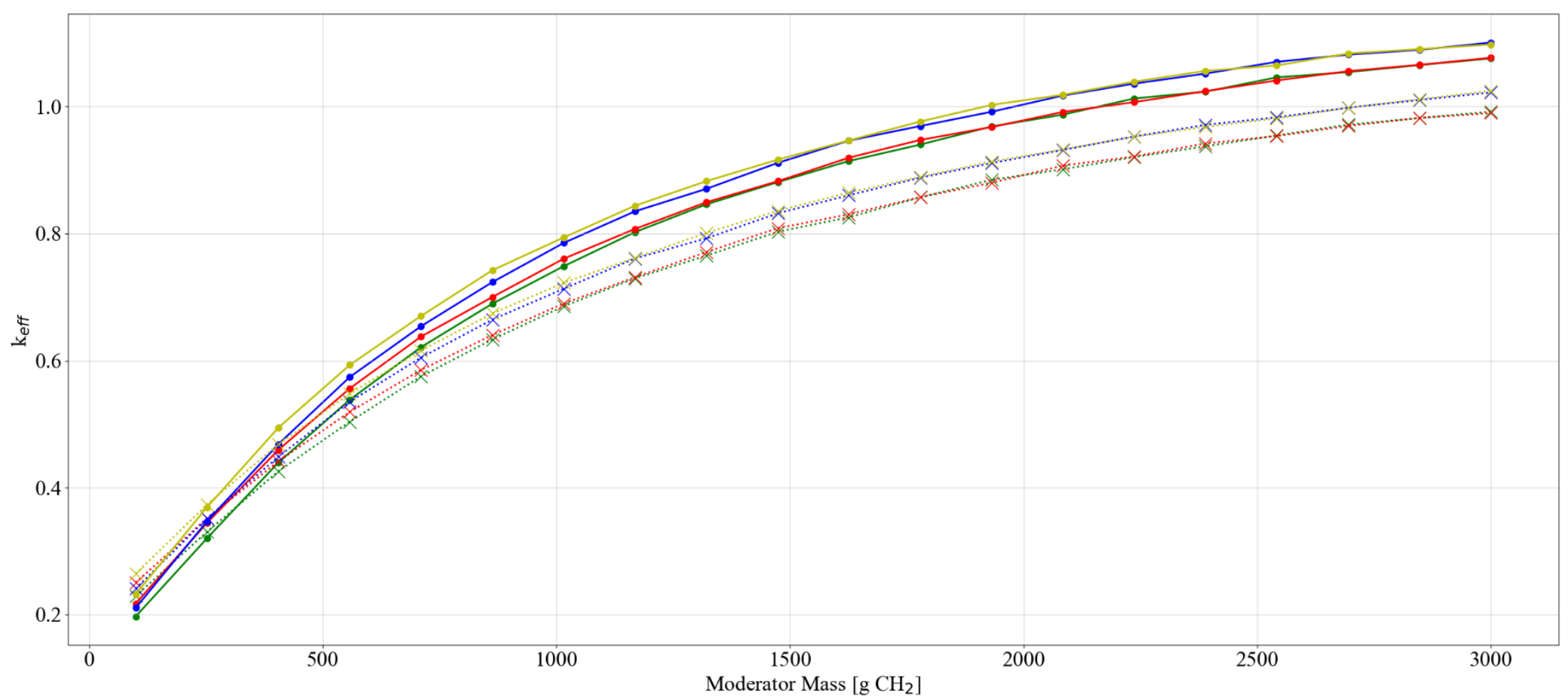
set-2-lh, $5 \mathrm{~kg}$ graphite/can, no Be, thin SS discrete reflector

$\ldots$ set-2-lh, no filler, $585 \mathrm{~g} \mathrm{Be}$, thin SS discrete reflector
$\ldots . \cdots \cdots$
set-2-lh, $5 \mathrm{~kg}$ graphite/can, $585 \mathrm{~g} \mathrm{Be}$, thin SS discrete reflector

$\longrightarrow$ set-2-lh, no filler, no Be, thick SS discrete reflector

........ set-2-lh, $5 \mathrm{~kg}$ graphite/can, no Be, thick SS discrete reflector

$\cdots$ set-2-lh, no filler, $585 \mathrm{~g} \mathrm{Be}$, thick SS discrete reflector

...... set-2-lh, $5 \mathrm{~kg}$ graphite/can, $585 \mathrm{~g} \mathrm{Be,} \mathrm{thick} \mathrm{SS} \mathrm{discrete} \mathrm{reflector}$

Figure D-10. Set-2-lh results, plot 9: reactivity effect of various parameters with $7.7 \mathrm{~cm}$ cylinder radius, graphite filler, poly moderated. 


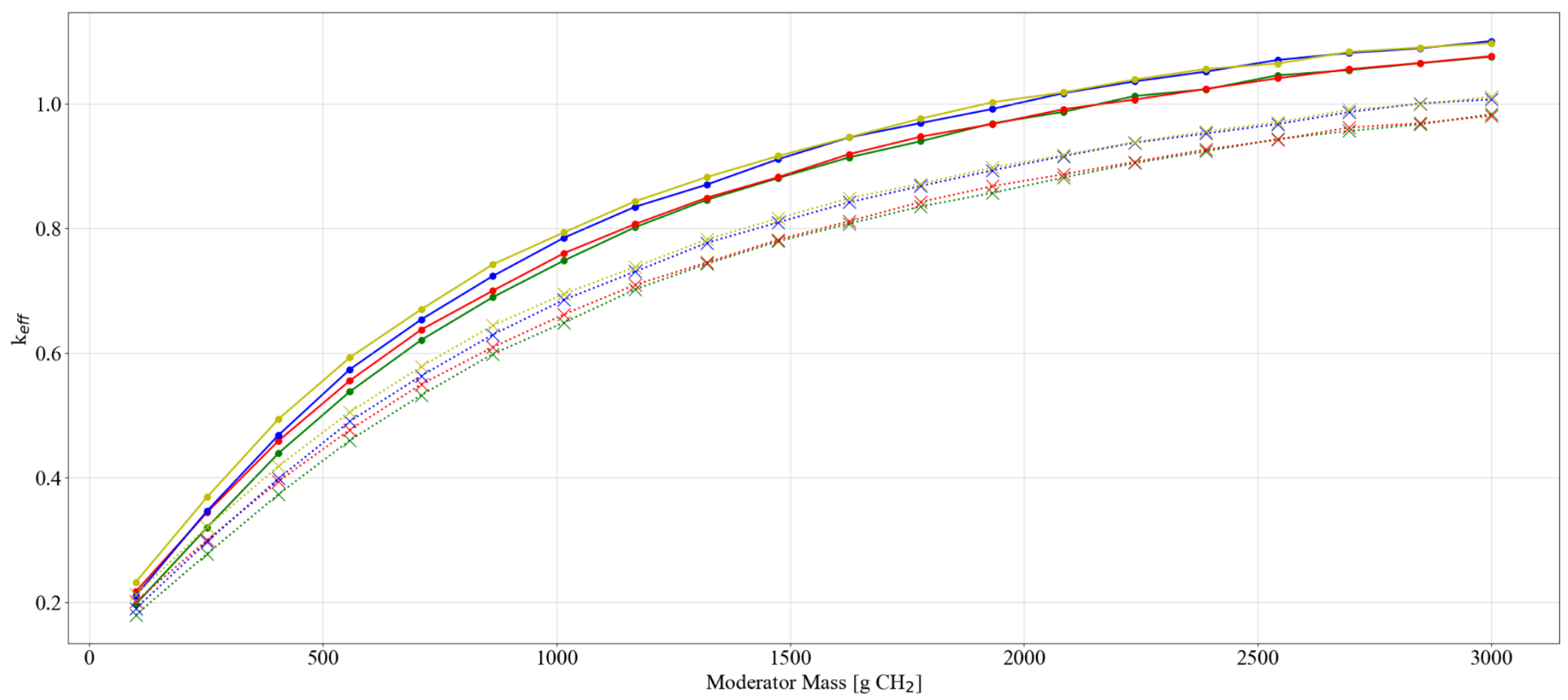
set-2-lh, $5 \mathrm{~kg}$ generic/can, no Be, thin SS discrete reflector

$\ldots$ set-2-lh, no filler, $585 \mathrm{~g} \mathrm{Be}$, thin SS discrete reflector

$\longrightarrow$ set-2-lh, no filler, no Be, thick SS discrete reflector

........ set-2-lh, $5 \mathrm{~kg}$ generic/can, no Be, thick SS discrete reflector

set-2-lh, $5 \mathrm{~kg}$ generic/can, $585 \mathrm{~g} \mathrm{Be}$, thin SS discrete $\mathrm{r}$ reflector

$\ldots$ set-2-lh, no filler, $585 \mathrm{~g} \mathrm{Be}$, thick SS discrete reflector

…… set-2-lh, $5 \mathrm{~kg}$ generic/can, $585 \mathrm{~g} \mathrm{Be}$, thick SS discrete reflector

Figure D-11. Set-2-lh results, plot 10: reactivity effect of various parameters with $7.7 \mathrm{~cm}$ cylinder radius, generic filler, poly moderated. 


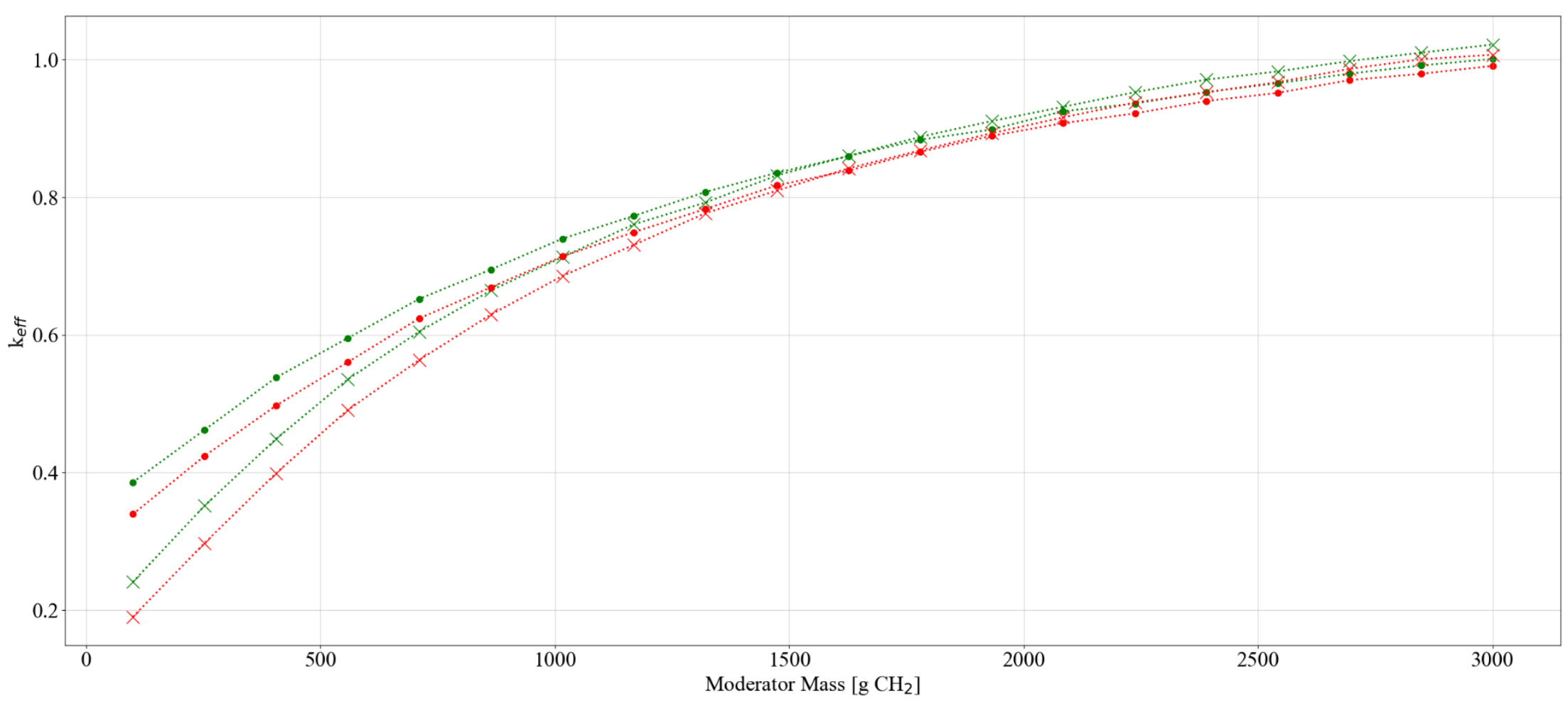

… set-2-lh, cylinder radius $=7.7 \mathrm{~cm}$, poly moderator, $5 \mathrm{~kg}$ graphite $/ \mathrm{can}$, no Be, thick SS discrete reflector

$\cdots \cdot \cdots$ set-2-lh, cylinder radius $=7.7 \mathrm{~cm}$, poly moderator, $5 \mathrm{~kg}$ graphite $/ \mathrm{can}, \mathrm{no} \mathrm{Be}$, thick poly discrete reflector

… set-2-lh, cylinder radius $=7.7 \mathrm{~cm}$, poly moderator, $5 \mathrm{~kg}$ generic $/ \mathrm{can}$, no Be, thick SS discrete reflector

$\cdots$ set-2-lh, cylinder radius $=7.7 \mathrm{~cm}$, poly moderator, $5 \mathrm{~kg}$ generic $/ \mathrm{can}$, no Be, thick poly discrete reflector

Figure D-12. Set-2-lh results, plot 11: comparison of graphite and generic filler with $7.7 \mathrm{~cm}$ cylinder radius, no Be, poly moderated, thick discrete reflector. 


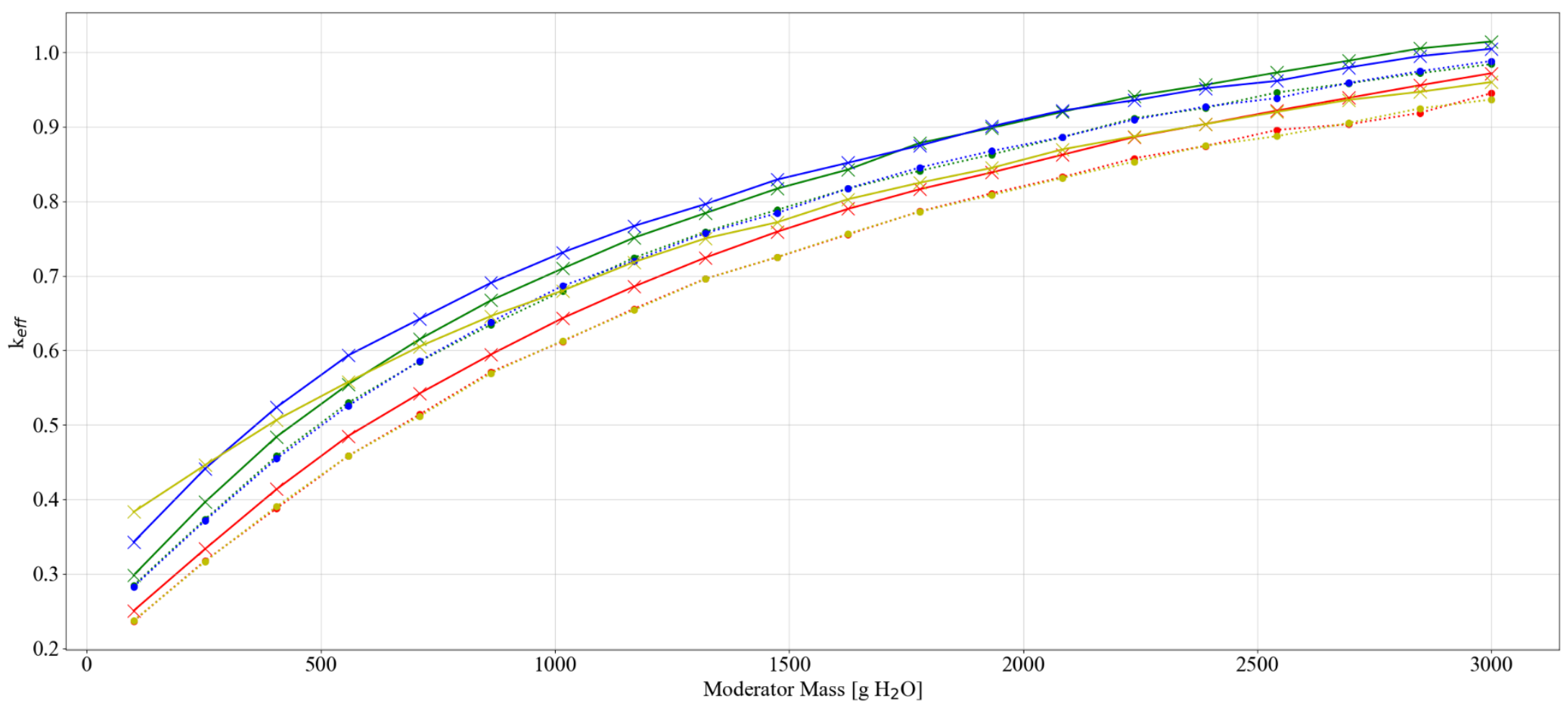

$\cdots \bullet \cdot$ set-2-lh, no filler, no Be, thin SS discrete reflector set-2-lh, no filler, no Be, thick SS discrete reflector

…… set-2-lh, $5 \mathrm{~kg}$ graphite/can, $585 \mathrm{~g} \mathrm{Be}$, thin SS discrete reflector

$\cdots \bullet \cdot$ set-2-lh, no filler, no Be, thin poly discrete reflector

* set-2-lh, no filler, no Be, thick poly discrete reflector

-... set-2-lh, $5 \mathrm{~kg}$ graphite/can, $585 \mathrm{~g} \mathrm{Be}$, thin poly discrete reflector

set-2-lh, 5 kg graphite/can, $585 \mathrm{~g} \mathrm{Be}$, thick SS discrete reflector

$\Varangle$ set-2-lh, $5 \mathrm{~kg}$ graphite/can, $585 \mathrm{~g} \mathrm{Be}$, thick poly discrete reflector

Figure D-13. Set-2-lh results, plot 12: reactivity effect of various parameters with spherical waste form geometry, graphite filler, water moderated. 


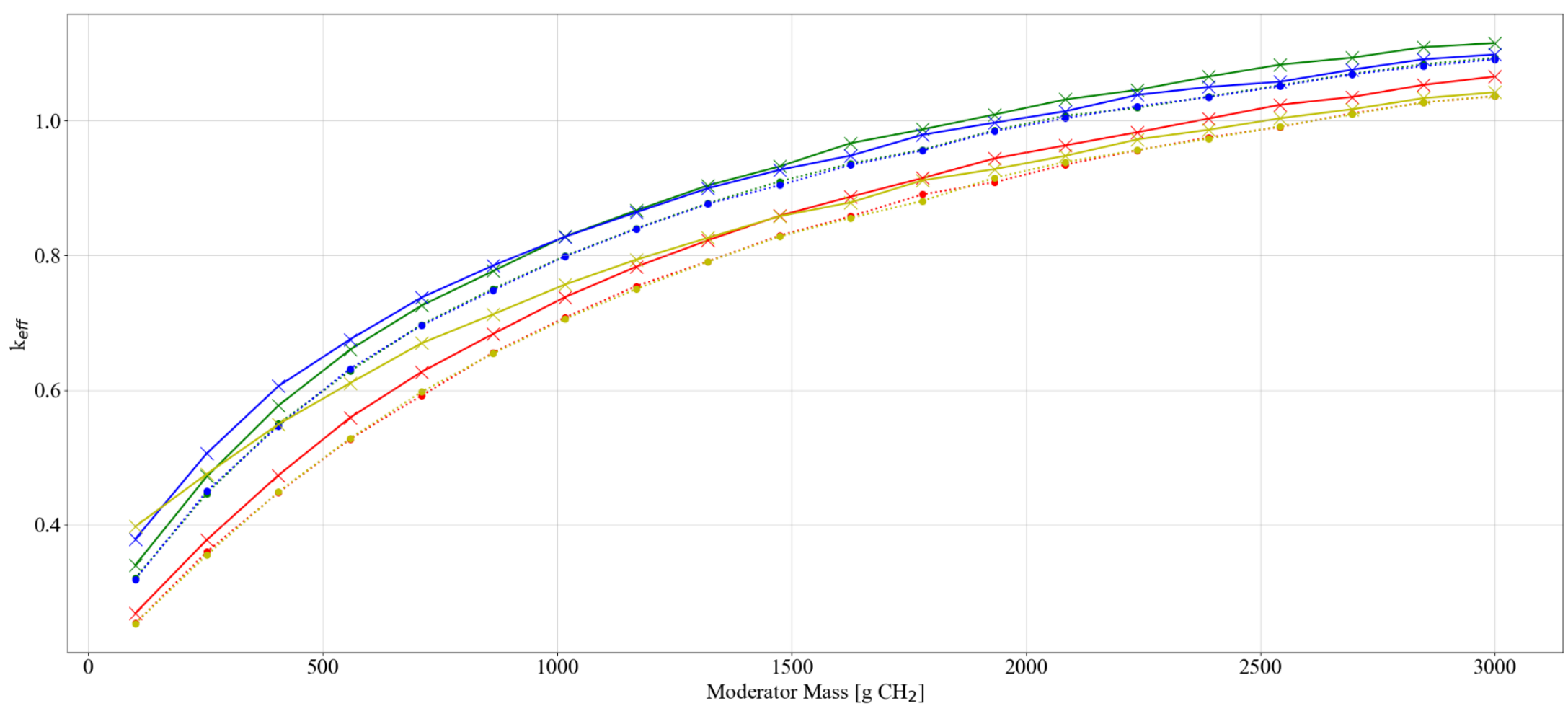

…... set-2-lh, no filler, no Be, thin SS discrete reflector set-2-lh, no filler, no Be, thick SS discrete reflector

…… set-2-lh, $5 \mathrm{~kg}$ graphite/can, $585 \mathrm{~g} \mathrm{Be}$, thin SS discrete reflector

….. set-2-lh, no filler, no Be, thin poly discrete reflector

set-2-lh, $5 \mathrm{~kg}$ graphite/can, $585 \mathrm{~g} \mathrm{Be}$, thick SS discrete reflector

* set-2-lh, no filler, no Be, thick poly discrete reflector

-... set-2-lh, $5 \mathrm{~kg}$ graphite/can, $585 \mathrm{~g} \mathrm{Be}$, thin poly discrete reflector

$\times$ set-2-lh, $5 \mathrm{~kg}$ graphite/can, $585 \mathrm{~g} \mathrm{Be}$, thick poly discrete reflector

Figure D-14. Set-2-lh results, plot 13: reactivity effect of various parameters with spherical waste form geometry, graphite filler, poly moderated. 


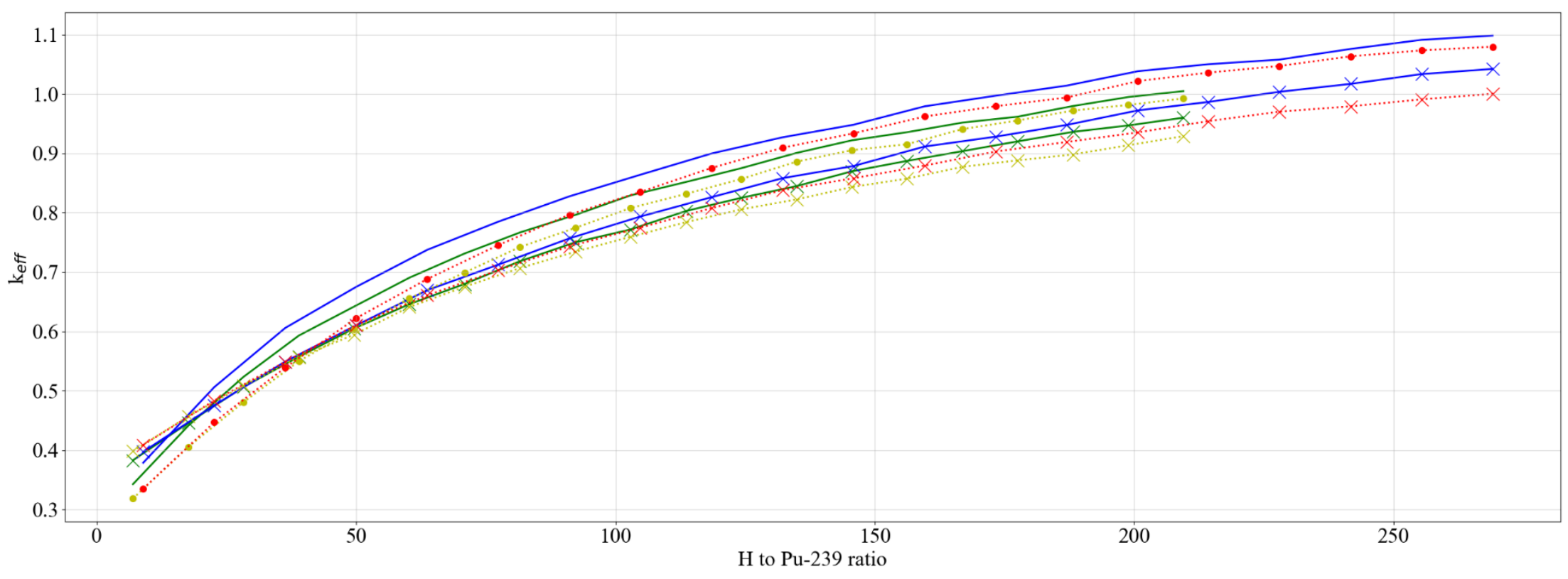

- set-2-lh, sphere, no filler, no Be, thick poly discrete reflector, water moderator

$\star \quad$ set-2-lh, sphere, $5 \mathrm{~kg}$ graphite/can, $585 \mathrm{~g} \mathrm{Be}$, thick poly discrete reflector, water moderator

- set-2-lh, sphere, no filler, no Be, thick poly discrete reflector, poly moderator

$\star$ set-2-lh, sphere, $5 \mathrm{~kg}$ graphite/can, $585 \mathrm{~g} \mathrm{Be}$, thick poly discrete reflector, poly moderator

$\cdots$ set-2-lh, cylinder with radius $=7.7 \mathrm{~cm}$, no filler, no Be, thick poly discrete reflector, water moderator

set-2-lh, cylinder with radius $=7.7 \mathrm{~cm}, 5 \mathrm{~kg}$ graphite $/ \mathrm{can}, 585 \mathrm{~g}$ Be, thick poly discrete reflector, water moderator

$\cdots \cdot \cdots$ set-2-lh, cylinder with radius $=7.7 \mathrm{~cm}$, no filler, no Be, thick poly discrete reflector, poly moderator

… $\ldots \ldots$ set-2-lh, cylinder with radius $=7.7 \mathrm{~cm}, 5 \mathrm{~kg}$ graphite $/ \mathrm{can}, 585 \mathrm{~g} \mathrm{Be}$, thick poly discrete reflector, poly moderator

Figure D-15. Set-2-lh results, plot 14: comparison of spherical and cylindrical geometries $(\mathbf{h} / \mathbf{x})$. 


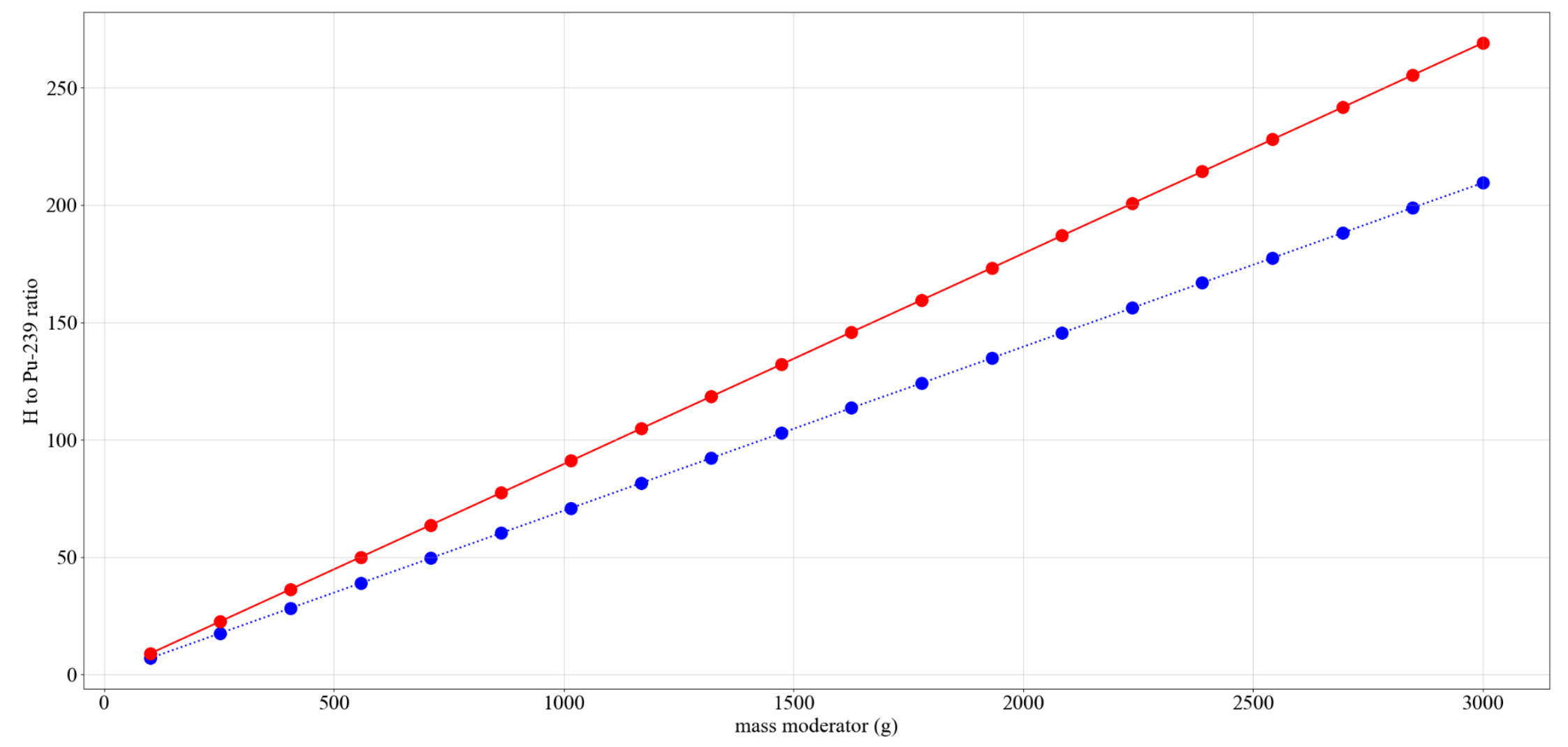

-... water moderator

$\longrightarrow$ poly moderator

Figure D-16. Set-2-lh results, plot 15: comparison of water and poly $\mathbf{h} / \mathbf{x}$. 


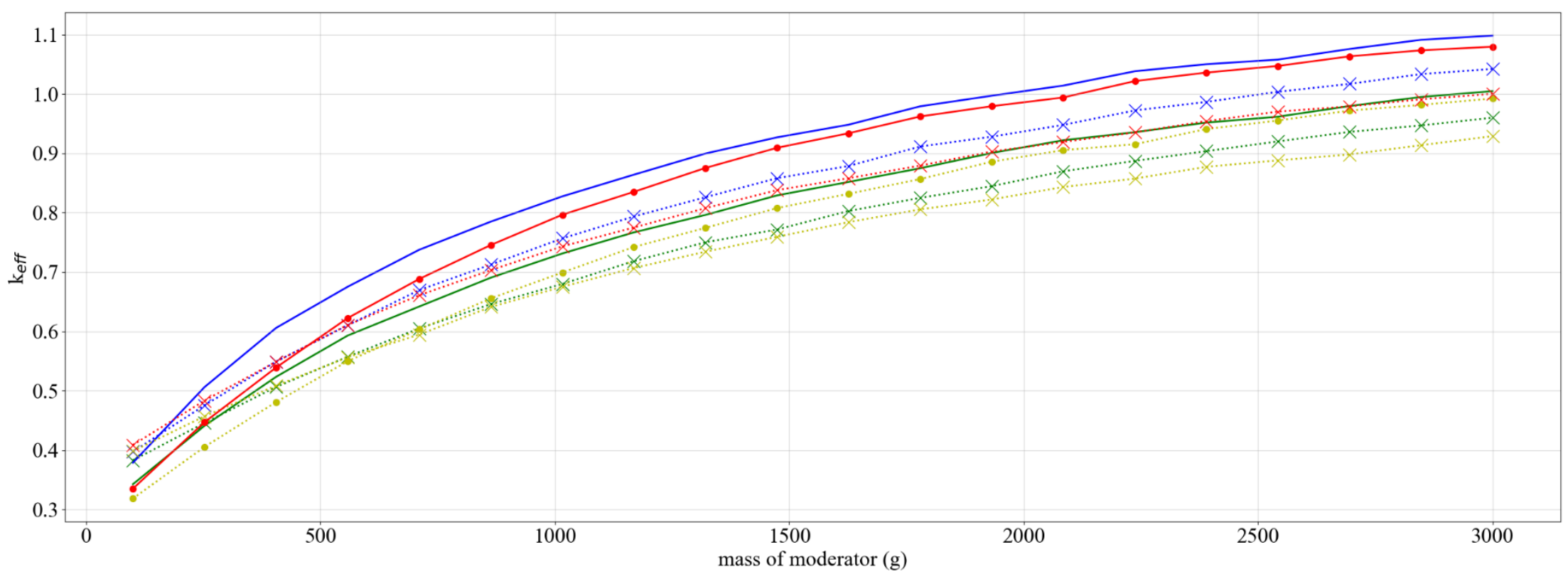

- set-2-lh, sphere, no filler, no Be, thick poly discrete reflector, water moderator

… ‘... set-2-lh, sphere, $5 \mathrm{~kg}$ graphite/can, $585 \mathrm{~g} \mathrm{Be}$, thick poly discrete reflector, water moderator

- set-2-lh, sphere, no filler, no Be, thick poly discrete reflector, poly moderator

......... set-2-lh, sphere, $5 \mathrm{~kg}$ graphite/can, $585 \mathrm{~g} \mathrm{Be}$, thick poly discrete reflector, poly moderator

$\cdots$ set-2-lh, cylinder with radius $=7.7 \mathrm{~cm}$, no filler, no Be, thick poly discrete reflector, water moderator

set-2-lh, cylinder with radius $=7.7 \mathrm{~cm}, 5 \mathrm{~kg}$ graphite $/ \mathrm{can}, 585 \mathrm{~g} \mathrm{Be}$, thick poly discrete reflector, water moderato

- set-2-lh, cylinder with radius $=7.7 \mathrm{~cm}$, no filler, no Be, thick poly discrete reflector, poly moderator

… $\ldots \ldots$ set-2-lh, cylinder with radius $=7.7 \mathrm{~cm}, 5 \mathrm{~kg}$ graphite $/ \mathrm{can}, 585 \mathrm{~g} \mathrm{Be}$, thick poly discrete reflector, poly moderator

Figure D-17. Set-2-lh results, plot 16: comparison of spherical and cylindrical geometries (mod mass). 


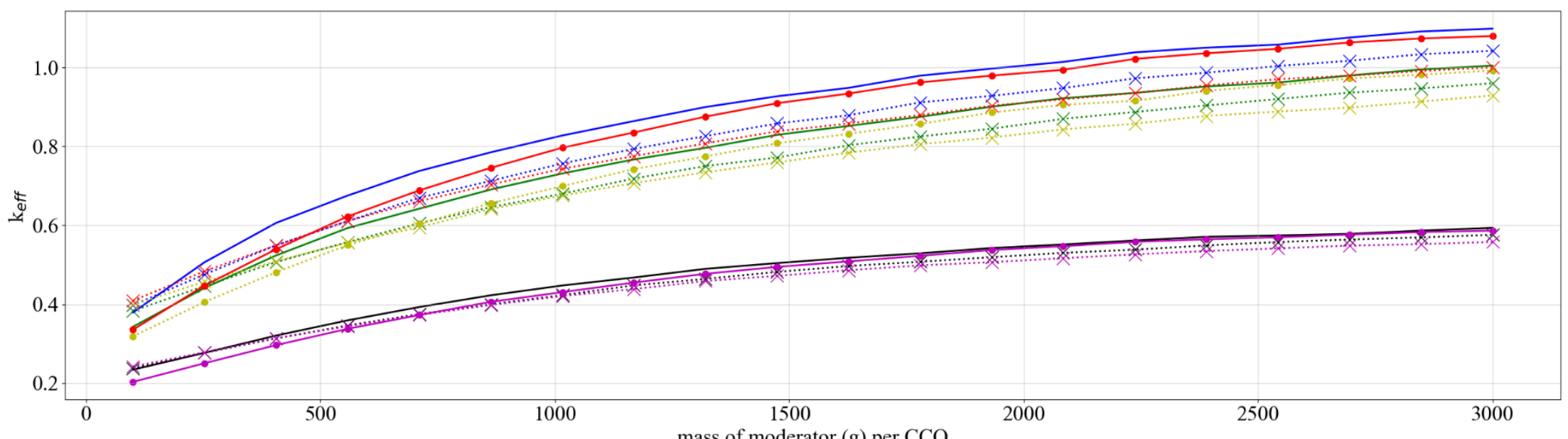

- set-2-lh, sphere, no filler, no Be, thick poly discrete reflector, water moderator

........ set-2-lh, sphere, $5 \mathrm{~kg}$ graphite/can, $585 \mathrm{~g} \mathrm{Be}$, thick poly discrete reflector, water moderator

- set-2-lh, sphere, no filler, no Be, thick poly discrete reflector, poly moderator

... ‘... set-2-lh, sphere, $5 \mathrm{~kg}$ graphite/can, $585 \mathrm{~g} \mathrm{Be}$, thick poly discrete reflector, poly moderator

$\cdots \cdots$ set-2-lh, cylinder with radius $=7.7 \mathrm{~cm}$, no filler, no Be, thick poly discrete reflector, water moderator

set-2-lh, cylinder with radius $=7.7 \mathrm{~cm}, 5 \mathrm{~kg}$ graphite $/ \mathrm{can}, 585 \mathrm{~g} \mathrm{Be}$, thick poly discrete reflector, water moderator

$\longrightarrow$ set-2-lh, cylinder with radius $=7.7 \mathrm{~cm}$, no filler, no Be, thick poly discrete reflector, poly moderator

....... set-2-lh, cylinder with radius $=7.7 \mathrm{~cm}, 5 \mathrm{~kg}$ graphite/can, $585 \mathrm{~g} \mathrm{Be}$, thick poly discrete reflector, poly moderator

- set-2-lh, sphere, $50 \mathrm{~g} \mathrm{~B} 4 \mathrm{C}$, no filler, no Be, thick poly discrete reflector, poly moderator

....... set-2-lh, sphere, $50 \mathrm{~g} \mathrm{~B} 4 \mathrm{C}, 5 \mathrm{~kg}$ graphite/can, $585 \mathrm{~g} \mathrm{Be}$, thick poly discrete reflector, poly moderator

$\longrightarrow$ set-2-lh, cylinder with radius $=7.7 \mathrm{~cm}, 50 \mathrm{~g} \mathrm{~B} 4 \mathrm{C}$, no filler, no Be, thick poly discrete reflector, poly moderator

… ‘... set-2-lh, cylinder with radius $=7.7 \mathrm{~cm}, 50 \mathrm{~g} \mathrm{~B} 4 \mathrm{C}, 5 \mathrm{~kg}$ graphite $/ \mathrm{can}, 585 \mathrm{~g} \mathrm{Be}$, thick poly discrete reflector, poly moderator

Figure D-18. Set-2-lh results, plot 17: comparison of $50 \mathrm{~g} \mathrm{~B}_{4} \mathrm{C}$ vs. no $\mathrm{B}_{4} \mathrm{C}$ for spherical and cylindrical geometries (mod mass). 
This page is intentionally blank 


\section{APPENDIX E. SET-2-TD: RESULTS OF THE TIME DEPENDENT}

NONUNIFORM ARRAY CALCULATIONS 
This page is intentionally blank 


\section{APPENDIX E. SET-2-TD: RESULTS OF THE TIME DEPENDENT NONUNIFORM ARRAY CALCULATIONS}

The analysis methodology for the time dependent nonuniform array studies is discussed in detail in Section 6.4 of the main report.

This appendix serves as a repository of the results for the time-dependent calculations.

The analysis model used for the calculations in this appendix is shown in Figure E-1 below. Additional discussions are provided in Section 6.3 of the main report.

The SAMPLER case sweeps presented in this appendix are summarized in Table E-1 below.

Results are presented in the following figures:

\section{LIST OF FIGURES}

Figure E-1. 3D Isometric view (top) and 2D top view (bottom) representations of the 1-unit

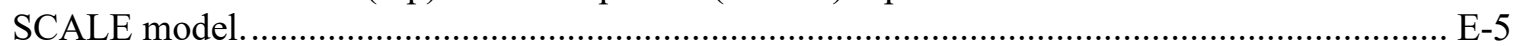

Figure E-2. 3D isometric (top) and 2D front (bottom) representations of the 2-unit SCALE model....... E-6

Figure E-3. Layout of the 1-unit, 0-year CCO locations in 3D isometric (top left), top view (right) and front (bottom left)........................................................................................................ E-8

Figure E-4. Distribution of the flux for the $\mathrm{x}-\mathrm{z}$ direction (top and bottom) with contour count of 10 for 0 years for the SNL upper horizon compaction results................................................ E-9

Figure E-5. Layout of the 1-unit, 50-year CCO locations in 3D isometric (top left), top view (right) and front (bottom left). ...................................................................................... E-10

Figure E-6. Distribution of the flux for the $\mathrm{x}-\mathrm{z}$ direction (top and bottom) with contour count of 10 for 50 years for the SNL upper horizon compaction results......................................... E-11

Figure E-7. Layout of the 1-unit, 100-year CCO locations in 3D isometric (top left), top view (right) and front (bottom left).

Figure E-8. Distribution of the flux for the $\mathrm{x}-\mathrm{z}$ direction (top and bottom) with contour count of

10 for 100 years for the SNL upper horizon compaction results......................................... E-13

Figure E-9. Layout of the 1-unit, 200-year CCO locations in 3D isometric (top left), top view (right) and front (bottom left). ................................................................................... E-14

Figure E-10. Distribution of the flux for the $\mathrm{x}-\mathrm{z}$ direction (top and bottom) with contour count of 10 for 200 years for the SNL upper horizon compaction results........................................ E-15

Figure E-11. Layout of the 1-unit, 300-year CCO locations in 3D isometric (top left), top view (right) and front (bottom left). .......................................................................................... E-16

Figure E-12. Distribution of the flux for the $\mathrm{x}-\mathrm{z}$ direction (top and bottom) with contour count of 10 for 300 years for the SNL upper horizon compaction results........................................ E-17

Figure E-13. Layout of the 1-unit, 400-year CCO locations in 3D isometric (top left), top view

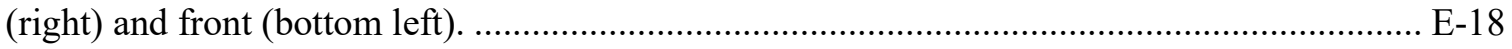

Figure E-14. Distribution of the flux for the $\mathrm{x}-\mathrm{z}$ direction (top and bottom) with contour count of 10 for 400 years for the SNL upper horizon compaction results....................................... E-19

Figure E-15. Layout of the 1-unit, 1,000-year CCO locations in 3D isometric (top left), top view (right) and front (bottom left).

Figure E-16. Distribution of the flux for the $\mathrm{x}-\mathrm{z}$ direction (top and bottom) with contour count of 10 for 1,000 years for the SNL upper horizon compaction results.

Figure E-17. Comparison of various small-step time-dependent compaction steps for the SNL upper horizon compaction results. E-22 
Figure E-18. Comparison of various large-step time-dependent compaction steps for the SNL upper horizon compaction results.

Figure E-19. Comparison of various small-step time-dependent compaction steps between 100 and 200 years for the SNL upper horizon compaction results.

Figure E-20. Comparison of the 1- (-1u-) to 2-unit SCALE models for various time dependent cases for the SNL upper horizon compaction results

Figure E-21. Comparison of the sphere and cylinder models for various time-dependent cases for the SNL upper horizon compaction results.

Figure E-22. Comparison of the reactivity effect of location of the reflective boundary conditions, KENO parametric block parameters for the 1- and 2-unit SCALE sphere, and cylinder models for the 1,000-year time-dependent case for the SNL upper horizon compaction results.

Figure E-23. Comparison of the reactivity effect of various boundary conditions for the 1- and 2unit SCALE sphere and cylinder models for the 1,000-year time-dependent case for the SNL upper horizon compaction results.

\section{LIST OF TABLES}

Table E-1. SAMPLER sweeps for the time-dependent studies 


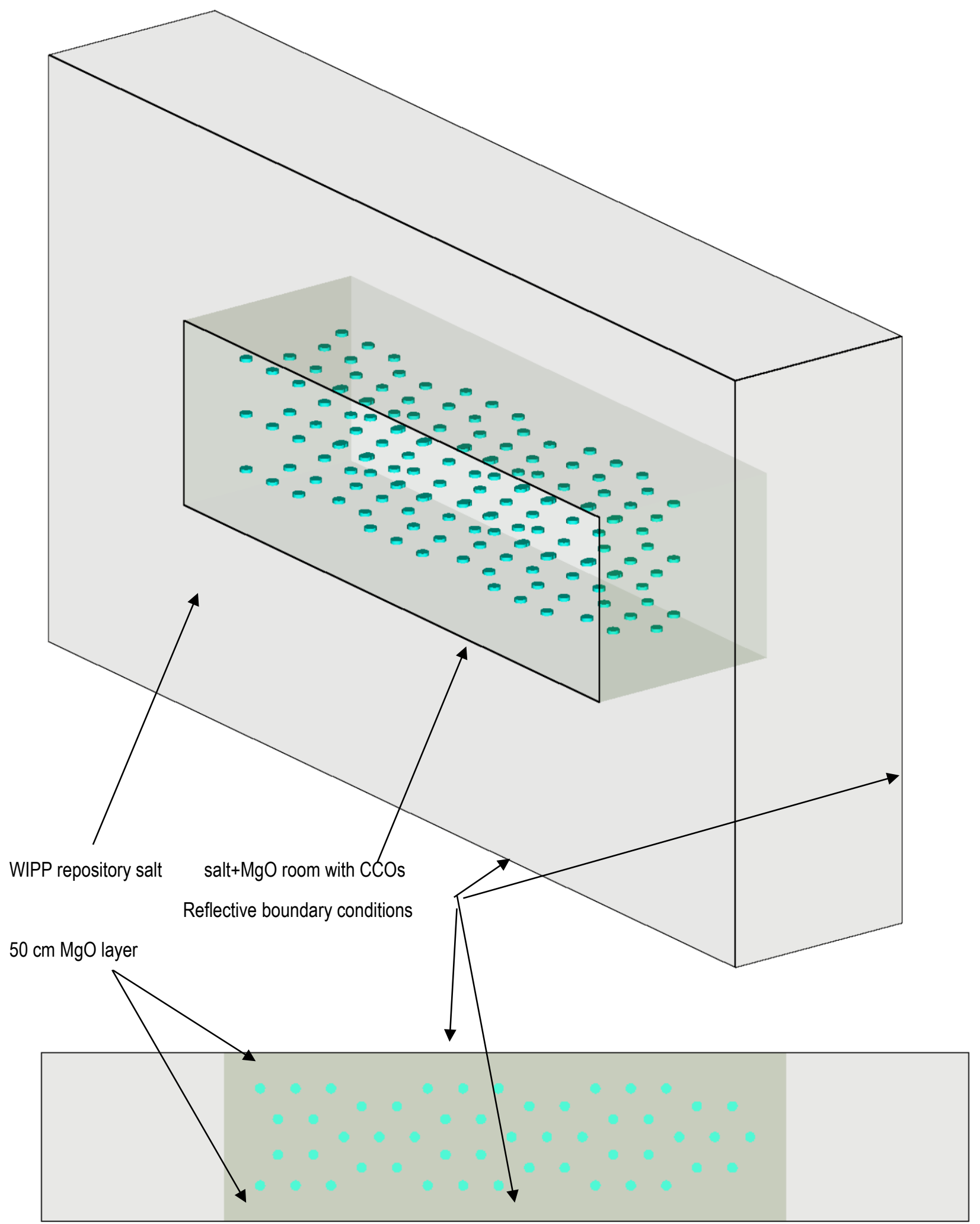

Figure E-1. 3D Isometric view (top) and 2D top view (bottom) representations of the 1-unit SCALE model. 


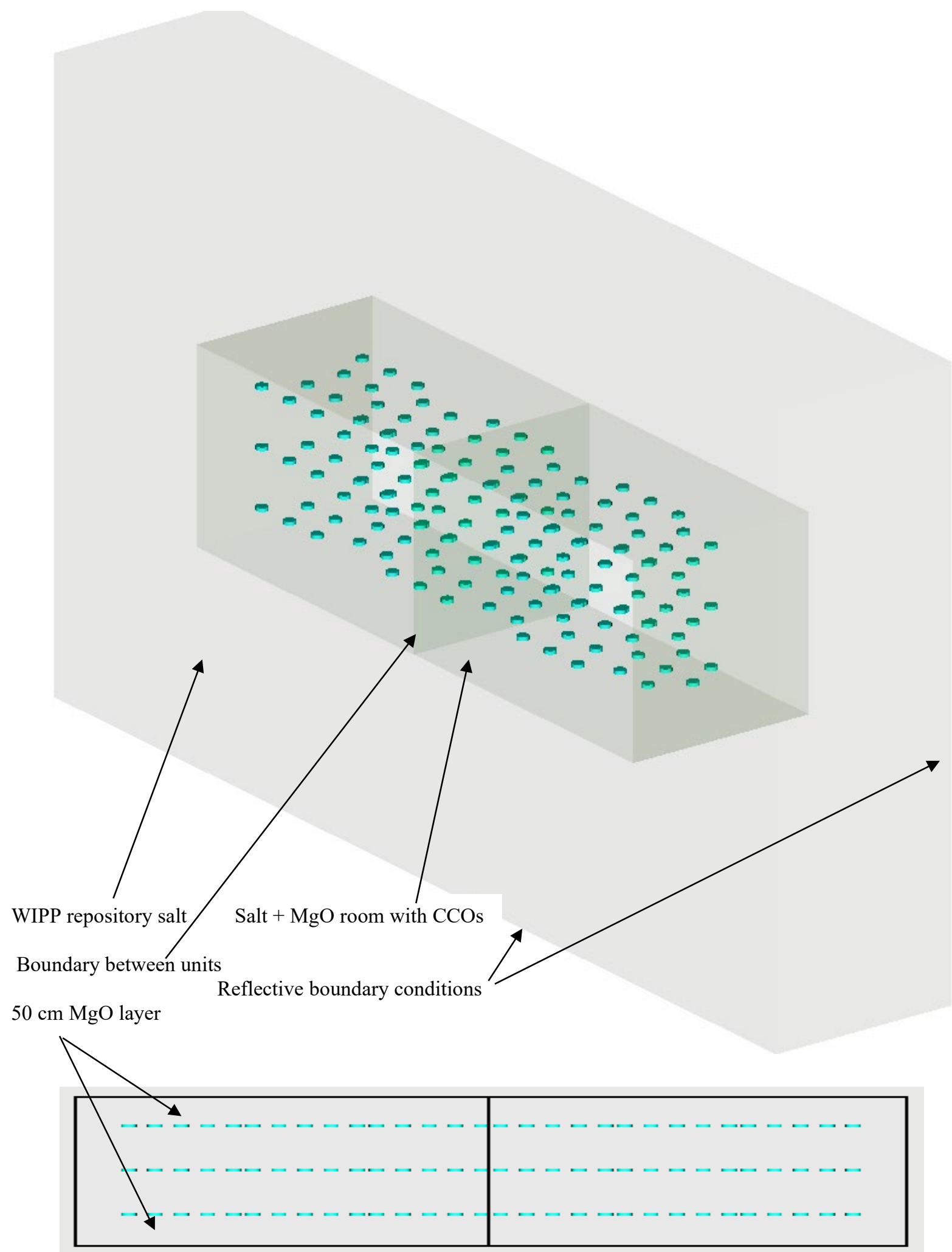

Figure E-2. 3D isometric (top) and 2D front (bottom) representations of the 2-unit SCALE model. 
Table E-1. SAMPLER sweeps for the time-dependent studies

\begin{tabular}{|c|c|c|c|c|c|c|c|c|}
\hline Case & Model type & Waste form shape & $\begin{array}{l}\text { Waste form } \\
\text { moderator }\end{array}$ & $\begin{array}{c}\text { Filler } \\
\text { material } \\
(0,2,000, \\
4,000 \mathrm{~g})\end{array}$ & $\begin{array}{l}\text { Metal in } \\
\text { filler }\end{array}$ & $\begin{array}{c}\text { Discrete reflector } \\
\text { (thin } 0.001 \text {, thick } \\
0.7112 \mathrm{~cm} \text { ) }\end{array}$ & $\mathrm{Be}(\mathrm{g})$ & $\begin{array}{c}\text { Subcase } \\
\text { (time-step } \\
\text { years) }\end{array}$ \\
\hline \multirow{10}{*}{ Set-2-4-td } & \multirow{9}{*}{$\begin{array}{l}\text { 2-unit nonuniform array with } \\
\text { centroids based on upper horizon } \\
\text { data. Centroid from pipe center is } \\
\text { used as the point in the center of the } \\
\text { cylinder bases. Reflective boundary } \\
\text { conditions are moved } 50 \mathrm{~cm} \text { off the } \\
\text { outermost centroid positions in the } \\
y \text {-direction. } 50 \mathrm{~cm} \text { is also used to } \\
\text { offset the remainder of the } \\
\text { reflective material box in the x and } \\
\text { z directions. } 1,000 \text { generations, } 100 \\
\text { skipped cycles, } \mathbf{1 0 , 0 0 0} \text { neutrons } \\
\text { per generation. }\end{array}$} & \multirow{8}{*}{$\begin{array}{l}\text { Cylinder (radius } \\
\text { range } 4.8,6.25,7.7 \\
\mathrm{~cm} \text { and height defined } \\
\text { by total volume of } \\
\text { mass) }\end{array}$} & water & $\mathrm{c} 12$ & \multirow{8}{*}{$\begin{array}{l}\text { SS from } \\
\text { can } \\
(0,500 \\
1,000 \mathrm{~g})\end{array}$} & steel & \multirow{8}{*}{$\begin{array}{l}0 \text { to } \\
585\end{array}$} & \multirow{8}{*}{0} \\
\hline & & & poly & $\mathrm{c} 12$ & & steel & & \\
\hline & & & water & $\mathrm{c} 12$ & & poly & & \\
\hline & & & poly & $\mathrm{c} 12$ & & poly & & \\
\hline & & & water & generic & & steel & & \\
\hline & & & poly & generic & & steel & & \\
\hline & & & water & generic & & poly & & \\
\hline & & & poly & generic & & poly & & \\
\hline & & $\begin{array}{l}\text { Cylinder with radius } \\
\text { of } 7.7 \mathrm{~cm} \text { and height } \\
\text { defined by total } \\
\text { volume of mass. }\end{array}$ & poly & none & 0 & $\begin{array}{l}\text { poly (thick } 0.7112 \\
\mathrm{~cm})\end{array}$ & 0 & $\begin{array}{c}10-300 \text { in } \\
10 \text {-year } \\
\text { increments; } \\
400-1,000 \\
\text { in } 100 \text {-year } \\
\text { increments }\end{array}$ \\
\hline & $\begin{array}{l}\text { 1-unit nonuniform array with } \\
\text { centroids based on upper horizon } \\
\text { data. Centroid from pipe center is } \\
\text { used as the point in the center of the } \\
\text { cylinder bases. Reflective boundary } \\
\text { conditions are directly adjacent to } \\
\text { the outermost waste form in the y- } \\
\text { direction, and the reflector box is } \\
\text { also directly adjacent to the waste } \\
\text { forms in the x and z directions. } 300 \\
\text { generations, } 50 \text { skipped cycles, } \\
\text { 1,000 neutrons per generation. }\end{array}$ & $\begin{array}{l}\text { Cylinder with radius } \\
\text { of } 7.7 \mathrm{~cm} \text { and height } \\
\text { defined by total } \\
\text { volume of mass. }\end{array}$ & poly & none & 0 & $\begin{array}{l}\text { poly (thick } 0.7112 \\
\mathrm{~cm})\end{array}$ & 0 & $\begin{array}{l}0,50,100 \\
200,300 \\
400,500 \\
1,000 \text { years }\end{array}$ \\
\hline
\end{tabular}




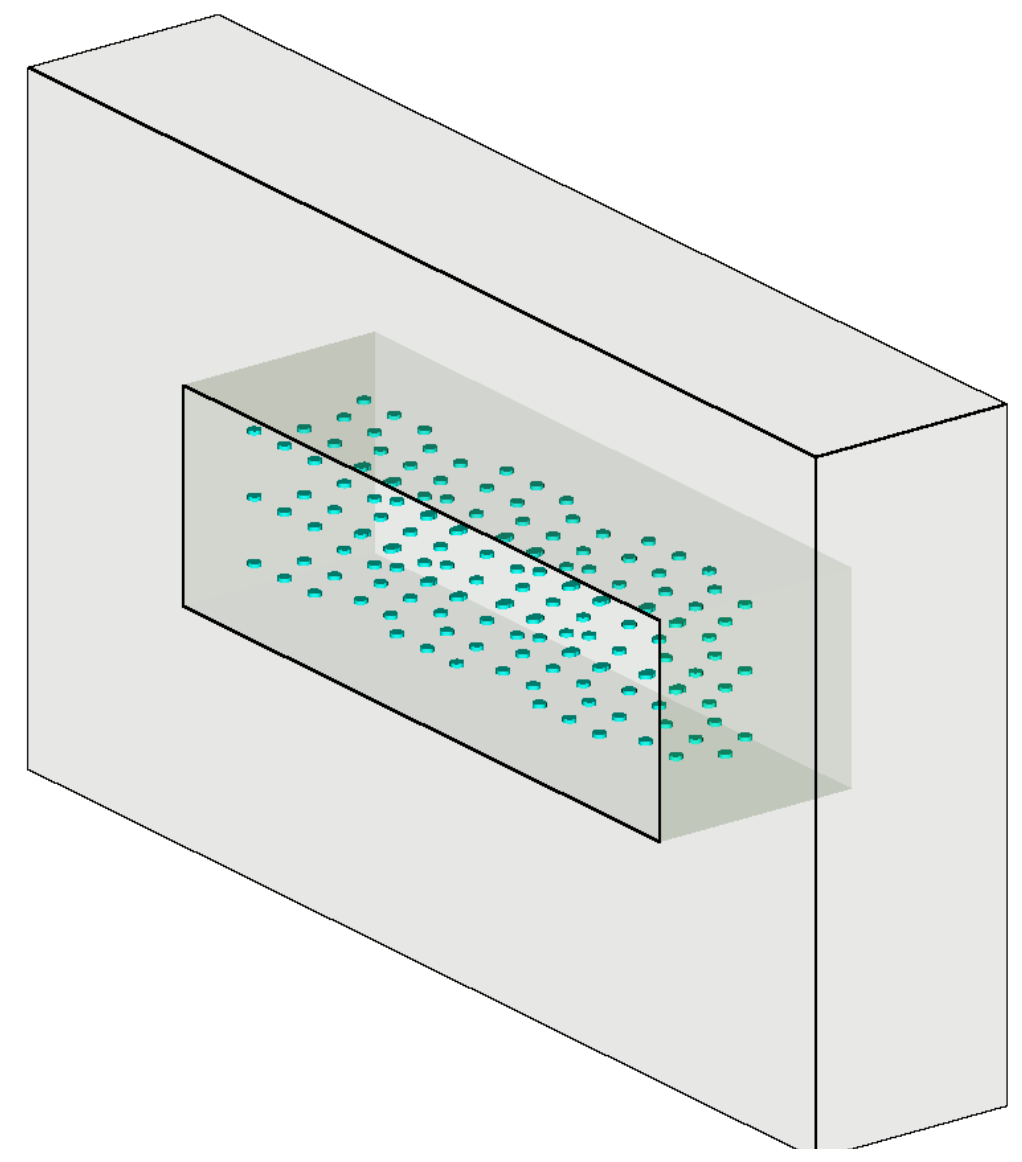

ดே

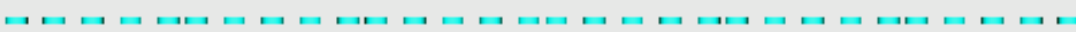

Figure E-3. Layout of the 1-unit, 0-year CCO locations in 3D isometric (top left), top view (right) and front (bottom left). 

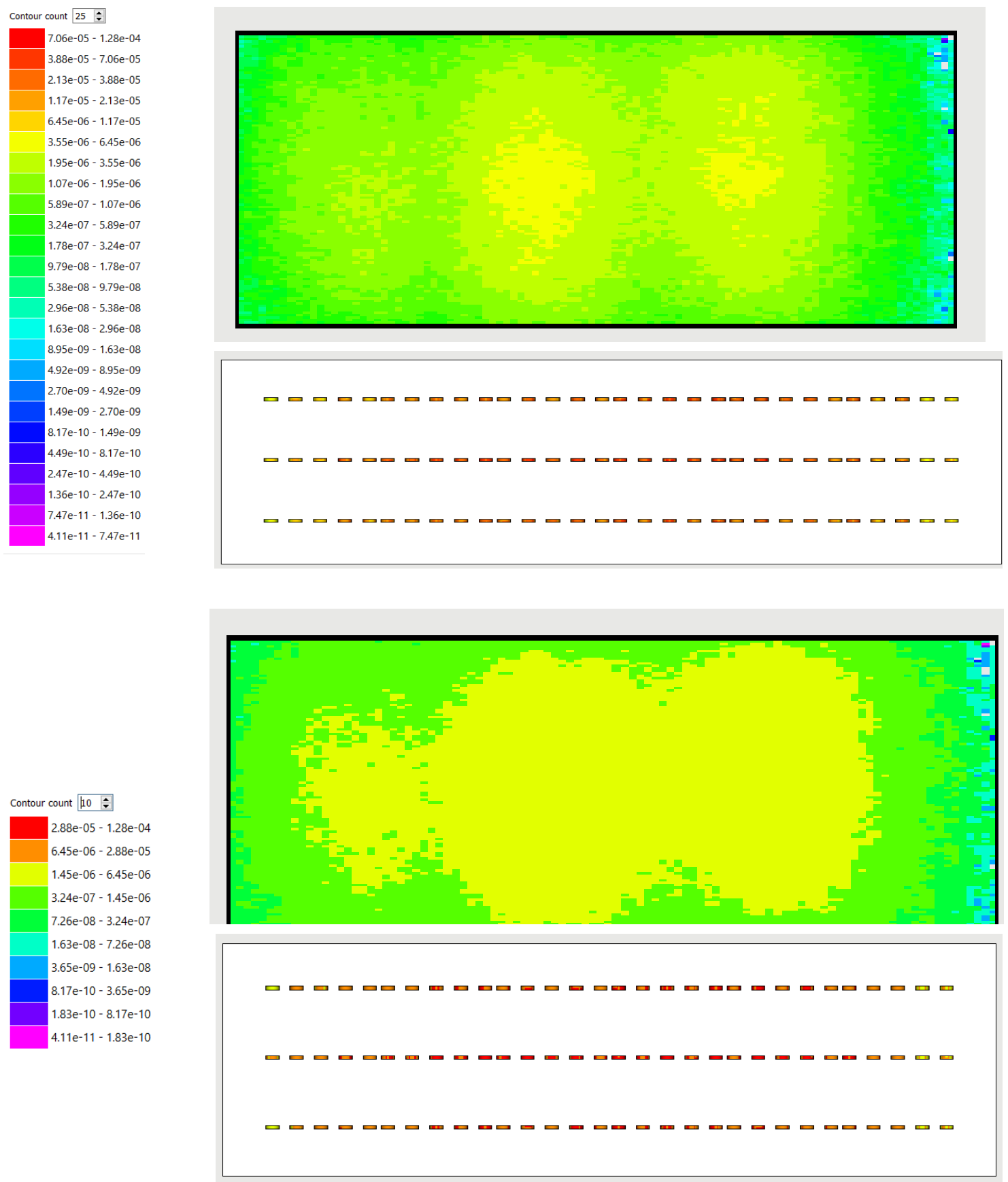

Figure E-4. Distribution of the flux for the $x-z$ direction (top and bottom) with contour count of 10 for 0 years for the SNL upper horizon compaction results. All cases use set-2-4 bounding conditions (cylindrical waste forms with no filler, no can, no Be, thick poly discrete reflector, and approximately $700 \mathrm{~g}$ poly moderator in the 1 unit model). 

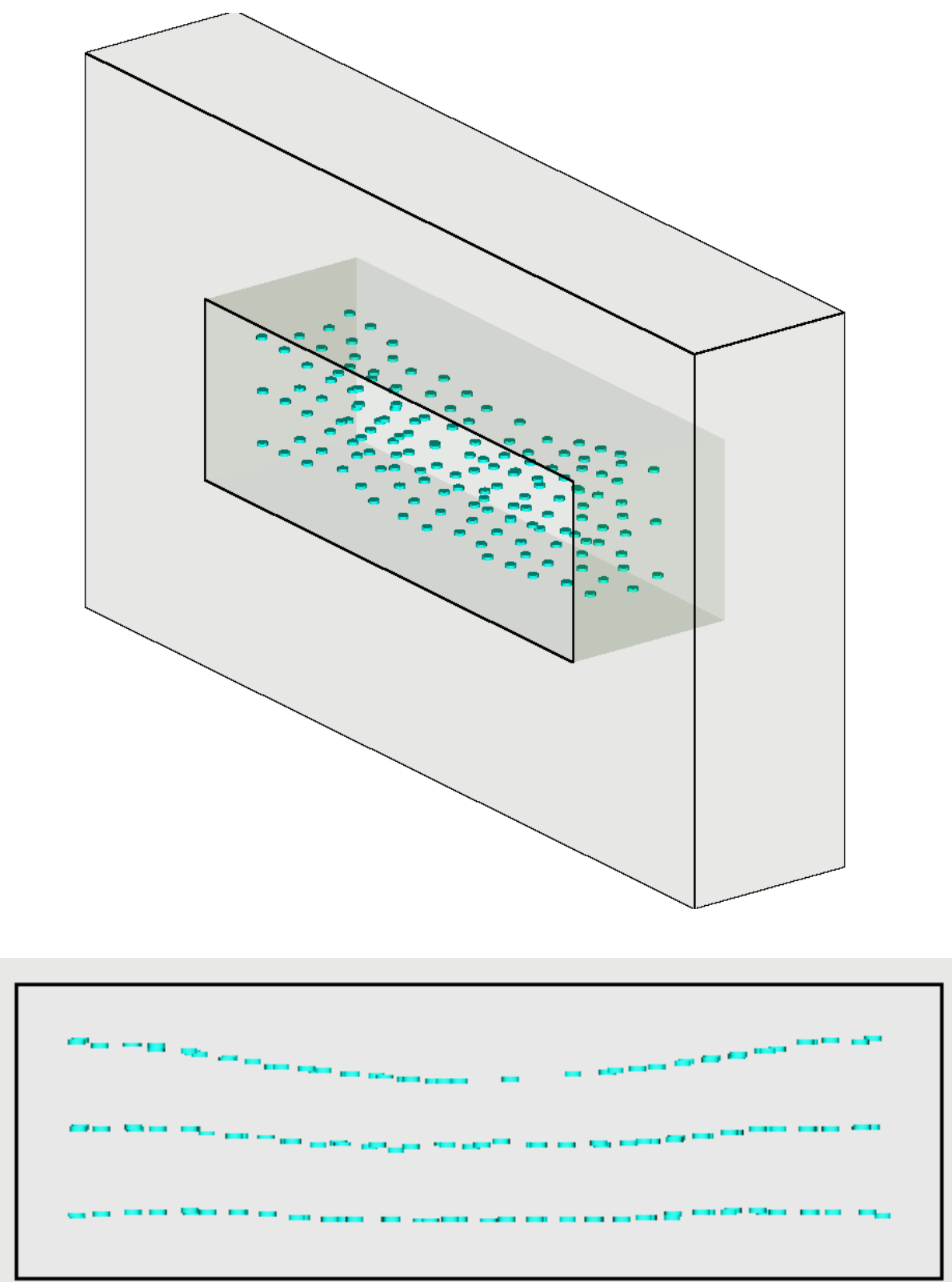

Figure E-5. Layout of the 1-unit, 50-year CCO locations in 3D isometric (top left), top view (right) and front (bottom left). 

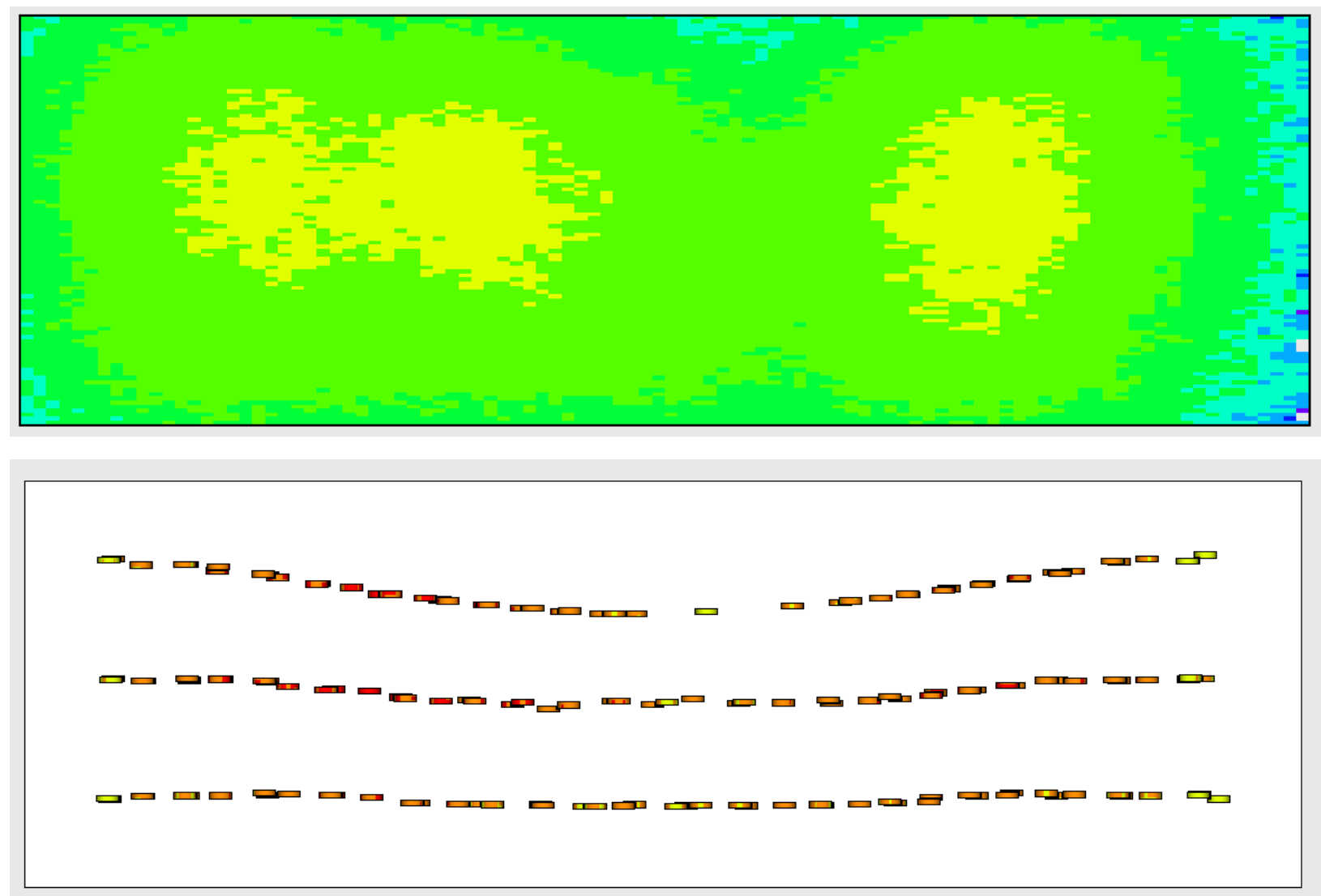

Figure E-6. Distribution of the flux for the $x-z$ direction (top and bottom) with contour count of 10 for 50 years for the SNL upper horizon compaction results. All cases use the set-2-4 bounding conditions (cylindrical waste forms with no filler, no can, no Be, thick poly discrete reflector, and approximately $700 \mathrm{~g}$ poly moderator in the 1-unit model). 

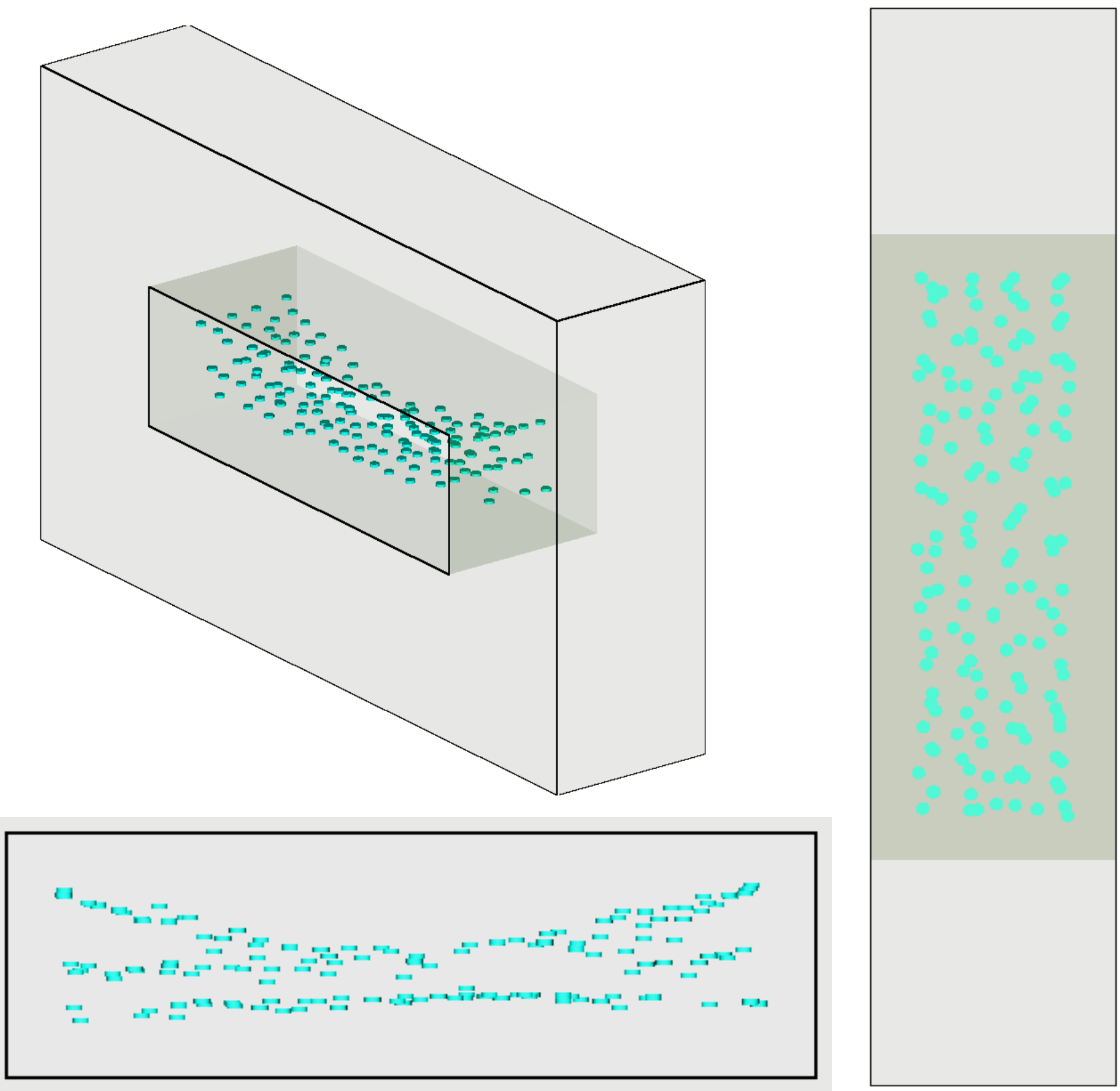

Figure E-7. Layout of the 1-unit, 100-year CCO locations in 3D isometric (top left), top view (right) and front (bottom left). 

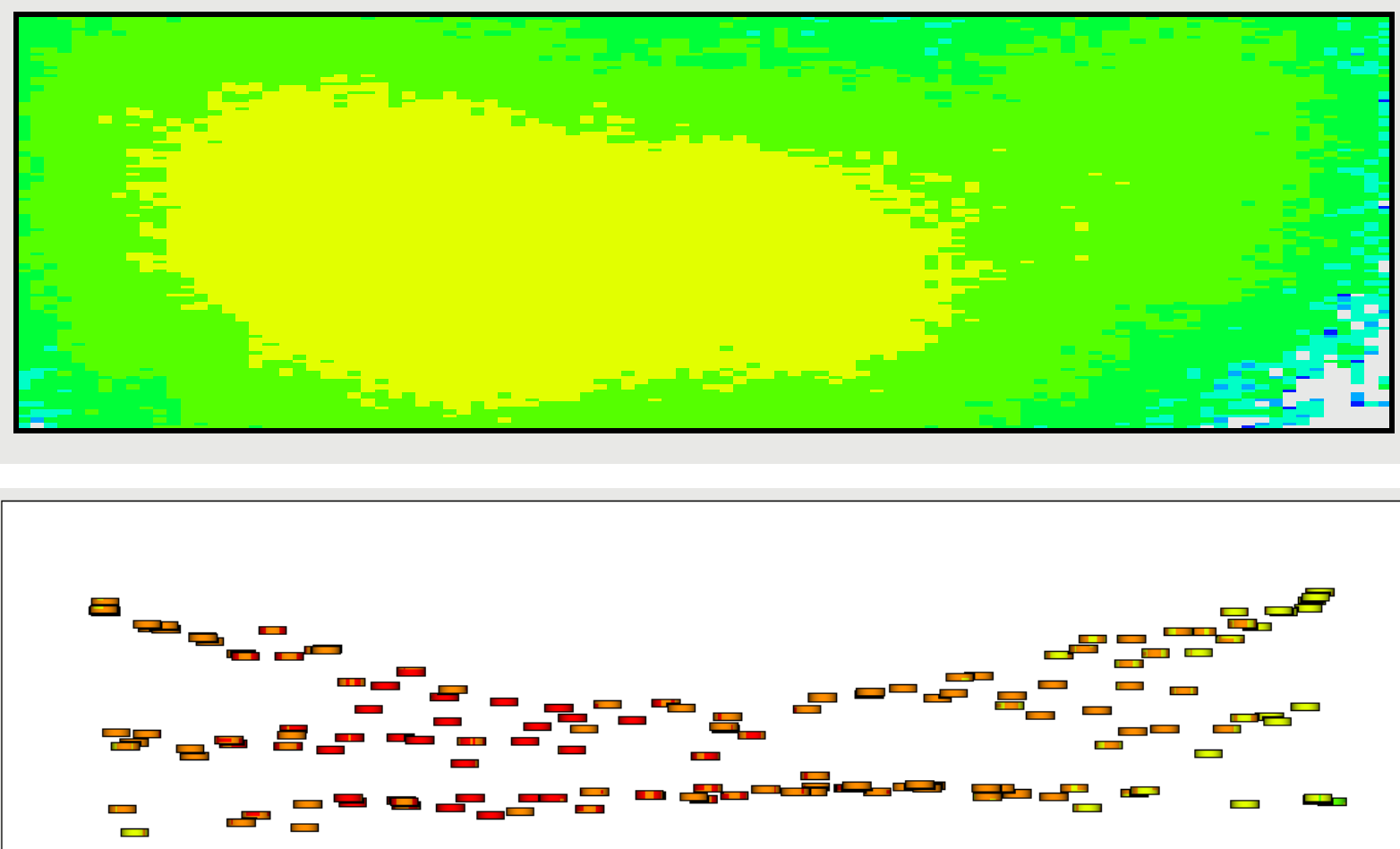

Figure E-8. Distribution of the flux for the $x-z$ direction (top and bottom) with contour count of 10 for 100 years for the SNL upper horizon compaction results. All cases use the set-2-4 bounding conditions (cylindrical waste forms with no filler, no can, no Be, thick poly discrete reflector, and approximately $700 \mathrm{~g}$ poly moderator in the 1-unit model). 

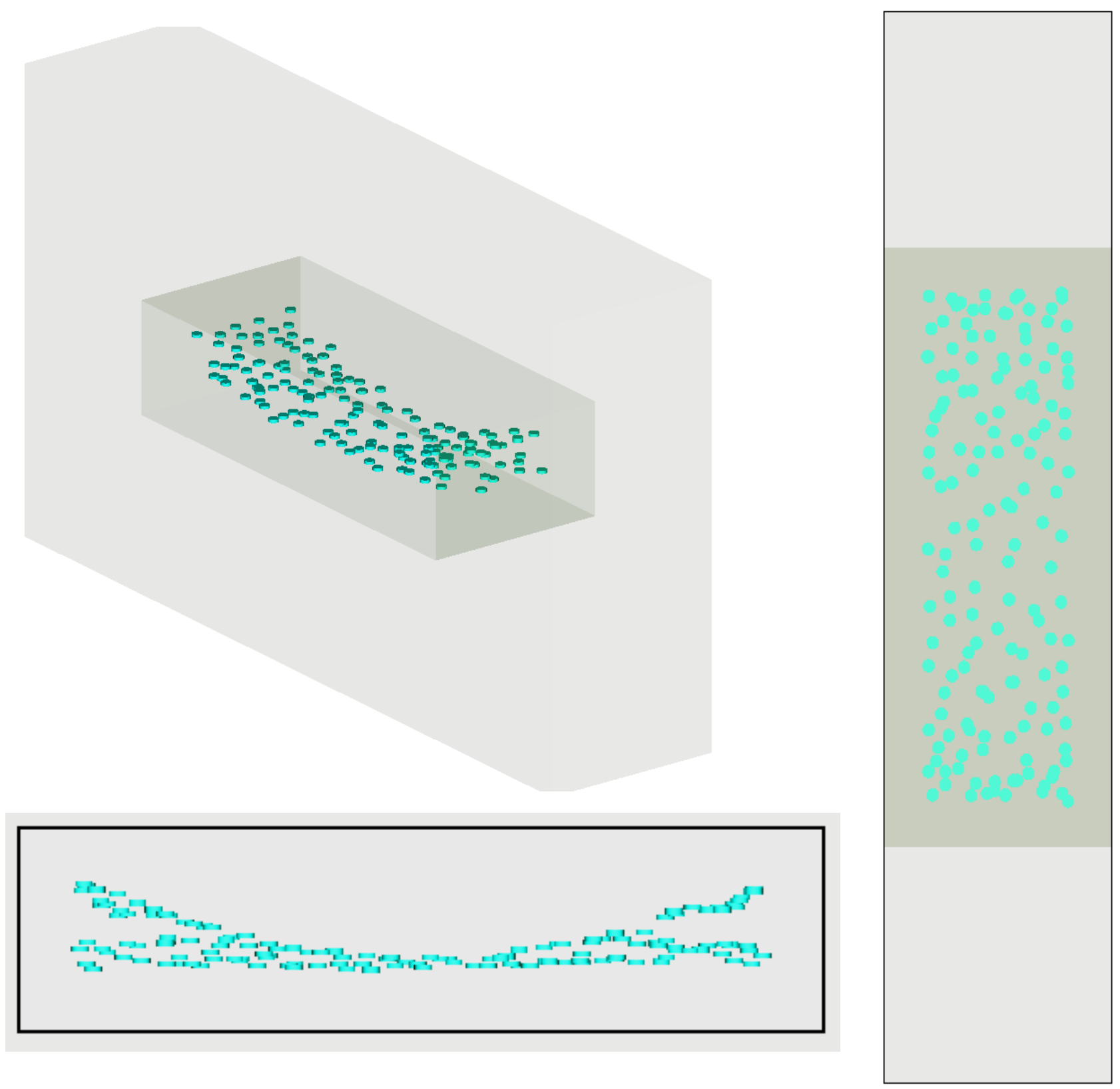

Figure E-9. Layout of the 1-unit, 200-year CCO locations in 3D isometric (top left), top view (right) and front (bottom left). 

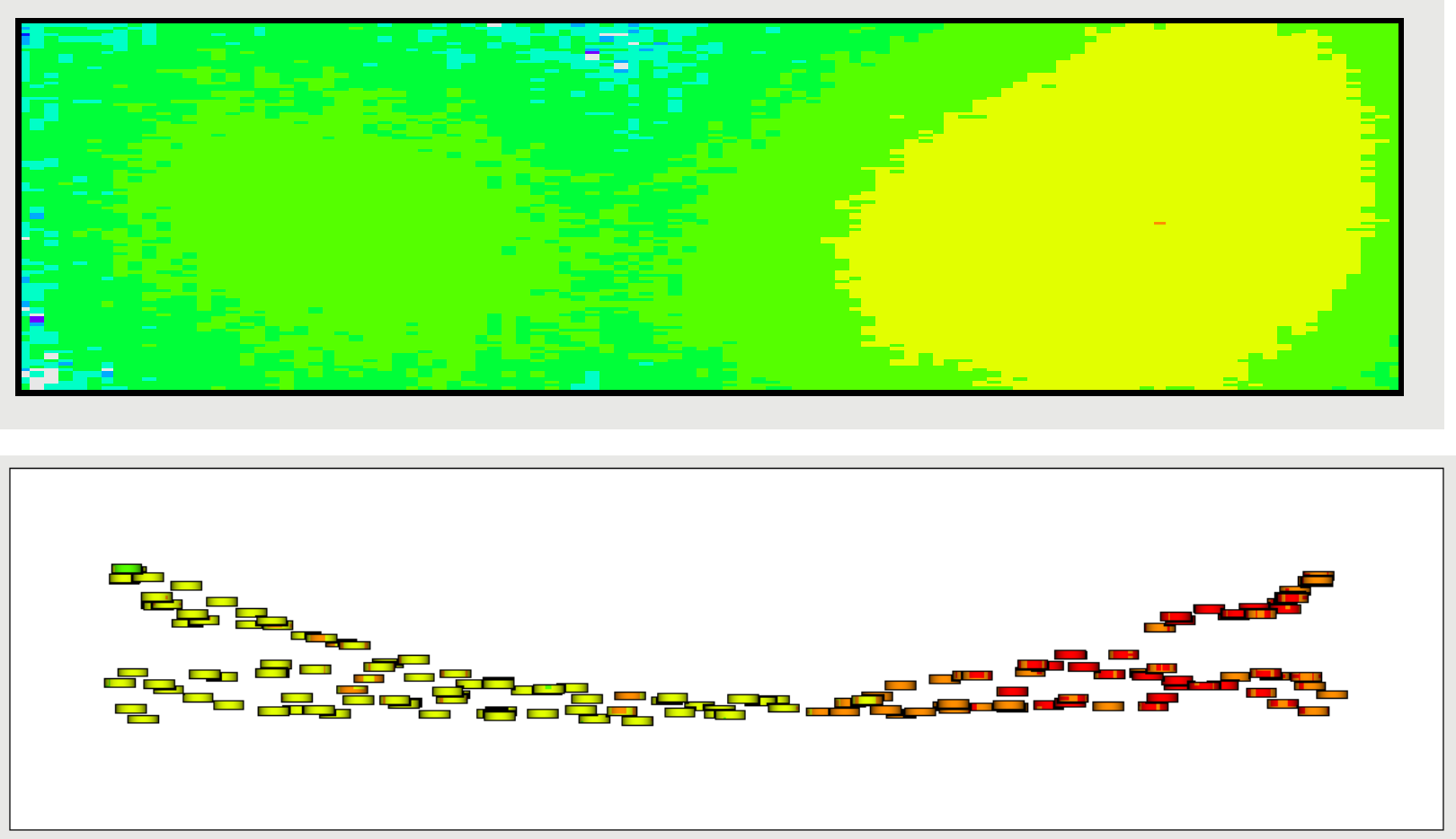

Figure E-10. Distribution of the flux for the $x-z$ direction (top and bottom) with contour count of 10 for 200 years for the SNL upper horizon compaction results. All cases use the set-2-4 bounding conditions (cylindrical waste forms with no filler, no can, no Be, thick poly discrete reflector, and approximately $700 \mathrm{~g}$ poly moderator in the 1-unit model) 


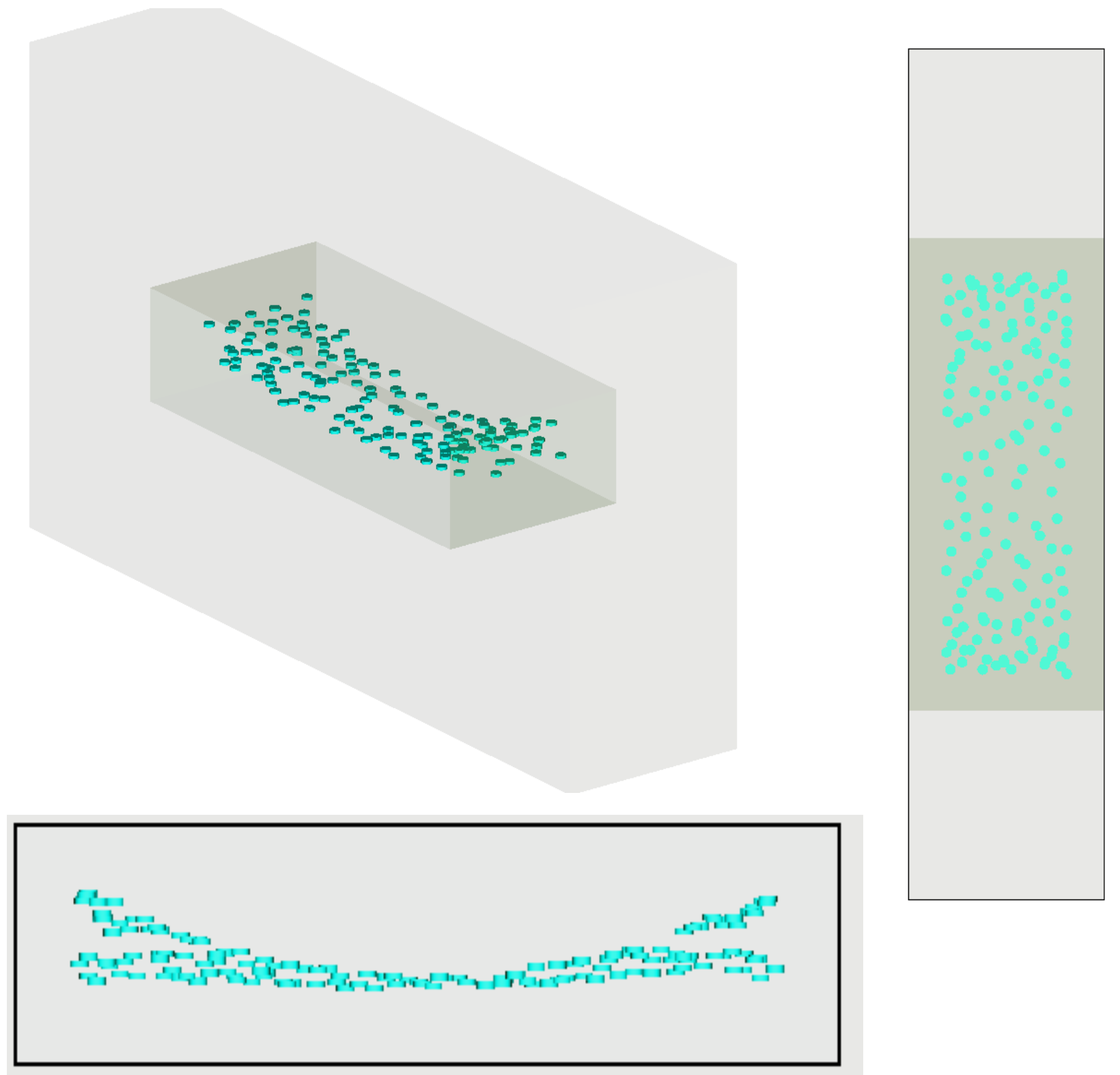

Figure E-11. Layout of the 1-unit, 300-year CCO locations in 3D isometric (top left), top view (right) and front (bottom left). 

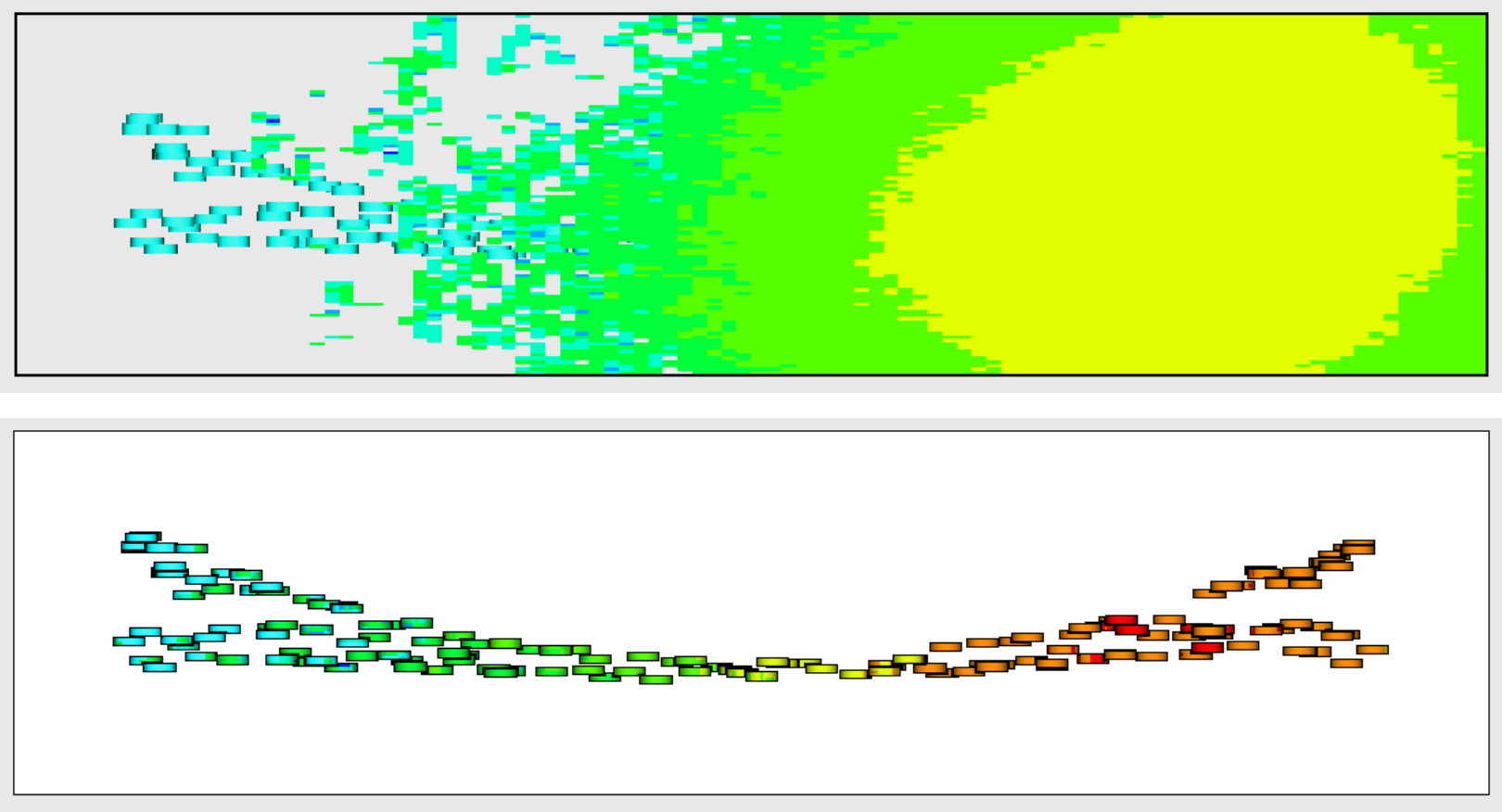

Figure E-12. Distribution of the flux for the $x-z$ direction (top and bottom) with contour count of 10 for 300 years for the SNL upper horizon compaction results. All cases use the set-2-4 bounding conditions (cylindrical waste forms with no filler, no can, no Be, thick poly discrete reflector, and approximately $700 \mathrm{~g}$ poly moderator in the 1-unit model) 

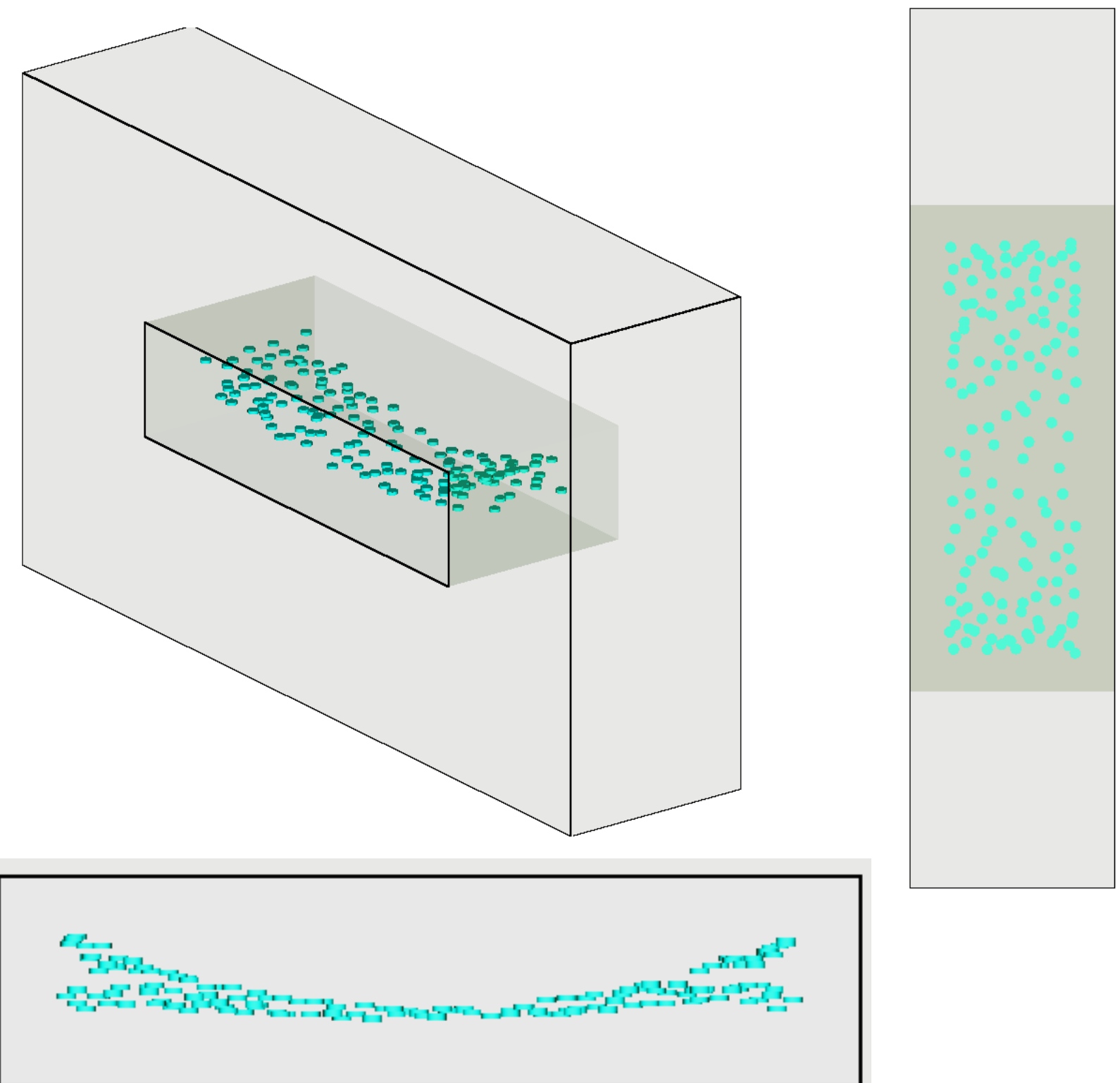

Figure E-13. Layout of the 1-unit, 400-year CCO locations in 3D isometric (top left), top view (right) and front (bottom left). 

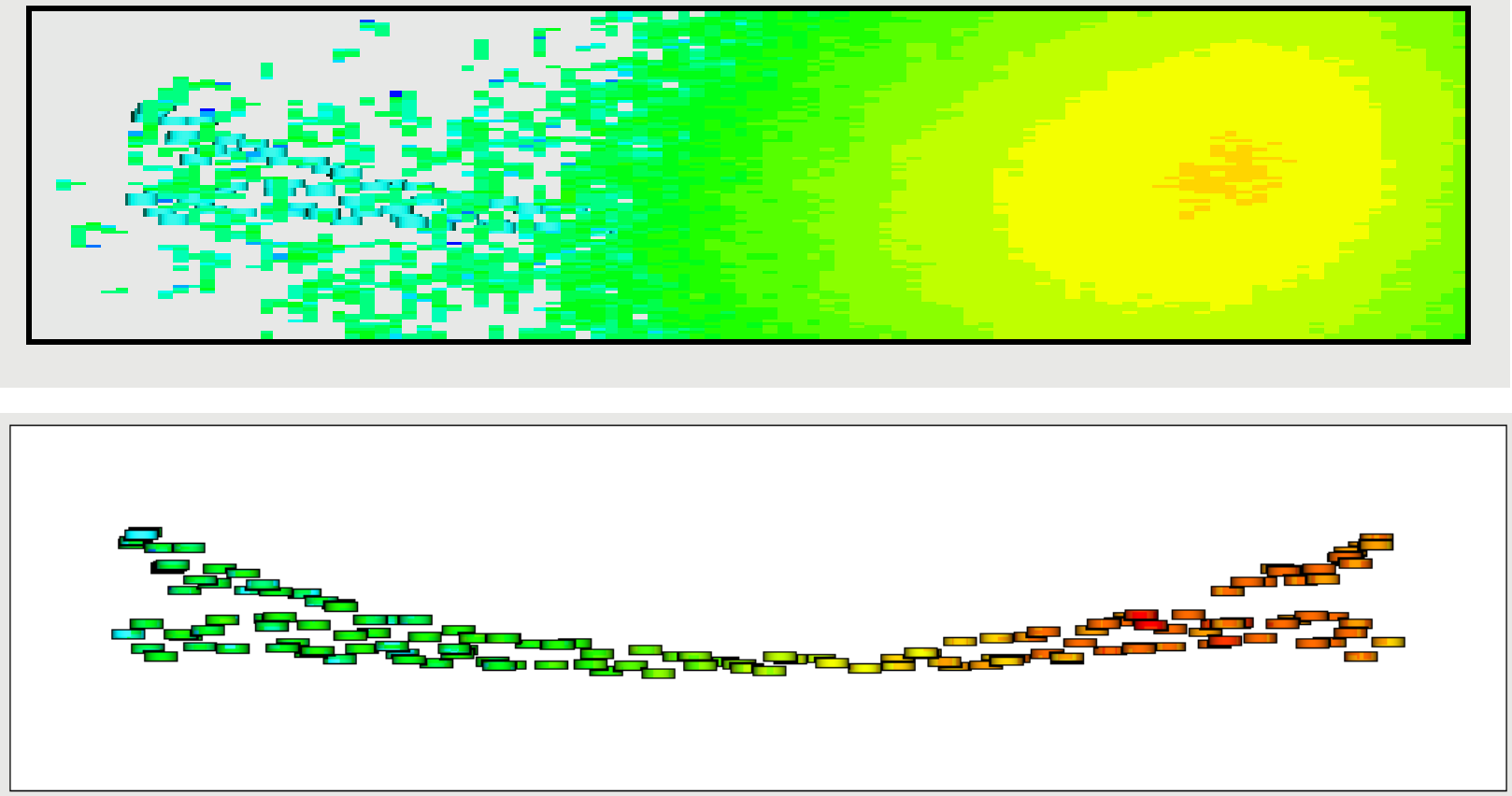

Figure E-14. Distribution of the flux for the $x-z$ direction (top and bottom) with contour count of 10 for 400 years for the SNL upper horizon compaction results. All cases use the set-2-4 bounding conditions (cylindrical waste forms with no filler, no can, no Be, thick poly discrete reflector, and approximately $700 \mathrm{~g}$ poly moderator in the 1-unit model) 


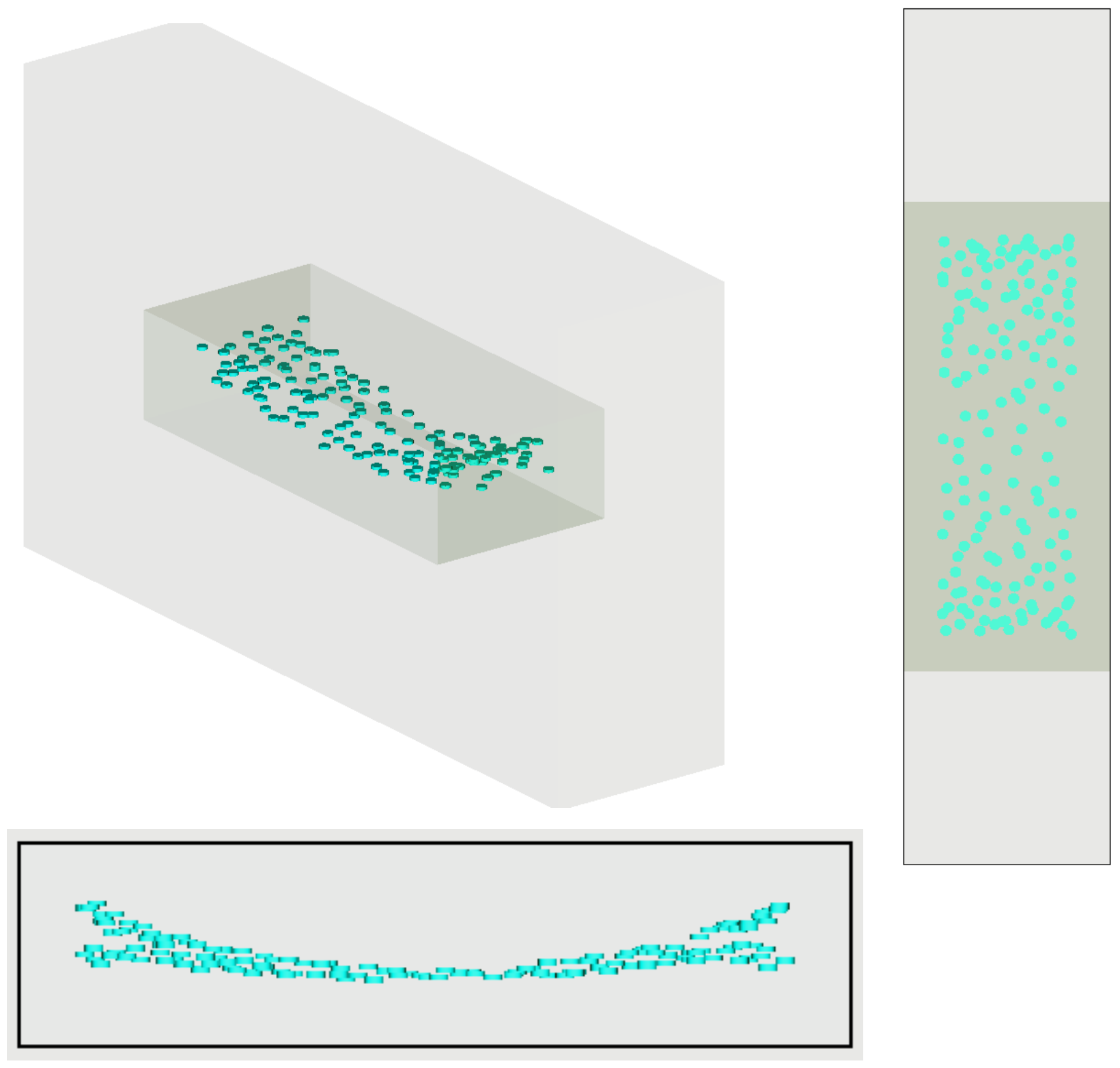

Figure E-15. Layout of the 1-unit, 1,000-year CCO locations in 3D isometric (top left), top view (right) and front (bottom left). 

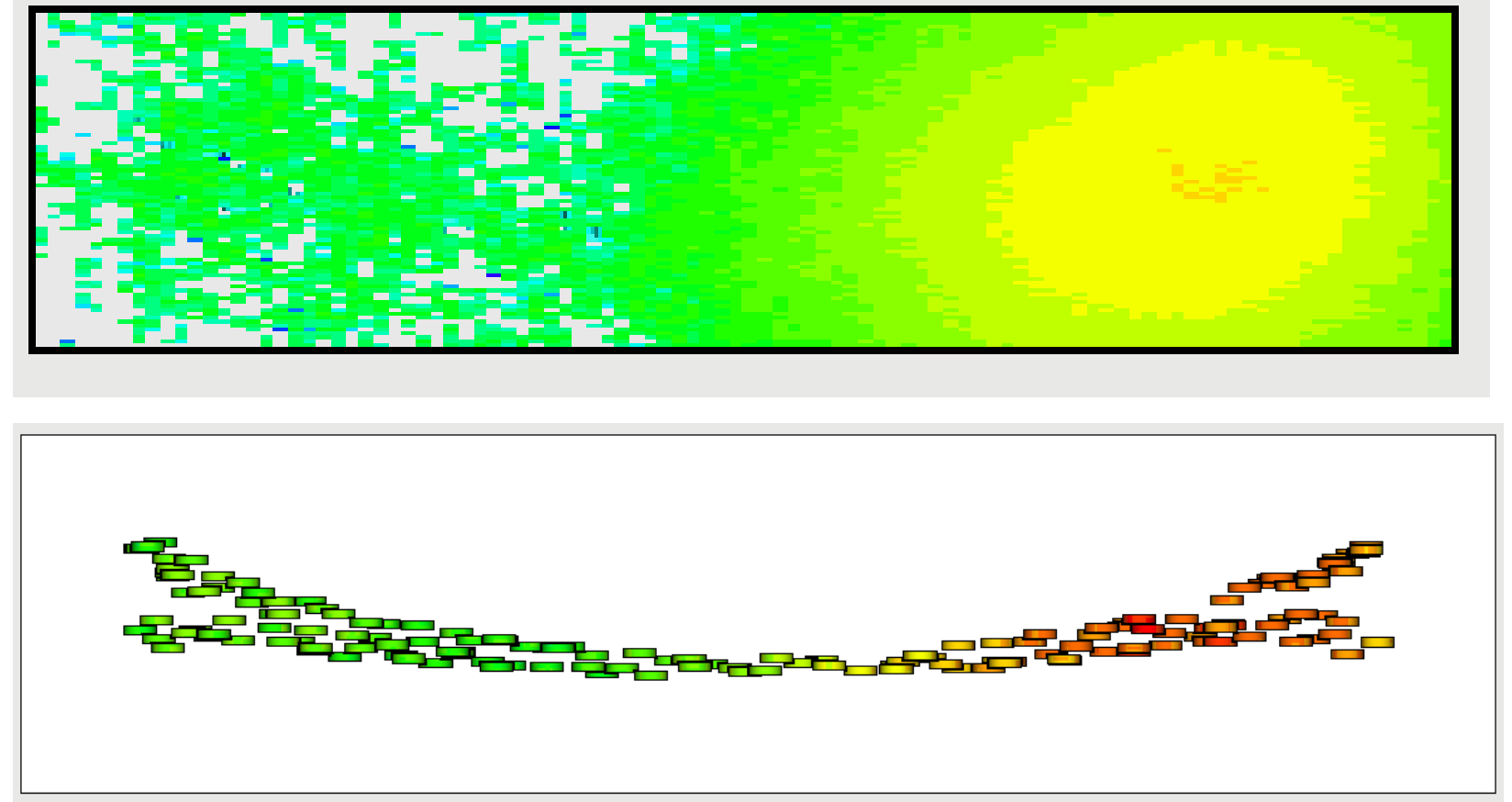

Figure E-16. Distribution of the flux for the $x-z$ direction (top and bottom) with contour count of 10 for 1,000 years for the SNL upper horizon compaction results. All cases use the set-2-4 bounding conditions (cylindrical waste forms with no filler, no can, no Be, thick poly discrete reflector, and approximately $700 \mathrm{~g}$ poly moderator in the 1-unit model) 


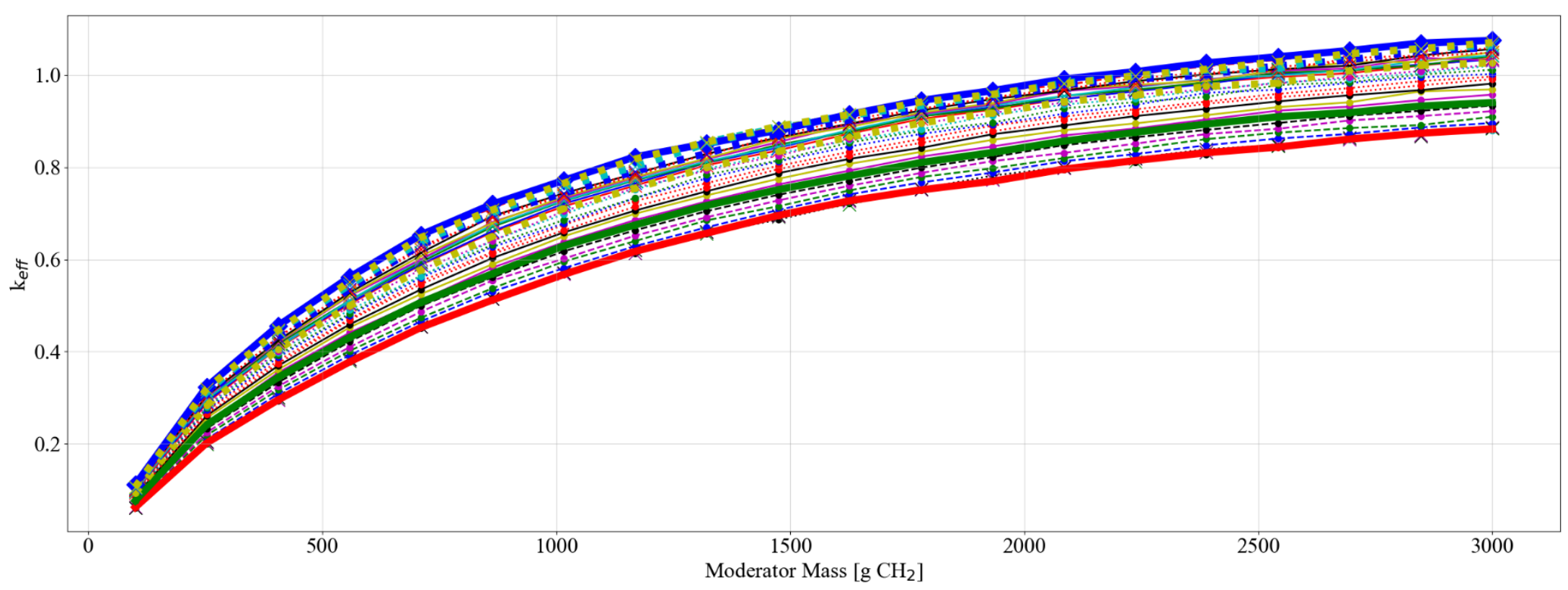

\begin{tabular}{|c|c|c|}
\hline & set-2-uh-td-0-yr & $\cdots \bullet \cdots \quad$ set-2-uh-td-150-yr \\
\hline & set-2-uh-td-1000-yr & $\cdots \bullet \cdots \quad$ set -2 \\
\hline & set-2-uh-td-100-yr & $\cdots \bullet \cdots \quad$ set- \\
\hline & set-2-uh-td-10-yr & $\cdots \bullet \cdots \quad$ set-2-uh-td-180-yr \\
\hline$\rightarrow$ & set-2-uh-td-110-yr & $\cdots \bullet \cdots \quad$ set-2-uh-td-190-yr \\
\hline 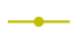 & set-2-uh-td-120-yr & " = " set-2-uh-td-200-yr \\
\hline 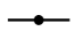 & set-2-uh-td-130-yr & $\cdots \bullet \cdots \quad$ set-2-uh-td-20-yr \\
\hline •.. & set-2-uh-td-140-yr & $\leftarrow$ set-2-uh-td-210-yr \\
\hline
\end{tabular}
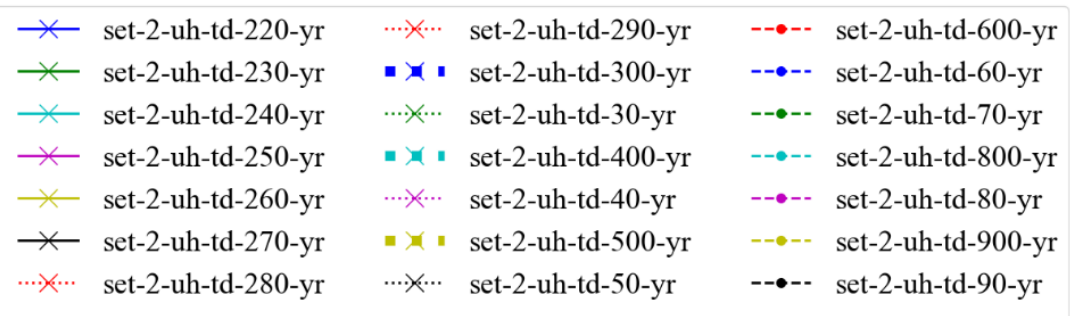

Figure E-17. Comparison of various small-step time-dependent compaction steps for the SNL upper horizon compaction results. All cases use the set-2-4 bounding conditions (cylindrical waste forms with no filler, no can, no Be, thick poly discrete reflector, and poly moderator). 


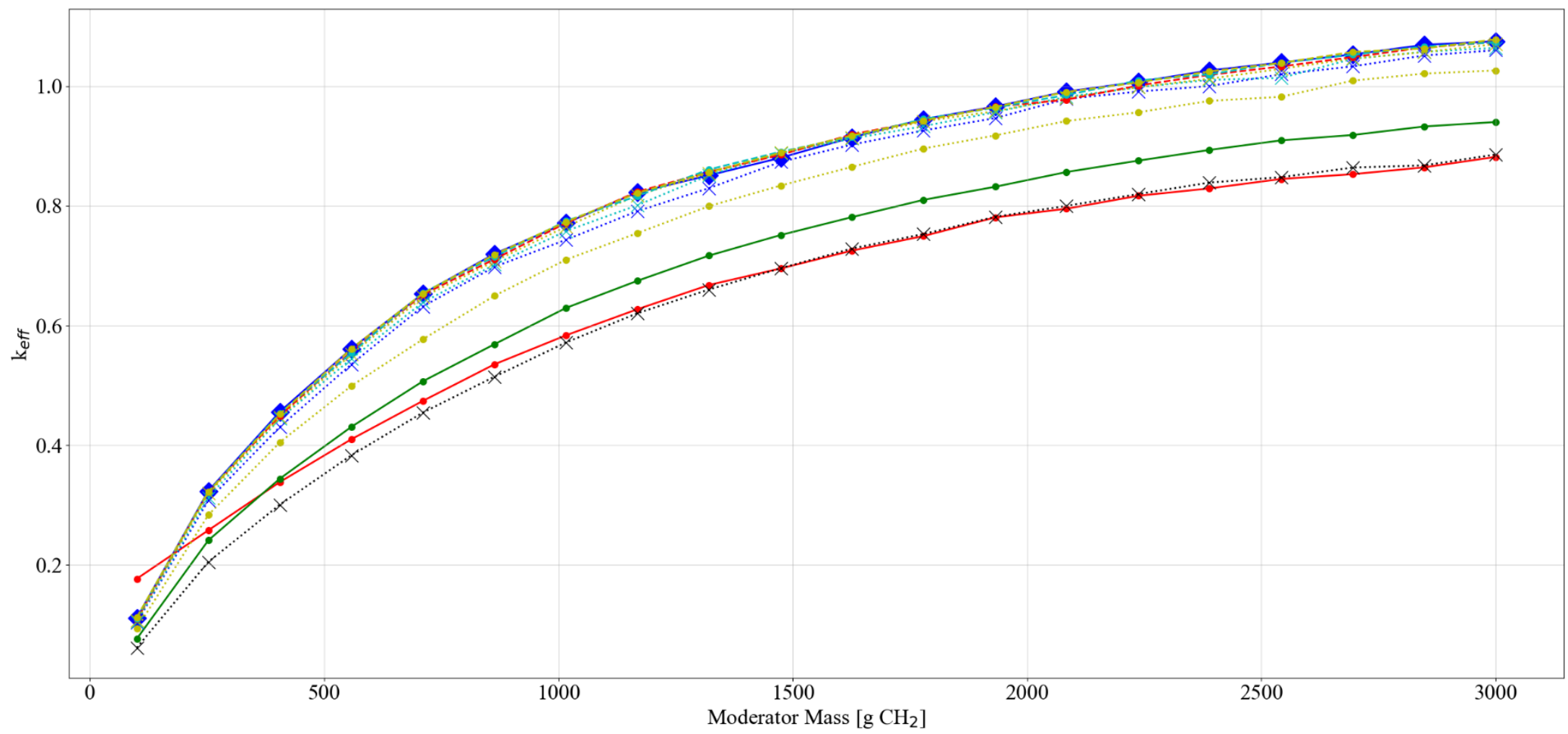

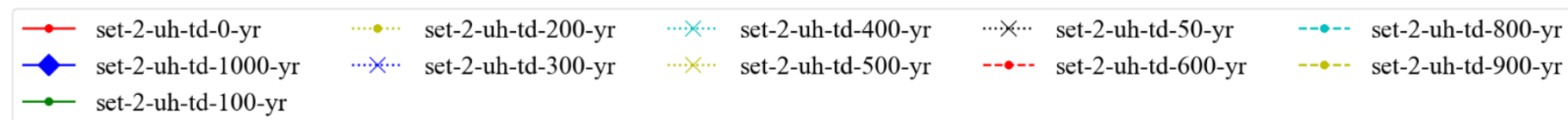

Figure E-18. Comparison of various large-step time-dependent compaction steps for the SNL upper horizon compaction results. All cases use the set-2-4 bounding conditions (cylindrical waste forms with no filler, no can, no Be, thick poly discrete reflector, and poly moderator). 


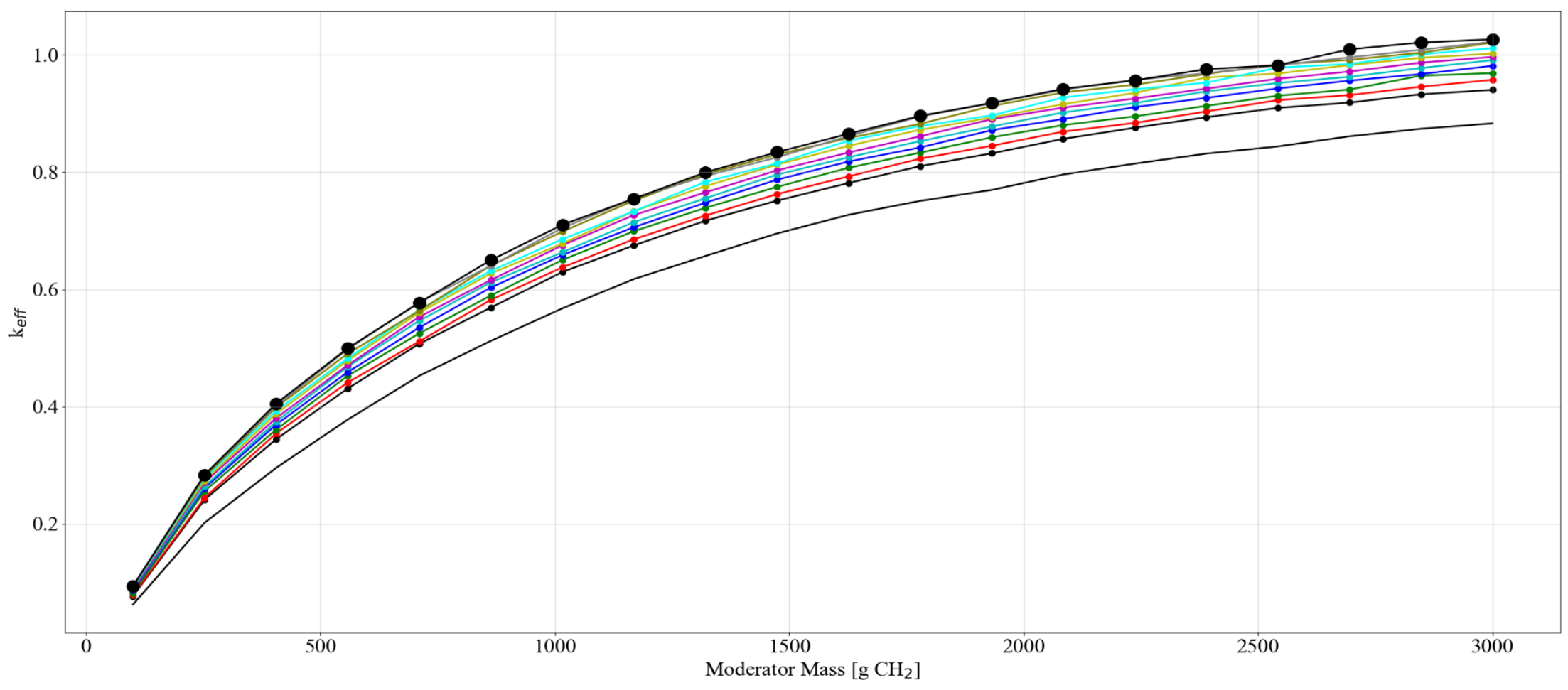

\begin{tabular}{|c|c|c|c|c|c|c|}
\hline & & $\rightarrow-$ & set-2-uh-td-120-yr & $\rightarrow$ & set-2-uh-td-150-yr & $\longrightarrow$ set- \\
\hline & & 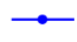 & set-2- & 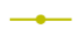 & -td-160-yr & $\longrightarrow$ se \\
\hline & -yr & 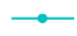 & set-2-uh-td-140-yr & $\because$ & set-2-uh-td-170-yr & set-2-uh-td-200-y \\
\hline
\end{tabular}

Figure E-19. Comparison of various small-step time-dependent compaction steps between 100 and 200 years for the SNL upper horizon compaction results. All cases use the set-2-4 bounding conditions (cylindrical waste forms with no filler, no can, no Be, thick poly discrete reflector, and poly moderator). 


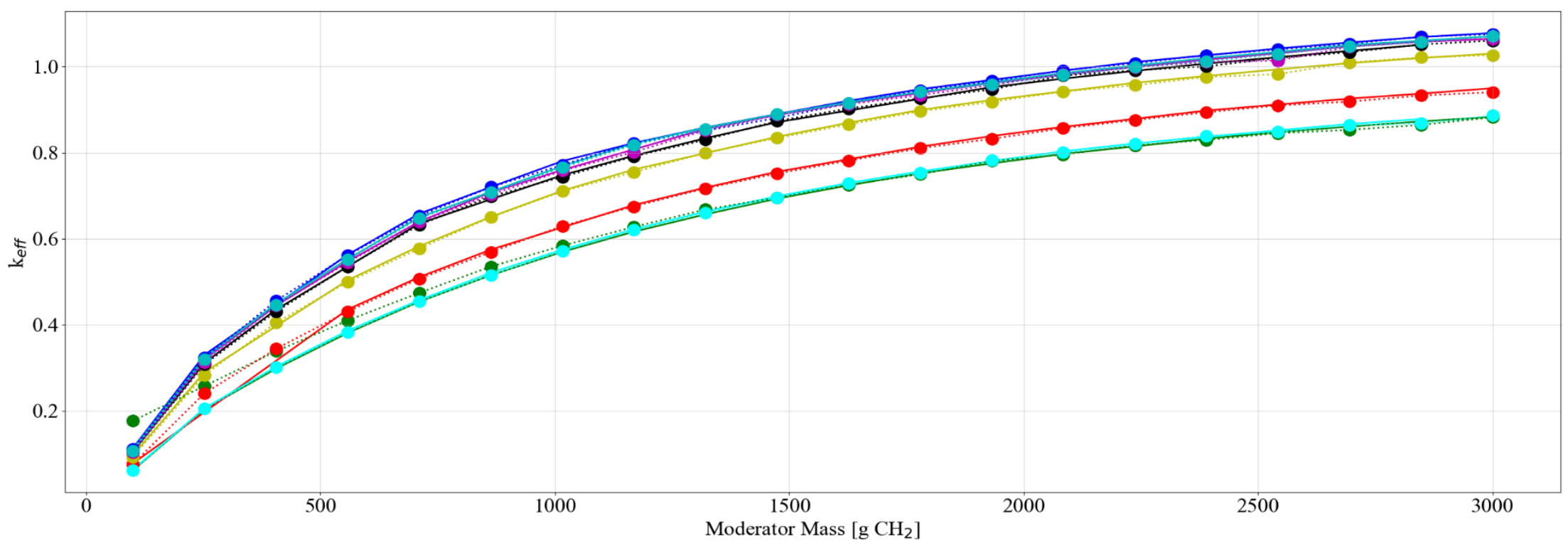

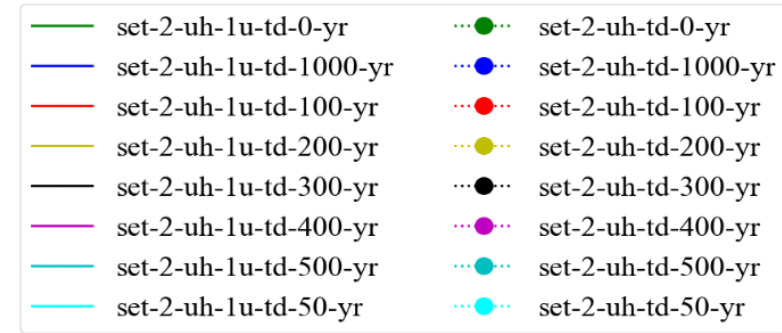

Figure E-20. Comparison of the 1- (-1u-) to 2-unit SCALE models for various time dependent cases for the SNL upper horizon compaction results. All cases use the set-2-4 bounding conditions (cylindrical waste forms with no filler, no can, no Be, thick poly discrete reflector, and poly moderator). 


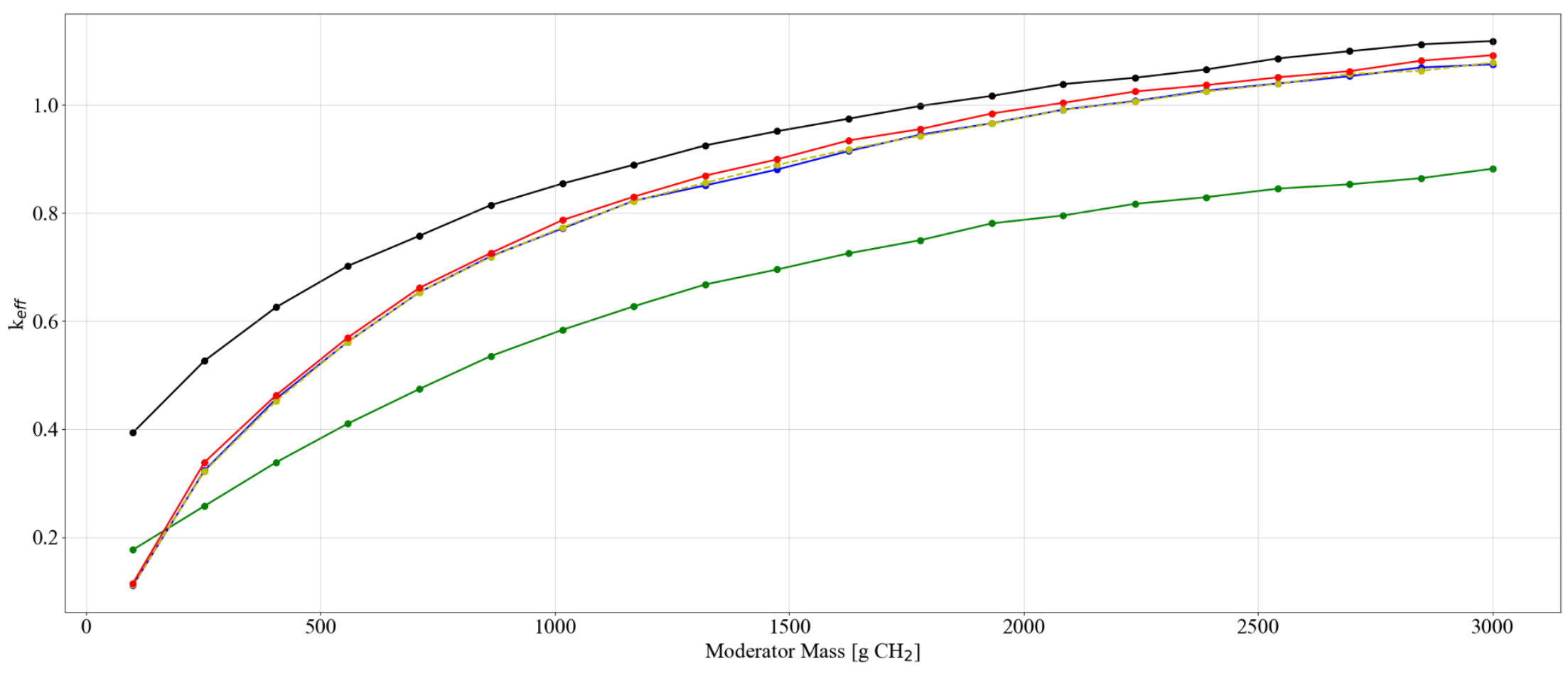

$$
\begin{array}{ll}
\longrightarrow & \text { set-2-uh-td-0-yr } \\
\longrightarrow & \text { set-2-uh-td-1000-yr } \\
-\bullet-- & \text { set-2-uh-td- } 900-\text { yr } \\
\longrightarrow & \text { set-2-uh, sphere } \\
\longrightarrow & \text { set-2-uh, cylinder with radius }=7.7 \mathrm{~cm}
\end{array}
$$

Figure E-21. Comparison of the sphere and cylinder models for various time-dependent cases for the SNL upper horizon compaction results. All cases use the set-2-4 bounding conditions (cylindrical or spherical waste forms with no filler, no can, no Be, thick poly discrete reflector, and poly moderator). 


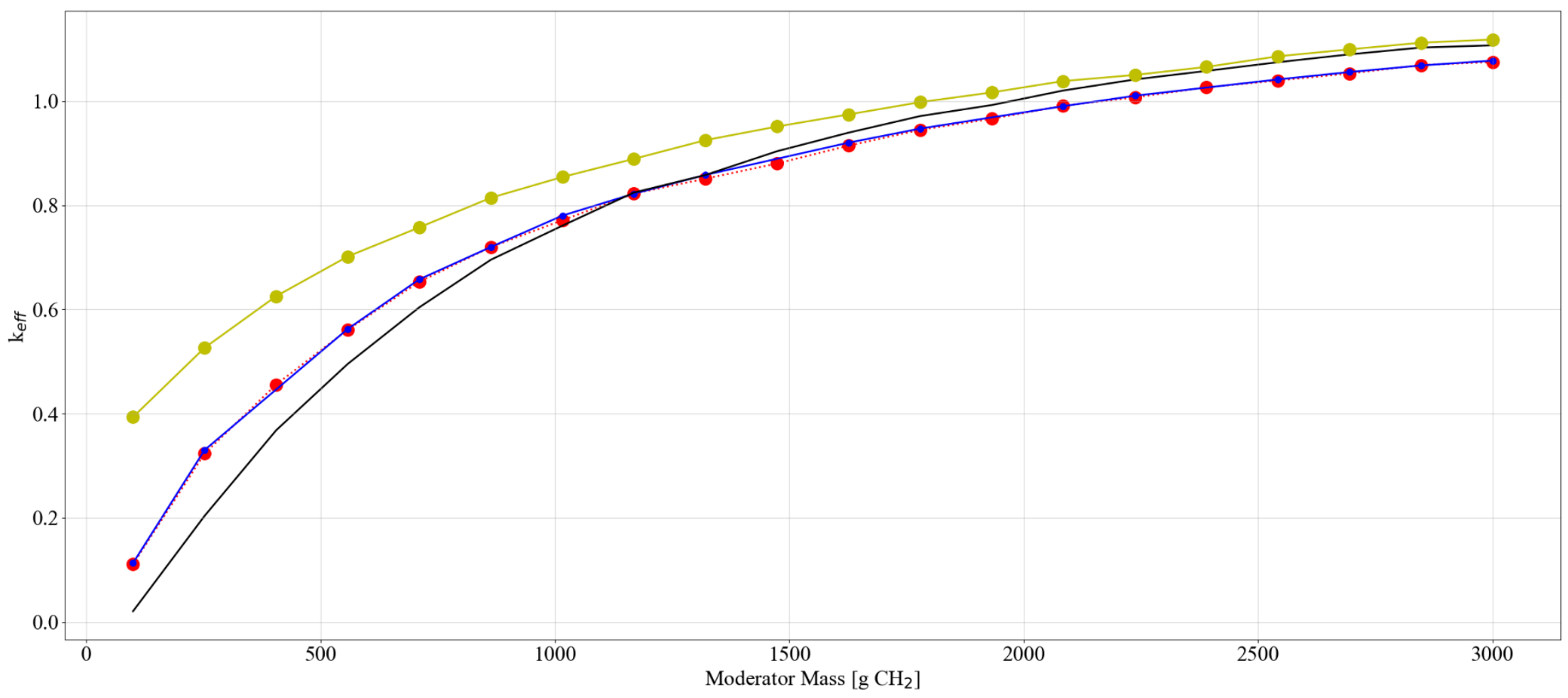

-.. set-2-uh-td-1000-yr, 2-unit, $50 \mathrm{~cm}$ before reflective boundary conditions

- - set-2-uh-td-1u-1000-yr, 1-unit, $50 \mathrm{~cm}$ before reflective boundary condition

— set-2-uh-td-1000-yr, 1-unit, $0 \mathrm{~cm}$ before reflective boundary conditions

- set-2-uh-td-1000-yr, 1-unit, $0 \mathrm{~cm}$ before reflective boundary conditions, spheres

Figure E-22. Comparison of the reactivity effect of location of the reflective boundary conditions, KENO parametric block parameters for the 1- and 2unit SCALE sphere, and cylinder models for the 1,000-year time-dependent case for the SNL upper horizon compaction results. All cases use the set-2-4 bounding conditions (cylindrical or spherical waste forms with no filler, no can, no Be, thick poly discrete reflector, and poly moderator). 


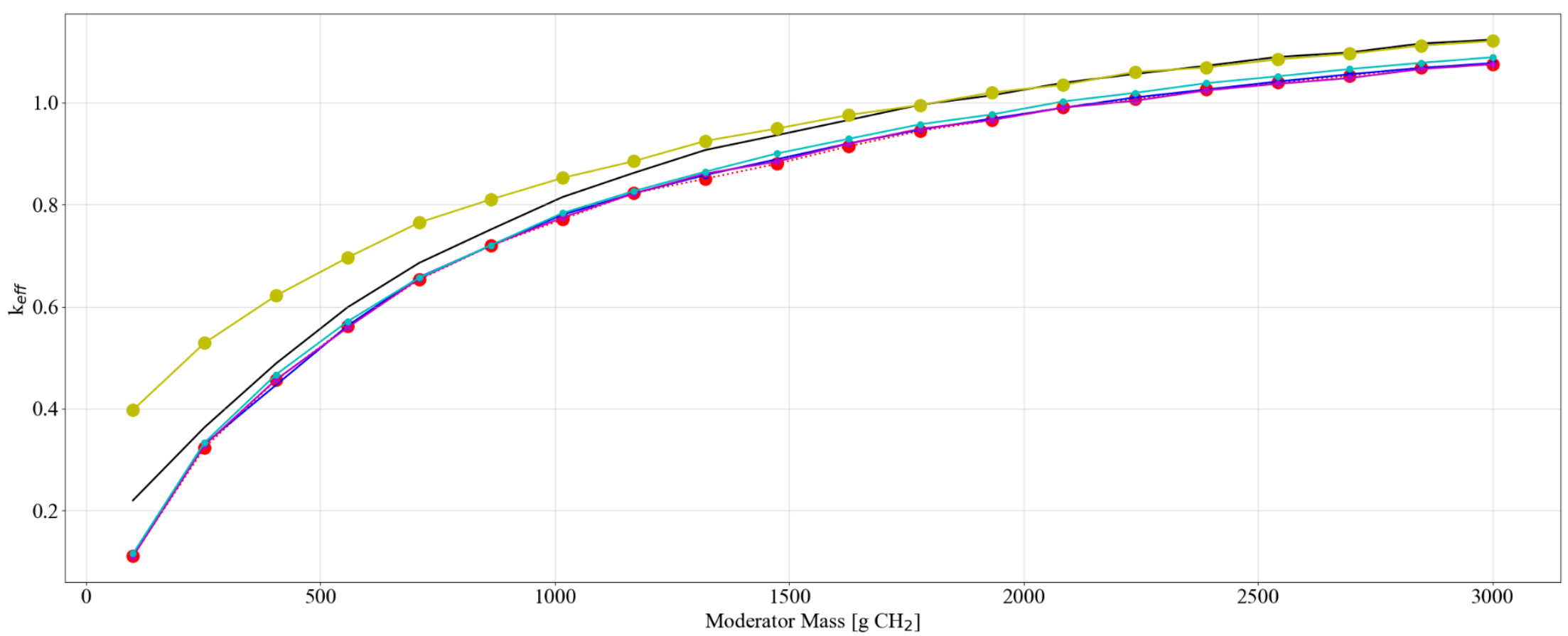

- $\cdots$ set-2-uh-td-1000-yr, 2-unit, $50 \mathrm{~cm}$ before reflective boundary conditions

$\rightarrow$ set-2-uh-td-1u-1000-yr, 1-unit, $50 \mathrm{~cm}$ before reflective boundary conditions

- set-2-uh-1000-yr, 2-unit, $0 \mathrm{~cm}$ before reflective boundary conditions

$\rightarrow$ set-2-uh-1000-yr, 2-unit, $0 \mathrm{~cm}$ before reflective boundary conditions, spheres

$\rightarrow$ set-2-uh-td-2up-1000-yr, 1-unit, $50 \mathrm{~cm}$ before periodic boundary conditions

$\longrightarrow$ set-2-uhp-1000-yr, 2-unit, set 2 model with periodic boundary conditions

Figure E-23. Comparison of the reactivity effect of various boundary conditions for the 1- and 2-unit SCALE sphere and cylinder models for the 1,000year time-dependent case for the SNL upper horizon compaction results. All cases use the set-2-4 bounding conditions (cylindrical or spherical waste forms with no filler, no can, no Be, thick poly discrete reflector, and poly moderator). 


\section{APPENDIX F. SET-3: RESULTS OF THE SIX-HIGH UNIFORM ARRAY WITH BOUNDING SPACING CALCULATIONS}


This page is intentionally blank 


\section{APPENDIX F. SET-3: RESULTS OF THE SIX-HIGH UNIFORM ARRAY WITH BOUNDING SPACING CALCULATIONS}

The analysis methodology for the uniform arrays is discussed in detail in Section 6.2 of the main report.

This appendix serves as a repository of those results for the set-1 calculations.

The complete results for all SAMPLER sweeps are provided in ADDENDUM 1.

The analysis model use for the calculations in this appendix is shown in Figure F-1 below.

The SAMPLER case sweeps presented in this appendix are summarized in Table F-1 below.

\section{LIST OF FIGURES}

Figure F-1. Diagram of the uniform array six-high model.

Figure F-2. Set-3 results, plot 1: reactivity effect of cylinder radius, pipe steel, no filler, no Be, water moderated.

Figure F-3. Set-3 results, plot 2: reactivity effect of cylinder radius, pipe steel, no filler, no Be, poly moderated.

Figure F-4. Set-3 results, plot 3: reactivity effect of cylinder radius, pipe poly, no filler, no Be, water moderated.

Figure F-5. Set-3 results, plot 4: reactivity effect of cylinder radius, pipe poly, no filler, no Be, poly moderated.

Figure F-6. Set-3 results, plot 5: reactivity effect of cylinder radius, pipe steel, $5 \mathrm{~kg}$ graphite/can, no $\mathrm{Be}$, water moderated.

Figure F-7. Set-3 results, plot 6: reactivity effect of cylinder radius, pipe steel, $5 \mathrm{~kg}$ graphite/can, no $\mathrm{Be}$, poly moderated.

Figure F-8. Set-3 results, plot 7: reactivity effect of cylinder radius, pipe poly, $5 \mathrm{~kg}$ graphite/can, no $\mathrm{Be}$, water moderated.

Figure F-9. Set-3 results, plot 8: reactivity effect of cylinder radius, pipe poly, $5 \mathrm{~kg}$ graphite/can, no Be, poly moderated.

Figure F-10. Set-3 results, plot 9: reactivity effect of various parameters with $7.7 \mathrm{~cm}$ cylinder radius, graphite filler, poly moderated.

Figure F-11. Set-3 results, plot 10: reactivity effect of various parameters with $7.7 \mathrm{~cm}$ cylinder radius, generic filler, poly moderated.

Figure F-12. Set-3 results, plot 11: comparison of graphite and generic filler with $7.7 \mathrm{~cm}$ cylinder radius, no Be, poly moderated, thick discrete reflector.....

Figure F-13. Set-3 results, plot 12: reactivity effect of various parameters with spherical waste form geometry, graphite filler, water moderated.

Figure F-14. Set-3 results, plot 13: reactivity effect of various parameters with spherical waste form geometry, graphite filler, poly moderated.

Figure F-15. Set-3 results, plot 14: comparison of spherical and cylindrical geometries (h/x)..............F-20

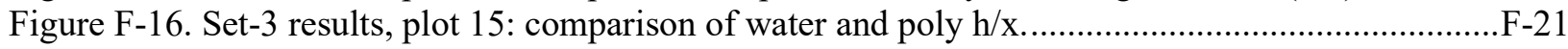

Figure F-17. Set-3 results, plot 16: comparison of spherical and cylindrical geometries (mod mass).

Figure F-18. Set-3 results, plot 17: Reactivity effect of $25 \mathrm{~g} \mathrm{~B} 4 \mathrm{C}$ per waste form (2 per CCO) ..............F-23 


\section{LIST OF TABLES}

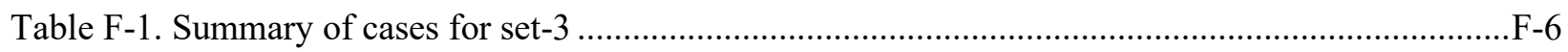




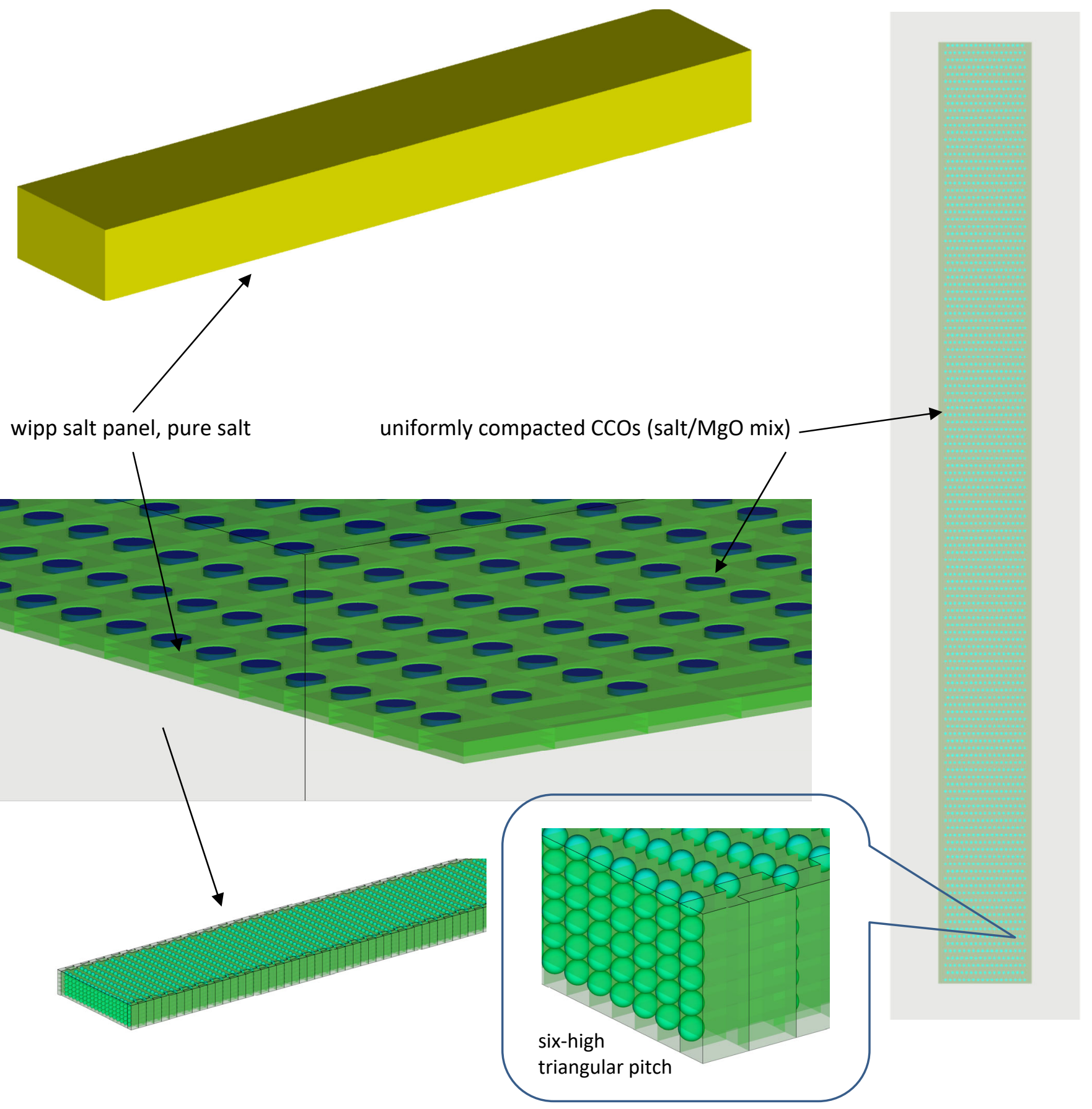

Figure F-1. Diagram of the uniform array six-high model. 
Table F-1. Summary of cases for set-3

\begin{tabular}{|c|c|c|c|c|c|c|c|c|}
\hline Case & Model type & Waste form shape & $\begin{array}{l}\text { Waste form } \\
\text { moderator }\end{array}$ & $\begin{array}{c}\text { Filler } \\
\text { material }(0, \\
2,000,4,000 \\
\text { g) }\end{array}$ & Metal in filler & $\begin{array}{l}\text { Discrete reflector } \\
\text { (thin } 0.001 \text { and } \\
\text { thick } 0.7112 \mathrm{~cm} \text { ) }\end{array}$ & be $(g)$ & Subcase \\
\hline \multirow{18}{*}{ Set-3 } & \multirow{18}{*}{$\begin{array}{c}\text { Uniform array stacked six- } \\
\text { high with CCO pitch } \\
\text { reduction } \mathrm{x}=25 \%, \mathrm{y}=10 \%, \\
\mathrm{z}=\text { no space }\end{array}$} & \multirow{8}{*}{$\begin{array}{c}\text { Cylinder (radius range } 4.8,6 \\
7.7 \text { and height defined by } \\
\text { total volume of mass) }\end{array}$} & water & c12 & \multirow{18}{*}{$\begin{array}{l}\text { SS from can } \\
(0,500,1,000 \\
\text { g) }\end{array}$} & steel & \multirow{18}{*}{$\begin{array}{l}0 \text { to } \\
585\end{array}$} & set-3-1 \\
\hline & & & poly & $\mathrm{c} 12$ & & steel & & set-3-2 \\
\hline & & & water & c12 & & poly & & set-3-3 \\
\hline & & & poly & $\mathrm{c} 12$ & & poly & & \begin{tabular}{|l|} 
set-3-4 \\
\end{tabular} \\
\hline & & & water & generic & & steel & & set-3-5 \\
\hline & & & poly & generic & & steel & & set-3-6 \\
\hline & & & water & generic & & poly & & \begin{tabular}{|l|} 
set-3-7 \\
\end{tabular} \\
\hline & & & poly & generic & & poly & & set-3-8 \\
\hline & & \multirow{8}{*}{$\begin{array}{l}\text { Sphere (radius defined by } \\
\text { total volume of mass) }\end{array}$} & water & $\mathrm{c} 12$ & & steel & & set-3-9 \\
\hline & & & poly & $\mathrm{c} 12$ & & steel & & set-3-10 \\
\hline & & & water & $\mathrm{c} 12$ & & poly & & set-3-11 \\
\hline & & & poly & $\mathrm{c} 12$ & & poly & & set-3-12 \\
\hline & & & water & generic & & steel & & set-3-13 \\
\hline & & & poly & generic & & steel & & set-3-14 \\
\hline & & & water & generic & & poly & & set-3-15 \\
\hline & & & poly & generic & & poly & & set-3-16 \\
\hline & & $\begin{array}{l}\text { Cylinder (radius range } 4.8,6, \\
7.7 \text { and height defined by } \\
\text { total volume of mass) with } \\
25 \mathrm{~g} \mathrm{~B}_{4} \mathrm{C}\end{array}$ & poly & $\mathrm{c} 12$ & & poly & & set-3-17 \\
\hline & & $\begin{array}{l}\text { Sphere (radius defined by } \\
\text { total volume of mass) with } \\
25 \mathrm{~g} \mathrm{~B}_{4} \mathrm{C}\end{array}$ & poly & $\mathrm{c} 12$ & & poly & & set-3-18 \\
\hline
\end{tabular}




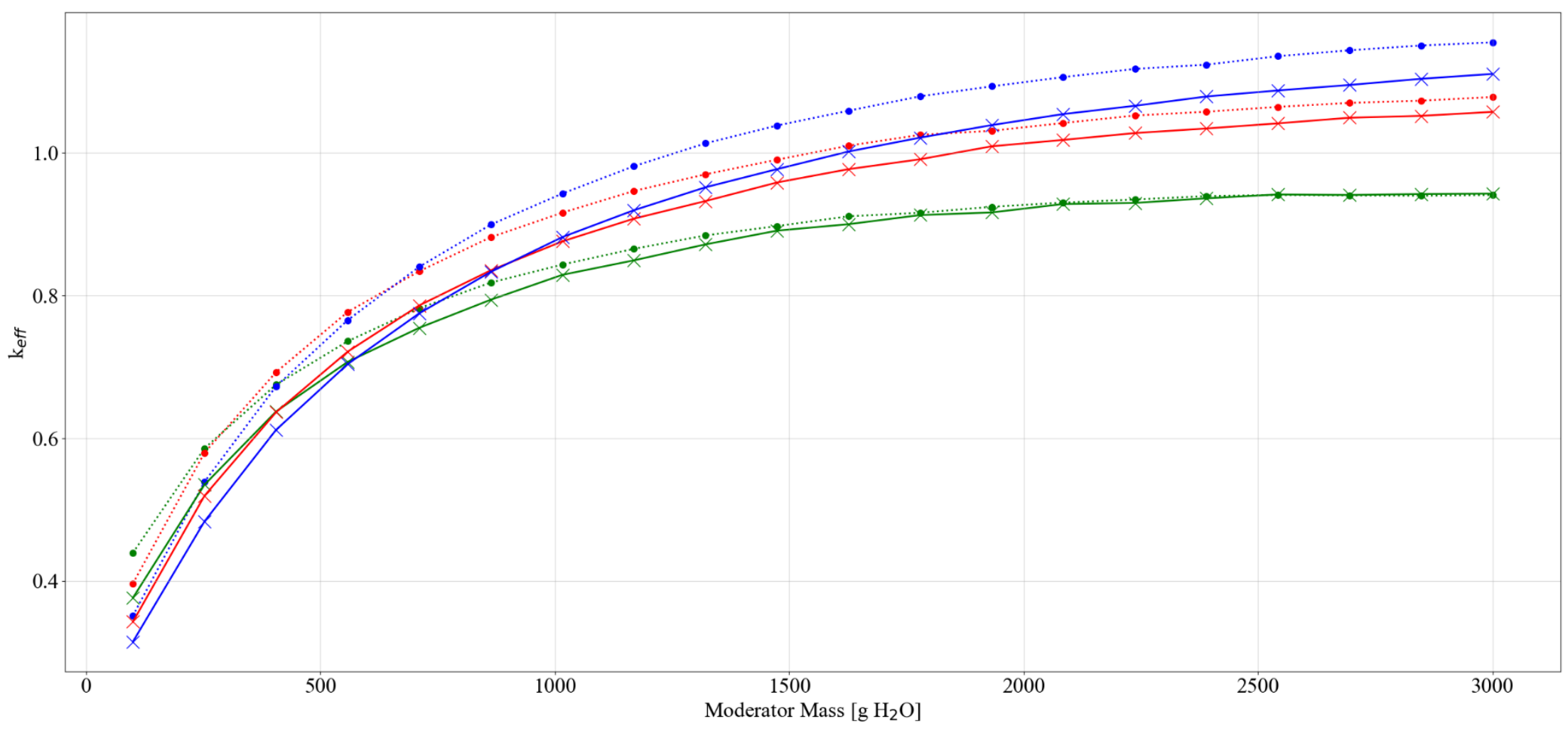

-.. set- 3 , cyl radius $=4.8 \mathrm{~cm}$, thin discrete reflector

$\begin{array}{ll}\bullet \cdots & \text { set- } 3 \text {, cyl radius }=6.25 \mathrm{~cm} \text {, thin discrete reflector } \\ \bullet \cdots & \text { set- } 3 \text {, cyl radius }=7.7 \mathrm{~cm} \text {, thin discrete reflector }\end{array}$

$\leftarrow$ set- 3 , cyl radius $=4.8 \mathrm{~cm}$, thick discrete reflector

$\leftarrow$ set- 3 , cyl radius $=6.25 \mathrm{~cm}$, thick discrete reflector

$\star$ set- 3 , cyl radius $=7.7 \mathrm{~cm}$, thick discrete reflector

Figure F-2. Set-3 results, plot 1: reactivity effect of cylinder radius,

pipe steel, no filler, no Be, water moderated. 


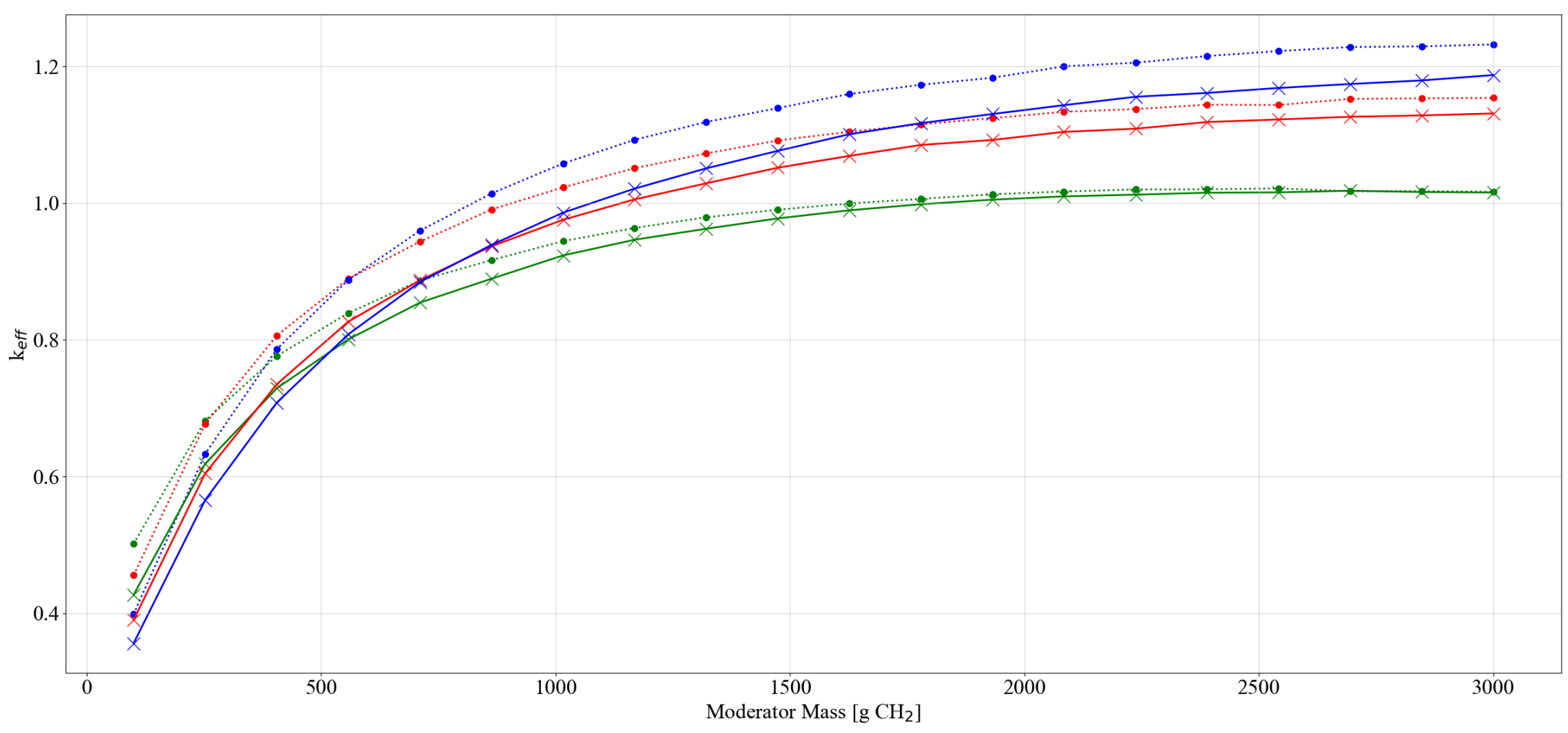

.... set -3 , cyl radius $=4.8 \mathrm{~cm}$, thin discrete reflector

$*$ set- 3 , cyl radius $=4.8 \mathrm{~cm}$, thick discrete reflector

... set- 3 , cyl radius $=6.25 \mathrm{~cm}$, thin discrete reflector

$\leftarrow$ set- 3 , cyl radius $=6.25 \mathrm{~cm}$, thick discrete reflector

$\cdots \cdots$ set- 3 , cyl radius $=7.7 \mathrm{~cm}$, thin discrete reflector

$\leftarrow$ set- 3 , cyl radius $=7.7 \mathrm{~cm}$, thick discrete reflector

Figure F-3. Set-3 results, plot 2: reactivity effect of cylinder radius, pipe steel, no filler, no Be, poly moderated. 


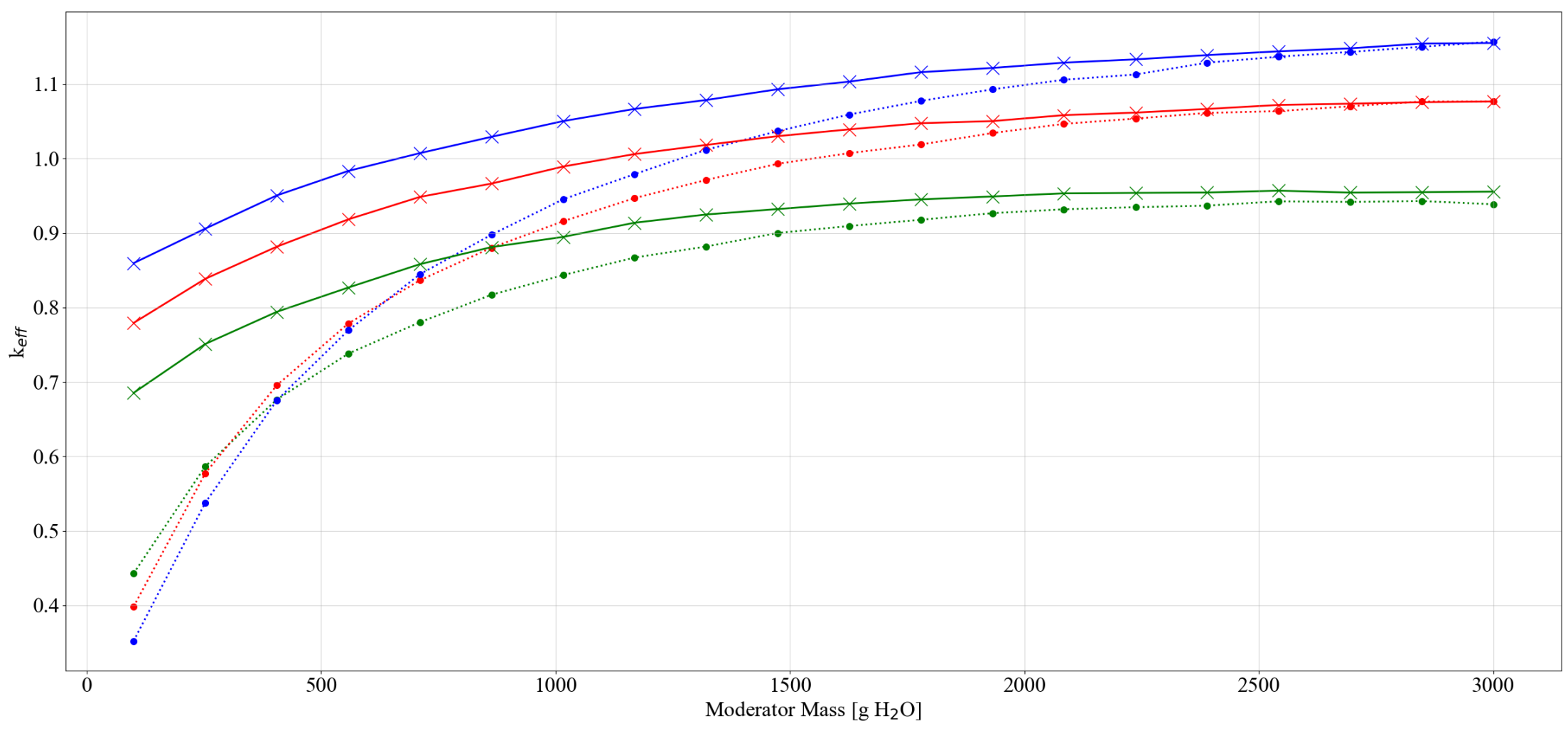

-... set-3, cyl radius $=4.8 \mathrm{~cm}$, thin discrete reflector

-... set-3, cyl radius $=6.25 \mathrm{~cm}$, thin discrete reflector

$\leftarrow$ set- 3 , cyl radius $=4.8 \mathrm{~cm}$, thick discrete reflector

$\star$ set- 3 , cyl radius $=6.25 \mathrm{~cm}$, thick discrete reflecto

$\cdots$ set- 3 , cyl radius $=7.7 \mathrm{~cm}$, thin discrete reflector

$\leftarrow$ set-3, cyl radius $=7.7 \mathrm{~cm}$, thick discrete reflector

Figure F-4. Set-3 results, plot 3: reactivity effect of cylinder radius, pipe poly, no filler, no Be, water moderated. 


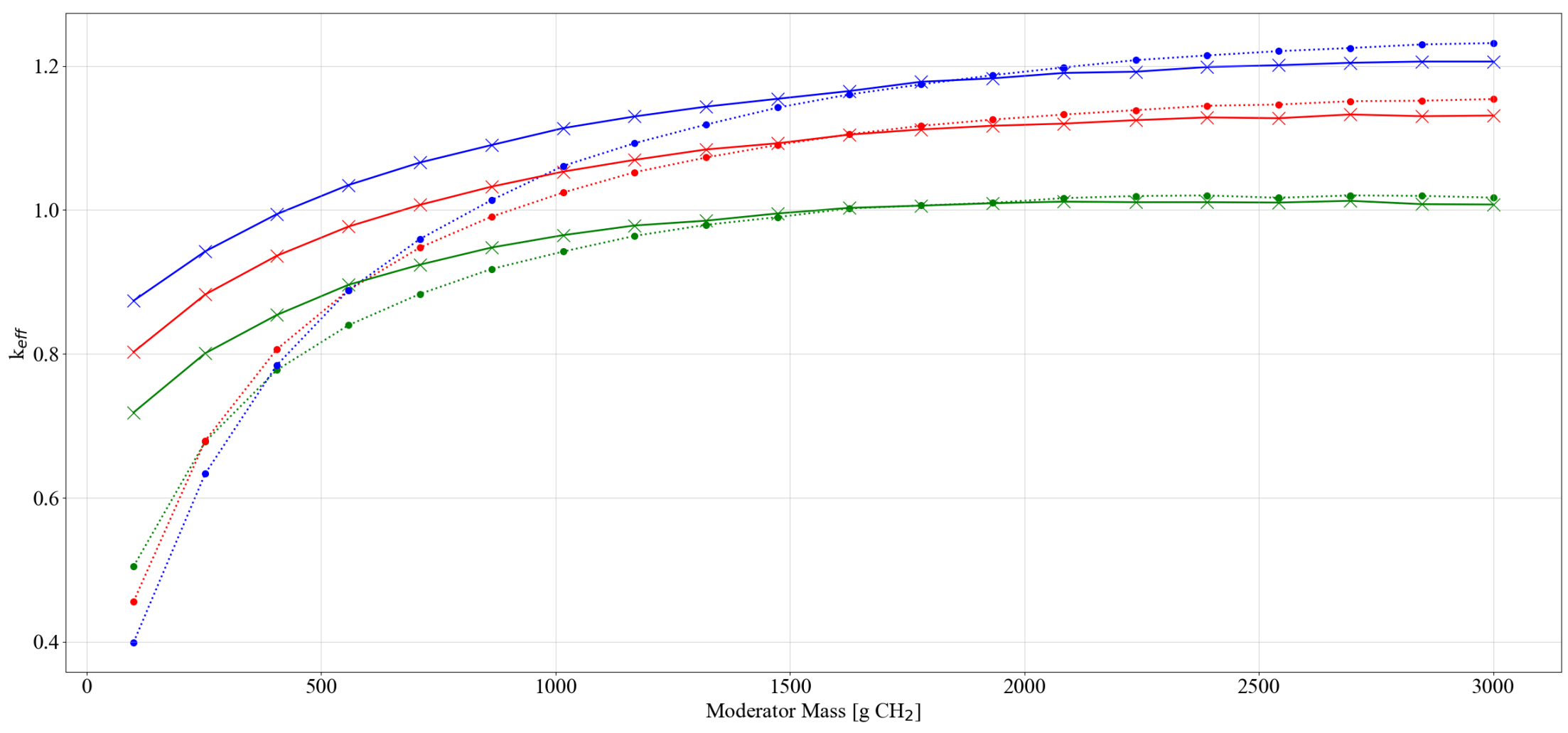

-... set-3, cyl radius $=4.8 \mathrm{~cm}$, thin discrete reflector

-... set-3, cyl radius $=6.25 \mathrm{~cm}$, thin discrete reflector

$\leftarrow$ set- 3 , cyl radius $=4.8 \mathrm{~cm}$, thick discrete reflector

$\leftarrow$ set- 3 , cyl radius $=6.25 \mathrm{~cm}$, thick discrete reflecto

$\cdots$ set- 3 , cyl radius $=7.7 \mathrm{~cm}$, thin discrete reflector

$\leftarrow$ set-3, cyl radius $=7.7 \mathrm{~cm}$, thick discrete reflector

Figure F-5. Set-3 results, plot 4: reactivity effect of cylinder radius, pipe poly, no filler, no Be, poly moderated. 


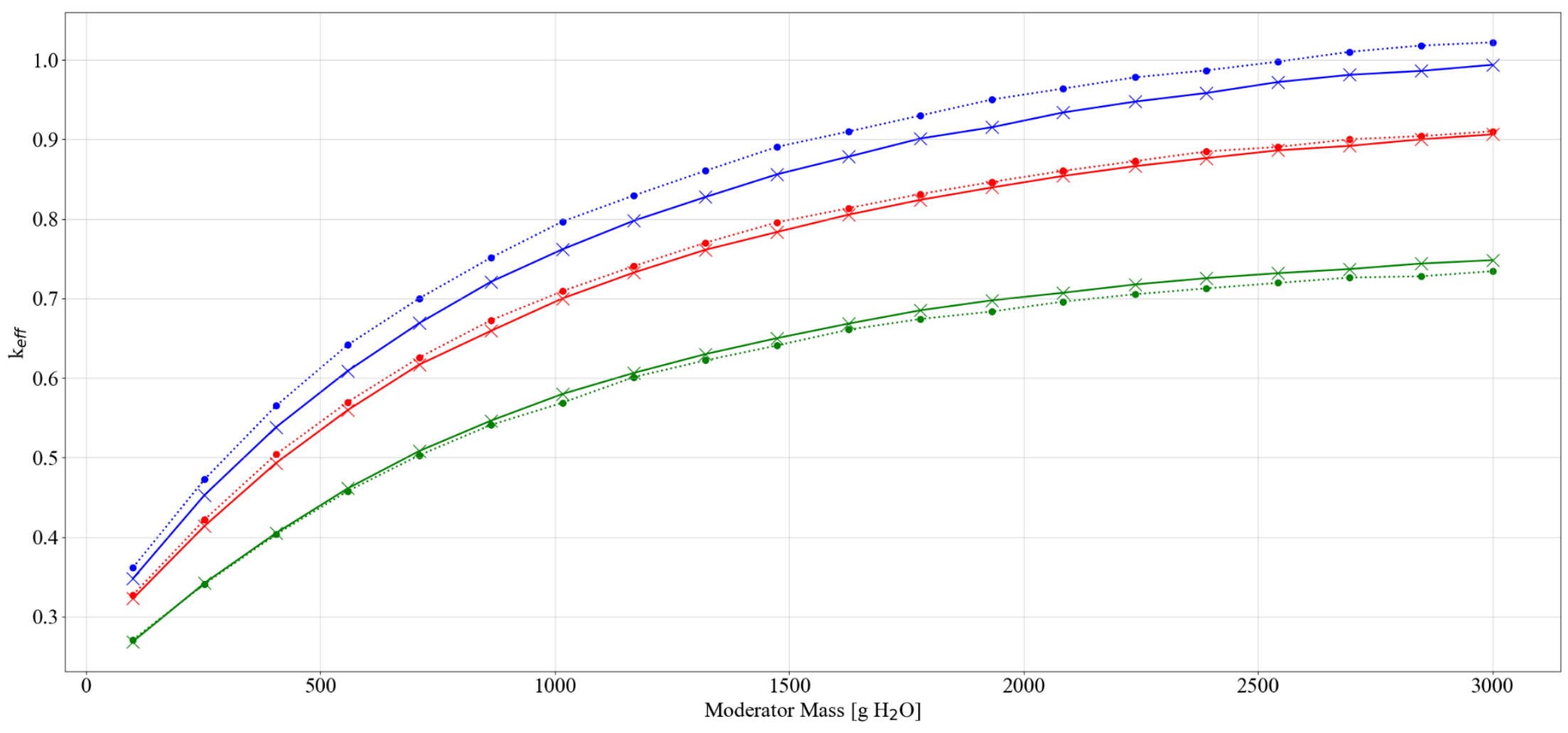

-... set-3, cyl radius $=4.8 \mathrm{~cm}$, thin discrete reflector

$\cdots$ set-3, cyl radius $=6.25 \mathrm{~cm}$, thin discrete reflector

$\star$ set- 3 , cyl radius $=4.8 \mathrm{~cm}$, thick discrete reflector

•.. set-3, cyl radius $=7.7 \mathrm{~cm}$, thin discrete reflector

* set- 3 , cyl radius $=6.25 \mathrm{~cm}$, thick discrete reflector

$\star$ set-3, cyl radius $=7.7 \mathrm{~cm}$, thick discrete reflector

Figure F-6. Set-3 results, plot 5: reactivity effect of cylinder radius, pipe steel, $5 \mathrm{~kg}$ graphite/can, no Be, water moderated. 


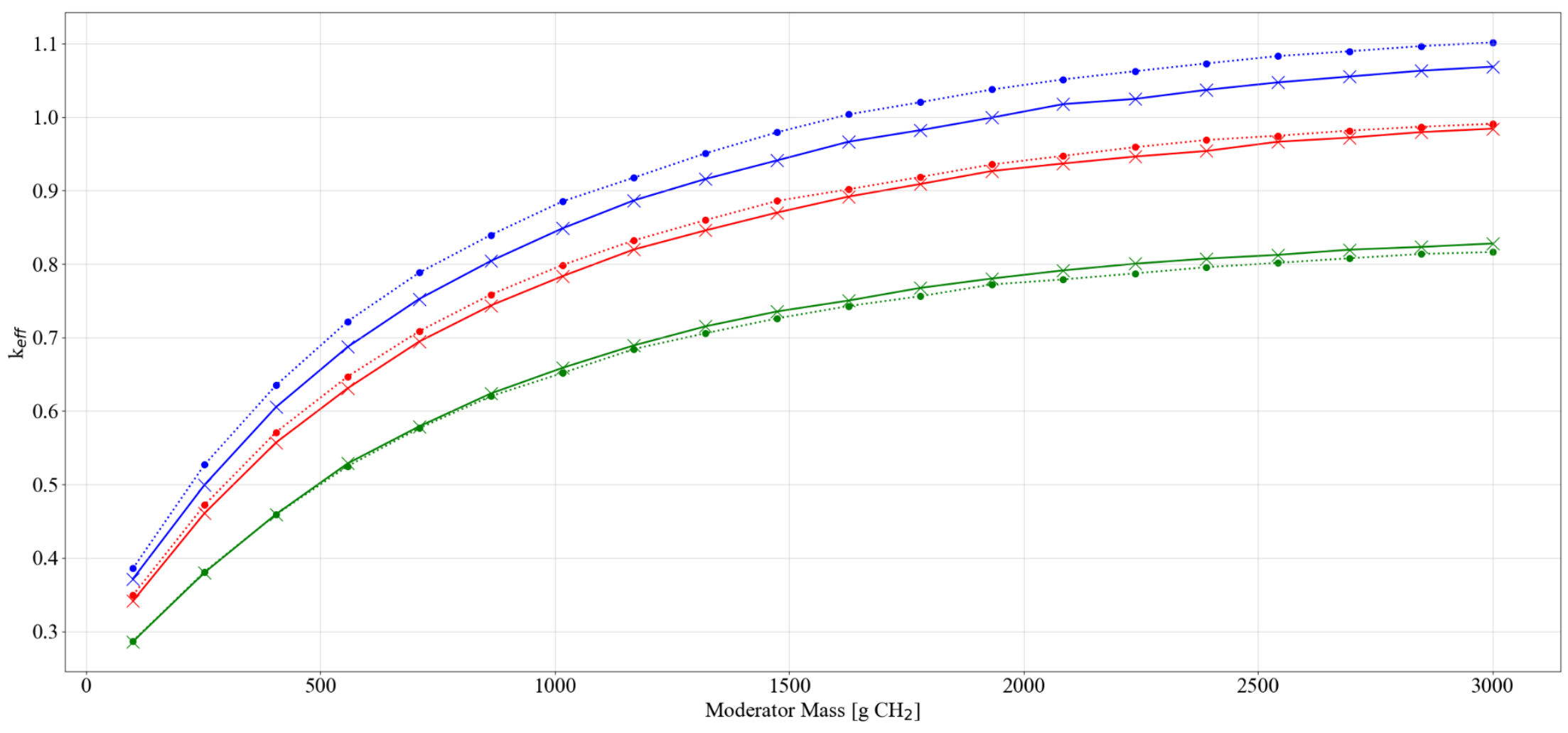

-... set-3, cyl radius $=4.8 \mathrm{~cm}$, thin discrete reflector

$\cdots$ set-3, cyl radius $=6.25 \mathrm{~cm}$, thin discrete reflector

$\star$ set- 3 , cyl radius $=4.8 \mathrm{~cm}$, thick discrete reflector

•.. set-3, cyl radius $=7.7 \mathrm{~cm}$, thin discrete reflector

* set-3, cyl radius $=6.25 \mathrm{~cm}$, thick discrete reflector

$\star$ set- 3 , cyl radius $=7.7 \mathrm{~cm}$, thick discrete reflector

Figure F-7. Set-3 results, plot 6: reactivity effect of cylinder radius, pipe steel, $5 \mathrm{~kg}$ graphite/can, no Be, poly moderated. 


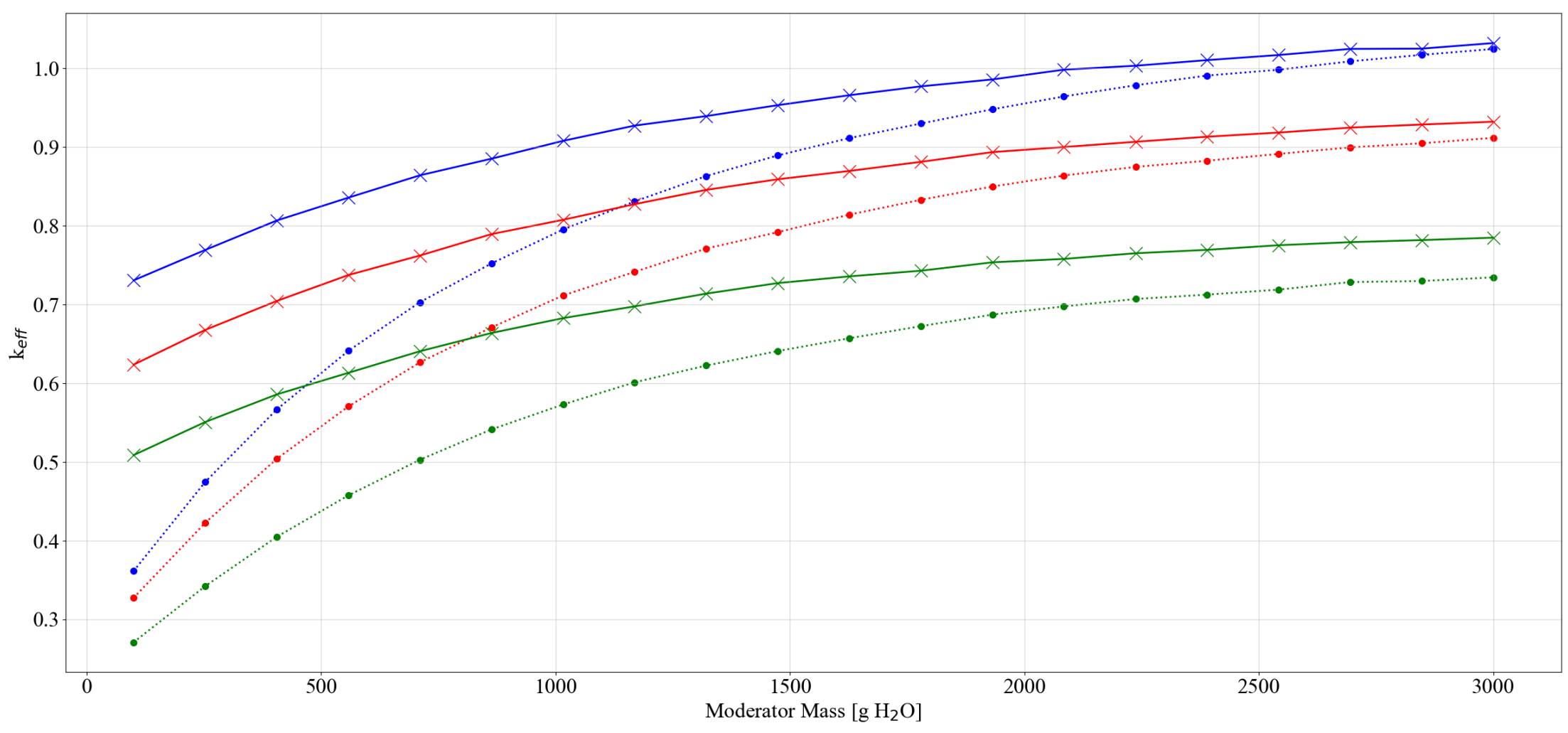

-... set-3, cyl radius $=4.8 \mathrm{~cm}$, thin discrete reflector

-... set-3, cyl radius $=6.25 \mathrm{~cm}$, thin discrete reflector

$\leftarrow$ set- 3 , cyl radius $=4.8 \mathrm{~cm}$, thick discrete reflector

set-3, cyl radius $=7.7 \mathrm{~cm}$, thin discrete reflector

* set-3, cyl radius $=6.25 \mathrm{~cm}$, thick discrete reflector

$\leftarrow$ set-3, cyl radius $=7.7 \mathrm{~cm}$, thick discrete reflector

Figure F-8. Set-3 results, plot 7: reactivity effect of cylinder radius, pipe poly, $5 \mathrm{~kg}$ graphite/can, no Be, water moderated. 


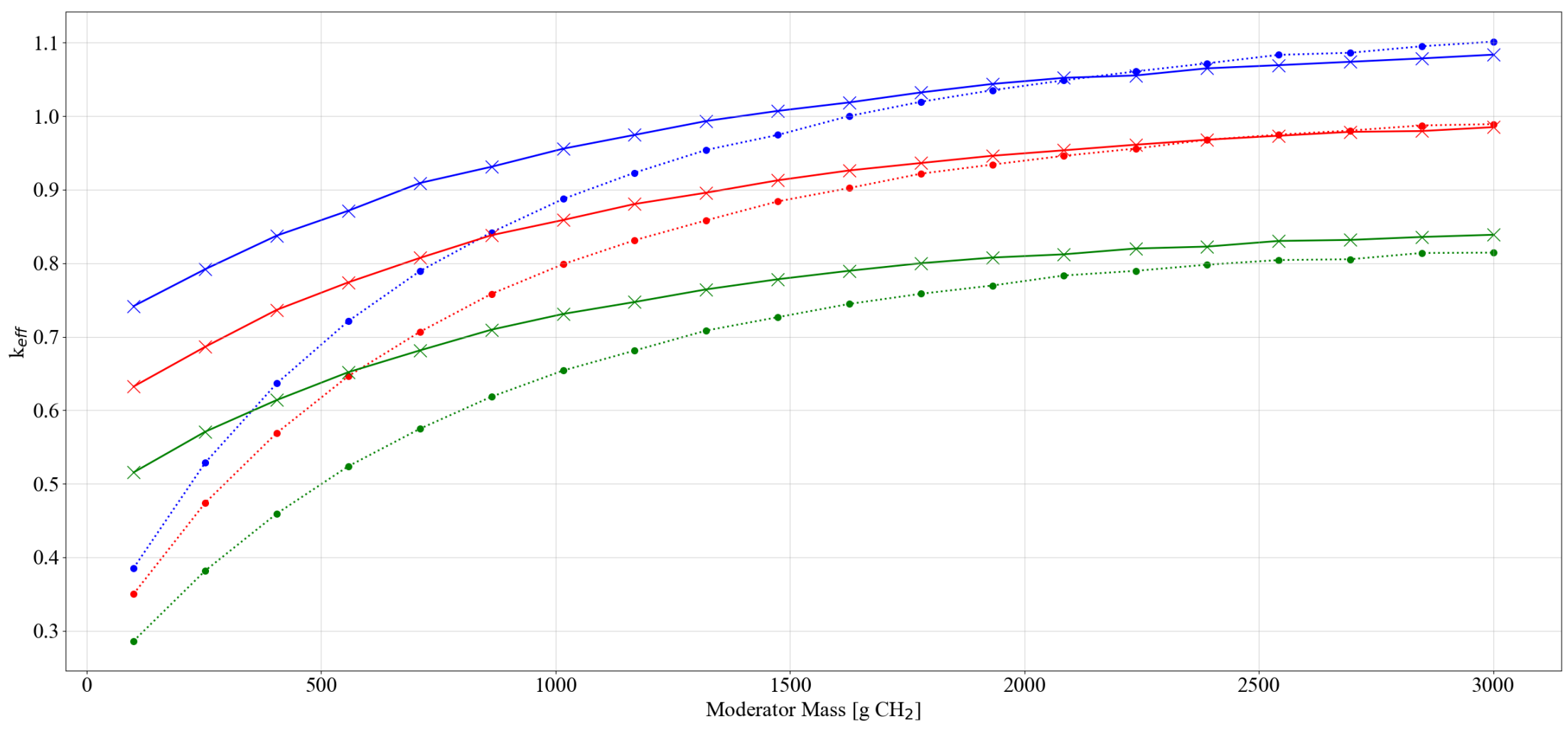

-... set-3, cyl radius $=4.8 \mathrm{~cm}$, thin discrete reflector

-.. set- 3 , cyl radius $=6.25 \mathrm{~cm}$, thin discrete reflector

$\leftarrow$ set- 3 , cyl radius $=4.8 \mathrm{~cm}$, thick discrete reflector

set- 3 , cyl radius $=7.7 \mathrm{~cm}$, thin discrete reflector

- set-3, cyl radius $=6.25 \mathrm{~cm}$, thick discrete reflecto

$\leftarrow$ set- 3 , cyl radius $=7.7 \mathrm{~cm}$, thick discrete reflector

Figure F-9. Set-3 results, plot 8: reactivity effect of cylinder radius, pipe poly, $5 \mathrm{~kg}$ graphite/can, no Be, poly moderated. 


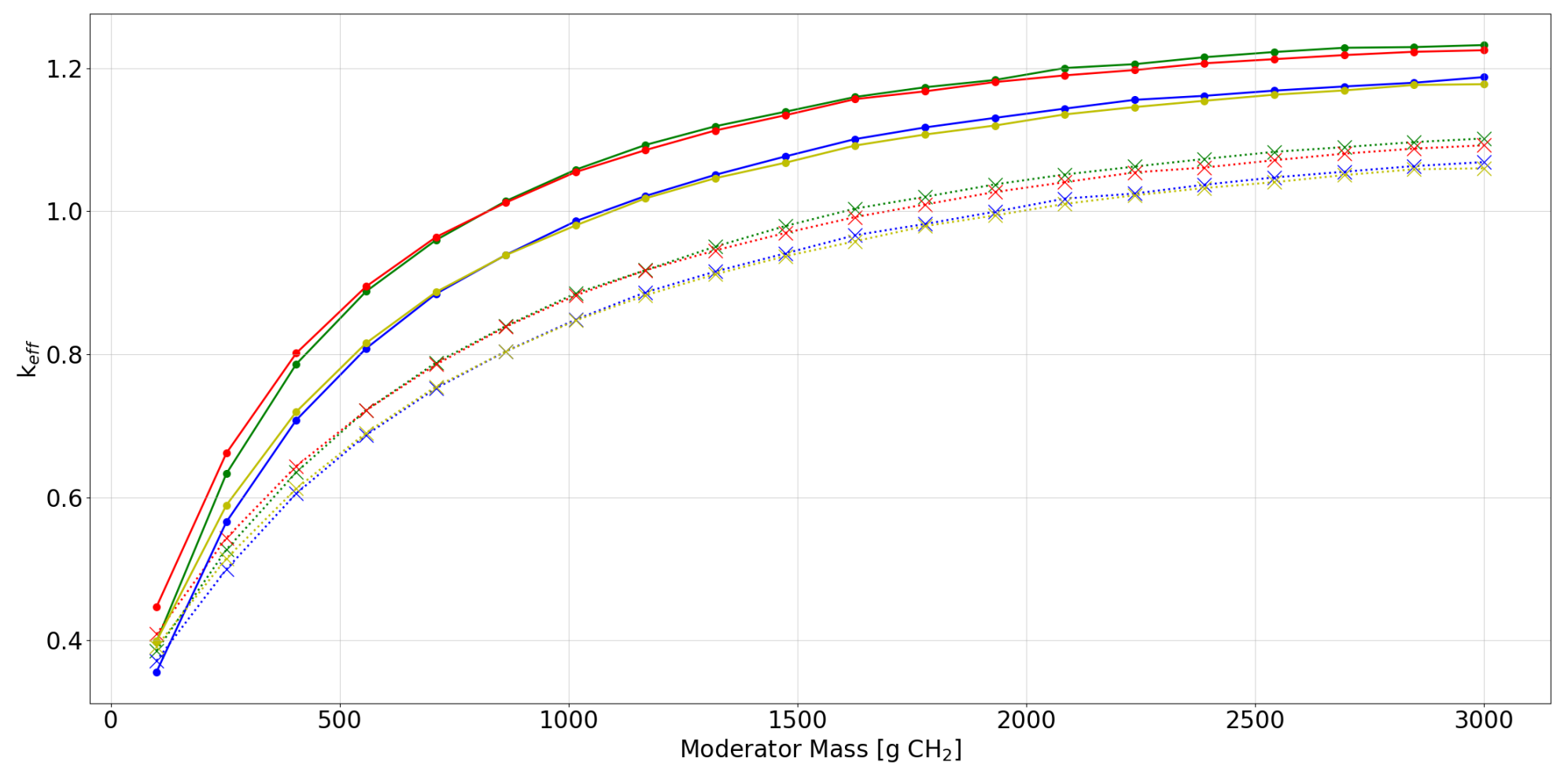

- set-3, no filler, no Be, thin SS discrete reflector

*... set-3, $5 \mathrm{~kg}$ graphite/can, no Be, thin SS discrete reflector

- set-3, no filler, $585 \mathrm{~g} \mathrm{Be}$, thin SS discrete reflector

- - set-3, no filler, no Be, thick SS discrete reflector

….... set-3, $5 \mathrm{~kg}$ graphite/can, no Be, thick SS discrete reflector

$\ldots$ set-3, no filler, $585 \mathrm{~g} \mathrm{Be}$, thick SS discrete reflector

...... set-3, $5 \mathrm{~kg}$ graphite/can, $585 \mathrm{~g} \mathrm{Be}$, thin SS discrete reflector

......... set-3, $5 \mathrm{~kg}$ graphite/can, $585 \mathrm{~g} \mathrm{Be}$, thick SS discrete reflector

Figure F-10. Set-3 results, plot 9: reactivity effect of various parameters with $7.7 \mathrm{~cm}$ cylinder radius, graphite filler, poly moderated. 


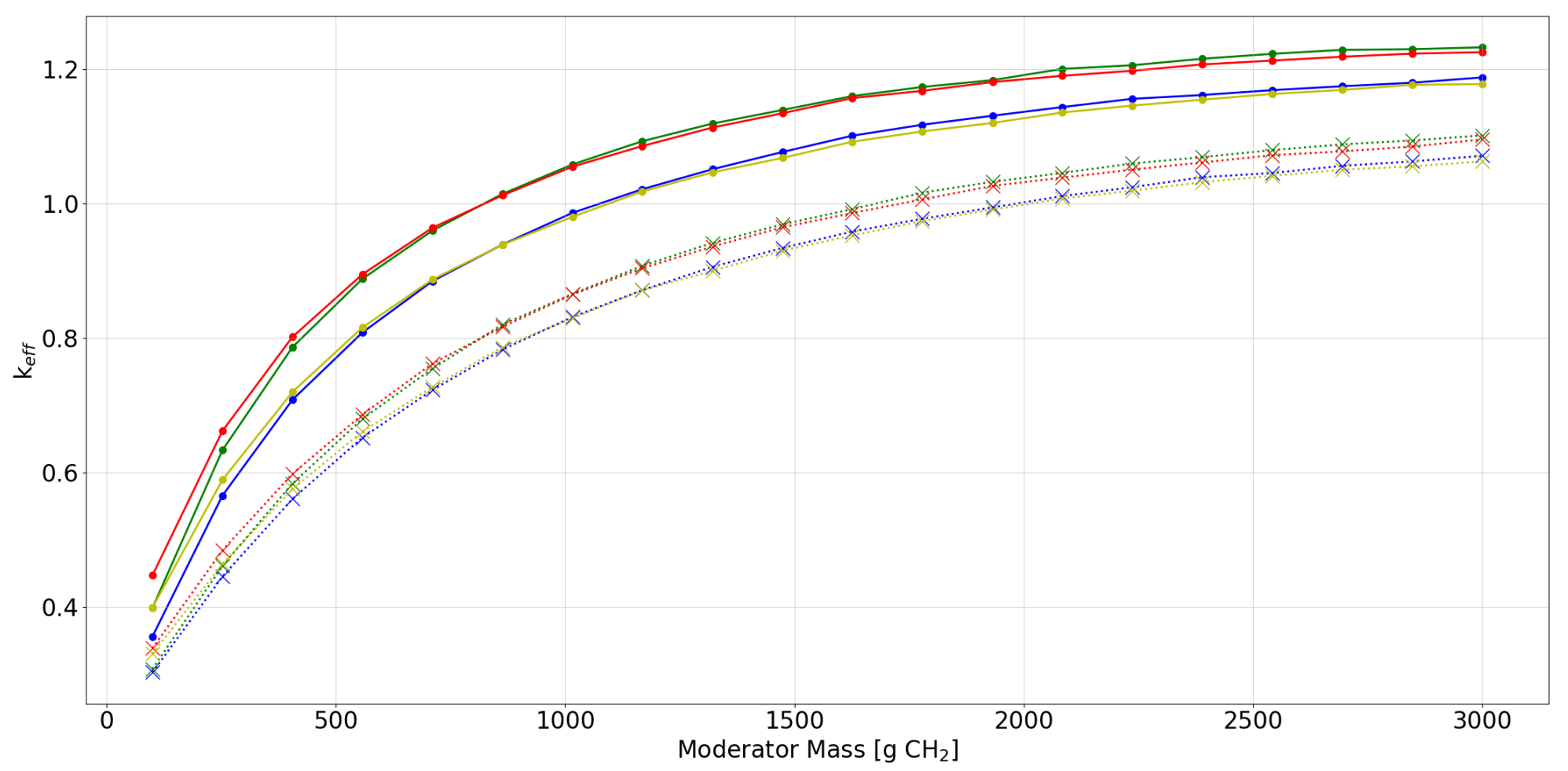

- set-3, no filler, no Be, thin SS discrete reflector

*... set-3, $5 \mathrm{~kg}$ generic/can, no Be, thin SS discrete reflector

- - set-3, no filler, $585 \mathrm{~g} \mathrm{Be}$, thin SS discrete reflector

- - set-3, no filler, no Be, thick SS discrete reflector

....... set-3, $5 \mathrm{~kg}$ generic/can, $585 \mathrm{~g} \mathrm{Be}$, thin SS discrete $r$ reflector

........ set-3, $5 \mathrm{~kg}$ generic/can, no Be, thick SS discrete reflector

- set-3, no filler, $585 \mathrm{~g} \mathrm{Be,} \mathrm{thick} \mathrm{SS} \mathrm{discrete} \mathrm{reflector}$

........ set-3, $5 \mathrm{~kg}$ generic/can, $585 \mathrm{~g} \mathrm{Be,} \mathrm{thick} \mathrm{SS} \mathrm{discrete} \mathrm{reflector}$

Figure F-11. Set-3 results, plot 10: reactivity effect of various parameters with $7.7 \mathrm{~cm}$ cylinder radius, generic filler, poly moderated. 


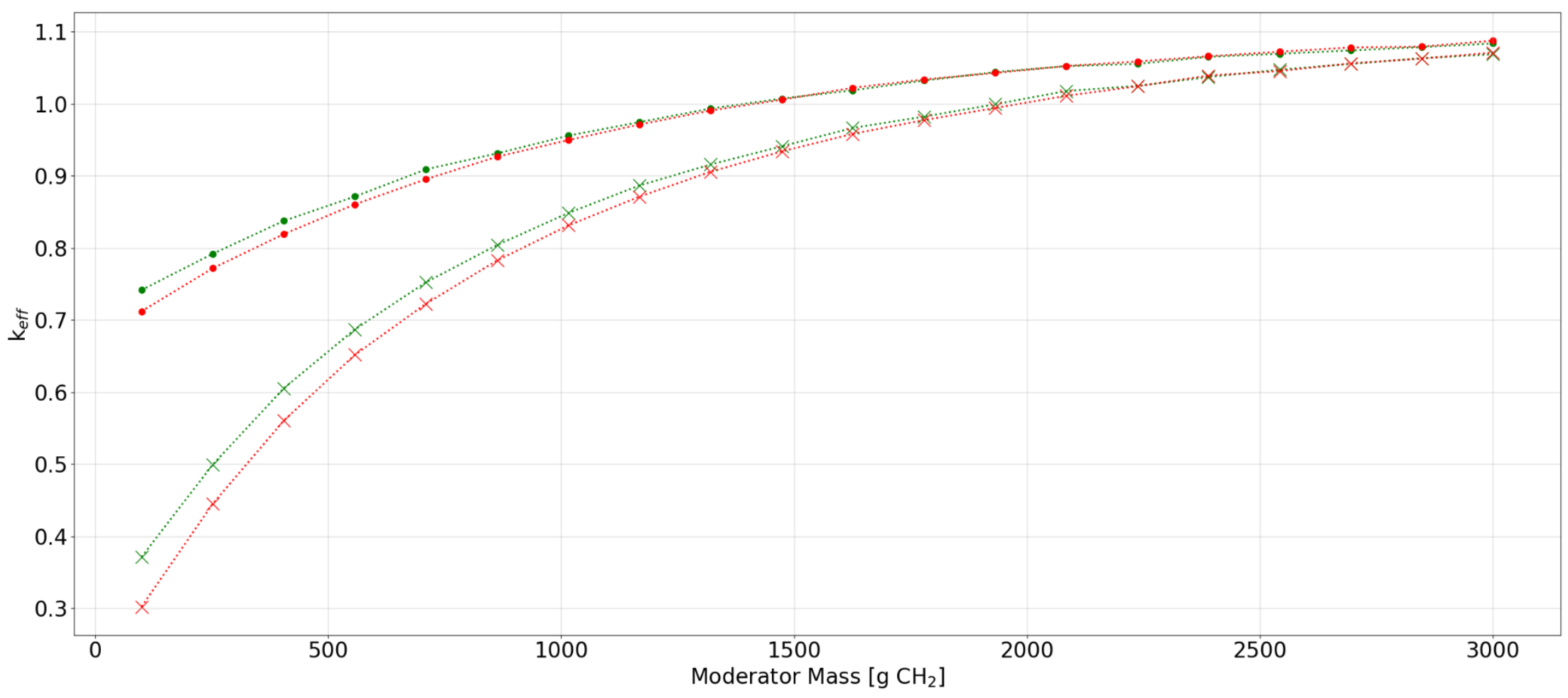

......... set-3, cylinder radius $=7.7 \mathrm{~cm}$, poly moderator, $5 \mathrm{~kg}$ graphite $/ \mathrm{can}$, no Be, thick SS discrete reflector

...... set-3, cylinder radius $=7.7 \mathrm{~cm}$, poly moderator, $5 \mathrm{~kg}$ graphite $/ \mathrm{can}$, no Be, thick poly discrete reflector

........ set-3, cylinder radius $=7.7 \mathrm{~cm}$, poly moderator, $5 \mathrm{~kg}$ generic $/ \mathrm{can}$, no Be, thick SS discrete reflector

$\ldots . \cdots$ set-3, cylinder radius $=7.7 \mathrm{~cm}$, poly moderator, $5 \mathrm{~kg}$ generic/can, no Be, thick poly discrete reflector

Figure F-12. Set-3 results, plot 11: comparison of graphite and generic filler with $7.7 \mathrm{~cm}$ cylinder radius, no Be, poly moderated, thick discrete reflector. 


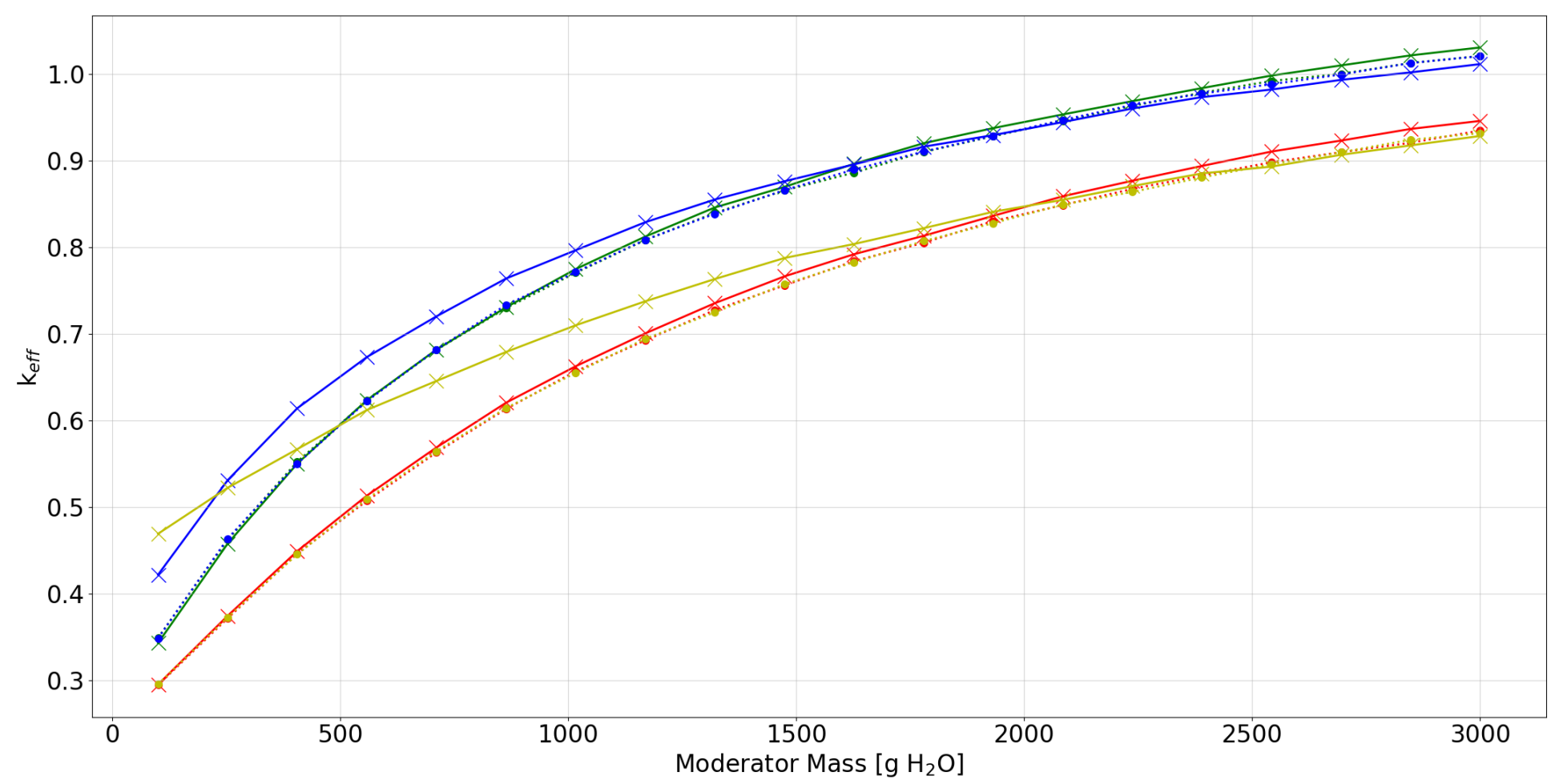

.... set-3, no filler, no Be, thin SS discrete reflector

$\star$ set-3, no filler, no Be, thick SS discrete reflector

..... set-3, $5 \mathrm{~kg}$ graphite/can, $585 \mathrm{~g} \mathrm{Be}$, thin SS discrete reflector set-3, $5 \mathrm{~kg}$ graphite/can, $585 \mathrm{~g} \mathrm{Be}$, thick SS discrete reflector
•... set-3, no filler, no Be, thin poly discrete reflector

$\star$ set-3, no filler, no Be, thick poly discrete reflector

-.... set-3, $5 \mathrm{~kg}$ graphite/can, $585 \mathrm{~g} \mathrm{Be}$, thin poly discrete reflector set-3, $5 \mathrm{~kg}$ graphite/can, $585 \mathrm{~g} \mathrm{Be}$, thick poly discrete reflector

Figure F-13. Set-3 results, plot 12: reactivity effect of various parameters with spherical waste form geometry, graphite filler, water moderated. 


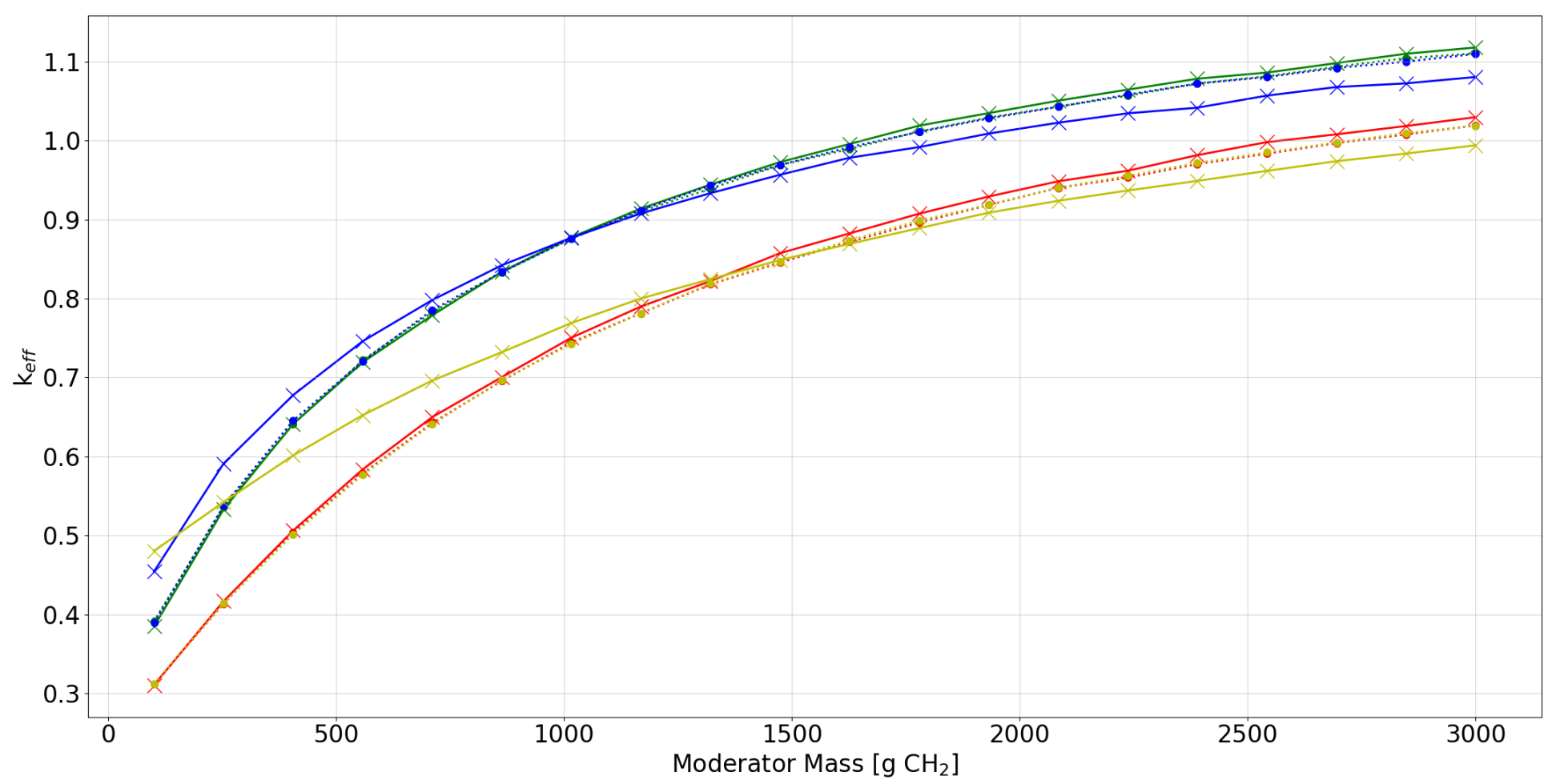

.... set-3, no filler, no Be, thin SS discrete reflector

$\star$ set-3, no filler, no Be, thick SS discrete reflector

..... set-3, $5 \mathrm{~kg}$ graphite/can, $585 \mathrm{~g} \mathrm{Be}$, thin SS discrete reflector set-3, $5 \mathrm{~kg}$ graphite/can, $585 \mathrm{~g}$ Be, thick SS discrete reflector
-... set-3, no filler, no Be, thin poly discrete reflector

$\star$ set-3, no filler, no Be, thick poly discrete reflector

-.... set-3, $5 \mathrm{~kg}$ graphite/can, $585 \mathrm{~g} \mathrm{Be}$, thin poly discrete reflector set-3, $5 \mathrm{~kg}$ graphite/can, $585 \mathrm{~g} \mathrm{Be}$, thick poly discrete reflector

Figure F-14. Set-3 results, plot 13: reactivity effect of various parameters with spherical waste form geometry, graphite filler, poly moderated. 


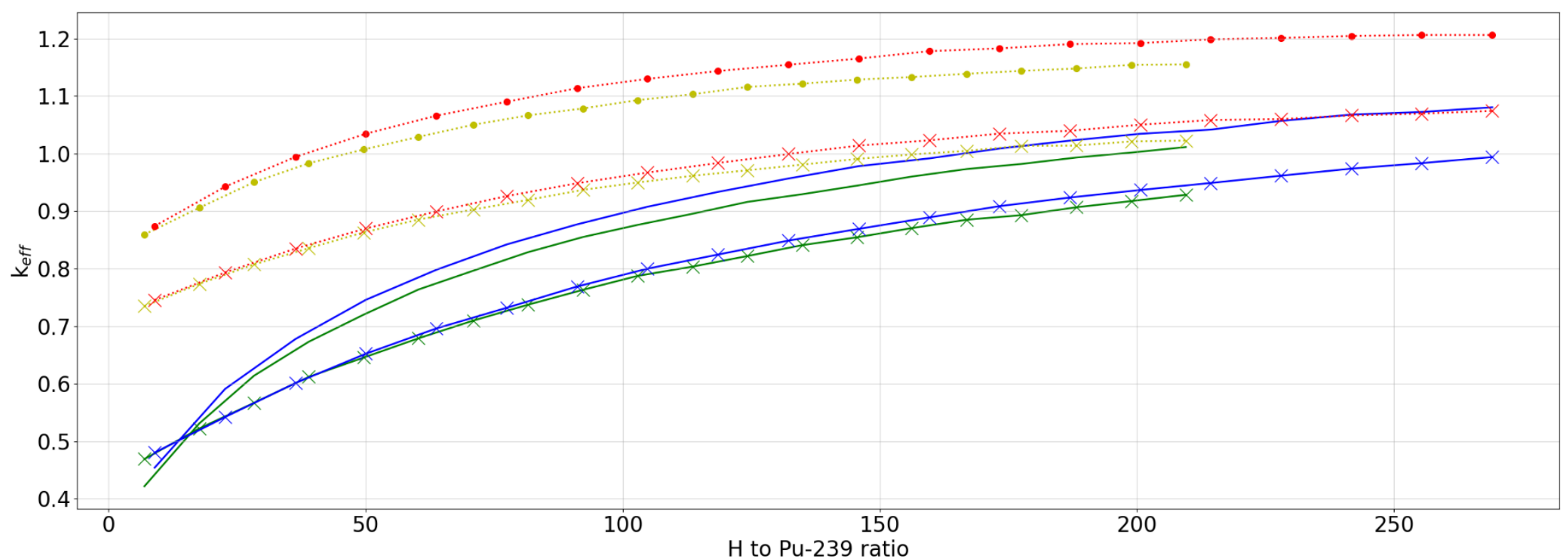

- set-3, sphere, no filler, no Be, thick poly discrete reflector, water moderator

$*$ set-3, sphere, $5 \mathrm{~kg}$ graphite/can, $585 \mathrm{~g} \mathrm{Be}$, thick poly discrete reflector, water moderator

— set-3, sphere, no filler, no Be, thick poly discrete reflector, poly moderator

$*$ set-3, sphere, $5 \mathrm{~kg}$ graphite/can, $585 \mathrm{~g} \mathrm{Be}$, thick poly discrete reflector, poly moderator

$\ldots . . .$. set-3, cylinder with radius $=7.7 \mathrm{~cm}$, no filler, no Be, thick poly discrete reflector, water moderator

X... set-3, cylinder with radius $=7.7 \mathrm{~cm}, 5 \mathrm{~kg}$ graphite/can, $585 \mathrm{~g} \mathrm{Be}$, thick poly discrete reflector, water moderator

..... set-3, cylinder with radius $=7.7 \mathrm{~cm}$, no filler, no Be, thick poly discrete reflector, poly moderator

... $\ldots$ set- 3 , cylinder with radius $=7.7 \mathrm{~cm}, 5 \mathrm{~kg}$ graphite $/ \mathrm{can}, 585 \mathrm{~g} \mathrm{Be}$, thick poly discrete reflector, poly moderator

Figure F-15. Set-3 results, plot 14: comparison of spherical and cylindrical geometries $(\mathrm{h} / \mathrm{x})$. 


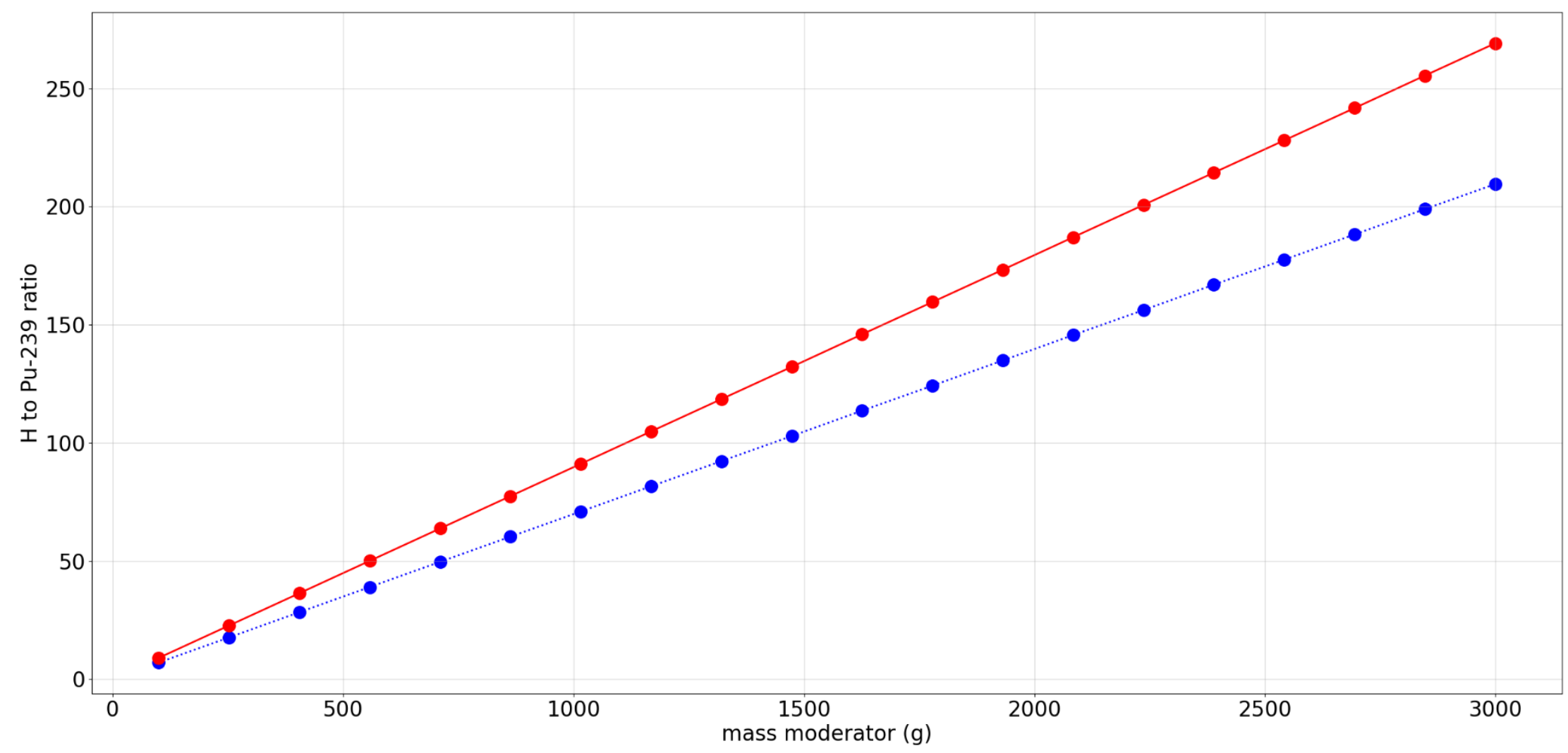

-.. water moderator

- poly moderator

Figure F-16. Set-3 results, plot 15: comparison of water and poly $h / x$. 


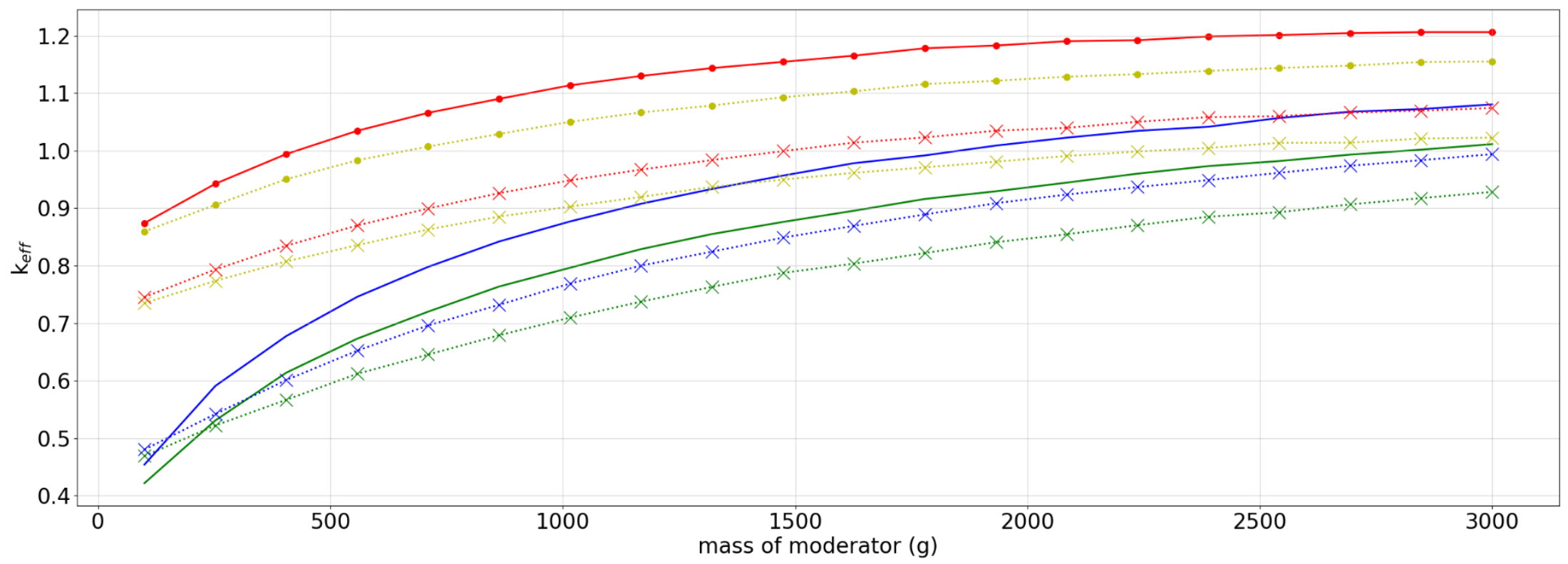

- set-3, sphere, no filler, no Be, thick poly discrete reflector, water moderator

....... set-3, sphere, $5 \mathrm{~kg}$ graphite/can, $585 \mathrm{~g} \mathrm{Be,} \mathrm{thick} \mathrm{poly} \mathrm{discrete} \mathrm{reflector,} \mathrm{water} \mathrm{moderator}$

- set-3, sphere, no filler, no Be, thick poly discrete reflector, poly moderator

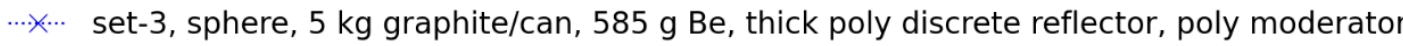

...... set-3, cylinder with radius $=7.7 \mathrm{~cm}$, no filler, no Be, thick poly discrete reflector, water moderator

.... set-3, cylinder with radius $=7.7 \mathrm{~cm}, 5 \mathrm{~kg}$ graphite/can, $585 \mathrm{~g} \mathrm{Be}$, thick poly discrete reflector, water moderator

$\rightarrow$ set-3, cylinder with radius $=7.7 \mathrm{~cm}$, no filler, no Be, thick poly discrete reflector, poly moderator

.... $\ldots$ set- 3 , cylinder with radius $=7.7 \mathrm{~cm}, 5 \mathrm{~kg}$ graphite/can, $585 \mathrm{~g} \mathrm{Be}$, thick poly discrete reflector, poly moderator

Figure F-17. Set-3 results, plot 16: comparison of spherical and cylindrical geometries (mod mass). 


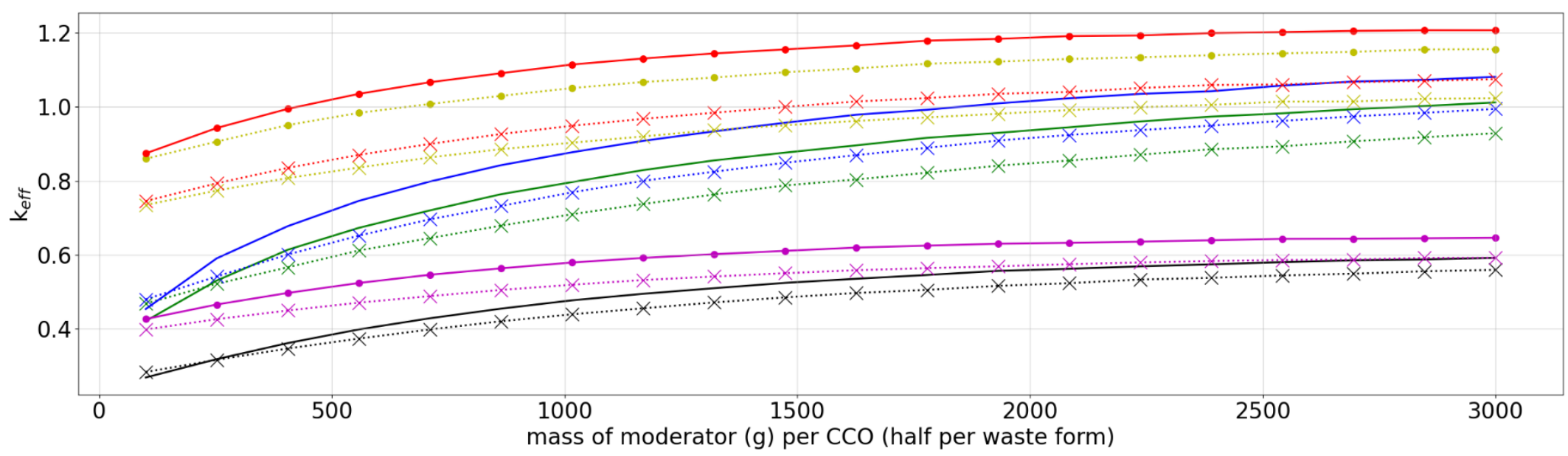

- set-3, sphere, no filler, no Be, thick poly discrete reflector, water moderator

........ set-3, sphere, $5 \mathrm{~kg}$ graphite/can, $585 \mathrm{~g}$ Be, thick poly discrete reflector, water moderator

- set-3, sphere, no filler, no Be, thick poly discrete reflector, poly moderator

........ set-3, sphere, $5 \mathrm{~kg}$ graphite/can, $585 \mathrm{~g}$ Be, thick poly discrete reflector, poly moderator

$\ldots . . .$. set-3, cylinder with radius $=7.7 \mathrm{~cm}$, no filler, no Be, thick poly discrete reflector, water moderator

$\ldots . . .$. set- 3 , cylinder with radius $=7.7 \mathrm{~cm}, 5 \mathrm{~kg}$ graphite/can, $585 \mathrm{~g} \mathrm{Be}$, thick poly discrete reflector, water moderator

$\rightarrow$ set-3, cylinder with radius $=7.7 \mathrm{~cm}$, no filler, no Be, thick poly discrete reflector, poly moderator

....... set-3, cylinder with radius $=7.7 \mathrm{~cm}, 5 \mathrm{~kg}$ graphite/can, $585 \mathrm{~g} \mathrm{Be}$, thick poly discrete reflector, poly moderator

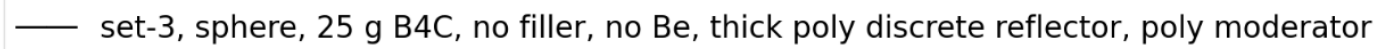

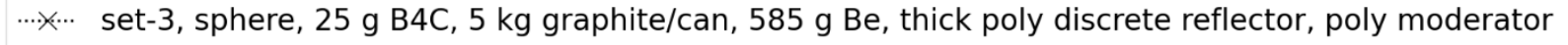

- - set-3, cylinder with radius $=7.7 \mathrm{~cm}, 25 \mathrm{~g} \mathrm{~B} 4 \mathrm{C}$, no filler, no Be, thick poly discrete reflector, poly moderator

........ set-3, cylinder with radius $=7.7 \mathrm{~cm}, 25 \mathrm{~g} \mathrm{~B} 4 \mathrm{C}, 5 \mathrm{~kg}$ graphite/can, $585 \mathrm{~g} \mathrm{Be}$, thick poly discrete reflector, poly moderator

Figure F-18. Set-3 results, plot 17: Reactivity effect of $25 \mathrm{~g} \mathrm{~B} 4 \mathrm{C}$ per waste form ( 2 per $\mathrm{CCO})$ 
This page is intentionally blank 
APPENDIX G. SET-2 UPPER HORIZON CENTROID LOCATION AND ORIENTATION STUDIES 
This page is intentionally blank 


\section{APPENDIX G. SET-2 UPPER HORIZON CENTROID LOCATION AND ORIENTATION STUDIES}

The analysis methodology for the nonuniform array studies is discussed in detail in Section 6.2 and Section 6.4 of the main report.

This appendix serves as a repository of the results for the centroid location and orientation study calculations.

The analysis model used for the calculations in this appendix is shown in Figure G-1 below. Additional discussions are provided in Section 6.4 of the main report.

The SAMPLER case sweeps presented in this appendix limited to those which have the same parameters as set-2-4 and with no filler material.

Results are presented in the following figures:

\section{LIST OF FIGURES}

Figure G-1. Representation of the compacted CCO pipe centerline "spaghetti model" drawn from

13 centroids per pipe centerline from [28] with permission. G-5

Figure G-2. Example of centroid locations within compacted pipes. .................................................... G-6

Figure G-3. 3D representation of the Appendix C and Appendix D 2-unit analysis model illustrating how pipe center centroid data were used to orient vertical cylinders......................... G-7

Figure G-4. A 3D python-generated illustration of how cylinder orientations follow pipe centerline orientations.

Figure G-5. Comparison of local and global orientations for centroid 1 using the isometric, top, and front view angles.

Figure G-6. Comparison of different visualizations of Centroid 6 shifted with global orientation case, $x-y$ plane (top view).

Figure G-7. Comparison of different visualizations of the Centroid 6 shifted with global orientation case, $x-z$ plane (front/back view).

Figure G-8. Results of the centroid studies for centroid 1.

Figure G-9. Results of the centroid studies for centroid 2. G-13

Figure G-10. Results of the centroid studies for centroid 3. G-14

Figure G-11. Results of the centroid studies for centroid 4. G-14

Figure G-12. Results of the centroid studies for centroid 5 . G-15

Figure G-13. Results of the centroid studies for centroid 6 . G-15

Figure G-14. Results of the centroid studies for centroid 7 . G-16

Figure G-15. Results of the centroid studies for centroid 8 . G-16

Figure G-16. Results of the centroid studies for centroid 9. G-17

Figure G-17. Results of the centroid studies for centroid 10. G-17

Figure G-18. Results of the centroid studies for centroid 11 . G-18

Figure G-19. Results of the centroid studies for centroid 12 . G-18

Figure G-20. Results of the centroid studies for centroid 13. G-19

Figure G-21. Results of the centroid studies for the "midpoint" centroid. G-19

Figure G-22 Comparison of all centroids and midpoint to various representative curves..... G-20

Figure G.23. Set-2-4 orientation study, random coordinate studies, results for 100 randomly selected centroid locations for each subset. G-22 
Figure G.24. Set-2-4 orientation study, random angle studies, results for 20 randomly selected angles for the 13 centroids plus the midpoint for each subset.

Figure G.25. Set-2-4 orientation study, random angle studies, results for 10 randomly selected angles for the 10 randomly selected centroids plus midpoint for each subset. G-25 
The analysis methodology for the nonuniform arrays, which are models that utilize the Reedlunn and Bean [7] data exclusively, was previously based on the work performed for the POCs by Brickner [4]. That previous work used the centroid positions provided by the SNL compaction studies by Reedlunn and Bean [7] to locate the waste forms in the model. Spheres and cylinders were used to geometrically represent the waste forms with ideal shapes. Both shapes were placed into the model based on the 153 individual pipe coordinate data. For the spheres, the coordinates defined the center point of the sphere. For the cylinders, the coordinates defined the center of a vertically oriented cylinder's base. These coordinate points for each pipe are termed centroids.

The results of the SNL compaction studies provide 153 pipe centerlines. A visualization of these pipe centerlines is shown in the figure below.
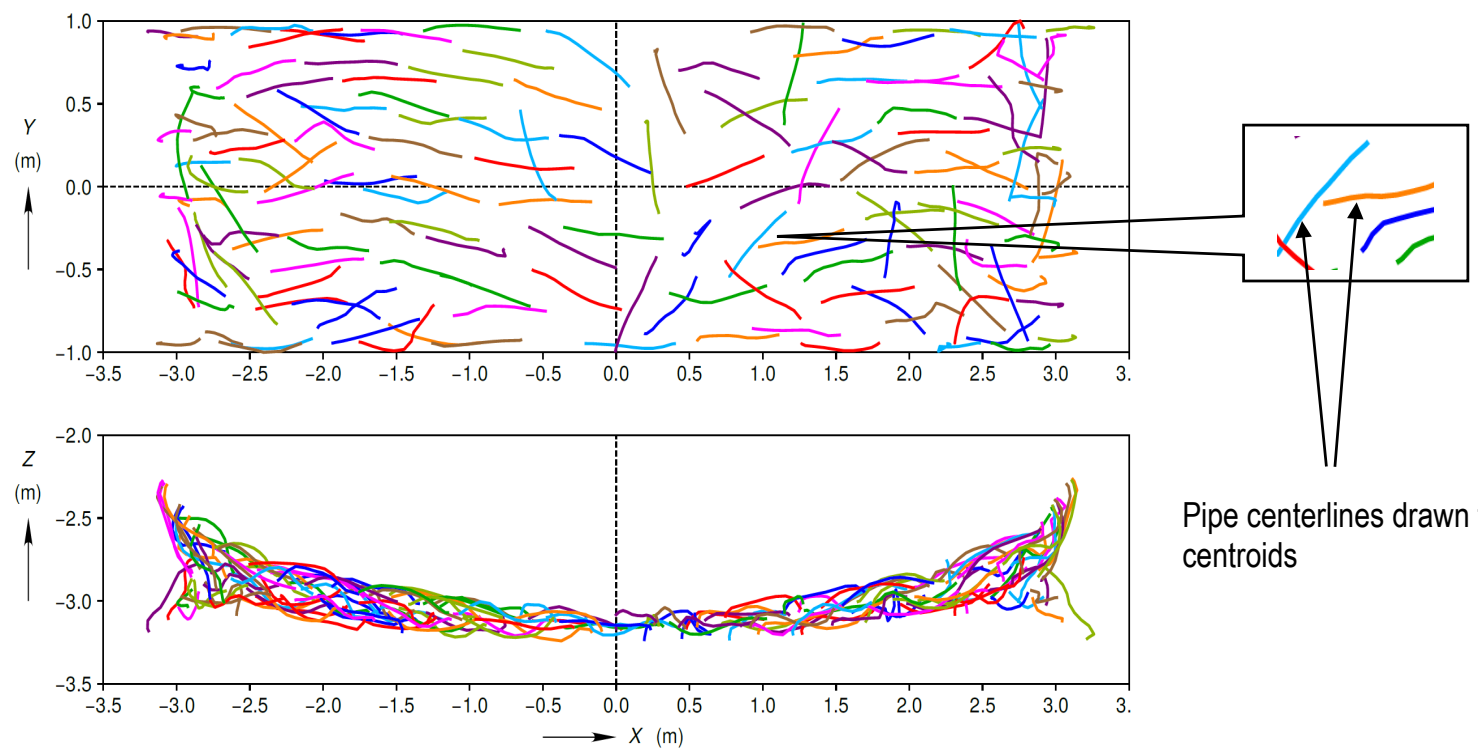

Pipe centerlines drawn from 13 centroids

Figure G-1. Representation of the compacted CCO pipe centerline "spaghetti model" drawn from 13 centroids per pipe centerline from [28] with permission. Note that this is not an actual analysis model; it was generated from the centroid dataset.

Each of the 153 pipe centerlines is constructed from 13 points, or centroids, spaced evenly along the compacted pipe centerline. Visualizations of these centroids are shown in the figure below. 


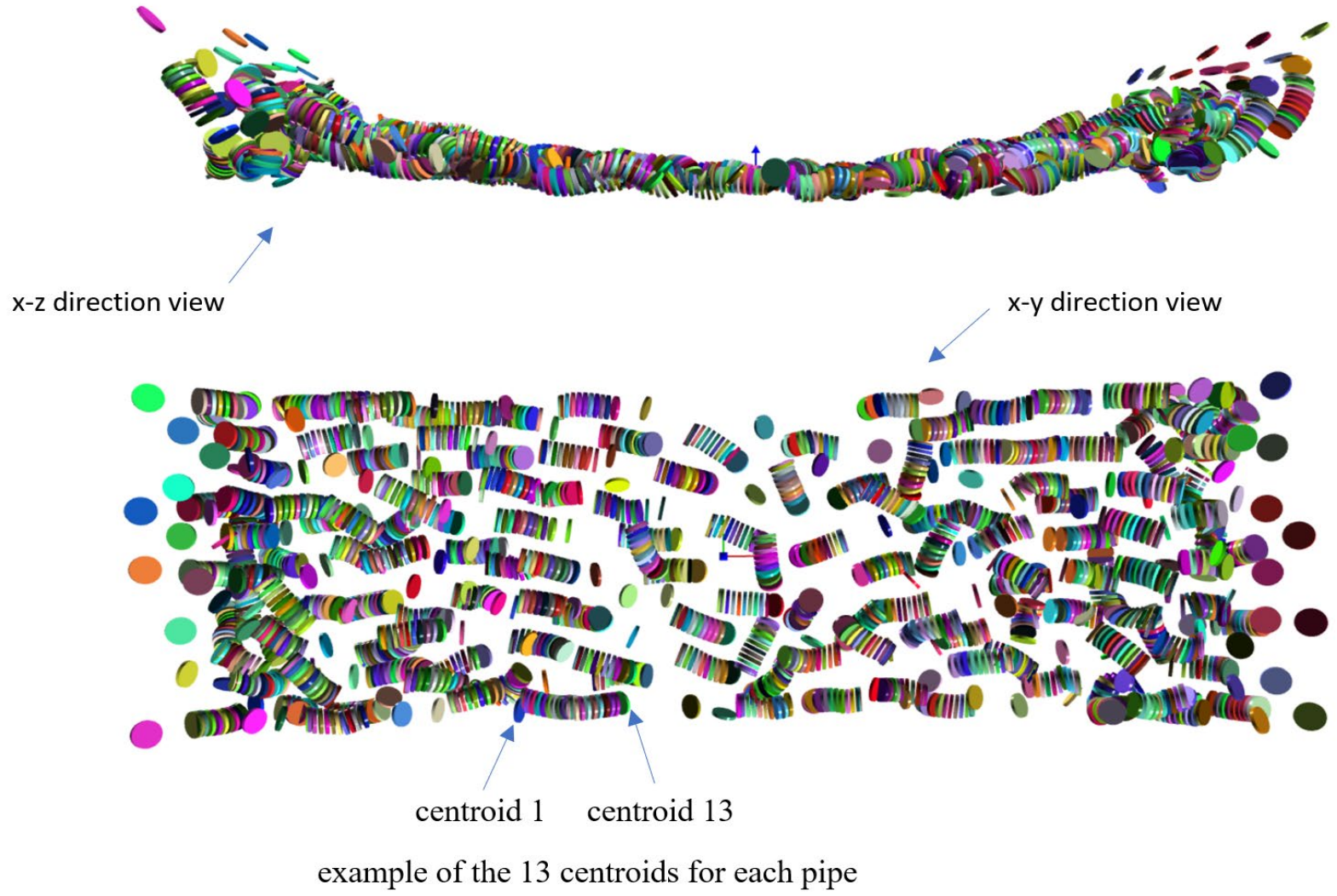

Figure G-2. Example of centroid locations within compacted pipes. This 3D representation of the compacted pipe centerline and centroid locations uses disks to show the locations of individual centroids. Note that this is not an actual analysis model but rather it is a python-generated representation of the centroid dataset.

One important assumption inherent to the previous analysis - as well as the calculations presented in this analysis for the set-2 upper horizon and lower horizon compaction results in APPENDIX C and APPENDIX D - is related to which of the 13 centroids along the compacted pipe centerlines is used to place the waste forms in the model relative to each other. For the calculations in APPENDIX $\mathrm{C}$ and APPENDIX D, a point close to the center centroid was used. Furthermore, the centroid was used to center the base of the upright cylinders, as shown in the figure below. 


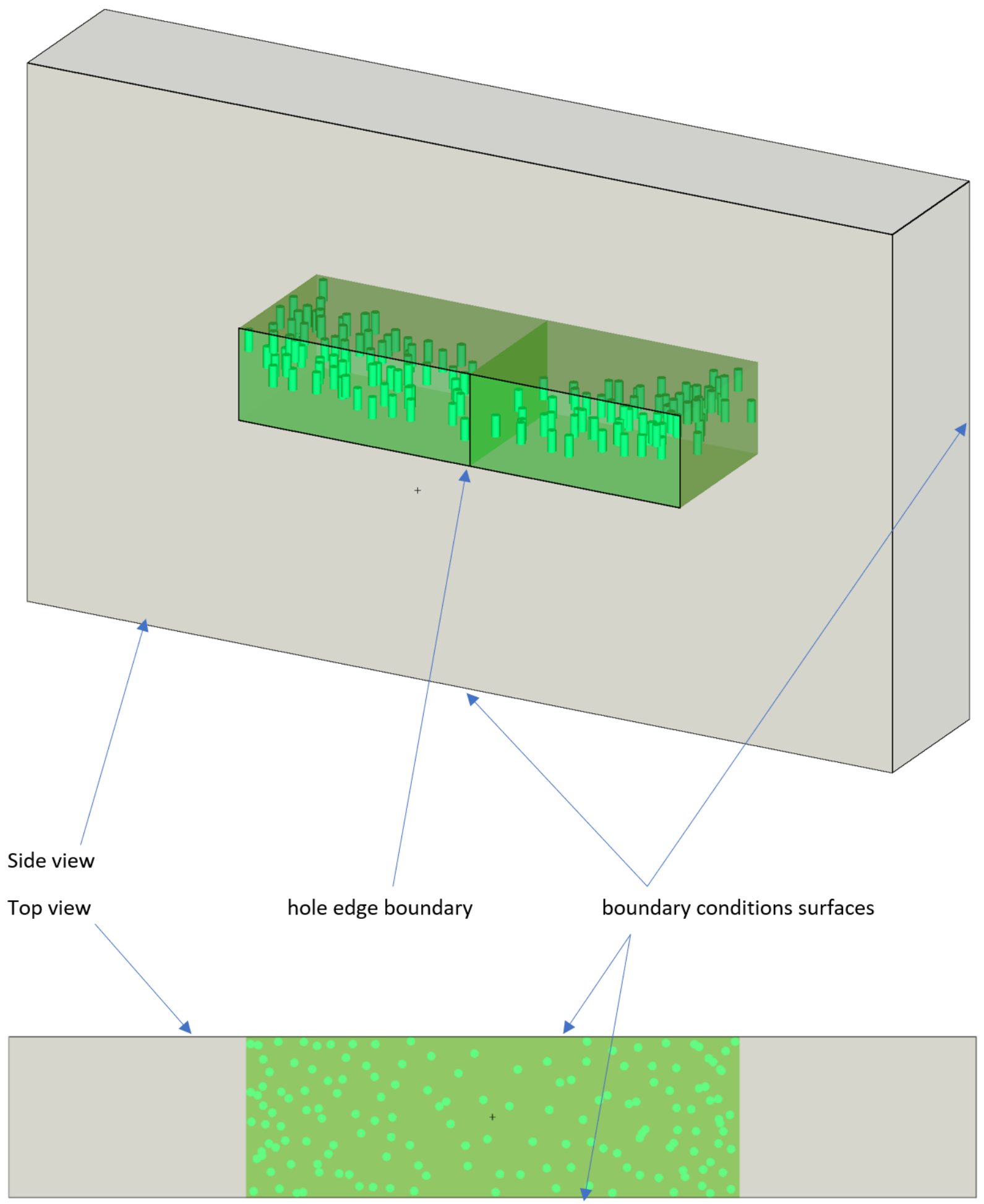

Figure G-3. 3D representation of the Appendix C and Appendix D 2-unit analysis model illustrating how pipe center centroid data were used to orient vertical cylinders. 
In the figure above, the model's reflective boundary conditions are conservatively placed just adjacent to the outmost edge of the waste forms along both boundaries. This assumption regarding the placement of the reflective boundary conditions is discussed further in APPENDIX E.

For the purpose of the studies presented in this appendix, many of which will change the orientation of the cylinders in the model, there will be instances in which this change in orientation will result in the cylinder extending far past the reflective boundary plane, thus causing the reflective boundary conditions to be farther away from the waste form than shown in this example. Rather than change the location of the reflective boundary condition for each sweep, it is more appropriate to move the reflective boundary condition to a fixed point for all cases so that the reactivity effect of the changes in orientation can be evaluated with like-for-like comparisons. Therefore, for all cases, the outermost centroid's coordinates are used to define the boundaries of the interstitial filler material $(\mathrm{MgO}+\mathrm{salt})$, and an additional $50 \mathrm{~cm}$ is applied so that all cases have a more uniform application of reflection. For these models, the $50 \mathrm{~cm}$ of reflector material was applied in all directions. The impact of this is expressly evaluated in APPENDIX E.

All cases in this appendix use similar models, and they also use the same set of conditions associated with set-2-4 for the upper horizon calculations presented in APPENDIX C. Specifically, these cases are for sweeps with poly moderator, graphite filler, and poly pipe material with cylinders.

It is possible that an alternate orientation configuration may yield a significant reactivity increase that could invalidate the conclusion that set-1 results are bounding of all nonuniform array results.

Furthermore, these calculations provide a methodology to conclude that under the CCO emplacement strategy used at WIPP, an insufficient amount of fissile material can be expected to collect in a given area of the room, thus significantly increasing the risk of criticality.

The specific nomenclature used for the studies in this appendix is provided below:

- centroid: location of a point defined by $(\mathrm{x}, \mathrm{y}, \mathrm{z})$ coordinates from SNL compaction studies. Each pipe in the dataset of 153 compacted pipes contains 13 centroids defined by their $(\mathrm{x}, \mathrm{y}, \mathrm{z})$ position along the pipe centerline.

- base case: the centroid defines the center of the cylinder base. The term base is not included in the case nomenclature, but in the absence of other case designations, it is considered consistent with the Appendix C and Appendix D calculations.

- shifted: the centroid defines the geometric center of the cylinder rather than the center of the bottom face.

- midpoint: the $(\mathrm{x}, \mathrm{y}, \mathrm{z})$ position calculated at the center of the vector between the endpoints (not a specific SNL-defined centroid, but calculated from the SNL data).

- orientation: direction of the cylinder relative to the $x-y$ plane. Following are specific orientation terms:

base case orientation is with the orientation in the vertical direction, and is a term which is not specifically used but is considered consistent with the Appendix C and Appendix D calculations. local orientation is determined by calculating the angles from a vector between two adjacent points (adjacent to the centroid). For endpoints (centroids at either end of the pipe), it is calculated from the endpoint to the adjacent centroid. For non-endpoints, it is calculated from the centroids adjacent to either side. For example, the local orientation for centroid 5 is calculated using a vector between centroids 4 and 6.

global orientation is taken from the vector between the two endpoints.

$\boldsymbol{r} \boldsymbol{A}$ is the random orientation taken by a python generated random angle pair $(-180<=$ phi $<=180,0<=$ theta $<=180$ ) for the two SCALE variables which control orientation.

relLocal is the local orientation provided for rcoord cases. 
Three sets of evaluations are performed, each having multiple subsets. These are centroid studies, rcoord studies and $r A$ studies. These are explained below.

The centroid studies evaluate the effect of using each of the 13 SNL centroids independently, along with a midpoint. For these cases, each pipe in the model uses the same centroid for that case. For example, if the case is centroid1, then each pipe in the model uses the centroid 1 coordinates.

The rcoord studies sets evaluate the effect of randomly selecting which of the 13 centroid coordinates is used. Each pipe in the model has its own unique random selection. For these studies, 100 rcoord cases are generated.

The following six subsets of calculations are evaluated for each of the centroid and rcoord sets:

- base case with no orientation of the cylinder: the centroid is at the center of the cylinder base.

- base-with-local-orientation case: a local orientation is applied as determined specifically for each pipe based on the relative local centroid vectors, with the centroid at the center of the cylinder base.

- base-with-global-orientation case: a global orientation is applied as determined specifically for each pipe based on the pipe endpoint centroid vectors, with the centroid at the center of the cylinder base.

- shifted case: no orientation of the cylinder, with the centroid at the geometric center of the cylinder.

- shifted-with-local-orientation case: a local orientation is applied as determined specifically for each pipe based on the relative local centroid vectors, with the centroid at the geometric center of the cylinder.

- shifted-with-global-orientation case: a global orientation is applied as determined specifically for each pipe based on the pipe endpoint centroid vectors, with the centroid at geometric center of the cylinder.

The rA studies sets evaluate the effect of randomly selecting both phi and theta for the orientation of the cylinders. Each of the pipes in the model has its own unique random phi and theta selection. For these studies, 20 random phi and theta cases were generated for each of 13 centroids plus the midpoint. rA studies were performed in conjunction with rcoord cases, considering a set of 10 rcoord cases with $10 \mathrm{rA}$ sets each.

The following illustration provides additional clarity for how the cylinder orientations were evaluated. For each pipe in the model, a pipe centerline was defined by the 13 centroids. For example, a local orientation or a global orientation was determined by a vector between either two centroids adjacent to the pipe or at the ends of the pipe, respectively. This is illustrated by the straight lines in the figure below. The cylinder's orientation is shown by how it follows the orientation of that line. 


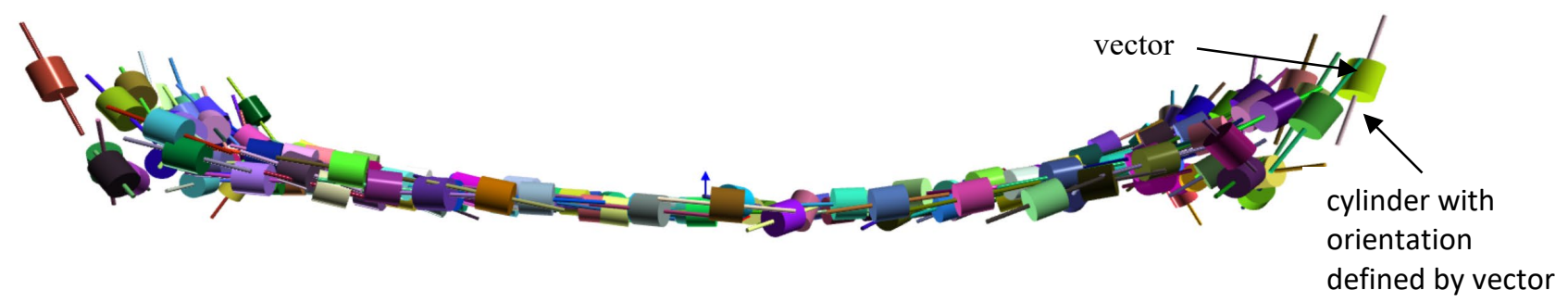

Figure G-4. A 3D python-generated illustration of how cylinder orientations follow pipe centerline orientations. Note that this illustration is not an actual analysis model.
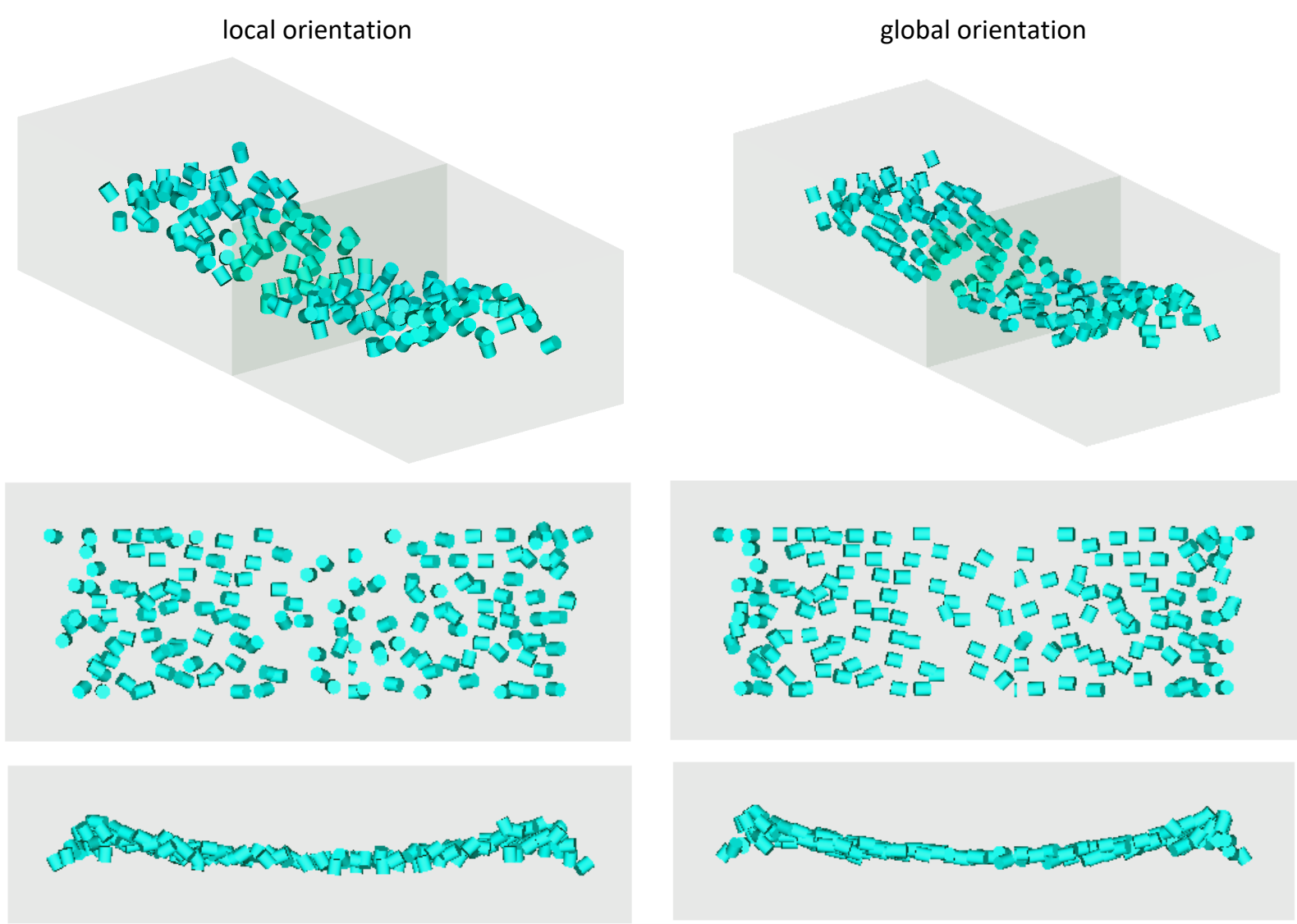

Figure G-5. Comparison of local and global orientations for centroid 1 using the isometric, top, and front view angles. 

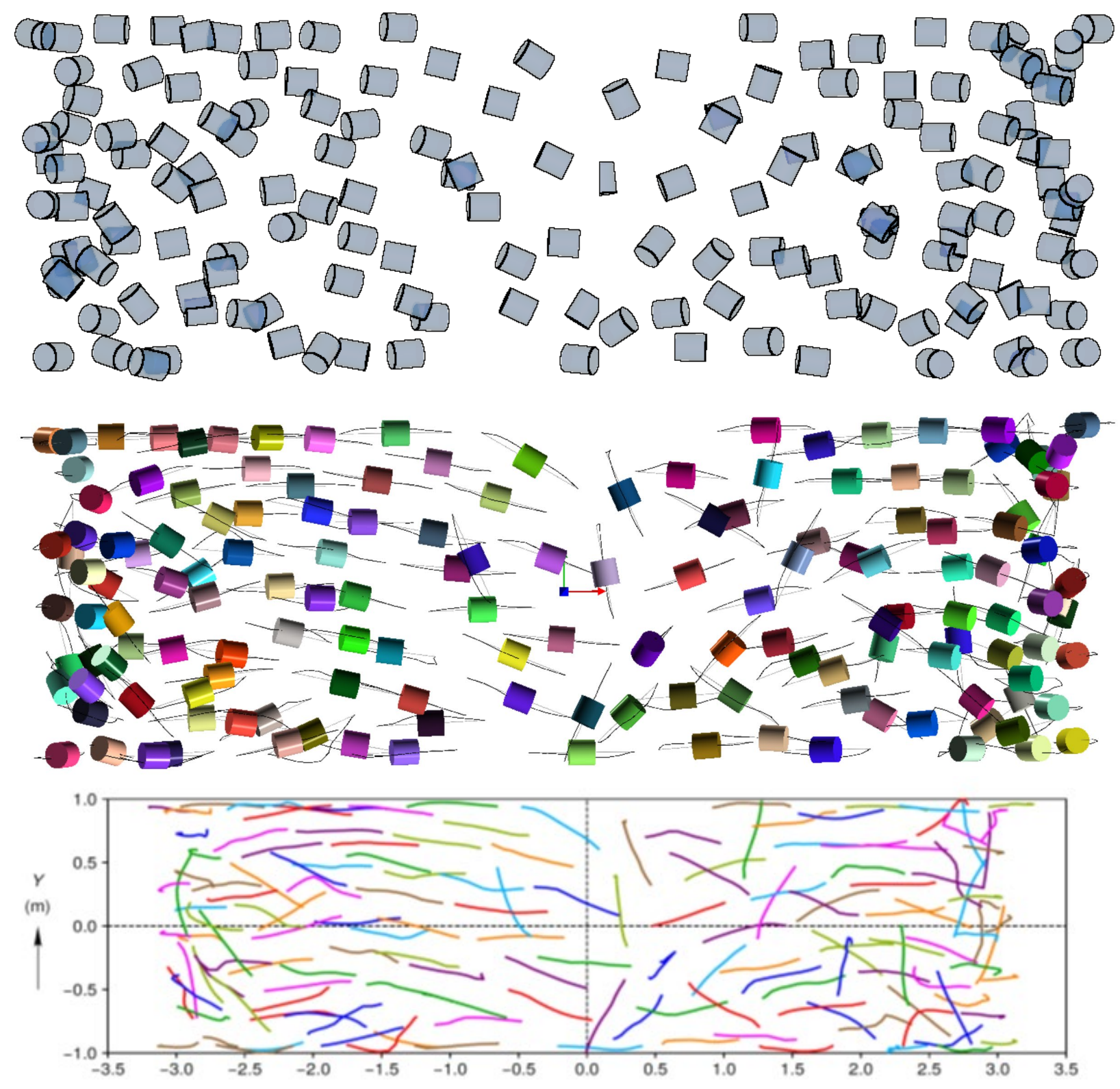

Figure G-6. Comparison of different visualizations of Centroid 6 shifted with global orientation case, $x-y$ plane (top view). 


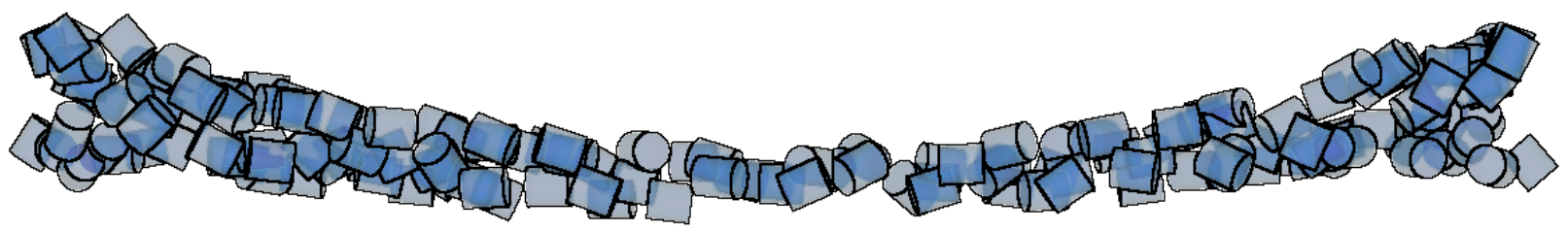

Front view rotated 180 degrees
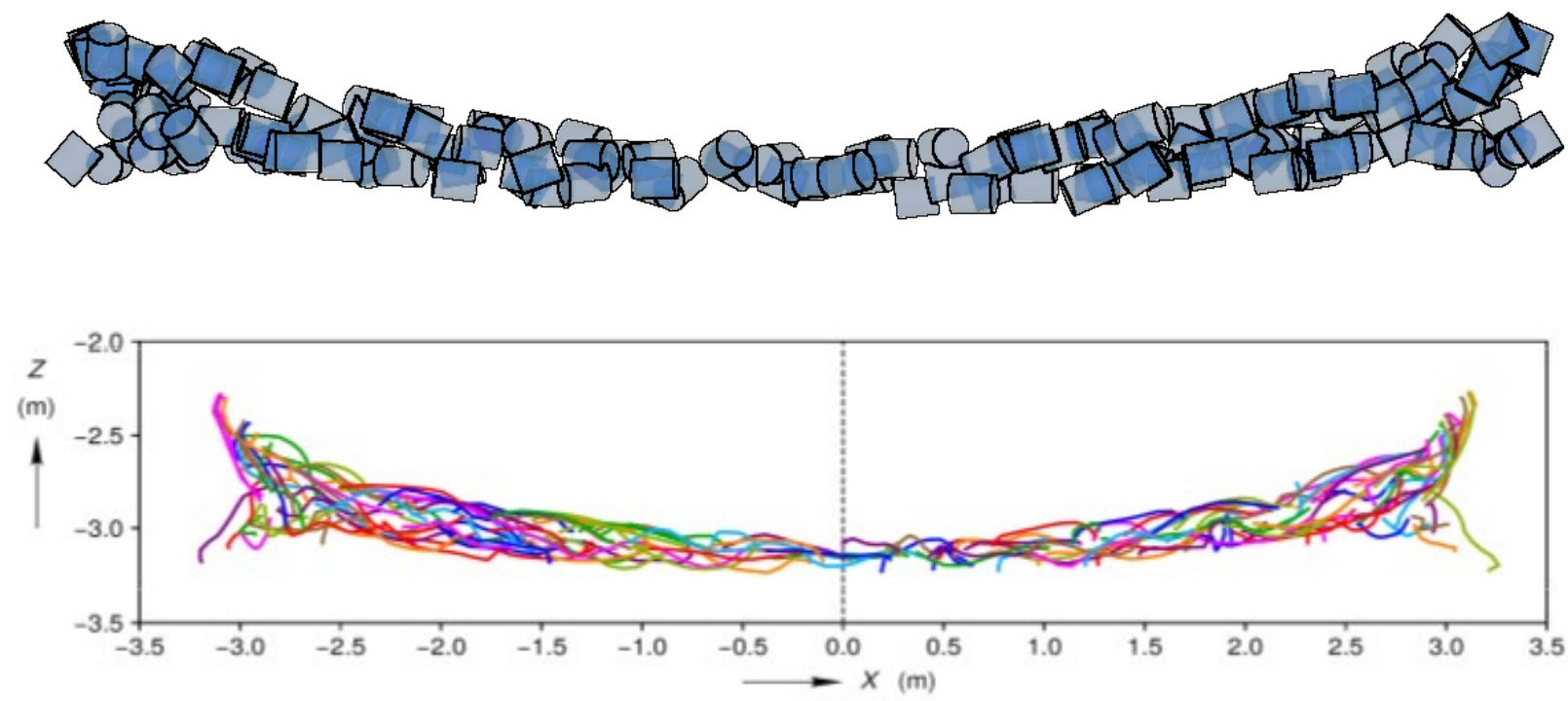

Figure G-7. Comparison of different visualizations of the Centroid 6 shifted with global orientation case, $x-z$ plane (front/back view).

The results of these studies are provided in the figures and lists below. The following nomenclature is used for the cases provided in the listings:

- centroid $n$ (where $\boldsymbol{n}$ is a number between 1 and 13): indicates which centroid location is used for each pipe in the model.

- _c1-1_: indicates that there is no orientation of the cylinder in the model, or the base case.

- _ _ $\mathbf{c n} \boldsymbol{m}$ : indicates that a local orientation is determined from a vector taken from centroid $n$ to $m$.

- _c1-13_: indicates a global orientation of the cylinders

- $\quad$ shifted: indicates that the geometric center of the cylinder is used for the centroid $(\mathrm{x}, \mathrm{y}, \mathrm{z})$ rather than the SCALE default center of the cylinder base.

- relLocal $n$ (where $\boldsymbol{n}$ is a number between 1 and 100): indicates which random number in the series of random coordinate sweeps is being used, and that the local orientation relative to that selection is being applied. 


\section{Centroid study results}

The following diagrams (Figures G-8 through G-21) show the results for centroid location and orientation for the base, local, and global orientations. Shifted cases are also included. The results show that there is little variation due to orientation for each centroid location, whereas some variation in reactivity due to centroid number exists. Centroids 8,10 and 11 are slightly more reactive than the other centroids. Figure G-22 shows all the data combined, along with the bounding set-2-4 curves (under same the conditions).

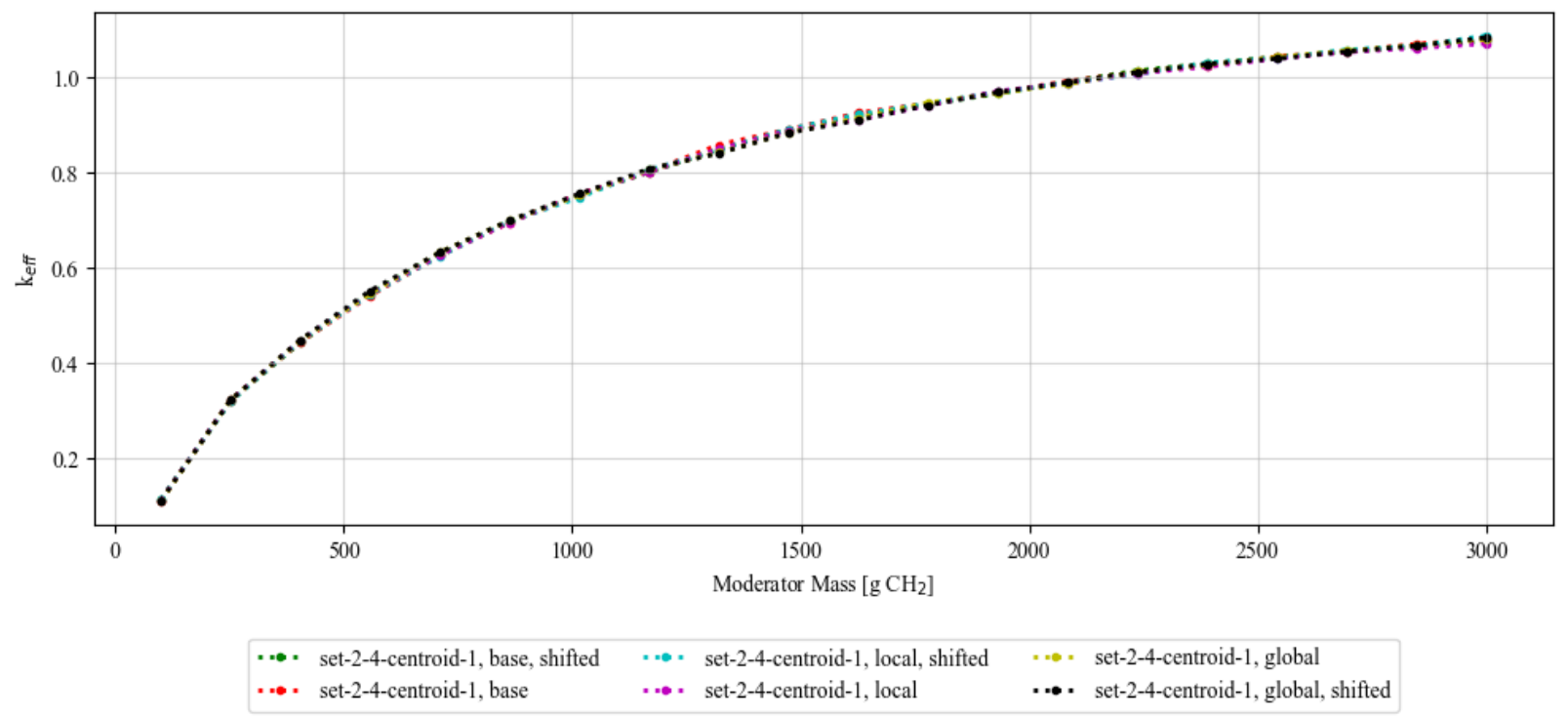

Figure G-8. Results of the centroid studies for centroid 1.

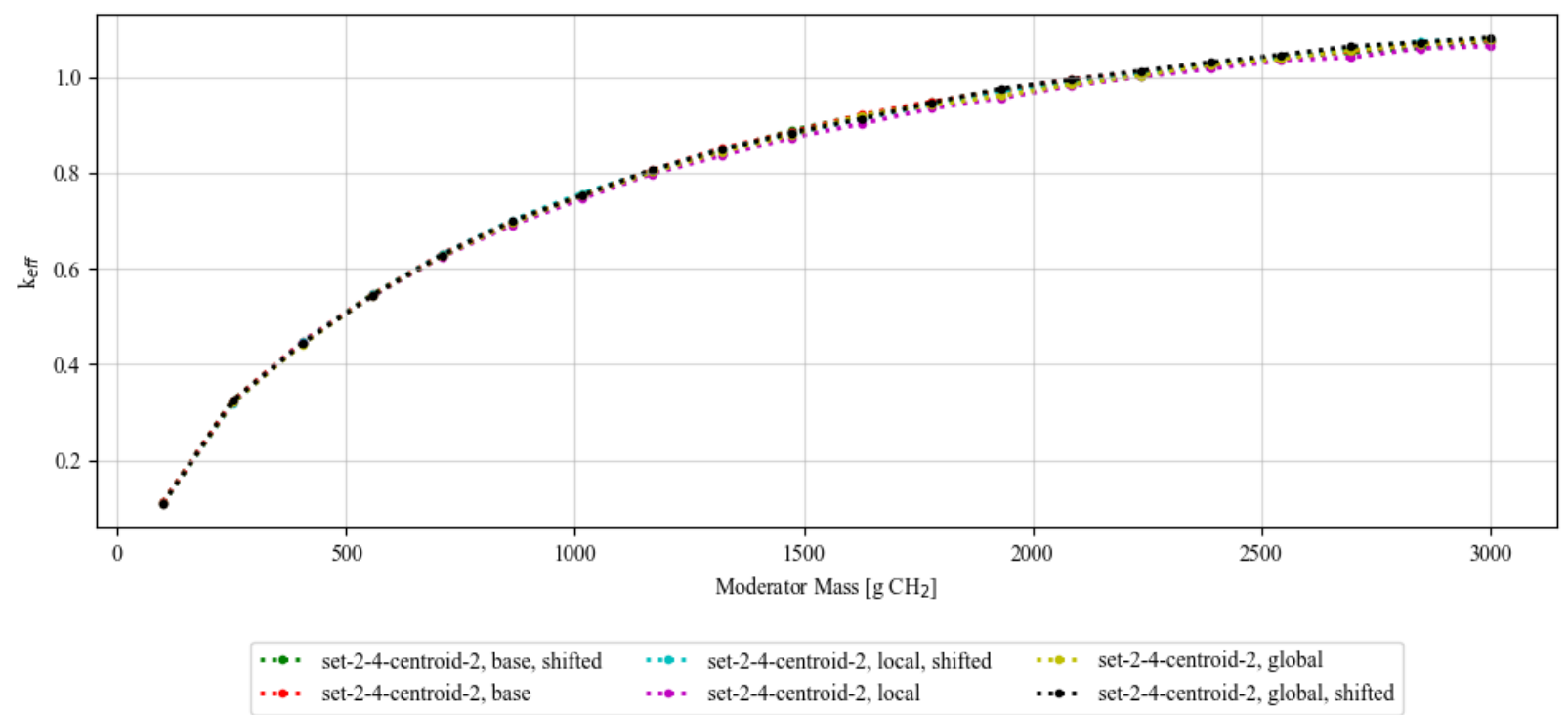

Figure G-9. Results of the centroid studies for centroid 2. 


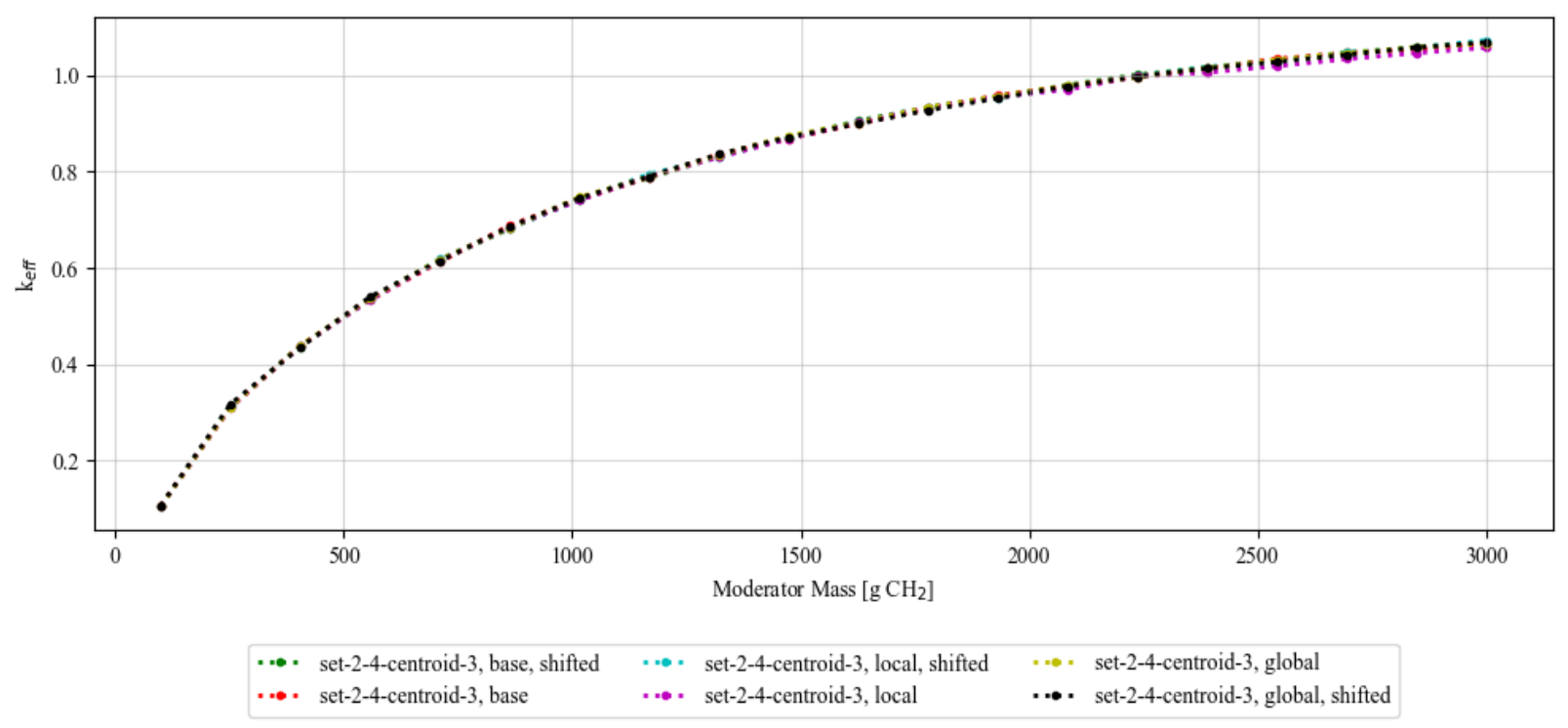

Figure G-10. Results of the centroid studies for centroid 3.

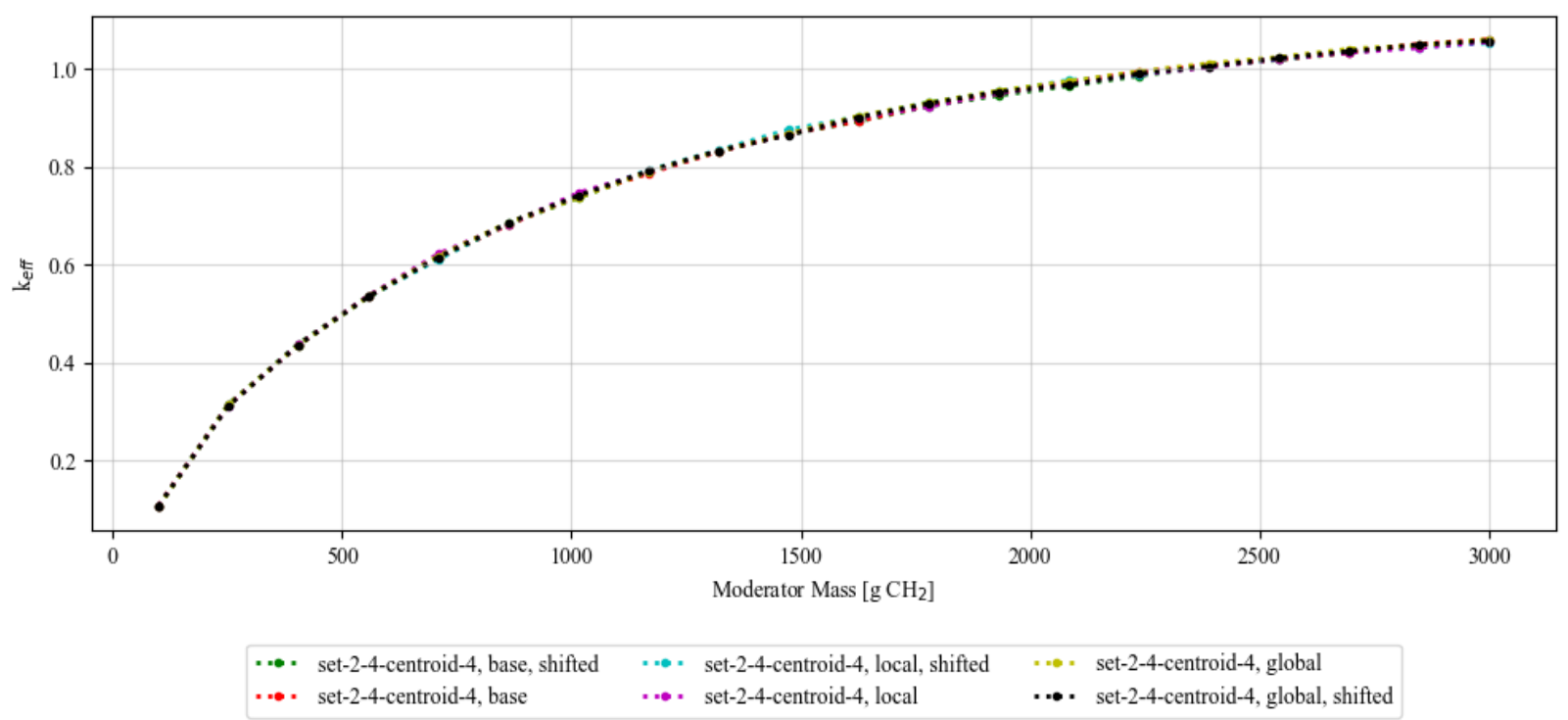

Figure G-11. Results of the centroid studies for centroid 4. 


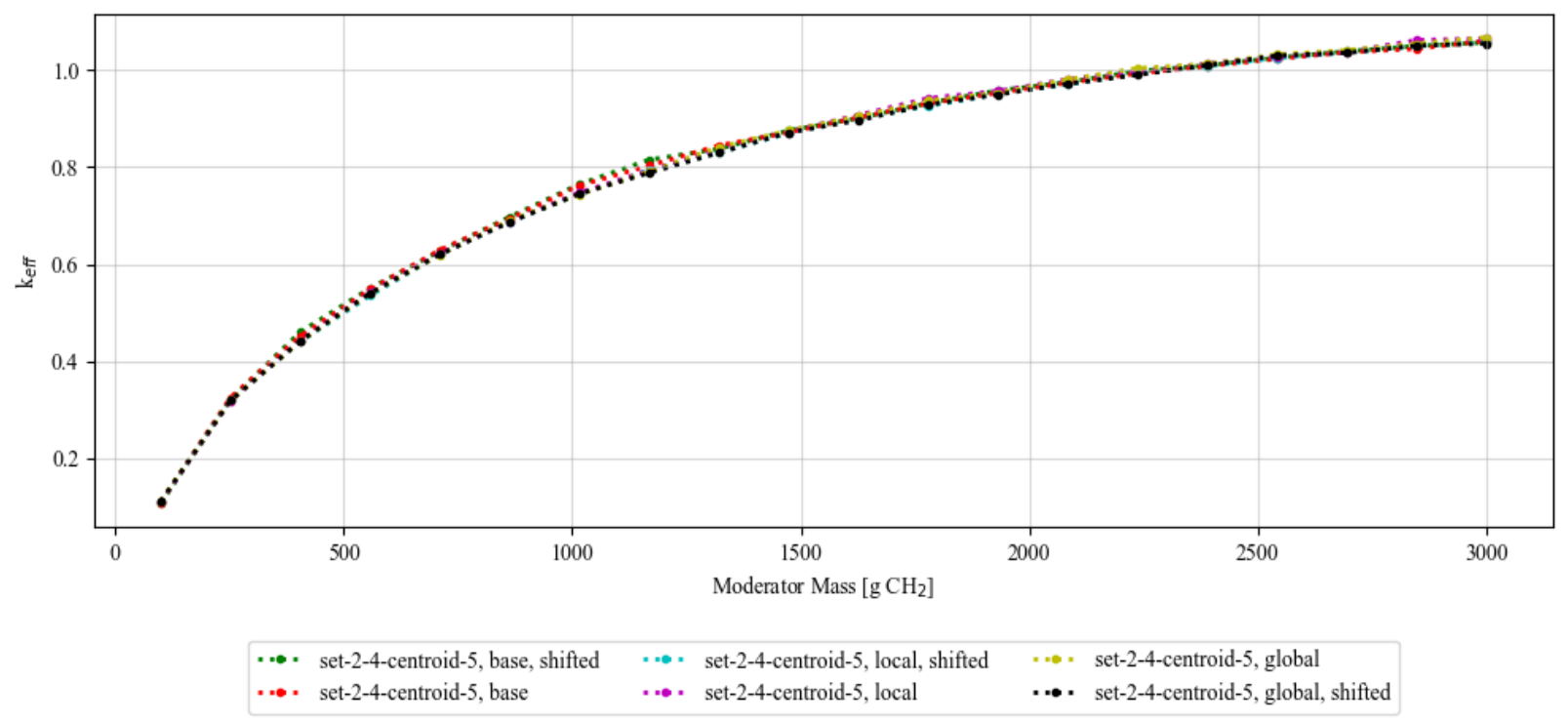

Figure G-12. Results of the centroid studies for centroid 5.

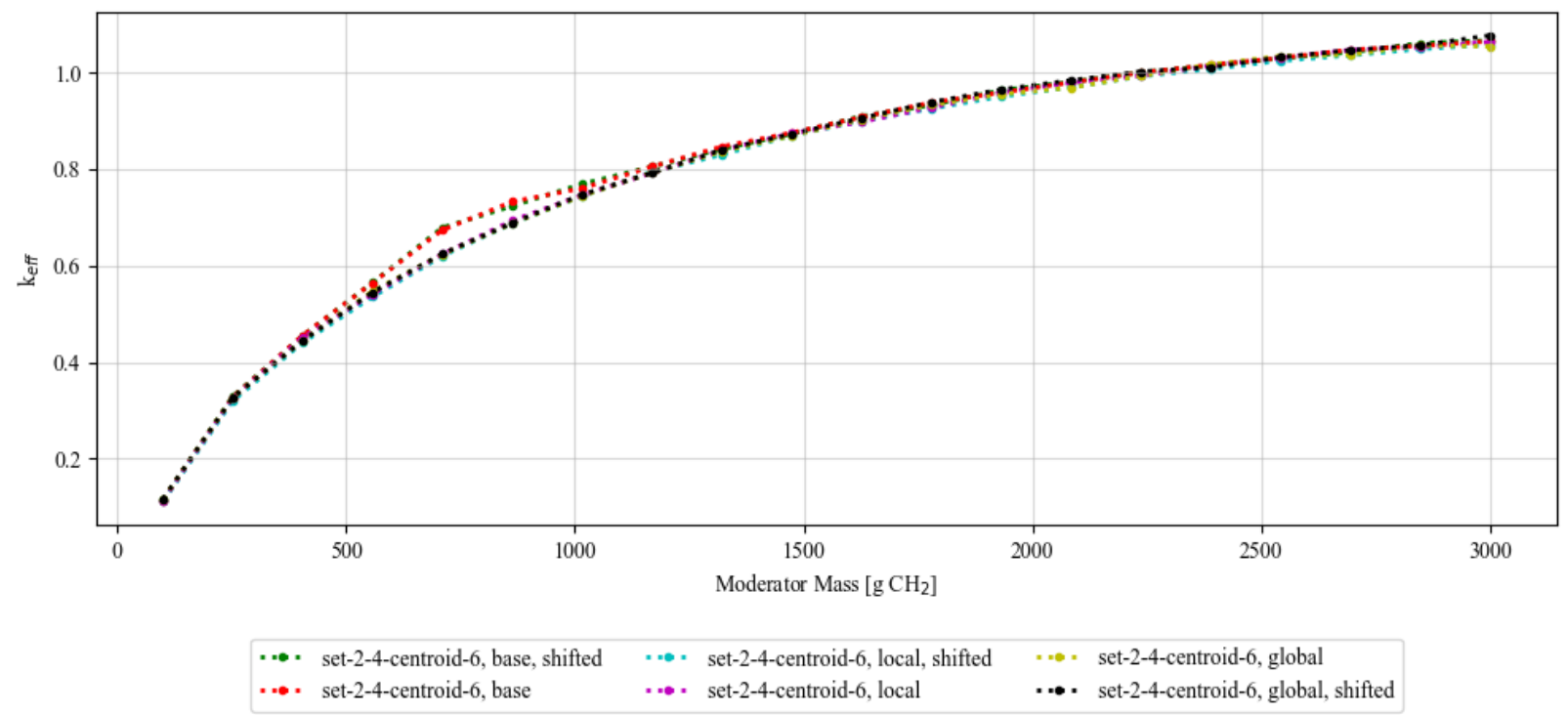

Figure G-13. Results of the centroid studies for centroid 6. 


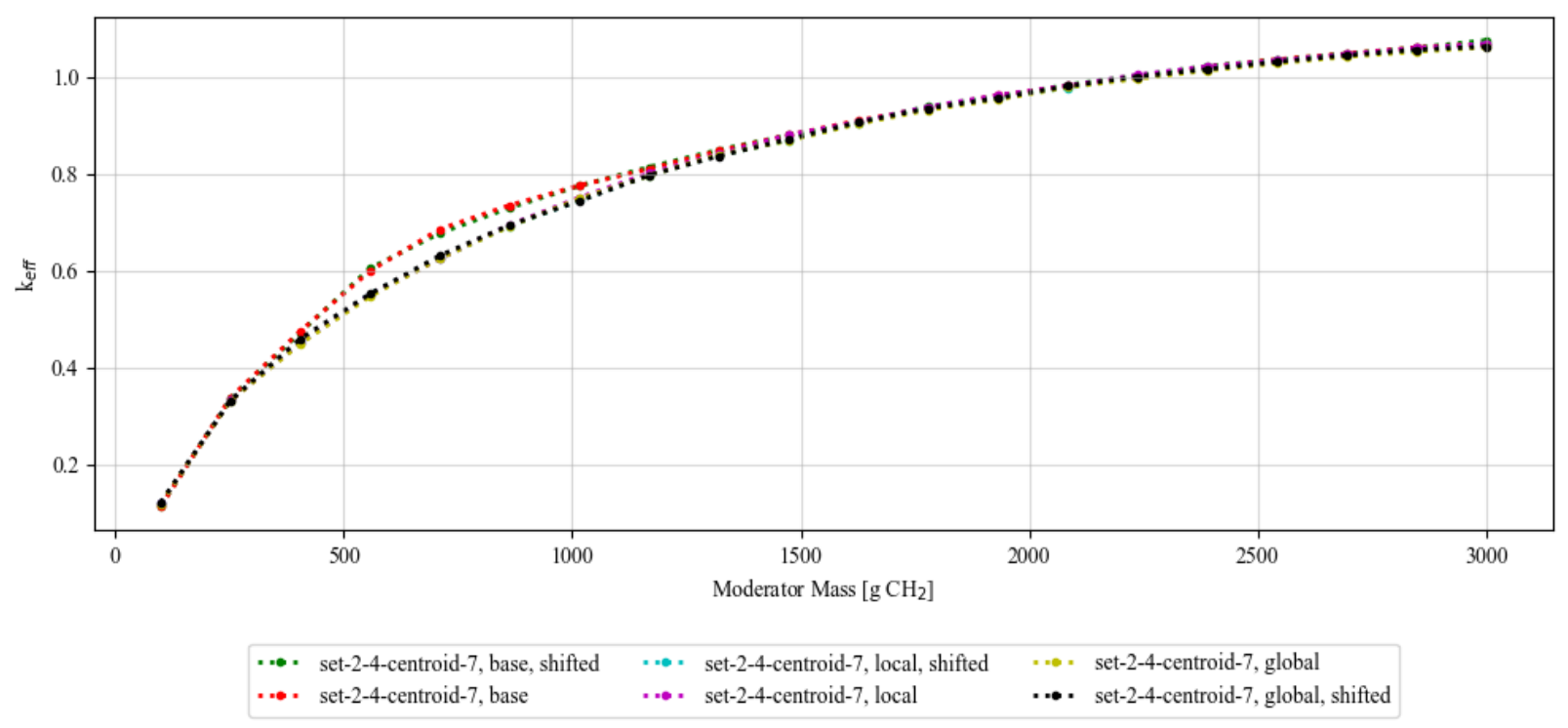

Figure G-14. Results of the centroid studies for centroid 7.

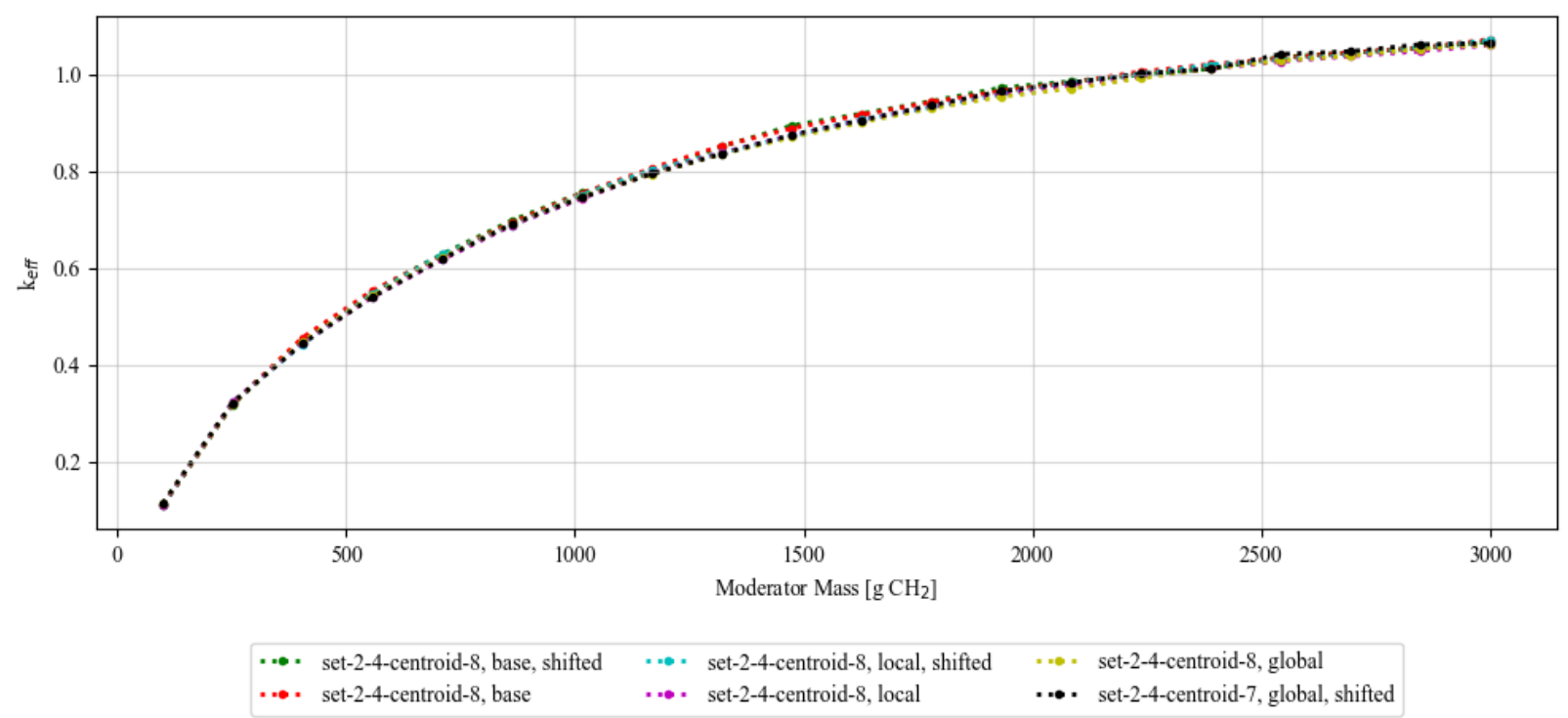

Figure G-15. Results of the centroid studies for centroid 8. 


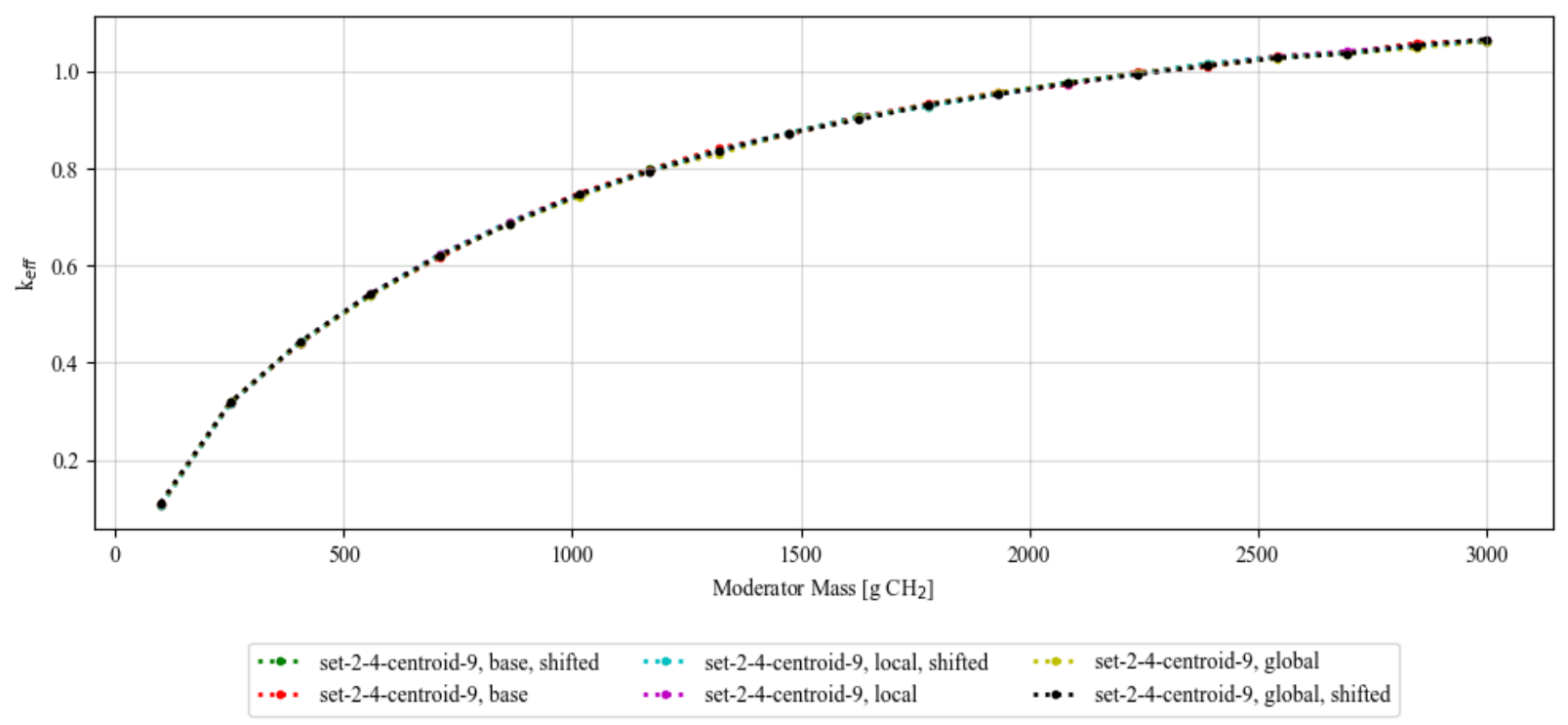

Figure G-16. Results of the centroid studies for centroid 9.

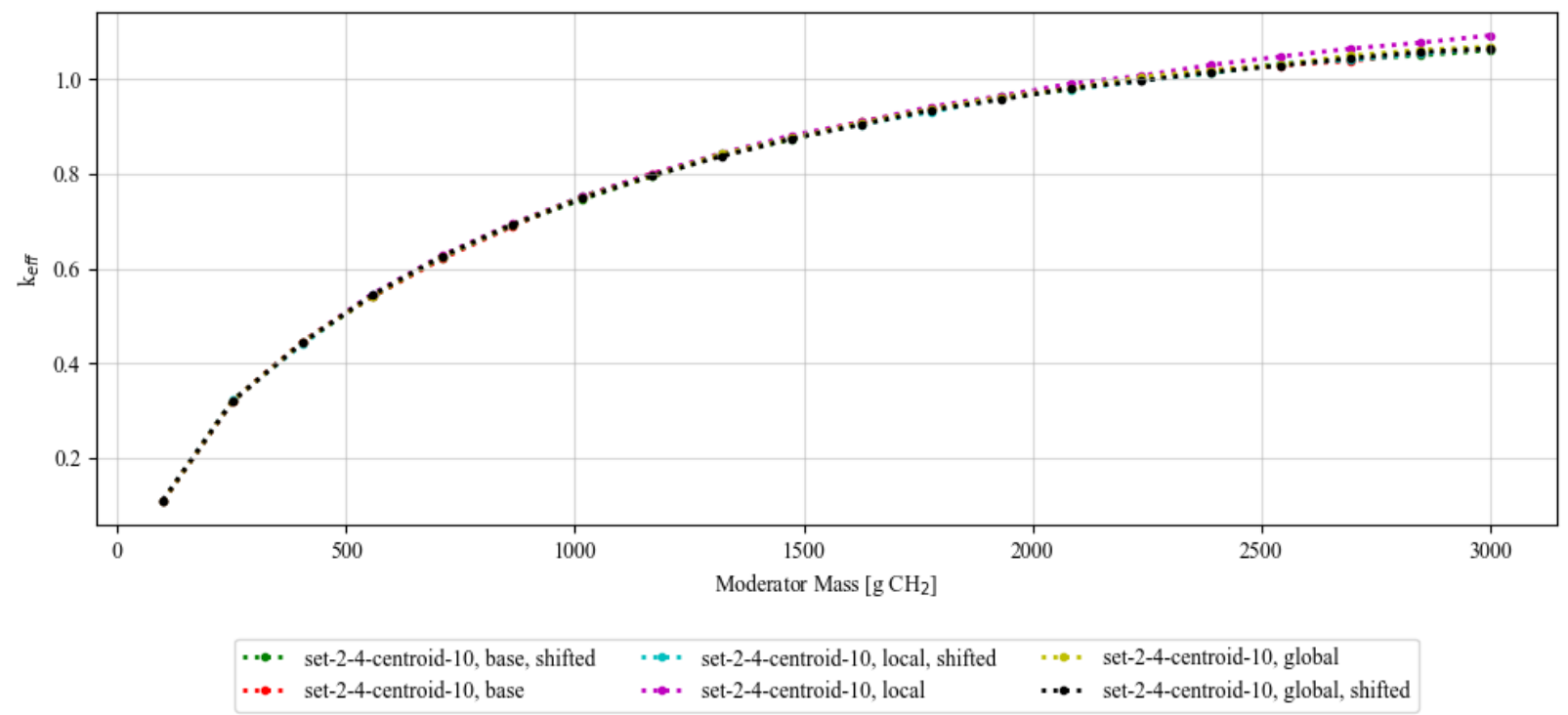

Figure G-17. Results of the centroid studies for centroid 10. 


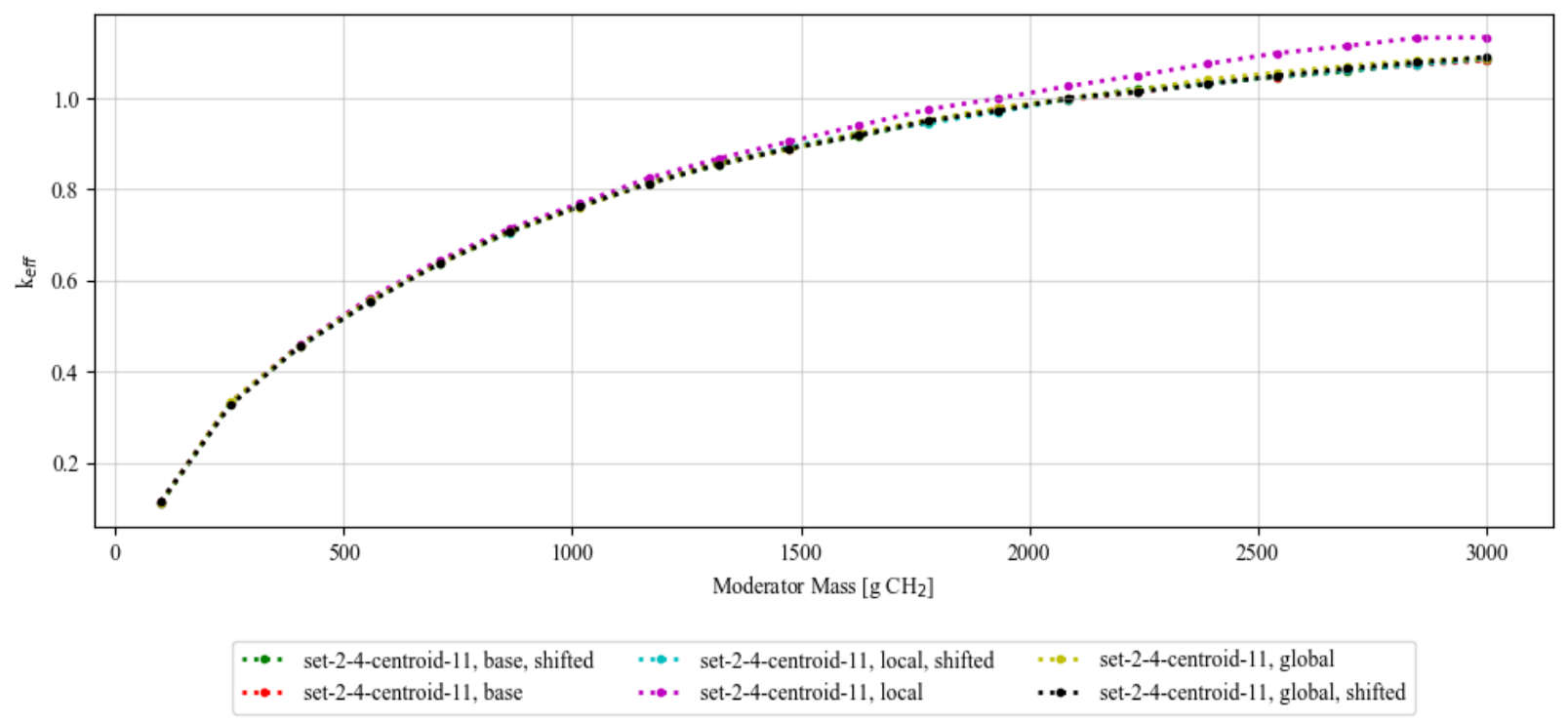

Figure G-18. Results of the centroid studies for centroid 11.

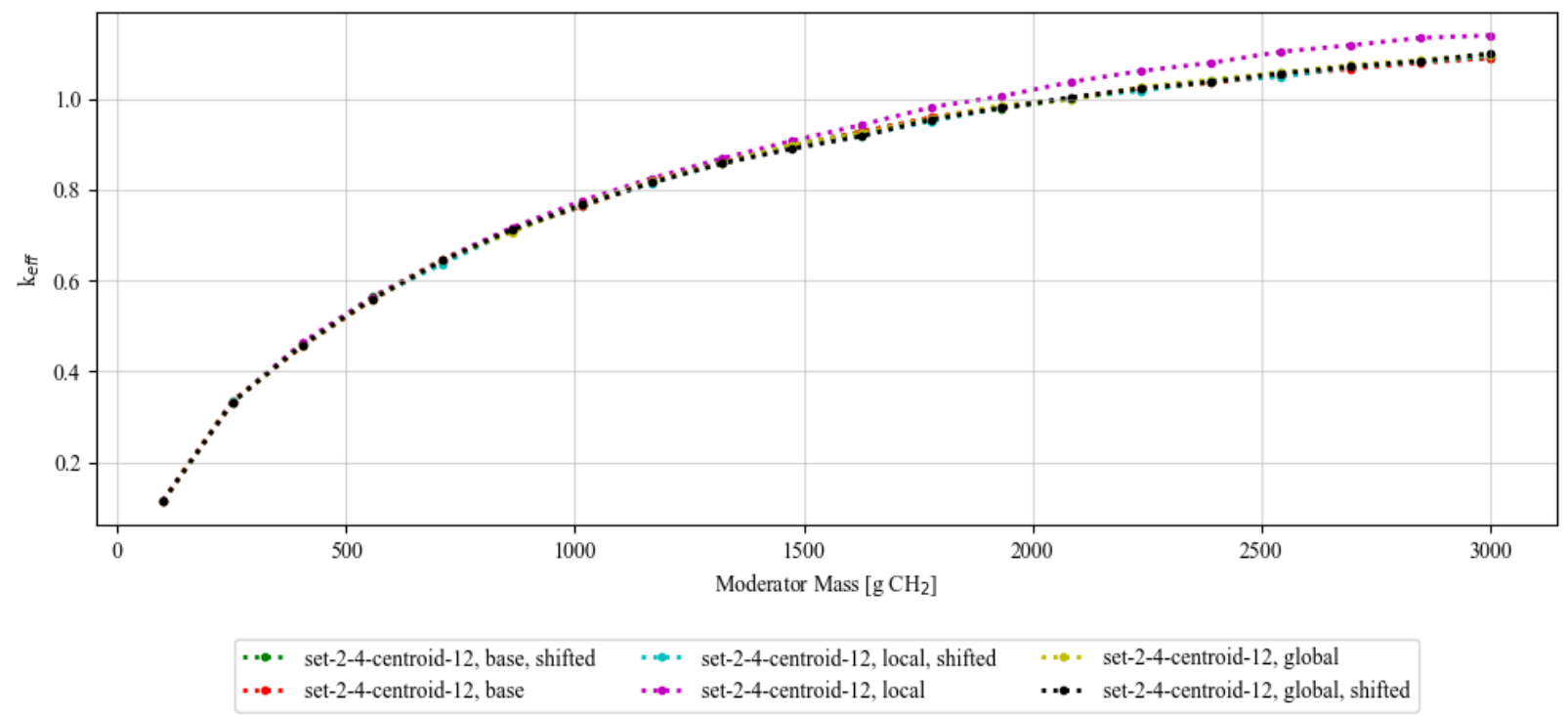

Figure G-19. Results of the centroid studies for centroid 12. 


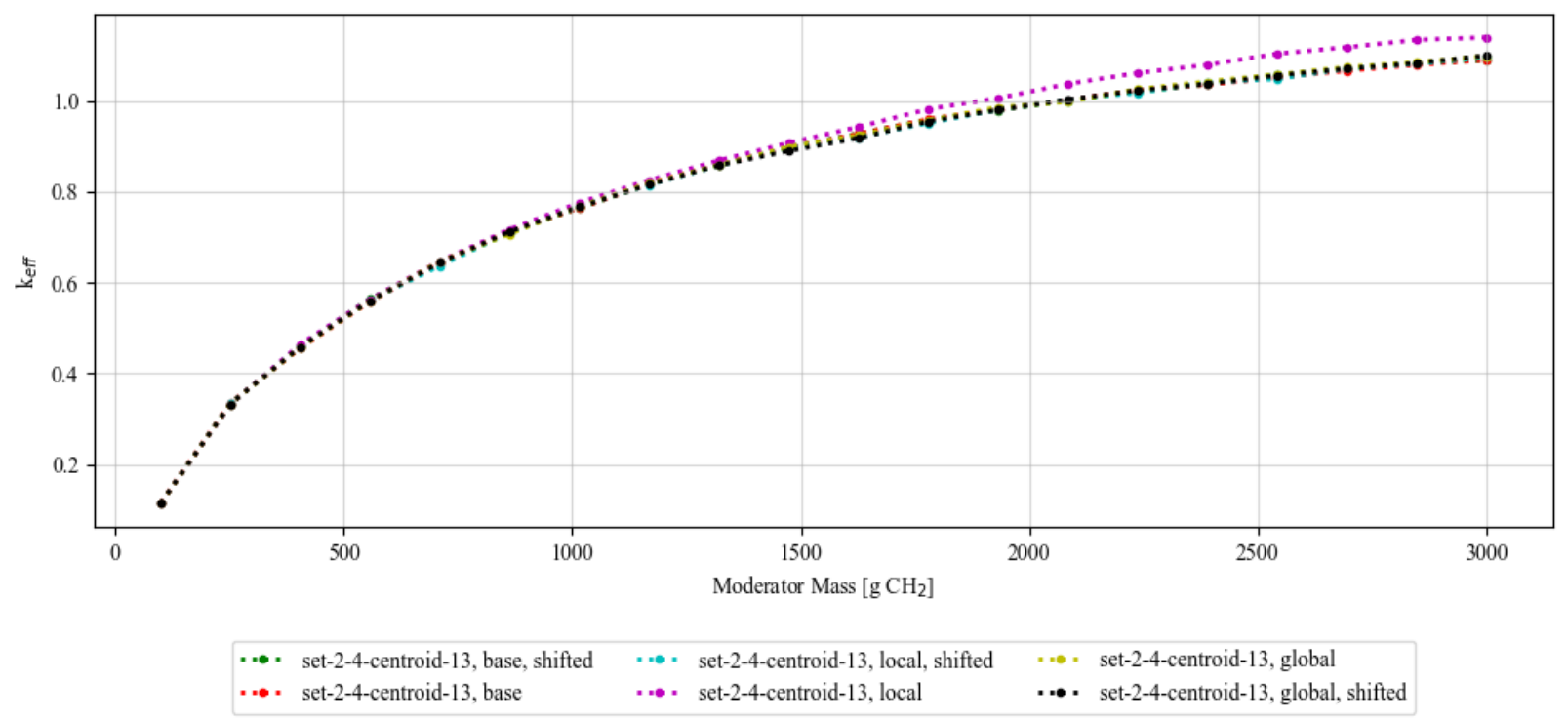

Figure G-20. Results of the centroid studies for centroid 13.

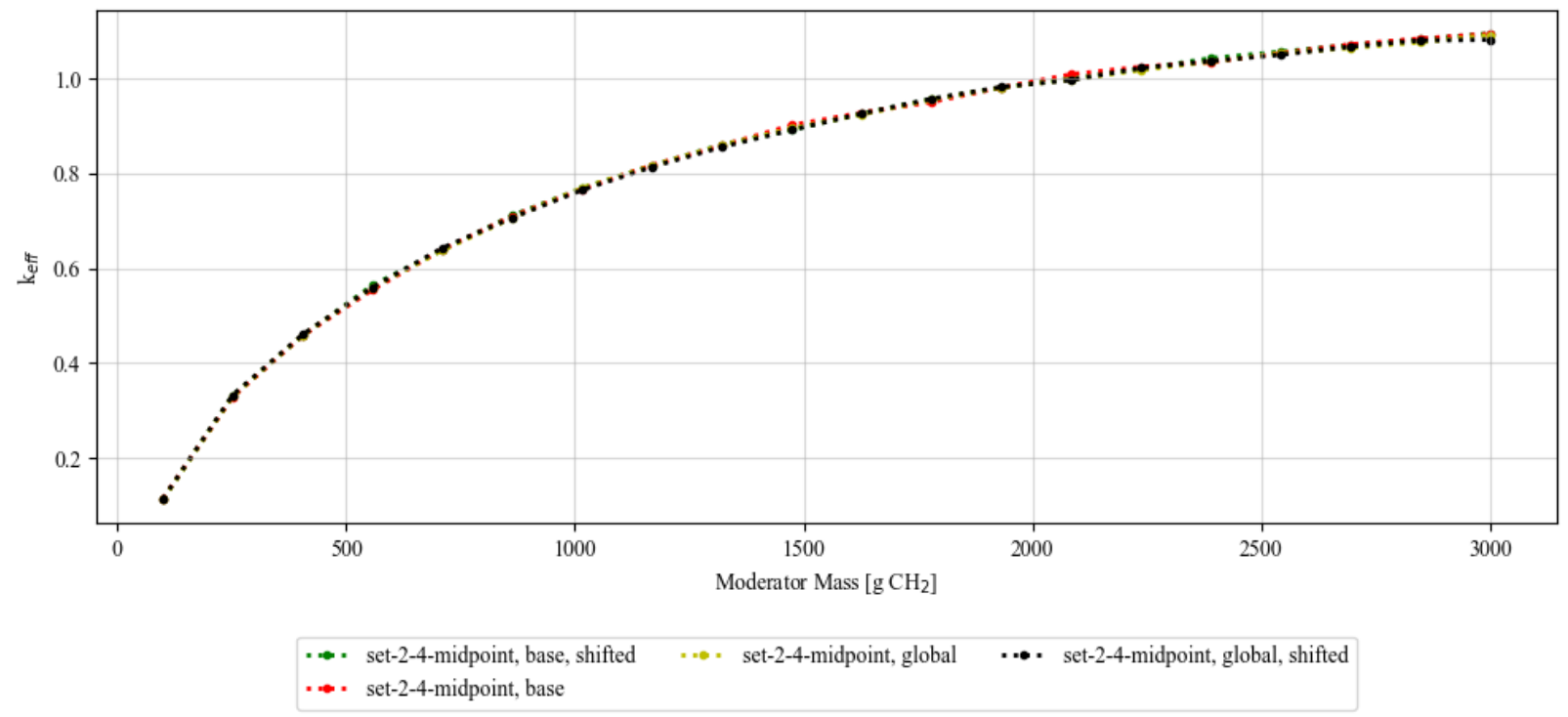

Figure G-21. Results of the centroid studies for the "midpoint" centroid. 


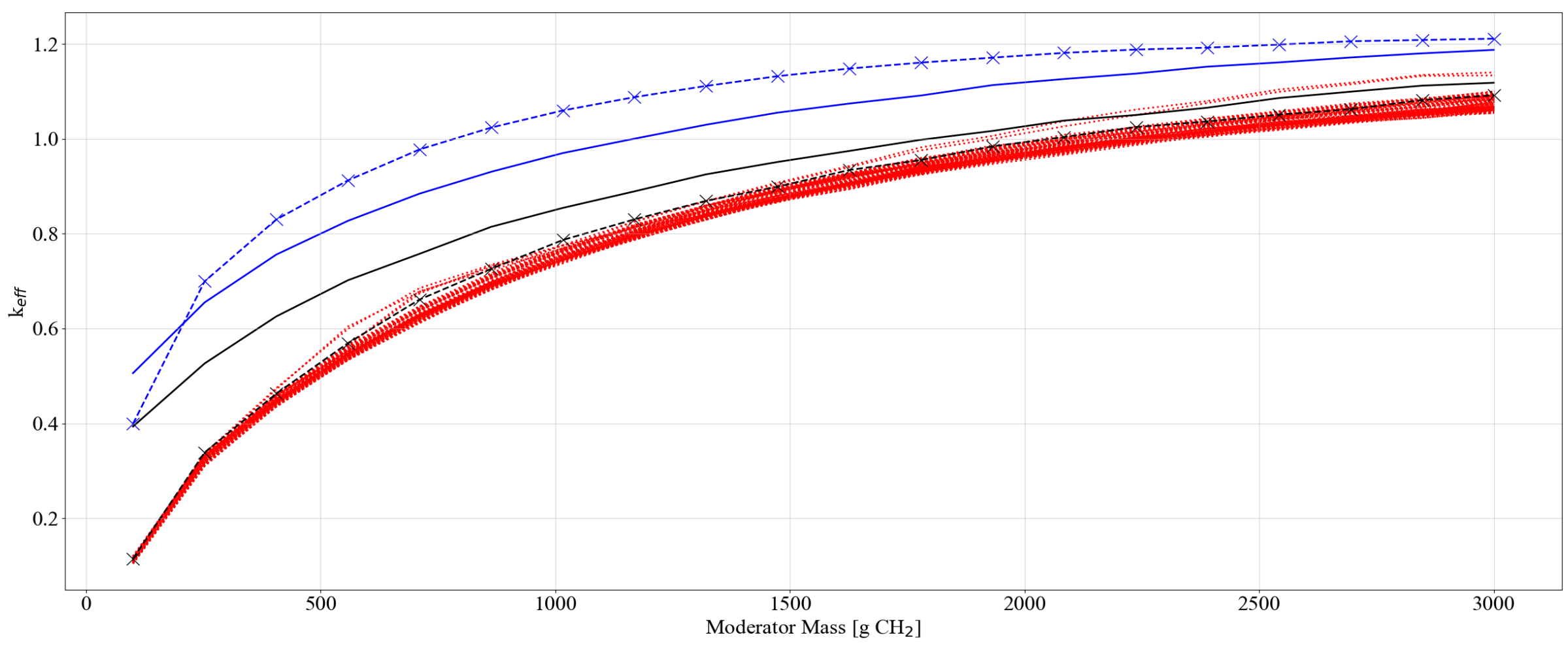

--※-- set-1, cylinder with radius $=7.7 \mathrm{~cm}$, no filler, no Be, thick poly discrete reflector, poly moderator

— set-2-uh, sphere, no filler, no Be, thick poly discrete reflector, poly moderator

$--\Varangle--$ set-2-uh, cylinder with radius $=7.7 \mathrm{~cm}$, no filler, no $\mathrm{Be}$, thick poly discrete reflector, poly moderator all centroids and midpoint

Figure G-22 Comparison of all centroids and midpoint to various representative curves. 
rcoord study results

Additional studies were performed to evaluate the impact of randomly selecting which centroid location each pipe in the model used. The results are summarized in the Figure G-23 below. 


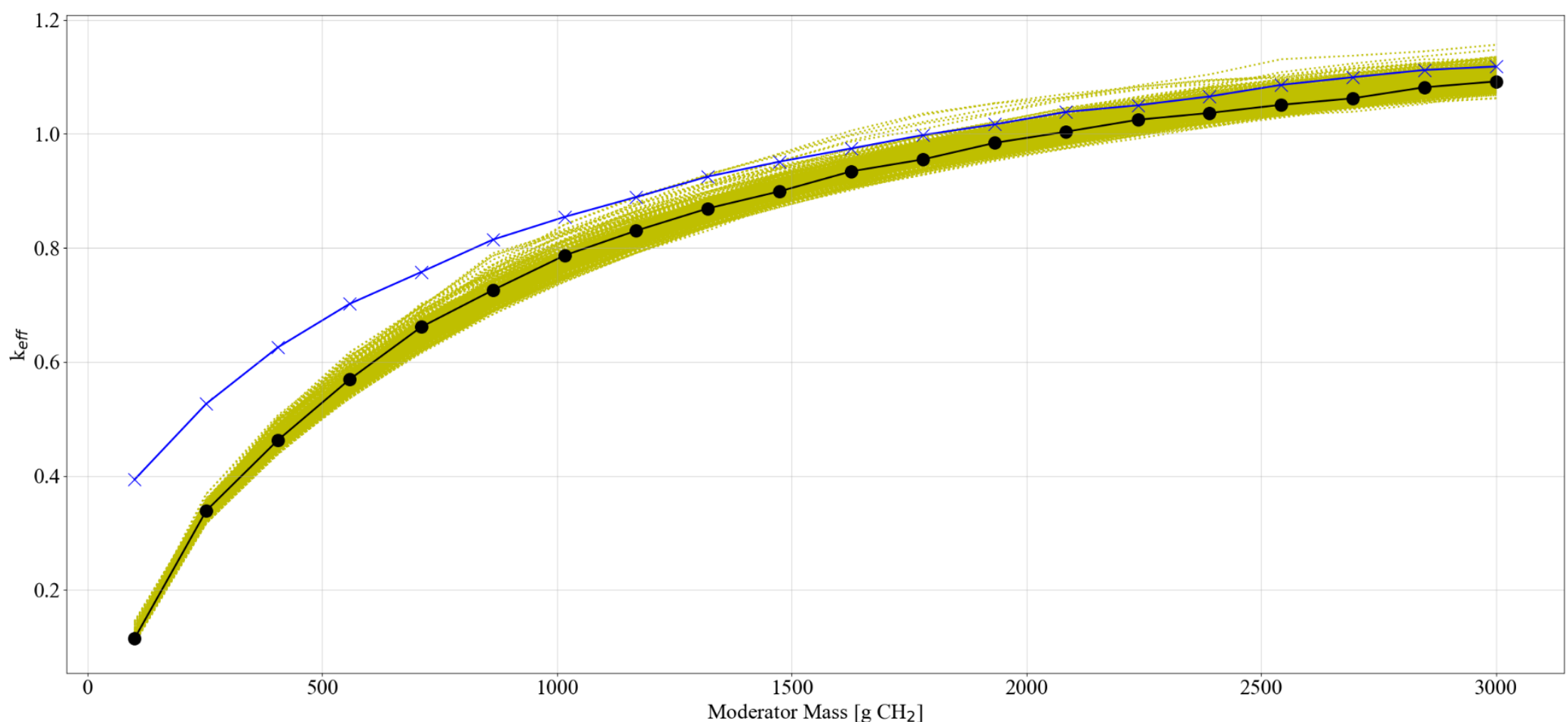

rcoord all

$\star$ set-2-uh, sphere, no filler, no Be, thick poly discrete reflector, poly moderator

$\longrightarrow$ set-2-uh, cylinder with radius $=7.7 \mathrm{~cm}$, no filler, no Be, thick poly discrete reflector, poly moderator

Figure G.23. Set-2-4 orientation study, random coordinate studies, results for 100 randomly selected centroid locations for each subset. 


\section{rA study results}

The final set of studies presented in this appendix were performed to randomly select the rotation angles for the orientation of the cylinders. The results are summarized in the Figure G-24 and G-25 below. 


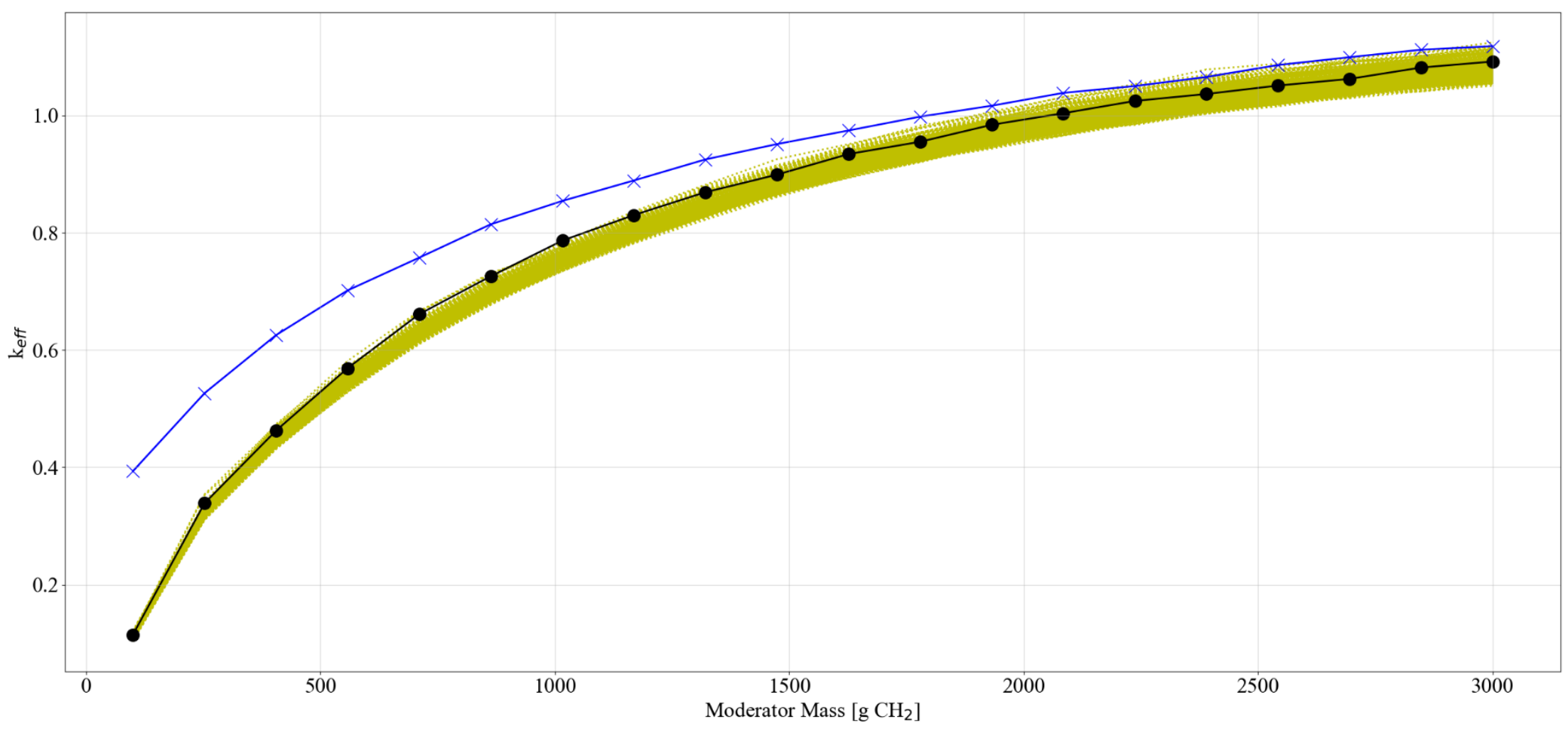

rA all

set-2-uh, sphere, no filler, no Be, thick poly discrete reflector, poly moderator

$\longrightarrow$ set-2-uh, cylinder with radius $=7.7 \mathrm{~cm}$, no filler, no Be, thick poly discrete reflector, poly moderator

Figure G.24. Set-2-4 orientation study, random angle studies, results for 20 randomly selected angles for the 13 centroids plus the midpoint for each subset. 


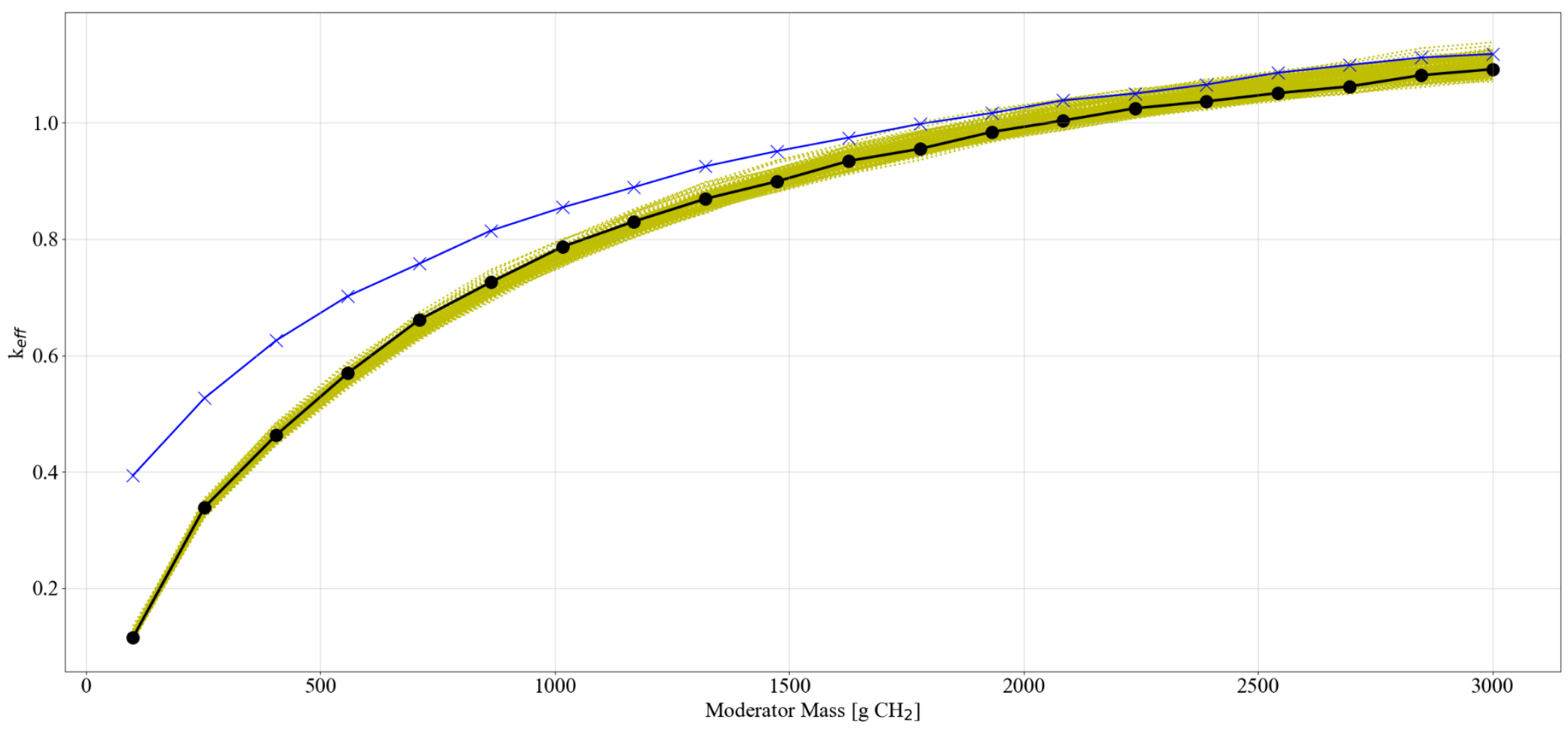

rcoord with $\mathrm{rA}$ all

set-2-uh, sphere, no filler, no Be, thick poly discrete reflector, poly moderator

set-2-uh, cylinder with radius $=7.7 \mathrm{~cm}$, no filler, no Be, thick poly discrete reflector, poly moderator

Figure G.25. Set-2-4 orientation study, random angle studies, results for 10 randomly selected angles for the $\mathbf{1 0}$ randomly selected centroids plus midpoint for each subset. 
This page is intentionally blank 
APPENDIX H. CALCULATIONAL VALIDATION 
This page is intentionally blank 


\section{CONTENTS}

H.1 SENSITIVITY COEFFICIENT GENERATION ................................................................ H-7

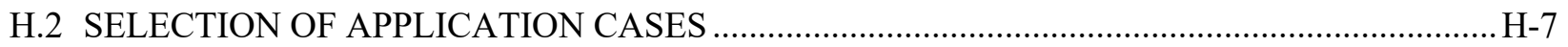

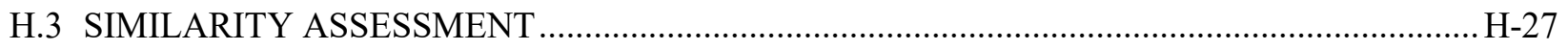

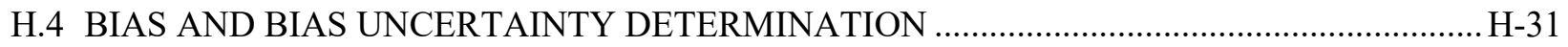

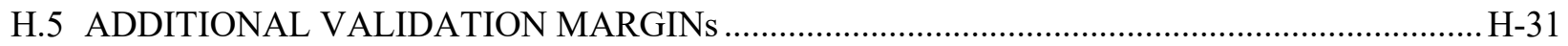

\section{LIST OF FIGURES}

Figure H-1. Top view of modified configuration. H-7

Figure H-2. Set-1-1 cases, water moderator, graphite filler, stainless steel pipe........................................

Figure H-3. Set-1-2 cases, poly moderator, graphite filler, stainless steel pipe..................................... H-8

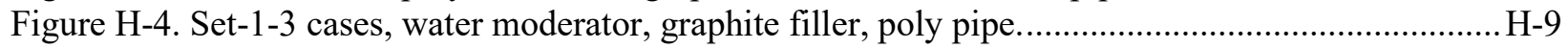

Figure H-5. Set-1-4 cases, poly moderator, graphite filler, poly pipe .............................................. H-9

Figure H-6. Set-1-5 cases, water moderator, generic filler, stainless steel pipe. ................................ H-10

Figure H-7. Set-1-6 cases, poly moderator, generic filler, stainless steel pipe. .................................. H-10

Figure H-8. Set-1-7 cases, water moderator, generic filler, poly pipe ............................................... H-11

Figure H-9. Set-1-8 cases, poly moderator, generic filler, poly pipe ................................................ H-11

\section{LIST OF TABLES}

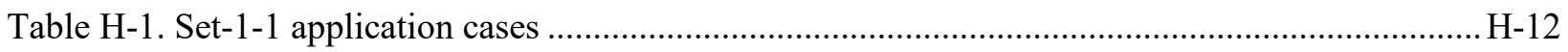

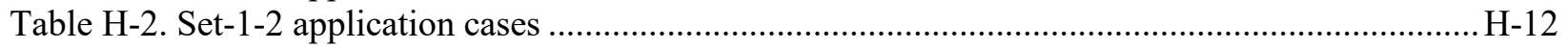

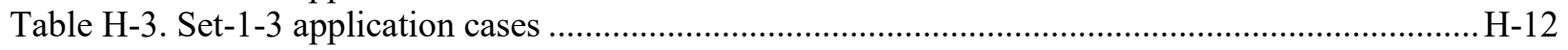

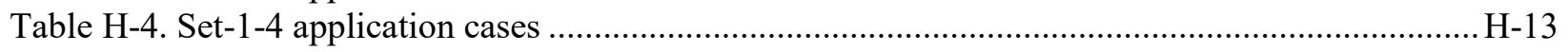

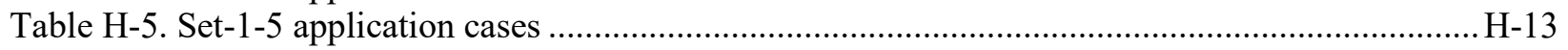

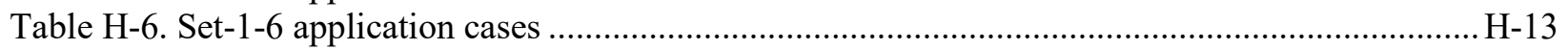

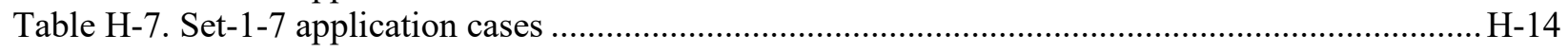

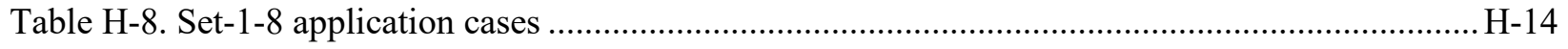

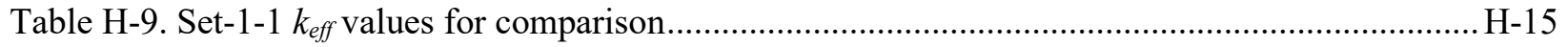

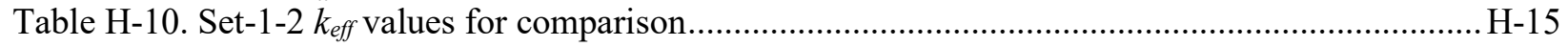

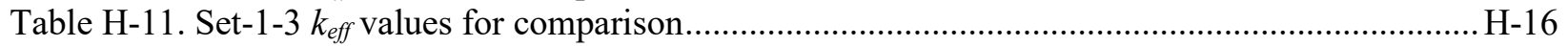

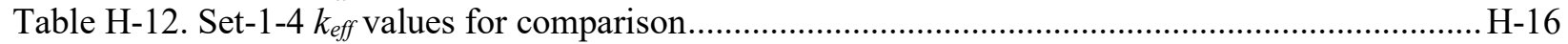

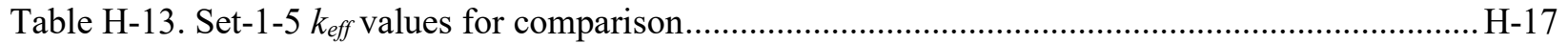

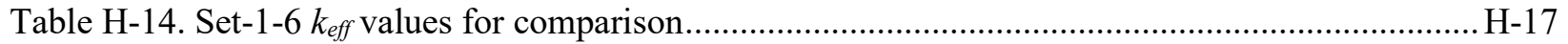

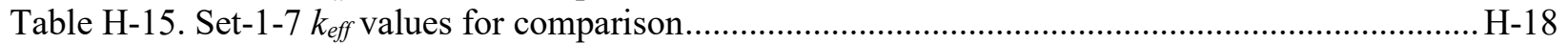

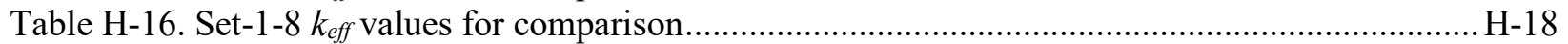

Table H-17. Nuclides with the highest sensitivities for set-1-1 cases.................................................

Table H-18. Nuclides with the highest sensitivities for set-1-2 cases........................................... H-20

Table H-19. Nuclides with the highest sensitivities for set-1-3 cases...................................................21

Table H-20. Nuclides with the highest sensitivities for set-1-4 cases...............................................22

Table H-21. Nuclides with the highest sensitivities for set-1-5 cases.................................................23

Table H-22. Nuclides with the highest sensitivities for set-1-6 cases................................................ 
Table H-23. Nuclides with the highest sensitivities for set-1-7 cases...............................................25

Table H-24. Nuclides with the highest sensitivities for set-1-8 cases.................................................26

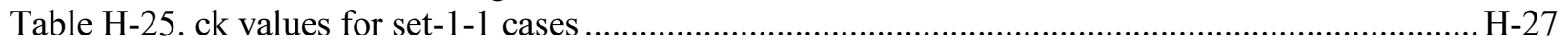

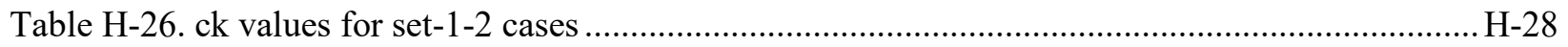

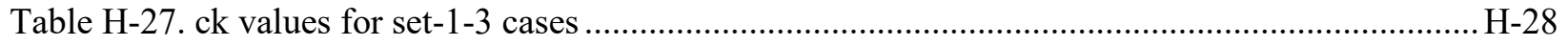

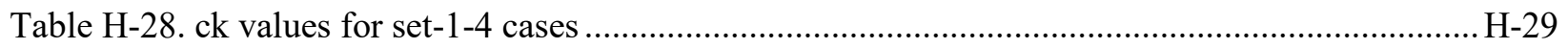

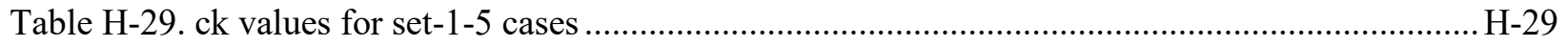

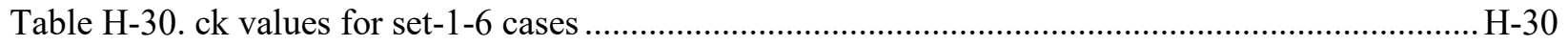

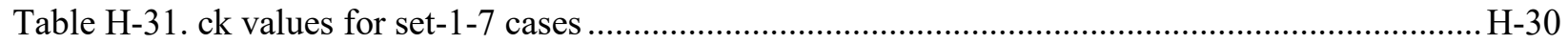

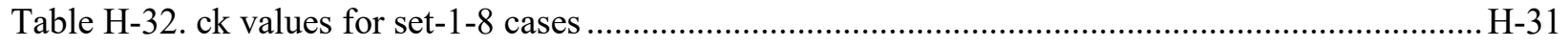

Table H-33. Bias, bias uncertainty, and data-induced uncertainty for set-1-1 cases ............................. H-32

Table H-34. Bias, bias uncertainty, and data-induced uncertainty for set-1-2 cases ..............................

Table H-35. Bias, bias uncertainty, and data-induced uncertainty for set-1-3 cases ..............................33

Table H-36. Bias, bias uncertainty, and data-induced uncertainty for set-1-4 cases ..............................-34

Table H-37. Bias, bias uncertainty, and data-induced uncertainty for set-1-5 cases ..............................-34

Table H-38. Bias, bias uncertainty, and data-induced uncertainty for set-1-6 cases ............................. H-35

Table H-39. Bias, bias uncertainty, and data-induced uncertainty for set-1-7 cases ............................ H-35

Table H-40. Bias, bias uncertainty, and data-induced uncertainty for set-1-8 cases ........................... H-36 


\section{APPENDIX H. CALCULATIONAL VALIDATION}

The calculations for this report were performed using the SCALE code system, version 6.2.4. The Criticality Safety Analysis Sequence (CSAS) with KENO-VI (CSAS6) was used to calculate effective neutron multiplication factors, or k-effective $\left(k_{\text {eff }}\right)$ values, for the various configurations analyzed. As with any computer code or calculation used in relation to safety analyses and assessments, the ability of the calculational methodology to prove a configuration subcritical is obtained through a validation process.

The validation process assesses how well a computational method predicts reality (e.g., whether a system that was calculated to be subcritical is in reality subcritical). Validation of computational methods used in criticality safety analyses is required by consensus standards $[34,35]$. These requirements are derived from the need for operational (notably worker) safety. Post-closure analyses of waste disposal sites do not fall directly under these requirements partly because there are no operations (other than static storage) or personnel presence. Post-closure analyses are also different because of the uncertainty in the waste composition/configuration due to the degradation of the waste and container materials over time. Even if it is not specifically required for post-closure analyses of waste disposal sites, the need for computational validation still exists.

The referenced consensus standards require validation to be conducted through comparisons of computed results with experimental data. Typically, well-documented critical experiments (critical benchmarks) are used for these comparisons. Documented critical experiments can be found in a variety of resources, including the International Handbook of Evaluated Criticality Safety Benchmark Experiments [36]. Ideally, calculational models of critical experiments would calculate results that are exactly equal to experimental results. In reality, calculational results do not exactly match experimental results because of simplifications and approximations made in the computational models to facilitate solutions on computer systems. Furthermore, the nuclear data used may include errors associated with the measurement, evaluation, and/or representation of the data. The validation process provides an understanding of the difference between calculated and experimental results, or bias, and the uncertainty in this difference, or bias uncertainty.

For a validation to yield an appropriate bias and bias uncertainty, the critical experiments used for comparison must be as similar as possible to the application being validated. Critical experiments are arrangements of fissile material and structural materials usually performed to support operational needs and processes. Validation of waste disposal operations can be challenging because of the difficulty in finding experiments similar in nature to waste disposal materials and operations.

The validation results (bias and bias uncertainty) are used to determine an upper subcritical limit (USL). Calculated results (including calculational uncertainty, $k_{\text {eff-calc }}+2 \sigma_{\text {calc }}$ ) below the USL are considered subcritical; results above the USL (even those below 1.0) are not considered to be subcritical. This number could also be considered as the maximum subcritical $k_{\text {eff }}$, referred to here as the MSk.

Determination of the MSk can also include an additional margin of subcriticality to account for dissimilarities between the experiments used and the application and identified gaps in the nuclear data. The MSk can be considered as the magnitude of the sum of the biases, uncertainties, and administrative and/or statistical margins applied to a set of critical benchmarks. Because a positive bias may be nonconservative, all positive biases are set to zero. An allowance to use a positive bias, if the cause of the positive bias is well understood and justified, has been established in ANSI/ANS-8.24-2017 [35], but this is not typical. The MSk can be represented by the following:

$$
\begin{gathered}
\text { MSk }=1.0+\text { bias }- \text { bias uncertainty }- \text { administrative margin } \\
k_{\text {eff-calc }}+2 \sigma_{\text {calc }}<\text { MSk. }
\end{gathered}
$$


Historically, the expected computational bias is established with the use of trending analyses of the bias for the critical experiments as a function of their physical characteristics such as $\mathrm{H} / \mathrm{X}$ or energy of average neutron lethargy causing fission (EALF). The bias uncertainty is then determined through a statistical analysis of the trend, accounting for the uncertainty in each $k_{\text {eff }}$ data point and the distribution of the data. The trending analysis can also be performed with sensitivity/uncertainty $(\mathrm{S} / \mathrm{U})$ tools. The $\mathrm{S} / \mathrm{U}$ tools are used to determine correlation coefficients $-\mathrm{c}_{\mathrm{k}}$ value or $\mathrm{c}(\mathrm{k})$ - for trending analysis. This report uses both $\mathrm{c}(\mathrm{k})$ and EALF values for trending analyses.

For the S/U method, the SCALE S/U analysis sequences, or TSUNAMI, is used. The TSUNAMI methods are based on the premise that the primary source of computational biases are the errors in the cross section data as bounded by their uncertainties, which can be tabulated in cross section covariance data.

TSUNAMI quantifies the predicted change in keff, reaction rates, or the reactivity differences that result from changes in the energy-dependent, nuclide-reaction-specific cross section data, whether CE or MG. The sensitivity data are useful because they indicate the differences in system $k_{\text {eff }}$ that would result from small changes in the underlying nuclear data. The sensitivity data can be used to quantify nuclear data uncertainties and to assess similarity between pairs of systems based on the shared nuclear data-induced uncertainty. This shared data uncertainty is expected to be a strong indicator of applicable benchmark experiments for use in validation of neutron transport methods. The two modules used in this report are TSUNAMI-3D and TSUNAMI-IP, both of which are described in more detail below.

The TSUNAMI-3D sequence is used for 3D cross section sensitivity generation in $\mathrm{S} / \mathrm{U}$ analysis. The sequence provides automated processing of material input and cross section data, neutron transport, calculation of sensitivity coefficients (i.e., sensitivity of $k_{\text {eff }}$ to nuclear data variation), and determination of uncertainty in $k_{\text {eff }}$ caused by cross section covariances. Sensitivities based on the fluxes calculated by KENO are written to a sensitivity data file (SDF) containing the nuclide-, energy-, and reaction-dependent $k_{\text {eff }}$ sensitivity coefficients. These energy-dependent sensitivities are determined for each nuclide in the model using first-order perturbation theory. SCALE 6.2.4 can generate sensitivity data using either CE or MG methods, but only MG calculations are used or reported in this work. Further details of the MG sensitivity calculation methodologies are available in Section 6 of the SCALE 6.2.4 manual [37].

TSUNAMI-IP is used to evaluate the similarity of critical experiments and application models and to determine uncertainties in system reactivity due to cross section covariance data. The similarity metric calculated here is $\mathrm{c}_{\mathrm{k}}$, which is the correlation coefficient of the effect of nuclear data uncertainty on $k_{\text {eff }}$ of the application and experiment. $c_{\mathrm{k}}$ can be determined by dividing the covariance between the experiment and application by the product of the uncertainties in the experiment and the application [5], as shown in Eq. (2),

$$
\mathrm{c}_{\mathrm{k}}=\frac{\sigma_{\mathrm{AppExp}}^{2}}{\sigma_{\mathrm{App}} \sigma_{\mathrm{Exp}}},
$$

where: $c_{k}$ is the similarity between an application and an experiment, $\sigma^{2}$ AppExp is the covariance between the application and the experiment, $\sigma_{\mathrm{App}}$ is the uncertainty in the application $k_{\text {eff }}$ resulting from cross section covariances (uncertainties), and $\sigma_{\text {Exp }}$ is the uncertainty in the experiment $k_{\text {eff }}$ caused by cross section covariances (uncertainties).

In essence, $c_{\mathrm{k}}$ is the fraction of the cross section-induced uncertainty in $k_{\mathrm{eff}}$ that is shared by two systems. A $c_{\mathrm{k}}$ value of 1 indicates that the $k_{\text {eff }}$ values for two compared systems would be affected identically by 
nuclear data errors, which are the primary contributors to the computational method's bias. Based on the assumption that computational biases are due primarily to nuclear data errors and that the nuclear data uncertainty values should indicate the potential for such nuclear data errors, two highly correlated systems should exhibit the same computational bias. A $c_{k}$ value $\geq 0.8$ is considered to have a high enough degree of similarity to be acceptable for use in validation studies [38]; this value is used as the cutoff for the acceptably similar experiments identified below.

TSUNAMI-IP can also generate additional inputs to be used with the Upper Subcritical Limit Statistics (USLSTATS) program, a statistical analysis program distributed with SCALE. USLSTATS can then be used to perform a trending analysis on the $\mathrm{c}_{\mathrm{k}}$ value (or any other parameter suitable for trending analysis, e.g., EALF) to calculate a bias and bias uncertainty.

\section{H.1 SENSITIVITY COEFFICIENT GENERATION}

The TSUNAMI-3D sequence discussed above was used to generate sensitivity data for several cases ("application" cases). Cases from the uniform array parameter studies were chosen to represent the different material/container configurations modeled in this report. All cases had $k_{\text {eff }}$ values close to 1.0, or slightly lower in similar material configuations. Limiting analysis conditions were used because the generation of SDFs requires significantly greater computational resources than the calculations of $k_{\text {eff. }}$ The MG TSUNAMI method is used, which is consistent with the use of MG data in the analyses.

The uniform array models were large arrays, and the calculations were run with multigroup cross sections. Running multigroup TSUNAMI-3D to generate sensitivity data files with these cases as-is required the definition of a grid mesh, and it also required an extremely large amount of computer memory, taking up an extremely large computer footprint. The finer the grid mesh, the larger the computer footprint. Direct perturbation calculations were used to verify the TSUNAMI-3D-generated sensitivities, which indicated the mesh size necessary to generate valid sensitivity data, required a memory footprint not available on the current computer system in the necessary time frame. Therefore, the cases were modified by decreasing the length of the room modeled and using mirror boundaries on the positive and negative $y$ faces. Figure $\mathrm{H}-1$ shows an example top view for these configurations.

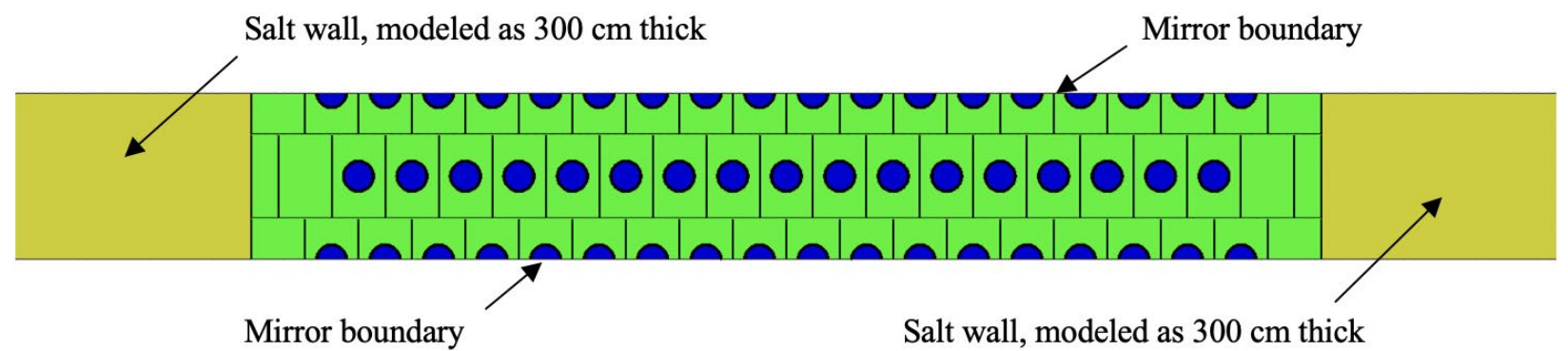

Figure H-1. Top view of modified configuration.

\section{H.2 SELECTION OF APPLICATION CASES}

The validation here considered only set- 1 cases: specifically set-1-1, set-1-2, set-1-3, set-1-4, set-1-5, set1-6, set-1-7, and set 1-8. The results were plotted by subset, as shown in Figures H-2 through H-9, to determine bounding cases to use as the application cases. The cases including Be were not included in the plots. For each set, the cases adding filler material and/or can material decrease the system $k_{\text {eff. }}$ The more material added, the larger the decrease. Cases chosen as application cases included those with no filler or can material, as well as cases with $2,000 \mathrm{~g}$ filler material and/or $500 \mathrm{~g}$ can material. The same cases including Be were also chosen. Tables $\mathrm{H}-1$ through $\mathrm{H}-8$ list the application cases. 


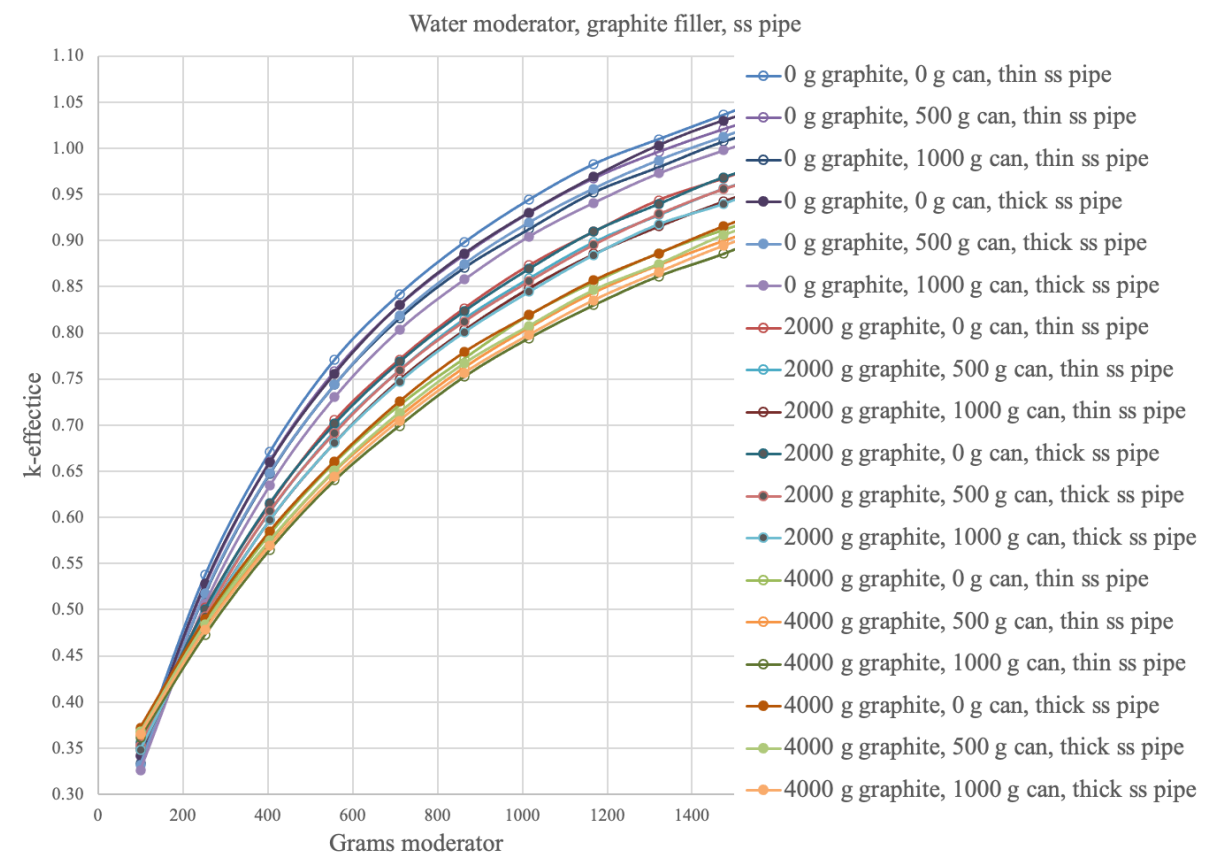

Figure H-2. Set-1-1 cases, water moderator, graphite filler, stainless steel pipe.

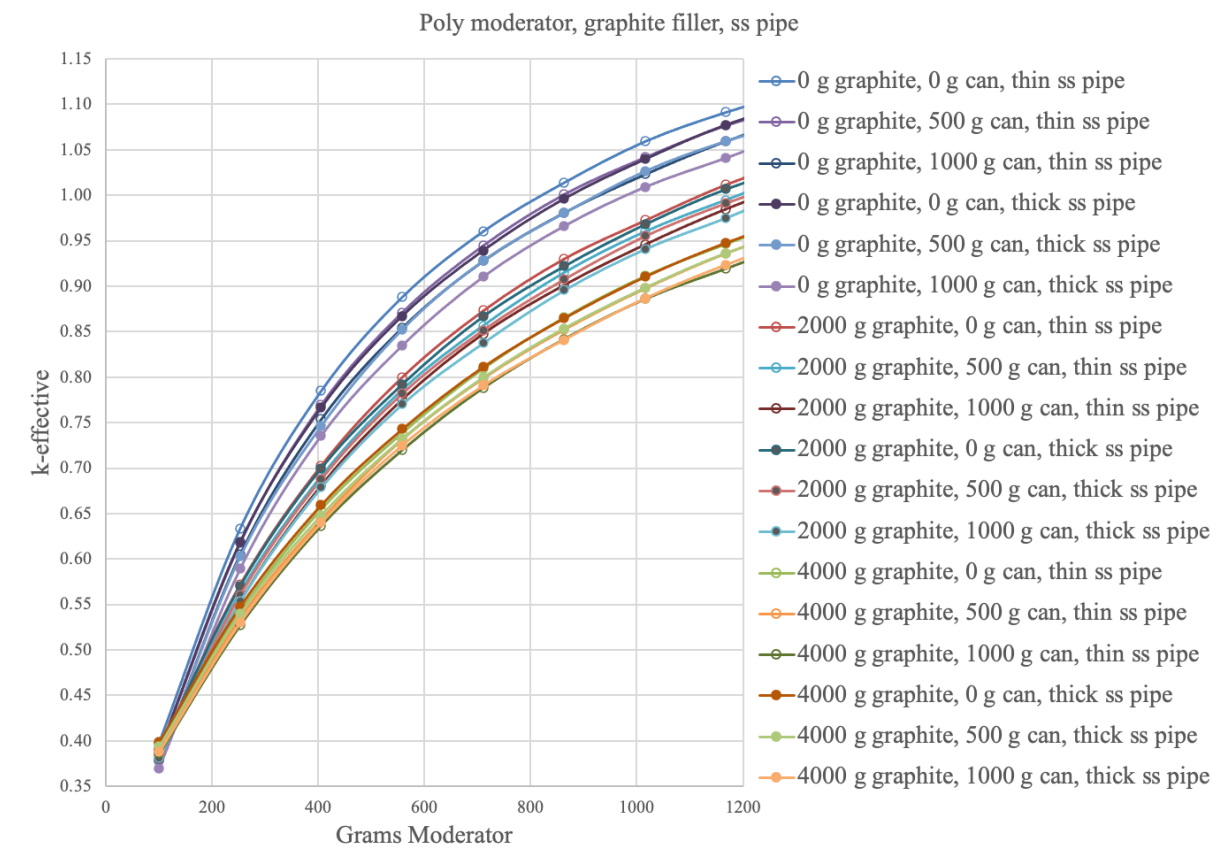

Figure H-3. Set-1-2 cases, poly moderator, graphite filler, stainless steel pipe. 


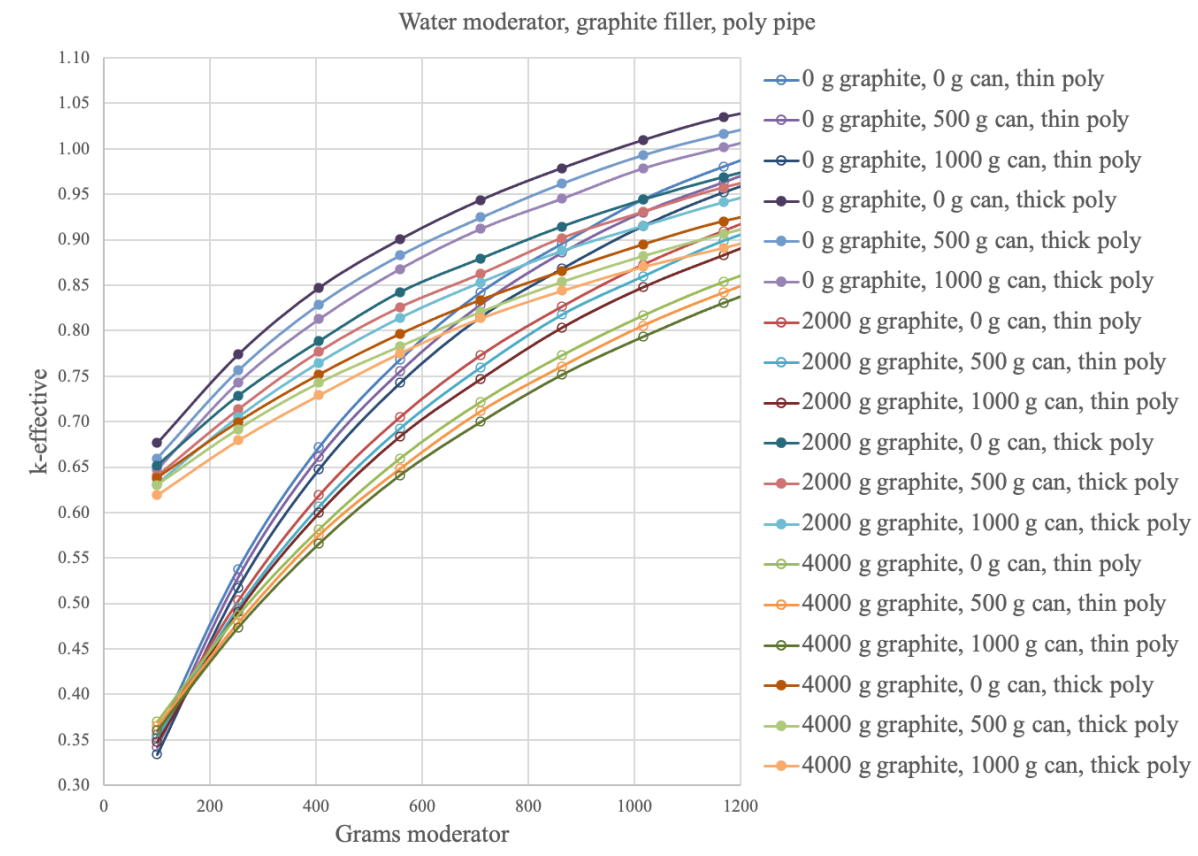

Figure H-4. Set-1-3 cases, water moderator, graphite filler, poly pipe.

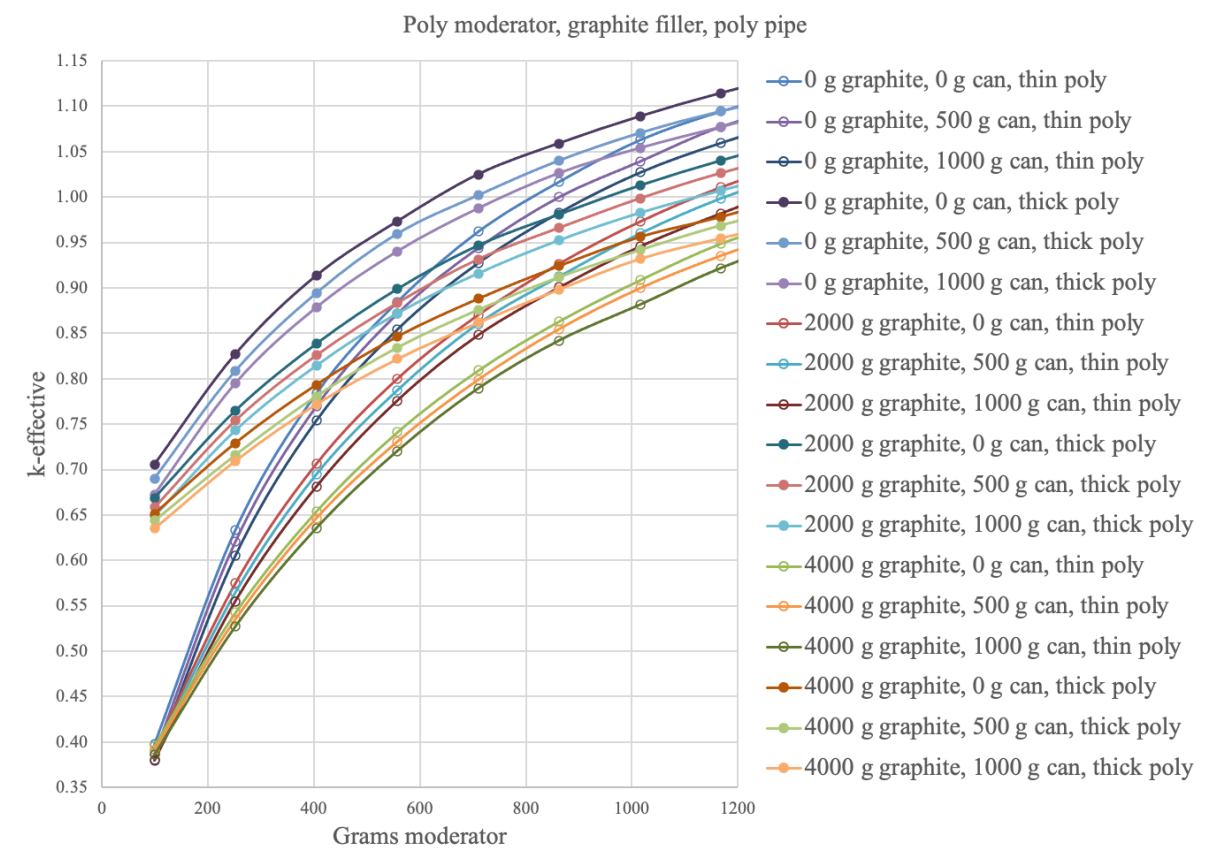

Figure H-5. Set-1-4 cases, poly moderator, graphite filler, poly pipe. 


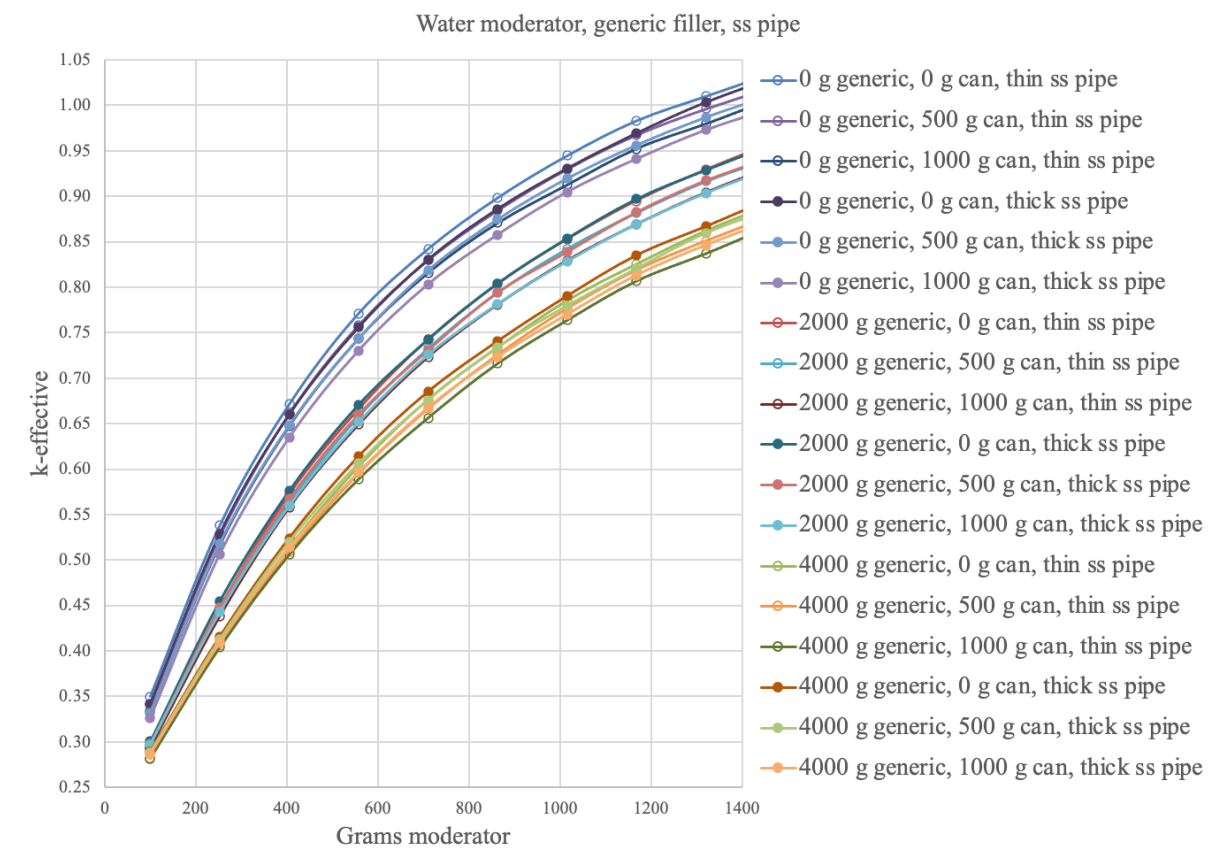

Figure H-6. Set-1-5 cases, water moderator, generic filler, stainless steel pipe.

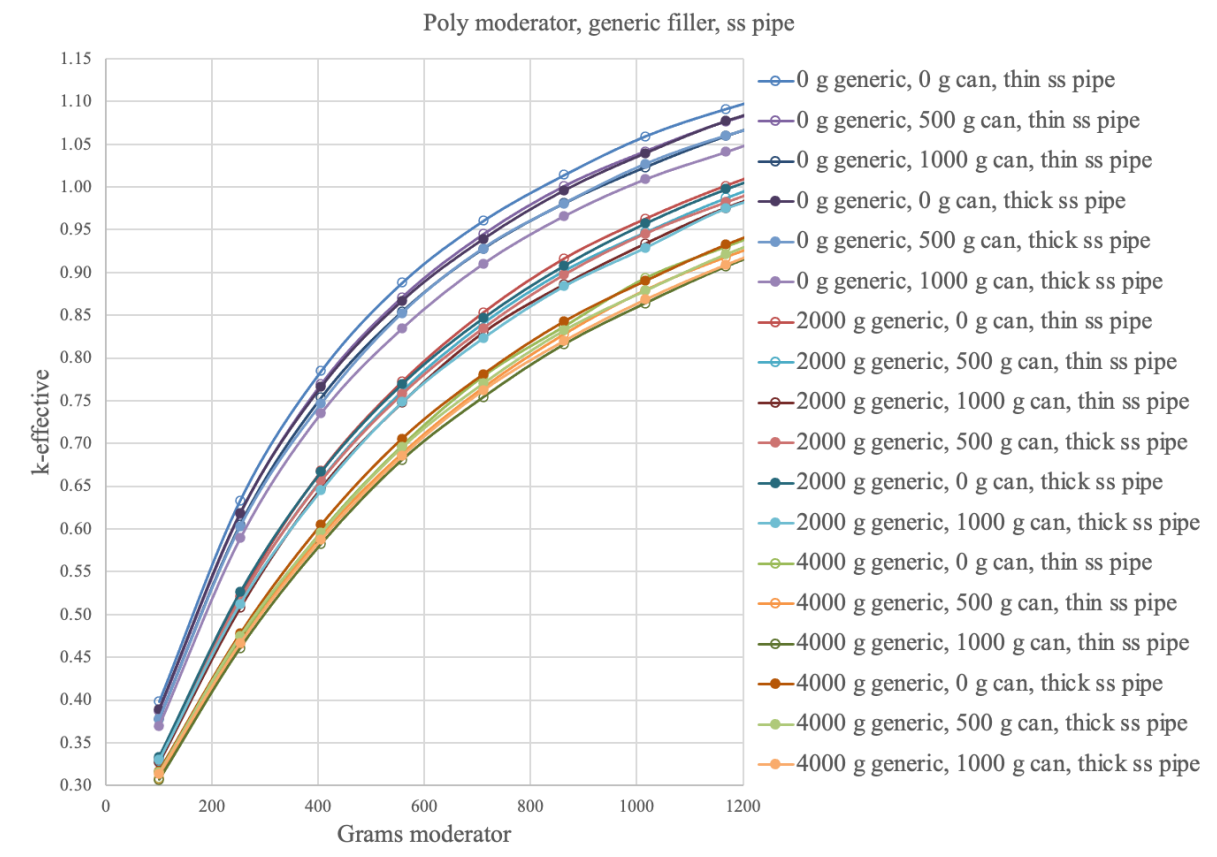

Figure H-7. Set-1-6 cases, poly moderator, generic filler, stainless steel pipe. 
Water moderator, generic filler, poly pipe

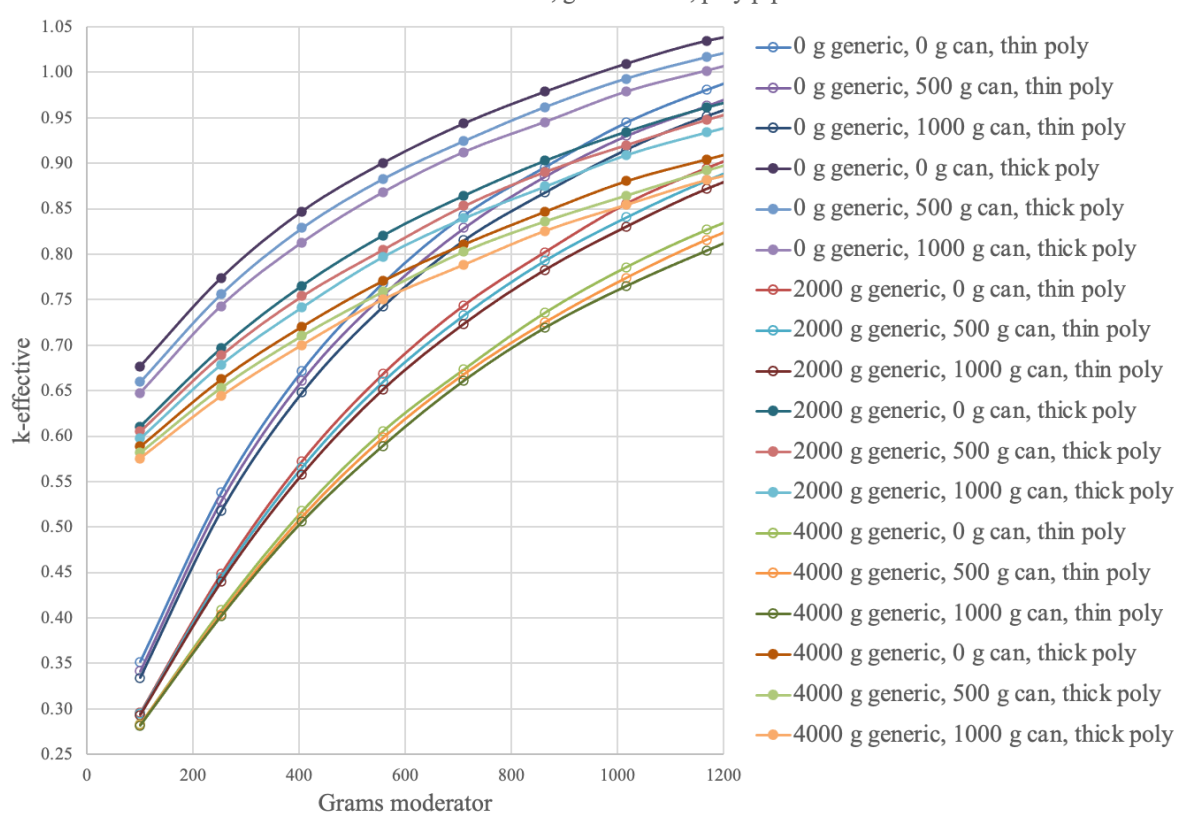

Figure H-8. Set-1-7 cases, water moderator, generic filler, poly pipe.

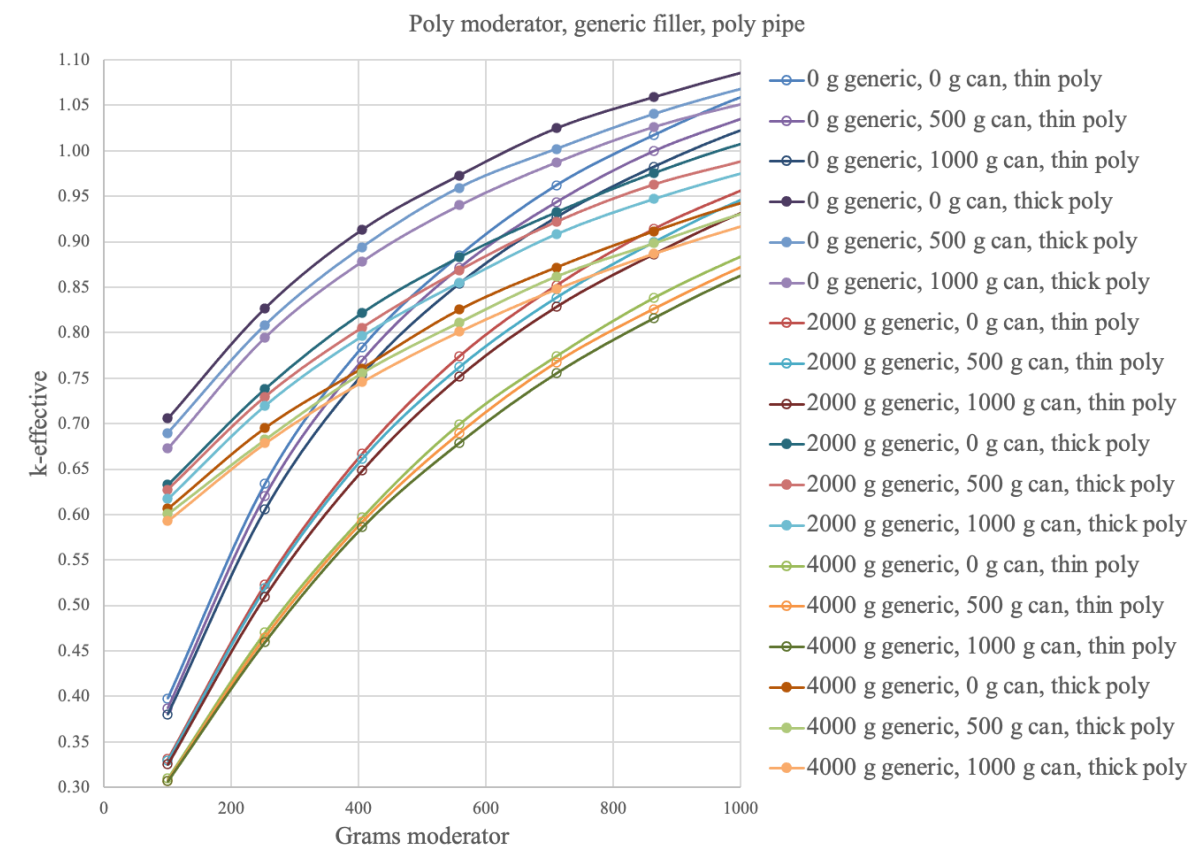

Figure H-9. Set-1-8 cases, poly moderator, generic filler, poly pipe. 
Table H-1. Set-1-1 application cases

\begin{tabular}{|c|c|c|c|c|c|c|c|c|}
\hline \multirow{2}{*}{ Case } & \multirow{2}{*}{ Short name } & \multicolumn{2}{|c|}{ Moderator } & \multicolumn{2}{|c|}{ Filler } & Can & \multicolumn{2}{|c|}{ Pipe Material } \\
\hline & & & grams & & grams & grams & & $\mathrm{cm}$ \\
\hline set-1-1-uac1_uh_m1_graphite_cyl_ps_1000_yr_para_00657 & set-1-1-00657 & \multirow{8}{*}{$\mathrm{H}_{2} \mathrm{O}$} & \multirow{8}{*}{1016} & \multirow{4}{*}{\multicolumn{2}{|c|}{ granhite }} & \multirow{2}{*}{0} & \multirow{8}{*}{ ss } & 0.0010 \\
\hline set-1-1-uac1_uh_m1_graphite_cyl_ps_1000_yr_para_00659 & set-1-1-00659 & & & & & & & 0.7112 \\
\hline set-1-1-uac1_uh_m1_graphite_cyl_ps_1000_yr_para_00669 & set-1-1-00669 & & & & & \multirow{2}{*}{500} & & 0.0010 \\
\hline set-1-1-uac1_uh_m1_graphite_cyl_ps_1000_yr_para_00671 & set-1-1-00671 & & & & & & & 0.7112 \\
\hline set-1-1-uac1_uh_m1_graphite_cyl_ps_1000_yr_para_00693 & set-1-1-00693 & & & \multirow{4}{*}{ graphite } & \multirow{4}{*}{2,000} & \multirow{2}{*}{0} & & 0.0010 \\
\hline set-1-1-uac1_uh_m1_graphite_cyl_ps_1000_yr_para_00695 & set-1-1-00695 & & & & & & & 0.7112 \\
\hline set-1-1-uac1_uh_m1_graphite_cyl_ps_1000_yr_para_00705 & set-1-1-00705 & & & & & \multirow{2}{*}{500} & & 0.0010 \\
\hline set-1-1-uac1 uh m1 graphite cyl ps 1000 yr para 00707 & set-1-1-00707 & & & & & & & 0.7112 \\
\hline \multicolumn{9}{|l|}{ With $\mathrm{Be}$} \\
\hline set-1-1-uac1_uh_m1_graphite_cyl_ps_1000_yr_para_00658 & set-1-1-00658 & \multirow{8}{*}{$\mathrm{H}_{2} \mathrm{O}$} & \multirow{8}{*}{1016} & \multirow{8}{*}{ graphite } & \multirow{4}{*}{0} & \multirow{2}{*}{0} & \multirow{8}{*}{ ss } & 0.0010 \\
\hline set-1-1-uac1_uh_m1_graphite_cyl_ps_1000_yr_para_00660 & set-1-1-00660 & & & & & & & 0.7112 \\
\hline set-1-1-uac1_uh_m1_graphite_cyl_ps_1000_yr_para_00670 & set-1-1-00670 & & & & & \multirow{2}{*}{500} & & 0.0010 \\
\hline set-1-1-uac1_uh_m1_graphite_cyl_ps_1000_yr_para_00672 & set-1-1-00672 & & & & & & & 0.7112 \\
\hline set-1-1-uac1_uh_m1_graphite_cyl_ps_1000_yr_para_00694 & set-1-1-00694 & & & & \multirow{4}{*}{2,000} & \multirow{2}{*}{0} & & 0.0010 \\
\hline set-1-1-uac1_uh_m1_graphite_cyl_ps_1000_yr_para_00696 & set-1-1-00696 & & & & & & & 0.7112 \\
\hline set-1-1-uac1_uh_m1_graphite_cyl_ps_1000_yr_para_00706 & set-1-1-00706 & & & & & \multirow{2}{*}{500} & & 0.0010 \\
\hline set-1-1-uac1 uh m1 graphite cyl ps 1000 yr para 00708 & set-1-1-00708 & & & & & & & 0.7112 \\
\hline
\end{tabular}

Table H-2. Set-1-2 application cases

\begin{tabular}{|c|c|c|c|c|c|c|c|c|}
\hline \multirow{2}{*}{ Case } & \multirow{2}{*}{ Short name } & \multicolumn{2}{|c|}{ Moderator } & \multicolumn{2}{|c|}{ Filler } & Can & \multicolumn{2}{|c|}{ Pipe Material } \\
\hline & & & grams & & grams & grams & & $\mathrm{cm}$ \\
\hline set-1-2-uac1_uh_m2_graphite_cyl_ps_1000_yr_para_00441 & set-1-2-00441 & \multirow{8}{*}{$\mathrm{CH}_{2}$} & \multirow{8}{*}{710.5} & \multirow{4}{*}{\multicolumn{2}{|c|}{ 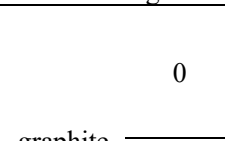 }} & 0 & \multirow{8}{*}{ ss } & 0.0010 \\
\hline set-1-2-uac1_uh_m2_graphite_cyl_ps_1000_yr_para_00443 & set-1-2-00443 & & & & & 0 & & 0.7112 \\
\hline set-1-2-uac1_uh_m2_graphite_cyl_ps_1000_yr_para_00453 & set-1-2-00453 & & & & & & & 0.0010 \\
\hline set-1-2-uac1_uh_m2_graphite_cyl_ps_1000_yr_para_00455 & set-1-2-00455 & & & & & 500 & & 0.7112 \\
\hline set-1-2-uac1_uh_m2_graphite_cyl_ps_1000_yr_para_00477 & set-1-2-00477 & & & \multirow{4}{*}{ hite } & \multirow{4}{*}{2,000} & & & 0.0010 \\
\hline set-1-2-uac1_uh_m2_graphite_cyl_ps_1000_yr_para_00479 & set-1-2-00479 & & & & & 0 & & 0.7112 \\
\hline set-1-2-uac1_uh_m2_graphite_cyl_ps_1000_yr_para_00489 & set-1-2-00489 & & & & & 500 & & 0.0010 \\
\hline set-1-2-uac1_uh_m2 graphite cyl_ps 1000 yr para 00491 & set-1-2-00491 & & & & & 500 & & 0.7112 \\
\hline \multicolumn{9}{|l|}{ (2) } \\
\hline set-1-2-uac1_uh_m2_graphite_cyl_ps_1000_yr_para_00442 & set-1-2-00442 & \multirow{8}{*}{$\mathrm{CH}_{2}$} & \multirow{8}{*}{710.5} & \multirow{8}{*}{ graphite } & \multirow{4}{*}{0} & \multirow{2}{*}{0} & \multirow{8}{*}{ ss } & 0.0010 \\
\hline set-1-2-uac1_uh_m2_graphite_cyl_ps_1000_yr_para_00444 & set-1-2-00444 & & & & & & & 0.7112 \\
\hline set-1-2-uac1_uh_m2_graphite_cyl_ps_1000_yr_para_00454 & set-1-2-00454 & & & & & 500 & & 0.0010 \\
\hline set-1-2-uac1_uh_m2_graphite_cyl_ps_1000_yr_para_00456 & set-1-2-00456 & & & & & 500 & & 0.7112 \\
\hline set-1-2-uac1_uh_m2_graphite_cyl_ps_1000_yr_para_00478 & set-1-2-00478 & & & & \multirow{4}{*}{2,000} & 0 & & 0.0010 \\
\hline set-1-2-uac1_uh_m2_graphite_cyl_ps_1000_yr_para_00480 & set-1-2-00480 & & & & & 0 & & 0.7112 \\
\hline set-1-2-uac1_uh_m2_graphite_cyl_ps_1000_yr_para_00490 & set-1-2-00490 & & & & & 500 & & 0.0010 \\
\hline set-1-2-uac1_uh_m2 graphite cyl_ps 1000 yr para 00492 & set-1-2-00492 & & & & & 500 & & 0.7112 \\
\hline
\end{tabular}

Table H-3. Set-1-3 application cases

\begin{tabular}{|c|c|c|c|c|c|c|c|}
\hline \multirow{2}{*}{ Case } & \multirow{2}{*}{ Short name } & \multicolumn{2}{|c|}{ Moderator } & Filler & Can & \multicolumn{2}{|c|}{ Pipe Material } \\
\hline & & & grams & grams & grams & & $\mathrm{cm}$ \\
\hline set-1-3-uac1_uh_m1_graphite_cyl_pp_1000_yr_para_00441 & set-1-3-00441 & \multirow{8}{*}{$\mathrm{H}_{2} \mathrm{O}$} & \multirow{8}{*}{710.5 graphite } & \multirow{4}{*}{0} & \multirow{2}{*}{0} & \multirow{8}{*}{ poly } & 0.0010 \\
\hline set-1-3-uac1_uh_m1_graphite_cyl_pp_1000_yr_para_00443 & set-1-3-00443 & & & & & & 0.7112 \\
\hline set-1-3-uac1 uh m1 graphite cyl pp 1000 yr para 00453 & set-1-3-00453 & & & & \multirow{2}{*}{500} & & 0.0010 \\
\hline set-1-3-uac1_uh_m1_graphite_cyl_pp_1000_yr_para_00455 & set-1-3-00455 & & & & & & 0.7112 \\
\hline set-1-3-uac1_uh_m1_graphite_cyl_pp_1000_yr_para_00477 & set-1-3-00477 & & & \multirow{4}{*}{2,000} & \multirow{2}{*}{0} & & 0.0010 \\
\hline set-1-3-uac1_uh_m1_graphite_cyl_pp_1000_yr_para_00479 & set-1-3-00479 & & & & & & 0.7112 \\
\hline set-1-3-uac1_uh_m1_graphite_cyl_pp_1000_yr_para_00489 & set-1-3-00489 & & & & \multirow{2}{*}{500} & & 0.0010 \\
\hline set-1-3-uac1 uh m1 graphite cyl pp 1000 yr para 00491 & set-1-3-00491 & & & & & & 0.7112 \\
\hline \multicolumn{8}{|l|}{ With Be } \\
\hline set-1-3-uac1_uh_m1_graphite_cyl_pp_1000_yr_para_00442 & set-1-3-00442 & \multirow{8}{*}{$\mathrm{H}_{2} \mathrm{O}$} & \multirow{8}{*}{710.5 graphite } & \multirow{4}{*}{0} & \multirow{2}{*}{0} & \multirow{8}{*}{ poly } & 0.0010 \\
\hline set-1-3-uac1_uh_m1_graphite_cyl_pp_1000_yr_para_00444 & set-1-3-00444 & & & & & & 0.7112 \\
\hline set-1-3-uac1_uh_m1_graphite_cyl_pp_1000_yr_para_00454 & set-1-3-00454 & & & & \multirow{2}{*}{500} & & 0.0010 \\
\hline set-1-3-uac1_uh_m1_graphite_cyl_pp_1000_yr_para_00456 & set-1-3-00456 & & & & & & 0.7112 \\
\hline set-1-3-uac1_uh_m1_graphite_cyl_pp_1000_yr_para_00478 & set-1-3-00478 & & & \multirow{4}{*}{2,000} & \multirow{2}{*}{0} & & 0.0010 \\
\hline set-1-3-uac1_uh_m1_graphite_cyl_pp_1000_yr_para_00480 & set-1-3-00480 & & & & & & 0.7112 \\
\hline set-1-3-uac1_uh_m1_graphite_cyl_pp_1000_yr_para_00490 & set-1-3-00490 & & & & \multirow{2}{*}{500} & & 0.0010 \\
\hline set-1-3-uac1 uh m1 graphite cyl pp 1000 yr para 00492 & set-1-3-00492 & & & & & & 0.7112 \\
\hline
\end{tabular}


Table H-4. Set-1-4 application cases

\begin{tabular}{|c|c|c|c|c|c|c|c|c|}
\hline \multirow{2}{*}{ Case } & \multirow{2}{*}{ Short name } & \multicolumn{2}{|c|}{ Moderator } & \multicolumn{2}{|c|}{ Filler } & Can & \multicolumn{2}{|c|}{ Pipe Material } \\
\hline & & & grams & & grams & grams & & $\mathrm{cm}$ \\
\hline set-1-4-uac1_uh_m2_graphite_cyl_pp_1000_yr_para_00333 & set-1-4-00333 & \multirow{8}{*}{$\mathrm{CH}_{2}$} & \multirow{8}{*}{557.9} & \multirow{8}{*}{ graphite } & \multirow{4}{*}{0} & \multirow[b]{2}{*}{0} & \multirow{8}{*}{ poly } & 0.0010 \\
\hline set-1-4-uac1_uh_m2_graphite_cyl_pp_1000_yr_para_00335 & set-1-4-00335 & & & & & & & 0.7112 \\
\hline set-1-4-uac1_uh_m2_graphite_cyl_pp_1000_yr_para_00345 & set-1-4-00345 & & & & & & & 0.0010 \\
\hline set-1-4-uac1_uh_m2_graphite_cyl_pp_1000_yr_para_00347 & set-1-4-00347 & & & & & 500 & & 0.7112 \\
\hline set-1-4-uac1_uh_m2_graphite_cyl_pp_1000_yr_para_00369 & set-1-4-00369 & & & & \multirow{4}{*}{2,000} & 0 & & 0.0010 \\
\hline set-1-4-uac1_uh_m2_graphite_cyl_pp_1000_yr_para_00371 & set-1-4-00371 & & & & & 0 & & 0.7112 \\
\hline set-1-4-uac1_uh_m2_graphite_cyl_pp_1000_yr_para_00381 & set-1-4-00381 & & & & & & & 0.0010 \\
\hline set-1-4-uac1 uh $\mathrm{m} 2$ graphite cyl pp 1000 yr para 00383 & set-1-4-00383 & & & & & 500 & & 0.7112 \\
\hline \multicolumn{9}{|l|}{ With Be } \\
\hline set-1-4-uac1 uh m2 graphite cyl pp 1000 yr para 00334 & set- & \multirow{8}{*}{$\mathrm{CH}_{2}$} & \multirow{8}{*}{557.9} & \multirow{8}{*}{ graphite } & \multirow{4}{*}{0} & \multirow{2}{*}{0} & \multirow{8}{*}{ poly } & 0.0010 \\
\hline set-1-4-uac1_uh_m2_graphite_cyl_pp_1000_yr_para_00336 & set-1-4-00336 & & & & & & & 0.7112 \\
\hline set-1-4-uac1_uh_m2_graphite_cyl_pp_1000_yr_para_00346 & set-1-4-00346 & & & & & \multirow{2}{*}{500} & & 0.0010 \\
\hline set-1-4-uac1_uh_m2_graphite_cyl_pp_1000_yr_para_00348 & set-1-4-00348 & & & & & & & 0.7112 \\
\hline set-1-4-uac1_uh_m2_graphite_cyl_pp_1000_yr_para_00370 & set-1-4-00370 & & & & \multirow{4}{*}{2,000} & 0 & & 0.0010 \\
\hline set-1-4-uac1_uh_m2_graphite_cyl_pp_1000_yr_para_00372 & set-1-4-00372 & & & & & 0 & & 0.7112 \\
\hline set-1-4-uac1_uh_m2_graphite_cyl_pp_1000_yr_para_00382 & set-1-4-00382 & & & & & 500 & & 0.0010 \\
\hline set-1-4-uac1_uh_m2 graphite cyl pp 1000 yr_para 00384 & set-1-4-00384 & & & & & 500 & & 0.7112 \\
\hline
\end{tabular}

Table H-5. Set-1-5 application cases

\begin{tabular}{|c|c|c|c|c|c|c|c|c|}
\hline \multirow{2}{*}{ Case } & \multirow{2}{*}{ Short name } & \multicolumn{2}{|c|}{ Moderator } & \multicolumn{2}{|c|}{ Filler } & Can & \multicolumn{2}{|c|}{ Pipe Material } \\
\hline & & & grams & & grams & grams & & $\mathrm{cm}$ \\
\hline set-1-5-uac1_uh_m1_generic_cyl_ps_1000_yr_para_00657 & set-1-5-00657 & \multirow{8}{*}{$\mathrm{H}_{2} \mathrm{O}$} & \multirow{8}{*}{1016} & \multirow{4}{*}{\multicolumn{2}{|c|}{ seneris }} & 0 & \multirow{8}{*}{ ss } & 0.0010 \\
\hline set-1-5-uac1_uh_m1_generic_cyl_ps_1000_yr_para_00659 & set-1-5-00659 & & & & & 0 & & 0.7112 \\
\hline set-1-5-uac1_uh_m1_generic_cyl_ps_1000_yr_para_00669 & set-1-5-00669 & & & & & & & 0.0010 \\
\hline set-1-5-uac1_uh_m1_generic_cyl_ps_1000_yr_para_00671 & set-1-5-00671 & & & & & 500 & & 0.7112 \\
\hline set-1-5-uac1_uh_m1_generic_cyl_ps_1000_yr_para_00693 & set-1-5-00693 & & & \multirow{4}{*}{ generic } & \multirow{4}{*}{2,000} & & & 0.0010 \\
\hline set-1-5-uac1_uh_m1_generic_cyl_ps_1000_yr_para_00695 & set-1-5-00695 & & & & & 0 & & 0.7112 \\
\hline set-1-5-uac1_uh_m1_generic_cyl_ps_1000_yr_para_00705 & set-1-5-00705 & & & & & 500 & & 0.0010 \\
\hline set-1-5-uac1_uh_m1_generic_cyl_ps 1000 yr para_00707 & set-1-5-00707 & & & & & 500 & & 0.7112 \\
\hline \multicolumn{9}{|l|}{ With Be } \\
\hline set-1-5-uac1_uh_m1_generic_cyl_ps_1000_yr_para_00658 & set-1-5-00658 & \multirow{8}{*}{$\mathrm{H}_{2} \mathrm{O}$} & \multirow{8}{*}{1016} & \multirow{8}{*}{ generic } & \multirow{4}{*}{0} & \multirow{2}{*}{0} & \multirow{8}{*}{ ss } & 0.0010 \\
\hline set-1-5-uac1_uh_m1_generic_cyl_ps_1000_yr_para_00660 & set-1-5-00660 & & & & & & & 0.7112 \\
\hline set-1-5-uac1_uh_m1_generic_cyl_ps_1000_yr_para_00670 & set-1-5-00670 & & & & & 500 & & 0.0010 \\
\hline set-1-5-uac1_uh_m1_generic_cyl_ps_1000_yr_para_00672 & set-1-5-00672 & & & & & 500 & & 0.7112 \\
\hline set-1-5-uac1_uh_m1_generic_cyl_ps_1000_yr_para_00694 & set-1-5-00694 & & & & \multirow{4}{*}{2,000} & 0 & & 0.0010 \\
\hline set-1-5-uac1_uh_m1_generic_cyl_ps_1000_yr_para_00696 & set-1-5-00696 & & & & & 0 & & 0.7112 \\
\hline set-1-5-uac1_uh_m1_generic_cyl_ps_1000_yr_para_00706 & set-1-5-00706 & & & & & 500 & & 0.0010 \\
\hline set-1-5-uac1_uh_m1_generic_cyl_ps 1000 yr para_00708 & set-1-5-00708 & & & & & 500 & & 0.7112 \\
\hline
\end{tabular}

Table H-6. Set-1-6 application cases

\begin{tabular}{|c|c|c|c|c|c|c|c|c|}
\hline \multirow{2}{*}{ Case } & \multirow{2}{*}{ Short name } & \multicolumn{2}{|c|}{ Moderator } & \multicolumn{2}{|c|}{ Filler } & Can & \multicolumn{2}{|c|}{ Pipe Material } \\
\hline & & & grams & & grams & grams & & $\mathrm{cm}$ \\
\hline set-1-6-uac1_uh_m2_generic_cyl_ps_1000_yr_para_00441 & set-1-6-00441 & \multirow{8}{*}{$\mathrm{CH}_{2}$} & \multirow{8}{*}{710.5} & \multirow{8}{*}{ generic } & \multirow{4}{*}{0} & \multirow[b]{2}{*}{0} & \multirow{8}{*}{ ss } & 0.0010 \\
\hline set-1-6-uac1_uh_m2_generic_cyl_ps_1000_yr_para_00443 & set-1-6-00443 & & & & & & & 0.7112 \\
\hline set-1-6-uac1_uh_m2_generic_cyl_ps_1000_yr_para_00453 & set-1-6-00453 & & & & & & & 0.0010 \\
\hline set-1-6-uac1_uh_m2_generic_cyl_ps_1000_yr_para_00455 & set-1-6-00455 & & & & & 500 & & 0.7112 \\
\hline set-1-6-uac1_uh_m2_generic_cyl_ps_1000_yr_para_00477 & set-1-6-00477 & & & & \multirow{4}{*}{2,000} & & & 0.0010 \\
\hline set-1-6-uac1_uh_m2_generic_cyl_ps_1000_yr_para_00479 & set-1-6-00479 & & & & & 0 & & 0.7112 \\
\hline set-1-6-uac1_uh_m2_generic_cyl_ps_1000_yr_para_00489 & set-1-6-00489 & & & & & & & 0.0010 \\
\hline set-1-6-uac1 uh m2 generic cyl ps 1000 yr para 00491 & set-1-6-00491 & & & & & 500 & & 0.7112 \\
\hline \multicolumn{9}{|l|}{ With Be } \\
\hline set-1-6-uac1_uh_m2_generic_cyl_ps_1000_yr_para_00442 & set-1-6-00442 & \multirow{8}{*}{$\mathrm{CH}_{2}$} & \multirow{8}{*}{710.5} & \multirow{8}{*}{ generic } & \multirow{4}{*}{0} & \multirow[b]{2}{*}{0} & \multirow{8}{*}{ ss } & 0.0010 \\
\hline set-1-6-uac1_uh_m2_generic_cyl_ps_1000_yr_para_00444 & set-1-6-00444 & & & & & & & 0.7112 \\
\hline set-1-6-uac1_uh_m2_generic_cyl_ps_1000_yr_para_00454 & set-1-6-00454 & & & & & & & 0.0010 \\
\hline set-1-6-uac1_uh_m2_generic_cyl_ps_1000_yr_para_00456 & set-1-6-00456 & & & & & 500 & & 0.7112 \\
\hline set-1-6-uac1_uh_m2_generic_cyl_ps_1000_yr_para_00478 & set-1-6-00478 & & & & \multirow{4}{*}{2,000} & 0 & & 0.0010 \\
\hline set-1-6-uac1_uh_m2_generic_cyl_ps_1000_yr_para_00480 & set-1-6-00480 & & & & & 0 & & 0.7112 \\
\hline set-1-6-uac1_uh_m2_generic_cyl_ps_1000_yr_para_00490 & set-1-6-00490 & & & & & & & 0.0010 \\
\hline set-1-6-uac1 uh $\mathrm{m} 2$ generic cyl ps 1000 yr para 00492 & set-1-6-00492 & & & & & 500 & & 0.7112 \\
\hline
\end{tabular}


Table H-7. Set-1-7 application cases

\begin{tabular}{|c|c|c|c|c|c|c|c|c|}
\hline \multirow{2}{*}{ Case } & \multirow{2}{*}{ Short name } & \multicolumn{2}{|c|}{ Moderator } & \multicolumn{2}{|c|}{ Filler } & Can & \multicolumn{2}{|c|}{ Pipe Material } \\
\hline & & & grams & & grams & grams & & $\mathrm{cm}$ \\
\hline set-1-7-uac1_uh_m1_generic_cyl_pp_1000_yr_para_00441 & set-1-7-00441 & \multirow{8}{*}{$\mathrm{H}_{2} \mathrm{O}$} & \multirow{8}{*}{710.5} & \multirow{8}{*}{ generic } & \multirow{4}{*}{0} & \multirow[b]{2}{*}{0} & \multirow{8}{*}{ poly } & 0.0010 \\
\hline set-1-7-uac1_uh_m1_generic_cyl_pp_1000_yr_para_00443 & set-1-7-00443 & & & & & & & 0.7112 \\
\hline set-1-7-uac1_uh_m1_generic_cyl_pp_1000_yr_para_00453 & set-1-7-00453 & & & & & & & 0.0010 \\
\hline set-1-7-uac1_uh_m1_generic_cyl_pp_1000_yr_para_00455 & set-1-7-00455 & & & & & 500 & & 0.7112 \\
\hline set-1-7-uac1_uh_m1_generic_cyl_pp_1000_yr_para_00477 & set-1-7-00477 & & & & \multirow{4}{*}{2,000} & 0 & & 0.0010 \\
\hline set-1-7-uac1_uh_m1_generic_cyl_pp_1000_yr_para_00479 & set-1-7-00479 & & & & & 0 & & 0.7112 \\
\hline set-1-7-uac1_uh_m1_generic_cyl_pp_1000_yr_para_00489 & set-1-7-00489 & & & & & & & 0.0010 \\
\hline set-1-7-uac1 uh $\mathrm{m} 1$ generic cyl pp 1000 yr para 00491 & set-1-7-00491 & & & & & 500 & & 0.7112 \\
\hline \multicolumn{9}{|l|}{ With Be } \\
\hline set-1-7-uac1_uh_m1_generic_cyl_pp_10 & set-1 & \multirow{8}{*}{$\mathrm{H}_{2} \mathrm{O}$} & \multirow{8}{*}{710.5} & \multirow{8}{*}{ generic } & \multirow{4}{*}{0} & \multirow{2}{*}{0} & \multirow{8}{*}{ poly } & 0.0010 \\
\hline set-1-7-uac1_uh_m1_generic_cyl_pp_1000_yr_para_00444 & -00444 & & & & & & & 0.7112 \\
\hline set-1-7-uac1_uh_m1_generic_cyl_pp_1000_yr_para_00454 & set-1-7-00454 & & & & & \multirow{2}{*}{500} & & 0.0010 \\
\hline set-1-7-uac1_uh_m1_generic_cyl_pp_1000_yr_para_00456 & set-1-7-00456 & & & & & & & 0.7112 \\
\hline set-1-7-uac1_uh_m1_generic_cyl_pp_1000_yr_para_00478 & set-1-7-00478 & & & & \multirow{4}{*}{2,000} & \multirow[b]{2}{*}{0} & & 0.0010 \\
\hline set-1-7-uac1_uh_m1_generic_cyl_pp_1000_yr_para_00480 & set-1-7-00480 & & & & & & & 0.7112 \\
\hline set-1-7-uac1_uh_m1_generic_cyl_pp_1000_yr_para_00490 & set-1-7-00490 & & & & & \multirow{2}{*}{500} & & 0.0010 \\
\hline set-1-7-uac1 uh $\mathrm{m} 1$ generic cyl pp 1000 yr para 00492 & set-1-7-00492 & & & & & & & 0.7112 \\
\hline
\end{tabular}

Table H-8. Set-1-8 application cases

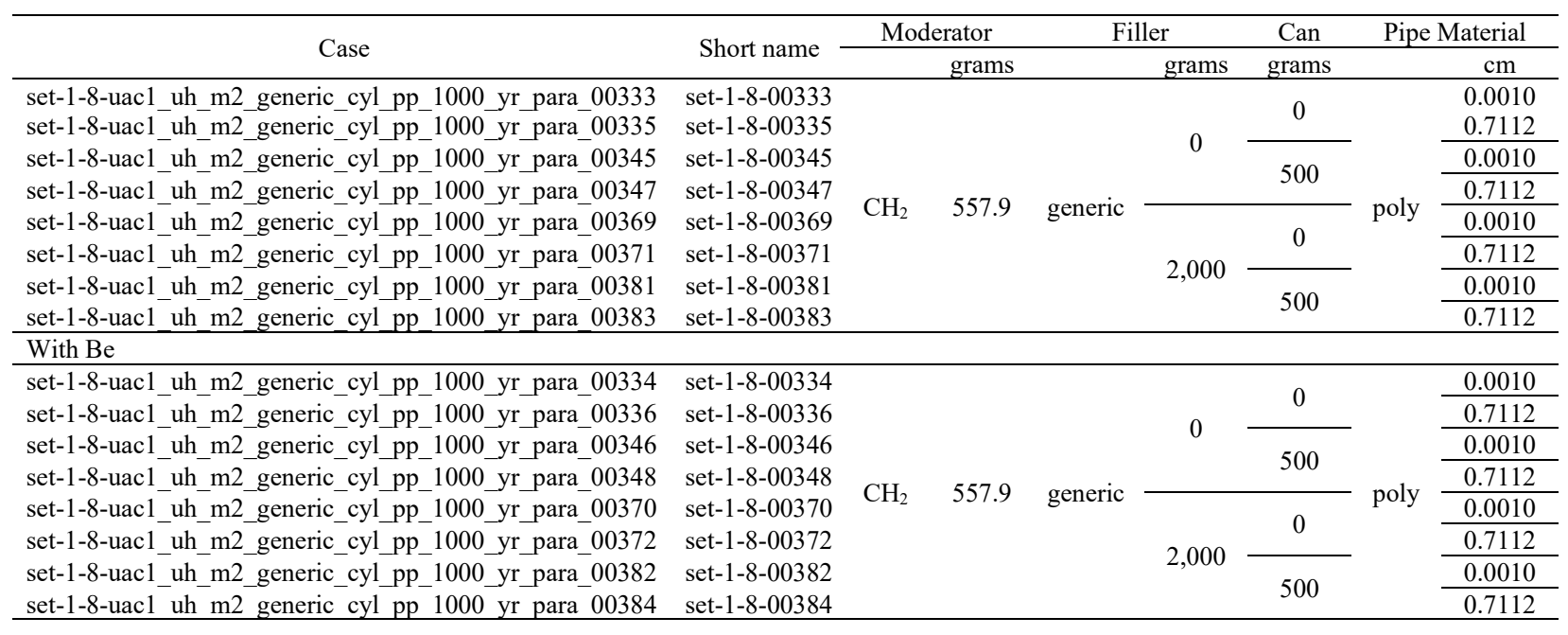

The TSUNAMI-3D sequence was used to generate SDFs for the modified versions of each of the application cases (shortened $y$-dimension with $y$-direction mirror boundaries, as shown in Figure $\mathrm{H}-1$ ). The KENO-VI sequence was also used to generate $k_{\text {eff }}$ values for the modified cases to compare with the original cases, as shown in Tables H-9 through H-16. The respective EALF values are also included for comparison. The nuclides with the highest magnitude sensitivities are listed in Tables H-17 through H-24. The sensitivities were confirmed through direct perturbation calculations. 
Table H-9. Set-1-1 $k_{\text {eff }}$ values for comparison

\begin{tabular}{|c|c|c|c|c|c|c|}
\hline \multirow{2}{*}{ Case } & \multicolumn{2}{|c|}{ Original } & \multicolumn{2}{|c|}{ Modified } & \multirow{2}{*}{$\begin{array}{c}\text { Original } \\
\text { EALF } \\
\end{array}$} & \multirow{2}{*}{$\begin{array}{c}\text { Modified } \\
\text { EALF }\end{array}$} \\
\hline & $\boldsymbol{k}_{\text {eff }}$ & sigma & $\boldsymbol{k}_{\text {eff }}$ & sigma & & \\
\hline set-1-1-00657 & 0.9445 & 0.0013 & 0.9437 & 0.0001 & 0.73 & 0.73 \\
\hline set-1-1-00658 & 0.9496 & 0.0015 & 0.9492 & 0.0001 & 0.69 & 0.69 \\
\hline set-1-1-00659 & 0.9303 & 0.0016 & 0.9323 & 0.0001 & 0.72 & 0.72 \\
\hline set-1-1-00660 & 0.9380 & 0.0014 & 0.9371 & 0.0001 & 0.69 & 0.69 \\
\hline set-1-1-00669 & 0.9298 & 0.0013 & 0.9290 & 0.0001 & 0.74 & 0.74 \\
\hline set-1-1-00670 & 0.9340 & 0.0016 & 0.9335 & 0.0001 & 0.71 & 0.71 \\
\hline set-1-1-00671 & 0.9194 & 0.0013 & 0.9180 & 0.0001 & 0.74 & 0.74 \\
\hline set-1-1-00672 & 0.9236 & 0.0015 & 0.9225 & 0.0001 & 0.71 & 0.71 \\
\hline set-1-1-00693 & 0.8732 & 0.0015 & 0.8724 & 0.0001 & 0.72 & 0.72 \\
\hline set-1-1-00694 & 0.8717 & 0.0013 & 0.8739 & 0.0001 & 0.70 & 0.69 \\
\hline set-1-1-00695 & 0.8694 & 0.0012 & 0.8702 & 0.0001 & 0.72 & 0.71 \\
\hline set-1-1-00696 & 0.8693 & 0.0013 & 0.8721 & 0.0001 & 0.68 & 0.69 \\
\hline set-1-1-00705 & 0.8590 & 0.0016 & 0.8598 & 0.0001 & 0.74 & 0.74 \\
\hline set-1-1-00706 & 0.8609 & 0.0012 & 0.8610 & 0.0001 & 0.71 & 0.71 \\
\hline set-1-1-00707 & 0.8559 & 0.0014 & 0.8580 & 0.0001 & 0.73 & 0.73 \\
\hline set-1-1-00708 & 0.8592 & 0.0013 & 0.8594 & 0.0001 & 0.70 & 0.70 \\
\hline
\end{tabular}

Table H-10. Set-1-2 $k_{\text {eff }}$ values for comparison

\begin{tabular}{|c|c|c|c|c|c|c|}
\hline \multirow{2}{*}{ Case } & \multicolumn{2}{|c|}{ Original } & \multicolumn{2}{|c|}{ Modified } & \multirow{2}{*}{$\begin{array}{c}\text { Original } \\
\text { EALF } \\
\end{array}$} & \multirow{2}{*}{$\begin{array}{c}\text { Modified } \\
\text { EALF } \\
\end{array}$} \\
\hline & $\boldsymbol{k}_{\text {eff }}$ & sigma & $\boldsymbol{k}_{\text {eff }}$ & sigma & & \\
\hline set-1-2-00441 & 0.9604 & 0.0013 & 0.9606 & 0.0001 & 0.88 & 0.88 \\
\hline set-1-2-00442 & 0.9653 & 0.0014 & 0.9617 & 0.0001 & 0.83 & 0.83 \\
\hline set-1-2-00443 & 0.9393 & 0.0013 & 0.9403 & 0.0001 & 0.88 & 0.88 \\
\hline set-1-2-00444 & 0.9406 & 0.0013 & 0.9418 & 0.0001 & 0.83 & 0.83 \\
\hline set-1-2-00453 & 0.9448 & 0.0014 & 0.9434 & 0.0001 & 0.90 & 0.90 \\
\hline set-1-2-00454 & 0.9434 & 0.0015 & 0.9444 & 0.0001 & 0.85 & 0.85 \\
\hline set-1-2-00455 & 0.9282 & 0.0013 & 0.9243 & 0.0001 & 0.90 & 0.90 \\
\hline set-1-2-00456 & 0.9234 & 0.0013 & 0.9258 & 0.0001 & 0.85 & 0.85 \\
\hline set-1-2-00477 & 0.8732 & 0.0013 & 0.8721 & 0.0001 & 0.86 & 0.87 \\
\hline set-1-2-00478 & 0.8729 & 0.0012 & 0.8725 & 0.0001 & 0.83 & 0.83 \\
\hline set-1-2-00479 & 0.8667 & 0.0013 & 0.8656 & 0.0001 & 0.86 & 0.86 \\
\hline set-1-2-00480 & 0.8655 & 0.0014 & 0.8670 & 0.0001 & 0.83 & 0.82 \\
\hline set-1-2-00489 & 0.8566 & 0.0021 & 0.8585 & 0.0001 & 0.89 & 0.89 \\
\hline set-1-2-00490 & 0.8589 & 0.0017 & 0.8591 & 0.0001 & 0.85 & 0.85 \\
\hline set-1-2-00491 & 0.8516 & 0.0013 & 0.8528 & 0.0001 & 0.88 & 0.88 \\
\hline set-1-2-00492 & 0.8548 & 0.0014 & 0.8542 & 0.0001 & 0.84 & 0.84 \\
\hline
\end{tabular}


Table H-11. Set-1-3 $k_{\text {eff }}$ values for comparison

\begin{tabular}{|c|c|c|c|c|c|c|}
\hline \multirow{2}{*}{ Case } & \multicolumn{2}{|c|}{ Original } & \multicolumn{2}{|c|}{ Modified } & \multirow{2}{*}{$\begin{array}{c}\text { Original } \\
\text { EALF }\end{array}$} & \multirow{2}{*}{$\begin{array}{c}\text { Modified } \\
\text { EALF }\end{array}$} \\
\hline & $\boldsymbol{k}_{\text {eff }}$ & sigma & $\boldsymbol{k}_{e f f}$ & sigma & & \\
\hline set-1-3-00441 & 0.8425 & 0.0013 & 0.8421 & 0.0001 & 1.45 & 1.45 \\
\hline set-1-3-00442 & 0.8561 & 0.0014 & 0.8555 & 0.0001 & 1.31 & 1.31 \\
\hline set-1-3-00443 & 0.9437 & 0.0013 & 0.9433 & 0.0001 & 0.57 & 0.57 \\
\hline set-1-3-00444 & 0.9504 & 0.0014 & 0.9498 & 0.0001 & 0.52 & 0.52 \\
\hline set-1-3-00453 & 0.8289 & 0.0013 & 0.8280 & 0.0001 & 1.48 & 1.48 \\
\hline set-1-3-00454 & 0.8399 & 0.0014 & 0.8403 & 0.0001 & 1.35 & 1.35 \\
\hline set-1-3-00455 & 0.9244 & 0.0013 & 0.9271 & 0.0001 & 0.57 & 0.57 \\
\hline set-1-3-00456 & 0.9313 & 0.0013 & 0.9333 & 0.0001 & 0.52 & 0.52 \\
\hline set-1-3-00477 & 0.7732 & 0.0012 & 0.7720 & 0.0001 & 1.39 & 1.38 \\
\hline set-1-3-00478 & 0.7790 & 0.0012 & 0.7789 & 0.0001 & 1.29 & 1.29 \\
\hline set-1-3-00479 & 0.8794 & 0.0014 & 0.8799 & 0.0001 & 0.49 & 0.49 \\
\hline set-1-3-00480 & 0.8819 & 0.0013 & 0.8827 & 0.0001 & 0.45 & 0.45 \\
\hline set-1-3-00489 & 0.7597 & 0.0012 & 0.7604 & 0.0001 & 1.41 & 1.41 \\
\hline set-1-3-00490 & 0.7692 & 0.0012 & 0.7671 & 0.0001 & 1.32 & 1.32 \\
\hline set-1-3-00491 & 0.8624 & 0.0014 & 0.8666 & 0.0001 & 0.49 & 0.49 \\
\hline set-1-3-00492 & 0.8702 & 0.0016 & 0.8691 & 0.0001 & 0.46 & 0.46 \\
\hline
\end{tabular}

Table H-12. Set-1-4 $k_{\text {eff }}$ values for comparison

\begin{tabular}{|c|c|c|c|c|c|c|}
\hline \multirow{2}{*}{ Case } & \multicolumn{2}{|c|}{ Original } & \multicolumn{2}{|c|}{ Modified } & \multirow{2}{*}{$\begin{array}{c}\text { Original } \\
\text { EALF }\end{array}$} & \multirow{2}{*}{$\begin{array}{c}\text { Modified } \\
\text { EALF }\end{array}$} \\
\hline & $\boldsymbol{k}_{\text {eff }}$ & sigma & $\boldsymbol{k}_{\text {eff }}$ & sigma & & \\
\hline set-1-4-00333 & 0.8848 & 0.0014 & 0.8871 & 0.0001 & 1.41 & 1.41 \\
\hline set-1-4-00334 & 0.8942 & 0.0013 & 0.8940 & 0.0001 & 1.28 & 1.28 \\
\hline set-1-4-00335 & 0.9730 & 0.0015 & 0.9742 & 0.0001 & 0.59 & 0.59 \\
\hline set-1-4-00336 & 0.9785 & 0.0013 & 0.9759 & 0.0001 & 0.54 & 0.54 \\
\hline set-1-4-00345 & 0.8713 & 0.0013 & 0.8705 & 0.0001 & 1.45 & 1.44 \\
\hline set-1-4-00346 & 0.8761 & 0.0015 & 0.8765 & 0.0001 & 1.31 & 1.31 \\
\hline set-1-4-00347 & 0.9594 & 0.0014 & 0.9566 & 0.0001 & 0.59 & 0.59 \\
\hline set-1-4-00348 & 0.9588 & 0.0013 & 0.9583 & 0.0001 & 0.54 & 0.54 \\
\hline set-1-4-00369 & 0.7999 & 0.0012 & 0.7999 & 0.0001 & 1.35 & 1.35 \\
\hline set-1-4-00370 & 0.8056 & 0.0013 & 0.8040 & 0.0001 & 1.26 & 1.26 \\
\hline set-1-4-00371 & 0.8985 & 0.0013 & 0.8975 & 0.0001 & 0.51 & 0.50 \\
\hline set-1-4-00372 & 0.8976 & 0.0013 & 0.8983 & 0.0001 & 0.47 & 0.47 \\
\hline set-1-4-00381 & 0.7870 & 0.0013 & 0.7869 & 0.0001 & 1.38 & 1.38 \\
\hline set-1-4-00382 & 0.7908 & 0.0013 & 0.7912 & 0.0001 & 1.29 & 1.29 \\
\hline set-1-4-00383 & 0.8834 & 0.0017 & 0.8835 & 0.0001 & 0.51 & 0.51 \\
\hline set-1-4-00384 & 0.8836 & 0.0015 & 0.8842 & 0.0001 & 0.47 & 0.48 \\
\hline
\end{tabular}


Table H-13. Set-1-5 $\boldsymbol{k}_{\text {eff }}$ values for comparison

\begin{tabular}{|c|c|c|c|c|c|c|}
\hline \multirow{2}{*}{ Case } & \multicolumn{2}{|c|}{ Original } & \multicolumn{2}{|c|}{ Modified } & \multirow{2}{*}{$\begin{array}{c}\text { Original } \\
\text { EALF }\end{array}$} & \multirow{2}{*}{$\begin{array}{c}\text { Modified } \\
\text { EALF }\end{array}$} \\
\hline & $\boldsymbol{k}_{\text {eff }}$ & sigma & $\boldsymbol{k}_{\text {eff }}$ & sigma & & \\
\hline set-1-5-00657 & 0.9445 & 0.0013 & 0.9437 & 0.0001 & 0.73 & 0.73 \\
\hline set-1-5-00658 & 0.9496 & 0.0015 & 0.9492 & 0.0001 & 0.69 & 0.69 \\
\hline set-1-5-00659 & 0.9303 & 0.0016 & 0.9323 & 0.0001 & 0.72 & 0.72 \\
\hline set-1-5-00660 & 0.9380 & 0.0014 & 0.9371 & 0.0001 & 0.69 & 0.69 \\
\hline set-1-5-00669 & 0.9298 & 0.0013 & 0.9290 & 0.0001 & 0.74 & 0.74 \\
\hline set-1-5-00670 & 0.9340 & 0.0016 & 0.9335 & 0.0001 & 0.71 & 0.71 \\
\hline set-1-5-00671 & 0.9194 & 0.0013 & 0.9180 & 0.0001 & 0.74 & 0.74 \\
\hline set-1-5-00672 & 0.9236 & 0.0015 & 0.9225 & 0.0001 & 0.71 & 0.71 \\
\hline set-1-5-00693 & 0.8526 & 0.0015 & 0.8531 & 0.0001 & 0.76 & 0.76 \\
\hline set-1-5-00694 & 0.8592 & 0.0013 & 0.8589 & 0.0001 & 0.73 & 0.73 \\
\hline set-1-5-00695 & 0.8535 & 0.0012 & 0.8533 & 0.0001 & 0.75 & 0.75 \\
\hline set-1-5-00696 & 0.8574 & 0.0014 & 0.8583 & 0.0001 & 0.72 & 0.72 \\
\hline set-1-5-00705 & 0.8421 & 0.0014 & 0.8414 & 0.0001 & 0.78 & 0.78 \\
\hline set-1-5-00706 & 0.8465 & 0.0015 & 0.8465 & 0.0001 & 0.75 & 0.74 \\
\hline set-1-5-00707 & 0.8386 & 0.0012 & 0.8417 & 0.0001 & 0.76 & 0.77 \\
\hline set- $1-5-00708$ & 0.8455 & 0.0012 & 0.8464 & 0.0001 & 0.74 & 0.73 \\
\hline
\end{tabular}

Table H-14. Set-1-6 $k_{\text {eff }}$ values for comparison

\begin{tabular}{|c|c|c|c|c|c|c|}
\hline \multirow{2}{*}{ Case } & \multicolumn{2}{|c|}{ Original } & \multicolumn{2}{|c|}{ Modified } & \multirow{2}{*}{$\begin{array}{c}\text { Original } \\
\text { EALF } \\
\end{array}$} & \multirow{2}{*}{$\begin{array}{c}\text { Modified } \\
\text { EALF } \\
\end{array}$} \\
\hline & $\boldsymbol{k}_{\text {eff }}$ & sigma & $\boldsymbol{k}_{\text {eff }}$ & sigma & & \\
\hline set-1-6-00441 & 0.9604 & 0.0013 & 0.9606 & 0.0001 & 0.88 & 0.88 \\
\hline set-1-6-00442 & 0.9653 & 0.0014 & 0.9617 & 0.0001 & 0.83 & 0.83 \\
\hline set-1-6-00443 & 0.9393 & 0.0013 & 0.9403 & 0.0001 & 0.88 & 0.88 \\
\hline set-1-6-00444 & 0.9406 & 0.0013 & 0.9418 & 0.0001 & 0.83 & 0.83 \\
\hline set-1-6-00453 & 0.9448 & 0.0014 & 0.9434 & 0.0001 & 0.90 & 0.90 \\
\hline set-1-6-00454 & 0.9434 & 0.0015 & 0.9444 & 0.0001 & 0.85 & 0.85 \\
\hline set-1-6-00455 & 0.9282 & 0.0013 & 0.9243 & 0.0001 & 0.90 & 0.90 \\
\hline set-1-6-00456 & 0.9234 & 0.0013 & 0.9258 & 0.0001 & 0.85 & 0.85 \\
\hline set-1-6-00477 & 0.8532 & 0.0013 & 0.8521 & 0.0001 & 0.92 & 0.92 \\
\hline set-1-6-00478 & 0.8552 & 0.0013 & 0.8571 & 0.0001 & 0.88 & 0.88 \\
\hline set-1-6-00479 & 0.8468 & 0.0012 & 0.8482 & 0.0001 & 0.91 & 0.91 \\
\hline set-1-6-00480 & 0.8537 & 0.0011 & 0.8530 & 0.0001 & 0.86 & 0.87 \\
\hline set-1-6-00489 & 0.8408 & 0.0012 & 0.8395 & 0.0001 & 0.95 & 0.94 \\
\hline set-1-6-00490 & 0.8436 & 0.0013 & 0.8440 & 0.0001 & 0.90 & 0.90 \\
\hline set-1-6-00491 & 0.8350 & 0.0015 & 0.8358 & 0.0001 & 0.93 & 0.93 \\
\hline set-1-6-00492 & 0.8407 & 0.0013 & 0.8403 & 0.0001 & 0.89 & 0.88 \\
\hline
\end{tabular}


Table H-15. Set-1-7 $k_{\text {eff }}$ values for comparison

\begin{tabular}{|c|c|c|c|c|c|c|}
\hline \multirow{2}{*}{ Case } & \multicolumn{2}{|c|}{ Original } & \multicolumn{2}{|c|}{ Modified } & \multirow{2}{*}{$\begin{array}{c}\text { Original } \\
\text { EALF }\end{array}$} & \multirow{2}{*}{$\begin{array}{c}\text { Modified } \\
\text { EALF }\end{array}$} \\
\hline & $\boldsymbol{k}_{\text {eff }}$ & sigma & $\boldsymbol{k}_{e f f}$ & sigma & & \\
\hline set-1-7-00441 & 0.8425 & 0.0013 & 0.8421 & 0.0001 & 1.45 & 1.45 \\
\hline set-1-7-00442 & 0.8561 & 0.0014 & 0.8555 & 0.0001 & 1.31 & 1.31 \\
\hline set-1-7-00443 & 0.9437 & 0.0013 & 0.9433 & 0.0001 & 0.57 & 0.57 \\
\hline set-1-7-00444 & 0.9504 & 0.0014 & 0.9498 & 0.0001 & 0.52 & 0.52 \\
\hline set-1-7-00453 & 0.8289 & 0.0013 & 0.8280 & 0.0001 & 1.48 & 1.48 \\
\hline set-1-7-00454 & 0.8399 & 0.0014 & 0.8403 & 0.0001 & 1.35 & 1.35 \\
\hline set-1-7-00455 & 0.9244 & 0.0013 & 0.9271 & 0.0001 & 0.57 & 0.57 \\
\hline set-1-7-00456 & 0.9313 & 0.0013 & 0.9333 & 0.0001 & 0.52 & 0.52 \\
\hline set-1-7-00477 & 0.7439 & 0.0011 & 0.7439 & 0.0001 & 1.53 & 1.52 \\
\hline set-1-7-00478 & 0.7577 & 0.0014 & 0.7569 & 0.0001 & 1.41 & 1.40 \\
\hline set-1-7-00479 & 0.8643 & 0.0013 & 0.8648 & 0.0001 & 0.51 & 0.51 \\
\hline set-1-7-00480 & 0.8703 & 0.0014 & 0.8717 & 0.0001 & 0.47 & 0.47 \\
\hline set-1-7-00489 & 0.7332 & 0.0012 & 0.7334 & 0.0001 & 1.56 & 1.56 \\
\hline set-1-7-00490 & 0.7454 & 0.0012 & 0.7456 & 0.0001 & 1.44 & 1.44 \\
\hline set-1-7-00491 & 0.8531 & 0.0012 & 0.8522 & 0.0001 & 0.52 & 0.52 \\
\hline set-1-7-00492 & 0.8598 & 0.0015 & 0.8584 & 0.0001 & 0.48 & 0.48 \\
\hline set-1-7-00444 & 0.9504 & 0.0014 & 0.8421 & 0.0001 & 1.45 & 1.45 \\
\hline
\end{tabular}

Table H-16. Set-1-8 $k_{\text {eff }}$ values for comparison

\begin{tabular}{|c|c|c|c|c|c|c|}
\hline \multirow{2}{*}{ Case } & \multicolumn{2}{|c|}{ Original } & \multicolumn{2}{|c|}{ Modified } & \multirow{2}{*}{$\begin{array}{c}\text { Original } \\
\text { EALF } \\
\end{array}$} & \multirow{2}{*}{$\begin{array}{c}\text { Modified } \\
\text { EALF } \\
\end{array}$} \\
\hline & $\boldsymbol{k}_{\text {eff }}$ & sigma & $\boldsymbol{k}_{\text {eff }}$ & sigma & & \\
\hline set-1-8-00333 & 0.8848 & 0.0014 & 0.8871 & 0.0001 & 1.41 & 1.41 \\
\hline set-1-8-00334 & 0.8942 & 0.0013 & 0.8940 & 0.0001 & 1.28 & 1.28 \\
\hline set-1-8-00335 & 0.9730 & 0.0015 & 0.9742 & 0.0001 & 0.59 & 0.59 \\
\hline set-1-8-00336 & 0.9785 & 0.0013 & 0.9759 & 0.0001 & 0.54 & 0.54 \\
\hline set-1-8-00345 & 0.8713 & 0.0013 & 0.8705 & 0.0001 & 1.45 & 1.44 \\
\hline set-1-8-00346 & 0.8761 & 0.0015 & 0.8765 & 0.0001 & 1.31 & 1.31 \\
\hline set-1-8-00347 & 0.9594 & 0.0014 & 0.9566 & 0.0001 & 0.59 & 0.59 \\
\hline set-1-8-00348 & 0.9588 & 0.0013 & 0.9583 & 0.0001 & 0.54 & 0.54 \\
\hline set-1-8-00369 & 0.7741 & 0.0014 & 0.7733 & 0.0001 & 1.48 & 1.48 \\
\hline set- $1-8-00370$ & 0.7823 & 0.0013 & 0.7836 & 0.0001 & 1.37 & 1.37 \\
\hline set-1-8-00371 & 0.8828 & 0.0014 & 0.8835 & 0.0001 & 0.53 & 0.53 \\
\hline set-1-8-00372 & 0.8887 & 0.0013 & 0.8885 & 0.0001 & 0.49 & 0.49 \\
\hline set-1-8-00381 & 0.7621 & 0.0013 & 0.7617 & 0.0001 & 1.52 & 1.51 \\
\hline set-1-8-00382 & 0.7714 & 0.0013 & 0.7713 & 0.0001 & 1.40 & 1.40 \\
\hline set-1-8-00383 & 0.8686 & 0.0012 & 0.8701 & 0.0001 & 0.54 & 0.53 \\
\hline set- $1-8-00384$ & 0.8755 & 0.0013 & 0.8747 & 0.0001 & 0.50 & 0.50 \\
\hline
\end{tabular}


Table H-17. Nuclides with the highest sensitivities for set-1-1 cases

\begin{tabular}{|c|c|c|c|c|c|c|c|c|c|}
\hline \multirow[t]{2}{*}{$\begin{array}{c}\text { Case } \\
\text { Number } \\
\end{array}$} & \multirow[t]{2}{*}{ Nuclide } & \multirow[t]{2}{*}{ Mixture } & \multirow[t]{2}{*}{$\begin{array}{l}\text { Sensitivity } \\
\text { coefficient }\end{array}$} & \multirow{2}{*}{$\begin{array}{l}\text { Total data- } \\
\text { induced } \\
\text { uncertainty } \\
\% \Delta \mathrm{k} / \mathrm{k} \\
\end{array}$} & \multirow{2}{*}{\begin{tabular}{|c} 
Case \\
Number
\end{tabular}} & \multirow[t]{2}{*}{ Nuclide } & \multirow[t]{2}{*}{ Mixture } & \multirow[t]{2}{*}{$\begin{array}{l}\text { Sensitivity } \\
\text { coefficient }\end{array}$} & \multirow{2}{*}{$\begin{array}{c}\text { Total data- } \\
\text { induced } \\
\text { uncertainty } \\
\% \Delta \mathrm{k} / \mathrm{k}\end{array}$} \\
\hline & & & & & & & & & \\
\hline 657 & $\begin{array}{l}\mathrm{h}-1 \\
\mathrm{pu}-239 \\
\mathrm{o}-16 \\
\mathrm{cl}-35\end{array}$ & $\begin{array}{l}\text { waste } \\
\text { waste } \\
\text { waste } \\
\mathrm{MgO} / \mathrm{NaCl}\end{array}$ & $\begin{array}{r}0.5017 \\
0.0932 \\
0.0405 \\
-0.0134\end{array}$ & 0.6705 & 658 & $\begin{array}{l}\text { h-1 } \\
\text { pu-239 } \\
\text { be } \\
\text { o-16 } \\
\text { cl-35 }\end{array}$ & $\begin{array}{l}\text { waste } \\
\text { waste } \\
\text { waste } \\
\text { waste } \\
\mathrm{MgO} / \mathrm{NaCl}\end{array}$ & $\begin{array}{l}0.4628 \\
0.0985 \\
0.0720 \\
0.0322 \\
-0.0201 \\
\end{array}$ & 0.6287 \\
\hline 659 & $\begin{array}{l}\text { h-1 } \\
\text { pu-239 } \\
\text { o-16 } \\
\text { fe-56 } \\
\text { cl-35 }\end{array}$ & $\begin{array}{l}\text { waste } \\
\text { waste } \\
\text { waste } \\
\text { ss pipe } \\
\mathrm{MgO} / \mathrm{NaCl}\end{array}$ & $\begin{array}{r}0.4727 \\
0.1057 \\
0.0315 \\
0.0220 \\
-0.0161\end{array}$ & 0.6627 & 660 & $\begin{array}{l}\text { h-1 } \\
\text { pu-239 } \\
\text { be } \\
\text { o-16 } \\
\text { cl-35 } \\
\text { fe-56 }\end{array}$ & $\begin{array}{l}\text { waste } \\
\text { waste } \\
\text { waste } \\
\text { waste } \\
\mathrm{MgO} / \mathrm{NaCl} \\
\text { ss pipe }\end{array}$ & $\begin{array}{l}0.4439 \\
0.1081 \\
0.0607 \\
0.0256 \\
-0.0216 \\
0.0206\end{array}$ & 0.6313 \\
\hline 669 & $\begin{array}{l}\text { h-1 } \\
\text { pu-239 } \\
\text { o-16 } \\
\text { cl-35 }\end{array}$ & $\begin{array}{l}\text { waste } \\
\text { waste } \\
\text { waste } \\
\mathrm{MgO} / \mathrm{NaCl}\end{array}$ & $\begin{array}{r}0.4986 \\
0.1030 \\
0.0388 \\
-0.0147\end{array}$ & 0.6685 & 670 & $\begin{array}{l}\text { h-1 } \\
\text { pu-239 } \\
\text { be } \\
\text { o-16 } \\
\text { cl-35 }\end{array}$ & $\begin{array}{l}\text { waste } \\
\text { waste } \\
\text { waste } \\
\text { waste } \\
\mathrm{MgO} / \mathrm{NaCl}\end{array}$ & $\begin{array}{l}0.4609 \\
0.1093 \\
0.0696 \\
0.0309 \\
-0.0216\end{array}$ & 0.6268 \\
\hline 671 & $\begin{array}{l}\text { h-1 } \\
\text { pu-239 } \\
\text { o-16 } \\
\text { fe-56 } \\
\text { cl-35 }\end{array}$ & $\begin{array}{l}\text { waste } \\
\text { waste } \\
\text { waste } \\
\text { ss pipe } \\
\mathrm{MgO} / \mathrm{NaCl}\end{array}$ & $\begin{array}{r}0.4745 \\
0.1128 \\
0.0304 \\
0.0219 \\
-0.0172\end{array}$ & 0.6698 & 672 & $\begin{array}{l}\text { h-1 } \\
\text { pu-239 } \\
\text { be } \\
\text { o-16 } \\
\text { cl-35 } \\
\text { fe-56 }\end{array}$ & $\begin{array}{l}\text { waste } \\
\text { waste } \\
\text { waste } \\
\text { waste } \\
\mathrm{MgO} / \mathrm{NaCl} \\
\text { ss pipe }\end{array}$ & $\begin{array}{l}0.4450 \\
0.1162 \\
0.0592 \\
0.0250 \\
-0.0228 \\
0.0205 \\
\end{array}$ & 0.6397 \\
\hline 693 & $\begin{array}{l}\text { h-1 } \\
\text { pu-239 } \\
\text { c-graphite } \\
\text { cl-35 } \\
\text { o-16 }\end{array}$ & $\begin{array}{l}\text { waste } \\
\text { waste } \\
\text { waste } \\
\mathrm{MgO} / \mathrm{NaCl} \\
\text { waste }\end{array}$ & $\begin{array}{r}0.4726 \\
0.1205 \\
0.0876 \\
-0.0361 \\
0.0255\end{array}$ & 0.6481 & 694 & $\begin{array}{l}\text { h-1 } \\
\text { pu-239 } \\
\text { c-graphite } \\
\text { be } \\
\text { cl-35 } \\
\text { o-16 } \\
\text { mg-24 }\end{array}$ & $\begin{array}{l}\text { waste } \\
\text { waste } \\
\text { waste } \\
\text { waste } \\
\mathrm{MgO} / \mathrm{NaCl} \\
\text { waste } \\
\mathrm{MgO} / \mathrm{NaCl}\end{array}$ & $\begin{array}{l}0.4483 \\
0.1266 \\
0.0758 \\
0.0529 \\
-0.0435 \\
0.0221 \\
0.0093 \\
\end{array}$ & 0.6224 \\
\hline 695 & $\begin{array}{l}\text { h-1 } \\
\text { pu-239 } \\
\text { c-graphite } \\
\text { cl-35 } \\
\text { fe-56 } \\
\text { o-16 }\end{array}$ & $\begin{array}{l}\text { waste } \\
\text { waste } \\
\text { waste } \\
\mathrm{MgO} / \mathrm{NaCl} \\
\text { ss pipe } \\
\text { waste }\end{array}$ & $\begin{array}{r}0.4524 \\
0.1278 \\
0.0737 \\
-0.0349 \\
0.0225 \\
0.0212\end{array}$ & 0.6513 & 696 & $\begin{array}{l}\text { h-1 } \\
\text { pu-239 } \\
\text { c-graphite } \\
\text { be } \\
\text { cl-35 } \\
\text { fe-56 } \\
\text { o-16 }\end{array}$ & $\begin{array}{l}\text { waste } \\
\text { waste } \\
\text { waste } \\
\text { waste } \\
\mathrm{MgO} / \mathrm{NaCl} \\
\text { ss pipe } \\
\text { waste }\end{array}$ & $\begin{array}{l}0.4331 \\
0.1315 \\
0.0649 \\
0.0466 \\
-0.0407 \\
0.0223 \\
0.0187 \\
\end{array}$ & 0.6319 \\
\hline 705 & $\begin{array}{l}\text { h-1 } \\
\text { pu-239 } \\
\text { c-graphite } \\
\text { cl-35 } \\
\text { o-16 }\end{array}$ & $\begin{array}{l}\text { waste } \\
\text { waste } \\
\text { waste } \\
\mathrm{MgO} / \mathrm{NaCl} \\
\text { waste }\end{array}$ & $\begin{array}{r}0.4726 \\
0.1294 \\
0.0857 \\
-0.0377 \\
0.0249\end{array}$ & 0.6464 & 706 & $\begin{array}{l}\text { h-1 } \\
\text { pu-239 } \\
\text { c-graphite } \\
\text { be } \\
\text { cl-35 } \\
\text { o-16 } \\
\text { mg-24 }\end{array}$ & $\begin{array}{l}\text { waste } \\
\text { waste } \\
\text { waste } \\
\text { waste } \\
\mathrm{MgO} / \mathrm{NaCl} \\
\text { waste } \\
\mathrm{MgO} / \mathrm{NaCl}\end{array}$ & $\begin{array}{l}0.4476 \\
0.1365 \\
0.0743 \\
0.0517 \\
-0.0449 \\
0.0217 \\
0.0092 \\
\end{array}$ & 0.6215 \\
\hline 707 & $\begin{array}{l}\text { h-1 } \\
\text { pu-239 } \\
\text { c-graphite } \\
\text { cl-35 } \\
\text { fe-56 } \\
\text { o-16 }\end{array}$ & $\begin{array}{l}\text { waste } \\
\text { waste } \\
\text { waste } \\
\mathrm{MgO} / \mathrm{NaCl} \\
\text { ss pipe } \\
\text { waste }\end{array}$ & $\begin{array}{r}0.4545 \\
0.1351 \\
0.0724 \\
-0.0362 \\
0.0226 \\
0.0207\end{array}$ & 0.6585 & 708 & $\begin{array}{l}\text { h-1 } \\
\text { pu-239 } \\
\text { c-graphite } \\
\text { be } \\
\text { cl-35 } \\
\text { fe-56 } \\
\text { o-16 }\end{array}$ & $\begin{array}{l}\text { waste } \\
\text { waste } \\
\text { waste } \\
\text { waste } \\
\mathrm{MgO} / \mathrm{NaCl} \\
\text { ss pipe } \\
\text { waste }\end{array}$ & $\begin{array}{l}0.4326 \\
0.1411 \\
0.0637 \\
0.0457 \\
-0.0419 \\
0.0222 \\
0.0183\end{array}$ & 0.6404 \\
\hline
\end{tabular}


Table H-18. Nuclides with the highest sensitivities for set-1-2 cases

\begin{tabular}{|c|c|c|c|c|c|c|c|c|c|}
\hline \multirow[t]{2}{*}{$\begin{array}{c}\text { Case } \\
\text { Number } \\
\end{array}$} & \multirow[t]{2}{*}{ Nuclide } & \multirow[t]{2}{*}{ Mixture } & \multirow[t]{2}{*}{$\begin{array}{l}\text { Sensitivity } \\
\text { coefficient }\end{array}$} & \multirow{2}{*}{$\begin{array}{c}\text { Total data- } \\
\text { induced } \\
\text { uncertainty } \\
\% \Delta \mathrm{k} / \mathrm{k} \\
\end{array}$} & $\begin{array}{c}\text { Case } \\
\text { Number } \\
\end{array}$ & Nuclide & Mixture & $\begin{array}{l}\text { Sensitivity } \\
\text { coefficient }\end{array}$ & \multirow{2}{*}{$\begin{array}{l}\text { Total data- } \\
\text { induced } \\
\text { uncertainty } \\
\% \Delta \mathrm{k} / \mathrm{k}\end{array}$} \\
\hline & & & & & With Be & & & & \\
\hline 441 & $\begin{array}{l}\text { h-poly } \\
\text { pu-239 } \\
\text { c }\end{array}$ & $\begin{array}{l}\text { waste } \\
\text { waste } \\
\text { waste }\end{array}$ & $\begin{array}{l}0.4969 \\
0.0890 \\
0.0505\end{array}$ & 0.6674 & 442 & $\begin{array}{l}\text { h-poly } \\
\text { pu-239 } \\
\text { be } \\
\text { c } \\
\text { cl-35 }\end{array}$ & $\begin{array}{l}\text { waste } \\
\text { waste } \\
\text { waste } \\
\text { waste } \\
\mathrm{MgO} / \mathrm{NaCl}\end{array}$ & $\begin{array}{r}0.4555 \\
0.0941 \\
0.0830 \\
0.0386 \\
-0.0137 \\
\end{array}$ & 0.6236 \\
\hline 443 & $\begin{array}{l}\text { h-poly } \\
\text { pu-239 } \\
\text { c } \\
\text { fe-56 } \\
\text { cl-35 }\end{array}$ & $\begin{array}{l}\text { waste } \\
\text { waste } \\
\text { waste } \\
\text { ss pipe } \\
\mathrm{MgO} / \mathrm{NaCl}\end{array}$ & $\begin{array}{r}0.4720 \\
0.1002 \\
0.0388 \\
0.0231 \\
-0.0110\end{array}$ & 0.6623 & 444 & $\begin{array}{l}\text { h-poly } \\
\text { pu-239 } \\
\text { be } \\
\text { c } \\
\text { fe-56 } \\
\text { cl-35 }\end{array}$ & $\begin{array}{l}\text { waste } \\
\text { waste } \\
\text { waste } \\
\text { waste } \\
\text { ss pipe } \\
\mathrm{MgO} / \mathrm{NaCl}\end{array}$ & $\begin{array}{r}0.4368 \\
0.1062 \\
0.0688 \\
0.0306 \\
0.0208 \\
-0.0165\end{array}$ & 0.6280 \\
\hline 453 & $\begin{array}{l}\text { h-poly } \\
\text { pu-239 } \\
\text { c } \\
\text { cl-35 }\end{array}$ & $\begin{array}{l}\text { waste } \\
\text { waste } \\
\text { waste } \\
\mathrm{MgO} / \mathrm{NaCl}\end{array}$ & $\begin{array}{r}0.4948 \\
0.0988 \\
0.0482 \\
-0.0089\end{array}$ & 0.6632 & 454 & $\begin{array}{l}\text { h-poly } \\
\text { pu-239 } \\
\text { be } \\
\text { c } \\
\text { cl-35 }\end{array}$ & $\begin{array}{l}\text { waste } \\
\text { waste } \\
\text { waste } \\
\text { waste } \\
\mathrm{MgO} / \mathrm{NaCl}\end{array}$ & $\begin{array}{r}0.4556 \\
0.1033 \\
0.0801 \\
0.0372 \\
-0.0152\end{array}$ & 0.6233 \\
\hline 455 & $\begin{array}{l}\text { h-poly } \\
\text { pu-239 } \\
\text { c } \\
\text { fe-56 } \\
\text { cl-35 }\end{array}$ & $\begin{array}{l}\text { waste } \\
\text { waste } \\
\text { waste } \\
\text { ss pipe } \\
\mathrm{MgO} / \mathrm{NaCl}\end{array}$ & $\begin{array}{r}0.4714 \\
0.1095 \\
0.0373 \\
0.0226 \\
-0.0122\end{array}$ & 0.6671 & 456 & $\begin{array}{l}\text { h-poly } \\
\text { pu-239 } \\
\text { be } \\
\text { c } \\
\text { fe-56 } \\
\text { cl-35 }\end{array}$ & $\begin{array}{l}\text { waste } \\
\text { waste } \\
\text { waste } \\
\text { waste } \\
\text { ss pipe } \\
\mathrm{MgO} / \mathrm{NaCl}\end{array}$ & $\begin{array}{r}0.4374 \\
0.1152 \\
0.0671 \\
0.0298 \\
0.0207 \\
-0.0176 \\
\end{array}$ & 0.6371 \\
\hline 477 & $\begin{array}{l}\text { h-poly } \\
\text { pu-239 } \\
\text { c-graphite } \\
\text { c } \\
\text { cl-35 } \\
\text { mg-24 }\end{array}$ & $\begin{array}{l}\text { waste } \\
\text { waste } \\
\text { waste } \\
\text { waste } \\
\mathrm{MgO} / \mathrm{NaCl} \\
\mathrm{MgO} / \mathrm{NaCl}\end{array}$ & $\begin{array}{r}0.4686 \\
0.1160 \\
0.1006 \\
0.0309 \\
-0.0296 \\
0.0081\end{array}$ & 0.6475 & 478 & $\begin{array}{l}\text { h-poly } \\
\text { pu-239 } \\
\text { c-graphite } \\
\text { be } \\
\text { cl-35 } \\
\text { c } \\
\text { mg-24 }\end{array}$ & $\begin{array}{l}\text { waste } \\
\text { waste } \\
\text { waste } \\
\text { waste } \\
\mathrm{MgO} / \mathrm{NaCl} \\
\text { waste } \\
\mathrm{MgO} / \mathrm{NaCl}\end{array}$ & $\begin{array}{r}0.4417 \\
0.1229 \\
0.0858 \\
0.0588 \\
-0.0373 \\
0.0264 \\
0.0085 \\
\end{array}$ & 0.6201 \\
\hline 479 & $\begin{array}{l}\text { h-poly } \\
\text { pu-239 } \\
\text { c-graphite } \\
\text { cl-35 } \\
\text { c } \\
\text { fe-56 }\end{array}$ & $\begin{array}{l}\text { waste } \\
\text { waste } \\
\text { waste } \\
\mathrm{MgO} / \mathrm{NaCl} \\
\text { waste } \\
\text { ss pipe }\end{array}$ & $\begin{array}{r}0.4492 \\
0.1251 \\
0.0839 \\
-0.0298 \\
0.0258 \\
0.0226\end{array}$ & 0.6501 & 480 & $\begin{array}{l}\text { h-poly } \\
\text { pu-239 } \\
\text { c-graphite } \\
\text { be } \\
\text { cl-35 } \\
\text { c } \\
\text { fe-56 }\end{array}$ & $\begin{array}{l}\text { waste } \\
\text { waste } \\
\text { waste } \\
\text { waste } \\
\mathrm{MgO} / \mathrm{NaCl} \\
\text { waste } \\
\text { ss pipe }\end{array}$ & $\begin{array}{r}0.4277 \\
0.1288 \\
0.0729 \\
0.0516 \\
-0.0360 \\
0.0224 \\
0.0221 \\
\end{array}$ & 0.6306 \\
\hline 489 & $\begin{array}{l}\text { h-poly } \\
\text { pu-239 } \\
\text { c-graphite } \\
\text { cl-35 } \\
\text { c } \\
\text { mg-24 }\end{array}$ & $\begin{array}{l}\text { waste } \\
\text { waste } \\
\text { waste } \\
\mathrm{MgO} / \mathrm{NaCl} \\
\text { waste } \\
\mathrm{MgO} / \mathrm{NaCl}\end{array}$ & $\begin{array}{r}0.4669 \\
0.1261 \\
0.0976 \\
-0.0313 \\
0.0300 \\
0.0083\end{array}$ & 0.6451 & 490 & $\begin{array}{l}\text { h-poly } \\
\text { pu-239 } \\
\text { c-graphite } \\
\text { be } \\
\text { cl-35 } \\
\text { c } \\
\text { mg-24 }\end{array}$ & $\begin{array}{l}\text { waste } \\
\text { waste } \\
\text { waste } \\
\text { waste } \\
\mathrm{MgO} / \mathrm{NaCl} \\
\text { waste } \\
\mathrm{MgO} / \mathrm{NaCl}\end{array}$ & $\begin{array}{r}0.4428 \\
0.1309 \\
0.0839 \\
0.0575 \\
-0.0389 \\
0.0258 \\
0.0089 \\
\end{array}$ & 0.6198 \\
\hline 491 & $\begin{array}{l}\text { h-poly } \\
\text { pu-239 } \\
\text { c-graphite } \\
\text { cl-35 } \\
\text { c } \\
\text { fe-56 }\end{array}$ & $\begin{array}{l}\text { waste } \\
\text { waste } \\
\text { waste } \\
\mathrm{MgO} / \mathrm{NaCl} \\
\text { waste } \\
\text { ss pipe }\end{array}$ & $\begin{array}{r}0.4470 \\
0.1358 \\
0.0818 \\
-0.0311 \\
0.0252 \\
0.0225\end{array}$ & 0.6574 & 492 & $\begin{array}{l}\text { h-poly } \\
\text { pu-239 } \\
\text { c-graphite } \\
\text { be } \\
\text { cl-35 } \\
\text { c } \\
\text { fe-56 }\end{array}$ & $\begin{array}{l}\text { waste } \\
\text { waste } \\
\text { waste } \\
\text { waste } \\
\mathrm{MgO} / \mathrm{NaCl} \\
\text { waste } \\
\text { ss pipe }\end{array}$ & $\begin{array}{r}0.4279 \\
0.1379 \\
0.0714 \\
0.0506 \\
-0.0374 \\
0.0220 \\
0.0219\end{array}$ & 0.6384 \\
\hline
\end{tabular}


Table H-19. Nuclides with the highest sensitivities for set-1-3 cases

\begin{tabular}{|c|c|c|c|c|c|c|c|c|c|}
\hline \multirow[t]{2}{*}{$\begin{array}{c}\text { Case } \\
\text { Number } \\
\end{array}$} & \multirow[t]{2}{*}{ Nuclide } & \multirow[t]{2}{*}{ Mixture } & \multirow[t]{2}{*}{$\begin{array}{l}\text { Sensitivity } \\
\text { coefficient }\end{array}$} & \multirow{2}{*}{$\begin{array}{c}\text { Total data- } \\
\text { induced } \\
\text { uncertainty } \\
\% \Delta \mathrm{k} / \mathrm{k}\end{array}$} & \multirow{2}{*}{\begin{tabular}{|c} 
Case \\
Number
\end{tabular}} & \multirow[t]{2}{*}{ Nuclide } & \multirow[t]{2}{*}{ Mixture } & \multirow[t]{2}{*}{$\begin{array}{l}\text { Sensitivity } \\
\text { coefficient }\end{array}$} & \multirow{2}{*}{$\begin{array}{c}\text { Total data- } \\
\text { induced } \\
\text { uncertainty } \\
\% \Delta \mathrm{k} / \mathrm{k}\end{array}$} \\
\hline & & & & & & & & & \\
\hline 441 & $\begin{array}{l}\text { h-1 } \\
\text { pu-239 } \\
\text { o-16 } \\
\text { na-23 }\end{array}$ & $\begin{array}{l}\text { waste } \\
\text { waste } \\
\text { waste } \\
\mathrm{NaCl} \text { walls }\end{array}$ & $\begin{array}{l}0.5632 \\
0.1138 \\
0.0456 \\
0.0109\end{array}$ & 0.6797 & 442 & $\begin{array}{l}\text { h-1 } \\
\text { pu-239 } \\
\text { be } \\
\text { o-16 } \\
\text { cl-35 }\end{array}$ & $\begin{array}{l}\text { waste } \\
\text { waste } \\
\text { waste } \\
\text { waste } \\
\mathrm{MgO} / \mathrm{NaCl}\end{array}$ & $\begin{array}{r}0.5026 \\
0.1185 \\
0.1039 \\
0.0349 \\
-0.0159\end{array}$ & 0.6258 \\
\hline 443 & $\begin{array}{l}\mathrm{h}-1 \\
\text { h-poly } \\
\text { pu-239 } \\
\text { o-16 } \\
\text { c } \\
\text { cl-35 }\end{array}$ & $\begin{array}{l}\text { waste } \\
\text { poly pipe } \\
\text { waste } \\
\text { waste } \\
\text { poly pipe } \\
\mathrm{MgO} / \mathrm{NaCl}\end{array}$ & $\begin{array}{r}0.3487 \\
0.1051 \\
0.0809 \\
0.0303 \\
0.0199 \\
-0.0107\end{array}$ & 0.6489 & 444 & $\begin{array}{l}\text { h-1 } \\
\text { h-poly } \\
\text { pu-239 } \\
\text { be } \\
\text { o-16 } \\
\text { c } \\
\text { cl-35 }\end{array}$ & $\begin{array}{l}\text { waste } \\
\text { poly pipe } \\
\text { waste } \\
\text { waste } \\
\text { waste } \\
\text { poly pipe } \\
\mathrm{MgO} / \mathrm{NaCl}\end{array}$ & $\begin{array}{r}0.3135 \\
0.0961 \\
0.0834 \\
0.0703 \\
0.0229 \\
0.0179 \\
-0.0161\end{array}$ & 0.6067 \\
\hline 453 & $\begin{array}{l}\text { h-1 } \\
\text { pu-239 } \\
\text { o-16 } \\
\text { na-23 } \\
\text { cl-35 }\end{array}$ & $\begin{array}{l}\text { waste } \\
\text { waste } \\
\text { waste } \\
\mathrm{NaCl} \text { walls } \\
\mathrm{MgO} / \mathrm{NaCl}\end{array}$ & $\begin{array}{r}0.5562 \\
0.1250 \\
0.0432 \\
0.0104 \\
-0.0101\end{array}$ & 0.6718 & 454 & $\begin{array}{l}\text { h-1 } \\
\text { pu-239 } \\
\text { be } \\
\text { o-16 } \\
\text { cl-35 }\end{array}$ & $\begin{array}{l}\text { waste } \\
\text { waste } \\
\text { waste } \\
\text { waste } \\
\mathrm{MgO} / \mathrm{NaCl}\end{array}$ & $\begin{array}{r}0.5001 \\
0.1289 \\
0.0997 \\
0.0335 \\
-0.0174\end{array}$ & 0.6231 \\
\hline 455 & $\begin{array}{l}\text { h-1 } \\
\text { h-poly } \\
\text { pu-239 } \\
\text { o-16 } \\
\text { c } \\
\text { cl-35 }\end{array}$ & $\begin{array}{l}\text { waste } \\
\text { poly pipe } \\
\text { waste } \\
\text { waste } \\
\text { poly pipe } \\
\mathrm{MgO} / \mathrm{NaCl}\end{array}$ & $\begin{array}{r}0.3459 \\
0.1043 \\
0.0904 \\
0.0288 \\
0.0195 \\
-0.0118\end{array}$ & 0.6446 & 456 & $\begin{array}{l}\text { h-1 } \\
\text { h-poly } \\
\text { pu-239 } \\
\text { be } \\
\text { o-16 } \\
\text { c } \\
\text { cl-35 }\end{array}$ & $\begin{array}{l}\text { waste } \\
\text { poly pipe } \\
\text { waste } \\
\text { waste } \\
\text { waste } \\
\text { poly pipe } \\
\mathrm{MgO} / \mathrm{NaCl}\end{array}$ & $\begin{array}{r}0.3133 \\
0.0954 \\
0.0916 \\
0.0680 \\
0.0221 \\
0.0177 \\
-0.0172 \\
\end{array}$ & 0.6077 \\
\hline 477 & $\begin{array}{l}\text { h-1 } \\
\text { pu-239 } \\
\text { c-graphite } \\
\text { cl-35 } \\
\text { o-16 } \\
\text { mg-24 }\end{array}$ & $\begin{array}{l}\text { waste } \\
\text { waste } \\
\text { waste } \\
\mathrm{MgO} / \mathrm{NaCl} \\
\text { waste } \\
\mathrm{MgO} / \mathrm{NaCl}\end{array}$ & $\begin{array}{r}0.5083 \\
0.1425 \\
0.1258 \\
-0.0345 \\
0.0263 \\
0.0103\end{array}$ & 0.6503 & 478 & $\begin{array}{l}\text { h-1 } \\
\text { pu-239 } \\
\text { c-graphite } \\
\text { be } \\
\text { cl-35 } \\
\text { o-16 } \\
\text { mg-24 }\end{array}$ & $\begin{array}{l}\text { waste } \\
\text { waste } \\
\text { waste } \\
\text { waste } \\
\mathrm{MgO} / \mathrm{NaCl} \\
\text { waste } \\
\mathrm{MgO} / \mathrm{NaCl} \\
\end{array}$ & $\begin{array}{r}0.4728 \\
0.1477 \\
0.1063 \\
0.0719 \\
-0.0433 \\
0.0223 \\
0.0109 \\
\end{array}$ & 0.6175 \\
\hline 479 & $\begin{array}{l}\text { h-1 } \\
\text { h-poly } \\
\text { pu-239 } \\
\text { c-graphite } \\
\text { cl-35 } \\
\text { c } \\
\text { o-16 }\end{array}$ & $\begin{array}{l}\text { waste } \\
\text { poly pipe } \\
\text { waste } \\
\text { waste } \\
\mathrm{MgO} / \mathrm{NaCl} \\
\text { poly pipe } \\
\text { waste }\end{array}$ & $\begin{array}{r}0.3016 \\
0.1030 \\
0.0967 \\
0.0776 \\
-0.0286 \\
0.0172 \\
0.0166\end{array}$ & 0.6249 & 480 & $\begin{array}{l}\text { h-1 } \\
\text { pu-239 } \\
\text { h-poly } \\
\text { c-graphite } \\
\text { be } \\
\text { cl-35 } \\
\text { c } \\
\text { o-16 }\end{array}$ & $\begin{array}{l}\text { waste } \\
\text { waste } \\
\text { poly pipe } \\
\text { waste } \\
\text { waste } \\
\mathrm{MgO} / \mathrm{NaCl} \\
\text { poly pipe } \\
\text { waste }\end{array}$ & $\begin{array}{r}0.2819 \\
0.0996 \\
0.0975 \\
0.0648 \\
0.0467 \\
-0.0341 \\
0.0165 \\
0.0139 \\
\end{array}$ & 0.6009 \\
\hline 489 & $\begin{array}{l}\text { h-1 } \\
\text { pu-239 } \\
\text { c-graphite } \\
\text { cl-35 } \\
\text { o-16 } \\
\text { mg-24 }\end{array}$ & $\begin{array}{l}\text { waste } \\
\text { waste } \\
\text { waste } \\
\mathrm{MgO} / \mathrm{NaCl} \\
\text { waste } \\
\mathrm{MgO} / \mathrm{NaCl}\end{array}$ & $\begin{array}{r}0.5048 \\
0.1530 \\
0.1218 \\
-0.0364 \\
0.0255 \\
0.0104\end{array}$ & 0.6483 & 490 & $\begin{array}{l}\text { h-1 } \\
\text { pu-239 } \\
\text { c-graphite } \\
\text { be } \\
\text { cl-35 } \\
\text { o-16 } \\
\text { mg-24 }\end{array}$ & $\begin{array}{l}\text { waste } \\
\text { waste } \\
\text { waste } \\
\text { waste } \\
\mathrm{MgO} / \mathrm{NaCl} \\
\text { waste } \\
\mathrm{MgO} / \mathrm{NaCl}\end{array}$ & $\begin{array}{r}0.4703 \\
0.1580 \\
0.1036 \\
0.0702 \\
-0.0449 \\
0.0217 \\
0.0112 \\
\end{array}$ & 0.6175 \\
\hline 491 & $\begin{array}{l}\text { h-1 } \\
\text { pu-239 } \\
\text { h-poly } \\
\text { c-graphite } \\
\text { cl-35 } \\
\text { c } \\
\text { o-16 }\end{array}$ & $\begin{array}{l}\text { waste } \\
\text { waste } \\
\text { poly pipe } \\
\text { waste } \\
\mathrm{MgO} / \mathrm{NaCl} \\
\text { poly pipe } \\
\text { waste }\end{array}$ & $\begin{array}{r}0.3001 \\
0.1069 \\
0.1013 \\
0.0753 \\
-0.0298 \\
0.0171 \\
0.0160\end{array}$ & 0.6241 & 492 & $\begin{array}{l}\text { h-1 } \\
\text { pu-239 } \\
\text { h-poly } \\
\text { c-graphite } \\
\text { be } \\
\text { cl-35 } \\
\text { c } \\
\text { o-16 }\end{array}$ & $\begin{array}{l}\text { waste } \\
\text { waste } \\
\text { poly pipe } \\
\text { waste } \\
\text { waste } \\
\mathrm{MgO} / \mathrm{NaCl} \\
\text { poly pipe } \\
\text { waste }\end{array}$ & $\begin{array}{r}0.2809 \\
0.1094 \\
0.0963 \\
0.0634 \\
0.0457 \\
-0.0352 \\
0.0163 \\
0.0135\end{array}$ & 0.6023 \\
\hline
\end{tabular}


Table H-20. Nuclides with the highest sensitivities for set-1-4 cases

\begin{tabular}{|c|c|c|c|c|c|c|c|c|c|}
\hline \multirow[t]{2}{*}{$\begin{array}{c}\text { Case } \\
\text { Number } \\
\end{array}$} & \multirow[t]{2}{*}{ Nuclide } & \multirow[t]{2}{*}{ Mixture } & \multirow[t]{2}{*}{$\begin{array}{l}\text { Sensitivity } \\
\text { coefficient }\end{array}$} & \multirow{2}{*}{$\begin{array}{c}\text { Total data- } \\
\text { induced } \\
\text { uncertainty } \\
\% \Delta \mathrm{k} / \mathrm{k} \\
\end{array}$} & \multirow{2}{*}{\begin{tabular}{|c} 
Case \\
Number
\end{tabular}} & \multirow[t]{2}{*}{ Nuclide } & \multirow[t]{2}{*}{ Mixture } & \multirow[t]{2}{*}{$\begin{array}{l}\text { Sensitivity } \\
\text { coefficient }\end{array}$} & \multirow{2}{*}{$\begin{array}{c}\text { Total data- } \\
\text { induced } \\
\text { uncertainty } \\
\% \Delta \mathrm{k} / \mathrm{k}\end{array}$} \\
\hline & & & & & & & & & \\
\hline 333 & $\begin{array}{l}\text { h-poly } \\
\text { pu-239 } \\
\text { c }\end{array}$ & $\begin{array}{l}\text { waste } \\
\text { waste } \\
\text { waste }\end{array}$ & $\begin{array}{l}0.5361 \\
0.1074 \\
0.0527\end{array}$ & 0.6703 & 334 & $\begin{array}{l}\text { h-poly } \\
\text { pu-239 } \\
\text { be } \\
\text { c } \\
\text { cl-35 }\end{array}$ & $\begin{array}{l}\text { waste } \\
\text { waste } \\
\text { waste } \\
\text { waste } \\
\mathrm{MgO} / \mathrm{NaCl}\end{array}$ & $\begin{array}{r}0.4854 \\
0.1071 \\
0.1058 \\
0.0394 \\
-0.0119 \\
\end{array}$ & 0.6217 \\
\hline 335 & $\begin{array}{l}\text { h-poly } \\
\text { h-poly } \\
\text { pu-239 } \\
\text { c } \\
\text { c } \\
\text { cl-35 }\end{array}$ & $\begin{array}{l}\text { waste } \\
\text { poly pipe } \\
\text { waste } \\
\text { waste } \\
\text { poly pipe } \\
\mathrm{MgO} / \mathrm{NaCl}\end{array}$ & $\begin{array}{r}0.3436 \\
0.0990 \\
0.0773 \\
0.0349 \\
0.0201 \\
-0.0081\end{array}$ & 0.6535 & 336 & $\begin{array}{l}\text { h-poly } \\
\text { h-poly } \\
\text { pu-239 } \\
\text { be } \\
\text { c } \\
\text { c } \\
\text { cl-35 }\end{array}$ & $\begin{array}{l}\text { waste } \\
\text { poly pipe } \\
\text { waste } \\
\text { waste } \\
\text { waste } \\
\text { poly pipe } \\
\mathrm{MgO} / \mathrm{NaCl}\end{array}$ & $\begin{array}{r}0.3096 \\
0.0901 \\
0.0797 \\
0.0729 \\
0.0261 \\
0.0179 \\
-0.0131\end{array}$ & 0.6124 \\
\hline 345 & $\begin{array}{l}\text { h-poly } \\
\text { pu-239 } \\
\text { c }\end{array}$ & $\begin{array}{l}\text { waste } \\
\text { waste } \\
\text { waste }\end{array}$ & $\begin{array}{l}0.5331 \\
0.1164 \\
0.0501\end{array}$ & 0.6648 & 346 & $\begin{array}{l}\text { h-poly } \\
\text { pu-239 } \\
\text { be } \\
\text { c } \\
\text { cl-35 }\end{array}$ & $\begin{array}{l}\text { waste } \\
\text { waste } \\
\text { waste } \\
\text { waste } \\
\mathrm{MgO} / \mathrm{NaCl}\end{array}$ & $\begin{array}{r}0.4835 \\
0.1179 \\
0.1017 \\
0.0378 \\
-0.0134\end{array}$ & 0.6197 \\
\hline 347 & $\begin{array}{l}\text { h-poly } \\
\text { h-poly } \\
\text { pu-239 } \\
\text { c } \\
\text { c }\end{array}$ & $\begin{array}{l}\text { waste } \\
\text { poly pipe } \\
\text { waste } \\
\text { waste } \\
\text { poly pipe }\end{array}$ & $\begin{array}{l}0.3418 \\
0.0978 \\
0.0861 \\
0.0332 \\
0.0196\end{array}$ & 0.6491 & 348 & $\begin{array}{l}\text { h-poly } \\
\text { pu-239 } \\
\text { h-poly } \\
\text { be } \\
\text { c } \\
\text { c } \\
\text { cl-35 }\end{array}$ & $\begin{array}{l}\text { waste } \\
\text { waste } \\
\text { poly pipe } \\
\text { waste } \\
\text { waste } \\
\text { poly pipe } \\
\mathrm{MgO} / \mathrm{NaCl}\end{array}$ & $\begin{array}{r}0.3088 \\
0.0889 \\
0.0877 \\
0.0700 \\
0.0250 \\
0.0176 \\
-0.0142 \\
\end{array}$ & 0.6112 \\
\hline 369 & $\begin{array}{l}\text { h-poly } \\
\text { pu-239 } \\
\text { c-graphite } \\
\text { c } \\
\text { cl-35 }\end{array}$ & $\begin{array}{l}\text { waste } \\
\text { waste } \\
\text { waste } \\
\text { waste } \\
\mathrm{MgO} / \mathrm{NaCl}\end{array}$ & $\begin{array}{r}0.4910 \\
0.1350 \\
0.1273 \\
0.0307 \\
-0.0292\end{array}$ & 0.6484 & 370 & $\begin{array}{l}\text { h-poly } \\
\text { pu-239 } \\
\text { c-graphite } \\
\text { be } \\
\text { cl-35 } \\
\text { c } \\
\end{array}$ & $\begin{array}{l}\text { waste } \\
\text { waste } \\
\text { waste } \\
\text { waste } \\
\mathrm{MgO} / \mathrm{NaCl} \\
\text { waste }\end{array}$ & $\begin{array}{r}0.4581 \\
0.1400 \\
0.1067 \\
0.0719 \\
-0.0379 \\
0.0257 \\
\end{array}$ & 0.6157 \\
\hline 371 & $\begin{array}{l}\text { h-poly } \\
\text { h-poly } \\
\text { pu-239 } \\
\text { c-graphite } \\
\text { cl-35 } \\
\text { c } \\
\text { c }\end{array}$ & $\begin{array}{l}\text { waste } \\
\text { poly pipe } \\
\text { waste } \\
\text { waste } \\
\mathrm{MgO} / \mathrm{NaCl} \\
\text { waste } \\
\text { poly pipe }\end{array}$ & $\begin{array}{r}0.3002 \\
0.0954 \\
0.0937 \\
0.0812 \\
-0.0252 \\
0.0197 \\
0.0171\end{array}$ & 0.6307 & 372 & $\begin{array}{l}\text { h-poly } \\
\text { pu-239 } \\
\text { h-poly } \\
\text { c-graphite } \\
\text { be } \\
\text { cl-35 } \\
\text { c } \\
\text { c }\end{array}$ & $\begin{array}{l}\text { waste } \\
\text { waste } \\
\text { poly pipe } \\
\text { waste } \\
\text { waste } \\
\mathrm{MgO} / \mathrm{NaCl} \\
\text { waste } \\
\text { poly pipe }\end{array}$ & $\begin{array}{r}0.2799 \\
0.0974 \\
0.0899 \\
0.0675 \\
0.0480 \\
-0.0307 \\
0.0164 \\
0.0162 \\
\end{array}$ & 0.6061 \\
\hline 381 & $\begin{array}{l}\text { h-poly } \\
\text { pu-239 } \\
\text { c-graphite } \\
\text { cl-35 } \\
\text { c }\end{array}$ & $\begin{array}{l}\text { waste } \\
\text { waste } \\
\text { waste } \\
\mathrm{MgO} / \mathrm{NaCl} \\
\text { waste }\end{array}$ & $\begin{array}{r}0.4923 \\
0.1422 \\
0.1238 \\
-0.0312 \\
0.0299\end{array}$ & 0.6458 & 382 & $\begin{array}{l}\text { h-poly } \\
\text { pu-239 } \\
\text { c-graphite } \\
\text { be } \\
\text { cl-35 } \\
\text { c } \\
\text { mg-24 }\end{array}$ & $\begin{array}{l}\text { waste } \\
\text { waste } \\
\text { waste } \\
\text { waste } \\
\mathrm{MgO} / \mathrm{NaCl} \\
\text { waste } \\
\mathrm{MgO} / \mathrm{NaCl}\end{array}$ & $\begin{array}{r}0.4596 \\
0.1476 \\
0.1048 \\
0.0705 \\
-0.0396 \\
0.0253 \\
0.0100 \\
\end{array}$ & 0.6162 \\
\hline 383 & $\begin{array}{l}\text { h-poly } \\
\text { pu-239 } \\
\text { h-poly } \\
\text { c-graphite } \\
\text { cl-35 } \\
\text { c } \\
\text { c }\end{array}$ & $\begin{array}{l}\text { waste } \\
\text { waste } \\
\text { poly pipe } \\
\text { waste } \\
\mathrm{MgO} / \mathrm{NaCl} \\
\text { waste } \\
\text { poly pipe }\end{array}$ & $\begin{array}{r}0.2979 \\
0.1048 \\
0.0947 \\
0.0788 \\
-0.0263 \\
0.0191 \\
0.0169\end{array}$ & 0.6311 & 384 & $\begin{array}{l}\text { h-poly } \\
\text { pu-239 } \\
\text { h-poly } \\
\text { c-graphite } \\
\text { be } \\
\text { cl-35 } \\
\text { c } \\
\text { c }\end{array}$ & $\begin{array}{l}\text { waste } \\
\text { waste } \\
\text { poly pipe } \\
\text { waste } \\
\text { waste } \\
\mathrm{MgO} / \mathrm{NaCl} \\
\text { poly pipe } \\
\text { waste }\end{array}$ & $\begin{array}{r}0.2797 \\
0.1063 \\
0.0884 \\
0.0659 \\
0.0469 \\
-0.0318 \\
0.0162 \\
0.0160\end{array}$ & 0.6071 \\
\hline
\end{tabular}


Table H-21. Nuclides with the highest sensitivities for set-1-5 cases

\begin{tabular}{|c|c|c|c|c|c|c|c|c|c|}
\hline \multirow[t]{2}{*}{$\begin{array}{c}\text { Case } \\
\text { Number } \\
\end{array}$} & \multirow[t]{2}{*}{ Nuclide } & \multirow[t]{2}{*}{ Mixture } & \multirow[t]{2}{*}{$\begin{array}{l}\text { Sensitivity } \\
\text { coefficient }\end{array}$} & \multirow{2}{*}{$\begin{array}{c}\text { Total data- } \\
\text { induced } \\
\text { uncertainty } \\
\% \Delta \mathrm{k} / \mathrm{k}\end{array}$} & $\begin{array}{c}\text { Case } \\
\text { Number }\end{array}$ & Nuclide & Mixture & $\begin{array}{l}\text { Sensitivity } \\
\text { coefficient }\end{array}$ & \multirow{2}{*}{$\begin{array}{c}\text { Total data- } \\
\text { induced } \\
\text { uncertainty } \\
\% \Delta \mathrm{k} / \mathrm{k}\end{array}$} \\
\hline & & & & & \multicolumn{3}{|l|}{ With Be } & & \\
\hline \multirow[t]{5}{*}{657} & h-1 & waste & 0.5017 & \multirow[t]{5}{*}{0.6705} & \multirow[t]{5}{*}{658} & h-1 & waste & 0.4628 & \multirow[t]{5}{*}{0.6287} \\
\hline & pu-239 & waste & 0.0932 & & & pu-239 & waste & 0.0985 & \\
\hline & $0-16$ & waste & 0.0405 & & & be & waste & 0.0720 & \\
\hline & $\mathrm{cl}-35$ & $\mathrm{MgO} / \mathrm{NaCl}$ & -0.0134 & & & $0-16$ & waste & 0.0322 & \\
\hline & na-23 & $\mathrm{NaCl}$ walls & 0.0080 & & & $\mathrm{cl}-35$ & $\mathrm{MgO} / \mathrm{NaCl}$ & -0.0201 & \\
\hline \multirow[t]{6}{*}{659} & h-1 & waste & 0.4727 & \multirow[t]{6}{*}{0.6627} & \multirow[t]{6}{*}{660} & h-1 & waste & 0.4439 & \multirow[t]{6}{*}{0.6313} \\
\hline & pu-239 & waste & 0.1057 & & & pu-239 & waste & 0.1081 & \\
\hline & o-16 & waste & 0.0315 & & & be & waste & 0.0607 & \\
\hline & fe-56 & ss pipe & 0.0220 & & & $0-16$ & waste & 0.0256 & \\
\hline & $\mathrm{cl}-35$ & $\mathrm{MgO} / \mathrm{NaCl}$ & -0.0161 & & & $\mathrm{cl}-35$ & $\mathrm{MgO} / \mathrm{NaCl}$ & -0.0216 & \\
\hline & & & & & & fe-56 & ss pipe & 0.0206 & \\
\hline \multirow[t]{5}{*}{669} & h-1 & waste & 0.4986 & \multirow[t]{5}{*}{0.6685} & \multirow[t]{5}{*}{670} & h-1 & waste & 0.4609 & \multirow[t]{5}{*}{0.6268} \\
\hline & pu-239 & waste & 0.1030 & & & pu-239 & waste & 0.1093 & \\
\hline & o-16 & waste & 0.0388 & & & be & waste & 0.0696 & \\
\hline & $\mathrm{cl}-35$ & $\mathrm{MgO} / \mathrm{NaCl}$ & -0.0147 & & & $0-16$ & waste & 0.0309 & \\
\hline & & & & & & cl-35 & $\mathrm{MgO} / \mathrm{NaCl}$ & -0.0216 & \\
\hline 671 & h-1 & waste & 0.4745 & 0.6698 & 672 & h-1 & waste & 0.4450 & 0.6397 \\
\hline & pu-239 & waste & 0.1128 & & & pu-239 & waste & 0.1162 & \\
\hline & $0-16$ & waste & 0.0304 & & & be & waste & 0.0592 & \\
\hline & fe-56 & ss pipe & 0.0219 & & & $0-16$ & waste & 0.0250 & \\
\hline & $\mathrm{cl}-35$ & $\mathrm{MgO} / \mathrm{NaCl}$ & -0.0172 & & & $\mathrm{cl}-35$ & $\mathrm{MgO} / \mathrm{NaCl}$ & -0.0228 & \\
\hline & & & & & & fe-56 & ss pipe & 0.0205 & \\
\hline 693 & h-1 & waste & 0.5104 & 0.6626 & 694 & h-1 & waste & 0.4798 & 0.6324 \\
\hline & pu-239 & waste & 0.1245 & & & pu-239 & waste & 0.1298 & \\
\hline & $0-16$ & waste & 0.0562 & & & be & waste & 0.0584 & \\
\hline & $\mathrm{cl}-35$ & $\mathrm{MgO} / \mathrm{NaCl}$ & -0.0345 & & & $0-16$ & waste & 0.0484 & \\
\hline & $m g-24$ & $\mathrm{MgO} / \mathrm{NaCl}$ & 0.0093 & & & $\mathrm{cl}-35$ & $\mathrm{MgO} / \mathrm{NaCl}$ & -0.0419 & \\
\hline & na-23 & $\mathrm{MgO} / \mathrm{NaCl}$ & 0.0083 & & & $\mathrm{mg}-24$ & $\mathrm{MgO} / \mathrm{NaCl}$ & 0.0101 & \\
\hline & na-23 & $\mathrm{NaCl}$ walls & 0.0080 & & & na-23 & $\mathrm{MgO} / \mathrm{NaCl}$ & 0.0081 & \\
\hline 695 & h-1 & waste & 0.4876 & 0.6624 & 696 & h-1 & waste & 0.4594 & 0.6387 \\
\hline & pu-239 & waste & 0.1298 & & & pu-239 & waste & 0.1360 & \\
\hline & $0-16$ & waste & 0.0464 & & & be & waste & 0.0508 & \\
\hline & $\mathrm{cl}-35$ & $\mathrm{MgO} / \mathrm{NaCl}$ & -0.0335 & & & $0-16$ & waste & 0.0404 & \\
\hline & fe-56 & ss pipe & 0.0241 & & & $\mathrm{cl}-35$ & $\mathrm{MgO} / \mathrm{NaCl}$ & -0.0394 & \\
\hline & & & & & & fe-56 & ss pipe & 0.0232 & \\
\hline 705 & h-1 & waste & 0.5061 & 0.6616 & 706 & h-1 & waste & 0.4785 & 0.6305 \\
\hline & pu-239 & waste & 0.1354 & & & pu-239 & waste & 0.1394 & \\
\hline & $0-16$ & waste & 0.0546 & & & be & waste & 0.0570 & \\
\hline & $\mathrm{cl}-35$ & $\mathrm{MgO} / \mathrm{NaCl}$ & -0.0359 & & & $0-16$ & waste & 0.0471 & \\
\hline & $\mathrm{mg}-24$ & $\mathrm{MgO} / \mathrm{NaCl}$ & 0.0094 & & & $\mathrm{cl}-35$ & $\mathrm{MgO} / \mathrm{NaCl}$ & -0.0434 & \\
\hline & na-23 & $\mathrm{MgO} / \mathrm{NaCl}$ & 0.0082 & & & $m g-24$ & $\mathrm{MgO} / \mathrm{NaCl}$ & 0.0101 & \\
\hline & & & & & & na-23 & $\mathrm{MgO} / \mathrm{NaCl}$ & 0.0080 & \\
\hline 707 & h-1 & waste & 0.4844 & 0.6688 & 708 & h-1 & waste & 0.4601 & 0.6488 \\
\hline & pu-239 & waste & 0.1404 & & & pu-239 & waste & 0.1437 & \\
\hline & $0-16$ & waste & 0.0451 & & & be & waste & 0.0500 & \\
\hline & $\mathrm{cl}-35$ & $\mathrm{MgO} / \mathrm{NaCl}$ & -0.0347 & & & $\mathrm{cl}-35$ & $\mathrm{MgO} / \mathrm{NaCl}$ & -0.0408 & \\
\hline & fe-56 & ss pipe & 0.0239 & & & $0-16$ & waste & 0.0396 & \\
\hline & & & & & & fe-56 & ss pipe & 0.0233 & \\
\hline
\end{tabular}


Table H-22. Nuclides with the highest sensitivities for set-1-6 cases

\begin{tabular}{|c|c|c|c|c|c|c|c|c|c|}
\hline \multirow[t]{2}{*}{$\begin{array}{c}\text { Case } \\
\text { Number } \\
\end{array}$} & \multirow[t]{2}{*}{ Nuclide } & \multirow[t]{2}{*}{ Mixture } & \multirow[t]{2}{*}{$\begin{array}{l}\text { Sensitivity } \\
\text { coefficient }\end{array}$} & \multirow{2}{*}{$\begin{array}{c}\text { Total data- } \\
\text { induced } \\
\text { uncertainty } \\
\% \Delta \mathrm{k} / \mathrm{k}\end{array}$} & \multirow{2}{*}{\begin{tabular}{|c|c}
$\begin{array}{c}\text { Case } \\
\text { Number }\end{array}$ \\
With Be
\end{tabular}} & \multirow[t]{2}{*}{ Nuclide } & \multirow[t]{2}{*}{ Mixture } & \multirow[t]{2}{*}{$\begin{array}{l}\text { Sensitivity } \\
\text { coefficient }\end{array}$} & \multirow{2}{*}{$\begin{array}{c}\text { Total data- } \\
\text { induced } \\
\text { uncertainty } \\
\% \Delta \mathrm{k} / \mathrm{k}\end{array}$} \\
\hline & & & & & & & & & \\
\hline 441 & $\begin{array}{l}\text { h-poly } \\
\text { pu-239 } \\
\text { c }\end{array}$ & $\begin{array}{l}\text { waste } \\
\text { waste } \\
\text { waste }\end{array}$ & $\begin{array}{l}0.4969 \\
0.0890 \\
0.0505\end{array}$ & 0.6674 & 442 & $\begin{array}{l}\text { h-poly } \\
\text { pu-239 } \\
\text { be } \\
\text { c } \\
\text { cl-35 }\end{array}$ & $\begin{array}{l}\text { waste } \\
\text { waste } \\
\text { waste } \\
\text { waste } \\
\mathrm{MgO} / \mathrm{NaCl}\end{array}$ & $\begin{array}{r}0.4555 \\
0.0941 \\
0.0830 \\
0.0386 \\
-0.0137\end{array}$ & 0.6236 \\
\hline 443 & $\begin{array}{l}\text { h-poly } \\
\text { pu-239 } \\
\text { c } \\
\text { fe-56 } \\
\text { cl-35 }\end{array}$ & $\begin{array}{l}\text { waste } \\
\text { waste } \\
\text { waste } \\
\text { ss pipe } \\
\mathrm{MgO} / \mathrm{NaCl}\end{array}$ & $\begin{array}{c}0.4720 \\
0.1002 \\
0.0388 \\
0.0231 \\
-0.0110\end{array}$ & 0.6623 & 444 & $\begin{array}{l}\text { h-poly } \\
\text { pu-239 } \\
\text { be } \\
\text { c } \\
\text { fe-56 } \\
\text { cl-35 }\end{array}$ & $\begin{array}{l}\text { waste } \\
\text { waste } \\
\text { waste } \\
\text { waste } \\
\text { ss pipe } \\
\mathrm{MgO} / \mathrm{NaCl}\end{array}$ & $\begin{array}{c}0.4368 \\
0.1062 \\
0.0688 \\
0.0306 \\
0.0208 \\
-0.0165\end{array}$ & 0.6280 \\
\hline 453 & $\begin{array}{l}\text { h-poly } \\
\text { pu-239 } \\
\text { c } \\
\text { cl-35 }\end{array}$ & $\begin{array}{l}\text { waste } \\
\text { waste } \\
\text { waste } \\
\mathrm{MgO} / \mathrm{NaCl}\end{array}$ & $\begin{array}{r}0.4948 \\
0.0988 \\
0.0482 \\
-0.0089\end{array}$ & 0.6632 & 454 & $\begin{array}{l}\text { h-poly } \\
\text { pu-239 } \\
\text { be } \\
\text { c } \\
\text { cl-35 }\end{array}$ & $\begin{array}{l}\text { waste } \\
\text { waste } \\
\text { waste } \\
\text { waste } \\
\mathrm{MgO} / \mathrm{NaCl}\end{array}$ & $\begin{array}{r}0.4556 \\
0.1033 \\
0.0801 \\
0.0372 \\
-0.0152 \\
\end{array}$ & 0.6233 \\
\hline 455 & $\begin{array}{l}\text { h-poly } \\
\text { pu-239 } \\
\text { c } \\
\text { fe-56 } \\
\text { cl-35 }\end{array}$ & $\begin{array}{l}\text { waste } \\
\text { waste } \\
\text { waste } \\
\text { ss pipe } \\
\mathrm{MgO} / \mathrm{NaCl}\end{array}$ & $\begin{array}{r}0.4714 \\
0.1095 \\
0.0373 \\
0.0226 \\
-0.0122\end{array}$ & 0.6671 & 456 & $\begin{array}{l}\text { h-poly } \\
\text { pu-239 } \\
\text { be } \\
\text { c } \\
\text { fe-56 } \\
\text { cl-35 }\end{array}$ & $\begin{array}{l}\text { waste } \\
\text { waste } \\
\text { waste } \\
\text { waste } \\
\text { ss pipe } \\
\mathrm{MgO} / \mathrm{NaCl}\end{array}$ & $\begin{array}{r}0.4374 \\
0.1152 \\
0.0671 \\
0.0298 \\
0.0207 \\
-0.0176 \\
\end{array}$ & 0.6371 \\
\hline 477 & $\begin{array}{l}\text { h-poly } \\
\text { pu-239 } \\
\text { o-16 } \\
\text { c } \\
\text { cl-35 } \\
\text { mg-24 } \\
\text { na-23 } \\
\text { si-28 }\end{array}$ & $\begin{array}{l}\text { waste } \\
\text { waste } \\
\text { waste } \\
\text { waste } \\
\mathrm{MgO} / \mathrm{NaCl} \\
\mathrm{MgO} / \mathrm{NaCl} \\
\mathrm{NaCl} \text { walls } \\
\text { waste }\end{array}$ & $\begin{array}{r}0.5068 \\
0.1223 \\
0.0361 \\
0.0349 \\
-0.0278 \\
0.0084 \\
0.0083 \\
0.0081 \\
\end{array}$ & 0.6564 & 478 & $\begin{array}{l}\text { h-poly } \\
\text { pu-239 } \\
\text { be } \\
\text { cl-35 } \\
\text { o-16 } \\
\text { c } \\
\text { mg-24 }\end{array}$ & $\begin{array}{l}\text { waste } \\
\text { waste } \\
\text { waste } \\
\mathrm{MgO} / \mathrm{NaCl} \\
\text { waste } \\
\text { waste } \\
\mathrm{MgO} / \mathrm{NaCl}\end{array}$ & $\begin{array}{r}0.4717 \\
0.1287 \\
0.0652 \\
-0.0353 \\
0.0304 \\
0.0292 \\
0.0092\end{array}$ & 0.6273 \\
\hline 479 & $\begin{array}{l}\text { h-poly } \\
\text { pu-239 } \\
\text { o-16 } \\
\text { c } \\
\text { cl-35 } \\
\text { fe-56 }\end{array}$ & $\begin{array}{l}\text { waste } \\
\text { waste } \\
\text { waste } \\
\text { waste } \\
\mathrm{MgO} / \mathrm{NaCl} \\
\text { ss pipe }\end{array}$ & $\begin{array}{r}0.4841 \\
0.1293 \\
0.0295 \\
0.0289 \\
-0.0283 \\
0.0240\end{array}$ & 0.6583 & 480 & $\begin{array}{l}\text { h-poly } \\
\text { pu-239 } \\
\text { be } \\
\text { cl-35 } \\
\text { o-16 } \\
\text { c } \\
\text { fe-56 }\end{array}$ & $\begin{array}{l}\text { waste } \\
\text { waste } \\
\text { waste } \\
\mathrm{MgO} / \mathrm{NaCl} \\
\text { waste } \\
\text { waste } \\
\text { ss pipe } \\
\end{array}$ & $\begin{array}{r}0.4565 \\
0.1328 \\
0.0566 \\
-0.0345 \\
0.0253 \\
0.0246 \\
0.0232 \\
\end{array}$ & 0.6349 \\
\hline 489 & $\begin{array}{l}\text { h-poly } \\
\text { pu-239 } \\
\text { o-16 } \\
\text { c } \\
\text { cl-35 } \\
\text { mg-24 } \\
\text { na-23 }\end{array}$ & $\begin{array}{l}\text { waste } \\
\text { waste } \\
\text { waste } \\
\text { waste } \\
\mathrm{MgO} / \mathrm{NaCl} \\
\mathrm{MgO} / \mathrm{NaCl} \\
\mathrm{NaCl} \text { walls }\end{array}$ & $\begin{array}{c}0.5059 \\
0.1307 \\
0.0352 \\
0.0339 \\
-0.0292 \\
0.0086 \\
0.0081 \\
\end{array}$ & 0.6548 & 490 & $\begin{array}{l}\text { h-poly } \\
\text { pu-239 } \\
\text { be } \\
\text { cl-35 } \\
\text { o-16 } \\
\text { c } \\
\text { mg-24 }\end{array}$ & $\begin{array}{l}\text { waste } \\
\text { waste } \\
\text { waste } \\
\mathrm{MgO} / \mathrm{NaCl} \\
\text { waste } \\
\text { waste } \\
\mathrm{MgO} / \mathrm{NaCl}\end{array}$ & $\begin{array}{r}0.4733 \\
0.1365 \\
0.0635 \\
-0.0371 \\
0.0296 \\
0.0285 \\
0.0093 \\
\end{array}$ & 0.6251 \\
\hline 491 & $\begin{array}{l}\text { h-poly } \\
\text { pu-239 } \\
\text { cl-35 } \\
\text { o-16 } \\
\text { c } \\
\text { fe-56 }\end{array}$ & $\begin{array}{l}\text { waste } \\
\text { waste } \\
\mathrm{MgO} / \mathrm{NaCl} \\
\text { waste } \\
\text { waste } \\
\text { ss pipe }\end{array}$ & $\begin{array}{r}0.4837 \\
0.1379 \\
-0.0295 \\
0.0288 \\
0.0282 \\
0.0241\end{array}$ & 0.6672 & 492 & $\begin{array}{l}\text { h-poly } \\
\text { pu-239 } \\
\text { be } \\
\text { cl-35 } \\
\text { o-16 } \\
\text { c } \\
\text { fe-56 }\end{array}$ & $\begin{array}{l}\text { waste } \\
\text { waste } \\
\text { waste } \\
\mathrm{MgO} / \mathrm{NaCl} \\
\text { waste } \\
\text { waste } \\
\text { ss pipe }\end{array}$ & $\begin{array}{r}0.4560 \\
0.1421 \\
0.0555 \\
-0.0359 \\
0.0248 \\
0.0241 \\
0.0231\end{array}$ & 0.6432 \\
\hline
\end{tabular}


Table H-23. Nuclides with the highest sensitivities for set-1-7 cases

\begin{tabular}{|c|c|c|c|c|c|c|c|c|c|}
\hline \multirow[t]{2}{*}{$\begin{array}{c}\text { Case } \\
\text { Number } \\
\end{array}$} & \multirow[t]{2}{*}{ Nuclide } & \multirow[t]{2}{*}{ Mixture } & \multirow[t]{2}{*}{$\begin{array}{l}\text { Sensitivity } \\
\text { coefficient }\end{array}$} & \multirow{2}{*}{$\begin{array}{c}\text { Total data- } \\
\text { induced } \\
\text { uncertainty } \\
\% \Delta \mathrm{k} / \mathrm{k} \\
\end{array}$} & \multirow{2}{*}{\begin{tabular}{|c} 
Case \\
Number
\end{tabular}} & \multirow[t]{2}{*}{ Nuclide } & \multirow[t]{2}{*}{ Mixture } & \multirow[t]{2}{*}{$\begin{array}{l}\text { Sensitivity } \\
\text { coefficient }\end{array}$} & \multirow{2}{*}{$\begin{array}{c}\text { Total data- } \\
\text { induced } \\
\text { uncertainty } \\
\% \Delta \mathrm{k} / \mathrm{k}\end{array}$} \\
\hline & & & & & & & & & \\
\hline 441 & $\begin{array}{l}\text { h-1 } \\
\text { pu-239 } \\
\text { o-16 } \\
\text { na-23 }\end{array}$ & $\begin{array}{l}\text { waste } \\
\text { waste } \\
\text { waste } \\
\mathrm{NaCl} \text { walls }\end{array}$ & $\begin{array}{l}0.5632 \\
0.1138 \\
0.0456 \\
0.0109\end{array}$ & 0.6797 & 442 & $\begin{array}{l}\text { h-1 } \\
\text { pu-239 } \\
\text { be } \\
\text { o-16 } \\
\text { cl-35 }\end{array}$ & $\begin{array}{l}\text { waste } \\
\text { waste } \\
\text { waste } \\
\text { waste } \\
\mathrm{MgO} / \mathrm{NaCl}\end{array}$ & $\begin{array}{r}0.5026 \\
0.1185 \\
0.1039 \\
0.0349 \\
-0.0159 \\
\end{array}$ & 0.6258 \\
\hline 443 & $\begin{array}{l}\text { h-1 } \\
\text { h-poly } \\
\text { pu-239 } \\
\text { o-16 } \\
\text { c } \\
\text { cl-35 }\end{array}$ & $\begin{array}{l}\text { waste } \\
\text { poly pipe } \\
\text { waste } \\
\text { waste } \\
\text { poly pipe } \\
\mathrm{MgO} / \mathrm{NaCl}\end{array}$ & $\begin{array}{l}0.3487 \\
0.1051 \\
0.0809 \\
0.0303 \\
0.0199 \\
-0.0107\end{array}$ & 0.6489 & 444 & $\begin{array}{l}\text { h-1 } \\
\text { h-poly } \\
\text { pu-239 } \\
\text { be } \\
\text { o-16 } \\
\text { c } \\
\text { cl-35 }\end{array}$ & $\begin{array}{l}\text { waste } \\
\text { poly pipe } \\
\text { waste } \\
\text { waste } \\
\text { waste } \\
\text { poly pipe } \\
\mathrm{MgO} / \mathrm{NaCl}\end{array}$ & $\begin{array}{r}0.3135 \\
0.0961 \\
0.0834 \\
0.0703 \\
0.0229 \\
0.0179 \\
-0.0161 \\
\end{array}$ & 0.6067 \\
\hline 453 & $\begin{array}{l}\text { h-1 } \\
\text { pu-239 } \\
\text { o-16 } \\
\text { na-23 } \\
\text { cl-35 }\end{array}$ & $\begin{array}{l}\text { waste } \\
\text { waste } \\
\text { waste } \\
\mathrm{NaCl} \text { walls } \\
\mathrm{MgO} / \mathrm{NaCl}\end{array}$ & $\begin{array}{r}0.5562 \\
0.1250 \\
0.0432 \\
0.0104 \\
-0.0101\end{array}$ & 0.6718 & 454 & $\begin{array}{l}\text { h-1 } \\
\text { pu-239 } \\
\text { be } \\
\text { o-16 } \\
\text { cl-35 }\end{array}$ & $\begin{array}{l}\text { waste } \\
\text { waste } \\
\text { waste } \\
\text { waste } \\
\mathrm{MgO} / \mathrm{NaCl}\end{array}$ & $\begin{array}{r}0.5001 \\
0.1289 \\
0.0997 \\
0.0335 \\
-0.0174\end{array}$ & 0.6231 \\
\hline 455 & $\begin{array}{l}\text { h-1 } \\
\text { h-poly } \\
\text { pu-239 } \\
\text { o-16 } \\
\text { c } \\
\text { cl-35 }\end{array}$ & $\begin{array}{l}\text { waste } \\
\text { poly pipe } \\
\text { waste } \\
\text { waste } \\
\text { poly pipe } \\
\mathrm{MgO} / \mathrm{NaCl}\end{array}$ & $\begin{array}{l}0.3459 \\
0.1043 \\
0.0904 \\
0.0288 \\
0.0195 \\
-0.0118\end{array}$ & 0.6446 & 456 & $\begin{array}{l}\text { h-1 } \\
\text { h-poly } \\
\text { pu-239 } \\
\text { be } \\
\text { o-16 } \\
\text { c } \\
\text { cl-35 }\end{array}$ & $\begin{array}{l}\text { waste } \\
\text { poly pipe } \\
\text { waste } \\
\text { waste } \\
\text { waste } \\
\text { poly pipe } \\
\mathrm{MgO} / \mathrm{NaCl}\end{array}$ & $\begin{array}{c}0.3133 \\
0.0954 \\
0.0916 \\
0.0680 \\
0.0221 \\
0.0177 \\
-0.0172\end{array}$ & 0.6077 \\
\hline 477 & $\begin{array}{l}\text { h-1 } \\
\text { pu-239 } \\
\text { o-16 } \\
\text { cl-35 } \\
\text { na-23 } \\
\text { mg-24 } \\
\text { si-28 } \\
\text { na-23 }\end{array}$ & $\begin{array}{l}\text { waste } \\
\text { waste } \\
\text { waste } \\
\mathrm{MgO} / \mathrm{NaCl} \\
\mathrm{NaCl} \text { walls } \\
\mathrm{MgO} / \mathrm{NaCl} \\
\text { waste } \\
\mathrm{MgO} / \mathrm{NaCl}\end{array}$ & $\begin{array}{r}0.5583 \\
0.1534 \\
0.0705 \\
-0.0326 \\
0.0111 \\
0.0109 \\
0.0099 \\
0.0097\end{array}$ & 0.6719 & 478 & $\begin{array}{l}\text { h-1 } \\
\text { pu-239 } \\
\text { be } \\
\text { o-16 } \\
\text { cl-35 } \\
\text { mg-24 } \\
\text { na-23 }\end{array}$ & $\begin{array}{l}\text { waste } \\
\text { waste } \\
\text { waste } \\
\text { waste } \\
\mathrm{MgO} / \mathrm{NaCl} \\
\mathrm{MgO} / \mathrm{NaCl} \\
\mathrm{MgO} / \mathrm{NaCl}\end{array}$ & $\begin{array}{r}0.5148 \\
0.1550 \\
0.0807 \\
0.0592 \\
-0.0414 \\
0.0119 \\
0.0100\end{array}$ & 0.6325 \\
\hline 479 & $\begin{array}{l}\mathrm{h}-1 \\
\text { h-poly } \\
\text { pu-239 } \\
\text { o-16 } \\
\text { cl-35 } \\
\text { c }\end{array}$ & $\begin{array}{l}\text { waste } \\
\text { poly pipe } \\
\text { waste } \\
\text { waste } \\
\mathrm{MgO} / \mathrm{NaCl} \\
\text { poly pipe }\end{array}$ & $\begin{array}{r}0.3218 \\
0.1128 \\
0.1014 \\
0.0435 \\
-0.0271 \\
0.0180\end{array}$ & 0.6348 & 480 & $\begin{array}{l}\text { h-1 } \\
\text { pu-239 } \\
\text { h-poly } \\
\text { be } \\
\text { o-16 } \\
\text { cl-35 } \\
\text { c }\end{array}$ & $\begin{array}{l}\text { waste } \\
\text { waste } \\
\text { poly pipe } \\
\text { waste } \\
\text { waste } \\
\mathrm{MgO} / \mathrm{NaCl} \\
\text { poly pipe }\end{array}$ & $\begin{array}{r}0.2971 \\
0.1037 \\
0.1036 \\
0.0512 \\
0.0359 \\
-0.0326 \\
0.0171 \\
\end{array}$ & 0.6068 \\
\hline 489 & $\begin{array}{l}\text { h-1 } \\
\text { pu-239 } \\
\text { o-16 } \\
\text { cl-35 } \\
\text { na-23 } \\
\text { mg-24 } \\
\text { na-23 } \\
\text { si-28 }\end{array}$ & $\begin{array}{l}\text { waste } \\
\text { waste } \\
\text { waste } \\
\mathrm{MgO} / \mathrm{NaCl} \\
\mathrm{NaCl} \text { walls } \\
\mathrm{MgO} / \mathrm{NaCl} \\
\mathrm{MgO} / \mathrm{NaCl} \\
\text { waste }\end{array}$ & $\begin{array}{r}0.5568 \\
0.1608 \\
0.0684 \\
-0.0343 \\
0.0112 \\
0.0111 \\
0.0099 \\
0.0095\end{array}$ & 0.6701 & 490 & $\begin{array}{l}\text { h-1 } \\
\text { pu-239 } \\
\text { be } \\
\text { o-16 } \\
\text { cl-35 } \\
\text { mg-24 } \\
\text { na-23 }\end{array}$ & $\begin{array}{l}\text { waste } \\
\text { waste } \\
\text { waste } \\
\text { waste } \\
\mathrm{MgO} / \mathrm{NaCl} \\
\mathrm{MgO} / \mathrm{NaCl} \\
\mathrm{MgO} / \mathrm{NaCl}\end{array}$ & $\begin{array}{r}0.5111 \\
0.1656 \\
0.0784 \\
0.0575 \\
-0.0433 \\
0.0120 \\
0.0103\end{array}$ & 0.6313 \\
\hline 491 & $\begin{array}{l}\text { h-1 } \\
\text { h-poly } \\
\text { pu-239 } \\
\text { o-16 } \\
\text { cl-35 } \\
\text { c }\end{array}$ & $\begin{array}{l}\text { waste } \\
\text { poly pipe } \\
\text { waste } \\
\text { waste } \\
\mathrm{MgO} / \mathrm{NaCl} \\
\text { poly pipe }\end{array}$ & $\begin{array}{r}0.3195 \\
0.1115 \\
0.1112 \\
0.0419 \\
-0.0283 \\
0.0178\end{array}$ & 0.6337 & 492 & $\begin{array}{l}\text { h-1 } \\
\text { pu-239 } \\
\text { h-poly } \\
\text { be } \\
\text { o-16 } \\
\text { cl-35 } \\
\text { c }\end{array}$ & $\begin{array}{l}\text { waste } \\
\text { waste } \\
\text { poly pipe } \\
\text { waste } \\
\text { waste } \\
\mathrm{MgO} / \mathrm{NaCl} \\
\text { poly pipe }\end{array}$ & $\begin{array}{r}0.2969 \\
0.1122 \\
0.1052 \\
0.0500 \\
0.0350 \\
-0.0339 \\
0.0170\end{array}$ & 0.6078 \\
\hline
\end{tabular}


Table H-24. Nuclides with the highest sensitivities for set-1-8 cases

\begin{tabular}{|c|c|c|c|c|c|c|c|c|c|}
\hline \multirow[t]{2}{*}{$\begin{array}{c}\text { Case } \\
\text { Number }\end{array}$} & \multirow[t]{2}{*}{ Nuclide } & \multirow[t]{2}{*}{ Mixture } & \multirow[t]{2}{*}{$\begin{array}{l}\text { Sensitivity } \\
\text { coefficient }\end{array}$} & \multirow{2}{*}{$\begin{array}{c}\text { Total data- } \\
\text { induced } \\
\text { uncertainty } \\
\% \Delta \mathrm{k} / \mathrm{k}\end{array}$} & \multirow{2}{*}{\begin{tabular}{|c|c}
$\begin{array}{c}\text { Case } \\
\text { Number }\end{array}$ \\
With Be
\end{tabular}} & \multirow[t]{2}{*}{ Nuclide } & \multirow[t]{2}{*}{ Mixture } & \multirow[t]{2}{*}{$\begin{array}{l}\text { Sensitivity } \\
\text { coefficient }\end{array}$} & \multirow{2}{*}{$\begin{array}{c}\text { Total data- } \\
\text { induced } \\
\text { uncertainty } \\
\% \Delta \mathrm{k} / \mathrm{k}\end{array}$} \\
\hline & & & & & & & & & \\
\hline 333 & $\begin{array}{l}\text { h-poly } \\
\text { pu-239 } \\
\text { c } \\
\text { na-23 }\end{array}$ & $\begin{array}{l}\text { waste } \\
\text { waste } \\
\text { waste } \\
\mathrm{NaCl} \text { walls }\end{array}$ & $\begin{array}{l}0.5361 \\
0.1074 \\
0.0527 \\
0.0097\end{array}$ & 0.6703 & 334 & $\begin{array}{l}\text { h-poly } \\
\text { pu-239 } \\
\text { be } \\
\text { c } \\
\text { cl-35 }\end{array}$ & $\begin{array}{l}\text { waste } \\
\text { waste } \\
\text { waste } \\
\text { waste } \\
\mathrm{MgO} / \mathrm{NaCl}\end{array}$ & $\begin{array}{r}0.4854 \\
0.1071 \\
0.1058 \\
0.0394 \\
-0.0119 \\
\end{array}$ & 0.6217 \\
\hline 335 & $\begin{array}{l}\text { h-poly } \\
\text { h-poly } \\
\text { pu-239 } \\
\text { c } \\
\text { c }\end{array}$ & $\begin{array}{l}\text { waste } \\
\text { poly pipe } \\
\text { waste } \\
\text { waste } \\
\text { poly pipe }\end{array}$ & $\begin{array}{l}0.3436 \\
0.0990 \\
0.0773 \\
0.0349 \\
0.0201\end{array}$ & 0.6535 & 336 & $\begin{array}{l}\text { h-poly } \\
\text { h-poly } \\
\text { pu-239 } \\
\text { be } \\
\text { c } \\
\text { c } \\
\text { cl-35 }\end{array}$ & $\begin{array}{l}\text { waste } \\
\text { poly pipe } \\
\text { waste } \\
\text { waste } \\
\text { waste } \\
\text { poly pipe } \\
\mathrm{MgO} / \mathrm{NaCl}\end{array}$ & $\begin{array}{r}0.3096 \\
0.0901 \\
0.0797 \\
0.0729 \\
0.0261 \\
0.0179 \\
-0.0131 \\
\end{array}$ & 0.6124 \\
\hline 345 & $\begin{array}{l}\text { h-poly } \\
\text { pu-239 } \\
\text { c } \\
\text { na-23 }\end{array}$ & $\begin{array}{l}\text { waste } \\
\text { waste } \\
\text { waste } \\
\mathrm{NaCl} \text { walls }\end{array}$ & $\begin{array}{l}0.5331 \\
0.1164 \\
0.0501 \\
0.0094\end{array}$ & 0.6648 & 346 & $\begin{array}{l}\text { h-poly } \\
\text { pu-239 } \\
\text { be } \\
\text { c } \\
\text { cl-35 }\end{array}$ & $\begin{array}{l}\text { waste } \\
\text { waste } \\
\text { waste } \\
\text { waste } \\
\mathrm{MgO} / \mathrm{NaCl}\end{array}$ & $\begin{array}{r}0.4835 \\
0.1179 \\
0.1017 \\
0.0378 \\
-0.0134\end{array}$ & 0.6197 \\
\hline 347 & $\begin{array}{l}\text { h-poly } \\
\text { h-poly } \\
\text { pu-239 } \\
\text { c } \\
\text { c } \\
\text { cl-35 }\end{array}$ & $\begin{array}{l}\text { waste } \\
\text { poly pipe } \\
\text { waste } \\
\text { waste } \\
\text { poly pipe } \\
\mathrm{MgO} / \mathrm{NaCl}\end{array}$ & $\begin{array}{l}0.3418 \\
0.0978 \\
0.0861 \\
0.0332 \\
0.0196 \\
-0.0091\end{array}$ & 0.6491 & 348 & $\begin{array}{l}\text { h-poly } \\
\text { pu-239 } \\
\text { h-poly } \\
\text { be } \\
\text { c } \\
\text { c } \\
\text { cl-35 }\end{array}$ & $\begin{array}{l}\text { waste } \\
\text { waste } \\
\text { poly pipe } \\
\text { waste } \\
\text { waste } \\
\text { poly pipe } \\
\mathrm{MgO} / \mathrm{NaCl}\end{array}$ & $\begin{array}{r}0.3088 \\
0.0889 \\
0.0877 \\
0.0700 \\
0.0250 \\
0.0176 \\
-0.0142\end{array}$ & 0.6112 \\
\hline 369 & $\begin{array}{l}\text { h-poly } \\
\text { pu-239 } \\
\text { o-16 } \\
\text { c } \\
\text { cl-35 } \\
\text { na-23 } \\
\text { si-28 }\end{array}$ & $\begin{array}{l}\text { waste } \\
\text { waste } \\
\text { waste } \\
\text { waste } \\
\mathrm{MgO} / \mathrm{NaCl} \\
\mathrm{NaCl} \text { walls } \\
\text { waste }\end{array}$ & $\begin{array}{r}0.5404 \\
0.1442 \\
0.0461 \\
0.0351 \\
-0.0272 \\
0.0105 \\
0.0103\end{array}$ & 0.6607 & 370 & $\begin{array}{l}\text { h-poly } \\
\text { pu-239 } \\
\text { be } \\
\text { o-16 } \\
\text { cl-35 } \\
\text { c } \\
\text { mg-24 }\end{array}$ & $\begin{array}{l}\text { waste } \\
\text { waste } \\
\text { waste } \\
\text { waste } \\
\mathrm{MgO} / \mathrm{NaCl} \\
\text { waste } \\
\mathrm{MgO} / \mathrm{NaCl}\end{array}$ & $\begin{array}{r}0.4998 \\
0.1454 \\
0.0811 \\
0.0385 \\
-0.0359 \\
0.0291 \\
0.0104\end{array}$ & 0.6261 \\
\hline 371 & $\begin{array}{l}\text { h-poly } \\
\text { h-poly } \\
\text { pu-239 } \\
\text { o-16 } \\
\text { cl-35 } \\
\text { c } \\
\text { c }\end{array}$ & $\begin{array}{l}\text { waste } \\
\text { poly pipe } \\
\text { waste } \\
\text { waste } \\
\mathrm{MgO} / \mathrm{NaCl} \\
\text { waste } \\
\text { poly pipe }\end{array}$ & $\begin{array}{r}0.3198 \\
0.1048 \\
0.0986 \\
0.0293 \\
-0.0236 \\
0.0220 \\
0.0180\end{array}$ & 0.6394 & 372 & $\begin{array}{l}\text { h-poly } \\
\text { pu-239 } \\
\text { h-poly } \\
\text { be } \\
\text { cl-35 } \\
\text { o-16 } \\
\text { c } \\
\text { c }\end{array}$ & $\begin{array}{l}\text { waste } \\
\text { waste } \\
\text { poly pipe } \\
\text { waste } \\
\mathrm{MgO} / \mathrm{NaCl} \\
\text { waste } \\
\text { waste } \\
\text { poly pipe }\end{array}$ & $\begin{array}{r}0.2966 \\
0.0992 \\
0.0970 \\
0.0528 \\
-0.0292 \\
0.0242 \\
0.0180 \\
0.0169 \\
\end{array}$ & 0.6107 \\
\hline 381 & $\begin{array}{l}\text { h-poly } \\
\text { pu-239 } \\
\text { o-16 } \\
\text { c } \\
\text { cl-35 } \\
\text { na-23 } \\
\text { si-28 }\end{array}$ & $\begin{array}{l}\text { waste } \\
\text { waste } \\
\text { waste } \\
\text { waste } \\
\mathrm{MgO} / \mathrm{NaCl} \\
\mathrm{NaCl} \text { walls } \\
\text { waste }\end{array}$ & $\begin{array}{r}0.5389 \\
0.1520 \\
0.0445 \\
0.0341 \\
-0.0289 \\
0.0103 \\
0.0099 \\
\end{array}$ & 0.6594 & 382 & $\begin{array}{l}\text { h-poly } \\
\text { pu-239 } \\
\text { be } \\
\text { cl-35 } \\
\text { o-16 } \\
\text { c } \\
\text { mg-24 }\end{array}$ & $\begin{array}{l}\text { waste } \\
\text { waste } \\
\text { waste } \\
\mathrm{MgO} / \mathrm{NaCl} \\
\text { waste } \\
\text { waste } \\
\mathrm{MgO} / \mathrm{NaCl}\end{array}$ & $\begin{array}{r}0.4963 \\
0.1561 \\
0.0785 \\
-0.0376 \\
0.0371 \\
0.0281 \\
0.0105 \\
\end{array}$ & 0.6240 \\
\hline 383 & $\begin{array}{l}\text { h-poly } \\
\text { pu-239 } \\
\text { h-poly } \\
\text { o-16 } \\
\text { cl-35 } \\
\text { c } \\
\text { c }\end{array}$ & $\begin{array}{l}\text { waste } \\
\text { waste } \\
\text { poly pipe } \\
\text { waste } \\
\mathrm{MgO} / \mathrm{NaCl} \\
\text { waste } \\
\text { poly pipe }\end{array}$ & $\begin{array}{r}0.3184 \\
0.1079 \\
0.1040 \\
0.0283 \\
-0.0248 \\
0.0213 \\
0.0178\end{array}$ & 0.6382 & 384 & $\begin{array}{l}\text { h-poly } \\
\text { pu-239 } \\
\text { h-poly } \\
\text { be } \\
\text { cl-35 } \\
\text { o-16 } \\
\text { c } \\
\text { c }\end{array}$ & $\begin{array}{l}\text { waste } \\
\text { waste } \\
\text { poly pipe } \\
\text { waste } \\
\mathrm{MgO} / \mathrm{NaCl} \\
\text { waste } \\
\text { waste } \\
\text { poly pipe }\end{array}$ & $\begin{array}{r}0.2954 \\
0.1096 \\
0.0977 \\
0.0514 \\
-0.0305 \\
0.0234 \\
0.0175 \\
0.0168\end{array}$ & 0.6106 \\
\hline
\end{tabular}




\section{H.3 SIMILARITY ASSESSMENT}

A subset of the critical experiments from The SCALE Verified, Archived Library of Inputs and Data$V A L I D$ [39] was used with TSUNAMI-IP to identify potentially applicable experiments for $k_{e f f}$ validation. Results from all of the chosen experiments are also included in the International Handbook of Evaluated Criticality Safety Benchmark Experiments [36]. The set of experiments includes 81 experiments from the Pu-solution-thermal category (PST), 49 from the mixed-composition-thermal category (MCT), and 10 from the mixed-solution-thermal category (MST), for a total of 140 experiments. The EALF range of the benchmarks is from 0.04 to $0.95 \mathrm{eV}$, with the majority being on the lower end of the spectrum. The EALF range from Tables H-9 through $\mathrm{H}-16$ is 0.45 to $1.56 \mathrm{eV}$. Finding benchmarks with EALFs in the energy range of $\sim 0.7$ to $2 \mathrm{eV}$ is a known challenge. The experiments are similar to the applications in the ${ }^{239} \mathrm{Pu}$ content, with an average of $>95 \%{ }^{239} \mathrm{Pu}$ in the $\mathrm{Pu}$ content. The $\mathrm{Pu}$ solution experiments are water moderated and are in the thermal energy region. The mixed composition and mixed solution experiment systems cover mostly the upper thermal into intermediate energy regions.

As discussed above, $c_{k}$ values are calculated by comparing the select applications to each of the benchmark cases. None of the benchmarks achieved the desired $c_{k}$ value of 0.8 or higher. This prompted consideration of experiments with smaller $c_{k}$ values, as well as the need for an additional margin because highly applicable benchmarks were lacking. Tables H-25 through H-32 list the numbers of benchmarks, with $c_{k}$ values in each range listed for each application case. Case specifics (e.g., moderator type, filler type) are also included for reference.

Table H-25. ck values for set-1-1 cases

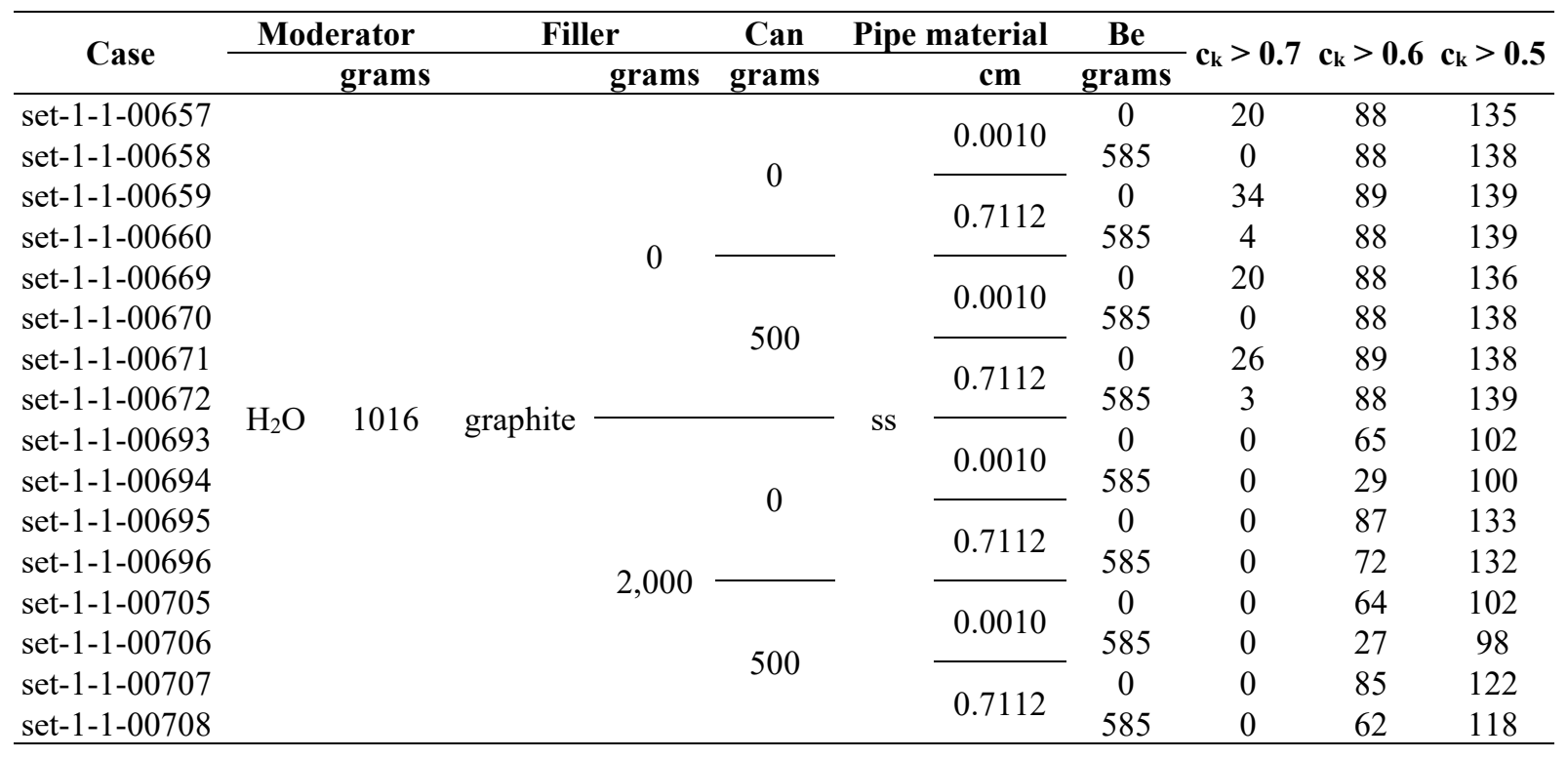


Table H-26. ck values for set-1-2 cases

\begin{tabular}{|c|c|c|c|c|c|c|c|c|c|c|c|}
\hline \multirow{2}{*}{ Case } & \multirow{2}{*}{\multicolumn{2}{|c|}{$\begin{array}{r}\text { Moderator } \\
\text { grams } \\
\end{array}$}} & \multicolumn{2}{|c|}{ Filler } & \multirow{2}{*}{$\begin{array}{c}\text { Can } \\
\text { grams } \\
\end{array}$} & \multicolumn{2}{|c|}{ Pipe material } & \multirow{2}{*}{$\begin{array}{c}\text { Be } \\
\text { grams } \\
\end{array}$} & \multirow{2}{*}{$c_{k}>0.7$} & \multirow{2}{*}{$c_{k}>0.6$} & \multirow{2}{*}{$c_{k}>0.5$} \\
\hline & & & & grams & & & $\mathbf{c m}$ & & & & \\
\hline set-1-2-00441 & \multirow{16}{*}{$\mathrm{CH}_{2}$} & \multirow{16}{*}{710.5} & \multirow{8}{*}{\multicolumn{2}{|c|}{ oraphite }} & \multirow{4}{*}{0} & \multirow{2}{*}{\multicolumn{2}{|c|}{0.0010}} & 0 & 0 & 87 & 105 \\
\hline set-1-2-00442 & & & & & & & & 585 & 0 & 50 & 105 \\
\hline set-1-2-00443 & & & & & & & & 0 & 3 & 88 & 127 \\
\hline set-1-2-00444 & & & & & & & 0.1112 & 585 & 0 & 68 & 131 \\
\hline set-1-2-00453 & & & & & \multirow{4}{*}{500} & & & 0 & 0 & 87 & 105 \\
\hline set-1-2-00454 & & & & & & & 0.0010 & 585 & 0 & 46 & 104 \\
\hline set-1-2-00455 & & & & & & & & 0 & 0 & 88 & 105 \\
\hline set-1-2-00456 & & & & & & & 0.7112 & 585 & 0 & 53 & 106 \\
\hline set-1-2-00477 & & & \multirow{8}{*}{ graphite } & \multirow{8}{*}{2,000} & \multirow{4}{*}{0} & SS & \multirow{2}{*}{0.0010} & 0 & 0 & 19 & 90 \\
\hline set-1-2-00478 & & & & & & & & 585 & 0 & 3 & 92 \\
\hline set-1-2-00479 & & & & & & & \multirow{2}{*}{0.7112} & 0 & 0 & 40 & 92 \\
\hline set-1-2-00480 & & & & & & & & 585 & 0 & 16 & 92 \\
\hline set-1-2-00489 & & & & & \multirow{4}{*}{500} & & \multirow{2}{*}{0.0010} & 0 & 0 & 19 & 90 \\
\hline set-1-2-00490 & & & & & & & & 585 & 0 & 3 & 92 \\
\hline set-1-2-00491 & & & & & & & \multirow{2}{*}{0.7112} & 0 & 0 & 31 & 92 \\
\hline set-1-2-00492 & & & & & & & & 585 & 0 & 9 & 92 \\
\hline
\end{tabular}

Table H-27. ck values for set-1-3 cases

\begin{tabular}{|c|c|c|c|c|c|c|c|c|c|c|c|}
\hline \multirow{2}{*}{ Case } & \multicolumn{2}{|c|}{ Moderator } & \multicolumn{2}{|c|}{ Filler } & \multirow{2}{*}{$\begin{array}{c}\text { Can } \\
\text { grams }\end{array}$} & \multirow{2}{*}{\multicolumn{2}{|c|}{$\begin{array}{c}\text { Pipe material } \\
\mathbf{c m}\end{array}$}} & \multirow{2}{*}{$\begin{array}{c}\text { Be } \\
\text { grams }\end{array}$} & \multirow{2}{*}{$\mathbf{c}_{\mathrm{k}}>0.7$} & \multirow{2}{*}{$c_{k}>0.6$} & \multirow{2}{*}{$c_{k}>0.5$} \\
\hline & & grams & & grams & & & & & & & \\
\hline set-1-3-00441 & \multirow{16}{*}{$\mathrm{H}_{2} \mathrm{O}$} & \multirow{16}{*}{710.5} & \multirow{8}{*}{\multicolumn{2}{|c|}{ oraphito }} & \multirow{4}{*}{0} & \multirow{2}{*}{\multicolumn{2}{|c|}{0.0010}} & 0 & 5 & 87 & 101 \\
\hline set-1-3-00442 & & & & & & & & 585 & 0 & 59 & 99 \\
\hline set-1-3-00443 & & & & & & & & 0 & 87 & 96 & 140 \\
\hline set-1-3-00444 & & & & & & & 0.7112 & 585 & 37 & 95 & 140 \\
\hline set-1-3-00453 & & & & & \multirow{4}{*}{500} & & 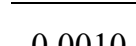 & 0 & 4 & 87 & 100 \\
\hline set-1-3-00454 & & & & & & & 0.0010 & 585 & 0 & 55 & 99 \\
\hline set-1-3-00455 & & & & & & & ? 7111 & 0 & 86 & 96 & 140 \\
\hline set-1-3-00456 & & & & & & noly & 0.1112 & 585 & 35 & 93 & 140 \\
\hline set-1-3-00477 & & & \multirow{8}{*}{ graphite } & \multirow{8}{*}{2,000} & \multirow{4}{*}{0} & рогу & 00010 & 0 & 0 & 20 & 89 \\
\hline set-1-3-00478 & & & & & & & 0.0010 & 585 & 0 & 3 & 89 \\
\hline set-1-3-00479 & & & & & & & ר 7111 & 0 & 9 & 88 & 140 \\
\hline set-1-3-00480 & & & & & & & 0.1112 & 585 & 0 & 88 & 140 \\
\hline set-1-3-00489 & & & & & \multirow{4}{*}{500} & & & 0 & 0 & 20 & 89 \\
\hline set-1-3-00490 & & & & & & & 0.0010 & 585 & 0 & 3 & 89 \\
\hline set-1-3-00491 & & & & & & & & 0 & 7 & 88 & 140 \\
\hline set-1-3-00492 & & & & & & & 12 & 585 & 0 & 89 & 140 \\
\hline
\end{tabular}


Table H-28. ck values for set-1-4 cases

\begin{tabular}{|c|c|c|c|c|c|c|c|c|c|c|c|}
\hline \multirow{2}{*}{ Case } & \multirow{2}{*}{\multicolumn{2}{|c|}{$\begin{array}{r}\text { Moderator } \\
\text { grams } \\
\end{array}$}} & \multicolumn{2}{|c|}{ Filler } & \multirow{2}{*}{$\begin{array}{c}\text { Can } \\
\text { grams } \\
\end{array}$} & \multirow{2}{*}{\multicolumn{2}{|c|}{$\begin{array}{c}\text { Pipe material } \\
\mathrm{cm}\end{array}$}} & \multirow{2}{*}{$\begin{array}{c}\text { Be } \\
\text { grams } \\
\end{array}$} & \multirow{2}{*}{$c_{k}>0.7$} & \multirow{2}{*}{$c_{k}>0.6$} & \multirow{2}{*}{$c_{k}>0.5$} \\
\hline & & & & grams & & & & & & & \\
\hline set-1-4-00333 & \multirow{16}{*}{$\mathrm{CH}_{2}$} & \multirow{16}{*}{557.9} & \multirow{8}{*}{\multicolumn{2}{|c|}{ Oro hit }} & \multirow{4}{*}{0} & \multirow{2}{*}{\multicolumn{2}{|c|}{0.0010}} & 0 & 0 & 62 & 90 \\
\hline set-1-4-00334 & & & & & & & & 585 & 0 & 19 & 92 \\
\hline set-1-4-00335 & & & & & & & & 0 & 38 & 90 & 140 \\
\hline set-1-4-00336 & & & & & & & 0.7112 & 585 & 3 & 91 & 140 \\
\hline set-1-4-00345 & & & & & \multirow{4}{*}{500} & & & 0 & 0 & 61 & 90 \\
\hline set-1-4-00346 & & & & & & & 0.0010 & 585 & 0 & 19 & 92 \\
\hline set-1-4-00347 & & & & & & & & 0 & 36 & 90 & 140 \\
\hline set- $1-4-00348$ & & & & & & & 0.7112 & 585 & 1 & 91 & 140 \\
\hline set-1-4-00369 & & & \multirow{8}{*}{ graphite } & \multirow{8}{*}{2,000} & \multirow{4}{*}{0} & poly & 00010 & 0 & 0 & 3 & 88 \\
\hline set- $1-4-00370$ & & & & & & & 0.0010 & 585 & 0 & 0 & 88 \\
\hline set-1-4-00371 & & & & & & & 07112 & 0 & 0 & 88 & 138 \\
\hline set-1-4-00372 & & & & & & & 0.1112 & 585 & 0 & 88 & 140 \\
\hline set-1-4-00381 & & & & & \multirow{4}{*}{500} & & ค $\cap \Omega 1 \Omega$ & 0 & 0 & 3 & 88 \\
\hline set-1-4-00382 & & & & & & & 0.0010 & 585 & 0 & 0 & 88 \\
\hline set-1-4-00383 & & & & & & & & 0 & 0 & 88 & 139 \\
\hline set-1-4-00384 & & & & & & & 0.1112 & 585 & 0 & 88 & 140 \\
\hline
\end{tabular}

Table H-29. ck values for set-1-5 cases

\begin{tabular}{|c|c|c|c|c|c|c|c|c|c|c|c|}
\hline \multirow{2}{*}{ Case } & \multicolumn{2}{|c|}{ Moderator } & \multicolumn{2}{|c|}{ Filler } & \multirow{2}{*}{$\begin{array}{c}\text { Can } \\
\text { grams }\end{array}$} & \multirow{2}{*}{\multicolumn{2}{|c|}{$\begin{array}{r}\text { Pipe material } \\
\mathrm{cm}\end{array}$}} & \multirow{2}{*}{$\begin{array}{c}\text { Be } \\
\text { grams }\end{array}$} & \multirow{2}{*}{$\mathbf{c}_{\mathrm{k}}>0.7$} & \multirow{2}{*}{$c_{k}>0.6$} & \multirow{2}{*}{$c_{k}>0.5$} \\
\hline & & grams & & grams & & & & & & & \\
\hline set-1-5-00657 & \multirow{16}{*}{$\mathrm{H}_{2} \mathrm{O}$} & \multirow{16}{*}{1016} & \multirow{8}{*}{\multicolumn{2}{|c|}{ generic }} & \multirow{4}{*}{0} & \multirow{2}{*}{\multicolumn{2}{|c|}{0.0010}} & 0 & 20 & 88 & 135 \\
\hline set-1-5-00658 & & & & & & & & 585 & 0 & 88 & 138 \\
\hline set-1-5-00659 & & & & & & & & 0 & 34 & 89 & 139 \\
\hline set-1-5-00660 & & & & & & & 0.7112 & 585 & 4 & 88 & 139 \\
\hline set-1-5-00669 & & & & & \multirow{4}{*}{500} & & م $0 \Omega 10$ & 0 & 20 & 88 & 136 \\
\hline set-1-5-00670 & & & & & & & 0.0010 & 585 & 0 & 88 & 138 \\
\hline set-1-5-00671 & & & & & & & ? 7111 & 0 & 26 & 89 & 138 \\
\hline set-1-5-00672 & & & & & & & 0.7112 & 585 & 3 & 88 & 139 \\
\hline set-1-5-00693 & & & \multirow{8}{*}{ generic } & \multirow{8}{*}{2,000} & \multirow{4}{*}{0} & SS & 0 & 0 & 0 & 56 & 96 \\
\hline set-1-5-00694 & & & & & & & 0.0010 & 585 & 0 & 29 & 98 \\
\hline set-1-5-00695 & & & & & & & ? 7111 & 0 & 0 & 87 & 105 \\
\hline set-1-5-00696 & & & & & & & 0.1112 & 585 & 0 & 72 & 128 \\
\hline set-1-5-00705 & & & & & \multirow{4}{*}{500} & & & 0 & 0 & 60 & 96 \\
\hline set-1-5-00706 & & & & & & & 0.0010 & 585 & 0 & 26 & 98 \\
\hline set-1-5-00707 & & & & & & & & 0 & 0 & 84 & 103 \\
\hline set-1-5-00708 & & & & & & & 2 & 585 & 0 & 61 & 104 \\
\hline
\end{tabular}


Table H-30. ck values for set-1-6 cases

\begin{tabular}{|c|c|c|c|c|c|c|c|c|c|c|c|}
\hline \multirow{2}{*}{ Case } & \multirow{2}{*}{\multicolumn{2}{|c|}{$\begin{array}{r}\text { Moderator } \\
\text { grams } \\
\end{array}$}} & \multicolumn{2}{|c|}{ Filler } & \multirow{2}{*}{$\begin{array}{c}\text { Can } \\
\text { grams }\end{array}$} & \multirow{2}{*}{\multicolumn{2}{|c|}{$\begin{array}{c}\text { Pipe material } \\
\mathrm{cm}\end{array}$}} & \multirow{2}{*}{$\begin{array}{c}\text { Be } \\
\text { grams }\end{array}$} & \multirow{2}{*}{$c_{k}>0.7$} & \multirow{2}{*}{$c_{k}>0.6$} & \multirow{2}{*}{$c_{k}>0.5$} \\
\hline & & & & grams & & & & & & & \\
\hline set-1-6-00441 & \multirow{16}{*}{$\mathrm{CH}_{2}$} & \multirow{16}{*}{710.5} & \multirow{8}{*}{\multicolumn{2}{|c|}{ generic }} & \multirow{4}{*}{0} & \multirow{2}{*}{\multicolumn{2}{|c|}{0.0010}} & 0 & 0 & 87 & 105 \\
\hline set-1-6-00442 & & & & & & & & 585 & 0 & 50 & 105 \\
\hline set-1-6-00443 & & & & & & & ( 7111 & 0 & 3 & 88 & 127 \\
\hline set-1-6-00444 & & & & & & & 0.1112 & 585 & 0 & 68 & 131 \\
\hline set-1-6-00453 & & & & & \multirow{4}{*}{500} & & & 0 & 0 & 87 & 105 \\
\hline set-1-6-00454 & & & & & & & 0.0010 & 585 & 0 & 46 & 104 \\
\hline set-1-6-00455 & & & & & & & & 0 & 0 & 88 & 105 \\
\hline set-1-6-00456 & & & & & & & 0.7112 & 585 & 0 & 53 & 106 \\
\hline set-1-6-00477 & & & \multirow{8}{*}{ generic } & \multirow{8}{*}{2,000} & \multirow{4}{*}{0} & SS & & 0 & 0 & 19 & 90 \\
\hline set-1-6-00478 & & & & & & & 0.0010 & 585 & 0 & 3 & 91 \\
\hline set-1-6-00479 & & & & & & & 07112 & 0 & 0 & 43 & 92 \\
\hline set-1-6-00480 & & & & & & & 0.7112 & 585 & 0 & 17 & 92 \\
\hline set-1-6-00489 & & & & & \multirow{4}{*}{500} & & & 0 & 0 & 19 & 90 \\
\hline set-1-6-00490 & & & & & & & 0.0010 & 585 & 0 & 3 & 90 \\
\hline set-1-6-00491 & & & & & & & 07112 & 0 & 0 & 36 & 92 \\
\hline set-1-6-00492 & & & & & & & 0.7112 & 585 & 0 & 14 & 92 \\
\hline
\end{tabular}

Table H-31. ck values for set-1-7 cases

\begin{tabular}{|c|c|c|c|c|c|c|c|c|c|c|c|}
\hline \multirow{2}{*}{ Case } & \multicolumn{2}{|c|}{ Moderator } & \multicolumn{2}{|c|}{ Filler } & \multirow{2}{*}{$\begin{array}{c}\text { Can } \\
\text { grams }\end{array}$} & \multirow{2}{*}{\multicolumn{2}{|c|}{$\begin{array}{r}\text { Pipe material } \\
\mathrm{cm}\end{array}$}} & \multirow{2}{*}{$\begin{array}{c}\text { Be } \\
\text { grams }\end{array}$} & \multirow{2}{*}{$\mathbf{c}_{\mathrm{k}}>0.7$} & \multirow{2}{*}{$c_{k}>0.6$} & \multirow{2}{*}{$c_{k}>0.5$} \\
\hline & & grams & & grams & & & & & & & \\
\hline set-1-7-00441 & \multirow{16}{*}{$\mathrm{H}_{2} \mathrm{O}$} & \multirow{16}{*}{710.5} & \multirow{8}{*}{\multicolumn{2}{|c|}{ Saneris }} & \multirow{4}{*}{0} & \multirow{2}{*}{\multicolumn{2}{|c|}{0.0010}} & 0 & 5 & 87 & 101 \\
\hline set-1-7-00442 & & & & & & & & 585 & 0 & 59 & 99 \\
\hline set-1-7-00443 & & & & & & & & 0 & 87 & 96 & 140 \\
\hline set-1-7-00444 & & & & & & & 0.7112 & 585 & 37 & 95 & 140 \\
\hline set-1-7-00453 & & & & & \multirow{4}{*}{500} & & 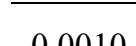 & 0 & 4 & 87 & 100 \\
\hline set-1-7-00454 & & & & & & & 0.0010 & 585 & 0 & 55 & 99 \\
\hline set-1-7-00455 & & & & & & & ( 7111 & 0 & 86 & 96 & 140 \\
\hline set-1-7-00456 & & & & & & noly & 0.1112 & 585 & 35 & 93 & 140 \\
\hline set-1-7-00477 & & & \multirow{8}{*}{ generic } & \multirow{8}{*}{2,000} & \multirow{4}{*}{0} & poly & 00010 & 0 & 0 & 17 & 88 \\
\hline set-1-7-00478 & & & & & & & 0.0010 & 585 & 0 & 2 & 88 \\
\hline set-1-7-00479 & & & & & & & & 0 & 7 & 88 & 139 \\
\hline set-1-7-00480 & & & & & & & 0.7112 & 585 & 0 & 88 & 140 \\
\hline set-1-7-00489 & & & & & \multirow{4}{*}{500} & & & 0 & 0 & 17 & 88 \\
\hline set-1-7-00490 & & & & & & & 0.0010 & 585 & 0 & 2 & 88 \\
\hline set-1-7-00491 & & & & & & & & 0 & 7 & 88 & 139 \\
\hline set-1-7-00492 & & & & & & & 2 & 585 & 0 & 88 & 140 \\
\hline
\end{tabular}


Table H-32. ck values for set-1-8 cases

\begin{tabular}{|c|c|c|c|c|c|c|c|c|c|c|c|}
\hline \multirow{2}{*}{ Case } & \multirow{2}{*}{\multicolumn{2}{|c|}{$\begin{array}{r}\text { Moderator } \\
\text { grams } \\
\end{array}$}} & \multicolumn{2}{|c|}{ Filler } & \multirow{2}{*}{$\begin{array}{c}\text { Can } \\
\text { grams }\end{array}$} & \multirow{2}{*}{\multicolumn{2}{|c|}{$\begin{array}{c}\text { Pipe material } \\
\text { cm }\end{array}$}} & \multirow{2}{*}{$\begin{array}{c}\text { Be } \\
\text { grams }\end{array}$} & \multirow{2}{*}{$c_{k}>0.7$} & \multirow{2}{*}{$c_{k}>0.6$} & \multirow{2}{*}{$c_{k}>0.5$} \\
\hline & & & & grams & & & & & & & \\
\hline set-1-8-00333 & \multirow{16}{*}{$\mathrm{CH}_{2}$} & \multirow{16}{*}{557.9} & \multirow{8}{*}{\multicolumn{2}{|c|}{ oromeri }} & \multirow{4}{*}{0} & \multirow{2}{*}{\multicolumn{2}{|c|}{0.0010}} & 0 & 0 & 62 & 90 \\
\hline set-1-8-00334 & & & & & & & & 585 & 0 & 19 & 92 \\
\hline set- $1-8-00335$ & & & & & & & ( 7111 & 0 & 38 & 90 & 140 \\
\hline set- $1-8-00336$ & & & & & & & 0.1112 & 585 & 3 & 91 & 140 \\
\hline set- $1-8-00345$ & & & & & \multirow{4}{*}{500} & & & 0 & 0 & 61 & 90 \\
\hline set- $1-8-00346$ & & & & & & & 0.0010 & 585 & 0 & 19 & 92 \\
\hline set-1-8-00347 & & & & & & & & 0 & 36 & 90 & 140 \\
\hline set-1-8-00348 & & & & & & noly & 0.7112 & 585 & 1 & 91 & 140 \\
\hline set-1-8-00369 & & & \multirow{8}{*}{ generic } & \multirow{8}{*}{2,000} & \multirow{4}{*}{0} & poly & 001 & 0 & 0 & 3 & 88 \\
\hline set- $1-8-00370$ & & & & & & & 0.0010 & 585 & 0 & 0 & 87 \\
\hline set-1-8-00371 & & & & & & & 07112 & 0 & 0 & 88 & 137 \\
\hline set-1-8-00372 & & & & & & & 0.7112 & 585 & 0 & 88 & 140 \\
\hline set-1-8-00381 & & & & & \multirow{4}{*}{500} & & & 0 & 0 & 3 & 88 \\
\hline set-1-8-00382 & & & & & & & 0.0010 & 585 & 0 & 0 & 87 \\
\hline set-1-8-00383 & & & & & & & 07112 & 0 & 0 & 88 & 137 \\
\hline set-1-8-00384 & & & & & & & 0.7112 & 585 & 0 & 88 & 140 \\
\hline
\end{tabular}

\section{H.4 BIAS AND BIAS UNCERTAINTY DETERMINATION}

Using the available $c_{\mathrm{k}}$ data, the USLSTATS program was implemented to determine bias and bias uncertainty by trending on the $c_{k}$ value. Tables $\mathrm{H}-33$ through $\mathrm{H}-40$ list the results by $\mathrm{c}_{\mathrm{k}}$ threshold value. For cases in which there were less than 20 experiments, bias and bias uncertainty are not listed, because there are not enough data points to determine a reliable value. There is a minimum of 26 experiments for each case with a $c_{\mathrm{k}}$ value $>0.5$, but there are no data to support using $c_{\mathrm{k}}$ values in this range. The bias and bias uncertainty values with $c_{k}$ values $>0.5$ are smaller than the bias and bias uncertainty values with $c_{k}$ values $>0.6$. Even though there more are smaller similarity values $\left(c_{k}\right.$ values $\left.<0.6\right)$, the bias and bias uncertainty values should be larger due to the smaller degree of similarity.

$\mathrm{S} / \mathrm{U}$ techniques provide tools for generating a quantitative, defensible estimate of what an appropriate margin might be. Examining the nuclides and reactions that contribute significantly to data-induced uncertainty will indicate the important processes to be validated. Elements such as Be and chlorine might be entirely absent from the validation set or only poorly represented. For these nuclides, the data-induced uncertainty provides an estimate for the magnitude of the bias that could occur in the application. This approach has been used in several other applications [40, 41, 42].

\section{H.5 ADDITIONAL VALIDATION MARGINS}

The magnitude of the data-induced uncertainty in the application also bounds the expected magnitude of the bias if there are no applicable benchmarks. Using this value might be more efficient than investing effort in developing and defending a lower additional margin. A demonstration that the data-induced uncertainty bounds the bias manifested for the most fast or thermal spectrum benchmark systems is provided in Section 3.2.5 of Scaglione et al. [43]. It is not clear if the bias of intermediate spectrum systems is also bounded by the nuclear data-induced uncertainty in $k_{\text {eff. }}$ It is likely that the most applicable benchmark experiments would be used to develop a bias and bias uncertainty, and this additional margin would be added to ensure sufficient conservatism in the USL. 
For set-1-1, case number 657 , the total data-induced uncertainty is $0.6705 \% \Delta k / k$. Given the system $k_{\text {eff }}$ value of 0.9437 (for the modified case), the absolute data-induced uncertainty is $0.6327 \% \Delta k$, which would be combined with the bias and bias uncertainty and any other applicable additional margins to determine the maximum subcritical $k_{\text {eff }}$ value. Tables H-33 through $\mathrm{H}-40$ also include the total datainduced uncertainty and the absolute data-induced uncertainty for each case.

A more complex approach could be to justify an additional margin based on the fact that the available benchmarks provide validation for some incident neutron energies. This approach could be useful for important nuclides that are present in the validation suite but that have energy-dependent sensitivity profiles that differ significantly from the application model. These profiles can be reviewed to determine whether validation exists for an energy range or perhaps multiple energy ranges. For example, it might be evident that high-energy cross sections are validated because fission neutrons are born at high energies in all systems. Thermal cross sections are generally well validated due to the many available thermal benchmark experiments. Therefore, an estimate of the unvalidated portion of the profile may be generated and used to estimate the magnitude of the remaining potential bias in the application in the energy ranges with weaker validation. In some cases, an energy range in the application might have significantly less sensitivity than that present in relevant benchmarks. An additional margin for these situations would not likely be needed because the low sensitivity directly indicates a low potential for bias.

Table H-33. Bias, bias uncertainty, and data-induced uncertainty for set-1-1 cases

\begin{tabular}{ccccccc}
\hline Case & $\begin{array}{c}\text { ck }>\mathbf{0 . 7} \\
\text { bias and } \\
\text { bias uncertainty }\end{array}$ & $\begin{array}{c}\text { ck }>\mathbf{0 . 6} \\
\text { bias and } \\
\text { bias uncertainty }\end{array}$ & $\begin{array}{c}\text { ck }>\mathbf{0 . 5} \\
\text { bias and } \\
\text { bias uncertainty }\end{array}$ & $\begin{array}{c}\text { Modified } \\
\boldsymbol{k}_{\text {eff }}\end{array}$ & $\begin{array}{c}\text { Total data- } \\
\text { induced } \\
\text { uncertainty }\end{array}$ & $\begin{array}{c}\text { Absolute data- } \\
\text { induced } \\
\text { uncertainty }\end{array}$ \\
\hline set-1-1-00657 & 0.0822 & 0.0178 & 0.0108 & 0.9437 & 0.006705 & 0.006327 \\
set-1-1-00658 & & 0.0216 & 0.0114 & 0.9492 & 0.006287 & 0.005968 \\
set-1-1-00659 & 0.0406 & 0.0168 & 0.0108 & 0.9323 & 0.006627 & 0.006178 \\
set-1-1-00660 & & 0.0211 & 0.0113 & 0.9371 & 0.006313 & 0.005916 \\
set-1-1-00669 & 0.0832 & 0.0179 & 0.0108 & 0.9290 & 0.006685 & 0.006211 \\
set-1-1-00670 & & 0.0220 & 0.0114 & 0.9335 & 0.006268 & 0.005851 \\
set-1-1-00671 & 0.0574 & 0.0172 & 0.0108 & 0.9180 & 0.006698 & 0.006148 \\
set-1-1-00672 & & 0.0214 & 0.0114 & 0.9225 & 0.006397 & 0.005901 \\
set-1-1-00693 & & 0.0316 & 0.0145 & 0.8724 & 0.006481 & 0.005654 \\
set-1-1-00694 & & 0.0854 & 0.0173 & 0.8739 & 0.006224 & 0.005439 \\
set-1-1-00695 & & 0.0226 & 0.0114 & 0.8702 & 0.006513 & 0.005668 \\
set-1-1-00696 & & 0.0315 & 0.0122 & 0.8721 & 0.006319 & 0.005511 \\
set-1-1-00705 & & 0.0325 & 0.0145 & 0.8598 & 0.006464 & 0.005558 \\
set-1-1-00706 & & 0.0913 & 0.0183 & 0.8610 & 0.006215 & 0.005351 \\
set-1-1-00707 & & 0.0236 & 0.0119 & 0.8580 & 0.006585 & 0.005650 \\
set-1-1-00708 & & 0.0370 & 0.0132 & 0.8594 & 0.006404 & 0.005503 \\
\hline
\end{tabular}


Table H-34. Bias, bias uncertainty, and data-induced uncertainty for set-1-2 cases

\begin{tabular}{ccccccc}
\hline Case & $\begin{array}{c}\text { ck }>\mathbf{0 . 7} \\
\text { bias and } \\
\text { bias uncertainty }\end{array}$ & $\begin{array}{c}\text { ck }>\mathbf{0 . 6} \\
\text { bias and } \\
\text { bias uncertainty }\end{array}$ & $\begin{array}{c}\text { ck }>\mathbf{0 . 5} \\
\text { bias and } \\
\text { bias uncertainty }\end{array}$ & $\begin{array}{c}\text { Modified } \\
\boldsymbol{k}_{\text {eff }}\end{array}$ & $\begin{array}{c}\text { Total data- } \\
\text { induced } \\
\text { uncertainty }\end{array}$ & $\begin{array}{c}\text { Absolute data- } \\
\text { induced } \\
\text { uncertainty }\end{array}$ \\
\hline set-1-2-00441 & & 0.0199 & 0.0128 & 0.9606 & 0.006674 & 0.006411 \\
set-1-2-00442 & 0.0415 & 0.0149 & 0.9617 & 0.006236 & 0.005998 \\
set-1-2-00443 & 0.0194 & 0.0113 & 0.9403 & 0.006623 & 0.006228 \\
set-1-2-00444 & 0.0290 & 0.0121 & 0.9418 & 0.006280 & 0.005914 \\
set-1-2-00453 & 0.0202 & 0.0128 & 0.9434 & 0.006632 & 0.006256 \\
set-1-2-00454 & 0.0432 & 0.0152 & 0.9444 & 0.006233 & 0.005886 \\
set-1-2-00455 & 0.0199 & 0.0129 & 0.9243 & 0.006671 & 0.006166 \\
set-1-2-00456 & 0.0362 & 0.0146 & 0.9258 & 0.006371 & 0.005898 \\
set-1-2-00477 & 0.1258 & 0.0234 & 0.8721 & 0.006475 & 0.005647 \\
set-1-2-00478 & & 0.0260 & 0.8725 & 0.006201 & 0.005411 \\
set-1-2-00479 & 0.0477 & 0.0206 & 0.8656 & 0.006501 & 0.005628 \\
set-1-2-00480 & & 0.0246 & 0.8670 & 0.006306 & 0.005468 \\
set-1-2-00489 & & 0.0237 & 0.8585 & 0.006451 & 0.005538 \\
set-1-2-00490 & & 0.0262 & 0.8591 & 0.006198 & 0.005325 \\
set-1-2-00491 & & 0.0211 & 0.8528 & 0.006574 & 0.005606 \\
set-1-2-00492 & & & 0.0250 & 0.8542 & 0.006384 & 0.005453 \\
\hline
\end{tabular}

Table H-35. Bias, bias uncertainty, and data-induced uncertainty for set-1-3 cases

\begin{tabular}{ccccccc}
\hline Case & $\begin{array}{c}\text { ck }>\mathbf{0 . 7} \\
\text { bias and } \\
\text { bias uncertainty }\end{array}$ & $\begin{array}{c}\text { ck }>\mathbf{0 . 6} \\
\text { bias and } \\
\text { bias uncertainty }\end{array}$ & $\begin{array}{c}\text { ck }>\mathbf{0 . 5} \\
\text { bias and } \\
\text { bias uncertainty }\end{array}$ & $\begin{array}{c}\text { Modified } \\
\boldsymbol{k}_{\text {eff }}\end{array}$ & $\begin{array}{c}\text { Total data- } \\
\text { induced } \\
\text { uncertainty }\end{array}$ & $\begin{array}{c}\text { Absolute data- } \\
\text { induced } \\
\text { uncertainty }\end{array}$ \\
\hline set-1-3-00441 & & 0.0177 & 0.0125 & 0.8421 & 0.006797 & 0.005724 \\
set-1-3-00442 & & 0.0308 & 0.0151 & 0.8555 & 0.006258 & 0.005354 \\
set-1-3-00443 & 0.0174 & 0.0127 & 0.0106 & 0.9433 & 0.006489 & 0.006121 \\
set-1-3-00444 & 0.0572 & 0.0159 & 0.0112 & 0.9498 & 0.006067 & 0.005762 \\
set-1-3-00453 & & 0.0180 & 0.0128 & 0.8280 & 0.006718 & 0.005562 \\
set-1-3-00454 & & 0.0335 & 0.0152 & 0.8403 & 0.006231 & 0.005235 \\
set-1-3-00455 & 0.0179 & 0.0128 & 0.0106 & 0.9271 & 0.006446 & 0.005976 \\
set-1-3-00456 & 0.0587 & 0.0171 & 0.0112 & 0.9333 & 0.006077 & 0.005672 \\
set-1-3-00477 & & 0.1054 & 0.0219 & 0.7720 & 0.006503 & 0.005020 \\
set-1-3-00478 & & & 0.0257 & 0.7789 & 0.006175 & 0.004809 \\
set-1-3-00479 & & 0.0238 & 0.0112 & 0.8799 & 0.006249 & 0.005499 \\
set-1-3-00480 & & 0.0303 & 0.0119 & 0.8827 & 0.006009 & 0.005304 \\
set-1-3-00489 & & 0.1070 & 0.0222 & 0.7604 & 0.006483 & 0.004930 \\
set-1-3-00490 & & 0.0242 & 0.0259 & 0.7671 & 0.006175 & 0.004737 \\
set-1-3-00491 & & 0.0284 & 0.0119 & 0.8666 & 0.006241 & 0.005409 \\
set-1-3-00492 & & & & 0.8691 & 0.006023 & 0.005234 \\
\hline
\end{tabular}


Table H-36. Bias, bias uncertainty, and data-induced uncertainty for set-1-4 cases

\begin{tabular}{ccccccc}
\hline Case & $\begin{array}{c}\text { ck }>\mathbf{0 . 7} \\
\text { bias and } \\
\text { bias uncertainty }\end{array}$ & $\begin{array}{c}\text { ck }>\mathbf{0 . 6} \\
\text { bias and } \\
\text { bias uncertainty }\end{array}$ & $\begin{array}{c}\text { ck }>\mathbf{0 . 5} \\
\text { bias and } \\
\text { bias uncertainty }\end{array}$ & $\begin{array}{c}\text { Modified } \\
\boldsymbol{k}_{\text {eff }}\end{array}$ & $\begin{array}{c}\text { Total data- } \\
\text { induced } \\
\text { uncertainty }\end{array}$ & $\begin{array}{c}\text { Absolute data- } \\
\text { induced } \\
\text { uncertainty }\end{array}$ \\
\hline set-1-4-00333 & & 0.0267 & 0.0185 & 0.8871 & 0.006703 & 0.005946 \\
set-1-4-00334 & & & 0.0214 & 0.8940 & 0.006217 & 0.005557 \\
set-1-4-00335 & 0.0449 & 0.0175 & 0.0108 & 0.9742 & 0.006535 & 0.006366 \\
set-1-4-00336 & & 0.0226 & 0.0116 & 0.9759 & 0.006124 & 0.005976 \\
set-1-4-00345 & & 0.0277 & 0.0188 & 0.8705 & 0.006648 & 0.005787 \\
set-1-4-00346 & & 0.0216 & 0.8765 & 0.006197 & 0.005432 \\
set-1-4-00347 & & 0.0178 & 0.0109 & 0.9566 & 0.006491 & 0.006209 \\
set-1-4-00348 & & 0.0229 & 0.0116 & 0.9583 & 0.006112 & 0.005857 \\
set-1-4-00369 & & 0.0252 & 0.7999 & 0.006484 & 0.005186 \\
set-1-4-00370 & & 0.0292 & 0.8040 & 0.006157 & 0.004950 \\
set-1-4-00371 & & 0.0260 & 0.0114 & 0.8975 & 0.006307 & 0.005660 \\
set-1-4-00372 & & 0.0325 & 0.0123 & 0.8983 & 0.006061 & 0.005444 \\
set-1-4-00381 & & & 0.0255 & 0.7869 & 0.006458 & 0.005082 \\
set-1-4-00382 & & 0.0292 & 0.7912 & 0.006162 & 0.004876 \\
set-1-4-00383 & & 0.0262 & 0.0114 & 0.8835 & 0.006311 & 0.005576 \\
set-1-4-00384 & & 0.0325 & 0.0123 & 0.8842 & 0.006071 & 0.005368 \\
\hline
\end{tabular}

Table H-37. Bias, bias uncertainty, and data-induced uncertainty for set-1-5 cases

\begin{tabular}{ccccccc}
\hline Case & $\begin{array}{c}\text { ck }>\mathbf{0 . 7} \\
\text { bias and } \\
\text { bias uncertainty }\end{array}$ & $\begin{array}{c}\text { ck }>\mathbf{0 . 6} \\
\text { bias and } \\
\text { bias uncertainty }\end{array}$ & $\begin{array}{c}\text { ck }>\mathbf{0 . 5} \\
\text { bias and } \\
\text { bias uncertainty }\end{array}$ & $\begin{array}{c}\text { Modified } \\
\boldsymbol{k}_{\text {eff }}\end{array}$ & $\begin{array}{c}\text { Total data- } \\
\text { induced } \\
\text { uncertainty }\end{array}$ & $\begin{array}{c}\text { Absolute data- } \\
\text { induced } \\
\text { uncertainty }\end{array}$ \\
\hline set-1-5-00657 & 0.0822 & 0.0178 & 0.0108 & 0.9437 & 0.006705 & 0.006327 \\
set-1-5-00658 & & 0.0216 & 0.0114 & 0.9492 & 0.006287 & 0.005968 \\
set-1-5-00659 & 0.0406 & 0.0168 & 0.0108 & 0.9323 & 0.006627 & 0.006178 \\
set-1-5-00660 & & 0.0211 & 0.0113 & 0.9371 & 0.006313 & 0.005916 \\
set-1-5-00669 & & 0.0179 & 0.0108 & 0.9290 & 0.006685 & 0.006211 \\
set-1-5-00670 & & 0.0220 & 0.0114 & 0.9335 & 0.006268 & 0.005851 \\
set-1-5-00671 & 0.0574 & 0.0172 & 0.0108 & 0.9180 & 0.006698 & 0.006148 \\
set-1-5-00672 & & 0.0214 & 0.0114 & 0.9225 & 0.006397 & 0.005901 \\
set-1-5-00693 & & 0.0361 & 0.0160 & 0.8531 & 0.006626 & 0.005653 \\
set-1-5-00694 & & 0.0858 & 0.0177 & 0.8589 & 0.006324 & 0.005432 \\
set-1-5-00695 & & 0.0219 & 0.0132 & 0.8533 & 0.006624 & 0.005652 \\
set-1-5-00696 & & 0.0308 & 0.0122 & 0.8583 & 0.006387 & 0.005481 \\
set-1-5-00705 & & 0.0342 & 0.0160 & 0.8414 & 0.006616 & 0.005567 \\
set-1-5-00706 & & 0.0968 & 0.0179 & 0.8465 & 0.006305 & 0.005337 \\
set-1-5-00707 & & 0.0234 & 0.0138 & 0.8417 & 0.006688 & 0.005629 \\
set-1-5-00708 & & 0.0367 & 0.0150 & 0.8464 & 0.006488 & 0.005492 \\
\hline
\end{tabular}


Table H-38. Bias, bias uncertainty, and data-induced uncertainty for set-1-6 cases

\begin{tabular}{ccccccc}
\hline Case & $\begin{array}{c}\text { ck }>\mathbf{0 . 7} \\
\text { bias and } \\
\text { bias uncertainty }\end{array}$ & $\begin{array}{c}\text { ck }>\mathbf{0 . 6} \\
\text { bias and } \\
\text { bias uncertainty }\end{array}$ & $\begin{array}{c}\text { ck }>\mathbf{0 . 5} \\
\text { bias and } \\
\text { bias uncertainty }\end{array}$ & $\begin{array}{c}\text { Modified } \\
\boldsymbol{k}_{\text {eff }}\end{array}$ & $\begin{array}{c}\text { Total data- } \\
\text { induced } \\
\text { uncertainty }\end{array}$ & $\begin{array}{c}\text { Absolute data- } \\
\text { induced } \\
\text { uncertainty }\end{array}$ \\
\hline set-1-6-00441 & & 0.0199 & 0.0128 & 0.9606 & 0.006674 & 0.006411 \\
set-1-6-00442 & 0.0415 & 0.0149 & 0.9617 & 0.006236 & 0.005998 \\
set-1-6-00443 & 0.0194 & 0.0113 & 0.9403 & 0.006623 & 0.006228 \\
set-1-6-00444 & 0.0290 & 0.0121 & 0.9418 & 0.006280 & 0.005914 \\
set-1-6-00453 & 0.0202 & 0.0128 & 0.9434 & 0.006632 & 0.006256 \\
set-1-6-00454 & 0.0432 & 0.0152 & 0.9444 & 0.006233 & 0.005886 \\
set-1-6-00455 & 0.0199 & 0.0129 & 0.9243 & 0.006671 & 0.006166 \\
set-1-6-00456 & 0.0362 & 0.0146 & 0.9258 & 0.006371 & 0.005898 \\
set-1-6-00477 & & 0.0225 & 0.8521 & 0.006564 & 0.005594 \\
set-1-6-00478 & & 0.0254 & 0.8571 & 0.006273 & 0.005376 \\
set-1-6-00479 & 0.0438 & 0.0195 & 0.8482 & 0.006583 & 0.005584 \\
set-1-6-00480 & & 0.0236 & 0.8530 & 0.006349 & 0.005415 \\
set-1-6-00489 & & 0.0227 & 0.8395 & 0.006548 & 0.005498 \\
set-1-6-00490 & & 0.0267 & 0.8440 & 0.006251 & 0.005276 \\
set-1-6-00491 & & 0.0198 & 0.8358 & 0.006672 & 0.005576 \\
set-1-6-00492 & & 0.0239 & 0.8403 & 0.006432 & 0.005405 \\
\hline
\end{tabular}

Table H-39. Bias, bias uncertainty, and data-induced uncertainty for set-1-7 cases

\begin{tabular}{ccccccc}
\hline Case & $\begin{array}{c}\text { ck }>\mathbf{0 . 7} \\
\text { bias and } \\
\text { bias uncertainty }\end{array}$ & $\begin{array}{c}\text { ck }>\mathbf{0 . 6} \\
\text { bias and } \\
\text { bias uncertainty }\end{array}$ & $\begin{array}{c}\text { ck }>\mathbf{0 . 5} \\
\text { bias and } \\
\text { bias uncertainty }\end{array}$ & $\begin{array}{c}\text { Modified } \\
\boldsymbol{k}_{\text {eff }}\end{array}$ & $\begin{array}{c}\text { Total data- } \\
\text { induced } \\
\text { uncertainty }\end{array}$ & $\begin{array}{c}\text { Absolute data- } \\
\text { induced } \\
\text { uncertainty }\end{array}$ \\
\hline set-1-7-00441 & & 0.0177 & 0.0125 & 0.8421 & 0.006797 & 0.005724 \\
set-1-7-00442 & & 0.0308 & 0.0151 & 0.8555 & 0.006258 & 0.005354 \\
set-1-7-00443 & 0.0174 & 0.0127 & 0.0106 & 0.9433 & 0.006489 & 0.006121 \\
set-1-7-00444 & 0.0572 & 0.0159 & 0.0112 & 0.9498 & 0.006067 & 0.005762 \\
set-1-7-00453 & & 0.0180 & 0.0128 & 0.8280 & 0.006718 & 0.005562 \\
set-1-7-00454 & & 0.0335 & 0.0152 & 0.8403 & 0.006231 & 0.005235 \\
set-1-7-00455 & 0.0179 & 0.0128 & 0.0106 & 0.9271 & 0.006446 & 0.005976 \\
set-1-7-00456 & 0.0587 & 0.0171 & 0.0112 & 0.9333 & 0.006077 & 0.005672 \\
set-1-7-00477 & & & 0.0227 & 0.7439 & 0.006719 & 0.004998 \\
set-1-7-00478 & & & 0.0262 & 0.7569 & 0.006325 & 0.004787 \\
set-1-7-00479 & & 0.0227 & 0.0111 & 0.8648 & 0.006348 & 0.005489 \\
set-1-7-00480 & & 0.0290 & 0.0118 & 0.8717 & 0.006068 & 0.005290 \\
set-1-7-00489 & & & 0.0228 & 0.7334 & 0.006701 & 0.004914 \\
set-1-7-00490 & & 0.0231 & 0.0264 & 0.7456 & 0.006313 & 0.004707 \\
set-1-7-00491 & & 0.0289 & 0.0118 & 0.8522 & 0.006337 & 0.005401 \\
set-1-7-00492 & & & & & & 0.006078 \\
\hline
\end{tabular}


Table H-40. Bias, bias uncertainty, and data-induced uncertainty for set-1-8 cases

\begin{tabular}{ccccccc}
\hline Case & $\begin{array}{c}\text { ck }>\mathbf{0 . 7} \\
\text { bias and } \\
\text { bias uncertainty }\end{array}$ & $\begin{array}{c}\text { ck }>\mathbf{0 . 6} \\
\text { bias and } \\
\text { bias uncertainty }\end{array}$ & $\begin{array}{c}\text { ck }>\mathbf{0 . 5} \\
\text { bias and } \\
\text { bias uncertainty }\end{array}$ & $\begin{array}{c}\text { Modified } \\
\boldsymbol{k}_{\text {eff }}\end{array}$ & $\begin{array}{c}\text { Total data- } \\
\text { induced } \\
\text { uncertainty }\end{array}$ & $\begin{array}{c}\text { Absolute data- } \\
\text { induced } \\
\text { uncertainty }\end{array}$ \\
\hline set-1-8-00333 & & 0.0267 & 0.0185 & 0.8871 & 0.006703 & 0.005946 \\
set-1-8-00334 & & & 0.0214 & 0.8940 & 0.006217 & 0.005557 \\
set-1-8-00335 & 0.0449 & 0.0175 & 0.0108 & 0.9742 & 0.006535 & 0.006366 \\
set-1-8-00336 & & 0.0226 & 0.0116 & 0.9759 & 0.006124 & 0.005976 \\
set-1-8-00345 & & 0.0277 & 0.0188 & 0.8705 & 0.006648 & 0.005787 \\
set-1-8-00346 & & & 0.0216 & 0.8765 & 0.006197 & 0.005432 \\
set-1-8-00347 & 0.0461 & 0.0178 & 0.0109 & 0.9566 & 0.006491 & 0.006209 \\
set-1-8-00348 & & 0.0229 & 0.0116 & 0.9583 & 0.006112 & 0.005857 \\
set-1-8-00369 & & & 0.0246 & 0.7733 & 0.006607 & 0.005109 \\
set-1-8-00370 & & 0.0285 & 0.7836 & 0.006261 & 0.004907 \\
set-1-8-00371 & & 0.0244 & 0.0114 & 0.8835 & 0.006394 & 0.005649 \\
set-1-8-00372 & & 0.0306 & 0.0122 & 0.8885 & 0.006107 & 0.005426 \\
set-1-8-00381 & & & 0.0247 & 0.7617 & 0.006594 & 0.005023 \\
set-1-8-00382 & & & 0.0288 & 0.7713 & 0.006240 & 0.004813 \\
set-1-8-00383 & & 0.0248 & 0.0114 & 0.8701 & 0.006382 & 0.005553 \\
set-1-8-00384 & & 0.0310 & 0.0122 & 0.8747 & 0.006106 & 0.005341 \\
\hline
\end{tabular}




\section{APPENDIX I. SET-3a: RESULTS OF THE SIX-HIGH UNIFORM ARRAY}

WITH ALTERNATIVE SPACING CALCULATIONS 
This page is intentionally blank 


\section{APPENDIX I. SET-3A: RESULTS OF THE SIX-HIGH UNIFORM ARRAY WITH BOUNDING SPACING CALCULATIONS}

The analysis methodology for the uniform arrays is discussed in detail in Section 6.2 of the main report.

This appendix serves as a repository of those results for the set-3a calculations.

The complete results for all SAMPLER sweeps are provided in Addendum 1.

The analysis model use for the calculations in this appendix is shown in Figure I-1 below.

The SAMPLER case sweeps presented in this appendix are summarized in Table I-1 below.

\section{LIST OF FIGURES}

Figure I-1. Diagram of the uniform array six-high model.

Figure I-2. Set-3a results, plot 1: reactivity effect of cylinder radius, pipe steel, no filler, no Be, water moderated.

Figure I-3. Set-3a results, plot 2: reactivity effect of cylinder radius, pipe steel, no filler, no Be, poly moderated.

Figure I-4. Set-3a results, plot 3: reactivity effect of cylinder radius, pipe poly, no filler, no Be, water moderated.

Figure I-5. Set-3a results, plot 4: reactivity effect of cylinder radius, pipe poly, no filler, no Be, poly moderated.

Figure I-6. Set-3a results, plot 5: reactivity effect of cylinder radius, pipe steel, $5 \mathrm{~kg}$ graphite/can, no $\mathrm{Be}$, water moderated.

Figure I-7. Set-3a results, plot 6: reactivity effect of cylinder radius, pipe steel, $5 \mathrm{~kg}$ graphite/can, no Be, poly moderated.

Figure I-8. Set-3a results, plot 7: reactivity effect of cylinder radius, pipe poly, $5 \mathrm{~kg}$ graphite/can, no Be, water moderated.

Figure I-9. Set-3a results, plot 8: reactivity effect of cylinder radius, pipe poly, $5 \mathrm{~kg}$ graphite/can, no Be, poly moderated.

Figure I-10. Set-3a results, plot 9: reactivity effect of various parameters with $7.7 \mathrm{~cm}$ cylinder radius, graphite filler, poly moderated.

Figure I-11. Set-3a results, plot 10: reactivity effect of various parameters with $7.7 \mathrm{~cm}$ cylinder radius, generic filler, poly moderated.

Figure I-12. Set-3a results, plot 11: comparison of graphite and generic filler with $7.7 \mathrm{~cm}$ cylinder radius, no Be, poly moderated, thick discrete reflector.

Figure I-13. Set-3a results, plot 12: reactivity effect of various parameters with spherical waste form geometry, graphite filler, water moderated.

Figure I-14. Set-3a results, plot 13: reactivity effect of various parameters with spherical waste form geometry, graphite filler, poly moderated.

Figure I-15. Set-3a results, plot 14: comparison of spherical and cylindrical geometries (h/x).............I-20

Figure I-16. Set-3a results, plot 15: comparison of water and poly $\mathrm{h} / \mathrm{x}$.

Figure I-17. Set-3a results, plot 16: comparison of spherical and cylindrical geometries (mod mass).

Figure I-18. Set-3a results, plot 17: Reactivity effect of $25 \mathrm{~g} \mathrm{~B} 4 \mathrm{C}$ per waste form (2 per CCO) I-23 


\section{LIST OF TABLES}

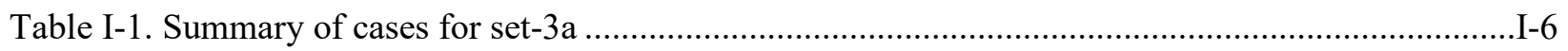




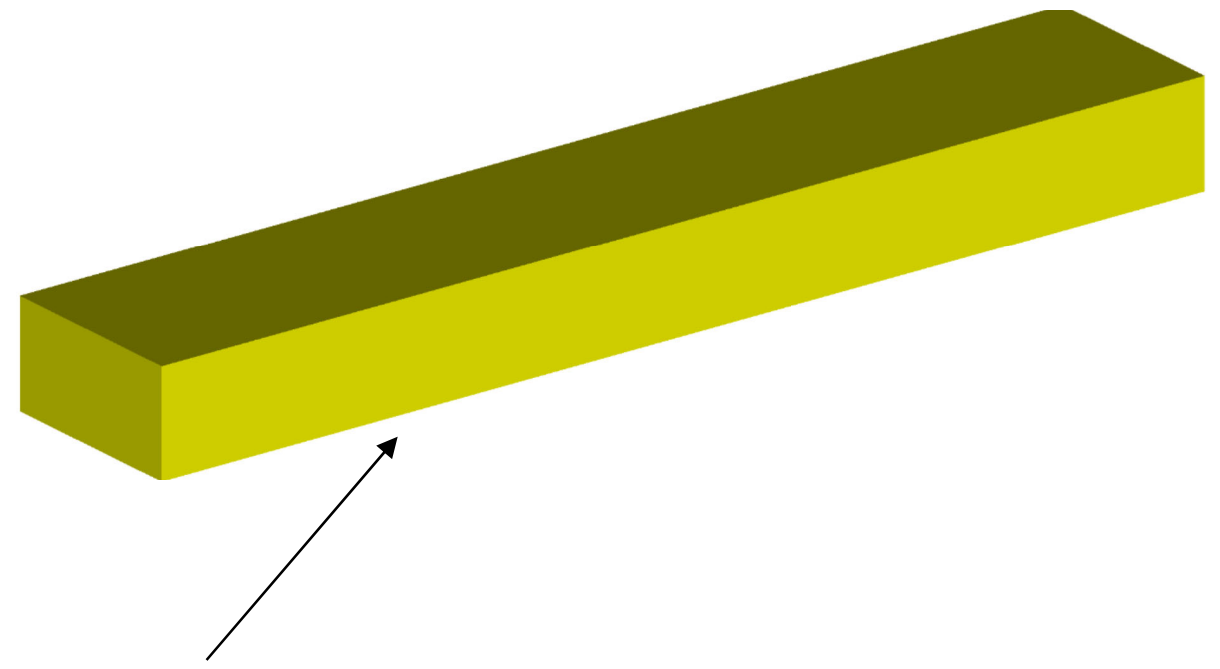

wipp salt panel, pure salt

uniformly compacted CCOs (salt/MgO mix)

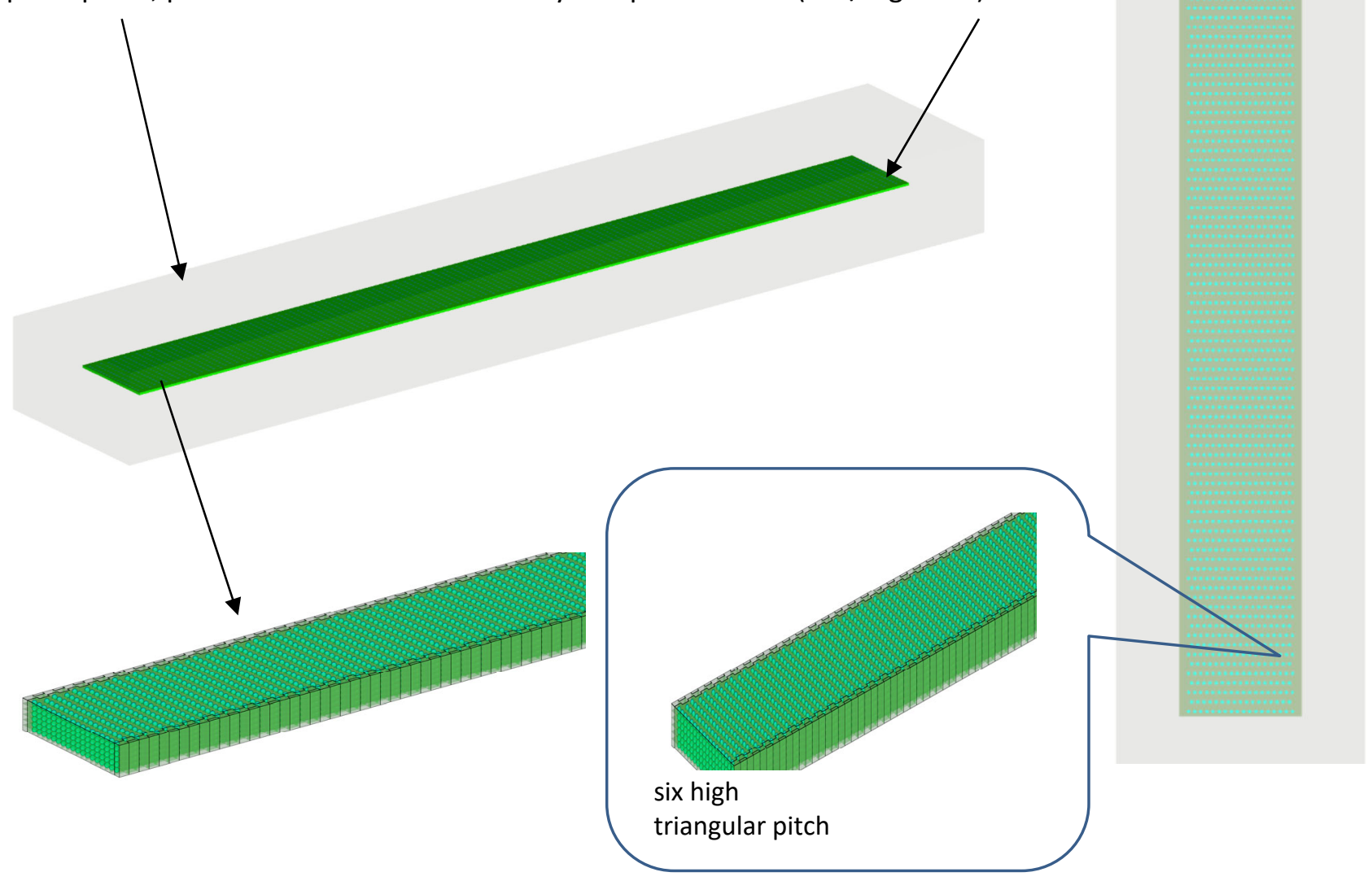

Figure I-1. Diagram of the uniform array six-high model. 
Table I-1. Summary of cases for set-3a

\begin{tabular}{|c|c|c|c|c|c|c|c|c|}
\hline Case & Model type & Waste form shape & $\begin{array}{l}\text { Waste form } \\
\text { moderator }\end{array}$ & $\begin{array}{c}\text { Filler } \\
\text { material }(0, \\
2,000,4,000 \\
\text { g) }\end{array}$ & Metal in filler & $\begin{array}{l}\text { Discrete reflector } \\
\text { (thin } 0.001 \text { and } \\
\text { thick } 0.7112 \mathrm{~cm} \text { ) }\end{array}$ & be $(\mathrm{g})$ & Subcase \\
\hline \multirow{18}{*}{ Set-3a } & \multirow{18}{*}{$\begin{array}{l}\text { Uniform array stacked six } \\
\text { high with CCO pitch } \\
\text { reduction } \mathrm{x}=25 \%, \mathrm{y}=10 \%, \\
\mathrm{z}=\text { no space }\end{array}$} & \multirow{8}{*}{$\begin{array}{c}\text { Cylinder (radius range } 4.8,6, \\
7.7 \text { and height defined by } \\
\text { total volume of mass) }\end{array}$} & water & $\mathrm{c} 12$ & \multirow{18}{*}{$\begin{array}{l}\text { SS from can } \\
(0,500,1,000 \\
\text { g) }\end{array}$} & steel & \multirow{18}{*}{$\begin{array}{l}0 \text { to } \\
585\end{array}$} & set-3a-1 \\
\hline & & & poly & $\mathrm{c} 12$ & & steel & & set-3a-2 \\
\hline & & & water & $\mathrm{c} 12$ & & poly & & set-3a-3 \\
\hline & & & poly & $\mathrm{c} 12$ & & poly & & set-3a-4 \\
\hline & & & water & generic & & steel & & set-3a-5 \\
\hline & & & poly & generic & & steel & & set-3a-6 \\
\hline & & & water & generic & & poly & & set-3a-7 \\
\hline & & & poly & generic & & poly & & set-3a-8 \\
\hline & & \multirow{8}{*}{$\begin{array}{l}\text { Sphere (radius defined by } \\
\text { total volume of mass) }\end{array}$} & water & $\mathrm{c} 12$ & & steel & & set-3a-9 \\
\hline & & & poly & $\mathrm{c} 12$ & & steel & & set-3a-10 \\
\hline & & & water & $\mathrm{c} 12$ & & poly & & set-3a-11 \\
\hline & & & poly & $\mathrm{c} 12$ & & poly & & set-3a-12 \\
\hline & & & water & generic & & steel & & set-3a-13 \\
\hline & & & poly & generic & & steel & & set-3a-14 \\
\hline & & & water & generic & & poly & & set-3a-15 \\
\hline & & & poly & generic & & poly & & set-3a-16 \\
\hline & & $\begin{array}{l}\text { Cylinder (radius range } 4.8,6 \text {, } \\
7.7 \text { and height defined by } \\
\text { total volume of mass) with } \\
25 \mathrm{~g} \mathrm{~B}_{4} \mathrm{C}\end{array}$ & poly & $\mathrm{c} 12$ & & poly & & set-3a-17 \\
\hline & & $\begin{array}{l}\text { Sphere (radius defined by } \\
\text { total volume of mass) with } \\
25 \mathrm{~g} \mathrm{~B}{ }_{4} \mathrm{C}\end{array}$ & poly & $\mathrm{c} 12$ & & poly & & set-3a-18 \\
\hline
\end{tabular}




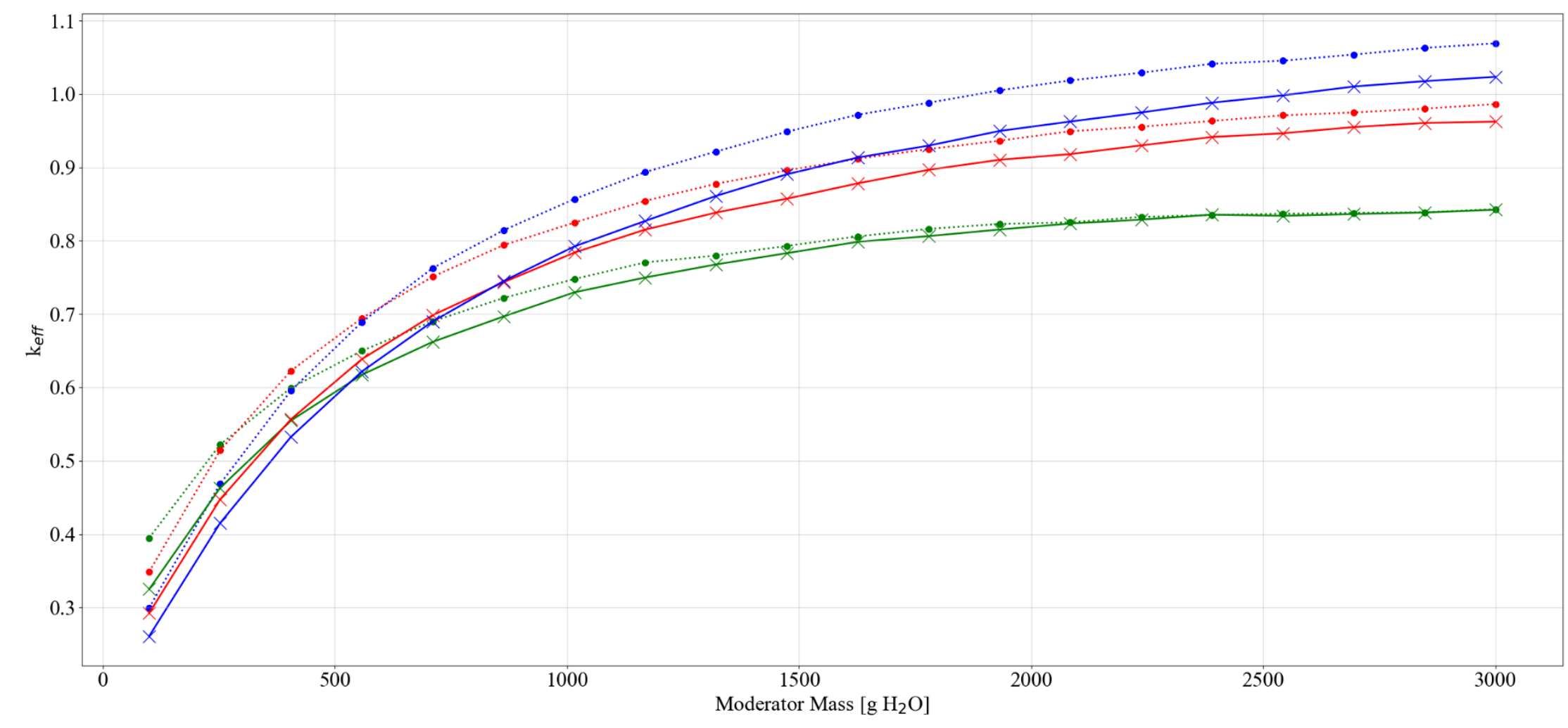

-... set-3a, cyl radius $=4.8 \mathrm{~cm}$, thin discrete reflector set- $3 \mathrm{a}$, cyl radius $=6.25 \mathrm{~cm}$, thin discrete reflecto

$\cdots \cdot \cdots$ set-3a, cyl radius $=7.7 \mathrm{~cm}$, thin discrete reflector

$*$ set-3a, cyl radius $=4.8 \mathrm{~cm}$, thick discrete reflector $\star$ set- 3 a, cyl radius $=6.25 \mathrm{~cm}$, thick discrete reflector $\leftarrow$ set-3a, cyl radius $=7.7 \mathrm{~cm}$, thick discrete reflector

Figure I-2. Set-3a results, plot 1: reactivity effect of cylinder radius, pipe steel, no filler, no Be, water moderated. 


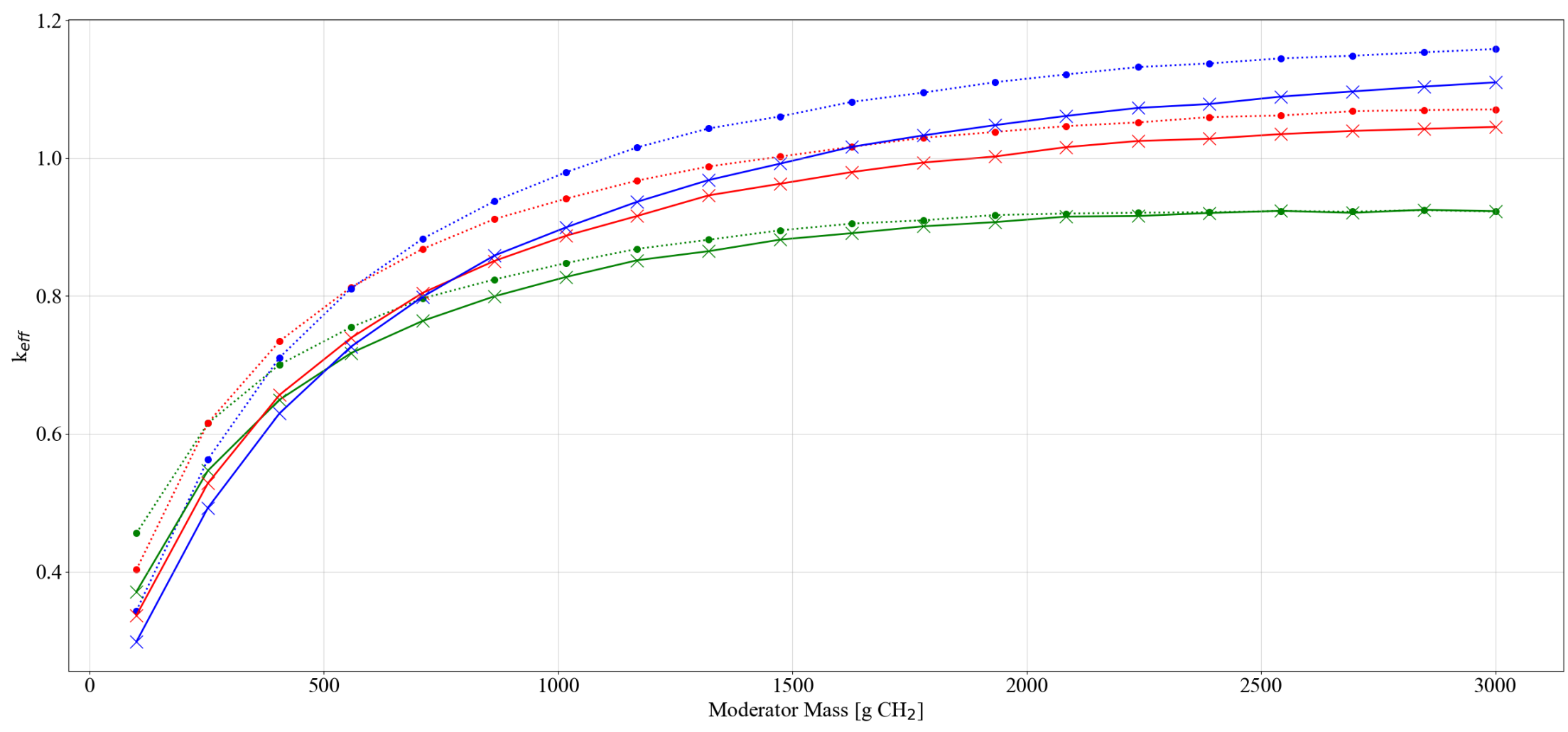

-... set-3a, cyl radius $=4.8 \mathrm{~cm}$, thin discrete reflector

-.. set-3a, cyl radius $=6.25 \mathrm{~cm}$, thin discrete reflector

$\star$ set- $3 \mathrm{a}$, cyl radius $=4.8 \mathrm{~cm}$, thick discrete reflector

.. set-3a, cyl radius $=7.7 \mathrm{~cm}$, thin discrete reflector

- set- $3 \mathrm{a}$, cyl radius $=6.25 \mathrm{~cm}$, thick discrete reflector

Figure I-3. Set-3a results, plot 2: reactivity effect of cylinder radius, pipe steel, no filler, no Be, poly moderated. 


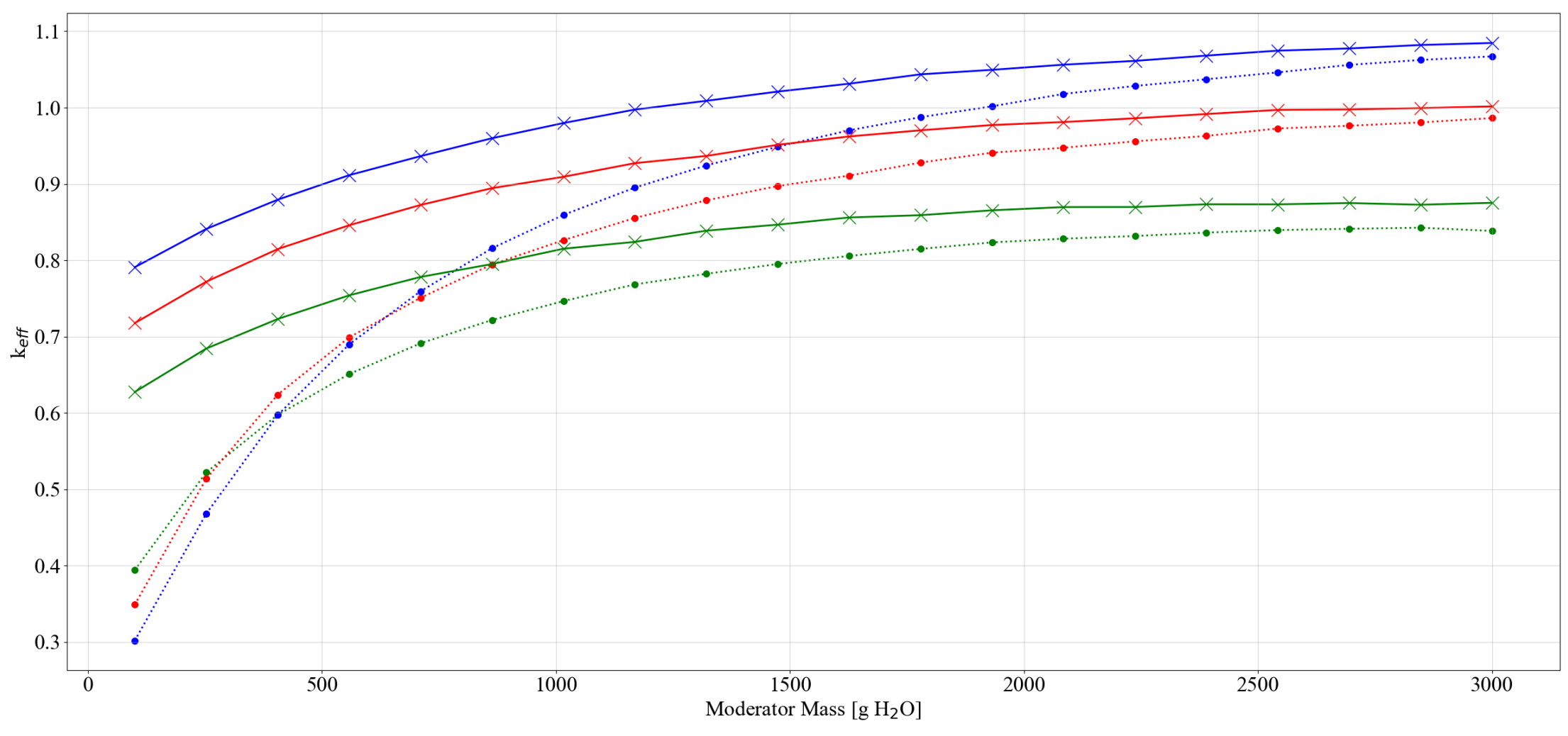

-... set-3a, cyl radius $=4.8 \mathrm{~cm}$, thin discrete reflector

- $\cdots$ set-3a, cyl radius $=6.25 \mathrm{~cm}$, thin discrete reflector

$\star \quad$ set-3a, cyl radius $=4.8 \mathrm{~cm}$, thick discrete reflector

$\leftarrow$ set- $3 \mathrm{a}$, cyl radius $=6.25 \mathrm{~cm}$, thick discrete reflector

- $\cdots$ set-3a, cyl radius $=7.7 \mathrm{~cm}$, thin discrete reflector

$\star$ set-3a, cyl radius $=7.7 \mathrm{~cm}$, thick discrete reflector

Figure I-4. Set-3a results, plot 3: reactivity effect of cylinder radius, pipe poly, no filler, no Be, water moderated. 


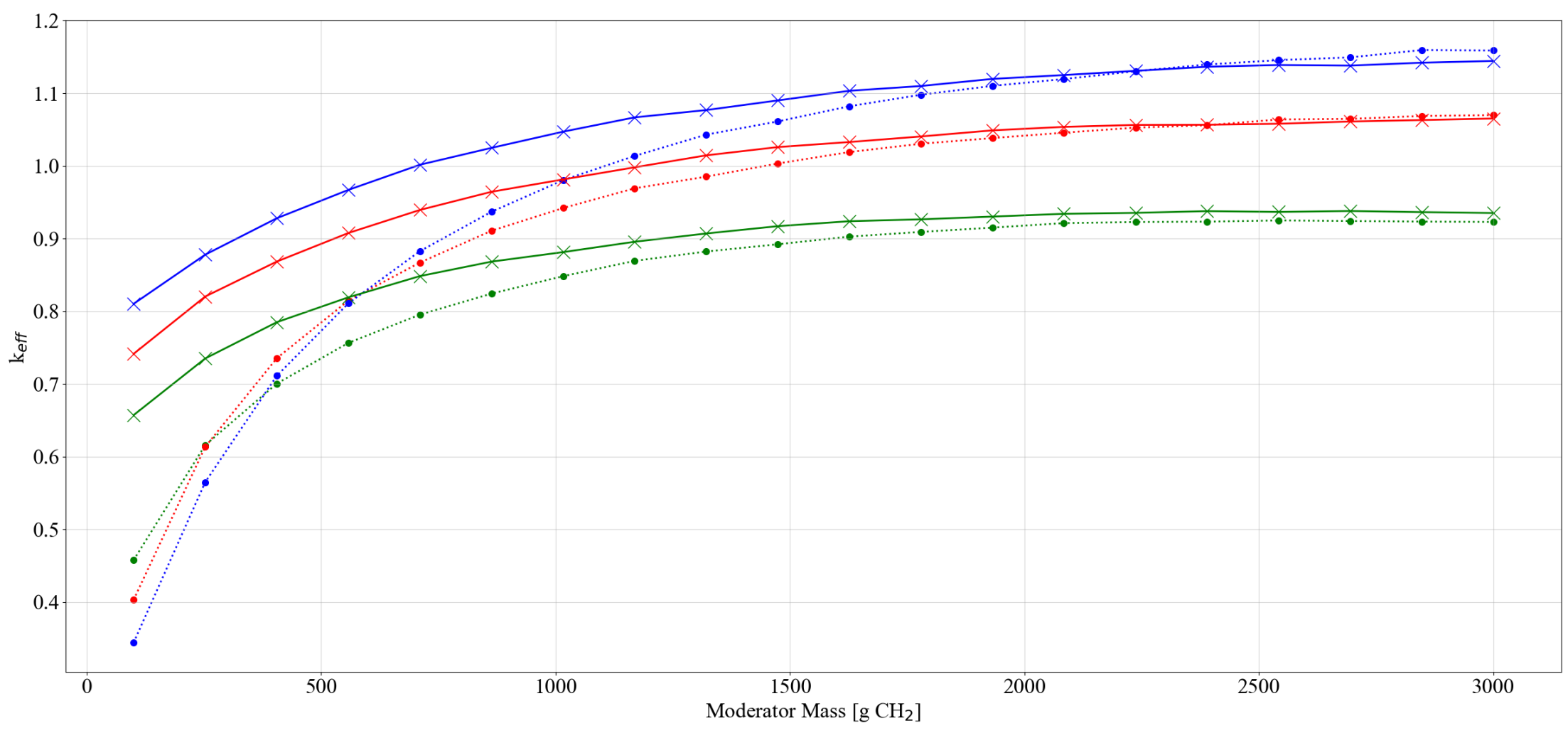

- $\cdots$ set-3a, cyl radius $=4.8 \mathrm{~cm}$, thin discrete reflector

-.. set-3a, cyl radius $=6.25 \mathrm{~cm}$, thin discrete reflector

* set-3a, cyl radius $=4.8 \mathrm{~cm}$, thick discrete reflector

$\leftarrow$ set-3a, cyl radius $=6.25 \mathrm{~cm}$, thick discrete reflector

-... set-3a, cyl radius $=7.7 \mathrm{~cm}$, thin discrete reflector

$\leftarrow$ set-3a, cyl radius $=7.7 \mathrm{~cm}$, thick discrete reflector

Figure I-5. Set-3a results, plot 4: reactivity effect of cylinder radius, pipe poly, no filler, no Be, poly moderated. 


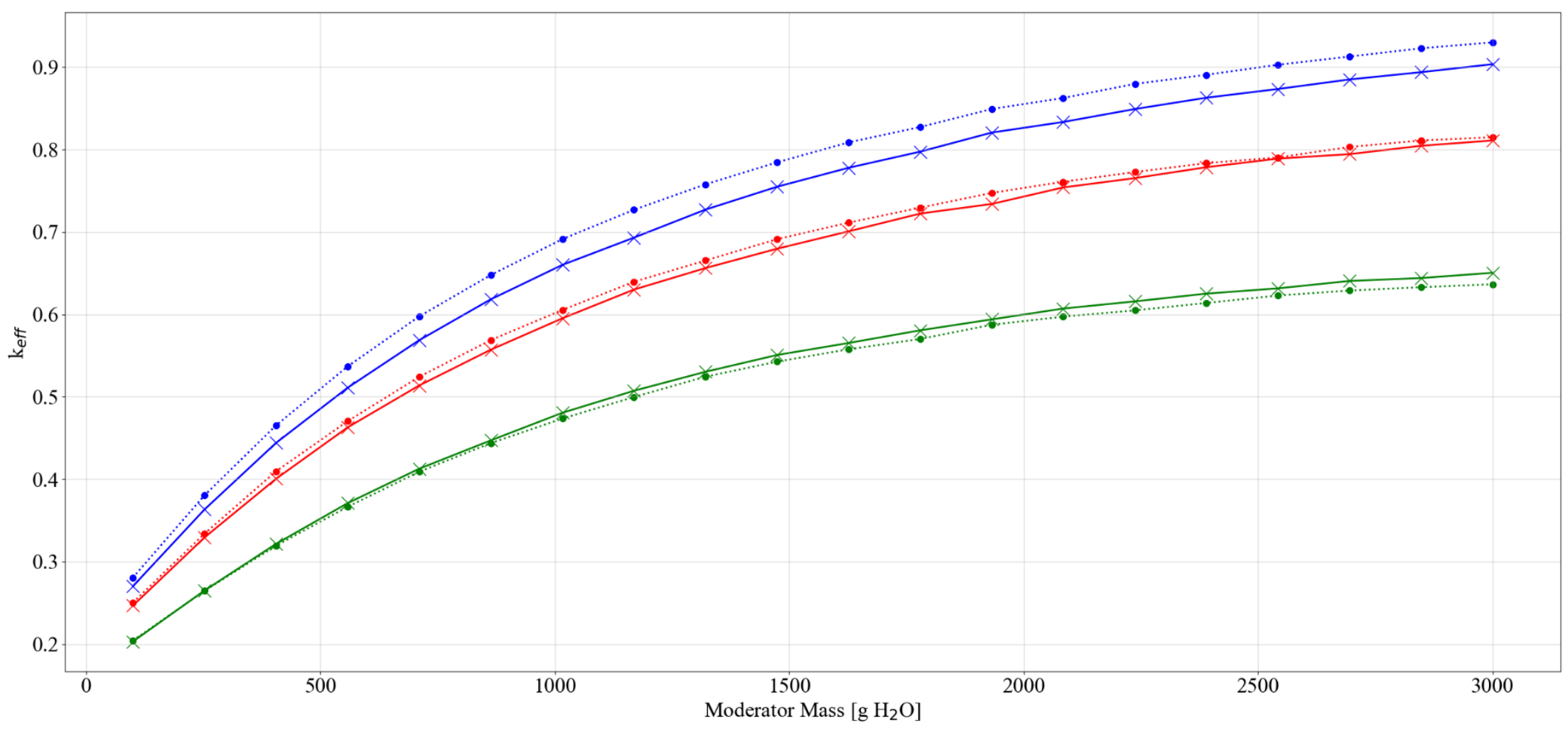

-... set-3a, cyl radius $=4.8 \mathrm{~cm}$, thin discrete reflector set- $3 \mathrm{a}$, cyl radius $=6.25 \mathrm{~cm}$, thin discrete reflecto set-3a, cyl radius $=7.7 \mathrm{~cm}$, thin discrete reflector

$$
\begin{aligned}
& * \text { set- } 3 \mathrm{a} \text {, cyl radius }=4.8 \mathrm{~cm} \text {, thick discrete reflector } \\
& \star \quad \text { set- } 3 \mathrm{a} \text {, cyl radius }=6.25 \mathrm{~cm} \text {, thick discrete reflector } \\
& \star \quad \text { set- } 3 \mathrm{a} \text {, cyl radius }=7.7 \mathrm{~cm} \text {, thick discrete reflector }
\end{aligned}
$$

Figure I-6. Set-3a results, plot 5: reactivity effect of cylinder radius, pipe steel, $5 \mathrm{~kg}$ graphite/can, no Be, water moderated. 


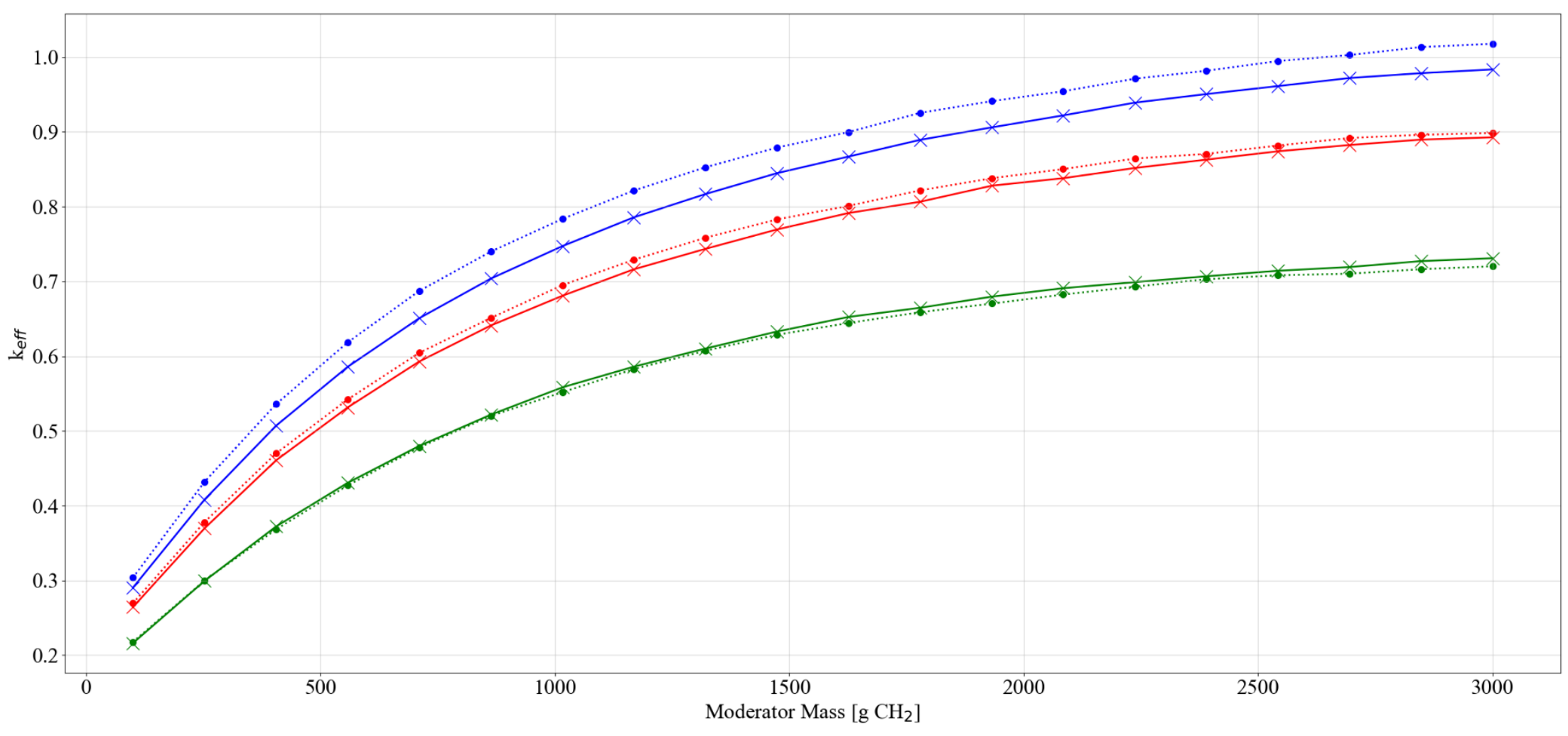

-... set-3a, cyl radius $=4.8 \mathrm{~cm}$, thin discrete reflector

set-3a, cyl radius $=6.25 \mathrm{~cm}$, thin discrete reflector $\leftarrow$ set- $3 \mathrm{a}$, cyl radius $=6.25 \mathrm{~cm}$, thick discrete reflector * set-3a, cyl radius $=4.8 \mathrm{~cm}$, thick discrete reflector

- $\cdots$ set- $3 \mathrm{a}$, cyl radius $=7.7 \mathrm{~cm}$, thin discrete reflector $\quad *$ set- $3 \mathrm{a}$, cyl radius $=7.7 \mathrm{~cm}$, thick discrete reflector

Figure I-7. Set-3a results, plot 6: reactivity effect of cylinder radius, pipe steel, $5 \mathrm{~kg}$ graphite/can, no Be, poly moderated. 


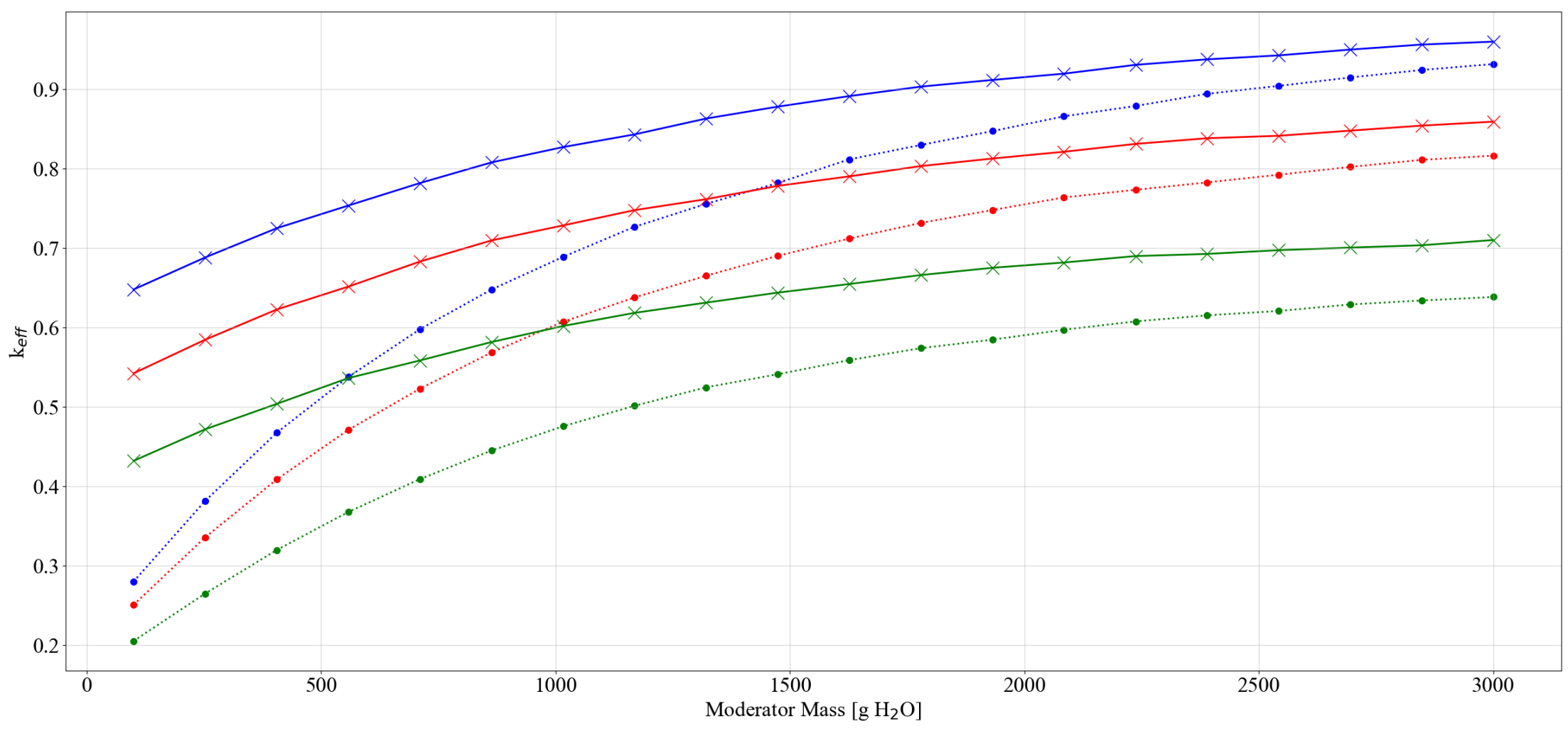

- $\cdots$ set-3a, cyl radius $=4.8 \mathrm{~cm}$, thin discrete reflector

- $\cdots$ set-3a, cyl radius $=6.25 \mathrm{~cm}$, thin discrete reflector

$\leftarrow$ set- 3 a, cyl radius $=4.8 \mathrm{~cm}$, thick discrete reflector

$\leftarrow$ set-3a, cyl radius $=6.25 \mathrm{~cm}$, thick discrete reflector

$\cdots \cdots$ set-3a, cyl radius $=7.7 \mathrm{~cm}$, thin discrete reflector

$\star$ set-3a, cyl radius $=7.7 \mathrm{~cm}$, thick discrete reflector

Figure I-8. Set-3a results, plot 7: reactivity effect of cylinder radius, pipe poly, $5 \mathrm{~kg}$ graphite/can, no Be, water moderated. 


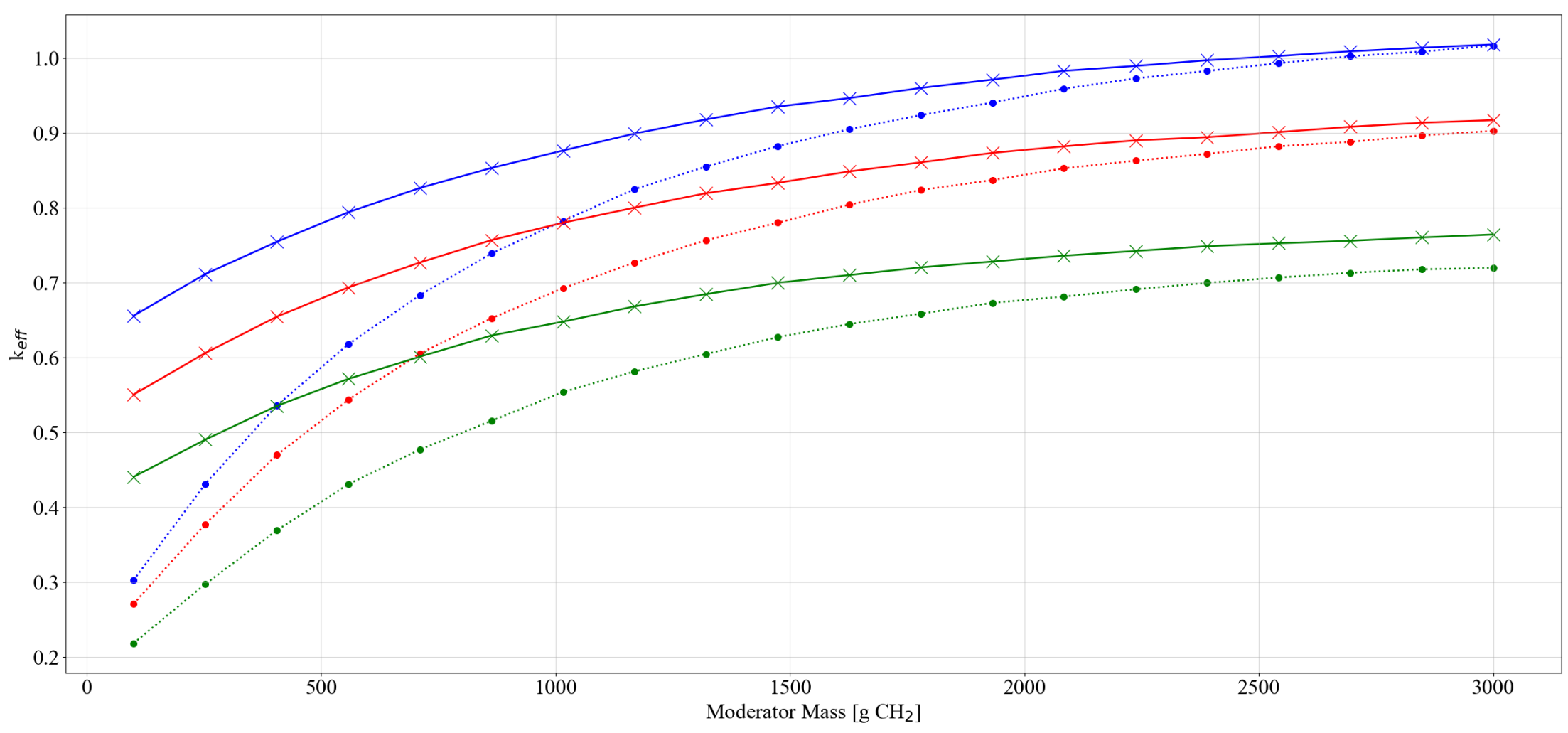

- $\cdots$ set- $3 \mathrm{a}$, cyl radius $=4.8 \mathrm{~cm}$, thin discrete reflector $\star$ set-3a, cyl radius $=4.8 \mathrm{~cm}$, thick discrete reflector

-... set-3a, cyl radius $=6.25 \mathrm{~cm}$, thin discrete reflecto $\star$ set- $3 \mathrm{a}$, cyl radius $=6.25 \mathrm{~cm}$, thick discrete reflector

•.. set-3a, cyl radius $=7.7 \mathrm{~cm}$, thin discrete reflector $\leftarrow$ set- 3 a, cyl radius $=7.7 \mathrm{~cm}$, thick discrete reflector

Figure I-9. Set-3a results, plot 8: reactivity effect of cylinder radius, pipe poly, $5 \mathrm{~kg}$ graphite/can, no Be, poly moderated. 


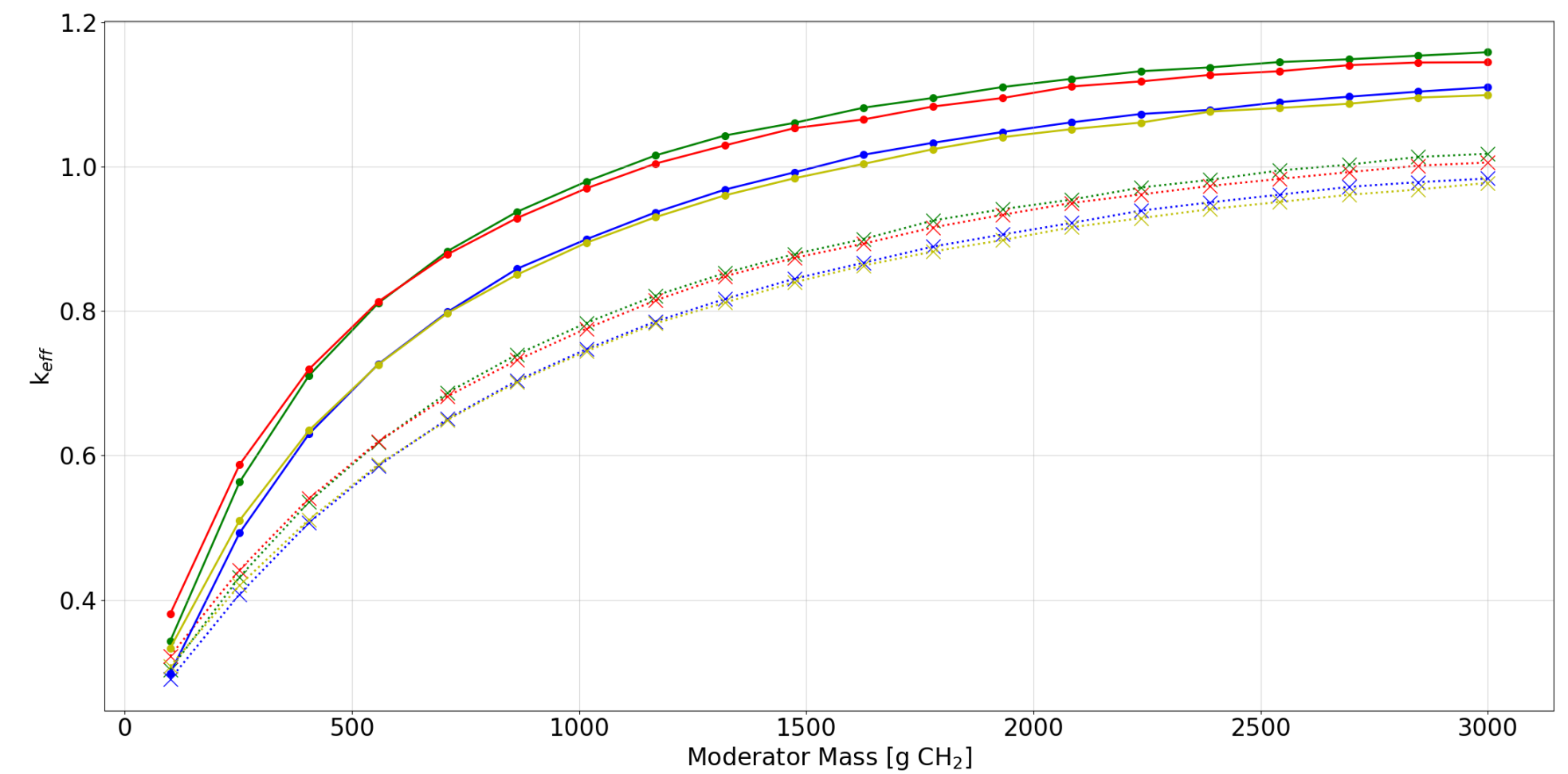

- set-3a, no filler, no Be, thin SS discrete reflector

........ set-3a, $5 \mathrm{~kg}$ graphite/can, no Be, thin SS discrete reflector

$\rightarrow$ set-3a, no filler, $585 \mathrm{~g} \mathrm{Be}$, thin SS discrete reflector

- - set-3a, no filler, no Be, thick SS discrete reflector

........ set-3a, $5 \mathrm{~kg}$ graphite/can, no Be, thick SS discrete reflector

- set-3a, no filler, $585 \mathrm{~g} \mathrm{Be}$, thick SS discrete reflector

set-3a, $5 \mathrm{~kg}$ graphite/can, $585 \mathrm{~g} \mathrm{Be}$, thin SS discrete reflector

set-3a, $5 \mathrm{~kg}$ graphite/can, $585 \mathrm{~g}$ Be, thick SS discrete reflector

Figure I-10. Set-3a results, plot 9: reactivity effect of various parameters with $7.7 \mathrm{~cm}$ cylinder radius, graphite filler, poly moderated. 


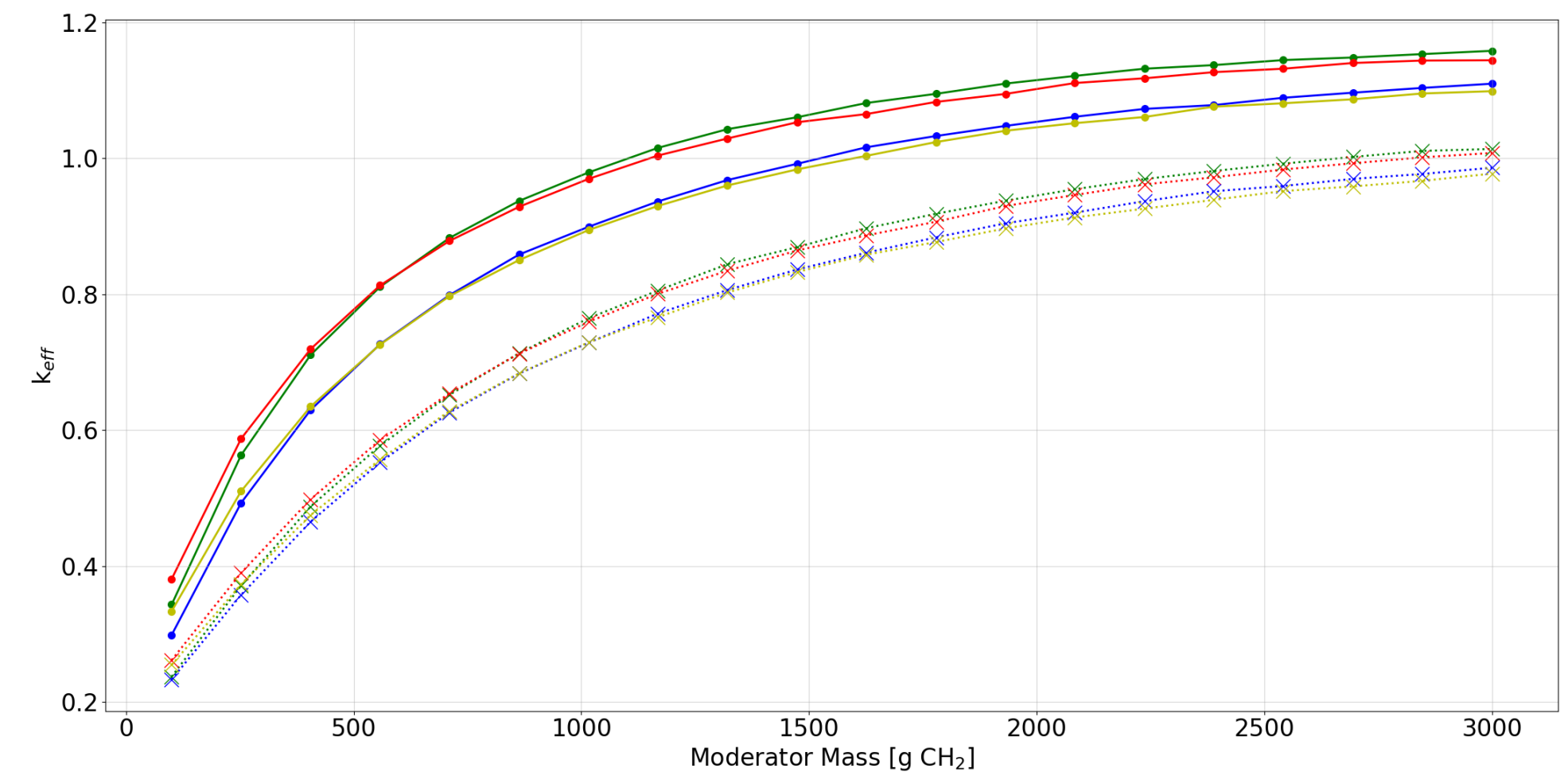

- set-3a, no filler, no Be, thin SS discrete reflector

........ set-3a, $5 \mathrm{~kg}$ generic/can, no Be, thin SS discrete reflector

- set-3a, no filler, $585 \mathrm{~g} \mathrm{Be}$, thin SS discrete reflector set-3a, $5 \mathrm{~kg}$ generic/can, $585 \mathrm{~g} \mathrm{Be}$, thin SS discrete $r$ reflector

- - set-3a, no filler, no Be, thick SS discrete reflector

... ‘.... set-3a, $5 \mathrm{~kg}$ generic/can, no Be, thick SS discrete reflector

- set-3a, no filler, $585 \mathrm{~g} \mathrm{Be}$, thick SS discrete reflector

......... set-3a, $5 \mathrm{~kg}$ generic/can, $585 \mathrm{~g} \mathrm{Be}$, thick SS discrete reflector

Figure I-11. Set-3a results, plot 10: reactivity effect of various parameters with $7.7 \mathrm{~cm}$ cylinder radius, generic filler, poly moderated. 


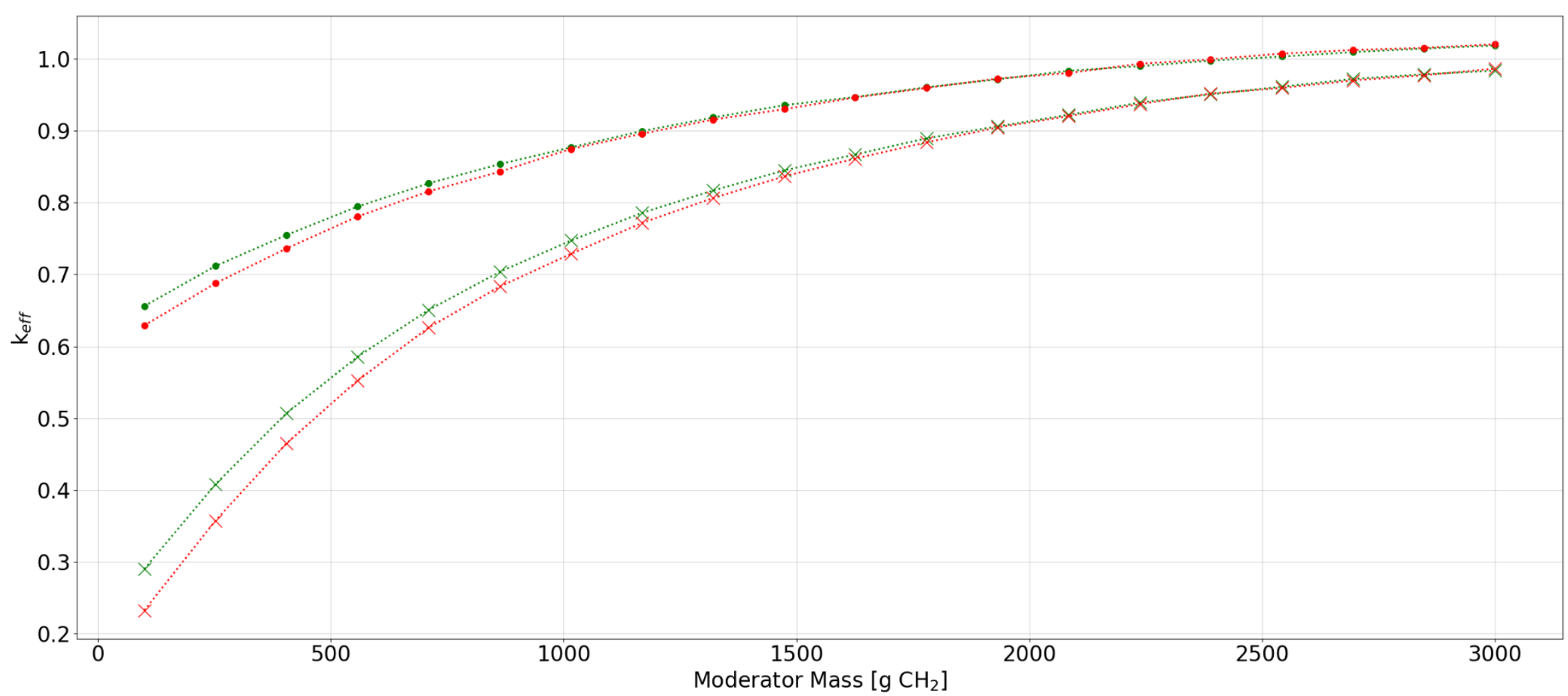

‘.. set-3a, cylinder radius $=7.7 \mathrm{~cm}$, poly moderator, $5 \mathrm{~kg}$ graphite/can, no Be, thick SS discrete reflector

-... set-3a, cylinder radius $=7.7 \mathrm{~cm}$, poly moderator, $5 \mathrm{~kg}$ graphite/can, no Be, thick poly discrete reflector

׳... set-3a, cylinder radius $=7.7 \mathrm{~cm}$, poly moderator, $5 \mathrm{~kg}$ generic/can, no Be, thick SS discrete reflector

$\cdots \cdots$ set-3a, cylinder radius $=7.7 \mathrm{~cm}$, poly moderator, $5 \mathrm{~kg}$ generic/can, no Be, thick poly discrete reflector

Figure I-12. Set-3a results, plot 11: comparison of graphite and generic filler with $7.7 \mathrm{~cm}$ cylinder radius, no Be, poly moderated, thick discrete reflector. 


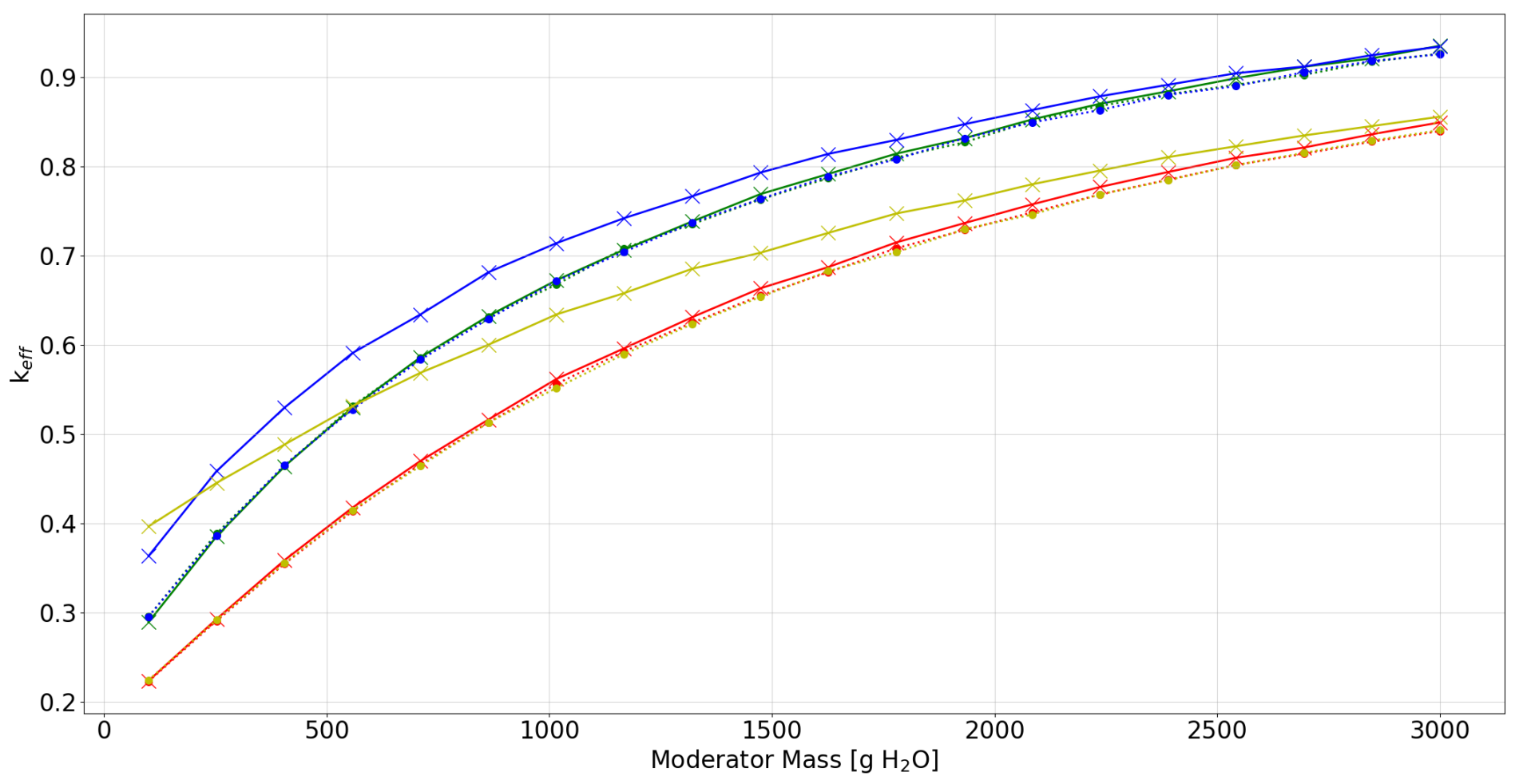

-... set-3a, no filler, no Be, thin SS discrete reflector

$\star$ set-3a, no filler, no Be, thick SS discrete reflector

....... set-3a, $5 \mathrm{~kg}$ graphite/can, $585 \mathrm{~g} \mathrm{Be}$, thin SS discrete reflector set-3a, $5 \mathrm{~kg}$ graphite/can, $585 \mathrm{~g}$ Be, thick SS discrete reflector
•... set-3a, no filler, no Be, thin poly discrete reflector

$\star$ set-3a, no filler, no Be, thick poly discrete reflector

-... set-3a, $5 \mathrm{~kg}$ graphite/can, $585 \mathrm{~g} \mathrm{Be}$, thin poly discrete reflector - set-3a, $5 \mathrm{~kg}$ graphite/can, $585 \mathrm{~g} \mathrm{Be}$, thick poly discrete reflector

Figure I-13. Set-3a results, plot 12: reactivity effect of various parameters with spherical waste form geometry, graphite filler, water moderated. 


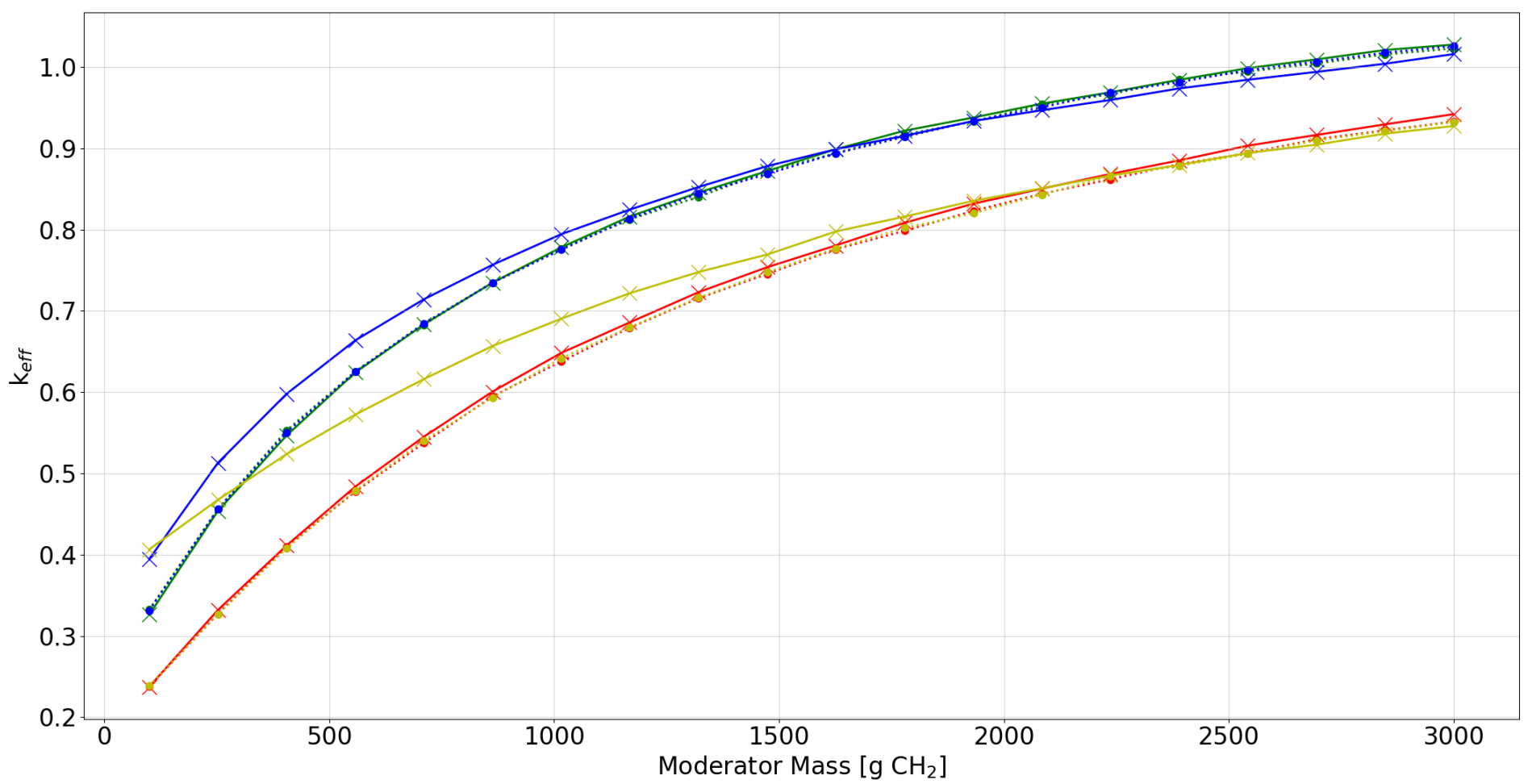

..... set-3a, no filler, no Be, thin SS discrete reflector

$\star$ set-3a, no filler, no Be, thick SS discrete reflector

..... set-3a, $5 \mathrm{~kg}$ graphite/can, $585 \mathrm{~g} \mathrm{Be}$, thin SS discrete reflector

$*$ set-3a, $5 \mathrm{~kg}$ graphite/can, $585 \mathrm{~g} \mathrm{Be}$, thick SS discrete reflector
-... set-3a, no filler, no Be, thin poly discrete reflector

$\star$ set-3a, no filler, no Be, thick poly discrete reflector

-... set-3a, $5 \mathrm{~kg}$ graphite/can, $585 \mathrm{~g} \mathrm{Be}$, thin poly discrete reflector - set-3a, $5 \mathrm{~kg}$ graphite/can, $585 \mathrm{~g} \mathrm{Be}$, thick poly discrete reflector

Figure I-14. Set-3a results, plot 13: reactivity effect of various parameters with spherical waste form geometry, graphite filler, poly moderated. 


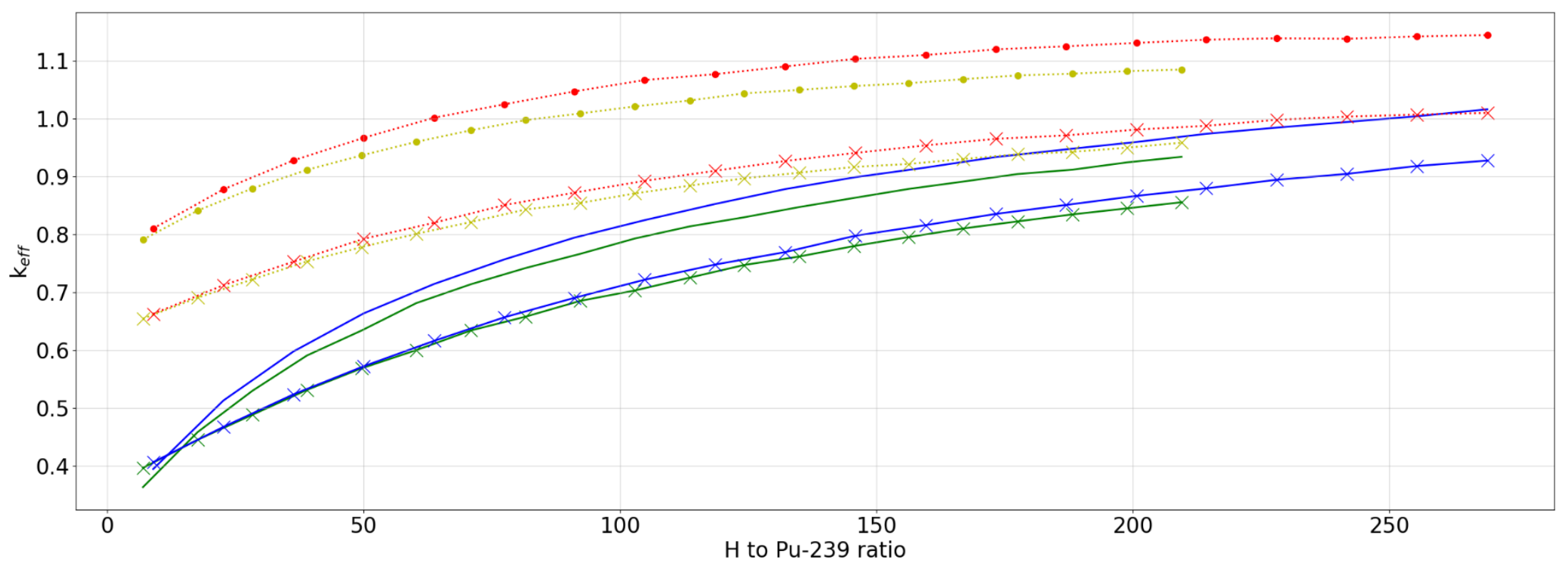

set-3a, sphere, no filler, no Be, thick poly discrete reflector, water moderator

set-3a, sphere, $5 \mathrm{~kg}$ graphite/can, $585 \mathrm{~g} \mathrm{Be}$, thick poly discrete reflector, water moderator

set-3a, sphere, no filler, no Be, thick poly discrete reflector, poly moderator

$*$ set-3a, sphere, $5 \mathrm{~kg}$ graphite/can, $585 \mathrm{~g} \mathrm{Be}$, thick poly discrete reflector, poly moderator

$\ldots . . .$. set-3a, cylinder with radius $=7.7 \mathrm{~cm}$, no filler, no $\mathrm{Be}$, thick poly discrete reflector, water moderator

.*... set-3a, cylinder with radius $=7.7 \mathrm{~cm}, 5 \mathrm{~kg}$ graphite $/ \mathrm{can}, 585 \mathrm{~g} \mathrm{Be}$, thick poly discrete reflector, water moderator

....... set-3a, cylinder with radius $=7.7 \mathrm{~cm}$, no filler, no Be, thick poly discrete reflector, poly moderator

........ set-3a, cylinder with radius $=7.7 \mathrm{~cm}, 5 \mathrm{~kg}$ graphite/can, $585 \mathrm{~g} \mathrm{Be}$, thick poly discrete reflector, poly moderator

Figure I-15. Set-3a results, plot 14: comparison of spherical and cylindrical geometries $(\mathbf{h} / \mathbf{x})$. 


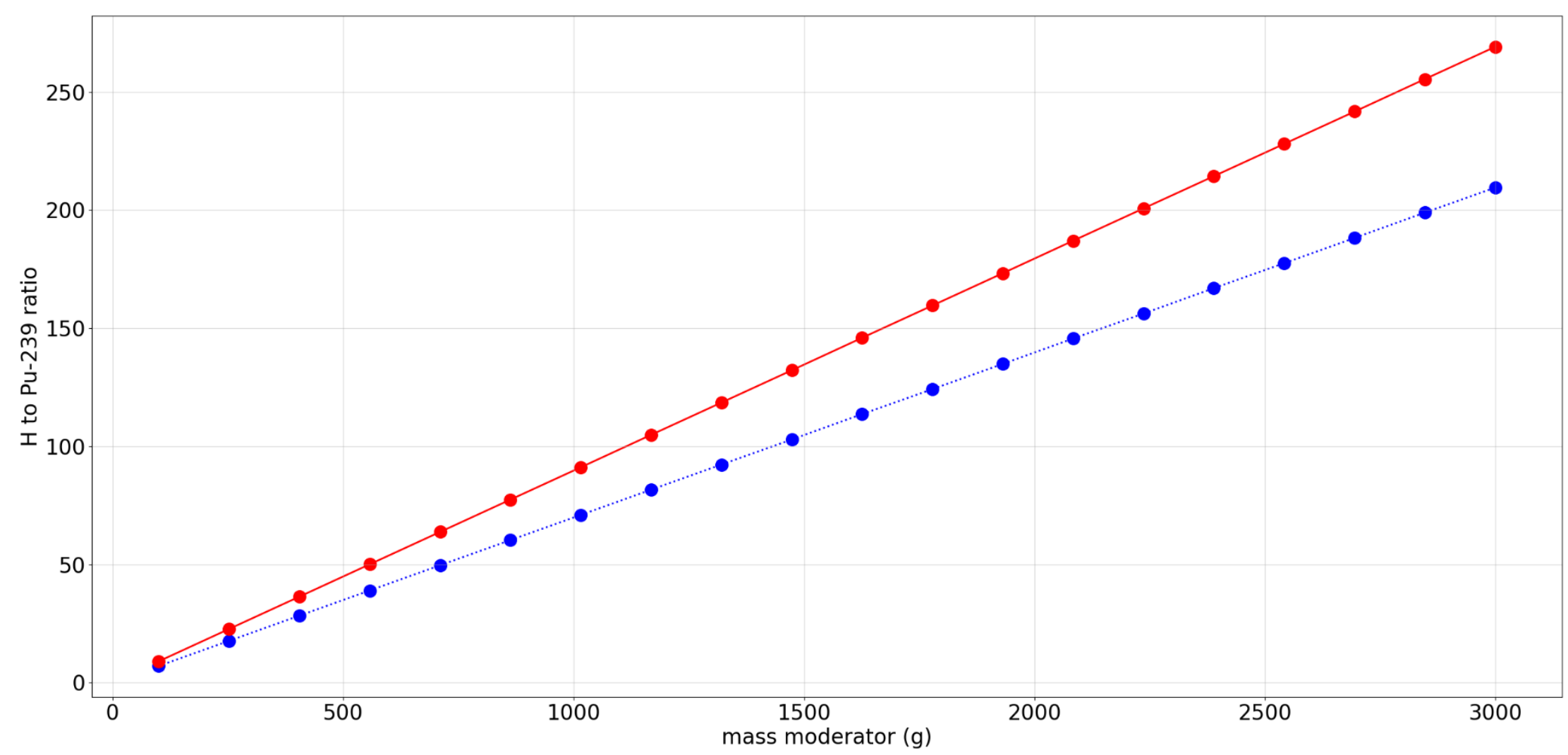

-.. water moderator

- poly moderator

Figure I-16. Set-3a results, plot 15: comparison of water and poly $\mathbf{h} / \mathbf{x}$. 


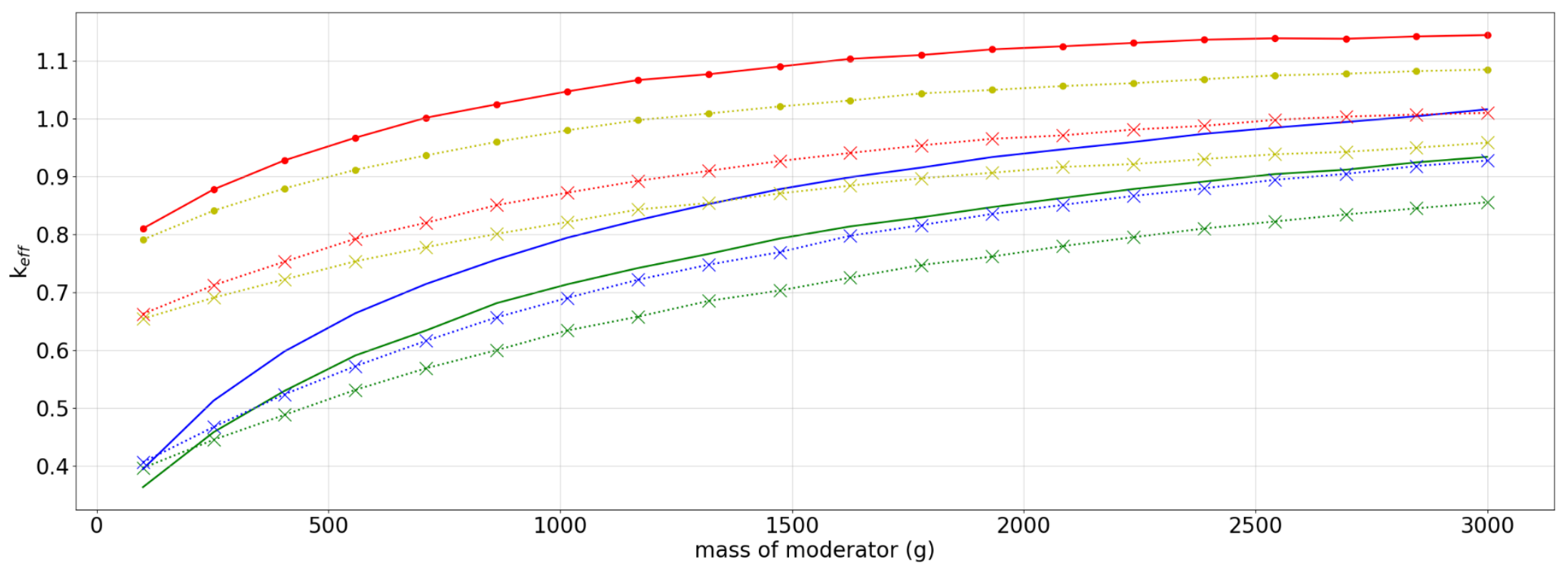

set-3a, sphere, no filler, no Be, thick poly discrete reflector, water moderator

......... set-3a, sphere, $5 \mathrm{~kg}$ graphite/can, $585 \mathrm{~g} \mathrm{Be}$, thick poly discrete reflector, water moderator

set-3a, sphere, no filler, no Be, thick poly discrete reflector, poly moderator

$\cdots \ldots \cdots$ set-3a, sphere, $5 \mathrm{~kg}$ graphite/can, $585 \mathrm{~g} \mathrm{Be}$, thick poly discrete reflector, poly moderator

...... set-3a, cylinder with radius $=7.7 \mathrm{~cm}$, no filler, no Be, thick poly discrete reflector, water moderator

... $\times$... set-3a, cylinder with radius $=7.7 \mathrm{~cm}, 5 \mathrm{~kg}$ graphite/can, $585 \mathrm{~g} \mathrm{Be}$, thick poly discrete reflector, water moderator

- - set-3a, cylinder with radius $=7.7 \mathrm{~cm}$, no filler, no Be, thick poly discrete reflector, poly moderator

.... $\ldots . .$. set-3a, cylinder with radius $=7.7 \mathrm{~cm}, 5 \mathrm{~kg}$ graphite $/ \mathrm{can}, 585 \mathrm{~g} \mathrm{Be}$, thick poly discrete reflector, poly moderator

Figure I-17. Set-3a results, plot 16: comparison of spherical and cylindrical geometries (mod mass). 


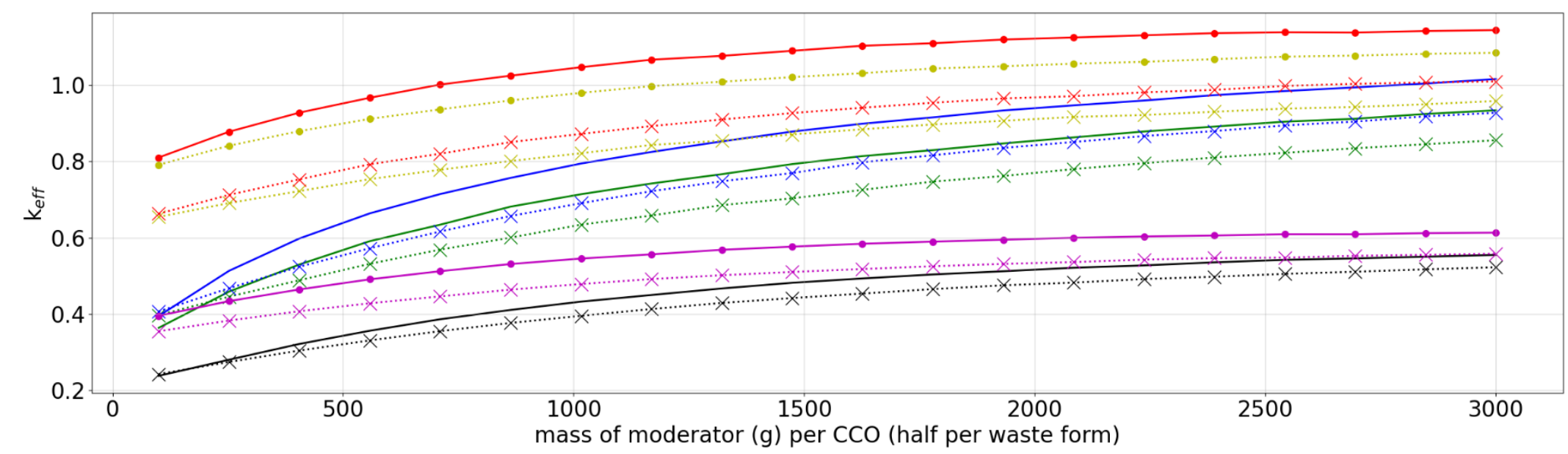

set-3a, sphere, no filler, no Be, thick poly discrete reflector, water moderator

........ set-3a, sphere, $5 \mathrm{~kg}$ graphite/can, $585 \mathrm{~g} \mathrm{Be}$, thick poly discrete reflector, water moderator

— set-3a, sphere, no filler, no Be, thick poly discrete reflector, poly moderator

........ set-3a, sphere, $5 \mathrm{~kg}$ graphite/can, $585 \mathrm{~g}$ Be, thick poly discrete reflector, poly moderator

$\ldots . . .$. set- $3 a$, cylinder with radius $=7.7 \mathrm{~cm}$, no filler, no $\mathrm{Be}$, thick poly discrete reflector, water moderator

....... set-3a, cylinder with radius $=7.7 \mathrm{~cm}, 5 \mathrm{~kg}$ graphite/can, $585 \mathrm{~g} \mathrm{Be}$, thick poly discrete reflector, water moderator

- set-3a, cylinder with radius $=7.7 \mathrm{~cm}$, no filler, no $\mathrm{Be}$, thick poly discrete reflector, poly moderator

........ set-3a, cylinder with radius $=7.7 \mathrm{~cm}, 5 \mathrm{~kg}$ graphite/can, $585 \mathrm{~g} \mathrm{Be}$, thick poly discrete reflector, poly moderator

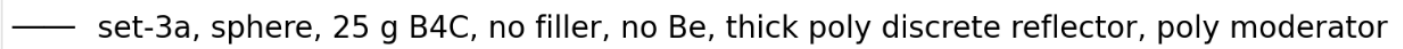

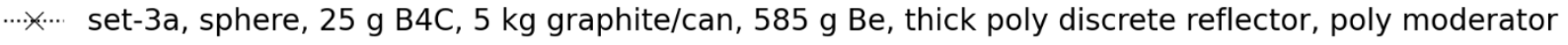

- - set-3a, cylinder with radius $=7.7 \mathrm{~cm}, 25 \mathrm{~g} \mathrm{~B} 4 \mathrm{C}$, no filler, no Be, thick poly discrete reflector, poly moderator

$\ldots . \ldots . .$. set-3a, cylinder with radius $=7.7 \mathrm{~cm}, 25 \mathrm{~g} \mathrm{~B} 4 \mathrm{C}, 5 \mathrm{~kg}$ graphite/can, $585 \mathrm{~g} \mathrm{Be}$, thick poly discrete reflector, poly moderator

Figure I-18. Set-3a results, plot 17: Reactivity effect of $25 \mathrm{~g} \mathrm{~B} 4 \mathrm{C}$ per waste form ( 2 per CCO) 
This page is intentionally blank 
APPENDIX J. SET-4: RESULTS OF HETEROGENOUS WASTE FORM MIXING STUDIES 
This page is intentionally blank 


\section{APPENDIX J: SET-4: RESULTS OF HETEROGENOUS WASTE FORM MIXING STUDIES}

\section{LIST OF FIGURES}

Figure J-1. Diagram of the various heterogenous sphere cases and spacings........................................ J-4

Figure J-2. Geometry diagram for a three-sphere case with spheres in centermost position..................... J-6

Figure J-3. Comparison of 1-sphere $k_{\text {eff }}$ to uniform $k_{\text {eff }}$ for cylinderical wasteforms of $7.7 \mathrm{~cm}$ diameter.

Figure J-4. Delta-k_eff when comparing highest reactivity 1 sphere case to a uniform case (positive values correspond to cases where the highest reactivity 1-sphere configuration has higher keff than uniform configuration.

Figure J-5. Comparison of 2-sphere $k_{\text {eff }}$ to uniform $k_{\text {eff }}$ for cylinderical wasteforms of $7.7 \mathrm{~cm}$ diameter.

Figure J-6 Delta-k_eff when comparing highest reactivity 2-sphere case to a uniform case (positive values correspond to cases where the highest reactivity 2-sphere configuration has higher $\mathrm{k}_{\text {eff }}$ than uniform configuration.

Figure J-7. Comparison of three-sphere $\mathrm{k}_{\text {eff }}$ to uniform $\mathrm{k}_{\mathrm{eff}}$ for cylinderical wasteforms of $7.7 \mathrm{~cm}$ diameter.

Figure J-8 Delta-keff when comparing highest reactivity three-sphere case to a uniform case (positive values correspond to cases where the highest reactivity three-sphere configuration has higher $\mathrm{k}_{\mathrm{eff}}$ than uniform configuration.

Figure J-9. Plot showing frequency of each radial position factor producing worst k-eff plotted for a given uniform case, plotted against moderator mass.

Figure J-10. Plot showing frequency of each sphere radius producing worst k-eff plotted for a given uniform case, plotted against moderator mass.

Figure $\mathrm{J}-11$. Plot showing upper values of $\mathrm{k}_{\mathrm{eff}}$ for various cylinder radii and polyethylene reflector combinations.

Figure J-12. Plot showing upper values of $\mathrm{k}_{\mathrm{eff}}$ for spheres with different polyethylene reflector thicknesses.

Figure J-13. Comparing $\mathrm{k}_{\mathrm{eff}}$ computed using multigroup and continuous energy. $\mathrm{J}-15$

\section{LIST OF TABLES}

Table J-1. Parameters varied in the heterogenous study. 
The purpose of this appendix is to evaluate the validity of the assumption that the $\mathrm{PuO}_{2}$ is uniformly mixed within the waste form. This evaluation examines the effect of locally increased fissile concentration in the waste from in terms of $\mathrm{k}_{\text {eff. }}$ This appendix is not intended to address all possible heterogeneous configurations, but only a selection of cases that correspond to the analyses performed and which cover a range of sizes which would allow larger particle sizes within a mixture to be acceptable.

This appendix evaluates the assumption that a uniform mixture for the waste form is acceptable by evaluating a unit cylindrical waste form (with set-2-uh subset 2-4 parameters, i.e., polyethylene waste form moderator, polyethylene discrete reflector and graphite filler material) with variations in the number, size, and location of pure $\mathrm{PuO}_{2}$ spheres (heterogenous spheres) embedded in a mixture of filler and $\mathrm{PuO}_{2}$. Spheres are used because the neutron leakage is bounding compared to similar sized chunks of FGE.

The mass limit of 380 FGE is maintained for all cases. The $\mathrm{PuO}_{2}$ sphere size diameter is parametrically swept over the range of 0.1 to $1.0 \mathrm{in}$. so that the mass of $\mathrm{PuO}_{2}$ in each heterogenous sphere is dictated by the diameter for each sweep. For each sweep of moderator content, every heterogenous sphere has the same size. The total mass of pure $\mathrm{PuO}_{2}$ in the heterogenous spheres does not exceed the overall 380 FGE limit. Therefore, to conserve mass, the remainder of $\mathrm{PuO}_{2}$ that is not contained in the heterogenous spheres is uniformly mixed with filler.

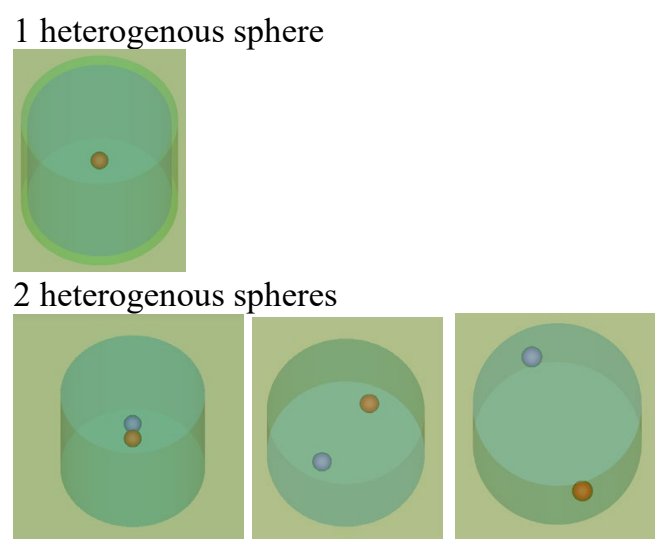

3 heterogenous spheres

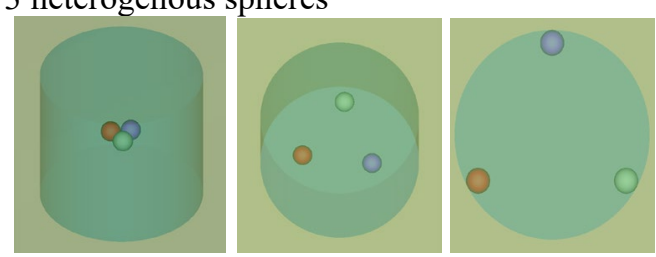

Figure J-1. Diagram of the various heterogenous sphere cases and spacings.

The number of heterogenous spheres ranges from 1 to 3 in the configurations evaluated. Two additional cases are considered with uniform mixtures as baselines against which to compare the heterogenous sphere cases: one with a cylindrical waste form shape and one with a spherical waste form shape.

The location of the spheres within the cylindrical waste form also is also evaluated as: centrally located, located midway between the center, or located at the edges, as shown in Figure J-1. A full listing of parameters evaluated for the study are listed in Table J-1.

To represent the interstitial reflector material used in the analysis, the waste form is embedded in a cube of " $50 / 50$ " $\mathrm{MgO}+\mathrm{NaCl}$ mixture with an edge length of $200 \mathrm{~cm}$ centered on the waste form. This itself is placed in the cube of $\mathrm{NaCl}$ with edge length of $800 \mathrm{~cm}$ to represent the WIPP repository, also consistent with the analysis. See Figure J-2. The boundaries are vacuum. Therefore, this study specifically evaluates changes in $\mathrm{k}_{\mathrm{eff}}$ and is not directly comparable to other evaluations in this analysis. The heterogeneous study most closely resembles set two because the cylinders are not in contact with other cylinders. No attempt is made with this study to evaluate neutron interactions between waste forms since the main impact of the uniform versus heterogenous mixing system is localized. 
Table J-1. Parameters varied in the heterogenous study.

\begin{tabular}{|c|c|c|}
\hline Parameter symbol & Description & Values \\
\hline fge_mass & $\begin{array}{l}\text { Mass of } \mathrm{PuO}_{2} \text { in waste form (both uniformly } \\
\text { mixed and lumped masses) }\end{array}$ & $\begin{array}{l}\text { Total is fixed at } 380 \text { per waste form; } \\
\text { heterogenous spheres varies by size }\end{array}$ \\
\hline n_hetro & Number of $\mathrm{PuO}_{2}$ lumped masses in waste form & $0,1,2,3$ \\
\hline sph/cyl & Shape of waste form & cyl \\
\hline puo2_het1_diameter & $\begin{array}{l}\text { Diameters (in.) of } \mathrm{PuO}_{2} \text { masses when } \\
\mathrm{n} \text { _hetro }>0\end{array}$ & $0.1,0.425,0.75$ \\
\hline radial_factor & $\begin{array}{l}\text { Fraction of distance from innermost position } \\
(0.0) \text { to outermost position }(1.0) \text {, tangent to } \\
\text { cylinder. This defines the position of the sphere } \\
\text { when } n \text { _hetro }>1\end{array}$ & $0.0,0.5,1.0$ \\
\hline can_mass & $\begin{array}{l}\text { Mass of stainless } 304 \text { can material mixed with } \\
\text { uniform portion of waste form }\end{array}$ & $0,500,1000$ \\
\hline thk_pipe & & $0.7112,0.001$ \\
\hline ch2_mass & Mass of polyethylene (g) & $\begin{array}{l}100.0,252.6,405.3,557.9,710.5,863.2, \\
1016.0,1168.0,1321.0,1474.0,1626.0, \\
1779.0,1932.0,2084.0,2237.0,2389.0, \\
2542.0,2695.0,2847.0,3000.0\end{array}$ \\
\hline graphite_mass & Mass of graphite filler $(\mathrm{g})$ & $0,1500,3000$ \\
\hline be_mass & Mass of Be $(\mathrm{g})$ & 585,0 \\
\hline r_cyl & Waste form radius & 7.7 \\
\hline
\end{tabular}




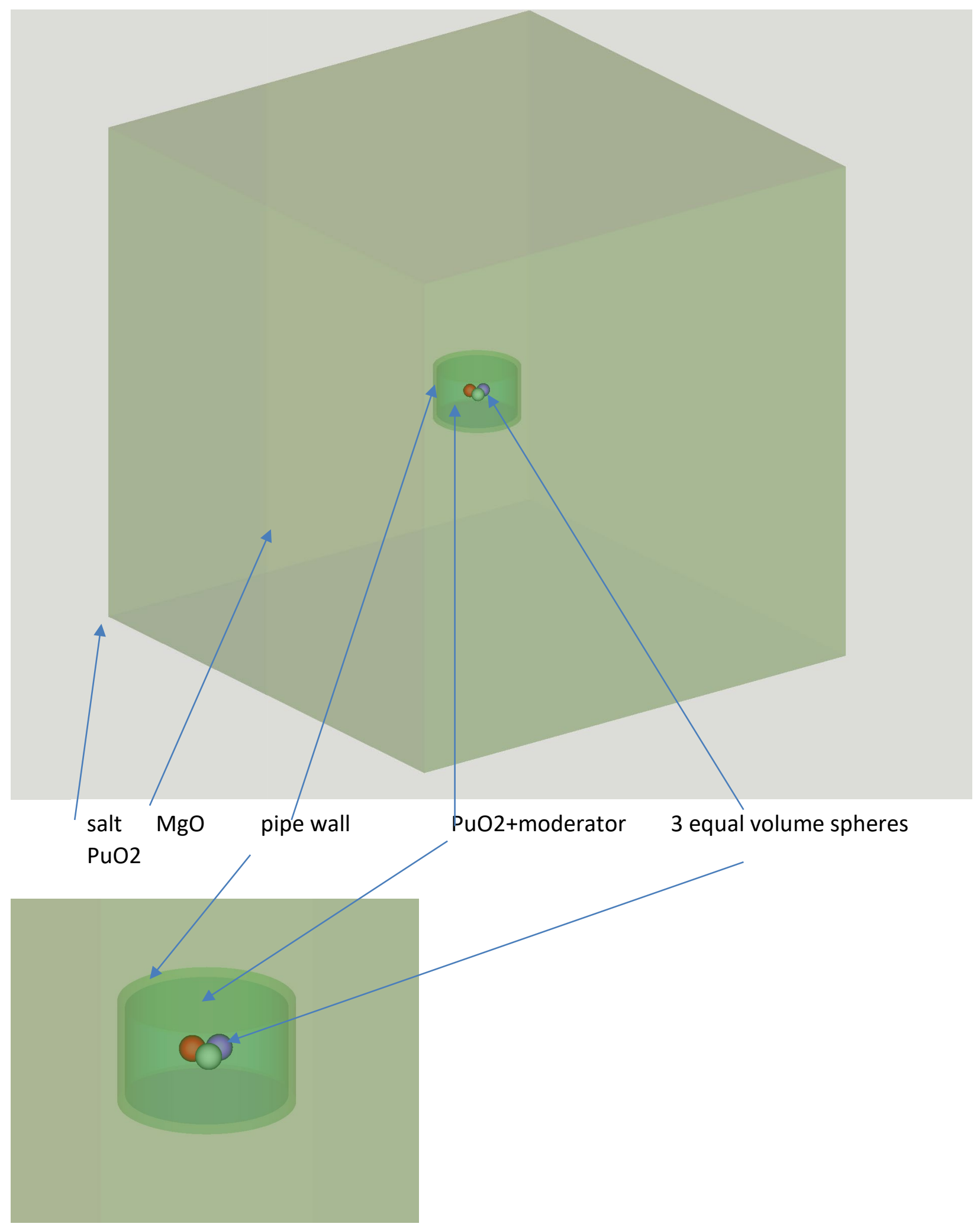

Figure J-2. Geometry diagram for a three-sphere case with spheres in centermost position. 


\section{Discussion of Results}

The reactivity of the various cases for heterogeneity are shown as $\mathrm{k}_{\mathrm{eff}}$ comparisons in Figures J-3, Figure J-5, and Figure J-7, which respectively compare 1-sphere, 2-sphere, and three-sphere (heterogenous spheres) to the uniform cylindrical case. In the cases plotted in Figure J-3 through Figure J-10, the waste forms being considered are cylinders with a radius of $7.7 \mathrm{~cm}$. Each heterogenous sphere case is selected to be the worst case for various sphere radii and radial factors that corresponds to a uniform case. The upper envelope of the uniform cases and the heterogenous sphere cases appear to be identical, except at low moderator. Regardless, the difference does not appear to substantially alter $k_{\text {eff. }}$ The differences in delta- $\mathrm{k}_{\text {eff }}$ between cases are more clearly seen in Figures J-4, Figure J-6, and Figure J-8. The cases with the largest increases in $\mathrm{k}_{\text {eff }}$ due to heterogeneity are at low moderator with a thin polyethylene discrete reflector. This suggests that the heterogeneity is not directly responsible for the change in $\mathrm{k}_{\mathrm{eff}}$, but rather the change in reflection may be changing $\mathrm{k}_{\mathrm{eff}}$ for cases with low moderator masses, or higher fissile concentrations.

The effect of reflection is also seen by examining which heterogeneous configurations are producing the worst case increases in $\mathrm{k}_{\text {eff. }}$ This is seen in Figures J-9 and J-10 which are scatter plots where darker points indicate a higher frequency of that combination. Figure J-9 shows the number of times a given sphere radius produces the worst delta-k vs a corresponding uniform case for a given moderator mass. To produce the figure, the heterogeneous cases are matched to the non-heterogeneous cases on every parameter except for sphere radius and radial factor. For sphere radius and radial factor, the combination producing the highest $\mathrm{k}_{\text {eff }}$ is selected, and the radius is plotted on Figure J-9. Figure J-10 is similar, except it shows the number of times a given radial factor produces the worst delta-k. For the three-sphere case, clear trends were only observable at low moderator, and they occurred when all three spheres were large and in contact at the center of the cylinder.

Three large spheres concentrated in the center of a disk-shaped waste from producing the largest jump in delta-k vs a uniformly mixed case is unsurprising. The cylinder configuration at low moderation has a very large leakage, so concentrating mass together in the center of the cylinder reduces the neutron leakage probability. In Figure 6-15 of the main text, similar behavior can be observed when changing between the cylindrical and spherical waste forms at low moderator. While the spherical waste form of Figure 6-15 is uniformly mixed and the configurations here consist of concentrated $\mathrm{Pu}$ in the center of a cylindrical waste form, the trend toward decreased leakage is the same in both cases. The worst cases in this section in Figure J-8 results in a delta-k increase of approximately 0.1 when concentrating the majority of the $\mathrm{Pu}$ is concentrated at the center of the waste form vs it being uniformly distributed in a disk shaped cylinder. Similarly, in Figure 6-15 of the main text also shows a similar delta-k of approximately 0.1 when transitioning from a cylindrical waste form to a spherical waste form at low moderator. Both times the delta-k correspond to a thin discrete reflector. When a thick discrete reflector is used, delta-k is reduced by about half in both figure 6-15 and J-8. Therefore it is suspected that the behavior being observed has more to do with leakage than non-homogeneity.

Finally, as a means to provide a fuller comparison of the vacuum boundary conditons in this appendix to the remainder of the report, $\mathrm{k}_{\text {eff }}$ for various cylinder radii are shown in Figure J-11. Comparing Figure J11 to Figure C-5 for the set-2-uh cases, it is seen that the vacuum boundary conditions result in lower $\mathrm{k}_{\text {eff. }}$. The vacuum boundary condition cases appear to have slightly exagerated trends vs Figure C-5. The $\mathrm{k}_{\text {eff }}$ values for spherical waste forms are shown in Figure J-12, and comparing to Figure C-13 for the set-2-uh cases also shows that the vaccum boundary conditions result in lower values of $k_{\text {eff. }}$ At a high level trends for set-2-uh and the baseline cases for set-4 appear to be similar overall with set- 4 having lower $k_{\text {eff }}$ values than set-2-uh. 
Various cases were compared to a continous energy baseline to deomonstrate that the cell data card and the multigroup approximation does not substantially alter the trends observed because it is on the order of the Monte Carlo uncertainty. This is shown in Figure J-13.

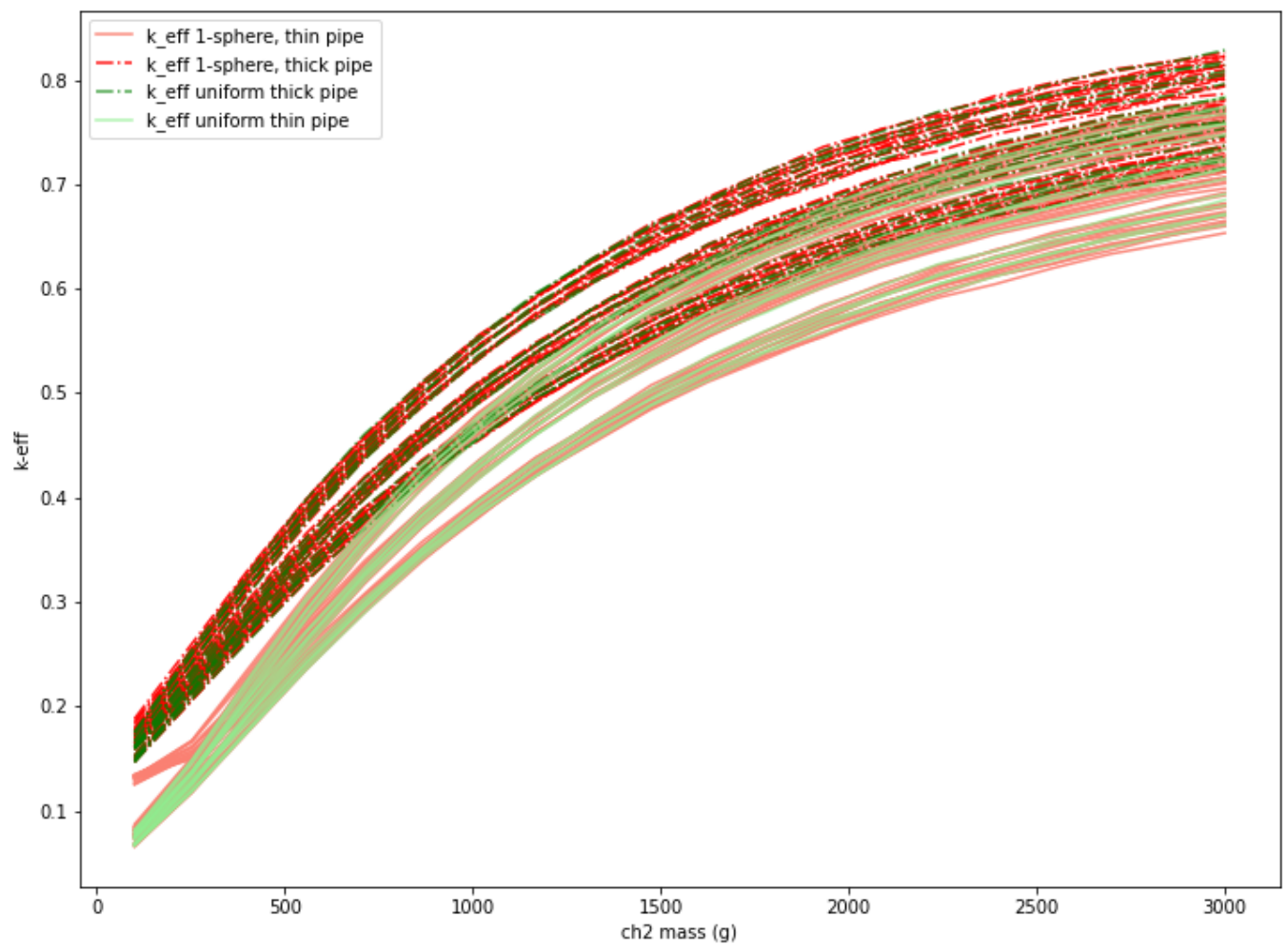

Figure J-3. Comparison of 1-sphere $k_{\text {eff }}$ to uniform $k_{\text {eff }}$ for cylinderical wasteforms of $7.7 \mathrm{~cm}$ diameter. 


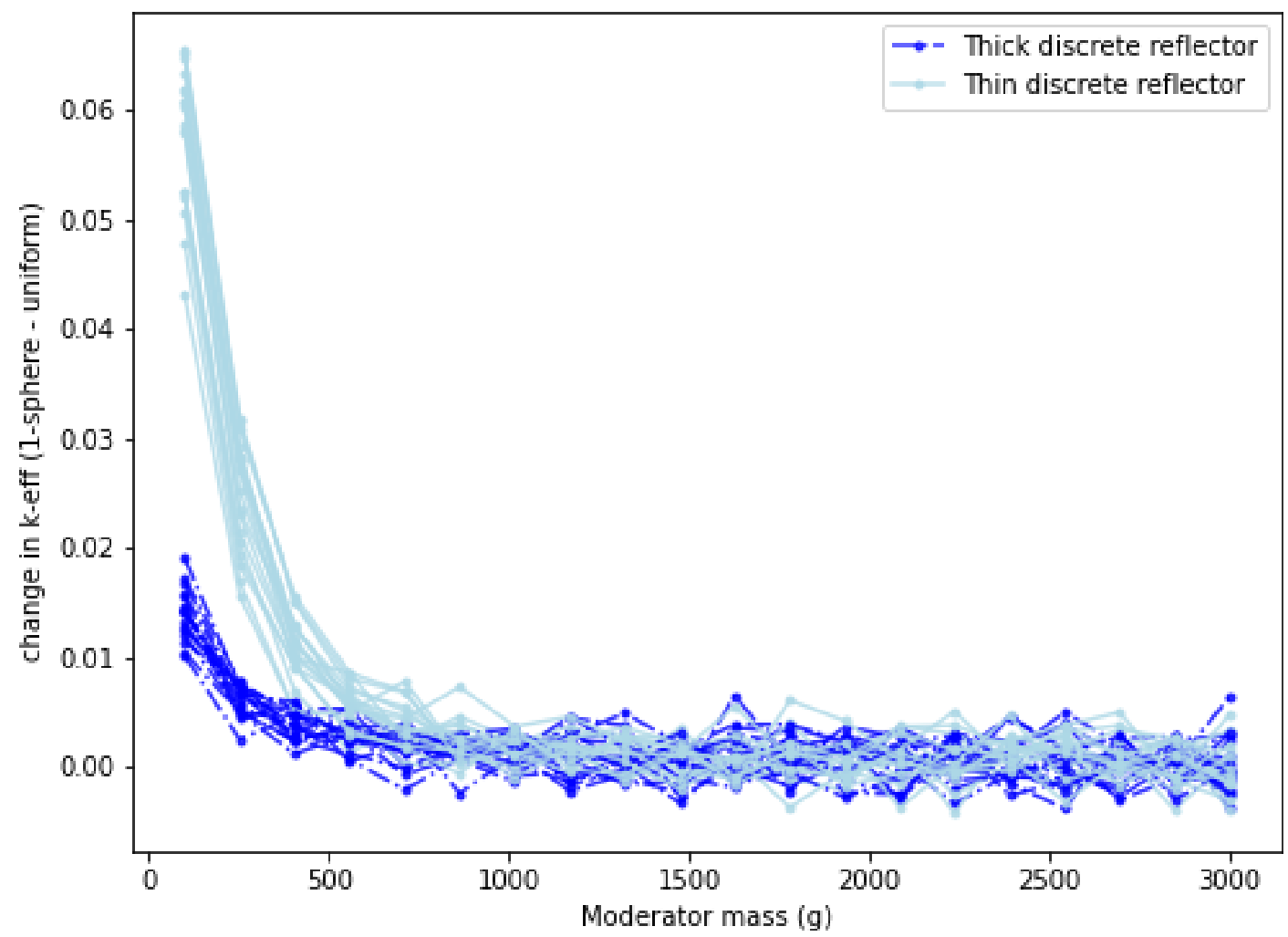

Figure J-4. Delta-k_eff when comparing highest reactivity 1 sphere case to a uniform case (positive values correspond to cases where the highest reactivity 1-sphere configuration has higher keff than uniform configuration. 


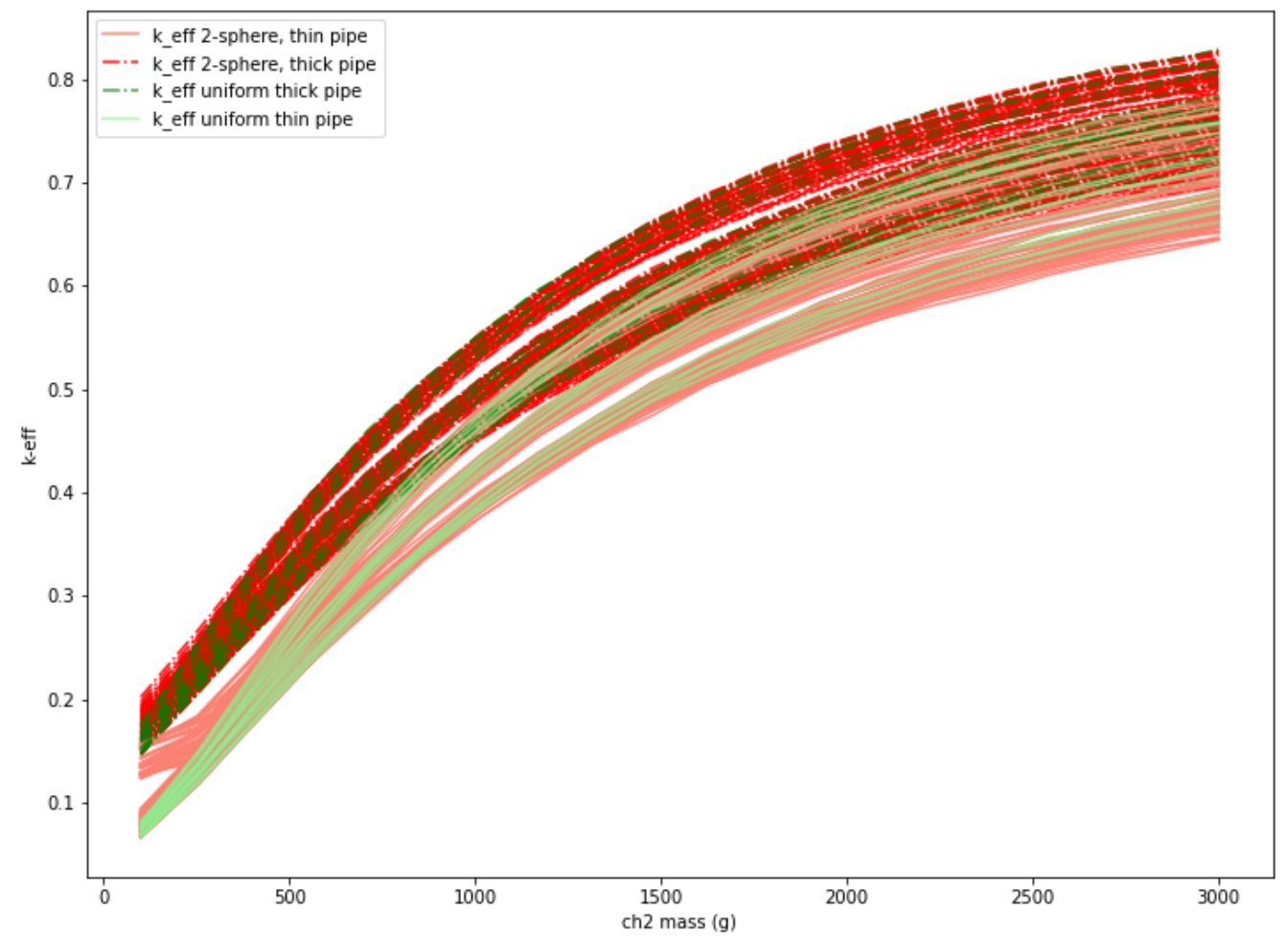

Figure J-5. Comparison of 2-sphere keff to uniform keff for cylinderical wasteforms of $7.7 \mathrm{~cm}$ diameter.

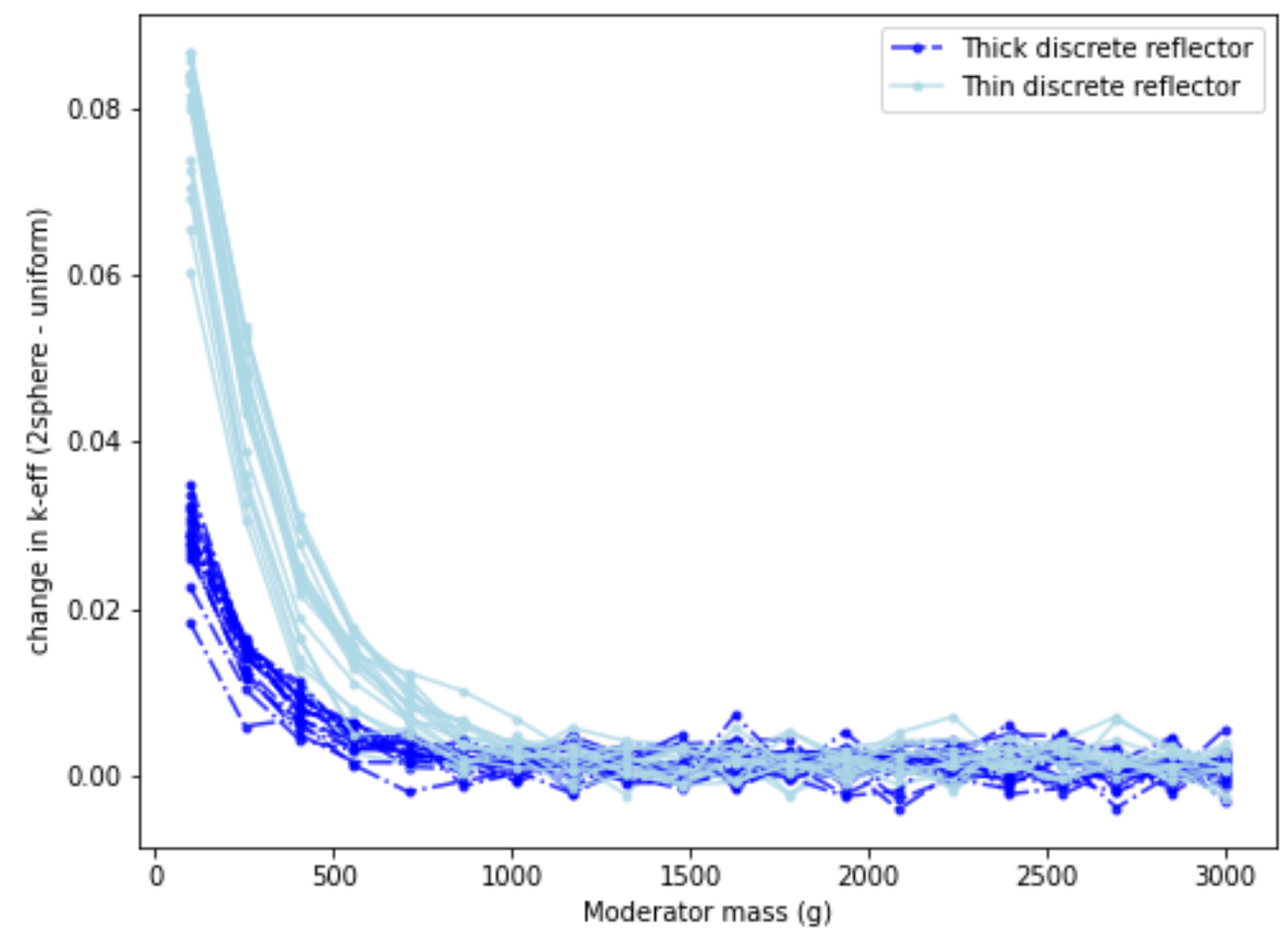

Figure J-6 Delta-k_eff when comparing highest reactivity 2-sphere case to a uniform case (positive values correspond to cases where the highest reactivity 2 -sphere configuration has higher $k_{\text {eff }}$ than uniform configuration. 


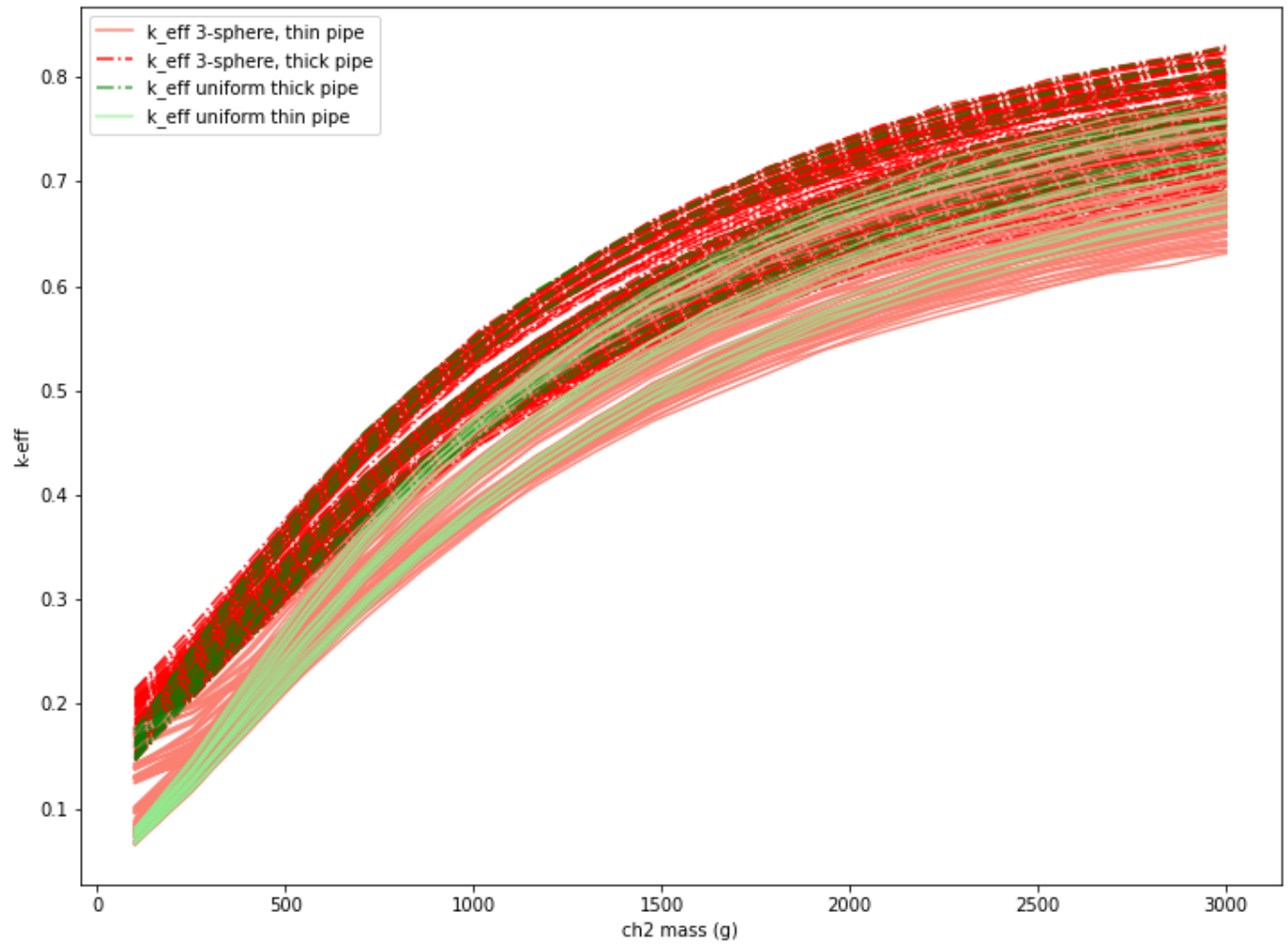

Figure J-7. Comparison of three-sphere $k_{\text {eff }}$ to uniform $k_{\text {eff }}$ for cylinderical wasteforms of $7.7 \mathrm{~cm}$ diameter. 


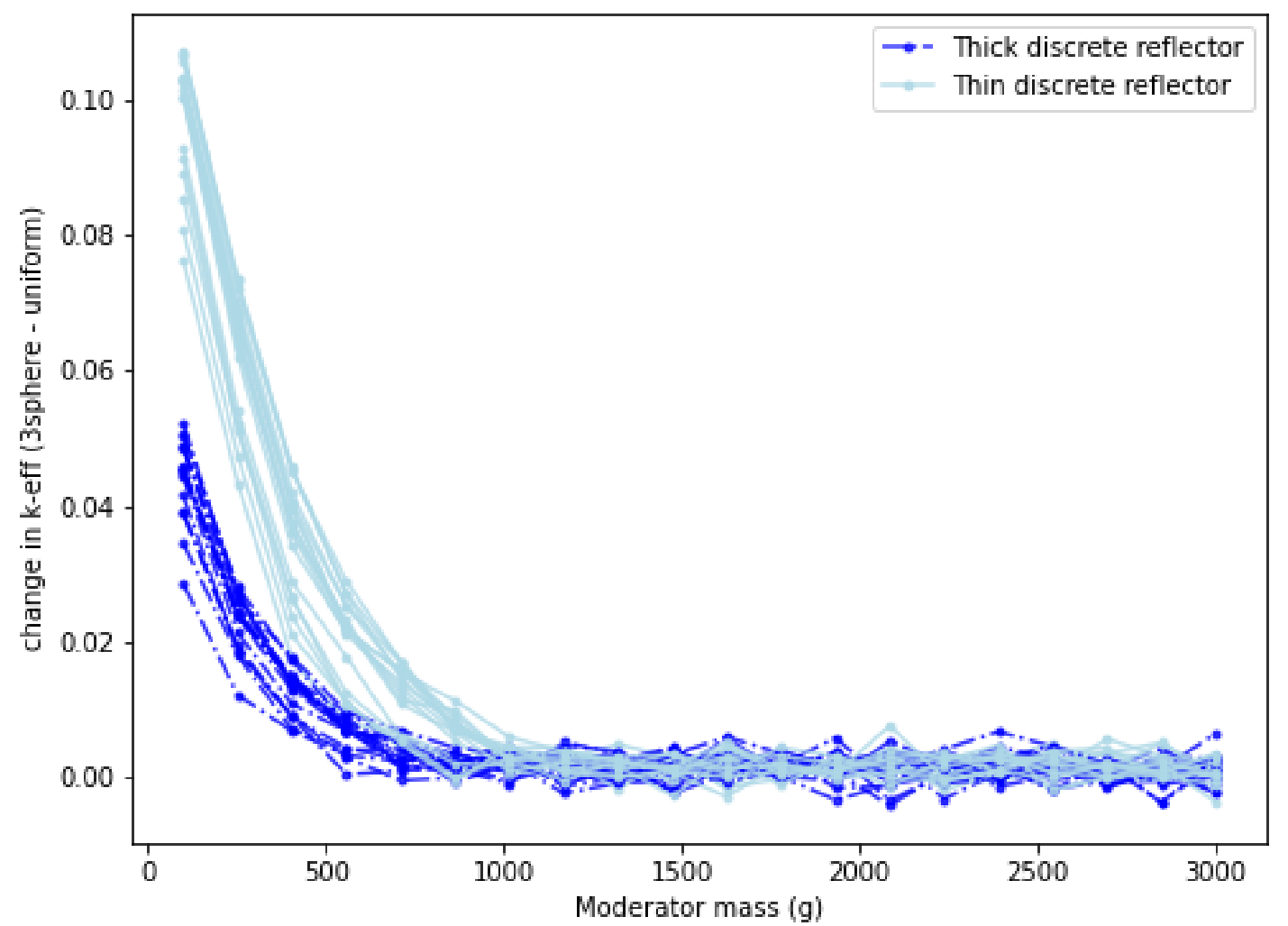

Figure J-8 Delta-keff when comparing highest reactivity three-sphere case to a uniform case (positive values correspond to cases where the highest reactivity three-sphere configuration has higher $k_{\text {eff }}$ than uniform configuration. 

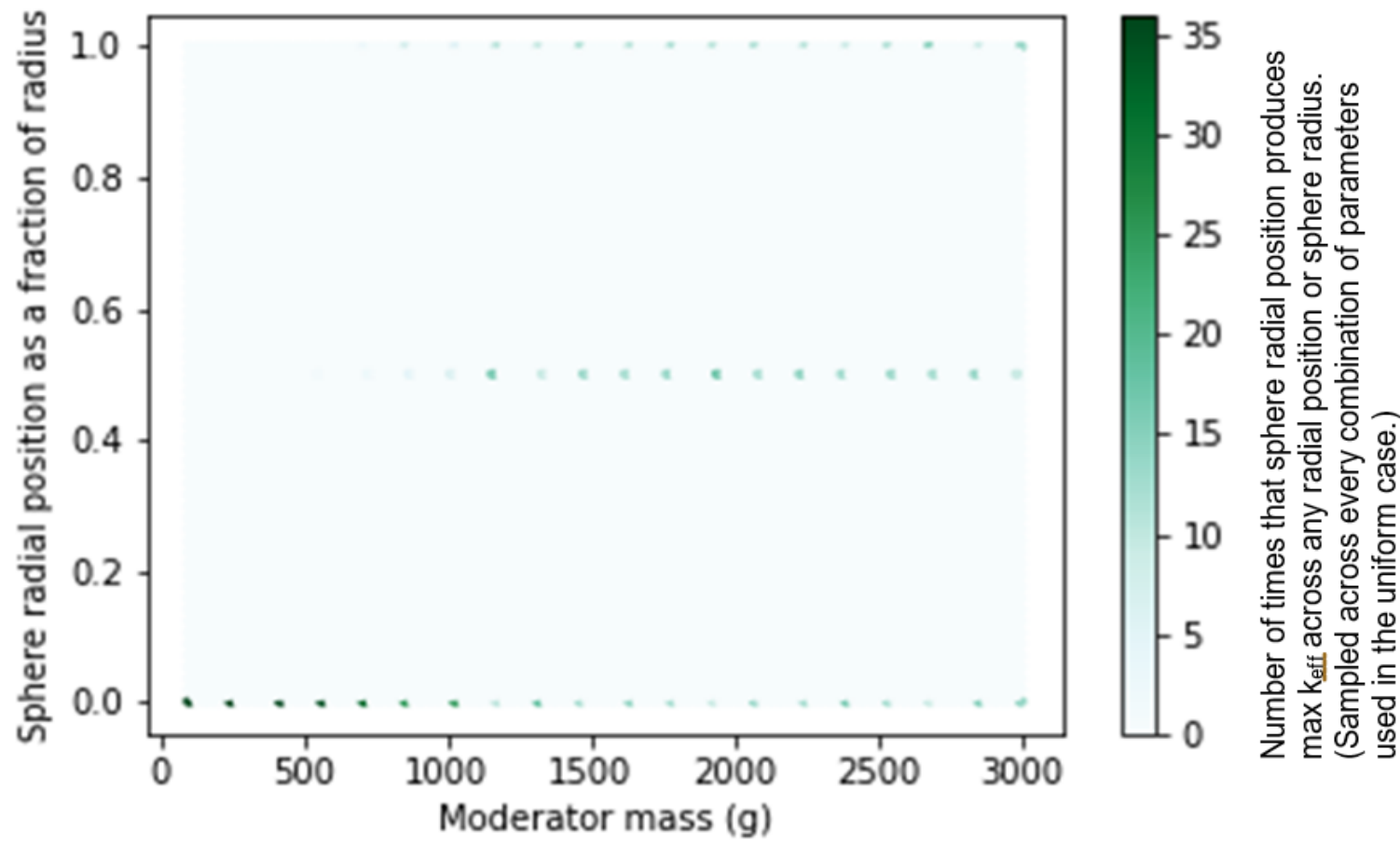

Figure J-9. Plot showing frequency of each radial position factor producing worst k-eff plotted for a given uniform case, plotted against moderator mass. Three sphere case. (Darker dots indicate higher frequency).

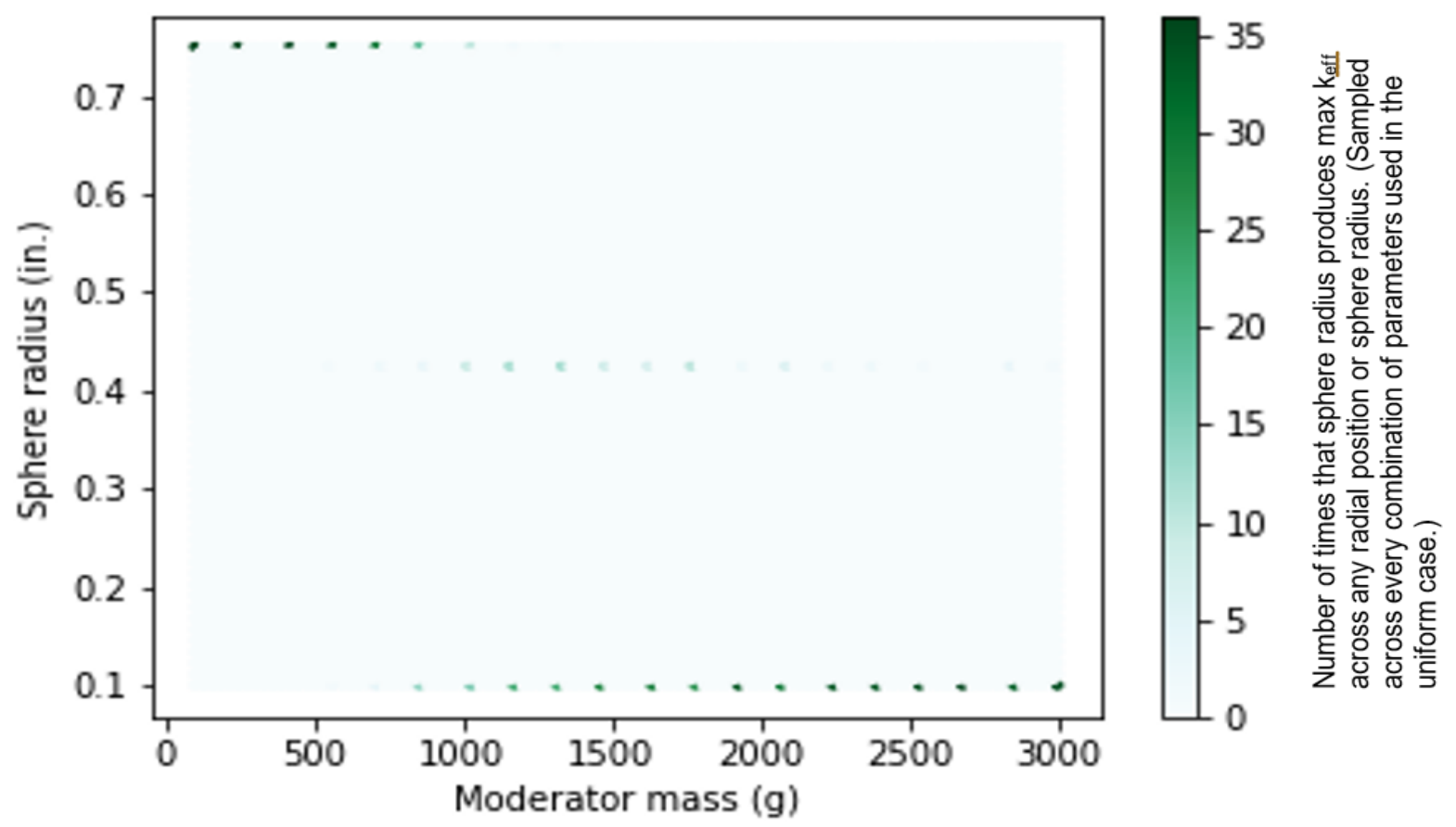

Figure J-10. Plot showing frequency of each sphere radius producing worst k-eff plotted for a given uniform case, plotted against moderator mass. Three sphere case. (Darker dots indicate higher frequency). 

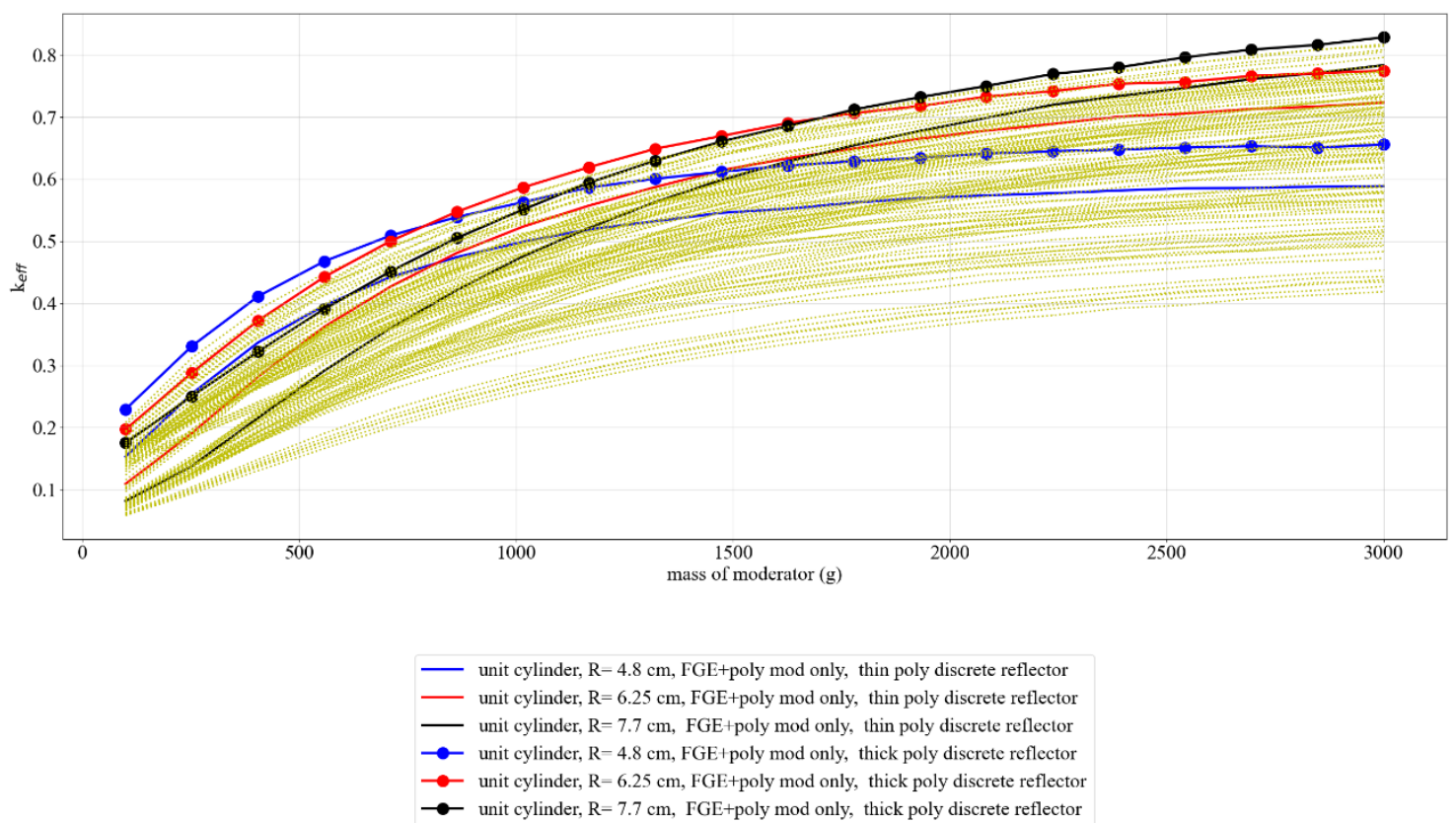

Figure J-11. Plot showing upper values of keff for various cylinder radii and polyethylene reflector combinations.

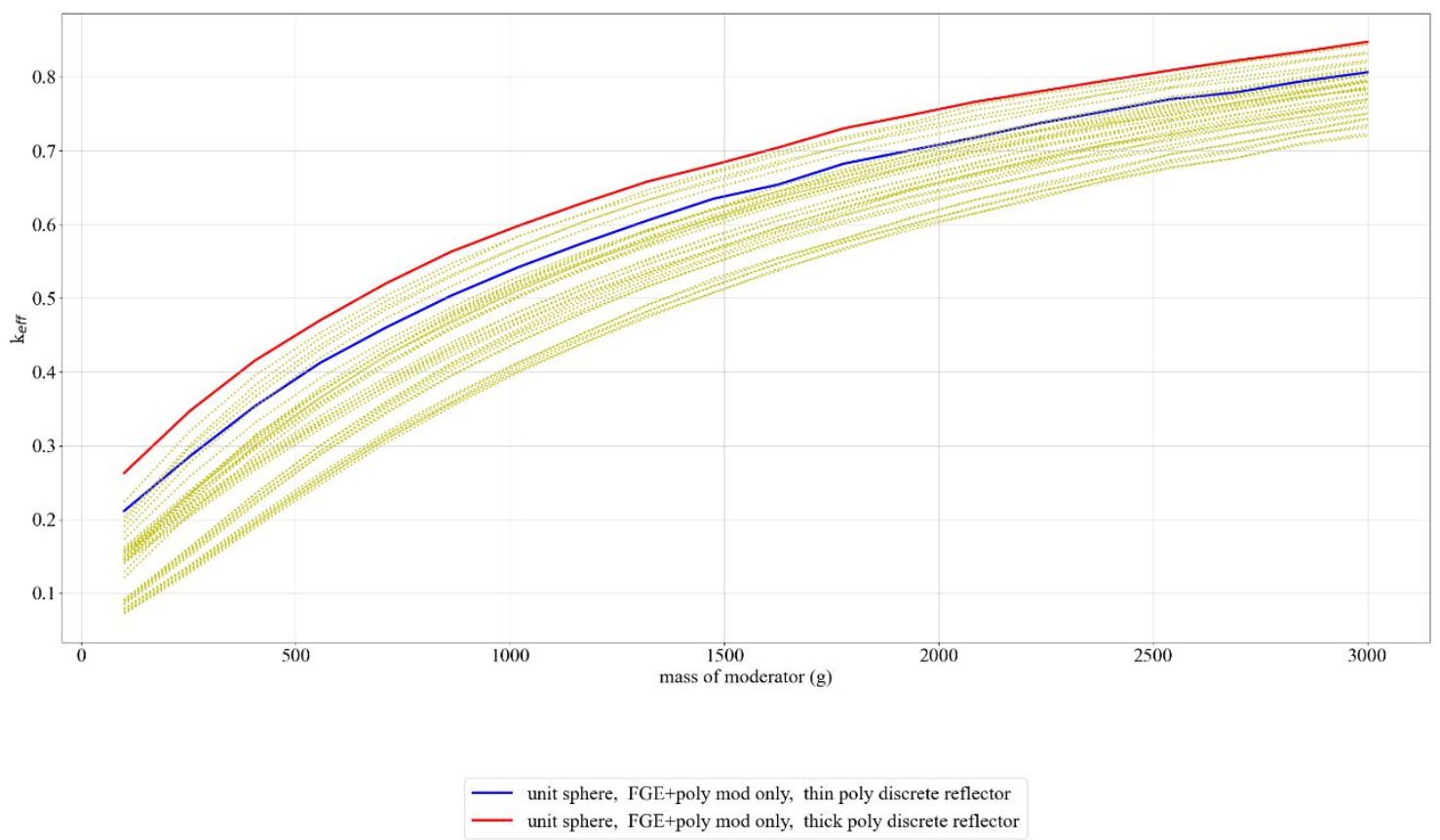

Figure J-12. Plot showing upper values of $k_{\text {eff }}$ for spheres with different polyethylene reflector thicknesses. 


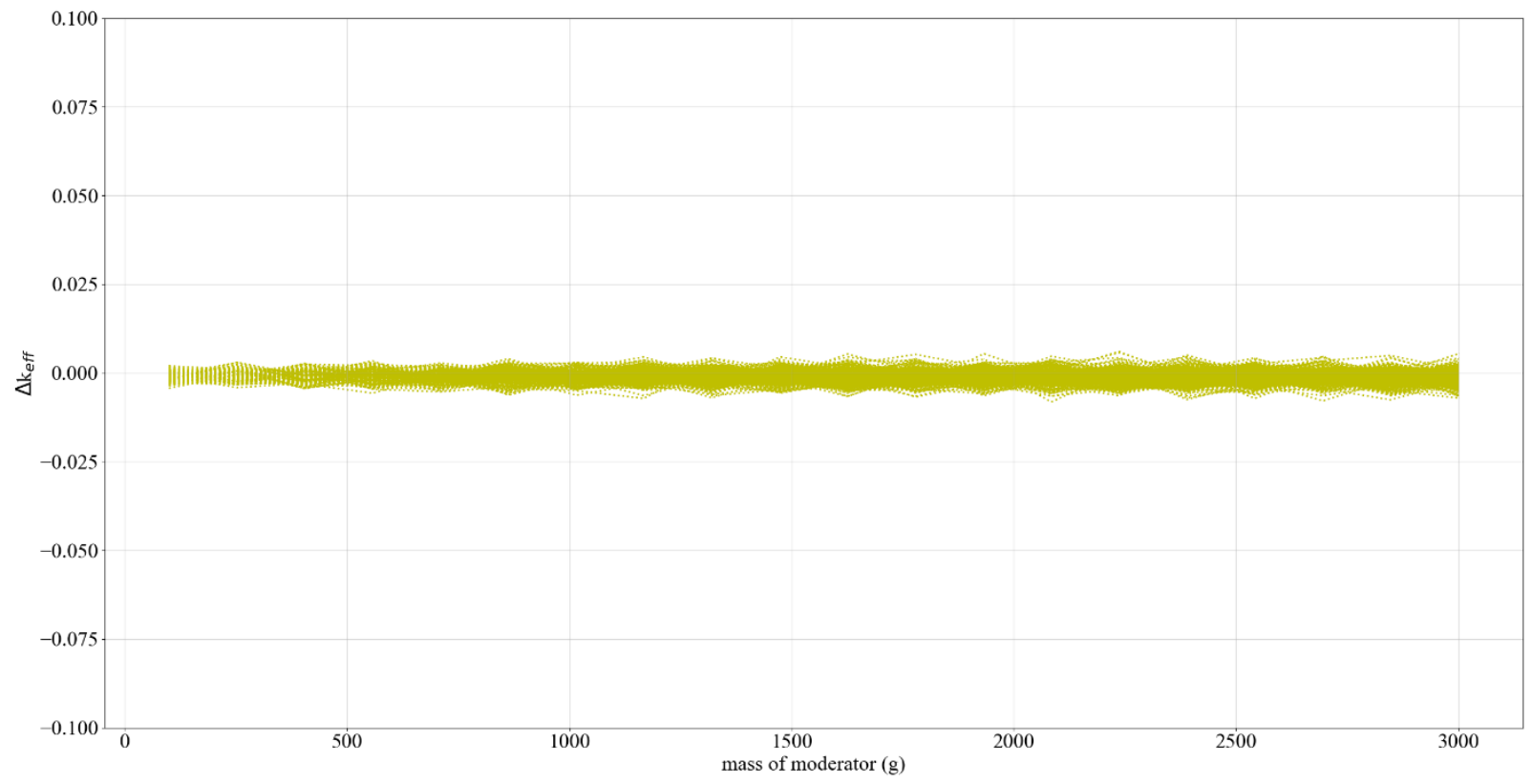

Figure J-13. Comparing $k_{\text {eff }}$ computed using multigroup and continuous energy. 
This page is intentionally blank 


\section{APPENDIX K. EVALUATION OF NEUTRON TRANSPORT FROM A}

CCO IN 50\%MGO 50\% NACL MIXTURE 
This page is intentionally blank 


\section{APPENDIX K. EVALUATION OF NEUTRON TRANSPORT FROM A CCO IN 50\%MGO 50\% NACL MIXTURE}

\section{LIST OF FIGURES}

Figure K-1. Geometry of MAVRIC model with vacuum boundary conditions. K-5

Figure $\mathrm{K}-2$. Neutron flux tallies at locations in $50 / 50 \mathrm{NaCl} / \mathrm{MgO}$ mixture. Values in neutrons $/ \mathrm{cm}^{2}$-s per source particle.

Figure K-3. Flux per source particle at various distances in the salt form the source (linear scale).......... K-7

Figure K-4. Flux per source particle at various distances in the salt form the source (log scale)............. K-7

Figure K-5. Flux spectra $\left(\mathrm{n} / \mathrm{cm}^{2} \mathrm{~s}\right.$ per source particle) at $10 \mathrm{~cm}$ from source (blue), $50 \mathrm{~cm}$ from source (red) and $100 \mathrm{~cm}$ from source (green).

\section{LIST OF TABLES}

Table K-1. Parameters of the CCO modeled. .................................................................................. K-4

Table K-2. Flux per source particle $\left({ }^{0} \mathrm{n} / \mathrm{cm}^{2} \mathrm{~s}\right)$ at various distances in the salt form the source ............... K-9 
This appendix documents a study related to neutron transport in the analysis interstitial mixtures of MGO and $\mathrm{NaCl}$.

\section{Shielding model of media surrounding a Pu fission source in a spherical CCC}

An exercise to determine the sphere of influence of a CCO was performed using the parameters in Table $\mathrm{K}-1$ for a spherical approximation. The CCO itself is not of particular importance, because the primary concern is the rate at which neutron flux tapers in the salt/MgO mixture. This exercise uses the MAVRIC shielding sequence in SCALE. The MAVRIC sequence uses the same input geometry and materials definition formats as KENO. For this calculation, the automated variance reduction feature was not needed and not used. Because the source was already simulating neutrons from fission of ${ }^{239} \mathrm{Pu}$, fission was turned off in the model. Continuous energy ENDF-7.1 cross sections were used, and tallies were taken using the 27-group energy structure used for some Scale cross section libraries.

Table K-1. Parameters of the CCO modeled.

\begin{tabular}{|c|c|}
\hline Parameter & Value \\
\hline ch2 mass & $252.6 \mathrm{~g}$ \\
\hline graphite_mass & $0 \mathrm{~g}$ \\
\hline can_mass & $0 \mathrm{~g}$ \\
\hline thk_pipe & $0.7112 \mathrm{~cm}$ \\
\hline be_mass & $0 \mathrm{~g}$ \\
\hline $\mathrm{h} / \mathrm{x}$ & 22.66 \\
\hline
\end{tabular}

Figure K-1 shows the assumed MAVRIC geometry, which is based on the KENO input set-1-12uac1_uh_m2_graphite_sph_pp_1000_yr_para_00039_single.inp, with characteristics listed in Table K-1. In Figure K-1, green corresponds to the interstitial reflector (typically a combination of salt and $\mathrm{MgO}$ ). The blue dot corresponds to the spherical waste form. The cuboid of salt has $600 \mathrm{~cm}$ edges with vacuum boundary conditions. A small point source is placed in the center of the sphere. This source is an arbitrary strength ${ }^{239} \mathrm{Pu}$ fission source having a Watt neutron energy spectrum. 


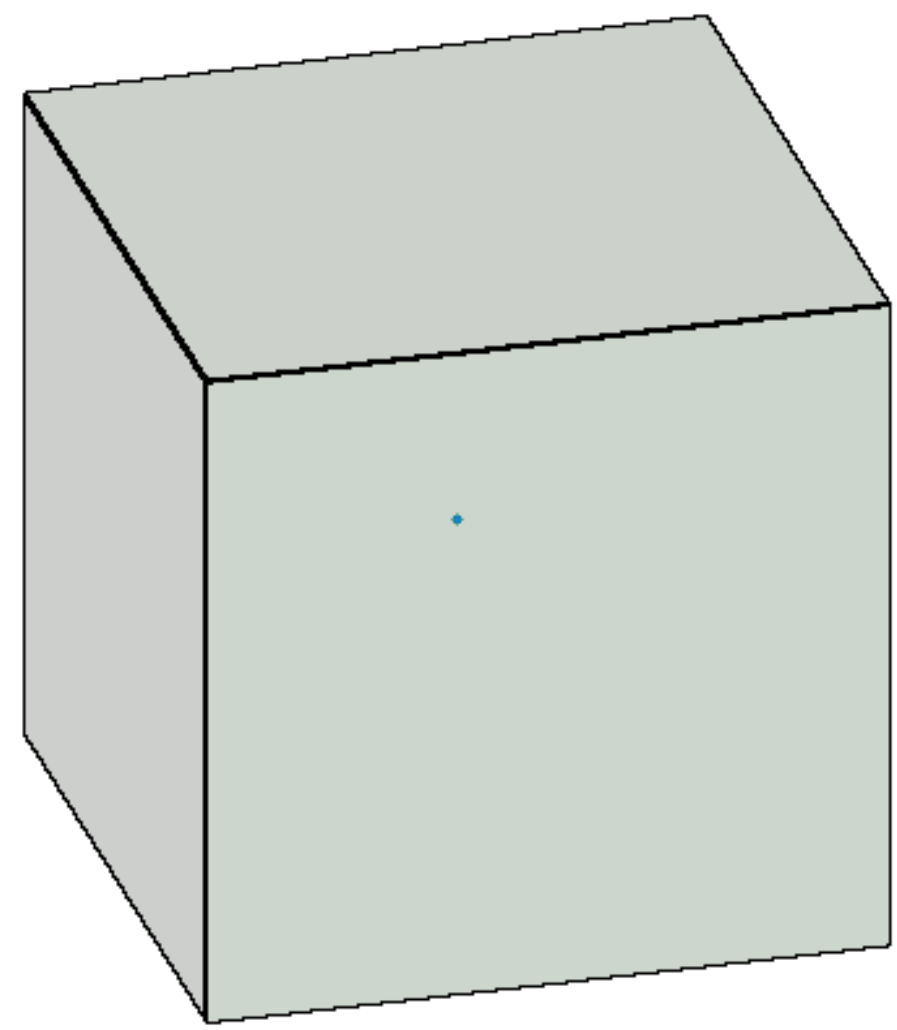

Figure K-1. Geometry of MAVRIC model with vacuum boundary conditions.

Figure K-2 shows neutron fluxes per source particle at varous distances from the source. 


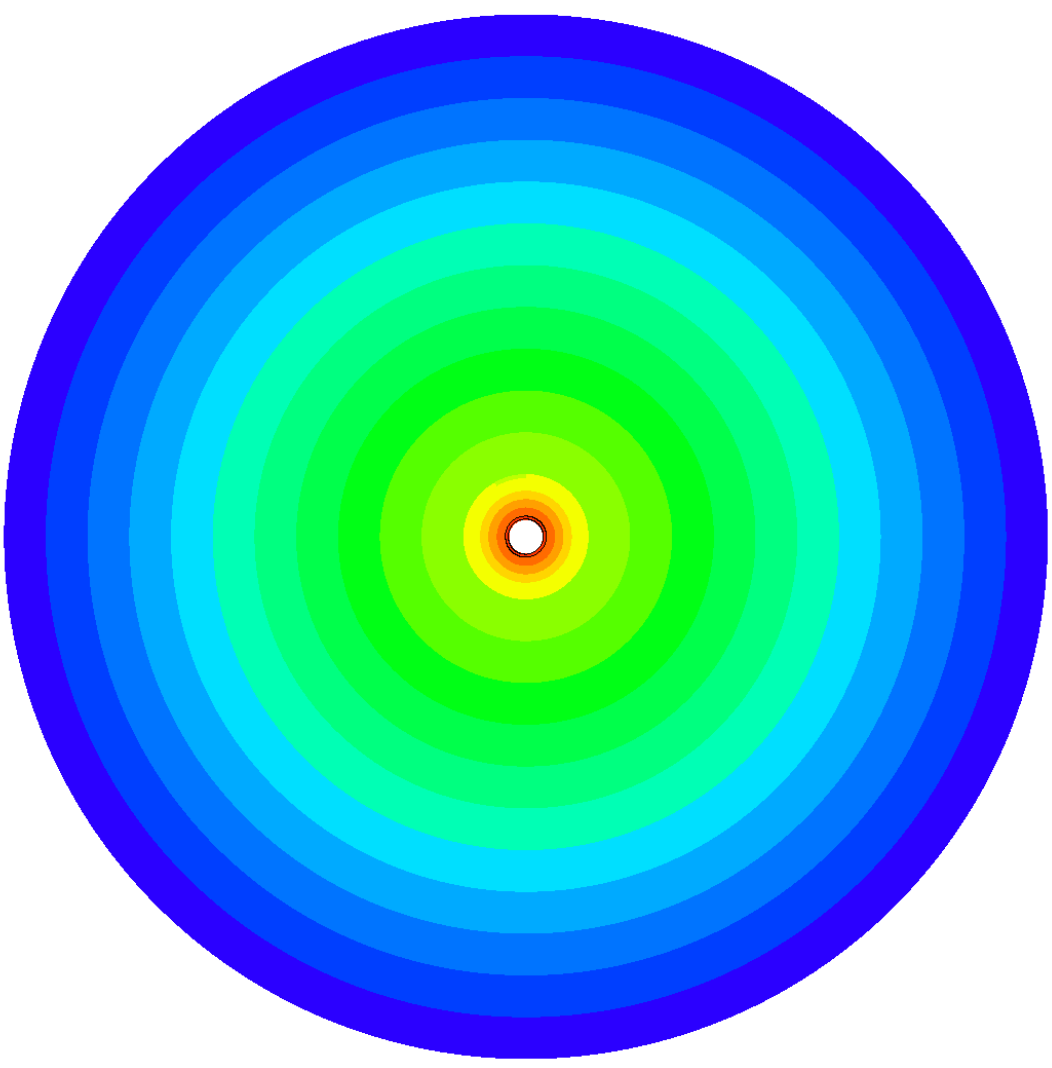

Figure K-2. Neutron flux tallies at locations in 50/50 $\mathrm{NaCl} / \mathrm{MgO}$ mixture. Values in neutrons $/ \mathrm{cm}^{2}$-s per source particle.

Figure K-3 show the neutron intensities at various distances for various types of interstitial reflector media. Each tally volume is a 30 degree azimuthal sector $10 \mathrm{~cm}$ in radial thickness. The innermost radial sector has an inner edge at a radius of $5 \mathrm{~cm}$, and an outer radius of $15 \mathrm{~cm}$. The outermost sector has an inner edge at a radius of $115 \mathrm{~cm}$ and an outer radius of $125 \mathrm{~cm}$. After leaving the CCO, the neutron intensity decreases exponentially, consistent with the solution to the diffusion equation for a point source in nonmultiplying media [15]. Table K-2 tabulates the values found in Figure K-3 and K-4. Note that the waste form is assumed to be approximately $10 \mathrm{~cm}$ in diameter, but that value will vary in reality. 


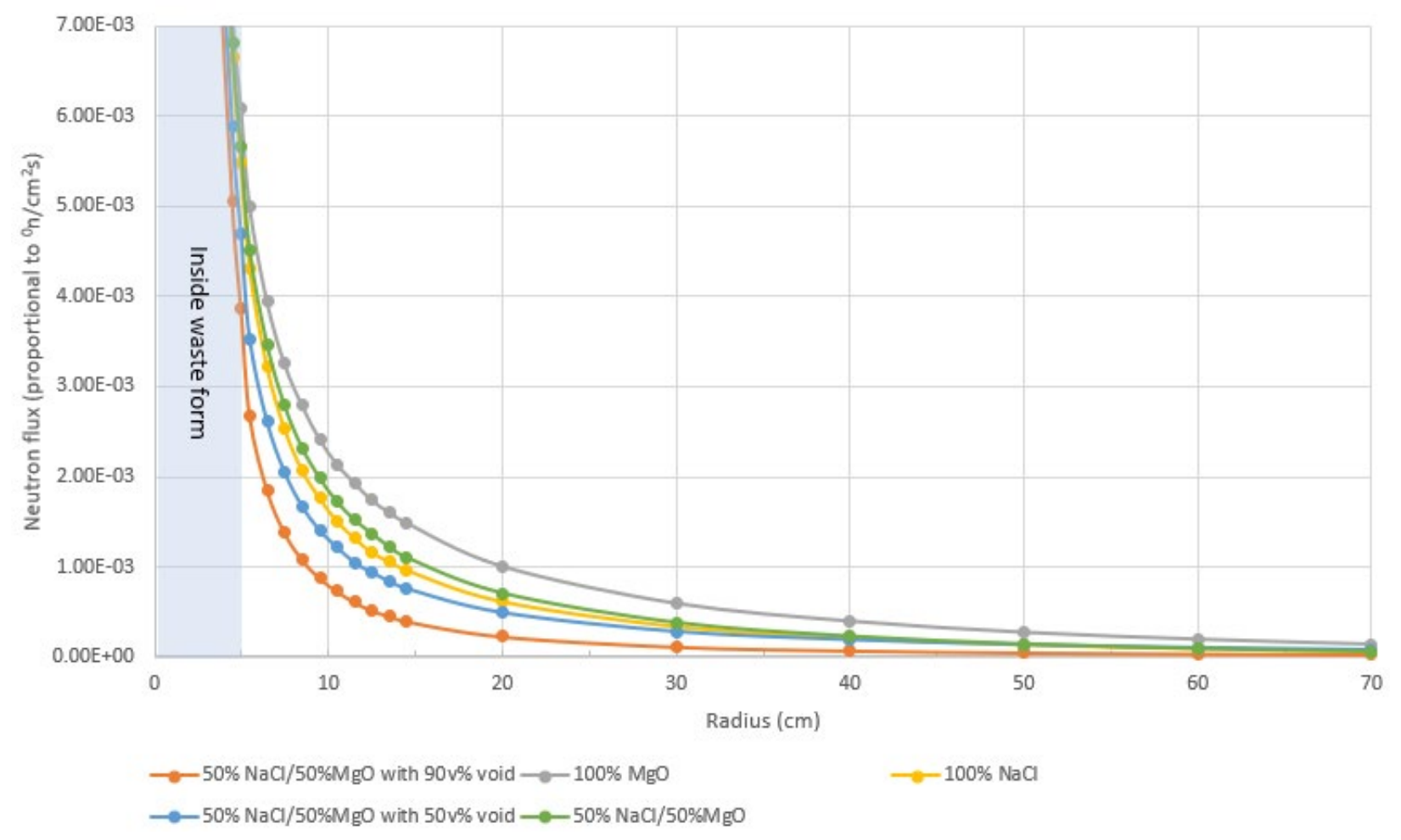

Figure K-3. Flux per source particle at various distances in the salt form the source (linear scale).

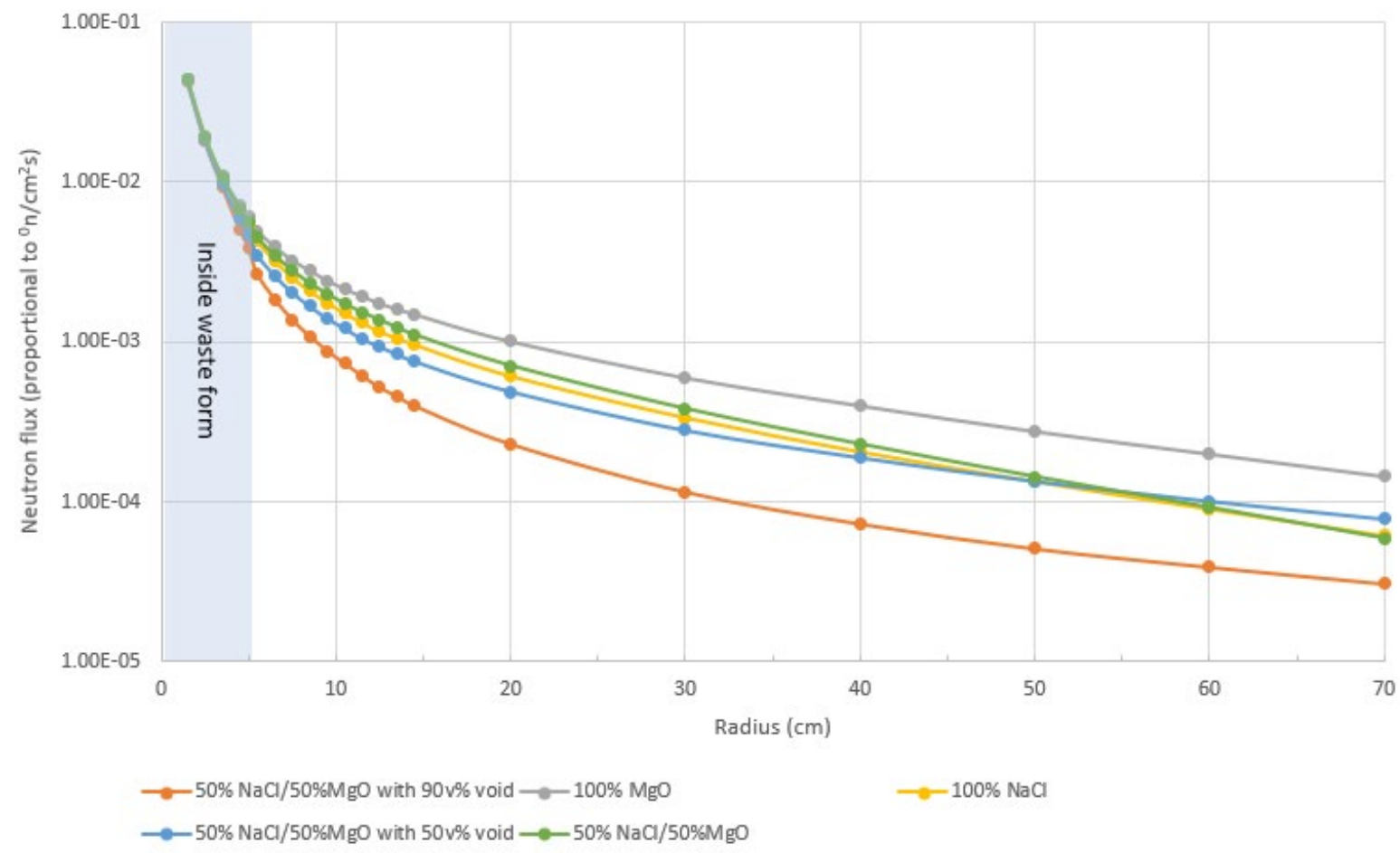

Figure K-4. Flux per source particle at various distances in the salt form the source (log scale). 
Figure K-5 shows energy spectra of the neutron flux at various distances from the source. Within $10 \mathrm{~cm}$ of sphere, the neutron flux is mostly fast. This is a low moderator case, so fast flux near the fission source is expected because there is no moderator to slow the fast flux down. Any thermal flux is attenuated by about 4 orders of magnitude between $10 \mathrm{~cm}$ and $50 \mathrm{~cm}$, with fluxes between $10 \mathrm{eV}$ and $1 \mathrm{MeV}$ penetrating farthest through the $\mathrm{NaCl} / \mathrm{MgO}$ mixture.

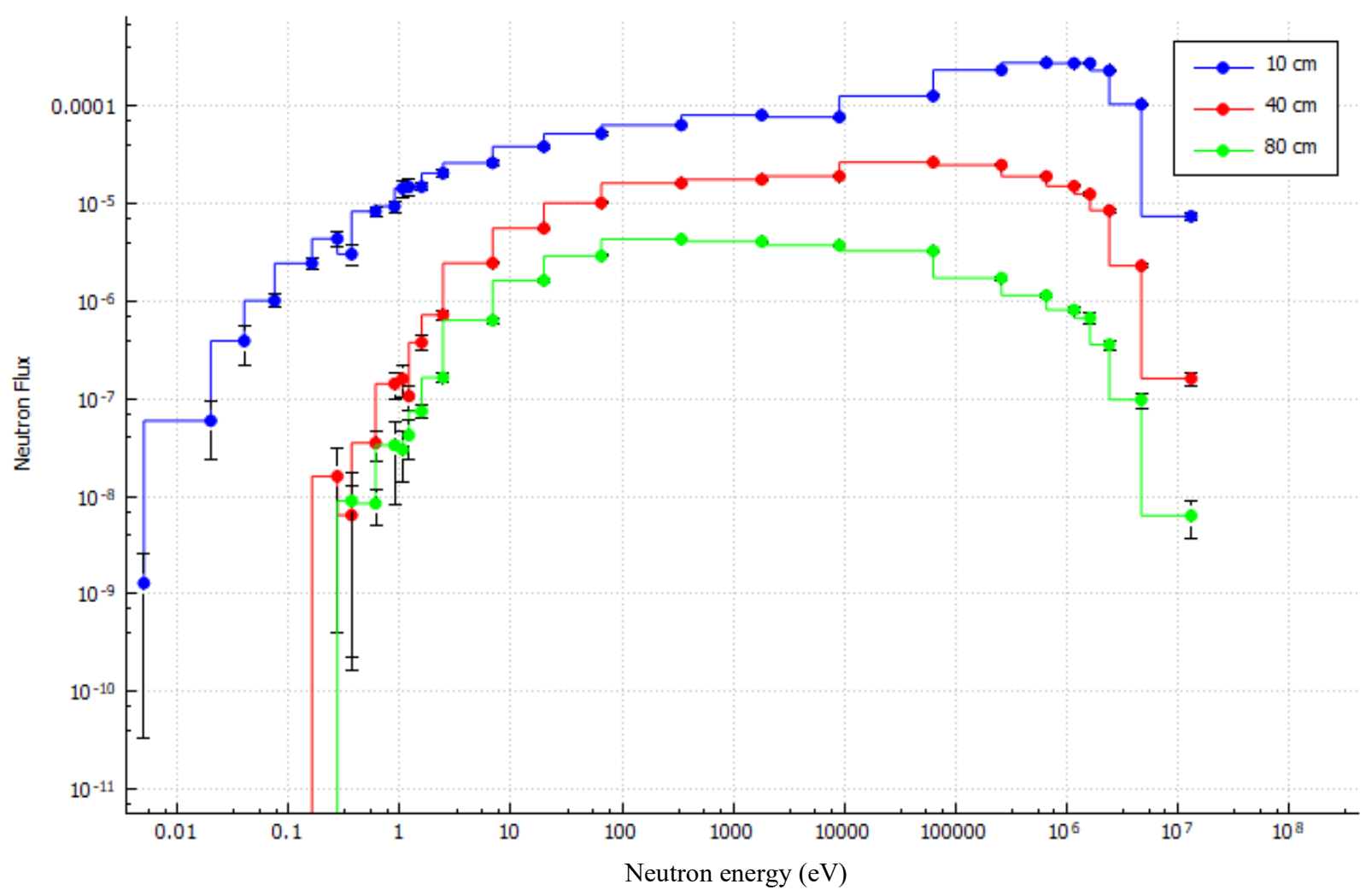

Figure K-5. Flux spectra (n/cm ${ }^{2} \mathrm{~s}$ per source particle) at $10 \mathrm{~cm}$ from source (blue), $50 \mathrm{~cm}$ from source (red) and $100 \mathrm{~cm}$ from source (green). 
Table K-2. Flux per source particle $\left({ }^{0} \mathrm{n} / \mathrm{cm}^{2} \mathrm{~s}\right)$ at various distances in the salt form the source

\begin{tabular}{|c|c|c|c|c|c|c|c|c|c|c|}
\hline 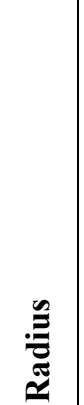 & 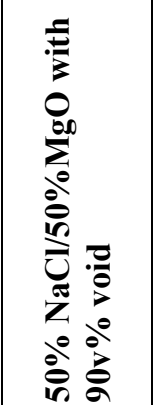 & 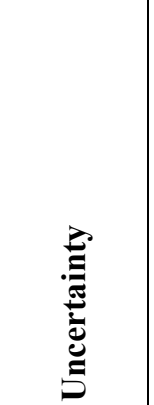 & 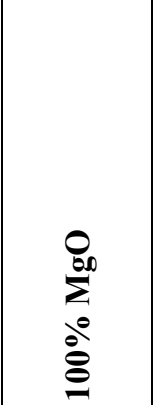 & 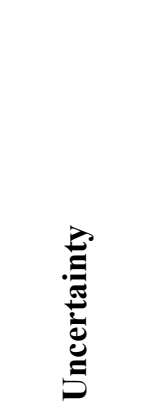 & $\begin{array}{l}\bar{\Xi} \\
\bar{z} \\
\stackrel{0}{0} \\
\stackrel{\theta}{0}\end{array}$ & 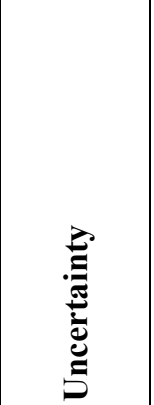 & 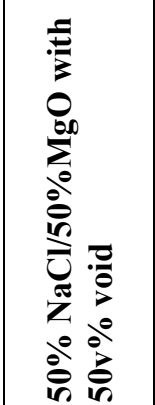 & 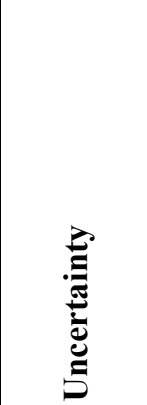 & 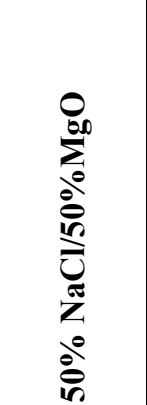 & 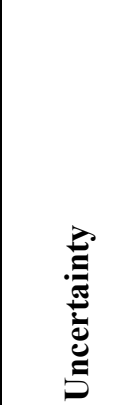 \\
\hline 1.5 & $4.30 \mathrm{E}-02$ & $3 \mathrm{E}-04$ & $3 E-02$ & $14 \mathrm{E}-04$ & $4.41 \mathrm{E}-02$ & $1.66 \mathrm{E}-04$ & $4.37 \mathrm{E}-02$ & 7.98E-05 & $4.42 \mathrm{E}-02$ & $1.55 \mathrm{E}-$ \\
\hline 2.5 & -02 & $4 \mathrm{E}-05$ & $4 \mathrm{E}-02$ & $7 \mathrm{E}-05$ & $3 \mathrm{E}-02$ & $8.85 \mathrm{E}-05$ & E-02 & 5 & 02 & \\
\hline 3.5 & 9.31E-03 & -05 & 9E-02 & -05 & $1.06 \mathrm{E}-02$ & 4.29 & 9.89E-03 & 2.6 & $1.06 \mathrm{E}-02$ & 3.8 \\
\hline 4.5 & $5.06 \mathrm{E}-03$ & $3.03 \mathrm{E}-05$ & 19E-03 & $5 \mathrm{E}-05$ & $6.65 \mathrm{E}-03$ & $3.39 \mathrm{E}-05$ & $5.87 \mathrm{E}-03$ & $2.82 \mathrm{E}-05$ & $6.82 \mathrm{E}-03$ & 2.93E-05 \\
\hline 5 & 3.87E-03 & $2.41 \mathrm{E}-05$ & 09E-03 & $42 \mathrm{E}-05$ & $5.48 \mathrm{E}-03$ & $2.87 \mathrm{E}-05$ & 4.70E-03 & $2.43 \mathrm{E}-05$ & $6 \mathrm{E}-03$ & $2.45 \mathrm{E}-05$ \\
\hline 5.5 & -03 & & & & & & & & & \\
\hline 6.5 & $1.85 \mathrm{E}-03$ & E-06 & 94E-03 & $\mathrm{E}-05$ & E-03 & E-05 & $2.61 \mathrm{E}-03$ & 1. & $3.47 \mathrm{E}-03$ & -05 \\
\hline 7.5 & $1.38 \mathrm{E}-03$ & $8.60 \mathrm{E}-06$ & $3.25 \mathrm{E}-03$ & $1.77 \mathrm{E}-05$ & $2.53 \mathrm{E}-03$ & $1.61 \mathrm{E}-05$ & $2.05 \mathrm{E}-03$ & $1.14 \mathrm{E}-05$ & $2.79 \mathrm{E}-03$ & $1.62 \mathrm{E}-05$ \\
\hline 8.5 & $1.09 \mathrm{E}-03$ & $8.54 \mathrm{E}-06$ & 79E-03 & $48 \mathrm{E}-05$ & E-03 & $\mathrm{E}-05$ & $1.67 \mathrm{E}-03$ & 1.1 & 03 & $\mathrm{E}-05$ \\
\hline 9.5 & $8.80 \mathrm{E}-04$ & & 2E-03 & & & & $1.40 \mathrm{E}-03$ & & $1.99 \mathrm{E}-03$ & \\
\hline 10.5 & 7.32E-04 & $5.60 \mathrm{E}-06$ & $14 \mathrm{E}-03$ & $41 \mathrm{E}-05$ & $1.51 \mathrm{E}-03$ & $1.06 \mathrm{E}-05$ & $1.22 \mathrm{E}-03$ & $8.73 \mathrm{E}-06$ & $1.72 \mathrm{E}-03$ & $1.08 \mathrm{E}-05$ \\
\hline 11.5 & $6.14 \mathrm{E}-04$ & $5.15 \mathrm{E}-06$ & $1.93 \mathrm{E}-03$ & $1.27 \mathrm{E}-05$ & $1.32 \mathrm{E}-03$ & 7.44E-06 & $1.05 \mathrm{E}-03$ & $8.11 \mathrm{E}-06$ & $1.52 \mathrm{E}-03$ & $9.41 \mathrm{E}-06$ \\
\hline 12.5 & 5.19E-04 & 4.41E-06 & $1.74 \mathrm{E}-03$ & $9.18 \mathrm{E}-06$ & $1.16 \mathrm{E}-03$ & 6.51E-06 & 9.34E-04 & 9.32E-06 & $1.37 \mathrm{E}-03$ & 9.91E-06 \\
\hline 13.5 & $4.56 \mathrm{E}-04$ & $4.06 \mathrm{E}-06$ & $1.60 \mathrm{E}-03$ & 11E-05 & $1.06 \mathrm{E}-03$ & 8.03E-06 & $8.36 \mathrm{E}-04$ & 5.42E-06 & $1.22 \mathrm{E}-03$ & 8.59E-06 \\
\hline 14.5 & $3.98 \mathrm{E}-04$ & $4.22 \mathrm{E}-06$ & 1.49E-03 & $71 \mathrm{E}-06$ & 9.61E-04 & 6.67E-06 & 7.56E-04 & 5.99E-06 & $1.10 \mathrm{E}-03$ & $4 \mathrm{E}-06$ \\
\hline 20 & $2.29 \mathrm{E}-04$ & $1.39 \mathrm{E}-06$ & $1.01 \mathrm{E}-03$ & $3.53 \mathrm{E}-06$ & $6.12 \mathrm{E}-04$ & $3.27 \mathrm{E}-06$ & 4.89E-04 & $1.84 \mathrm{E}-06$ & 7.07E-04 & 2.42E-06 \\
\hline 30 & $1.15 \mathrm{E}-04$ & $8.83 \mathrm{E}-07$ & 5.97E-04 & $2.23 \mathrm{E}-06$ & $3.37 \mathrm{E}-04$ & $1.30 \mathrm{E}-06$ & 2.79E-04 & $1.67 \mathrm{E}-06$ & $3.80 \mathrm{E}-04$ & $1.42 \mathrm{E}-06$ \\
\hline 40 & 7.25E-05 & $6.90 \mathrm{E}-07$ & $4.00 \mathrm{E}-04$ & $1.80 \mathrm{E}-06$ & $2.06 \mathrm{E}-04$ & $1.27 \mathrm{E}-06$ & $1.88 \mathrm{E}-04$ & $1.08 \mathrm{E}-06$ & $2.28 \mathrm{E}-04$ & $1.40 \mathrm{E}-06$ \\
\hline 50 & $5.07 \mathrm{E}-05$ & $5.07 \mathrm{E}-07$ & $2.76 \mathrm{E}-04$ & $1.48 \mathrm{E}-06$ & $1.35 \mathrm{E}-04$ & $1.12 \mathrm{E}-06$ & $1.34 \mathrm{E}-04$ & 9.35E-07 & $1.43 \mathrm{E}-04$ & $1.12 \mathrm{E}-06$ \\
\hline 60 & 3.89E-05 & $5.88 \mathrm{E}-07$ & 1.99E-04 & 9.41E-07 & 9.04E-05 & 6.80E-07 & $1.00 \mathrm{E}-04$ & 6.44E-07 & $9.14 \mathrm{E}-05$ & 8.03E-07 \\
\hline 70 & $3.06 \mathrm{E}-05$ & $3.88 \mathrm{E}-07$ & $1.44 \mathrm{E}-04$ & $8.90 \mathrm{E}-07$ & $6.15 \mathrm{E}-05$ & $3.95 \mathrm{E}-07$ & 7.77E-05 & 3.93E-07 & $5.87 \mathrm{E}-05$ & $4.86 \mathrm{E}-\mathrm{C}$ \\
\hline
\end{tabular}


This page is intentionally blank K-10 
APPENDIX L. SET-5: RESULTS OF THE NONUNIFORM ARRAY FOR THE UPPER HORIZON CALCULATIONS WITH ALTERNATIVE INTERSTITIAL REFLECTOR MATERIALS 
This page is intentionally blank 


\section{APPENDIX L. SET-5: RESULTS OF THE NONUNIFORM ARRAY FOR THE UPPER HORIZON CALCULATIONS WITH ALTERNATIVE INTERSTITIAL REFLECTOR MATERIALS}

The purpose of this Appendix is to document the studies performed to evaluate the reactivity effect of various interstitial material assumptions for the material density of the $\mathrm{MgO}$ and salt interstitial material as well as the reactivity effect of a brine intrusion. Since the important parameter being evaluated is the interstitial material the model is modified in some cases to include more interstitial material by moving the location of the y-direction reflective boundary conditions by $50 \mathrm{~cm}$ (see also Appendix E). The full summary of the cases evaluated in this Appendix are shown in Table L-1.

The analysis model for the studies in this appendix is based on the set-2-uh nonuniform array model discussed in Section 6.3. For each of the cases listed in Table L-1 the full set of parametric sweeps is provided in Table L-2. For the case with the brine intrusion into the waste form mixture itself (case $5 \mathrm{~h}$ ), the brine mixture replaces the filler mass (graphite is replaced by brine).

\section{LIST OF FIGURES}

Figure L-1. Delta-k between set-2 and set-5 base case (set-2 minus set-5) to evaluate location of reflective boundary conditions.

Figure L-2. Delta-k between set-5 base case and set-5a (set-5 minus set-5a) to evaluate the effect of half density interstitial reflective material. ................................................................. L-10

Figure L-3. Delta-k between set-5 base case and set-5b (set-5 minus set-5b) to evaluate the effect of zero density (void) interstitial reflective material............................................................. L-11

Figure L-4. Delta-k between set-5 base case and set-5c to evaluate the effect of pure salt as interstitial reflective material. ....................................................................................... L-12

Figure L-5. Delta-k between set-5 base case and set-5d to evaluate the effect of brine (no MgO)

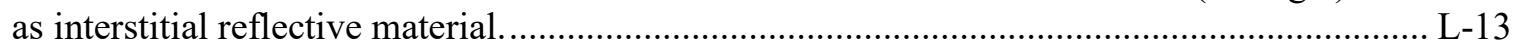

Figure L-6. Reactivity of set-5d compared to a single set- 2 representative curve to evaluate the effect of brine (no $\mathrm{MgO}$ ) as interstitial reflective material.

Figure L-7. Delta-k between set-2 and set-5e to evaluate the effect of brine (no $\mathrm{MgO}$ ) as interstitial reflective material but with the additional $50 \mathrm{~cm}$ of space to locate the $\mathrm{y}$ direction reflective boundary conditions removed (similar to set-2).

Figure L-8. Reactivity of set-5e compared to a single set-2 representative curve to evaluate the effect of brine (no $\mathrm{MgO}$ ) as interstitial reflective material but with the additional $50 \mathrm{~cm}$ of space to locate the y-direction reflective boundary conditions removed (similar to set-2). ...... L-16

Figure L-9. Delta-k between set-2 and set- $5 \mathrm{f}$ to evaluate the effect of brine (no $\mathrm{MgO}$ ) as interstitial reflective material but with the additional $50 \mathrm{~cm}$ of space to locate the $y$ direction reflective boundary conditions removed (similar to set-2) and brine in the external salt box.

Figure L-10. Delta-k between set- 2 and set- $5 \mathrm{~g}$ to evaluate the effect of brine in a $50 \%$ mixture with $\mathrm{MgO}$ as interstitial reflective material but with the additional $50 \mathrm{~cm}$ of space to locate the y-direction reflective boundary conditions removed (similar to set-2) and brine in the external salt box.....

Figure L-11. Delta-k between set- 2 and set- $5 \mathrm{~h}$ to evaluate the effect of brine in a $50 \%$ mixture with $\mathrm{MgO}$ as interstitial reflective material but with the additional $50 \mathrm{~cm}$ of space to locate the y-direction reflective boundary conditions removed (similar to set-2) and brine in the external salt box. The graphite filler, when present, is replaced by an equivalent mass of brine in a $50 \%$ mixture with $\mathrm{MgO}$. 
Figure L-12. $\mathrm{k}_{\text {eff }}$ of set-5h compared to a single set-2 representative curve to evaluate the effect of brine in a $50 \%$ mixture with $\mathrm{MgO}$ as interstitial reflective material but with the additional $50 \mathrm{~cm}$ of space to locate the y-direction reflective boundary conditions removed (similar to set-2) and brine in the external salt box. The graphite filler, when present, is replaced by an equivalent mass of brine in a $50 \%$ mixture with $\mathrm{MgO}$

\section{LIST OF TABLES}

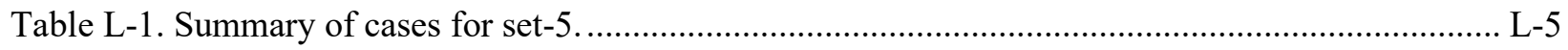

Table L-2. Summary of parametric sweeps for set-5 (applicable to set-5 and versions a through h)....... L-6 
Table L-1. Summary of cases for set-5.

\begin{tabular}{|c|c|c|c|c|c|c|}
\hline Case & Purpose & $\begin{array}{c}\text { Interstitial } \\
\text { material } \\
\text { mixture }\end{array}$ & $\begin{array}{c}\text { Interstitial } \\
\text { material } \\
\text { density }\end{array}$ & $\begin{array}{l}\text { External } \\
\text { salt box } \\
\text { material }\end{array}$ & $\begin{array}{l}\text { y-direction } \\
\text { reflective } \\
\text { boundary } \\
\text { condition } \\
\text { location }\end{array}$ & $\begin{array}{l}\text { Waste form } \\
\text { filler } \\
\text { material }\end{array}$ \\
\hline $\begin{array}{l}\text { Set- } 5 \text { base } \\
\text { case }\end{array}$ & $\begin{array}{l}\text { Same as set-2-uh but } \\
\text { with alternative } \\
\text { reflective boundary } \\
\text { condition location }\end{array}$ & $\mathrm{Mgo}+$ salt & Full density & Pure salt & $+50 \mathrm{~cm}$ & Graphite \\
\hline Set-5a & $\begin{array}{l}\text { Evaluates the } \\
\text { assumption that full } \\
\text { density is appropriate }\end{array}$ & $\begin{array}{c}50 \% \text { Mgo } \\
50 \% \text { salt }\end{array}$ & Half density & Pure salt & $+50 \mathrm{~cm}$ & Graphite \\
\hline Set-5b & $\begin{array}{l}\text { Evaluates the } \\
\text { assumption that full } \\
\text { density is appropriate }\end{array}$ & Void & 0 density & Pure salt & $+50 \mathrm{~cm}$ & Graphite \\
\hline Set-5c & $\begin{array}{l}\text { Evaluates the } \\
\text { assumption that } \\
\mathrm{MgO} \text { in the mixture } \\
\text { is appropriate }\end{array}$ & $100 \%$ salt & Full density & Pure salt & $+50 \mathrm{~cm}$ & Graphite \\
\hline Set-5d & $\begin{array}{l}\text { Shows the reactivity } \\
\text { effect of brine } \\
\text { without } \mathrm{MgO}\end{array}$ & $\begin{array}{c}100 \% \text { brine } \\
\text { (no Mgo) }\end{array}$ & Full density & Pure salt & $+50 \mathrm{~cm}$ & Graphite \\
\hline Set-5e & $\begin{array}{l}\text { Shows the reactivity } \\
\text { effect of brine with } \\
\mathrm{MgO}\end{array}$ & $\begin{array}{c}100 \% \text { brine } \\
\text { (no Mgo) }\end{array}$ & Full density & Pure salt & $+0 \mathrm{~cm}$ & Graphite \\
\hline Set-5f & $\begin{array}{l}\text { Shows the reactivity } \\
\text { effect of including } \\
\text { brine in the outer salt } \\
\text { box }\end{array}$ & $\begin{array}{c}100 \% \text { brine } \\
\text { (no Mgo) }\end{array}$ & Full density & Brine & $+0 \mathrm{~cm}$ & Graphite \\
\hline Set-5g & $\begin{array}{l}\text { Shows the reactivity } \\
\text { effect of including } \\
\mathrm{MgO} \text { in the brine }\end{array}$ & $\begin{array}{l}50 \% \text { brine } \\
50 \% \mathrm{MgO}\end{array}$ & Full density & Brine & $+0 \mathrm{~cm}$ & Graphite \\
\hline Set-5h & $\begin{array}{l}\text { Shows the reactivity } \\
\text { effect of including } \\
\text { brine in the waste } \\
\text { form }\end{array}$ & $\begin{array}{l}50 \% \text { brine } \\
50 \% \mathrm{MgO}\end{array}$ & Full density & Brine & $+0 \mathrm{~cm}$ & $\begin{array}{l}50 \% \text { brine } \\
50 \% \mathrm{MgO}\end{array}$ \\
\hline
\end{tabular}


Table L-2. Summary of parametric sweeps for set-5 (applicable to set-5 and versions a through $\mathbf{h}^{\mathbf{9}}$ )

\begin{tabular}{|c|c|c|c|c|c|c|c|c|}
\hline Case & Model type & Waste form shape & $\begin{array}{l}\text { Waste form } \\
\text { moderator }\end{array}$ & $\begin{array}{c}\text { Filler } \\
\text { material }(0, \\
2,000,4,000 \\
\text { g) }\end{array}$ & Metal in filler & $\begin{array}{l}\text { Discrete reflector } \\
\text { (thin } 0.001 \text { and } \\
\text { thick } 0.7112 \mathrm{~cm} \text { ) }\end{array}$ & be (g) & Subcase \\
\hline \multirow{18}{*}{ Set-5 } & \multirow{18}{*}{$\begin{array}{l}\text { Uniform array stacked six } \\
\text { high with CCO pitch } \\
\text { reduction } \mathrm{x}=25 \%, \mathrm{y}=10 \%, \\
\mathrm{z}=\text { no space }\end{array}$} & \multirow{8}{*}{$\begin{array}{c}\text { Cylinder (radius range } 4.8,6, \\
7.7 \text { and height defined by } \\
\text { total volume of mass) }\end{array}$} & water & $\mathrm{c} 12$ & \multirow{18}{*}{$\begin{array}{l}\text { SS from can } \\
(0,500,1,000 \\
\mathrm{g})\end{array}$} & steel & \multirow{18}{*}{$\begin{array}{l}0 \text { to } \\
585\end{array}$} & set-5-1 \\
\hline & & & poly & $\mathrm{c} 12$ & & steel & & set-5-2 \\
\hline & & & water & $\mathrm{c} 12$ & & poly & & set-5-3 \\
\hline & & & poly & $\mathrm{c} 12$ & & poly & & set-5-4 \\
\hline & & & water & generic & & steel & & set-5-5 \\
\hline & & & poly & generic & & steel & & set-5-6 \\
\hline & & & water & generic & & poly & & set-5-7 \\
\hline & & & poly & generic & & poly & & set-5-8 \\
\hline & & \multirow{8}{*}{$\begin{array}{l}\text { Sphere (radius defined by } \\
\text { total volume of mass) }\end{array}$} & water & $\mathrm{c} 12$ & & steel & & set-5-9 \\
\hline & & & poly & $\mathrm{c} 12$ & & steel & & set-5-10 \\
\hline & & & water & $\mathrm{c} 12$ & & poly & & set-5-11 \\
\hline & & & poly & $\mathrm{c} 12$ & & poly & & set-5-12 \\
\hline & & & water & generic & & steel & & set-5-13 \\
\hline & & & poly & generic & & steel & & set-5-14 \\
\hline & & & water & generic & & poly & & set-5-15 \\
\hline & & & poly & generic & & poly & & set-5-16 \\
\hline & & $\begin{array}{l}\text { Cylinder (radius range } 4.8,6 \text {, } \\
7.7 \text { and height defined by } \\
\text { total volume of mass) with } \\
25 \mathrm{~g} \mathrm{~B}_{4} \mathrm{C}\end{array}$ & poly & $\mathrm{c} 12$ & & poly & & set-5-17 \\
\hline & & $\begin{array}{l}\text { Sphere (radius defined by } \\
\text { total volume of mass) with } \\
25 \mathrm{~g} \mathrm{~B}_{4} \mathrm{C}\end{array}$ & poly & $\mathrm{c} 12$ & & poly & & set-5-18 \\
\hline
\end{tabular}

\footnotetext{
${ }^{9}$ set-5h does not include sub-cases 17 or 18 with $\mathrm{B}_{4} \mathrm{C}$.
} 


\section{Summary of Results}

- Figure L-1: The delta-k between set-2 and set- 5 shows that adding $50 \mathrm{~cm}$ of interstitial material between the waste forms and the reflective boundary conditions in the y-direction always yields a decrease in $\mathrm{k}_{\text {eff. }}$. However, the reactivity effect is relatively small, typically less than $5 \%$ delta-k and typically less than $2.5 \%$ delta-k for the representative curves.

- Figure L-2. The delta-k between set-5 base and set-5a shows that reducing the material density by $50 \%$ for the interstitial material between the waste forms always yields a moderate decrease in $\mathrm{k}_{\text {eff. }}$ The reactivity effect is moderate, typically less than $7.5 \%$ delta-k overall. These results show that full density interstitial material is appropriate.

- Figure L-3. The delta-k between set-5 base and set-5b shows that reducing the material density by $100 \%$ for the interstitial material between the waste forms (void) typically yields a significant decrease in $\mathrm{k}_{\text {eff. }}$ The reactivity effect is significant for under moderated cases, typically less than $22 \%$ delta-k overall. These results show that the trend seen for the half density set-5a cases continues and that full density interstitial material is appropriate.

- Figure L-4: The delta-k between set-5 base and set-5c shows that removing the $\mathrm{MgO}$ from the interstitial material between the waste forms always yields a decrease in $\mathrm{k}_{\text {eff. }}$. However, the reactivity effect is relatively small, typically less than $6 \%$ delta-k and typically less than $4 \%$ delta-k for the representative curves. These results show that the assumption of using $\mathrm{MgO}$ is appropriate but not overly conservative because the overall impact is relatively small.

- Figure L-5: The delta-k between set-5 base and set-5d shows that the reactivity effect of the brine in the interstitial material between the waste forms is highly dependent upon whether the waste form is under moderated or over moderated. For cases where the waste form is under moderated, up to about $0.9 \mathrm{~kg}$ of moderator, the brine can provide additional moderation and thus increases $\mathrm{k}_{\text {eff }}$ moderately. Otherwise, when the waste form is not under moderated, the brine reduces reactivity. However, cases in set- 2 under $1 \mathrm{~kg}$ of moderator are well below subcritical.

- Figure L-6: The plot provides $\mathrm{k}_{\text {eff }}$ curves for set-5d results compared to a set- 2 representative curve. The results shown in Figure L-5 and Figure L-6 show that while for under moderated waste form cases the brine may increase reactivity slightly, the slight increase in reactivity remains well subcritical.

- Figure L-7: The delta-k between set-2 and set-5e shows that the reactivity effect of the brine in the interstitial material between the waste forms is dependent upon the amount of moderator in the waste form. This effect is increased when the amount of brine is decreased (the additional $50 \mathrm{~cm}$ of interstitial material in the y-direction is removed). For cases where the waste form is under moderated, up to about $1.5 \mathrm{~kg}$ of moderator, the brine can provide additional moderation and thus increases $k_{\text {eff }}$ moderately. Otherwise, when the waste form is not under moderated, the brine reduces reactivity.

- Figure L-8: The plot provides $\mathrm{k}_{\text {eff }}$ curves for set-5e results compared to a set-2 representative curve. The results shown in Figure L-7 and Figure L-8 show that while for under moderated waste form cases the brine may increase reactivity slightly, the slight increase in reactivity remains well subcritical. 
- Figure L-9: The delta-k between set- 2 and set-5f shows that, similar to set-5e, in which the brine is in the interstitial material between the waste forms, and it is also included in the exterior salt box, the effect of the additional brine is negligible compared to when it is not included (Figure L-7).

- Figure L-10: The delta-k between set-2 and set-5g shows an impact that is similar to that of set-5e, but when the brine in the interstitial material between the waste forms is a $50 \%$ mixture with $\mathrm{MgO}$, the positive reactivity effect of the brine is reduced for under moderated cases.

- Figure L-11: The delta-k between set-2 and set-5h shows that when the brine and MgO mixture replaces the graphite filler material in waste form there is a significant positive reactivity effect for under moderated cases.

- Figure L-12: The plot provides $\mathrm{k}_{\text {eff }}$ curves for set-5h results compared to a set-2 representative curve. The results shown in Figure L-11 and Figure L-12 show that while for under moderated waste form cases the brine and $\mathrm{MgO}$ mixture replacing the graphite filler may increase reactivity significantly, the increase in reactivity remains well subcritical. 


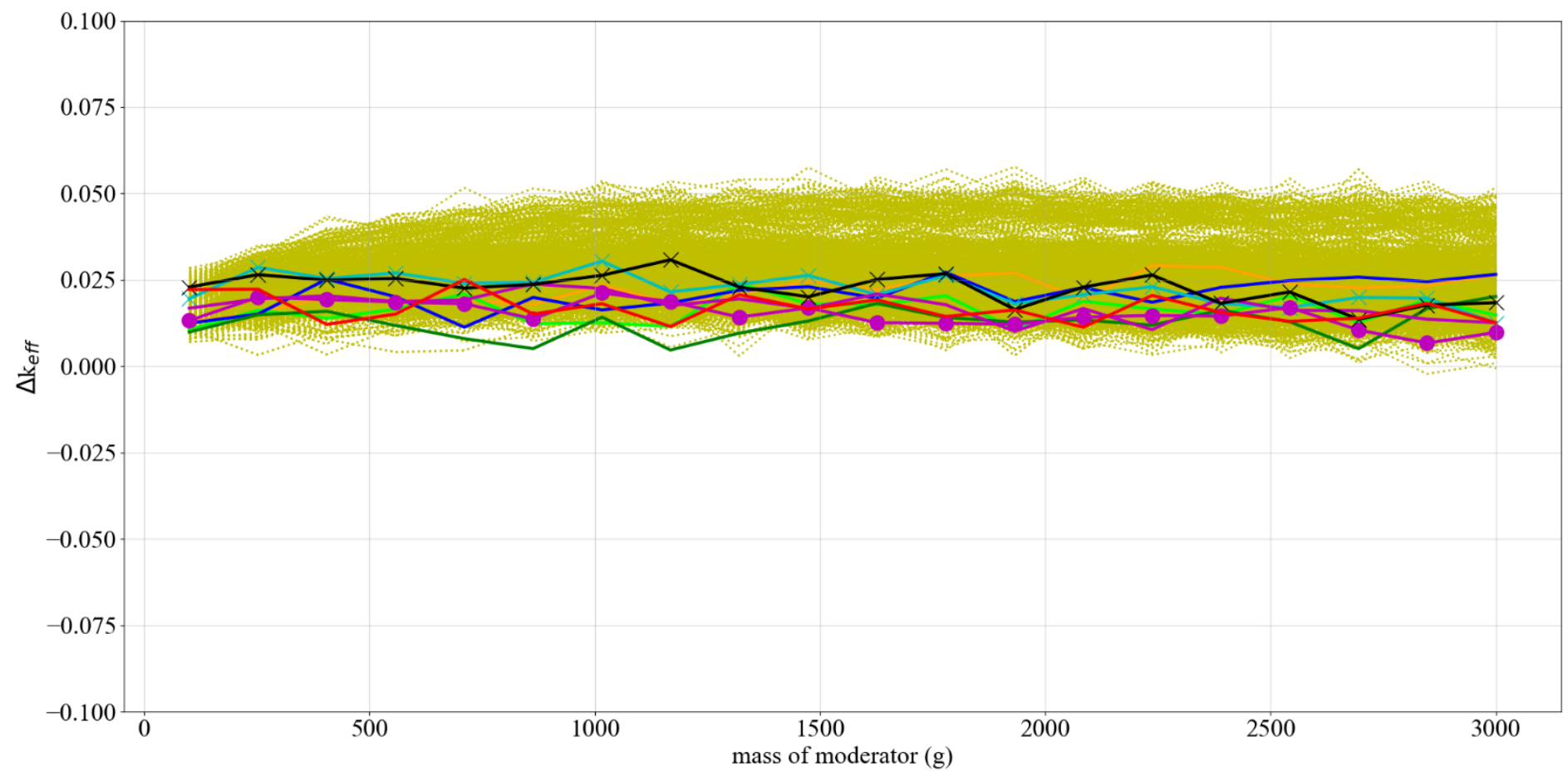

\section{delta all data}

$\leftarrow$ waste form sphere, FGE+poly mod only, thin steel discrete reflector

waste form $\mathrm{R}=7.7 \mathrm{~cm}$ cylinder, $\mathrm{FGE}+$ poly mod only, thin steel discrete reflector waste form $\mathrm{R}=7.7 \mathrm{~cm}$ cylinder, FGE+ poly mod only, thick steel discrete reflector waste form $\mathrm{R}=7.7 \mathrm{~cm}$ cylinder, $\mathrm{FGE}+$ poly mod only, thin poly discrete reflector - waste form sphere, FGE+poly mod only, thick steel discrete reflecto - waste form sphere, FGE + poly mod $+4 \mathrm{~kg}$ graphite, thick steel discrete reflector

$\star$ waste form sphere, FGE+poly mod only, thin poly discrete reflector waste form $\mathrm{R}=7.7 \mathrm{~cm}$ cylinder, $\mathrm{FGE}+$ poly mod only, thick poly discrete reflector

Figure L-1. Delta-k between set-2 and set-5 base case (set-2 minus set-5) to evaluate location of reflective boundary conditions. 


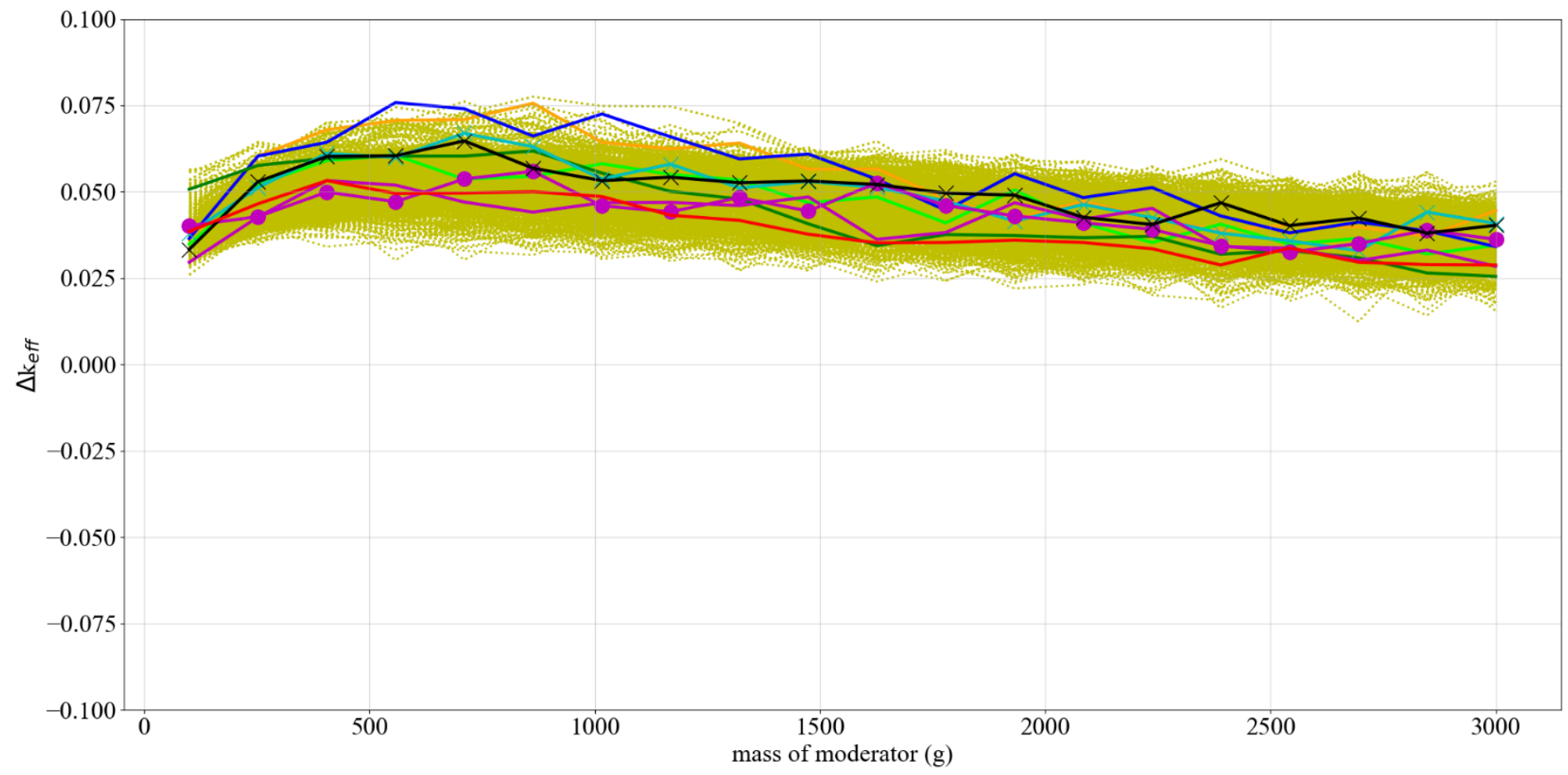

delta all data

waste form $\mathrm{R}=7.7 \mathrm{~cm}$ cylinder, $\mathrm{FGE}+$ poly mod only, thin steel discrete reflector waste form $\mathrm{R}=7.7 \mathrm{~cm}$ cylinder, FGE+poly mod only, thick steel discrete reflector waste form $\mathrm{R}=7.7 \mathrm{~cm}$ cylinder, $\mathrm{FGE}$ +poly mod only, thin poly discrete reflector waste form $\mathrm{R}=7.7 \mathrm{~cm}$ cylinder, FGE+poly mod only, thick poly discrete reflector

$\leftarrow$ waste form sphere, $\mathrm{FGE}+$ poly mod only, thin steel discrete reflector — waste form sphere, FGE+ poly mod only, thick steel discrete reflector - waste form sphere, $\mathrm{FGE}+$ poly mod $+4 \mathrm{~kg}$ graphite, thick steel discrete reflector

* waste form sphere, FGE+ poly mod only, thin poly discrete reflector - waste form sphere, FGE+poly mod only, thick poly discrete reflector

\section{Figure L-2. Delta-k between set-5 base case and set-5a (set-5 minus set-5a) to evaluate the effect of half density interstitial reflective material.}




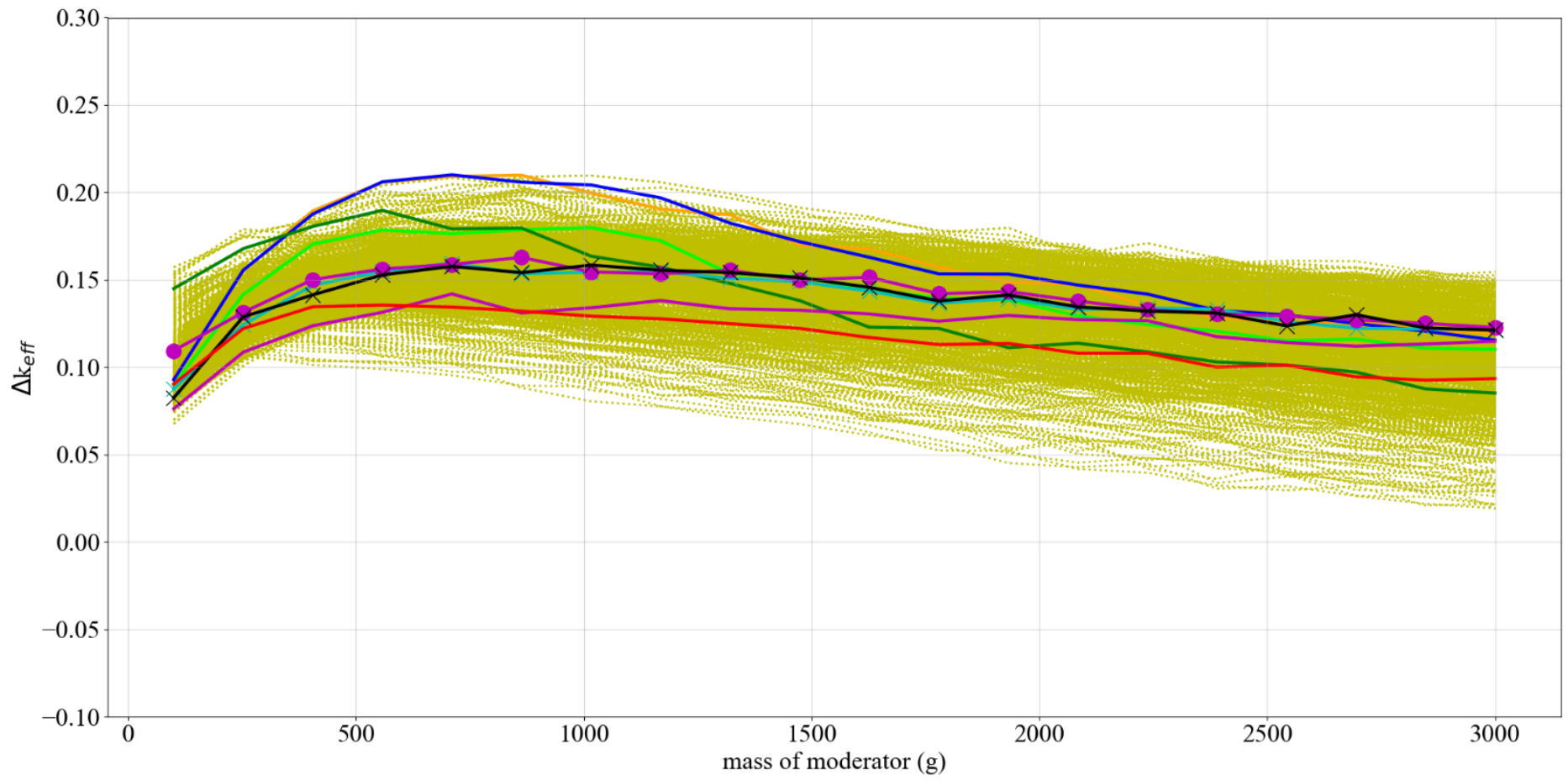

delta all data

waste form $\mathrm{R}=7.7 \mathrm{~cm}$ cylinder, $\mathrm{FGE}+$ poly mod only, thin steel discrete reflector waste form $\mathrm{R}=7.7 \mathrm{~cm}$ cylinder, $\mathrm{FGE}+$ poly mod only, thick steel discrete reflector waste form $\mathrm{R}=7.7 \mathrm{~cm}$ cylinder, $\mathrm{FGE}$ +poly mod only, thin poly discrete reflector waste form $\mathrm{R}=7.7 \mathrm{~cm}$ cylinder, FGE+poly mod only, thick poly discrete reflector

$\leftarrow$ waste form sphere, $\mathrm{FGE}+$ poly mod only, thin steel discrete reflector — waste form sphere, FGE+ poly mod only, thick steel discrete reflector - waste form sphere, $\mathrm{FGE}+$ poly mod $+4 \mathrm{~kg}$ graphite, thick steel discrete reflector

* waste form sphere, FGE+ poly mod only, thin poly discrete reflector — waste form sphere, FGE+poly mod only, thick poly discrete reflector

\section{Figure L-3. Delta-k between set-5 base case and set-5b (set-5 minus set-5b) to evaluate the effect of zero density (void) interstitial reflective material.}




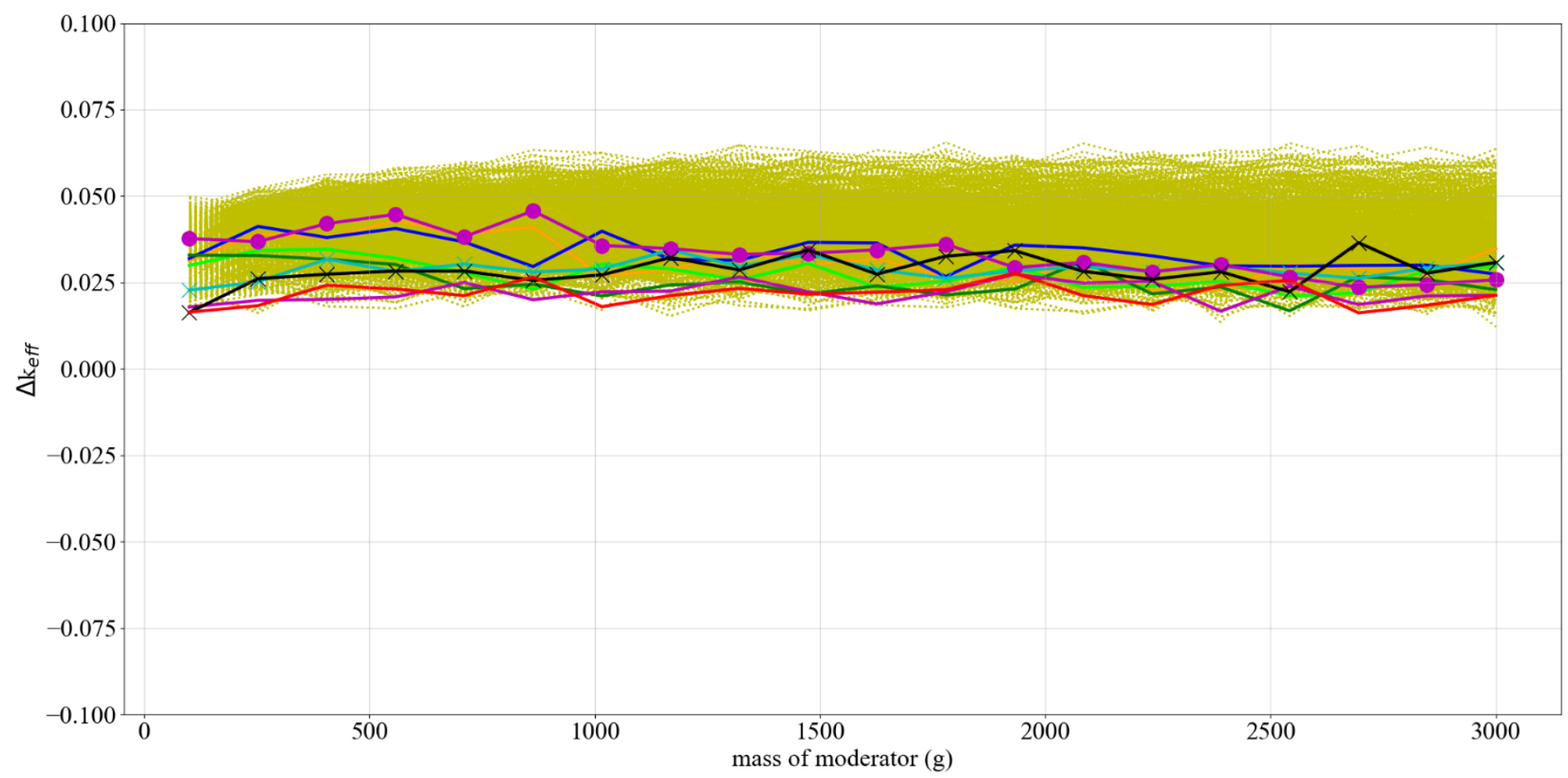

delta all data

waste form $\mathrm{R}=7.7 \mathrm{~cm}$ cylinder, $\mathrm{FGE}+$ poly mod only, thin steel discrete reflector waste form $\mathrm{R}=7.7 \mathrm{~cm}$ cylinder, $\mathrm{FGE}+$ poly mod only, thick steel discrete reflector waste form $\mathrm{R}=7.7 \mathrm{~cm}$ cylinder, $\mathrm{FGE}$ +poly mod only, thin poly discrete reflector waste form $\mathrm{R}=7.7 \mathrm{~cm}$ cylinder, FGE+poly mod only, thick poly discrete reflector

$\leftarrow$ waste form sphere, $\mathrm{FGE}+$ poly mod only, thin steel discrete reflector — waste form sphere, FGE+ poly mod only, thick steel discrete reflector - waste form sphere, FGE+poly mod $+4 \mathrm{~kg}$ graphite, thick steel discrete reflector

* waste form sphere, $\mathrm{FGE}+$ poly mod only, thin poly discrete reflector - waste form sphere, FGE+poly mod only, thick poly discrete reflector

Figure L-4. Delta-k between set-5 base case and set-5c to evaluate the effect of pure salt as interstitial reflective material. 


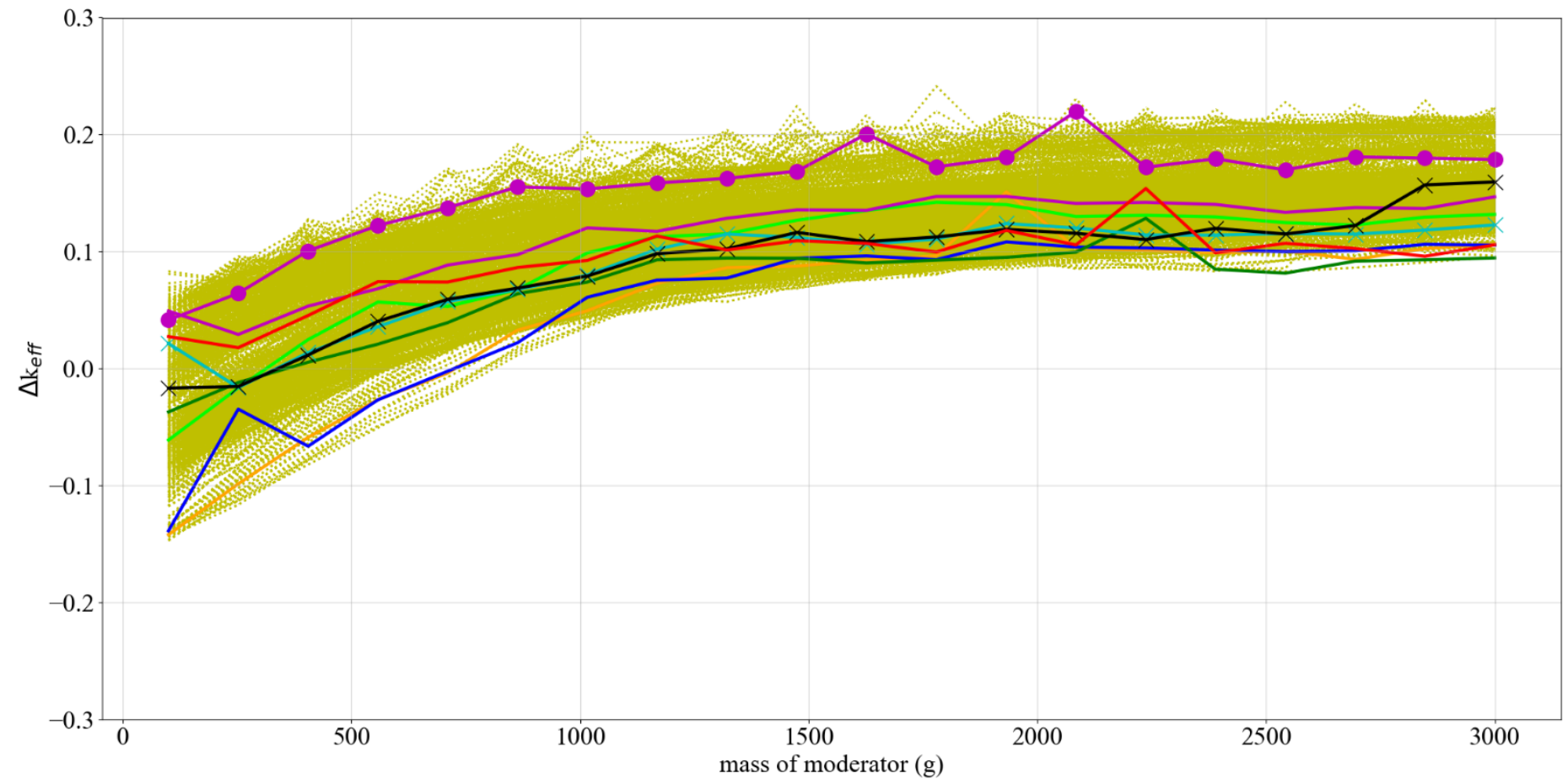

delta all data

waste form $\mathrm{R}=7.7 \mathrm{~cm}$ cylinder, $\mathrm{FGE}+$ poly mod only, thin steel discrete reflector waste form $\mathrm{R}=7.7 \mathrm{~cm}$ cylinder, $\mathrm{FGE}+$ poly mod only, thick steel discrete reflector waste form $\mathrm{R}=7.7 \mathrm{~cm}$ cylinder, $\mathrm{FGE}$ +poly mod only, thin poly discrete reflector waste form $\mathrm{R}=7.7 \mathrm{~cm}$ cylinder, FGE+poly mod only, thick poly discrete reflector

$\leftarrow$ waste form sphere, FGE+poly mod only, thin steel discrete reflector — waste form sphere, FGE+ poly mod only, thick steel discrete reflector - waste form sphere, FGE+poly mod $+4 \mathrm{~kg}$ graphite, thick steel discrete reflector

* waste form sphere, FGE+ poly mod only, thin poly discrete reflector — waste form sphere, FGE+poly mod only, thick poly discrete reflector

Figure L-5. Delta-k between set-5 base case and set-5d to evaluate the effect of brine (no MgO) as interstitial reflective material. 


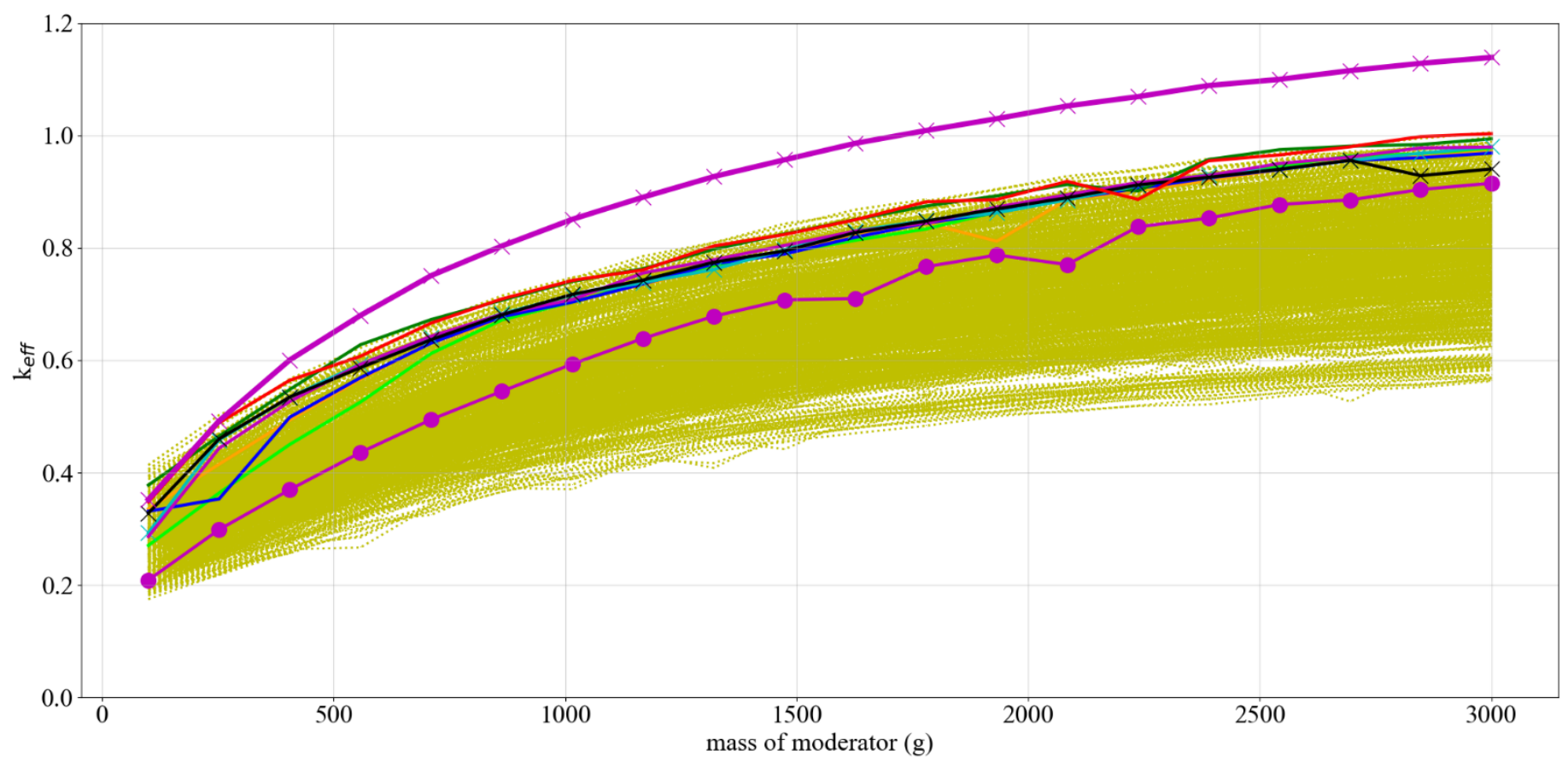

delta all data

waste form $\mathrm{R}=7.7 \mathrm{~cm}$ cylinder, FGE+poly mod only, thin steel discrete reflector waste form $\mathrm{R}=7.7 \mathrm{~cm}$ cylinder, $\mathrm{FGE}+$ poly mod only, thick steel discrete reflector waste form $\mathrm{R}=7.7 \mathrm{~cm}$ cylinder, $\mathrm{FGE}+$ poly mod only, thin poly discrete reflector waste form $\mathrm{R}=7.7 \mathrm{~cm}$ cylinder, $\mathrm{FGE}+$ poly mod only, thick poly discrete reflector waste form sphere, FGE+poly mod only, thin steel discrete reflector
— waste form sphere, FGE+poly mod only, thick steel discrete reflector - waste form sphere, FGE + poly mod $+4 \mathrm{~kg}$ graphite, thick steel discrete reflector * waste form sphere, FGE+poly mod only, thin poly discrete reflector — waste form sphere, FGE+poly mod only, thick poly discrete reflector $\leftarrow$ set-2, waste form sphere, FGE+poly mod only, thick steel discrete reflector

Figure L-6. Reactivity of set-5d compared to a single set-2 representative curve to evaluate the effect of brine (no $\mathrm{MgO}$ ) as interstitial reflective material. 


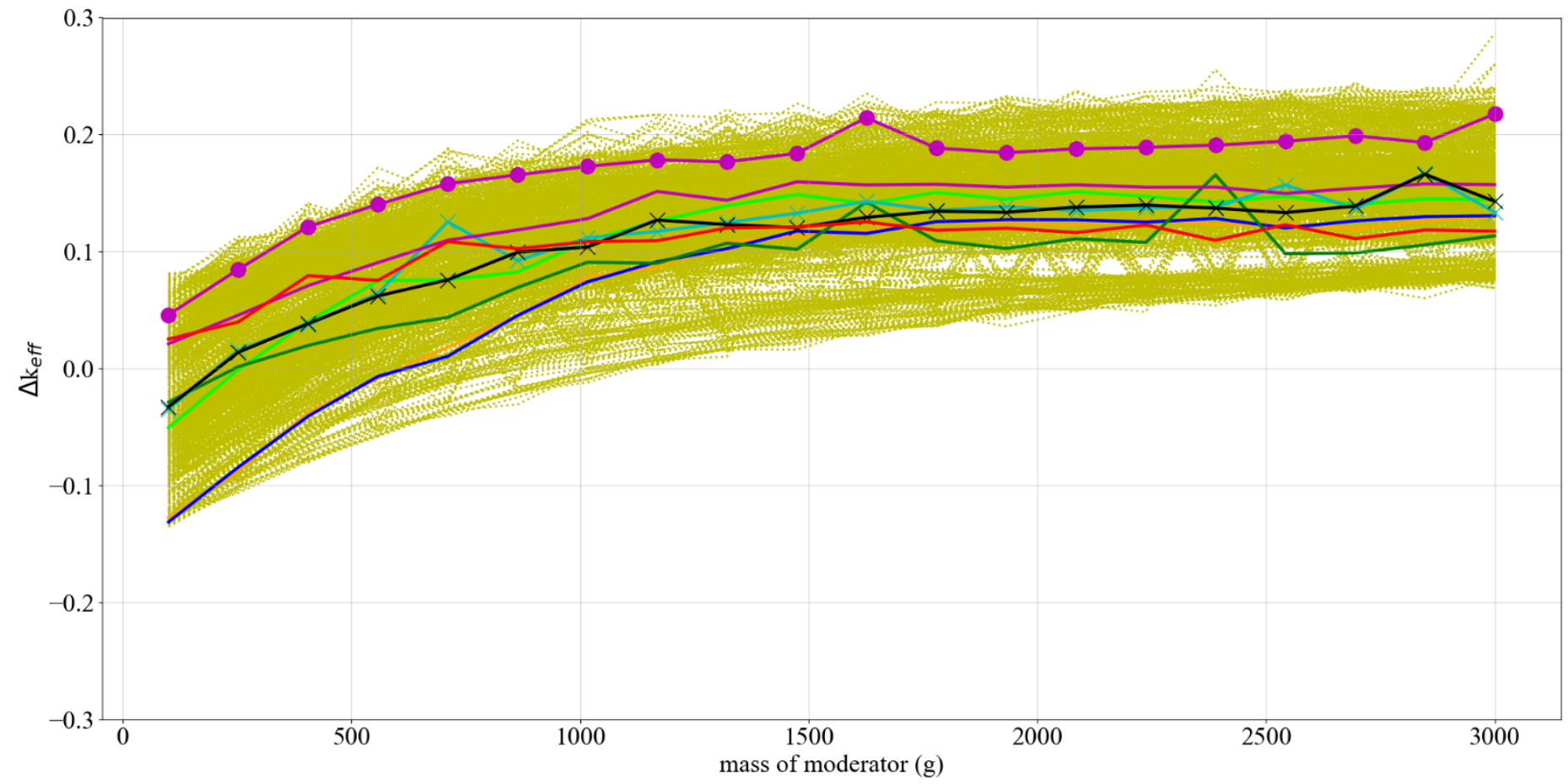

delta all data

waste form $\mathrm{R}=7.7 \mathrm{~cm}$ cylinder, $\mathrm{FGE}+$ poly mod only, thin steel discrete reflector waste form $\mathrm{R}=7.7 \mathrm{~cm}$ cylinder, $\mathrm{FGE}+$ poly mod only, thick steel discrete reflector waste form $\mathrm{R}=7.7 \mathrm{~cm}$ cylinder, $\mathrm{FGE}$ +poly mod only, thin poly discrete reflector waste form $\mathrm{R}=7.7 \mathrm{~cm}$ cylinder, $\mathrm{FGE}+$ poly mod only, thick poly discrete reflector

$\leftarrow$ waste form sphere, FGE+poly mod only, thin steel discrete reflector — waste form sphere, FGE+ poly mod only, thick steel discrete reflector - waste form sphere, FGE+poly mod $+4 \mathrm{~kg}$ graphite, thick steel discrete reflector * waste form sphere, $\mathrm{FGE}+$ poly mod only, thin poly discrete reflector — waste form sphere, FGE+poly mod only, thick poly discrete reflector

Figure L-7. Delta-k between set-2 and set-5e to evaluate the effect of brine (no MgO) as interstitial reflective material but with the additional $50 \mathrm{~cm}$ of space to locate the y-direction reflective boundary conditions removed (similar to set-2). 


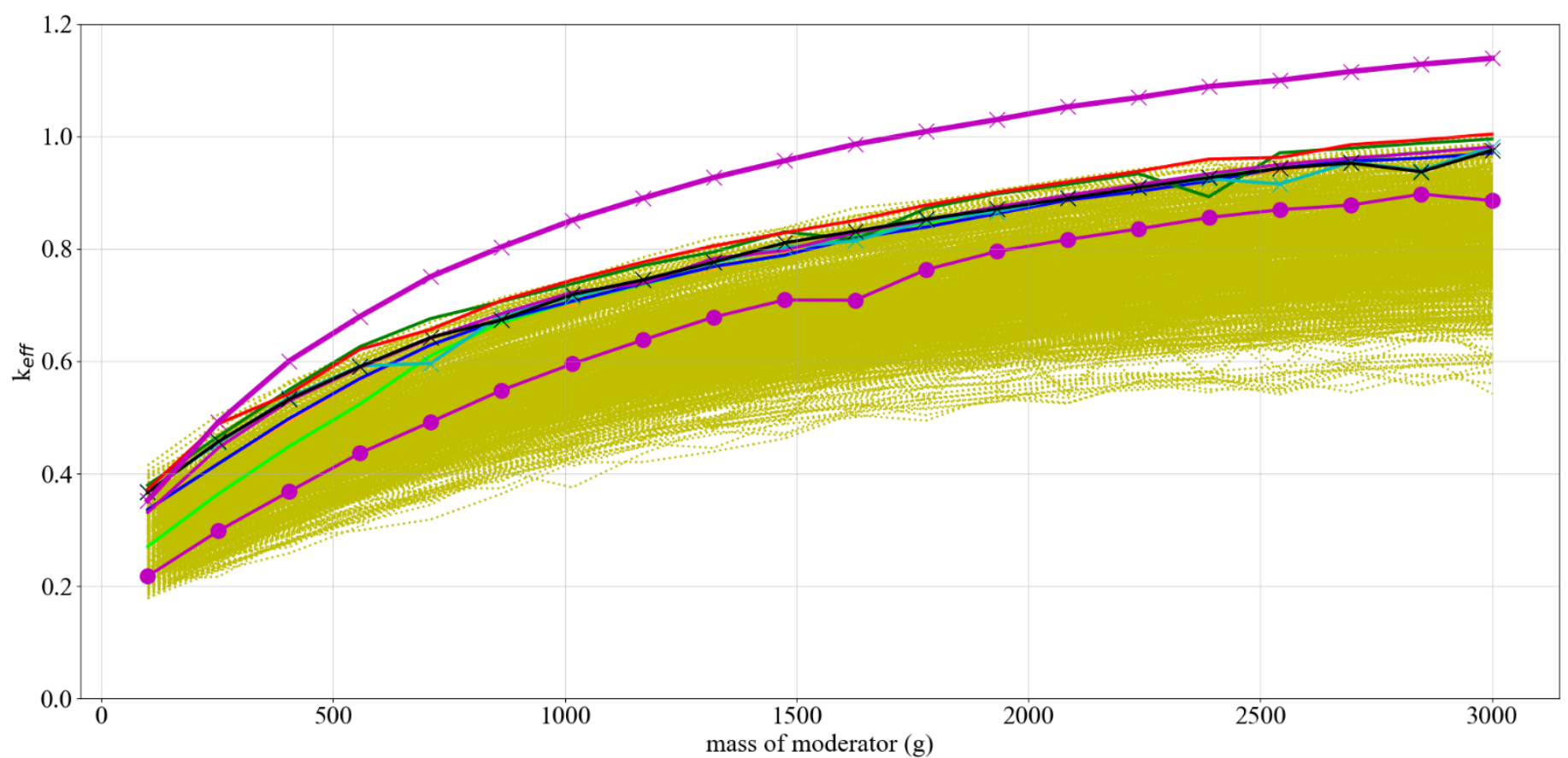

delta all data

waste form $\mathrm{R}=7.7 \mathrm{~cm}$ cylinder, FGE+poly mod only, thin steel discrete reflector waste form $\mathrm{R}=7.7 \mathrm{~cm}$ cylinder, $\mathrm{FGE}+$ poly mod only, thick steel discrete reflector waste form $\mathrm{R}=7.7 \mathrm{~cm}$ cylinder, $\mathrm{FGE}+$ poly mod only, thin poly discrete reflector waste form $\mathrm{R}=7.7 \mathrm{~cm}$ cylinder, $\mathrm{FGE}+$ poly mod only, thick poly discrete reflector waste form sphere, FGE+poly mod only, thin steel discrete reflector
— waste form sphere, FGE+poly mod only, thick steel discrete reflector

$\longrightarrow$ waste form sphere, FGE + poly mod $+4 \mathrm{~kg}$ graphite, thick steel discrete reflector * waste form sphere, FGE+poly mod only, thin poly discrete reflector

— waste form sphere, FGE+ poly mod only, thick poly discrete reflector $\leftarrow$ set-2, waste form sphere, FGE+poly mod only, thick steel discrete reflector

Figure L-8. Reactivity of set-5e compared to a single set-2 representative curve to evaluate the effect of brine (no MgO) as interstitial reflective material but with the additional $50 \mathrm{~cm}$ of space to locate the y-direction reflective boundary conditions removed (similar to set-2). 


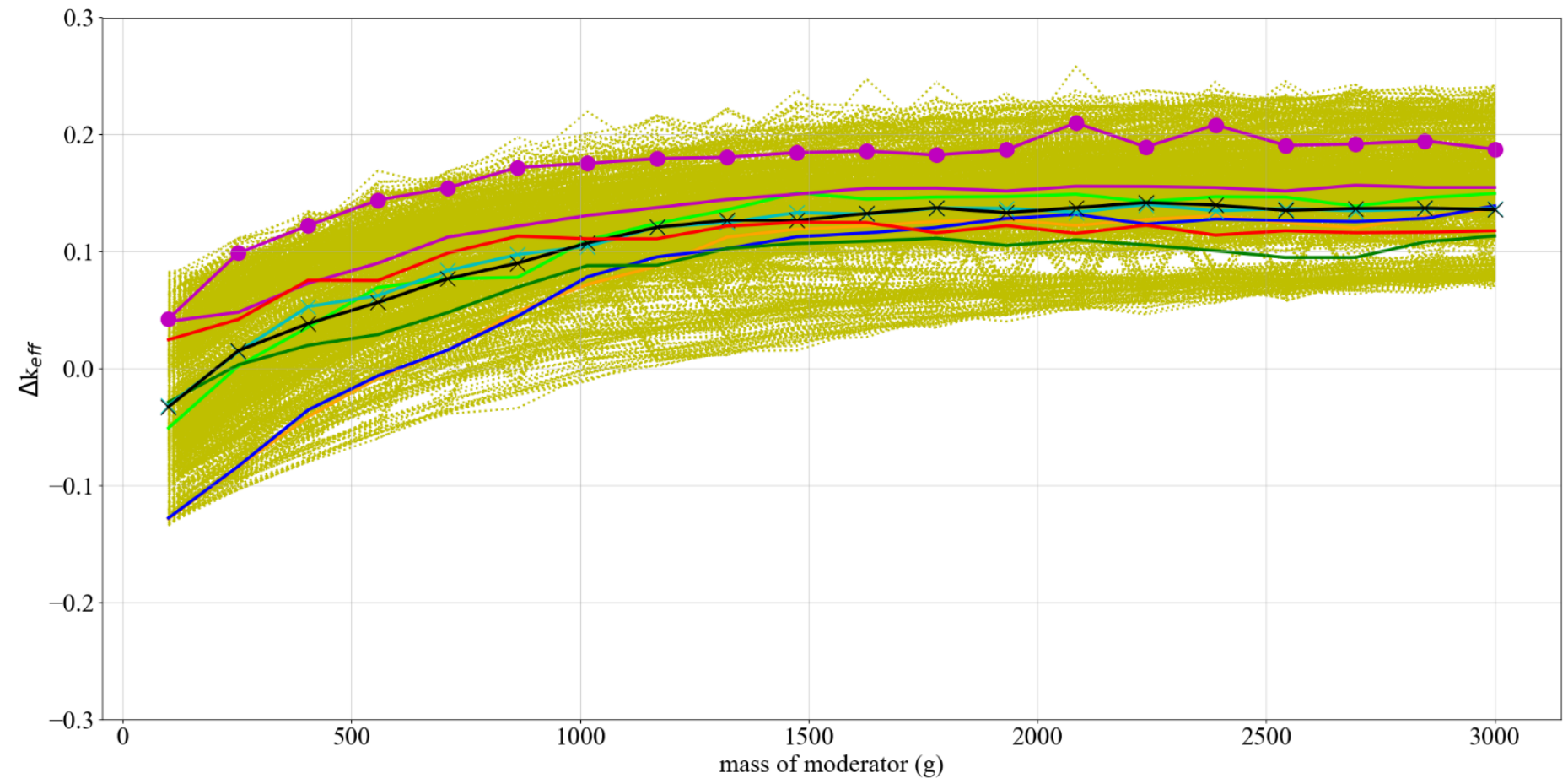

delta all data

waste form $\mathrm{R}=7.7 \mathrm{~cm}$ cylinder, $\mathrm{FGE}+$ poly mod only, thin steel discrete reflector waste form $\mathrm{R}=7.7 \mathrm{~cm}$ cylinder, $\mathrm{FGE}+$ poly mod only, thick steel discrete reflector waste form $\mathrm{R}=7.7 \mathrm{~cm}$ cylinder, $\mathrm{FGE}+$ poly mod only, thin poly discrete reflector waste form $\mathrm{R}=7.7 \mathrm{~cm}$ cylinder, FGE+poly mod only, thick poly discrete reflector

$\leftarrow$ waste form sphere, FGE+poly mod only, thin steel discrete reflector — waste form sphere, FGE+ poly mod only, thick steel discrete reflector - waste form sphere, FGE+poly mod $+4 \mathrm{~kg}$ graphite, thick steel discrete reflector

* waste form sphere, FGE+ poly mod only, thin poly discrete reflector — waste form sphere, FGE+poly mod only, thick poly discrete reflector

Figure L-9. Delta-k between set-2 and set-5f to evaluate the effect of brine (no MgO) as interstitial reflective material but with the additional $50 \mathrm{~cm}$ of space to locate the y-direction reflective boundary conditions removed (similar to set-2) and brine in the external salt box. 


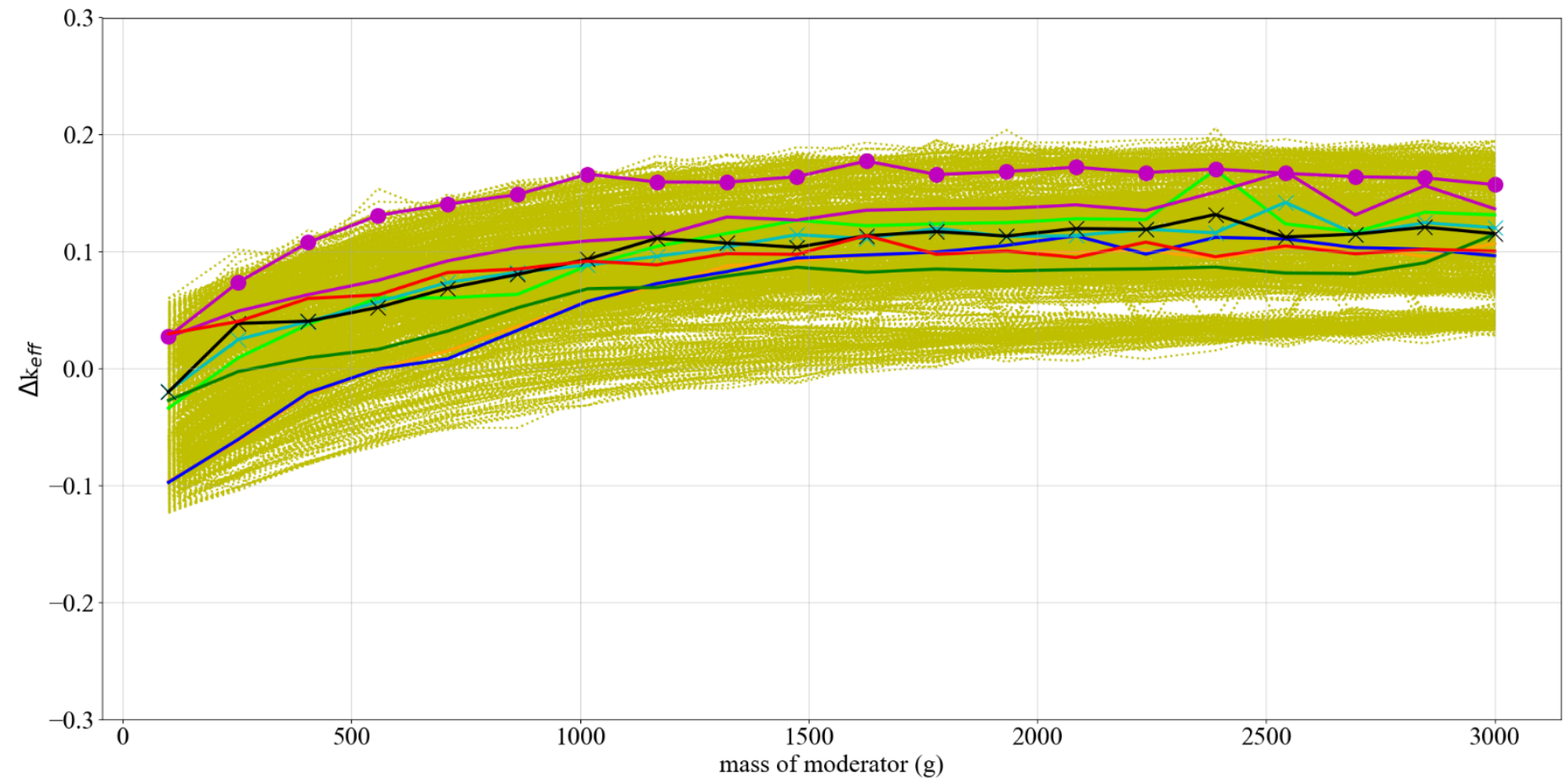

delta all data

waste form $\mathrm{R}=7.7 \mathrm{~cm}$ cylinder, $\mathrm{FGE}+$ poly mod only, thin steel discrete reflector waste form $\mathrm{R}=7.7 \mathrm{~cm}$ cylinder, $\mathrm{FGE}+$ poly mod only, thick steel discrete reflector waste form $\mathrm{R}=7.7 \mathrm{~cm}$ cylinder, FGE+ poly mod only, thin poly discrete reflector waste form $\mathrm{R}=7.7 \mathrm{~cm}$ cylinder, FGE+poly mod only, thick poly discrete reflector

$\leftarrow$ waste form sphere, FGE+poly mod only, thin steel discrete reflector - waste form sphere, FGE+ poly mod only, thick steel discrete reflector - waste form sphere, FGE+poly mod + 4kg graphite, thick steel discrete reflector * waste form sphere, FGE+ poly mod only, thin poly discrete reflector — waste form sphere, FGE+poly mod only, thick poly discrete reflector

Figure L-10. Delta-k between set-2 and set-5g to evaluate the effect of brine in a $50 \%$ mixture with $\mathrm{MgO}$ as interstitial reflective material but with the additional $50 \mathrm{~cm}$ of space to locate the y-direction reflective boundary conditions removed (similar to set-2) and brine in the external salt box. 


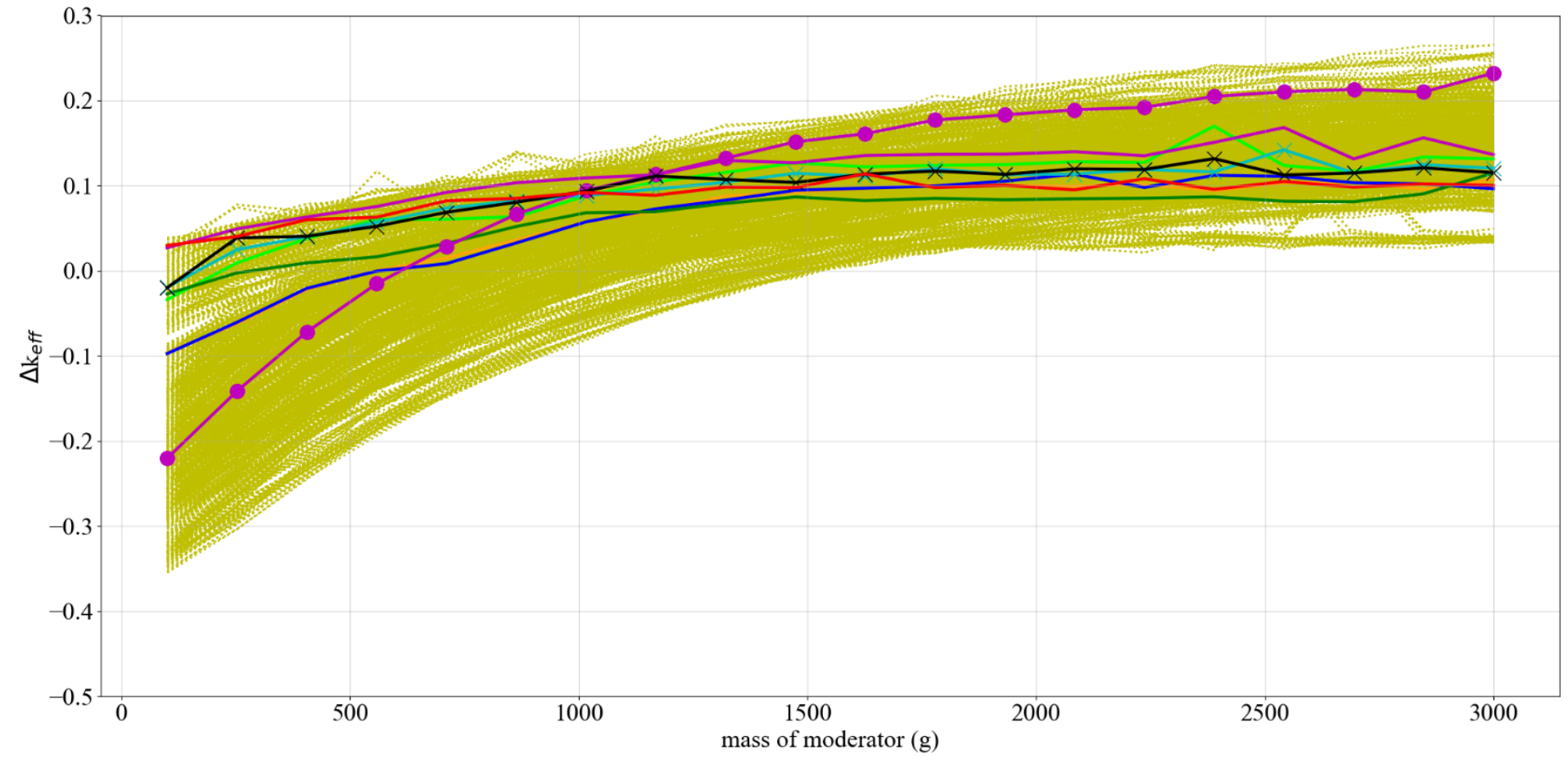

delta all data

_ waste form $\mathrm{R}=7.7 \mathrm{~cm}$ cylinder, $\mathrm{FGE}+$ poly mod only, thin steel discrete reflector

— waste form $\mathrm{R}=7.7 \mathrm{~cm}$ cylinder, $\mathrm{FGE}+$ poly mod only, thick steel discrete reflector

- waste form $\mathrm{R}=7.7 \mathrm{~cm}$ cylinder, $\mathrm{FGE}$ +poly mod only, thin poly discrete reflector

* waste form sphere, FGE+poly mod only, thin steel discrete reflector

— waste form sphere, FGE+ poly mod only, thick steel discrete reflector

$\longrightarrow$ waste form sphere, FGE+poly mod $+4 \mathrm{~kg}$ mix, thick steel discrete reflector

$\star$ waste form sphere, FGE+poly mod only, thin poly discrete reflector

waste form $\mathrm{R}=7.7 \mathrm{~cm}$ cylinder, $\mathrm{FGE}+$ poly mod only, thick poly discrete reflector

- waste form sphere, FGE+poly mod only, thick poly discrete reflecto

Figure L-11. Delta-k between set-2 and set-5h to evaluate the effect of brine in a $50 \%$ mixture with $\mathrm{MgO}$ as interstitial reflective material but with the additional $50 \mathrm{~cm}$ of space to locate the y-direction reflective boundary conditions removed (similar to set-2) and brine in the external salt box. The graphite filler, when present, is replaced by an equivalent mass of brine in a 50\% mixture with $\mathrm{MgO}$. 


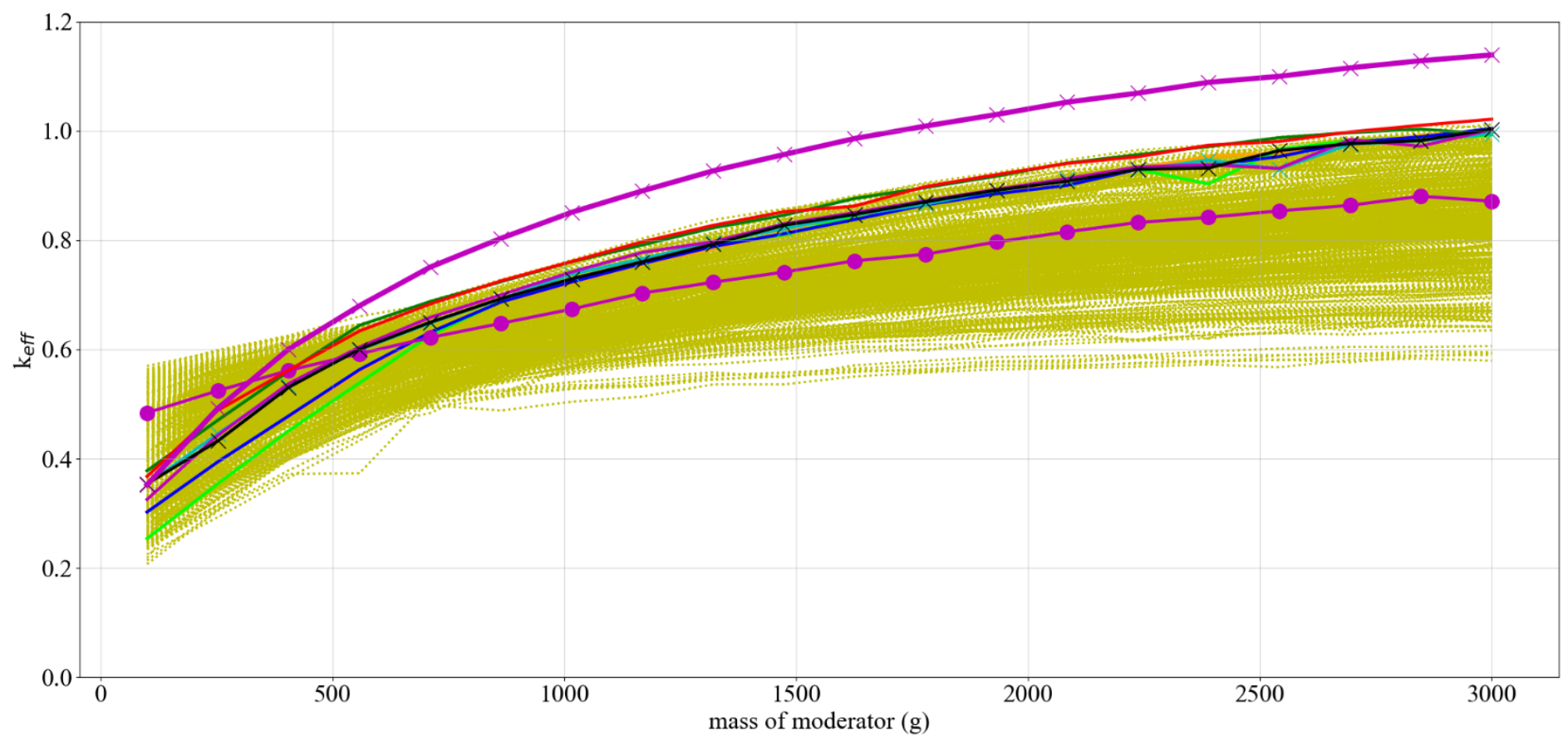

delta all data

waste form $\mathrm{R}=7.7 \mathrm{~cm}$ cylinder, $\mathrm{FGE}+$ poly mod only, thin steel discrete reflector waste form $\mathrm{R}=7.7 \mathrm{~cm}$ cylinder, $\mathrm{FGE}+$ poly mod only, thick steel discrete reflector waste form $\mathrm{R}=7.7 \mathrm{~cm}$ cylinder, $\mathrm{FGE}+$ poly mod only, thin poly discrete reflector waste form $\mathrm{R}=7.7 \mathrm{~cm}$ cylinder, FGE+poly mod only, thick poly discrete reflector waste form sphere, FGE+poly mod only, thin steel discrete reflector

Figure L-12. $\mathrm{K}_{\text {eff }}$ of set-5h compared to a single set-2 representative curve to evaluate the effect of brine in a $50 \%$ mixture with $\mathrm{MgO}$ as interstitial reflective material but with the additional $50 \mathrm{~cm}$ of space to locate the y-direction reflective boundary conditions removed (similar to set-2) and brine in the external salt box. The graphite filler, when present, is replaced by an equivalent mass of brine in a $50 \%$ mixture with $\mathrm{MgO}$. 
APPENDIX M. SET-6: RESULTS OF THE TRIANGULAR PITCHED CCO NONUNIFORM ARRAY CENTROID LOCATION STUDIES AND THE POP HEXAGONAL AND TRIANGULAR PITCHED NONUNIFORM ARRAY CENTROID LOCATION STUDIES. 
This page intentionally blank. 


\section{APPENDIX M. SET-6: RESULTS OF THE TRIANGULAR PITCHED CCO NONUNIFORM ARRAY CENTROID LOCATION STUDIES AND THE POP HEXAGONAL AND TRIANGULAR PITCHED NONUNIFORM ARRAY CENTROID LOCATION STUDIES.}

This appendix serves as a repository of the results for the triangular pitched Criticality Control Overpack (CCO) nonuniform array centroid location studies and the Standard Pipe Overpack (POP) hexagonal and triangular pitched nonuniform array centroid location studies. POP containers were evaluated in Brickner [4]; however, in this appendix, they are treated in the same manner as the CCO (same parametric sweeps) with the exception of the FGE limit, which is $200 \mathrm{~g}$.

The analysis models used in this appendix for set- 6 are based on the set- 2 models discussed in detail in Appendix $\mathrm{C}$ and Appendix D. The model changes to the calculations provided in this appendix compared to Appendix $\mathrm{C}$ and Appendix $\mathrm{D}$ are related to the centroid locations, which changed based on new data provided by Sandia National Laboratory (SNL) [46] including six new centroid location datasets. The SNL data also provide centroid locations for simulations that begin as a triangular pitch rather than a hexagonal pitch (like Appendix C and Appendix D) for both the CCO and POP containers, as well as new hexagonal pitch information for the POP containers. The SNL data are for both lower horizon and upper horizon simulations. For all datasets, the models are constructed in the same fashion as the set-2 models, including the additional centroids for the triangular pitch data, and cases are also added with mirror boundary conditions as well as the periodic boundary conditions, as considered in Appendix $\mathrm{C}$ and Appendix D (the mirror boundary conditions are also evaluated in Appendix E).

This appendix (1) expands the studies from the other appendices with new datasets to provide additional technical support of the conclusions in the main report, (2) shows the reactivity trend for additional containers like POP that also have a FGE limit of $200 \mathrm{~g}$, and (3) shows the reactivity trend associated with the boundary condition in a comprehensive manner.

The cases in this appendix are summarized in Table N-1. Note that although six new centroid datasets are evaluated in this appendix, the parametric sweeps are consistent and therefore the subcases are the same for each dataset.

The data provided in Reedlunn and Bean [46] used in this appendix for the 12 new datasets are presented in Table $\mathrm{M}-1$ for the $\mathrm{CCO}$ in the lower horizon and upper horizon in a triangular pitched array (two datasets); Table M-2 for the 6 in. POP in the upper horizon in a triangular pitched array and the lower horizon in a hexagonal pitched array (two datasets); and Table M-3 for the $12 \mathrm{in.} \mathrm{POP} \mathrm{in} \mathrm{the} \mathrm{upper}$ horizon in a triangular pitched array and the lower horizon in a hexagonal pitched array (two datasets).

For all the datasets, both 0 year and 1,000 year data are provided and used. A summary of the calculations is presented in Table M-5. Figure M-1 through Figure M-6 compare container arrangement (hexagonal vs. triangular pitched) and boundary conditions (periodic vs. mirror) for $\mathrm{CCO}$ containers. Figure M-7 through M-12 present the $k_{\text {eff }}$ results for 6 in. POP containers, whereas Figure M-13 through Figure M-15 compare container arrangement (hexagonal vs. triangular pitched) and boundary conditions (periodic vs. mirror) for 6 in. POP containers. Figure M-16 through Figure M-19 present the $k_{\text {eff }}$ results for 12 in. POP containers, whereas Figure M-20 through Figure M-22 compare container arrangement (hexagonal vs. triangular pitched) and boundary conditions (periodic vs. mirror) for 6 in. POP containers. Figure M-23 displays the maximum occurring $\mathrm{k}_{\mathrm{eff}}$ given the moderator for each of the evaluated container configurations when in storage for 1,000 years. Figure M-24 through Figure M-39 display the maximum occurring $k_{\text {eff }}$ of each subset given the moderator for each of the evaluated container configurations with the maximum occurring $\mathrm{k}_{\mathrm{eff}}$ for all subsets of set-2-uh for comparison. 
Additional calculations were run with the datasets to create flux map plots that can be used to illustrate the most reactive regions of the model. As seen in Appendix E - in which flux maps were provided for the cylindrical waste form models from set-2-uh calculations (Appendix C) - the flux in the model is a function of multiple competing parameters (see discussion in Section 6.4 of the main report). Moreover, when the room is essentially divided in half by the very low reactivity region in the center of the model, the maximum reactivity on either side can be very close to the maximum reactivity on the other side-but as the criticality calculation proceeds in KENO, the code eventually focuses on the region with the highest value. The flux maps created to illustrate the maximum reactivity regions in these models are presented in Figure M-40 to M-47.

\section{LIST OF FIGURES}

Figure M-1. Comparison of CCO upper horizon datasets, set-2-uh hexagonal pitch results (mirror boundary conditions) with set-6-uh-tp triangular pitch results (mirror boundary conditions) as delta- $k_{\text {eff }}$ vs. moderator mass, time $=0$ years.

Figure M-2. Comparison of CCO upper horizon datasets, set-2-uh hexagonal pitch results (mirror boundary conditions) with set-6-uh-tp triangular pitch results (mirror boundary conditions) as delta- $k_{e f f}$ vs. moderator mass, time $=1,000$ years.

Figure M-3. Comparison of CCO lower horizon datasets, set-2-lh hexagonal pitch results (mirror boundary conditions) with set-6-lh-tp triangular pitch results (mirror boundary conditions) as delta- $k_{\text {eff }}$ Vs. moderator mass, time $=1,000$ years.

Figure M-4. Comparison of CCO upper horizon datasets, set6-uh-tp triangular pitch results (mirror boundary conditions) results with set6a-uh-tp triangular pitch results (periodic boundary conditions) as delta- $k_{\text {eff }}$ vs. moderator mass, time $=0$ years.

Figure M-5. Comparison of CCO upper horizon datasets, set6-uh-tp triangular pitch results (mirror boundary conditions) results with set6a-uh-tp triangular pitch results (periodic boundary conditions) as delta- $k_{\text {eff }}$ vs. moderator mass, time $=1,000$ years.

Figure M-6. Comparison of CCO lower horizon datasets, set6-lh-tp triangular pitch results (mirror boundary conditions) results with set6a-lh-tp triangular pitch results (periodic boundary conditions) as delta- $k_{\text {eff }}$ vs. moderator mass, 1,000 years.

Figure M-7. Summary of CCO upper horizon datasets, set6a-uh-tp triangular pitch results (periodic boundary conditions) as $k_{\text {eff }}$ vs. moderator mass, time $=0$ years.

Figure M-8. Summary of CCO upper horizon datasets, set6a-uh-tp triangular pitch results (periodic boundary conditions) as $k_{\text {eff }}$ vs. moderator mass, time $=1,000$ years.

Figure M-9. Summary of 6 in. POP upper horizon datasets, set6-uhpop6-tp triangular pitch results (mirror boundary conditions) as $k_{\text {eff }}$ vs. moderator mass, time $=0$ years

Figure M-10. Summary of 6 in. POP upper horizon datasets, set6-uhpop6-tp triangular pitch results (mirror boundary conditions) as $k_{\text {eff }}$ vs. moderator mass, time $=1,000$ years.

Figure M-11. Summary of 6 in. POP lower horizon datasets, set6-lhpop6-hp hexagonal pitch results (mirror boundary conditions) as $k_{\text {eff }}$ vs. moderator mass, time $=0$ years.

Figure M-12. Summary of 6 in. POP lower horizon datasets, set6-lhpop6-hp hexagonal pitch results (mirror boundary conditions) as $k_{\text {eff }}$ Vs. moderator mass, time $=1,000$ years.

Figure M-13. Comparison of 6 in. POP upper horizon datasets set6-uhpop6-tp triangular pitch results (mirror boundary conditions) results with set6a-uhpop6-tp triangular pitch results (periodic boundary conditions) as delta- $k_{\text {eff }}$ vs. moderator mass, time $=1,000$ years.

Figure M-14. Comparison of 6 in. POP lower horizon datasets set6-lhpop6-hp hexagonal pitch results (mirror boundary conditions) results with set6a-lhpop6-hp hexagonal pitch results (periodic boundary conditions) as delta- $k_{\text {eff }}$ vs. moderator mass, time $=1,000$ years. 
Figure M-15. Comparison of 6 in. POP lower horizon datasets set6-lhpop6-hp hexagonal pitch results (mirror boundary conditions) results with upper horizon set6-uhpop6-tp triangular pitch results (mirror boundary conditions) as delta- $k_{\text {eff }}$ Vs. moderator mass, time $=1,000$ years.

Figure M-16. Summary of 12 in. POP upper horizon datasets, set6-uhpop12-tp triangular pitch results (mirror boundary conditions) as $k_{\text {eff }}$ vs. moderator mass, time $=0$ years.

Figure M-17. Summary of 12 in. POP upper horizon datasets, set6-uhpop12-tp triangular pitch results (mirror boundary conditions) as $k_{\text {eff }} \mathrm{vs.}$ moderator mass, time $=1,000$ years.

Figure M-18. Summary of 12 in. POP lower horizon datasets, set6-lhpop12-hp hexagonal pitch results (mirror boundary conditions) as $k_{\text {eff }} v$ vs. moderator mass, time $=0$ years.

Figure M-19. Summary of 12 in. POP lower horizon datasets, set6-lhpop12-hp hexagonal pitch results (mirror boundary conditions) as $k_{\text {eff }}$ vs. moderator mass, time $=1,000$ years.

Figure M-20. Comparison of 12 in. POP upper horizon datasets set6-uhpop12-tp triangular pitch results (mirror boundary conditions) results with set6a-uhpop12-tp triangular pitch results (periodic boundary conditions) as delta- $k_{\text {eff }} \mathrm{vs}$. moderator mass, time $=1,000$ years.

Figure M-21. Comparison of 12 in. POP lower horizon datasets set6-lhpop12-hp hexagonal pitch results (mirror boundary conditions) results with set6a-lhpop12-hp hexagonal pitch results (periodic boundary conditions) as delta- $k_{\text {eff }}$ vs. moderator mass, time $=1,000$ years.

Figure M-22. Comparison of 12 in. POP lower horizon datasets set6-lhpop12-hp hexagonal pitch results (mirror boundary conditions) results with upper horizon set6-uhpop12-tp triangular pitch results (mirror boundary conditions) as delta- $k_{\text {eff }}$ vs. moderator mass, time $=1,000$ years.

Figure M-23. Max $k_{\text {eff }}$ of all subsets of all CCO and POP centroid configurations at time $=1,000$ years compared with max $k_{\text {eff }}$ of all subsets of set-2-uh hexagonal pitch (mirror boundary conditions) as $k_{\text {eff }}$ vs. moderator mass, time $=1,000$ years.

Figure M-24. Max $k_{\text {eff }}$ of all subcase- 1 of all $\mathrm{CCO}$ and POP centroid configurations at time = 1,000 years compared with max $k_{\text {eff }}$ of all subsets of set-2-uh hexagonal pitch (mirror boundary conditions) as $k_{\text {eff }} \mathrm{vs}$. moderator mass, time $=1,000$ years.

Figure M-25. Max $k_{\text {eff }}$ of all subcase- 2 of all $\mathrm{CCO}$ and POP centroid configurations at time = 1,000 years compared with max $k_{\text {eff }}$ of all subsets of set-2-uh hexagonal pitch (mirror boundary conditions) as $k_{\text {eff }} \mathrm{vs}$. moderator mass, time $=1,000$ years.

Figure M-26. Max $k_{\text {eff }}$ of all subcase-3 of all CCO and POP centroid configurations at time = 1,000 years compared with max $k_{\text {eff }}$ of all subsets of set-2-uh hexagonal pitch (mirror boundary conditions) as $k_{\text {eff }} \mathrm{Vs}$. moderator mass, time $=1,000$ years.

Figure M-27. Max $k_{\text {eff }}$ of all subcase- 4 of all $\mathrm{CCO}$ and POP centroid configurations at time $=$ 1,000 years compared with max $k_{\text {eff }}$ of all subsets of set-2-uh hexagonal pitch (mirror boundary conditions) as $k_{\text {eff }} \mathrm{Vs}$. moderator mass, time $=1,000$ years.

Figure M-28. Max $k_{\text {eff }}$ of all subcase- 5 of all CCO and POP centroid configurations at time $=$ 1,000 years compared with max $k_{\text {eff }}$ of all subsets of set-2-uh hexagonal pitch (mirror boundary conditions) as $k_{\text {eff }} \mathrm{vs}$. moderator mass, time $=1,000$ years.

Figure M-29. Max $k_{\text {eff }}$ of all subcase- 6 of all CCO and POP centroid configurations at time = 1,000 years compared with max $k_{\text {eff }}$ of all subsets of set-2-uh hexagonal pitch (mirror boundary conditions) as $k_{\text {eff }} \mathrm{vs}$. moderator mass, time $=1,000$ years.

Figure M-30. Max $k_{\text {eff }}$ of all subcase-7 of all CCO and POP centroid configurations at time = 1,000 years compared with max $k_{\text {eff }}$ of all subsets of set-2-uh hexagonal pitch (mirror boundary conditions) as $k_{\text {eff }} \mathrm{vs}$. moderator mass, time $=1,000$ years.

Figure M-31. Max $k_{\text {eff }}$ of all subcase- 8 of all CCO and POP centroid configurations at time = 1,000 years compared with max $k_{\text {eff }}$ of all subsets of set-2-uh hexagonal pitch (mirror boundary conditions) as $k_{\text {eff }} \mathrm{vs}$. moderator mass, time $=1,000$ years. 
Figure M-32. Max $k_{\text {eff }}$ of all subcase- 9 of all $\mathrm{CCO}$ and POP centroid configurations at time = 1,000 years compared with max $k_{\text {eff }}$ of all subsets of set-2-uh hexagonal pitch (mirror boundary conditions) as $k_{\text {eff }} \mathrm{vs}$. moderator mass, time $=1,000$ years.

Figure M-33. Max $k_{\text {eff }}$ of all subcase-10 of all CCO and POP centroid configurations at time $=$ 1,000 years compared with max $k_{\text {eff }}$ of all subsets of set-2-uh hexagonal pitch (mirror boundary conditions) as $k_{\text {eff }} \mathrm{vs}$. moderator mass, time $=1,000$ years.

Figure M-34. Max $k_{\text {eff }}$ of all subcase-11 of all CCO and POP centroid configurations at time = 1,000 years compared with max $k_{\text {eff }}$ of all subsets of set-2-uh hexagonal pitch (mirror boundary conditions) as $k_{\text {eff }} \mathrm{vs}$. moderator mass, time $=1,000$ years.

Figure M-35. Max $k_{\text {eff }}$ of all subcase-12 of all CCO and POP centroid configurations at time $=$ 1,000 years compared with max $k_{\text {eff }}$ of all subsets of set-2-uh hexagonal pitch (mirror boundary conditions) as $k_{\text {eff }} \mathrm{vs}$. moderator mass, time $=1,000$ years.

Figure M-36. Max $k_{\text {eff }}$ of all subcase-13 of all CCO and POP centroid configurations at time $=$ 1,000 years compared with max $k_{\text {eff }}$ of all subsets of set-2-uh hexagonal pitch (mirror boundary conditions) as $k_{\text {eff }} \mathrm{vs}$. moderator mass, time $=1,000$ years.

Figure M-37. Max $k_{\text {eff }}$ of all subcase-14 of all CCO and POP centroid configurations at time = 1,000 years compared with max $k_{\text {eff }}$ of all subsets of set-2-uh hexagonal pitch (mirror boundary conditions) as $k_{\text {eff }} \mathrm{vs}$. moderator mass, time $=1,000$ years.

Figure M-38. Max $k_{\text {eff }}$ of all subcase-15 of all CCO and POP centroid configurations at time = 1,000 years compared with max $k_{\text {eff }}$ of all subsets of set-2-uh hexagonal pitch (mirror boundary conditions) as $k_{\text {eff }} \mathrm{vs}$. moderator mass, time $=1,000$ years.

Figure M-39. Max $k_{\text {eff }}$ of all subcase-16 of all CCO and POP centroid configurations at time = 1,000 years compared with max $k_{\text {eff }}$ of all subsets of set-2-uh hexagonal pitch (mirror boundary conditions) as $k_{\text {eff }} \mathrm{vs}$. moderator mass, time $=1,000$ years.

Figure M-40. Diagram of the neutron flux for subcase-10 in the CCO with no filler material and thin SS discrete reflector (sweep 470), upper horizon with hexagonal pitch (set-2-uh) with a maximum $k_{\text {eff }}$ of 1.0134 and moderator mass of 2,084 g, comparable to Figure 29 in [46]

Figure M-41. Diagram of the neutron flux for subcase-10 in the CCO with $4 \mathrm{~kg}$ graphite filler material and thin SS discrete reflector (sweep 494), upper horizon with hexagonal pitch (set-2-uh) with a maximum $k_{\text {eff }}$ of 0.9649 and moderator mass of 2,084 $\mathrm{g}$, comparable to Figure 29 in [46].

Figure M-42. Diagram of the neutron flux for subcase-10 in the CCO with no filler material and thick SS discrete reflector (sweep 472), upper horizon with hexagonal pitch (set-2-uh) with a maximum $k_{\text {eff }}$ of 1.047 and moderator mass of 2,084 g, comparable to Figure 29 in [46].

Figure M-43. Diagram of the neutron flux for subcase-10 in the CCO with $4 \mathrm{~kg}$ graphite filler material and thick SS discrete reflector (sweep 496), upper horizon with hexagonal pitch (set-2-uh) with a maximum $k_{\text {eff }}$ of 1.047 and moderator mass of 2,084 g, comparable to Figure 29 in [46].

Figure M-44. Diagram of the neutron flux for subcase-10 in the CCO with no filler material and thin SS discrete reflector (sweep 470), lower horizon with hexagonal pitch (set-2-lh) with a maximum $k_{\text {eff }}$ of 1.0428 and moderator mass of 2,084 g, comparable to Figure 27 in [46]......

Figure M-45. Diagram of the neutron flux for subcase-10 in the 12 in. POP with no filler material and thick SS discrete reflector (sweep 688), lower horizon with hexagonal pitch with a maximum $k_{\text {eff }}$ of 0.9816 and moderator mass of $3,000 \mathrm{~g}$, comparable to Figure 37 in [46]..... M-75

Figure M-46. Diagram of the neutron flux for subcase-10 in the CCO with no filler material and thin SS discrete reflector (sweep 470), lower horizon with triagonal pitch with a maximum $k_{\text {eff }}$ of 1.0227 and moderator mass of 2,084 g, comparable to Figure 33 in [46]...... M-76 
Figure M-47. Diagram of the neutron flux for subcase-10 in the 6 in. POP with no filler material and thin SS discrete reflector (sweep 470), lower horizon with hexagonal pitch with a maximum $k_{\text {eff }}$ of 1.0227 and moderator mass of 2,084 g, comparable to Figure 35 in [46]

\section{LIST OF TABLES}

Table M-1. Summary of cases for all set- 6 models. M-8

Table M-2. Centroid locations from [46] for the CCO containers in a triangular pitch. ............................. M-9

Table M-3. Centroid locations from [46] for the 6 in. POP containers. ..............................................

Table M-4. Centroid locations from [46] for the 12 in. POP containers..............................................23

Table M-5. Summary of the calculations in this appendix. M-30 
Table M-1. Summary of cases for all set-6 models.

\begin{tabular}{|c|c|c|c|c|c|c|c|c|}
\hline Case & $\begin{array}{l}\text { Model } \\
\text { type }\end{array}$ & Waste form shape & $\begin{array}{l}\text { Waste form } \\
\text { moderator }\end{array}$ & $\begin{array}{c}\text { Filler material } \\
(0,2,000 \\
\mathbf{4 , 0 0 0} \mathbf{g})\end{array}$ & $\begin{array}{c}\text { Metal } \\
\text { in filler }\end{array}$ & $\begin{array}{c}\text { Discrete reflector } \\
\text { (thin } 0.001 \mathrm{~cm} \text { and } \\
\text { thick } 0.7112 \mathrm{~cm} \text { ) }\end{array}$ & Be (g) & Subcase \\
\hline \multirow{16}{*}{ Set-6 } & \multirow{16}{*}{$\begin{array}{l}\text { Models } \\
\text { similar to } \\
\text { set-2 }\end{array}$} & \multirow{8}{*}{$\begin{array}{c}\text { Cylinder (radius range } 4.8,6 \text {, } \\
7.7 \text { and height defined by total } \\
\text { volume of mass) }\end{array}$} & water & $\mathrm{c} 12$ & \multirow{16}{*}{$\begin{array}{c}\text { SS from } \\
\text { can } \\
(0,500 \\
1,000 \mathrm{~g})\end{array}$} & steel & \multirow{16}{*}{$\begin{array}{l}0 \text { to } \\
585\end{array}$} & set-6-1 \\
\hline & & & poly & $\mathrm{c} 12$ & & steel & & set-6-2 \\
\hline & & & water & $\mathrm{c} 12$ & & poly & & set-6-3 \\
\hline & & & poly & $\mathrm{c} 12$ & & poly & & set-6 -4 \\
\hline & & & water & generic & & steel & & set-6-5 \\
\hline & & & poly & generic & & steel & & set-6-6 \\
\hline & & & water & generic & & poly & & set-6-7 \\
\hline & & & poly & generic & & poly & & set-6-8 \\
\hline & & \multirow{8}{*}{$\begin{array}{l}\text { Sphere (radius defined by total } \\
\text { volume of mass) }\end{array}$} & water & $\mathrm{c} 12$ & & steel & & set-6-9 \\
\hline & & & poly & $\mathrm{c} 12$ & & steel & & set-6-10 \\
\hline & & & water & $\mathrm{c} 12$ & & poly & & set-6-11 \\
\hline & & & poly & $\mathrm{c} 12$ & & poly & & \begin{tabular}{|l|} 
set-6-12 \\
\end{tabular} \\
\hline & & & water & generic & & steel & & set-6-13 \\
\hline & & & poly & generic & & steel & & set-6-14 \\
\hline & & & water & generic & & poly & & set-6-15 \\
\hline & & & poly & generic & & poly & & set-6-16 \\
\hline
\end{tabular}


Table M-2. Centroid locations from [46] for the CCO containers in a triangular pitch.

\begin{tabular}{|c|c|c|c|c|c|c|c|c|c|c|c|c|}
\hline \multicolumn{13}{|c|}{ CCO containers } \\
\hline \multirow{3}{*}{ Centroid } & \multicolumn{6}{|c|}{ Lower horizon triangular with triangular pitch array } & \multicolumn{6}{|c|}{ Upper horizon triangular with triangular pitch array } \\
\hline & \multicolumn{3}{|c|}{$\mathrm{t}=\mathbf{0}$ year } & \multicolumn{3}{|c|}{$t=1,000$ years } & \multicolumn{3}{|c|}{$t=0$ year } & \multicolumn{3}{|c|}{$t=1,000$ years } \\
\hline & $\mathbf{x}(\mathbf{m})$ & $\mathbf{y}(\mathbf{m})$ & $\mathrm{z}(\mathrm{m})$ & $\mathbf{x}(\mathbf{m})$ & $\mathbf{y}(\mathbf{m})$ & $\mathbf{z}(\mathbf{m})$ & $\mathbf{x}(\mathbf{m})$ & $\mathbf{y}(\mathbf{m})$ & $\mathbf{z}(\mathbf{m})$ & $\mathbf{x}(\mathbf{m})$ & $\mathbf{y}(\mathbf{m})$ & $\mathbf{z}(\mathbf{m})$ \\
\hline 0 & -3.910681 & -0.752791 & -5.965605 & -2.90163 & -0.91488 & -5.36427 & -3.91082 & -0.75279 & -3.53563 & -3.03254 & -0.37986 & -3.05446 \\
\hline 1 & -3.911674 & -0.752792 & -5.139917 & -2.83411 & -0.75901 & -5.5154 & -3.9117 & -0.75279 & -2.70994 & -3.03983 & -0.87336 & -2.87072 \\
\hline 2 & -3.912668 & -0.752795 & -4.314184 & -2.91043 & -0.78327 & -4.91443 & -3.91257 & -0.7528 & -1.88421 & -3.18882 & -0.83341 & -2.2964 \\
\hline 3 & -3.331431 & -0.752792 & -5.965029 & -2.09153 & -0.84417 & -5.66998 & -3.33159 & -0.75279 & -3.53513 & -2.4117 & -0.71271 & -3.07672 \\
\hline 4 & -3.332166 & -0.752792 & -5.139339 & -2.70562 & -0.68503 & -5.53604 & -3.33218 & -0.75279 & -2.70944 & -2.98234 & -0.9332 & -2.92463 \\
\hline 5 & -3.332899 & -0.752794 & -4.313606 & -2.81508 & -0.63186 & \begin{tabular}{|l|}
-5.11287 \\
\end{tabular} & -3.33277 & \begin{tabular}{|l|}
-0.75279 \\
\end{tabular} & -1.88371 & -3.0758 & -0.69539 & -2.46973 \\
\hline 6 & -2.752036 & -0.75279 & -5.964558 & -1.71718 & -0.58932 & -5.6277 & -2.75221 & -0.75279 & -3.53476 & -1.92937 & -0.66768 & -3.09643 \\
\hline 7 & -2.75265 & -0.752792 & -5.13887 & -2.34186 & -0.87558 & -5.43192 & -2.75266 & -0.75279 & -2.70907 & -2.53207 & -0.94059 & -3.02164 \\
\hline 8 & -2.753264 & -0.752795 & -4.313135 & -2.59381 & -0.45859 & \begin{tabular}{|l|}
-5.22233 \\
\end{tabular} & -2.75312 & -0.75279 & -1.88334 & -2.86678 & -0.6792 & -2.59926 \\
\hline 9 & -2.172671 & -0.752791 & -5.964178 & -1.34539 & -0.6985 & -5.62668 & -2.17281 & -0.75279 & -3.53448 & -1.50153 & -0.93716 & -3.09943 \\
\hline 10 & -2.17315 & -0.752792 & -5.138488 & -1.96158 & -0.92523 & -5.50984 & -2.17315 & -0.75279 & -2.7088 & -2.15002 & -0.86723 & -2.85505 \\
\hline 11 & -2.173628 & -0.752794 & -4.312755 & -2.55639 & -0.71459 & -5.3019 & -2.1735 & -0.7528 & -1.88306 & -2.71353 & -0.69317 & -2.689 \\
\hline 12 & -1.59327 & -0.75279 & -5.963884 & -0.91533 & -0.68409 & \begin{tabular}{|l|}
-5.69717 \\
\end{tabular} & -1.59341 & -0.75279 & -3.53428 & -1.24346 & -0.88506 & -3.02233 \\
\hline 13 & -1.593633 & -0.752792 & -5.138194 & -1.53364 & -0.92069 & -5.49581 & -1.59364 & -0.75279 & -2.7086 & -1.91845 & -0.69897 & -2.94898 \\
\hline 14 & -1.593999 & -0.752796 & -4.312461 & -2.2069 & -0.86025 & -5.20043 & -1.59388 & -0.75279 & -1.88286 & -2.57222 & -0.77731 & -2.86477 \\
\hline 15 & -1.013915 & -0.752792 & -5.963681 & -0.37342 & -0.59707 & \begin{tabular}{|l|}
-5.74613 \\
\end{tabular} & -1.01399 & \begin{tabular}{|l|}
-0.75279 \\
\end{tabular} & -3.53415 & -0.88569 & -0.77434 & -3.12777 \\
\hline 16 & -1.014137 & -0.752792 & -5.13799 & -1.06297 & -0.94637 & -5.58051 & -1.01414 & -0.75279 & -2.70846 & -1.58704 & -0.79807 & -2.84665 \\
\hline 17 & -1.014358 & -0.752794 & -4.312257 & -1.75793 & -0.74029 & -5.37522 & -1.01428 & -0.7528 & -1.88273 & -2.23934 & -0.94098 & -2.73115 \\
\hline 18 & -0.434519 & -0.75279 & -5.963566 & 0.013811 & -0.57371 & -5.53996 & -0.43457 & -0.75279 & -3.53408 & -0.47188 & -0.87367 & -3.16148 \\
\hline 19 & -0.434623 & -0.752792 & -5.137877 & -0.59212 & -0.81901 & -5.49691 & -0.43463 & -0.75279 & -2.70839 & -0.58075 & -0.67582 & -3.00091 \\
\hline 20 & -0.43473 & -0.752795 & -4.312143 & -1.29909 & -0.64262 & -5.4346 & -0.43469 & -0.7528 & -1.88265 & 0.346985 & -0.70783 & -3.00543 \\
\hline 21 & 0.144834 & -0.752791 & -5.963544 & 0.411219 & -0.53124 & -5.52756 & 0.14485 & -0.75279 & -3.53406 & -0.34495 & -0.888 & -3.05578 \\
\hline 22 & 0.144872 & -0.752792 & -5.137854 & -0.07078 & -0.92379 & -5.64527 & 0.144873 & -0.75279 & -2.70837 & 0.476231 & -0.53544 & -3.02281 \\
\hline 23 & 0.144911 & -0.752794 & -4.31212 & -0.55154 & -0.48765 & -5.5025 & 0.144898 & -0.75279 & -1.88264 & 1.229786 & -0.60485 & -2.89042 \\
\hline 24 & 0.724228 & -0.75279 & -5.963611 & 0.506316 & -0.7523 & -5.52825 & 0.724281 & -0.75279 & -3.5341 & 0.445383 & -0.80163 & -3.06482 \\
\hline
\end{tabular}


Table M-2. Centroid locations from [46] for the CCO containers in a triangular pitch (continued).

\begin{tabular}{|c|c|c|c|c|c|c|c|c|c|c|c|c|}
\hline \multicolumn{13}{|c|}{ CCO containers } \\
\hline \multirow{3}{*}{ Centroid } & \multicolumn{6}{|c|}{ Lower horizon triangular with triangular pitch array } & \multicolumn{6}{|c|}{ Upper horizon triangular with triangular pitch array } \\
\hline & \multicolumn{3}{|c|}{$\mathbf{t}=\mathbf{0}$ year } & \multicolumn{3}{|c|}{$t=1,000$ years } & \multicolumn{3}{|c|}{$\mathbf{t}=\mathbf{0}$ year } & \multicolumn{3}{|c|}{$t=1,000$ years } \\
\hline & $x(m)$ & $y(m)$ & $\mathrm{z}(\mathrm{m})$ & $x(m)$ & $y(m)$ & $\mathrm{z}(\mathrm{m})$ & $x(m)$ & $y(m)$ & $\mathrm{z}(\mathrm{m})$ & $x(m)$ & $y(m)$ & $\mathrm{z}(\mathrm{m})$ \\
\hline 25 & 0.724386 & -0.752792 & -5.137922 & 1.266119 & -0.94356 & -5.5341 & 0.724384 & -0.75279 & -2.70842 & 1.095588 & -0.85754 & -2.9138 \\
\hline 26 & 0.724539 & -0.752795 & -4.312189 & 0.702078 & -0.61499 & -5.54147 & 0.724484 & -0.75279 & -1.88268 & 1.771318 & -0.92091 & -2.79517 \\
\hline 27 & 1.303584 & -0.752792 & -5.963771 & 0.983463 & -0.75424 & -5.68954 & 1.303694 & -0.75279 & -3.53421 & 0.906266 & -0.66142 & -3.16066 \\
\hline 28 & 1.303882 & -0.752792 & -5.13808 & 1.658848 & -0.68004 & -5.40967 & 1.303885 & -0.75279 & -2.70852 & 1.570999 & -0.86082 & -2.9473 \\
\hline 29 & 1.30418 & -0.752794 & -4.312347 & 2.150832 & -0.89539 & -5.26032 & .30408 & -0.7528 & -1.88279 & 2.235273 & -0.59693 & -2.79569 \\
\hline 30 & 1.882982 & -0.75279 & -5.964019 & 1.30921 & -0.89122 & -5.65918 & 1.883112 & -0.75279 & -3.53438 & 1.410753 & -0.77626 & -3.14802 \\
\hline 31 & 1.883399 & -0.752791 & -5.13833 & 1.943917 & -0.92092 & -5.51457 & 1.883398 & \begin{tabular}{|l|}
-0.75279 \\
\end{tabular} & -2.70869 & 2.026407 & -0.94765 & -2.98119 \\
\hline 32 & 1.88381 & -0.752796 & -4.312598 & 2.494824 & -0.67201 & -5.37577 & 1.883682 & -0.75279 & -1.88295 & 2.568517 & -0.71637 & -2.65583 \\
\hline 33 & 2.462347 & -0.752792 & -5.964358 & 1.522499 & -0.73278 & -5.63065 & 2.462522 & -0.75279 & -3.53461 & 1.853539 & -0.89701 & -3.09003 \\
\hline 34 & 2.462897 & -0.752792 & -5.138668 & 2.153964 & -0.9297 & -5.46098 & 2.462913 & -0.75279 & -2.70892 & 2.440916 & -0.6837 & -2.81359 \\
\hline 35 & 2.463446 & -0.752794 & -4.312934 & 2.624686 & -0.77956 & -5.18251 & 2.463299 & -0.7528 & -1.88319 & 2.89419 & -0.72366 & -2.55665 \\
\hline 36 & 3.041741 & -0.75279 & -5.964781 & 1.881795 & -0.62726 & -5.67045 & 3.041886 & -0.75279 & -3.53493 & 2.165982 & -0.57386 & -3.07024 \\
\hline 37 & 3.042408 & -0.752791 & -5.139093 & 2.531813 & -0.92494 & -5.62109 & 3.042417 & -0.75279 & -2.70924 & 2.737982 & -0.84306 & -2.8545 \\
\hline 38 & 3.043068 & -0.752795 & & 2.76334 & -0.80368 & \begin{tabular}{|l|}
-5.10918 \\
\end{tabular} & 3.042953 & -0.7528 & -1.88351 & 2.993375 & -0.89953 & -2.4846 \\
\hline 39 & 3.621093 & -0.752792 & -5.965302 & 2.569047 & -0.58884 & -5.58595 & 3.621253 & -0.75279 & -3.53536 & 2.736165 & -0.88471 & -2.91345 \\
\hline 40 & 3.621922 & -0.752791 & -5.139613 & 2.872611 & -0.9546 & -5.3911 & 3.621934 & -0.75279 & -2.70967 & 3.076498 & -0.77613 & -2.87468 \\
\hline 41 & 3.622749 & -0.752794 & -4.31388 & 2.939747 & -0.60464 & -5.00139 & 3.622611 & -0.75279 & -1.88393 & 3.122722 & -0.60842 & -2.40351 \\
\hline 42 & -3.621093 & -0.250931 & -5.965302 & -2.27593 & -0.36609 & -5.60556 & -3.62125 & -0.25093 & -3.53536 & -2.60278 & -0.14046 & -3.02947 \\
\hline 43 & -3.621922 & -0.250931 & -5.139612 & -2.90453 & -0.50545 & -5.50074 & -3.62193 & -0.25093 & -2.70967 & -2.98984 & -0.46938 & -2.88682 \\
\hline 44 & -3.622749 & -0.250931 & -4.313879 & -2.91594 & -0.26364 & -4.9536 & -3.62261 & -0.25093 & -1.88393 & -3.1574 & -0.28211 & -2.40037 \\
\hline 45 & -3.041746 & -0.250931 & -5.964781 & -1.86056 & -0.0217 & -5.64067 & -3.04189 & -0.25093 & -3.53493 & -2.06814 & -0.36996 & -3.08149 \\
\hline 46 & -3.042408 & -0.250931 & -5.139092 & -2.45227 & -0.32864 & -5.5006 & -3.04242 & -0.25093 & -2.70924 & -2.68489 & -0.47516 & -3.03338 \\
\hline 47 & -3.043065 & -0.250931 & -4.313358 & -2.84788 & -0.13519 & -5.1589 & -3.04295 & -0.25093 & -1.88351 & -2.97401 & -0.32629 & -2.54036 \\
\hline 48 & -2.462347 & -0.250931 & -5.964358 & -1.5614 & -0.15471 & -5.61835 & -2.46253 & -0.25093 & -3.53461 & -1.69963 & -0.09423 & -3.10173 \\
\hline 49 & -2.462898 & -0.250931 & -5.138667 & -2.19708 & -0.34489 & -5.49944 & -2.46291 & -0.25093 & -2.70892 & -2.37075 & -0.13276 & -2.99957 \\
\hline 50 & -2.463445 & -0.25093 & -4.312934 & -2.762 & -0.04692 & -5.24929 & -2.4633 & -0.25093 & -1.88319 & -2.78173 & -0.43965 & -2.61629 \\
\hline
\end{tabular}

M-10 
Table M-2. Centroid locations from [46] for the CCO containers in a triangular pitch (continued).

\begin{tabular}{|c|c|c|c|c|c|c|c|c|c|c|c|c|}
\hline \multicolumn{13}{|c|}{ CCO containers } \\
\hline \multirow{3}{*}{ Centroid } & \multicolumn{6}{|c|}{ Lower horizon triangular with triangular pitch array } & \multicolumn{6}{|c|}{ Upper horizon triangular with triangular pitch array } \\
\hline & \multicolumn{3}{|c|}{$\mathbf{t}=\mathbf{0}$ year } & \multicolumn{3}{|c|}{$t=1,000$ years } & \multicolumn{3}{|c|}{$\mathbf{t}=\mathbf{0}$ year } & \multicolumn{3}{|c|}{$t=1,000$ years } \\
\hline & $x(m)$ & $y(m)$ & $\mathrm{z}(\mathrm{m})$ & $x(m)$ & $y(m)$ & $\mathrm{z}(\mathrm{m})$ & $x(m)$ & $y(m)$ & $\mathrm{z}(\mathrm{m})$ & $x(m)$ & $y(m)$ & $\mathrm{z}(\mathrm{m})$ \\
\hline 51 & -1.882987 & -0.250931 & -5.964019 & -1.17337 & -0.38888 & -5.71742 & -1.88311 & -0.25093 & -3.53438 & -1.3274 & -0.15707 & -3.0605 \\
\hline 52 & -1.883399 & -0.250931 & -5.138329 & -1.84055 & -0.45972 & -5.47792 & -1.8834 & -0.25093 & -2.70869 & -2.01178 & -0.21326 & -2.84592 \\
\hline 53 & -1.883807 & -0.250931 & -4.312596 & -2.42057 & -0.23672 & -5.23784 & -1.88368 & -0.25093 & -1.88295 & -2.66954 & -0.20023 & -2.74259 \\
\hline 54 & -1.303584 & -0.250931 & -5.963771 & -0.71945 & -0.24618 & -5.72996 & -1.30369 & -0.25093 & -3.53421 & -1.04843 & -0.14959 & -3.06315 \\
\hline 55 & -1.303882 & -0.250931 & -5.138081 & -1.39634 & -0.13213 & -5.58268 & -1.30388 & -0.25093 & -2.70852 & -1.6725 & -0.41786 & -2.97043 \\
\hline 56 & -1.304179 & -0.250931 & -4.312347 & -2.04033 & -0.18782 & -5.32498 & -1.30408 & -0.25093 & -1.88279 & -2.29255 & -0.42989 & -2.83326 \\
\hline 57 & -0.724234 & -0.250931 & -5.963611 & -0.26265 & -0.12615 & -5.7231 & -0.72428 & -0.25093 & -3.5341 & -0.91177 & -0.23761 & -3.05195 \\
\hline 58 & -0.724387 & -0.250931 & -5.137921 & -0.91377 & -0.35643 & -5.59889 & -0.72438 & -0.25093 & -2.70842 & -1.19835 & -0.52436 & -2.95329 \\
\hline 59 & -0.724537 & -0.250931 & -4.312188 & -1.5855 & -0.41856 & -5.46494 & -0.72449 & -0.25093 & -1.88268 & -1.95467 & -0.45514 & -2.7943 \\
\hline 60 & -0.144833 & -0.250931 & -5.963544 & 0.141201 & -0.29516 & -5.53718 & -0.14485 & -0.25093 & -3.53406 & -0.57928 & -0.42216 & -3.12582 \\
\hline 61 & -0.144873 & -0.250931 & -5.137853 & -0.51291 & -0.0586 & -5.49902 & -0.14487 & -0.25093 & -2.70837 & 0.350669 & -0.26969 & -2.95735 \\
\hline 62 & -0.14491 & -0.25093 & -4.312119 & -1.20347 & -0.20346 & -5.44826 & -0.1449 & -0.25093 & -1.88264 & 1.081882 & -0.13426 & -2.90276 \\
\hline 63 & 0.434514 & -0.250931 & -5.963566 & 0.305451 & 0.006486 & -5.66397 & 0.434566 & -0.25093 & -3.53408 & 0.164447 & -0.17345 & -3.11892 \\
\hline 64 & 0.434622 & -0.250931 & -5.137876 & & & \begin{tabular}{|l|}
-5.62184 \\
\end{tabular} & 0.434626 & -0.25093 & -2.70839 & 0.807891 & -0.22681 & -3.12133 \\
\hline 65 & 0.434732 & -0.250931 & -4.312142 & 0.524582 & 0.064025 & -5.55876 & 0.434692 & -0.25093 & -1.88265 & 1.459983 & -0.01117 & -2.99928 \\
\hline 66 & 1.013915 & -0.250931 & -5.963681 & 0.888984 & -0.19572 & -5.56381 & 1.013989 & -0.25093 & -3.53415 & 0.687277 & -0.28919 & -3.18503 \\
\hline 67 & 1.014137 & -0.250931 & -5.13799 & 1.455011 & -0.49211 & -5.41555 & 1.014136 & -0.25093 & -2.70846 & 1.348835 & -0.4377 & -2.90366 \\
\hline 68 & 1.014358 & -0.250931 & -4.312256 & 2.096351 & -0.33026 & -5.27875 & 1.014284 & -0.25093 & -1.88273 & 2.052539 & -0.40291 & -2.79158 \\
\hline 69 & 1.593265 & -0.250931 & -5.963883 & 1.160565 & -0.27796 & -5.58742 & 1.593405 & -0.25093 & -3.53428 & 1.154792 & -0.2581 & -3.16166 \\
\hline 70 & 1.593632 & -0.250931 & -5.138193 & 1.813709 & -0.50842 & -5.53922 & 1.593642 & -0.25093 & -2.70859 & 1.809401 & -0.21587 & -2.9116 \\
\hline 71 & 1.594002 & -0.250931 & -4.31246 & 2.426963 & -0.22124 & -5.39324 & 1.593876 & -0.25093 & -1.88286 & 2.434813 & -0.09709 & -2.84374 \\
\hline 72 & 2.172672 & -0.250931 & -5.964178 & 1.35735 & -0.23775 & -5.58942 & 2.172813 & -0.25093 & -3.53448 & 1.608329 & -0.20359 & -3.04232 \\
\hline 73 & 2.17315 & -0.250931 & -5.138487 & 2.005729 & -0.48909 & -5.56268 & 2.173153 & -0.25093 & -2.70879 & 2.253144 & -0.40133 & -2.88652 \\
\hline 74 & 2.173628 & -0.250931 & -4.312754 & 2.59934 & -0.3406 & -5.22988 & 2.173496 & -0.25093 & -1.88306 & 2.740789 & -0.12856 & -2.75294 \\
\hline 75 & 2.75203 & -0.250931 & -5.964558 & 1.650364 & -0.14252 & -5.67396 & 2.752209 & -0.25093 & -3.53476 & 2.031273 & -0.11501 & -3.07677 \\
\hline 76 & 2.752648 & -0.250931 & -5.138869 & 2.336199 & -0.2915 & -5.51217 & 2.752663 & -0.25093 & -2.70907 & 2.635922 & -0.44175 & -2.87558 \\
\hline
\end{tabular}

M-11 
Table M-2. Centroid locations from [46] for the CCO containers in a triangular pitch (continued).

\begin{tabular}{|c|c|c|c|c|c|c|c|c|c|c|c|c|}
\hline \multicolumn{13}{|c|}{ CCO containers } \\
\hline \multirow{3}{*}{ Centroid } & \multicolumn{6}{|c|}{ Lower horizon triangular with triangular pitch array } & \multicolumn{6}{|c|}{ Upper horizon triangular with triangular pitch array } \\
\hline & \multicolumn{3}{|c|}{$\mathbf{t}=0$ year } & \multicolumn{3}{|c|}{$t=1,000$ years } & \multicolumn{3}{|c|}{$\mathbf{t}=0$ year } & \multicolumn{3}{|c|}{$t=1,000$ years } \\
\hline & $\mathbf{x}(\mathrm{m})$ & y (m) & $\mathrm{z}(\mathrm{m})$ & $\mathrm{x}(\mathrm{m})$ & y (m) & $\mathrm{z}(\mathrm{m})$ & $\mathbf{x}(\mathrm{m})$ & y (m) & $\mathrm{z}(\mathrm{m})$ & $\mathbf{x}(\mathrm{m})$ & $\mathbf{y}(\mathrm{m})$ & $\mathrm{z}(\mathrm{m})$ \\
\hline 77 & 2.753268 & -0.250931 & -4.313134 & 2.693863 & -0.55599 & -5.20362 & 2.753114 & -0.25093 & -1.88334 & 2.862431 & -0.19575 & -2.67176 \\
\hline 78 & 3.331432 & -0.250931 & -5.965029 & 2.047828 & -0.05314 & -5.64528 & 3.331588 & -0.25093 & -3.53513 & 2.380388 & -0.41047 & -3.09083 \\
\hline 79 & 3.332166 & -0.250931 & -5.139338 & 2.717821 & -0.13859 & -5.52119 & 3.332182 & -0.25093 & -2.70944 & 2.974955 & -0.59153 & -2.97979 \\
\hline 80 & 3.332899 & -0.250931 & -4.313605 & 2.827418 & -0.35186 & -5.10162 & 3.332773 & -0.25093 & -1.88371 & 2.99987 & -0.22719 & -2.51851 \\
\hline 81 & 3.910678 & -0.250931 & -5.965605 & 2.610284 & 0.047978 & -5.54154 & 3.910824 & -0.25093 & -3.53563 & 3.063602 & 0.142025 & -3.03179 \\
\hline 82 & 3.911673 & -0.250931 & -5.139918 & 2.87013 & -0.3224 & -5.49971 & 3.911694 & -0.25093 & -2.70994 & 3.046717 & -0.49012 & -2.85716 \\
\hline 83 & 3.912668 & -0.250931 & -4.314184 & 2.930382 & -0.23711 & -4.95818 & 3.912568 & -0.25093 & -1.88421 & 3.148911 & -0.29648 & -2.37315 \\
\hline 84 & -3.910679 & 0.250931 & -5.965605 & -2.69741 & 0.034009 & -5.57097 & -3.91082 & 0.250931 & -3.53563 & -2.94076 & 0.481496 & -2.85572 \\
\hline 85 & -3.911673 & 0.250931 & -5.139918 & -2.93088 & 0.561359 & -5.43104 & -3.9117 & 0.250931 & -2.70994 & -2.93797 & -0.17431 & -2.88276 \\
\hline 86 & -3.912667 & 0.250931 & -4.314184 & -2.88907 & 0.156537 & -4.9902 & -3.91257 & 0.250931 & -1.88421 & -3.15322 & 0.055127 & -2.40987 \\
\hline 87 & -3.331432 & 0.250931 & -5.965029 & -2.11592 & 0.440845 & -5.63841 & -3.33159 & 0.250931 & -3.53513 & -2.49249 & 0.474328 & -3.01817 \\
\hline 88 & -3.332166 & 0.250931 & -5.139339 & -2.63405 & 0.037576 & -5.53679 & -3.33218 & 0.250931 & -2.70944 & -2.76523 & 0.049542 & -3.02146 \\
\hline 89 & -3.332899 & 0.250931 & -4.313605 & -2.76871 & 0.21948 & -5.17274 & -3.33277 & 0.250931 & -1.88371 & -3.00916 & 0.050994 & -2.54658 \\
\hline 90 & -2.75203 & & -5.964558 & -1.81793 & 0.409623 & -5.62 & & 0.250931 & -3.53476 & -1.8 & 20788 & -3.07979 \\
\hline 91 & -2.752648 & 0.250931 & -5.138869 & -2.35768 & -0.00131 & -5.45509 & -2.75266 & 0.250931 & -2.70907 & -2.51015 & 0.18157 & -3.02103 \\
\hline 92 & -2.753268 & 0.250931 & -4.313134 & -2.67789 & 0.466532 & -5.22112 & -2.75311 & 0.250931 & -1.88334 & -2.79387 & 0.017696 & -2.56154 \\
\hline 93 & -2.172672 & 0.250931 & -5.964178 & -1.37832 & 0.368461 & -5.72034 & -2.17281 & 0.250931 & -3.53448 & -1.57249 & 0.316793 & -3.02845 \\
\hline 94 & -2.17315 & 0.250931 & -5.138487 & -1.97761 & 0.154697 & -5.42129 & -2.17315 & 0.250931 & -2.70879 & -2.23891 & 0.272387 & -2.96523 \\
\hline 95 & -2.173628 & 0.250931 & -4.312754 & -2.55302 & 0.316112 & -5.35602 & -2.1735 & 0.250931 & -1.88306 & -2.69363 & 0.272768 & -2.7115 \\
\hline 96 & -1.593265 & 0.250931 & -5.963883 & -0.94791 & 0.365318 & -5.61155 & -1.59341 & 0.250931 & -3.53428 & -1.13312 & 0.22839 & -3.09005 \\
\hline 97 & -1.593632 & 0.250931 & -5.138193 & -1.57916 & 0.312935 & -5.4969 & -1.59364 & 0.250931 & -2.70859 & -1.78776 & 0.358452 & -2.82966 \\
\hline 98 & -1.594002 & 0.250931 & -4.31246 & -2.22389 & 0.31015 & -5.25762 & -1.59388 & 0.250931 & -1.88286 & -2.46216 & 0.339946 & -2.74376 \\
\hline 99 & -1.013915 & 0.250931 & -5.963681 & -0.51968 & 0.196313 & -5.7111 & -1.01399 & 0.250931 & -3.53415 & -0.70116 & 0.289163 & -3.00472 \\
\hline 100 & -1.014136 & 0.250931 & -5.13799 & -1.21737 & 0.178976 & -5.5188 & -1.01414 & 0.250931 & -2.70846 & -1.3371 & 0.158978 & -2.89355 \\
\hline 101 & -1.014358 & 0.250931 & -4.312256 & -1.79514 & 0.451472 & -5.32575 & -1.01428 & 0.250931 & -1.88273 & -2.10767 & 0.061665 & -2.79626 \\
\hline 102 & -0.434514 & 0.250931 & -5.963566 & -0.0992 & 0.241545 & -5.64028 & -0.43457 & 0.250931 & -3.53408 & -0.3187 & 0.174023 & -3.01928 \\
\hline
\end{tabular}

M-12 
Table M-2. Centroid locations from [46] for the CCO containers in a triangular pitch (continued).

\begin{tabular}{|c|c|c|c|c|c|c|c|c|c|c|c|c|}
\hline \multicolumn{13}{|c|}{ CCO containers } \\
\hline \multirow{3}{*}{ Centroid } & \multicolumn{6}{|c|}{ Lower horizon triangular with triangular pitch array } & \multicolumn{6}{|c|}{ Upper horizon triangular with triangular pitch array } \\
\hline & \multicolumn{3}{|c|}{$\mathbf{t}=\mathbf{0}$ year } & \multicolumn{3}{|c|}{$t=1,000$ years } & \multicolumn{3}{|c|}{$\mathbf{t}=\mathbf{0}$ year } & \multicolumn{3}{|c|}{$t=1,000$ years } \\
\hline & $x(m)$ & $y(m)$ & $\mathrm{z}(\mathrm{m})$ & $x(m)$ & $y(m)$ & $\mathrm{z}(\mathrm{m})$ & $x(m)$ & $y(m)$ & $\mathrm{z}(\mathrm{m})$ & $x(m)$ & $y(m)$ & $\mathrm{z}(\mathrm{m})$ \\
\hline 103 & -0.434622 & 0.250931 & -5.137876 & -0.75593 & 0.091724 & -5.51233 & -0.43463 & 0.250931 & -2.70839 & -0.98941 & 0.009907 & -2.96817 \\
\hline 104 & -0.434732 & 0.250931 & -4.312142 & -1.50217 & 0.025454 & -5.43357 & -0.43469 & 0.250931 & -1.88265 & 0.77203 & 0.109828 & -2.93362 \\
\hline 105 & 0.144833 & 0.250931 & -5.963544 & 0.312724 & 0.278738 & -5.61426 & 0.14485 & 0.250931 & -3.53406 & -0.01906 & 0.288524 & -3.07031 \\
\hline 106 & 0.144873 & 0.250931 & -5.137853 & -0.35417 & 0.43779 & -5.52382 & 0.144873 & 0.250931 & -2.70837 & 0.603315 & 0.022482 & -3.13293 \\
\hline 107 & 14491 & 0.250931 & -4.312119 & -1.0672 & 0.388367 & -5.44826 & 0.144898 & 0.250931 & -1.88264 & 1.207432 & 0.452809 & -2.93661 \\
\hline 108 & 0.724234 & 0.250931 & -5.963611 & 0.703202 & 0.319221 & -5.57559 & 0.724282 & 0.250931 & -3.5341 & 0.414521 & 0.341257 & -3.06594 \\
\hline 109 & 0.724387 & 0.250931 & -5.137921 & 0.968222 & 0.02463 & -5.5126 & 0.724384 & 0.250931 & -2.70842 & 1.077825 & 0.315685 & -3.00488 \\
\hline 110 & 0.724536 & 0.250931 & -4.312188 & 1.669049 & 0.129907 & -5.38472 & 0.724485 & 0.250931 & -1.88268 & 1.779156 & 0.316164 & -2.80221 \\
\hline 111 & 1.303584 & 0.250931 & -5.963771 & 0.904639 & 0.34647 & -5.54455 & 1.303691 & 0.250931 & -3.53421 & 0.917503 & 0.271858 & -3.18671 \\
\hline 112 & 1.303883 & 0.250931 & -5.138081 & 1.483856 & 0.207134 & \begin{tabular}{|l|}
-5.44857 \\
\end{tabular} & 1.303884 & 0.250931 & -2.70852 & 1.620341 & 0.418823 & -3.01763 \\
\hline 113 & 1.304179 & 0.250931 & -4.312347 & 2.025226 & 0.17975 & -5.36093 & 1.304082 & 0.250931 & -1.88279 & 2.154816 & 0.136642 & -2.79648 \\
\hline 114 & 1.882987 & 0.250931 & -5.964019 & 1.23055 & 0.239141 & -5.69719 & 1.883112 & 0.250931 & -3.53438 & 1.428994 & 0.166618 & -3.09898 \\
\hline 115 & 1.883399 & 0.250931 & -5.138329 & 1.86977 & -0.03532 & -5.52962 & 1.883398 & 0.250931 & -2.70869 & 2.07848 & 0.389502 & -2.95697 \\
\hline 116 & & 0.250931 & -4.312596 & 2.46334 & 0.147153 & -5.2166 & 1.883683 & 0.250931 & -1.88295 & 2.605636 & 0.295323 & -2.81093 \\
\hline 117 & 2.462347 & 0.250931 & -5.964358 & 1.44848 & 0.388578 & -5.63721 & 2.462528 & 0.250931 & -3.53461 & 1.894424 & 0.069549 & -3.05378 \\
\hline 118 & 2.462897 & 0.250931 & -5.138667 & 2.111696 & 0.229082 & -5.48866 & 2.462914 & 0.250931 & -2.70892 & 2.560842 & 0.171722 & -2.97675 \\
\hline 119 & 2.463445 & 0.250931 & -4.312934 & 2.665974 & 0.080124 & -5.19768 & 2.463296 & 0.250931 & -1.88319 & 2.814673 & 0.12854 & -2.61904 \\
\hline 120 & 3.041746 & 0.250931 & -5.964781 & 1.758802 & 0.530698 & -5.55004 & 3.041886 & 0.250931 & -3.53493 & 2.207336 & 0.392462 & -2.91714 \\
\hline 121 & 3.042408 & 0.250931 & -5.139092 & 2.430604 & 0.351202 & -5.47465 & 3.042418 & 0.250931 & -2.70924 & 2.787861 & 0.403343 & -3.03886 \\
\hline 122 & 3.043065 & 0.250931 & -4.313358 & 2.80301 & -0.06078 & -5.15664 & 3.042953 & 0.250931 & -1.88351 & 2.974685 & 0.006764 & -2.61813 \\
\hline 123 & 3.621093 & 0.250931 & -5.965301 & 2.208283 & 0.542522 & -5.6183 & 3.621254 & 0.250931 & -3.53536 & 2.820667 & 0.068947 & -3.02552 \\
\hline 124 & 3.621922 & 0.250931 & -5.139612 & 2.779644 & 0.323879 & -5.46967 & 3.621933 & 0.250931 & -2.70967 & 2.907405 & 0.44998 & -2.84936 \\
\hline 125 & 3.622749 & 0.250931 & -4.313879 & 2.914363 & 0.006705 & -5.0095 & 3.622611 & 0.250931 & -1.88393 & 3.097351 & 0.122288 & -2.47876 \\
\hline 126 & -3.621093 & 0.752792 & -5.965302 & -2.58063 & 0.581499 & -5.56683 & -3.62125 & 0.752791 & -3.53536 & -3.06648 & 0.933946 & -2.9091 \\
\hline 127 & -3.621922 & 0.752792 & -5.139612 & -2.96656 & 0.818151 & -5.39721 & -3.62193 & 0.752792 & -2.70967 & -2.72483 & 0.682488 & -2.86591 \\
\hline 128 & -3.622748 & 0.752794 & -4.31388 & -2.87284 & 0.543105 & -5.03024 & -3.62261 & 0.752794 & -1.88393 & -3.13054 & 0.409741 & -2.43082 \\
\hline
\end{tabular}

M-13 
Table M-2. Centroid locations from [46] for the CCO containers in a triangular pitch (continued).

\begin{tabular}{|c|c|c|c|c|c|c|c|c|c|c|c|c|}
\hline \multicolumn{13}{|c|}{ CCO containers } \\
\hline \multirow{3}{*}{ Centroid } & \multicolumn{6}{|c|}{ Lower horizon triangular with triangular pitch array } & \multicolumn{6}{|c|}{ Upper horizon triangular with triangular pitch array } \\
\hline & \multicolumn{3}{|c|}{$\mathbf{t}=\mathbf{0}$ year } & \multicolumn{3}{|c|}{$t=1,000$ years } & \multicolumn{3}{|c|}{$\mathbf{t}=\mathbf{0}$ year } & \multicolumn{3}{|c|}{$t=1,000$ years } \\
\hline & $x(m)$ & $y(m)$ & $\mathrm{z}(\mathrm{m})$ & $x(m)$ & $y(m)$ & $\mathrm{z}(\mathrm{m})$ & $x(m)$ & $y(m)$ & $\mathrm{z}(\mathrm{m})$ & $x(m)$ & $y(m)$ & $\mathrm{z}(\mathrm{m})$ \\
\hline 129 & -3.041741 & 0.75279 & -5.964781 & -2.06969 & 0.827013 & -5.66061 & -3.04189 & 0.752791 & -3.53493 & -2.27169 & 0.922325 & -3.08952 \\
\hline 130 & -3.042408 & 0.752792 & -5.139093 & -2.66728 & 0.89082 & -5.58258 & -3.04242 & 0.752792 & -2.70924 & -2.87725 & 0.913511 & -2.8763 \\
\hline 131 & -3.043068 & 0.752795 & -4.313359 & -2.71212 & 0.817717 & -5.16005 & -3.04295 & 0.752794 & -1.88351 & -3.00616 & 0.520713 & -2.55933 \\
\hline 132 & -2.462347 & 0.752792 & -5.964358 & -1.66973 & 0.86551 & -5.63689 & -2.46252 & 0.75279 & -3.53461 & -1.93197 & 0.665972 & -3.05803 \\
\hline 133 & -2.462897 & 0.752792 & -5.138668 & -2.27298 & 0.667151 & -5.43572 & -2.46291 & 0.752792 & -2.70892 & -2.44092 & 0.945383 & -2.87202 \\
\hline 134 & -2.463446 & 0.752794 & -4.312934 & -2.65095 & 0.980043 & \begin{tabular}{|l|}
-5.19575 \\
\end{tabular} & -2.4633 & 0.752795 & -1.88319 & -2.75865 & 0.662173 & -2.57971 \\
\hline 135 & -1.882981 & 75279 & -5.96402 & -1.23994 & 0.863263 & -5.65156 & -1.88311 & 0.752791 & -3.53438 & -1.51664 & 0.476365 & -3.01618 \\
\hline 136 & -1.883398 & 0.752791 & -5.13833 & -1.90459 & 0.905378 & -5.47272 & -1.8834 & 0.752792 & -2.70869 & -2.02709 & 0.936722 & -2.8438 \\
\hline 137 & -1.88381 & 0.752796 & -4.312597 & -2.48232 & 0.605465 & -5.3613 & -1.88368 & 0.752794 & -1.88295 & -2.55016 & 0.662086 & -2.68255 \\
\hline 138 & -1.303584 & 0.752792 & -5.963771 & -0.83369 & 0.734503 & -5.70986 & -1.30369 & 0.75279 & -3.53421 & -0.99908 & 0.618354 & -3.11922 \\
\hline 139 & -1.303882 & 0.752792 & -5.13808 & -1.46874 & 0.925826 & -5.47908 & -1.30389 & 0.752792 & -2.70852 & -1.61179 & 0.841436 & -3.01353 \\
\hline 140 & -1.30418 & 0.752794 & -4.312347 & -2.1379 & 0.92774 & -5.26097 & -1.30408 & 0.752795 & -1.88279 & -2.19449 & 0.565108 & -2.89942 \\
\hline 141 & -0.724228 & 0.75279 & -5.963611 & -0.22874 & 0.804903 & -5.74547 & -0.72428 & 0.752791 & -3.5341 & -0.83732 & 0.779193 & -3.06881 \\
\hline 142 & -0.724386 & 0.752792 & -5.137922 & -0.96491 & 0.622604 & \begin{tabular}{|l|}
-5.67835 \\
\end{tabular} & -0.72438 & 0.752792 & -2.70842 & -1.19275 & 0.599147 & -2.91593 \\
\hline 143 & -0.72454 & 0.752795 & -4.312189 & -1.62593 & 0.62544 & -5.39074 & -0.72449 & 0.752795 & -1.88268 & -1.8605 & 0.780675 & -2.80682 \\
\hline 144 & -0.144834 & 0.752792 & -5.963544 & 0.22644 & 0.788729 & -5.63729 & -0.14485 & 0.752791 & -3.53406 & -0.42508 & 0.745575 & -3.04563 \\
\hline 145 & -0.144873 & 0.752792 & -5.137854 & -0.42404 & 0.938576 & -5.53113 & -0.14487 & 0.752792 & -2.70837 & 0.253248 & 0.493745 & -3.00516 \\
\hline 146 & -0.144911 & 0.752794 & -4.31212 & -1.13341 & 0.801822 & -5.43238 & -0.1449 & 0.752794 & -1.88264 & 0.921231 & 0.73219 & -2.91944 \\
\hline 147 & 0.434519 & 0.75279 & -5.963566 & 0.548746 & 0.626162 & -5.65255 & 0.434566 & 0.752791 & -3.53408 & 0.204115 & 0.780457 & -3.03954 \\
\hline 148 & 0.434623 & 0.752792 & -5.137877 & 0.063484 & 0.833442 & -5.51176 & 0.434627 & 0.752792 & -2.70839 & 0.778168 & 0.924761 & -3.02625 \\
\hline 149 & 0.43473 & 0.752795 & -4.312143 & -0.62143 & 0.622957 & -5.48109 & 0.434691 & 0.752795 & -1.88265 & 1.448677 & 0.752576 & -2.84628 \\
\hline 150 & 1.013914 & 0.752791 & -5.963681 & 0.709884 & 0.822319 & -5.58049 & 1.01399 & 0.752791 & -3.53415 & 0.591139 & 0.688836 & -3.17378 \\
\hline 151 & 1.014136 & 0.752792 & -5.13799 & 1.262835 & 0.594437 & -5.44393 & 1.014136 & 0.752792 & -2.70846 & 1.291674 & 0.711607 & -2.9916 \\
\hline 152 & 1.014359 & 0.752794 & -4.312257 & 1.803017 & 0.495589 & -5.36065 & 1.014284 & 0.752795 & -1.88273 & 1.924553 & 0.745502 & -2.78298 \\
\hline 153 & 1.59327 & 0.75279 & -5.963884 & 1.143655 & 0.652482 & -5.51145 & 1.593405 & 0.752791 & -3.53428 & 1.125323 & 0.785728 & -3.17257 \\
\hline 154 & 1.593633 & 0.752791 & -5.138194 & 1.698649 & 0.871221 & -5.36652 & 1.593643 & 0.752792 & -2.70859 & 1.774273 & 0.702412 & -2.9515 \\
\hline
\end{tabular}

M-14 
Table M-2. Centroid locations from [46] for the CCO containers in a triangular pitch (continued).

\begin{tabular}{|c|c|c|c|c|c|c|c|c|c|c|c|c|}
\hline \multicolumn{13}{|c|}{ CCO containers } \\
\hline \multirow{3}{*}{ Centroid } & \multicolumn{6}{|c|}{ Lower horizon triangular with triangular pitch array } & \multicolumn{6}{|c|}{ Upper horizon triangular with triangular pitch array } \\
\hline & \multicolumn{3}{|c|}{$\mathbf{t}=\mathbf{0}$ year } & \multicolumn{3}{|c|}{$t=1,000$ years } & \multicolumn{3}{|c|}{$\mathbf{t}=0$ year } & \multicolumn{3}{|c|}{$t=1,000$ years } \\
\hline & $\mathbf{x}(\mathrm{m})$ & $\mathrm{y}(\mathrm{m})$ & $\mathrm{z}(\mathrm{m})$ & $\mathbf{x}(\mathrm{m})$ & y (m) & $\mathrm{z}(\mathrm{m})$ & $\mathbf{x}(\mathrm{m})$ & y (m) & $\mathrm{z}(\mathrm{m})$ & $\mathbf{x}(\mathrm{m})$ & y (m) & $\mathrm{z}(\mathrm{m})$ \\
\hline 155 & 1.593999 & 0.752796 & -4.312461 & 2.31254 & 0.53717 & -5.32034 & 1.593876 & 0.752795 & -1.88286 & 2.401345 & 0.889491 & -2.7084 \\
\hline 156 & 2.172671 & 0.752791 & -5.964178 & 1.385721 & 0.796297 & -5.6133 & 2.172814 & 0.752791 & -3.53448 & 1.693158 & 0.902982 & -3.06847 \\
\hline 157 & 2.17315 & 0.752792 & -5.138488 & 2.033557 & 0.823406 & -5.53022 & 2.173152 & 0.752792 & -2.7088 & 2.215579 & 0.676911 & -2.88669 \\
\hline 158 & 2.173628 & 0.752795 & -4.312755 & 2.575101 & 0.776472 & -5.10254 & 2.173495 & 0.752795 & -1.88306 & 2.775491 & 0.865881 & -2.66968 \\
\hline 159 & & & & & & & & & & & & \\
\hline 160 & 2.752649 & 0.752792 & -5.13887 & 2.270513 & 0.92132 & -5.40265 & 2.752663 & 0.752792 & -2.70907 & 2.668941 & 0.921929 & -2.90293 \\
\hline 161 & 2.753264 & 0.752795 & -4.313135 & 2.725721 & 0.541925 & -5.16865 & 2.753115 & 0.752794 & -1.88334 & 2.737126 & 0.527551 & -2.56726 \\
\hline 162 & 3.331431 & 0.752792 & -5.965029 & 2.01376 & 0.684832 & -5.63457 & 3.331588 & 0.752791 & -3.53513 & 2.475437 & 0.706887 & -3.0388 \\
\hline 163 & 3.332166 & 0.752792 & -5.139339 & 2.587849 & 0.950717 & -5.40044 & 3.332182 & 0.752792 & -2.70944 & 2.951343 & 0.972697 & -2.84771 \\
\hline 164 & 3.332899 & 0.752794 & -4.313606 & 2.857304 & 0.344833 & -5.15884 & 3.332773 & 0.752794 & -1.88371 & 3.013397 & 0.547619 & -2.53693 \\
\hline 165 & 3.910681 & 0.752791 & -5.965605 & 2.807992 & 0.428743 & -5.53663 & 3.910824 & 0.752791 & -3.53563 & 3.059556 & 0.86021 & -2.86028 \\
\hline 166 & 3.911674 & 0.752792 & & 2.786013 & 0.965766 & -5.29039 & & 0.752792 & -2.70994 & 2.845196 & 0.592625 & -2.85096 \\
\hline 167 & 3.912668 & 0.752795 & -4.314184 & 2.916078 & 0.536843 & -5.05188 & 3.912567 & 0.752795 & -1.88421 & 3.083926 & 0.750927 & -2.38355 \\
\hline
\end{tabular}


Table M-3. Centroid locations from [46] for the 6 in. POP containers.

\begin{tabular}{|c|c|c|c|c|c|c|c|c|c|c|c|c|}
\hline \multicolumn{13}{|c|}{6 in. POP containers } \\
\hline \multirow[b]{3}{*}{ Centroid } & \multicolumn{6}{|c|}{ Upper horizon triangular array } & \multicolumn{6}{|c|}{ Lower horizon hexagonal array } \\
\hline & \multicolumn{3}{|c|}{$t=0$ year } & \multicolumn{3}{|c|}{$t=1,000$ years } & \multicolumn{3}{|c|}{$t=0$ year } & \multicolumn{3}{|c|}{$t=1,000$ years } \\
\hline & $\mathbf{x}(\mathbf{m})$ & $\mathbf{y}(\mathbf{m})$ & $\mathbf{z}(\mathbf{m})$ & $\mathbf{x}(\mathbf{m})$ & $\mathbf{y}(\mathbf{m})$ & $\mathbf{z}(\mathbf{m})$ & $\mathbf{x}(\mathbf{m})$ & $\mathbf{y}(\mathbf{m})$ & $\mathbf{z}(\mathbf{m})$ & $\mathbf{x}(\mathbf{m})$ & $\mathbf{y}(\mathbf{m})$ & $\mathbf{z}(\mathbf{m})$ \\
\hline 0 & -3.91231 & -0.75278 & -3.58444 & -3.12238 & -0.74717 & -3.10268 & -3.42855 & -0.7916 & -6.01389 & -2.73344 & -0.65793 & -5.56198 \\
\hline 1 & -3.91319 & -0.7528 & -2.75915 & -2.92592 & -0.84039 & -2.83478 & -2.84898 & -0.7916 & -6.01341 & -2.06434 & -0.60245 & -5.55401 \\
\hline 2 & -3.91407 & -0.75281 & -1.93343 & -3.08746 & -0.78173 & -2.27553 & -3.13877 & -0.28975 & -6.01364 & -2.64677 & -0.3011 & -5.56224 \\
\hline 3 & -3.33271 & -0.75278 & -3.58393 & -2.80602 & -0.78022 & -3.04358 & -3.71833 & -0.28975 & -6.01418 & -3.00605 & -0.28624 & -5.56601 \\
\hline 4 & -3.33334 & -0.75281 & -2.75865 & -2.58945 & -0.90328 & -2.81877 & -4.00811 & -0.79161 & -6.0145 & -3.0308 & -0.74871 & -5.6152 \\
\hline 5 & -3.33396 & -0.75282 & -1.93293 & -2.81305 & -0.59291 & -2.47221 & -3.42933 & -0.79162 & -5.1886 & -2.53294 & -0.94268 & -5.40394 \\
\hline 6 & -2.75312 & -0.75279 & -3.58357 & -2.39734 & -0.80979 & -3.00609 & -2.84963 & -0.79162 & -5.18811 & -2.31914 & -1.0235 & -5.34378 \\
\hline 7 & -2.7536 & -0.7528 & \begin{tabular}{|l}
-2.75828 \\
\end{tabular} & -2.22995 & -0.53351 & -2.7915 & -3.13948 & -0.28975 & -5.18834 & -2.42802 & -0.50178 & -5.3435 \\
\hline 8 & -2.75407 & -0.7528 & \begin{tabular}{|l|}
-1.93256 \\
\end{tabular} & -2.58233 & -0.89197 & -2.69927 & -3.7192 & -0.28975 & -5.18888 & -2.57751 & -0.48689 & -5.33321 \\
\hline 9 & -2.17353 & -0.75278 & \begin{tabular}{|l|}
-3.58329 \\
\end{tabular} & -1.99075 & -0.77734 & -2.99884 & -4.00913 & -0.79162 & -5.1892 & -2.77577 & -0.69737 & -5.28899 \\
\hline 10 & -2.17388 & -0.7528 & -2.758 & -1.72669 & -0.90769 & -2.86113 & -3.43011 & -0.79162 & -4.36287 & -2.83803 & -0.76936 & -4.93277 \\
\hline 11 & -2.17422 & -0.75281 & -1.93228 & -2.34292 & -0.83734 & -2.77207 & -2.85027 & -0.79163 & -4.36239 & -2.42816 & -0.72184 & -5.35454 \\
\hline 12 & -1.59392 & -0.75278 & -3.58309 & -1.33588 & -0.53457 & -3.00016 & -3.14018 & -0.28975 & -4.36262 & -2.74406 & -0.03926 & -5.27082 \\
\hline 13 & -1.59417 & -0.7528 & -2.7578 & -1.32285 & -0.92532 & -2.97199 & -3.72006 & -0.28975 & -4.36315 & -2.91209 & -0.28119 & -4.92069 \\
\hline 14 & -1.59442 & -0.75281 & -1.93208 & -1.71157 & -0.55328 & -2.95865 & -4.01014 & -0.79162 & -4.36348 & -3.04666 & -0.76465 & -4.77055 \\
\hline 15 & -1.01432 & -0.75279 & -3.58295 & -0.92224 & -0.92262 & -3.10214 & -2.05722 & 0 & -6.01289 & -1.87327 & -0.23016 & -5.53429 \\
\hline 16 & -1.01447 & -0.75279 & -2.75767 & -0.79803 & -0.52918 & -3.10468 & -1.47758 & 0 & -6.01261 & -1.36327 & -0.07286 & -5.50711 \\
\hline 17 & -1.01461 & -0.75279 & -1.93194 & -1.11882 & -0.69246 & -3.04625 & -1.76741 & 0.501872 & -6.01274 & -1.77763 & 0.600827 & -5.50232 \\
\hline 18 & -0.43471 & -0.75279 & -3.58288 & -0.44453 & -0.94004 & -3.15345 & -2.34703 & 0.501863 & -6.01306 & -1.82058 & 0.353039 & -5.57899 \\
\hline 19 & -0.43478 & -0.7528 & -2.75759 & -0.38534 & -0.63515 & -3.13365 & -2.63685 & 0 & -6.01325 & -2.28469 & 0.020458 & -5.473 \\
\hline 20 & -0.43484 & -0.75281 & -1.93187 & -0.77033 & -0.75814 & -3.0693 & -2.34703 & -0.50186 & -6.01306 & -2.19962 & -0.52669 & -5.41206 \\
\hline 21 & 0.14491 & -0.75279 & -3.58287 & 0.190665 & -0.58996 & -3.09238 & -1.76741 & -0.50187 & -6.01274 & -1.79175 & -0.50012 & -5.60377 \\
\hline 22 & 0.144922 & -0.7528 & -2.75758 & -0.27202 & -0.79091 & -3.01951 & -2.05771 & 0 & -5.18759 & -2.43117 & -0.18431 & -5.36619 \\
\hline 23 & 0.14494 & -0.7528 & -1.93186 & 0.218245 & -0.86035 & -3.06506 & -1.47794 & $-1 \mathrm{E}-06$ & -5.18732 & -2.04167 & -0.18681 & -5.39586 \\
\hline 24 & 0.724506 & -0.75278 & -3.58291 & 0.692335 & -0.58857 & -3.07871 & -1.76782 & 0.501855 & -5.18744 & -1.75529 & 0.186115 & -5.47261 \\
\hline 25 & 0.724624 & -0.7528 & -2.75762 & 0.662188 & -0.99915 & -3.01888 & -2.34758 & 0.501858 & -5.18776 & -2.4079 & 0.330124 & -5.53825 \\
\hline 26 & 0.724734 & -0.75281 & -1.9319 & 1.137311 & -0.62432 & -3.12435 & -2.63744 & 0 & -5.18796 & -2.48274 & -0.31278 & -5.36034 \\
\hline 27 & 1.304122 & -0.75278 & -3.58301 & 1.449741 & -0.88738 & -2.97116 & -2.34758 & -0.50186 & -5.18776 & -2.08714 & -0.85607 & -5.35015 \\
\hline 28 & 1.304321 & -0.7528 & -2.75773 & 1.16765 & -0.8155 & -2.97846 & -1.76782 & -0.50186 & -5.18744 & -1.90246 & -0.8996 & -5.45117 \\
\hline
\end{tabular}




\begin{tabular}{|c|c|c|c|c|c|c|c|c|c|c|c|c|}
\hline \multicolumn{13}{|c|}{6 in. POP containers } \\
\hline \multirow[b]{3}{*}{ Centroid } & \multicolumn{6}{|c|}{ Upper horizon triangular array } & \multicolumn{6}{|c|}{ Lower horizon hexagonal array } \\
\hline & \multicolumn{3}{|c|}{$t=0$ year } & \multicolumn{3}{|c|}{$t=1,000$ years } & \multicolumn{3}{|c|}{$t=0$ year } & \multicolumn{3}{|c|}{$t=1,000$ years } \\
\hline & $\mathbf{x}(\mathrm{m})$ & $y(m)$ & $\mathrm{z}(\mathrm{m})$ & $\mathbf{x}(\mathbf{m})$ & $y(m)$ & $\mathrm{z}(\mathbf{m})$ & $\mathbf{x}(\mathbf{m})$ & $y(m)$ & $\mathrm{z}(\mathbf{m})$ & $\mathbf{x}(\mathbf{m})$ & $\mathbf{y}(\mathbf{m})$ & $\mathrm{z}(\mathbf{m})$ \\
\hline 29 & 1.304517 & -0.75281 & -1.932 & 1.919734 & -0.87205 & -2.95109 & -2.05818 & 0 & -4.36187 & -2.13097 & 0.005771 & -5.49978 \\
\hline
\end{tabular}


Table M-3. Centroid locations from [46] for the 6 in. POP containers (continued).

\begin{tabular}{|c|c|c|c|c|c|c|c|c|c|c|c|c|}
\hline \multicolumn{13}{|c|}{6 in. POP containers } \\
\hline \multirow[b]{3}{*}{ Centroid } & \multicolumn{6}{|c|}{ Upper horizon triangular array } & \multicolumn{6}{|c|}{ Lower horizon hexagonal array } \\
\hline & \multicolumn{3}{|c|}{$t=0$ year } & \multicolumn{3}{|c|}{$t=1,000$ years } & \multicolumn{3}{|c|}{$t=0$ year } & \multicolumn{3}{|c|}{$t=1,000$ years } \\
\hline & $\mathbf{x}(\mathbf{m})$ & $\mathbf{y}(\mathbf{m})$ & $\mathbf{z}(\mathbf{m})$ & $\mathbf{x}(\mathbf{m})$ & $\mathbf{y}(\mathbf{m})$ & $\mathbf{z}(\mathbf{m})$ & $\mathbf{x}(\mathbf{m})$ & $\mathbf{y}(\mathbf{m})$ & $\mathbf{z}(\mathbf{m})$ & $\mathbf{x}(\mathbf{m})$ & $\mathbf{y}(\mathbf{m})$ & $\mathbf{z}(\mathbf{m})$ \\
\hline 30 & 1.883724 & -0.75279 & -3.58318 & 1.528693 & -0.91196 & -3.07825 & -1.47828 & $-1 \mathrm{E}-06$ & -4.36159 & -1.57353 & 0.091387 & -5.59896 \\
\hline 31 & 1.884021 & -0.75279 & -2.75789 & 2.031372 & -0.60465 & -3.02527 & -1.76823 & 0.501847 & -4.36172 & -1.9514 & 0.644844 & -5.49318 \\
\hline 32 & 1.884311 & -0.75278 & -1.93217 & 2.13425 & -0.94374 & -2.87101 & -2.34812 & 0.501859 & -4.36204 & -2.08441 & 0.616447 & -5.39108 \\
\hline 33 & 2.463326 & -0.75278 & -3.58342 & 2.271243 & -0.69924 & -2.90024 & -2.63802 & 0 & -4.36223 & -2.42868 & 0.141489 & -5.29792 \\
\hline 34 & 2.463736 & -0.7528 & -2.75813 & 2.355915 & -1.01578 & -2.80514 & -2.34812 & -0.50186 & -4.36204 & -2.30251 & -0.46659 & -5.40993 \\
\hline 35 & 2.464138 & -0.75281 & -1.93241 & 2.580745 & -0.56984 & -2.80446 & -1.76823 & -0.50185 & -4.36172 & -1.92642 & -0.40247 & -5.48779 \\
\hline 36 & 3.042924 & -0.75278 & -3.58374 & 2.448614 & -0.85634 & -2.87116 & -0.68574 & -0.79161 & -6.01238 & -0.48572 & -0.67719 & -5.52085 \\
\hline 37 & 3.043466 & -0.7528 & -2.75845 & 2.553397 & -0.56051 & -2.93605 & -0.10609 & -0.7916 & -6.01232 & -0.30927 & -0.96884 & -5.51524 \\
\hline 38 & 3.044004 & -0.75281 & -1.93273 & 2.788536 & -0.81337 & -2.5901 & -0.39592 & -0.28975 & -6.01234 & -0.39051 & -0.25103 & -5.67276 \\
\hline 39 & 3.622497 & -0.75279 & -3.58416 & 2.921292 & -0.61877 & -3.01878 & -0.97559 & -0.28975 & -6.01245 & -1.03638 & -0.12206 & -5.57677 \\
\hline 40 & 3.623213 & -0.7528 & -2.75888 & 2.697535 & -0.95396 & -2.77041 & -1.26542 & -0.7916 & -6.01254 & -1.37693 & -1.04304 & -5.49382 \\
\hline 41 & 3.623916 & -0.75281 & -1.93316 & 3.021178 & -0.80224 & -2.3924 & -0.68592 & -0.79162 & -5.18708 & -1.38897 & -0.77131 & -5.6358 \\
\hline 42 & -3.6225 & -0.25093 & -3.58417 & -2.98313 & -0.26258 & -3.07911 & -0.10611 & -0.79162 & -5.18702 & -0.17197 & -0.46673 & -5.62083 \\
\hline 43 & -3.62321 & -0.25093 & -2.75888 & -2.91267 & -0.45487 & -2.78546 & -0.39602 & -0.28975 & -5.18704 & -0.79741 & -0.33769 & -5.57405 \\
\hline 44 & -3.62391 & -0.25094 & -1.93316 & -2.94319 & -0.15813 & -2.47429 & -0.97583 & -0.28975 & -5.18715 & -1.52988 & -0.46639 & -5.64155 \\
\hline 45 & -3.04292 & -0.25093 & -3.58374 & -2.3889 & -0.13252 & -2.90314 & -1.26573 & -0.79162 & -5.18724 & -1.59653 & -0.66167 & -5.50589 \\
\hline 46 & -3.04347 & -0.25093 & -2.75845 & -2.66237 & -0.46802 & -2.90197 & -0.68609 & -0.79162 & -4.36136 & -1.00347 & -0.93268 & -5.60277 \\
\hline 47 & -3.04401 & -0.25093 & -1.93273 & -2.80021 & -0.0466 & -2.73862 & -0.10614 & -0.79163 & -4.3613 & 0.312279 & -0.90859 & -5.53795 \\
\hline 48 & -2.46333 & -0.25093 & -3.58342 & -1.93399 & -0.18199 & -2.82474 & -0.39611 & -0.28976 & -4.36132 & -0.2099 & -0.27537 & -5.64602 \\
\hline 49 & -2.46374 & -0.25094 & -2.75813 & -2.36825 & 0.061672 & -2.85918 & -0.97607 & -0.28975 & -4.36142 & -1.15932 & -0.36798 & -5.53288 \\
\hline 50 & -2.46414 & -0.25094 & -1.93241 & -2.6356 & -0.32081 & -2.8232 & -1.26603 & -0.79163 & -4.36151 & -1.62151 & -0.76009 & -5.37193 \\
\hline 51 & -1.88373 & -0.25093 & -3.58318 & -1.66677 & -0.0039 & -2.94066 & 0.685738 & 0 & -6.01238 & 0.734495 & -0.0022 & -5.59357 \\
\hline 52 & -1.88402 & -0.25093 & -2.75789 & -1.80095 & -0.35891 & -3.01499 & 1.265415 & 0 & -6.01253 & 1.24762 & -0.247 & -5.48223 \\
\hline 53 & -1.88432 & -0.25094 & -1.93217 & -2.17594 & -0.22472 & -2.88616 & 0.975599 & 0.501865 & -6.01245 & 0.919442 & 0.545228 & -5.53854 \\
\hline 54 & -1.30412 & -0.25093 & -3.58301 & -1.29476 & -0.13498 & -3.03279 & 0.395916 & 0.501862 & -6.01234 & 0.355871 & 0.519446 & -5.5878 \\
\hline 55 & -1.30432 & -0.25093 & -2.75772 & -1.16226 & -0.29674 & -2.92219 & 0.106099 & 0 & -6.01232 & 0.221935 & -0.0497 & -5.64791 \\
\hline 56 & -1.30452 & -0.25094 & -1.932 & -1.81983 & -0.4075 & -2.91278 & 0.395917 & -0.50186 & -6.01234 & 0.274748 & -0.76918 & -5.51875 \\
\hline 57 & -0.72451 & -0.25093 & -3.58291 & -0.80939 & -0.22833 & -3.12002 & 0.975599 & -0.50187 & -6.01245 & 1.041745 & -0.52277 & -5.54486 \\
\hline 58 & -0.72462 & -0.25093 & -2.75762 & -0.60867 & 0.063407 & -3.08388 & 0.68592 & 0 & -5.18708 & 0.817841 & -0.34449 & -5.57219 \\
\hline
\end{tabular}


Table M-3. Centroid locations from [46] for the 6 in. POP containers (continued).

\begin{tabular}{|c|c|c|c|c|c|c|c|c|c|c|c|c|}
\hline \multicolumn{13}{|c|}{6 in. POP containers } \\
\hline \multirow[b]{3}{*}{ Centroid } & \multicolumn{6}{|c|}{ Upper horizon triangular array } & \multicolumn{6}{|c|}{ Lower horizon hexagonal array } \\
\hline & \multicolumn{3}{|c|}{$t=0$ year } & \multicolumn{3}{|c|}{$t=1,000$ years } & \multicolumn{3}{|c|}{$t=0$ year } & \multicolumn{3}{|c|}{$t=1,000$ years } \\
\hline & $\mathbf{x}(\mathbf{m})$ & $\mathbf{y}(\mathbf{m})$ & $\mathbf{z}(\mathbf{m})$ & $\mathbf{x}(\mathbf{m})$ & $\mathbf{y}(\mathbf{m})$ & $\mathbf{z}(\mathbf{m})$ & $\mathbf{x}(\mathbf{m})$ & $\mathbf{y}(\mathbf{m})$ & $\mathbf{z}(\mathbf{m})$ & $\mathbf{x}(\mathbf{m})$ & $\mathbf{y}(\mathbf{m})$ & $\mathbf{z}(\mathbf{m})$ \\
\hline 59 & -0.72472 & -0.25093 & -1.9319 & -0.74958 & -0.20289 & -3.04183 & 1.265722 & 0 & -5.18724 & 1.380338 & 0.34395 & -5.5574 \\
\hline 60 & -0.14491 & -0.25093 & -3.58287 & 0.061575 & -0.37365 & -3.02039 & 0.975826 & 0.501859 & -5.18715 & 1.277287 & 0.646196 & -5.63161 \\
\hline 61 & -0.14492 & -0.25093 & -2.75758 & -0.0201 & 0.163553 & -3.12378 & 0.396007 & 0.501856 & -5.18704 & 0.750051 & 0.765752 & -5.57473 \\
\hline 62 & -0.14494 & -0.25093 & -1.93185 & -0.40441 & -0.21401 & -3.0522 & 0.106107 & 0 & -5.18702 & -0.24007 & -0.05024 & -5.55342 \\
\hline 63 & 0.434708 & -0.25093 & -3.58288 & 0.396466 & -0.26203 & -3.0579 & 0.396008 & -0.50186 & -5.18704 & 0.893084 & -0.68576 & -5.61648 \\
\hline 64 & 0.434775 & -0.25093 & -2.75759 & 0.453635 & 0.069774 & -2.95058 & 0.975826 & -0.50186 & -5.18715 & 0.817638 & -0.96957 & -5.52519 \\
\hline 65 & 0.434842 & -0.25094 & -1.93187 & 0.968699 & -0.40806 & -3.00029 & 0.686085 & 0.000001 & -4.36136 & 1.116529 & 0.186939 & -5.59822 \\
\hline 66 & 1.014321 & -0.25093 & -3.58296 & 0.955218 & -0.39562 & -3.0593 & 1.266019 & $-1 \mathrm{E}-06$ & -4.36151 & 1.550795 & -0.15555 & -5.57089 \\
\hline 67 & 1.01447 & -0.25093 & -2.75766 & 1.134386 & -0.03174 & -3.0524 & 0.976054 & 0.501858 & -4.36143 & 1.130051 & 0.723777 & -5.56489 \\
\hline 68 & 1.014619 & -0.25093 & -1.93194 & 1.187652 & -0.49433 & -3.0773 & 0.396097 & 0.501851 & -4.36132 & 0.805696 & 0.455416 & -5.64714 \\
\hline 69 & 1.593922 & -0.25093 & -3.58309 & 1.661345 & -0.34911 & -3.00617 & 0.106125 & 0 & -4.3613 & 0.2759 & -0.27686 & -5.59871 \\
\hline 70 & 1.594171 & -0.25093 & -2.7578 & 1.459601 & -0.30481 & -2.89375 & 0.396097 & -0.50185 & -4.36132 & 0.271063 & -0.51021 & -5.64219 \\
\hline 71 & 1.594413 & -0.25094 & -1.93208 & 1.975618 & -0.56727 & -2.97584 & 0.976053 & -0.50186 & -4.36143 & 1.377869 & -0.51622 & -5.51581 \\
\hline 72 & 2.173529 & -0.25093 & -3.58329 & 2.07904 & -0.25428 & -3.01806 & 2.057221 & -0.7916 & -6.01289 & 1.906264 & -0.93159 & -5.49001 \\
\hline 73 & 2.173882 & -0.25093 & -2.758 & 1.92805 & -0.19733 & -2.80325 & 2.636842 & -0.79161 & -6.01325 & 2.145236 & -0.96944 & -5.48435 \\
\hline 74 & 2.174229 & -0.25094 & -1.93228 & 2.378876 & -0.18397 & -2.76063 & 2.34703 & -0.28975 & -6.01306 & 2.055996 & -0.34456 & -5.39083 \\
\hline 75 & 2.753123 & -0.25093 & -3.58357 & 2.390618 & -0.26881 & -2.98865 & 1.767409 & -0.28975 & -6.01274 & 1.70538 & -0.56577 & -5.45305 \\
\hline 76 & 2.753592 & -0.25093 & -2.75828 & 2.284355 & 0.009273 & -2.82018 & 1.477581 & -0.79162 & -6.01262 & 1.54453 & -0.77671 & -5.43455 \\
\hline 77 & 2.75405 & -0.25093 & -1.93256 & 2.746802 & -0.19985 & -2.76533 & 2.057703 & -0.79162 & -5.18759 & 2.350791 & -0.54894 & -5.57722 \\
\hline 78 & 3.332713 & -0.25093 & -3.58394 & 2.722765 & 0.005382 & -3.03041 & 2.637433 & -0.79162 & -5.18795 & 2.540043 & -1.00492 & -5.37453 \\
\hline 79 & 3.333341 & -0.25093 & -2.75865 & 2.633769 & -0.301 & -2.90781 & 2.347572 & -0.28975 & -5.18776 & 2.184308 & -0.01856 & -5.52228 \\
\hline 80 & 3.33396 & -0.25094 & -1.93293 & 2.87217 & -0.2948 & -2.5077 & 1.767828 & -0.28975 & -5.18744 & 1.629219 & -0.11971 & -5.42255 \\
\hline 81 & 3.912311 & -0.25093 & -3.58444 & 3.089804 & -0.21903 & -3.11357 & 1.477936 & -0.79161 & -5.18731 & 1.113931 & -0.97708 & -5.51156 \\
\hline 82 & 3.913197 & -0.25093 & -2.75915 & 2.888612 & -0.12156 & -2.87294 & 2.058173 & -0.79163 & -4.36187 & 2.172858 & -0.89286 & -5.41562 \\
\hline 83 & 3.914073 & -0.25093 & -1.93343 & 3.065469 & -0.25998 & -2.2992 & 2.638024 & -0.79162 & -4.36223 & 2.425588 & -0.56545 & -5.32606 \\
\hline 84 & -3.91231 & 0.250928 & -3.58444 & -3.09741 & 0.153762 & -3.13725 & 2.348106 & -0.28976 & -4.36204 & 2.311724 & -0.49401 & -5.42057 \\
\hline 85 & -3.9132 & 0.250934 & -2.75915 & -2.96214 & 0.047263 & -2.77815 & 1.768241 & -0.28975 & -4.36172 & 1.928692 & -0.56745 & -5.4989 \\
\hline 86 & -3.91407 & 0.250933 & -1.93343 & -2.98574 & 0.326728 & -2.4169 & 1.478282 & -0.79161 & -4.36159 & 1.772879 & -0.90862 & -5.57245 \\
\hline 87 & -3.33271 & 0.25093 & -3.58394 & -2.74687 & 0.453296 & -2.9702 & 3.428558 & 0 & -6.01389 & 2.702754 & -0.13408 & -5.52898 \\
\hline
\end{tabular}


Table M-3. Centroid locations from [46] for the 6 in. POP containers (continued).

\begin{tabular}{|c|c|c|c|c|c|c|c|c|c|c|c|c|}
\hline \multicolumn{13}{|c|}{6 in. POP containers } \\
\hline \multirow[b]{3}{*}{ Centroid } & \multicolumn{6}{|c|}{ Upper horizon triangular array } & \multicolumn{6}{|c|}{ Lower horizon hexagonal array } \\
\hline & \multicolumn{3}{|c|}{$t=0$ year } & \multicolumn{3}{|c|}{$t=1,000$ years } & \multicolumn{3}{|c|}{$t=0$ year } & \multicolumn{3}{|c|}{$t=1,000$ years } \\
\hline & $\mathbf{x}(\mathbf{m})$ & $\mathbf{y}(\mathbf{m})$ & $\mathbf{z}(\mathbf{m})$ & $\mathbf{x}(\mathbf{m})$ & $\mathbf{y}(\mathbf{m})$ & $\mathbf{z}(\mathbf{m})$ & $\mathbf{x}(\mathbf{m})$ & $\mathbf{y}(\mathbf{m})$ & $\mathbf{z}(\mathbf{m})$ & $\mathbf{x}(\mathbf{m})$ & $\mathbf{y}(\mathbf{m})$ & $\mathbf{z}(\mathbf{m})$ \\
\hline 88 & -3.33334 & 0.250933 & -2.75865 & -2.79111 & 0.05204 & -2.93207 & 4.008103 & 0 & -6.0145 & 2.989456 & 0.133445 & -5.57923 \\
\hline 89 & -3.33396 & 0.250938 & -1.93293 & -2.78933 & 0.233862 & -2.51239 & 3.71833 & 0.501875 & -6.01417 & 2.868203 & 0.523661 & -5.46902 \\
\hline 90 & -2.75312 & 0.250926 & -3.58357 & -2.51373 & 0.141499 & -2.91687 & 3.138772 & 0.501857 & -6.01364 & 2.356034 & 0.544058 & -5.53781 \\
\hline 91 & -2.75359 & 0.250933 & -2.75828 & -2.42076 & 0.503264 & -2.80189 & 2.848975 & 0 & -6.0134 & .899889 & 0.063451 & -5.43051 \\
\hline 92 & -2.75405 & 0.250933 & -1.93256 & -2.76518 & 0.153695 & -2.82515 & 3.138772 & -0.50186 & -6.01364 & .498321 & -0.47441 & -5.39994 \\
\hline 93 & -2.17353 & 0.250928 & -3.58329 & -2.15434 & 0.38274 & -2.99426 & 3.71833 & -0.50188 & -6.01417 & .759735 & -0.53978 & -5.42106 \\
\hline 94 & -2.17388 & 0.250934 & -2.758 & -1.86577 & 0.381673 & -2.91674 & 3.429327 & 0 & -5.1886 & 2.557723 & 0.142509 & -5.38022 \\
\hline 95 & -2.17423 & 0.250936 & -1.93228 & -2.32295 & 0.23251 & -2.72767 & 4.009133 & 0 & -5.18921 & 2.769696 & -0.18676 & -5.29701 \\
\hline 96 & -1.59392 & 0.250929 & -3.58309 & -1.56192 & 0.244654 & -3.02778 & 3.719204 & 0.501849 & -5.18888 & 2.804169 & 0.918748 & -5.27974 \\
\hline 97 & -1.59417 & 0.250934 & -2.7578 & -1.44921 & 0.54361 & -2.89479 & 3.13948 & 0.501864 & -5.18834 & 2.666773 & 0.779192 & -5.39418 \\
\hline 98 & -1.59441 & 0.250935 & -1.93208 & -1.98849 & 0.159264 & -2.887 & 2.849636 & 0 & -5.18811 & 2.303499 & 0.131164 & -5.42796 \\
\hline 99 & -1.01432 & 0.250931 & -3.58296 & -0.97123 & 0.206914 & -3.13268 & 3.13948 & -0.50186 & -5.18834 & 2.574044 & -0.92715 & -5.38173 \\
\hline 100 & -1.01447 & 0.25093 & -2.75766 & -0.79194 & 0.38867 & -2.95236 & 3.719205 & -0.50185 & -5.18888 & 2.701623 & -0.97307 & -5.30427 \\
\hline 101 & -1.01462 & 0.25093 & -1.93194 & -1.13943 & 0.382096 & -3.08243 & 3.430097 & 0 & -4.36287 & 2.768989 & -0.25935 & -5.02199 \\
\hline 102 & -0.43471 & 0.25093 & -3.58288 & -0.38857 & 0.327744 & -3.11849 & 4.010144 & 0 & -4.36348 & 2.971555 & 0.13224 & -4.86498 \\
\hline 103 & -0.43478 & 0.250932 & -2.75759 & -0.10675 & 0.440043 & -3.00446 & 3.720068 & 0.501839 & -4.36316 & 2.879486 & 0.47972 & -4.9826 \\
\hline 104 & -0.43484 & 0.250939 & -1.93187 & -0.66671 & 0.207027 & -3.08903 & 3.140181 & 0.501867 & -4.36262 & 2.613226 & 0.327092 & -5.29179 \\
\hline 105 & 0.144909 & 0.25093 & -3.58287 & 0.244752 & 0.050921 & -3.13765 & 2.85028 & 0 & -4.36239 & 2.448363 & -0.31988 & -5.25046 \\
\hline 106 & 0.144924 & 0.250931 & -2.75758 & 0.366742 & 0.465772 & -2.97776 & 3.140181 & -0.50187 & -4.36262 & 2.6712 & -0.46382 & -5.26397 \\
\hline 107 & 0.144943 & 0.250931 & -1.93186 & 0.046161 & -0.13398 & -3.08397 & 3.720069 & -0.50184 & -4.36316 & 2.792762 & -0.52891 & -5.02587 \\
\hline 108 & 0.724507 & 0.250929 & -3.58291 & 0.43689 & 0.319515 & -3.0473 & -3.42855 & 0.791602 & -6.01389 & -2.66436 & 0.694983 & -5.54963 \\
\hline 109 & 0.724619 & 0.250932 & -2.75762 & 0.990124 & 0.169254 & -3.02583 & -2.84898 & 0.791603 & -6.01341 & -2.10607 & 0.747501 & -5.51927 \\
\hline 110 & 0.724725 & 0.250933 & -1.9319 & 0.810122 & 0.586188 & -2.98012 & -4.00811 & 0.791611 & -6.0145 & -2.99111 & 0.737713 & -5.55717 \\
\hline 111 & 1.304122 & 0.250928 & -3.58301 & 1.320439 & 0.186248 & -2.95677 & -3.71833 & 0.289747 & -6.01417 & -2.87378 & 0.104276 & -5.46149 \\
\hline 112 & 1.304324 & 0.250933 & -2.75772 & 1.274853 & 0.387764 & -2.91104 & -3.13877 & 0.289748 & -6.01364 & -2.31996 & 0.609228 & -5.52785 \\
\hline 113 & 1.304523 & 0.250936 & -1.932 & 1.731657 & 0.258591 & -2.96553 & -3.42933 & 0.791616 & -5.1886 & -2.41573 & 0.936345 & -5.37868 \\
\hline 114 & 1.883726 & 0.250928 & -3.58318 & 1.790322 & 0.040219 & -2.99087 & -2.84963 & 0.791618 & -5.18811 & -1.87918 & 0.941417 & -5.31962 \\
\hline 115 & 1.884023 & 0.250932 & -2.75789 & 1.807353 & 0.357946 & -2.98153 & -4.00913 & 0.791615 & -5.1892 & -2.7577 & 0.873623 & -5.28247 \\
\hline 116 & 1.884315 & 0.250934 & -1.93217 & 2.243284 & 0.130556 & -2.88986 & -3.7192 & 0.289753 & -5.18888 & -2.67157 & 0.532796 & -5.33969 \\
\hline
\end{tabular}


Table M-3. Centroid locations from [46] for the 6 in. POP containers (continued).

\begin{tabular}{|c|c|c|c|c|c|c|c|c|c|c|c|c|}
\hline \multicolumn{13}{|c|}{6 in. POP containers } \\
\hline \multirow[b]{3}{*}{ Centroid } & \multicolumn{6}{|c|}{ Upper horizon triangular array } & \multicolumn{6}{|c|}{ Lower horizon hexagonal array } \\
\hline & \multicolumn{3}{|c|}{$t=0$ year } & \multicolumn{3}{|c|}{$t=1,000$ years } & \multicolumn{3}{|c|}{$t=0$ year } & \multicolumn{3}{|c|}{$t=1,000$ years } \\
\hline & $\mathbf{x}(\mathbf{m})$ & $\mathbf{y}(\mathbf{m})$ & $\mathbf{z}(\mathbf{m})$ & $\mathbf{x}(\mathbf{m})$ & $\mathbf{y}(\mathbf{m})$ & $\mathbf{z}(\mathbf{m})$ & $\mathbf{x}(\mathbf{m})$ & $\mathbf{y}(\mathbf{m})$ & $\mathbf{z}(\mathbf{m})$ & $\mathbf{x}(\mathbf{m})$ & $\mathbf{y}(\mathbf{m})$ & $\mathbf{z}(\mathbf{m})$ \\
\hline 117 & 2.463326 & 0.250928 & -3.58342 & 2.267997 & 0.165975 & -3.03259 & -3.13948 & 0.289751 & -5.18834 & -2.39818 & 0.122204 & -5.4808 \\
\hline 118 & 2.463737 & 0.250935 & -2.75813 & 2.145541 & 0.432488 & -2.74952 & -3.43011 & 0.791623 & -4.36287 & -2.78512 & 0.634907 & -4.95416 \\
\hline 119 & 2.464138 & 0.250938 & -1.93241 & 2.569876 & 0.291732 & -2.83075 & -2.85027 & 0.791625 & -4.36239 & -2.37159 & 0.656688 & -5.26414 \\
\hline 120 & 3.042924 & 0.25093 & -3.58374 & 2.495227 & 0.237684 & -2.9286 & -4.01014 & 0.791618 & -4.36348 & -3.02804 & 0.900095 & -4.74833 \\
\hline 121 & 3.043473 & 0.250934 & -2.75845 & 2.508612 & 0.603304 & -2.84634 & -3.72006 & 0.289755 & -4.36315 & -2.92091 & 0.18129 & -4.93766 \\
\hline 122 & 3.044013 & 0.250933 & -1.93273 & 2.674045 & 0.199694 & -2.68353 & -3.14018 & 0.289751 & -4.36262 & -2.64401 & 0.266038 & -5.11477 \\
\hline 123 & 3.622498 & 0.250928 & -3.58417 & 2.807103 & 0.26227 & -3.06795 & -0.68574 & 0.791607 & -6.01238 & -0.55806 & 0.951115 & -5.57731 \\
\hline 124 & 3.62321 & 0.250934 & -2.75888 & 2.944939 & 0.335295 & -2.81406 & -0.10609 & 0.791604 & -6.01232 & 0.006759 & 1.061035 & -5.54795 \\
\hline 125 & 3.623908 & 0.250937 & -1.93316 & 2.939778 & 0.232438 & -2.46151 & -1.26542 & 0.791601 & -6.01254 & -1.22655 & 0.846087 & -5.61512 \\
\hline 126 & -3.6225 & 0.752784 & -3.58416 & -2.91366 & 0.654929 & -2.98319 & -0.97559 & 0.289747 & -6.01245 & -1.14163 & 0.298007 & -5.61312 \\
\hline 127 & -3.62321 & 0.752799 & -2.75888 & -2.87503 & 0.950187 & -2.82127 & -0.39592 & 0.289745 & -6.01234 & -0.4115 & 0.456737 & -5.60561 \\
\hline 128 & -3.62392 & 0.752806 & -1.93316 & -2.9908 & 0.67995 & -2.37742 & -0.68592 & 0.791617 & -5.18708 & -1.17377 & 1.028937 & -5.52392 \\
\hline 129 & -3.04292 & 0.752784 & -3.58374 & -2.63235 & 0.692569 & -2.92111 & -0.10611 & 0.791619 & -5.18702 & -0.22967 & 0.617616 & -5.55758 \\
\hline 130 & -3.04347 & 0.7528 & -2.75845 & -2.52328 & 0.965475 & -2.90043 & -1.26573 & 0.791622 & -5.18724 & -1.59886 & 0.830601 & -5.50241 \\
\hline 131 & -3.04401 & 0.752808 & -1.93273 & -2.78117 & 0.656755 & -2.67289 & -0.97583 & 0.289751 & -5.18715 & -1.49005 & 0.499568 & -5.50518 \\
\hline 132 & -2.46333 & 0.75278 & -3.58342 & -2.29774 & 0.693713 & -2.99743 & -0.39602 & 0.289751 & -5.18704 & -0.80984 & 0.348033 & -5.56667 \\
\hline 133 & -2.46374 & 0.752801 & -2.75813 & -2.25443 & 0.928677 & -2.72564 & -0.68609 & 0.791622 & -4.36136 & -0.8562 & 0.667061 & -5.5831 \\
\hline 134 & -2.46414 & 0.752811 & -1.93241 & -2.52127 & 0.629617 & -2.81537 & -0.10614 & 0.791627 & -4.3613 & 0.349544 & 0.696358 & -5.59788 \\
\hline 135 & -1.88372 & 0.752794 & -3.58318 & -1.92565 & 0.919216 & -2.87973 & -1.26603 & 0.791631 & -4.36151 & -1.64695 & 0.549872 & -5.45051 \\
\hline 136 & -1.88402 & 0.752789 & -2.75789 & -1.74594 & 0.616501 & -2.942 & -0.97606 & 0.289753 & -4.36142 & -1.07476 & 0.25814 & -5.55291 \\
\hline 137 & -1.88431 & 0.752784 & -1.93217 & -2.00258 & 0.80116 & -2.8081 & -0.39611 & 0.289755 & -4.36132 & -0.34896 & 0.16145 & -5.69586 \\
\hline 138 & -1.30412 & 0.75278 & -3.58301 & -1.25906 & 0.643007 & -3.04944 & 2.057221 & 0.791599 & -6.01289 & 1.88021 & 0.648041 & -5.47579 \\
\hline 139 & -1.30432 & 0.752802 & -2.75773 & -1.07267 & 0.899952 & -2.92565 & 2.636842 & 0.791605 & -6.01325 & 2.252306 & 0.761214 & -5.3851 \\
\hline 140 & -1.30452 & 0.752811 & -1.932 & -1.48643 & 0.836181 & -3.00115 & 1.477581 & 0.791617 & -6.01262 & 1.656394 & 0.942845 & -5.50276 \\
\hline 141 & -0.72451 & 0.752784 & -3.58291 & -0.80155 & 0.800576 & -3.08415 & 1.767409 & 0.289747 & -6.01274 & 1.540706 & 0.141387 & -5.46951 \\
\hline 142 & -0.72462 & 0.752799 & -2.75762 & -0.28579 & 0.837456 & -3.00761 & 2.34703 & 0.289747 & -6.01306 & 1.918346 & 0.318784 & -5.3543 \\
\hline 143 & -0.72473 & 0.752809 & -1.9319 & -0.81185 & 0.6538 & -3.00581 & 2.057703 & 0.791622 & -5.18759 & 2.201324 & 1.029696 & -5.33687 \\
\hline 144 & -0.14491 & 0.752789 & -3.58287 & -0.2545 & 0.656702 & -3.13591 & 2.637434 & 0.791618 & -5.18795 & 2.532902 & 1.028219 & -5.42864 \\
\hline 145 & -0.14492 & 0.752796 & -2.75758 & 0.138265 & 0.972489 & -2.95962 & 1.477936 & 0.791609 & -5.18731 & 1.053869 & 0.904511 & -5.47865 \\
\hline
\end{tabular}


Table M-3. Centroid locations from [46] for the 6 in. POP containers (continued).

\begin{tabular}{|c|c|c|c|c|c|c|c|c|c|c|c|c|}
\hline \multicolumn{13}{|c|}{6 in. POP containers } \\
\hline \multirow[b]{3}{*}{ Centroid } & \multicolumn{6}{|c|}{ Upper horizon triangular array } & \multicolumn{6}{|c|}{ Lower horizon hexagonal array } \\
\hline & \multicolumn{3}{|c|}{$\mathbf{t}=\mathbf{0}$ year } & \multicolumn{3}{|c|}{$t=1,000$ years } & \multicolumn{3}{|c|}{$\mathrm{t}=\mathbf{0}$ year } & \multicolumn{3}{|c|}{$t=1,000$ years } \\
\hline & $\mathbf{x}(\mathbf{m})$ & $\mathbf{y}(\mathbf{m})$ & $\mathbf{z}(\mathbf{m})$ & $\mathbf{x}(\mathbf{m})$ & $\mathbf{y}(\mathbf{m})$ & $\mathbf{z}(\mathbf{m})$ & $\mathbf{x}(\mathbf{m})$ & $\mathbf{y}(\mathbf{m})$ & $\mathbf{z}(\mathbf{m})$ & $\mathbf{x}(\mathbf{m})$ & $\mathbf{y}(\mathbf{m})$ & $\mathbf{z}(\mathbf{m})$ \\
\hline 146 & -0.14494 & 0.7528 & -1.93186 & -0.03111 & 0.614136 & -3.03327 & 1.767828 & 0.289752 & -5.18744 & 1.828943 & 0.581206 & -5.39108 \\
\hline 147 & 0.434711 & 0.752786 & -3.58288 & 0.242697 & 0.88485 & -3.10876 & 2.347572 & 0.289754 & -5.18776 & 2.182465 & 0.48664 & -5.50438 \\
\hline 148 & 0.434775 & 0.7528 & -2.75759 & 0.700076 & 0.91009 & -3.11373 & 2.058173 & 0.79163 & -4.36187 & 2.37724 & 0.700741 & -5.36926 \\
\hline 149 & 0.43484 & 0.752808 & -1.93187 & 0.429908 & 0.713551 & -3.15746 & 2.638024 & 0.791623 & -4.36223 & 2.541214 & 0.715279 & -5.28838 \\
\hline 150 & 1.01432 & 0.752793 & -3.58295 & 1.060789 & 0.647593 & -2.9845 & 1.478281 & 0.791607 & -4.36159 & 1.803836 & 0.86741 & -5.48695 \\
\hline 151 & 1.014468 & 0.752793 & -2.75767 & 0.982485 & 0.922335 & -2.93716 & 1.768241 & 0.289753 & -4.36172 & 2.023802 & 0.026891 & -5.31925 \\
\hline 152 & 1.014611 & 0.752795 & -1.93194 & 1.38683 & 0.572603 & -2.95819 & 2.348106 & 0.289755 & -4.36204 & 2.491562 & 0.208756 & -5.28205 \\
\hline 153 & 1.593922 & 0.752782 & -3.58309 & 1.572962 & 0.631092 & -3.06307 & & & & & & \\
\hline 154 & 1.594174 & 0.752803 & -2.7578 & 1.389791 & 0.958604 & -2.99083 & & & & & & \\
\hline 155 & 1.594414 & 0.752815 & -1.93208 & 1.977935 & 0.598896 & -2.98487 & & & & & & \\
\hline 156 & 2.17353 & 0.75278 & -3.58329 & 2.04346 & 0.604497 & -3.02278 & & & & & & \\
\hline 157 & 2.173876 & 0.752803 & -2.758 & 1.892546 & 0.931721 & -2.90148 & & & & & & \\
\hline 158 & 2.174221 & 0.752815 & -1.93228 & 2.235544 & 0.633227 & -2.76293 & & & & & & \\
\hline 159 & 2.753121 & 0.752791 & -3.58357 & 2.35051 & 0.627318 & -2.96602 & & & & & & \\
\hline 160 & 2.753597 & 0.752797 & -2.75828 & 2.213935 & 0.927412 & -2.83138 & & & & & & \\
\hline 161 & 2.754067 & 0.752799 & -1.93256 & 2.585767 & 0.543909 & -2.72115 & & & & & & \\
\hline 162 & 3.332712 & 0.752782 & -3.58393 & 2.802007 & 0.605801 & -3.06741 & & & & & & \\
\hline 163 & 3.333338 & 0.752806 & -2.75865 & 2.608336 & 0.940514 & -2.88407 & & & & & & \\
\hline 164 & 3.333956 & 0.752815 & -1.93293 & 2.841664 & 0.601991 & -2.48087 & & & & & & \\
\hline 165 & 3.912311 & 0.752783 & -3.58444 & 3.143062 & 0.663047 & -3.13894 & & & & & & \\
\hline 166 & 3.913192 & 0.752801 & -2.75915 & 2.924898 & 0.887031 & -2.81574 & & & & & & \\
\hline 167 & 3.914066 & 0.752806 & -1.93343 & 3.096061 & 0.660652 & -2.29168 & & & & & & \\
\hline
\end{tabular}


Table M-4. Centroid locations from [46] for the 12 in. POP containers.

\begin{tabular}{|c|c|c|c|c|c|c|c|c|c|c|c|c|}
\hline \multicolumn{13}{|c|}{12 in. POP containers } \\
\hline \multirow[b]{3}{*}{ Centroid } & \multicolumn{6}{|c|}{ Upper horizon triangular array } & \multicolumn{6}{|c|}{ Lower horizon hexagonal array } \\
\hline & \multicolumn{2}{|c|}{$t=0$ year } & \multirow[b]{2}{*}{$\mathrm{z}(\mathrm{m})$} & \multicolumn{3}{|c|}{$t=1,000$ years } & \multicolumn{2}{|c|}{$\mathbf{t}=\mathbf{0}$ year } & \multirow[b]{2}{*}{$\mathrm{z}(\mathrm{m})$} & \multicolumn{3}{|c|}{$t=1,000$ years } \\
\hline & $\mathbf{x}(\mathrm{m})$ & $\mathbf{y}(\mathbf{m})$ & & $\mathbf{x}(\mathrm{m})$ & $\mathbf{y}(\mathbf{m})$ & $\mathrm{z}(\mathrm{m})$ & $\mathbf{x}(\mathrm{m})$ & y (m) & & $\mathbf{x}(\mathrm{m})$ & $\mathbf{y}(\mathbf{m})$ & $\mathrm{z}(\mathrm{m})$ \\
\hline 0 & -3.91236 & -0.75279 & -3.54958 & -3.12169 & -0.71012 & -3.16098 & -3.42859 & -0.7916 & -5.97904 & -2.66045 & & \\
\hline 1 & -3.91324 & -0.7528 & -2.72436 & & & & -2.8 & & -5.97855 & -2.24666 & & \\
\hline 2 & -3.91412 & 0.75281 & -1.89864 & 569 & 0.7681 & -2.14626 & -3.13881 & -0.28975 & -5.97 & 26 & & \\
\hline 3 & -3.33275 & -0.75278 & -3.54908 & -2.75044 & -0.8472 & -3.01219 & -3.71837 & -0.28975 & -5.97932 & -2.96957 & -0.22511 & -5.5 \\
\hline 4 & -3.33337 & -0.75281 & -2.72386 & -2.74386 & -0.74247 & -2.57811 & -4.00816 & -0.79161 & -5.97964 & -3.00482 & -0.7 & \\
\hline 5 & -3.33 & -0.75282 & -1.8 & -3.0 & -0.6 & -2 & -3 & -0.7 & -5 & -2.5 & & \\
\hline 6 & -2.7 & -0.7 & -3 . & & -0.7 & & -2.8 & -0 & & & & \\
\hline 7 & -2.7 & & -2. & & & & -3.13 & & & 79 & & \\
\hline 8 & -2.75408 & -0.7528 & -1.89777 & -2.51791 & -0.52181 & -2 . & -3.7 & -0.28975 & -5 . & -2.7 & & \\
\hline 9 & -2.17 & -0.75278 & -3.54 & -1.8068 & -0.75783 & -3.0 & -4.00 & -0.7 & -5 . & & & \\
\hline 10 & -2.17389 & -0.75281 & -2.72321 & -2.19497 & -0.90476 & -2 & -3.4 & -0 . & -4 . & -2 . & & -4 \\
\hline 11 & & & -1 . & & & & -2 & & & & & \\
\hline 12 & -1.5 & & -3 . & & -0 . & & -3.1 & -0 & 32 & & & \\
\hline 13 & -1.59418 & -0.75281 & -2.72 & -1.90 & -0.5104 & -2.72417 & -3.72 & -0.28975 & -4.3 & -3. & & -4.7 \\
\hline 14 & -1.59443 & -0.75282 & -1.89729 & -1.74514 & -0.94518 & -2.65205 & -4.01 & & -4.32 & -3.1 & & \\
\hline 15 & -1.0 & & & & & & -2.0 & 0 & & & & \\
\hline 16 & -1.0 & & -2. & & 58 & & -1. & 0 & -5 . & & & \\
\hline 17 & -1.0 & -0 . & -1.8 & -1.0 & -0.82 & -2 & -1.7 & 871 & -5 . & 47 & 268 & 27 \\
\hline 18 & -0.43471 & -0.75279 & -3.54802 & -0.1907 & -0.681 & -2.98563 & -2.34706 & 0.501862 & -5.97821 & -2.0651 & 0.428444 & -5.60533 \\
\hline 19 & -0.43478 & -0.7528 & -2.7228 & -0.56792 & -1.0182 & -2.81299 & -2.63688 & 0 & -5.9784 & -2.10175 & 0.236863 & -5.62588 \\
\hline 20 & -0.43 & 75281 & -1.8 & & .5571 & & -2.34 & 186 & 21 & -1 & & \\
\hline 21 & 0.14 & -0.75279 & -3.54 & -0.07548 & -0.86744 & -3.00907 & -1.76744 & 187 & $-5.9^{\prime}$ & -1.4 & -0 & -5 \\
\hline 22 & 0.144925 & -0.7528 & -2.72279 & 0.346023 & -0.9468 & -2.95021 & -2.05773 & 0 & -5.15281 & -1.77429 & 0.169107 & -5.3 \\
\hline 23 & 0.144944 & -0.7528 & -1.89707 & 0.235886 & -0.53603 & -2.91957 & -1.47796 & 0 & -5.15253 & -1.56743 & 0.255982 & -5.46807 \\
\hline 24 & 0.724511 & -0.75278 & -3.54805 & 0.769029 & -0.88126 & -3.04569 & -1.76784 & 0.501853 & -5.15266 & -1.73324 & 0.884671 & -5.3121 \\
\hline 25 & & & -2.72 & & & & & & & & & \\
\hline 26 & 0.724737 & -0.75281 & -1.89711 & 0.660288 & -0.61214 & -2.90741 & -2.63747 & $-1 \mathrm{E}-06$ & -5.15317 & -2.29009 & -0.14847 & -5.43753 \\
\hline 27 & 1.304137 & -0.75278 & -3.54816 & 1.222922 & -0.6656 & -3.00436 & -2.34761 & -0.50186 & -5.15298 & -2.06032 & -0.29746 & -5.3849 \\
\hline 28 & 1.304331 & -0.7528 & -2.72293 & 1.382151 & -0.93775 & -2.67087 & -1.76784 & -0.50185 & -5.15266 & -1.85058 & -0.18147 & -5.38606 \\
\hline
\end{tabular}




\begin{tabular}{|c|c|c|c|c|c|c|c|c|c|c|c|c|}
\hline \multicolumn{13}{|c|}{12 in. POP containers } \\
\hline \multirow[b]{3}{*}{ Centroid } & \multicolumn{6}{|c|}{ Upper horizon triangular array } & \multicolumn{6}{|c|}{ Lower horizon hexagonal array } \\
\hline & \multicolumn{2}{|c|}{$t=0$ year } & & \multicolumn{3}{|c|}{$t=1,000$ years } & \multicolumn{2}{|c|}{$t=0$ year } & \multicolumn{4}{|c|}{$t=1,000$ years } \\
\hline & $\mathbf{x}(\mathbf{m})$ & $y(m)$ & $\mathrm{z}(\mathbf{m})$ & $\mathbf{x}(\mathrm{m})$ & $y(m)$ & $\mathrm{z}(\mathbf{m})$ & $\mathbf{x}(\mathbf{m})$ & $y(m)$ & $\mathrm{z}(\mathbf{m})$ & $\mathbf{x}(\mathbf{m})$ & $y(m)$ & $\mathrm{z}(\mathbf{m})$ \\
\hline 29 & 1.304527 & -0.75281 & -1.89721 & 1.674747 & -0.70761 & -2.66778 & -2.0582 & 0.000002 & -4.32707 & -2.13539 & 0.033748 & -5.14946 \\
\hline
\end{tabular}


Table M-4. Centroid locations from [46] for the 12 in. POP containers (continued).

\begin{tabular}{|c|c|c|c|c|c|c|c|c|c|c|c|c|}
\hline \multicolumn{13}{|c|}{12 in. POP containers } \\
\hline \multirow[b]{3}{*}{ Centroid } & \multicolumn{6}{|c|}{ Upper horizon triangular array } & \multicolumn{6}{|c|}{ Lower horizon hexagonal array } \\
\hline & \multicolumn{2}{|c|}{$t=0$ year } & & \multicolumn{3}{|c|}{$t=1,000$ years } & \multicolumn{2}{|c|}{$\mathbf{t}=\mathbf{0}$ year } & \multicolumn{4}{|c|}{$t=1,000$ years } \\
\hline & $\mathbf{x}(\mathbf{m})$ & $\mathbf{y}(\mathbf{m})$ & $\mathbf{z}(\mathbf{m})$ & $\mathbf{x}(\mathbf{m})$ & $\mathbf{y}(\mathbf{m})$ & $\mathbf{z}(\mathbf{m})$ & $\mathbf{x}(\mathbf{m})$ & y (m) & $\mathbf{z}(\mathbf{m})$ & $\mathbf{x}(\mathbf{m})$ & y (m) & $\mathbf{z}(\mathbf{m})$ \\
\hline 30 & 1.883742 & -0.7528 & -3.54832 & 1.655704 & -0.80199 & -2.8387 & -1.4783 & 0 & -4.3268 & -1.40413 & -0.23416 & -5.32997 \\
\hline 31 & 1.884033 & -0.75279 & -2.7231 & 2.202358 & -0.79516 & -3.08576 & -1.76825 & 0.501843 & -4.32692 & -1.79956 & 0.470528 & -5.26112 \\
\hline 32 & 1.884326 & -0.75279 & -1.89738 & 2.031082 & -0.87263 & -2.62744 & -2.34815 & 0.501857 & -4.32724 & -2.32389 & 0.379396 & -5.12191 \\
\hline 33 & 2.463347 & -0.75278 & -3.54856 & 2.458787 & -0.64892 & -3.06956 & -2.63807 & $-1 \mathrm{E}-06$ & -4.32744 & -2.48341 & 0.057502 & -5.02471 \\
\hline 34 & 2.463752 & -0.75281 & -2.72334 & 2.433864 & -0.91434 & -2.67755 & -2.34815 & -0.50186 & -4.32724 & -2.28974 & -0.47604 & -5.08747 \\
\hline 35 & 2.464159 & -0.75281 & -1.89762 & 2.351707 & -0.67635 & -2.49462 & -1.76825 & -0.50184 & -4.32692 & -1.76574 & -0.62318 & -5.30671 \\
\hline 36 & 3.042958 & -0.75278 & -3.54888 & 2.689908 & -0.68311 & -2.97788 & -0.68576 & -0.79161 & -5.97753 & -0.45825 & -0.79518 & -5.50834 \\
\hline 37 & 3.04349 & -0.7528 & -2.72366 & 2.626242 & -0.89515 & -2.5278 & -0.1061 & -0.7916 & -5.97747 & 0.150351 & -0.8071 & -5.51402 \\
\hline 38 & 3.04403 & -0.75281 & -1.89794 & 2.841625 & -0.60094 & -2.32349 & -0.39593 & -0.28975 & -5.97749 & -0.36206 & -0.18277 & -5.52576 \\
\hline 39 & 3.622536 & -0.75279 & -3.54931 & 2.884299 & -0.85956 & -2.99822 & -0.97562 & -0.28975 & -5.9776 & -0.73587 & -0.30114 & -5.53523 \\
\hline 40 & 3.623245 & -0.7528 & -2.72409 & 3.021945 & -0.83256 & -2.71156 & -1.26544 & -0.7916 & -5.97768 & -1.13303 & -0.6503 & -5.47088 \\
\hline 41 & 3.623956 & -0.75281 & -1.89837 & 3.101355 & -0.74449 & -2.19215 & -0.68593 & -0.79162 & -5.1523 & -0.97315 & -0.99921 & -5.39529 \\
\hline 42 & -3.62254 & -0.25093 & -3.54931 & -3.13444 & -0.25795 & -3.12413 & -0.10611 & -0.79162 & -5.15223 & -0.03671 & -0.41183 & -5.55513 \\
\hline 43 & -3.62324 & -0.25094 & -2.72409 & -2.9341 & -0.34883 & -2.68619 & -0.39602 & -0.28975 & -5.15225 & -0.79066 & 0.080148 & -5.45667 \\
\hline 44 & -3.62396 & -0.25094 & -1.89837 & -3.14288 & -0.23275 & -2.25546 & -0.97584 & -0.28975 & -5.15236 & -1.18374 & 0.043004 & -5.52197 \\
\hline 45 & -3.04296 & -0.25093 & -3.54888 & -2.69708 & -0.24813 & -3.01024 & -1.26574 & -0.79162 & -5.15245 & -1.56277 & -1.00868 & -5.40384 \\
\hline 46 & -3.0435 & -0.25094 & -2.72366 & -2.74096 & -0.33767 & -2.67301 & -0.68609 & -0.79162 & -4.32656 & -0.84886 & -0.62399 & -5.4537 \\
\hline 47 & -3.04404 & -0.25094 & -1.89794 & -2.74047 & -0.20051 & -2.34344 & -0.10613 & -0.79163 & -4.3265 & -0.26762 & -0.92926 & -5.50626 \\
\hline 48 & -2.46335 & -0.25093 & -3.54856 & -2.19243 & -0.25933 & -3.08078 & -0.39611 & -0.28976 & -4.32652 & -0.4489 & -0.4941 & -5.39689 \\
\hline 49 & -2.46375 & -0.25094 & -2.72334 & -2.49327 & -0.56007 & -2.84673 & -0.97607 & -0.28976 & -4.32663 & -1.11058 & -0.36349 & -5.44978 \\
\hline 50 & -2.46416 & -0.25094 & -1.89762 & -2.35697 & -0.15481 & -2.56562 & -1.26603 & -0.79164 & -4.32672 & -1.36177 & -0.57358 & -5.31442 \\
\hline 51 & -1.88374 & -0.25093 & -3.54832 & -1.55336 & -0.17829 & -2.91606 & 0.685749 & 0 & -5.97753 & 0.416251 & 0.181325 & -5.49574 \\
\hline 52 & -1.88404 & -0.25094 & -2.7231 & -2.06299 & -0.34837 & -2.95411 & 1.265441 & 0 & -5.97768 & 1.31229 & 0.017544 & -5.64537 \\
\hline 53 & -1.88433 & -0.25094 & -1.89738 & -1.98919 & -0.17968 & -2.66378 & 0.975621 & 0.501866 & -5.97759 & 1.112264 & 0.625149 & -5.49708 \\
\hline 54 & -1.30414 & -0.25093 & -3.54816 & -1.02879 & -0.11062 & -3.04091 & 0.395924 & 0.501866 & -5.97749 & 0.43624 & 0.646254 & -5.49063 \\
\hline 55 & -1.30433 & -0.25093 & -2.72293 & -1.53873 & -0.12729 & -2.87457 & 0.106103 & 0 & -5.97746 & 0.060221 & 0.116966 & -5.65622 \\
\hline 56 & -1.30453 & -0.25093 & -1.89721 & -1.5639 & -0.33488 & -2.61102 & 0.395924 & -0.50187 & -5.97749 & 0.486118 & -0.73141 & -5.61383 \\
\hline 57 & -0.72451 & -0.25093 & -3.54806 & -0.57698 & -0.12899 & -2.95595 & 0.975621 & -0.50187 & -5.97759 & 0.920857 & -0.53434 & -5.4854 \\
\hline 58 & -0.72462 & -0.25093 & -2.72283 & -0.96251 & -0.53479 & -2.98338 & 0.685928 & 0 & -5.1523 & 1.085968 & -0.13843 & -5.53514 \\
\hline
\end{tabular}


Table M-4. Centroid locations from [46] for the 12 in. POP containers (continued).

\begin{tabular}{|c|c|c|c|c|c|c|c|c|c|c|c|c|}
\hline \multicolumn{13}{|c|}{12 in. $P O P$ containers } \\
\hline \multirow[b]{3}{*}{ Centroid } & \multicolumn{6}{|c|}{ Upper horizon triangular array } & \multicolumn{6}{|c|}{ Lower horizon hexagonal array } \\
\hline & \multicolumn{2}{|c|}{$\mathrm{t}=\mathbf{0}$ year } & \multirow[b]{2}{*}{$\mathbf{z}(\mathbf{m})$} & \multicolumn{3}{|c|}{$t=1,000$ years } & \multicolumn{2}{|c|}{$\mathbf{t}=\mathbf{0}$ year } & \multirow[b]{2}{*}{$\mathbf{z}(\mathbf{m})$} & \multicolumn{3}{|c|}{$t=1,000$ years } \\
\hline & $\mathbf{x}(\mathbf{m})$ & $\mathbf{y}(\mathbf{m})$ & & $\mathbf{x}(\mathbf{m})$ & $\mathbf{y}(\mathbf{m})$ & $\mathbf{z}(\mathbf{m})$ & $\mathbf{x}(\mathbf{m})$ & $\mathbf{y}(\mathbf{m})$ & & $\mathbf{x}(\mathbf{m})$ & $\mathbf{y}(\mathbf{m})$ & $\mathbf{z}(\mathbf{m})$ \\
\hline 59 & -0.72473 & -0.25093 & -1.89711 & -0.92115 & -0.18269 & -2.77471 & 1.265732 & 0 & -5.15245 & 1.516822 & -0.3412 & -5.32357 \\
\hline 60 & -0.14491 & -0.25093 & -3.54801 & -0.01599 & -0.40295 & -2.93399 & 0.975833 & 0.501856 & -5.15236 & 1.298201 & 0.280059 & -5.40016 \\
\hline 61 & -0.14493 & -0.25093 & -2.72279 & -0.45526 & -0.35477 & -2.82364 & 0.396009 & 0.501852 & -5.15225 & 0.902006 & 0.801115 & -5.53241 \\
\hline 62 & -0.14495 & -0.25093 & -1.89707 & 0.026034 & -0.08748 & -2.87611 & 0.106108 & 0 & -5.15224 & 0.330125 & -0.27696 & -5.52664 \\
\hline 63 & 0.434713 & -0.25093 & -3.54803 & 0.472103 & -0.4282 & -3.05045 & 0.396007 & -0.50185 & -5.15225 & 0.904208 & -0.95483 & -5.43665 \\
\hline 64 & 0.434781 & -0.25093 & -2.7228 & 0.204102 & -0.20198 & -2.81096 & 0.975832 & -0.50186 & -5.15236 & 1.361483 & -0.6685 & -5.25979 \\
\hline 65 & 0.434846 & -0.25093 & -1.89708 & 0.644938 & -0.14819 & -2.87681 & 0.686086 & 0.000001 & -4.32656 & 0.717916 & 0.064012 & -5.47077 \\
\hline 66 & 1.014332 & -0.25093 & -3.5481 & 0.931435 & -0.31768 & -2.88799 & 1.266025 & 0 & -4.32672 & 1.202849 & 0.019799 & -5.26197 \\
\hline 67 & 1.014479 & -0.25093 & -2.72287 & 1.022021 & -0.09249 & -2.67501 & 0.976057 & 0.501852 & -4.32663 & 0.901535 & 0.427393 & -5.36124 \\
\hline 68 & 1.014629 & -0.25093 & -1.89715 & 1.617822 & -0.19747 & -2.75382 & 0.396093 & 0.501847 & -4.32652 & 0.242528 & 0.495601 & -5.50702 \\
\hline 69 & 1.593937 & -0.25093 & -3.54823 & 1.156296 & -0.3287 & -2.86236 & 0.106126 & 0 & -4.3265 & 0.001143 & 0.180805 & -5.46508 \\
\hline 70 & 1.594179 & -0.25093 & -2.72301 & 1.797167 & -0.34679 & -2.94253 & 0.396093 & -0.50185 & -4.32652 & 0.420438 & -0.39856 & -5.51768 \\
\hline 71 & 1.594423 & -0.25094 & -1.89729 & 1.936065 & -0.47257 & -2.53365 & 0.976058 & -0.50185 & -4.32663 & 0.737592 & -0.33634 & -5.47857 \\
\hline 72 & 2.173551 & -0.25093 & -3.54844 & 1.955452 & -0.11432 & -3.04477 & 2.057251 & -0.7916 & -5.97804 & 1.713645 & -0.84601 & -5.56193 \\
\hline 73 & 2.173895 & -0.25093 & -2.72321 & 2.458473 & -0.42627 & -2.89281 & 2.636879 & -0.79161 & -5.9784 & 2.323647 & -0.49468 & -5.51515 \\
\hline 74 & 2.174241 & -0.25094 & -1.89749 & 2.163383 & -0.34681 & -2.60979 & 2.347062 & -0.28975 & -5.97821 & 1.947333 & -0.48105 & -5.61581 \\
\hline 75 & 2.753148 & -0.25093 & -3.54871 & 2.48637 & -0.08547 & -2.9734 & 1.767441 & -0.28975 & -5.97789 & 1.473046 & -0.34578 & -5.57099 \\
\hline 76 & 2.753612 & -0.25094 & -2.72349 & 2.690075 & -0.13212 & -2.65433 & 1.47761 & -0.79162 & -5.97776 & 1.250347 & -0.80572 & -5.49017 \\
\hline 77 & 2.754078 & -0.25094 & -1.89777 & 2.492678 & -0.39595 & -2.45262 & 2.057727 & -0.79162 & -5.15281 & 2.200328 & -0.88027 & -5.5208 \\
\hline 78 & 3.332748 & -0.25093 & -3.54908 & 2.781039 & -0.35136 & -3.03727 & 2.63747 & -0.79162 & -5.15317 & 2.443994 & -0.98587 & -5.44012 \\
\hline 79 & 3.333368 & -0.25094 & -2.72386 & 2.774961 & -0.15828 & -2.65351 & 2.347604 & -0.28976 & -5.15298 & 2.064545 & -0.17242 & -5.35263 \\
\hline 80 & 3.333992 & -0.25094 & -1.89814 & 2.970317 & -0.27416 & -2.25546 & 1.76785 & -0.28975 & -5.15266 & 1.878651 & -0.54812 & -5.39788 \\
\hline 81 & 3.912359 & -0.25093 & -3.54958 & 3.077755 & -0.00885 & -3.13467 & 1.477952 & -0.79161 & -5.15253 & 1.709485 & -0.96984 & -5.28554 \\
\hline 82 & 3.913238 & -0.25094 & -2.72436 & 3.050907 & -0.26484 & -2.76841 & 2.058201 & -0.79164 & -4.32707 & 2.018609 & -0.75137 & -5.15254 \\
\hline 83 & 3.91412 & -0.25094 & -1.89864 & 3.172256 & -0.25688 & -2.1575 & 2.638068 & -0.79163 & -4.32744 & 2.448851 & -0.77692 & -5.03867 \\
\hline 84 & -3.91236 & 0.250928 & -3.54958 & -3.06125 & 0.192525 & -3.18749 & 2.348141 & -0.28976 & -4.32724 & 2.279582 & -0.50433 & -5.06519 \\
\hline 85 & -3.91324 & 0.250936 & -2.72436 & -3.08003 & 0.177958 & -2.75432 & 1.768262 & -0.28976 & -4.32692 & 1.761915 & -0.18123 & -5.24418 \\
\hline 86 & -3.91412 & 0.250935 & -1.89864 & -3.13211 & 0.264656 & -2.21189 & 1.478297 & -0.7916 & -4.3268 & 1.557701 & -0.55173 & -5.15323 \\
\hline 87 & -3.33275 & 0.250927 & -3.54908 & -2.78157 & 0.477429 & -2.97214 & 3.428595 & 0 & -5.97904 & 2.682308 & 0.022021 & -5.54087 \\
\hline
\end{tabular}


Table M-4. Centroid locations from [46] for the 12 in. POP containers (continued).

\begin{tabular}{|c|c|c|c|c|c|c|c|c|c|c|c|c|}
\hline \multicolumn{13}{|c|}{12 in. POP containers } \\
\hline \multirow[b]{3}{*}{ Centroid } & \multicolumn{6}{|c|}{ Upper horizon triangular array } & \multicolumn{6}{|c|}{ Lower horizon hexagonal array } \\
\hline & \multicolumn{2}{|c|}{$\mathrm{t}=\mathbf{0}$ year } & & \multicolumn{3}{|c|}{$t=1,000$ years } & \multicolumn{2}{|c|}{$\mathbf{t}=\mathbf{0}$ year } & \multicolumn{4}{|c|}{$t=1,000$ years } \\
\hline & $\mathbf{x}(\mathbf{m})$ & $\mathbf{y}(\mathbf{m})$ & $\mathbf{z}(\mathbf{m})$ & $\mathbf{x}(\mathbf{m})$ & $\mathbf{y}(\mathbf{m})$ & $\mathbf{z}(\mathbf{m})$ & $\mathbf{x}(\mathbf{m})$ & $\mathbf{y}(\mathbf{m})$ & $\mathbf{z}(\mathbf{m})$ & $\mathbf{x}(\mathbf{m})$ & $\mathbf{y}(\mathbf{m})$ & $\mathbf{z}(\mathbf{m})$ \\
\hline 88 & -3.33337 & 0.250936 & -2.72386 & -2.84419 & -0.0031 & -2.71586 & 4.008152 & 0 & -5.97964 & 2.985804 & 0.019081 & -5.5332 \\
\hline 89 & -3.33399 & 0.250935 & -1.89814 & -2.91229 & 0.297618 & -2.40701 & 3.718369 & 0.501866 & -5.97932 & 2.905028 & 0.490525 & -5.45706 \\
\hline 90 & -2.75315 & 0.250928 & -3.54871 & -2.47819 & 0.468301 & -2.93111 & 3.138808 & 0.501857 & -5.97878 & 2.625752 & 0.380749 & -5.45023 \\
\hline 91 & -2.75361 & 0.250937 & -2.72349 & -2.60457 & 0.006326 & -2.77111 & 2.84901 & 0 & -5.97855 & 2.359 & 0.036794 & -5.57522 \\
\hline 92 & -2.75408 & 0.250936 & -1.89777 & -2.54882 & 0.394392 & -2.51488 & 3.138808 & -0.50186 & -5.97878 & 2.510952 & -0.33055 & -5.4542 \\
\hline 93 & -2.17355 & 0.250928 & -3.54844 & -1.96009 & 0.523689 & -2.92498 & 3.71837 & -0.50187 & -5.97932 & 2.865059 & -0.40524 & -5.47591 \\
\hline 94 & -2.17389 & 0.250934 & -2.72321 & -2.34122 & -0.06433 & -2.95736 & 3.429376 & 0 & -5.15381 & 2.513751 & -0.08001 & -5.22955 \\
\hline 95 & -2.17424 & 0.250937 & -1.89749 & -2.16515 & 0.273794 & -2.6583 & 4.009198 & 0 & -5.15442 & 2.880893 & -0.05623 & -5.1763 \\
\hline 96 & -1.59394 & 0.250929 & -3.54823 & -1.3454 & 0.329858 & -3.00747 & 3.719264 & 0.501852 & -5.15409 & 2.78676 & 0.898975 & -5.16707 \\
\hline 97 & -1.59418 & 0.250933 & -2.72301 & -1.66665 & 0.392476 & -2.9834 & 3.139526 & 0.501866 & -5.15355 & 2.460111 & 0.505793 & -5.15004 \\
\hline 98 & -1.59442 & 0.250936 & -1.89729 & -1.90679 & 0.134361 & -2.69866 & 2.849679 & 0 & -5.15333 & 2.163522 & 0.036029 & -5.27461 \\
\hline 99 & -1.01433 & 0.250931 & -3.5481 & -1.0053 & 0.514422 & -3.01902 & 3.139527 & -0.50187 & -5.15355 & 2.599956 & -0.80871 & -5.33163 \\
\hline 100 & -1.01448 & 0.250934 & -2.72287 & -1.1105 & 0.107577 & -2.8037 & 3.719264 & -0.50185 & -5.15409 & 2.865276 & -0.88881 & -5.19225 \\
\hline 101 & -1.01463 & 0.25093 & -1.89715 & -1.57207 & 0.194376 & -2.67222 & 3.430157 & 0 & -4.32808 & 2.780665 & 0.110989 & -4.85741 \\
\hline 102 & -0.43471 & 0.250929 & -3.54803 & -0.4469 & 0.265057 & -3.10278 & 4.010225 & 0 & -4.32869 & 3.076107 & 0.053241 & -4.71186 \\
\hline 103 & -0.43478 & 0.250933 & -2.7228 & -0.85219 & 0.239825 & -2.83585 & 3.720149 & 0.501845 & -4.32836 & 3.018568 & 0.595085 & -4.74759 \\
\hline 104 & -0.43485 & 0.250932 & -1.89708 & -0.30745 & 0.228736 & -2.88357 & 3.14024 & 0.501871 & -4.32782 & 2.800643 & 0.470171 & -4.93066 \\
\hline 105 & 0.144913 & 0.25093 & -3.54801 & 0.413574 & 0.073732 & -3.00712 & 2.850333 & 0 & -4.32759 & 2.547663 & 0.02674 & -4.92595 \\
\hline 106 & 0.144928 & 0.250933 & -2.72279 & -0.16472 & 0.236572 & -2.97996 & 3.14024 & -0.50187 & -4.32782 & 2.650004 & -0.40801 & -4.97136 \\
\hline 107 & 0.144946 & 0.25093 & -1.89707 & 0.200818 & 0.369639 & -2.95904 & 3.720149 & -0.50184 & -4.32836 & 2.962743 & -0.4677 & -4.81178 \\
\hline 108 & 0.724511 & 0.250928 & -3.54806 & 0.90383 & 0.083363 & -2.95174 & -3.42859 & 0.791604 & -5.97904 & -2.67989 & 0.706953 & -5.56056 \\
\hline 109 & 0.72462 & 0.250933 & -2.72283 & 0.506805 & 0.393262 & -2.87103 & -2.84901 & 0.791603 & -5.97855 & -2.26555 & 0.712144 & -5.56036 \\
\hline 110 & 0.724731 & 0.250933 & -1.89711 & 1.096664 & 0.408727 & -2.8506 & -4.00816 & 0.791609 & -5.97964 & -2.9979 & 0.761044 & -5.55994 \\
\hline 111 & 1.304136 & 0.250928 & -3.54816 & 1.158867 & 0.088853 & -3.00701 & -3.71837 & 0.28975 & -5.97932 & -2.79418 & 0.122936 & -5.61837 \\
\hline 112 & 1.304334 & 0.250934 & -2.72293 & 1.613132 & 0.26578 & -3.08628 & -3.13881 & 0.289749 & -5.97878 & -2.48544 & 0.454814 & -5.50527 \\
\hline 113 & 1.304529 & 0.250934 & -1.89721 & 1.468625 & 0.232186 & -2.78717 & -3.42938 & 0.79162 & -5.15381 & -2.5568 & 0.9558 & -5.25929 \\
\hline 114 & 1.883744 & 0.250929 & -3.54832 & 1.961509 & 0.421396 & -2.92237 & -2.84967 & 0.791619 & -5.15332 & -2.19968 & 0.939638 & -5.22101 \\
\hline 115 & 1.884034 & 0.250936 & -2.7231 & 1.931244 & -0.12489 & -2.72712 & -4.00919 & 0.791617 & -5.15442 & -2.92093 & 0.771261 & -5.15947 \\
\hline 116 & 1.884328 & 0.250934 & -1.89738 & 1.913564 & 0.381098 & -2.67499 & -3.71926 & 0.28975 & -5.15409 & -2.93933 & 0.337345 & -5.16316 \\
\hline
\end{tabular}


Table M-4. Centroid locations from [46] for the 12 in. POP containers (continued).

\begin{tabular}{|c|c|c|c|c|c|c|c|c|c|c|c|c|}
\hline \multicolumn{13}{|c|}{12 in. $P O P$ containers } \\
\hline \multirow[b]{3}{*}{ Centroid } & \multicolumn{6}{|c|}{ Upper horizon triangular array } & \multicolumn{6}{|c|}{ Lower horizon hexagonal array } \\
\hline & \multicolumn{2}{|c|}{$\mathrm{t}=\mathbf{0}$ year } & & \multicolumn{3}{|c|}{$t=1,000$ years } & \multicolumn{2}{|c|}{$\mathbf{t}=\mathbf{0}$ year } & \multicolumn{4}{|c|}{$t=1,000$ years } \\
\hline & $\mathbf{x}(\mathbf{m})$ & $\mathbf{y}(\mathbf{m})$ & $\mathbf{z}(\mathbf{m})$ & $\mathbf{x}(\mathbf{m})$ & $\mathbf{y}(\mathbf{m})$ & $\mathbf{z}(\mathbf{m})$ & $\mathbf{x}(\mathbf{m})$ & $\mathbf{y}(\mathbf{m})$ & $\mathbf{z}(\mathbf{m})$ & $\mathbf{x}(\mathbf{m})$ & $\mathbf{y}(\mathbf{m})$ & $\mathbf{z}(\mathbf{m})$ \\
\hline 117 & 2.463349 & 0.250926 & -3.54856 & 2.318782 & 0.246136 & -2.88479 & -3.13953 & 0.289752 & -5.15355 & -2.51551 & 0.138164 & -5.36313 \\
\hline 118 & 2.46375 & 0.250936 & -2.72334 & 2.383524 & 0.477025 & -2.59156 & -3.43017 & 0.791629 & -4.32808 & -2.72783 & 0.781352 & -4.85486 \\
\hline 119 & 2.464155 & 0.250937 & -1.89762 & 2.292436 & 0.020472 & -2.52188 & -2.85032 & 0.791631 & -4.32759 & -2.54323 & 0.673603 & -4.99949 \\
\hline 120 & 3.042958 & 0.250928 & -3.54888 & 2.548386 & 0.189475 & -2.90063 & -4.01021 & 0.79162 & -4.32869 & -3.12577 & 0.786447 & -4.6859 \\
\hline 121 & 3.043501 & 0.250935 & -2.72366 & 2.725856 & 0.369299 & -2.60705 & -3.72014 & 0.289753 & -4.32836 & -3.05758 & 0.298216 & -4.6684 \\
\hline 122 & 3.044037 & 0.250935 & -1.89794 & 2.791171 & 0.247124 & -2.30777 & -3.14024 & 0.289755 & -4.32782 & -2.69927 & 0.314759 & -4.94159 \\
\hline 123 & 3.622539 & 0.250928 & -3.54931 & 2.93996 & 0.432671 & -3.14074 & -0.68576 & 0.791606 & -5.97753 & -0.44113 & 0.857951 & -5.53853 \\
\hline 124 & 3.623244 & 0.250937 & -2.72409 & 2.974567 & 0.240715 & -2.69751 & -0.1061 & 0.791604 & -5.97747 & 0.137899 & 0.811433 & -5.55086 \\
\hline 125 & 3.623955 & 0.250938 & -1.89837 & 3.138872 & 0.230508 & -2.19455 & -1.26544 & 0.7916 & -5.97768 & -1.21591 & 0.644822 & -5.57744 \\
\hline 126 & -3.62254 & 0.752787 & -3.54931 & -2.94433 & 0.878782 & -2.98235 & -0.97562 & 0.289747 & -5.9776 & -0.82325 & 0.53944 & -5.61371 \\
\hline 127 & -3.62325 & 0.752805 & -2.72409 & -3.05533 & 0.768002 & -2.67605 & -0.39593 & 0.289749 & -5.97749 & -0.28066 & 0.192112 & -5.64104 \\
\hline 128 & -3.62396 & 0.75281 & -1.89837 & -3.0664 & 0.718952 & -2.13976 & -0.68593 & 0.791616 & -5.1523 & -0.9837 & 1.018876 & -5.41533 \\
\hline 129 & -3.04296 & 0.752784 & -3.54888 & -2.80371 & 0.710526 & -2.93403 & -0.10611 & 0.791621 & -5.15224 & 0.016602 & 0.470308 & -5.52827 \\
\hline 130 & -3.04349 & 0.752804 & -2.72366 & -2.70575 & 0.966254 & -2.55358 & -1.26574 & 0.791623 & -5.15245 & -1.44785 & 1.025177 & -5.42478 \\
\hline 131 & -3.04403 & 0.752811 & -1.89794 & -2.75601 & 0.644666 & -2.36602 & -0.97584 & 0.289753 & -5.15236 & -1.45987 & 0.362982 & -5.50702 \\
\hline 132 & -2.46335 & 0.752778 & -3.54856 & -2.16419 & 0.83137 & -2.96171 & -0.39602 & 0.289753 & -5.15225 & -0.56769 & 0.64082 & -5.46128 \\
\hline 133 & -2.46375 & 0.752805 & -2.72334 & -2.55196 & 0.839225 & -2.9262 & -0.68609 & 0.791623 & -4.32656 & -0.83994 & 0.624155 & -5.37588 \\
\hline 134 & -2.46416 & 0.752813 & -1.89762 & -2.4081 & 0.74881 & -2.50917 & -0.10613 & 0.791629 & -4.3265 & -0.25699 & 0.922007 & -5.46696 \\
\hline 135 & -1.88374 & 0.752795 & -3.54832 & -1.45613 & 0.801935 & -2.84946 & -1.26603 & 0.791635 & -4.32672 & -1.27999 & 0.521797 & -5.27569 \\
\hline 136 & -1.88403 & 0.752787 & -2.7231 & -2.03785 & 0.879039 & -2.83202 & -0.97607 & 0.289756 & -4.32663 & -0.97758 & 0.296017 & -5.41937 \\
\hline 137 & -1.88433 & 0.752788 & -1.89738 & -2.11082 & 0.648579 & -2.60179 & -0.39611 & 0.289758 & -4.32652 & -0.3762 & 0.189984 & -5.36535 \\
\hline 138 & -1.30414 & 0.752782 & -3.54816 & -1.14308 & 0.849718 & -2.89256 & 2.057251 & 0.7916 & -5.97804 & 1.701194 & 0.835923 & -5.60037 \\
\hline 139 & -1.30433 & 0.752803 & -2.72293 & -1.5001 & 0.853414 & -2.68016 & 2.636878 & 0.791606 & -5.9784 & 2.322519 & 0.676432 & -5.56328 \\
\hline 140 & -1.30453 & 0.752809 & -1.89721 & -1.75672 & 0.526568 & -2.73674 & 1.47761 & 0.791615 & -5.97776 & 1.280974 & 0.786964 & -5.58466 \\
\hline 141 & -0.72451 & 0.752784 & -3.54805 & -0.74747 & 0.885863 & -2.92984 & 1.767441 & 0.28975 & -5.97789 & 1.529722 & 0.21706 & -5.49019 \\
\hline 142 & -0.72463 & 0.752802 & -2.72283 & -1.04079 & 0.561187 & -2.74413 & 2.347062 & 0.289747 & -5.97821 & 1.687639 & 0.315408 & -5.48318 \\
\hline 143 & -0.72474 & 0.75281 & -1.89711 & -0.57742 & 0.734919 & -2.82557 & 2.057727 & 0.791624 & -5.15281 & 2.229523 & 0.916176 & -5.55109 \\
\hline 144 & -0.14491 & 0.75279 & -3.54801 & -0.22229 & 0.600576 & -3.05275 & 2.637471 & 0.791619 & -5.15317 & 2.605339 & 0.961959 & -5.37441 \\
\hline 145 & -0.14493 & 0.752798 & -2.72279 & -0.19661 & 0.95655 & -2.91029 & 1.477952 & 0.791607 & -5.15253 & 1.752532 & 0.948267 & -5.35006 \\
\hline
\end{tabular}


Table M-4. Centroid locations from [46] for the 12 in. POP containers (continued).

\begin{tabular}{|c|c|c|c|c|c|c|c|c|c|c|c|c|}
\hline \multicolumn{13}{|c|}{12 in. POP containers } \\
\hline \multirow[b]{3}{*}{ Centroid } & \multicolumn{6}{|c|}{ Upper horizon triangular array } & \multicolumn{6}{|c|}{ Lower horizon hexagonal array } \\
\hline & \multicolumn{2}{|c|}{$t=0$ year } & \multirow[b]{2}{*}{$\mathbf{z}(\mathbf{m})$} & \multicolumn{3}{|c|}{$t=1,000$ years } & \multicolumn{2}{|c|}{$\mathbf{t}=\mathbf{0}$ year } & \multicolumn{4}{|c|}{\begin{tabular}{l|l} 
& $t=1,000$ years \\
\end{tabular}} \\
\hline & $\mathbf{x}(\mathrm{m})$ & $\mathbf{y}(\mathbf{m})$ & & $\mathbf{x}(\mathrm{m})$ & $\mathbf{y}(\mathbf{m})$ & $\mathrm{z}(\mathbf{m})$ & $\mathbf{x}(\mathbf{m})$ & $\mathbf{y}(\mathbf{m})$ & $\mathrm{z}(\mathbf{m})$ & $\mathbf{x}(\mathrm{m})$ & $\mathbf{y}(\mathbf{m})$ & $\mathrm{z}(\mathrm{m})$ \\
\hline 146 & -0.14494 & 0.752802 & -1.89707 & -0.36098 & 0.513449 & -2.84643 & 1.767849 & 0.289754 & -5.15266 & 1.788434 & 0.602688 & -5.26204 \\
\hline 147 & 0.434714 & 0.752787 & -3.54802 & 0.483656 & 0.835606 & -3.038 & 2.347604 & 0.289755 & -5.15298 & 2.171804 & 0.483955 & -5.52161 \\
\hline 148 & 0.434777 & 0.7528 & -2.7228 & 0.073481 & 0.77627 & -2.91596 & 2.058201 & 0.791635 & -4.32707 & 2.010708 & 0.802043 & -5.19885 \\
\hline 149 & 0.434843 & 0.752806 & -1.89708 & 0.636502 & 0.773352 & -2.84094 & 2.638068 & 0.791628 & -4.32744 & 2.394844 & 0.776964 & -5.04736 \\
\hline 150 & 1.01433 & 0.752793 & -3.5481 & 1.03232 & 0.76913 & -3.02287 & 1.478296 & 0.791604 & -4.3268 & 1.273444 & 0.764656 & -5.27997 \\
\hline 151 & 1.014474 & 0.752793 & -2.72287 & 0.896123 & 0.815816 & -2.73602 & 1.768262 & 0.289756 & -4.32692 & 1.767326 & 0.111603 & -5.18417 \\
\hline 152 & 1.014626 & 0.752793 & -1.89715 & 1.382086 & 0.698374 & -2.74872 & 2.348141 & 0.289758 & -4.32724 & 2.186319 & 0.34296 & -5.08883 \\
\hline 153 & 1.593935 & 0.75278 & -3.54823 & 1.454874 & 0.767377 & -2.97204 & & & & & & \\
\hline 154 & 1.594184 & 0.752806 & -2.72301 & 1.862781 & 0.845661 & -3.06569 & & & & & & \\
\hline 155 & 1.594431 & 0.752815 & -1.89729 & 1.759203 & 0.632823 & -2.72129 & & & & & & \\
\hline 156 & 2.173551 & 0.75278 & -3.54843 & 2.233855 & 0.695485 & -2.95875 & & & & & & \\
\hline 157 & 2.173893 & 0.752807 & -2.72321 & 2.031724 & 0.909775 & -2.72813 & & & & & & \\
\hline 158 & 2.174238 & 0.752814 & -1.89749 & 2.149959 & 0.648437 & -2.55292 & & & & & & \\
\hline 159 & 2.753147 & 0.752792 & -3.54871 & 2.415224 & 0.809595 & -2.89912 & & & & & & \\
\hline 160 & 2.753617 & 0.752794 & -2.72349 & 2.50545 & 0.897433 & -2.56129 & & & & & & \\
\hline 161 & 2.754082 & 0.752797 & -1.89777 & 2.633536 & 0.625728 & -2.38836 & & & & & & \\
\hline 162 & 3.332746 & 0.752779 & -3.54908 & 2.578598 & 0.867686 & -2.9613 & & & & & & \\
\hline 163 & 3.333366 & 0.752808 & -2.72386 & 2.787421 & 0.692669 & -2.68058 & & & & & & \\
\hline 164 & 3.333987 & 0.752815 & -1.89814 & 2.969734 & 0.764581 & -2.30927 & & & & & & \\
\hline 165 & 3.912361 & 0.752786 & -3.54958 & 3.039836 & 0.663804 & -3.15321 & & & & & & \\
\hline 166 & 3.913236 & 0.752804 & -2.72436 & 3.04233 & 0.835564 & -2.71542 & & & & & & \\
\hline 167 & 3.914116 & 0.752811 & -1.89864 & 3.158888 & 0.735983 & -2.17246 & & & & & & \\
\hline
\end{tabular}


Table M-5. Summary of the calculations in this appendix.

\begin{tabular}{|c|c|c|c|c|c|c|}
\hline Set & $\begin{array}{l}\text { Condition at the } \\
y \text {-axis boundary }\end{array}$ & Horizon & Container & Arrangement & Mass of FGE (g) & $\begin{array}{c}\text { Time of } \\
\text { compaction } \\
\text { (years) }\end{array}$ \\
\hline set6-lhpop12-hp & Mirror & lower & \multirow{4}{*}{12 in. pop } & Hexagonal nitch & \multirow{8}{*}{200} & \multirow{12}{*}{$0,1,000$} \\
\hline set6a-lhpop12-hp & Periodic & lower & & Hexagonal pitcn & & \\
\hline set6-uhpop12-tp & Mirror & unner & & Trianoular nitch & & \\
\hline set6a-uhpop12-tp & Periodic & upper & & 11rangural piten & & \\
\hline set6-lhpop6-hp & Mirror & lawror & \multirow{4}{*}{6 in. pop } & Horrom 1 nita & & \\
\hline set6a-lhpop6-hp & Periodic & IOWEC & & & & \\
\hline set6-uhpop6-tp & Mirror & \multirow{2}{*}{ upper } & & Trianoular nitch & & \\
\hline set6a-uhpop6-tp & Periodic & & & 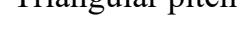 & & \\
\hline set6-lh-tp & Mirror & \multirow{2}{*}{ lower } & \multirow{4}{*}{$\mathrm{COC}$} & \multirow{2}{*}{ Triangular pitch } & \multirow{4}{*}{380} & \\
\hline set6a-lh-tp & Periodic & & & & & \\
\hline set6-uh-tp & Mirror & \multirow{2}{*}{ upper } & & \multirow{2}{*}{ Triangular pitch } & & \\
\hline set6a-uh-tp & Periodic & & & & & \\
\hline
\end{tabular}




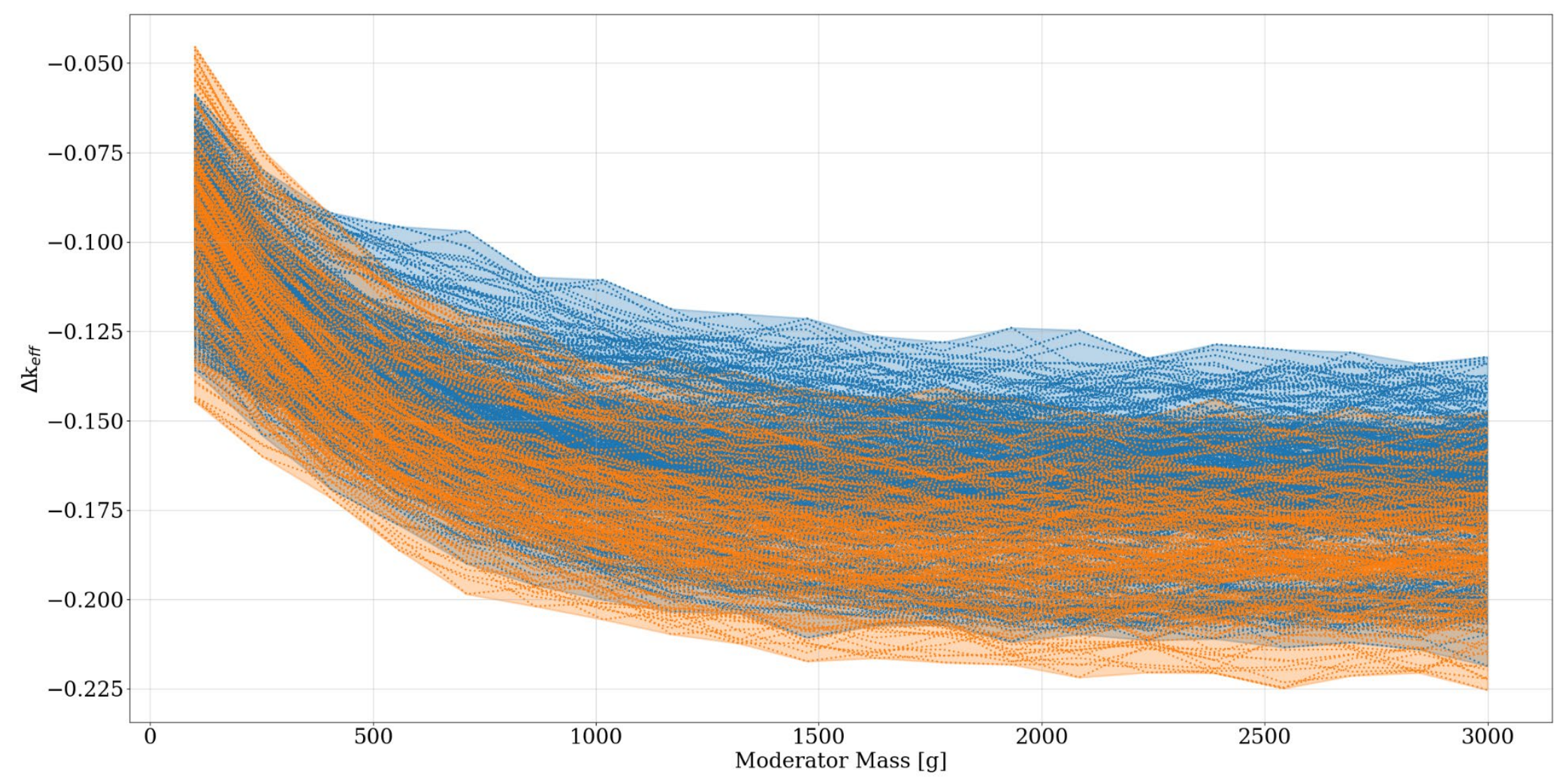

Figure M-1. Comparison of CCO upper horizon datasets, set-2-uh hexagonal pitch results (mirror boundary conditions) with set-6-uh-tp triangular pitch results (mirror boundary conditions) as delta- $k_{e f f}$ vs. moderator mass, time $=0$ years. 


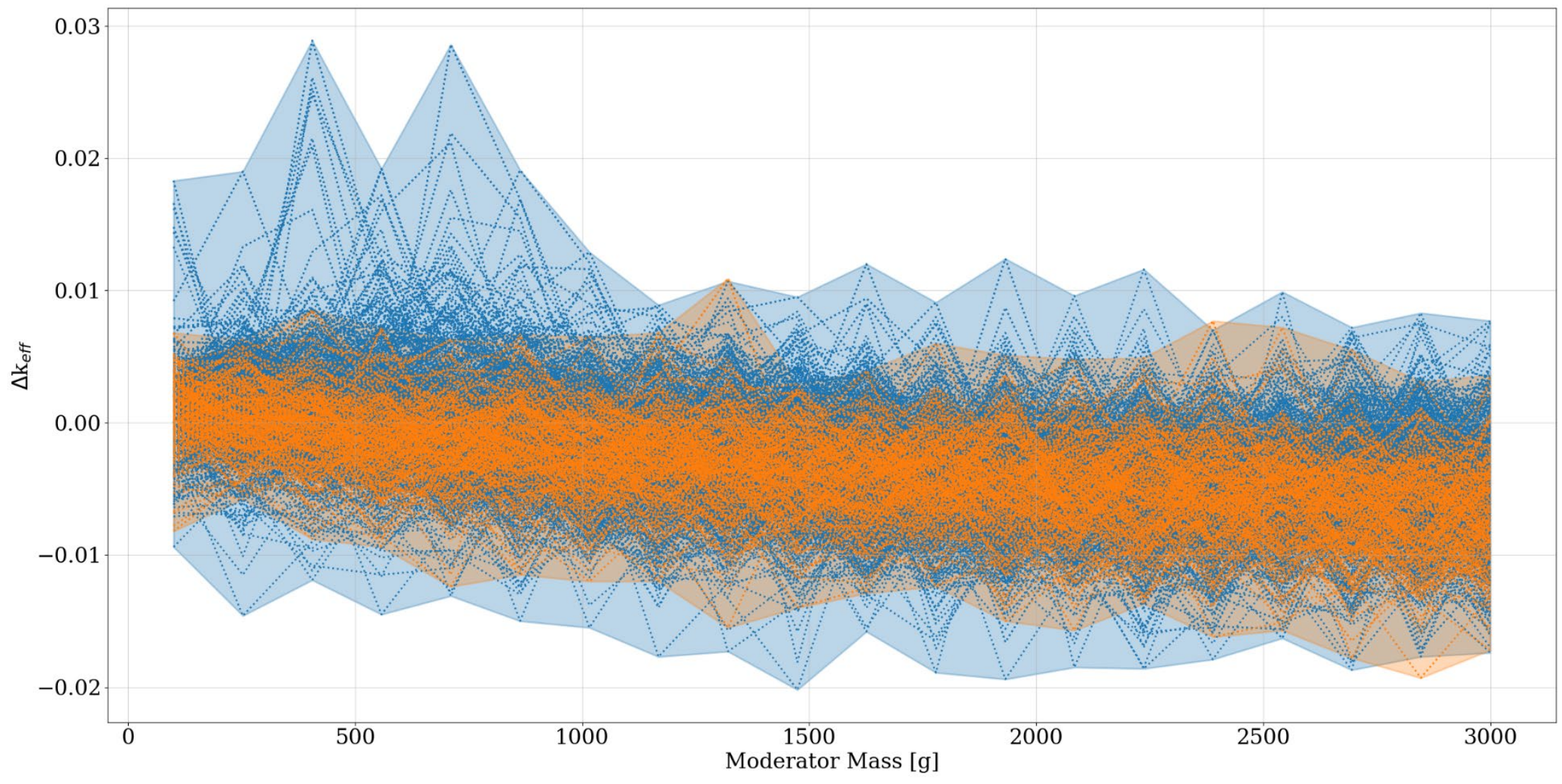

Figure M-2. Comparison of CCO upper horizon datasets, set-2-uh hexagonal pitch results (mirror boundary conditions) with set-6-uh-tp triangular pitch results (mirror boundary conditions) as delta- $k_{e f f}$ vs. moderator mass, time $=1,000$ years. 


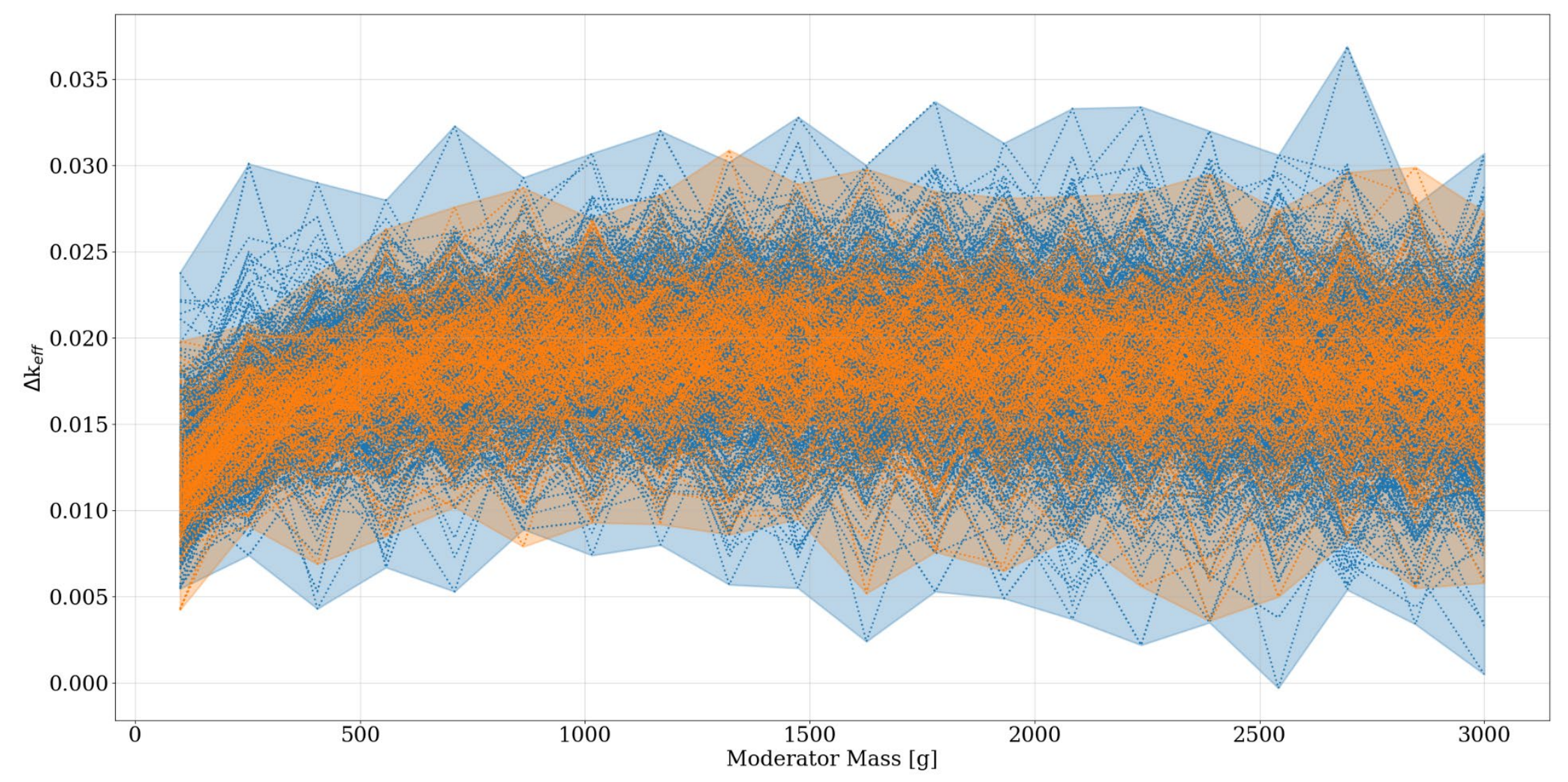

Figure M-3. Comparison of CCO lower horizon datasets, set-2-lh hexagonal pitch results (mirror boundary conditions) with set-6-lh-tp triangular pitch results (mirror boundary conditions) as delta- $\boldsymbol{k}_{\text {eff }}$ vs. moderator mass, time $=1,000$ years. 


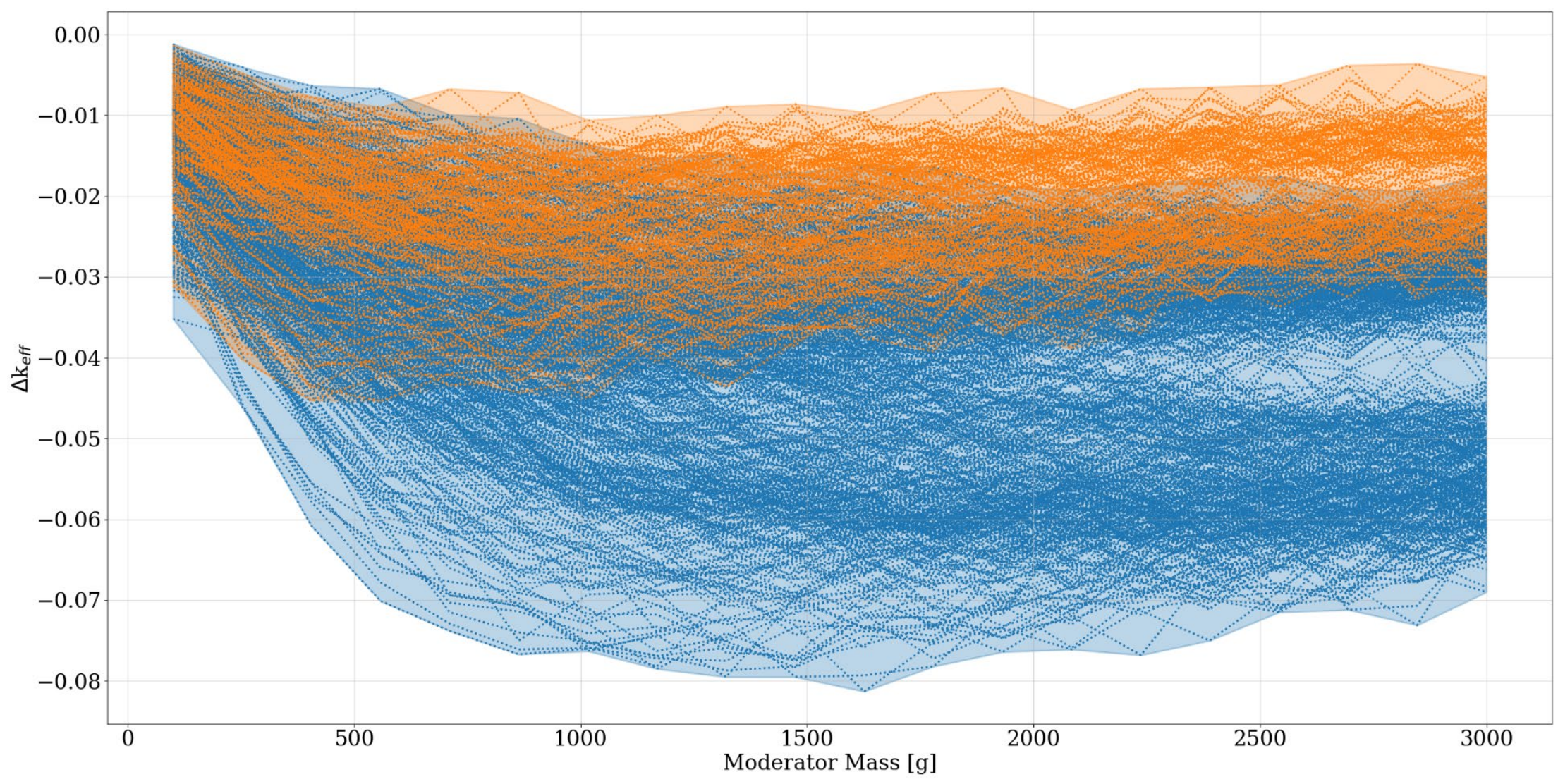

Figure M-4. Comparison of CCO upper horizon datasets, set6-uh-tp triangular pitch results (mirror boundary conditions) results with set6a-uh-tp triangular pitch results (periodic boundary conditions) as delta- $k_{e f f}$ vs. moderator mass, time $=0$ years. 


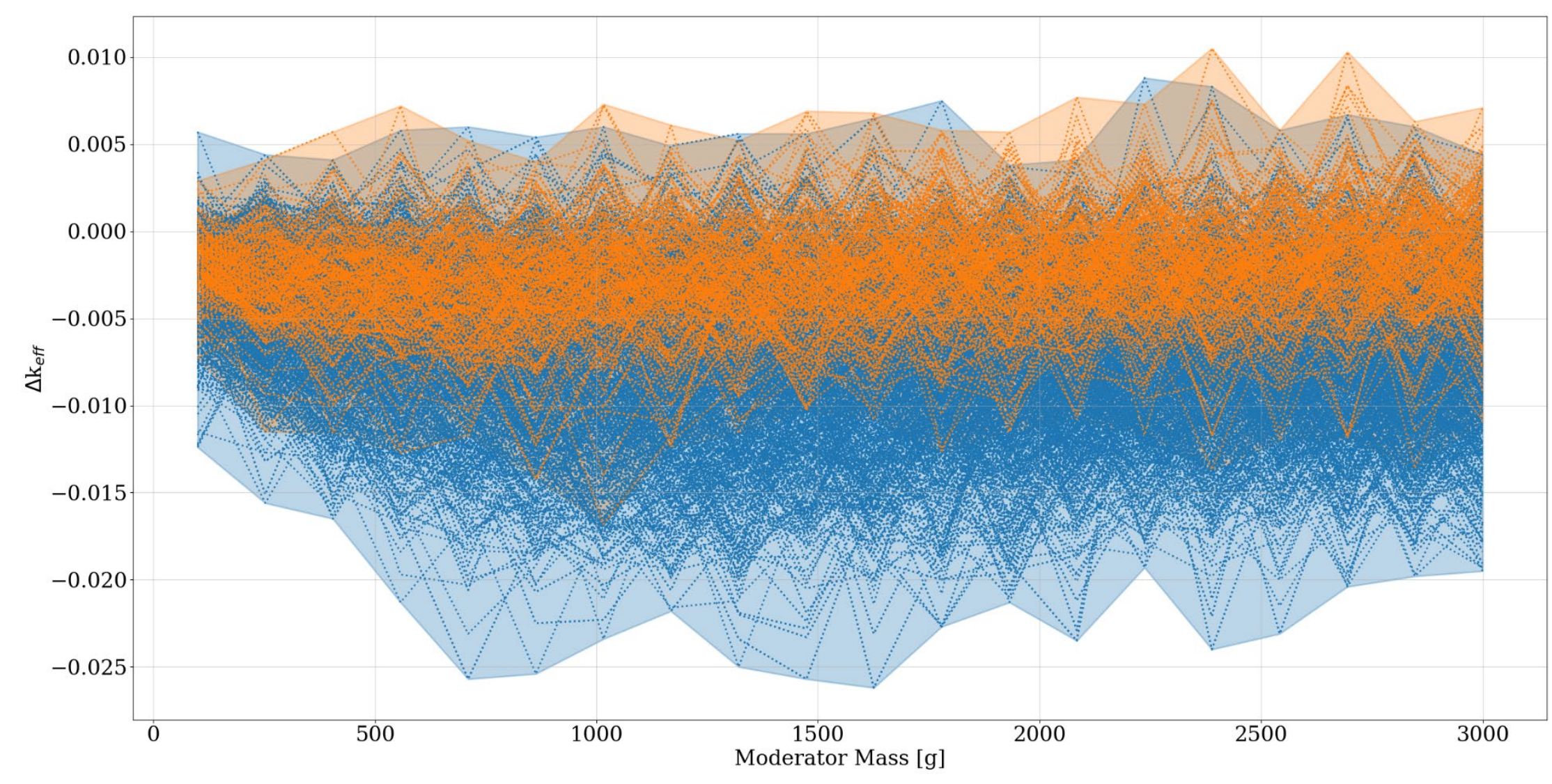

Figure M-5. Comparison of CCO upper horizon datasets, set6-uh-tp triangular pitch results (mirror boundary conditions) results with set6a-uh-tp triangular pitch results (periodic boundary conditions) as delta- $k_{\text {eff }}$ vs. moderator mass, time $=1,000$ years. 


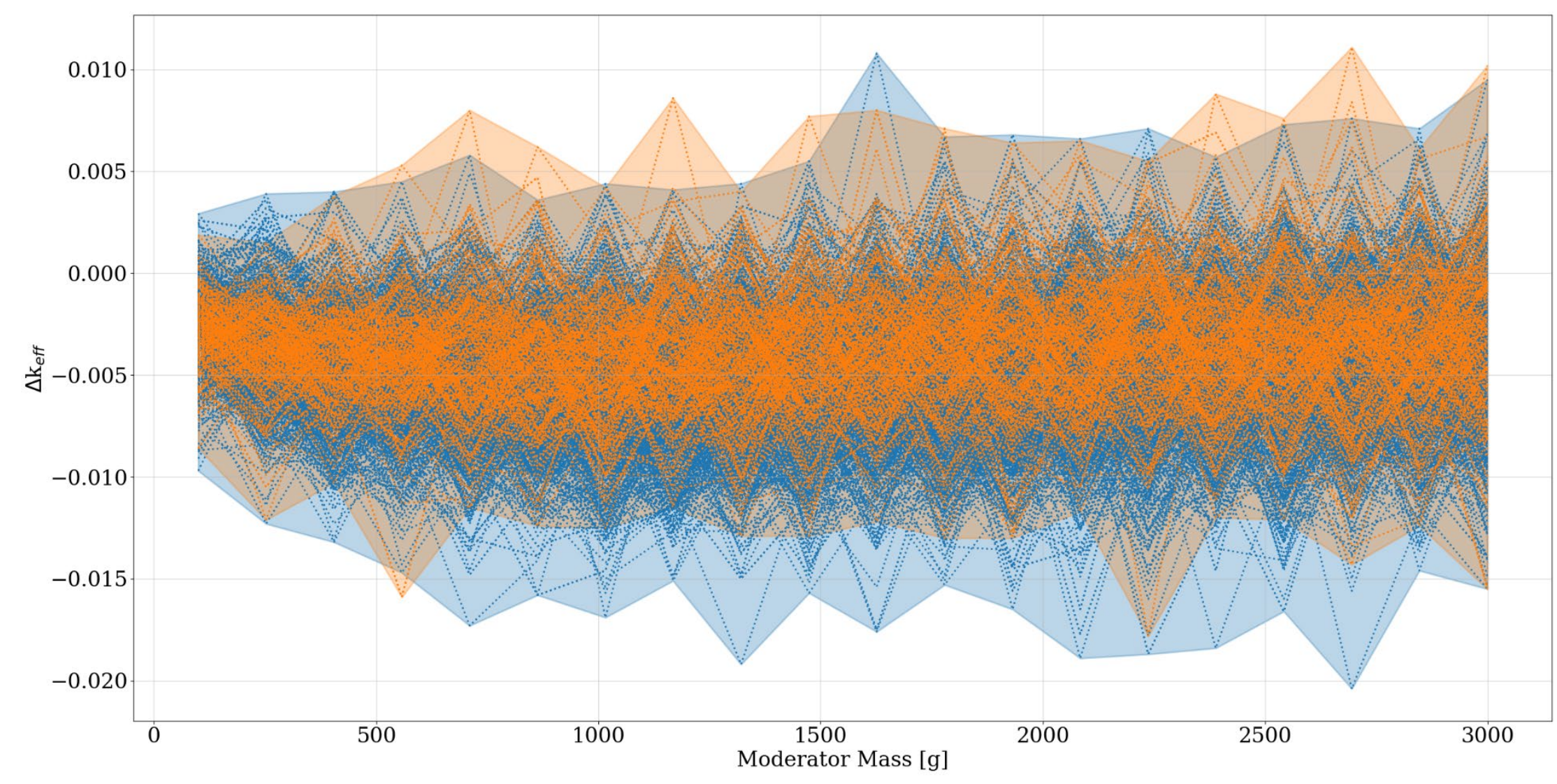

Figure M-6. Comparison of CCO lower horizon datasets, set6-lh-tp triangular pitch results (mirror boundary conditions) results with set6a-lh-tp triangular pitch results (periodic boundary conditions) as delta- $k_{\text {eff }}$ vs. moderator mass, 1,000 years. 


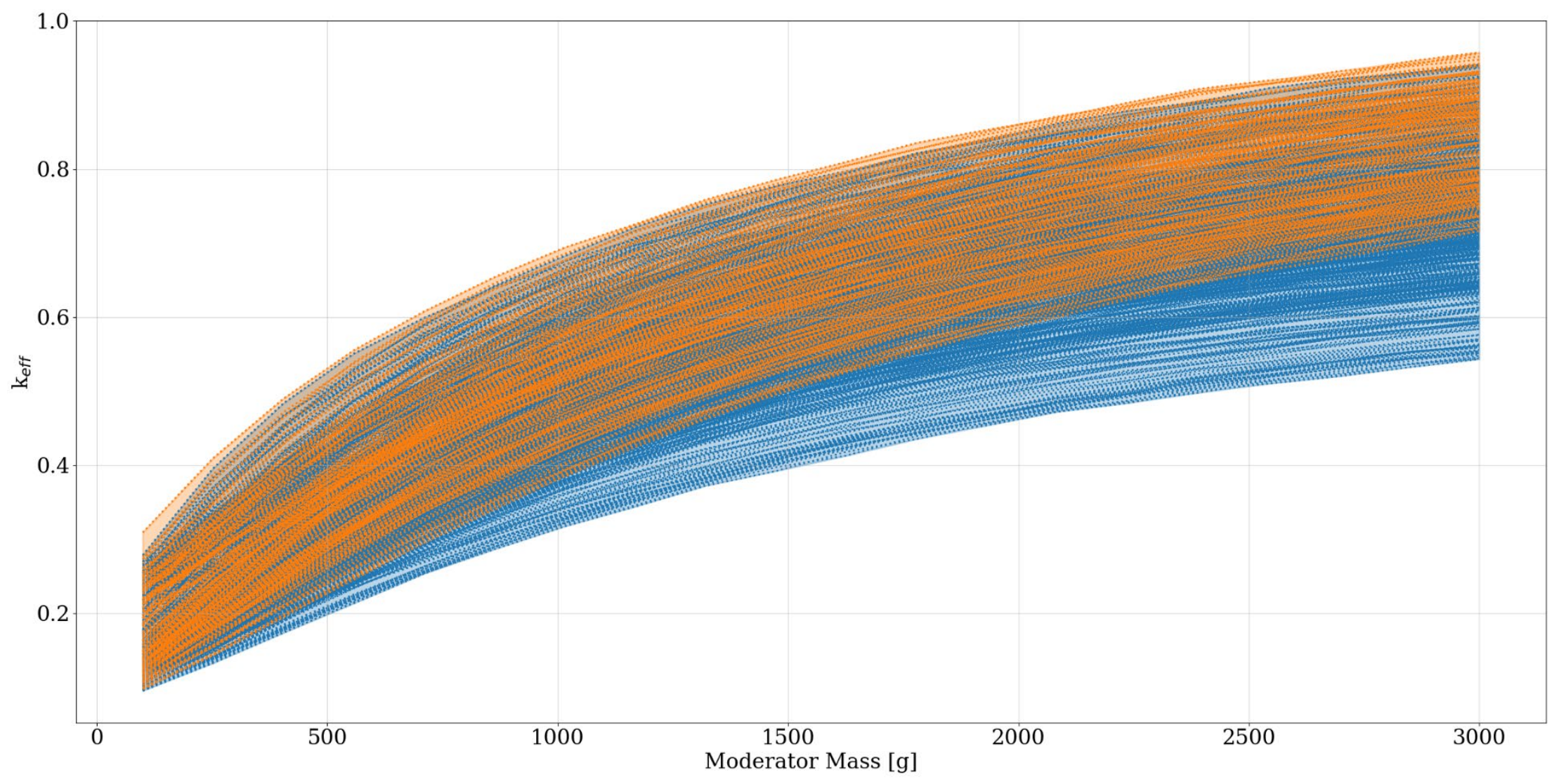

set-6a-uh-tp-0 cylinders $\square$ set-6a-uh-tp-0 spheres

Figure M-7. Summary of CCO upper horizon datasets, set6a-uh-tp triangular pitch results (periodic boundary conditions) as $\boldsymbol{k}_{\text {eff }}$ vs. moderator mass, time $=0$ years. 


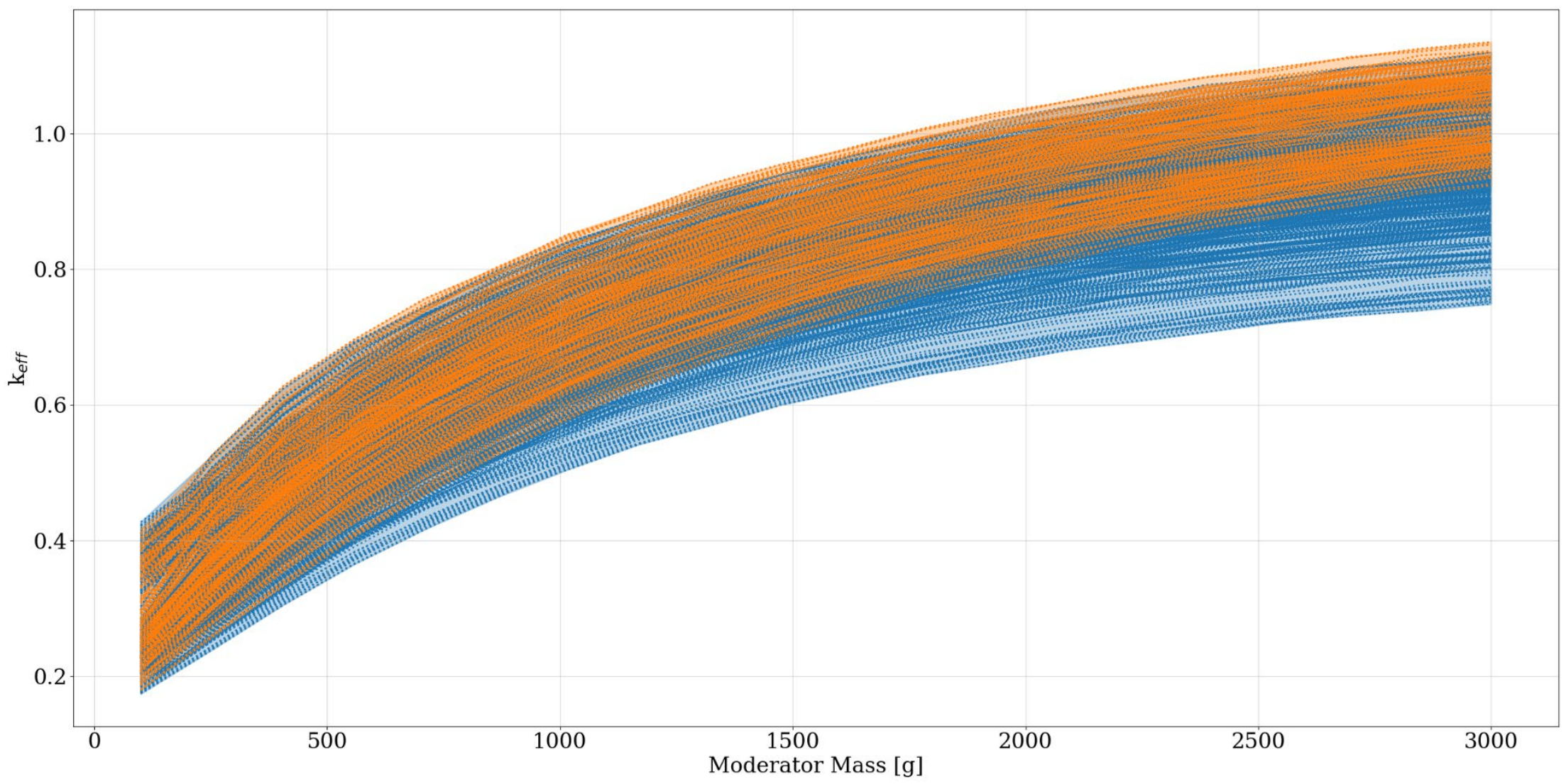

Figure M-8. Summary of CCO upper horizon datasets, set6a-uh-tp triangular pitch results (periodic boundary conditions) as $\boldsymbol{k}_{e f f}$ vs. moderator mass, time $=1,000$ years. 


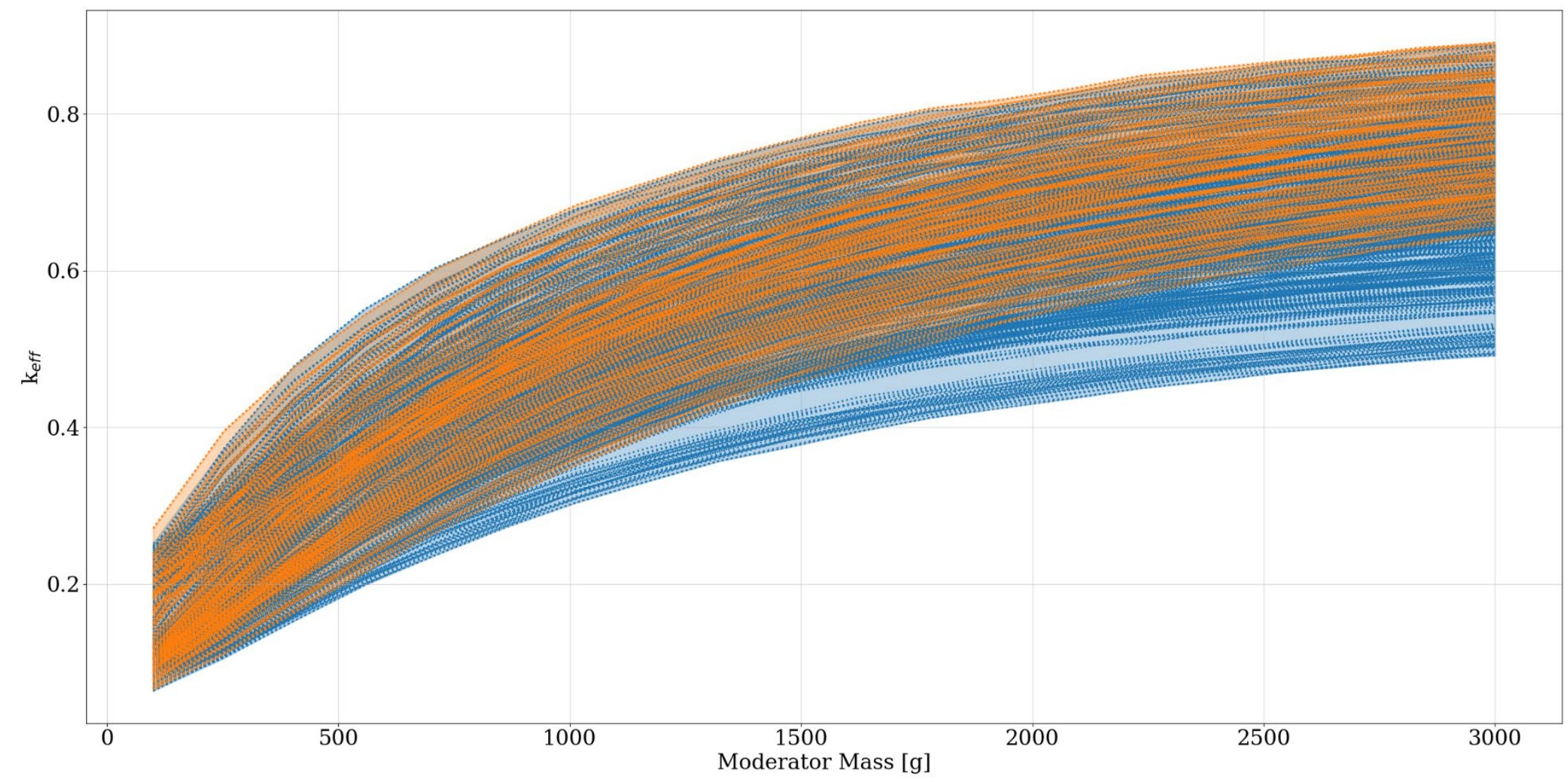

Figure M-9. Summary of 6 in. POP upper horizon datasets, set6-uhpop6-tp triangular pitch results (mirror boundary conditions) as $k_{e f f}$ vs. moderator mass, time $=0$ years. 


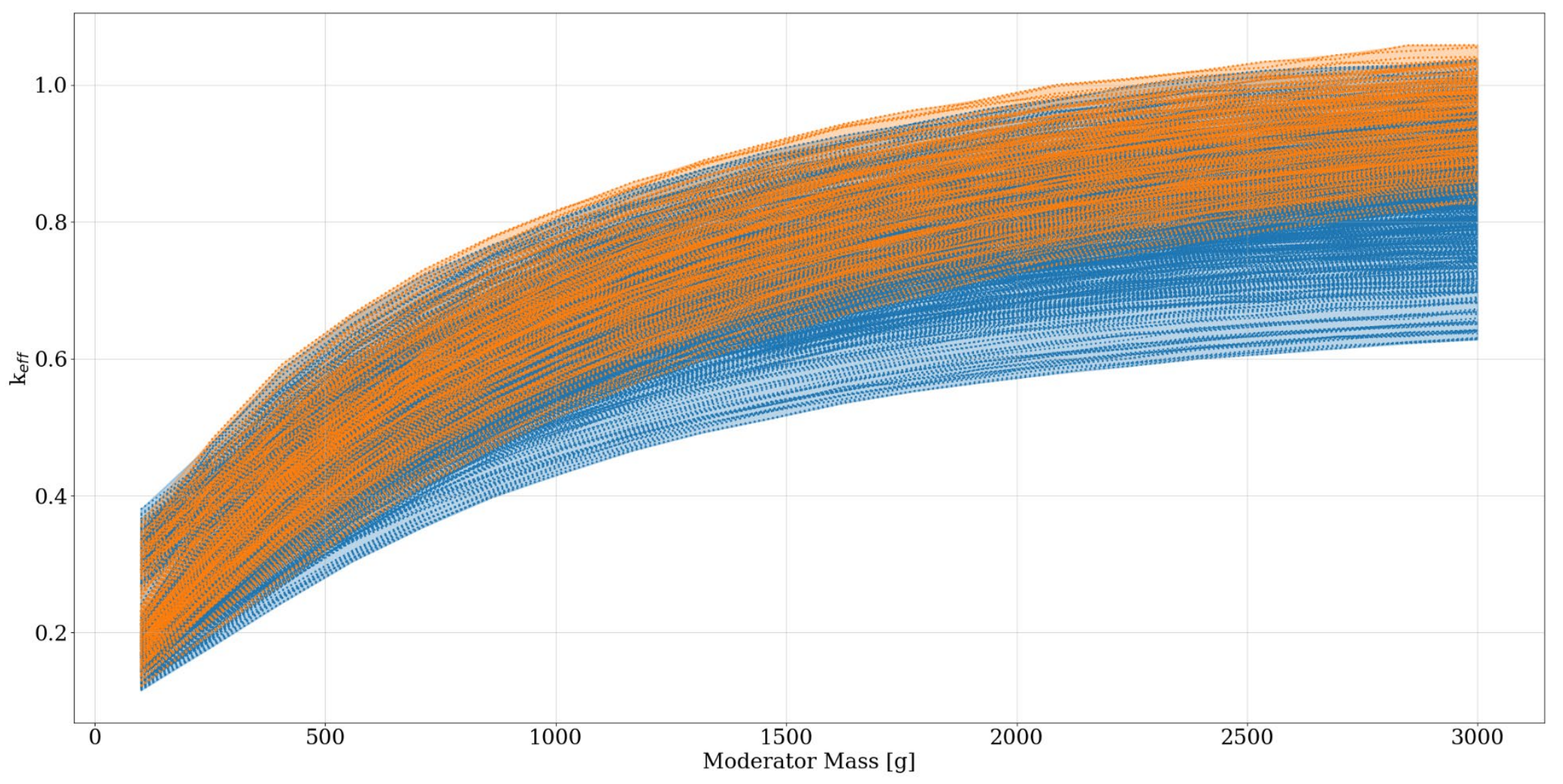

Figure M-10. Summary of 6 in. POP upper horizon datasets, set6-uhpop6-tp triangular pitch results (mirror boundary conditions) as $k_{e f f}$ vs. moderator mass, time $=1,000$ years. 


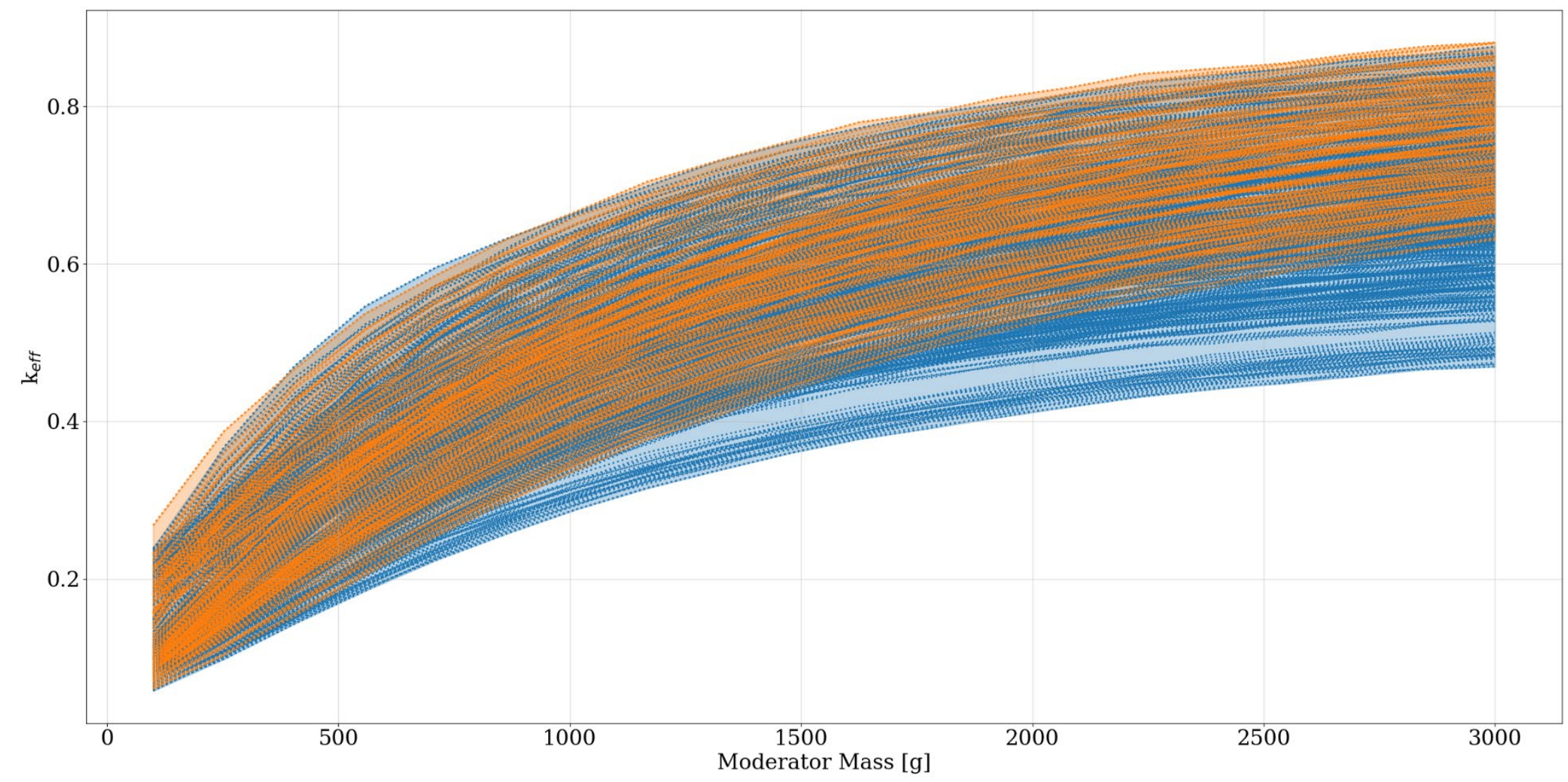

Figure M-11. Summary of 6 in. POP lower horizon datasets, set6-lhpop6-hp hexagonal pitch results (mirror boundary conditions) as $k_{e f f}$ vs. moderator mass, time $=0$ years. 


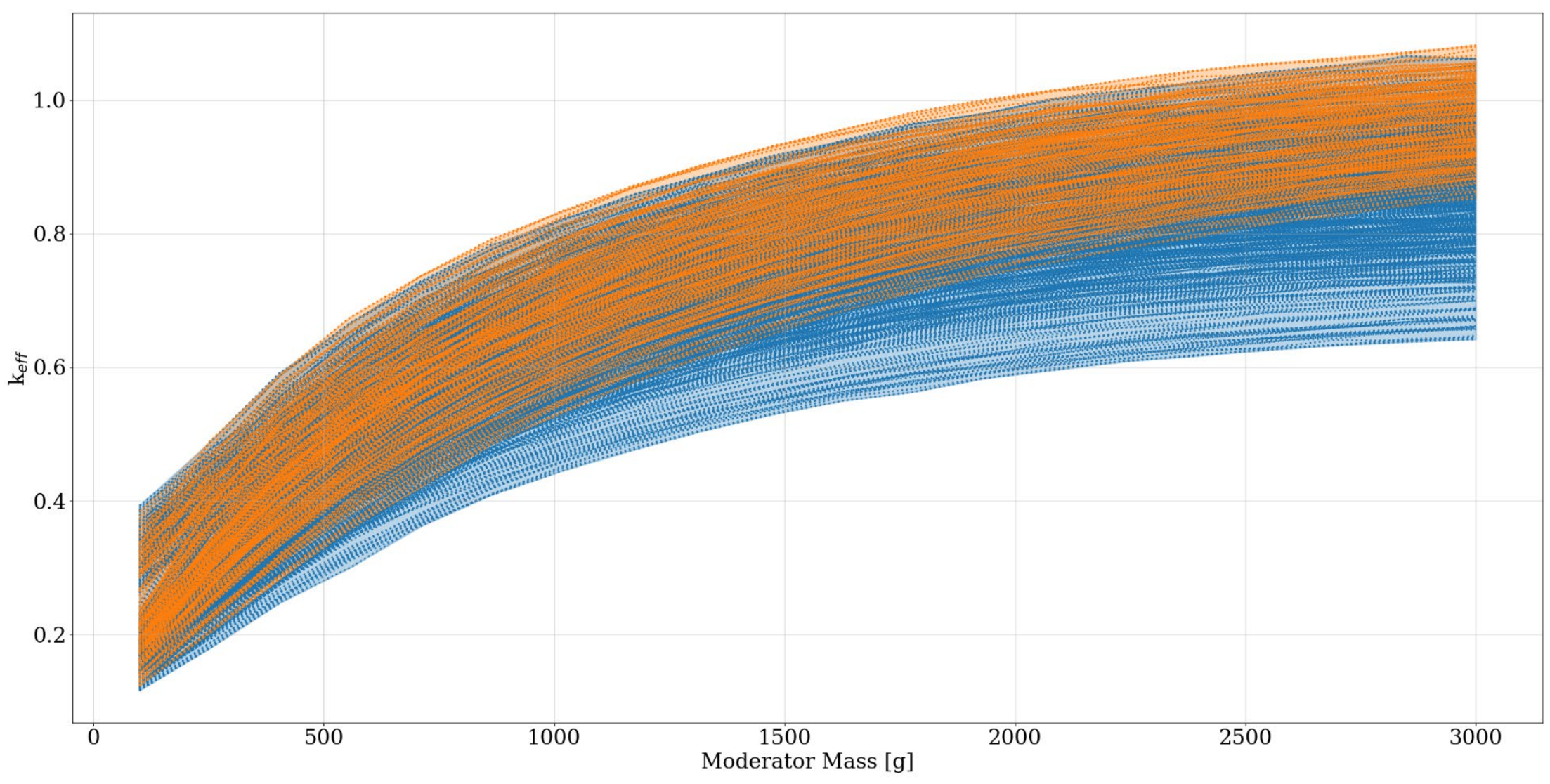

Figure M-12. Summary of 6 in. POP lower horizon datasets, set6-lhpop6-hp hexagonal pitch results (mirror boundary conditions) as $k_{e f f}$ vs. moderator mass, time $=1,000$ years. 


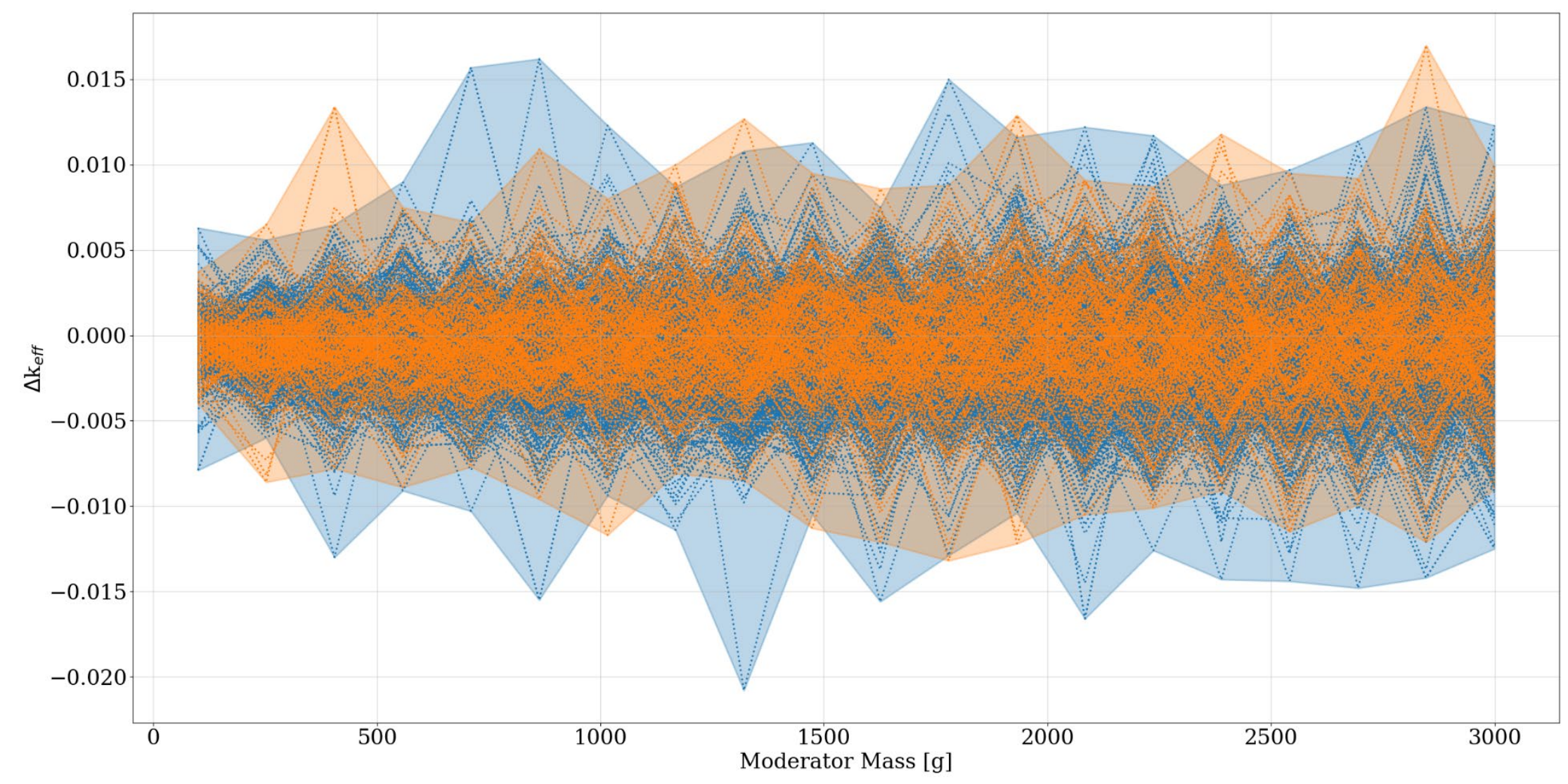

Figure M-13. Comparison of 6 in. POP upper horizon datasets set6-uhpop6-tp triangular pitch results (mirror boundary conditions) results with set6auhpop6-tp triangular pitch results (periodic boundary conditions) as delta- $\boldsymbol{k}_{\text {eff }}$ vs. moderator mass, time $=1,000$ years. 


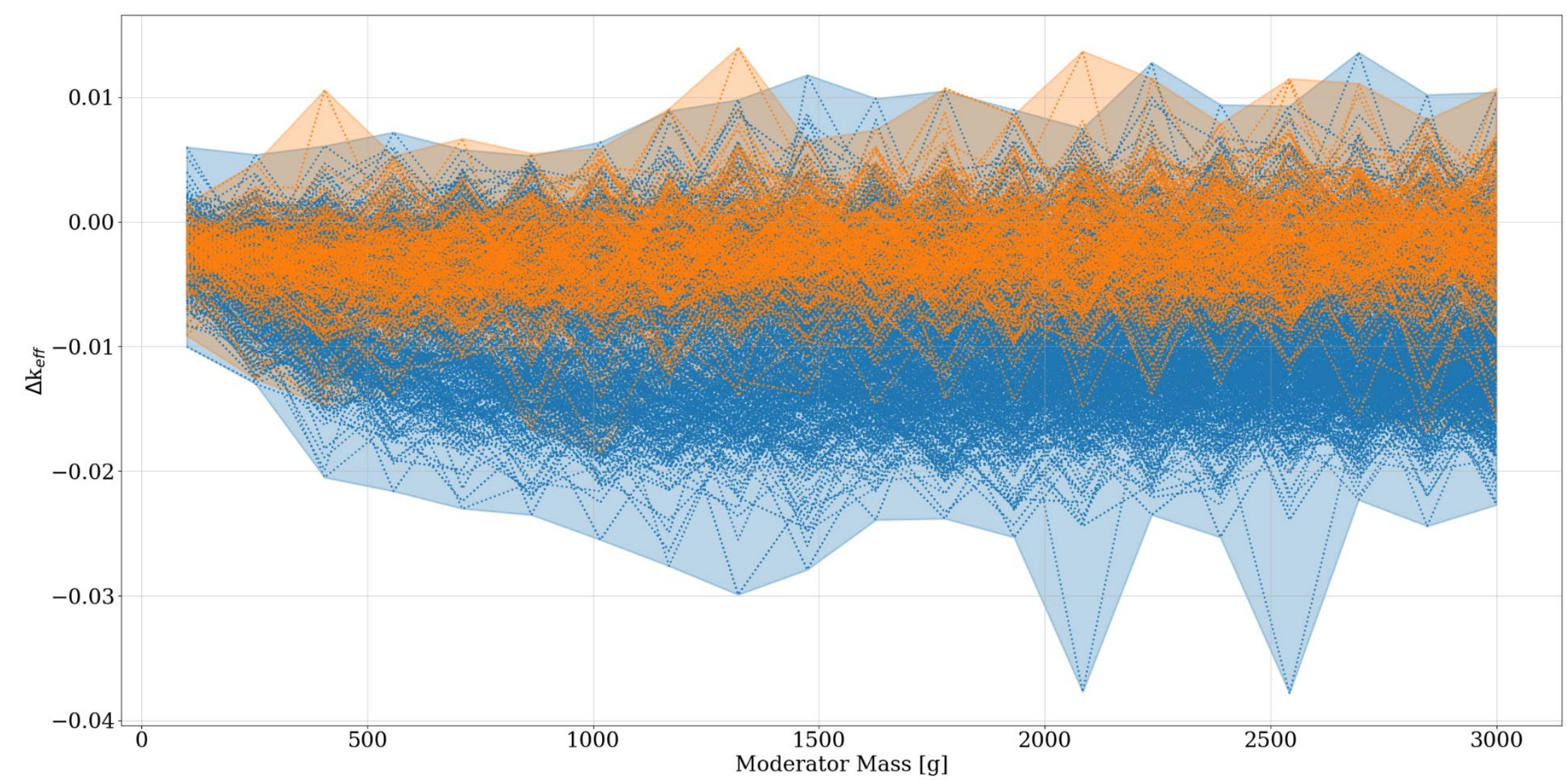

Figure M-14. Comparison of 6 in. POP lower horizon datasets set6-lhpop6-hp hexagonal pitch results (mirror boundary conditions) results with set6alhpop6-hp hexagonal pitch results (periodic boundary conditions) as delta- $\boldsymbol{k}_{e f f}$ vs. moderator mass, time $=1,000$ years. 


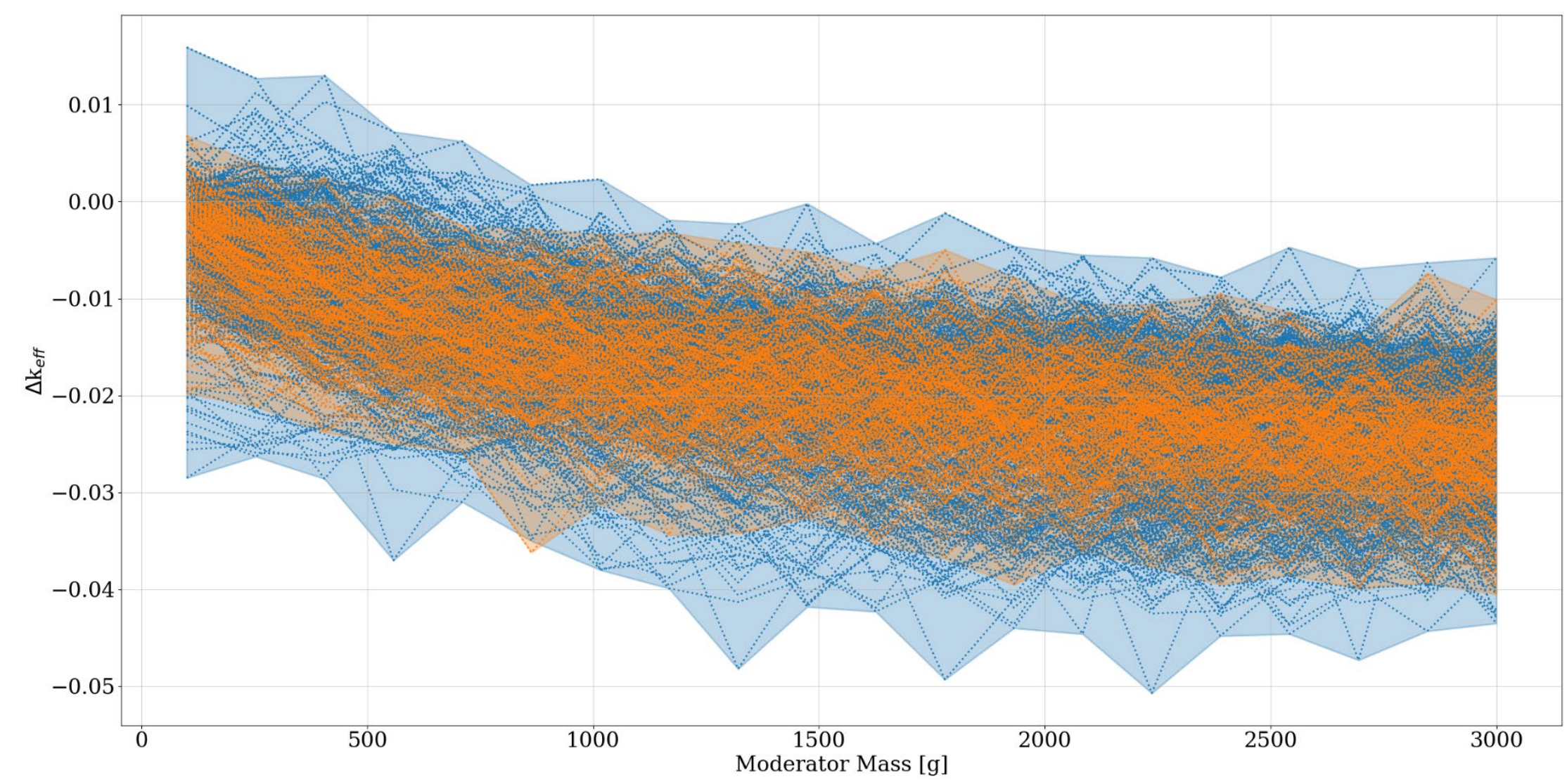

Figure M-15. Comparison of 6 in. POP lower horizon datasets set6-lhpop6-hp hexagonal pitch results (mirror boundary conditions) results with upper horizon set6-uhpop6-tp triangular pitch results (mirror boundary conditions) as delta- $k_{\text {eff }}$ vs. moderator mass, time $=1,000$ years. 


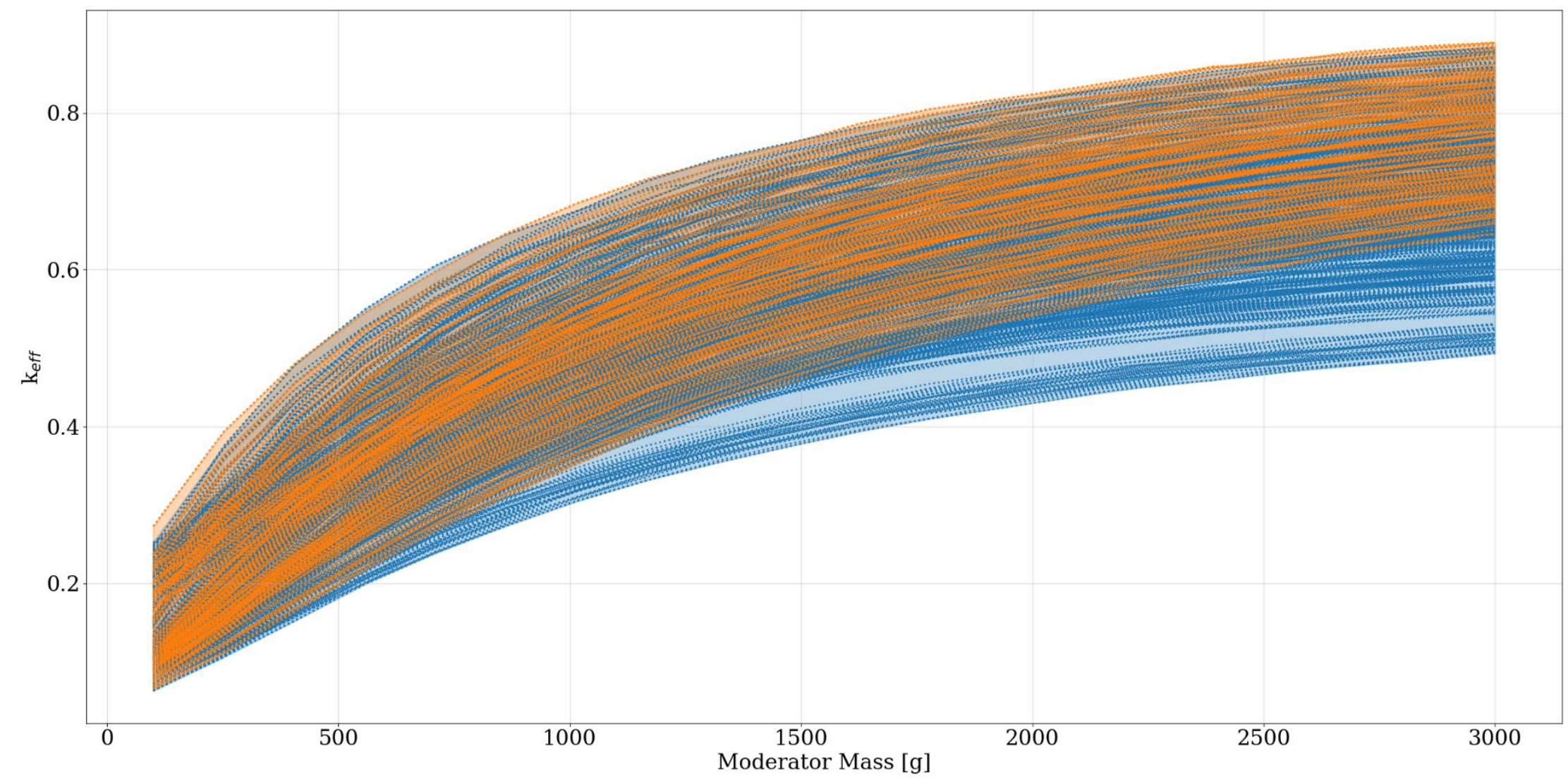

\section{set-6-uhpop12-tp-0 cylinders $\square$ set-6-uhpop12-tp-0 spheres}

Figure M-16. Summary of 12 in. POP upper horizon datasets, set6-uhpop12-tp triangular pitch results (mirror boundary conditions) as $k_{e f f}$ vs. moderator mass, time $=0$ years. 


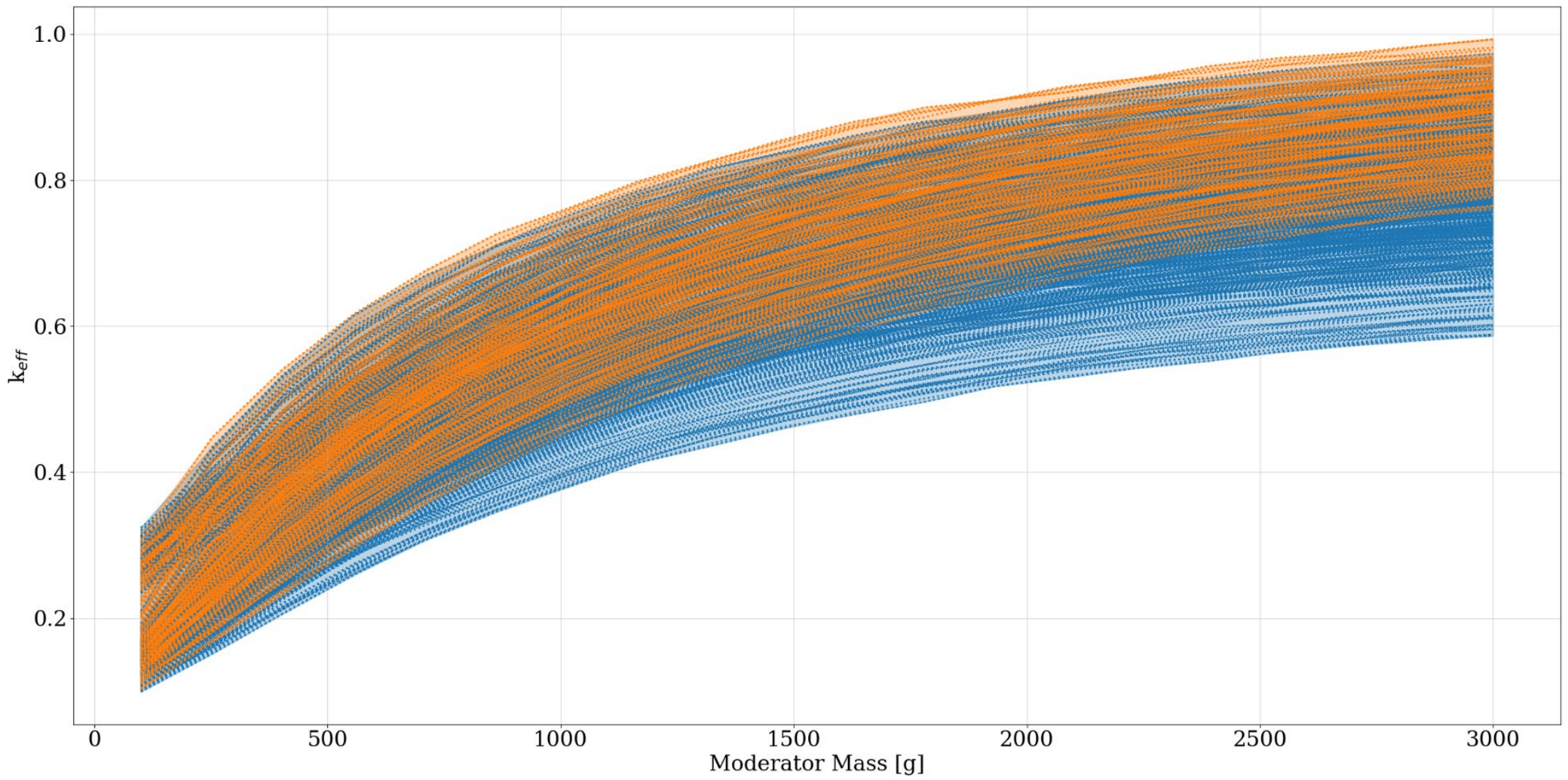

Figure M-17. Summary of 12 in. POP upper horizon datasets, set6-uhpop12-tp triangular pitch results (mirror boundary conditions) as $k_{e f f}$ vs. moderator mass, time $=1,000$ years. 


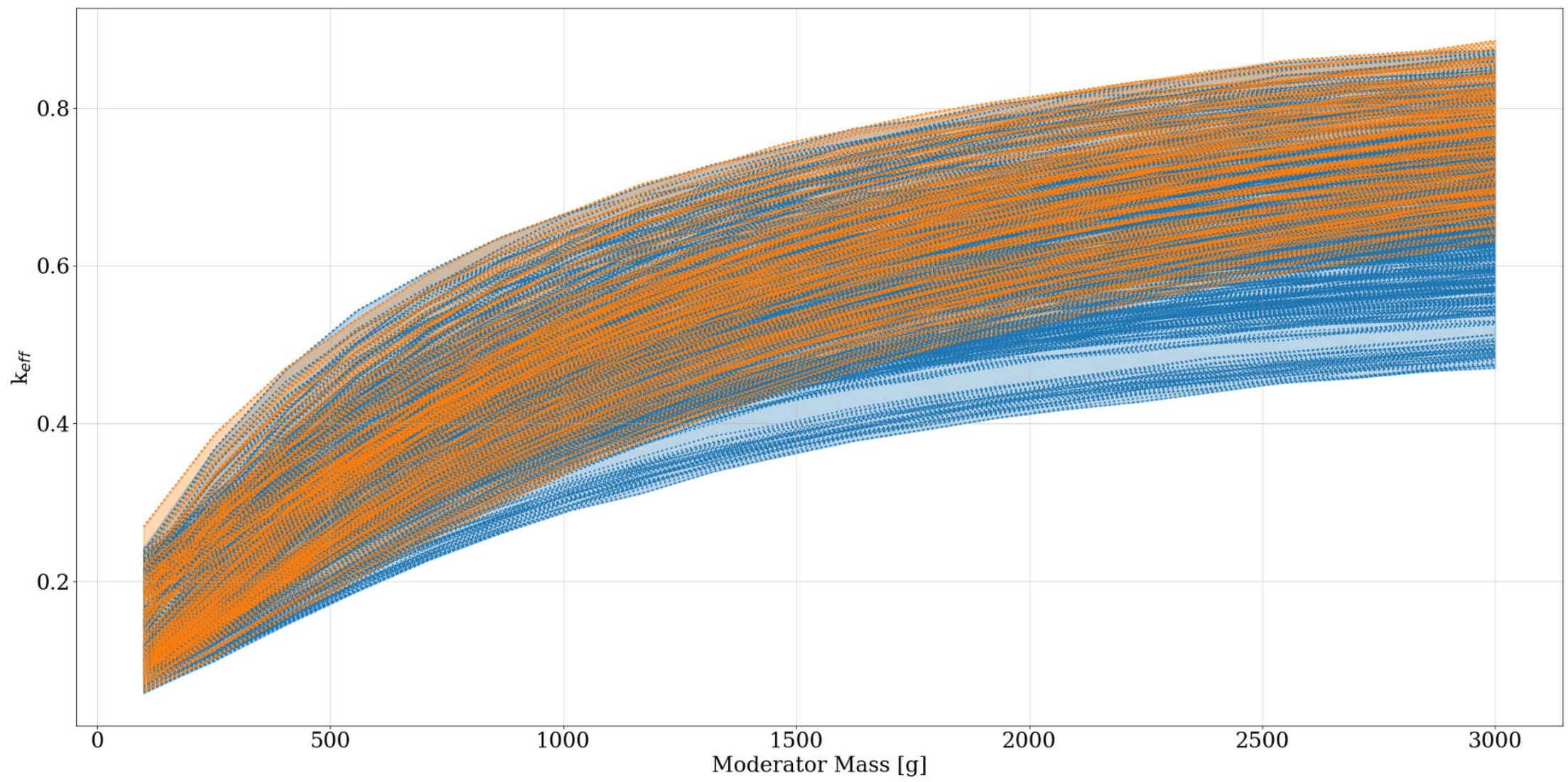

Figure M-18. Summary of 12 in. POP lower horizon datasets, set6-lhpop12-hp hexagonal pitch results (mirror boundary conditions) as $k_{\text {eff }}$ vs. moderator mass, time $=0$ years. 


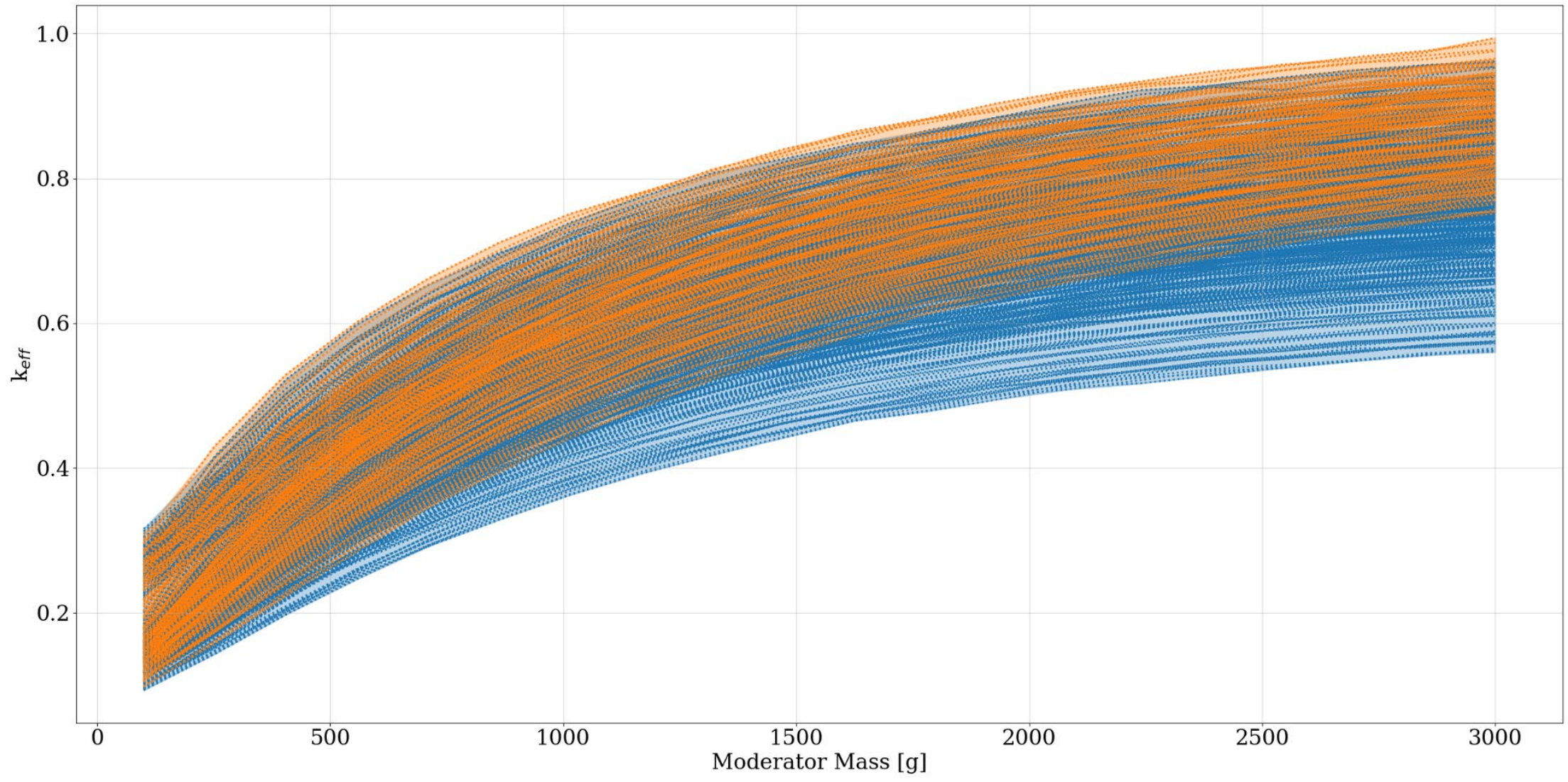

Figure M-19. Summary of 12 in. POP lower horizon datasets, set6-lhpop12-hp hexagonal pitch results (mirror boundary conditions) as $k_{\text {eff }}$ vs. moderator mass, time $=1,000$ years. 


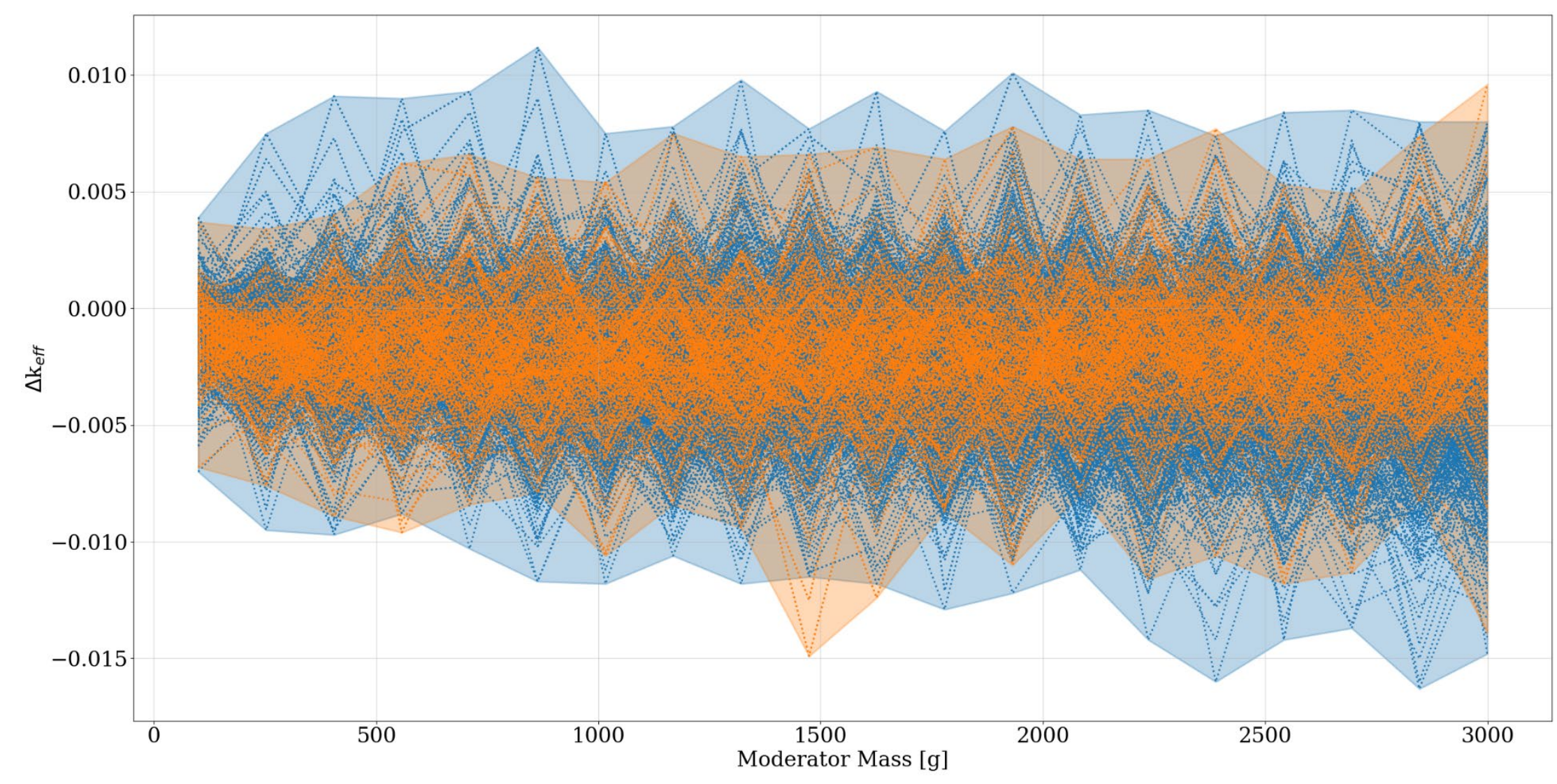

Figure M-20. Comparison of 12 in. POP upper horizon datasets set6-uhpop12-tp triangular pitch results (mirror boundary conditions) results with set6a-uhpop12-tp triangular pitch results (periodic boundary conditions) as delta- $k_{e f f}$ vs. moderator mass, time $=1,000$ years. 


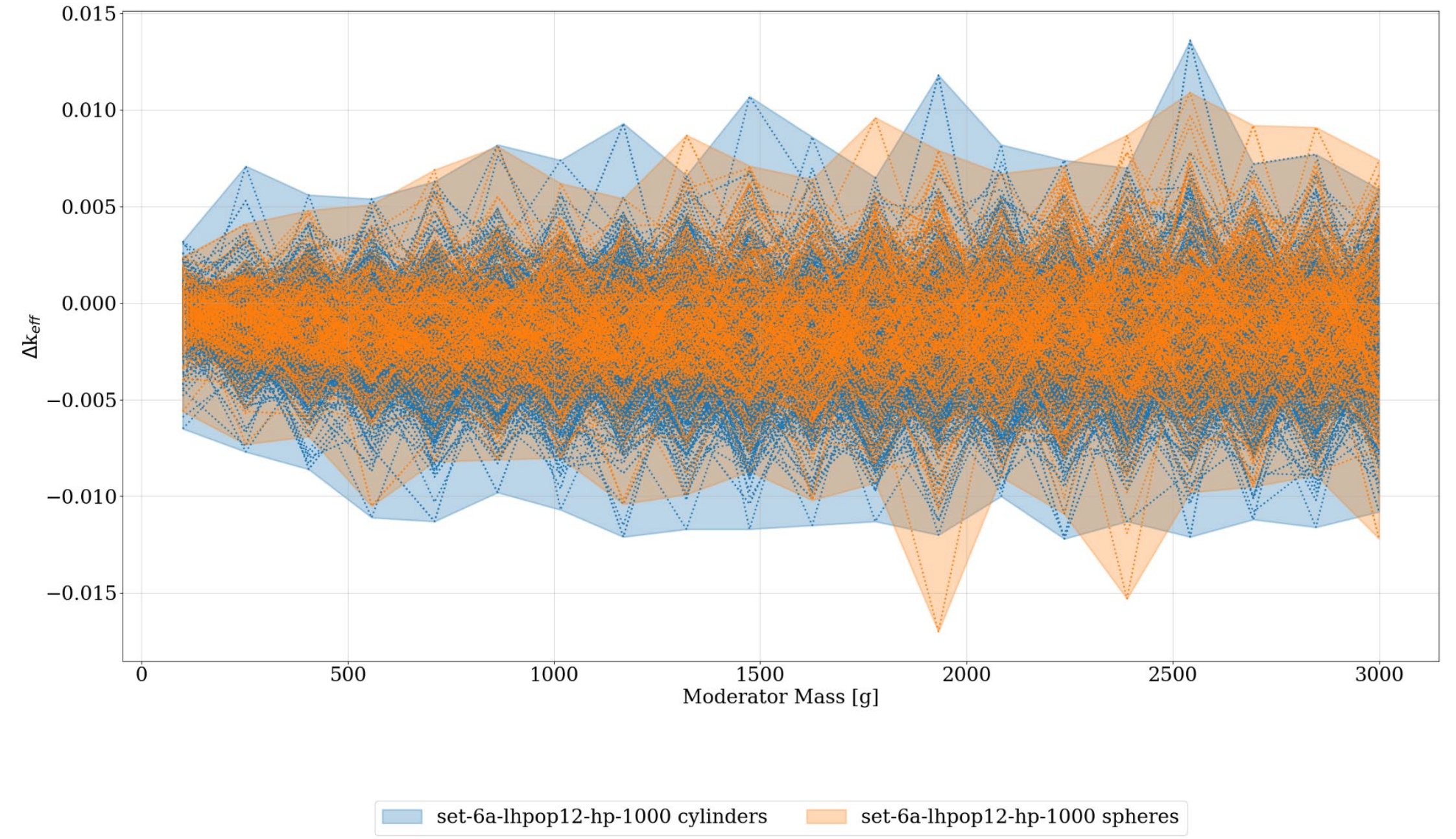

Figure M-21. Comparison of 12 in. POP lower horizon datasets set6-lhpop12-hp hexagonal pitch results (mirror boundary conditions) results with set6a-lhpop12-hp hexagonal pitch results (periodic boundary conditions) as delta- $\boldsymbol{k}_{\text {eff }}$ vs. moderator mass, time $=1,000$ years. 


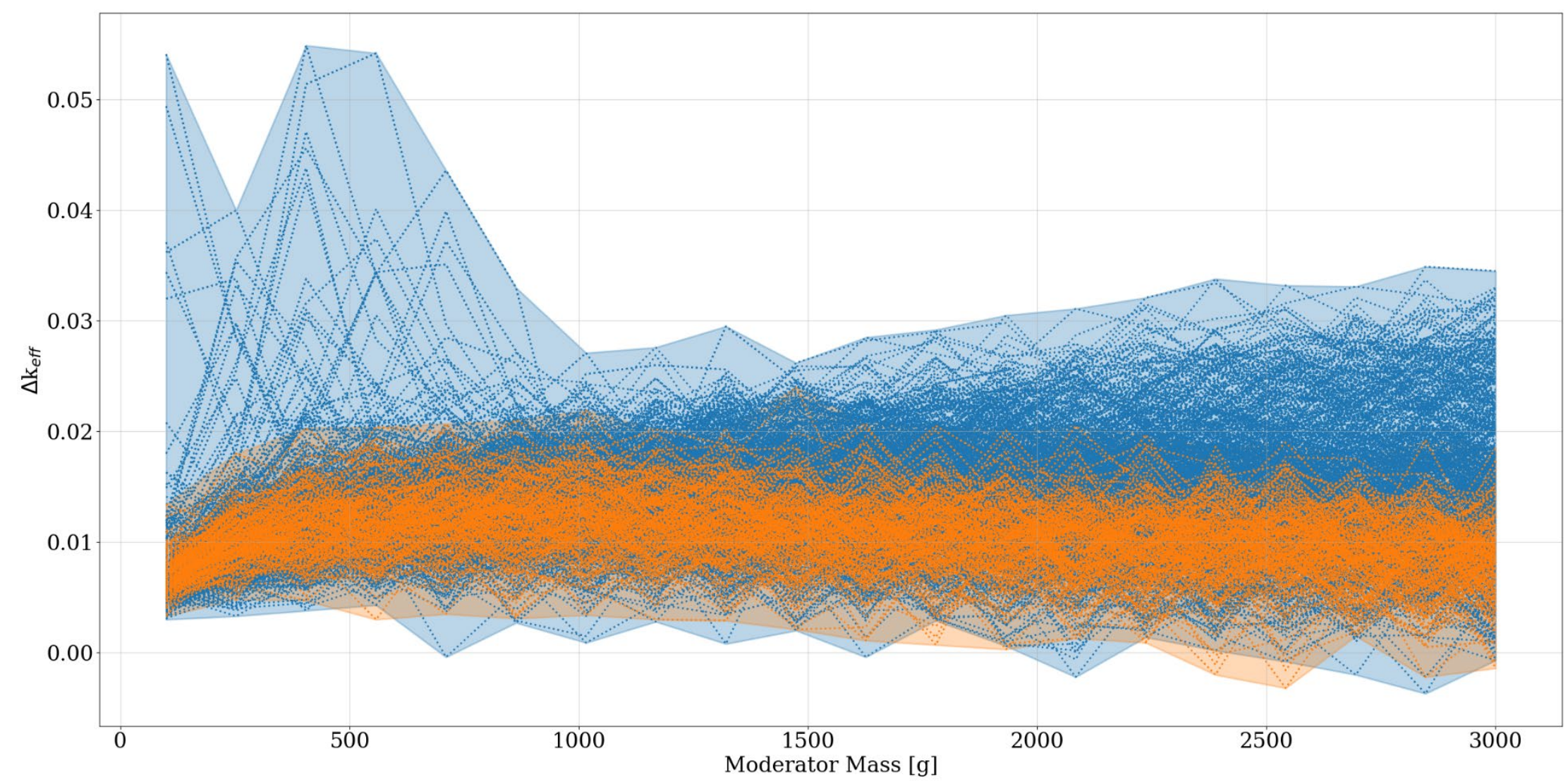

Figure M-22. Comparison of 12 in. POP lower horizon datasets set6-lhpop12-hp hexagonal pitch results (mirror boundary conditions) results with upper horizon set6-uhpop12-tp triangular pitch results (mirror boundary conditions) as delta- $k_{e f f}$ vs. moderator mass, time $=1,000$ years. 


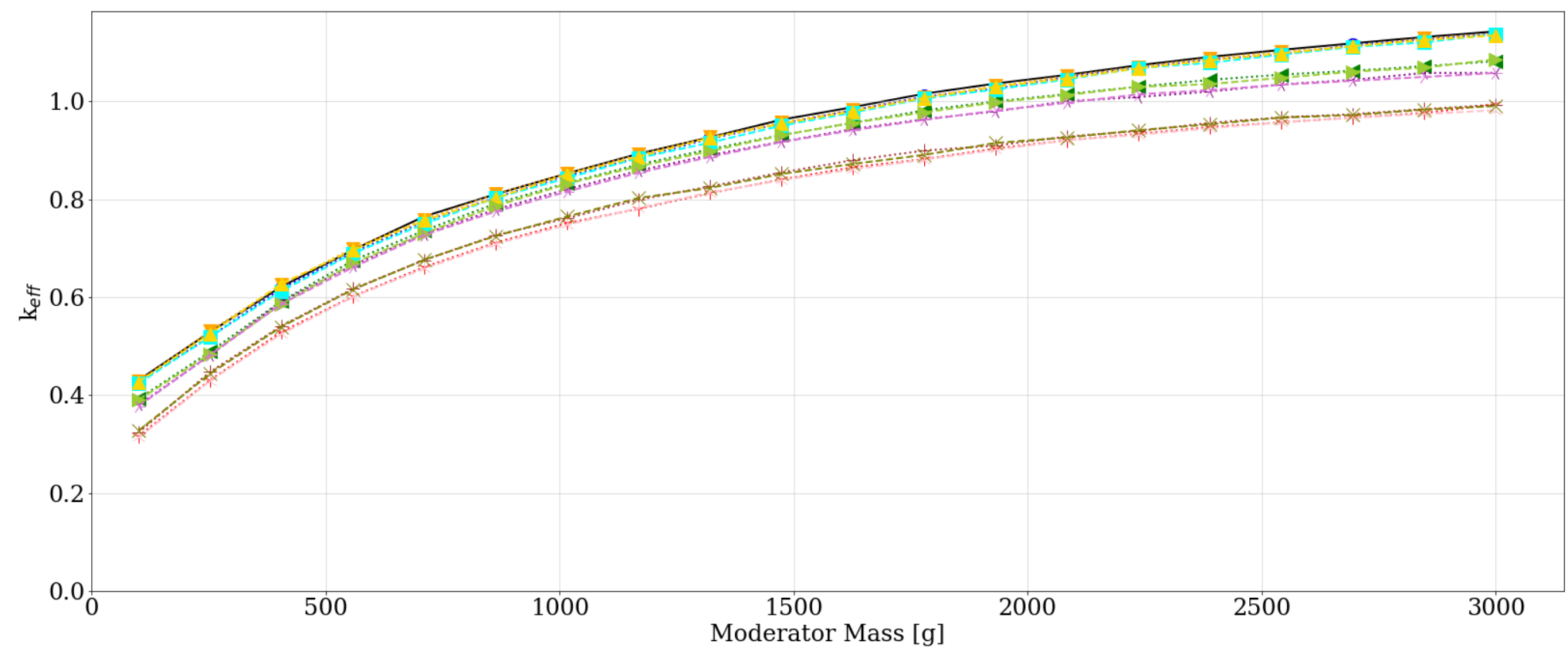

- Max Upper Horizon Reference (Set-2)

-... Max Lower Horizon, CCO Tri Pitch Array, Mirror BC

$\checkmark$... Max Upper Horizon, CCO Tri Pitch Array, Mirror BC

4. Max Lower Horizon, 6" POP Hex Pitch Array, Mirror BC

..... Max Lower Horizon, 12" POP Hex Pitch Array, Mirror BC

....... Max Upper Horizon, 6" POP Tri Pitch Array, Mirror BC

...+... Max Upper Horizon, 12" POP Tri Pitch Array, Mirror BC

Figure M-23. Max $k_{\text {eff }}$ of all subsets of all CCO and POP centroid configurations at time = 1,000 years compared with max $k_{e f f}$ of all subsets of set-2-uh hexagonal pitch (mirror boundary conditions) as $k_{e f f} v s$. moderator mass, time $=1,000$ years. 


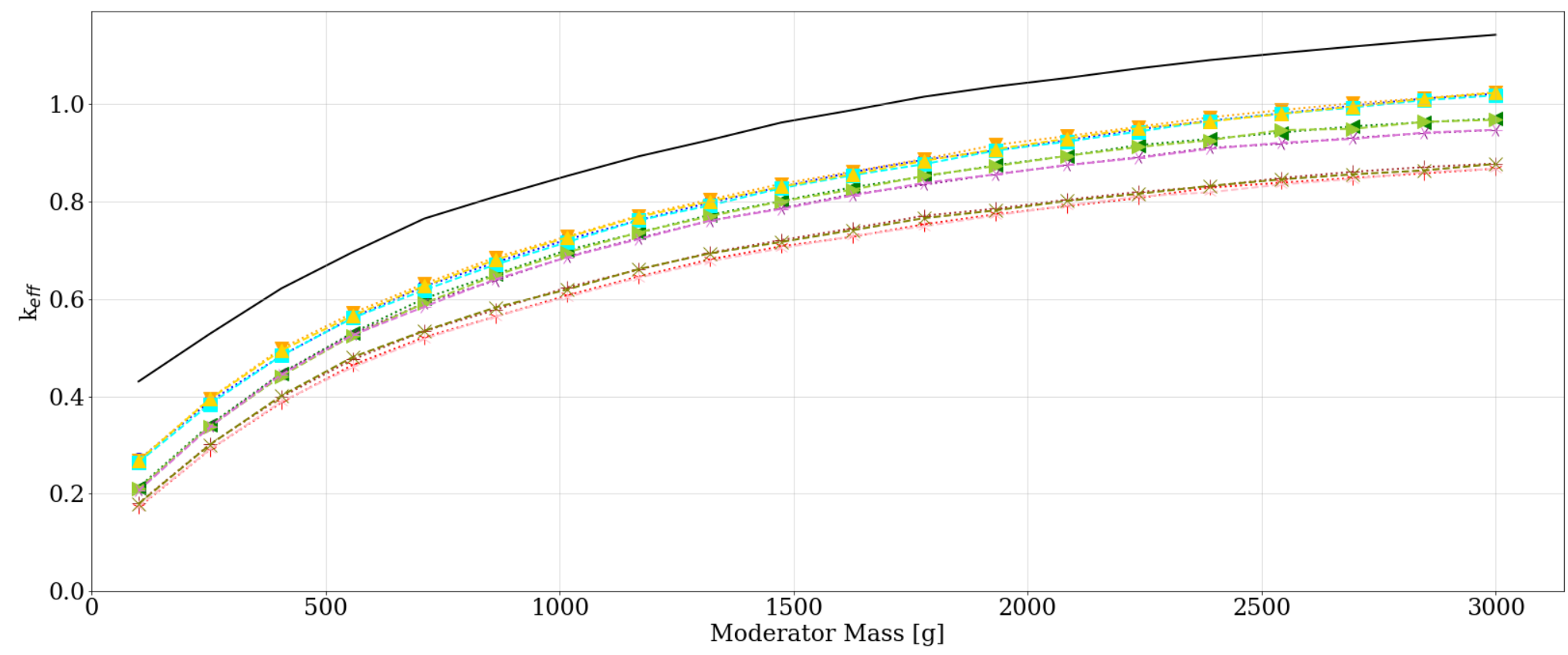

- Max Upper Horizon Reference (Set-2)

-... Max Lower Horizon, CCO Tri Pitch Array, Mirror BC

$\checkmark$... Max Upper Horizon, CCO Tri Pitch Array, Mirror BC

4. Max Lower Horizon, 6" POP Hex Pitch Array, Mirror BC

..... Max Lower Horizon, 12" POP Hex Pitch Array, Mirror BC

«.... Max Upper Horizon, 6" POP Tri Pitch Array, Mirror BC

...+... Max Upper Horizon, 12" POP Tri Pitch Array, Mirror BC

Figure M-24. Max $k_{\text {eff }}$ of all subcase-1 of all CCO and POP centroid configurations at time = 1,000 years compared with max $k_{\text {eff }}$ of all subsets of set-2uh hexagonal pitch (mirror boundary conditions) as $k_{\text {eff }} \mathrm{vs}$. moderator mass, time $=1,000$ years. 


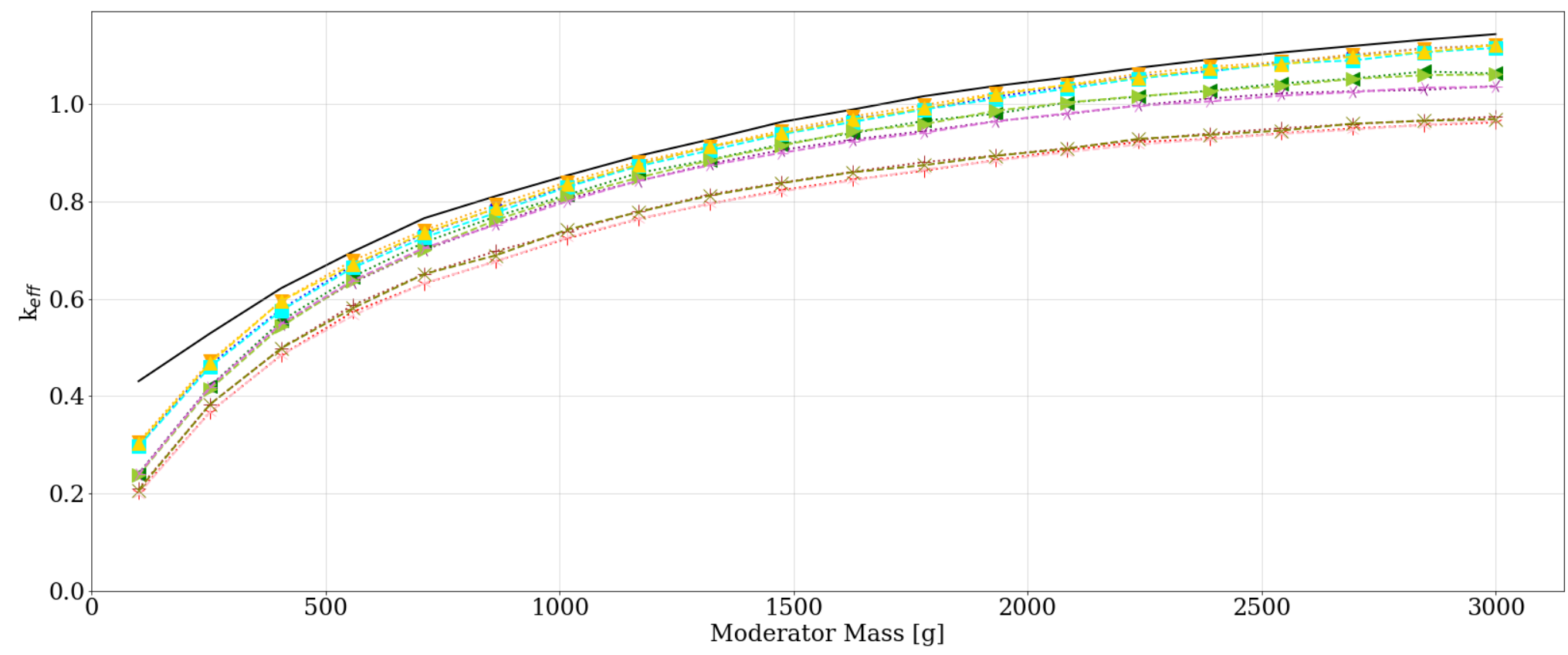

- Max Upper Horizon Reference (Set-2)

-... Max Lower Horizon, CCO Tri Pitch Array, Mirror BC

$\checkmark$... Max Upper Horizon, CCO Tri Pitch Array, Mirror BC

4. Max Lower Horizon, 6" POP Hex Pitch Array, Mirror BC

..... Max Lower Horizon, 12" POP Hex Pitch Array, Mirror BC

«.... Max Upper Horizon, 6" POP Tri Pitch Array, Mirror BC

...+... Max Upper Horizon, 12" POP Tri Pitch Array, Mirror BC

Figure M-25. Max $k_{e f f}$ of all subcase-2 of all CCO and POP centroid configurations at time = 1,000 years compared with max $k_{e f f}$ of all subsets of set-2uh hexagonal pitch (mirror boundary conditions) as $k_{\text {eff }} \mathrm{vs}$. moderator mass, time $=1,000$ years. 


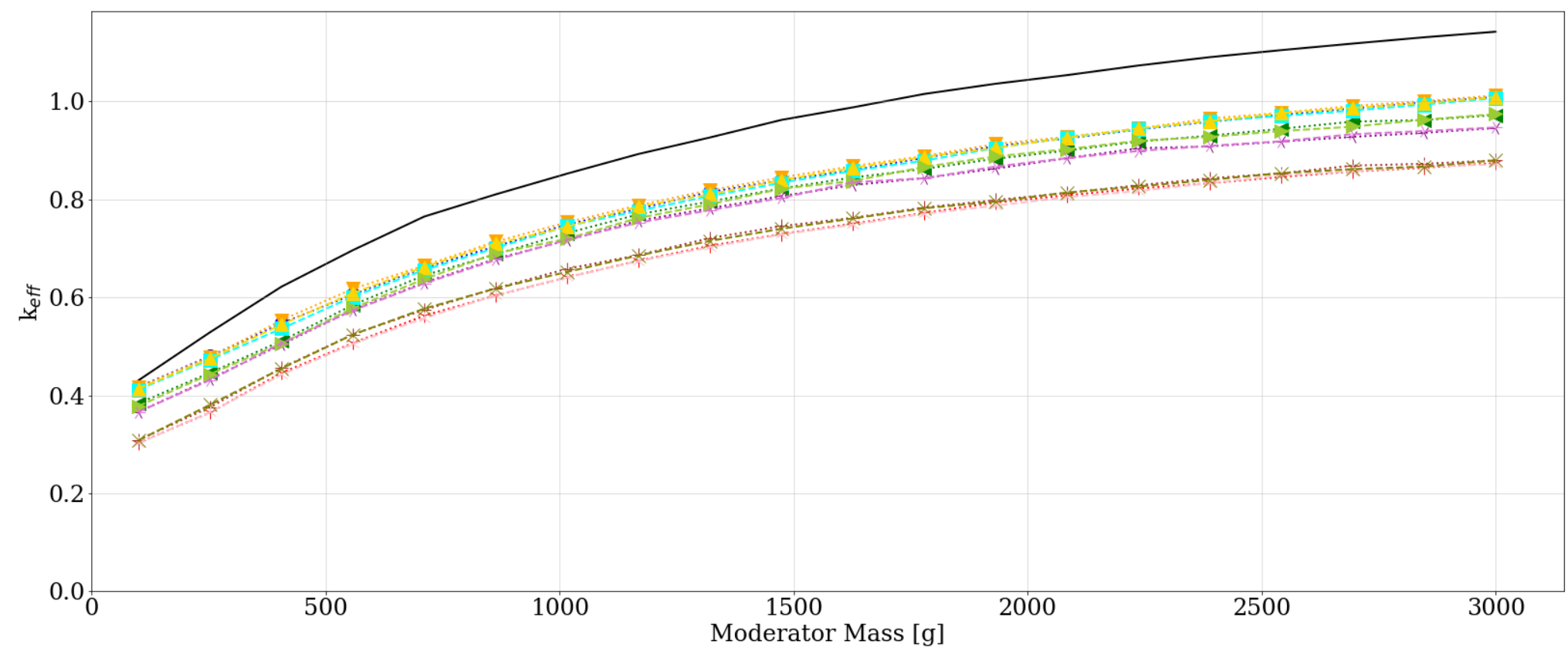

- Max Upper Horizon Reference (Set-2)

-... Max Lower Horizon, CCO Tri Pitch Array, Mirror BC

$\checkmark$... Max Upper Horizon, CCO Tri Pitch Array, Mirror BC

4. Max Lower Horizon, 6" POP Hex Pitch Array, Mirror BC

..... Max Lower Horizon, 12" POP Hex Pitch Array, Mirror BC

«.... Max Upper Horizon, 6" POP Tri Pitch Array, Mirror BC

...+... Max Upper Horizon, 12" POP Tri Pitch Array, Mirror BC

Figure M-26. Max $k_{\text {eff }}$ of all subcase-3 of all CCO and POP centroid configurations at time = 1,000 years compared with max $k_{e f f}$ of all subsets of set-2uh hexagonal pitch (mirror boundary conditions) as $k_{\text {eff }} \mathrm{vs}$. moderator mass, time $=1,000$ years. 


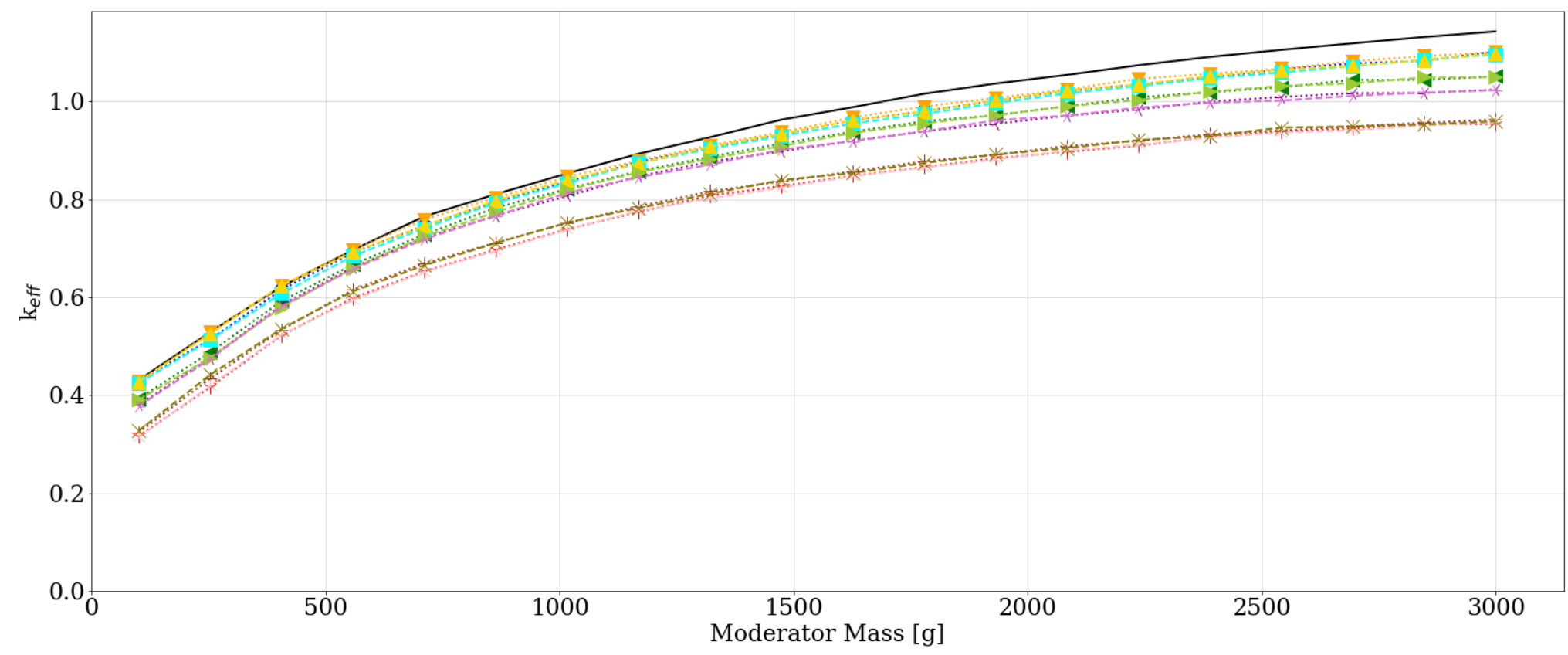

- Max Upper Horizon Reference (Set-2)

-... Max Lower Horizon, CCO Tri Pitch Array, Mirror BC

$\checkmark$... Max Upper Horizon, CCO Tri Pitch Array, Mirror BC

4. Max Lower Horizon, 6" POP Hex Pitch Array, Mirror BC

..... Max Lower Horizon, 12" POP Hex Pitch Array, Mirror BC

«.... Max Upper Horizon, 6" POP Tri Pitch Array, Mirror BC

...+... Max Upper Horizon, 12" POP Tri Pitch Array, Mirror BC

Figure M-27. Max $k_{e f f}$ of all subcase-4 of all CCO and POP centroid configurations at time = 1,000 years compared with max $k_{e f f}$ of all subsets of set-2uh hexagonal pitch (mirror boundary conditions) as $k_{\text {eff }} \mathrm{vs}$. moderator mass, time $=1,000$ years. 


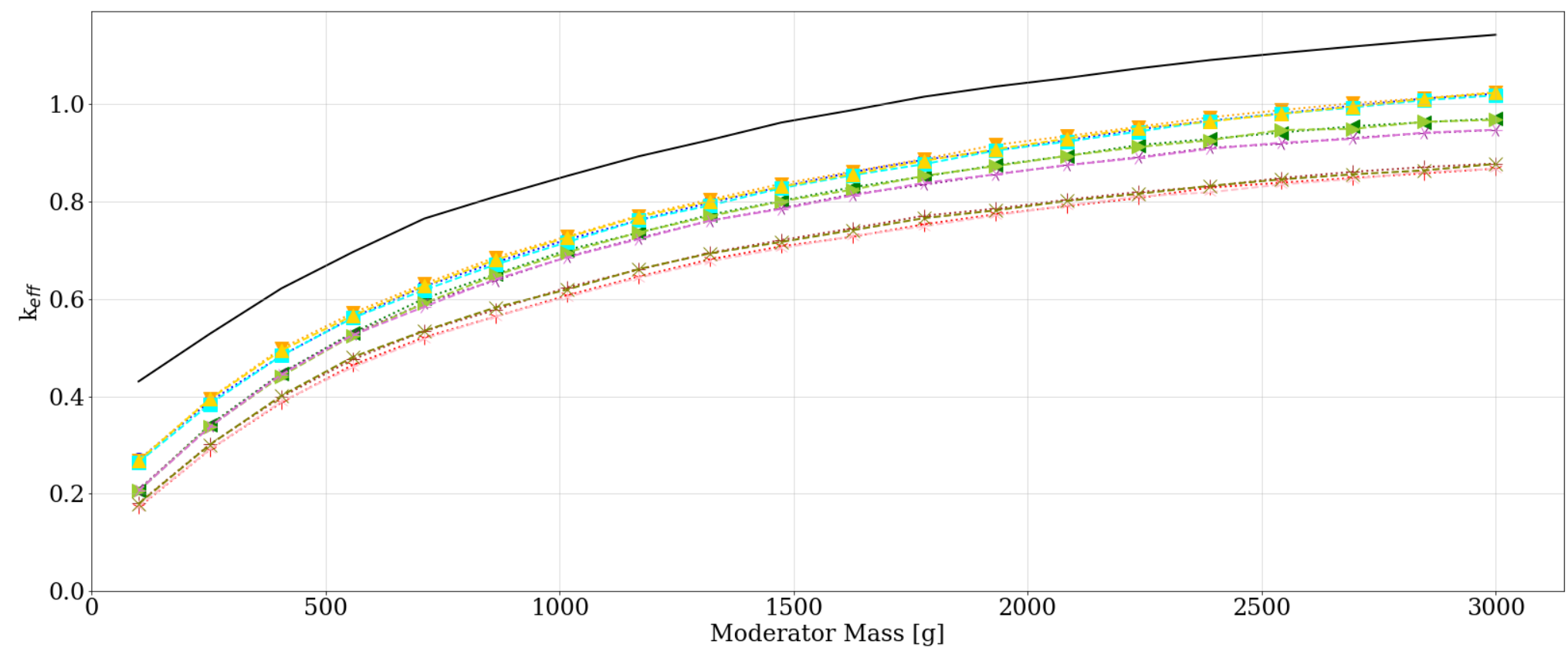

- Max Upper Horizon Reference (Set-2)

-.. Max Lower Horizon, CCO Tri Pitch Array, Mirror BC

$\checkmark$... Max Upper Horizon, CCO Tri Pitch Array, Mirror BC

4. Max Lower Horizon, 6" POP Hex Pitch Array, Mirror BC

..... Max Lower Horizon, 12" POP Hex Pitch Array, Mirror BC

....... Max Upper Horizon, 6" POP Tri Pitch Array, Mirror BC

...+... Max Upper Horizon, 12" POP Tri Pitch Array, Mirror BC

Figure M-28. Max $k_{\text {eff }}$ of all subcase-5 of all CCO and POP centroid configurations at time = 1,000 years compared with max $k_{e f f}$ of all subsets of set-2uh hexagonal pitch (mirror boundary conditions) as $k_{\text {eff }} \mathrm{vs}$. moderator mass, time $=1,000$ years. 


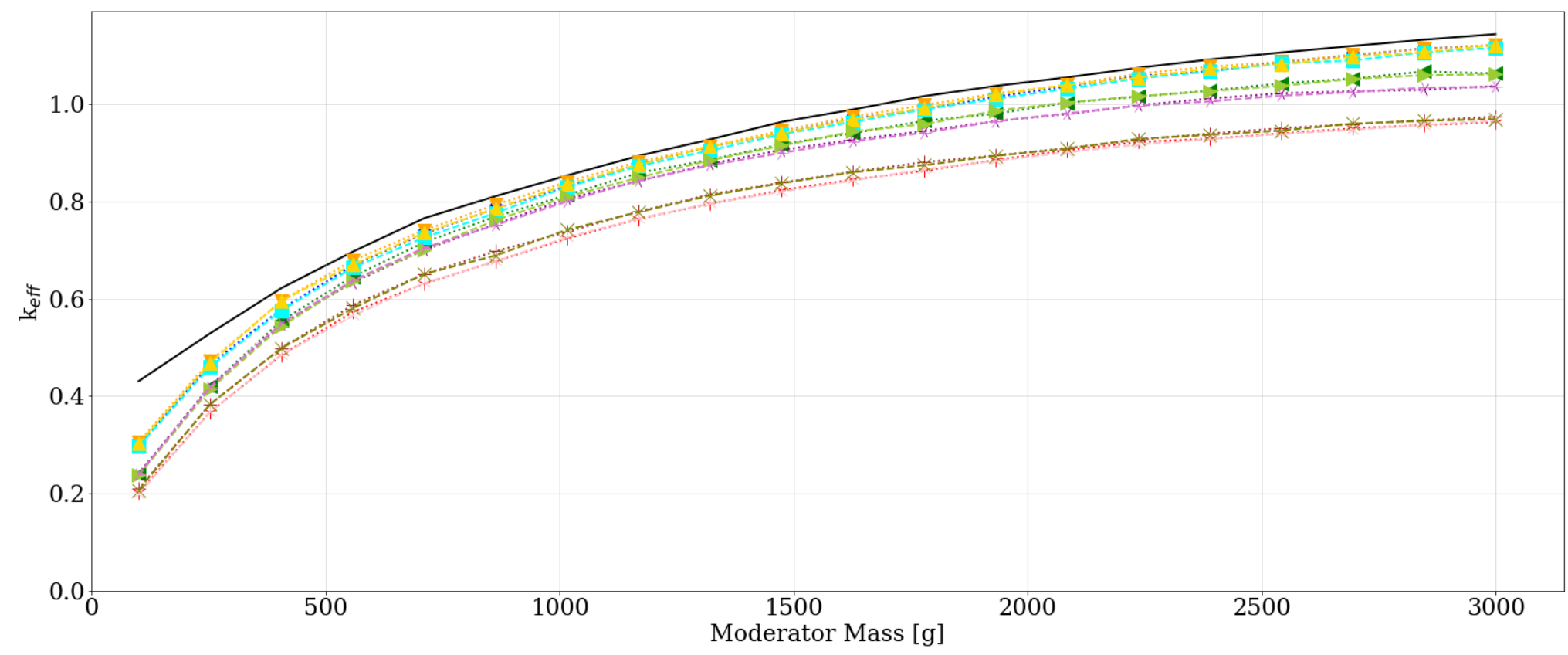

- Max Upper Horizon Reference (Set-2)

-... Max Lower Horizon, CCO Tri Pitch Array, Mirror BC

$\checkmark$... Max Upper Horizon, CCO Tri Pitch Array, Mirror BC

4. Max Lower Horizon, 6" POP Hex Pitch Array, Mirror BC

..... Max Lower Horizon, 12" POP Hex Pitch Array, Mirror BC

«.... Max Upper Horizon, 6" POP Tri Pitch Array, Mirror BC

...+... Max Upper Horizon, 12" POP Tri Pitch Array, Mirror BC

Figure M-29. Max $k_{e f f}$ of all subcase-6 of all CCO and POP centroid configurations at time = 1,000 years compared with max $k_{e f f}$ of all subsets of set-2uh hexagonal pitch (mirror boundary conditions) as $k_{\text {eff }} \mathrm{vs}$. moderator mass, time $=1,000$ years. 


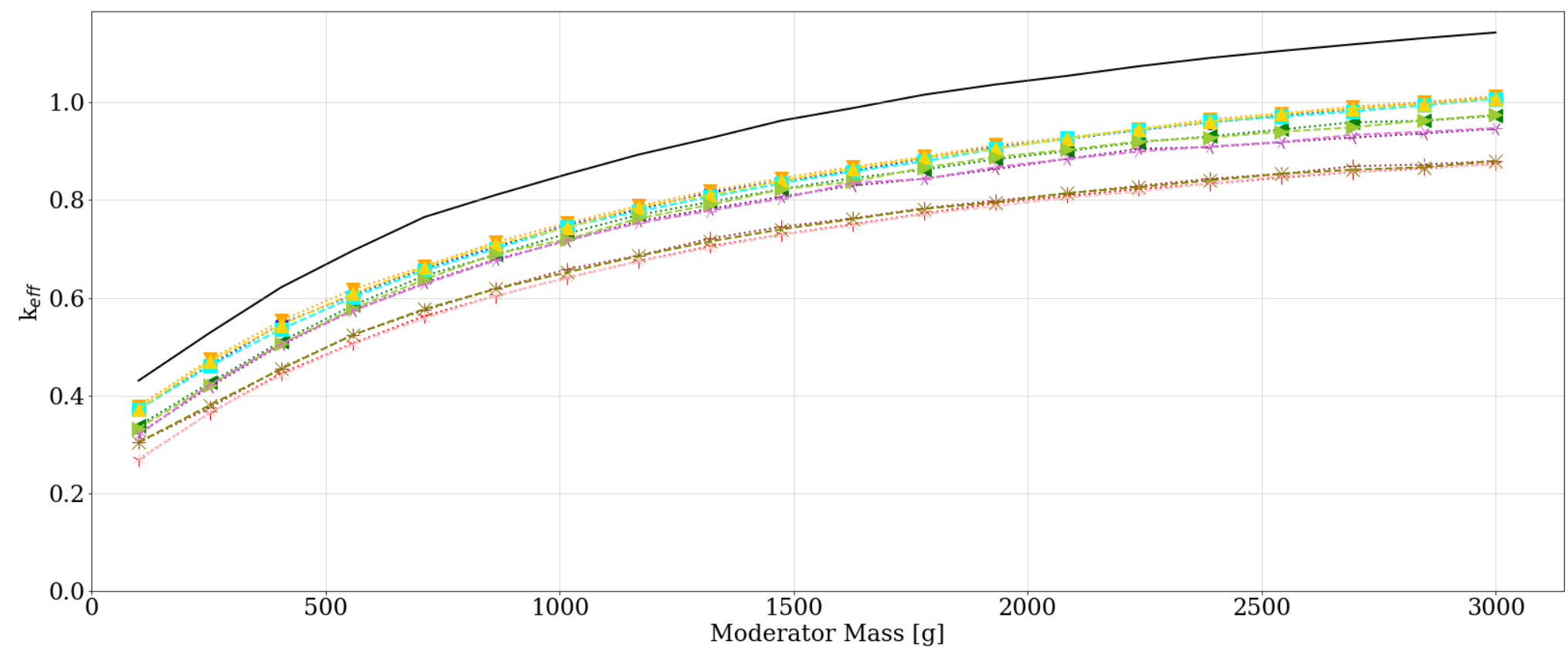

- Max Upper Horizon Reference (Set-2)

-... Max Lower Horizon, CCO Tri Pitch Array, Mirror BC

$\checkmark$... Max Upper Horizon, CCO Tri Pitch Array, Mirror BC

4. Max Lower Horizon, 6" POP Hex Pitch Array, Mirror BC

..... Max Lower Horizon, 12" POP Hex Pitch Array, Mirror BC

«.... Max Upper Horizon, 6" POP Tri Pitch Array, Mirror BC

...+... Max Upper Horizon, 12" POP Tri Pitch Array, Mirror BC

Figure M-30. Max $k_{\text {eff }}$ of all subcase-7 of all CCO and POP centroid configurations at time = 1,000 years compared with max $k_{e f f}$ of all subsets of set-2uh hexagonal pitch (mirror boundary conditions) as $k_{\text {eff }} \mathrm{vs}$. moderator mass, time $=1,000$ years. 


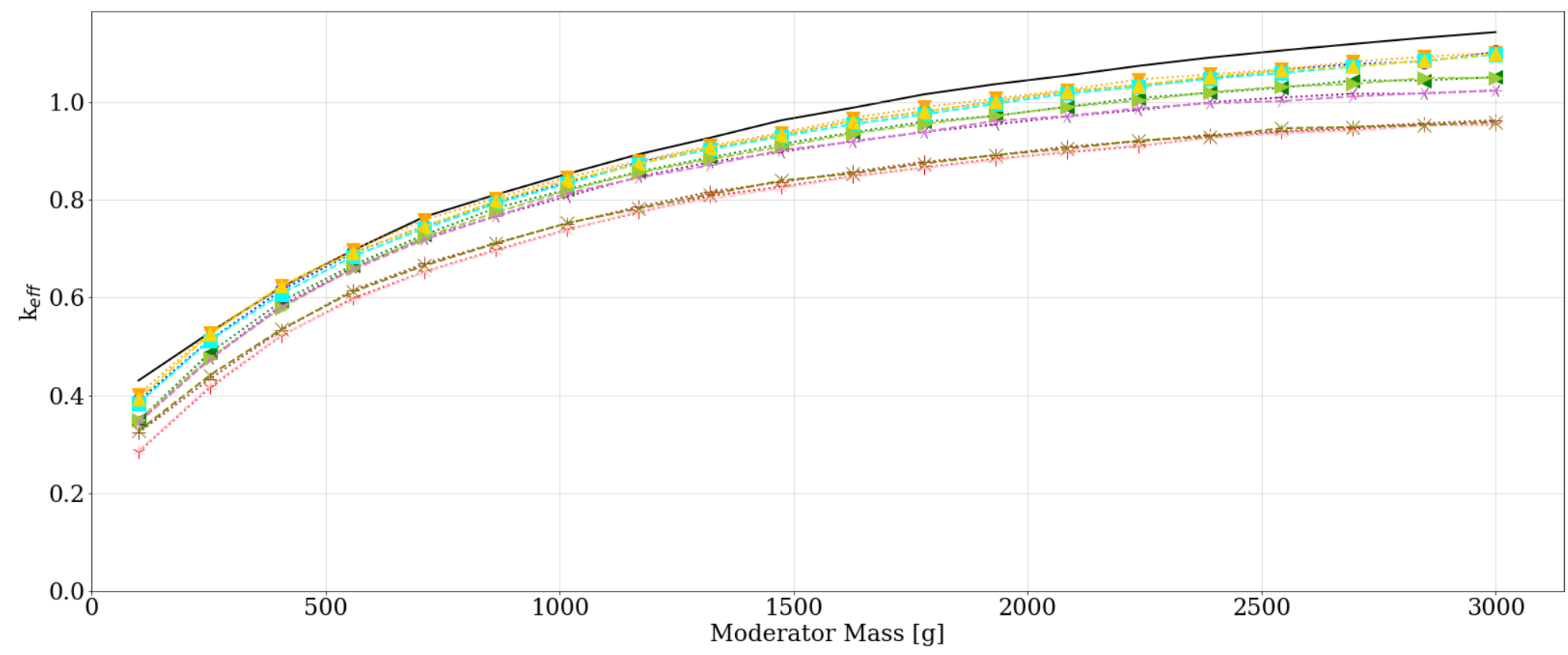

- Max Upper Horizon Reference (Set-2)

-... Max Lower Horizon, CCO Tri Pitch Array, Mirror BC

$\checkmark$... Max Upper Horizon, CCO Tri Pitch Array, Mirror BC

4. Max Lower Horizon, 6" POP Hex Pitch Array, Mirror BC

..... Max Lower Horizon, 12" POP Hex Pitch Array, Mirror BC

«.... Max Upper Horizon, 6" POP Tri Pitch Array, Mirror BC

...+... Max Upper Horizon, 12" POP Tri Pitch Array, Mirror BC

Figure M-31. Max $k_{\text {eff }}$ of all subcase-8 of all CCO and POP centroid configurations at time = 1,000 years compared with max $k_{\text {eff }}$ of all subsets of set-2uh hexagonal pitch (mirror boundary conditions) as $k_{\text {eff }} \mathrm{vs}$. moderator mass, time $=1,000$ years. 


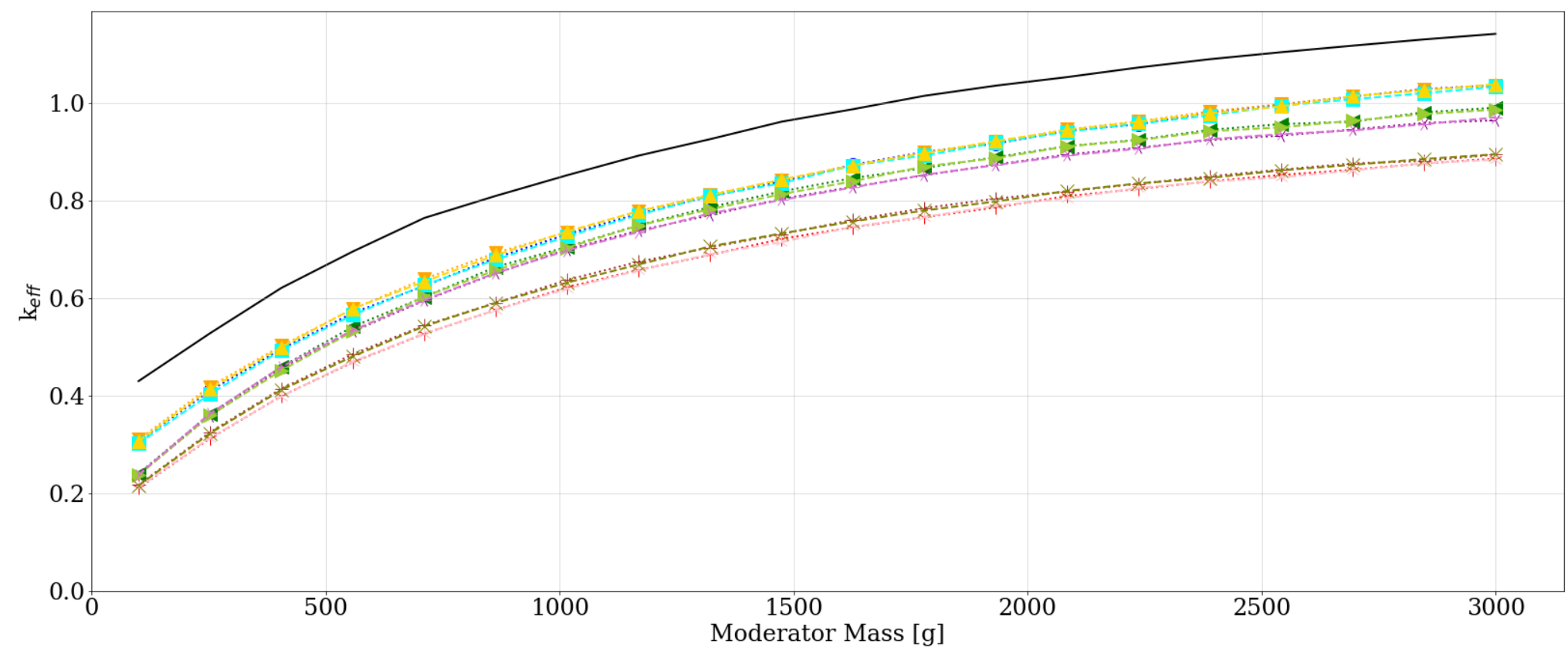

- Max Upper Horizon Reference (Set-2)

-... Max Lower Horizon, CCO Tri Pitch Array, Mirror BC

$\checkmark$... Max Upper Horizon, CCO Tri Pitch Array, Mirror BC

4. Max Lower Horizon, 6" POP Hex Pitch Array, Mirror BC

..... Max Lower Horizon, 12" POP Hex Pitch Array, Mirror BC

«.... Max Upper Horizon, 6" POP Tri Pitch Array, Mirror BC

...+... Max Upper Horizon, 12" POP Tri Pitch Array, Mirror BC

Figure M-32. Max $k_{\text {eff }}$ of all subcase-9 of all CCO and POP centroid configurations at time = 1,000 years compared with max $k_{e f f}$ of all subsets of set-2uh hexagonal pitch (mirror boundary conditions) as $k_{\text {eff }} \mathrm{vs}$. moderator mass, time $=1,000$ years. 


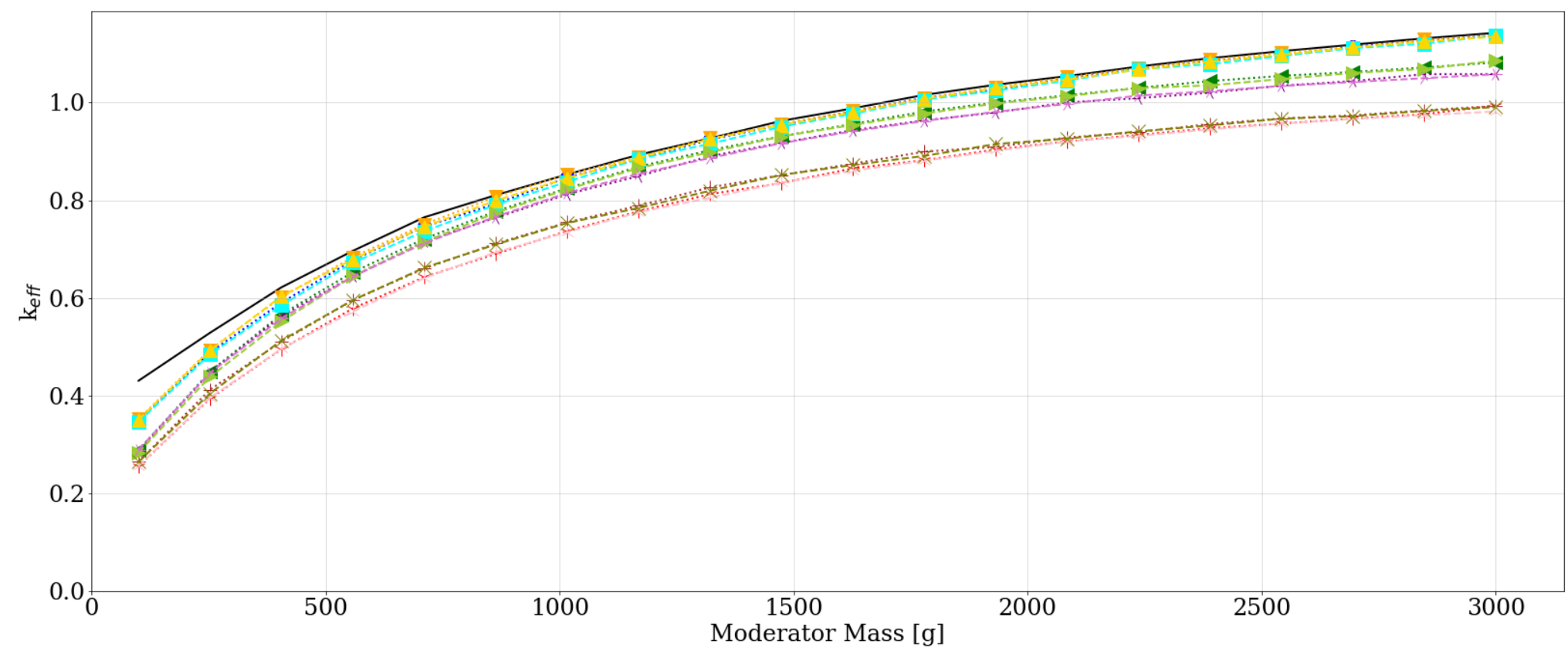

- Max Upper Horizon Reference (Set-2)

-... Max Lower Horizon, CCO Tri Pitch Array, Mirror BC

$\checkmark$... Max Upper Horizon, CCO Tri Pitch Array, Mirror BC

4. Max Lower Horizon, 6" POP Hex Pitch Array, Mirror BC

..... Max Lower Horizon, 12" POP Hex Pitch Array, Mirror BC

«.... Max Upper Horizon, 6" POP Tri Pitch Array, Mirror BC

...+... Max Upper Horizon, 12" POP Tri Pitch Array, Mirror BC

Figure M-33. Max $k_{\text {eff }}$ of all subcase-10 of all CCO and POP centroid configurations at time = 1,000 years compared with max $k_{\text {eff }}$ of all subsets of set-2uh hexagonal pitch (mirror boundary conditions) as $k_{\text {eff }} \mathrm{vs}$. moderator mass, time $=1,000$ years. 


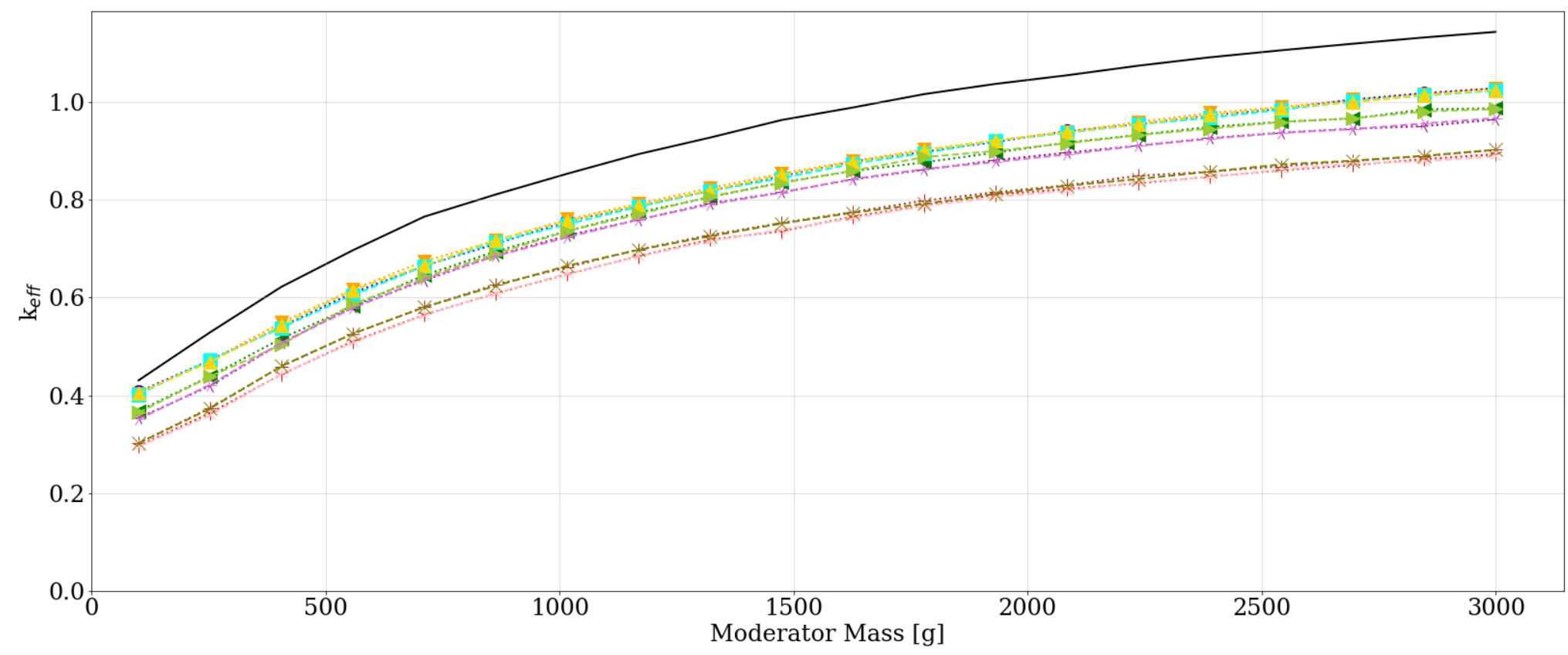

- Max Upper Horizon Reference (Set-2)

-... Max Lower Horizon, CCO Tri Pitch Array, Mirror BC

$\checkmark$... Max Upper Horizon, CCO Tri Pitch Array, Mirror BC

4. Max Lower Horizon, 6" POP Hex Pitch Array, Mirror BC

..... Max Lower Horizon, 12" POP Hex Pitch Array, Mirror BC

«.... Max Upper Horizon, 6" POP Tri Pitch Array, Mirror BC

...+... Max Upper Horizon, 12" POP Tri Pitch Array, Mirror BC

Figure M-34. Max $k_{\text {eff }}$ of all subcase-11 of all CCO and POP centroid configurations at time = 1,000 years compared with max $k_{\text {eff }}$ of all subsets of set-2uh hexagonal pitch (mirror boundary conditions) as $k_{\text {eff }} \mathrm{vs}$. moderator mass, time $=1,000$ years. 


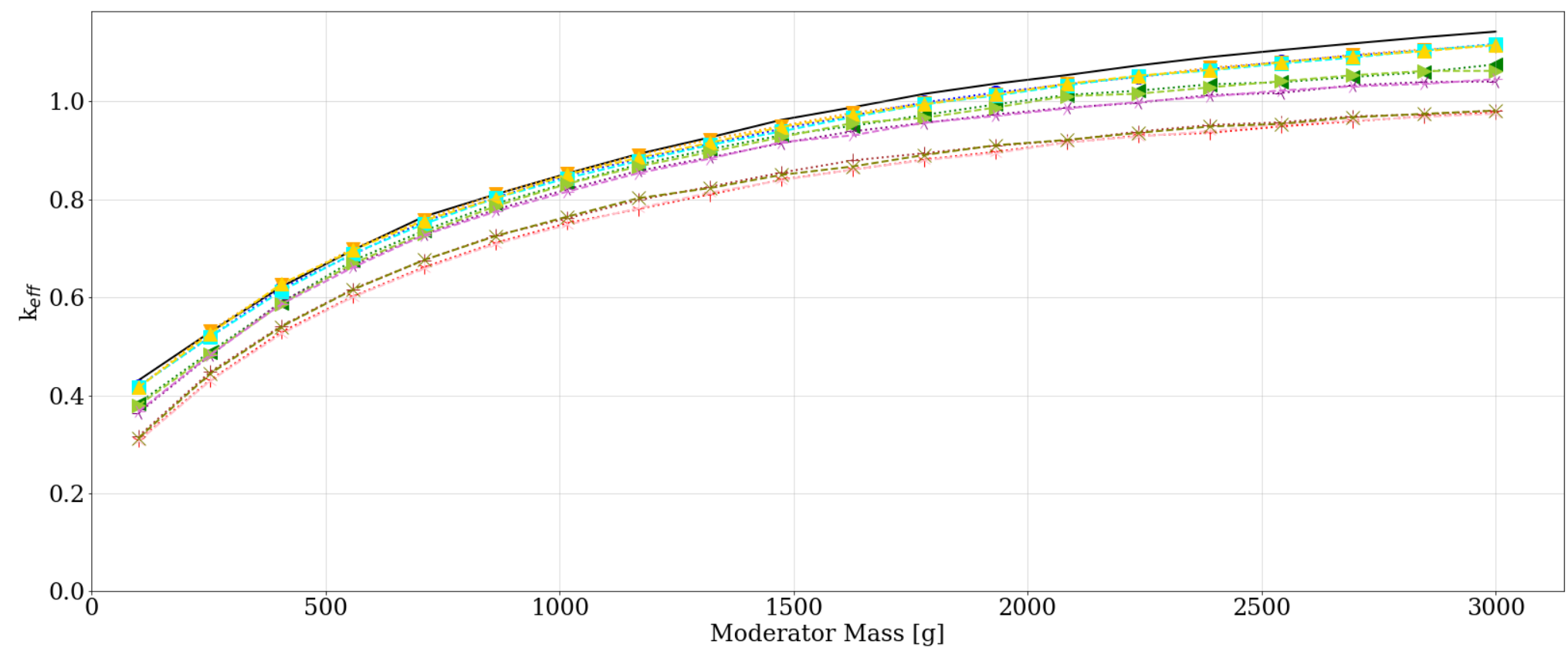

- Max Upper Horizon Reference (Set-2)

-... Max Lower Horizon, CCO Tri Pitch Array, Mirror BC

$\checkmark$... Max Upper Horizon, CCO Tri Pitch Array, Mirror BC

4. Max Lower Horizon, 6" POP Hex Pitch Array, Mirror BC

..... Max Lower Horizon, 12" POP Hex Pitch Array, Mirror BC

«.... Max Upper Horizon, 6" POP Tri Pitch Array, Mirror BC

...+... Max Upper Horizon, 12" POP Tri Pitch Array, Mirror BC

Figure M-35. Max $k_{\text {eff }}$ of all subcase-12 of all CCO and POP centroid configurations at time = 1,000 years compared with max $k_{\text {eff }}$ of all subsets of set-2uh hexagonal pitch (mirror boundary conditions) as $k_{\text {eff }} \mathrm{vs}$. moderator mass, time $=1,000$ years. 


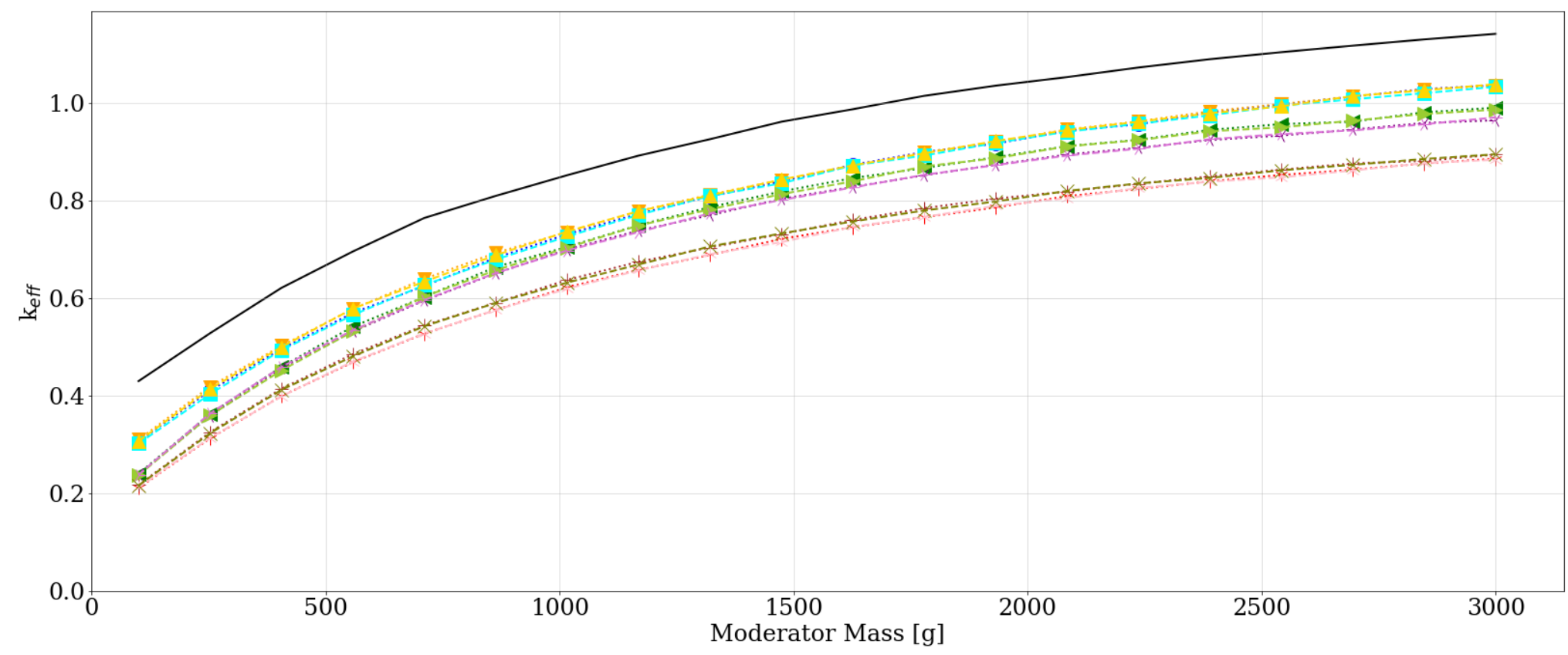

- Max Upper Horizon Reference (Set-2)

-.. Max Lower Horizon, CCO Tri Pitch Array, Mirror BC

$\checkmark$... Max Upper Horizon, CCO Tri Pitch Array, Mirror BC

4. Max Lower Horizon, 6" POP Hex Pitch Array, Mirror BC

..... Max Lower Horizon, 12" POP Hex Pitch Array, Mirror BC

«.... Max Upper Horizon, 6" POP Tri Pitch Array, Mirror BC

...+... Max Upper Horizon, 12" POP Tri Pitch Array, Mirror BC

Figure M-36. Max $k_{\text {eff }}$ of all subcase-13 of all CCO and POP centroid configurations at time = 1,000 years compared with max $k_{\text {eff }}$ of all subsets of set-2uh hexagonal pitch (mirror boundary conditions) as $k_{\text {eff }} \mathrm{vs}$. moderator mass, time $=1,000$ years. 


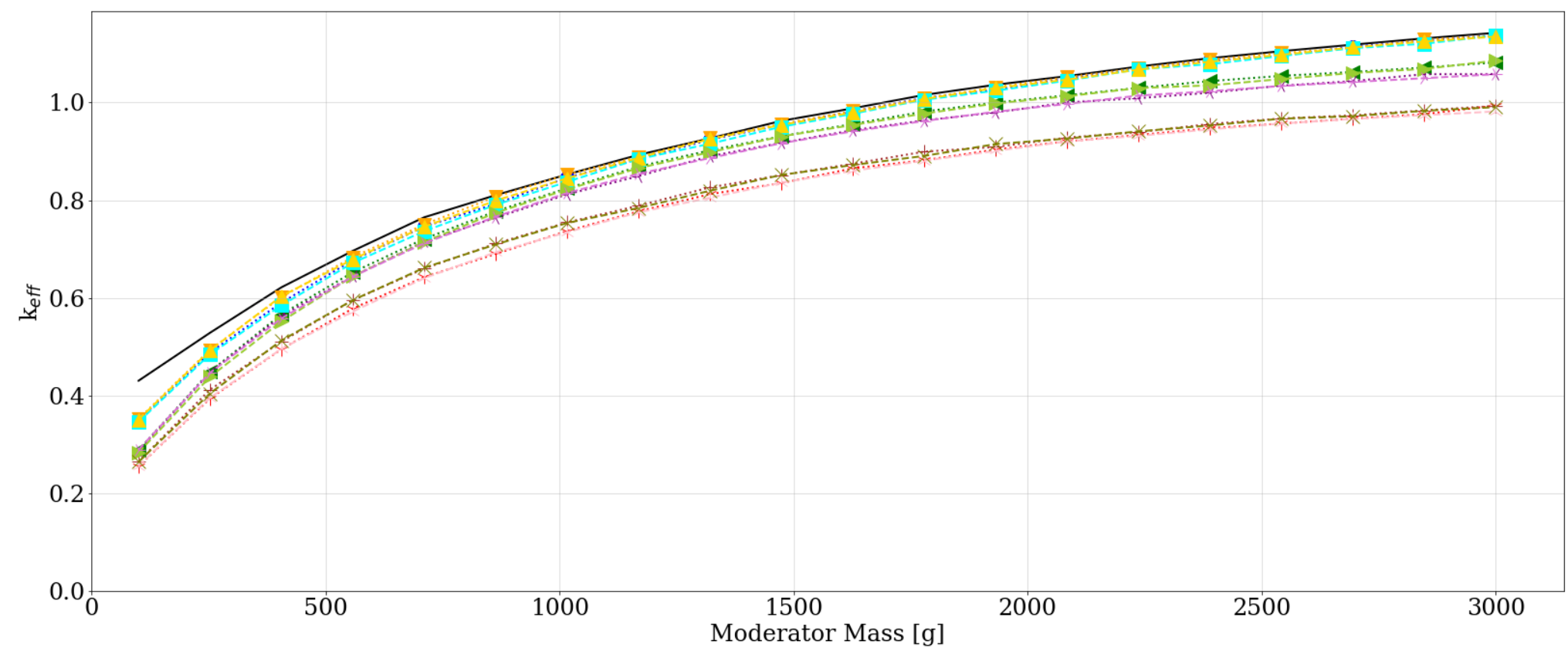

- Max Upper Horizon Reference (Set-2)

-... Max Lower Horizon, CCO Tri Pitch Array, Mirror BC

$\checkmark$... Max Upper Horizon, CCO Tri Pitch Array, Mirror BC

4. Max Lower Horizon, 6" POP Hex Pitch Array, Mirror BC

..... Max Lower Horizon, 12" POP Hex Pitch Array, Mirror BC

«.... Max Upper Horizon, 6" POP Tri Pitch Array, Mirror BC

...+... Max Upper Horizon, 12" POP Tri Pitch Array, Mirror BC

Figure M-37. Max $k_{\text {eff }}$ of all subcase-14 of all CCO and POP centroid configurations at time = 1,000 years compared with max $k_{\text {eff }}$ of all subsets of set-2uh hexagonal pitch (mirror boundary conditions) as $k_{\text {eff }} \mathrm{vs}$. moderator mass, time $=1,000$ years. 


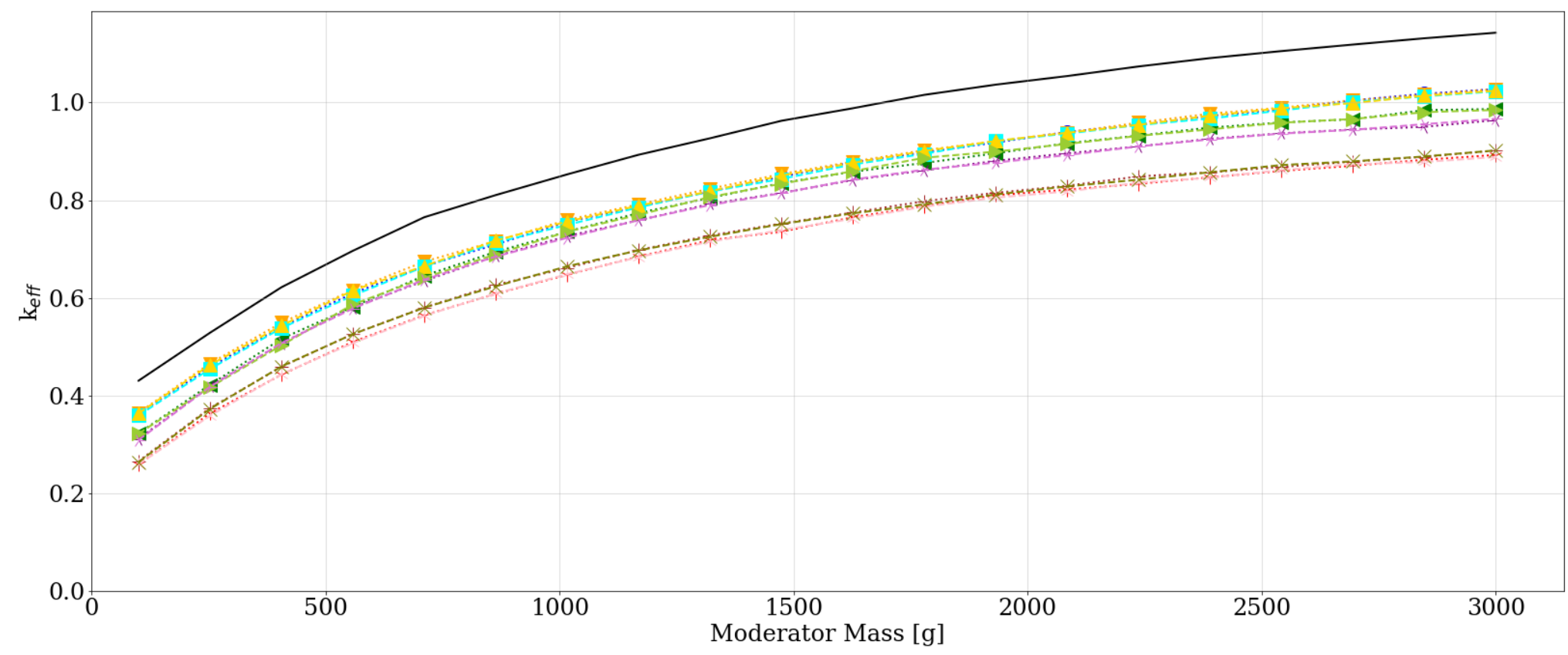

- Max Upper Horizon Reference (Set-2)

-... Max Lower Horizon, CCO Tri Pitch Array, Mirror BC

$\checkmark$... Max Upper Horizon, CCO Tri Pitch Array, Mirror BC

4. Max Lower Horizon, 6" POP Hex Pitch Array, Mirror BC

..... Max Lower Horizon, 12" POP Hex Pitch Array, Mirror BC

«.... Max Upper Horizon, 6" POP Tri Pitch Array, Mirror BC

...+... Max Upper Horizon, 12" POP Tri Pitch Array, Mirror BC

Figure M-38. Max $k_{\text {eff }}$ of all subcase-15 of all CCO and POP centroid configurations at time = 1,000 years compared with max $k_{\text {eff }}$ of all subsets of set-2uh hexagonal pitch (mirror boundary conditions) as $k_{\text {eff }} \mathrm{vs}$. moderator mass, time $=1,000$ years. 


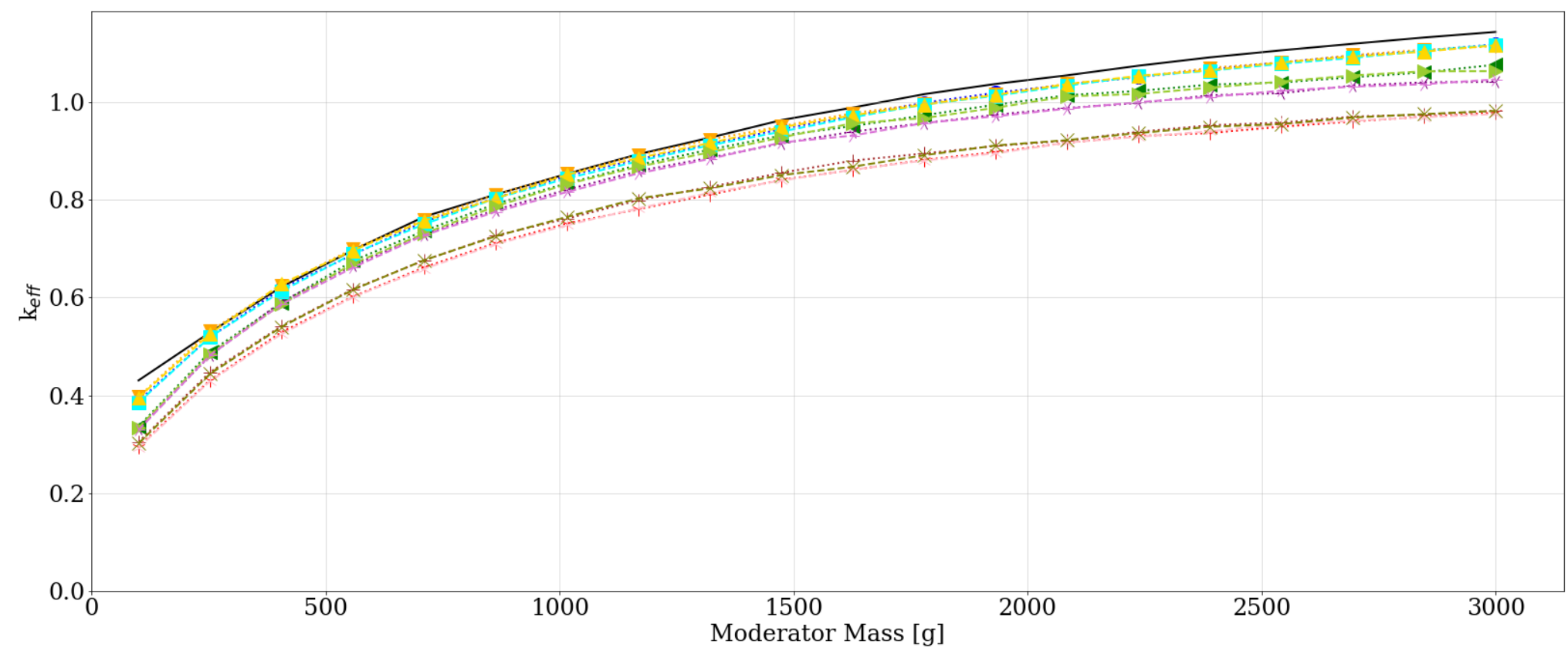

- Max Upper Horizon Reference (Set-2)

-... Max Lower Horizon, CCO Tri Pitch Array, Mirror BC

$\checkmark$... Max Upper Horizon, CCO Tri Pitch Array, Mirror BC

4. Max Lower Horizon, 6" POP Hex Pitch Array, Mirror BC

..... Max Lower Horizon, 12" POP Hex Pitch Array, Mirror BC

«.... Max Upper Horizon, 6" POP Tri Pitch Array, Mirror BC

...+... Max Upper Horizon, 12" POP Tri Pitch Array, Mirror BC

Figure M-39. Max $k_{\text {eff }}$ of all subcase-16 of all CCO and POP centroid configurations at time = 1,000 years compared with max $k_{\text {eff }}$ of all subsets of set-2uh hexagonal pitch (mirror boundary conditions) as $k_{\text {eff }} \mathrm{vs}$. moderator mass, time $=1,000$ years. 
Max in centroid-34 at $(-2.8,-0.4,-2.8)$
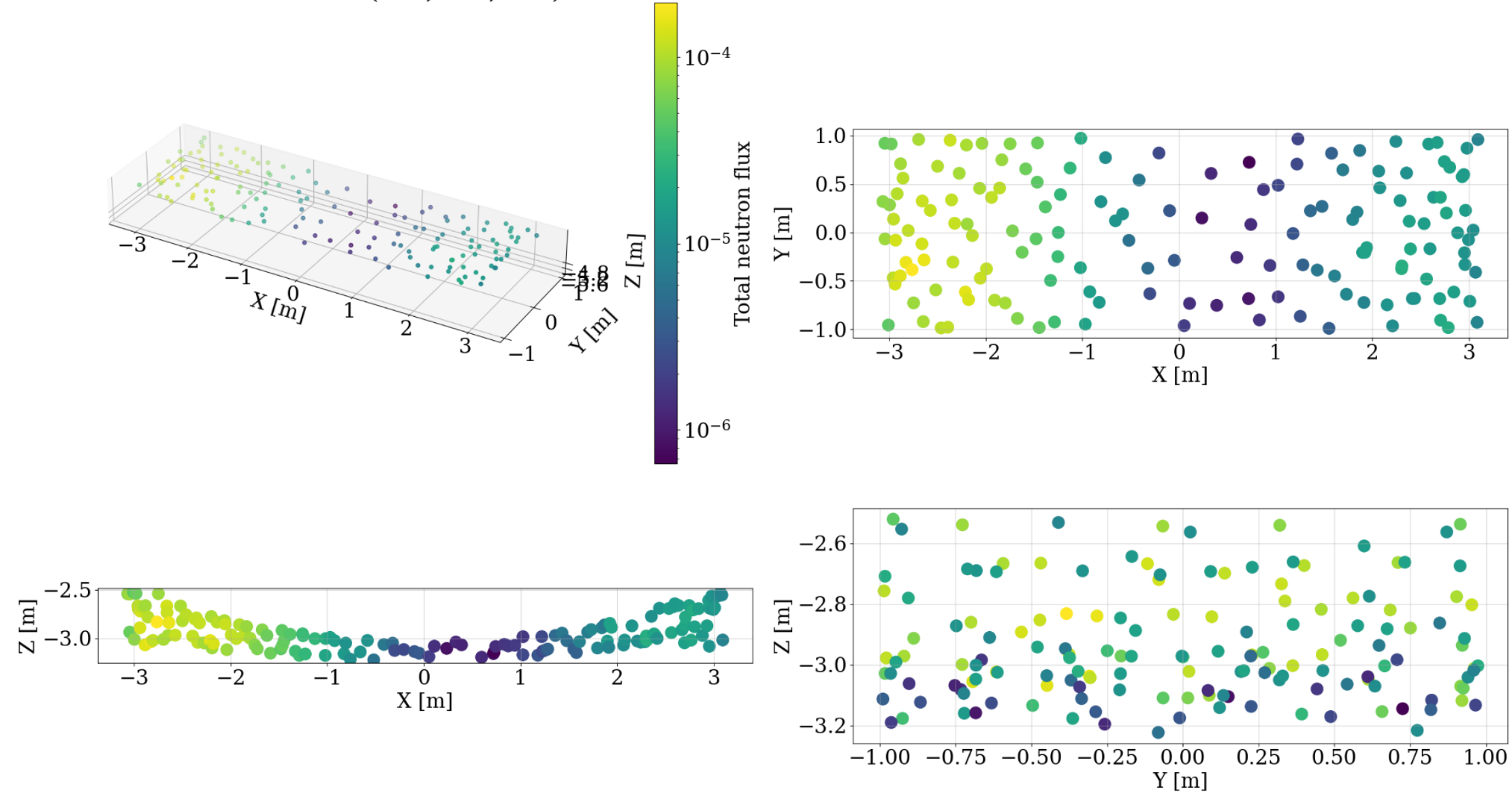

Figure M-40. Diagram of the neutron flux for subcase-10 in the CCO with no filler material and thin SS discrete reflector (sweep 470), upper horizon with hexagonal pitch (set-2-uh) with a maximum $k_{\text {eff }}$ of 1.0134 and moderator mass of 2,084 g, comparable to Figure 29 in [46]. 
Max in centroid-34 at $(-2.8,-0.4,-2.8)$

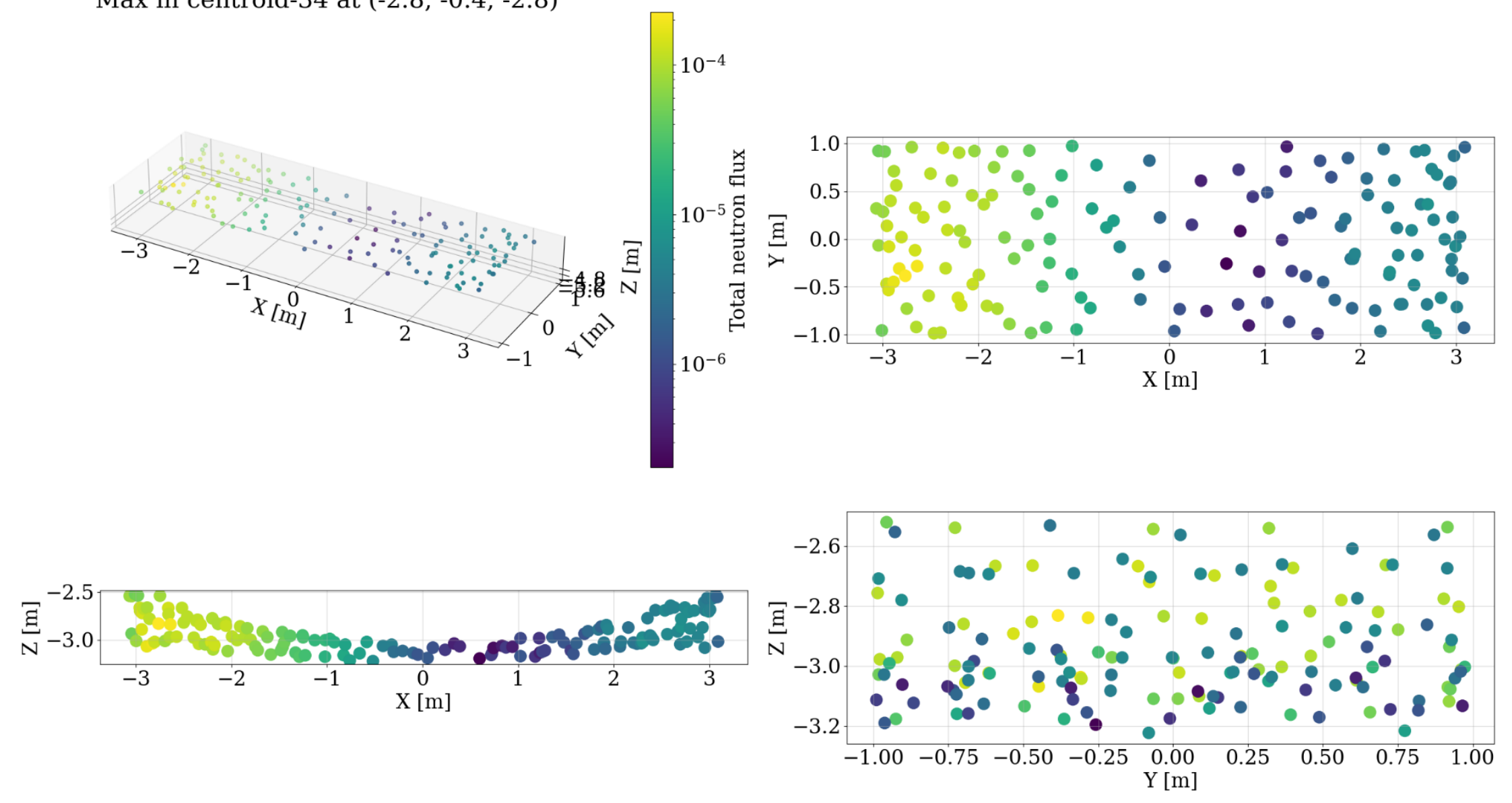

Figure M-41. Diagram of the neutron flux for subcase-10 in the CCO with $4 \mathrm{~kg}$ graphite filler material and thin SS discrete reflector (sweep 494 ), upper horizon with hexagonal pitch (set-2-uh) with a maximum $k_{\text {eff }}$ of 0.9649 and moderator mass of 2,084 g, comparable to Figure 29 in [46]. 
Max in centroid-34 at $(-2.8,-0.4,-2.8)$
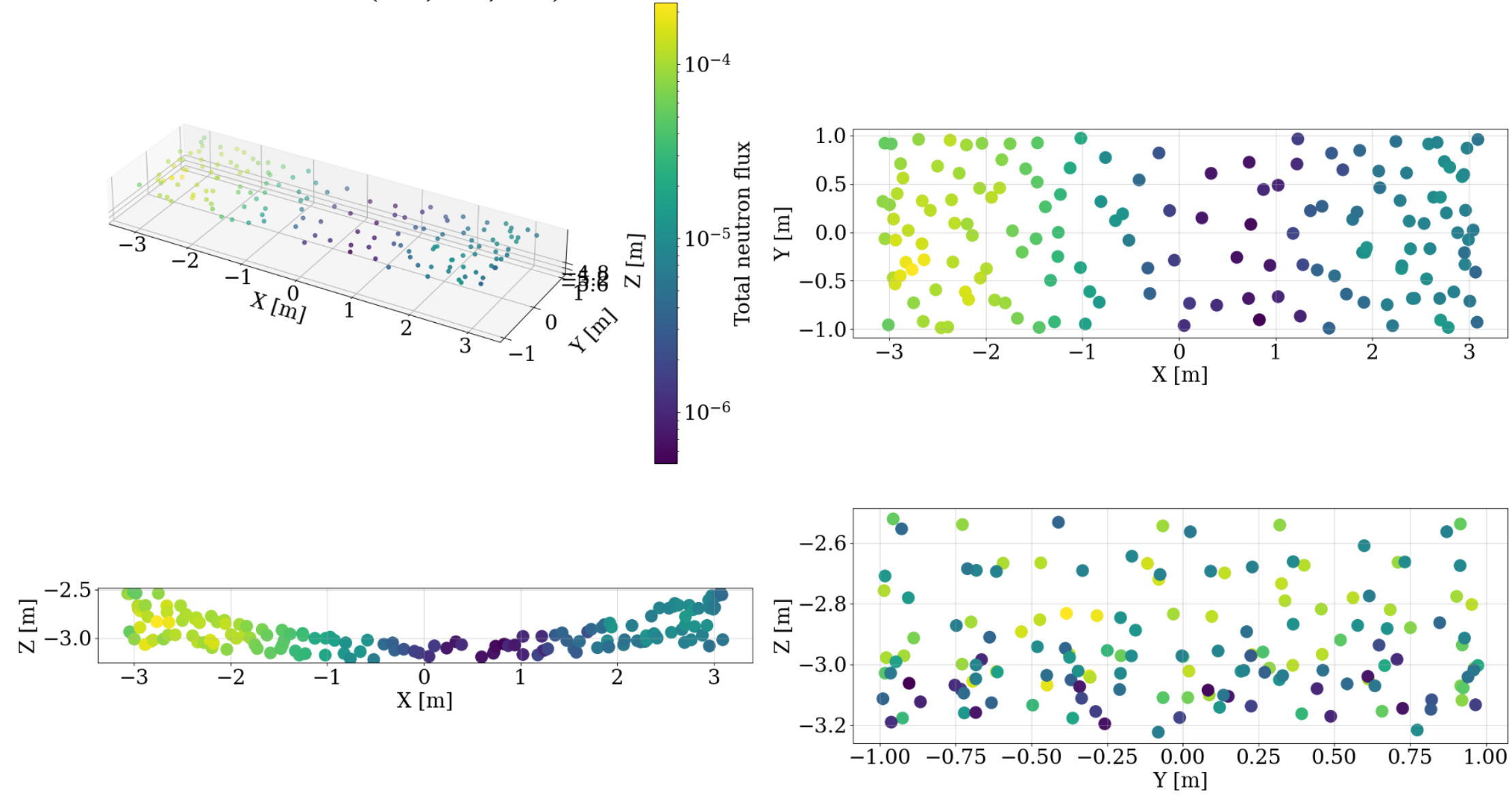

Figure M-42. Diagram of the neutron flux for subcase-10 in the CCO with no filler material and thick SS discrete reflector (sweep 472), upper horizon with hexagonal pitch (set-2-uh) with a maximum $k_{\text {eff }}$ of 1.047 and moderator mass of 2,084 g, comparable to Figure 29 in [46]. 
Max in centroid-34 at $(-2.8,-0.4,-2.8)$
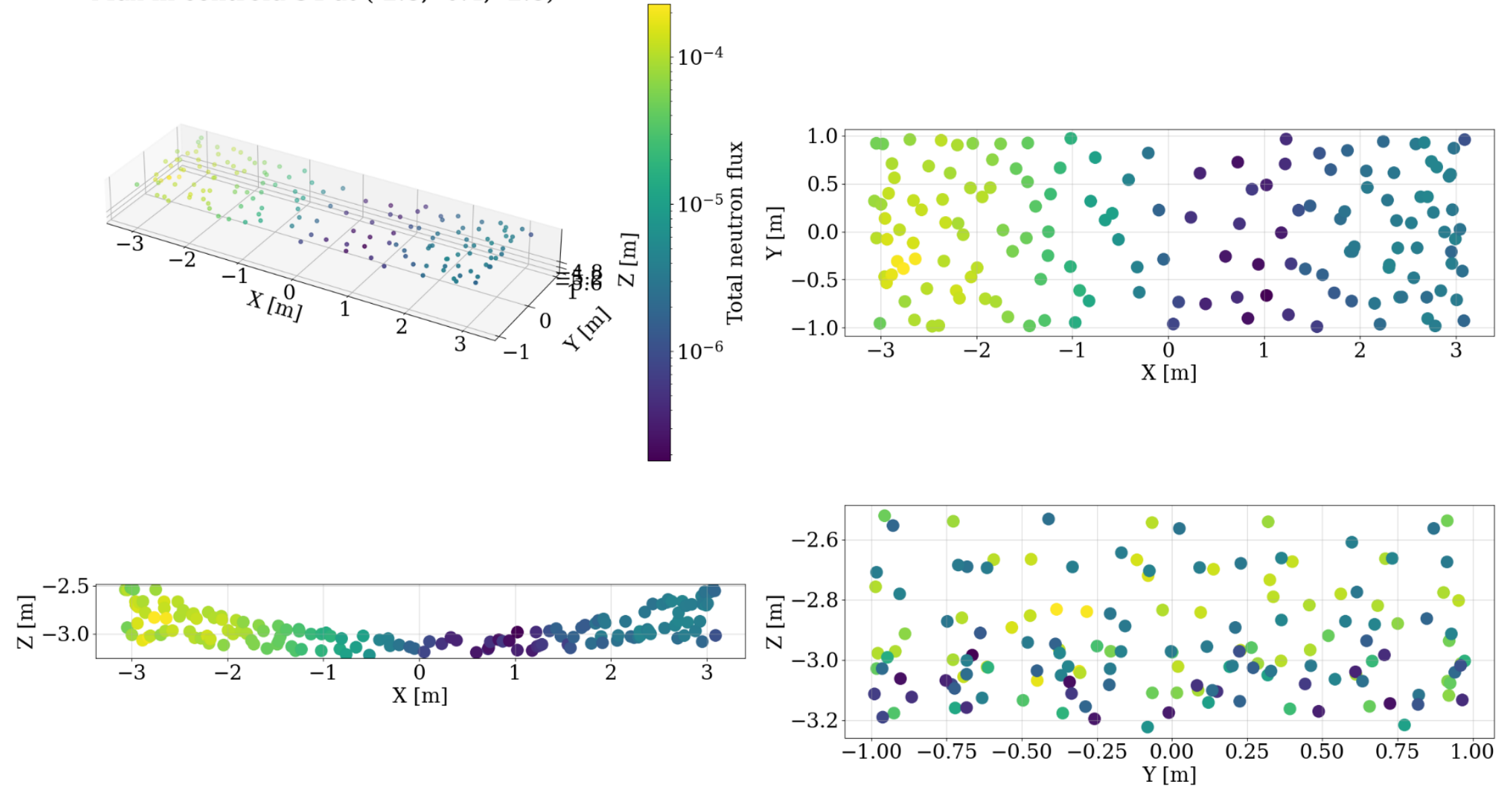

Figure M-43. Diagram of the neutron flux for subcase-10 in the CCO with $4 \mathrm{~kg}$ graphite filler material and thick SS discrete reflector (sweep 496), upper horizon with hexagonal pitch (set-2-uh) with a maximum $k_{\text {eff }}$ of 1.047 and moderator mass of 2,084 g, comparable to Figure 29 in [46]. 
Max in centroid-117 at $(-2.3,0.2,-5.4)$
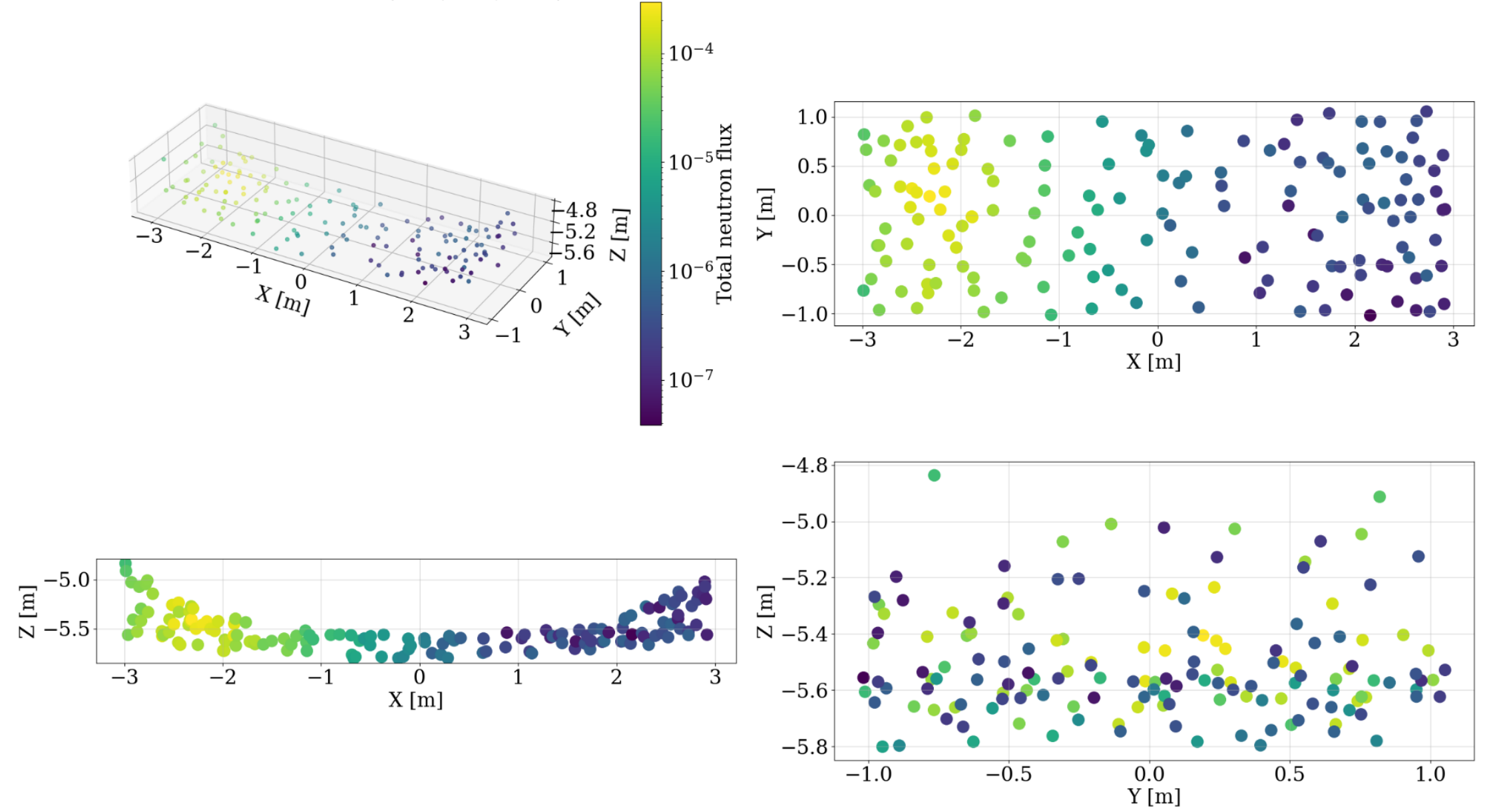

Figure M-44. Diagram of the neutron flux for subcase-10 in the CCO with no filler material and thin SS discrete reflector (sweep 470), lower horizon with hexagonal pitch (set-2-lh) with a maximum $k_{\text {eff }}$ of 1.0428 and moderator mass of 2,084 g, comparable to Figure 27 in [46]. 
Max in centroid-131 at $(-1.5,0.4,-5.5)$
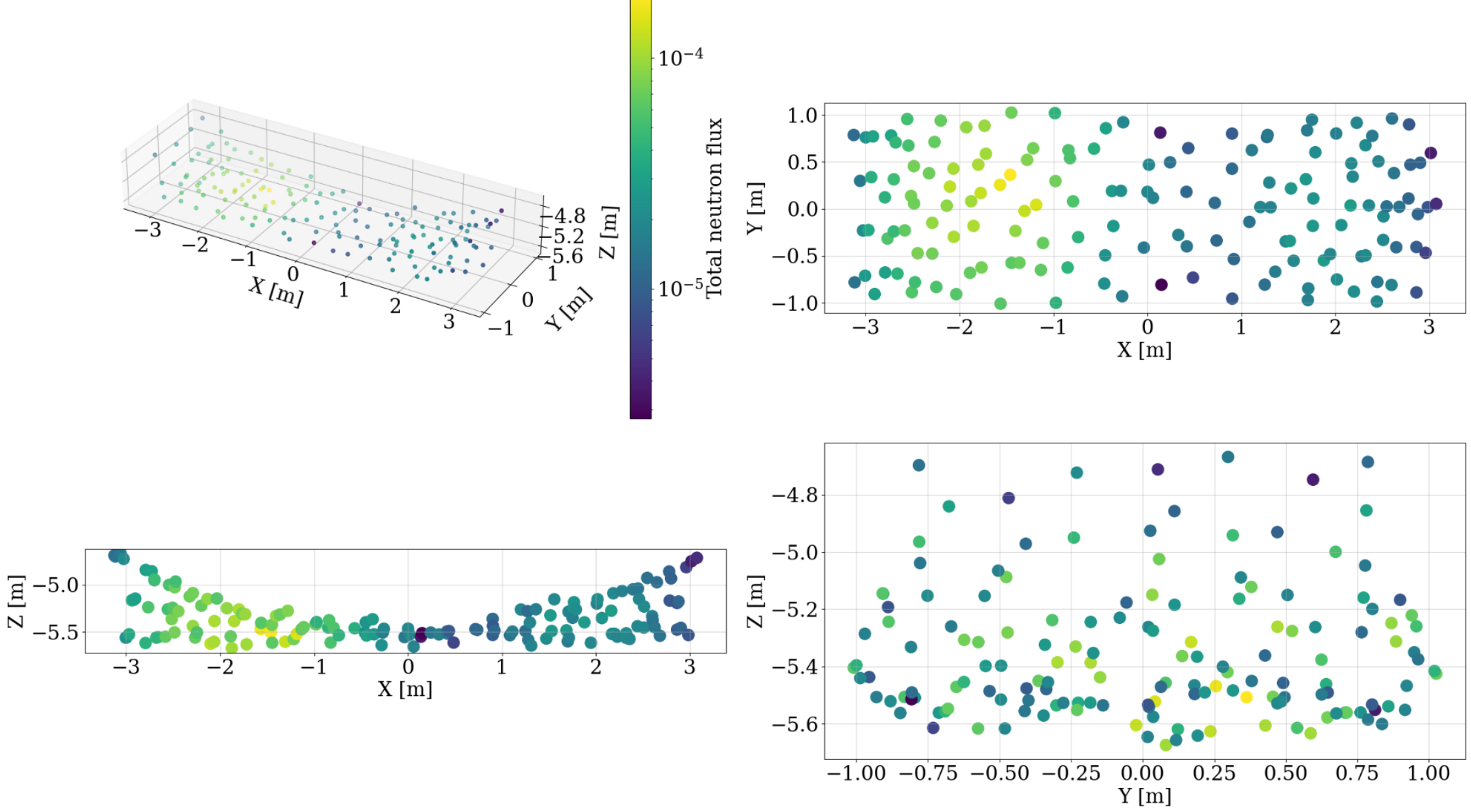

Figure M-45. Diagram of the neutron flux for subcase-10 in the 12 in. POP with no filler material and thick SS discrete reflector (sweep 688), lower horizon with hexagonal pitch with a maximum $k_{\text {eff }}$ of 0.9816 and moderator mass of $3,000 \mathrm{~g}$, comparable to Figure 37 in [46]. 
Max in centroid-37 at $(2.7,-0.8,-2.9)$
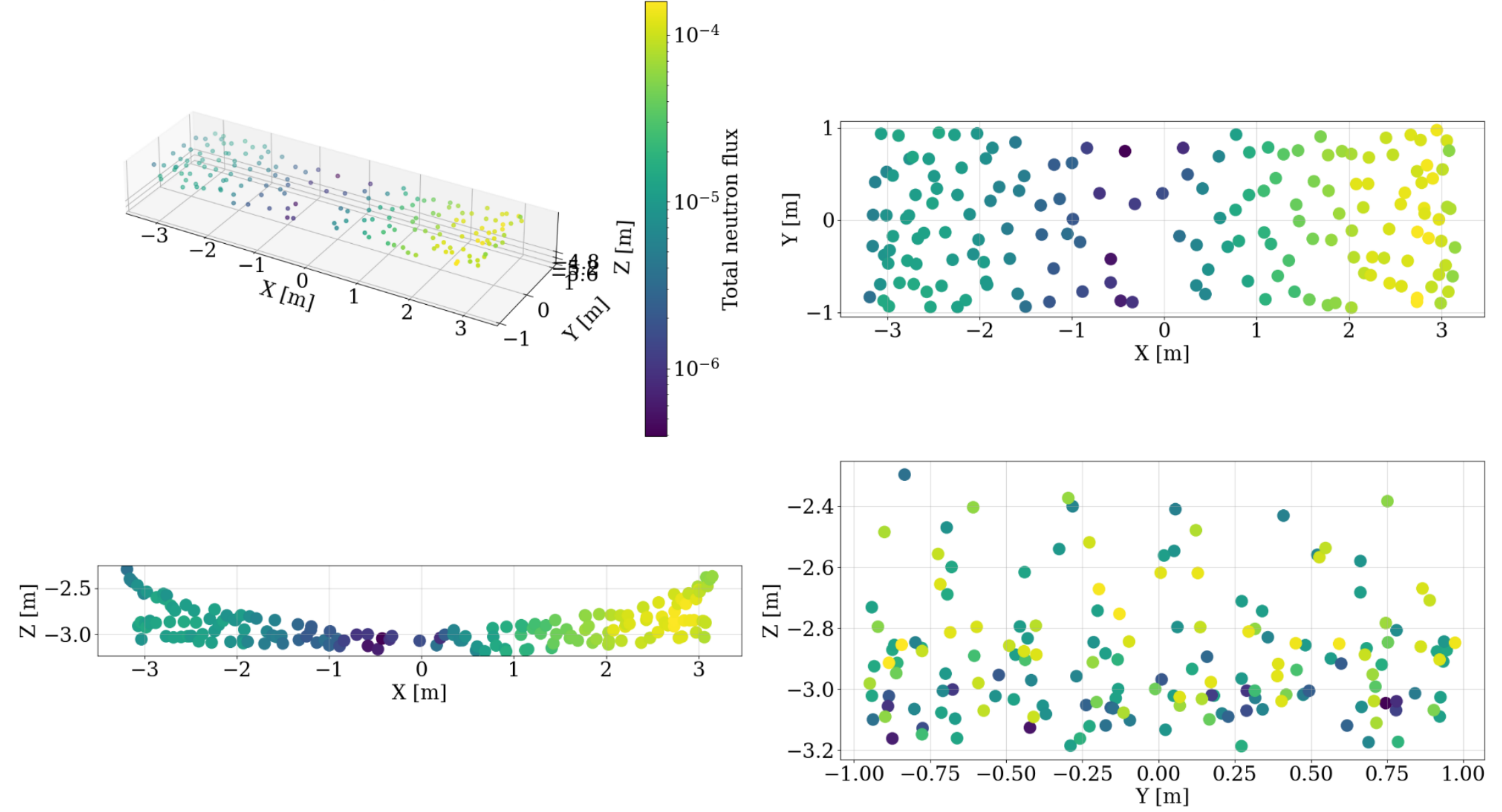

Figure M-46. Diagram of the neutron flux for subcase-10 in the CCO with no filler material and thin SS discrete reflector (sweep 470), lower horizon with triagonal pitch with a maximum $k_{\text {eff }}$ of 1.0227 and moderator mass of $2,084 \mathrm{~g}$, comparable to Figure 33 in [46]. 
Max in centroid-34 at $(-2.3,-0.5,-5.4)$

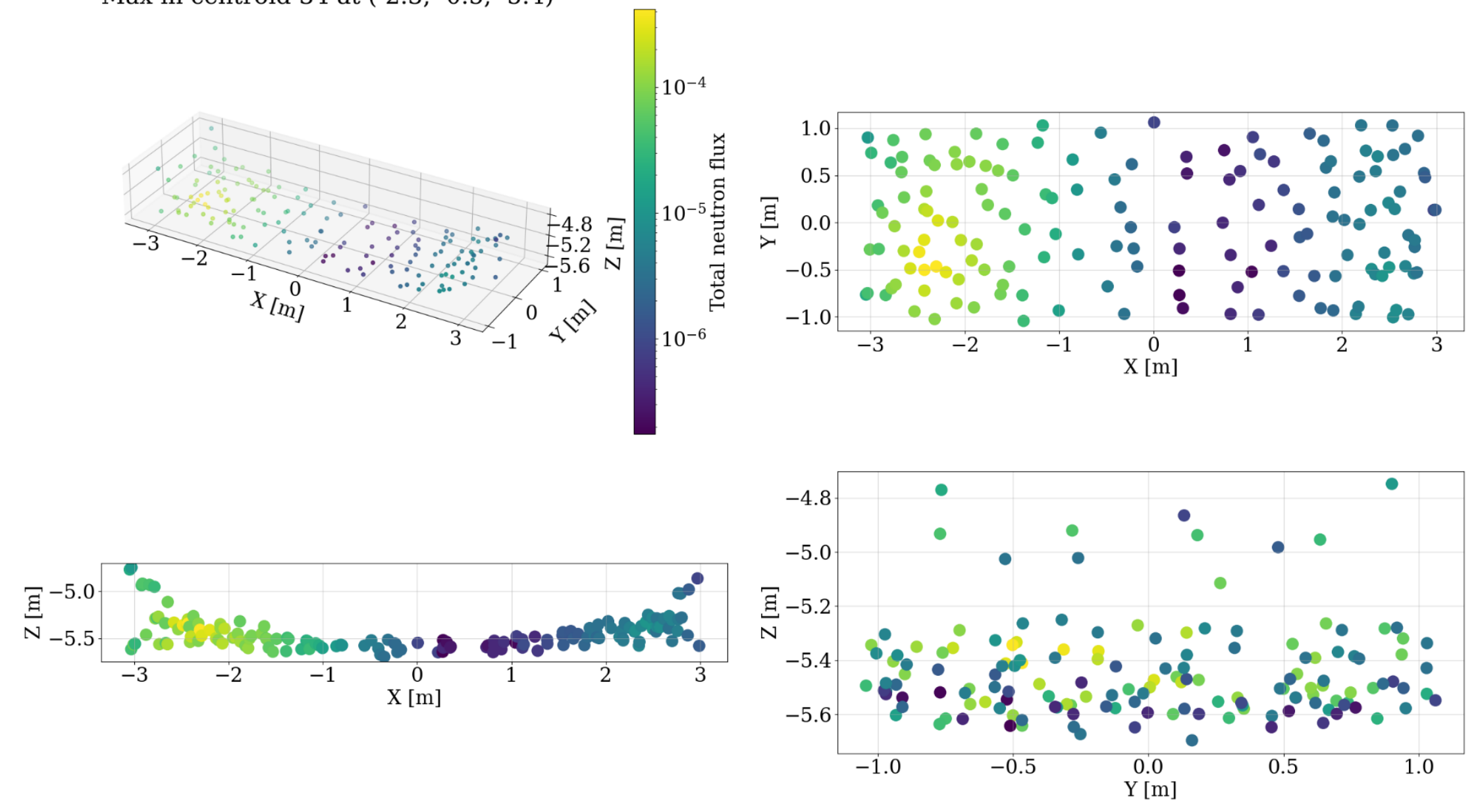

Figure M-47. Diagram of the neutron flux for subcase-10 in the 6 in. POP with no filler material and thin SS discrete reflector (sweep 470), lower horizon with hexagonal pitch with a maximum $k_{e f f}$ of 1.0227 and moderator mass of 2,084 g, comparable to Figure 35 in [46]. 
This page is intentionally blank 
APPENDIX N. SET-7: RESULTS OF THE CALCULATIONS TO SHOW THE REACTIVITY EFFECT OF VARIOUS AMOUNTS OF B $\mathrm{B}_{4} \mathrm{C}$ UNIFORMLY MIXED IN THE WASTE FORM 
This page intentionally blank. 


\section{APPENDIX N. SET-7: RESULTS OF THE CALCULATIONS TO SHOW THE REACTIVITY EFFECT OF VARIOUS AMOUNTS OF B ${ }_{4} C$ UNIFORMLY MIXED IN THE WASTE FORM.}

This appendix serves as a repository of the results for additional $\mathrm{B}_{4} \mathrm{C}$ calculations to provide technical support for the use of $\mathrm{B}_{4} \mathrm{C}$ as a control.

The analysis models used in this appendix for set-7a are the same as the set- 1 models discussed in detail in Appendix A, the analysis models used for set- $7 \mathrm{~b}$ are the same as the set- 3 models discussed in detail in Appendix F, and the analysis models used for set-7c are the same as the set-2-uh models discussed in detail in Appendix $\mathrm{C}$. The model changes to the calculations provided in this appendix compared with Appendices A, F, and $\mathrm{C}$ are related to changes made to include the addition of various amounts of $\mathrm{B}_{4} \mathrm{C}$ uniformly mixed in the waste form and to increase the total amount of moderator mass in the waste form up to $6 \mathrm{~kg}$ (subcase-10 only) (Tables N-1, N-2, and N-3).

The purpose of this appendix is to (1) expand the studies from the other appendices to the full set of subcases with $\mathrm{B}_{4} \mathrm{C}$ uniformly mixed in the waste form because only a small set of subcases was previously evaluated, (2) show the reactivity trend for additional lower masses of $\mathrm{B}_{4} \mathrm{C}$ because only $50 \mathrm{~g}$ were previously evaluated, and (3) provide sufficient detail to support technical justification for a potential $\mathrm{B}_{4} \mathrm{C}<50 \mathrm{~g}$ control by specifically using subcase-10 parametric sweeps only (Tables $\mathrm{N}-1, \mathrm{~N}-2$, and N-3).

The cases in this appendix are summarized in Tables N-1, N-2, and N-3 for set-7a, set-7b, and set-7c, respectively.

The results for the first two stated purposes - showing the complete dataset (i.e., all parametric sweeps) reactivity trends as a delta $k_{\text {eff }}$ to their reference case (set- 1 for set-7a, set- 3 for set-7b, and set-2-uh for set$7 \mathrm{c}$ ) as a function of waste form moderator mass when including 10,30 , or $50 \mathrm{~g}$ of $\mathrm{B}_{4} \mathrm{C}$ - are provided in Figures N-1, N-2, and N-3 for set-7a, set-7b, and set-7c, respectively. The results shown for the reactivity trends associated with all subsets of parametric sweeps show similar reactivity trends, as expected, because of how the ${ }^{10} \mathrm{~B}$ neutron absorption affects the system. Additionally, the same set of results is plotted as $k_{\text {eff }}$ as a function of waste form moderator mass, as provided in Figures N-4, N-5, and N-6 for set-7a, set-7b, and set-7c, respectively, and as $k_{\text {eff }}$ as a function of $\mathrm{B}_{4} \mathrm{C}$ mass, as provided in Figures N-7, $\mathrm{N}-8$, and $\mathrm{N}-9$. As expected, the results show that the reactivity effect of the ${ }^{10} \mathrm{~B}$ decreases with decreasing mass. Depending on the model, the system can remain subcritical with only the addition of uniformly mixed $\mathrm{B}_{4} \mathrm{C}$ within the waste. The summary of these results is provided in Table $\mathrm{N}-4$.

However, because of the current lack of moderator mass limits on the CCO transportation requirements ( $2 \mathrm{~kg}$ plastic limit, no water limit), additional studies are required to provide more robust technical justification for a single minimum $\mathrm{B}_{4} \mathrm{C}$ mass limit (significantly less than $50 \mathrm{~g}$ ) with no restriction on moderator mass. This appendix presents a study to provide this additional technical justification.

The results for the third aforementioned purpose - showing the subcase-10 parametric sweeps with additional $\mathrm{B}_{4} \mathrm{C}$ masses and additional moderator masses to show the peak reactivity mass conclusivelyare shown as $k_{\text {eff }}$ as a function of moderator mass in Figures N-10, N-11, and N-12 for set-7a, set-7b, and set-7c, respectively, and as $k_{\text {eff }}$ as a function of $\mathrm{B}_{4} \mathrm{C}$ mass in Figures N-13, N-14, and N-15 for set-7a, set$7 \mathrm{~b}$, and set-7c, respectively. The results show that although $k_{\text {eff }}$ continues to increase with additional moderator, this trend tapers with increasing $\mathrm{B}_{4} \mathrm{C}$ mass. Reviewing additional quantities of $\mathrm{B}_{4} \mathrm{C}$ within the waste material (i.e., 15, 20, and $25 \mathrm{~g} \mathrm{~B}_{4} \mathrm{C}$ ) also demonstrates that even though adding $\mathrm{B}_{4} \mathrm{C}$ is not a linear trend, a linear trend would be conservative. Based on these results for all of set-7 (i.e., all model designs, all subcases), $30 \mathrm{~g}$ of $\mathrm{B}_{4} \mathrm{C}$ are considered sufficient as a control with no additional controls (i.e., no moderator mass control). 
Additionally, for any waste form limit that is based at least partly on some mass of $\mathrm{B}_{4} \mathrm{C}$ uniformly mixed in the waste form, it is important to establish that it is possible (albeit very unlikely) for the ${ }^{10} \mathrm{~B}$ to be depleted because of neutron absorption over the lifetime of the repository. The following discussion provides the technical justification related to the possibility of ${ }^{10} \mathrm{~B}$ depletion.

\section{Discussion}

Criticality can be prevented by adding isotopes that have a large neutron absorption cross section. The absorption of neutrons via neutron capture by the ${ }^{10} \mathrm{~B}$ isotope component of $\mathrm{B}_{4} \mathrm{C}$ is an example material used as a parasitic neutron absorber. This discussion assumes that the waste stream mixture uses naturally occurring B with the relative abundance of ${ }^{10} \mathrm{~B}$ of $\sim 19$ atom $\%$. Neutrons are not a significant source of naturally occurring radiation and primarily occur from the interaction of cosmic rays and solar particle events with the atmosphere. Because the system is in a deep repository, there is no neutron source other than nuclear reactions within the waste. In this system, the neutron production within the waste can occur from either the spontaneous fission of the fissile material or the $\alpha$-Be reactions in which the alpha particles are emitted from the natural decay of $\mathrm{Pu}$ (the assumption is that all fissile material are $\mathrm{Pu}$ ). Essentially, fissile material will always decay via $\alpha$-decay, so spontaneous fission is not a credible neutron source. Therefore, the discussion focuses on the secondary reaction of $\alpha$-Be reactions.

The quantity of alpha-emitting isotopes is evaluated to show the number of alpha decays needed to deplete all $\mathrm{B}$ in the package, assuming that all neutrons are absorbed by $\mathrm{B}$. The assumption that all neutrons emitted via $\alpha$-Be reactions is grossly conservative because the probability of an alpha produced from decay interacting with $\mathrm{Be}$ is low, and most neutrons produced from this reaction will leak from the waste form.

In this discussion, the PuBe source was engineered to maximize neutron production efficiency from $\mathrm{Pu}$. The PuBe source produces only $2 \times 10^{6}$ neutrons/s per curie of ${ }^{238} \mathrm{Pu}$ [44]. Because all $\mathrm{Pu}$ isotopes also produce alphas with similar energies between 5 and $6 \mathrm{MeV}$ [45], this number is likely sufficient for all $\mathrm{Pu}$ isotopes. However, for conservatism, an alpha-to-neutron conversion efficiency of $10^{7}$ neutrons/s per Curie is assumed. This is five times worse than the PuBe system engineered for neutron production. Applying the definition of a Curie as $3.7 \times 10^{10}$ decays per second per Curie, the conversion efficiency of $\mathrm{Be}$ in a PuBe source is $2.7 \times 10^{-4}$ neutrons per alpha. Assuming that every neutron produced is absorbed by ${ }^{10} \mathrm{~B}, 3,700$ times more Pu nuclei would be needed than ${ }^{10} \mathrm{~B}$ nuclei to deplete all the ${ }^{10} \mathrm{~B}$. Because of Pu's large mass, the Pu mass must be roughly 88,000 times larger than the ${ }^{10} \mathrm{~B}$ mass. Given that each package has at most $380 \mathrm{~g}$ of $\mathrm{Pu}, 0.0043 \mathrm{~g}$ of ${ }^{10} \mathrm{~B}$ would be depleted if all the $\mathrm{Pu}$ decays over all time interact ideally with Be to produce neutrons, all of which are then absorbed by ${ }^{10} \mathrm{~B}$.

For perspective, $10 \mathrm{~g}$ of $\mathrm{B}_{4} \mathrm{C}$ contains $7.8 \mathrm{~g}$ of $\mathrm{B}, 19.65 \%(1.53 \mathrm{~g})$ of which is ${ }^{10} \mathrm{~B}$, and only $0.28 \%$ of this would be depleted by neutrons from $(\alpha, n)$ interactions under the grossly conservative assumptions used in this discussion. Therefore, the likelihood of ${ }^{10} \mathrm{~B}$ being depleted beyond what is needed to perform the function as evaluated is considered unrealistic. 


\section{LIST OF FIGURES}

Figure N-1. Set-7a results (three-high uniform array model) for 10,30 , and $50 \mathrm{~g}$ of $\mathrm{B}_{4} \mathrm{C}$ for all subcases.

Figure N-2. Set-7b results (six-high uniform array model) for 10,30 , and $50 \mathrm{~g}$ of $\mathrm{B}_{4} \mathrm{C}$ for all subcases.

Figure N-3. Set-7c results (three-high uniform array model) for 10, 30, and $50 \mathrm{~g}$ of $\mathrm{B}_{4} \mathrm{C}$ for all subcases.

Figure N-4. Set-7a results (three-high uniform array model) for $0,10,30$, and $50 \mathrm{~g}$ of $\mathrm{B}_{4} \mathrm{C}$ for all subcases.

Figure N-5. Set-7b results (six-high uniform array model) for $0,10,30$, and $50 \mathrm{~g}$ of $\mathrm{B}_{4} \mathrm{C}$ for all subcases.

Figure N-6. Set-7c results (nonuniform upper horizon array model) for $0,10,30$, and $50 \mathrm{~g}$ of $\mathrm{B}_{4} \mathrm{C}$ for all subcases.

Figure N-7. Set-7a results (three-high uniform array model) for 10, 30, and $50 \mathrm{~g}$ of $\mathrm{B}_{4} \mathrm{C}$ for all subcases.

Figure N-8. Set-7b results (six-high uniform array model) for 10,30 , and $50 \mathrm{~g}$ of $\mathrm{B}_{4} \mathrm{C}$ for all subcases.

Figure N-9. Set-7c results (nonuniform array model) for 10,30 , and $50 \mathrm{~g}$ of $\mathrm{B}_{4} \mathrm{C}$ for all subcases....... N-17

Figure N-10. Set-7a results (three-high uniform array model) for 10, 15, 20, 25, 30, and $50 \mathrm{~g}$ of $\mathrm{B}_{4} \mathrm{C}$ for subcase- 10 only.

Figure N-11. Set-7b results (six-high uniform array model) for 10, 15, 20, 25, 30, and $50 \mathrm{~g}$ of $\mathrm{B}_{4} \mathrm{C}$ for subcase-10 only.

Figure N-12 Set-7c results (nonuniform array model) for 10, 15, 20, 25, 30, and $50 \mathrm{~g}$ of $\mathrm{B}_{4} \mathrm{C}$ for subcase-10 only.

Figure N-13. Set-7a results (three-high uniform array model) for 10, 15, 20, 25, 30, and $50 \mathrm{~g}$ of $\mathrm{B}_{4} \mathrm{C}$ for all subcases.

Figure N-14. Set-7b results (six-high uniform array model) for 10, 15, 20, 25, 30, and $50 \mathrm{~g}$ of $\mathrm{B}_{4} \mathrm{C}$ for all subcases.

Figure N-15. Set-7c results (nonuniform array model) for 10, 15, 20, 25, 30, and $50 \mathrm{~g}$ of $\mathrm{B}_{4} \mathrm{C}$ for up to a $6 \mathrm{~kg}$ moderator.

\section{LIST OF TABLES}

Table N-1. Summary of cases for set-7a for the three-high uniform array model................................ N-6

Table N-2. Summary of cases for set-7b for the six-high uniform array model. .................................... N-7

Table N-3. Summary of cases for set-7c for the nonuniform array model. ......................................... N-8

Table N-4. Summary of results for the addition of 10,30 , or $50 \mathrm{~g}$ of $\mathrm{B}_{4} \mathrm{C}$ with a waste form moderator mass up to $3 \mathrm{~kg}$ for all subcases. N-8 
Table N-1. Summary of cases for set-7a for the three-high uniform array model.

\begin{tabular}{|c|c|c|c|c|c|c|c|c|c|}
\hline Case & $\begin{array}{l}\text { Model } \\
\text { type }\end{array}$ & Waste form shape & $\begin{array}{l}\text { Waste form } \\
\text { moderator }\end{array}$ & $\begin{array}{c}\text { Filler material } \\
(0,2,000 \\
4,000 \mathrm{~g})\end{array}$ & $\begin{array}{l}\text { Metal in } \\
\text { filler }\end{array}$ & $\begin{array}{l}\text { Discrete reflector } \\
\text { (thin } 0.001 \mathrm{~cm} \text { and } \\
\text { thick } 0.7112 \mathrm{~cm} \text { ) }\end{array}$ & $\begin{array}{l}\mathrm{Be} \\
(\mathrm{g})\end{array}$ & $\begin{array}{c}\mathrm{B}_{4} \mathrm{C} \\
(\mathrm{g})\end{array}$ & Subcase \\
\hline \multirow{16}{*}{ Set-7a } & \multirow{16}{*}{$\begin{array}{l}\text { Models } \\
\text { from } \\
\text { set-1 }\end{array}$} & \multirow{8}{*}{$\begin{array}{l}\text { Cylinder (radius range } 4.8,6 \text {, } \\
\text { and } 7.7 \text {, and height defined by } \\
\text { total volume of mass) }\end{array}$} & Water & $\mathrm{c} 12$ & \multirow{16}{*}{$\begin{array}{l}\text { SS from can } \\
\quad(0,500 \\
1,000 \mathrm{~g})\end{array}$} & Steel & \multirow{16}{*}{$0-585$} & \multirow{16}{*}{$10,30,50$} & set-7a-1 \\
\hline & & & Poly & $\mathrm{c} 12$ & & Steel & & & set-7a-2 \\
\hline & & & Water & $\mathrm{c} 12$ & & Poly & & & set-7a-3 \\
\hline & & & Poly & $\mathrm{c} 12$ & & Poly & & & set- $7 \mathrm{a}-4$ \\
\hline & & & Water & Generic & & Steel & & & set-7a -5 \\
\hline & & & Poly & Generic & & Steel & & & set-7a -6 \\
\hline & & & Water & Generic & & Poly & & & set-7a -7 \\
\hline & & & Poly & Generic & & Poly & & & set- $7 \mathrm{a}-8$ \\
\hline & & \multirow{8}{*}{$\begin{array}{l}\text { Sphere (radius defined by total } \\
\text { volume of mass) }\end{array}$} & Water & $\mathrm{c} 12$ & & Steel & & & set- $7 a-9$ \\
\hline & & & Poly & $\mathrm{c} 12$ & & Steel & & & set- $7 \mathrm{a}-10$ \\
\hline & & & Water & $\mathrm{c} 12$ & & Poly & & & set- $7 \mathrm{a}-11$ \\
\hline & & & Poly & $\mathrm{c} 12$ & & Poly & & & set- $7 \mathrm{a}-12$ \\
\hline & & & Water & Generic & & Steel & & & set- $7 a-13$ \\
\hline & & & Poly & Generic & & Steel & & & set-7a-14 \\
\hline & & & Water & Generic & & Poly & & & set- $7 \mathrm{a}-15$ \\
\hline & & & Poly & Generic & & Poly & & & set- $7 \mathrm{a}-16$ \\
\hline
\end{tabular}


Table N-2. Summary of cases for set-7b for the six-high uniform array model.

\begin{tabular}{|c|c|c|c|c|c|c|c|c|c|}
\hline Case & $\begin{array}{l}\text { Model } \\
\text { type }\end{array}$ & Waste form shape & $\begin{array}{l}\text { Waste form } \\
\text { moderator }\end{array}$ & $\begin{array}{c}\text { Filler material } \\
(\mathbf{0}, \mathbf{2 , 0 0 0}, \\
\mathbf{4 , 0 0 0} \mathbf{g})\end{array}$ & $\begin{array}{l}\text { Metal in } \\
\text { filler }\end{array}$ & $\begin{array}{c}\text { Discrete reflector } \\
\text { (thin } 0.001 \mathrm{~cm} \text { and } \\
\text { thick } 0.7112 \mathrm{~cm})\end{array}$ & $\begin{array}{l}\text { Be } \\
(g)\end{array}$ & $\begin{array}{r}\mathrm{B}_{4} \mathrm{C} \\
(\mathrm{g})\end{array}$ & Subcase \\
\hline \multirow{16}{*}{ Set- $7 b$} & \multirow{16}{*}{$\begin{array}{l}\text { Models } \\
\text { from set-3 }\end{array}$} & \multirow{8}{*}{$\begin{array}{c}\text { Cylinder (radius range } 4.8, \\
6, \text { and } 7.7 \text {, and height } \\
\text { defined by total volume of } \\
\text { mass) }\end{array}$} & Water & $\mathrm{c} 12$ & \multirow{16}{*}{$\begin{array}{l}\text { SS from can } \\
(0,500 \\
1,000 \mathrm{~g})\end{array}$} & Steel & \multirow{16}{*}{$0-585$} & \multirow{16}{*}{$10,30,50$} & set-7b-1 \\
\hline & & & Poly & $\mathrm{c} 12$ & & Steel & & & set- $7 b-2$ \\
\hline & & & Water & $\mathrm{c} 12$ & & Poly & & & set-7b-3 \\
\hline & & & Poly & $\mathrm{c} 12$ & & Poly & & & set- $7 b-4$ \\
\hline & & & Water & Generic & & Steel & & & set-7b -5 \\
\hline & & & Poly & Generic & & Steel & & & set-7b -6 \\
\hline & & & Water & Generic & & Poly & & & set-7b-7 \\
\hline & & & Poly & Generic & & Poly & & & set-7b -8 \\
\hline & & \multirow{8}{*}{$\begin{array}{l}\text { Sphere (radius defined by } \\
\text { total volume of mass) }\end{array}$} & Water & $\mathrm{c} 12$ & & Steel & & & \begin{tabular}{|l|} 
set- $7 b-9$ \\
\end{tabular} \\
\hline & & & Poly & $\mathrm{c} 12$ & & Steel & & & set- $7 b-10$ \\
\hline & & & Water & $\mathrm{c} 12$ & & Poly & & & set-7b-11 \\
\hline & & & Poly & $\mathrm{c} 12$ & & Poly & & & \begin{tabular}{|ll} 
set- $7 b-12$ \\
\end{tabular} \\
\hline & & & Water & Generic & & Steel & & & set- $7 b-13$ \\
\hline & & & Poly & Generic & & Steel & & & \begin{tabular}{|ll} 
set- $7 b-14$ \\
\end{tabular} \\
\hline & & & Water & Generic & & Poly & & & set- $7 b-15$ \\
\hline & & & Poly & Generic & & Poly & & & set- $7 b-16$ \\
\hline
\end{tabular}


Table N-3. Summary of cases for set-7c for the nonuniform array model.

\begin{tabular}{|c|c|c|c|c|c|c|c|c|c|}
\hline Case & Model type & Waste form shape & $\begin{array}{l}\text { Waste form } \\
\text { moderator }\end{array}$ & $\begin{array}{c}\text { Filler material } \\
(\mathbf{0}, \mathbf{2 , 0 0 0} \\
\mathbf{4 , 0 0 0} \mathbf{g})\end{array}$ & $\begin{array}{l}\text { Metal in } \\
\text { filler }\end{array}$ & \begin{tabular}{|c|} 
Discrete reflector (thin \\
$0.001 \mathrm{~cm}$ and thick \\
$0.7112 \mathrm{~cm})$
\end{tabular} & $\begin{array}{l}\text { Be } \\
\text { (g) }\end{array}$ & $\begin{array}{r}\mathrm{B}_{4} \mathrm{C} \\
(\mathrm{g})\end{array}$ & Subcase \\
\hline \multirow{16}{*}{ Set-7c } & \multirow{16}{*}{$\begin{array}{c}\text { Models } \\
\text { from set-2- } \\
\text { uh }\end{array}$} & \multirow{8}{*}{$\begin{array}{l}\text { Cylinder (radius range } 4.8,6 \text {, } \\
\text { and } 7.7 \text {, and height defined by } \\
\text { total volume of mass) }\end{array}$} & Water & $\mathrm{c} 12$ & \multirow{16}{*}{$\begin{array}{c}\text { SS from } \\
\text { can } \\
(0,500 \\
1,000 \mathrm{~g})\end{array}$} & Steel & \multirow{16}{*}{$0-585$} & \multirow{16}{*}{$10,30,50$} & set-7c-1 \\
\hline & & & Poly & $\mathrm{c} 12$ & & Steel & & & set-7c-2 \\
\hline & & & Water & $\mathrm{c} 12$ & & Poly & & & \begin{tabular}{|l|} 
set-7c-3 \\
\end{tabular} \\
\hline & & & Poly & $\mathrm{c} 12$ & & Poly & & & set-7c -4 \\
\hline & & & Water & Generic & & Steel & & & set-7c -5 \\
\hline & & & Poly & Generic & & Steel & & & set-7c -6 \\
\hline & & & Water & Generic & & Poly & & & set-7c -7 \\
\hline & & & Poly & Generic & & Poly & & & set-7c -8 \\
\hline & & \multirow{8}{*}{$\begin{array}{l}\text { Sphere (radius defined by total } \\
\text { volume of mass) }\end{array}$} & Water & $\mathrm{c} 12$ & & Steel & & & set-7c -9 \\
\hline & & & Poly & $\mathrm{c} 12$ & & Steel & & & set-7c -10 \\
\hline & & & Water & $\mathrm{c} 12$ & & Poly & & & set-7c -11 \\
\hline & & & Poly & $\mathrm{c} 12$ & & Poly & & & set-7c -12 \\
\hline & & & Water & Generic & & Steel & & & set-7c -13 \\
\hline & & & Poly & Generic & & Steel & & & set-7c -14 \\
\hline & & & Water & Generic & & Poly & & & set-7c -15 \\
\hline & & & Poly & Generic & & Poly & & & set- $7 \mathrm{c}-16$ \\
\hline
\end{tabular}

Table N-4. Summary of results for the addition of 10,30 , or $50 \mathrm{~g}$ of $\mathrm{B}_{4} \mathrm{C}$ with a waste form moderator mass up to $3 \mathrm{~kg}$ for all subcases.

\begin{tabular}{|l|c|}
\hline \multicolumn{1}{|c|}{ Model } & Amount of $\mathbf{B}_{\mathbf{4}} \mathbf{C}$ \\
\hline $\begin{array}{l}\text { Three-high uniform array model (set-1 and set- } \\
\text { 7a) }\end{array}$ & $30 \mathrm{~g} \mathrm{~B}{ }_{4} \mathrm{C}$ \\
\hline Six-high uniform array model (set-3 and set-7b) & $30 \mathrm{~g} \mathrm{~B} \mathrm{C}_{4}$ \\
\hline $\begin{array}{l}\text { Nonuniform upper-horizon array model (set-2- } \\
\text { uh and set-7c) }\end{array}$ & $10 \mathrm{~g} \mathrm{~B}{ }_{4} \mathrm{C}$ \\
\hline
\end{tabular}




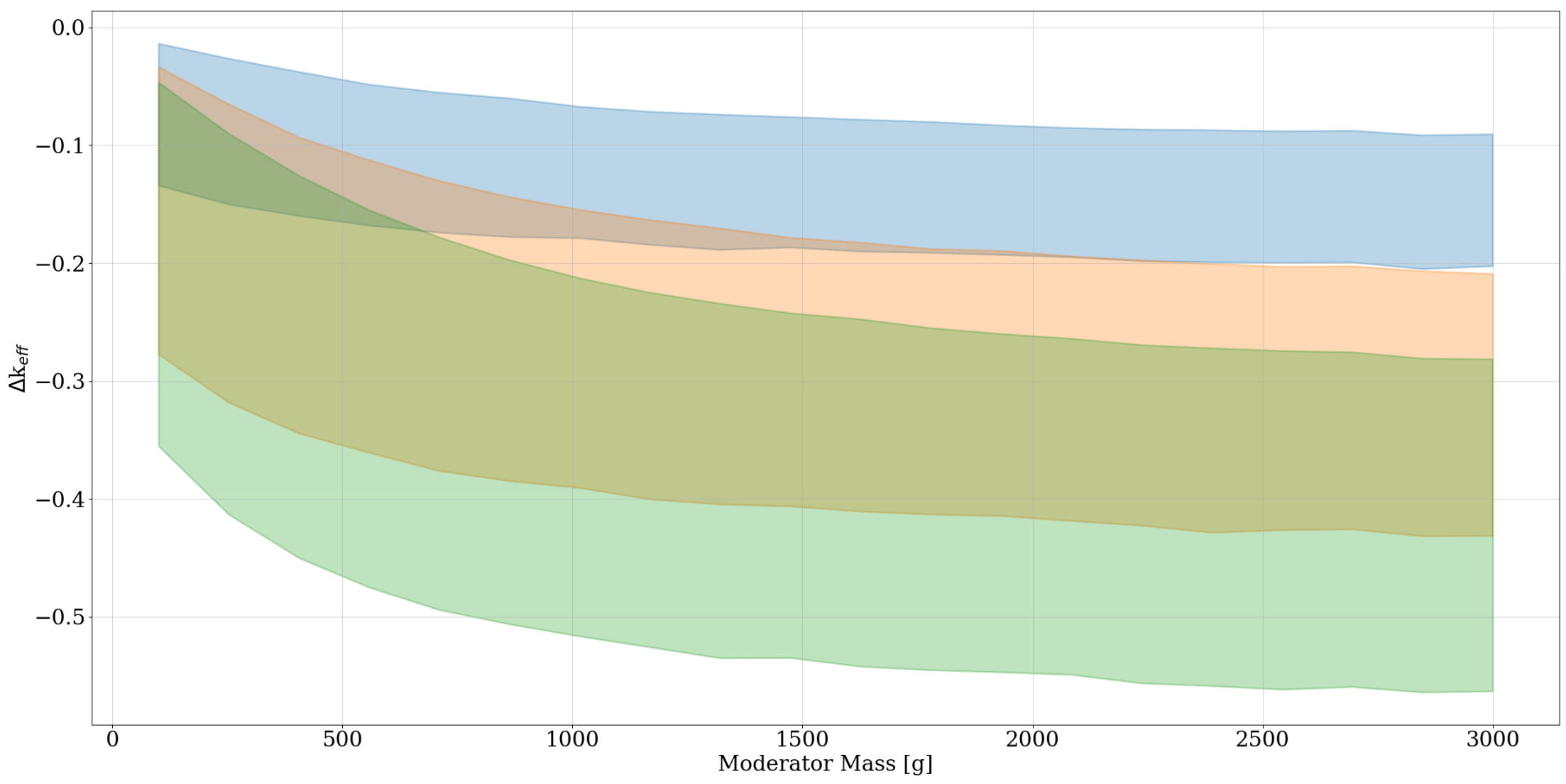

$10 \mathrm{~g} \mathrm{~B}_{4} \mathrm{C} \square 30 \mathrm{~g} \mathrm{~B}_{4} \mathrm{C} \square 50 \mathrm{~g} \mathrm{~B}_{4} \mathrm{C}$

Figure N-1. Set-7a results (three-high uniform array model) for 10,30 , and $50 \mathrm{~g} \mathrm{of} \mathrm{B}_{4} \mathrm{C}$ for all subcases. Reactivity trends of all subcases as a delta $k_{\text {eff }}$ as a function of moderator mass. 


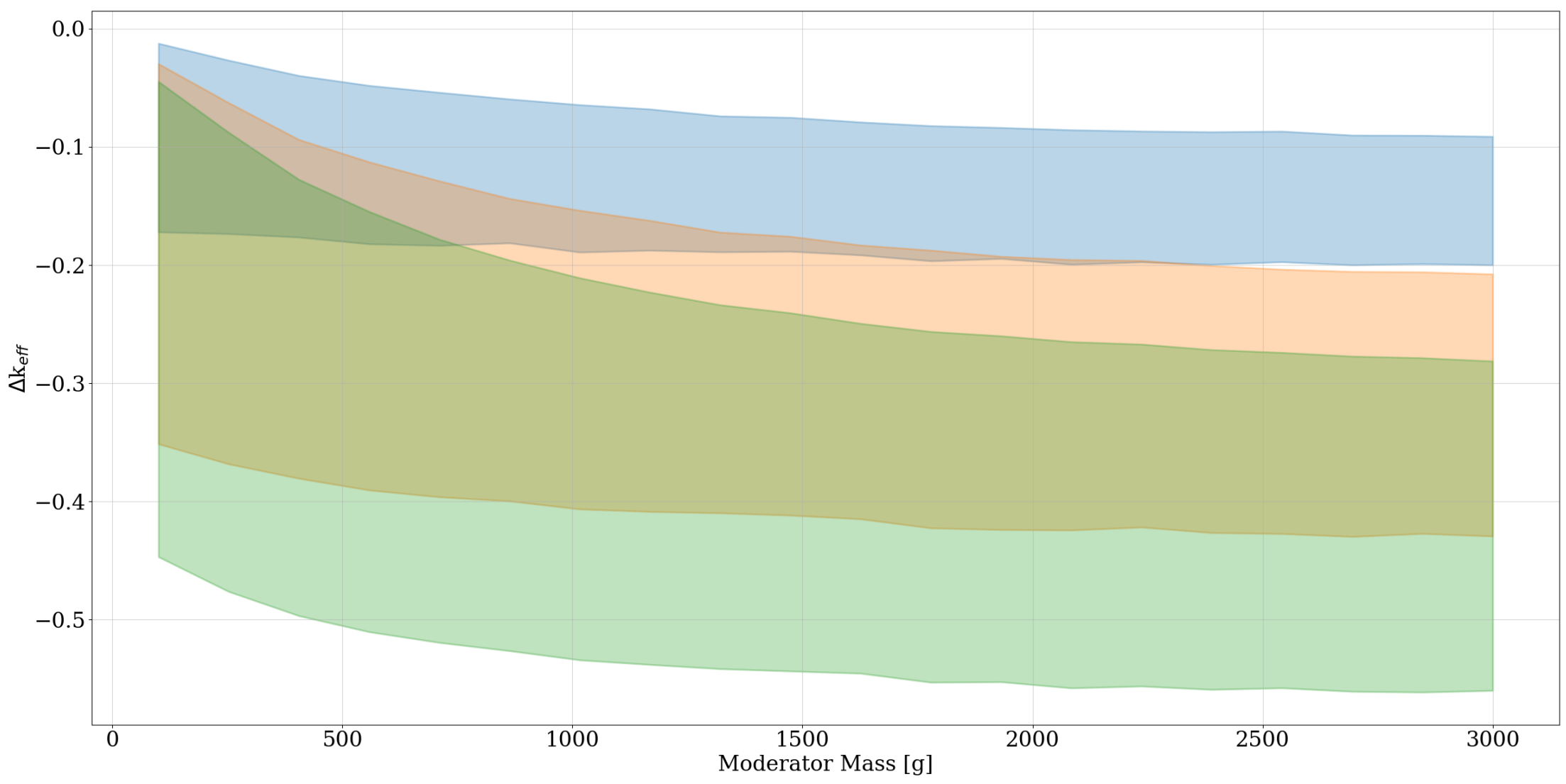

$10 \mathrm{~g} \mathrm{~B}_{4} \mathrm{C} \square 30 \mathrm{~g} \mathrm{~B}_{4} \mathrm{C} \square 50 \mathrm{~g} \mathrm{~B}_{4} \mathrm{C}$

Figure N-2. Set-7b results (six-high uniform array model) for 10,30 , and $50 \mathrm{~g}$ of $\mathrm{B}_{4} \mathrm{C}$ for all subcases. Reactivity trends of all subcases as a delta $k_{\text {eff }}$ as a function of moderator mass. 


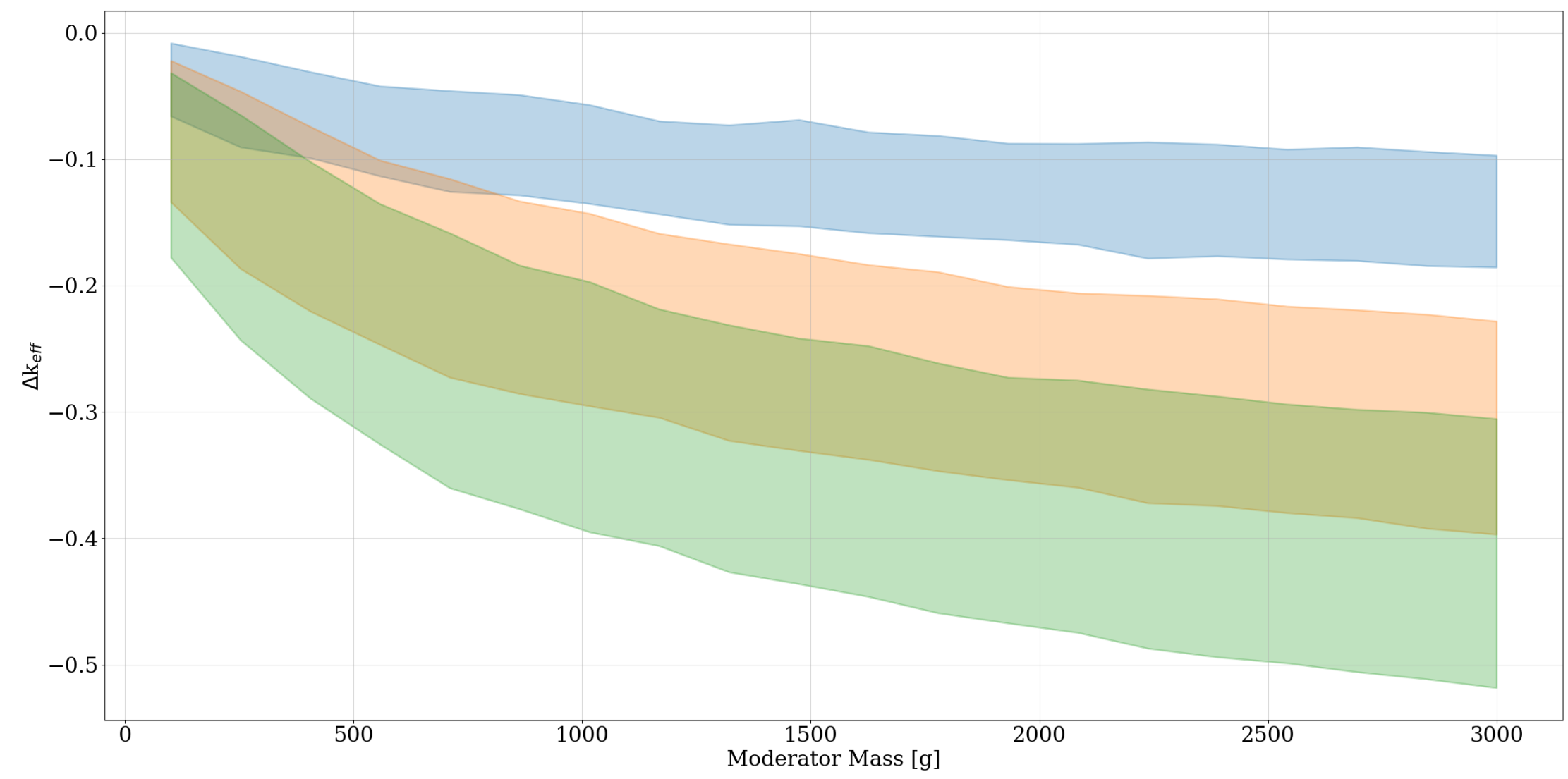

$10 \mathrm{~g} \mathrm{~B}_{4} \mathrm{C} \square 30 \mathrm{~g} \mathrm{~B}_{4} \mathrm{C} \square 50 \mathrm{~g} \mathrm{~B}_{4} \mathrm{C}$

Figure N-3. Set-7c results (three-high uniform array model) for 10,30 , and $50 \mathrm{~g} \mathrm{of} \mathrm{B}_{4} \mathrm{C}$ for all subcases. Reactivity trends of all subcases as a delta $k_{\text {eff }}$ as a function of moderator mass. 


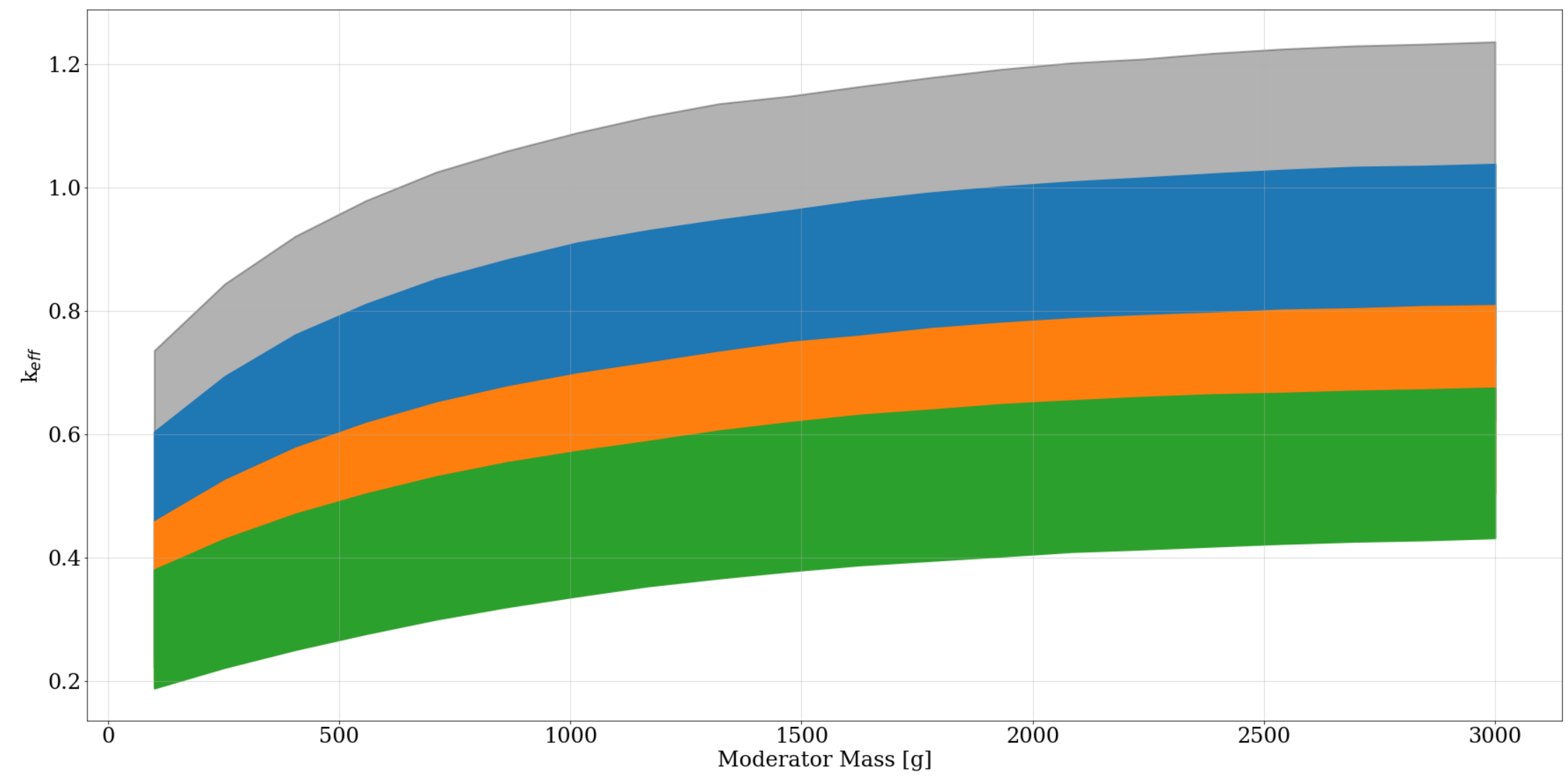

$\square 0 \mathrm{~g} \mathrm{~B}_{4} \mathrm{C} \square 10 \mathrm{~g} \mathrm{~B}_{4} \mathrm{C} \quad 30 \mathrm{~g} \mathrm{~B}_{4} \mathrm{C} \quad 50 \mathrm{~g} \mathrm{~B}_{4} \mathrm{C}$

Figure N-4. Set-7a results (three-high uniform array model) for $0,10,30$, and $50 \mathrm{~g}$ of $\mathrm{B}_{4} \mathrm{C}$ for all subcases. Reactivity trends of all subcases as a $k_{\text {eff }}$ as a function of moderator mass. 


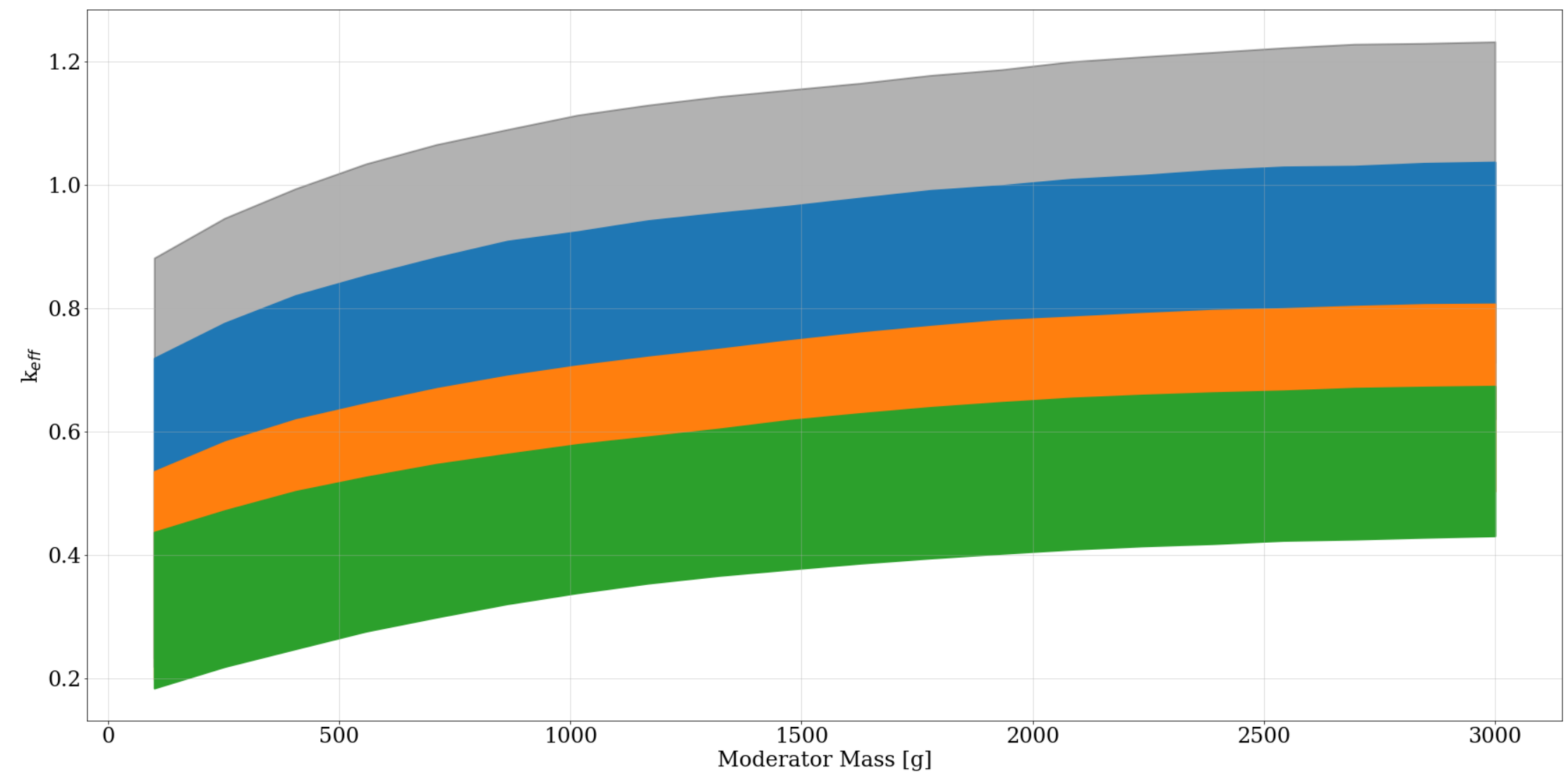

$\square 0 \mathrm{~g} \mathrm{~B}_{4} \mathrm{C} \square 10 \mathrm{~g} \mathrm{~B}_{4} \mathrm{C} \square 30 \mathrm{~g} \mathrm{~B}_{4} \mathrm{C} \square 50 \mathrm{~g} \mathrm{~B}_{4} \mathrm{C}$

Figure $\mathrm{N}-5$. Set-7b results (six-high uniform array model) for $0,10,30$, and $50 \mathrm{~g}$ of $\mathrm{B}_{4} \mathrm{C}$ for all subcases. Reactivity trends of all subcases as $k_{\text {eff }}$ as a function of moderator mass. 


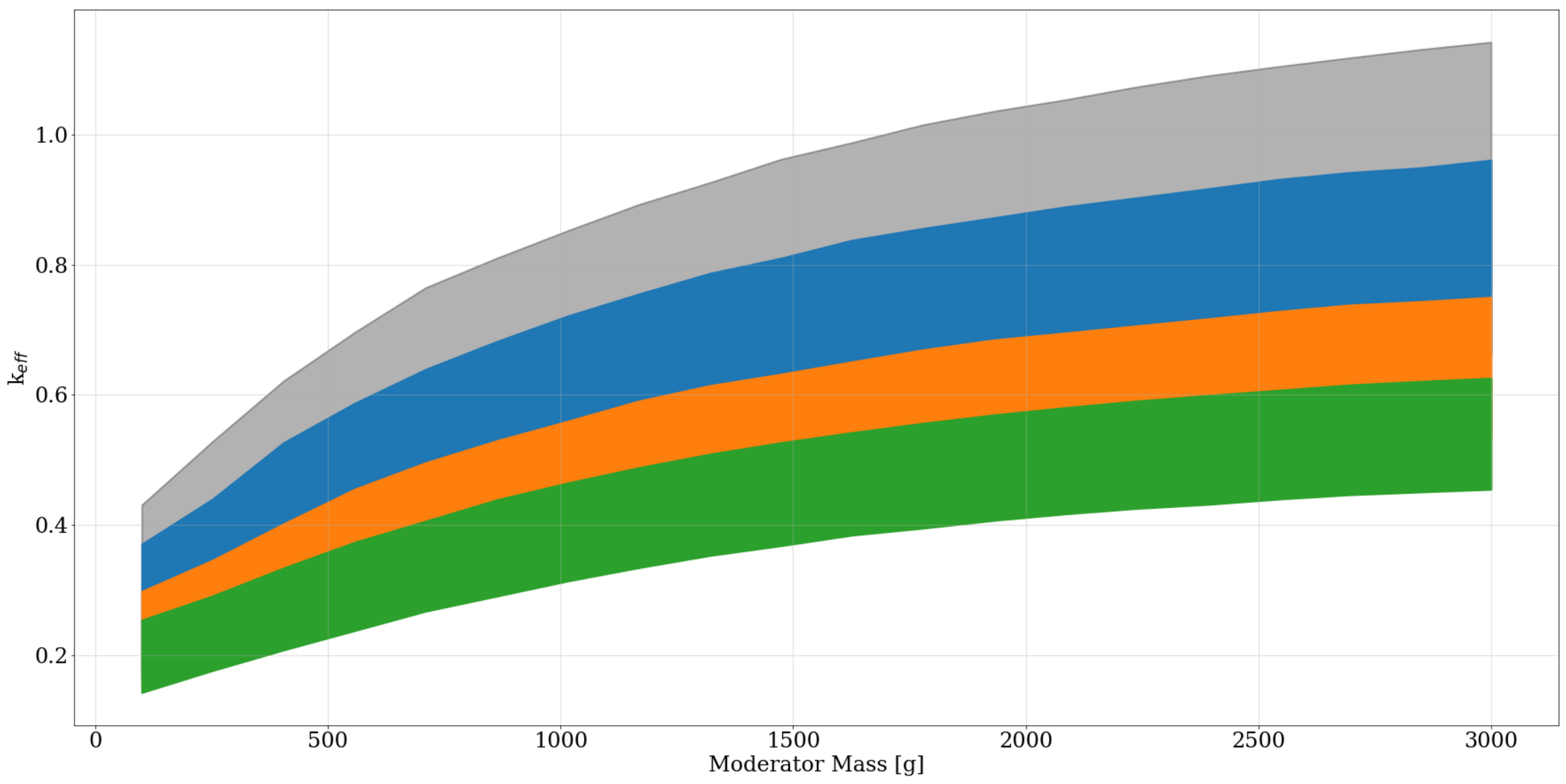

$\square 0 \mathrm{~g} \mathrm{~B}_{4} \mathrm{C} \square 10 \mathrm{~g} \mathrm{~B}_{4} \mathrm{C} \quad 30 \mathrm{~g} \mathrm{~B}_{4} \mathrm{C} \quad \square 0 \mathrm{~g} \mathrm{~B}_{4} \mathrm{C}$

Figure N-6. Set-7c results (nonuniform upper horizon array model) for $0,10,30$, and $50 \mathrm{~g}$ of $\mathrm{B}_{4} \mathrm{C}$ for all subcases. Reactivity trends of all subcases as a delta $k_{\text {eff }}$ as a function of moderator mass. 


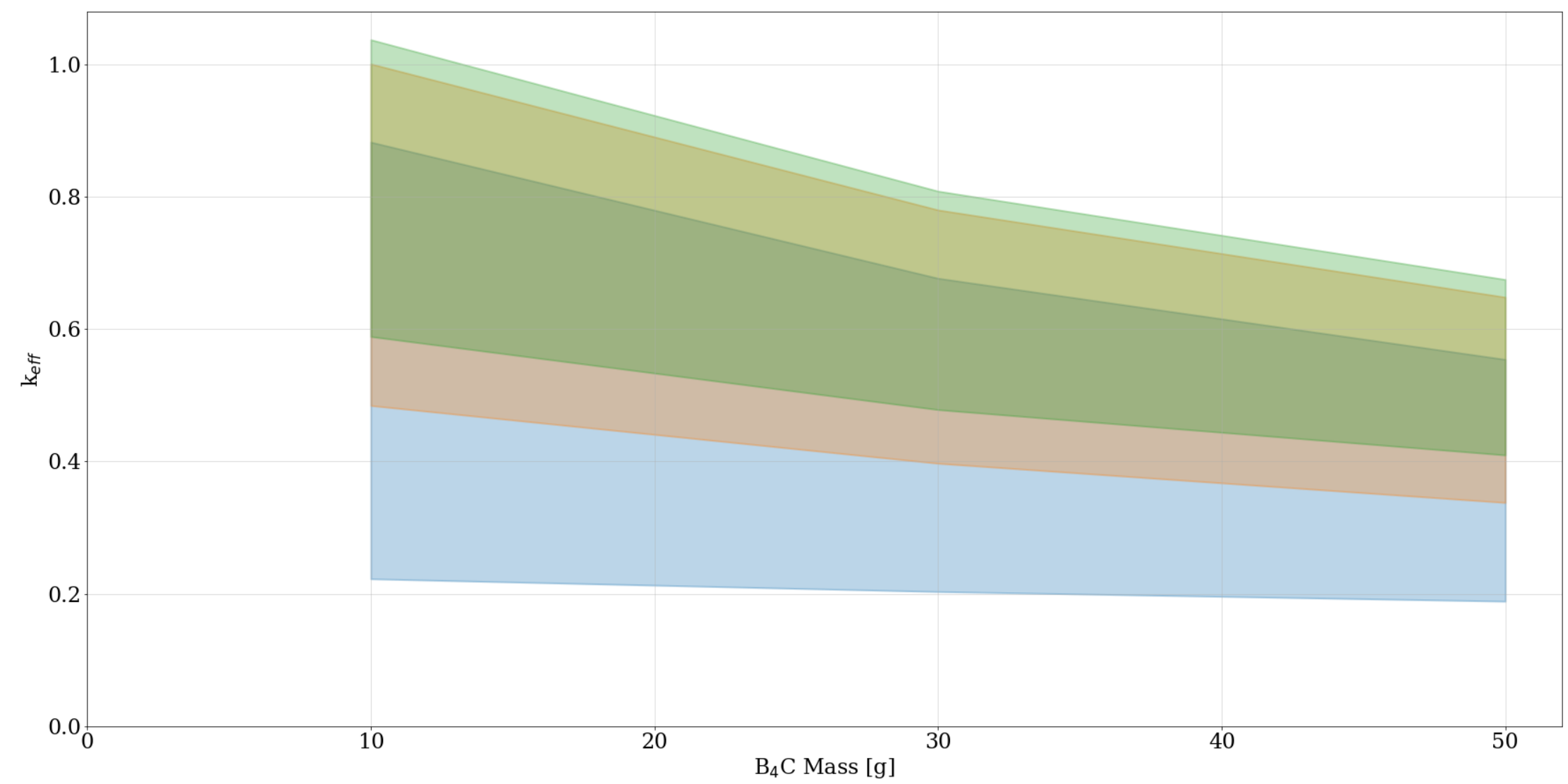

Figure N-7. Set-7a results (three-high uniform array model) for 10,30 , and $50 \mathrm{~g} \mathrm{of} \mathrm{B}_{4} \mathrm{C}$ for all subcases. Reactivity trends of all subcases as $k_{\text {eff }}$ as a function of $\mathrm{B}_{4} \mathrm{C}$ mass. 


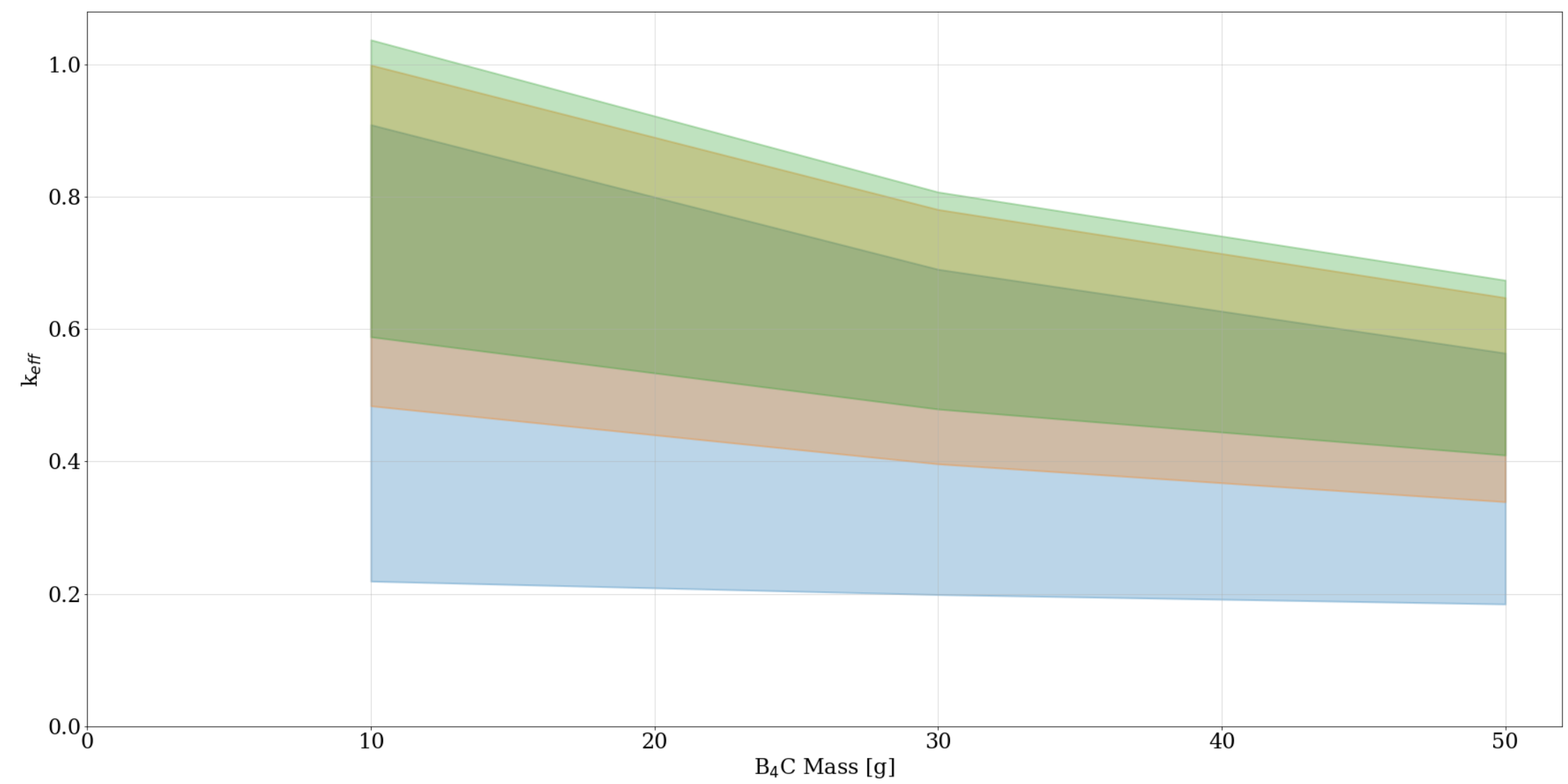

Figure N-8. Set-7b results (six-high uniform array model) for 10,30 , and $50 \mathrm{~g}$ of $\mathrm{B}_{4} \mathrm{C}$ for all subcases. Reactivity trends of all subcases as $k_{\text {eff }}$ as a function of $\mathrm{B}_{4} \mathrm{C}$ mass. 


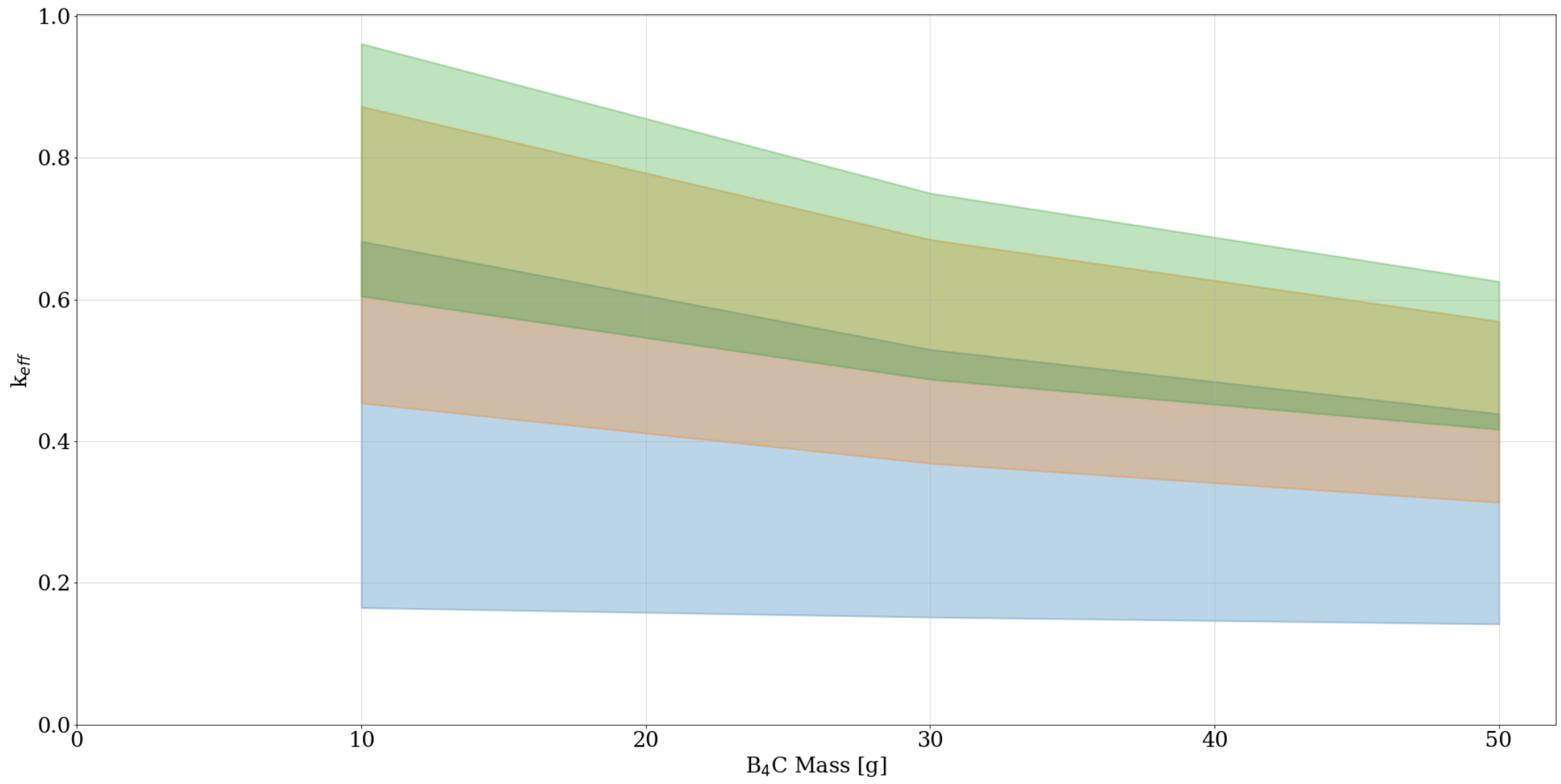

Figure N-9. Set-7c results (nonuniform array model) for 10,30 , and $50 \mathrm{~g}$ of $\mathrm{B}_{4} \mathrm{C}$ for all subcases. Reactivity trends of all subcases as $k_{\text {eff }}$ as a function of $\mathrm{B}_{4} \mathrm{C}$ mass. 


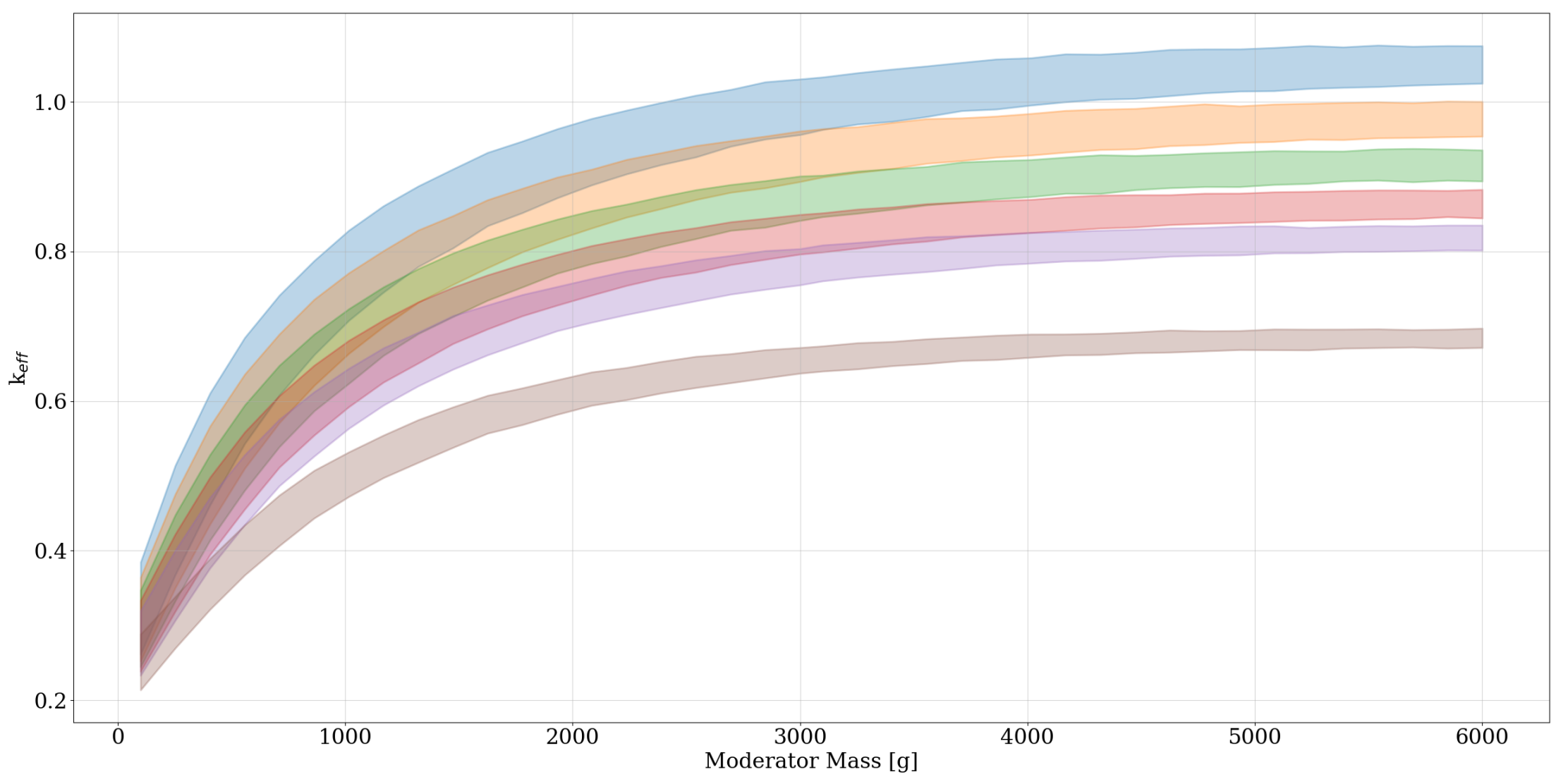

$\square 10 \mathrm{~g} \mathrm{~B}_{4} \mathrm{C} \square 15 \mathrm{~g} \mathrm{~B}_{4} \mathrm{C} \square 20 \mathrm{~g} \mathrm{~B}_{4} \mathrm{C} \square 25 \mathrm{~g} \mathrm{~B}_{4} \mathrm{C} \square 30 \mathrm{~g} \mathrm{~B}_{4} \mathrm{C} \quad \square \quad \square 0 \mathrm{~g} \mathrm{~B}{ }_{4} \mathrm{C}$

Figure N-10. Set-7a results (three-high uniform array model) for $10,15,20,25,30$, and $50 \mathrm{~g}$ of $\mathrm{B}_{4} \mathrm{C}$ for subcase-10 only. Reactivity trends of all subcases as $k_{\text {eff }}$ as a function of moderator mass up to $6 \mathrm{~kg}$. 


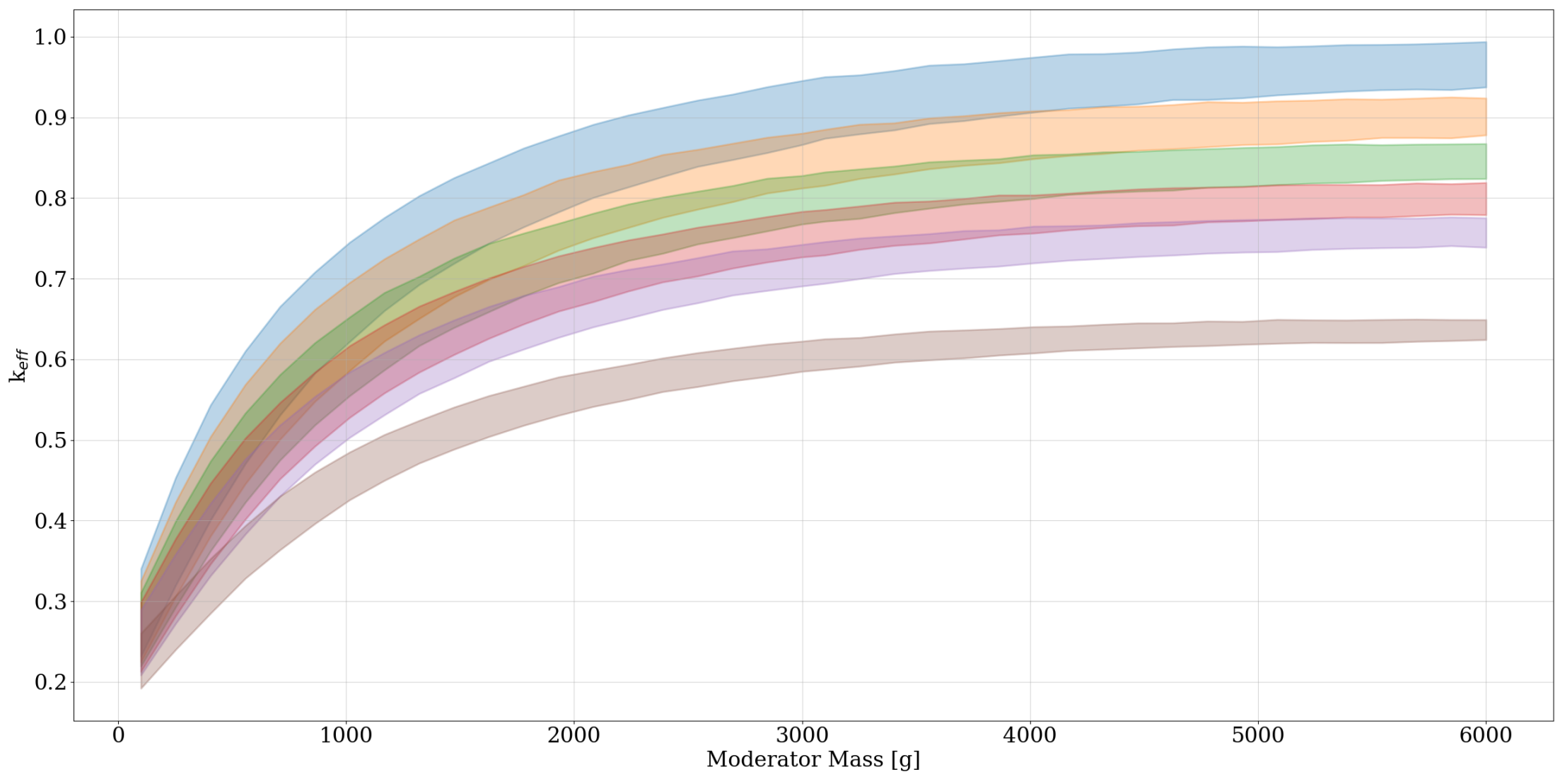

$\square 10 \mathrm{~g} \mathrm{~B}_{4} \mathrm{C} \square 15 \mathrm{~g} \mathrm{~B}_{4} \mathrm{C} \square 20 \mathrm{~g} \mathrm{~B}_{4} \mathrm{C} \square 25 \mathrm{~g} \mathrm{~B}_{4} \mathrm{C} \square 30 \mathrm{~g} \mathrm{~B}_{4} \mathrm{C} \quad \square \quad \square 0 \mathrm{~g} \mathrm{~B}{ }_{4} \mathrm{C}$

Figure N-11. Set-7b results (six-high uniform array model) for $10,15,20,25,30$, and $50 \mathrm{~g}$ of $\mathrm{B}_{4} \mathrm{C}$ for subcase-10 only. Reactivity trends of subcase- 10 as $k_{\text {eff }}$ as a function of moderator mass up to $6 \mathrm{~kg}$. 


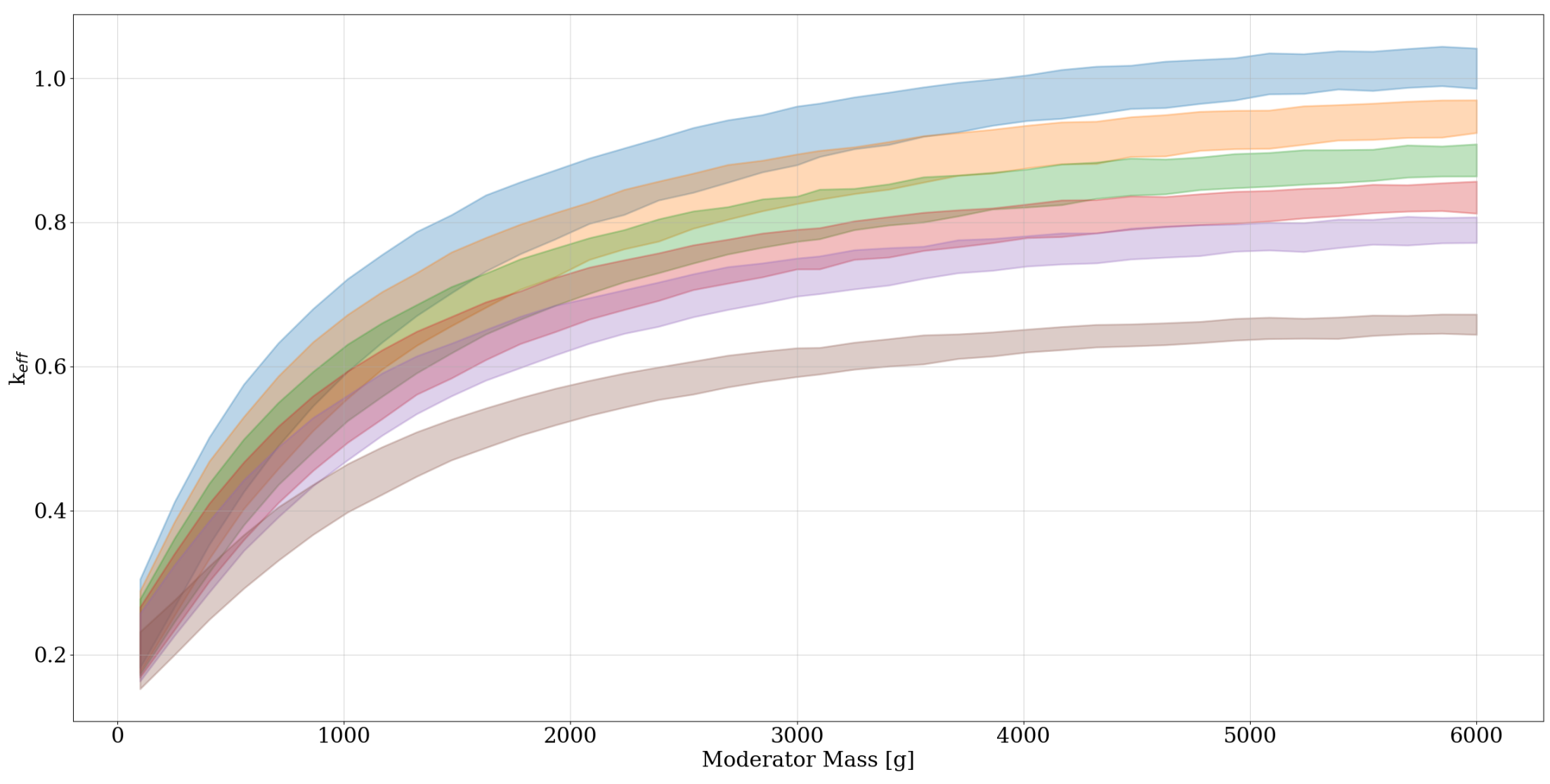

$\square 10 \mathrm{~g} \mathrm{~B}_{4} \mathrm{C} \square 15 \mathrm{~g} \mathrm{~B}_{4} \mathrm{C} \square 20 \mathrm{~g} \mathrm{~B}_{4} \mathrm{C} \square 25 \mathrm{~g} \mathrm{~B}_{4} \mathrm{C} \quad \square \quad \square 0 \mathrm{~g} \mathrm{~B}_{4} \mathrm{C} \quad \square \quad \square 0 \mathrm{~g} \mathrm{~B}{ }_{4} \mathrm{C}$

Figure N-12 Set-7c results (nonuniform array model) for $10,15,20,25,30$, and $50 \mathrm{~g}$ of $\mathrm{B}_{4} \mathrm{C}$ for subcase-10 only. Reactivity trends of subcase- 10 as $k_{\text {eff }}$ as a function of moderator mass up to $6 \mathrm{~kg}$. 


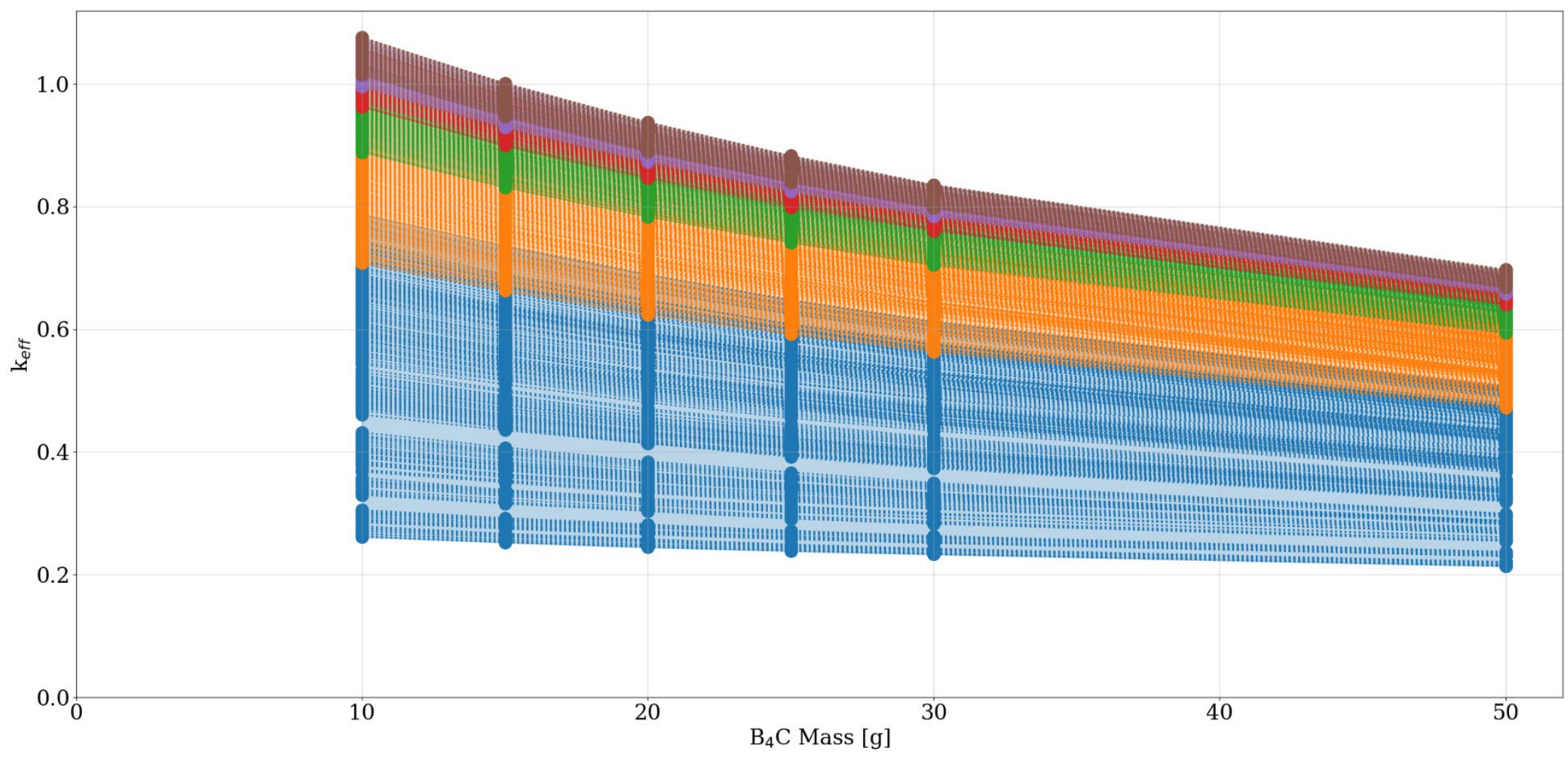
Reactivity trends of all subcase- 10 as $k_{\text {eff }}$ as a function of $\mathrm{B}_{4} \mathrm{C}$ mass. 


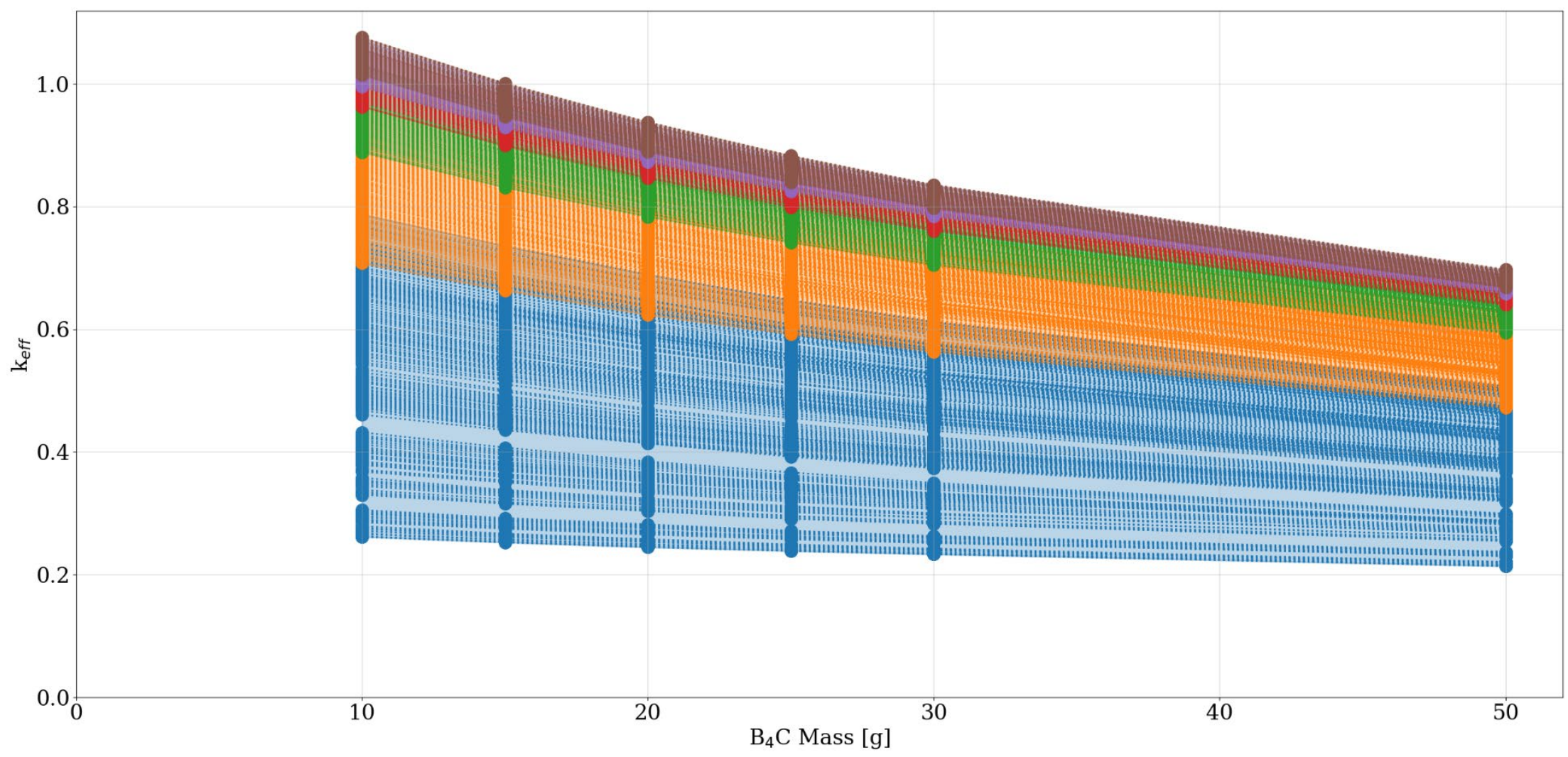
Reactivity trends of all subcase- 10 as $k_{\text {eff }}$ as a function of $\mathrm{B}_{4} \mathrm{C}$ mass. 


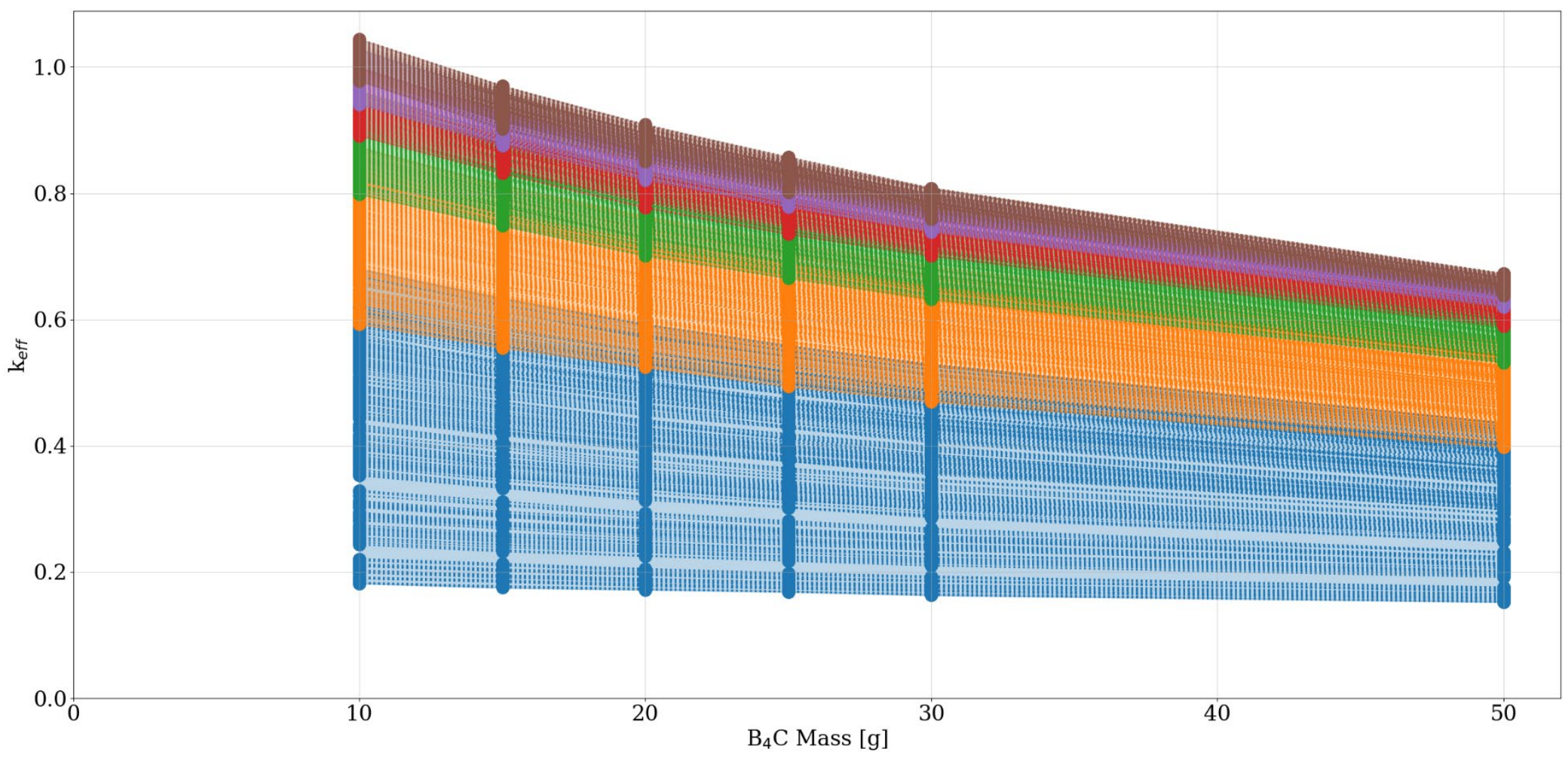
Reactivity trends of subcase- 10 as $k_{\text {eff }}$ as a function of $\mathrm{B}_{4} \mathrm{C}$ mass. 
This page is intentionally blank 
APPENDIX O. SET-8: RESULTS OF THE CALCULATIONS TO SHOW THE REACTIVITY EFFECT OF GREATER THICKNESSES OF THE DISCRETE REFLECTOR MATERIAL AROUND THE WASTE FORM 
This page is intentionally blank 


\section{APPENDIX O. SET-8: RESULTS OF THE CALCULATIONS TO SHOW THE REACTIVITY EFFECT OF GREATER THICKNESSES OF THE DISCRETE REFLECTOR MATERIAL AROUND THE WASTE FORM}

This appendix serves as a repository of the results for additional thicker thicknesses of the discrete reflector material around the waste form.

The analysis models used in this appendix for set- $8 \mathrm{a}$ are the same as those used for the set- 1 models discussed in detail in Appendix A, and those used for set- $8 \mathrm{~b}$ are the same as those used the set-2-uh models discussed in detail in Appendix C. The model changes to the calculations in this appendix compared with Appendix A and Appendix $\mathrm{C}$ are for the increase in the discrete reflector thickness around each waste form.

This appendix expands upon how $k_{\text {eff }}$ is affected when the thickness of the discrete reflector is increased from what was already evaluated. The source of the discrete reflector material is expected to be mainly steel from the pipes and CCO drums, and although the evaluation already considered in the main report uses a thin and a thick thickness to account for this material, the maximum thickness evaluated is equivalent to the thickness of the inner CCO pipe only. Because additional material is known to exist, additional calculations are considered by increasing the thickness to multiples of the inner CCO pipe thickness of $0.7112: 1.422$ and $2.134 \mathrm{~cm}$. Note that for set- $8 \mathrm{~b}$, the maximum thickness used is limited to $1.422 \mathrm{~cm}$ because of the close proximity of the centroids.

The cases in this appendix are summarized in Table O-1 and Table O-2 for set-8a and set-8b, respectively.

The results in this appendix are presented first as a summary of the reactivity trends related to discrete reflector thickness and then as a delta $k_{\text {eff }}$ for each sublisting. For the summary evaluations, the results to demonstrate the reactivity effect of the increasing discrete reflector thickness are provided in Figures O-1 through O-5 for set-8a and in Figures O-6 through O-10 for set-8b. Specifically, these results summaries are provided to demonstrate the impact of discrete reflector thickness on the system at its maximum subcritical moderator mass for the two waste form geometries and the two discrete reflector materials considered, or four total combinations.

For each combination of waste form geometry and discrete reflector material composition, the maximum moderator mass over all sublistings was determined for set-1 calculations as follows.

- The maximum subcritical moderator mass for cylindrical waste forms with a poly discrete reflector is a $557.9 \mathrm{~g}$ moderator mass.

- The maximum subcritical moderator mass for spherical waste forms with a poly discrete reflector is a $1,016 \mathrm{~g}$ moderator.

- The maximum subcritical moderator mass for cylindrical waste forms with a stainless-steel discrete reflector is a $710.5 \mathrm{~g}$ moderator.

- The maximum subcritical moderator mass for spherical waste forms with a stainless-steel discrete reflector is a $1,016 \mathrm{~g}$ moderator. 
For each combination of waste form geometry and discrete reflector material composition, the maximum moderator mass over all sublistings was determined for set-2-uh calculations as follows.

- The maximum subcritical moderator mass for cylindrical waste forms with a poly discrete reflector is a $1,779 \mathrm{~g}$ moderator mass.

- The maximum subcritical moderator mass for spherical waste forms with a poly discrete reflector is a 1,779 g moderator.

- The maximum subcritical moderator mass for cylindrical waste forms with a stainless-steel discrete reflector is a $1,626 \mathrm{~g}$ moderator.

- The maximum subcritical moderator mass for spherical waste forms with a stainless-steel discrete reflector is a $1,474 \mathrm{~g}$ moderator.

The set of parameter sweeps was identified for each of these identified maximum subcritical moderator masses for the discrete reflector thickness of $0.001 \mathrm{~cm}$. Using these same set of parameter sweeps for the $0.001 \mathrm{~cm}$ thickness, the datasets for the additional discrete reflector thicknesses were identified so that the only difference in the plotted results is the discrete reflector thickness. These four datasets for each of the four thickness are then plotted against the complete set of data results in Figures O-1 through O-4 for set- 1 and set-8a and in Figures O-6 through O-9 for set-2-uh and set-8b. As expected, there is much variation in the results, showing the interdependence of the system parameters on $k_{\text {eff }}$.

Alternatively, the results are also provided as $k_{\text {eff }}$ vs. discrete reflector thickness in Figure O-5 for set-1 and set-8a and in Figure O-10 for set-2-uh and set-8b. For these plots, the maximum $k_{\text {eff }}$ is provided, regardless of the parameter sweeps that yield the maximum result.

To provide more detailed results that specifically evaluate the reactivity trends seen in the summary figures, additional $k_{\text {eff }}$ plots are presented for each sublisting. The sublisting results for the $k_{\text {eff }}$ from set- 1 to set-8a are presented in Figures O-11 through O-26, and the $k_{\text {eff }}$ from set-2-uh to set-8b are presented in Figures O-27 through O-42.

\section{LIST OF FIGURES}

Figure O-1. Set-8a and set- $1 k_{\text {eff }}$ results (three-high uniform array model) for the cylindrical waste forms with a poly discrete reflector and a discrete reflector thickness comparison between the sublisting parameters, which yield a maximum subcritical moderator mass for a discrete reflector thickness of $0.001 \mathrm{~cm}$.

Figure O-2. Set-8a and set-1 $k_{\text {eff }}$ results (three-high uniform array model) for the spherical waste forms with a poly discrete reflector, and a discrete reflector thickness comparison between the sublisting parameters, which yield a maximum subcritical moderator mass for a discrete reflector thickness of $0.001 \mathrm{~cm}$.

Figure O-3. Set-8a and set- $1 k_{\text {eff }}$ results (three-high uniform array model) for the cylindrical waste forms with a stainless-steel discrete reflector and a discrete reflector thickness comparison between the sublisting parameters, which yield a maximum subcritical moderator mass for a discrete reflector thickness of $0.001 \mathrm{~cm}$.

Figure O-4. Set-8a and set-1 $k_{\text {eff }}$ results (three-high uniform array model) for the spherical waste forms with a stainless-steel discrete reflector and a discrete reflector thickness comparison between the sublisting parameters, which yield a maximum subcritical moderator mass for a discrete reflector thickness of $0.001 \mathrm{~cm}$. 
Figure O-5. Set-8a and set-1 maximum $k_{\text {eff }}$ results (three-high uniform array model) overall sublistings as a function of discrete reflector thickness.

Figure O-6. Set-8b and set-2-uh $k_{\text {eff }}$ results (nonuniform array model) for the cylindrical waste forms with a poly discrete reflector and a discrete reflector thickness comparison between the sublisting parameters, which yield a maximum subcritical moderator mass for a discrete reflector thickness of $0.001 \mathrm{~cm}$.

Figure O-7. Set-8b and set-2-uh $k_{\text {eff }}$ results (nonuniform array model) for the spherical waste forms with a poly discrete reflector and a discrete reflector thickness comparison between the sublisting parameters, which yield a maximum subcritical moderator mass for a discrete reflector thickness of $0.001 \mathrm{~cm}$

Figure O-8. Set-8b and set-2-uh $k_{\text {eff }}$ results (nonuniform array model) for the cylindrical waste forms with a stainless-steel discrete reflector and a discrete reflector thickness comparison between the sublisting parameters, which yield a maximum subcritical moderator mass for a discrete reflector thickness of $0.001 \mathrm{~cm}$.

Figure 0-9. Set-8b and set-2-uh $k_{\text {eff }}$ results (nonuniform array model) for the spherical waste forms with a stainless-steel discrete reflector and a discrete reflector thickness comparison between the sublisting parameters, which yield a maximum subcritical moderator mass for a discrete reflector thickness of $0.001 \mathrm{~cm}$.

Figure O-10. Set- $8 b$ and set-2-uh maximum $k_{\text {eff }}$ results (nonuniform array model) overall sublistings as a function of discrete reflector thickness.

Figure O-11. Set-8a sublisting-1 results (three-high uniform array model): $k_{\text {eff }}$ for set-1 and set-8a by discrete reflector thicknesses.

Figure O-12. Set-8a sublisting-2 results (three-high uniform array model): $k_{\text {eff }}$ for set- 1 and set-8a by discrete reflector thicknesses.

Figure O-13. Set-8a sublisting-3 results (three-high uniform array model): ): $k_{\text {eff }}$ for set- 1 and set8 a by discrete reflector thicknesses.

Figure O-14. Set-8a sublisting-4 results (three-high uniform array model): $k_{\text {eff }}$ for set- 1 and set-8a by discrete reflector thicknesses.

Figure O-15. Set-8a sublisting-5 results (three-high uniform array model): $k_{\text {eff }}$ for set- 1 and set-8a by discrete reflector thicknesses.

Figure O-16. Set-8a sublisting-6 results (three-high uniform array model): $k_{\text {eff }}$ for set- 1 and set-8a by discrete reflector thicknesses.

Figure 0-17. Set-8a sublisting-7 results (three-high uniform array model): $k_{\text {eff }}$ for set-1 and set-8a by discrete reflector thicknesses.

Figure O-18. Set-8a sublisting-8 results (three-high uniform array model): $k_{\text {eff }}$ for set- 1 and set-8a by discrete reflector thicknesses.

Figure O-19. Set-8a sublisting-9 results (three-high uniform array model): $k_{\text {eff }}$ for set- 1 and set-8a by discrete reflector thicknesses.

Figure O-20. Set-8a sublisting-10 results (three-high uniform array model): $k_{\text {eff }}$ for set- 1 and set-

8 a by discrete reflector thicknesses.

Figure O-21. Set-8a sublisting-11 results (three-high uniform array model): $k_{\text {eff }}$ for set- 1 and set8 a by discrete reflector thicknesses.

Figure O-22. Set-8a sublisting-12 results (three-high uniform array model): $k_{\text {eff }}$ for set- 1 and set8 a by discrete reflector thicknesses.

Figure O-23. Set-8a sublisting-13 results (three-high uniform array model): $k_{\text {eff }}$ for set-1 and set8 a by discrete reflector thicknesses.

Figure O-24. Set-8a sublisting-14 results (three-high uniform array model): $k_{\text {eff }}$ for set-1 and set8 a by discrete reflector thicknesses.

Figure O-25. Set-8a sublisting-15 results (three-high uniform array model): $k_{\text {eff }}$ for set- 1 and set8 a by discrete reflector thicknesses. O-26 
Figure O-26. Set-8a sublisting-16 results (three-high uniform array model): $k_{\text {eff }}$ for set- 1 and set8 a by discrete reflector thicknesses.

Figure O-27. Set-8b sublisting-1 results (nonuniform array model): $k_{\text {eff }}$ for set-2-uh and set-8a by discrete reflector thicknesses.

Figure O-28. Set-8b sublisting-2 results (nonuniform array model): $k_{\text {eff }}$ for set-2-uh and set-8a by discrete reflector thicknesses.

Figure O-29. Set-8b sublisting-3 results (nonuniform array model): $k_{\text {eff }}$ for set-2-uh and set-8a by discrete reflector thicknesses.

Figure O-30. Set-8b sublisting-4 results (three-high uniform array model): $k_{\text {eff }}$ for set-2-uh and set-8a by discrete reflector thicknesses.

Figure O-31. Set-8b sublisting-5 results (nonuniform array model): $k_{\text {eff }}$ for set-2-uh and set-8a by discrete reflector thicknesses.

Figure 0-32. Set-8b sublisting-6 results (nonuniform array model): $k_{\text {eff }}$ for set-2-uh and set-8a by discrete reflector thicknesses.

Figure 0-33. Set-8b sublisting-7 results (nonuniform array model): $k_{\text {eff }}$ for set-2-uh and set-8a by discrete reflector thicknesses.

Figure O-34. Set-8b sublisting-8 results (nonuniform array model): $k_{\text {eff }}$ for set-2-uh and set-8a by discrete reflector thicknesses.

Figure O-35. Set-8a sublisting-9 results (nonuniform array model): $k_{\text {eff }}$ for set-2-uh and set-8a by discrete reflector thicknesses.

Figure O-36. Set-8a sublisting-10 results (nonuniform array model): $k_{\text {eff }}$ for set-2-uh and set-8a by discrete reflector thicknesses.

Figure 0-37. Set-8b sublisting-11 results (nonuniform array model): $k_{\text {eff }}$ for set-2-uh and set-8a by discrete reflector thicknesses.

Figure O-38. Set-8b sublisting-12 results (nonuniform array model): $k_{\text {eff }}$ for set-2-uh and set-8a by discrete reflector thicknesses.

Figure O-39. Set-8b sublisting-13 results (nonuniform array model): $k_{\text {eff }}$ for set-2-uh and set-8a by discrete reflector thicknesses.

Figure O-40. Set-8b sublisting-14 results (nonuniform array model): $k_{\text {eff }}$ for set-2-uh and set-8a by discrete reflector thicknesses.

Figure O-41. Set-8b sublisting-15 results (nonuniform array model): $k_{\text {eff }}$ for set-2-uh and set-8a by discrete reflector thicknesses.

Figure O-42. Set-8b sublisting-16 results (nonuniform array model): $k_{\text {eff }}$ for set-2-uh and set- $8 \mathrm{a}$ by discrete reflector thicknesses.

\section{LIST OF TABLES}

Table O-1. Summary of cases for set-8a for three-high uniform array model O-7

Table O-2. Summary of cases for set- 8 b for the nonuniform array model. O-8 
Table 0-1. Summary of cases for set-8a for three-high uniform array model.

\begin{tabular}{|c|c|c|c|c|c|c|c|c|}
\hline Case & Model type & Waste form shape & $\begin{array}{l}\text { Waste form } \\
\text { moderator }\end{array}$ & $\begin{array}{c}\text { Filler material }(0, \\
\mathbf{2 , 0 0 0 , 4 , 0 0 0} \mathrm{g})\end{array}$ & $\begin{array}{c}\text { Metal in } \\
\text { filler }\end{array}$ & $\begin{array}{c}\text { Discrete reflector }(1.4224 \mathrm{~cm} \\
\text { thick and } 2.1336 \mathrm{~cm} \text { thick) }\end{array}$ & $\begin{array}{l}\text { Be } \\
(\mathrm{g})\end{array}$ & $\begin{array}{l}\text { Sublistin } \\
\mathrm{g}\end{array}$ \\
\hline \multirow{16}{*}{$\begin{array}{l}\text { Set- } \\
8 \mathrm{a}\end{array}$} & \multirow{16}{*}{$\begin{array}{l}\text { Models } \\
\text { from Set-1 }\end{array}$} & \multirow{8}{*}{$\begin{array}{c}\text { Cylinder (radius range } 4.8,6.25,7.7 \\
\text { and height defined by total volume } \\
\text { of mass) }\end{array}$} & Water & $\mathrm{c} 12$ & \multirow{16}{*}{$\begin{array}{c}\text { SS from } \\
\text { can } \\
(0,500 \\
1,000 \mathrm{~g})\end{array}$} & Steel & \multirow{16}{*}{$\begin{array}{l}0 \text { to } \\
585\end{array}$} & set-8a-1 \\
\hline & & & Poly & $\mathrm{c} 12$ & & Steel & & set- $8 \mathrm{a}-2$ \\
\hline & & & Water & $\mathrm{c} 12$ & & Poly & & set-8a-3 \\
\hline & & & Poly & $\mathrm{c} 12$ & & Poly & & set- $8 \mathrm{a}-4$ \\
\hline & & & Water & Generic & & Steel & & set-8a -5 \\
\hline & & & Poly & Generic & & Steel & & set-8a -6 \\
\hline & & & Water & Generic & & Poly & & set-8a -7 \\
\hline & & & Poly & Generic & & Poly & & set- $8 \mathrm{a}-8$ \\
\hline & & \multirow{8}{*}{$\begin{array}{l}\text { Sphere (radius defined by total } \\
\text { volume of mass) }\end{array}$} & Water & $\mathrm{c} 12$ & & Steel & & set-8a -9 \\
\hline & & & Poly & $\mathrm{c} 12$ & & Steel & & set- $8 \mathrm{a}-10$ \\
\hline & & & Water & $\mathrm{c} 12$ & & Poly & & set-8a-11 \\
\hline & & & Poly & $\mathrm{c} 12$ & & Poly & & set-8a-12 \\
\hline & & & Water & Generic & & Steel & & set- $8 a-13$ \\
\hline & & & Poly & Generic & & Steel & & set-8a-14 \\
\hline & & & Water & Generic & & Poly & & set- $8 \mathrm{a}-15$ \\
\hline & & & Poly & Generic & & Poly & & set- $8 \mathrm{a}-16$ \\
\hline
\end{tabular}


Table 0-2. Summary of cases for set-8b for the nonuniform array model.

\begin{tabular}{|c|c|c|c|c|c|c|c|c|}
\hline Case & Model type & Waste form shape & $\begin{array}{l}\text { Waste form } \\
\text { moderator }\end{array}$ & $\begin{array}{c}\text { Filler material }(0, \\
\mathbf{2 , 0 0 0 , 4 , 0 0 0} \mathrm{g})\end{array}$ & $\begin{array}{l}\text { Metal in } \\
\text { filler }\end{array}$ & $\begin{array}{l}\text { Discrete reflector } \\
(1.4224 \mathrm{~cm} \text { thick })\end{array}$ & $\begin{array}{l}\text { Be } \\
(g)\end{array}$ & $\begin{array}{l}\text { Sublisti } \\
\text { ng }\end{array}$ \\
\hline \multirow{16}{*}{$\begin{array}{l}\text { Set- } \\
8 b\end{array}$} & \multirow{16}{*}{$\begin{array}{l}\text { Models from } \\
\text { Set-2-uh }\end{array}$} & \multirow{8}{*}{$\begin{array}{l}\text { Cylinder (radius range } 4.8,6.25,7.7 \text { and } \\
\text { height defined by total volume of mass) }\end{array}$} & Water & $\mathrm{c} 12$ & \multirow{16}{*}{$\begin{array}{c}\text { SS from } \\
\text { can } \\
(0,500 \\
1,000 \mathrm{~g})\end{array}$} & Steel & \multirow{16}{*}{$\begin{array}{l}0 \text { to } \\
585\end{array}$} & set- $8 b-1$ \\
\hline & & & Poly & $\mathrm{c} 12$ & & Steel & & set- $8 b-2$ \\
\hline & & & Water & $\mathrm{c} 12$ & & Poly & & set- $8 b-3$ \\
\hline & & & Poly & $\mathrm{c} 12$ & & Poly & & set- $8 \mathrm{~b}-4$ \\
\hline & & & Water & Generic & & Steel & & set- $8 b-5$ \\
\hline & & & Poly & Generic & & Steel & & set- $8 b-6$ \\
\hline & & & Water & Generic & & Poly & & set- $8 b-7$ \\
\hline & & & Poly & Generic & & Poly & & set- $8 b-8$ \\
\hline & & \multirow{8}{*}{$\begin{array}{l}\text { Sphere (radius defined by total volume } \\
\text { of mass) }\end{array}$} & Water & $\mathrm{c} 12$ & & Steel & & set-8b-9 \\
\hline & & & Poly & $\mathrm{c} 12$ & & Steel & & set- $8 \mathrm{~b}-10$ \\
\hline & & & Water & $\mathrm{c} 12$ & & Poly & & set- $8 b-11$ \\
\hline & & & Poly & $\mathrm{c} 12$ & & Poly & & set- $8 b-12$ \\
\hline & & & Water & Generic & & Steel & & set- $8 b-13$ \\
\hline & & & Poly & Generic & & Steel & & set- $8 b-14$ \\
\hline & & & Water & Generic & & Poly & & set- $8 b-15$ \\
\hline & & & Poly & Generic & & Poly & & set- $8 \mathrm{~b}-16$ \\
\hline
\end{tabular}




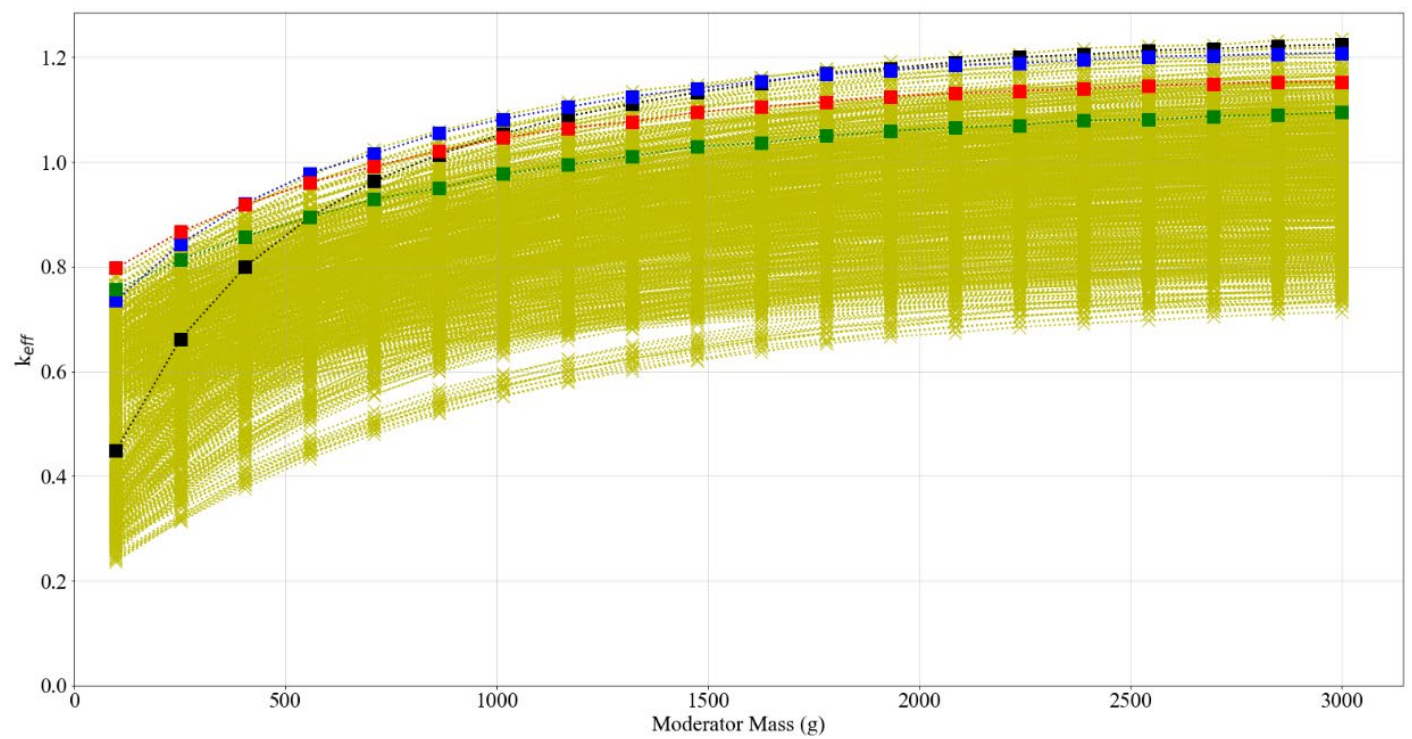

set- 1 and set-8a poly cylinders

… set-1, no filler, 7.7-cm radius cylinder, $585 \mathrm{~g}-\mathrm{Be}, 1.422-\mathrm{cm}$ discrete reflector

set-1, no filler, $7.7-\mathrm{cm}$ radius cylinder, $585 \mathrm{~g}-\mathrm{Be}, 0.001-\mathrm{cm}$ discrete reflector

set-1, no filler, $7.7-\mathrm{cm}$ radius cylinder, $585 \mathrm{~g}-\mathrm{Be}, 0.7112-\mathrm{cm}$ discrete reflector

…… set-1, no filler, $7.7-\mathrm{cm}$ radius cylinder, $585 \mathrm{~g}-\mathrm{Be}, 2.134-\mathrm{cm}$ discrete reflector

Figure 0-1. Set-8a and set-1 $\boldsymbol{k}_{\text {eff }}$ results (three-high uniform array model) for the cylindrical waste forms with a poly discrete reflector and a discrete reflector thickness comparison between the sublisting parameters, which yield a maximum subcritical moderator mass for a discrete reflector thickness of $0.001 \mathrm{~cm}$. 


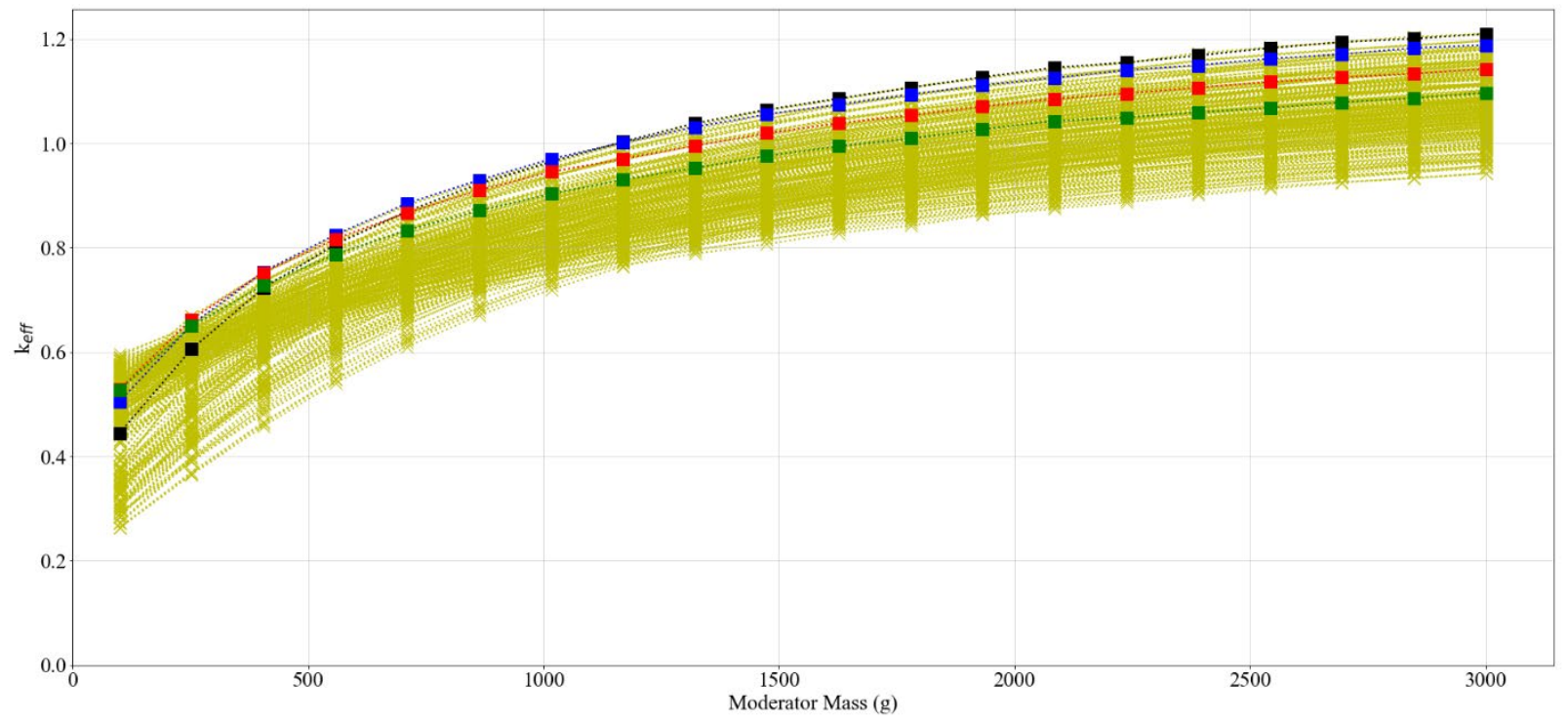

set- 1 and set- 8 a poly spheres
set- 1 , no filler, $0 \mathrm{~g}-\mathrm{Be}, 0.001-\mathrm{cm}$ discrete reflector
set- 1 , no filler, $0 \mathrm{~g}-\mathrm{Be}, 0.7112-\mathrm{cm}$ discrete reflecto

…ㄹ. set-8a, no filler, $0 \mathrm{~g}-\mathrm{Be}, 1.422-\mathrm{cm}$ discrete reflector

를 set-8a, no filler, 0g-Be, 2.134-cm discrete reflector

Figure 0-2. Set-8a and set-1 $\boldsymbol{k}_{\text {eff }}$ results (three-high uniform array model) for the spherical waste forms with a poly discrete reflector, and a discrete reflector thickness comparison between the sublisting parameters, which yield a maximum subcritical moderator mass for a discrete reflector thickness of $0.001 \mathrm{~cm}$. 


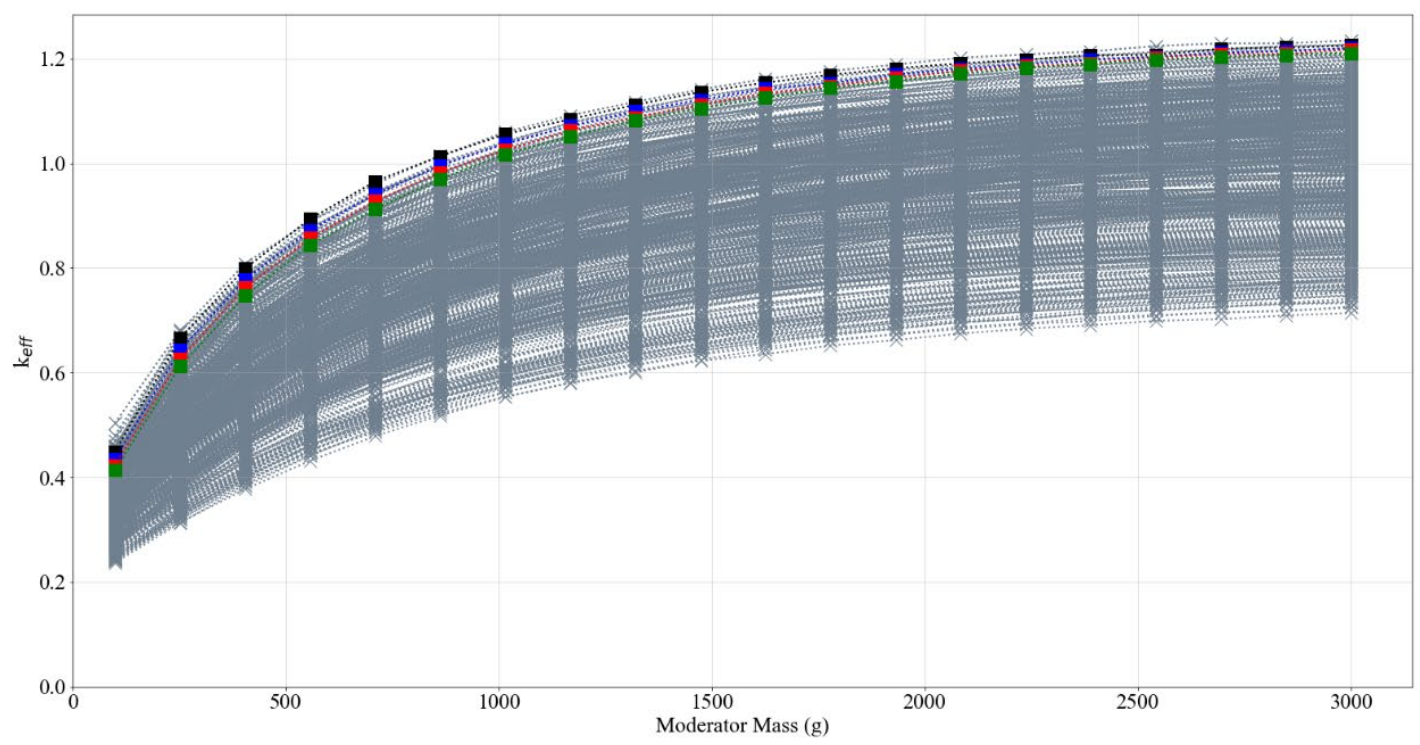

set- 1 and set-8a SS cylinders

ㄷ. set-1, no filler, 7.7-cm radius cylinder, $585 \mathrm{~g}-\mathrm{Be}, 1.422-\mathrm{cm}$ discrete reflector

set- 1 , no filler, $7.7-\mathrm{cm}$ radius cylinder, $585 \mathrm{~g}-\mathrm{Be}, 0.001-\mathrm{cm}$ discrete reflector

- - set-1, no filler, 7.7-cm radius cylinder, $585 \mathrm{~g}-\mathrm{Be}, 0.7112$-cm discrete reflector

Figure 0-3. Set-8a and set-1 $\boldsymbol{k}_{\text {eff }}$ results (three-high uniform array model) for the cylindrical waste forms with a stainless-steel discrete reflector and a discrete reflector thickness comparison between the sublisting parameters, which yield a maximum subcritical moderator mass for a discrete reflector thickness of $0.001 \mathrm{~cm}$. 


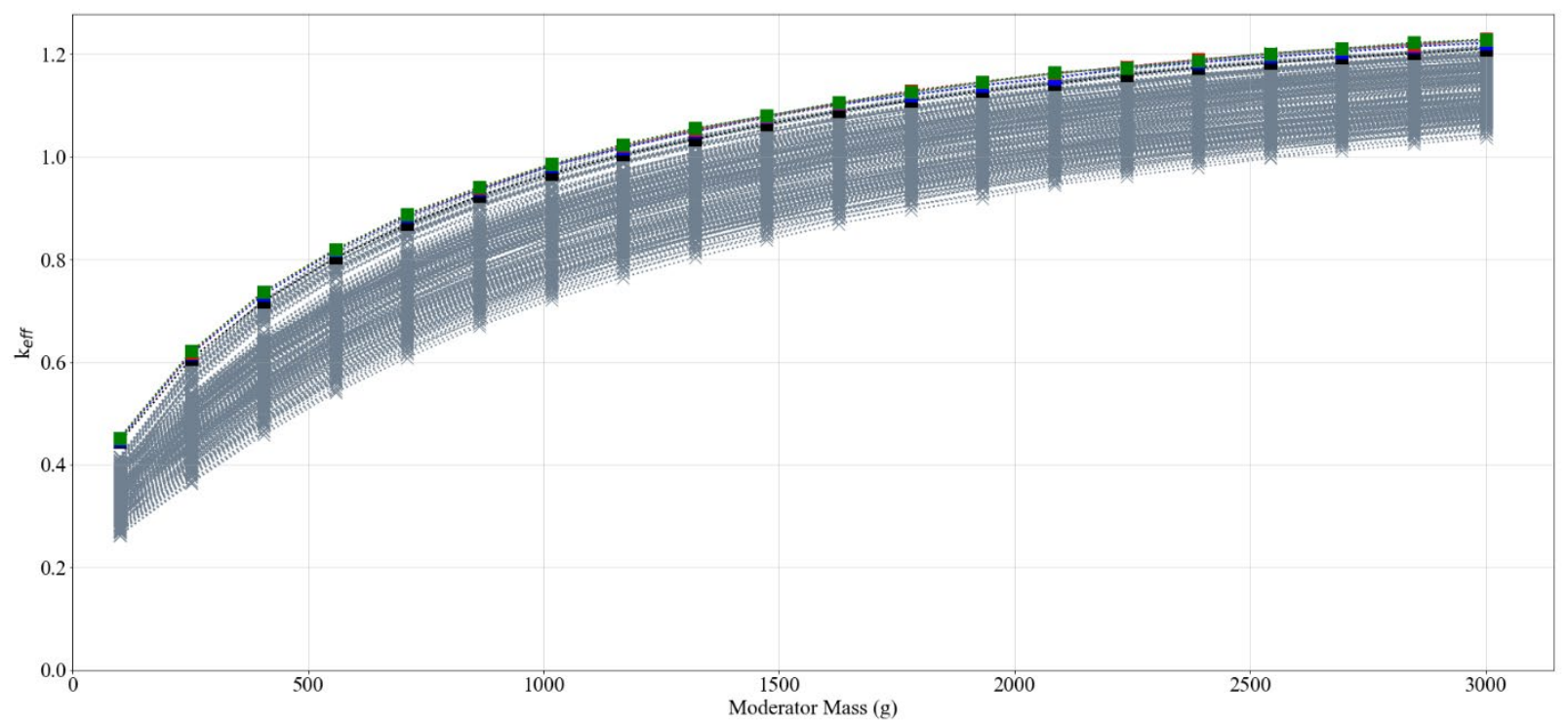

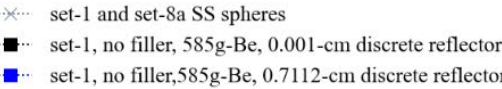

ㄹ.

를." set-8a, no filler, $585 \mathrm{~g}-\mathrm{Be}, 2.134-\mathrm{cm}$ discrete reflector

Figure 0-4. Set-8a and set-1 $\boldsymbol{k}_{\text {eff }}$ results (three-high uniform array model) for the spherical waste forms with a stainless-steel discrete reflector and a discrete reflector thickness comparison between the sublisting parameters, which yield a maximum subcritical moderator mass for a discrete reflector thickness of $0.001 \mathrm{~cm}$. 


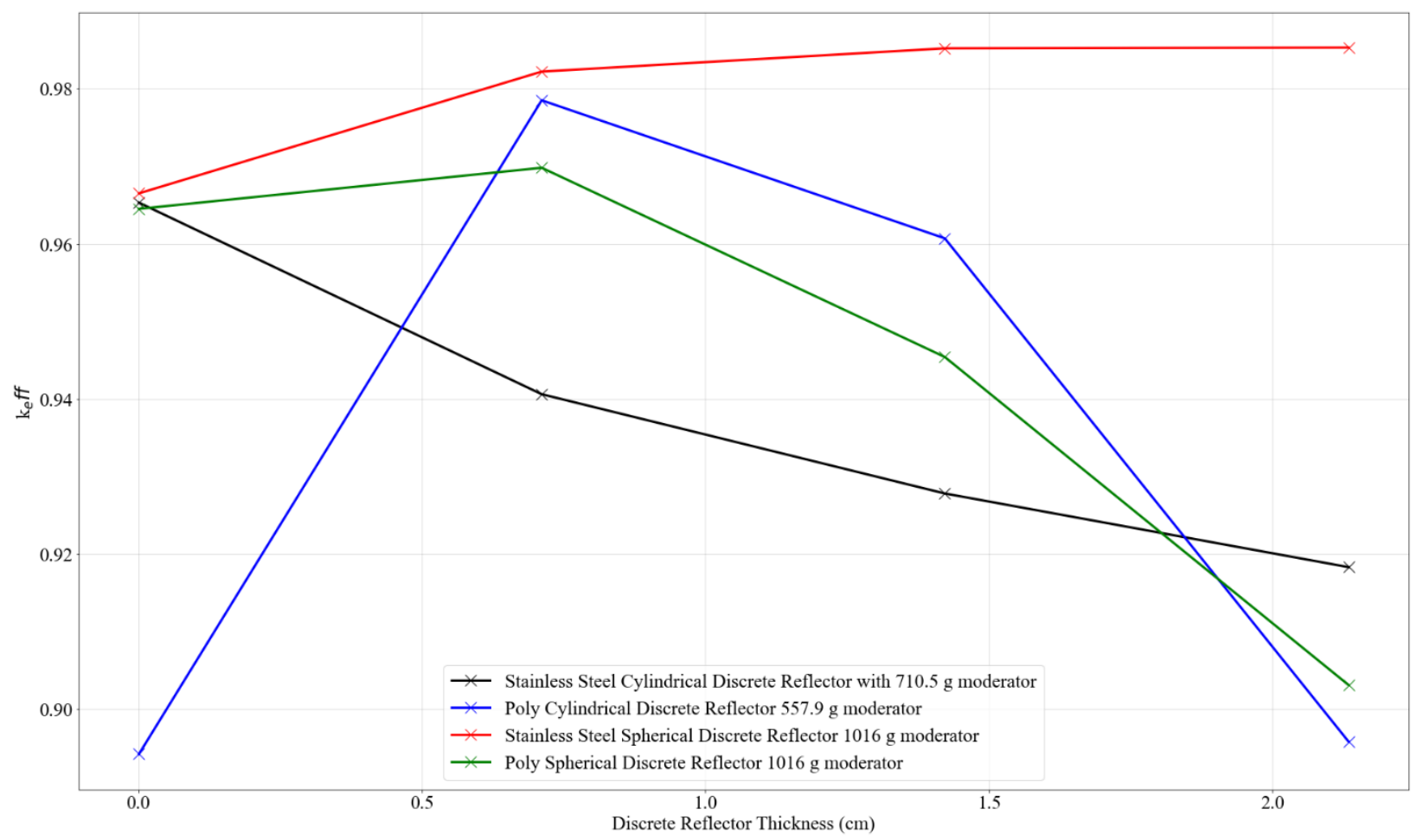

Figure $0-5$. Set-8a and set-1 maximum $\boldsymbol{k}_{\text {eff }}$ results (three-high uniform array model) overall sublistings as a function of discrete reflector thickness.

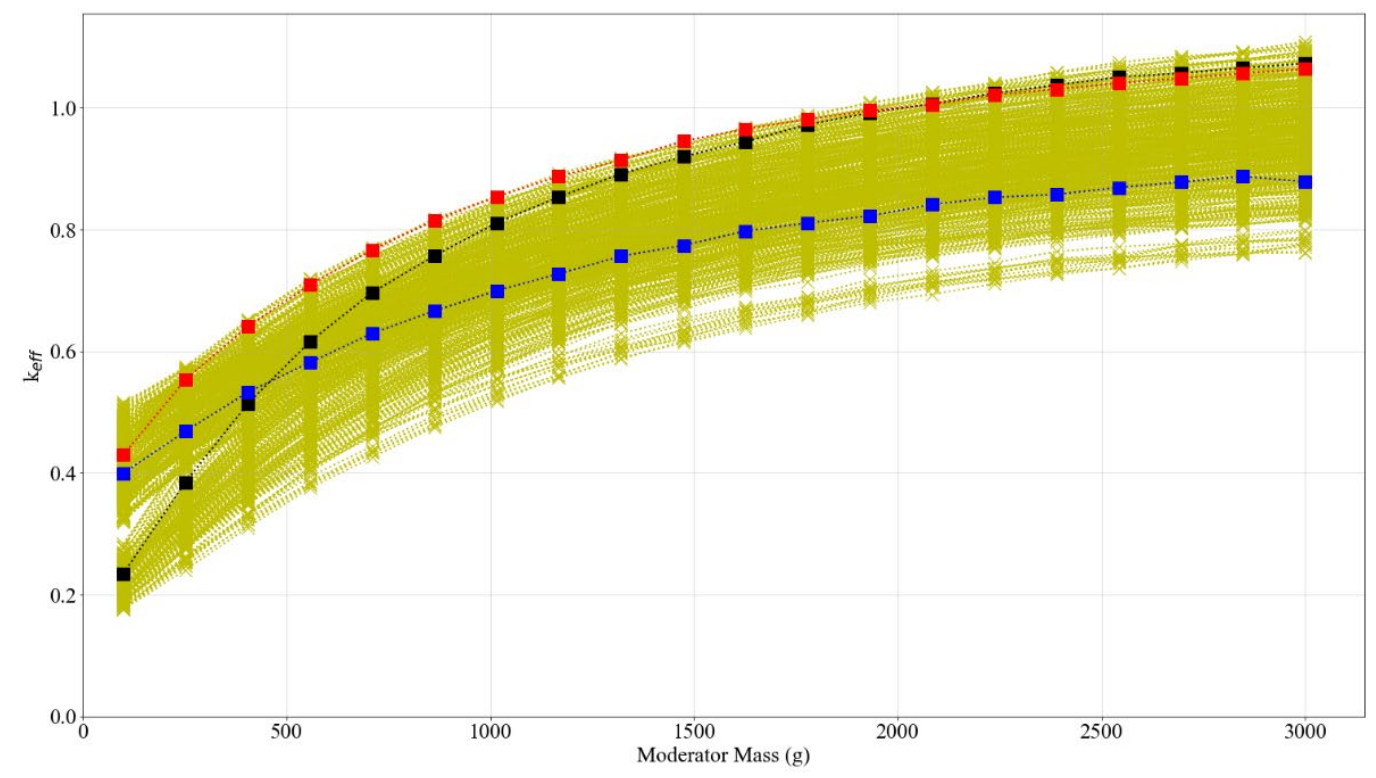

Figure 0-6. Set-8b and set-2-uh $k_{\text {eff }}$ results (nonuniform array model) for the cylindrical waste forms with a poly discrete reflector and a discrete reflector thickness comparison between the sublisting parameters, which yield a maximum subcritical moderator mass for a discrete reflector thickness of $0.001 \mathrm{~cm}$. 


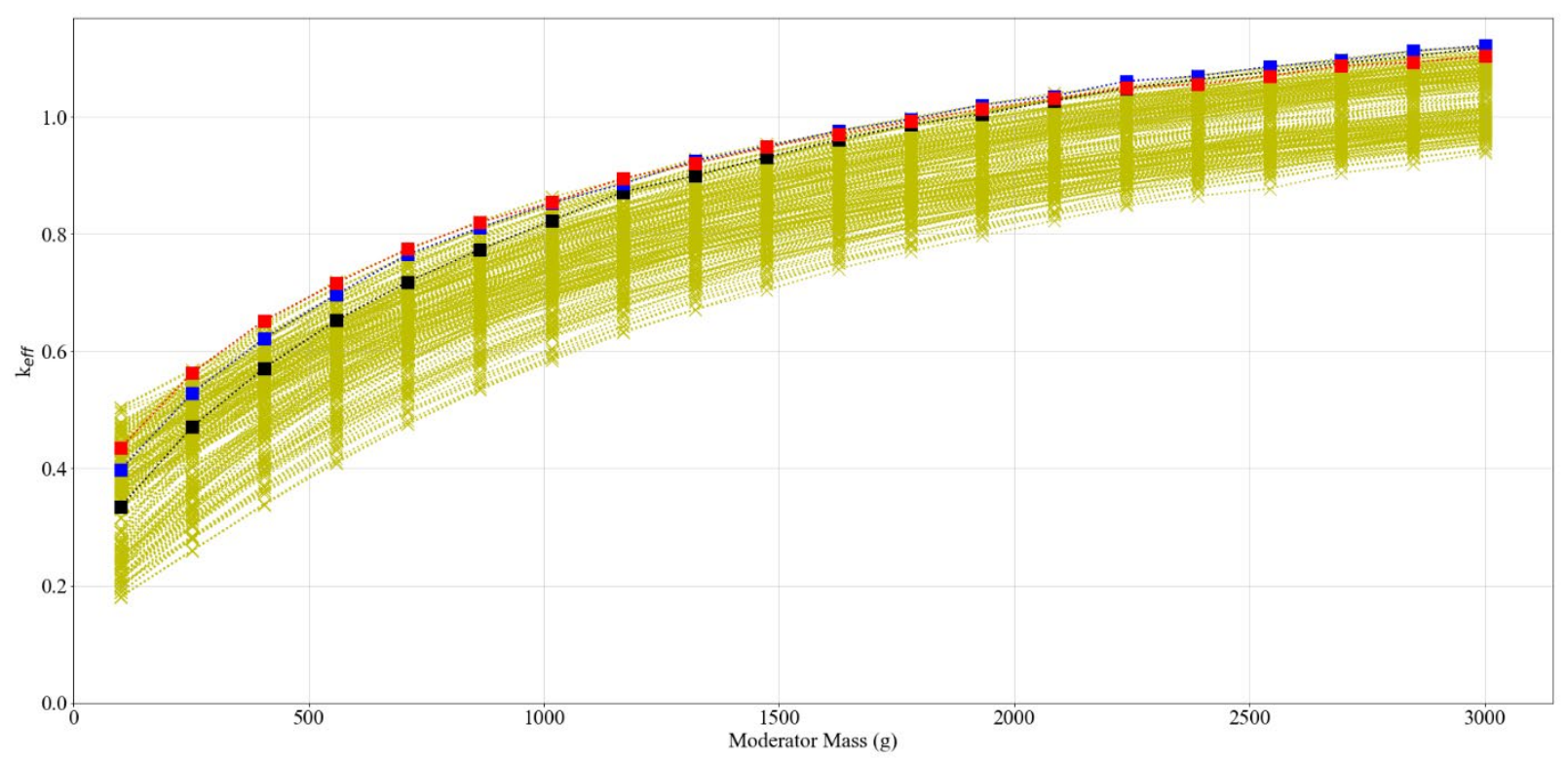

set-2-uh and set-8b-uh poly spheres

- set-2-uh, no filler, $0 \mathrm{~g}-\mathrm{Be}, 0.001-\mathrm{cm}$ discrete reflector
-.. set-2-uh, no filler, $0 \mathrm{~g}$-Be, 0.7112 -cm discrete reflector

=. set-8b-uh, no filler, $0 \mathrm{~g}-\mathrm{Be}, 1.4224-\mathrm{cm}$ discrete reflector

Figure 0-7. Set-8b and set-2-uh $\boldsymbol{k}_{\text {eff }}$ results (nonuniform array model) for the spherical waste forms with a poly discrete reflector and a discrete reflector thickness comparison between the sublisting parameters, which yield a maximum subcritical moderator mass for a discrete reflector thickness $0 \mathrm{of} 0.001 \mathrm{~cm}$. 


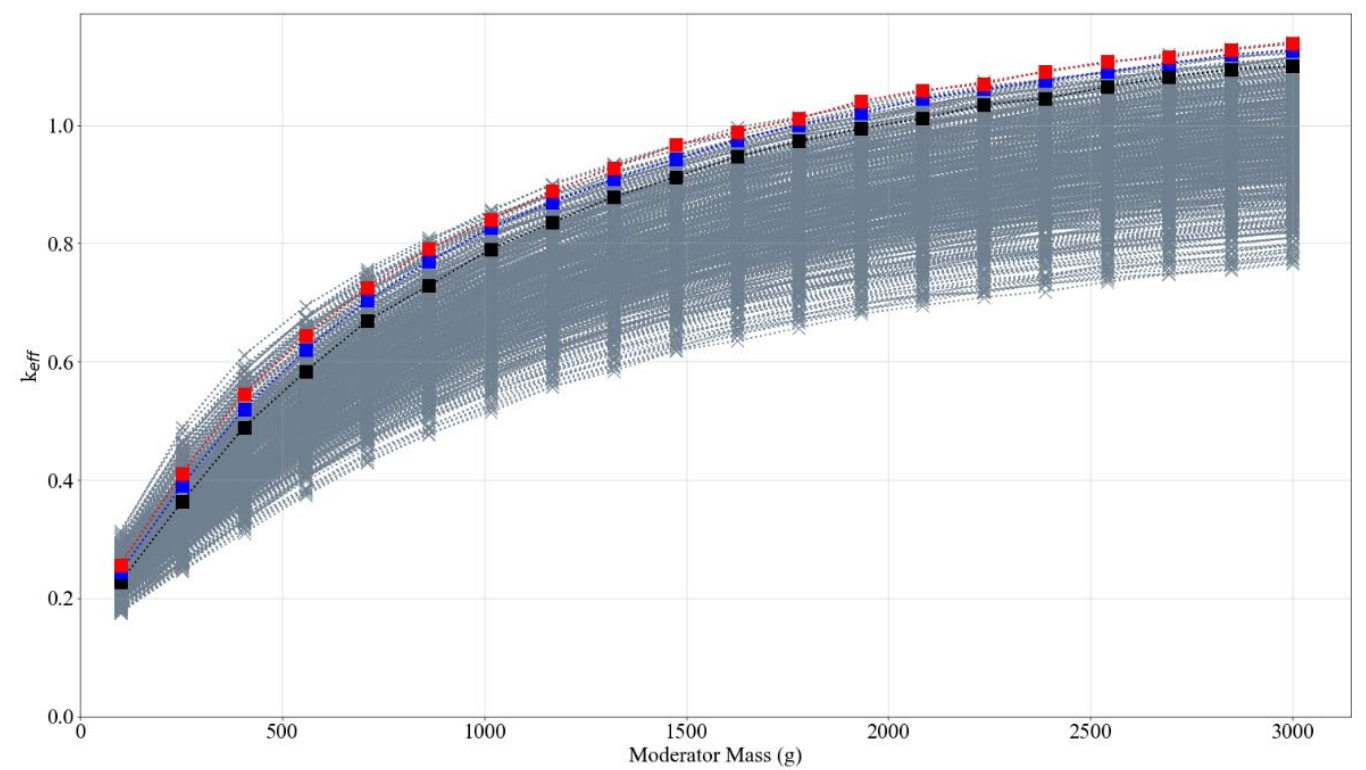

(w.. set-2-uh and set-8b-uh SS cylinders

- set-2-uh, no filler, 7.7-cm radius cylinder, $585 \mathrm{~g}-\mathrm{Be}, 0.7112$-cm discrete reflector

- set-2-uh, no filler, 7.7 -cm radius cylinder, $585 \mathrm{~g}-\mathrm{Be}, 0.001-\mathrm{cm}$ discrete reflector

ㄹ. set- $8 \mathrm{~b}$-uh, no filler, $7.7-\mathrm{cm}$ radius cylinder, $585 \mathrm{~g}-\mathrm{Be}, 1.4224-\mathrm{cm}$ discrete reflector

Figure 0-8. Set-8b and set-2-uh $\boldsymbol{k}_{\text {eff }}$ results (nonuniform array model) for the cylindrical waste forms with a stainless-steel discrete reflector and a discrete reflector thickness comparison between the sublisting parameters, which yield a maximum subcritical moderator mass for a discrete reflector thickness of $0.001 \mathrm{~cm}$. 


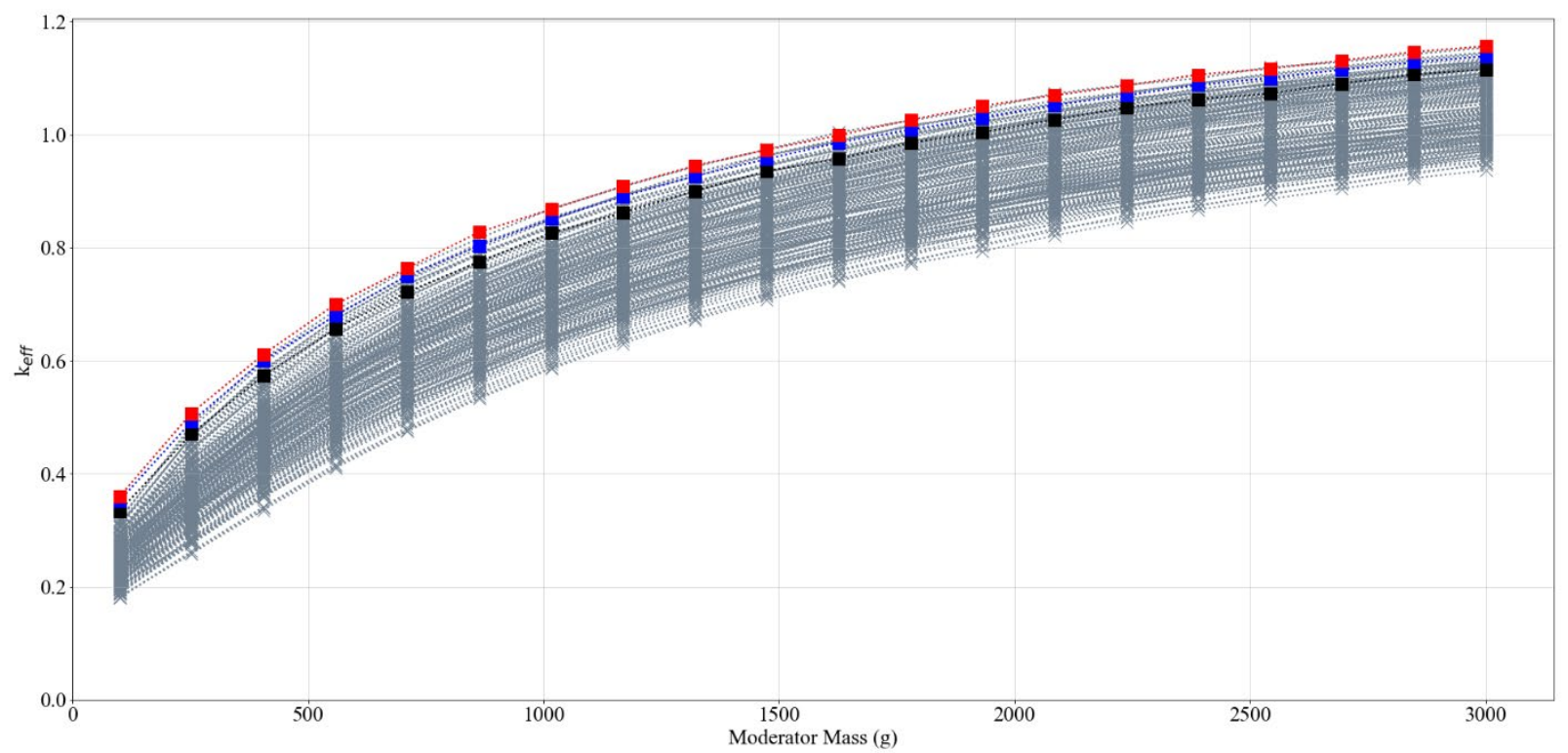

set-2-uh and set-8b-uh SS spheres

E. set-2-uh, no filler, $0 \mathrm{~g}-\mathrm{Be}, 0.001-\mathrm{cm}$ discrete reflector set-2-uh, no filler, $0 \mathrm{~g}$-Be, 0.7112 -cm discrete reflector

-.. set-8b-uh, no filler, 0g-Be, 1.4224-cm discrete reflector

Figure 0-9. Set-8b and set-2-uh $k_{\text {eff }}$ results (nonuniform array model) for the spherical waste forms with a stainless-steel discrete reflector and a discrete reflector thickness comparison between the sublisting parameters, which yield a maximum subcritical moderator mass for a discrete reflector thickness of $0.001 \mathrm{~cm}$. 


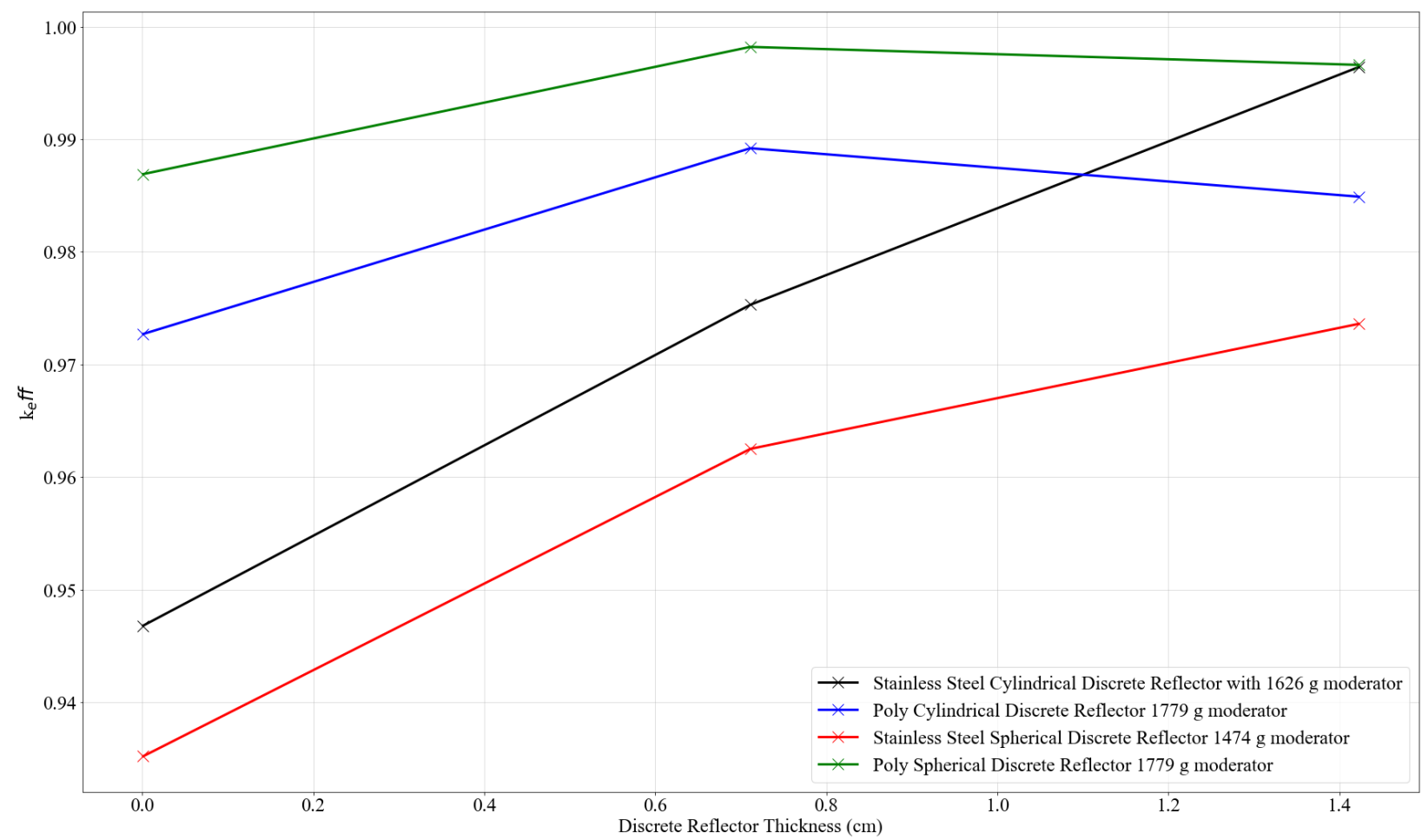

Figure 0-10. Set-8b and set-2-uh maximum $\boldsymbol{k}_{\text {eff }}$ results (nonuniform array model) overall sublistings as a function of discrete reflector thickness.

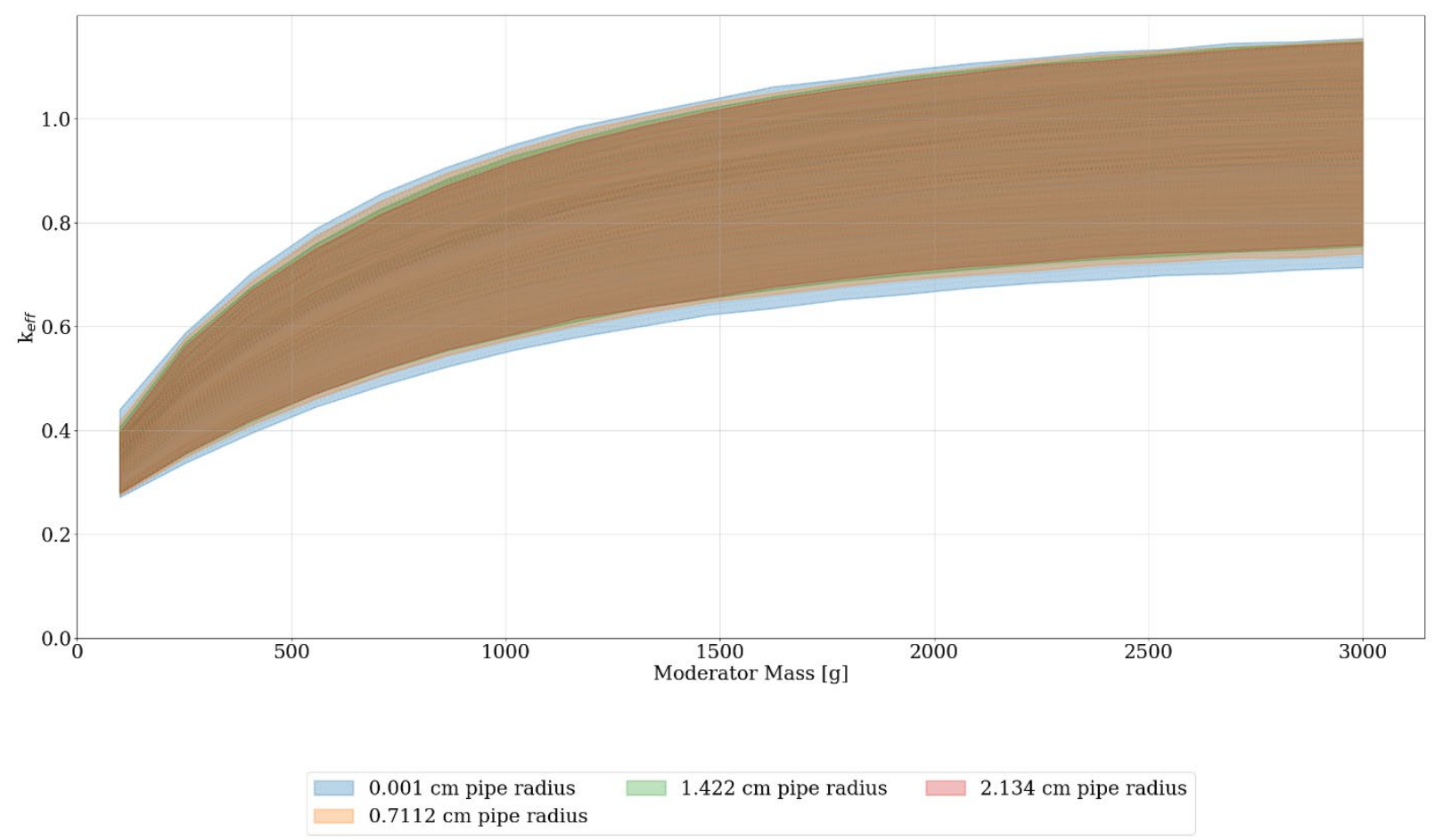

Figure 0-11. Set-8a sublisting-1 results (three-high uniform array model): $\boldsymbol{k}_{\text {eff }}$ for set-1 and set-8a by discrete reflector thicknesses. 


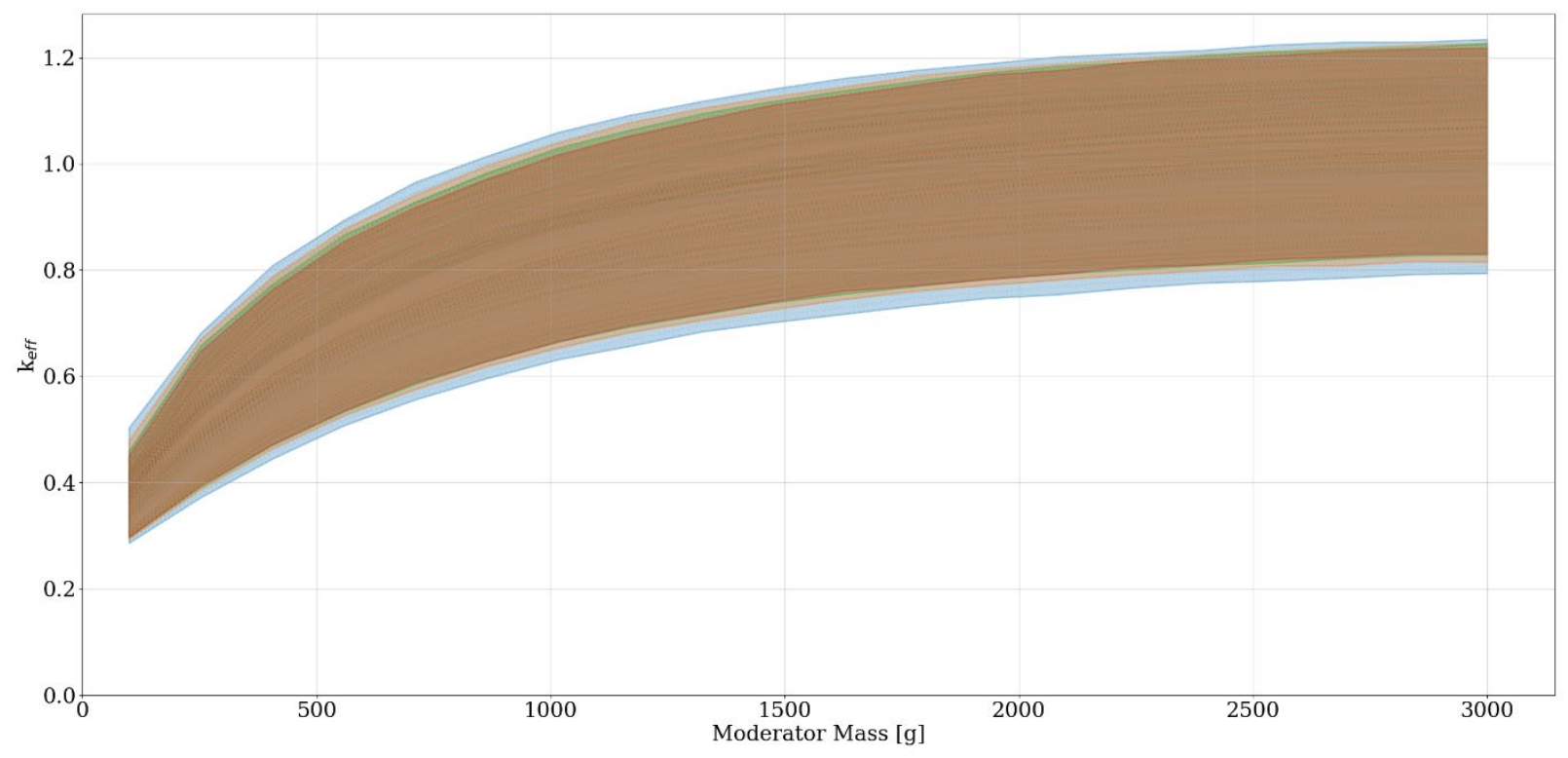

$0.001 \mathrm{~cm}$ pipe radius $\square 1.422 \mathrm{~cm}$ pipe radius $\square 2.134 \mathrm{~cm}$ pipe radius $0.7112 \mathrm{~cm}$ pipe radius

Figure 0-12. Set-8a sublisting-2 results (three-high uniform array model): $\boldsymbol{k}_{\text {eff }}$ for set-1 and set-8a by discrete reflector thicknesses.

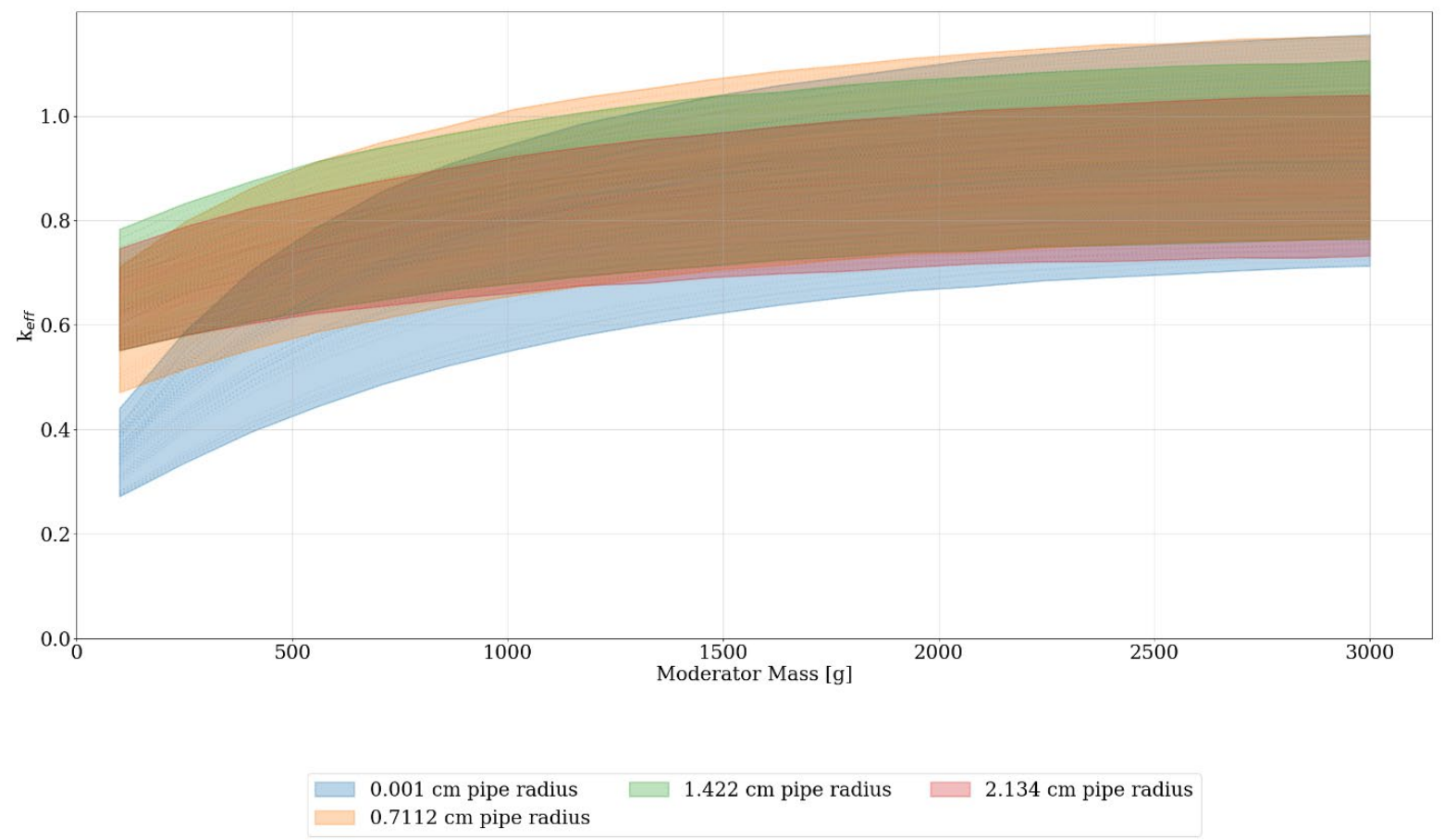

Figure 0-13. Set-8a sublisting-3 results (three-high uniform array model): ): $\boldsymbol{k}_{\text {eff }}$ for set-1 and set-8a by discrete reflector thicknesses. 


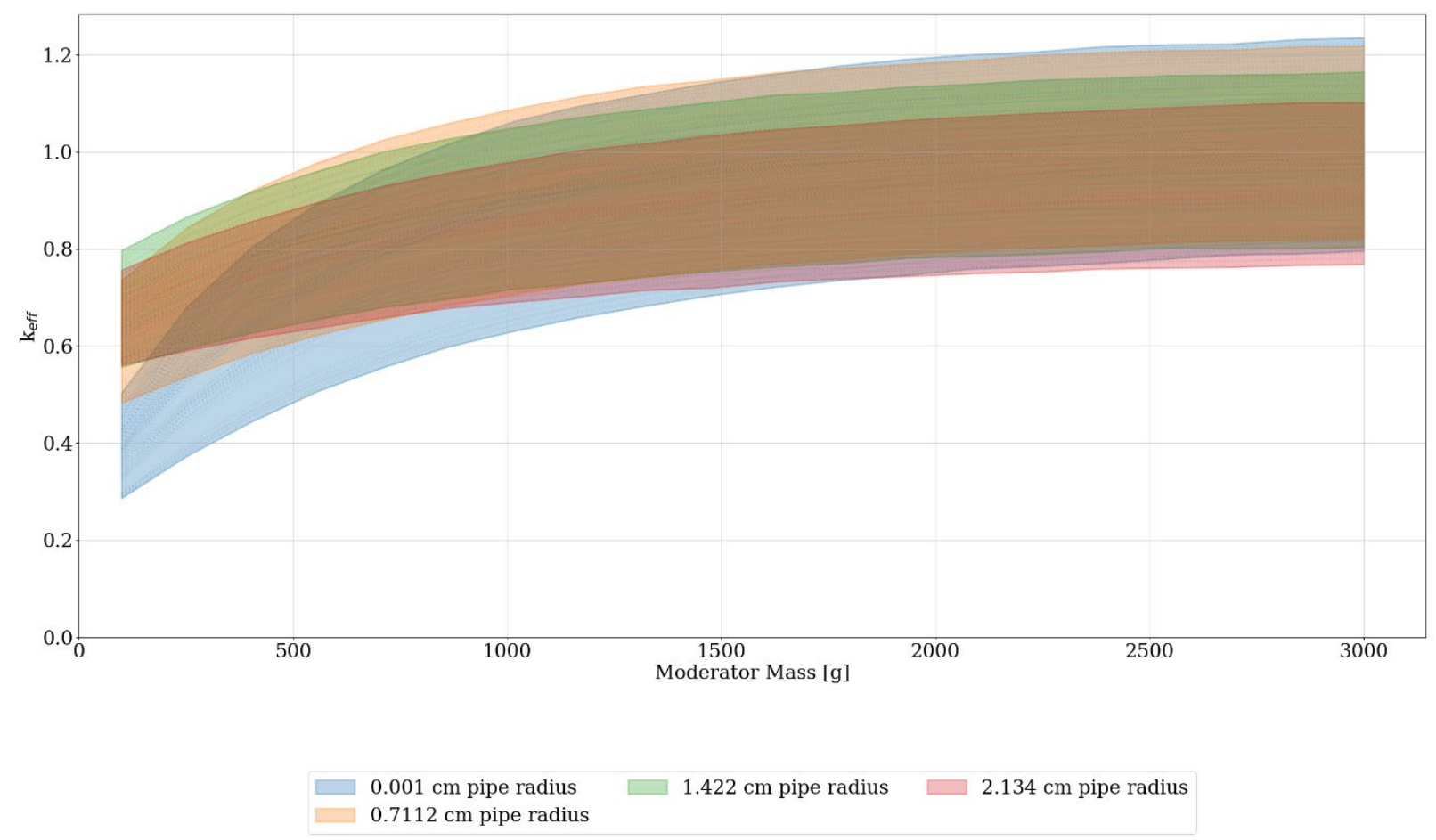

Figure 0-14. Set-8a sublisting-4 results (three-high uniform array model): $\boldsymbol{k}_{\text {eff }}$ for set-1 and set-8a by discrete reflector thicknesses. 


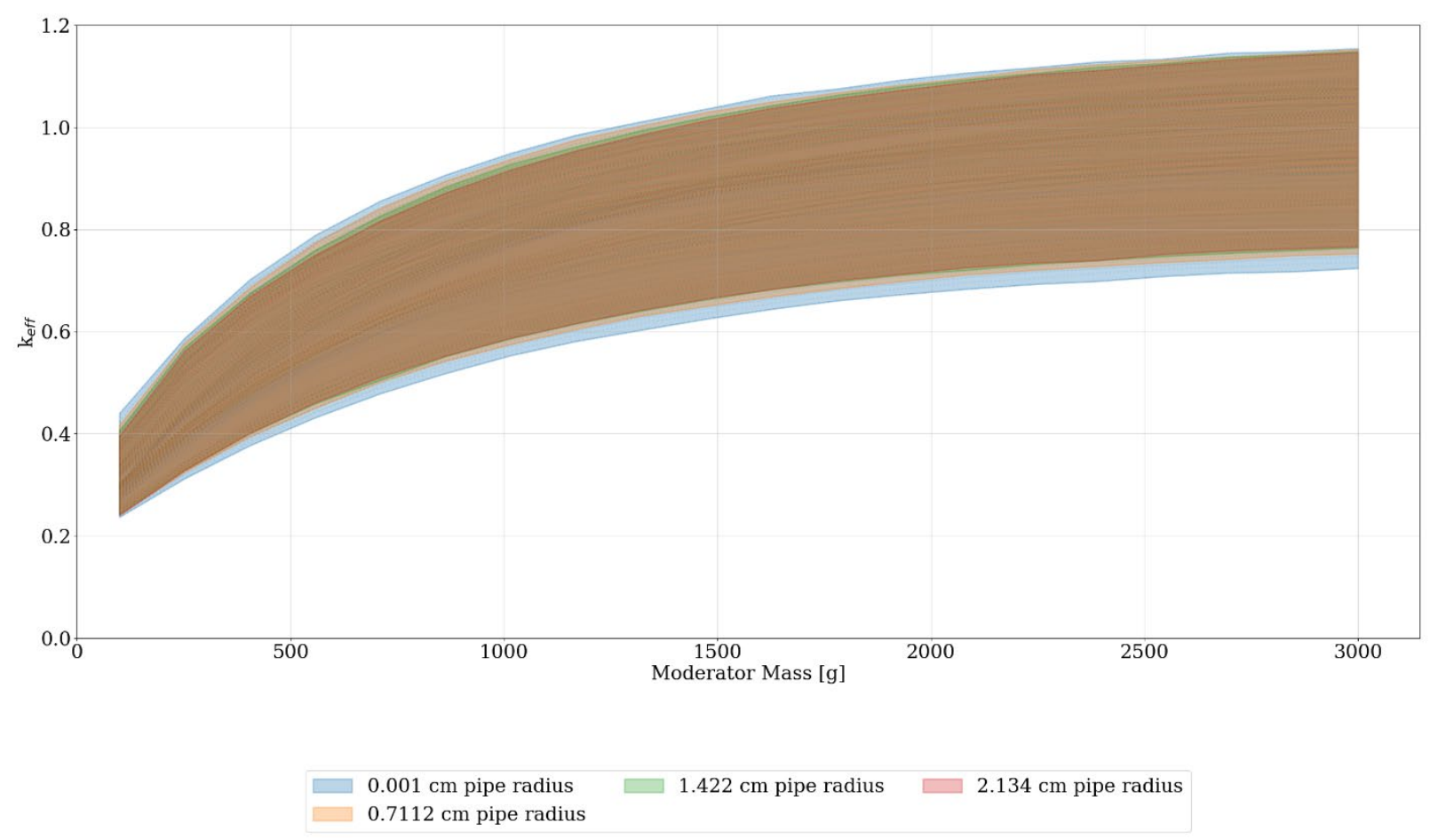

Figure 0-15. Set-8a sublisting-5 results (three-high uniform array model): $\boldsymbol{k}_{\text {eff }}$ for set-1 and set-8a by discrete reflector thicknesses.

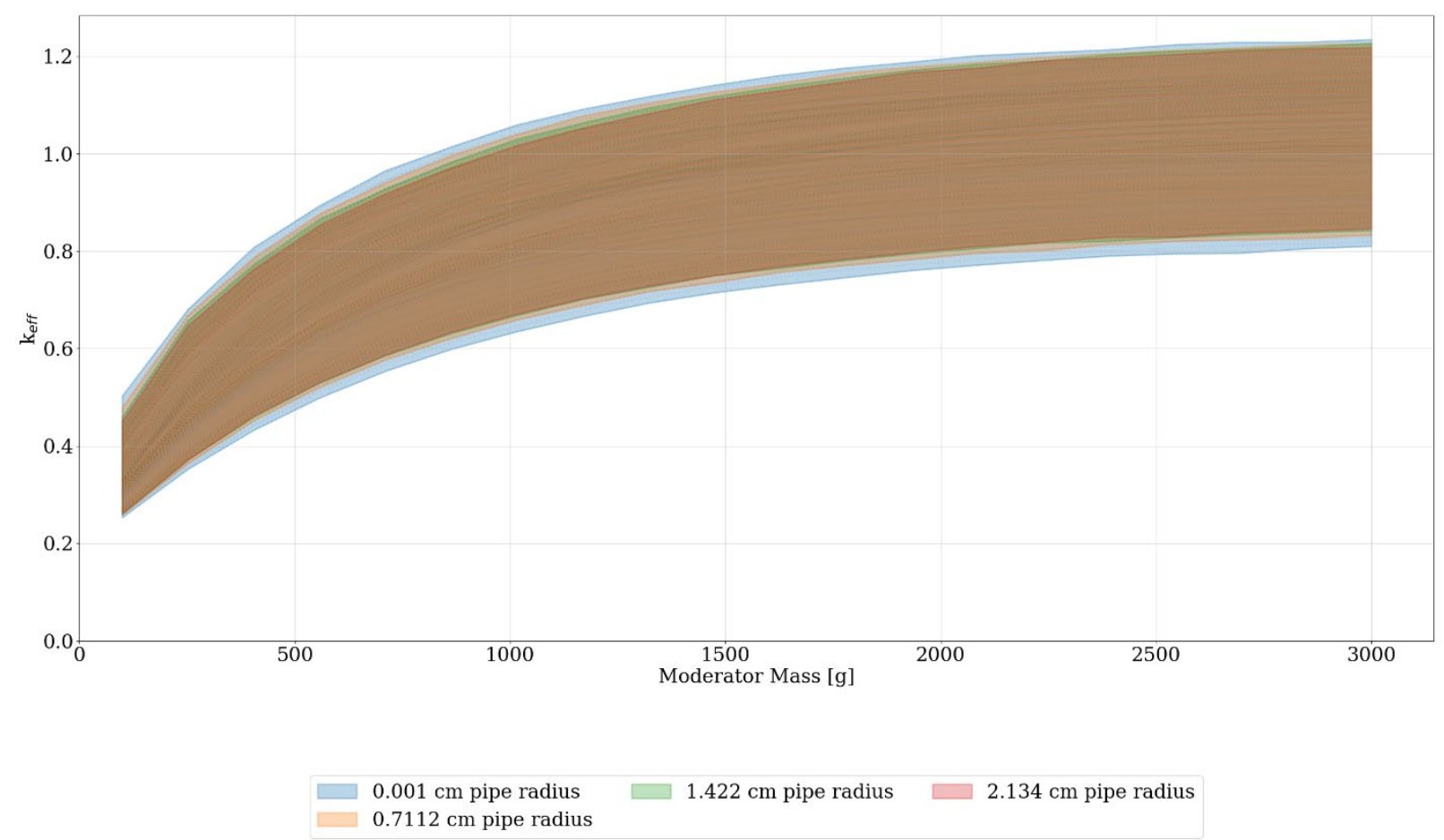

Figure 0-16. Set-8a sublisting-6 results (three-high uniform array model): $\boldsymbol{k}_{\text {eff }}$ for set-1 and set-8a by discrete reflector thicknesses. 


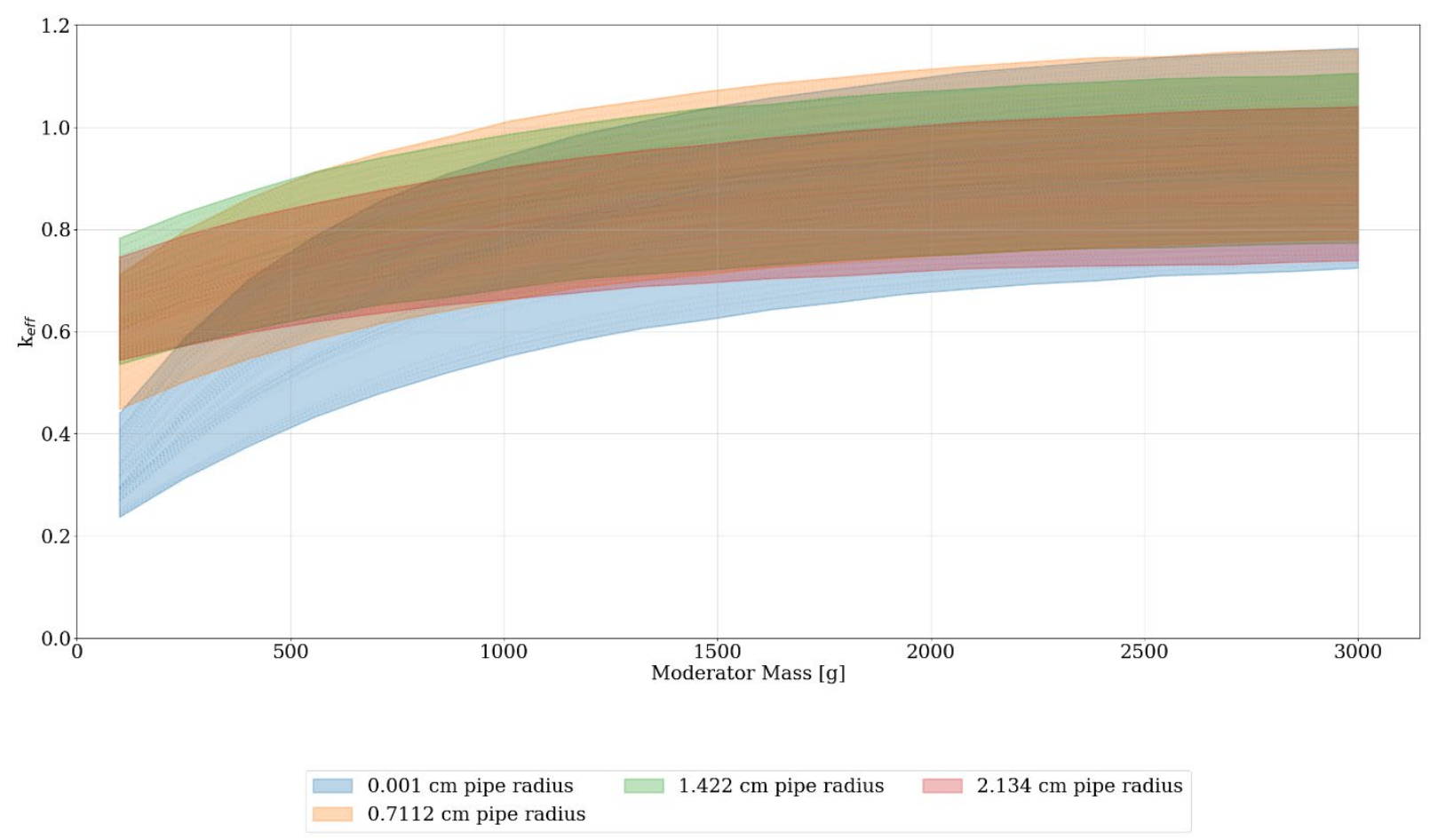

Figure 0-17. Set-8a sublisting-7 results (three-high uniform array model): $\boldsymbol{k}_{\text {eff }}$ for set-1 and set-8a by discrete reflector thicknesses.

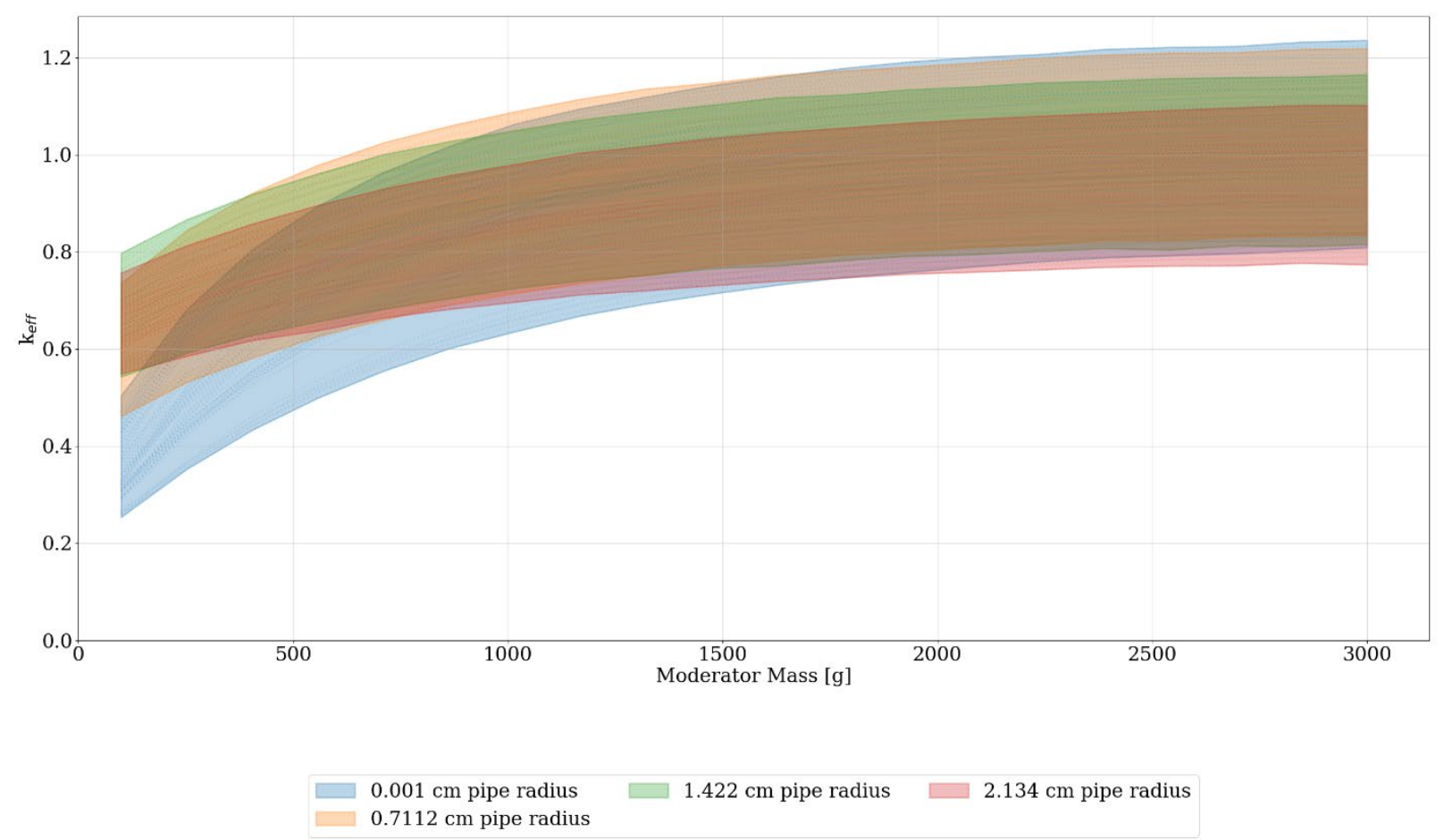

Figure 0-18. Set-8a sublisting-8 results (three-high uniform array model): $\boldsymbol{k}_{\text {eff }}$ for set-1 and set-8a by discrete reflector thicknesses. 


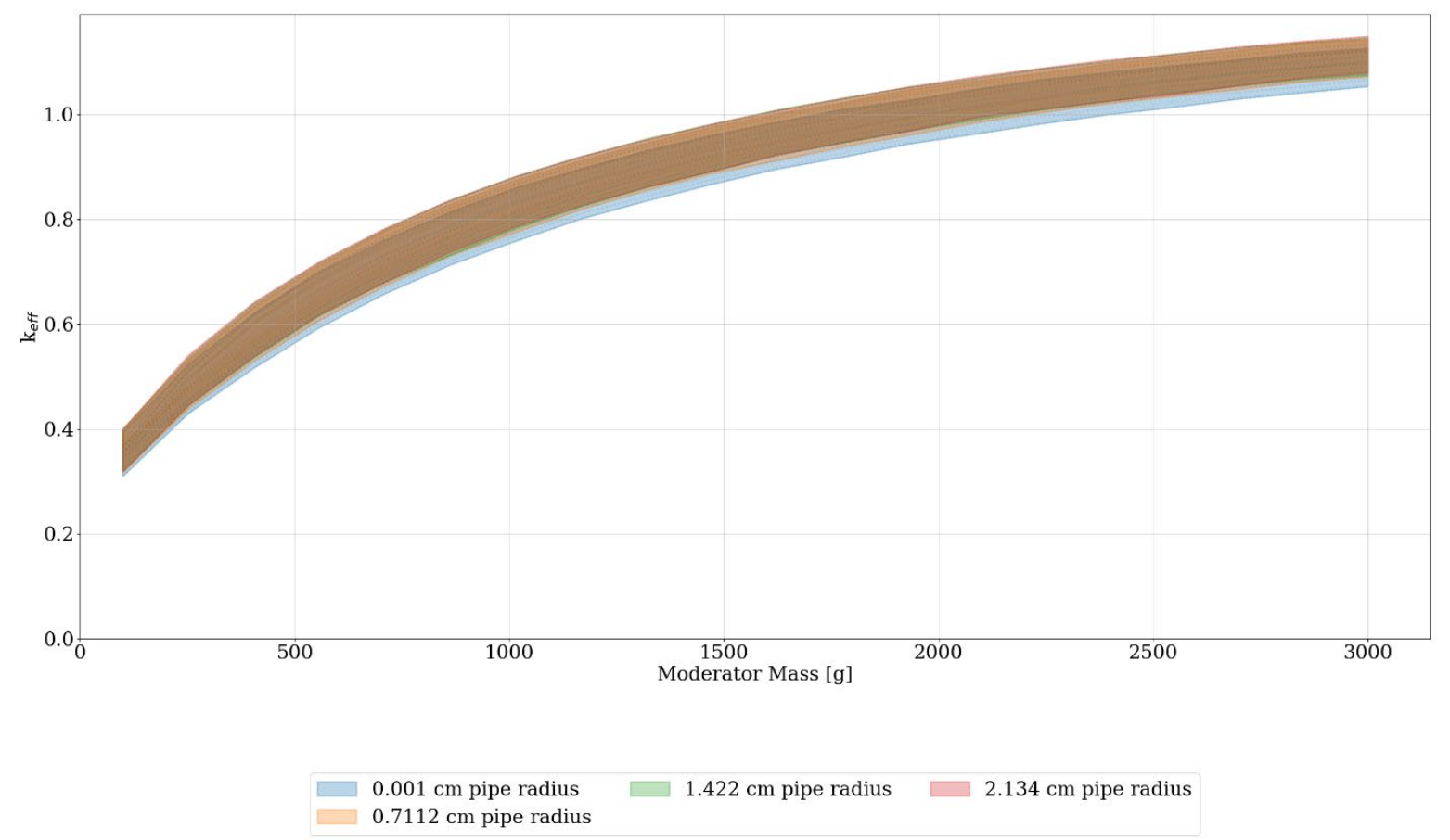

Figure 0-19. Set-8a sublisting-9 results (three-high uniform array model): $\boldsymbol{k}_{\text {eff }}$ for set-1 and set-8a by discrete reflector thicknesses.

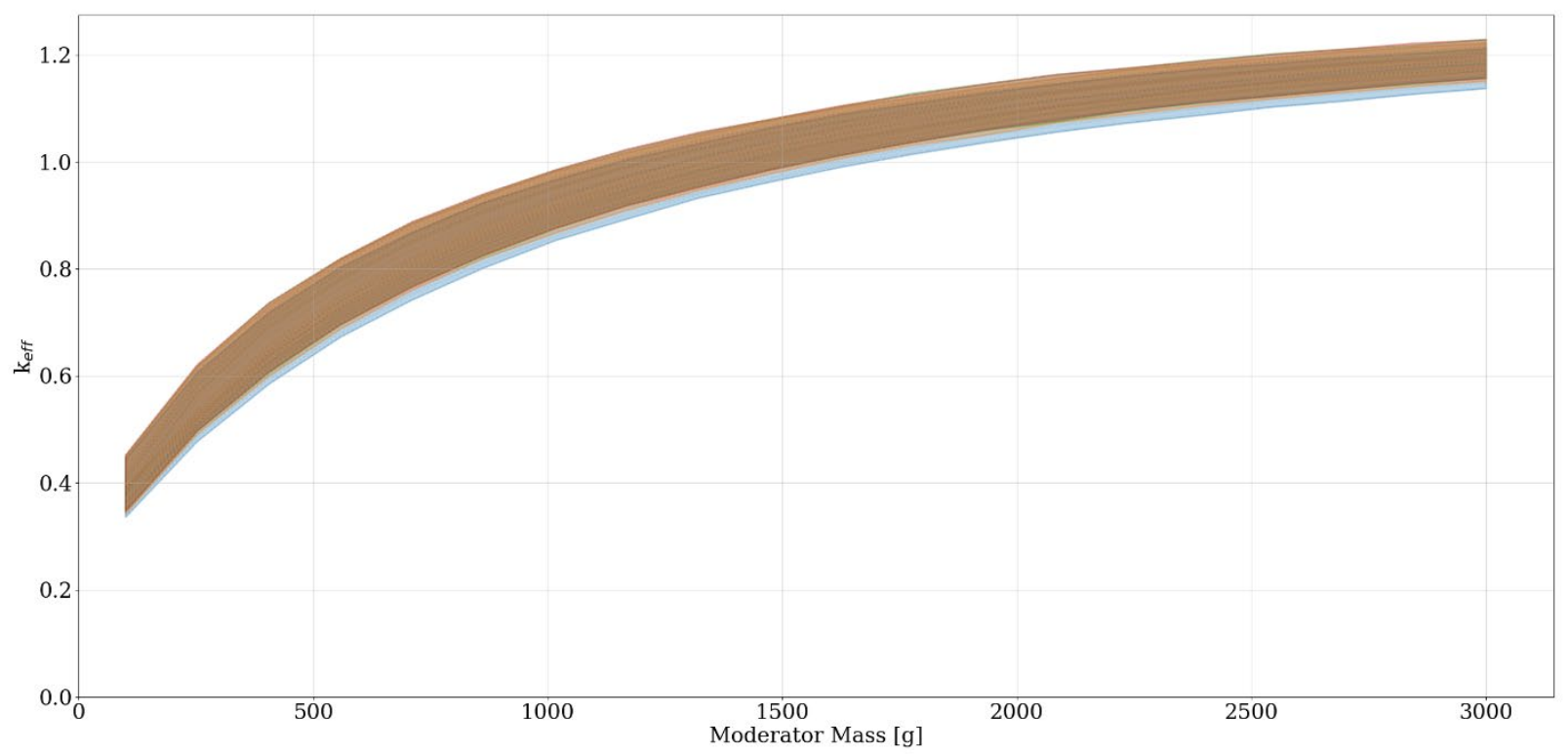

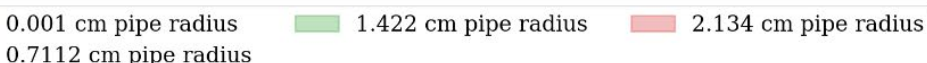

Figure 0-20. Set-8a sublisting-10 results (three-high uniform array model): $k_{\text {eff }}$ for set-1 and set-8a by discrete reflector thicknesses. 
O-23 


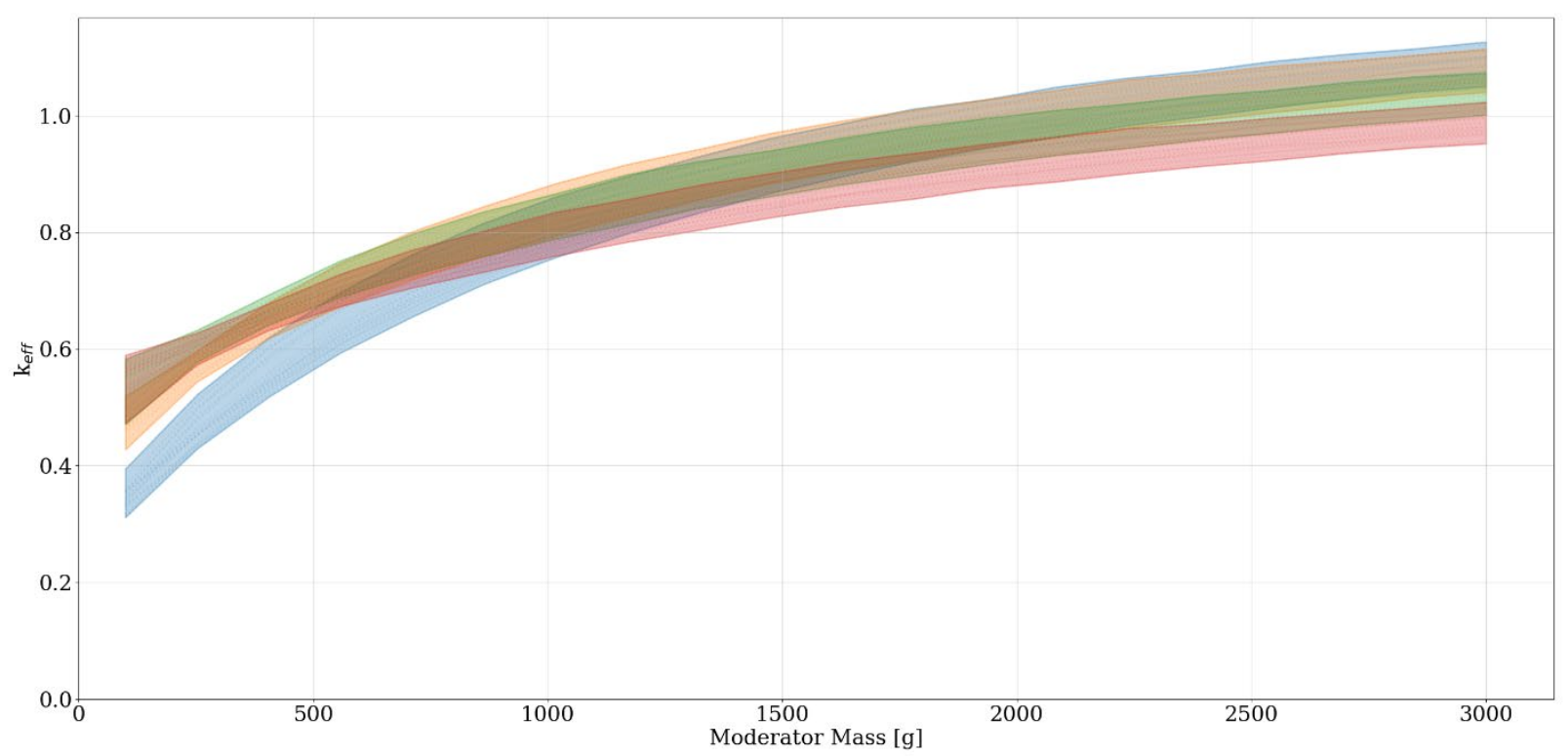

$0.001 \mathrm{~cm}$ pipe radius $\square 1.422 \mathrm{~cm}$ pipe radius $\square 2.134 \mathrm{~cm}$ pipe radius
$0.7112 \mathrm{~cm}$ pipe radius

Figure 0-21. Set-8a sublisting-11 results (three-high uniform array model): $\boldsymbol{k}_{\text {eff }}$ for set-1 and set-8a by discrete reflector thicknesses.

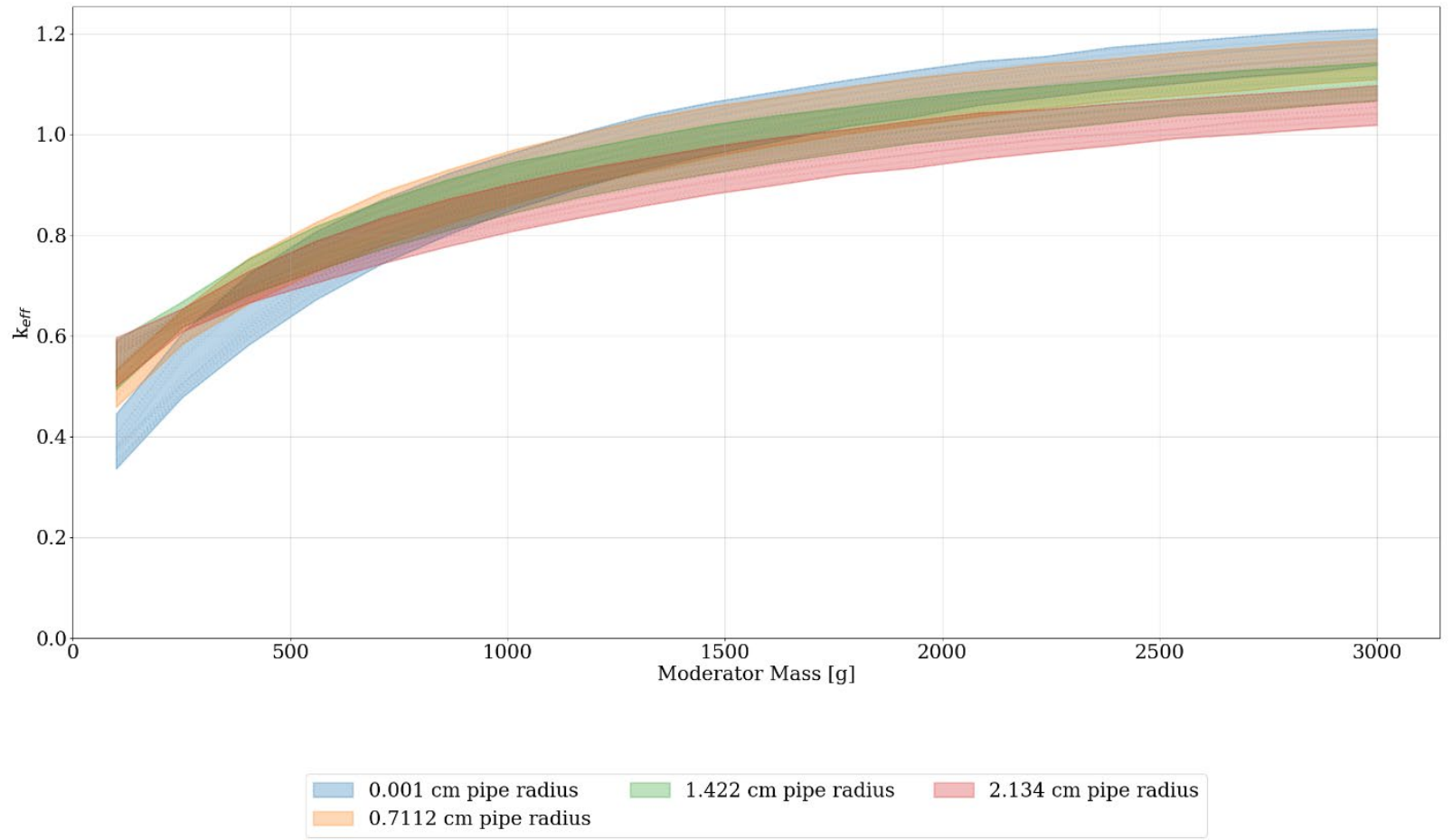

Figure 0-22. Set-8a sublisting-12 results (three-high uniform array model): $k_{e f f}$ for set-1 and set-8a by discrete reflector thicknesses. 

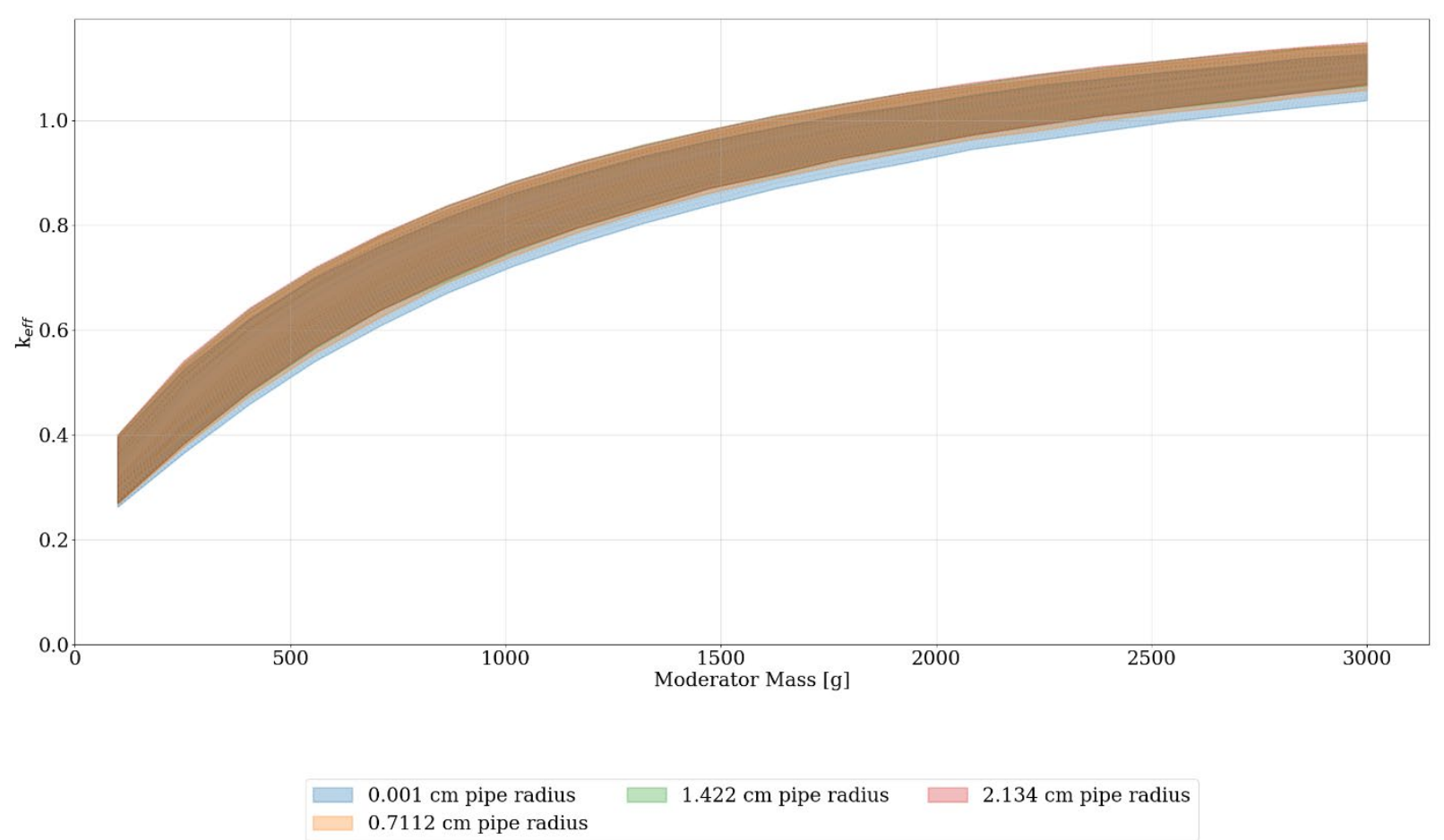

Figure 0-23. Set-8a sublisting-13 results (three-high uniform array model): $\boldsymbol{k}_{\text {eff }}$ for set-1 and set-8a by discrete reflector thicknesses.

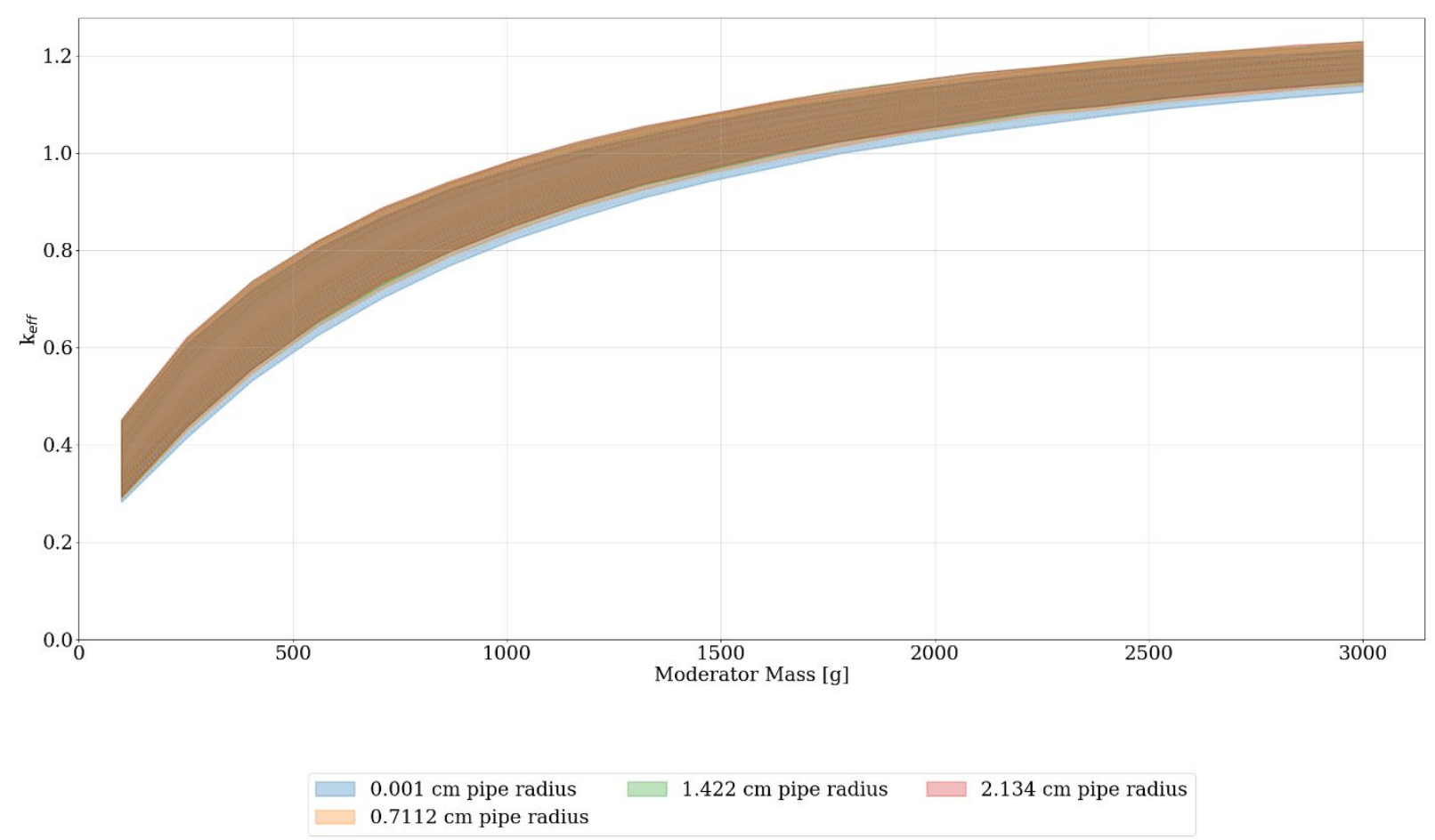

Figure 0-24. Set-8a sublisting-14 results (three-high uniform array model): $k_{\text {eff }}$ for set-1 and set-8a by discrete reflector thicknesses. 


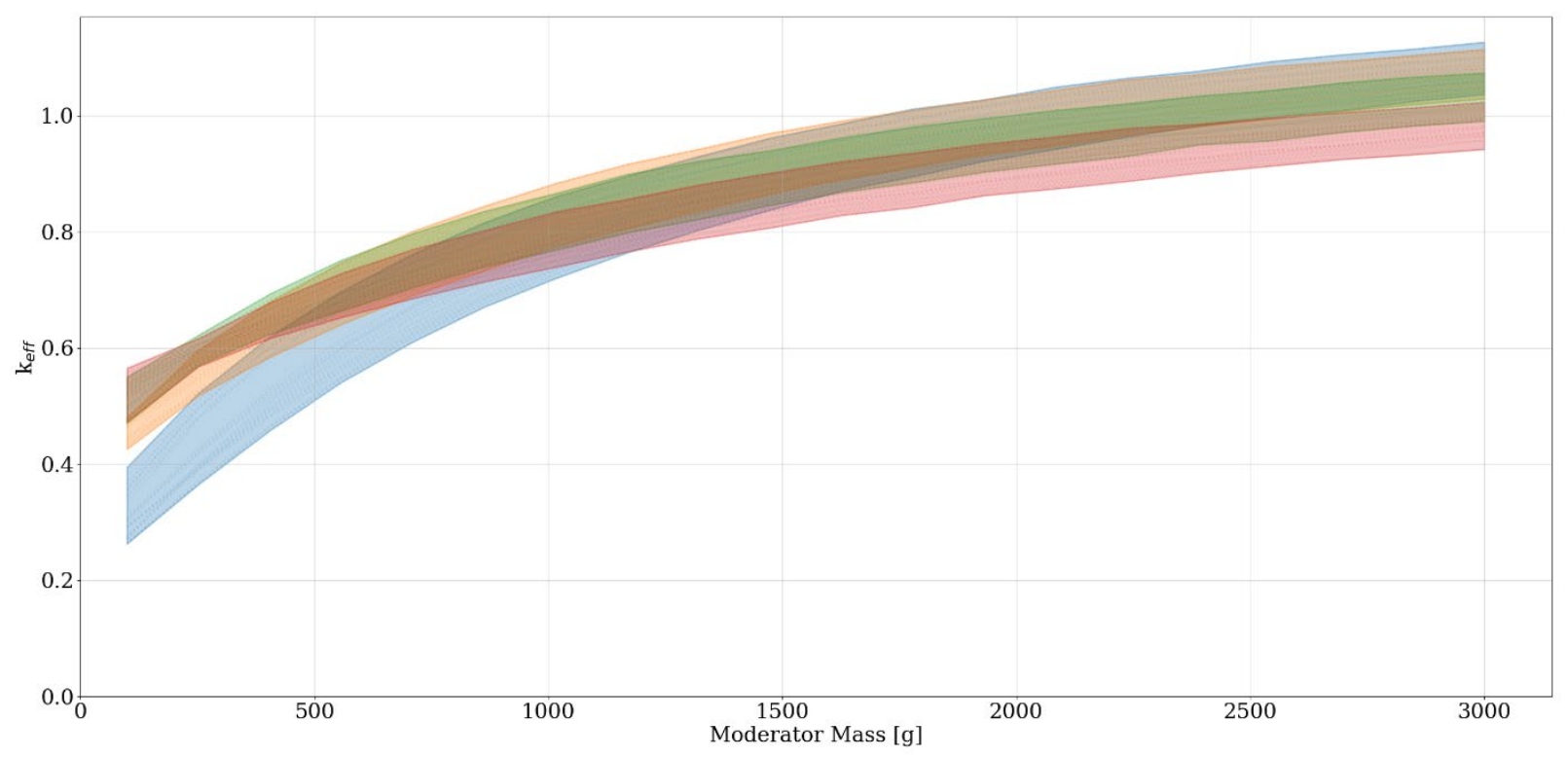

$0.001 \mathrm{~cm}$ pipe radius $\square 1.422 \mathrm{~cm}$ pipe radius $\square 2.134 \mathrm{~cm}$ pipe radius
$0.7112 \mathrm{~cm}$ pipe radius

Figure O-25. Set-8a sublisting-15 results (three-high uniform array model): $\boldsymbol{k}_{\text {eff }}$ for set-1 and set-8a by discrete reflector thicknesses.

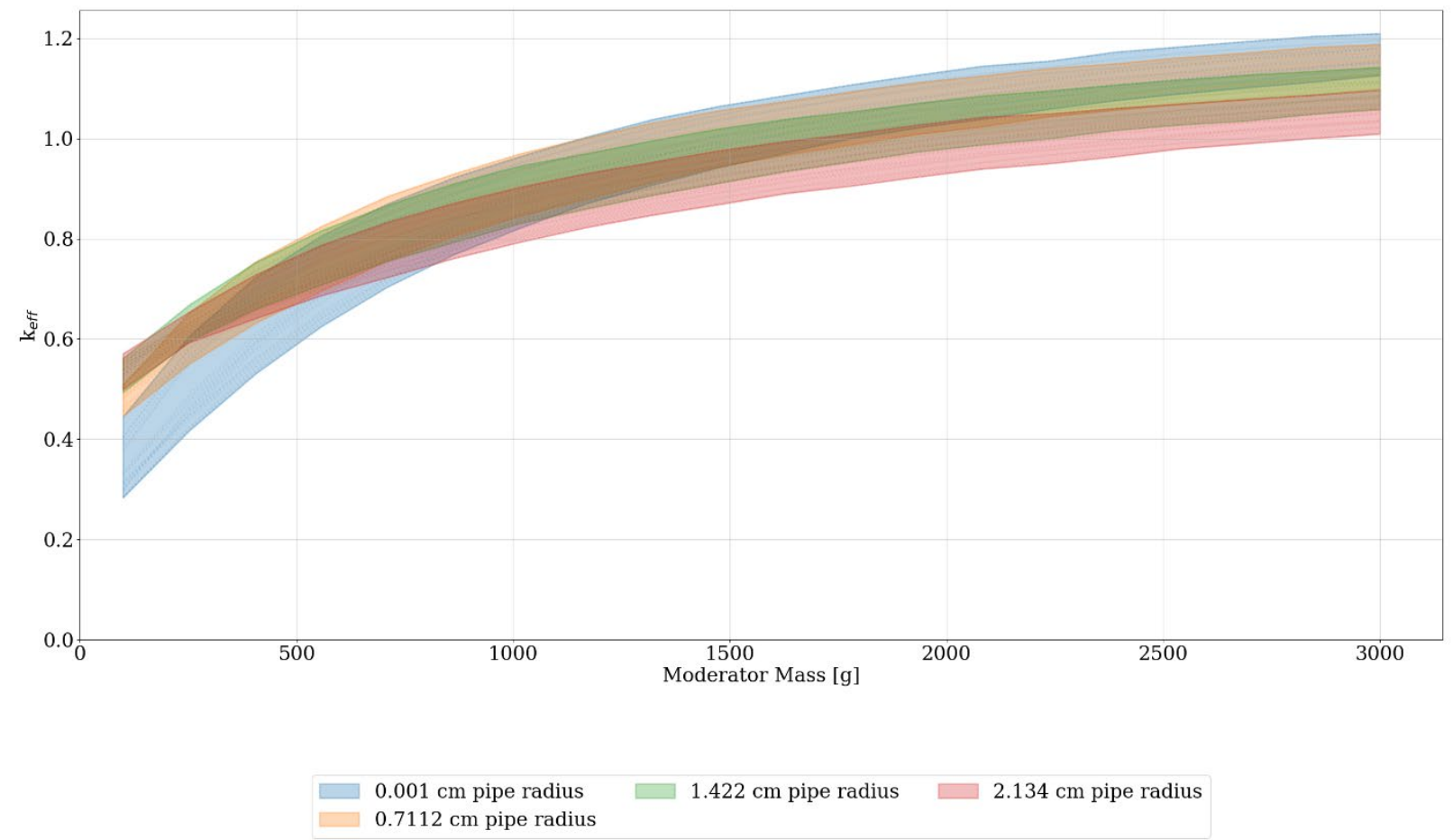

Figure 0-26. Set-8a sublisting-16 results (three-high uniform array model): $k_{\text {eff }}$ for set-1 and set-8a by discrete reflector thicknesses. 


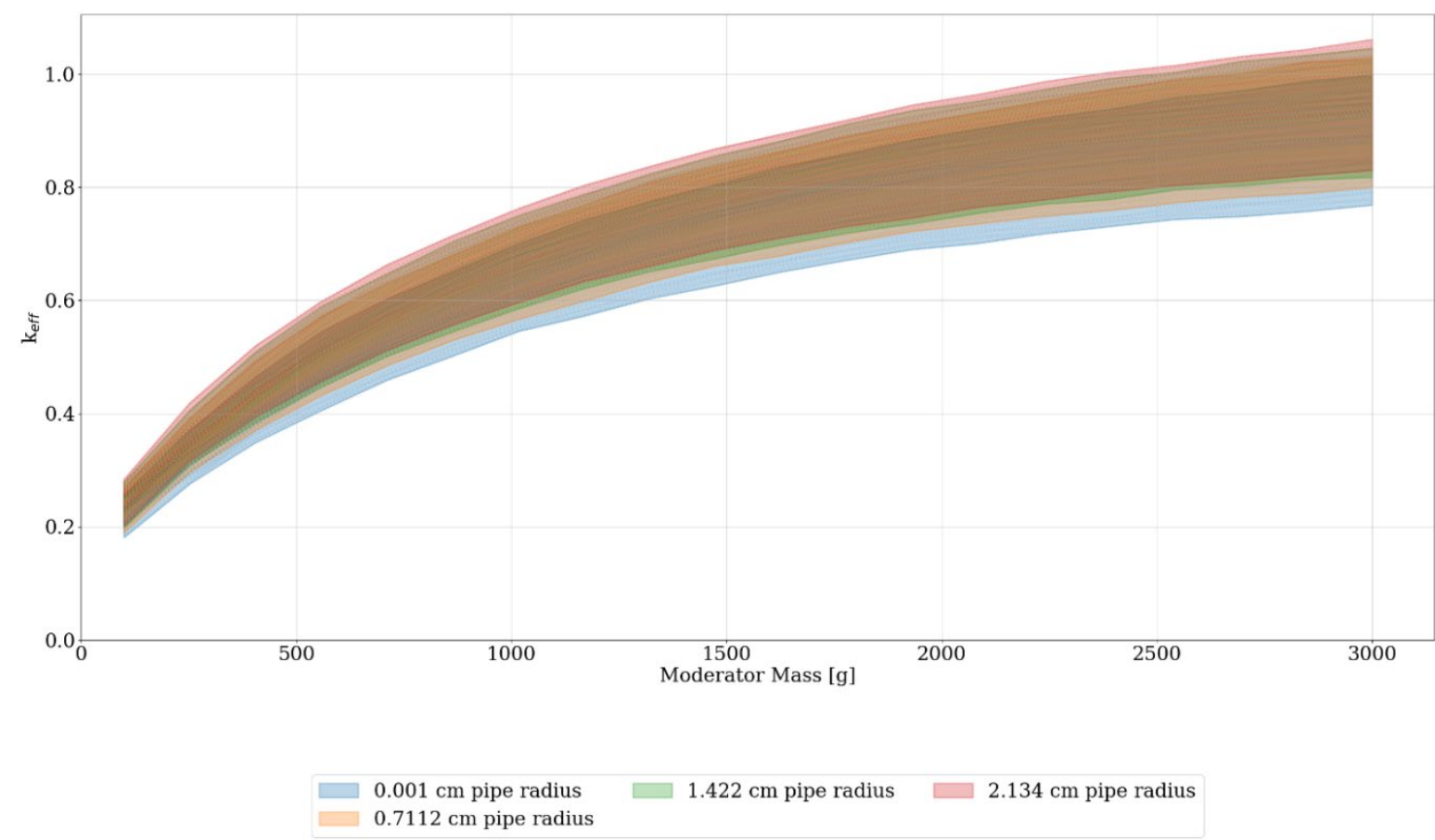

Figure 0-27. Set-8b sublisting-1 results (nonuniform array model): $\boldsymbol{k}_{\text {eff }}$ for set-2-uh and set-8a by discrete reflector thicknesses.

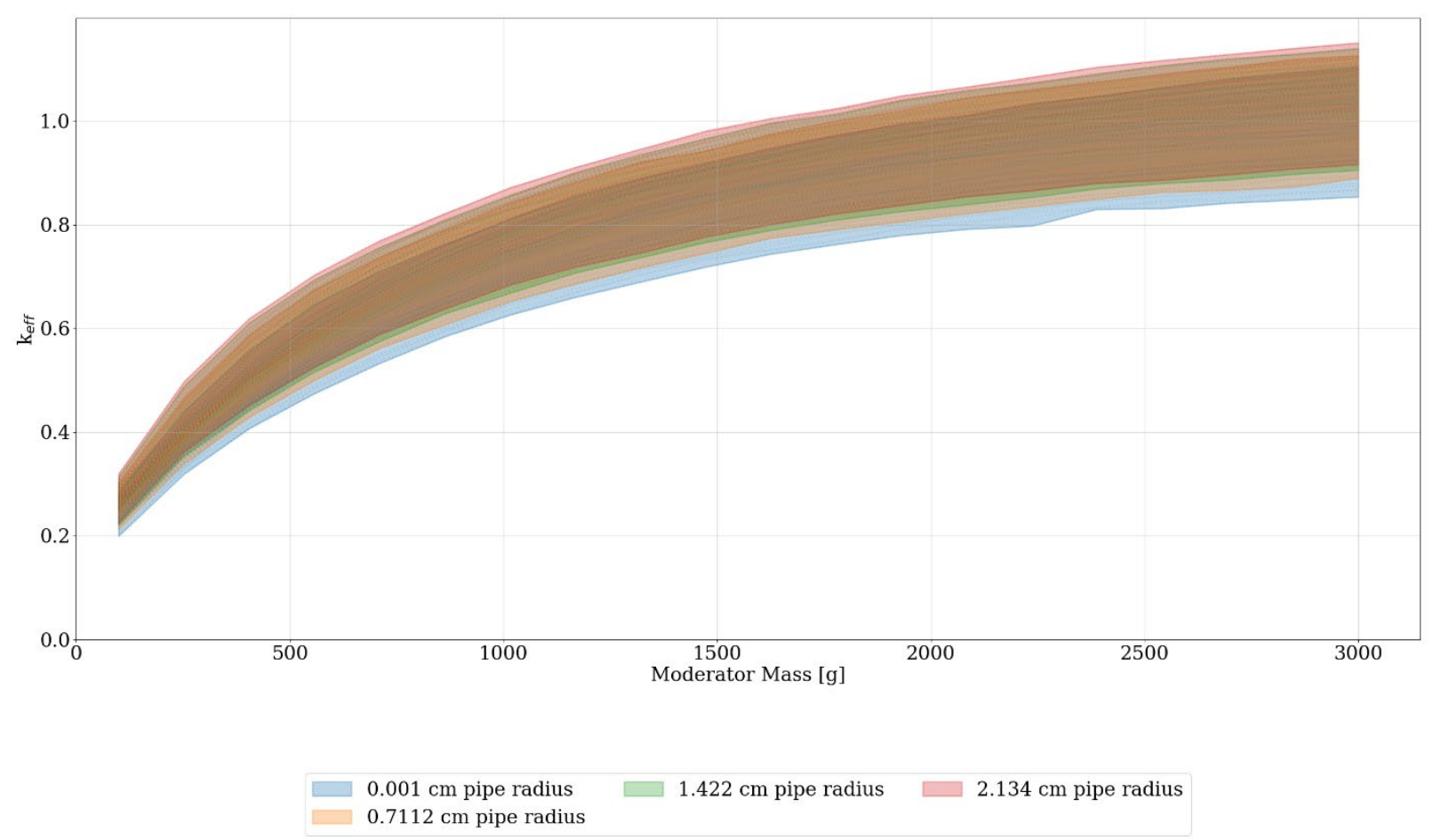

Figure 0-28. Set-8b sublisting-2 results (nonuniform array model): $\boldsymbol{k}_{\text {eff }}$ for set-2-uh and set-8a by discrete reflector thicknesses. 


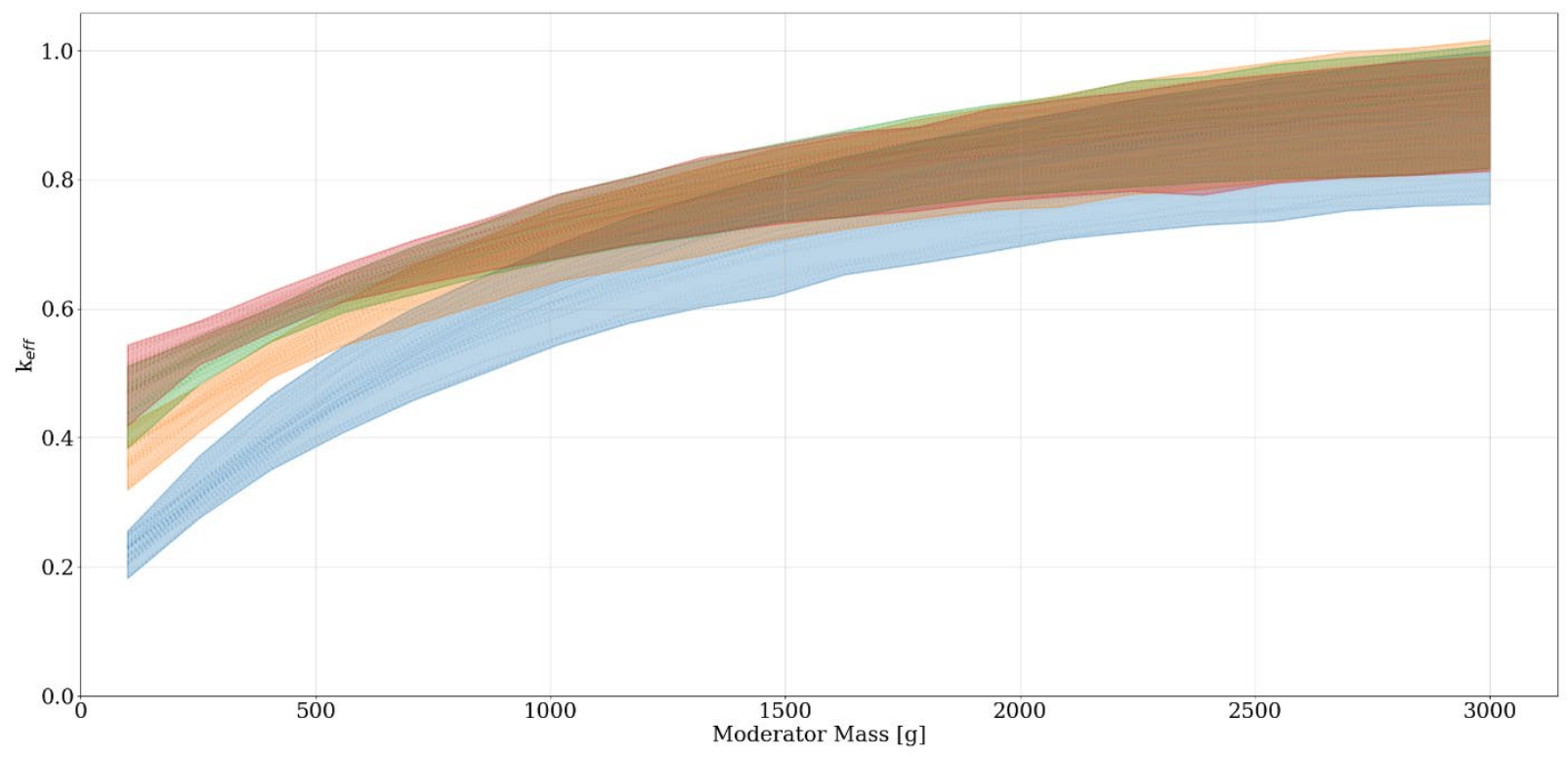

\begin{tabular}{|c|c|c|}
\hline $.001 \mathrm{~cm}$ pipe radius & $\square$ & $1.422 \mathrm{~cm}$ pipe radius \\
\hline
\end{tabular}

Figure O-29. Set-8b sublisting-3 results (nonuniform array model): $\boldsymbol{k}_{\text {eff }}$ for set-2-uh and set-8a by discrete reflector thicknesses. 


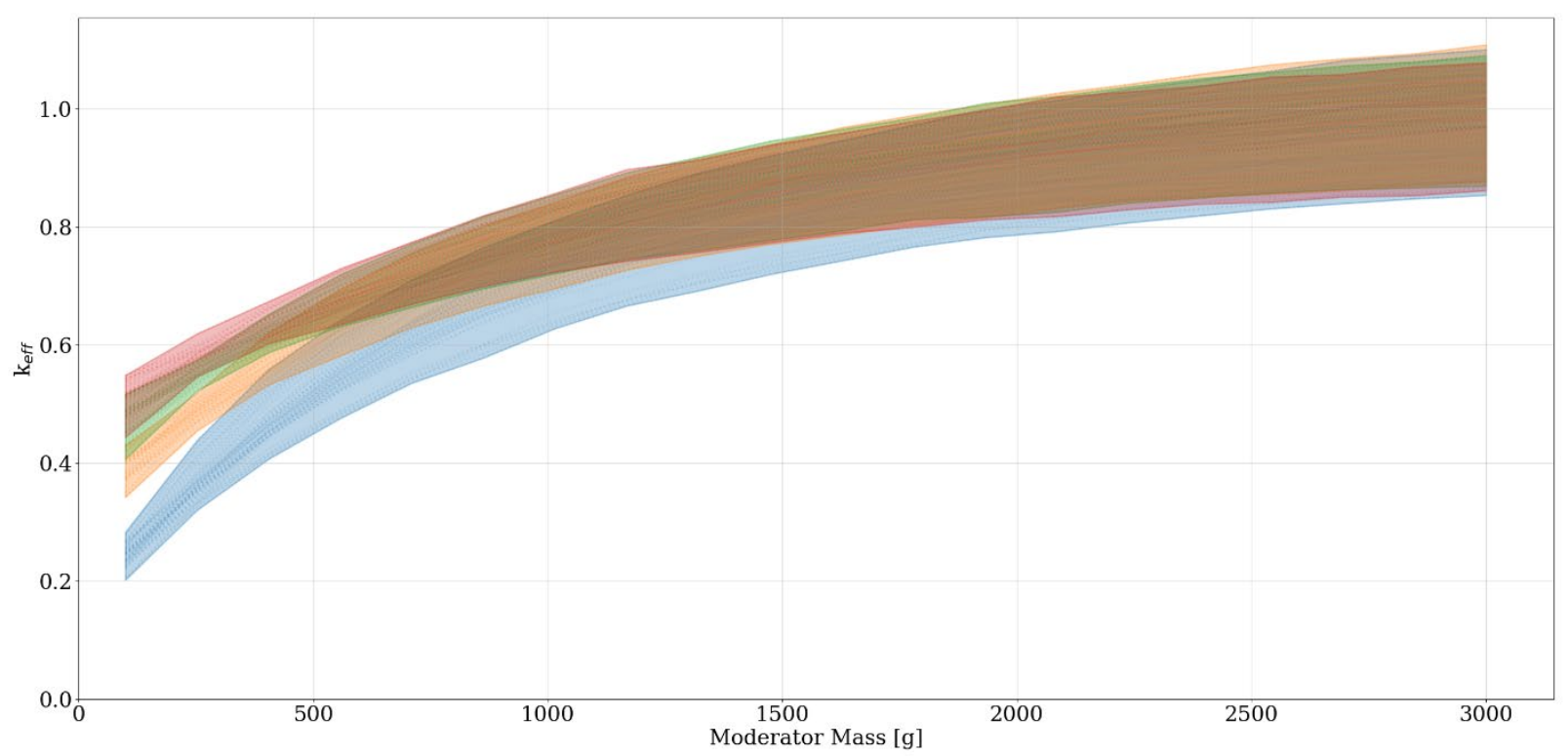

$0.001 \mathrm{~cm}$ pipe radius $\square 1.422 \mathrm{~cm}$ pipe radius $\square 2.134 \mathrm{~cm}$ pipe radius
$0.7112 \mathrm{~cm}$ pipe radius

Figure 0-30. Set-8b sublisting-4 results (three-high uniform array model): $\boldsymbol{k}_{\text {eff }}$ for set-2-uh and set-8a by discrete reflector thicknesses.

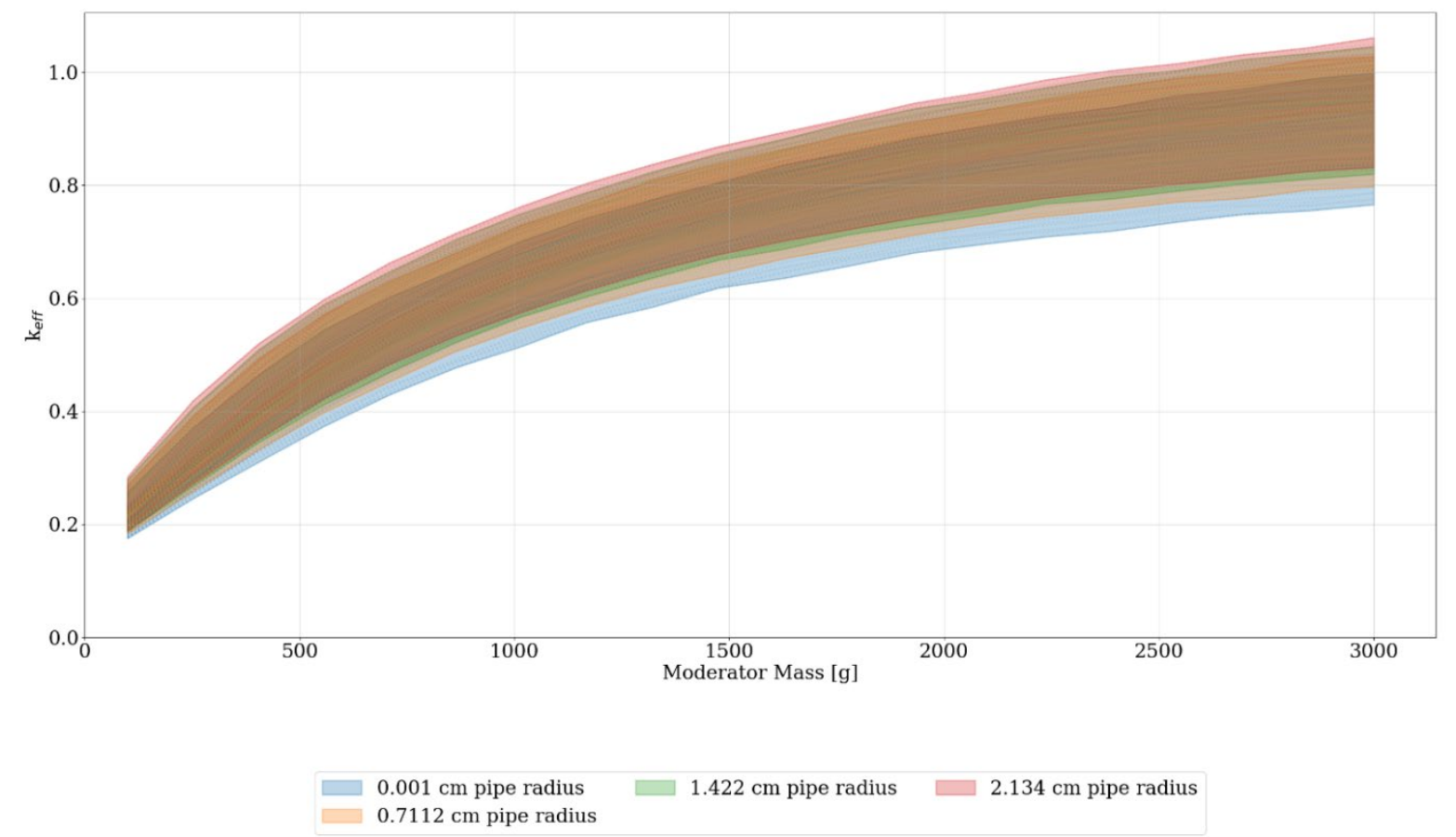

Figure O-31. Set-8b sublisting-5 results (nonuniform array model): $\boldsymbol{k}_{\text {eff }}$ for set-2-uh and set-8a by discrete reflector thicknesses. 


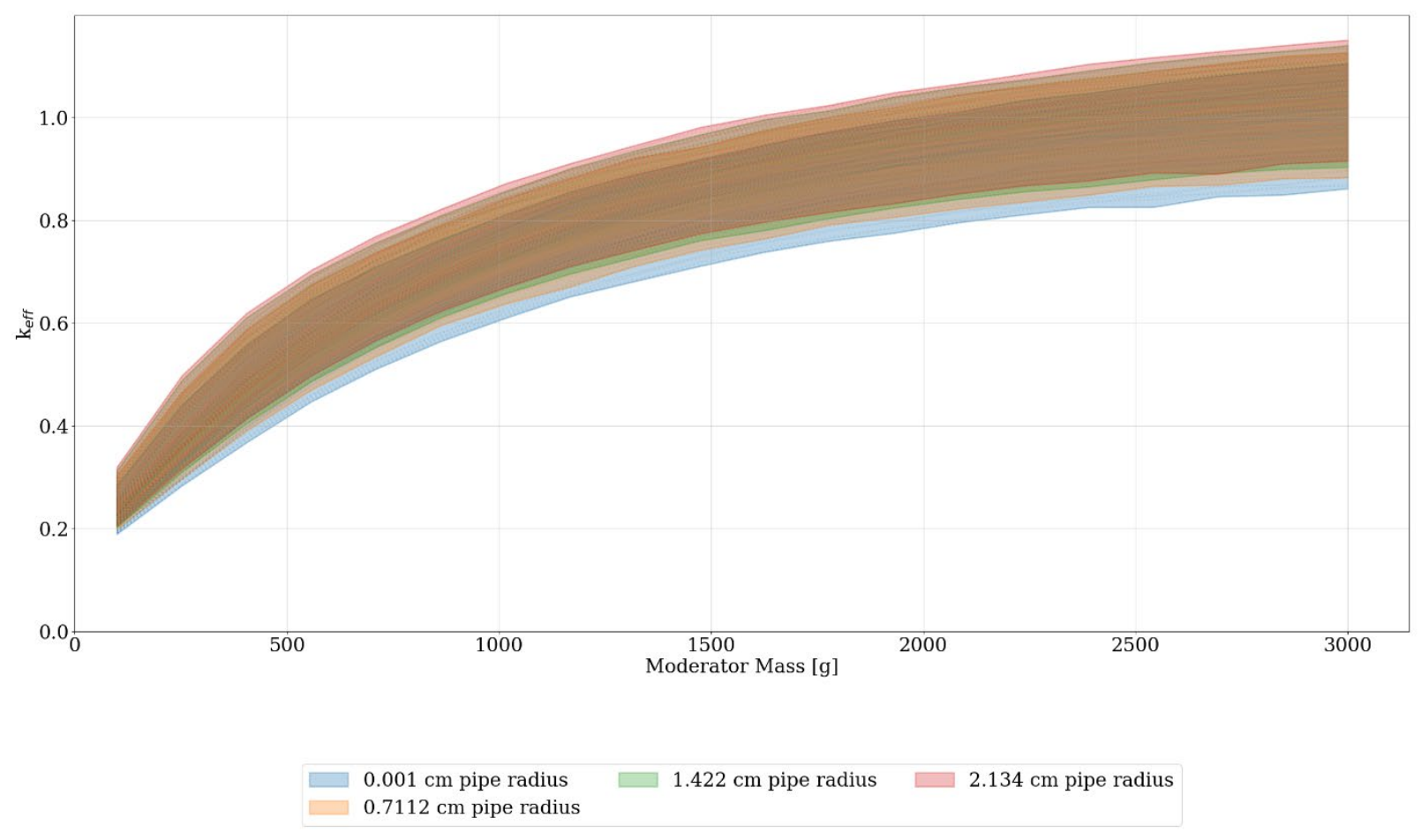

Figure 0-32. Set-8b sublisting-6 results (nonuniform array model): $\boldsymbol{k}_{\text {eff }}$ for set-2-uh and set-8a by discrete reflector thicknesses. 


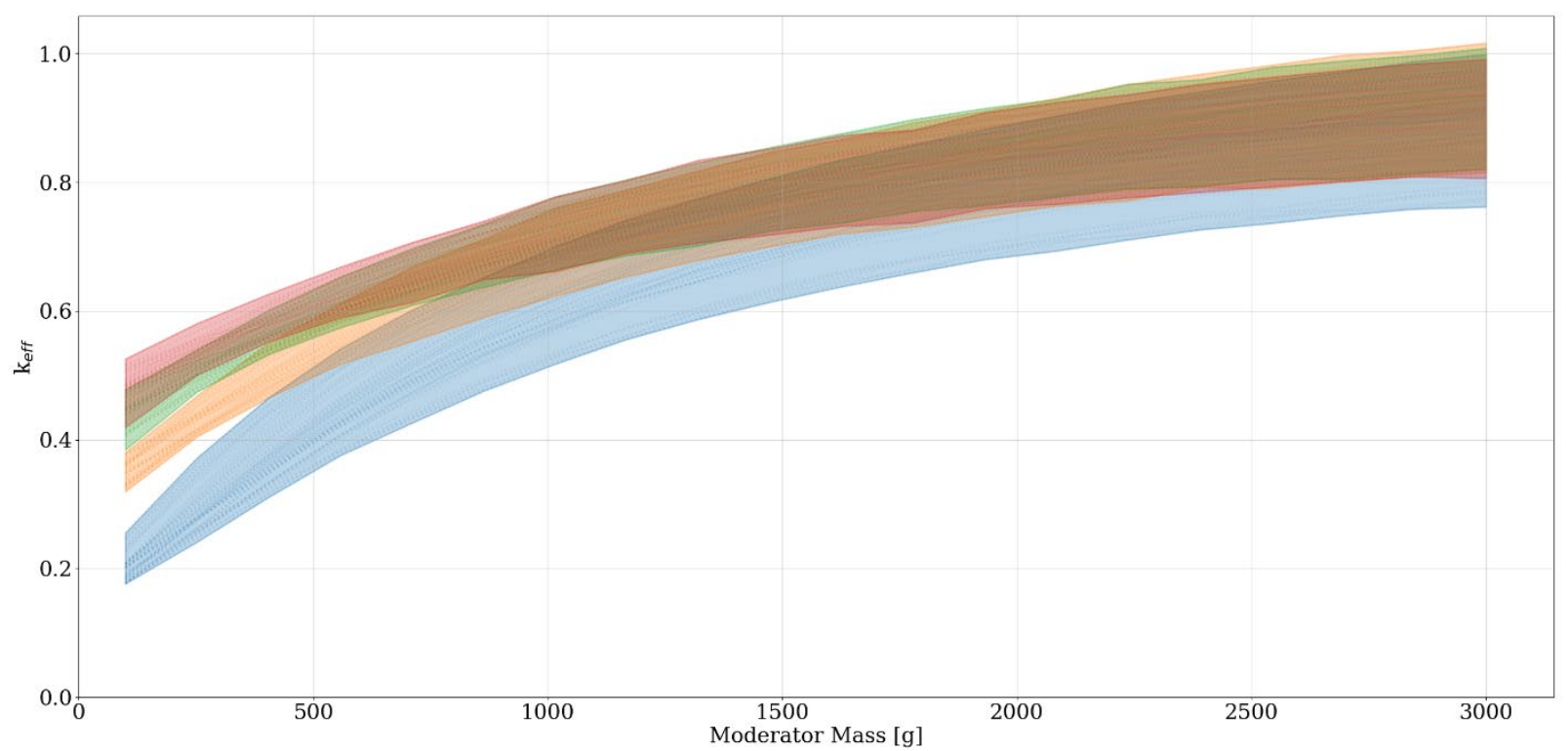

$0.001 \mathrm{~cm}$ pipe radius $\square 1.422 \mathrm{~cm}$ pipe radius $\square 2.134 \mathrm{~cm}$ pipe radius
$0.7112 \mathrm{~cm}$ pipe radius

Figure O-33. Set-8b sublisting-7 results (nonuniform array model): $\boldsymbol{k}_{\text {eff }}$ for set-2-uh and set-8a by discrete reflector thicknesses.

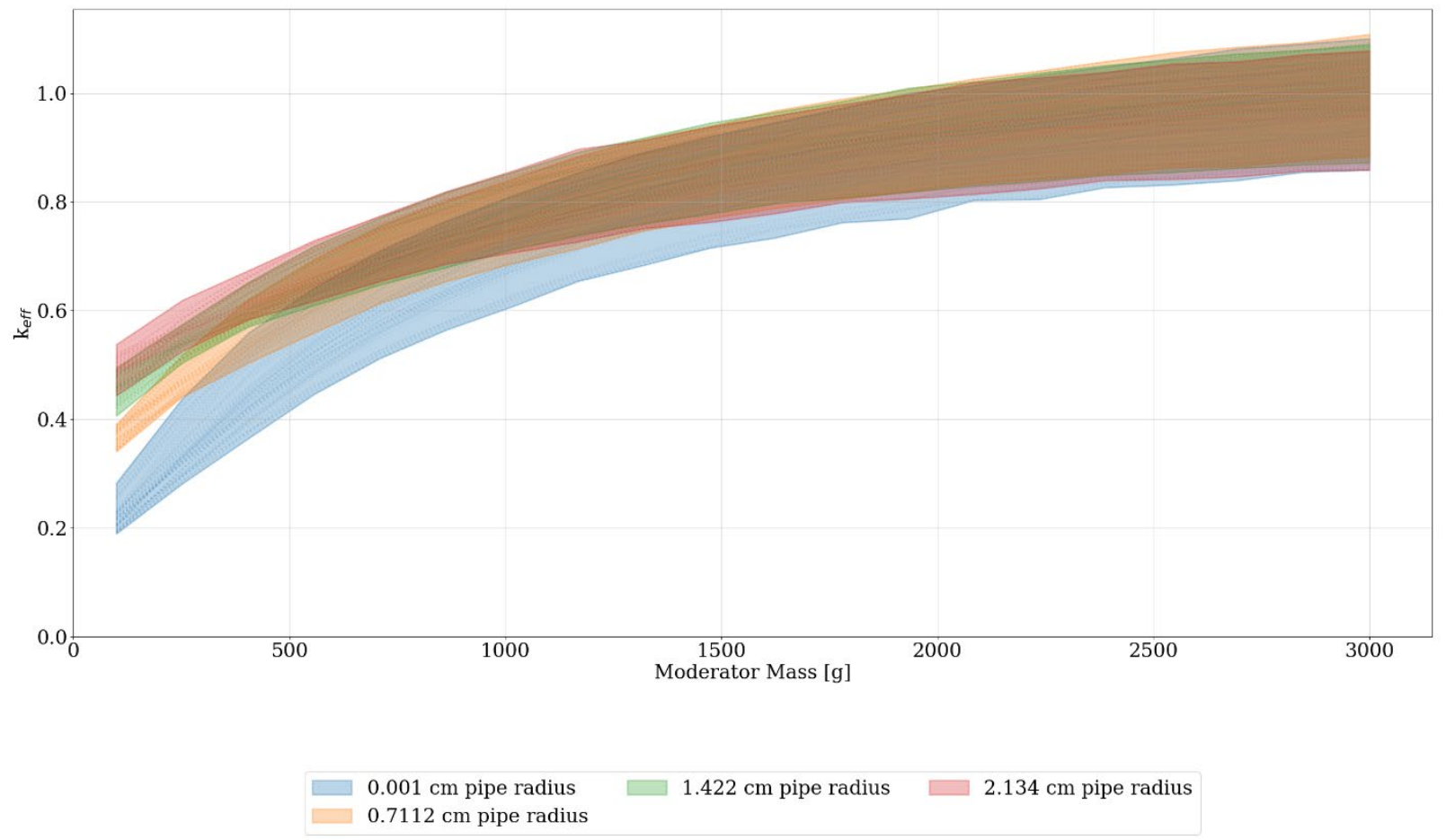

Figure 0-34. Set-8b sublisting-8 results (nonuniform array model): $\boldsymbol{k}_{\text {eff }}$ for set-2-uh and set-8a by discrete reflector thicknesses. 


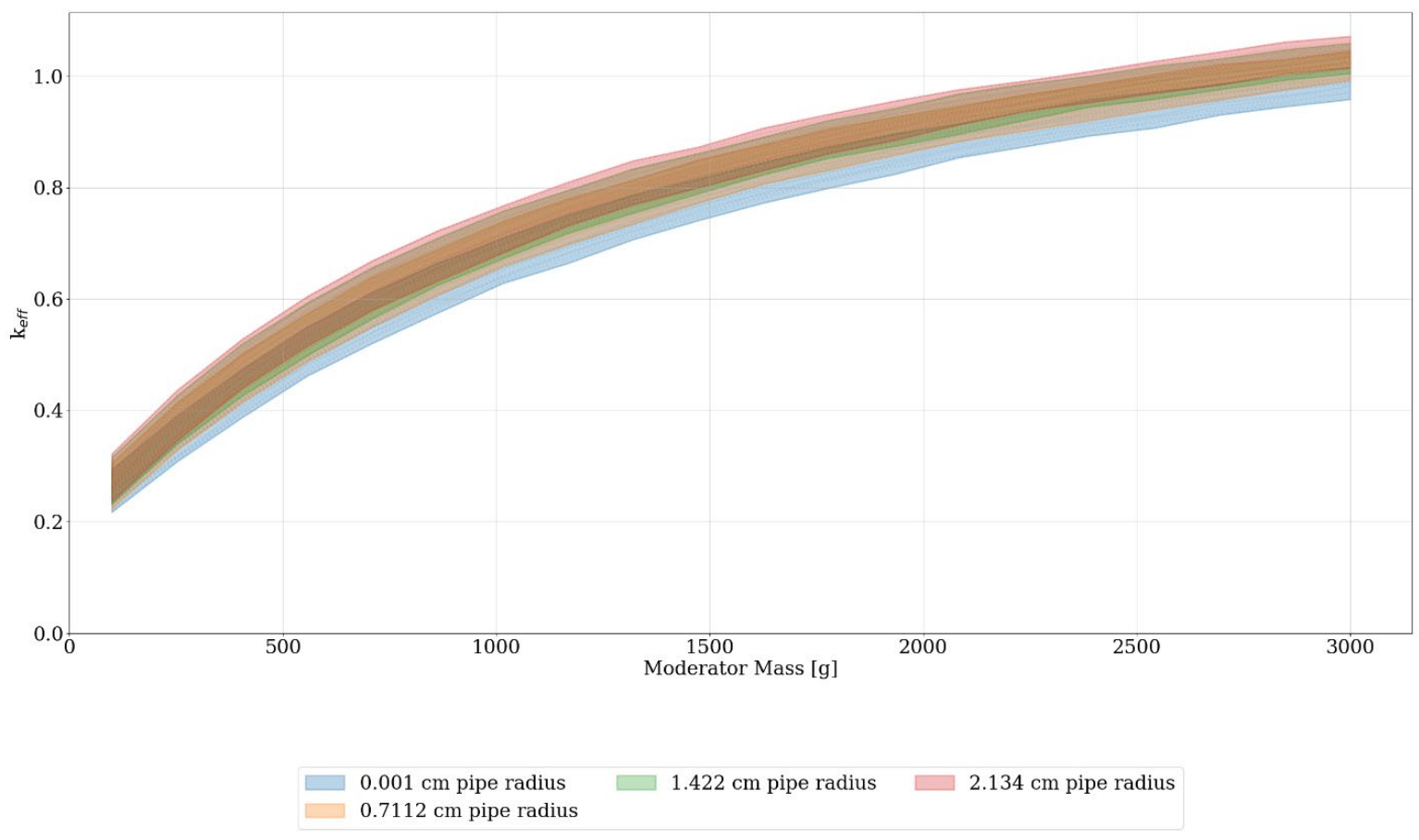

Figure O-35. Set-8a sublisting-9 results (nonuniform array model): $\boldsymbol{k}_{\text {eff }}$ for set-2-uh and set-8a by discrete reflector thicknesses.

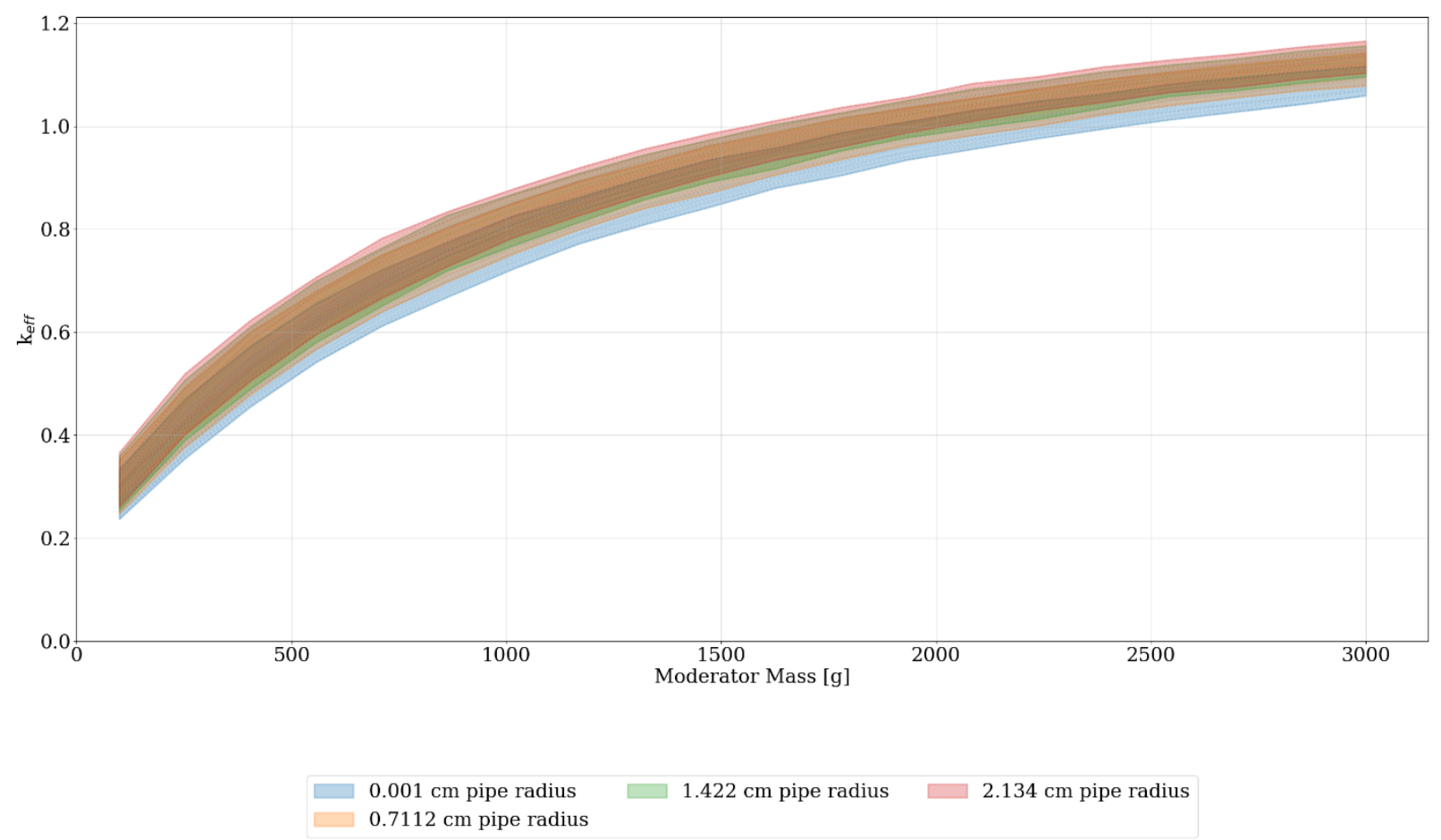

Figure 0-36. Set-8a sublisting-10 results (nonuniform array model): $\boldsymbol{k}_{\text {eff }}$ for set-2-uh and set-8a by discrete reflector thicknesses. 


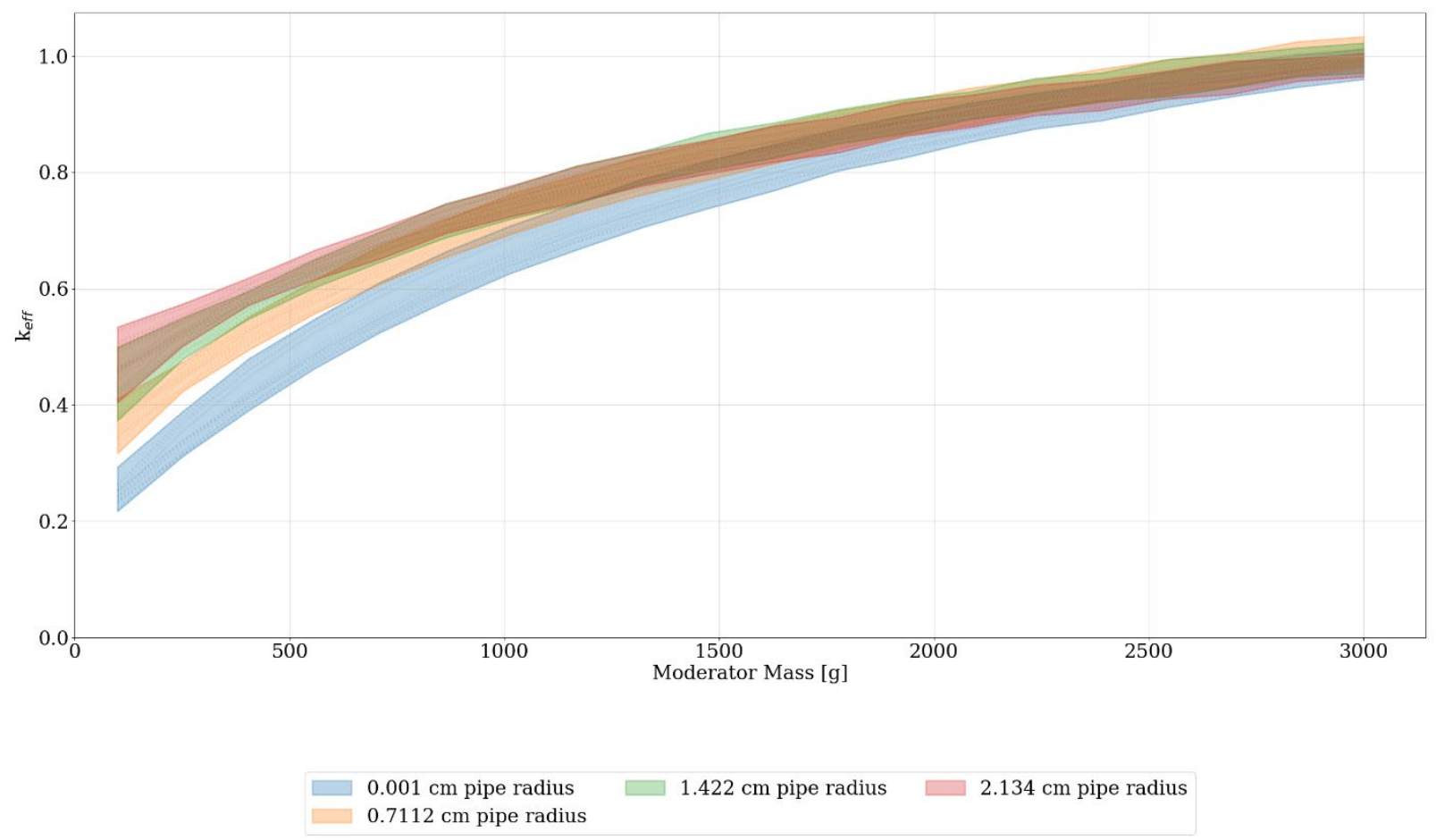

Figure O-37. Set-8b sublisting-11 results (nonuniform array model): $\boldsymbol{k}_{\text {eff }}$ for set-2-uh and set-8a by discrete reflector thicknesses. 


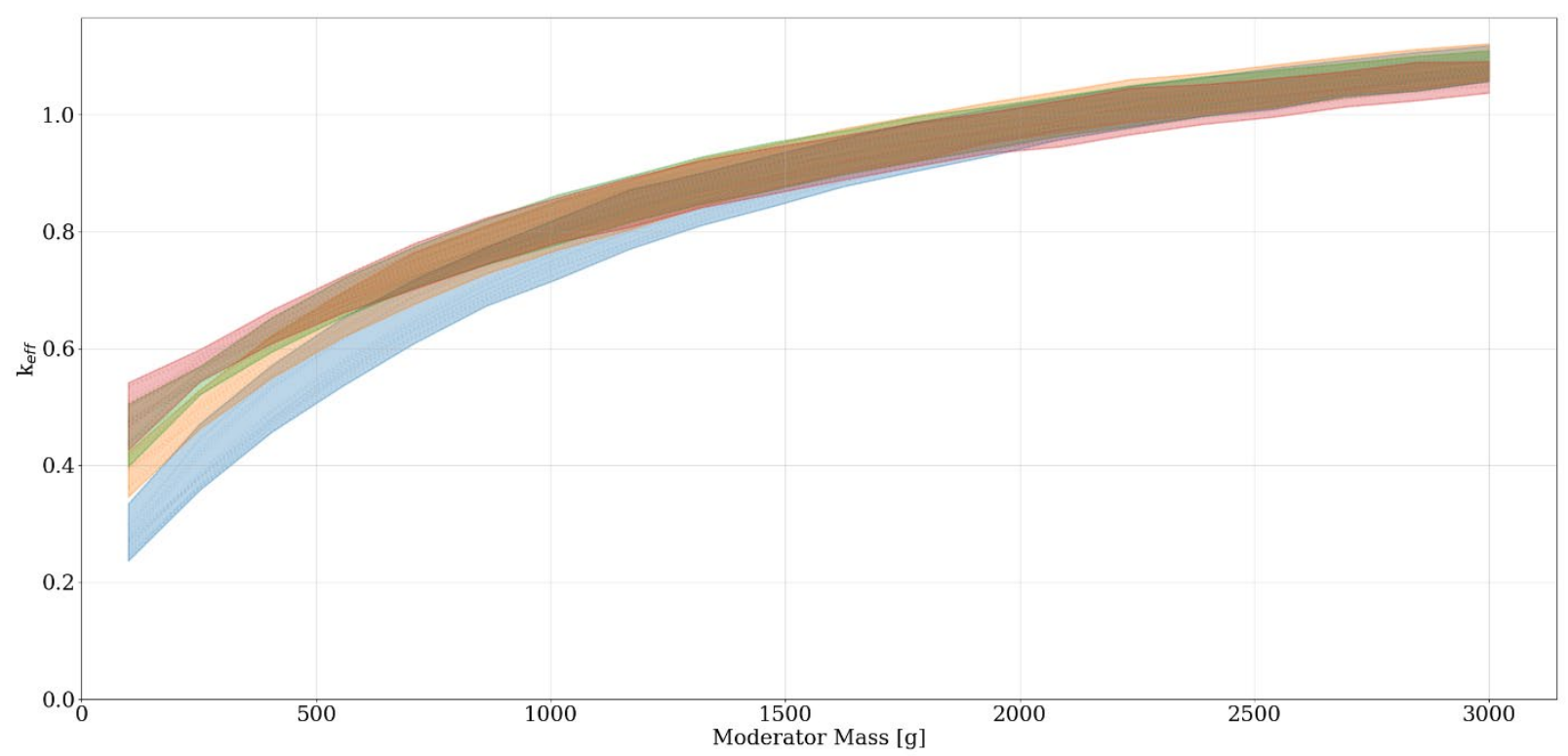

$0.001 \mathrm{~cm}$ pipe radius $\square 1.422 \mathrm{~cm}$ pipe radius $\square 2.134 \mathrm{~cm}$ pipe radius
$0.7112 \mathrm{~cm}$ pipe radius

Figure 0-38. Set-8b sublisting-12 results (nonuniform array model): $\boldsymbol{k}_{\text {eff }}$ for set-2-uh and set-8a by discrete reflector thicknesses.

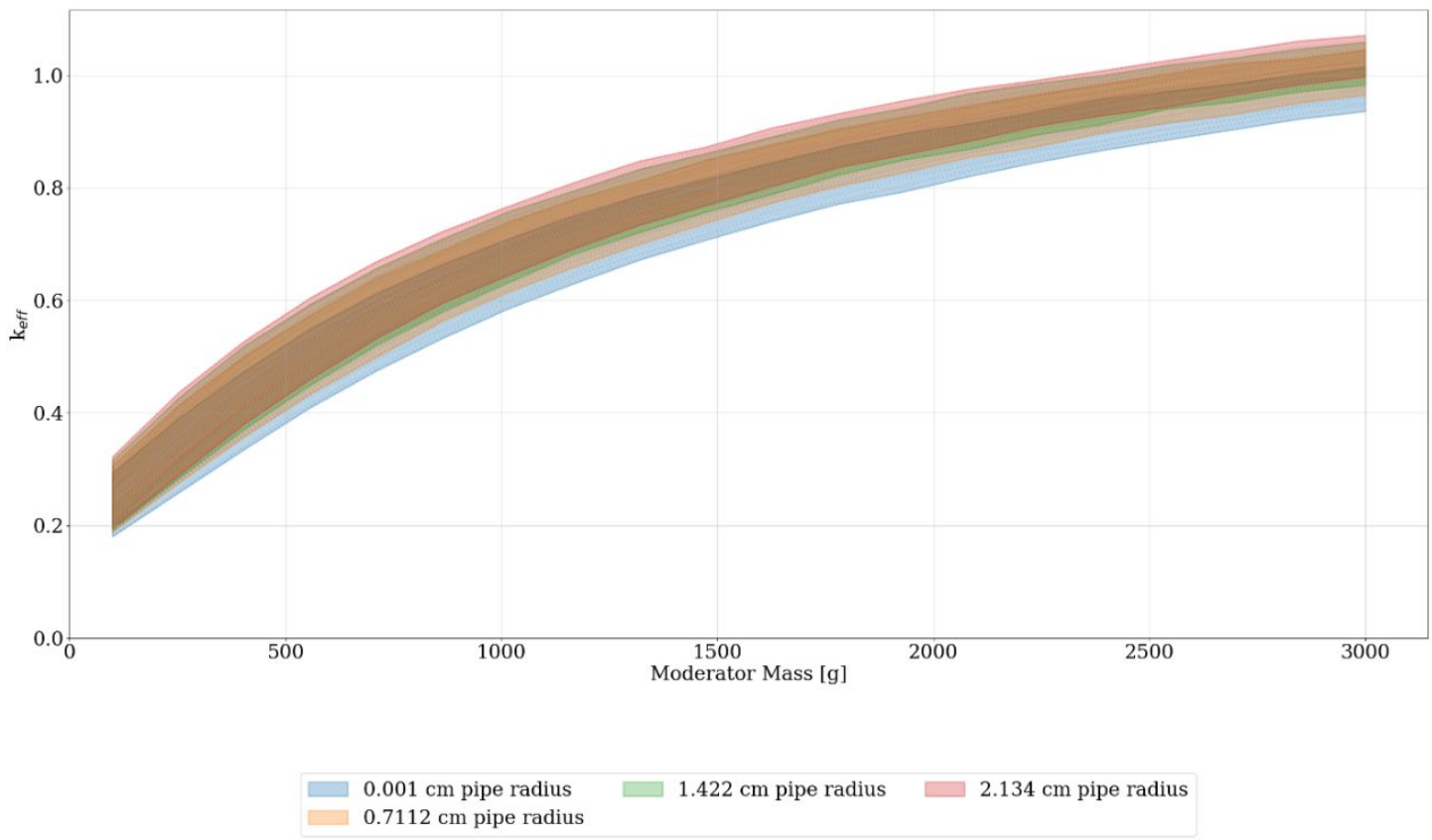

Figure O-39. Set-8b sublisting-13 results (nonuniform array model): $\boldsymbol{k}_{\text {eff }}$ for set-2-uh and set-8a by discrete reflector thicknesses. 


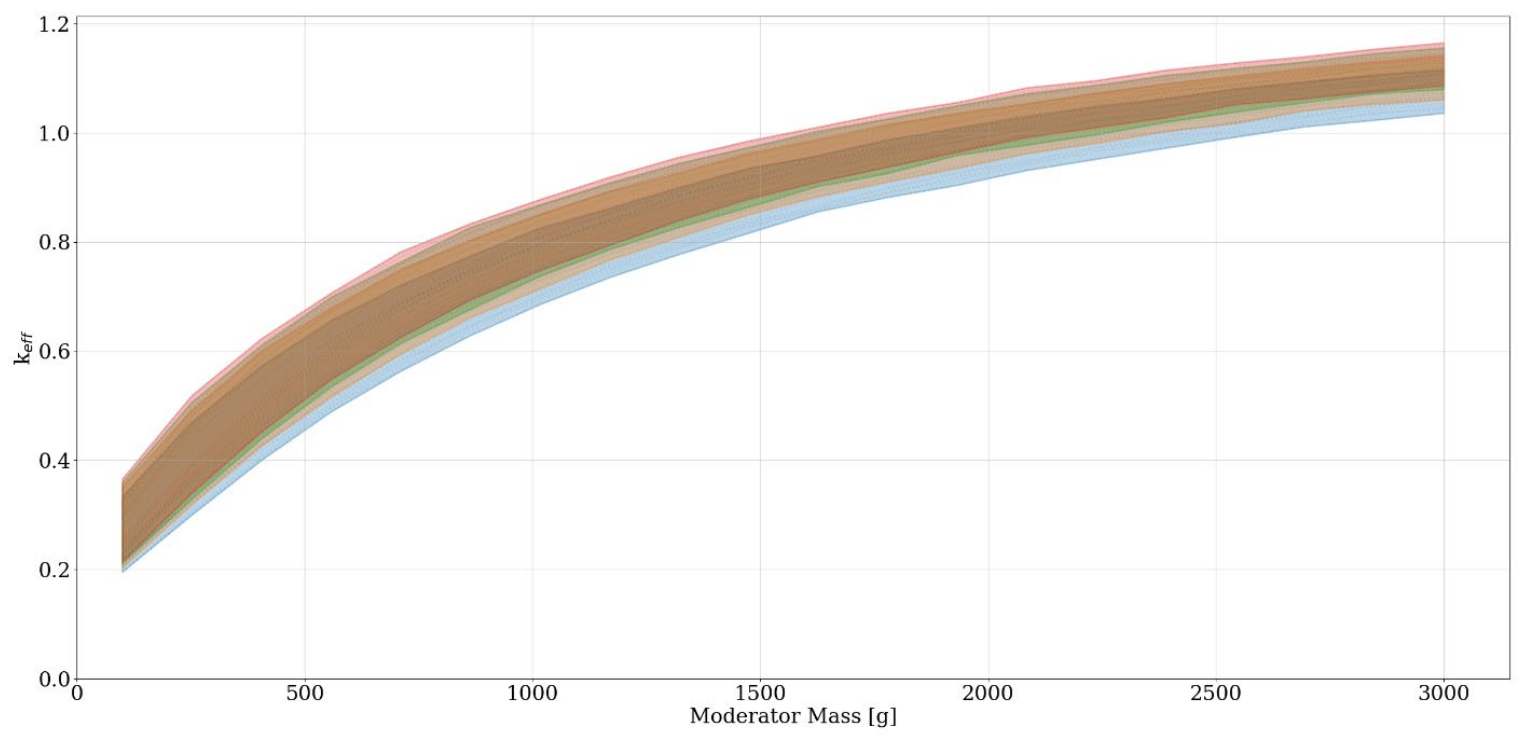

$\square .001 \mathrm{~cm}$ pipe radius $\square$
$0.7112 \mathrm{~cm}$ pipe radius $\square .422 \mathrm{~cm}$ pipe radius $\square 2.134 \mathrm{~cm}$ pipe radius
$\square$

Figure 0-40. Set-8b sublisting-14 results (nonuniform array model): $\boldsymbol{k}_{\text {eff }}$ for set-2-uh and set-8a by discrete reflector thicknesses.

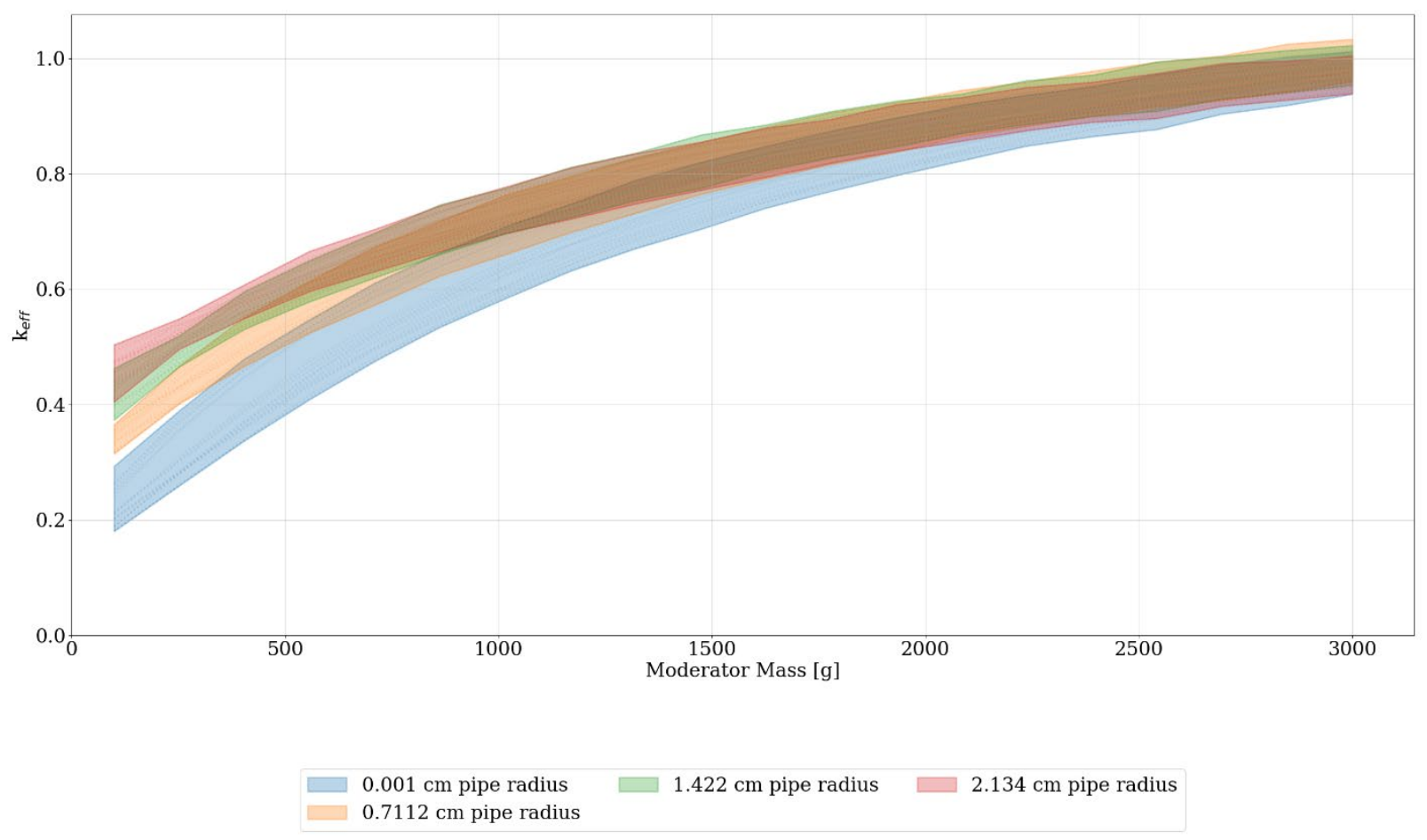

Figure O-41. Set-8b sublisting-15 results (nonuniform array model): $\boldsymbol{k}_{\text {eff }}$ for set-2-uh and set-8a by discrete reflector thicknesses. 


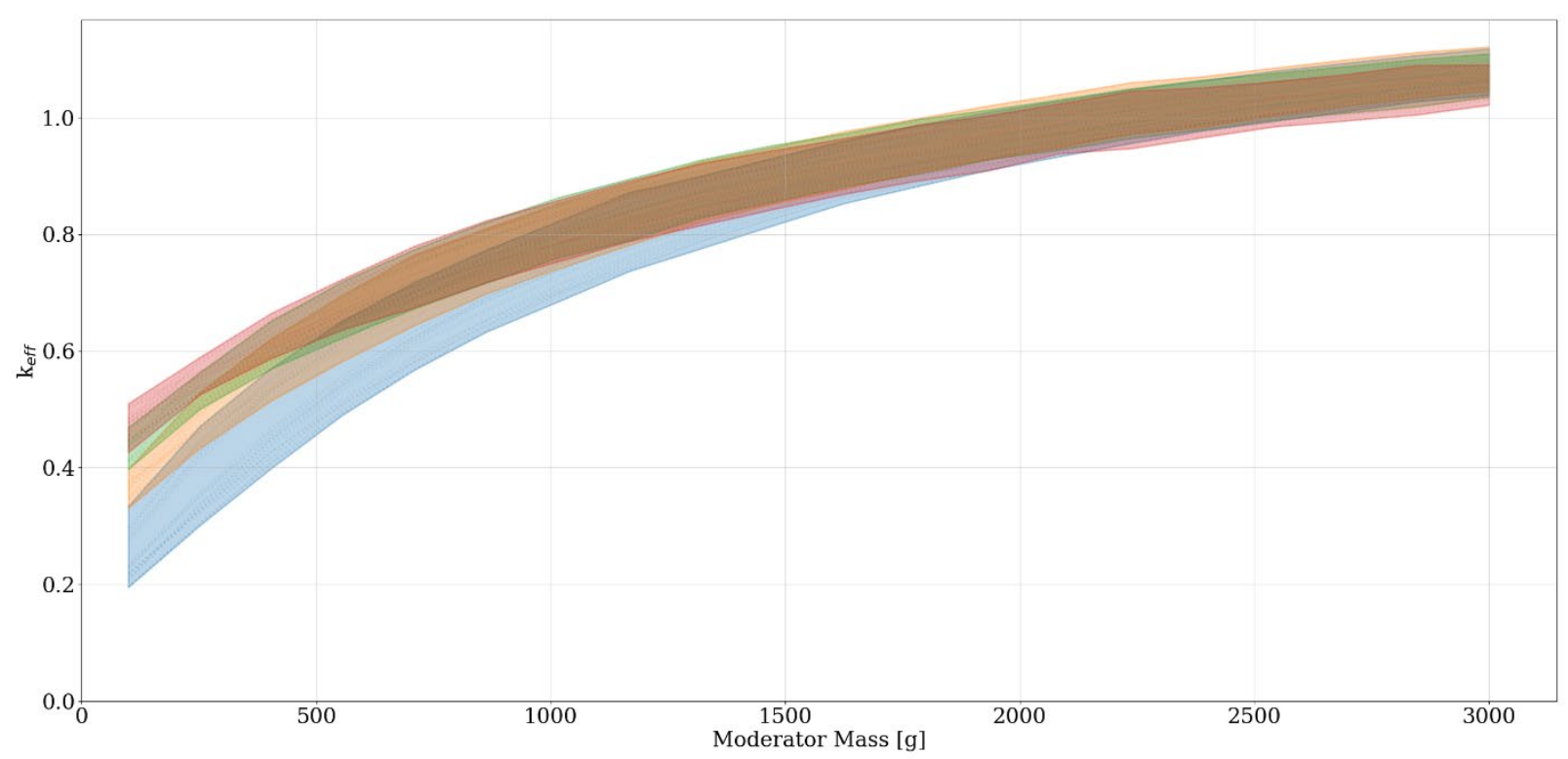

$\square .001 \mathrm{~cm}$ pipe radius $\square 1.422 \mathrm{~cm}$ pipe radius $\square 2.134 \mathrm{~cm}$ pipe radius
$0.7112 \mathrm{~cm}$ pipe radius

Figure 0-42. Set-8b sublisting-16 results (nonuniform array model): $\boldsymbol{k}_{\text {eff }}$ for set-2-uh and set-8a by discrete reflector thicknesses. 
ADDENDUM 1 SUMMARY OF ANALYSIS RESULTS 
This page is intentionally blank 


\section{ADDENDUM 1. SUMMARY OF ANALYSIS RESULTS}

Addendum 1 is the repository of the full set of analysis results. The analysis results have been postprocessed into various sets of "reorder" files so that the data can be evaluated in various ways. These data files have been placed on the Constellation Portal at ORNL and may be accessed by emailing bricknerbd@ornl.gov for more information. 
This page is intentionally blank 